\section{Pacific Northwest}

National Laboratory
Operated by Battelle for the

U.S. Department of Energy

\title{
Retained Gas Sampling Results for the Flammable Gas Program
}

L. A. Mahoney

Z. I. Antoniak

J. M. Bates

M. E. Dahl

\section{RECFIVFD \\ NOV 291999 \\ OSTI}

November 1999

Prepared for the U.S. Department of Energy under Contract DE-AC06-76RLO 1830 


\section{DISCLAIMIER}

This report was prepared as an account of work sponsored by an agency of the United States Government. Neither the United States Government nor any agency thereof, nor Battelle Memorial Institute, nor any of their employees, makes any warranty, express or implied, or assumes any legal liability or responsibility for the accuracy, completeness, or usefulness of any information, apparatus, product, or process disclosed, or represents that its use would not infringe privately owned rights. Reference herein to any specific commercial product, process, or service by trade name, trademark, manufacturer, or otherwise does not necessarily constitute or imply its endorsement, recommendation, or favoring by the United States Govermment or any agency thereof, or Battelle Memorial Institute. The views and opinions of authors expressed herein do not necessarily state or reflect those of the United States Government or any agency thereof.

\section{PACIFIC NORTHWEST NATIONAL LABORATORY operated by \\ BATTELLE for the \\ UNITED STATES DEPARTMENT OF ENERGY - under Contract DE-AC06-76RLO 1830}

\section{Printed in the United States of America}

Available to DOE and DOE contractors from the

Office of Scientific and Technical Information, P.O. Box 62, Oak Ridge, TN 37831; prices available from (615) 576-8401.

Available to the public from the National Technical Information Service, U.S. Department of Commerce, 5285 Port Royal Rd., Springfield, VA 22161 


\section{DISCLAIMER}

Portions of this document may be illegible in electronic image products. Images are produced from the best available original document. 


\title{
Retained Gas Sampling Results
} for the Flammable Gas Program

\author{
L. A. Mahoney \\ Z. I. Antoniak \\ J. M. Bates \\ M.E. Dahl(a)
}

October 1999

Prepared for the U.S. Department of Energy under Contract DE-AC06-76RLO 1830

Pacific Northwest National Laboratory Richland, Washington 99352
(a) COGEMA 


\section{Preface}

This report was prepared as part of a task supporting the deployment of the retained gas sampler (RGS) system in Flammable Gas Watch List Tanks. The emphasis of this report is on presenting the measurements resulting from retained gas sampling of Tanks 241-AW-101, A-101, AN-105, AN-104, AN-103, U-103, S-106, BY-101, BY-109, SX-106, AX-101, S-102, S-111, U-109, and SY-101. The retained gas sampling information is a direct measurement of the local gas volume fraction and composition of gas retained in these tanks. This information can then be combined with information from other sources to develop a better understanding of the mechanisms for gas generation, retention, and release, which will assist in resolution of the Flammable Gas Safety Issue and provide a more detailed understanding of tank behavior to support retrieval operations. 


\section{Executive Summary}

This report provides the results obtained for the tanks sampled with the retained gas sampler (RGS): Tanks 241-AW-101, 241-AN-105, 241-A-101, 241-AN-104, 241-AN-103, 241-U-103, 241-S-106, 241-BY-101, 241-BY-109, 241-SX-106, 241-AX-101, 241-S-102, 241-S-111, 241-U-109, and 241-SY-101. (Hereafter, these tanks will be referred to without the prefix $241-$, following standard practice.)

The RGS is a modified version of the universal core sampler used to core-sample Hanford tanks. It is designed specifically to be used in concert with the gas extraction equipment in the hot cell to capture and extrude a gas-containing waste sample in a hermetically sealed system. The retained gases are then extracted and stored in small gas canisters. The composition of the gases contained in the canisters is measured by mass spectroscopy. The total gas volume in the sample is obtained from analyzing pressure, volume, and temperature data from the extraction process. The details of the RGS calculations and the inputs to the calculations can be found in the calculation spreadsheets on the CD supplied with this report. The raw data from extraction are also on the CD in text file and spreadsheet form. These files contain more detail than is appropriate for this report and are provided to support reproducible ancillary calculations.

There have been two earlier reports covering RGS results. The first, Shekarriz et al. (1997), covered Tanks AW-101, AN-105, A-101, AN-104, and AN-103. The second, Mahoney et al. (1997), covered Tanks U-103, S-106, BY-101, and BY-109. This report provides final documentation of the RGS sampling results between late 1997 and early 1999 (SX-106, AX-101, S-102, S-111, U-109, and SY-109). It also re-examines the earlier results by applying insights gained from later work and by performing additional calculations.

The primary change has been in interpreting ammonia data, evaluating their reliability, and comparing RGS ammonia measurements with other information. Early RGS ammonia measurements were insufficient to determine ammonia concentrations because of measurement challenges. Our later work shows that the ammonia concentrations reported in Shekarriz et al. (1997) and Mahoney et al. (1997) for Tanks AW-101, AN-105, A-101, AN-104, AN-103, U-103, S-106, and BY-109 were underestimated and should not be used. The new procedures (used on Tanks SX-106, AX-101, S-102, S-111, U-109, and SY-101) are believed to be more reliable, though uncertainty remains high. This report contains ammonia measurements from RGS extractions, ammonia partial pressure measurements, ammonia measured by chemical analyses of RGS samples after extraction, and ammonia data from sources other than RGS samples and measurement techniques. These collected data (not all of which were drawn upon in earlier reports) allow us to state ammonia concentrations and assess their reliability by comparing the values found by different measurement techniques.

Other changes include accounting for hydrostatic head fluid intrusion on the first five tanks (Shekarriz et al, 1997), more accurate water vapor estimates, improved data inputs for solubility calculations, and more comparisons between the gas measured by extraction and that visible in the $x$-ray, among others. The aggregate impact of these changes on composition and gas volume fraction generally ranged between 10 and $30 \%$ of the previously reported values.

The remainder of this summary is arranged in the following manner: First are bulleted items that summarize the central findings of this research; these are followed by an overview table (Table S.1) that describes the gas inventories and compositions for the significant gas-retaining wastes in all the tanks. Further supporting information is then presented as tank-by-tank discussions. 
- The retained gas inventories calculated from local measurements of gas volume fraction made by the RGS often differed significantly from the total gas inventories estimated by the void fraction instrument (VFI) or barometric pressure effect (BPE) method. These differences occurred when the waste has irregular layers and there are other strong indicators of lateral inhomogeneity. Because RGS samples were localized and frequently few in number, they captured little of the lateral variation and in some cases missed some of the vertical variation. Therefore, auxiliary information such as BPE inventories and $x$-rays and extrusion lengths of non-RGS samples were used to supplement RGS measurements in estimating the gas inventories in tanks with inhomogeneous waste.

- The RGS method provided retained gas volume fraction measurements with low uncertainty. The uncertainty caused by gas solubility and extraction measurement uncertainty was usually less than $\pm 15 \%$ of the measurement for samples from nonconvective waste (Table S.1). There was typically good agreernent between RGS and VFI data (Tanks AW-101, AN-105, AN-104, and AN-103), validating the low calculated uncertainty.

- The convective waste consistently contained less than 0.01 volume fraction of free (undissolved) gas while almost all wet nonconvective waste contained gas volume fractions of 0.02 to 0.3 or more. No high-gas samples were taken from liquid waste, although SY-101 mechanically mixed slurry samples contained volume fractions of 0.02 to 0.03 .

- For wastes with gas volume fractions of 0.02 or more, such as nonconvective wastes, more than $45 \%$ of the $\mathrm{N}_{2} \mathrm{O}$ (often much more) and more than $90 \%$ of the $\mathrm{H}_{2}, \mathrm{~N}_{2}$, and $\mathrm{CH}_{4}$ were calculated to be present in the free (undissolved) gas phase. In convective wastes (with less than 0.01 gas volume fraction), as much as $90 \%$ of the $\mathrm{N}_{2} \mathrm{O}$ and $25 \%$ of the $\mathrm{H}_{2}$, $\mathrm{N}_{2}$, and $\mathrm{CH}_{4}$ were calculated to be dissolved in the liquid.

- Unusually high gas volume fractions (of 0.3 or greater) were found in samples from Tanks U-103, SX-106, S-102, U-109, and SY-101. These measurements were confirmed by large gas volumes shown in the RGS sampler $x$-rays. The high-gas samples were all taken from nonconvective layers, but there was no consistent rule as to whether the high gas volume fractions were found in the top, middle, or bottom of the layer.

- The RGS gave retained gas compositions with low uncertainty for samples containing more than 0.02 gas volume fraction (Table S.1). In those, mole fraction uncertainties were typically within $\pm 20 \%$ of the measured value. Drillstring sample and domespace $\mathrm{H}_{2} / \mathrm{N}_{2} \mathrm{O}$ ratios frequently matched the ratios from RGS data, providing some corroboration.

- The gas composition in convective and nonconvective wastes was consistently distinctly different; the nonconvective waste gas contained more $\mathrm{H}_{2}$ and less $\mathrm{N}_{2}$. Substantial tank to tank variation existed in the composition of gases retained in the nonconvective wastes. The gas composition was typically fairly uniform within the nonconvective waste in a tank.

- Nitrogen, an inert gas, was a major component of the retained gas (from 15 to 67 vol\%) in the gas retained in nonconvective wastes. Only A-101, SX-106, and S-111 contained nonconvecting wastes with mole fractions of $\mathrm{N}_{2}$ less than $20 \mathrm{~mol} \%$ in the retained gas.

- The composition of retained gases in the gas-retaining layers of RGS-sampled tanks showed considerable diversity. Some nonconvective wastes retained gas that was more than 60 mol\% hydrogen, such as the wastes in A-101, AN-105, AN-103, S-106, AX-101, and S-111. Other wastes retained gas that contained $20 \mathrm{~mol} \%$ or more of $\mathrm{N}_{2} \mathrm{O}: \mathrm{AN}-104$, U-103, SX-106, S-102, U-109, and SY-101. AW-101 was the only tank in which high $\mathrm{N}_{2}$ (50 mol\% or more) was measured in the gas in the nonconvective waste. 
- Substantial gas fractions can be retained in wastes after salt-well pumping. Samples taken below the interstitial liquid level (ILL) from BY-109, a tank that had been pumped not long before RGS sampling, had in situ gas fractions of roughly 0.10 .

- Recent findings suggest that early RGS reports (Shekarriz et al. 1997; Mahoney et al. 1997) considerably underestimated ammonia concentrations for AW-101, A-101, AN-105, AN-104, AN-103, U-103, S-106, and BY-109. Improvements in the methodology indicate that the ammonia concentrations found for some wastes sampled later (the SX-106 con- . vective layer, AX-101, U-109, and SY-101) are more reliable. Some justifiable doubt remains in these results because the ammonia concentrations measured by the isotopic solution method frequently disagree with other measurements (which themselves can be subject to question). Ongoing standards tests should resolve many of these questions.

Table S.1. Overview of Tank Gas Contents Based on RGS Data (all values derived from RGS data alone)

\begin{tabular}{|c|c|c|c|c|c|c|}
\hline & $\begin{array}{c}\text { Average } \\
\text { In Situ Gas } \\
\text { Volume } \\
(\%)\end{array}$ & $\begin{array}{c}\text { Maximum } \\
\text { In Situ Gas } \\
\text { Volume } \\
(\%)\end{array}$ & $\begin{array}{l}\text { Average } \\
\text { Mol\% } \\
\mathrm{H}_{2} \text { (a) }\end{array}$ & $\begin{array}{l}\text { Average } \\
\text { Mol\% } \\
\mathrm{N}_{2} \text { (a) }\end{array}$ & $\begin{array}{c}\text { Average } \\
\text { Mol\% } \\
\mathrm{N}_{2} \mathrm{O} \text { (a) }\end{array}$ & $\begin{array}{l}\text { Total STP } \\
\text { Free Gas } \\
\quad\left(\mathrm{m}^{3}\right)\end{array}$ \\
\hline AW-101 (one convective sample) & $-\cdots$ & $0.8 \pm 0.1$ & $26 \pm 9.4$ & $67 \pm 32$ & $2.3 \pm 0.8$ & $29 \pm 10$ \\
\hline AW-101 (nonconvective layer) & $3.7 \pm 1.8(b)$ & $5.2 \pm 0.5$ & $32 \pm 3.2$ & $55 \pm 6.2$ & $7.5 \pm 0.8$ & $86 \pm 42(b)$ \\
\hline AN-105 (nonconvective layer) & $5.2 \pm 2.6(\mathrm{~b})$ & $12 \pm 0.8$ & $59 \pm 5.4$ & $24 \pm 4.0$ & $15 \pm 1.5$ & $186 \pm 93(\mathrm{~b})$ \\
\hline A-101 (nonconvective layer) & $18 \pm 9.0(b)$ & $22 \pm 2.1$ & $70 \pm 7.3$ & $19 \pm 4.9$ & $5.7 \pm 0.6$ & $344 \pm 172^{(b)}$ \\
\hline AN-104 (nonconvective layer) & $8.1 \pm 4.0(b)$ & $17 \pm 1.9$ & $45 \pm 6.9$ & $29 \pm 4.8$ & $23 \pm 3.8$ & $257 \pm 128(\mathrm{~b})$ \\
\hline AN-103 (one crust sample) & $-\cdots$ & $16 \pm 1.4$ & $62 \pm 6.4$ & $29 \pm 3.2$ & $6.9 \pm 0.7$ & $54 \pm 27(b)$ \\
\hline AN-103 (nonconvective layer) & $9.2 \pm 4.6(b)$ & $12 \pm 1.5$ & $61 \pm 7.7$ & $33 \pm 4.3$ & $4.2 \pm 0.6$ & $260 \pm 130(b)$ \\
\hline U-103 (nonconvective layer) & $19 \pm 9.6(\mathrm{~b})$ & $42 \pm 2.7$ & $23 \pm 1.4$ & $36 \pm 2.3$ & $40 \pm 2.4$ & $330 \pm 165(b)$ \\
\hline S-106 (nonconvective layer) & $10 \pm 5.2(\mathrm{~b})$ & $14 \pm 1.2$ & $63 \pm 5.7$ & $25 \pm 3.7$ & $11 \pm 1.0$ & $224 \pm 112^{(b)}$ \\
\hline BY-101 & \multicolumn{6}{|c|}{ No results are available for this tank. } \\
\hline BY-109 (layer below ILL) & $9.5 \pm 4.7(b)$ & $12 \pm 1.0$ & $50 \pm 5.5$ & $28 \pm 5.0$ & $18 \pm 2.5$ & $122 \pm 61^{(b)}$ \\
\hline SX-106 (nonconvective layer) & $26 \pm 13(b)$ & $35 \pm 2.2$ & $51 \pm 4.7$ & $20 \pm 4.0$ & $24 \pm 2.7$ & $426 \pm 213^{(b)}$ \\
\hline AX-101 (one nonconvective sample) & $-\cdots$ & $17 \pm 1.3$ & $60 \pm 5.5$ & $16 \pm 2.6$ & $11 \pm 1.0$ & $--\cdot$ \\
\hline S-102 (all the waste) & $26 \pm 13(b)$ & $33 \pm 4.3$ & $33 \pm 3.0$ & $32 \pm 4.3$ & $33 \pm 3.3$ & $601 \pm 300$ (b) \\
\hline S-111 (one convective sample) & $\ldots$ & $0.8 \pm 0.3$ & $6.4 \pm 3.4$ & $90 \pm 68$ & $2.2 \pm 1.3$ & $0.3 \pm 0.1$ \\
\hline S-111 (nonconvective layer) & $15 \pm 7.5(b)$ & $23 \pm 3.2$ & $66 \pm 10$ & $21 \pm 5.6$ & $11 \pm 1.8$ & $382 \pm 196(b)$ \\
\hline U-109 (nonconvective layer) & $22 \pm 11^{(b)}$ & $30 \pm 1.8$ & $25 \pm 3.0$ & $46 \pm 7.7$ & $27 \pm 3.6$ & $441 \pm 220$ (b) \\
\hline SY-101 (crust and bubble slurry) & $40 \pm 20(b)$ & $72 \pm 7$ & $34 \pm 4.4$ & $27 \pm 4.5$ & $19 \pm 2.5$ & $245 \pm 122(b)$ \\
\hline SY-101 (mixed slurry layer) & $2.9 \pm 1.4(\mathrm{~b})$ & $3.6 \pm 1.2$ & $26 \pm 8.1$ & $40 \pm 14$ & $26 \pm 7.5$ & $108 \pm 54(b)$ \\
\hline \multicolumn{7}{|c|}{$\begin{array}{l}\text { (a) The uncertainty bands on the average composition represent only the instrument error and solubility uncertainty. } \\
\text { There were too few samples to define the spatial variability of gas compositions. Lateral variation is not included. } \\
\text { (b) The RGS gas inventories and average gas volume fractions are assigned a } 50 \% \text { uncertainty because of the limited } \\
\text { number of samples on which the estimates are based. }\end{array}$} \\
\hline
\end{tabular}




\section{Overall Summary for All Tanks}

Table S.1 summarizes the gas volume fractions, gas volumes, and hydrogen fractions of the significant gas-retaining waste regions in tanks discussed in this report. The values in the table are derived from RGS data alone. In most tanks, the free gas inventory in the convective layer was less than $10 \%$ of the inventory in the other layer or layers. In addition, the compositions of gases in convective wastes were consistent (relative to nonconvective waste gas compositions): 55 to 70 mol\% $\mathrm{N}_{2}, 10$ to $25 \mathrm{~mol} \% \mathrm{H}_{2}$, and the remainder predominantly $\mathrm{N}_{2} \mathrm{O}$. Only the convective layers that were exceptions to these rules are included in Table S.1, for the sake of brevity.

The remaining sections of this summary describe the RGS findings for the tanks sampled during the RGS program. The results are described in the order in which the tanks were sampled (AW-101, AN-105, A-101, AN-104, AN-103, U-103, S-106, BY-101, BY-109, SX-106, AX-101, S-102, S-111, U-109, and SY-101) to reflect the increasing experience on which RGS methods were based.

\section{Tank 241-AW-101 (AW-101) Results}

Tank AW-101 was the first tank, and the first double-shell tank (DST), sampled using the RGS system to measure retained gases. At the time of sampling, the waste in this tank consisted of a nonconvective layer $286 \pm 30 \mathrm{~cm}$ (112 \pm 12 in.) deep, a surface crust $80 \pm 30 \mathrm{~cm}$ ( $31 \pm$ 12 in.) thick, and a $674 \pm 37 \mathrm{~cm}(266 \pm 14 \mathrm{in}$.) deep layer of supernatant liquid between the crust and nonconvective layer. Total depth of waste was $1040 \mathrm{~cm}$ (409 in.). Six segments were taken with the RGS from two risers in this tank, both of which were located toward the periphery of the tank. The waste in the nonconvective layer was not laterally homogeneous; observations of nonRGS core sample segments described the waste as salt in one riser and sludge in the other.

Retained gas measurements and estimated solubilities showed three major constituents in the gas phase (free gas) of the nonconvective layer: $55 \mathrm{~mol} \%$ nitrogen, $32 \mathrm{~mol} \%$ hydrogen, and $7 \mathrm{~mol} \%$ nitrous oxide. In the convective layer, the three major constituents of the free gas were $67 \mathrm{~mol} \%$ nitrogen, $26 \mathrm{~mol} \%$ hydrogen, and $2 \mathrm{~mol} \%$ nitrous oxide. The remainder of the gas content in the tank comprised ammonia, methane, and other hydrocarbons. The ammonia concentration was not conclusively determined by RGS measurements, but the lower bound was found to be 0.02 to $0.03 \mathrm{~mol} \mathrm{NH}_{3} / \mathrm{L}$ waste.

The extraction results showed that the insoluble gases (other than those in the crust, which RGS did not sample) were primarily retained in the lower $170 \mathrm{~cm}$ (70 in.) of the tank, or the lower two-thirds of the nonconvective layer. Based on estimated solubilities and RGS measurements of gas concentrations, about $3.7 \%$ by volume (in situ) of the nonconvective layer was filled with free gas, while $0.8 \%$ by volume (in situ) of the convective (upper) layer was free gas. The in situ gas volume fraction in the nonconvective layer ranged between 2.0 and $5.2 \%$ by volume, the maximum being somewhat lower than the maxima reported for VFI measurements in this tank. Some lateral nonuniformity in gas retention and possibly in gas composition was apparent from differences in measurements between the two risers at similar elevations.

Because of the waste variability from one riser to another and the availability of only four RGS samples from any one riser, the best estimate of the total free gas inventory (including the crust gas) was considered to be that based on a combination of VFI and RGS data, $95 \pm 16 \mathrm{~m}^{3}$ of gas at in situ conditions (153 $\pm 19 \mathrm{~m}^{3}$ at STP). The VFI\&RGS free gas inventory was made up of $45 \pm 5 \mathrm{~m}^{3}$ of in situ gas in the nonconvective layer ( $97 \pm 11 \mathrm{~m}^{3}$ at STP), $10 \pm 2 \mathrm{~m}^{3}$ of in situ gas in the convective layer ( $15 \pm 3 \mathrm{~m}^{3}$ at STP), and $40 \pm 15 \mathrm{~m}^{3}$ of in situ gas in the crust ( $41 \pm 15 \mathrm{~m}^{3}$ at STP). By comparison, the inventory estimated from the RGS data alone was $115 \pm 43 \mathrm{~m}^{3}$ at STP, excluding crust gas. 
Mostly round bubbles ranging from 1 to $7 \mathrm{~mm}$ in diameter were observed in the $\mathrm{x}$-ray images of several of the segments taken from the nonconvective layer. However, most of the gas volume was found to be smaller than the detection threshold $(<\sim 1 \mathrm{~mm})$ of the current $\mathrm{x}$-ray imaging system. Thus, based on our examination of the $\mathrm{x}$-ray images taken from various points in the nonconvective layer, the structure of the retained gases in this tank is speculated to be a bubbly mixture.

\section{Tank 241-AN-105 (AN-105) Results}

Tank AN-105 was the second tank and the second DST sampled for retained gases. The RGS was used in two risers within this tank to obtain eight segments. At the time of sampling the total depth of waste was $1041 \mathrm{~cm}$ ( $410 \mathrm{in}$.), of which $450 \pm 40 \mathrm{~cm}$ (177 $\pm 16 \mathrm{in}$.) was the nonconvective layer at the bottom of the tank, $45 \pm 10 \mathrm{~cm}(18 \pm 4 \mathrm{in}$.) was the crust on the surface, and the remainder was supernatant liquid [ $546 \pm 43 \mathrm{~cm}(215 \pm 17 \mathrm{in}$.) thick]. One riser was located near the center of the tank, the other about halfway between the center and the tank wall.

Retained gas measurements and estimated solubilities showed three major constituents in the gas phase (free gas) of the nonconvective layer: $24 \mathrm{~mol} \%$ nitrogen, $59 \mathrm{~mol} \%$ hydrogen, and $15 \mathrm{~mol} \%$ nitrous oxide. In the convective layer, the three major constituents of the free gas were $57 \mathrm{~mol} \%$ nitrogen, $25 \mathrm{~mol} \%$ hydrogen, and $13 \mathrm{~mol} \%$ nitrous oxide. The remainder of the gas in the tank was ammonia, methane, and other hydrocarbons. The ammonia concentration was not conclusively determined by RGS measurements, but RGS ammonia partial pressure measurements made on AN-105 samples suggested concentrations about the same as in Tank AW-101.

The extraction results showed that the insoluble gases were retained primarily in the lower, nonconvective layer. Based on the estimated solubilities and RGS measurements of gas concentrations, about $5.2 \%$ by volume (in situ) of the nonconvective layer was filled with free gas; $0.6 \%$ by volume (in situ) of the convective layer was free gas. Local calculated gas volume fractions based on RGS data were in close agreement with the VFI results for the segments above $200 \mathrm{~cm}$ ( $80 \mathrm{in}$.) elevation. While the lower two RGS segments did not have VFI measurements to compare, the trends pointed to reasonable estimates of the gas volume fraction. The maximum gas volume fraction measured with RGS was $12 \%$. Although the gas volume fractions appeared consistent for both risers, there could have been some difference in the gas composition.

The best estimate of the total gas inventory (including crust gas) was considered to be based on a combination of VFI and RGS data, $109 \pm 19 \mathrm{~m}^{3}$ of gas at in situ conditions (171 \pm 41 $\mathrm{m}^{3}$ at STP). The VFI\&RGS free gas inventory was made up of $80 \pm 16 \mathrm{~m}^{3}$ of in situ gas in the nonconvective layer (140 $\pm 40 \mathrm{~m}^{3}$ at STP), $4 \pm 2 \mathrm{~m}^{3}$ of in situ gas in the convective layer $(6 \pm 2$ $\mathrm{m}^{3}$ at STP), and $25 \pm 10 \mathrm{~m}^{3}$ of in situ gas in the crust (25 $\pm 10 \mathrm{~m}^{3}$ at STP). By comparison, the inventory estimated from the RGS data alone was $204 \pm 93 \mathrm{~m}^{3}$ at STP, excluding crust gas.

The $\mathrm{x}$-ray images showed large gas pockets that accounted for a large portion of the measured gas volume fraction. This contrasts with the observations made for some other wastes, where the major portion of the gas was observed to be smaller than the detection threshold of the $\mathrm{x}$-ray imaging system $(<0.5 \mathrm{~mm})$. No fractures or irregularly shaped bubbles were observed in samples from this tank.

\section{Tank 241-A-101 (A-101) Results}

Tank A-101 was the third tank and the first SST sampled for measurement of the retained gases in the waste. The tank was on the Flammable Gas Watch List (FGWL). The RGS was used in two risers within this tank to sample seven segments. The waste consisted of two distinct 
layers. At the time of sampling the lower convective layer was $472 \mathrm{~cm}$ (186 in.) thick and the upper nonconvective layer $411 \mathrm{~cm}$ (162 in.) thick. Both risers were closer to the periphery than the center of the tank. Core extrusions showed little or no evidence of lateral inhomogeneities in either layer.

Retained gas measurements and estimated solubilities showed three major low-solubility constituents in the gas phase (free gas) of the nonconvective layer: $19 \mathrm{~mol} \%$ nitrogen, $70 \mathrm{~mol} \%$ hydrogen, and $6 \mathrm{~mol} \%$ nitrous oxide. In the convective layer, the three major constituents of the free gas were $60 \mathrm{~mol} \%$ nitrogen, $14 \mathrm{~mol} \%$ hydrogen, and $15 \mathrm{~mol} \%$ nitrous oxide. The remainder of the gas content in the tank was ammonia, methane, and other hydrocarbons. The ammonia concentration was not conclusively determined by RGS measurements, but RGS ammonia partial pressure measurements made on A-101 samples suggested concentrations two to five times those as in Tank AW-101.

The extraction results showed that the insoluble gases were primarily retained in the upper layer, with the gas fraction consistently increasing from the top to the bottom of the layer. Based on the estimated solubilities and RGS measurements of gas concentrations, about $18 \%$ by volume (in situ) of the upper nonconvective layer was filled with free gas, and $0.8 \%$ by volume (in situ) of the lower layer was free gas. The in situ gas volume fraction in the upper layer ranged between 16 and $22 \%$ by volume. The compositions and gas volumes were consistent between the two risers.

Because there was little indication of lateral variability, the best estimate of the total gas inventory was considered to be that based on the RGS method, $334 \pm 159 \mathrm{~m}^{3}$ of gas at in situ conditions ( $368 \pm 172 \mathrm{~m}^{3}$ at STP). The RGS free gas inventory was made up of $318 \pm 159 \mathrm{~m}^{3}$ of in situ gas in the nonconvective layer ( $344 \pm 172 \mathrm{~m}^{3}$ at STP) and $16 \pm 5 \mathrm{~m}^{3}$ of in situ gas in the convective layer ( $24 \pm 8 \mathrm{~m}^{3}$ at STP). By comparison, the total gas inventory estimated by the BPE method was $270 \pm 67 \mathrm{~m}^{3}$ at STP.

Images of the sampler taken with the X-ray imaging system revealed that the lower waste layer was primarily a uniform (homogeneous) mixture, possibly a dense liquid. On the other hand, the upper layer was made up of gas pockets, fractures, and phase heterogeneities. In one sample from the nonconvective layer, there was a gas gap at the bottom of the sample as well as the top, indicating that the waste cohered to itself and adhered to the sampler walls so strongly as to support its own weight. This behavior was unusual.

\section{Tank 241-AN-104 (AN-104) Results}

Tank AN-104 was the fourth tank and the third DST to be sampled for retained gases. At the time of sampling the waste in this tank consisted of a nonconvective layer $410 \pm 40 \mathrm{~cm}$ (161 \pm 16 in.) deep, a surface crust $41 \pm 7 \mathrm{~cm}$ (16 \pm 3 in.) thick, and $526 \pm 41 \mathrm{~cm}(207 \pm 16 \mathrm{in}$.) of supernatant liquid between the crust and nonconvective layer. Total depth of the waste was $979 \mathrm{~cm}$ (385 in.). The RGS was used in two risers within this tank to obtain seven segments. One was near the center of the tank, the other closer to the tank periphery than to the center.

Retained gas measurements and estimated solubilities showed three major constituents in the gas phase (free gas) of the nonconvective layer: $29 \mathrm{~mol} \%$ nitrogen, $45 \mathrm{~mol} \%$ hydrogen, and $23 \mathrm{~mol} \%$ nitrous oxide. In the convective layer, the three major constituents of the free gas were $55 \mathrm{~mol} \%$ nitrogen, $24 \mathrm{~mol} \%$ hydrogen, and $15 \mathrm{~mol} \%$ nitrous oxide. The remainder of the gas in the tank was ammonia, methane, and other hydrocarbons. The ammonia concentration was not conclusively determined by RGS measurements, but RGS ammonia partial pressure measurements made on AN-104 samples suggested concentrations about the same as in Tank AW-101. 
The extraction results showed that the insoluble gases were primarily retained in the lower, nonconvective layer. Based on the estimated solubilities and RGS measurements of gas concentrations, about $8.1 \%$ by volume (in situ) of the nonconvective layer was filled with free gas, while $0.5 \%$ by volume (in situ) of the convective (upper) layer was free gas. The nonconvective layer gas volume fractions calculated from RGS data ranged from $2 \%$ to $17 \%$ with a monotonic increase as the tank bottom was approached. With the exception of the bottom RGS segment, with which there were no VFI data to compare, all the RGS measurements agreed closely with the VFI results. Because of scheduling constraints, there were insufficient data from both risers to form any conclusions about lateral uniformity in gas volume fraction and gas composition in this tank.

The best estimate of the total gas inventory (including crust gas) was considered to be that based on a combination of VFI and RGS data, $138 \pm 14 \mathrm{~m}^{3}$ of gas at in situ conditions (217 \pm $15 \mathrm{~m}^{3}$ at STP). The VFI\&RGS free gas inventory was made up of $110 \pm 12 \mathrm{~m}^{3}$ of in situ gas in the nonconvective layer (186 $\pm 13 \mathrm{~m}^{3}$ at STP), $9 \pm 2 \mathrm{~m}^{3}$ of in situ gas in the convective layer (12 $\pm 2 \mathrm{~m}^{3}$ at STP), and $19 \pm 7 \mathrm{~m}^{3}$ of in situ gas in the crust (19 $\pm 7 \mathrm{~m}^{3}$ at STP). By comparison, the inventory estimated from the RGS data was $272 \pm 128 \mathrm{~m}^{3}$ at STP, excluding crust gas.

The X-ray images showed large gas pockets that accounted for a large portion of the measured gas volume fraction. No fractures and few irregularly shaped bubbles were observed in samples from this tank.

\section{Tank 241-AN-103 (AN-103) Results}

Tank AN-103 was the fifth tank and the fourth DST sampled for retained gases. At the time of sampling the total depth of waste was $1041 \mathrm{~cm}$ (410 in.), of which $378 \pm 7 \mathrm{~cm}$ (149 \pm $3 \mathrm{in}$.) was the nonconvective layer at the bottom of the tank, $89 \pm 16 \mathrm{~cm}$ (35 $\pm 6 \mathrm{in}$.) was the crust on the surface, and the remainder was supernatant liquid $417 \pm 18 \mathrm{~cm}(164 \pm 7 \mathrm{in}$.$) . Seven seg-$ ments were taken with the RGS in two risers within this tank. One riser was near the center of the tank, the other near the periphery.

Retained gas measurements and estimated solubilities showed three major constituents in the gas phase (free gas) of the nonconvective and convective layers: $33 \mathrm{~mol} \%$ nitrogen, $61 \mathrm{~mol} \%$ hydrogen, and $4 \mathrm{~mol} \%$ nitrous oxide. The remainder of the gas comprised ammonia, methane, and other hydrocarbons. The composition of the crust free gas was very similar: $29 \%$ nitrogen, $62 \%$ hydrogen, and $7 \%$ nitrous oxide. In the convective layer, the three major constituents of the free gas were $68 \mathrm{~mol} \%$ nitrogen, $19 \mathrm{~mol} \%$ hydrogen, and $8 \mathrm{~mol} \%$ nitrous oxide. The ammonia concentration was not conclusively determined by RGS measurements, but RGS ammonia partial pressure measurements made on $\mathrm{AN}-103$ samples suggested concentrations about the same as in Tank AW-101.

The extraction results show that the insoluble gases were primarily retained in the lower, nonconvective layer; about one-sixth of the gas in the tank was in the crust. Based on the estimated solubilities and RGS measurements of gas concentrations, about $9.2 \%$ by volume (in situ) of the nonconvective layer was filled with free gas, while $0.7 \%$ by volume (in situ) of the convective layer was free gas. One crust sample was taken; its gas volume fraction was $16 \%$. The nonconvective layer gas volume fractions calculated from RGS data were $7 \%$ and $12 \%$. These measurements were lower than those measured with VFI. Because some samples were lost due to sampler valve problems, there were insufficient data from both risers to form any conclusions about lateral uniformity in gas volume fraction and gas composition in this tank.

Because only five RGS samples were available from any one riser, the best estimate of the total gas inventory (including crust gas) was considered to be that based on a combination of VFI and RGS data, $230 \pm 24 \mathrm{~m}^{3}$ of gas at in situ conditions ( $421 \pm 28 \mathrm{~m}^{3}$ at STP). The VFI\&RGS 
free gas inventory was made up of $170 \pm 10 \mathrm{~m}^{3}$ of in situ gas in the nonconvective layer (356 \pm $16 \mathrm{~m}^{3}$ at STP), $8 \pm 2 \mathrm{~m}^{3}$ of in situ gas in the convective layer $\left(11 \pm 3 \mathrm{~m}^{3}\right.$ at STP), and $52 \pm 22 \mathrm{~m}^{3}$ of in situ gas in the crust (54 $\pm 23 \mathrm{~m}^{3}$ at STP). By comparison, the inventory estimated from the RGS data was $329 \pm 133 \mathrm{~m}^{3}$ at STP, including the crust gas.

The x-ray images showed large gas pockets that accounted for a large portion of the measured gas volume fraction. One thin fracture bubble was observed in samples from AN-103. One sample contained two bullet-shaped voids; the arched top and flat bottom suggested some liquid separation within the pockets.

\section{Tank 241-U-103 (U-103) Results}

Tank U-103 was the sixth tank and the second SST sampled using the RGS system to measure the retained gases. It was on the FGWL. At the time of sampling, the waste in this tank was $424 \mathrm{~cm}$ (167 in.) deep and showed substantial variation between the two risers. Four RGS samples were taken from riser 7 in this tank. Further samples from riser 2 had been planned, but the drill string was blocked by an obstacle or hard waste. One RGS sample was taken from riser 13 but was lost because the sampler valve could not be opened for extrusion.

Retained gas measurements and estimated solubilities showed three major constituents in the gas phase (free gas) of the nonconvective layer: $36 \mathrm{~mol} \%$ nitrogen, $23 \mathrm{~mol} \%$ hydrogen, and $40 \mathrm{~mol} \%$ nitrous oxide. The remainder of the gas comprised ammonia, methane, and other hydrocarbons. The ammonia concentration was not conclusively determined by RGS measurements, but RGS ammonia partial pressure measurements made on U-103 samples suggested concentrations about twice those in Tank AW-101.

The extraction results, combined with $\mathrm{x}$-rays and core extrusions, showed that a significant amount of gas was stored near the top of the waste. The high-gas waste was not confined to the waste under the riser sampled by RGS but was not present in all samples taken near the top of the waste. The in situ gas volume fraction ranged from 7.9 to $42 \%$. The high value was a single measurement for the waste just under the surface; further down in the waste, the range was 7.9 to $11 \%$. According to the usual RGS data integration method, which assumed the high-gas measurement was uniform across the whole tank area at the measurement elevation, about $19 \%$ by volume (in situ) of the waste (excluding the supernatant) was filled with free gas. This estimate was expected to be an overestimate based on other observations that showed the high gas content did not extend across the tank.

Because the evidence showed that the single high-gas measurement did not represent a continuous layer in the waste, the best estimate of the total gas inventory was considered to be that based on the BPE method, $180 \pm 58 \mathrm{~m}^{3}$ of gas at in situ conditions, or $196 \pm 64 \mathrm{~m}^{3}$ (STP). By comparison, the nonconvective layer inventory estimated from the RGS data was $330 \pm 165 \mathrm{~m}^{3}$ (STP). The RGS inventory was larger than the BPE inventory, probably because the RGS inventory was biased by the high-gas measurement. The gas in the supernatant was not measured by RGS, but was negligible (based on the small supernatant volume and low gas concentration typical of supernatant).

The exceptionally high gas content (42\%) of the topmost sample (segment 7-2) was confirmed by observing a large gas space in the $\mathrm{x}$-ray of the sampler. It was also noted that in most of the samples the waste was strong enough and cohesive enough to form a "stalactite" hanging from the piston at the top of the sampler. 


\section{Tank 241-S-106 (S-106) Results}

Tank S-106 was the seventh tank and the third SST sampled for measurement of the retained gases in the waste. The waste consisted of two distinct layers, a supernatant liquid layer and a lower nonconvective saltcake layer, both of varying thickness. The waste surface depth was about $460 \mathrm{~cm}$ (181 in.) at the sampling locations at the time of sampling. There was also a thick, dry crust around the perimeter of the tank. The appearance of core extrusions from the two risers was not consistent, suggesting that the waste was not laterally uniform in the tank.

The RGS was used in risers 7 and 8 of S-106 to sample four segments, two from each riser. Both of these risers were near the tank center. There were no RGS samples from either the perimeter crust or the supernatant layer; such sampling was attempted but was not successful because of hard waste and sampler valve closure problems.

Retained gas measurements and estimated solubilities showed three major low-solubility constituents in the gas phase (free gas) of the nonconvective layer: $25 \mathrm{~mol} \%$ nitrogen, $63 \mathrm{~mol} \%$ hydrogen, and $11 \mathrm{~mol} \%$ nitrous oxide. The remainder of the gas content comprised ammonia, methane, and other hydrocarbons. The ammonia concentration was not conclusively determined by RGS measurements, but RGS ammonia partial pressure measurements made on S-106 samples suggested concentrations about half of those in Tank AW-101.

Based on the estimated solubilities and RGS measurements of gas concentrations, about $10 \%$ by volume (in situ) of the nonconvective layer was filled with free gas. The in situ gas volume fraction ranged between 7.7 and 14\%. Because of the waste and level variability from one riser to another, the availability of only two RGS samples from each riser, and doubts about the appropriateness of using the BPE method on level data from a tank that lacked a continuous supernatant layer, no "best estimate" of the total gas inventory was recommended. The BPE method gave a total gas inventory of $411 \pm 166 \mathrm{~m}^{3}$ at in situ conditions, or $543 \pm 219 \mathrm{~m}^{3}$ (STP). By comparison, the nonconvective layer inventory estimated from the RGS data was $169 \pm 85 \mathrm{~m}^{3}$ at in situ conditions, or $224 \pm 112 \mathrm{~m}^{3}$ (STP). Further data would be needed to choose between these inventories.

$X$-ray images of the samplers revealed that the nonconvective layer contained a number of gas pockets and gas-filled fractures. The largest bubbles, ranging from $1 \mathrm{~cm}$ to more than $2.5 \mathrm{~cm}$ across, were found in the sample taken nearest the bottom. Some of the largest bubbles in this sample were bullet-shaped, with separated liquid layers at the bottom. Another sample contained $12 \%$ gas, of which all was less than the detection threshold $(<\sim 1 \mathrm{~mm})$ of the current $\mathrm{x}$-ray imaging system.

\section{Tank 241-BY-101 (BY-101) Results}

Tank BY-101 was the eighth tank and the fourth SST sampled for retained gases. One RGS sample was taken at each of two risers, but further sampling was impossible because of obstructions at both risers. The samplers contained no waste that was visible in $\mathrm{X}$-ray images, and upon extraction the samples proved to contain no retained gas, only air. The samples provided no useful information on the composition or quantity of the gas retained in BY-101.

\section{Tank 241-BY-109 (BY-109) Results}

Tank BY-109 was the ninth tank and the fifth SST sampled for retained gases. The total depth of waste in BY-109 was 310 to $343 \mathrm{~cm}$ (122 to 135 in.); it was composed of saltcake and sludge. The interstitial liquid level (ILL) was below the surface because of salt-well pumping. 
The ILL was $279 \mathrm{~cm}$ (110 in.). The waste varied between the two risers that were sampled; at riser $12 \mathrm{C}$, most of the waste was sludge or salt/sludge slurry under a thin $(20 \mathrm{~cm})$ layer of moist saltcake, while at riser $10 \mathrm{~B}$ the top $150 \mathrm{~cm}$ of waste was all saltcake and the remaining lower layer sludge slurry or sludge.

Three segments were taken with the RGS in two risers within this tank. Both risers were about halfway between the tank center and the tank wall, on opposite sides of the tank from each other. No RGS samples were taken above the ILL; two had been planned but were lost because of damaged piston seals. One other sample at the bottona of riser $12 \mathrm{C}$ had also been planned but was blocked by impenetrable waste.

Retained gas measurements and estimated solubilities showed three major constituents in the gas phase (free gas) of the waste below the IIL: $28 \mathrm{~mol} \%$ nitrogen, 50 mol\% hydrogen, and $18 \mathrm{~mol} \%$ nitrous oxide. The remainder of the gas was composed of ammonia, methane, and other hydrocarbons. The ammonia concentration was not conclusively determined by RGS measurements, but RGS ammonia partial pressure measurements made on BY-109 samples suggested concentrations between one-third and one-half of those in Tank AW-101.

Based on the estimated solubilities and RGS measurements of gas concentrations, about $9.5 \%$ by volume (in situ) of the waste below the ILL was filled with free gas. The gas volume fractions based on RGS data ranged between 6.4 and 12\%. The total gas inventory, based on the RGS data alone, was $101 \pm 50 \mathrm{~m}^{3}$ at in situ conditions, or $122 \pm 61 \mathrm{~m}^{3}$ (STP). (The BPE method could not be used because the liquid in this tank was all subsurface.)

$X$-ray images of the samplers showed both small bubbles (a few mm in diameter) and large bubbles (more than $2.5 \mathrm{~cm}$ across). The waste characteristically contained bands and swirls of denser material, indicating that the solids did not all have the same composition.

\section{Tank 241-SX-106 (SX-106) Results}

Tank SX-106 was the tenth tank and the sixth SST sampled for retained gases. The tank was on the FGWL. At the time of sampling the total depth of waste in SX-106 was $518 \mathrm{~cm}$ (204 in.) with a crust $16 \mathrm{~cm}$ (6.5 in.) thick, $182 \mathrm{~cm}$ (72 in.) of supernatant liquid, and $320 \mathrm{~cm}$ (126 in.) of a nonconvective layer. Most of the nonconvective material was salt or salt slurry, though sludge or sludge/salt slurry was present in the top half of the nonconvective layer in both risers. The wastes from the two risers were noticeably different, suggesting lateral inhomogeneity. Six RGS segments were taken in two risers in this tank; both risers were closer to the tank center than the tank wall. No crust samples were planned or taken.

RGS sampling showed that the gas phase (free gas) in the nonconvective layer of SX-106 had an average composition of $20 \mathrm{~mol} \%$ nitrogen, $51 \mathrm{~mol} \%$ hydrogen, $24 \mathrm{~mol} \%$ nitrous oxide, and $4 \mathrm{~mol} \%$ ammonia. The free gas in the convective supernatant layer had an average composition of $61 \mathrm{~mol} \%$ nitrogen, $21 \mathrm{~mol} \%$ hydrogen, $15 \mathrm{~mol} \%$ nitrous oxide, and $0.6 \mathrm{~mol} \%$ ammonia. Minor components included methane and other hydrocarbons. RGS and non-RGS ammonia measurements in the supernatant layer disagreed, varying from $0.0011 \mathrm{~mol} \mathrm{NH}_{3} / \mathrm{L}$ of liquid in an non-RGS grab sample to $0.025 \mathrm{~mol} / \mathrm{L}$ of liquid in RGS samples. The measured ammonia levels in the nonconvective layer were more consistent, falling between 0.06 and $0.11 \mathrm{~mol} \mathrm{NH} / \mathrm{L}$ of waste (averaging $0.18 \mathrm{wt} \% \mathrm{NH}_{3}$ in the bulk waste, or $2500 \mu \mathrm{g} \mathrm{NH} / \mathrm{mL}$ liquid, or $0.15 \mathrm{M}$ in the liquid).

Based on the estimated solubilities and RGS measurements of gas concentrations, about $26 \%$ by volume (in situ) of the nonconvective layer and $0.2 \%$ by volume of the supernatant layer were filled with free gas. The in situ gas volume fraction in the nonconvective layer ranged from 8.9 to $35 \%$. The gas volume fraction was equal to or greater than $30 \%$ in the three deepest 
samples. Because the high RGS gas volume fractions were confirmed by non-RGS core extrusion observations, the best estimate of the total gas inventory was considered to be that based on the RGS method, $327 \pm 163 \mathrm{~m}^{3}$ at in situ conditions, or $427 \pm 213 \mathrm{~m}^{3}$ (STP); of this, only $1.2 \pm$ $0.6 \mathrm{~m}^{3}$ at in situ conditions, or $1.2 \pm 0.6 \mathrm{~m}^{3}$ (STP), was in the supernatant. By comparison, the total gas inventory estimated by the BPE method was $252 \pm 68 \mathrm{~m}^{3}$ (STP).

$\mathrm{X}$-ray images of the samples showed that gas was present in a variety of forms. More often than not, gas was visible as 2- to 3-mm-diameter bubbles. In two samples, however, waste was observed to form "stalactites" in the sampler, large chunks of waste held up by adhesion to the piston and walls.

\section{Tank 241-AX-101 (AX-101) Results}

Tank AX-101, the eleventh tank and seventh SST sampled for retained gases, was on the FGWL. At the time of sampling, the total depth of waste in AX-101 was about $703 \mathrm{~cm}(277 \mathrm{in}$.), with $343 \mathrm{~cm}$ (135 in.) of a nonconvective layer above $360 \mathrm{~cm}$ (142 in.) of a dense convective liquid layer. The wastes in the two risers were very similar. Cores were taken from two risers, but only one RGS segment was taken; it came from an elevation near the bottom of the upper layer of this tank. The riser was close to the tank wall.

RGS sampling showed that the gas phase (free gas) in the single AX-101 sample had a composition of $16 \mathrm{~mol} \%$ nitrogen, $60 \mathrm{~mol} \%$ hydrogen, $11 \mathrm{~mol} \%$ nitrous oxide, and $9 \mathrm{~mol} \%$ ammonia, with minor components including methane and other hydrocarbons. The RGS ammonia measurement was between 0.08 and $0.14 \mathrm{~mol} \mathrm{NH}_{3} / \mathrm{L}$ of waste, while salt-well grab-sample measurements were in the range of 0.04 to $0.07 \mathrm{~mol} \mathrm{NH}_{3} / \mathrm{L}$ of waste.

Based on the estimated solubilities and RGS measurements of gas concentrations, about $17 \%$ by volume (in situ) of the single sample was filled with free gas. Because there was only a single RGS sample and there were no data that were appropriate to calculate an inventory by the $\mathrm{BPE}$ method, the gas volume fractions visible in $\mathrm{x}$-rays were used to calculate a minimum gas inventory of $186 \mathrm{~m}^{3}$ in situ (195 m3 STP) for the nonconvective layer. The inventory must be considered a minimum value because $\mathrm{x}$-ray gas volume fractions (such as those used to calculate the inventory) were typically found to underestimate the gas measured by RGS extraction.

$X$-ray images of the RGS and non-RGS samples showed that gas was present in a variety of forms, including bubbles up to $6 \mathrm{~mm}$ in diameter and fractures across most or all of the sampler diameter. The $\mathrm{x}$-ray observations gave no reason to believe that any of the $\mathrm{x}$-rayed samples from the nonconvective layer contained much less gas than the RGS sample.

\section{Tank 241-S-102 (S-102) Results}

Tank S-102 was the twelfth tank, and the eighth SST, sampled for retained gases. The tank was on the FGWL. At the time of sampling, the total depth of waste in Tank S-102 was 521 cm (205 in.). The tank contained no supernatant. Many differences in appearance were seen between the waste sampled at three risers during this and an earlier sampling campaign. Five RGS segments were taken in one riser in this tank; one of these could not be processed because the sampler valve jammed closed, and one came from re-sampling the same location. The riser was closer to the periphery than to the center of the tank.

RGS sampling showed that the gas phase (free gas) in S-102 waste had an average composition of $32 \mathrm{~mol} \%$ nitrogen, $33 \mathrm{~mol} \%$ hydrogen, and $33 \mathrm{~mol} \%$ nitrous oxide, with minor components including ammonia, methane, and other hydrocarbons. RGS and non-RGS ammonia 
measurements disagreed and there was unusual scatter among the RGS measurements. However, there were no plausible data that suggested ammonia concentrations outside the range of 0.01 to $0.07 \mathrm{~mol} \mathrm{NH}_{3} / \mathrm{L}$ waste.

Based on estimated solubilities and RGS measurements of gas concentrations, about $26 \%$ by volume (in situ) of the waste was filled with free gas. The in situ gas volume fraction in the waste ranged from 12 to $33 \%$. Because the high RGS gas volume fractions were confirmed by non-RGS x-ray and core extrusion observations, and the level data came from a Food Instrument Corporation (FIC) rather than an Enraf instrument, the best estimate of the total gas inventory was considered to be that based on the RGS method, $544 \pm 272 \mathrm{~m}^{3}$ at in situ conditions, or $601 \pm 300$ $\mathrm{m}^{3}$ (STP). By comparison, the inventory estimated by the BPE method was. $294 \pm 83 \mathrm{~m}^{3}$ (STP).

X-ray images of the RGS and non-RGS samples showed that gas was present in a variety of forms. More often than not, gas was visible as $1-$ to $5-\mathrm{mm}$ diameter bubbles. However, two samples from the lower part of the waste displayed fracture bubbles spanning the entire sampler diameter. In one of these samples, waste also was observed to form a stalactite in the sampler, a large chunk of waste supported by adhesion to the piston and walls. In several samples, the liquid and solids in the waste seemed to have separated into two layers.

\section{Tank 241-S-111 (S-111) Results}

Tank S-111 was the thirteenth tank, and the ninth SST, sampled for retained gases. The tank was on the FGWL. At the time of sampling, the total depth of waste in Tank S-111 was $515 \mathrm{~cm}$ (203 in.) at the sampled riser. A supernatant layer overlay part of the waste. Its average depth was $10 \mathrm{~cm}$ (4 in.), and that of the nonconvective layer, which made up the rest of the waste, was $505 \mathrm{~cm}$ (199 in.). The nonconvective layer was almost entirely salt. Many differences in appearance were seen between the waste sampled at three risers during this and an earlier sampling campaign. Five segments were taken with the RGS in one riser within this tank. The riser was located closer to the tank center than to the tank wall.

RGS sampling showed that the gas phase (free gas) in the nonconvective layer in S-111 had an average composition of $21 \mathrm{~mol} \%$ nitrogen, $66 \mathrm{~mol} \%$ hydrogen, and $11 \mathrm{~mol} \%$ nitrous oxide. The free gas in the convective supernatant layer had an average composition of $90 \mathrm{~mol} \%$ nitrogen, $6 \mathrm{~mol} \%$ hydrogen, and $2 \mathrm{~mol} \%$ nitrous oxide. Minor components included methane and other hydrocarbons. RGS and non-RGS ammonia measurements disagreed and there was unusual scatter among the RGS measurements. However, there were no plausible data that suggested ammonia concentrations outside the range of 0.004 to $0.08 \mathrm{~mol} \mathrm{NH}_{3} / \mathrm{L}$ waste.

Based on the estimated solubilities and RGS measurements of gas concentrations, about $15 \%$ by volume (in situ) of the nonconvective layer and $0.8 \%$ of the supernatant layer was filled with free gas. The in situ gas volume fraction in the nonconvective layer ranged between 7 and $23 \%$, increasing monotonically toward the bottom of the waste. The best estimate of the nonconvective layer gas inventory was considered to be that based on the RGS method, $293 \pm 146 \mathrm{~m}^{3}$ at in situ conditions, or $382 \pm 196 \mathrm{~m}^{3}$ (STP); of this, only $0.3 \pm 0.1 \mathrm{~m}^{3}$ at in situ conditions, or 0.3 $\pm 0.1 \mathrm{~m}^{3}$ (STP), was in the supernatant. The total gas inventory estimated by the BPE method was nearly the same, $393 \pm 88 \mathrm{~m}^{3}$ (STP).

$\mathrm{X}$-ray images of the RGS samples showed that gas was present in a variety of forms. More often than not, gas was visible as bubbles from 1 to $5 \mathrm{~mm}$ in size. However, the bottommost sample displayed two fracture bubbles spanning the entire sampler diameter as well as smaller bubbles up to $10 \mathrm{~mm}$ in diameter. Little or no waste was attached to the sampler pistons. In the bottom-most sample, the liquid and solids in the waste seemed to have separated into two layers. 


\section{Tank 241-U-109 (U-109) Results}

Tank U-109 was the fourteenth tank, and the tenth SST, sampled for retained gases. The tank is on the FGWL. At the time of sampling, the total depth of waste at the sampled riser in Tank U-109 was $450 \mathrm{~cm}$ (177 in.). Neutron scans indicated liquid at the waste surface, and a supernatant layer was present over part of the waste surface. The average depth of the supernatant layer was $17 \mathrm{~cm}$ ( 7 in.). Many differences in appearance were seen between the waste sampled at four risers during this and an earlier sampling campaign. Four segments were taken with the RGS in one riser within this tank. No samples were taken from the supernatant. The riser was located close to the tank wall.

RGS sampling showed that the gas phase (free gas) in the U-109 nonconvective layer had an average composition of $46 \mathrm{~mol} \%$ nitrogen, $25 \mathrm{~mol} \%$ hydrogen, $27 \mathrm{~mol} \%$ nitrous oxide, and $0.9 \mathrm{~mol} \%$ ammonia, with minor components including methane and other hydrocarbons. RGS ammonia measurements in the nonconvective layer were consistent, falling between 0.02 and $0.05 \mathrm{~mol} \mathrm{NH} / \mathrm{L}$ of waste (averaging $0.077 \mathrm{wt} \% \mathrm{NH}_{3}$ in the bulk waste, or $1100 \mu \mathrm{g} \mathrm{NH} / \mathrm{mL}$ liquid, or $0.065 \mathrm{M}$ in the liquid).

Based on the estimated solubilities and RGS measurements of gas concentrations, about $22 \%$ by volume (in situ) of the nonconvective layer was filled with free gas. The in situ gas volume fraction ranged from 15 to $30 \%$. Because the RGS high gas fractions were backed up by core extrusion observations from other risers, and because of doubts about the appropriateness of using the BPE method on data from a tank that lacked a continuous supernatant layer, the best estimate of the total gas inventory was considered to be that based on the RGS method, $377 \pm 189 \mathrm{~m}^{3}$ at in situ conditions or $441 \pm 220 \mathrm{~m}^{3}$ (STP). By comparison, the total gas inventory estimated by the $\mathrm{BPE}$ method was $178 \pm 78 \mathrm{~m}^{3}$ (STP). The gas in the supernatant was not measured by RGS but was negligible (based on the small supernatant volume and low gas concentration typical of supernatant).

$\mathrm{X}$-ray images of the RGS samples indicated that $10 \%$ by volume or more of gas could be present in forms that were not visible in the images. In some cases, however, gas was visible as bubbles between 1 and $4 \mathrm{~mm}$ in size. Little or no waste was attached to the sampler pistons. In the two uppermost samples, the liquid and solids seemed to have separated into two layers.

\section{Tank 241-SY-101 (SY-101) Results}

Tank SY-101 was the fifteenth tank, and the fifth DST, sampled for retained gases.

SY-101 was sampled as part of a program to determine the causes and significance of recent unexplained level rises that had resulted in declaration of a new Unreviewed Safety Question (USQ) (Waste Surface Level Growth in 241-SY-101, USQ No. TF-97-0975). Twelve samples were taken from two risers, one near the center of the tank and one closer to the tank periphery than to the center. In addition, a single sample was taken from a third riser.

The waste level was $1046 \mathrm{~cm}$ (412 in.) at the time and location of the first set of samples and $1080 \mathrm{~cm}$ (425 in.) at the time and location of the second set of samples. A thick crust layer lay above a mixed slurry layer (mixed by periodic pumping). Between the crust and the mixed slurry was a high-gas region often postulated to be a "bubble slurry" layer, which was categorized as part of the crust for tabulation purposes. There was no evidence in the cores of a settled solids layer that retained a higher gas fraction than the mixed slurry.

The RGS samples from riser 23A (taken in November and December 1998) showed that the gas phase (free gas) in the SY-101 crust, roughly two-thirds of the gas in the tank, had an average composition of $27 \mathrm{~mol} \%$ nitrogen, $34 \mathrm{~mol} \%$ hydrogen, $19 \mathrm{~mol} \%$ nitrous oxide, and 
$19 \mathrm{~mol} \%$ ammonia, with minor components including methane and other hydrocarbons. The gas phase (free gas) in the mixed slurry layer had an average composition of $40 \mathrm{~mol} \%$ nitrogen, $26 \mathrm{~mol} \%$ hydrogen, $24 \mathrm{~mol} \%$ nitrous oxide, and $7 \mathrm{~mol} \%$ ammonia, with the same minor components. Similar gas compositions were found in risers 22A and 4A, sampled in January 1999 and March 1999 respectively. Most of the measured ammonia levels in the waste fell between 0.14 and $0.20 \mathrm{~mol} \mathrm{NH}_{3} / \mathrm{L}$ of bulk waste volume (including gas, liquid, and solids). The average total concentration of ammonia was equivalent to $0.24 \mathrm{wt} \% \mathrm{NH}_{3}$ in the bulk waste, which compared well with Window $\mathrm{E}$ measurements from 1991. The average dissolved ammonia was $4600 \mu \mathrm{g}$ $\mathrm{NH}_{3} / \mathrm{mL}$ liquid $(0.27 \mathrm{M})$.

Based on the estimated solubilities and RGS measurements of gas concentrations, about $40 \%$ by volume (in situ) of the crust layer and $2.9 \%$ by volume of the mixed slurry layer was filled with free gas. The in situ gas volume fraction ranged from 2.3 to $72 \%$; the latter value was from the high-gas region at the bottom of the crust. The total gas inventory was calculated from RGS data to be $353 \pm 133 \mathrm{~m}^{3}$ at in situ conditions in November 1998 and $540 \pm 220 \mathrm{~m}^{3}$ at in situ conditions in January 1999. The corresponding STP gas inventories were $402 \pm 143 \mathrm{~m}^{3}$ and $605 \pm$ $229 \mathrm{~m}^{3}$. The November 1998 value is considered the better estimate of the gas inventory in SY-101 between November 1998 and January 1999.

An opportunistic organic speciation analysis was performed on one of the SY-101 samples from the "bubble slurry." The results suggest that the individual organic compounds that were most concentrated in the retained gas were probably at concentrations of $300 \mathrm{ppmv}$ or less in the SY-101 crust, or 2000 ppmv or less in the SY-101 mixed slurry. The most concentrated of the firmly identified compounds included ethanol, methanol, butane, 1-butanol, and 1,3-butadiene, and the most concentrated of the tentatively identified compounds included propene, methylamine, 2-butene and propane.

X-ray images of the RGS samples showed large amounts of gas in the crust. The forms varied from large blobs of gas 3 to $6 \mathrm{~mm}$ in size, which appeared to be pressed against the sampler wall, to plumes and swirls of bubbles so fine as to not be resolvable as individual bubbles. In many cases, however, gas was visible as bubbles between 1 and $3 \mathrm{~mm}$ in size. Waste was often attached to the sampler pistons in crust samples but not in mixed slurry samples. Small bubbles were sometimes visible in the mixed slurry samples.

\section{References}

Mahoney LA, ZI Antoniak, and JM Bates. 1997. Composition and Quantities of Retained Gas Measured in Hanford Waste Tanks 241-U-103, S-106, BY-101, and BY-109. PNNL-11777, Pacific Northwest National Laboratory, Richland, WA 99352.

Shekarriz A, DR Rector, LA Mahoney, MA Chieda, JM Bates, RE Bauer, NS Cannon, BE Hey, CG Linschooten, FJ Reitz, and ER Siciliano. 1997. Composition and Quantities of Retained Gas Measured in Hanford Waste Tanks 241-AW-101, A-101, AN-105, AN-104, and AN-103. PNNL-11450 Rev. 1, Pacific Northwest National Laboratory, Richland, Washington. 


\section{Acknowledgments}

The authors would especially like to acknowledge the instrumental role played in implementing the Retained Gas Sampler System by the following Project Hanford Management Contract staff: Dennis Graves, Jerry Johnson, Jim Person, Scott Cannon, Bob Kowitz, and Jim Weber. A special thank you is extended to Roger Bauer for his overall management of the RGS efforts and his encouragement of a truly team approach to meeting the challenges of the sampling and subsequent interpretation of results. Bruce Hey carried out the extraction procedures under frequently trying conditions, and Dave Hedengren has provided a number of valuable insights. Many thanks to Milt Goheen, Stan Bos, and Larry Pederson at Pacific Northwest National Laboratory and Bill Kubic at the Los Alamos National Laboratory. Also, we would like to thank Chuck Stewart and Joe Brothers for reviewing the document and Reza Shekarriz for his management and guidance in the early part of the program. 


\section{Contents}

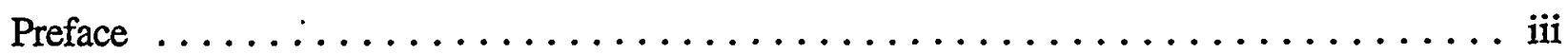

Executive Summary $\ldots \ldots \ldots \ldots \ldots \ldots \ldots \ldots \ldots \ldots \ldots \ldots \ldots \ldots \ldots \ldots$

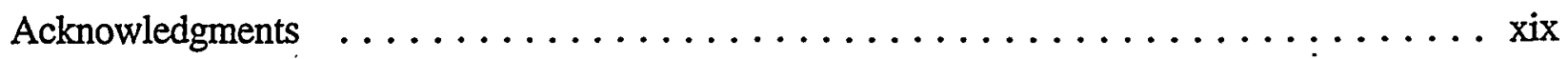

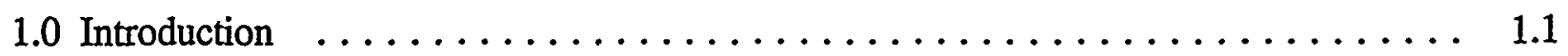

2.0 Retained Gas Sampler Processing $\ldots \ldots \ldots \ldots \ldots \ldots \ldots \ldots \ldots \ldots \ldots \ldots \ldots \ldots \ldots \ldots$

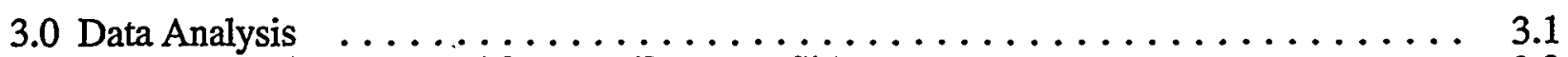

3.1 Material Removed from the Extractor Side $\ldots \ldots \ldots \ldots \ldots \ldots \ldots \ldots, 3.3$

3.2 Material Added to the Collector Side $\ldots \ldots \ldots \ldots \ldots \ldots \ldots \ldots \ldots \ldots .7$

3.3 Phase Partitioning in the Collector Side $\ldots \ldots \ldots \ldots \ldots \ldots \ldots \ldots . .7$

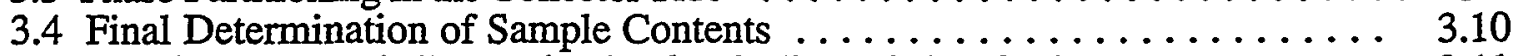

3.4.1 Ammonia Determination by the Isotopic Method ............. 3.11

3.4.2 Ammonia Determination from Post-RGS Concentration Measurements 3.12

3.4.3 Ammonia Determination from the Partial Pressure $\ldots \ldots \ldots \ldots .3 .13$

3.4.4 Choice of Ammonia Determination Method $\ldots \ldots \ldots \ldots \ldots \ldots . .14$

3.5 Corrections for Contamination $\ldots \ldots \ldots \ldots \ldots \ldots \ldots \ldots \ldots \ldots \ldots \ldots \ldots . .15$

3.6 Phase Distribution and Gas Volume Fraction $\ldots \ldots \ldots \ldots \ldots \ldots \ldots \ldots . .17$

3.6 .1 In Situ Solubility Model $\ldots \ldots \ldots \ldots \ldots \ldots \ldots \ldots \ldots \ldots \ldots \ldots \ldots$

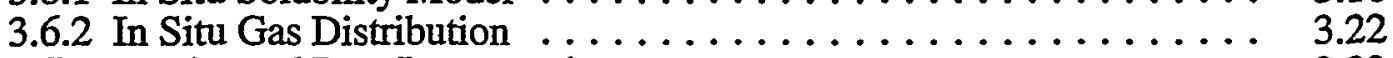

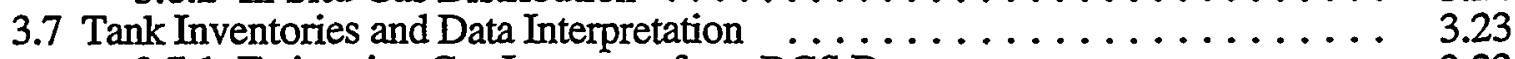

3.7.1 Estimating Gas Inventory from RGS Data $\ldots \ldots \ldots \ldots \ldots \ldots . \quad 3.23$

3.7.2 Best-Estimate Inventory and Uncertainty $\ldots \ldots \ldots \ldots \ldots \ldots \ldots .6 \ldots$

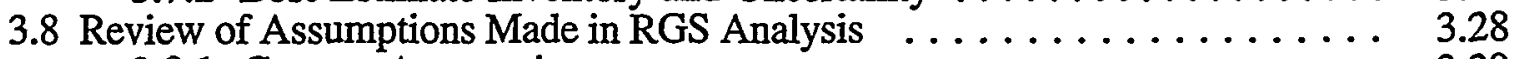

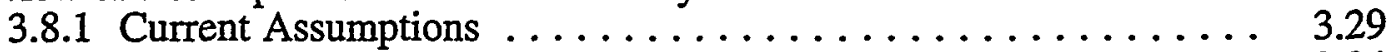

3.8.2 Assumptions Used in Previous RGS Analyses $\ldots \ldots \ldots \ldots \ldots .3 .34$

3.9 X-Ray Image Analysis $\ldots \ldots \ldots \ldots \ldots \ldots \ldots \ldots \ldots \ldots . . \ldots \ldots$

3.9.1 General Background on X-Ray Image Processing $\ldots \ldots \ldots \ldots .3 .36$

3.9.2 Guide for Viewing X-Ray Images $\ldots \ldots \ldots \ldots \ldots \ldots \ldots \ldots .3 .37$

4.0 Tank-by-Tank RGS Results $\ldots \ldots \ldots \ldots \ldots \ldots \ldots \ldots \ldots \ldots \ldots \ldots \ldots \ldots \ldots \ldots \ldots \ldots$

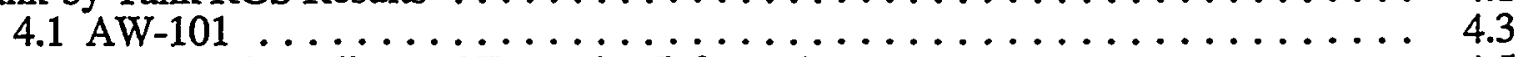

4.1.1 Sampling and Extraction Information $\ldots \ldots \ldots \ldots \ldots \ldots \ldots .4$

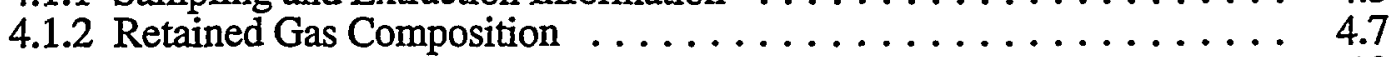

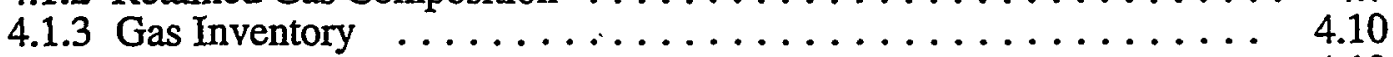

4.1 .4 X-Ray Results $\ldots \ldots \ldots \ldots \ldots \ldots \ldots \ldots \ldots \ldots \ldots \ldots \ldots$

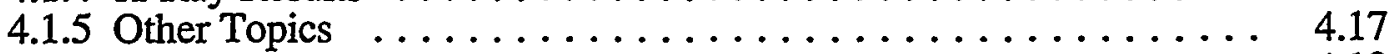

4.2 AN-105 .................................. 4.19

4.2.1 Sampling and Extraction Information $\ldots \ldots \ldots \ldots \ldots \ldots \ldots .21$

4.2 .2 Retained Gas Composition $\ldots \ldots \ldots \ldots \ldots \ldots \ldots \ldots . .22$

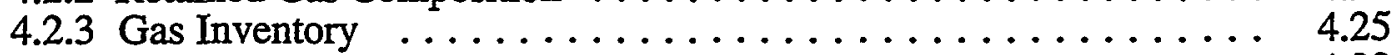

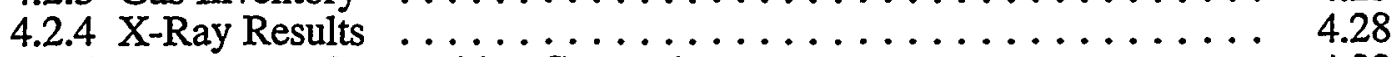

4.2.5 Domespace Composition Comparison $\ldots \ldots \ldots \ldots \ldots \ldots \ldots .32$

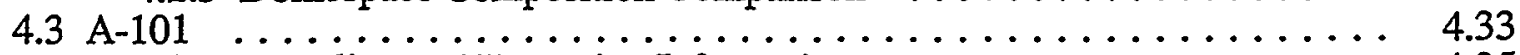

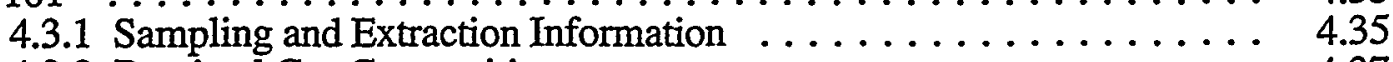

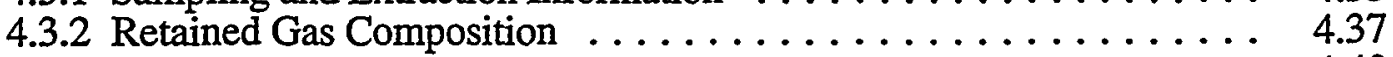

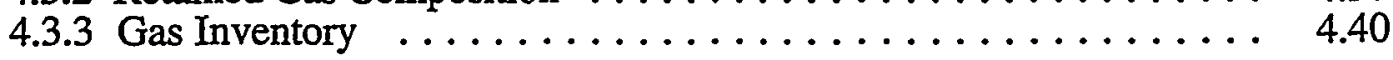




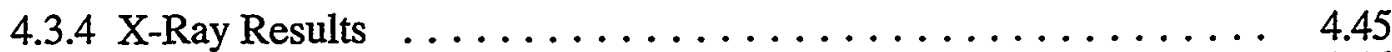

4.3.5 Domespace Composition Comparison $\ldots \ldots \ldots \ldots \ldots \ldots .4 .46$

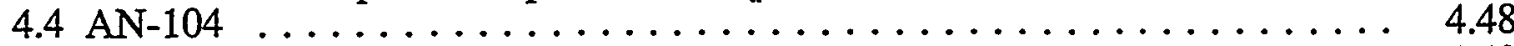

4.4.1 Sampling and Extraction Information $\ldots \ldots \ldots \ldots \ldots \ldots \ldots .4 .49$

4.4 .2 Retained Gas Composition $\ldots \ldots \ldots \ldots \ldots \ldots \ldots \ldots . \ldots .51$

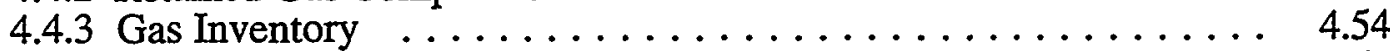

4.4 .4 X-Ray Results . . . . . . . . . . . . . . 4.57

4.4.5 Drillstring and Domespace Composition Comparison $\ldots \ldots \ldots .4 .62$

4.5 AN-103 .............................. 4.62

4.5.1 Sampling and Extraction Information $\ldots \ldots \ldots \ldots \ldots \ldots \ldots .64$

4.5.2 Retained Gas Composition $\ldots \ldots \ldots \ldots \ldots \ldots \ldots \ldots . \ldots \ldots 6$

4.5 .3 Gas Inventory $\ldots \ldots \ldots \ldots \ldots \ldots \ldots \ldots \ldots \ldots \ldots .6 .6 . \ldots \ldots$

4.5.4 X-Ray Results $\ldots \ldots \ldots \ldots \ldots \ldots \ldots \ldots \ldots \ldots . .71$

4.5.5 Drillstring and Domespace Composition Comparison ...... 4.76

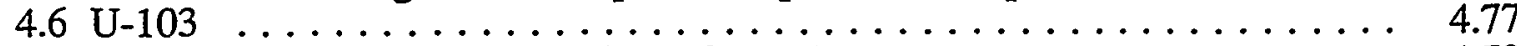

4.6:1 Sampling and Extraction Information $\ldots \ldots \ldots \ldots \ldots \ldots \ldots .79$

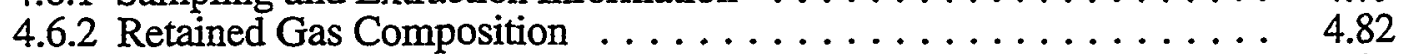

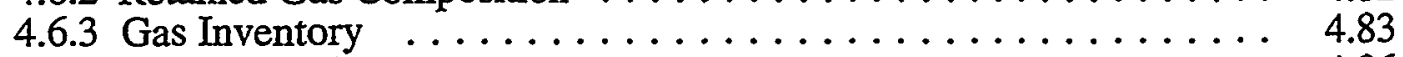

4.6 .4 X-Ray Results $\ldots \ldots \ldots \ldots \ldots \ldots \ldots \ldots \ldots \ldots \ldots \ldots \ldots \ldots \ldots$

4.6 .5 Other Discussions $\ldots \ldots \ldots \ldots \ldots \ldots \ldots \ldots \ldots \ldots \ldots . \ldots \ldots . \ldots \ldots$

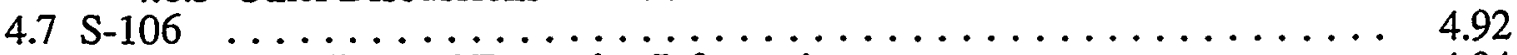

4.7.1 Sampling and Extraction Information $\ldots \ldots \ldots \ldots \ldots \ldots \ldots .9 .94$

4.7.2 Retained Gas Composition $\ldots \ldots \ldots \ldots \ldots \ldots \ldots \ldots . \ldots \ldots$

4.7 .3 Gas Inventory $\ldots \ldots \ldots \ldots \ldots \ldots \ldots \ldots \ldots \ldots . \ldots \ldots . . \ldots 9$

4.7 .4 X-Ray Results . . . . . . . . . . . . . . . 4.102

4.7.5 Drillstring Composition Comparison $\ldots \ldots \ldots \ldots \ldots \ldots .4 .106$

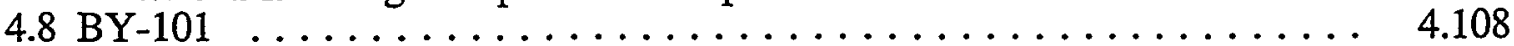

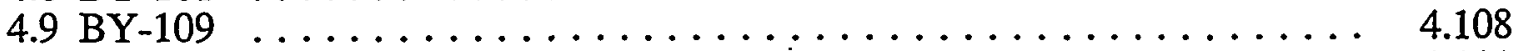

4.9.1 Sampling and Extraction Information $\ldots \ldots \ldots \ldots \ldots \ldots .111$

4.9 .2 Retained Gas Composition $\ldots \ldots \ldots \ldots \ldots \ldots \ldots \ldots \ldots \ldots \ldots \ldots \ldots \ldots \ldots \ldots \ldots .114$

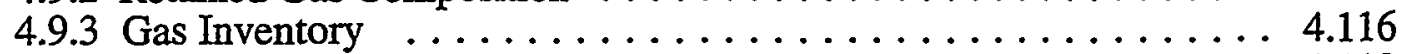

4.9 .4 X-Ray Results $\ldots \ldots \ldots \ldots \ldots \ldots \ldots \ldots \ldots \ldots \ldots \ldots \ldots \ldots \ldots \ldots \ldots \ldots .118$

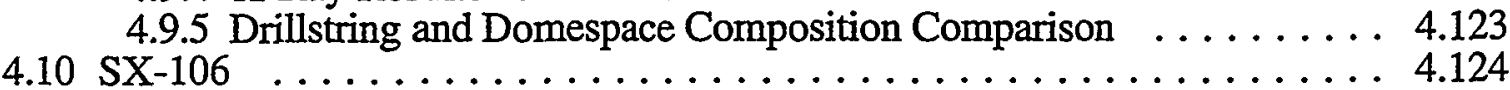

4.10 .1 Sampling and Extraction Information $\ldots \ldots \ldots \ldots \ldots \ldots .126$

4.10 .2 Retained Gas Composition $\ldots \ldots \ldots \ldots \ldots \ldots \ldots \ldots \ldots .128$

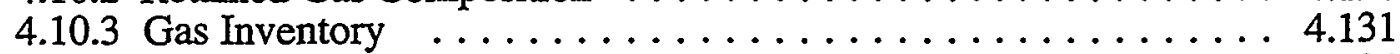

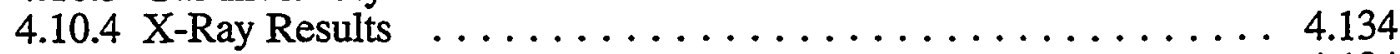

4.10 .5 High Gas Fractions $\ldots \ldots \ldots \ldots \ldots \ldots \ldots \ldots . \ldots \ldots .134$

4.10.6 Drillstring and Domespace Composition Comparison . . . . . . 4.139

$4.11 \mathrm{AX}-101 \ldots \ldots \ldots \ldots \ldots \ldots \ldots \ldots \ldots \ldots \ldots \ldots \ldots \ldots \ldots \ldots \ldots \ldots .140$

4.11 .1 Sampling and Extraction Information $\ldots \ldots \ldots \ldots \ldots \ldots .4 .142$

4.11 .2 Retained Gas Composition $\ldots \ldots \ldots \ldots \ldots \ldots \ldots \ldots .4 .143$

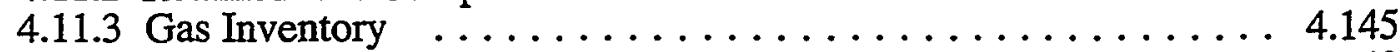

4.11 .4 X-Ray Results $\ldots \ldots \ldots \ldots \ldots \ldots \ldots \ldots \ldots . \ldots \ldots .148$

4.11.5 Domespace Composition Comparison .............. 4.152

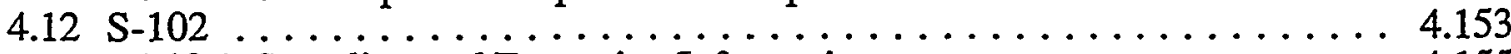

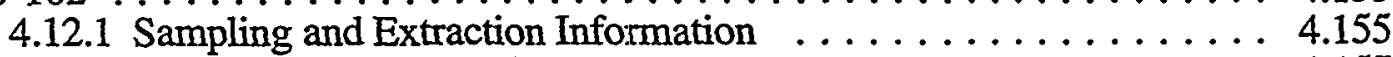

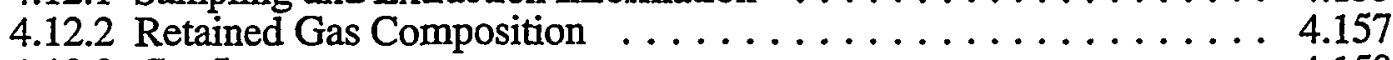

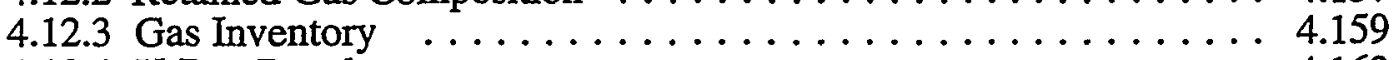

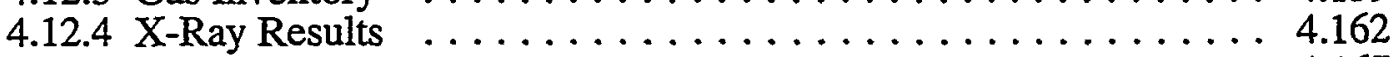

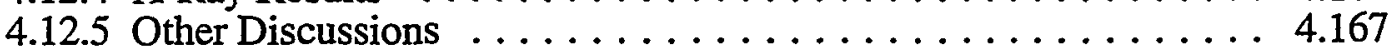

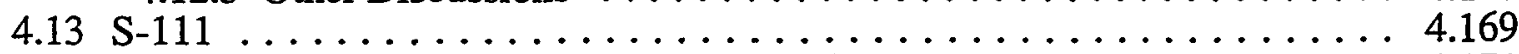

4.13.1 Sampling and Extraction Information $\ldots \ldots \ldots \ldots \ldots \ldots . .171$ 
4.13.2 Retained Gas Composition $\ldots \ldots \ldots \ldots \ldots \ldots \ldots \ldots .173$

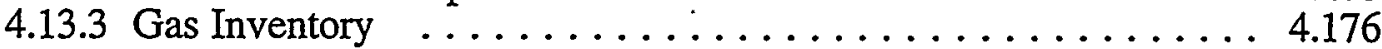

4.13 .4 X-Ray Results $\ldots \ldots \ldots \ldots \ldots \ldots \ldots \ldots \ldots \ldots \ldots .178$

4.13.5 Other Discussions $\ldots \ldots \ldots \ldots \ldots \ldots \ldots \ldots \ldots \ldots .182$

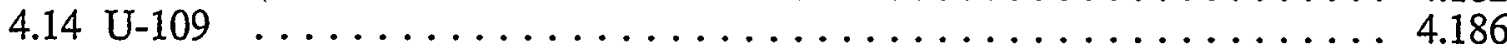

4.14.1 Sampling and Extraction Information $\ldots \ldots \ldots \ldots \ldots \ldots .4 .188$

4.14.2 Retained Gas Composition . . . . . . . . . . . . . . . 4.189

4.14 .3 Gas Inventory $\ldots \ldots \ldots \ldots \ldots \ldots \ldots \ldots \ldots . . \ldots \ldots . \ldots \ldots 2$

4.14.4 X-Ray-Results . . . . . . . . . . . . . . . . . . . . 4.194

4.14.5 Drillstring and Domespace Composition Comparison ....... 4.198

4.15 SY-101 ................................. 4.199

4.15.1 Sampling and Extraction Information $\ldots \ldots \ldots \ldots \ldots \ldots .4 .203$

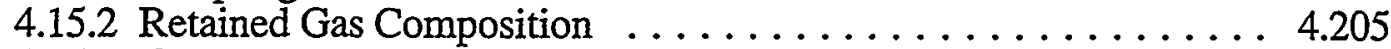

4.15 .3 Gas Inventory $\ldots \ldots \ldots \ldots \ldots \ldots \ldots \ldots \ldots \ldots . \ldots \ldots . \ldots \ldots$

4.15 .4 X-Ray Results $\ldots \ldots \ldots \ldots \ldots \ldots \ldots \ldots \ldots \ldots . \ldots \ldots .214$

4.15 .5 Other Discussions $\ldots \ldots \ldots \ldots \ldots \ldots \ldots \ldots \ldots . \ldots \ldots .214$

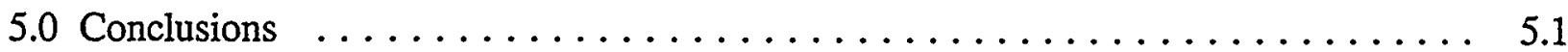

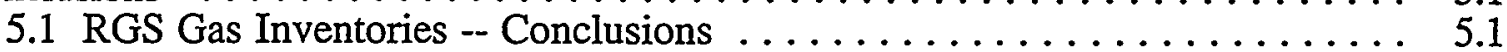

5.2 RGS Gas Volume Fractions-Conclusions $\ldots \ldots \ldots \ldots \ldots \ldots \ldots \ldots . \quad 5.2$

5.3 RGS Gas Volume Fractions-Key Observations $\ldots \ldots \ldots \ldots \ldots \ldots \ldots$. 5.2

5.4 RGS Gas Compositions-Conclusions $\ldots \ldots \ldots \ldots \ldots \ldots \ldots \ldots . \ldots \ldots .2$

5.5 RGS Gas Compositions-Key Observations $\ldots \ldots \ldots \ldots \ldots \ldots \ldots \ldots . . \ldots . \ldots \ldots$

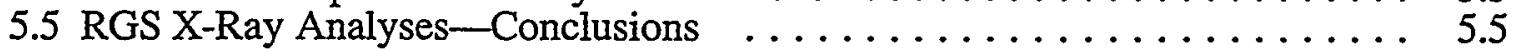

5.6 RGS General Conclusions . . . . . . . . . . . . . . . . 5.5

5.7 Summary of RGS Results $\ldots \ldots \ldots \ldots \ldots \ldots \ldots \ldots \ldots \ldots . \ldots \ldots$

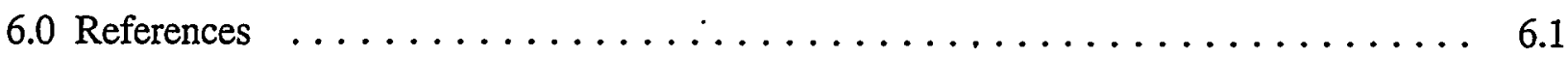

Appendix A: Field Data for RGS Tanks $\ldots \ldots \ldots \ldots \ldots \ldots \ldots \ldots \ldots \ldots$ A.1

Appendix B: Organic Speciation $\ldots \ldots \ldots \ldots \ldots \ldots \ldots \ldots \ldots \ldots \ldots \ldots \ldots \ldots \ldots \ldots \ldots$ B.1

Appendix C: Detailed RGS Ammonia Results ${ }^{2} \ldots \ldots \ldots \ldots \ldots \ldots \ldots \ldots \ldots$ C.1

Supplemental Data and Calculations (compact disc) $\ldots \ldots \ldots \ldots \ldots$ inside back cover 


\section{Figures}

$2.1 \quad$ RGS Process Flow Diagram $\ldots \ldots \ldots \ldots \ldots \ldots \ldots \ldots \ldots \ldots \ldots \ldots \ldots \ldots \ldots .2$

2.2 Photograph of the RGS Extrusion and Extraction System $\ldots \ldots \ldots \ldots \ldots$

3.1 Schematic of the RGS Extraction System $\ldots \ldots \ldots \ldots \ldots \ldots \ldots \ldots \ldots \ldots \ldots$

3.2 Test for Significant $\mathrm{O}_{2}$ Reaction in RGS Samples $\ldots \ldots \ldots \ldots \ldots \ldots \ldots$

3.3 Comparison of the Schumpe Solubility Model with Simulant Data $\ldots \ldots \ldots 3.21$

$3.4 \quad$ Example of Integration Scheme for Averaging RGS Concentrations $\ldots \ldots \ldots 3.25$

3.5 Schematic Diagram of X-Ray Subsegments $\ldots \ldots \ldots \ldots \ldots \ldots \ldots .3 .39$

4.1.1 Schematic Diagram of Riser Locations in Tank AW-101 . . . . . . . . . 4.4

4.1.2 Diagram of Waste Layering and RGS Sample Elevations for AW-101 . . . . . 4.4

4.1.3 Gas Volume Fractions Measured by RGS and VFI $\ldots \ldots \ldots \ldots \ldots .12$

4.1.4 Gas Volume Fractions, Temperatures, Compositions, and Observations

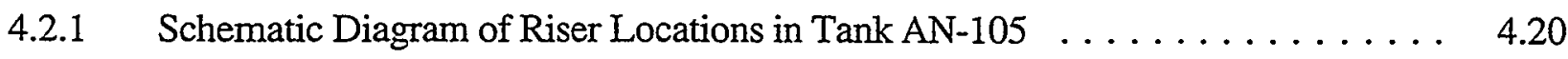

4.2.2 Diagram of Expected Waste Layering and RGS Sample Elevations for AN-105 4.20

4.2.3 Gas Volume Fractions Measured by RGS and VFI $\ldots \ldots \ldots \ldots \ldots \ldots .27$

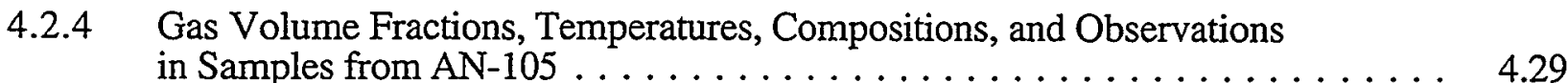

4.3.1 Schematic Diagram of Riser Locations in Tank A-101 . . . . . . . . . . 4.34

4.3.2 Diagram of Expected Waste Layering and RGS Sample Elevations for A-101 .. 4.34

4.3.3 Gas Volume Fractions, Temperatures, Compositions, and Observations in Samples from A-101 ........................... 4.43

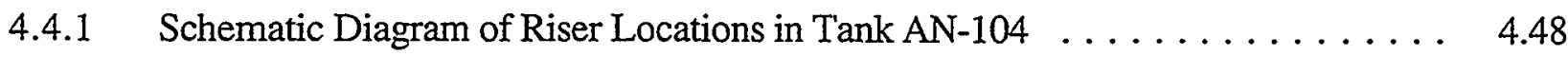

4.4.2 Diagram of Waste Layering and RGS Sample Elevations for AN-104 . . . . 4.49

4.4.3 Gas Volume Fractions Measured by RGS and VFI $\ldots \ldots \ldots \ldots \ldots . \ldots 4 . \ldots$

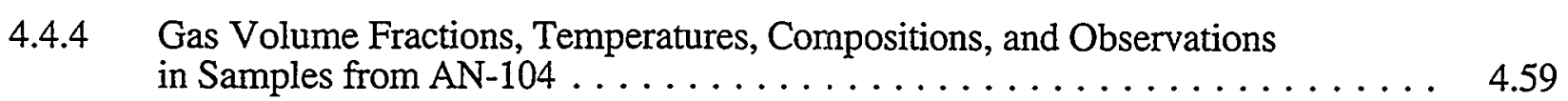

4.5.1 Schematic Diagram of Riser Locations in Tank AN-103 . . . . . . . . 4.63 
4.5.2 Diagram of Waste Layering and RGS Sample Elevations for AN-103 . . . . . 4.63

4.5.3 Gas Volume Fractions Measured by RGS and VFI $\ldots \ldots \ldots \ldots \ldots \ldots .70$

4.6.1 Schematic Diagram of Riser Locations in Tank U-103 f . . . . . . . . . . . 4.77

4.6.2 Diagram of Waste Layering and RGS Sample Elevations for U-103 $\ldots \ldots \ldots 4.78$

4.6.3 Gas Volume Fractions, Temperatures, Compositions, and Observations in Samples from $\mathrm{U}-103 \quad \ldots \ldots \ldots \ldots \ldots \ldots \ldots \ldots \ldots \ldots . \ldots \ldots . \ldots \ldots$

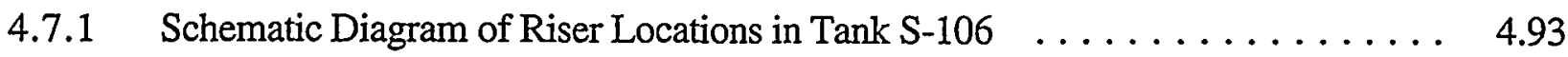

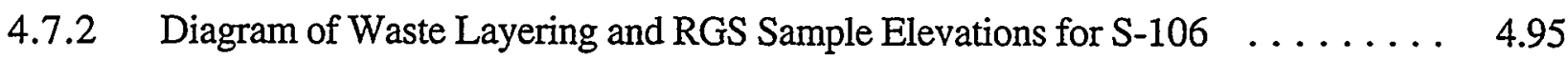

4.7.3 Recent Core Profile for Selected Push-Mode Sampling Risers in Tank S-106 . . 4.95

4.7.4 Gas Volume Fractions, Temperatures, Compositions, and Observations

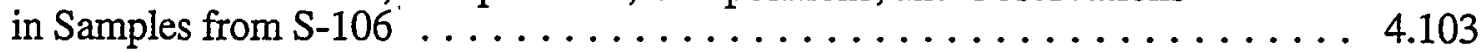

4.9.1 Schematic Diagram of Riser Locations in Tank BY-109 . . . . . . . . . . . 4.109

4.9.2 Diagram of Waste Layering and RGS Sample Elevations for BY-109 . . . . 4.110

4.9.3 Recent Core Profiles for the Push-Mode Sampling Risers in Tank BY-109 . . . . 4.111

4.9.4 Gas Volume Fractions, Temperatures, Compositions, and Observations in Samples from BY-109 ......................... 4.119

4.10.1 Schematic Diagram of Riser Locations in Tank SX-106 . . . . . . . . . 4.124

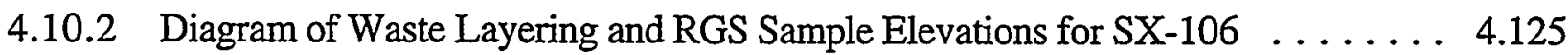

4.10.3 Gas Volume Fractions, Temperatures, Compositions, and Observations in Samples from SX $-106 \ldots \ldots \ldots \ldots \ldots \ldots \ldots \ldots \ldots . \ldots \ldots \ldots$

4.11.1 Schematic Diagram of Riser Locations in Tank AX-101 . . . . . . . . . . . 4.140

4.11.2 Diagram of Waste Layering and RGS Sample Elevations for AX-101 . . . . 4.141

4.11.3 Gas Volume Fractions, Temperatures, Compositions, and Observations in Samples from AX-101 ....................... 4.149

4.12.1 Schematic Diagram of Riser Locations in Tank S-1.02 . . . . . . . . . 4.154

4.12.2 Diagram of Waste Layering and RGS Sample Elevations for S-102 $\ldots \ldots \ldots 4.154$

4.12.3 Gas Volume Fractions, Temperatures, Compositions, and Observations in Samples from S-102 . . . . . . . . . . . . . . . . . 4.163

4.13.1 Schematic Diagram of Riser Locations in Tank S-111 . . . . . . . . . . 4.169

4.13.2 Diagram of Waste Layering and RGS Sample Elevations for S-111 . . . . . 4.170 
4.13.3 Gas Volume Fractions, Temperatures, Compositions, and Observations in Samples from S-111 . . . . . . . . . . . . . . . . . . . . . . 4.179

4.14.1 Schematic Diagram of Riser Locations in Tank U-109 . . . . . . . . . . . 4.186

4.14.2 Diagram of Waste Layering and RGS Sample Elevations for U-109 . . . . . . 4.187

4.14.3 Gas Volume Fractions, Temperatures, Compositions, and Observations in Samples from U-109 . . . . . . . . . . . . . . . . . . . . . . 4.195

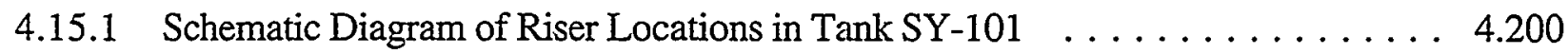

4.15.2 Waste Level Data from Tank SY-101 . . . . . . . . . . . . . . . 4.201

4.15.3 Tank 241-SY-101 Estimated Waste Layer Structure and Chart of RGS Sample Elevations for Risers 22A, 23A, and 4A. . . . . . . . . . . . 4.202

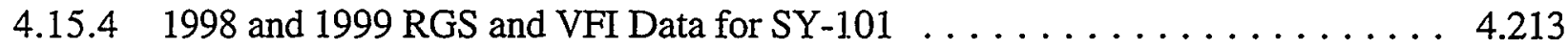

4.15.5 Gas Volume Fractions, Temperatures, Compositions, and Observations in Samples from SY-101 Riser 23 . . . . . . . . . . . . . . . . . . . 4.215

4.15.6 Gas Volume Fractions, Temperatures, Compositions, and Observations in Samples from SY-101 Risers $22 \mathrm{~A}$ and $4 \mathrm{~A} \ldots \ldots \ldots \ldots \ldots \ldots . \ldots .217$ 


\section{Tables}

3.1 Possible RGS Extraction Steps $\ldots \ldots \ldots \ldots \ldots \ldots \ldots \ldots \ldots \ldots \ldots \ldots$

3.2 Comparison of NH3 Partial Pressures Measured by Two Methods . . . . . . . 3.14

3.3 Schumpe Model Coefficients Used in RGS $\ldots \ldots \ldots \ldots \ldots \ldots \ldots \ldots$

3.4 Comparison of RGS and VFI Nonconvective Layer Gas Inventory in Four DSTs 3.26

3.5 Summary of Major Assumptions in RGS Calculations of Sample Gas

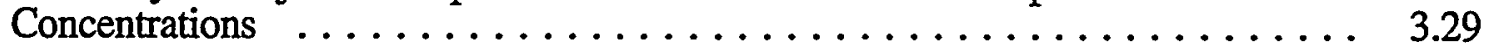

3.6 Differences Between Original and Final X-Ray Image Analysis Techniques $\ldots \quad \ldots .38$

$4.1 \quad$ Old and New Riser Numbers and Core Numbers for DSTs $\ldots \ldots \ldots \ldots \ldots . . . .4$

$4.2 \quad$ Riser Numbers and Core Numbers for SSTs $\ldots \ldots \ldots \ldots \ldots \ldots \ldots . \ldots \ldots$

4.1.1 Lag Times for Processing RGS Samples from Tank 241-AW-101 … . . . 4.5

4.1.2 HHF Contamination in AW-101 RGS Samples $\ldots \ldots \ldots \ldots \ldots \ldots \ldots . \ldots .6$

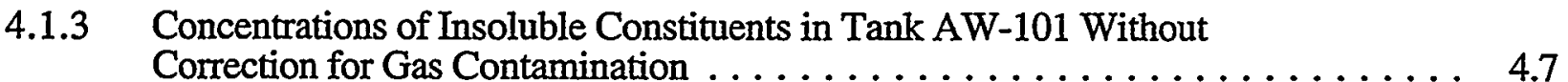

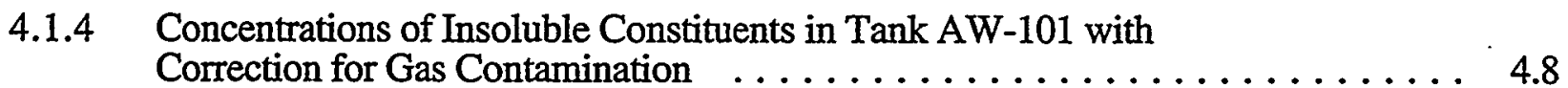

4.1.5 Ammonia Data from Tank AW-101 Samples $\ldots \ldots \ldots \ldots \ldots \ldots \ldots . \ldots . . \ldots$

4.1.6. Sample and Overall Average Compositions of Retained Gas in Tank AW-101

with Correction for Gas Contamination $\ldots \ldots \ldots \ldots \ldots \ldots \ldots \ldots . . \ldots$

4.1.7 In Situ Gas Volume Fractions and Conditions in Tank AW-101 . . . . . . 4.10

4.1.8 AW-101 Gas Inventory Estimates $\ldots \ldots \ldots \ldots \ldots \ldots \ldots \ldots \ldots \ldots . .11$

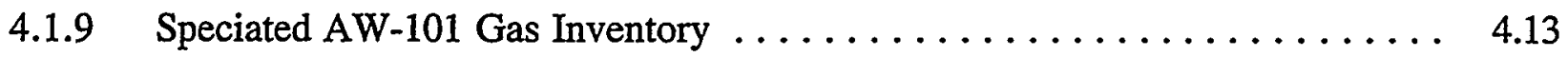

4.1.10 Summary of Observations from X-Ray Images of Tank AW-101 . . . . . . . 4.14

4.1.11 Densities of AW-101 Samples from Radiography and Core Samples . . . . . 4.17

4.1.12 Comparison to Drillstring and Domespace Data $\ldots \ldots \ldots \ldots \ldots \ldots \ldots$

4.2.1 Lag Times for Processing RGS Samples from Tank AN-105 $\ldots \ldots \ldots \ldots 4.21$

4.2.2 Hydrostatic Head Fluid Contamination in AN-105 RGS Samples . . . . . . . 4.22

4.2.3 Concentrations of Insoluble Constituents in Tank AN-105
Without Correction for Gas Contamination $\ldots \ldots \ldots \ldots \ldots \ldots$ 4. 23 
4.2.4 Concentrations of Insoluble Constituents in Tank AN-105

with Correction for Gas Contamination ................ 4. 23

4.2.5 Ammonia Data from Tank AN-105 Samples . . . . . . . . . . . . . . . 4.24

4.2.6 Sample and Overall Average Compositions of Retained Gas in

Tank AN-105 with Correction for Gas Contamination ............ . . 4. 25

4.2.7 In Situ Gas Volume Fractions and Conditions in Tank AN-105 . . . . . . . . 4.26

$4.2 .8 \quad$ AN-105 Gas Inventory Estimates $\ldots \ldots \ldots \ldots \ldots \ldots \ldots \ldots \ldots$

$4.2 .9 \quad$ Speciated $\mathrm{AN}-105$ Gas Inventory $\ldots \ldots \ldots \ldots \ldots \ldots \ldots$

4.2.10 Summary of Observations from X-Ray Images of Tank AN-105 $\ldots \ldots$. . . . 4.31

4.2.11 Densities of AN-105 Samples from Radiography and Core Samples . . . . . 4.32

4.2.12 Comparison with Domespace Data $\ldots \ldots \ldots \ldots \ldots \ldots \ldots$

4.3.1 Lag Times for Processing RGS Samples from Tank A-101 . . . . . . . . . 4.35

4.3.2 Hydrostatic Head Fluid Contamination in A-101 RGS Samples . . . . . . . 4.36

4.3.3 Concentrations of Insoluble Constituents in Tank A-101
Without Correction for Gas Contamination $\ldots \ldots \ldots \ldots \ldots . \ldots \ldots$

4.3.4 Concentrations of Insoluble Constituents in A-101 with
Correction for Gas Contamination $\ldots \ldots \ldots \ldots \ldots \ldots$

4.3.5 Ammonia Data from Tank A-101 Samples . . . . . . . . . . . . . . . 4.39

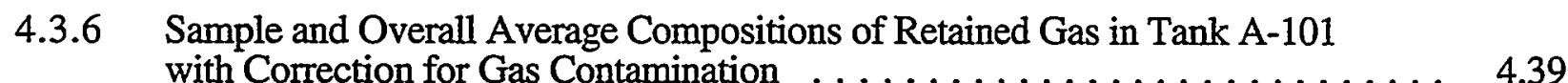

4.3.7 In Situ Gas Volume Fractions and Conditions in Tank A-101 $\ldots \ldots \ldots 41$

$4.3 .8 \quad$ A-101 Gas Inventory Estimates $\ldots \ldots \ldots \ldots \ldots \ldots \ldots \ldots$

$4.3 .9 \quad$ Speciated A-101 Gas Inventory $\ldots \ldots \ldots \ldots \ldots \ldots . \ldots \ldots$

4.3.10 Summary of Observations from X-Ray Images of Tank A-101 $\ldots \ldots .45$

4.3.11 Dènsities of A-101 Samples from Radiography and Core Samples $\ldots$. . . . . 4.47

4.3 .12 Comparison with Domespace Data $\ldots \ldots \ldots \ldots \ldots$. . . . . . . 4.47

4.4.1 Lag Times for Processing RGS Samples from Tank AN-104 _ . . . . . . . 4.50

4.4.2 Hydrostatic Head Fluid Contamination in AN-104 RGS Samples . . . . . . . 4.51

4.4.3 Concentrations of Insoluble Constituents in

Tank AN-104 Without Correction for Gas Contamination $\ldots \ldots \ldots \ldots . \ldots . . \ldots 2$ 
4.4.4 Concentrations of Insoluble Constituents in Tank AN-104

with Correction for Gas Contamination $\ldots \ldots \ldots \ldots \ldots \ldots \ldots \ldots . .52$

4.4.5 Ammonia Data from Tank AN-104 Samples ................ 4.53

4.4.6. Sample and Overall Average Compositions of Retained Gas in Tank AN-104

with Correction for Gas Contamination $\ldots \ldots \ldots \ldots \ldots \ldots \ldots \ldots . .54$

4.4.7 In Situ Gas Volume Fractions and Conditions in Tank AN-104 . . . . . . . 4.55

4.4.8 AN-104 Gas Inventory Estimates $\ldots \ldots \ldots \ldots \ldots \ldots \ldots \ldots \ldots \ldots \ldots$

4.4.9 Speciated AN-104 Gas Inventory $\ldots \ldots \ldots \ldots \ldots \ldots \ldots \ldots \ldots \ldots . \ldots \ldots$

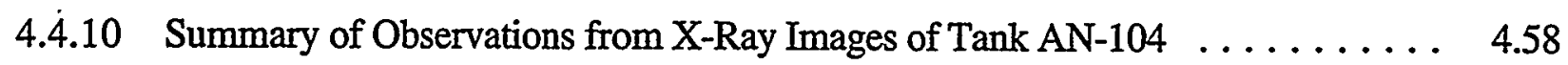

4.4.11 Densities of AN-104 Samples from Radiography and Core Samples . . . . . . . 4.61

4.4.12 Comparison with Drillstring and Domespace Data $\ldots \ldots \ldots \ldots \ldots \ldots . \ldots 2$

4.5.1. Lag Times for Processing RGS Samples from Tank AN-103 f . . . . . . 4.65

4.5.2 Hydrostatic Head Fluid Contamination in AN-103 RGS Samples. . . . . . . . 4.65

4.5.3 Concentrations of Insoluble Constituents in

Tank AN-103 Without Correction for Gas Contamination $\ldots \ldots \ldots \ldots \ldots .66$

4.5.4 Concentrations of Insoluble Constituents in

Tank AN-103 With Correction for Gas Contamination $\ldots \ldots \ldots \ldots \ldots .4 .66$

4.5.5 Ammonia Data from Tank AN-103 Samples . . . . . . . . . . . . . . 4.67

4.5.6. Sample and Overall Average Compositions of Retained Gas
in Tank AN-103 with Correction for Gas Contamination $\ldots \ldots \ldots \ldots \ldots \ldots$

4.5.7 In Situ Gas Volume Fractions and Conditions in Tank AN-103 . . . . . . . . 4.69

4.5.8 AN-103 Gas Inventory Estimates $\ldots \ldots \ldots \ldots \ldots \ldots \ldots \ldots \ldots \ldots \ldots$

4.5.9 Speciated AN-103 Gas Inventory $\ldots \ldots \ldots \ldots \ldots \ldots \ldots \ldots \ldots \ldots . \ldots \ldots$

4.5.10 Summary of Observations from X-Ray Images of Tank AN-103 $\ldots \ldots \ldots \ldots 4.72$

4.5.11 Densities of AN-103 Samples from Radiography and Core Samples . . . . . . 4.75

4.5.12 Comparison to Drillstring and Domespace Data $\ldots \ldots \ldots \ldots \ldots \ldots . \ldots . \ldots$

4.6.1 Lag Times for Processing RGS Samples from Tank U-103 . . . . . . . . . 4.79

4.6.2 Hydrostatic Head Fluid Contamination in U-103 RGS Samples . . . . . . . 4.80

4.6.3 Concentrations of Insoluble Constituents in

Tank U-103 Without Correction for Gas Contamination $\ldots \ldots \ldots \ldots \ldots .81$ 
4.6.4. Concentrations of Insoluble Constituents in

Tank U-103 with Correction for Gas Contarnination $\ldots \ldots \ldots \ldots \ldots . . . .41$

4.6.5 Ammonia Data from Tank U-103 Samples $\ldots \ldots \ldots \ldots \ldots \ldots \ldots . . .42$

4.6.6. Sample and Overall Average Compositions of Retained Gas

in Tank U-103 with Correction for Gas Contamination ............ 4.83

4.6.7 In Situ Gas Volume Fractions and Conditions in Tank U-103 . . . . . . . . 4.84

4.6.8 U-103 Gas Inventory Estimates $\ldots \ldots \ldots \ldots \ldots \ldots \ldots \ldots \ldots \ldots \ldots \ldots \ldots$

4.6.9 Speciated U-103 Gas Inventory $\ldots \ldots \ldots \ldots \ldots \ldots \ldots \ldots \ldots \ldots \ldots$

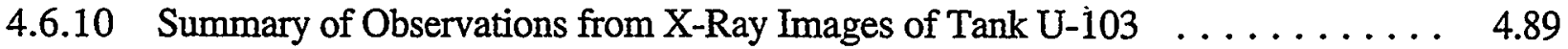

4.6.11 Densities of U-103 Samples from Radiography and Core Samples . . . . . . 4.90

4.6.12 Comparison with Drillstring and Domespace Data $\ldots \ldots \ldots \ldots . \ldots . \ldots . . \ldots 2$

4.7.1 Lag Times for Processing RGS Samples from Tank S-106 . . . . . . . . 4.95

4.7.2 Hydrostatic Head Fluid Contamination in S-106 Non-RGS Samples . . . . . 4.96

4.7.3 Concentrations of Insoluble Constituents in

Tank S-106 Without Correction for Gas Contamination $\ldots \ldots \ldots \ldots \ldots .4 .97$

4.7.4 Concentrations of Insoluble Constituents in

Tank S-106 with Correction for Gas Contamination ............ 4.97

4.7.5 Ammonia Data from Tank S-106 Samples ... . . . . . . . . . . . 4.98

4.7.6 Sample and Overall Average Compositions of Retained Gas

in Tank S-106 with Correction for Gas Contamination . . . . . . . . . . 4.99

4.7.7 In Situ Gas Volume Fractions and Conditions in Tank S-106 . . . . . . . . 4.100

$4.7 .8 \quad$ S-106 Gas Inventory Estimates $\ldots \ldots \ldots \ldots \ldots \ldots \ldots \ldots \ldots \ldots \ldots \ldots$

4.7.9 Speciated $\mathrm{S}-106$ Gas Inventory $\ldots \ldots \ldots \ldots \ldots \ldots \ldots \ldots \ldots . \ldots \ldots$

4.7.10 Summary of Observations from X-Ray Images of Tank S-106 . . . . . . . 4.105

4.7.11 Densities of S-106 Samples from Radiography and Core Samples . . . . . 4. 4.106

4.7.12 Comparison to Drillstring Data . . . . . . . . . . . . . . . 4.107

4.9.1 Lag Times for Processing RGS Samples from Tank BY-109 . . . . . . . . 4.112

4.9.2 Hydrostatic Head Fluid Contamination in BY-109 Samples . . . . . . . . . 4.112

4.9.3 Concentrations of Insoluble Constituents in
Tank BY-109 Without Correction for Gas Contamination $\ldots \ldots \ldots \ldots \ldots .4 .114$ 
4.9.4 Concentrations of Insoluble Constituents in

Tank BY-109 with Correction for Gas Contamination . . . . . . . . . . 4.114

4.9.5 Ammonia Data from Tank BY-109 Samples . . . . . . . . . . . . . . . 4.115

4.9.6 Sample and Overall Average Compositions of Retained Gas

in Tank BY-109 with Correction for Gas Contamination . . . . . . . . . 4.116

4.9.7 In Situ Gas Volume Fractions and Conditions in Tank BY-109 . . . . . . . 4.117

4.9.8 BY-109 Gas Inventory Estimates $\ldots \ldots \ldots \ldots \ldots \ldots \ldots \ldots \ldots \ldots \ldots \ldots$

4.9.9 Speciated BY-109 Gas Inventory $\ldots \ldots \ldots \ldots \ldots \ldots \ldots \ldots \ldots \ldots \ldots \ldots \ldots$

4.9.10 Summary of Observations from X-Ray Images of Tank BY-109 . . . . . . . 4.121

4.9.11 Densities of BY-109 Samples, from Radiography and Core Samples . . . . . 4.122

4.9.12 Comparison to Drillstring and Domespace Data $\ldots \ldots \ldots \ldots \ldots \ldots .123$

4.10.1 Lag Times for Processing RGS Samples from Tank SX-106 . . . . . . . . 4.126

4.10.2 Hydrostatic Head Fluid Contamination in SX-106 RGS Samples . . . . . . 4.127

4.10.3 Concentrations of Insoluble Constituents in

Tank SX-106 Without Correction for Gas Contamination $\ldots \ldots \ldots \ldots . . .4 .128$

4.10.4 Concentrations of Insoluble Constituents in

Tank SX-106 with Correction for Gas Contamination . . . . . . . . . . . 4.129

4.10.5 Ammonia Data from Tank SX-106 Samples . . . . . . . . . . . 4.130

4.10.6 Sample and Overall Average Compositions of Retained Gas in

SX-106 with Correction for Gas Contamination $\ldots \ldots \ldots \ldots \ldots \ldots \ldots .130$

4.10.7 In Situ Gas Volume Fractions and Conditions in Tank SX-106 . . . . . . 4.132

4.10 .8 SX-106 Gas Inventory Estimates $\ldots \ldots \ldots \ldots \ldots \ldots \ldots \ldots \ldots \ldots .133$

4.10 .9 Speciated SX-106 Gas Inventory $\ldots \ldots \ldots \ldots \ldots \ldots \ldots \ldots \ldots . .133$

4.10.10 Summary of Observations from X-Ray Images of Tank SX-106 . . . . . . 4.137

4.10.11 Densities of SX-106 Samples from Radiography and Core Samples . . . . . 4.138

4.10.12 Comparison to Drillstring and Domespace Data . . . . . . . . . . . 4.139

4.11.1 Lag Times for Processing RGS Sample from Tank AX-101 . . . . . . . 4.142

4.11.2 Hydrostatic Head Fluid Contamination in AX-101 RGS Sample . . . . . . 4.143

4.11.3 Concentrations of Insoluble Constituents in Tank AX-101

Without Correction for Gas Contamination 
4.11.4 Concentrations of Insoluble Constituents in

Tank AX-101 with Correction for Gas Contamination . . . . . . . . . . . . 4.144

4.11.5 Ammonia Data from Tank AX-101 Sample $\ldots \ldots \ldots \ldots \ldots \ldots \ldots . .4 .144$

4.11.6 Sample Composition of Retained Gas in Tank AX-101 with

Correction for Gas Contamination . . . . . . . . . . . . . . . 4.145

4.11.7 In Situ Gas Volume Fraction and Conditions in Tank AX-101 . . . . . . . 4.146

4.11.8 AX-101 Minimum Gas Inventory Estimates . . . . . . . . . . . . . . . . . 4.147

4.11.9 Speciated Minimum AX-101 Gas Inventory $\ldots \ldots \ldots \ldots \ldots \ldots \ldots .4 .147$

4.11.10 Summary of Observations from X-Ray Images of Tank AX-101 . . . . . . . 4.151

4.11.11 Density of AX-101 Sample from Radiography and Core Samples . . . . . . . 4.152

4.11.12 Comparison with Drillstring and Domespace Data $\ldots \ldots \ldots \ldots \ldots . .153$

4.12.1 Lag Times for Processing RGS Samples from Tank S-102 . . . . . . . . . 4.155

4.12.2 Hydrostatic Head Fluid Contamination in S-102 RGS Samples . . . . . . . 4.156

4.12.3 Concentrations of Insoluble Constituents in

Tank S-102 Without Correction for Gas Contamination . . . . . . . . . . 4.157

4.12.4 Concentrations of Insoluble Constituents in

Tank S-102 with Correction for. Gas Contamination ............. 4.158

4.12.5 Ammonia Data from Tank S-102 Samples . . . . . . . . . . . . 4.158

4.12.6 Sample and Overall Average Compositions of Retained Gas in Tank

S-102 with Correction for Gas Contamination $\ldots \ldots \ldots \ldots \ldots \ldots . . . .4 .159$

4.12.7 In Situ Gas Volume Fractions and Conditions in Tank S-102 . . . . . . . 4.160

$4.12 .8 \quad \mathrm{~S}-102$ Gas Inventory Estimates $\ldots \ldots \ldots \ldots \ldots \ldots \ldots \ldots \ldots \ldots \ldots \ldots \ldots \ldots$

4.12.9 Speciated S-102 Gas Inventory $\ldots \ldots \ldots \ldots \ldots \ldots \ldots \ldots \ldots \ldots .162$

4.12.10 Summary of Observations from X-Ray Images of Tank S-102 . . . . . . 4.165

4.12.11 Densities of S-102 Samples from Radiography $\ldots \ldots \ldots \ldots \ldots \ldots \ldots .166$

4.12.12 Comparison to Drillstring and Domespace Data . . . . . . . . . . 4.168

4.13.1 Lag Times for Processing RGS Samples from Tank 241-S-111 . . . . . . 4.171

4.13.2 Concentrations of Insoluble Constituents in

Tank S-111 Without Correction for Gas Contamination . . . . . . . . 4.173

4.13.3 Concentrations of Insoluble Constituents in

Tank S-111 with Correction for Gas Contamination .............. 4.174 
4.13.4 Ammonia Data from Tank S-111 Samples ... . . . . . . . . . . . . 4.174

4.13.5 Sample and Overall Average Compositions of Retained Gas in

Tank S-111 with Correction for Gas Contamination .............. 4.175

4.13.6 In Situ Gas Volume Fractions and Conditions in Tank S-111 . . . . . . . 4.176

$4.13 .7 \mathrm{~S}-111 \mathrm{Gas}$ Inventory Estimates $\ldots \ldots \ldots \ldots \ldots \ldots \ldots \ldots \ldots \ldots \ldots \ldots \ldots .177$

4.13 .8 Speciated $S-111$ Gas Inventory $\ldots \ldots \ldots \ldots \ldots \ldots \ldots \ldots \ldots \ldots \ldots \ldots \ldots$

4.13.9 Summary of Observations from X-Ray Images of Tank S-111 $\ldots \ldots \ldots . .181$

4.13.10 Densities of S-111 Samples from Radiography $\ldots \ldots \ldots \ldots \ldots \ldots . . \ldots 4$

4.13.11 Comparison to Domespace Data . . . . . . . . . . . . . 4.185

4.14.1 Lag Times for Processing RGS Samples from Tank U-109 ............ 4.188

4.14.2 Concentrations of Insoluble Constituents in

Tank U-109 Without Correction for Gas Contamination $\ldots \ldots \ldots \ldots \ldots \ldots . . . .190$

4.14.3 Concentrations of Insoluble Constituents in

Tank U-109 with Correction for Gas Contamination $\ldots \ldots \ldots \ldots \ldots \ldots \ldots . .190$

4.14.4 Ammonia Data from Tank U-109 Samples .................... 4.191

4.14.5 Sample and Overall Average Compositions of Retained Gas in
Tank U-109 with Correction for Gas Contamination $\ldots \ldots \ldots \ldots \ldots \ldots \ldots \ldots .4 .191$

4.14.6 In Situ Gas Volume Fractions and Conditions in Tank U-109 . . . . . . . . . 4.192

4.14.7 U-109 Gas Inventory Estimates .............................. 4.193

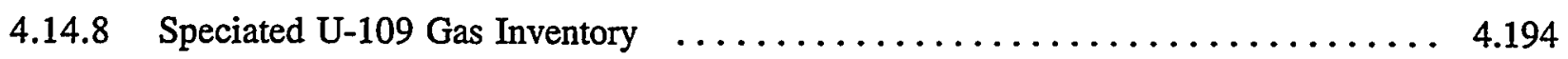

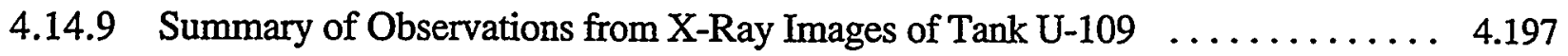

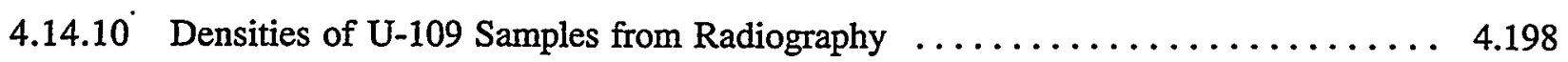

4.14.11 Comparison with Drillstring and Domespace Data ................. 4.199

4.15.1 Acquisition and Processing Schedule for RGS Samples from Tank SY-101 . . . 4.203

4.15.2 Concentrations of Insoluble Constituents in

Tank SY-101 Without Correction for Gas Contamination ............. 4.206

4.15.3 Concentrations of Insoluble Constituents in

Tank SY-101 with Correction for Gas Contamination $\ldots \ldots \ldots \ldots \ldots \ldots . \ldots .206$

4.15.4 Ammonia Data from Tank SY-101 Samples ..................... 4.207

4.15.5 Sample and Overall Average Compositions of Retained Gas in
Tank SY-101 with Correction for Gas Contamination $\ldots \ldots \ldots \ldots \ldots \ldots, 4.208$

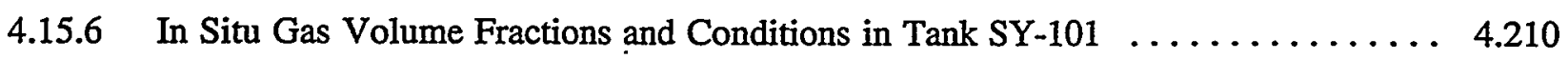


4.15.7 SY-101 Gas Inventory Estimates $\ldots \ldots \ldots \ldots \ldots \ldots \ldots \ldots \ldots \ldots \ldots \ldots \ldots \ldots$

4.15 .8 Speciated $S Y-101$ Gas Inventories $\ldots \ldots \ldots \ldots \ldots \ldots \ldots \ldots \ldots \ldots \ldots \ldots \ldots \ldots$

4.15.9 Summary of Observations from X-Ray Images of Tank SY-101 $\ldots \ldots \ldots$. . . . 4.219

4.15.10 Densities of SY-101 Samples from Radiography $\ldots \ldots \ldots \ldots \ldots \ldots$. . . . . 4.221

4.15.11 Calculated and Observed Piston Retraction . . . . . . . . . . . 4.223

4.15 .12 Comparison to Domespace Data $\ldots \ldots \ldots \ldots \ldots \ldots \ldots \ldots \ldots$

$5.1 \quad$ Comparison with BPE Retained Gas Inventories $\ldots \ldots \ldots \ldots \ldots \ldots \ldots \ldots \ldots$

5.2 In Situ Compositions and Volume Fractions of Retained Gas from RGS Data . . . . 5.6 


\section{Abbreviations and Acronyms .}

\begin{tabular}{ll} 
BPE & barometric pressure effect \\
BSVD & bulk sample volume determiniation \\
CCD & charge-coupled device \\
CD & compact disk \\
CGM & combustible gas meter \\
DST & double-shell tank \\
FGWL & Flammable Gas Watch List \\
FIC & Food Instrument Corporation \\
GRE & gas release event \\
HHF & hydrostatic head fluid \\
HTCE & historical tank contents estimate \\
ILL & interstitial liquid level \\
ISE & ion-specific electrode \\
LFL & lower flammability limit \\
LOW & liquid observation well \\
MDL & minimum detection limit \\
MIT & multifunctional instrument tree \\
NIH & National Institutes of Health \\
PNNL & Pacific Northwest National Laboratory \\
ppm & parts per million \\
RGS & retained gas sampler \\
rms & root-mean-square \\
SHMS & standard hydrogen monitoring system \\
SLR & surface level rise \\
SST & single-shell tank \\
\hline
\end{tabular}


STP

TC

TOC

USQ

VFI standard temperature and pressure

thermocouple

total organic carbon

unreviewed safety question

void fraction instrument 


\subsection{Introduction}

The key phenomena of the Flammable Gas Safety Issue are generation of the gas mixture, the modes of gas retention, and the mechanisms causing release of the gas. An understanding of the mechanisms of these processes is required for final resolution of the safety issue. Central to understanding is gathering information from such sources as historical records, tank sampling data, tank process data (temperatures, ventilation rates, etc.), and laboratory evaluations conducted on tank waste samples.

Gas generation processes must be understood well enough to estimate the generation rate and gas composition. The generation rates of the major fuel (hydrogen, ammonia, methane) and diluent species (nitrogen) determine the minimum tank ventilation rate required to prevent the buildup of a flammable mixture in the head space of a tank. A knowledge of gas generation processes also aids in assessing the long-term behavior of tank wastes and will support analyses of potential changes in waste storage conditions. Knowledge of the composition is needed to assess postulated deflagrations. The presence of such gases as ammonia, methane, and nitrous oxide can have a significant influence on the burn characteristics of a gas mixture. RGS data have provided valuable composition information.

Gas retention processes must also be understood before gas releases can be predicted. Experimental observations for actual waste samples and most of the simulants studied have suggested the predominant mode of gas retention is dominated by yield strength, producing particledisplacing bubbles. (The other, less observed mode of retention is dominated by capillary forces and produces bubbles that displace interstitial liquid but not particles.) The amounts of gas found in RGS samples and, to some extent, the forms in which gas appear in the x-ray images of the samples, provide useful information on gas retention in Hanford wastes.

Evaluation of the stored gas to date has been through analysis of tank level data and temperature profiles, detailed modeling activities, and in situ measurements using the void fraction instrument (VFI) and retained gas sampler (RGS). VFI measurements have been conducted on double-shell tanks (DSTs), but that method does not work for the single-shell tanks (SSTs), where the waste is either sludge or saltcake. Therefore, the RGS was used in selected SSTs to directly determine the gas composition and gas volume fraction. This report provides the results for the SSTs sampled with RGS during the RGS program: 241-AW-101, 241-AN-105, 241-A-101, 241-AN-104, 241-AN-103, 241-U-103, 241-S-106, 241-BY-101, 241-BY-109, 241-SX-106, 241-AX-101, 241-S-102, 241-S-111, 241-U-109, and 241-SY-101.(a)

The tanks that were sampled represented several different types of waste behavior associated with flammable gas. The DSTs were AW-101, AN-105, AN-104, AN-103, and SY-101. The first four of these DSTs have exhibited continuing gas releases associated with buoyancyinduced displacement events, and so were chosen for sampling. Tank SY-101, in the months before its sampling, had shown an unprecedented continuing level rise whose causes could in part be investigated by RGS sampling.

The SSTs were sampled for a variety of reasons. Tanks A-101, U-103, SX-106, AX-101, S-102, S-111, and U-109 are on the Flammable Gas Watch List (FGWL). Tanks BY-101 and BY-109 were not on the FGWL but were chosen to test the effect of salt-well pumping on gas retention. U-103, SX-106, S-102, and U-109 are members of the highest-priority group of SSTs, which showed evidence of significant gas retention (Stewart et al. 1996a). Tank S-111 (like S-106) is part of the second highest-priority group of SSTs. Tank S-106, though not a FGWL

(a) Hanford waste tanks are numbered beginning with the prefix 241-. Hereafter, in this report, the prefix will not be used, as is common practice when referring to the tanks at Hanford. 
tank, had a uniquely high barometric pressure response and continuing rapid surface level rise, indicating a large and increasing volume of retained gas. Tanks A-101 and AX-101 were considered important because of their level oscillations and their unusual waste structure (a gasretaining nonconvective layer floating on a dense liquid layer). In addition, high hydrogen and ammonia were observed in the dome space of A-101. RGS sampling was also intended to assist in characterizing tanks prior to salt-well pumping.

-There have been two earlier reports covering RGS results. The first, Shekarriz et al. (1997), covered Tanks AW-101, A-101, AN-105, AN-104, and AN-103. The second, Mahoney et al. (1997), covered Tanks U-103, S-106, BY-101, and BY-109. This report provides final documentation of the RGS sampling results between late 1997 and early 1999 (SX-106, AX-101, S-102, S-111, U-109, and SY-109). It also returns to the earlier tanks to apply insights gained from later work and add data that were not employed in the earlier reports.

The primary change has been in the handling of ammonia data. Throughout the RGS program, the data pertaining to ammonia have been the most difficult to interpret. Our later work has led us to believe that the ammonia concentrations reported in Shekarriz et al. (1997) and Mahoney et al. (1997) were for the most part underestimates and should not be used. More recent results were derived from changed measurement procedures and are believed to be more reliable. This report contains ammonia data from RGS extractions, ammonia partial pressure measurements, chemical analyses of RGS samples after extraction, and non-RGS ammonia data. These various data (which were not used in earlier reports) are used to provide estimates of ammonia concentrations and to assess the reliability of the estimates by comparing the values found by different measurement techniques.

Other changes include accounting for hydrostatic head fluid intrusion on the first five tanks (Shekarriz et al. 1997), more accurate water vapor estimates, improved data inputs for solubility calculations, more comparisons between the gas measured by extraction and that visible in the $\mathrm{X}$-ray, among many others. The aggregate impact of these changes on composition and gas volume fraction was generally 10 to $30 \%$ of the previously reported values.

Section 2 of this report provides an overview of the process by which retained gases in the Hanford tanks are sampled and analyzed. A detailed description of the procedure used to reduce and analyze the data is provided in Section 3. Tank-by-tank results are covered in Section 4 (with the data presented in the order in which the tanks were sampled), and Section 5 presents our conclusions from these analyses. The cited references are listed in Section 6. Appendix A gives field sampling data and Appendix B contains organic speciation information for an SY-101 sample. 


\subsection{Retained Gas Sampler Processing}

The RGS is a version of the universal core sampler specifically designed for sampling waste that contains gas. An overview of the procedure by which the data obtained using the RGS were analyzed and interpreted is provided in this section. Figure 2.1 provides a graphical diagram of the flow of material and information from the tanks sampled to the end product (a report). The RGS system; the sampler preparation, deployment, and retrieval; and the analytical procedure are detailed in Appendix A of Shekarriz et al. (1997). Details on the core sampler and modifications and operational constraints of the RGS are contained in Webb (1994).

To obtain an RGS sample, an RGS is installed in the drill string, lowered into the correct vertical position in the tank, and the sample taken by holding the sampler piston in position while pushing the rest of the sampler and drill string down through the waste. When the piston contacts the top end of the sampler, a spring is triggered that closes the sampler ball valve-hermetically sealing the waste sample solids, liquids, and gases in a chamber approximately $2.8 \mathrm{~cm}(1.125 \mathrm{in}$.) in diameter and $46 \mathrm{~cm}$ (18.2 in.) long.

The sampler is then removed from the tank and transferred to the $\mathrm{x}$-ray cart for imaging. The $x$-ray images provide information about the gas structure (bubble shape, etc.) and density of the waste and also allow a semi-quantitative estimate of the amount of gas present. After being. $\mathrm{x}$-rayed, the sampler is placed in a transport cask for delivery to the hot cell facilities for extrusion and gas extraction. To extract the gases, the sampler is loaded into the hot cell and installed on a previously evacuated extraction system. (A photograph of the extrusion and extraction system is shown in Figure 2.2.) The sampler ball valve is opened, and the extruder's hydraulic ram pushes the sampler piston all the way forward to move the sampler contents into the extractor. The sample waste is then stirred, after which sample gas is transferred from the extractor to collector gas sample canisters using a mercury transfer pump. In some cases the sample is heated to $50^{\circ} \mathrm{C}$ and cooled to hot cell temperature to force gas release. A tracer standard containing $15 \mathrm{NH}_{3}$ may be added before the final extraction steps to measure the sample ammonia concentration. During this process, the sampler and canister pressures and temperatures are monitored and recorded in computer files for further data reduction and analysis. The extracted gases are analyzed for composition and $15 \mathrm{~N} / 14 \mathrm{~N}$ ratio with a mass spectrometry system.(a) After gas extraction is complete, an inert gas is introduced to measure the gasless volume of the sample, and the sample may then be analyzed to measure ammonia and bromide (a tracer of hydrostatic head fluid intrusion during sample acquisition).

A data analysis procedure (derived in Section 3) was formulated that uses as input the temperature, pressure, and volumes during extraction as well as the mass spectrometry data on species concentration and isotopic ratio. The model outputs are the mole fraction of gas constituents, total volume of each gas, vapor phase versus dissolved percentages for all species, and gas volume fraction for each elevation at which the gas was sampled. The analysis also provides an estimate of the gas inventory in the sampled layers within the tank. The $\mathrm{x}$-ray images are analyzed

(a) The mass spectrometer is used in to determine the hydrocarbons present in the RGS gas samples. When small amounts of $\mathrm{C}_{2}$ are present, the speciation methodology uses either the 27 or 28 mass peaks. If there are many peaks detected, the 28 peak from a high resolution scan is used. In those instances when there are very small amounts of $\mathrm{C}_{\mathrm{x}} \mathrm{H}_{\mathrm{y}}$, the 41,43 , and 58 peaks are used with n-butane as a reference, and adjustments are made for other mass peaks $\left(C_{1}\right.$ by the 15 peak or a high resolution scan of the 16 mass peak). Higher amounts of hydrocarbons necessitate a matrix data reduction with methane, ethane, propane, and butane in the reference data base. 


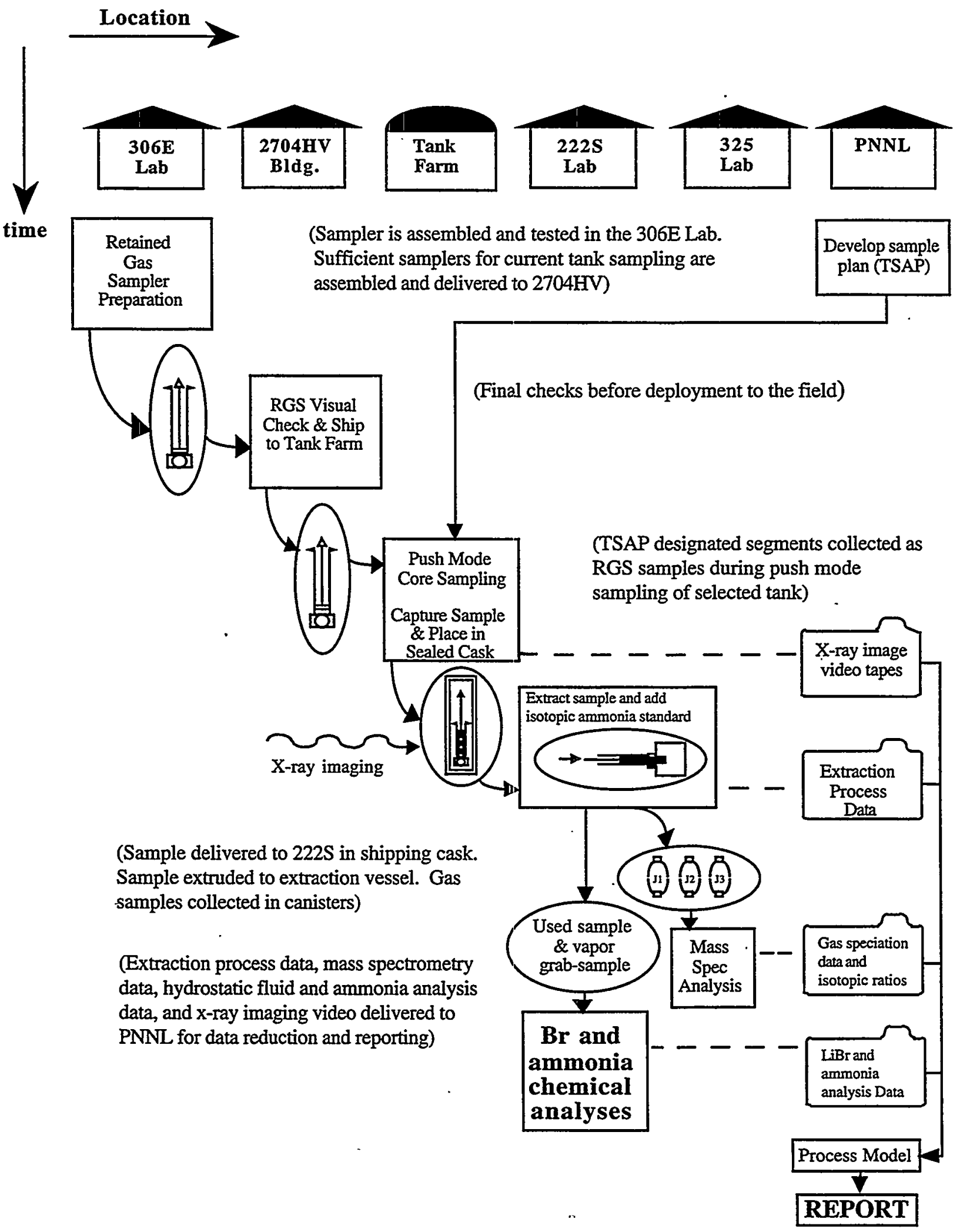

Figure 2.1. RGS Process Flow Diagram 


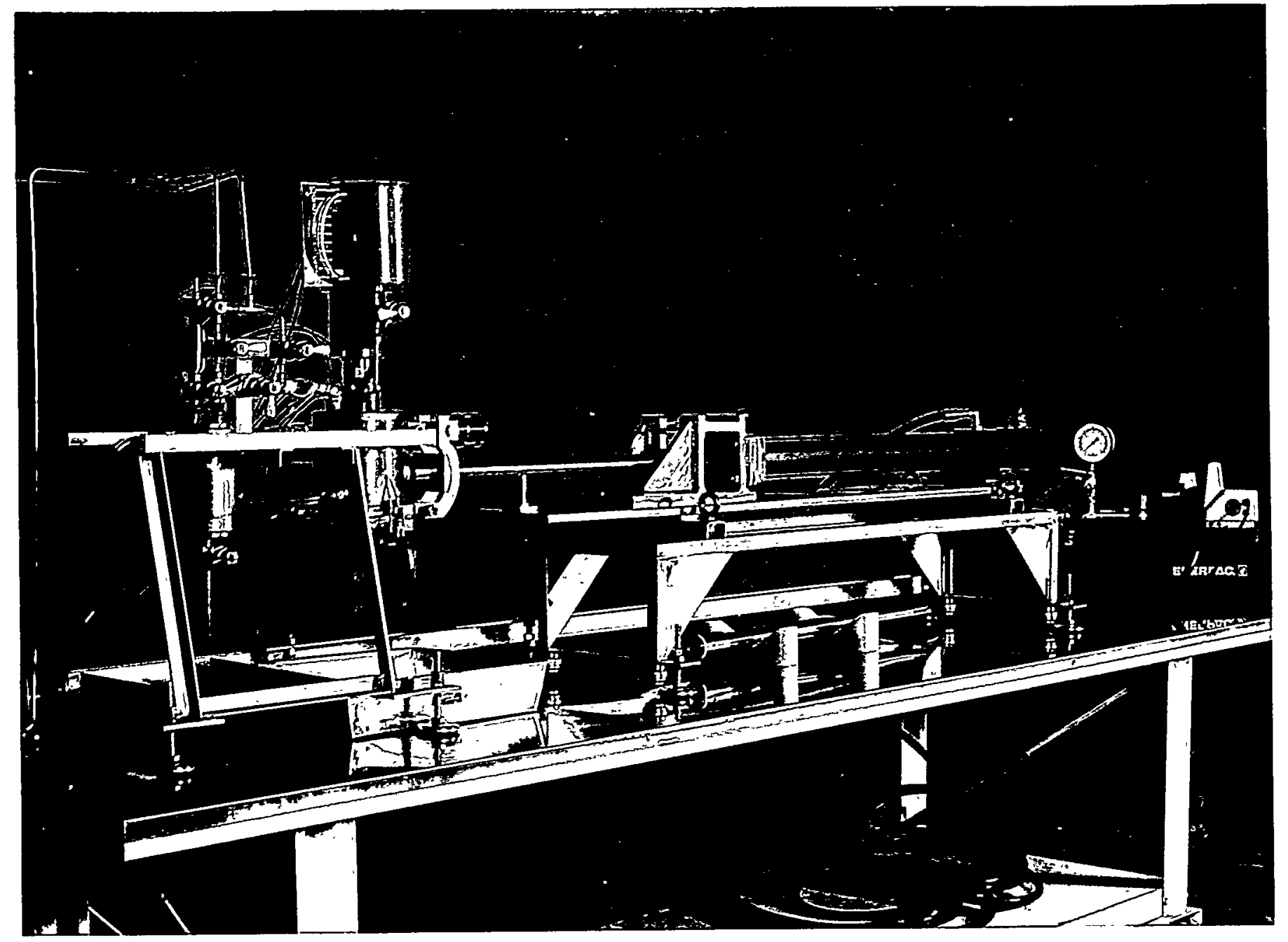

Figure 2.2. Photograph of the RGS Extrusion and Extraction System 
to provide, in addition to qualitative observations, approximate quantitative information on the density and distribution of the phases in the sampler immediately after the sample is removed from the tank (see Section 3.8).

Shekarriz (1994) performed a series of flow visualization tests to examine the effectiveness of the RGS drill bit in capturing bubbles in the sludge during sampling. A transparent simulant with rheological properties similar to the sludges in the DSTs was used for these tests. Based on these tests, we concluded that a $60^{\circ}$ drill bit provides the optimal conditions for minimizing disturbance of the bubbles and the waste during sampling. Comprehensive studies were performed to quantify the uncertainty in measuring retained gases using the RGS (Cannon and Knight 1995; Cannon 1996). In these acceptance tests, the procedures were calibrated for sampling both insoluble (low solubility) gases and ammonia. We found that, while the insoluble components could be measured with high accuracy, ammonia was more problematic because it is absorbed within the system. The tank-by-tank results are given in the order in which sampling was carried out to reflect the increasing experience on which RGS methods were based. The details of the difficulties with ammonia measurements are given in Section 3.7 and discussed elsewhere in the report as well. 


\subsection{Data Analysis}

This section sets forth the data analysis method that is used on the raw data to determine the total concentrations of low-solubility gas ("insoluble gas") and ammonia in an RGS waste sample, to calculate the in-tank gas volume fraction at each sampled elevation, and to estimate the total tank gas inventory. The section describes the inputs, assumptions, and procedures related to each of the analysis tasks. A tank-by-tank discussion of the analysis results is presented in Section 4.

- The objective of the derivation in this section is to summarize how the concentration and composition of gases in each waste sample is calculated from the pressure and temperature measurements made during RGS extraction and from the composition measurements made on the extracted gas. The calculations are sufficiently complicated-chiefly because details of the extraction procedure changed over the course of the RGS program-that this section cannot provide enough information to allow the calculations to be duplicated. The remainder of the information can be obtained from the calculation spreadsheets on the CD that accompanies this report. These spreadsheets contain more detail than is appropriate for this report and are provided to support reproducible ancillary calculations.

RGS samples are obtained by push-mode core sampling. An RGS sample consists of one 46-cm (18.2-in.) waste segment (the words segment and sample are used interchangeably in this report). The stroke length for core sampling is 19 in., and the segment length is 19 in. for nonRGS core samples. However, an RGS sample is only 18.2 in. long because the RGS sampler piston is 0.8 in. longer than the universal sampler piston. An RGS sample, as received, may contain not only native tank waste but hydrostatic head fluid (HHF) and entrained gases (potentially a combination of air with the argon gas used to purge the drillstring). These possible contaminants are discussed in Section 3.5.

The basics of the RGS extraction system are shown in the schematic in Figure 3.1; note that the actual valves and lines are more complex and have been simplified for this brief discussion of the extraction procedure. More details of the extraction procedure are given on a tank-by-tank basis in Section 4, and details of the system volumes and other input data may be found in the calculation spreadsheets on the CD that accompanies this report.)

An RGS gas extraction begins with the sample fully extruded into the previously evacuated extractor vessel, which is connected (via open valves $B$ and $T$ ) to the extractor lines. After extrusion is complete, the extractor vessel and lines are opened to the second volume vessel (opening valve R) so that the whole extractor side of the system is open to the sample. Finally, just before pumping begins, valve $F$ is opened and the extractor vessel is valved into the lines that connect it to the collector side of the system, made up of the collection canister(s) and collector lines. This allows the sample to approach equilibrium with the entire gas volume of the extraction system, though equilibrium may not be reached before the next step begins.

Next, the extractor and collector sides are disconnected from one another by closing valve F. Once that has been done, gas can be transferred from the extractor side of the system to the collector side only by cycling the mercury pump (which is a displacement vacuum pump). First, the three-way valve $G$ is turned to connect the pump displacement chamber to the extractor side and the mercury level is dropped to its minimum, fully opening the chamber. Then valve $G$ is turned to connect the pump displacement chamber to the collector side and the mercury level is raised to its maximum, compressing the chamber contents into the collector side. The compression resulting from pumping causes water to condense in the collector side, and ammonia dissolves in the condensate. The pump is cycled for $\mathrm{N}$ pump strokes before the canisters are closed off (valves $\mathrm{H}$ and $\mathrm{J})$ and the canisters disconnected. 


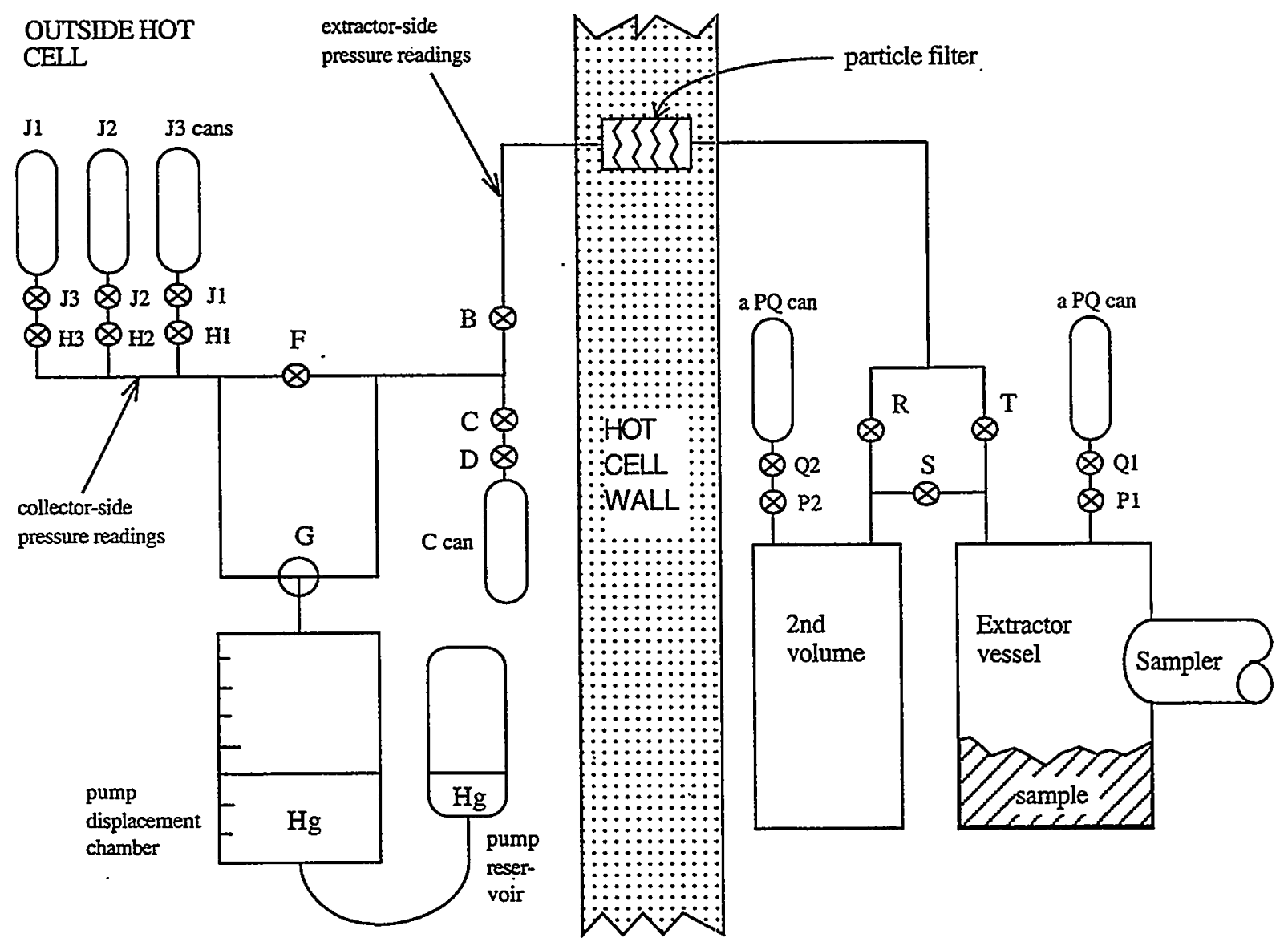

The "extractor side" is everything to the right of valves $F$ and $G$, excluding the pump; the "collector side", everything to the left, excluding the pump.

Many details are not shown, including the pump sight glass and lines for evacuating the system and injecting $\mathrm{Ar}, \mathrm{He}$, and isotopic standards.

\section{Figure 3.1. Schematic of the RGS Extraction System}

A typical RGS extraction procedure extracts gas into a sequence of canisters, or sets of canisters, using the kind of procedure summarized above. The collection canisters (or "J canisters") may be unpumped-filled without compression during the initial approach to equilibrium while collector side and extractor side are connected - or pumped (that is, filled by a series of gas additions that are compressed by pumping). Other "grab sample" canisters ("PQ" or "C" canisters, depending on the sampling point) may also be filled by connecting previously evacuated canisters to various valves upstream of the collector canisters. The PQ canisters are washed with acid solution to capture the ammonia in the canister, which is measured and used to calculate the ammonia vapor pressure in the extractor side. The collection and grab sample canisters are subjected to mass spectrometry to determine the dry mole fractions of the insoluble gases and ammonia. Mass spectrometry also supplies isotopic ratios $(15 \mathrm{~N} / 14 \mathrm{~N})$, for those samples in which an isotopic standard was added to determine residual ammonia (Section 3.4.1).

Measured extractor pressures and temperatures and system volumes are used to calculate the total moles of insoluble gas plus ammonia plus water extracted from the sample. Collector pressures and temperatures, system volumes, and composition data from mass spectrometry are used to determine the amount and composition of the extracted insoluble gas and ammonia vapor 
and the amount of ammonia in the collector-side condensate. Isotopic ratios (measured by mass spectrometry) and known quantities of added standards are used (in some samples) to find the amount of ammonia remaining in the extractor side, both in solution and in vapor.

Table 3.1 summarizes the kinds of extraction steps that can be used during an RGS extraction and lists the data that are measured and used to account for the moles removed from the RGS system by each step. The procedures used varied considerably over the program (see Section 4 for sample-by-sample descriptions) but were generally made up of these elements. The total mass balance for the sample includes all the separate portions of removed gas, plus the gas remaining in the extractor side at the end of extraction (typically negligible), plus the ammonia still dissolved in the sample.

Section 3.1 derives expressions, in terms of extractor-side pressures, that describe the amounts of insoluble gas, ammonia vapor, and water vapor that are removed from the extractor by pumping. Section 3.2 completes the extractor-collector mass balance by deriving expressions, in terms of collector pressures, for the gas-phase and condensed moles in the collector. Section 3.3 derives the equations used to calculate the partitioning of ammonia and water between the vapor and liquid phases in the collector, based on the collector pressure and temperature and the dry gas/vapor composition determined by mass spectrometry. Section 3.4 ties the derivations from the preceding sections together to get the total amounts of retained gas and ammonia in the pumped sample. These methods vary depending on exactly what extraction procedure was used (for example, whether an isotopic ammonia standard was added to the system to determine residual ammonia). Section 3.4 (Equation 3.4.3) also shows how the insoluble gas is speciated using the mass-spectrometric data.

The concentrations of insoluble gases and ammonia in the sample, which are determined by the methods given in Sections 3.1 through 3.4, are then corrected (as discussed in Section 3.5) to remove air entrained during sample acquisition and to account for contamination by HHF. The corrected concentrations are used to find the distribution of constituents between the gas and liquid phases under in-tank conditions using methods described in Section 3.6. These results, which include the in-tank gas volume fraction, are then used to determine the total tank inventory of gases in the liquid and vapor phases, as described in Section 3.7. A description of the procedure for analyzing $\mathrm{x}$-ray images is presented in Section 3.9.

The assumptions used in the analysis are stated briefly in the derivation sections, Sections 3.1 through 3.7. The justifications of the assumptions and the estimates of their significance are not given in those sections but are reserved for Section 3.8 to avoid interrupting the flow of the derivation.

\subsection{Material Removed from the Extractor Side}

We begin by calculating the moles removed from the extractor side with each stroke of the mercury pump. This calculation is the first part of the mass balance because the moles removed from the extractor side equal those added to the collector side. Each stroke of the RGS mercury pump ( $\mathrm{i}$ is the pump stroke counter) removes $\mathrm{P}_{\mathrm{xi}} \mathrm{V}_{\mathrm{s}} / \mathrm{RT}$ moles (of water plus $\mathrm{NH}_{3}$ plus insoluble gas) from the extractor side. The extractor-side pressure, $\mathrm{P}_{\mathrm{xi}}$, can be expressed as

$$
P_{x i}=P_{x w i}+P_{x A i}+P_{x g i}
$$

where 
Table 3.1. Possible RGS Extraction Steps

\begin{tabular}{|c|c|c|}
\hline $\begin{array}{c}\text { Type of extraction } \\
\text { step }\end{array}$ & $\begin{array}{l}\text { Status of } \\
\text { extraction step }\end{array}$ & Mass balance inputs and outputs \\
\hline $\begin{array}{l}\text { Analyze PQ canister } \\
\text { from extractor side }\end{array}$ & not always done & $\begin{array}{l}\text { Inputs: canister volume, extractor-side pressure and } \\
\text { temperature during collection, composition and water vapor } \\
\text { pressure from the first canister with a mass-spec analysis. } \\
\text { Calcs: no condensate, so ideal gas law can be used to calculate } \\
\text { gas in canister. }\end{array}$ \\
\hline $\begin{array}{l}\text { Analyze unpumped 1st } \\
\text { canister from } \mathrm{C} \text { or } \mathrm{J} 1 \\
\text { valve }\end{array}$ & not always done & $\begin{array}{l}\text { Inputs: canister volume, collector-side pressure and } \\
\text { temperature during collection, mass-spec dry composition of the } \\
\text { canister after collection, water vapor pressure over sample as } \\
\text { calculated for the first pumped canister. } \\
\text { Calcs: no condensate, so ideal gas law can be used to calculate } \\
\text { gas in canister. }\end{array}$ \\
\hline $\begin{array}{l}\text { Analyze set of pumped } \\
\text { canister(s) from J } \\
\text { valve(s) }\end{array}$ & $\begin{array}{l}\text { always at least } \\
\text { one pumped } \\
\text { canister set, } \\
\text { usually more }\end{array}$ & $\begin{array}{l}\text { Inputs: canister and other system volumes, extractor-side and } \\
\text { collector-side pressures and temperatures during collection, mass- } \\
\text { spec dry composition of the canister set after collection. } \\
\text { If the collector lines were not evacuated after the previous } \\
\text { canister(s) were removed, inputs also include the collector line } \\
\text { contents calculated for the previous pumped canister set. } \\
\text { If the canisters are taken after adding the isotopic standard, mass- } \\
\text { spec isotopic ratio measurements are used to calculate the total } \\
\text { ammonia (vapor and dissolved) remaining in the extractor side. } \\
\text { Calcs: condensate is present in the collector, so Section } 3.3 \\
\text { phase-partitioning calcs are used to provide water and ammonia } \\
\text { liquid moles (and the sample water vapor pressure). }\end{array}$ \\
\hline $\begin{array}{l}\text { Add isotopic standard } \\
\text { to extractor vessel }\end{array}$ & not always done & $\begin{array}{l}\text { Inputs: moles of ammonia in the standard, volume occupied } \\
\text { by the standard (if it is in solution). }\end{array}$ \\
\hline Evacuate collector side & $\begin{array}{l}\text { frequently done } \\
\text { after a collector } \\
\text { canister set is } \\
\text { removed }\end{array}$ & $\begin{array}{l}\text { Inputs: the collector line contents calculated for the previous } \\
\text { pumped canister set, canister and collector line volumes. } \\
\text { Calcs: scale the canister contents by the ratio of line to } \\
\text { canister volume to obtain the collector-line contents lost to } \\
\text { evacuation. }\end{array}$ \\
\hline $\begin{array}{l}\text { Evacuate part of the } \\
\text { extractor side (not the } \\
\text { extractor vessel) }\end{array}$ & not always done & $\begin{array}{l}\text { Inputs: system volumes, extractor-side pressure and } \\
\text { temperature during collection, mass-spec dry composition of the } \\
\text { next canister after the evacuation, water vapor pressure over } \\
\text { sample as calculated for the next pumped canister after the } \\
\text { evacuation. } \\
\text { Calcs: no condensate, so ideal gas law can be used to obtain } \\
\text { the gas and vapor lost to evacuation. }\end{array}$ \\
\hline
\end{tabular}




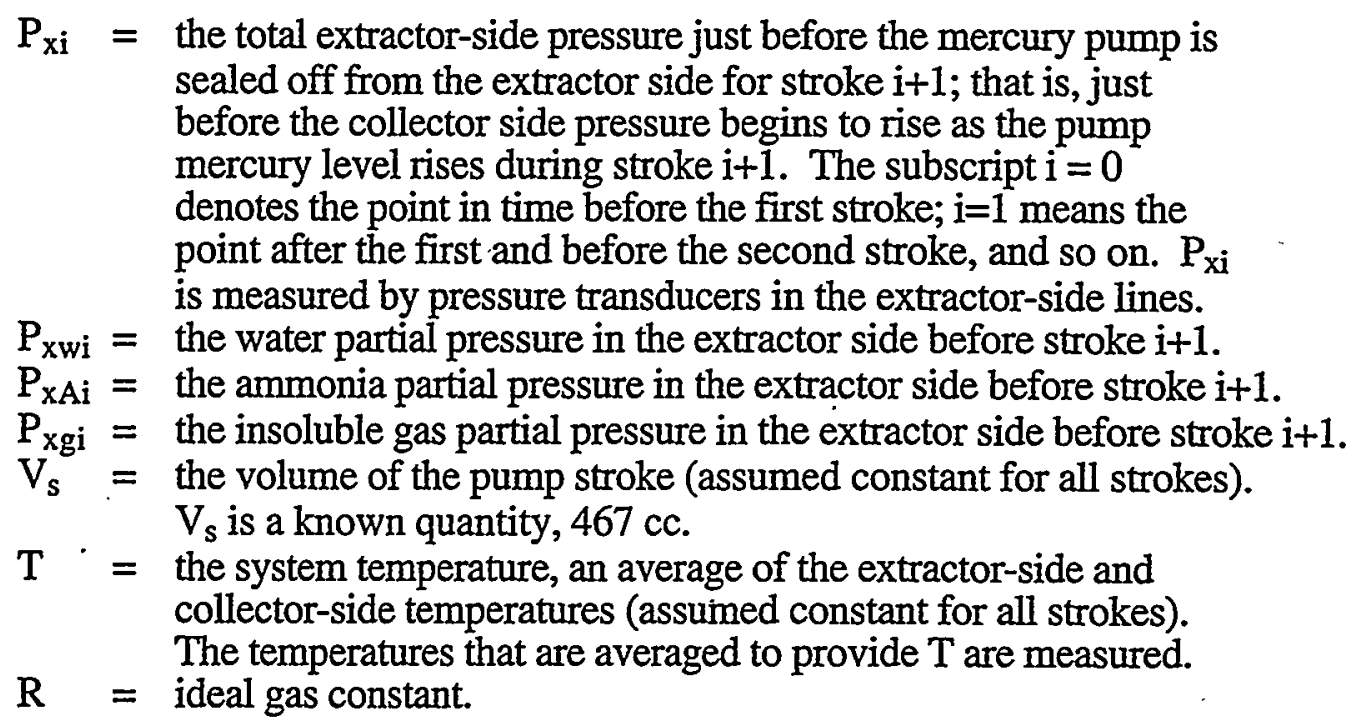

The stroke-by-stroke water partial pressure, $\mathrm{P}_{\mathrm{xwi}}$, can be assumed constant for all strokes if $T$ and the liquid water concentration in the sample are constant. For the latter to be a good assumption, the water evaporated from the sample must be a negligible part of the total water content, and the extraction of ammonia and insoluble gas must have a negligible effect on the water vapor pressure. In addition, the water in the sample and in the vapor volume must be in equilibrium, with no diffusional mass transfer limitations imposed by drying of the surface of the sample. With these assumptions, we can say

$$
\mathrm{P}_{\mathrm{xwi}}=\mathrm{P}_{\mathrm{xw}} \text {. }
$$

where $P_{x w}=$ the water vapor pressure over the waste sample. This is an unknown to be solved for (see Equation 3.4.1). The moles of water removed from the extractor by each pump stroke are therefore equal to

$$
\mathrm{n}_{\mathrm{xwi}}-\mathrm{n}_{\mathrm{xwi}-1}=\mathrm{P}_{\mathrm{xw}} \mathrm{V}_{\mathrm{s}} / \mathrm{RT}
$$

To find the insoluble gas partial pressure, $\mathrm{P}_{\mathrm{xgi}}$, assume that the solubility of all the constituents $\left(\mathrm{H}_{2}, \mathrm{~N}_{2}, \mathrm{~N}_{2} \mathrm{O}, \mathrm{CH}_{4}\right.$, etc.) is so low that the amount dissolved in the waste is negligible. Also assume that no gas pockets are trapped in the waste and that the gas is uniformly mixed throughout the extractor side. Finally, assume that all of the gas in the pump goes to the collector side and none of it leaks back to the extractor side at any time. Given these assumptions, each pump stroke removes a fraction $\left[V_{s} /\left(V_{s}+V_{x}\right)\right]$ of the gas present in the extractor side and transfers all of it to the collector side. Then the moles of insoluble gas removed from the extractor side by stroke $i$ are equal to

$$
n_{x g i}-n_{x g i-1}=n_{x g 0} F_{v}\left(1-F_{v}\right)^{i-1} \quad \text { for } i=1,2, \ldots
$$

and the moles of insoluble gas remaining on the extractor side are

$$
\mathrm{n}_{\mathrm{xgi}}=\mathrm{n}_{\mathrm{xg} 0}\left(1-\mathrm{F}_{\mathrm{v}}\right)^{\mathrm{i}} \quad \text { for } \mathrm{i} \geq 0
$$

where

$$
F_{v}=V_{s} /\left(V_{s}+V_{x}+V_{2}\right) \text {, the fractional gas removal per stroke. }
$$




$$
\begin{aligned}
& V_{x}=\text { the effective volume of the extractor vessel. This equals the } \\
& \text { volume of the empty extractor minus the liquid/solid volume of } \\
& \text { the sample. The latter (usually between } 200 \text { and } 300 \mathrm{cc} \text { ) is equal } \\
& \text { to the sampler volume minus the fraction of the sampler that is } \\
& \text { occupied by insoluble gas and vapor under in situ conditions. } \\
& \text { The empty extractor volume is a known quantity (about } 1300 \mathrm{cc} \\
& \text { but varies with equipment changes over the project history). } \mathrm{V}_{\mathrm{x}} \\
& \text { must be calculated iteratively: a value assumed, the sample gas } \\
& \text { content calculated, and from that gas content the in situ gas } \\
& \text { volume fraction derived (per Section 3.6). The gas volume } \\
& \text { fraction is then used to obtain a new value for } V_{x} \text {. This volume } \\
& \text { determination required the sampler to be completely filled with } \\
& \text { tank waste ( } 100 \% \text { recovery of sample gas, liquids, and solids). } \\
& \mathrm{V}_{2}=\text { the volume of the second-volume vessel and the lines associated } \\
& \text { with the extractor. This is a known quantity, which, like other } \\
& \text { system volumes, is determined by pressurizing the system with a } \\
& \text { known quantity of gas. The volume of this vessel was } 321 \mathrm{cc} \text {, but } \\
& \text { it was set to zero for cases in which the second volume was } \\
& \text { kept closed off during extraction. } \\
& \mathrm{n}_{\mathrm{xg} 0}=\text { the initial moles of insoluble gas in the extractor side: extractor } \\
& \text { vessel, second volume, and associated line volume. This is an } \\
& \text { unknown to be solved for (see Equation 3.4.2). It is less than } \\
& \text { the sample insoluble gas content because it does not include } \\
& \text { insoluble gas in the collector side or in any grab sample canisters } \\
& \text { that may be mounted. However, once } n_{\mathrm{xg} 0} \text { has been deter- } \\
& \text { mined, the sample insoluble gas content can be back-calculated } \\
& \text { using the ideal gas law and ratios of known volumes. }
\end{aligned}
$$

It follows from Equation (3.1.5) that the extractor-side insoluble gas pressure after stroke i

$$
P_{x g i}=\frac{n_{x g 0}\left(1-F_{v}\right)^{i} R T}{V_{s}+V_{x}+V_{2}} \quad \text { for } i \geq 0
$$

Having modeled the water and insoluble gas partial pressures in the extractor side, the ammonia partial pressure, $\mathrm{P}_{\mathrm{xAi}}$, can be calculated as

$$
P_{x A i}=P_{x i}-P_{x g i}-P_{x w}
$$

In some cases, the post-extrusion ammonia vapor pressure, $\mathrm{P}_{\mathrm{XAE}}$, was not only calculated as the pressure difference by Equation(3.1.7) but was directly measured. "PQ canisters" (so called after the valves to which they were attached) were connected to the second vessel and used to take grab samples of the extractor-side gas just before connecting the extractor side to the collector side. The PQ canisters were then washed with a known volume of $0.1 \mathrm{M}$ sulfuric acid to quantitatively capture the ammonia, and the ammonia concentration in the wash solution was measured to find the moles of ammonia in the PQ canister. The measured moles of PQ ammonia, as well as the PQ canister volume and the pressure at the time the extractor side was grab-sampled, were used to calculate the partial pressure of ammonia in the PQ canister (and so in the extractor side) at the time the grab sample was taken. 


\subsection{Material Added to the Collector Side}

The total moles of all the species that have been pumped to the collector side, when pumping is completed after stroke $\mathrm{N}$, can be found by summing the pumped insoluble gas and vapor over all pump strokes from stroke i to stroke $\mathrm{N}$ :

$$
\mathrm{n}_{\mathrm{cN}}=\mathrm{n}_{\mathrm{cw0}}+\mathrm{n}_{\mathrm{cA0} 0}+\mathrm{n}_{\mathrm{cg} 0}+\sum_{\mathrm{i}=1}^{\mathrm{N}} \frac{\mathrm{P}_{\mathrm{xi}} \mathrm{V}_{\mathrm{s}}}{\mathrm{RT}}
$$

where

$$
\begin{aligned}
& \mathrm{n}_{\mathrm{cw} 0}= \text { the initial moles of water in the collector side (before any pumping } \\
& \text { is done). This value is not an independent unknown; it can be found } \\
& \text { in terms of } \mathrm{n}_{\mathrm{xw0}} \text { by performing a full process mass balance that } \\
& \text { takes into account the sequence in which the collector side and the } \mathrm{PQ} \\
& \text { and/or C canisters were opened to the sample, which parts of the } \\
& \text { system were evacuated before this canister was opened, and other } \\
& \text { details that depend on the specific extraction procedure. } \\
& \mathrm{n}_{\mathrm{cA} 0}=\text { the initial moles of ammonia in the collector side (before any pumping } \\
& \text { is done). Like } \mathrm{n}_{\mathrm{cw} 0} \text {, this value is not an independent unknown (see } \\
& \text { above). } \\
& \mathrm{n}_{\mathrm{cg} 0=} \text { the initial moles of insoluble gas in the collector side (before any } \\
& \text { pumping is done). Like } \mathrm{n}_{\mathrm{cw} 0} \text {, this value is not an independent } \\
& \text { unknown (see above). }
\end{aligned}
$$

Not all of the moles in the collector side are in the gas phase; some moles of ammonia and water condense in the J canister(s) and the collector lines. The presence of condensation is reflected in the fact that the pressure measured in the collector side at the end of pumping is much lower than that which would be measured if all the ammonia and water were in the gas phase. A measure of the extent of condensation is the parameter $r$, which is defined as the fraction of moles in the collector side that are in the gas phase. This fraction is expressed by

$$
\mathrm{r}=\frac{\mathrm{P}_{\mathrm{cN}}}{\left(\mathrm{n}_{\mathrm{cN}} \mathrm{RT} /\left(\mathrm{V}_{\mathrm{cl}}+\mathrm{V}_{\mathrm{J}}\right)\right)}
$$

where

$$
\begin{aligned}
& \mathrm{V}_{\mathrm{cl}}=\text { the volume of the collector lines. This is a known quantity that } \\
& \text { varied with small system changes made over the course of the program. } \\
& \mathrm{V}_{\mathrm{J}}=\text { the volume of the } \mathrm{J} \text { canister(s). This is a known quantity that varied } \\
& \text { with the canister(s) used. } \\
& \left.\mathrm{P}_{\mathrm{cN}}=\text { the final collector side pressure (after stroke } \mathrm{N}\right) .
\end{aligned}
$$

The quantity (1-r) is the fraction of moles in the condensate. It has been assumed that the volume of condensate is negligible compared to the collector-side volume, $V_{c}$, where $V_{c}=V_{J}+$ $V_{c l .}$ The ratio $r$ is used in Section 3.3 (Equation 3.3.14) to determine how much of the ammonia and water in the collector side is present as liquid (condensate) and how much as vapor.

\subsection{Phase Partitioning in the Collector Side}

The amounts of water and ammonia that condense in the collector side are not measured but must be known before the water, ammonia, and insoluble gas content of the sample can be 
determined. The condensation can be calculated based on solution thermodynamics. Among the known quantities are the following:

$\mathrm{P}_{\mathrm{cN}}=$ the total pressure in the $\mathrm{J}$ canister after pumping is complete, which is the same as the total pressure in the collector side at large

$\mathrm{T}=$ the system temperature (absolute)

$\mathrm{Y}_{\mathrm{A}}=$ the mole fraction of ammonia in the gas phase in the $\mathrm{J}$ canister (collector side) on a dry basis (water excluded). This is the ammonia mole fraction that is measured by mass spectrometry. The temperature at which the mole fraction is measured is assumed to be negligibly different from that at which the gas sample was collected (room temperature), meaning that the equilibrium concentrations are the same.

The unknowns include

$\mathrm{y}_{\mathrm{A}}=$ the mole fraction of ammonia in the gas in the collector side on a wet basis

$\mathrm{y}_{\mathrm{w}}=$ the mole fraction of water in the collector-side gas on a wet basis

$\mathrm{x}_{\mathrm{A}}=$ the mole fraction of ammonia in the collector-side condensate

$\mathrm{x}_{\mathrm{w}}=$ the mole fraction of water in the collector-side condensate

$\mathrm{v}_{\mathrm{A}}=$ the moles of ammonia in the gas phase in the collector side

$\mathrm{v}_{\mathrm{w}}=$ the moles of water in the collector-side gas phase

$\mathrm{L}_{\mathrm{A}}=$ the moles of ammonia in the collector-side condensate

$\mathrm{L}_{\mathrm{w}}=$ the moles of water in the collector-side condensate.

It is clear from the definitions of the variables that

$\mathrm{y}_{\mathrm{A}}=\mathrm{Y}_{\mathrm{A}}\left(1-\mathrm{y}_{\mathrm{w}}\right)$

Assuming that other dissolved gases (including $\mathrm{N}_{2} \mathrm{O}$ ) can be neglected, we can also say

that

$$
\mathrm{x}_{\mathrm{w}}=1-\mathrm{x}_{\mathrm{A}}
$$

If the ammonia and water vapor in the canister (collector) are in equilibrium with the condensate, then

$$
\begin{aligned}
& x_{A} P_{A} \gamma_{A}=y_{A} P_{c N}=Y_{A}\left(1-y_{w}\right) P_{c N} \\
& \left(1-x_{A}\right) p_{w} \gamma_{w}=y_{w} P_{c N}
\end{aligned}
$$

where

$\mathrm{p}_{\mathrm{A}}=$ the vapor pressure of pure ammonia at temperature $\mathrm{T}$

$\mathrm{p}_{\mathrm{w}}=$ the vapor pressure of pure water at temperature $T$

$\gamma_{\mathrm{A}}=$ the activity coefficient of ammonia in the ammonia-water condensate

$\gamma_{\mathrm{w}}=$ the activity coefficient of water in the ammonia-water condensate.

The following expressions are used to find the pure component vapor pressures (in units of $\mathrm{kPa}$ ) and the solution activity coefficients:

$$
\mathrm{p}_{\mathrm{A}}=100 \exp \left(45.327-4104.67 / \mathrm{T}-5.146 \ln \mathrm{T}+6.15 \mathrm{p}_{\mathrm{A}} / \mathrm{T}^{2}\right)
$$




$$
\begin{aligned}
& \mathrm{p}_{\mathrm{w}}=22120 \exp \left[( 1 / \mathrm { T } _ { \mathrm { r } } ) \left(-7.76451\left(1-\mathrm{T}_{\mathrm{r}}\right)+1.45838\left(1-\mathrm{T}_{\mathrm{r}}\right)^{1.5}-2.7758\left(1-\mathrm{T}_{\mathrm{r}}\right)^{3}\right.\right. \\
& \left.\left.-1.23303\left(1-\mathrm{T}_{\mathrm{r}}\right)^{6}\right)\right] \\
& \ln \gamma_{\mathrm{A}}=\left(1-\mathrm{x}_{\mathrm{A}}\right)\left(\frac{\Lambda_{\mathrm{Aw}}}{\mathrm{x}_{\mathrm{A}}+\Lambda_{\mathrm{Aw}}\left(1-\mathrm{x}_{\mathrm{A}}\right)}-\frac{\Lambda_{\mathrm{wA}}}{\mathrm{x}_{\mathrm{A}} \Lambda_{\mathrm{wA}}+\left(1-\mathrm{x}_{\mathrm{A}}\right)}\right)-\ln \left(\mathrm{x}_{\mathrm{A}}+\Lambda_{\mathrm{Aw}}\left\{1-\mathrm{x}_{\mathrm{A}}\right\}\right) \\
& \ln \gamma_{\mathrm{w}}=-\mathrm{x}_{\mathrm{A}}\left(\frac{\Lambda_{\mathrm{Aw}}}{\mathrm{x}_{\mathrm{A}}+\Lambda_{\mathrm{Aw}}\left(1-\mathrm{x}_{\mathrm{A}}\right)}-\frac{\Lambda_{\mathrm{wA}}}{\mathrm{x}_{\mathrm{A}} \Lambda_{\mathrm{wA}}+\left(1-\mathrm{x}_{\mathrm{A}}\right)}\right)-\ln \left(\left\{1-\mathrm{x}_{\mathrm{A}}\right\}+\Lambda_{\mathrm{wA}} \mathrm{x}_{\mathrm{A}}\right)
\end{aligned}
$$

where

$$
\begin{aligned}
\mathrm{T}_{\mathrm{r}}= & \text { the reduced collector temperature, } \mathrm{T} / 647.3 \text { (the critical temperature for } \\
& \text { water is } 647.3 \mathrm{~K}) \\
\Lambda_{\mathrm{Aw}}= & 0.6777 / \exp \left(\left[-908.30+2.0723 \mathrm{x}_{\mathrm{A}} \mathrm{T} / \mathrm{x}_{\mathrm{w}}\right] / \mathrm{T}\right) \text {, the correlating parameter } \\
& \text { for ammonia in an ammonia/water system } \\
\Lambda_{\mathrm{wA}}= & 1.476 / \exp \left(\left[77.584+0.041975 \mathrm{x}_{\mathrm{w}} \mathrm{T} / \mathrm{x}_{\mathrm{A}}\right] / \mathrm{T}\right) \text {, the correlating parameter } \\
& \text { for water in an ammonia/water system. }
\end{aligned}
$$

The pure component vapor pressures came from Reid et al. (1987), while the activity coefficients were estimated by calculating activity coefficients from ammonia/water composition/ vapor pressure data found in $\mathrm{AIChE}$ (1984) and fitting the coefficients to a variant of the Wilson equation (Prausnitz et al. 1986, p. 234). A least-squares fit was used to find the optimum parameters over 40 data points that fell in a range of dissolved ammonia concentrations between 0 and $40 \mathrm{~mol} \%$ and temperatures between 15 and $40^{\circ} \mathrm{C}$. In this range, the correlation predicts ammonia mole fractions within $7 \%$ error and water mole fractions within $10 \%$ error.

Equations (3.3.3) and (3.3.4) constitute two equations in two unknowns $\left(x_{A}\right.$ and $\left.y_{w}\right)$. These equations can be solved iteratively, allowing the compositions of the phases, $\mathrm{y}_{\mathrm{A}}, \mathrm{y}_{\mathrm{w}}, \mathrm{x}_{\mathrm{A}}$, and $\mathrm{x}_{\mathrm{w}}$, to be determined. First, the collector-side moles of ammonia, water, and insoluble gas in the gas phase after stroke $N\left(v_{A}, v_{w}\right.$, and $n_{\text {cgN }}$, respectively) must be stated in terms of the unknowns:

$$
\begin{aligned}
& \mathrm{v}_{\mathrm{A}}=\mathrm{y}_{\mathrm{A}} \mathrm{P}_{\mathrm{cN}}\left(\mathrm{V}_{\mathrm{cl}}+\mathrm{V}_{\mathrm{J}}\right) / \mathrm{RT} \\
& \mathrm{v}_{\mathrm{w}}=\mathrm{y}_{\mathrm{w}} \mathrm{P}_{\mathrm{cN}}\left(\mathrm{V}_{\mathrm{cl}}+\mathrm{V}_{\mathrm{J}}\right) / \mathrm{RT} \\
& \mathrm{n}_{\mathrm{cgN}}=\left(1-\mathrm{y}_{\mathrm{w}}-\mathrm{y}_{\mathrm{A}}\right) \mathrm{P}_{\mathrm{cN}}\left(\mathrm{V}_{\mathrm{cl}}+\mathrm{V}_{\mathrm{J}}\right) / \mathrm{RT}
\end{aligned}
$$

The next step is to find the amounts of ammonia and water in the gas and condensed phases. By definition, the total collected moles of ammonia and water are the sum of the gas and liquid contributions:

$$
\begin{aligned}
& \mathrm{n}_{\mathrm{cAN}}=\mathrm{v}_{\mathrm{A}}+\mathrm{L}_{\mathrm{A}} \\
& \mathrm{n}_{\mathrm{cwN}}=\mathrm{v}_{\mathrm{w}}+\mathrm{L}_{\mathrm{w}}
\end{aligned}
$$

The ratio $r$ was defined in Section 3.2 as the fraction of moles in the collector side that are in the gas phase. It can be calculated from measurements using Equation (3.2.2). The ratio is used in the collector-side equilibrium calculations of this section by way of the following equation: 


$$
r=\frac{n_{c g N}+v_{A}+v_{w}}{n_{c g N}+v_{A}+v_{w}+L_{A}+L_{w}}
$$

where

$\mathrm{n}_{\mathrm{CAN}}=$ total moles ammonia in the collector side at the end of pumping

$\mathrm{n}_{\mathrm{cwN}}=$ total moles water in the collector side at the end of pumping.

From Equations (3.3.9), (3.3.10), (3.3.11), and (3.3.14), it follows that

$$
\mathrm{r}=\frac{\mathrm{P}_{\mathrm{cN}}\left(\mathrm{V}_{\mathrm{cl}}+\mathrm{V}_{\mathrm{J}}\right) / \mathrm{RT}}{\mathrm{P}_{\mathrm{cN}}\left(\mathrm{V}_{\mathrm{cl}}+\mathrm{V}_{\mathrm{J}}\right) / \mathrm{RT}+\mathrm{L}_{\mathrm{A}}\left(1+\left(1-\mathrm{x}_{\mathrm{A}}\right) / \mathrm{x}_{\mathrm{A}}\right)}=\frac{1}{1+\mathrm{L}_{\mathrm{A}}\left(\mathrm{RT} / \mathrm{x}_{\mathrm{A}} \mathrm{P}_{\mathrm{cN}}\left(\mathrm{V}_{\mathrm{cl}}+\mathrm{V}_{\mathrm{J}}\right)\right)}
$$

From this it can be shown, by substituting Equation (3.2.2) into Equation (3.3.15), that

$$
\mathrm{L}_{\mathrm{A}}=(1-\mathrm{r}) \mathrm{x}_{\mathrm{A}} \mathrm{n}_{\mathrm{cN}}
$$

This finding can be carried through to find $L_{w}, n_{c A N}$, and $n_{c w N}$ :

$$
\begin{aligned}
& \mathrm{L}_{\mathrm{w}}=\mathrm{x}_{\mathrm{w}}(1-\mathrm{r}) \mathrm{n}_{\mathrm{cN}} \\
& \mathrm{n}_{\mathrm{cAN}}=\mathrm{y}_{\mathrm{A}} \mathrm{P}_{\mathrm{cN}}\left(\mathrm{V}_{\mathrm{cl}}+\mathrm{V}_{\mathrm{J}}\right) / \mathrm{RT}+\mathrm{L}_{\mathrm{A}} \\
& \mathrm{n}_{\mathrm{cwN}}=\mathrm{y}_{\mathrm{w}} \mathrm{P}_{\mathrm{cN}}\left(\mathrm{V}_{\mathrm{cl}}+\mathrm{V}_{\mathrm{J}}\right) / \mathrm{RT}+\mathrm{L}_{\mathrm{w}}
\end{aligned}
$$

\subsection{Final Determination of Sample Contents}

At this point, we have three unknown variables: $\mathrm{P}_{\mathrm{xw}}$ (water vapor pressure in the extractor side), $\mathrm{n}_{\mathrm{xg} 0}$ (initial "insoluble" gas moles in the extractor side), and $\mathrm{n}_{\mathrm{xA0}}$ (initial ammonia moles in the extractor side). Two of these variables are easy to find via the results of Section 3.3:

$$
\begin{aligned}
& \mathrm{P}_{\mathrm{xw}}=\frac{\left(\mathrm{n}_{\mathrm{cwN}}-\mathrm{n}_{\mathrm{cw0} 0}\right) \mathrm{RT}}{N V_{\mathrm{s}}} \\
& \mathrm{n}_{\mathrm{xg} 0}=\frac{\mathrm{n}_{\mathrm{cgN}}-\mathrm{n}_{\mathrm{cg} 0}}{\mathrm{~F}_{\mathrm{v}} \sum_{i=1}^{\mathrm{N}}\left(1-\mathrm{F}_{\mathrm{v}}\right)^{i-1}}
\end{aligned}
$$

As was already noted, once $\mathrm{n}_{\mathrm{xg0}}$ is known, the original sample insoluble gas content $\mathrm{n}_{\mathrm{Sg}}$ is easily back-calculated by adding in whatever insoluble gas was removed in grab sample canisters and partial system evacuations. The residual insoluble gas left in the extractor after pumping can also be calculated (with Equation 3.1.5).

The moles of each insoluble gas constituent are equal to

$$
n_{S i}=\left(\sum_{j=1}^{M} \frac{n_{d g N, j} Y_{i, j}}{1-Y_{A, j}}\right)+\frac{n_{R g} Y_{i, M}}{1-Y_{A, M}}
$$

where 


$$
\begin{aligned}
& \mathrm{n}_{\mathrm{Si}}=\text { original sample moles of constituent } \mathrm{i} \text {. } \\
& \mathrm{n}_{\mathrm{dgN}, \mathrm{j}}=\text { moles of insoluble gas removed from the system in each con- } \\
& \text { tainer } \mathrm{j} \text {, where there are a total of } \mathrm{M} \text { containers. Most of the } \\
& \text { gas is in collection canisters whose pressure, temperature, } \\
& \text { and composition are measured so the moles of insoluble gas } \\
& \text { can be calculated as in Sections 3.1-3.3. A small amount of } \\
& \text { gas leaves the system in grab sample canisters and system } \\
& \text { evacuations, as noted in Table 3.1. In these cases, where } \\
& \text { there is no pumping to compress the gas and cause conden- } \\
& \text { sation, calculations use measured system pressures and } \\
& \text { volumes and the measured composition of the gas in the } \\
& \text { collection canister taken just before or after the grab sample } \\
& \text { (or evacuation). } \\
& Y_{i, j}=\text { the dry mole fraction of constituent } i \text { in container } j \text { as } \\
& \text { measured by mass spectrometry. } \\
& \mathrm{Y}_{\mathrm{A}, \mathrm{j}}=\text { the dry mole fraction of ammonia vapor in container } \mathrm{j} \text { as } \\
& \text { measured by mass spectrometry. } \\
& \mathrm{n}_{\mathrm{Rg}}=\text { moles of insoluble gas present in the system when the } \\
& \text { extraction is complete. This quantity is found by calculating } \\
& \mathrm{n}_{\mathrm{XgN}, \mathrm{M}} \text { for the last pumped canister using Equation (3.1.5). }
\end{aligned}
$$

There is more than one way to complete the ammonia calculation to find $\mathrm{n}_{\mathrm{xA} 0}$. The choice of method depends heavily on the details of the extraction procedure. In particular, some samples were analyzed using an injection of isotopically labeled ammonia to estimate the residual ammonia (that which is left on the extractor side when gas extraction is complete). The different methods that may be used to finish the ammonia determination are described in Sections 3.4.1 and 3.4.2.

The original sample contents of each constituent are then divided by the sampler volume to give the concentration of each insoluble gas species per liter of waste under in-tank conditions (see Section 4 for results). This assumes that the sampler is filled up to the piston with tank waste (gas, liquid, and solids) and that the piston is fully retracted.

\subsubsection{Ammonia Determination by the Isotopic Method}

This section describes the laboratory technique used for the isotopic method. After gas extraction is complete and the gas collection canisters valved out, new collection canisters are attached and the whole system evacuated (except for the extractor system and sometimes the extractor lines). The ammonia remaining in the unevacuated part of the extractor side is called the residual ammonia and includes both ammonia vapor and ammonia dissolved in the waste sample.

The isotopically labeled ammonia was in some cases (U-103, S-106, and BY-109) added as vapor and in other cases (SX-106, AX-101, S-102, S-111, U-109, and SY-101) as $15 \mathrm{NH}_{4} \mathrm{OH}$ solution. After the isotopic ammonia is added, it is given some time to approach equilibrium with the residual ammonia, whose nitrogen is quantitatively the $14 \mathrm{~N}$ isotope. The ammonia vapor is then extracted via mercury pumping. Mass spectrometry is used to determine the molar ratio, $15 \mathrm{~N} / 14 \mathrm{~N}$. (This ratio, provided to us by the mass-spectrometry analysts, is corrected for the small fraction of $15 \mathrm{~N}$ that is naturally present in the sample ammonia so that the ratio is equal to injected/residual ammonia moles.)

The isotopic method assumes that both the $15 \mathrm{~N}$ and $14 \mathrm{~N}$ ammonia have reached phase equilibrium, so the isotopic ratio in the pumped vapor is the same as that in the liquid, and that the ammonia holdover (ammonia sorbed on the RGS system and mass-spectrometer walls) is not large enough to skew the isotopic ratio if desorbed. 
The amount of labeled ammonia (15N ammonia) that is added is known, so the residual ammonia is found by

$$
\mathrm{n}_{\mathrm{RA}}=\mathrm{n}_{\mathrm{LA}} / \mathrm{Q}
$$

where

$$
\begin{aligned}
& \mathrm{n}_{\mathrm{RA}}= \text { the moles of residual } 14 \mathrm{~N} \text { ammonia (both the vapor and dissolved } \\
& \text { ammonia that is in the extractor side after gas extraction). } \\
& \mathrm{Q}=\text { the molar ratio (15N/14N ratio corrected for natural } 15 \mathrm{~N}) . \\
& \mathrm{n}_{\mathrm{LA}}=\text { the moles of isotopically labeled ammonia. }
\end{aligned}
$$

The initial ammonia in the extractor side, $\mathrm{n}_{\mathrm{xA} 0}$, is found by using mass balance methods to account for all the ammonia that was removed from the extractor side between the time the extractor side was sealed off from the collector side (before the first pump stroke) and the time when the residual ammonia was determined. These moles of ammonia removed are added to the residual to obtain $\mathrm{n}_{\mathrm{xA} 0}$. The isotopic ammonia determination method has not been fully tested with standards, so its results should be used according to the cautions given on a sample-by-sample basis in Section 4.

\subsubsection{Ammonia Determination from Post-RGS Concentration Measurements}

Some RGS samples were subjected to ion-specific electrode (ISE) analysis after the RGS extraction was complete. The extractor vessel was opened and a portion of the sample scraped or poured into jars, which were loaded out (sometimes after some storage time) and sent for ammonia and bromide analysis. Some loss of ammonia would be expected from sample handling and exposure to air, and data exist to confirm that losses were measurable. In one case, a standard solution of $\mathrm{NH}_{4} \mathrm{OH}$ was sent for ISE analysis. The solution was made up containing $0.04 \mathrm{M}$ $\mathrm{NH}_{3}$, or $680 \mu \mathrm{g} / \mathrm{mL} \mathrm{NH}_{3}$. The ammonia measured in the sample was $483 \mu \mathrm{g} / \mathrm{mL} \mathrm{NH}, 29 \%$ lower than the standard concentration.(a)

Ammonia losses could also be inferred from some of the sample results. In five samples from SX-106 and S-102, the ammonia measured in post-RGS samples was 7, 76, 33, 13, and $2 \%$ less than the amount that had been added in the isotopic standard. (These calculations can be seen in the RGS calculation spreadsheets on the CD that accompanies this report.) These losses could not be accounted for by the amount of ammonia that had been extracted between the time the isotopic ammonia was added and the time the extraction was completed. The smaller discrepancies might be accounted for by scatter, but the large values must have been caused by losses not only of the isotopic ammonia but of the sample ammonia. For these reasons, the post-RGS ISE results should be viewed as lower bounds on the true dissolved ammonia concentration after extraction. (The same holds for non-RGS core extrusions, which also are exposed to hot cell air during handling. A study by Herting(b) addressed possible losses from core extrusions. Stirred ammonia/salt solutions in 15-mL vials were exposed to the air and the change in ammonia concentration tracked. As much as $20 \%$ of the original ammonia could be lost in 10 minutes, up to $35 \%$ in an hour, and up to $80 \%$ in eight hours. Considering the differences between the test solutions and real waste samples and the differences in their handling, Herting concluded that core samples could lose "up to, but probably less than, one-half of the total ammonia present at the time of extrusion."

(a) RGS log-sheet for sample 237-10, August 20 1998, BE Hey, Numatec Hanford Corporation. (b) Herting DL. October 7, 1994. "Rate of Ammonia Loss from Laboratory Samples." Letter report to GD Johnson, Westinghouse Hanford Company, Richland, Washington. 
It is probably accurate to conclude that ammonia measured in core extrusions is a lower bound, but there may be exceptions. As discussed in Appendix C, a few ammonia measurements from an SY-101 core composite were high enough to be physically unlikely and were much higher than the ammonia measurements for the samples from which the composite was created. This observation suggests that other core extrusion ammonia measurements might be overestimates rather than underestimates.

In the cases where post-RGS ISE measurements are available for samples to which no standard was added, the measured ammonia can be used directly as a lower bound on the residual ammonia in the sample. (The residual ammonia actually included both the extractor-side ammonia vapor and the dissolved ammonia after extraction, and ISE measures only the latter, but the vapor is negligible compared to the dissolved ammonia.)

In cases where an isotopic standard was added, the amount of ammonia added must be accounted for in calculating the sample's residual ammonia. Assume that an unknown fraction $f$ of the dissolved ammonia was lost during sample handling, and that the loss fraction was the same for the sample ammonia and the standard ammonia. Then the measured moles of ammonia, $\mathrm{n}_{\text {meas }}$, can be expressed as

$$
\mathrm{n}_{\text {meas }}=\mathrm{f}\left(\mathrm{n}_{\mathrm{RA}}+\mathrm{n}_{\mathrm{LA}}\right)
$$

Applying Equation (3.4.4), the residual ammonia moles can be found as

$$
\mathrm{n}_{\mathrm{RA}}=\frac{\mathrm{n}_{\text {meas }}}{\mathrm{f}(\mathrm{Q}+1)}
$$

The loss fraction $f$ is unknown but is less than or equal to unity; setting $f=1$ gives the lower-bound estimate of $n_{R A}$. The residual ammonia calculated in this way is added to the ammonia extracted from the sample (found in Section 3.3) to obtain the original sample ammonia content.

\subsubsection{Ammonia Determination from the Partial Pressure}

In theory, once an ammonia partial pressure in the extractor has been calculated from direct PQ canister measurement or by using Equation (3.1.7), Henry's Law can be used to find the total ammonia in the sample:

$$
\mathrm{n}_{\mathrm{xAE}}=\mathrm{P}_{\mathrm{xA}}\left(\mathrm{V}_{\mathrm{w}} \mathrm{K}_{\mathrm{HA}}+\mathrm{V}_{\mathrm{gas}} / \mathrm{RT}\right)
$$

Here $V_{\text {gas }}$ is the gas volume with which the sample is connected, which may include the extractor lines and/or second volume as well as the extractor vessel headspace, depending on the point in the procedure at which the ammonia partial pressure is measured. The residual ammonia can be found by subtracting the extracted ammonia (calculated as detailed in Section 3.3) from the initial total ammonia that was calculated from the initial partial pressure.

This approach depends on being able to determine the ammonia partial pressure and the Henry's Law constant and on the assumption that equilibrium is reached (that the partial pressure of ammonia equals the equilibrium vapor pressure). Because there are questions about the accuracy of the Schumpe gas solubility model (discussed in Section 3.6.1), this report does not present the ammonia concentrations calculated (using the Schumpe model) from partial pressures. This method of ammonia determination is discussed here only for completeness. 


\subsubsection{Choice of Ammonia Determination Method}

The isotopic method is the preferred ammonia determination method. All the methods, including the isotopic, depend on the assumption that ammonia has reached phase equilibrium at some point in the extraction process. The isotopic method, in theory, requires fewer (and less constraining) additional assumptions than the partial pressure method and, in practice, generally allows more time for equilibrium to be reached.

In the cases in which the isotopic method was not yet employed (Tanks AW-101, AN-105, A-101, AN-104, and AN-103) or was used only with short equilibration times (Tanks U-103, S-106, and BY-109), the total ammonia in the sample is not known but the ammonia partial pressure is reported. In these cases, the partial pressure measured by the PQ canister, a direct measurement, is preferred to that calculated from the difference in post-extrusion pressures even though the uncertainty of the $\mathrm{PQ}$ canister measurement method is not known. The post-extrusion partial pressure calculation depends on the accuracy of the calculated insoluble gas and water vapor pressures rather than being measured directly. However, the PQ canister data are available only for some of the samples from Tanks AW-101, AN-105, A-101, AN-104, and AN-103. For all other samples from these tanks, the post-extrusion ammonia partial pressure was used.

Table 3.2 lists the averages of the ammonia partial pressures found by PQ canisters and post-extrusion calculation for those tanks where a large number of PQ canister data were taken. Values were averaged for each waste layer, and the number of data in each average is given in parentheses. Post-extrusion partial pressures that are suspected of inaccuracy because of air inleakage or unusual extractor pressure behavior were excluded from the averages.

In most cases, the average values of the post-extrusion and $P Q$ canister partial pressures are more than a standard deviation apart, and the average post-extrusion partial pressures are higher (as much as twice the PQ canister values). Calibration studies with ammonia standards would be needed to check the relative accuracy of the two types of ammonia partial-pressure determination.

Table 3.2. Comparison of $\mathrm{NH}_{3}$ Partial Pressures Measured by Two Methods

\begin{tabular}{||l|r|rl||}
\hline \multicolumn{1}{|c|}{ Tank/Layer } & $\begin{array}{c}\text { Average partial pressure of } \mathrm{NH}_{3} \\
\text { from post-extrusion calculation } \\
\text { made using Equation (3.1.7) } \\
\text { (kPa) }\end{array}$ & $\begin{array}{r}\text { Average partial pressure of } \mathrm{NH}_{3} \\
\text { from PQ canister analysis } \\
\text { (kPa) }\end{array}$ \\
\hline AW-101 Convective & $0.64 \quad$ (1 sample) & 0.39 (1 sample) \\
\hline AW-101 Nonconvective & $0.92 \pm 0.14$ (over 5 samples) & $0.60 \pm 0.19$ (over 3 samples) \\
\hline AN-105 Convective & 0.60 (1 sample) & 0.49 (1 sample) \\
\hline AN-105 Nonconvective & $0.72 \pm 0.16 \quad$ (over 5 samples) & $0.56 \pm 0.10$ (over 5 samples) \\
\hline A-101 Upper Layer & $2.46 \pm 0.41$ (over 2 samples) & $1.29 \pm 0.40$ (over 4 samples) \\
\hline A-101 Lower Layer & $2.04 \pm 0.16$ (over 3 samples) & $2.67 \pm 0.23$ (over 3 samples) \\
\hline AN-104 Convective & 0.63 (1 sample) & 0.57 (1 sample) \\
\hline AN-104 Nonconvective & $0.93 \pm 0.22$ (over 4 samples) & $1.03 \pm 0.90$ (over 2 samples) \\
\hline
\end{tabular}


In theory, the $\mathrm{PQ}$ canister vapor pressure, being measured at a greater system volume than that immediately after extrusion, should be slightly lower than the ammonia vapor pressure after extrusion that is calculated from Equation (3.1.7). The expected difference in vapor pressure results from the slight depletion of ammonia in solution by evaporation into the expanded vapor space and should be quite small at equilibrium.

In practice, however, the difference between post-extrusion and $P Q$ canister ammonia partial pressures is increased because the ammonia partial pressure does not reach a new equilibrium after the extractor and lines are opened to the second volume (where the PQ canister is connected). The approach to equilibrium is probably closer after extrusion than after any subsequent expansion because extrusion is accompanied by sample shear and by fresh surface exposed when gas bubbles pop, having expanded from in situ pressure (1 to $2.5 \mathrm{~atm}$ ) to the extractor vacuum (less than $0.1 \mathrm{~atm}$ ).

Because there are questions about the accuracy of the Schumpe gas solubility model (discussed in Section 3.6.1), this report does not present any ammonia concentrations calculated (using the Schumpe model solubilities) from partial pressures. Instead, the measured partial pressures are reported (on a tank-by-tank basis, in Section 4). The calculated in situ ammonia partial pressures are found by scaling the measured partial pressures from laboratory temperature to in situ temperature using the Schumpe model to provide the reported compositions and in situ gas volume fractions. To account for the scatter in the data and the possibility that the equilibrium vapor pressure was not reached, an uncertainty of $\pm 50 \%$ was assigned to the ammonia partial pressure data when they were used as the bases for calculating in situ ammonia pressures.

\subsection{Corrections for Contamination}

Shekarriz et al. (1997) found concentrations of oxygen and argon that were higher than the essentially zero values that were anticipated. This contamination was believed to occur during the sampling process because the nosepiece (leading end) of the sampler trapped air and argon (used as a drillstring purge gas).

A separate laboratory measurement of the maximum air volume at standard temperature and pressure (STP) suggested that a maximum of $6.1 \mathrm{cc}$ of air/argon could be trapped in the nose piece (Cannon 1997). To reduce this source of contamination, procedural changes were instituted in sampler preparation, beginning with Tank U-103. (Sampling of Tanks AW-101, A-101, and the AN tanks preceded these changes.) Each sampler's ball valve assembly was backfilled with helium and sealed with Dow Corning ${ }^{\circledR} 111$ lubricant/sealant before the sampler was deployed, filling the potential entrainment volume with sealant and helium (Cannon 1997). This step decreased the contamination from gas entrainment considerably (Mahoney et al. 1997, Section 5.2.1). However, contamination by air from RGS system inleakage and from isotopic ammonia standard additions has been apparent. The air contamination results are discussed in Section 4 on a tank-by-tank basis.

The gas concentration results measured by RGS extraction are corrected to in-tank concentrations based on the assumption that all the argon and oxygen in the sample are from the entrained drillstring gases and air inleakage. The argon and oxygen are removed, and the nitrogen is reduced based on the nitrogen-to-oxygen ratio for standard atmospheric air, $\mathrm{N}_{2} / \mathrm{O}_{2}=3.73$.

It has been suggested that inert argon be used as an air tracer gas to avoid the complication of oxygen's possible reaction with and consumption by the waste (leading to overestimation of sample $\mathrm{N}_{2}$ ). However, in at least a third of the samples, less nitrogen was found than was calculated by multiplying the measured argon by the nitrogen-to-argon ratio for standard atmospheric 
air, $\mathrm{N}_{2} / \mathrm{Ar}=83.6$. The excess argon may have come from drillstring purge gas. Records do not always tell when the drillstring was purged, so we cannot be sure the argon is from air alone.

In any case, inleaked or entrained oxygen is unlikely to have reacted away to any significant extent in most RGS samples. (Here "significant" means a change that is large compared with the measurement uncertainty.) Experiments with waste from Tank AN-105 found that the half-life for $\mathrm{O}_{2}$ removal at $25^{\circ} \mathrm{C}$ was 210 days (Person 1998). Some samples from SY-101, U-109, and S-111 had delays of 100 to 150 days between sample acquisition and extraction and might have reacted as much as $40 \%$. One would expect these samples to have shown an increase in the ratio of air calculated from argon to air calculated from oxygen, compared with samples with short delays, if oxygen were reacting significantly. No such trend is visible in Figure 3.2, which shows the (air from Ar)/(air from $\mathrm{O}_{2}$ ) ratio for all the successful RGS samples. The scatter in the ratio is too large to permit any statements about trends. Because there was no clear evidence of significant oxygen reaction and because argon might have come not merely from air but from drillstring purges, we used $\mathrm{O}_{2}$ as the air tracer gas.

Another type of sample contamination could result from the HHF, the fluid used during the sampling procedures to balance hydrostatic head, seeping into the sampler and replacing some of the waste volume. This fluid is water marked with trace amounts of lithium bromide (standard practice), so a chemical analysis could indicate whether contamination has occurred (neither $\mathrm{Li}^{+}$nor $\mathrm{Br}^{-}$are present in tank waste). If such contamination occurred, the RGS measurements would misrepresent pure waste in four ways (in order of dirninishing importance):

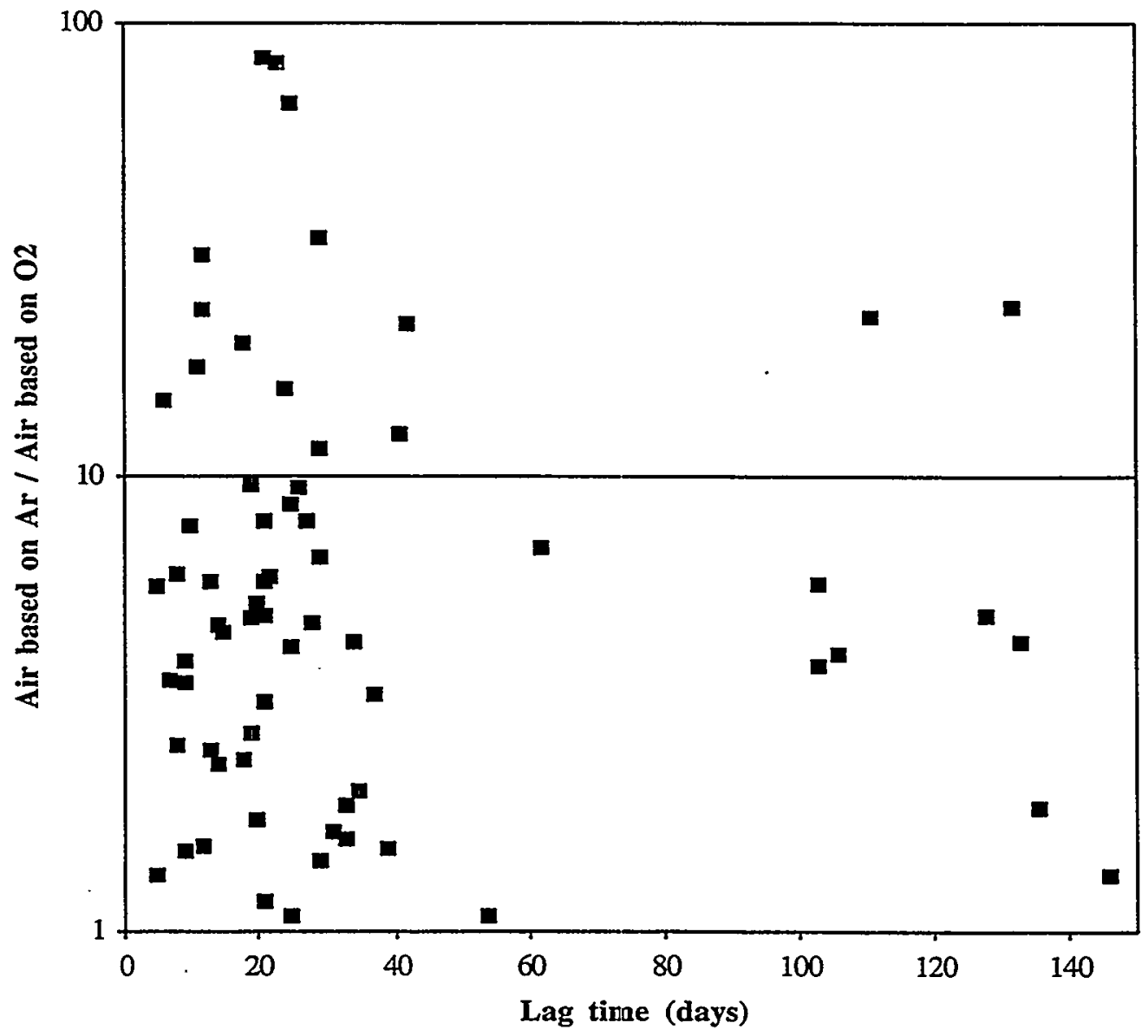

Figure 3.2. Test for Significant $\mathrm{O}_{2}$ Reaction in RGS Samples 
1) for a given volume percent of HHF the calculated gas content of the waste is reduced by about the same percentage, just as if incomplete sample recovery had occurred; 2) the HHF contains dissolved air constituents with which it contaminates the waste gas; 3) the HHF dilutes the ammonia in the sample, reducing its measured partial pressure (measured by the PQ canister grab sample during extraction) below that of pure waste; 4) the HHF may decrease the ionic strength of the waste liquid, increasing the solubility of ammonia and leading to a further underestimate of the partial pressure of ammonia. However, this depends on whether the solids in the waste are still present and in equilibrium with the solution; if so, the solution concentration will be essentially the same, as if no HHF had been added.

Chemical analysis results (further discussed on a tank-by-tank.basis in Section 4) show that the HHFF tracer, when it was detectible at all, was present in the waste in concentrations that usually corresponded to HHF contamination of $10 \%$ or less. (The bromide ion, rather than the lithium ion, is used as the tracer because bromide interacts less with the waste than lithium.) Concentrations were often below the $\mathrm{Br}^{-}$detection limit, which ranged from 3 to $15 \mathrm{vol} \%$ depending on the amount of solids in the waste (the amount of dilution required to allow analysis); in most cases, the detection limit was less than $8 \mathrm{vol} \%$. The average contamination of samples was typically about $7 \mathrm{vol} \% \mathrm{HHF}$ when detection limits were included in the average as if they were measurements. The actual contamination could be substantially less because the detection limit represents the maximum amount of HHF that could be present without being measurable, not the actual amount.

\subsection{Phase Distribution and Gas Volume Fraction}

Once the concentrations of gases have been determined, the distribution of the different components between the liquid and gas/vapor phases must be determined for each waste sample under in-tank conditions. The quantity of gases in the gas phase determines the in situ gas volume fraction of the sample.

The phase distribution of the gas constituents is based on the effective Henry's Law constants that are calculated for in situ conditions using the Schumpe gas solubility model, which is described in Section 3.6.1. The in-tank pressure for each sample is calculated as hydrostatic pressure based on the waste densities measured from core extrusions or by ball theometry. An iterative procedure (described in Section 3.6.2) is used that matches the sum of partial pressures of all the gas constituents with the in-tank pressure.

The analysis procedures described in this section require input from a variety of sources. The total gas concentrations are determined using the procedures described in Sections 3.1 through 3.5. Several tank waste properties are used in the analysis; these were obtained from a variety of sources, including the Tank Characterization Reports (tank-specific references are given in Sections 4 and 6). These properties include

- Molar ion concentrations in the waste solution, used to calculate Henry's Law constants

- Solid volume fractions and weight fraction of water in solution, used to calculate effective Henry's Law constants per liter of waste

- Average bulk densities of the different layers, used to calculate the in-tank hydrostatic pressures at each elevation.

Other inputs include

- Elevations from which the samples were taken (from the sampling plan) 
- Location and thickness of the different layers and crust

- Temperature at each sample elevation

- Water vapor pressure at each sample location.

\subsubsection{In Situ Solubility Model}

The distribution of low-solubility gases and ammonia between the vapor and liquid phases of the waste is determined by a parameter referred to as a Henry's Law constant, $\mathrm{K}_{\mathrm{H}}$, which is defined as

$$
\mathrm{K}_{\mathrm{H}}=\mathrm{c}_{\mathrm{i}} / \mathrm{p}_{\mathrm{i}}
$$

where $p_{i}$ is the partial pressure of a component in atmospheres, and $c_{i}$ is the concentration of the component expressed in terms of moles per liter of waste. This equation differs from the normal definition of the Henry's Law constant because it is in terms of unit volume of degassed waste rather than mass of solvent (water) in the waste. The distribution of a component between both the vapor and liquid phases can be determined under any set of conditions if the total moles of component and the effective Henry's Law expression have been determined.

The Henry's Law constant for a gas depends on several variables, including the solution temperature and ion concentrations. Several expressions have been developed to calculate the Henry's Law constant and have been reviewed by Norton and Pederson (1994, 1995). (A word of caution: there are two different conventions for expressing the Henry's Law constant; one is the inverse of the other. Our discussion uses the expression that is defined in Equation [3.6.1] and is consistent with Norton and Pederson [1994, 1995].) The Schumpe model is given by

$$
\log \left(\mathrm{c}_{\mathrm{G}, \mathrm{o}} / \mathrm{c}_{\mathrm{G}}\right)=\log \left(\frac{\mathrm{K}_{\mathrm{H}, \mathrm{G}} \text { (water) }}{\left.\mathrm{K}_{\mathrm{H}, \mathrm{G}} \text { (solution) }\right)}\right)=\sum_{\mathrm{i}}\left(\mathrm{h}_{\mathrm{i}}+\mathrm{h}_{\mathrm{G}}\right) \mathrm{s}_{\mathrm{i}}
$$

where

$c_{G, 0}$ and $c_{G}=$ the gas solubility of gas $G$ in pure water and salt solution, respectively

$h_{i}$ and $h G=$ the ion and gas-specific coefficients, respectively

$$
\begin{aligned}
& s_{i}=\text { the concentration of ion } i \text { in the salt solution. The gas-specific constant, } h_{G} \text {, is } \\
& \text { assumed to be a linear function of temperature } \\
& h_{G}=h_{G, o}+h_{T}(T-298.15 K)
\end{aligned}
$$

where $h_{G, o}$ is the reference value, and $h_{T}$ is the temperature-specific coefficient. The values of $h_{i}$, $h_{G, 0}$, and $h_{T}$ used to produce this report were the same as those used by Norton and Pederson (1995, Appendix A) and originating with Hermann et al. (1995). These values were used in this report because they were found (Norton and Pederson 1995) to give reasonable agreement with the solubilities of low-solubility gases and ammonia in a waste simulant at about half the salt concentration found in the tank wastes that have been sampled using RGS. Table 3.3 shows the Schumpe model coefficients that were used.

For many tanks (AW-101, AN-105, A-101, AN-104, AN-103, S-111, U-109, and SY-101), there were data available for the concentrations of all the major salt ions (those listed in Table 3.3). In these cases, the ion concentrations in the supernatant or in drainable liquid were used directly. (The salt concentrations are given in the tank calculation spreadsheets on the $\mathrm{CD}$ that accompanies 
Table 3.3. Schumpe Model Coefficients Used in RGS(a)

\begin{tabular}{|c|c|c|c|c|}
\hline Ion & $\begin{array}{c}\mathrm{h}_{\mathrm{i}} \\
\left(\mathrm{m}^{3} / \mathrm{kmole}\right)\end{array}$ & Gas & $\begin{array}{c}\mathrm{h}_{\mathrm{G}, 0} \\
(\mathrm{~m} 3 / \mathrm{kmole})\end{array}$ & $\begin{array}{c}\mathrm{h}_{\mathrm{T}} \\
\left(\mathrm{m}^{3} / \mathrm{kmole} \mathrm{K}\right)\end{array}$ \\
\hline $\mathrm{Na}^{+}$ & 0.1079 & $\mathrm{~N}_{2} \mathrm{O}$ & -0.011 & 0 \\
\hline $\mathrm{Al}^{3+}\left(\right.$ as $\left.\mathrm{AlO}_{2}^{-}\right)$ & 0.1 & $\mathrm{O}_{2}$ & 0 & 0 \\
\hline $\mathrm{Fe}^{3+}$ & 0.0957 & $\mathrm{H}_{2}$ & -0.0176 & 0 \\
\hline $\mathrm{Cr}^{2+}$ & 0.0578 & $\mathrm{CH}_{4}$ & 0.0028 & 0 \\
\hline $\mathrm{Ni}^{2+}$ & 0.1556 & $\mathrm{~N}_{2}$ & 0.0002 & 0 \\
\hline $\mathrm{K}^{+}$ & 0.0929 & $\mathrm{NH}_{3}$ & -0.0506 & 0 \\
\hline $\mathrm{OH}^{-}$ & 0.0918 & \multirow{8}{*}{\multicolumn{3}{|c|}{$\begin{array}{l}\text { Other gases ( } \mathrm{Ar}, \mathrm{He}, \mathrm{C}_{2} \mathrm{H}_{\mathrm{x}}, \mathrm{C}_{3} \mathrm{H}_{\mathrm{x}}, \mathrm{NO}_{\mathrm{x}} \text {, and } \\
\text { other hydrocarbons) were treated as } \\
\text { completely insoluble. }\end{array}$}} \\
\hline $\mathrm{NO}_{3}^{-}$ & 0.0136 & & & \\
\hline $\mathrm{NO}_{2}^{-}$ & 0.0726 & & & \\
\hline $\mathrm{CO}_{3}^{2-}$ & 0.1558 & & & \\
\hline $\mathrm{PO}_{4}^{3-}$ & 0.2243 & & & \\
\hline $\mathrm{SO}_{4}^{2-}$ & 0.1164 & & & \\
\hline $\mathrm{F}^{-}$ & 0.1058 & & & \\
\hline $\mathrm{Cl}^{-}$ & 0.0381 & & & \\
\hline
\end{tabular}

this report.) The remaining tanks lacked hydroxide ion measurements for the tank liquid. The hydroxide concentrations were roughly approximated by a two-step process. First, the nitrite concentration in solution (which was always available) was multiplied by the ratio of the hydroxide inventory to the nitrite inventory (as given in the TWINS Best-Basis Inventory database). That result (the scaled concentration) had four times the $\mathrm{Al}^{3+}$ concentration subtracted from it to account for the incorporation of hydroxide in aluminate ion. In three cases (BY-109, SX-106, S-102) the approximated hydroxide concentrations fell outside the 2- to 6-M range that held for the measured hydroxide concentrations. Spot checks indicated that the effect of uncertainty in the hydroxide approximation was less than that in the solubility model itself (which is discussed later)

The solubility of ammonia in waste salt solution is at least four orders of magnitude greater than that of the next-highest solubility species, nitrous oxide. In turn, nitrous oxide is 10 to 100 times as soluble in salt solution as hydrogen, nitrogen, and the other low-solubility gases. The Henry's Law constants that were calculated from the Schumpe model to determine phase distributions in the tanks can be found in the "In Situ" worksheets of the RGS calculation spreadsheets included on the $\mathrm{CD}$ that accompanies this report.

The Henry's Law constant obtained using Equation (3.6.2) and (3.6.3) must be converted from a molal basis, moles of solute per $\mathrm{kg}$ of solvent (water) in solution, to a basis of moles per volume of gas-free waste (both solution and solids). The conversion is accomplished by calculating

$$
\mathrm{K}_{\mathrm{H}}, \mathrm{L} \text { waste basis }=\left(\mathrm{K}_{\mathrm{H}}, \mathrm{kg} \text { water basis }\right)\left(1-\mathrm{x}_{\mathrm{s}}\right) \rho_{\mathrm{L}} \omega_{\mathrm{L}}
$$


where $\mathrm{x}_{\mathrm{S}}$ is the volume fraction of solids in the gas-free waste, $\omega_{\mathrm{L}}$ is the weight fraction of water in the solution, and $\rho_{L}$ is the solution density. Solids volume fractions are either taken from core sample data (when available) or estimated based on solution density, intrinsic solid density $\rho_{S}$ (also known as particle density), and degassed bulk waste density, $\rho_{\mathrm{b}}$ :

$$
x_{s}=\frac{\rho_{b}-\rho_{L}}{\rho_{S}-\rho_{L}}
$$

Values for the solids fractions and densities used in Equations (3.6.4) and (3.6.5) are given in the "In-situ" worksheets of the RGS calculation spreadsheets on the CD that accompanies this report. The weight fraction of water in the solution is taken from analyses of core extrusions that were obtained in the same sampling campaign as the RGS samples.

Uncertainties and bias in the Schumpe model, as used for calculating in situ gas compositions and volume fractions, stem from uncertainties both in the parameters and in the theoretical basis of the model itself. The liquid fraction in the waste (calculated as $1-\mathrm{x}_{\dot{\mathbf{s}}}$ ) contains the most uncertainty and has a proportional effect on the solubility of gases in the bulk waste because the gases are soluble only in the liquid part of the waste. The liquid density and water fraction (both measured quantities) affect the solubility via Equation (3.6.4), and the salt concentrations of course also affect the solubility through Equation (3.6.2). Probably the greatest uncertainty in all of these inputs comes from that fact that concentrations, densities, and so on are typically measured at laboratory temperature, which may be much lower than in situ temperature. However, the uncertainty in these inputs is expected to have less effect than the uncertainty in the model itself.

The Schumpe model itself was intended for use at low salt concentrations and was therefore based on a linearized relationship between the activity of dissolved gas in water and in salt solution. The linear assumption is not expected to be accurate at higher salt concentrations (deviations can occur at concentrations as low as $1 \mathrm{M}$ or as high as $8 \mathrm{M}$, depending on the dissolved species). The result of linearization inaccuracy is that the effect of salt on solubility is overestimated, which leads to an underestimation of gas solubility. This in turn causes an overestimation of the equilibrium gas or vapor pressure and underestimation of the amount of gas dissolved in the liquid.

Figure 3.3 shows a comparison of ammonia vapor pressure (VP) data from Norton and Pederson (1994) with predictions made by the Schumpe model. Norton and Pederson measured the ammonia vapor pressures over a homogeneous (liquid-only) simulant and a heterogeneous (liquid and solids) simulant at a range of ammonia concentrations and pressures. The heterogeneous simulant had a total $\mathrm{Na}^{+}$concentration of $12.6 \mathrm{M}$, about the same as in SY-101 liquid; the homogeneous simulant contained $6.0 \mathrm{M} \mathrm{Na}^{+}$. (For lack of other information, the Schumpe model calculations assumed that all the salt in the heterogeneous simulant was dissolved at all temperatures, even though some precipitate was evident. This input assumption would have overstated the effect of salt on solubility, causing overprediction of vapor pressure beyond what was implicit in the model itself.) The data presented in Figure 3.3 include only those for the lowest ammonia concentration, $0.7 \mathrm{M}$ (which is higher than any ammonia concentration measured in tank waste). The figure plots the percent error in the predicted vapor pressure:

$$
\text { percent error }=100 *(\text { predicted VP } \text { - measured VP) } / \text { measured VP }
$$




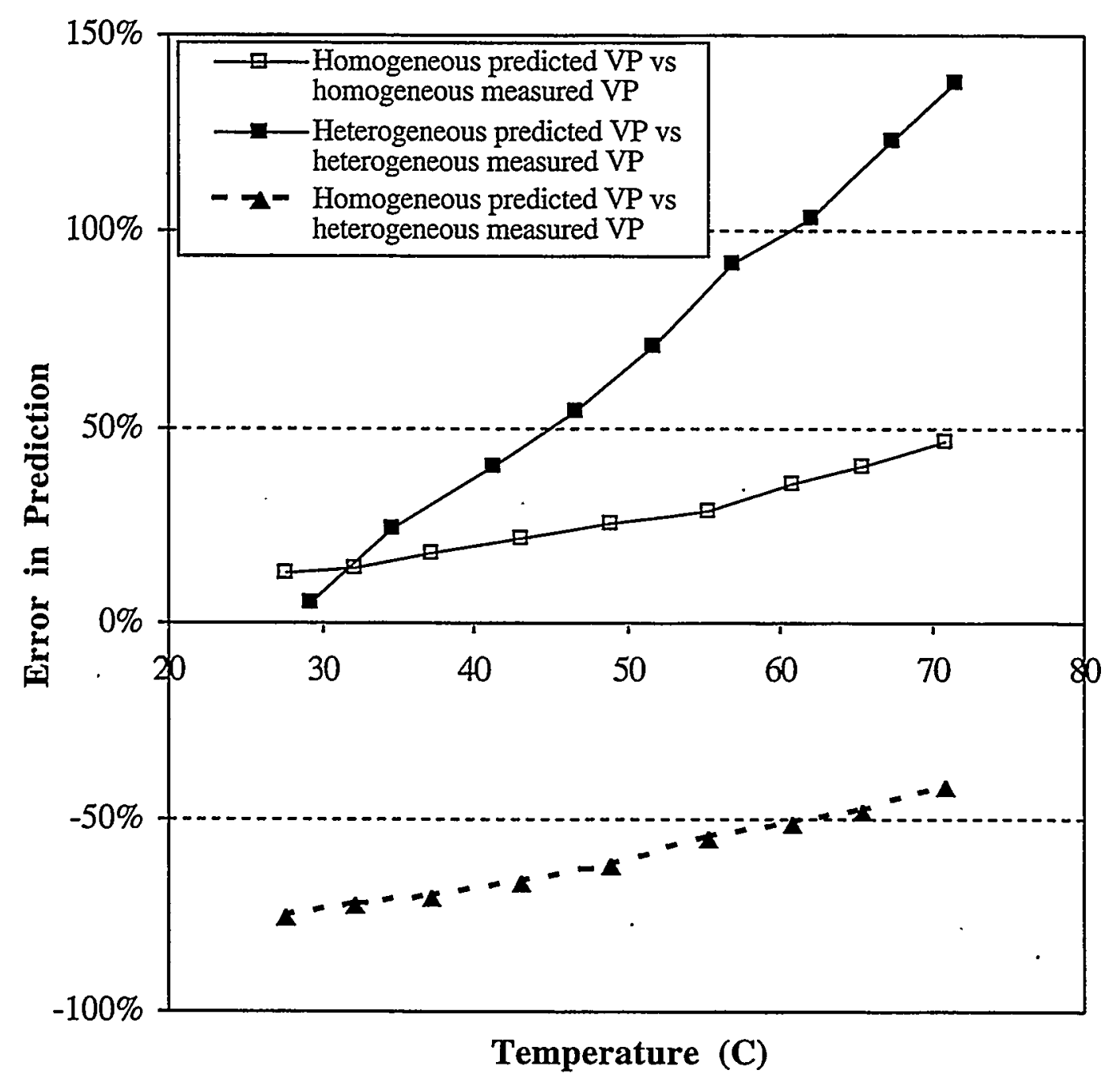

Figure 3.3. Comparison of the Schumpe Solubility Model with Simulant Data

The figure shows that the Schumpe model overpredicted the ammonia vapor pressure by 15 to $50 \%$ at the moderate concentration of the homogeneous simulant, with a consistent trend to greater overprediction at higher temperatures. The model's overprediction was less than $10 \%$ at laboratory temperatures $\left(20\right.$ to $\left.25^{\circ} \mathrm{C}\right)$ for the heterogeneous simulant but increased more with temperature than it did for the homogeneous simulant. At $70^{\circ} \mathrm{C}$, the Schumpe model overpredicted the vapor pressure by $140 \%$ (the predicted vapor pressure is 2.4 times the measured). Part of the difference in the predictability of the homogeneous and heterogeneous simulants may be the change in liquid composition caused by the solution and dissolution of solids as the temperature changes.

Thus the Schumpe model must be considered to overpredict the ammonia vapor pressure (and the gas volume contributed by ammonia) under in situ conditions. (However, for ammonia, this overprediction may be small at laboratory temperatures or in low-temperature tanks. The small overprediction at laboratory conditions suggests that, if ammonia estimates were made by the partial pressure method described in Section 3.4.3, the Schumpe model might not introduce substantial error.)

This underprediction of gas solubility probably holds for all the dissolved gases, not only ammonia, though the temperature dependence of the model error could be quite different for other. 
gases. The extent of solubility underprediction is not known for $\mathrm{H}_{2}, \mathrm{~N}_{2}, \mathrm{~N}_{2} \mathrm{O}, \mathrm{CH}_{4}$, and other low-solubility gases but has little effect on RGS calculations. The solubilities of most of the gases are so low that the composition and quantity of most of the low-solubility portion of the gas is about the same as it would be for zero solubility.

This report brackets the uncertainty range associated with gas solubility prediction in the following way. To obtain the upper end of the range of ammonia (and other gas) contributions to the vapor, we use the Schumpe model with the salt concentrations measured for the drainable liquid in the waste. Based on Figure 3.3, this upper-end value may overestimate ammonia by up to $140 \%$ at high temperatures. The lower end of the range is obtained by decreasing all the measured salt concentrations by the factor that reduces the $\mathrm{Na}^{+}$to $6 \mathrm{M}$, the same concentration as in Norton and Pederson's homogeneous simulant. The reduction factor is typically about 2 ; in effect, a 1:1 dilution. The reduced concentrations are then used to calculate the lower-end ammonia (and other gas) contributions.

The dotted line in Figure 3.3 shows what happens when the homogeneous salt concentrations were used to predict vapor pressures over the heterogeneous solution. In this case, the ammonia vapor pressures were underpredicted by 40 to $80 \%$, with less underprediction at higher temperature. Thus the "diluted-solution" approach gives an extreme lower bound on the extent to which the solubility model uncertainty affects the in situ gas quantity and composition. At low temperatures $\left(25\right.$ to $\left.35^{\circ} \mathrm{C}\right)$, the true vapor pressure is probably much closer to the upper bound than the lower. At high temperatures $\left(60\right.$ to $\left.70^{\circ} \mathrm{C}\right)$, the opposite is expected, as shown in Figure 3.3.

As shown in various tables in Section 4, for example, Table 4.3.6, the composition of retained gas in liquid layers is often significantly different at the low end of the solubility uncertainty range than at the high end. (Here "significant" means a change that is large compared with the measurement uncertainty.) $\mathrm{NH}_{3}$ and $\mathrm{N}_{2} \mathrm{O}$ are the most affected constituents, with their mole fractions showing a change of a factor of 2 or more. When there is more than about 0.02 volume fraction gas, the solubility uncertainty has less effect on composition because a smaller fraction of the gas moves into or out of solution. At these higher gas volume fractions, only the $\mathrm{NH}_{3}$ mole fraction shows a large difference between the two ends of the solubility uncertainty range.

There are few cases in which the solubility uncertainty causes an uncertainty in the gas volume fraction that is greater than the instrument uncertainty. The exceptions are cases in which both the insoluble gas and ammonia concentrations are high. In such cases ammonia makes up a significant part of the gas volume, and changes in the solubility of ammonia can have significant effects on the gas volume fraction. This effect was most noticeable in some of the SY-101 waste (Table 4.15.7).

\subsubsection{In Situ Gas Distribution}

The gas volume fraction (phase distribution) analysis begins by determining the in-tank gas pressure, $\mathrm{p}_{\mathrm{z}}$, at each sample elevation. It is assumed to be equal to the hydrostatic pressure, which is obtained by multiplying the average waste density for each layer by the thickness of each layer above that elevation and summing. Next, the effective Henry's law constant at the in-tank temperature is calculated for each sample elevation using the procedure described in Section 3.6.1.

The distribution of each gas constituent between the liquid and vapor phases can be determined using Henry's Law if the concentration of the gas constituent, the total gas volume fraction, and the effective Henry's Law constant for that constituent are known. The portion of each gas constituent, $i$, in the vapor phase is given by the expression 


$$
\frac{n_{i, v}}{n_{i, t o t}}=\frac{\alpha / R T}{\alpha / R T+(1-\alpha) K_{H, i}}
$$

where $\alpha$ is the gas volume fraction. The partial pressure for each gas constituent is given by the expression

$$
\mathrm{p}_{\mathrm{i}}=\frac{\mathrm{n}_{\mathrm{i}, \mathrm{tot}} / \mathrm{V}_{\mathrm{tot}}}{\left[\frac{\alpha}{\mathrm{RT}}+(1-\alpha) \cdot \mathrm{K}_{\mathrm{H}, \mathrm{i}}\right]}
$$

The system is constrained by the fact that the sum of all the gas constituent partial pressures must equal the dry hydrostatic pressure in the tank at that elevation:

$$
\mathrm{p}_{\mathrm{hyd}}=\mathrm{p}_{\mathrm{H}_{2} \mathrm{O}}+\sum \mathrm{p}_{\mathrm{i}}
$$

Here $\mathrm{pH}_{2 \mathrm{O}}$ is the vapor pressure of water over the salt solution, found by using salt concentrations and temperatures as inputs to Equation 6.2 of Mahoney and Trent (1995), a correlation for water vapor pressure over concentrated homogeneous and non-homogeneous waste simulants.

The gas volume fraction can be calculated using the ideal gas law:

$$
\alpha=\frac{\sum \mathrm{n}_{\mathrm{i}, \mathrm{v}} \mathrm{RT}}{\mathrm{p}_{\mathrm{hyd}} \mathrm{V}}
$$

Note that the in situ gas volume fraction is not known a priori. It is required to calculate the phase distributions using Equation (3.6.6). As a result, an iterative procedure has been developed that begins with an estimated total gas volume fraction. The phase distribution for each gas component is calculated, and the partial pressures are summed. This sum is compared with the in-tank hydrostatic pressure, and the gas volume fraction is adjusted accordingly. This iteration continues until the sum of partial pressures is within $0.001 \%$ of the specified in-tank pressure.

\subsection{Tank Inventories and Data Interpretation}

The data analysis tasks in Sections 3.1 through 3.6 provide gas concentration data and in situ gas volume fraction values at the points where samples were successfully taken. These results require further interpretation to provide overall tank waste properties such as gas inventories.

\subsubsection{Estimating Gas Inventory from RGS Data}

Once the phase distribution of each of the gas constituents has been determined, the total tank inventory of free and dissolved gases can be calculated. The analysis consists of calculating the average in situ gas volume fraction in each of the layers from which samples were taken, calculating the average gas composition in each layer, estimating the total number of moles of each gas constituent in both layers, and summing to determine the total gas inventory. The analysis also provides the average gas location and pressure in the tank. 
The analysis procedures described in this section require input from a variety of sources. The free and dissolved gas concentrations at the various sampling locations are determined using the procedure described in Sections 3.1 through 3.6. Other inputs include

- Location, thickness, and volume of the different layers, including estimated variation (uncertainty) in layer thicknesses and location; layers are distinguished by their temperature profiles, core sample physical properties, and gas retention capacities.

- Elevations at which samples were taken.

- Temperature at each sample elevation (obtained from multifunction instrument trees [MTTs] or thermocouple [TC] profiles).

- Pressure at each sample elevation (calculated in Section 3.3).

In generating RGS inventories, three calculation methods may be used on RGS concentration data. Where only one RGS sample is available in a layer, its data are used directly. The species concentrations of the single sample are assumed to extend throughout the layer. The gas volume for the layer is calculated from the single gas concentration and the average pressure in the layer (usually the hydrostatic pressure at the layer midpoint).

When more than one sample is available for a convective layer, the layer is assumed to be well mixed. An arithmetic average of the samples' concentrations is used to determine the average gas concentrations (mol/L) for the layer. In this case, too, the in situ gas volume is based on the average pressure in the layer.

When more than one sample is available for a nonconvecting layer, the concentrations are integrated over depth to find the average. In addition, the mass-average pressure and temperature of the gas in the layer are found from integrating the temperatures and pressures at sample locations (multiplied by gas concentration). The STP gas volurne for the layer is calculated from the averaged concentration, temperature, and pressure using the ideal gas law to adjust from tank conditions to standard conditions.

The integration method assumes that the concentrations of all the gases are piece-wise linear continuous between samples within a layer. (The discontinuities between layers are preserved so that, for example, the low gas concentration in a convective layer does not "pull down" the higher average concentration in a nonconvecting layer.) The assumption of piece-wise linearity allows Simpson's Rule to be used for integration, with the concentrations between sample centers linearly interpolated. Figure 3.4 shows an example integration for one layer of waste from which three samples (segments 5,6, and 10, the closed circles) have been taken. The concentrations (of whatever constituent) at the bottom of the layer are set equal to the concentrations from the lowest sample within the layer and similarly for the top of the layer. The four integration intervals are unequal in size, reflecting the different distances between data locations.

\subsubsection{Best-Estimate Inventory and Uncertainty}

The RGS extraction system provides sufficient data to determine the total gas volume in most samples to within about $10 \%$. However, because only three to seven segments were successfully extracted in any of the tanks studied (except SY-101) and core extrusions often show high lateral nonuniformity in the gas-retaining waste layers (crust and nonconvective layers), there is a large uncertainty in using these sparse data to derive the total tank gas inventory. A statistically sound estimate of the uncertainty cannot be made because there are too few samples to quantify the spatial variability of gas retention. 


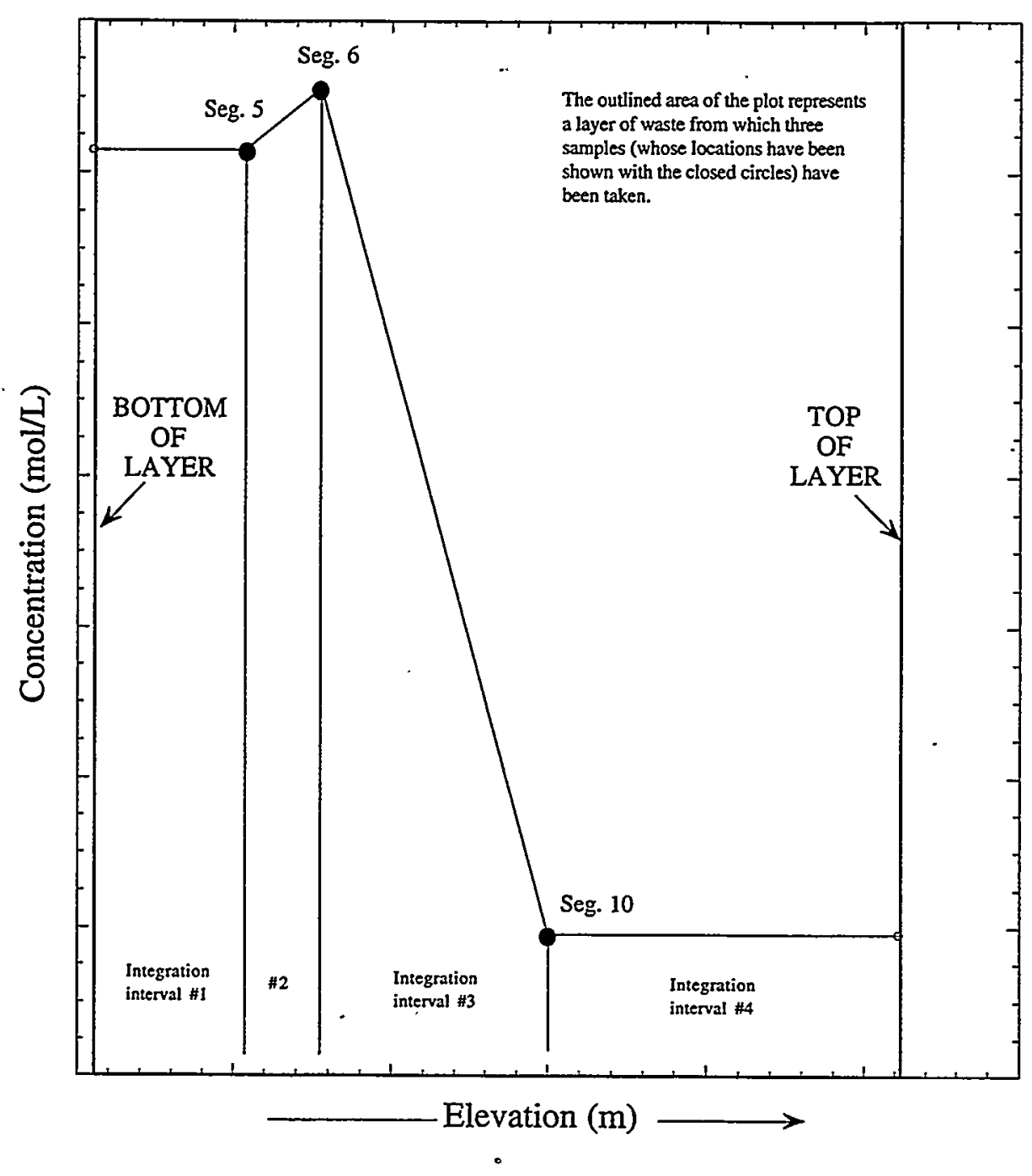

Figure 3.4. Example of Integration Scheme for Averaging RGS Concentrations

However, methods other than the RGS have sometimes been used in the same tanks and provide an independent estimate of the retained gas volume and a way to assess the uncertainty in RGS-derived inventories. This assessment was originally detailed in Mahoney et al. (1997) and is summarized here with the RGS-based gas inventory values updated to match those in this report.

Local gas fractions were measured in several DSTs with both the RGS and the VFI in 1995-96 (Schienbein et al. 1999). The VFI provides many more measurements and therefore defines the gas fraction profile in much greater detail than the RGS. Gas inventories (STP) in the nonconvective layers of the four tanks were calculated with RGS data alone and with both VFI and RGS data. The comparison is summarized in Table 3.4 along with references to the tables in Section 4 where the RGS inventories can be found. The "VFI\&RGS" inventories were taken from Table 2.7.3 of Schienbein et al. (1999).

The volume derived from RGS measurements was 30 to $40 \%$ high in AN-104 and AN-105 and 10 to $30 \%$ low in AW-101 and AN-103. The root-mean-square (rms) average of the differences is $29 \%$, which represents the difference between the inventories calculated from RGS data alone and those calculated from all the available data (reducing the effect of lateral variability). 
Table 3.4. Comparison of RGS and VFI Nonconvective Layer Gas Inventory in Four DSTs

\begin{tabular}{||c|c|c|c|c||}
\hline \multirow{2}{*}{ Tank } & \multirow{2}{*}{$\begin{array}{c}\text { Number of } \\
\text { RGS } \\
\text { Nonconvective } \\
\text { Layer Samples }\end{array}$} & $\begin{array}{c}\text { RGS } \\
\left(\mathrm{STP} \mathrm{m}^{3}\right)\end{array}$ & $\begin{array}{c}\text { VFI\&RGS } \\
\left(\mathrm{STP} \mathrm{m}^{3}\right)\end{array}$ & (VFI\&RGS - RGS) / VFI\&RGS \\
\hline \hline $\begin{array}{c}\text { AN-103 } \\
\text { (Table 4.5.8) }\end{array}$ & 2 & 260 & $356 \pm 16$ & $27 \%$ \\
\hline $\begin{array}{c}\text { AN-104 } \\
\text { (Table 4.4.8) }\end{array}$ & 5 & 257 & $186 \pm 13$ & $-38 \%$ \\
\hline $\begin{array}{c}\text { AN-105 } \\
\text { (Table 4.2.8) }\end{array}$ & 6 & 186 & $140 \pm 40$ & $-33 \%$ \\
\hline $\begin{array}{c}\text { AW-101 } \\
\text { (Table 4.1.8) }\end{array}$ & 5 & 86 & $97 \pm 11$ & $11 \%$ \\
\hline
\end{tabular}

The representativeness of a single RGS sample might be comparable to that of a single VFI measurement. The standard error of a single VFI gas fraction measurement with respect to the average is quite high even in DSTs. Statistical analysis of the VFI data from five DSTs gives uncertainties from $25 \%$ to over $60 \%$ of the mean (Schienbein et al. 1999) with an rms average of $48 \%$.(a) This implies that the uncertainty of an average of four such measurements would be at least $24 \%$.

The gas inventory derived from the VFI data set is also uncertain because it represents the waste in the immediate vicinity of just two risers. However, where it can be applied, the barometric pressure effect (BPE) method can be used to compute the total gas inventory directly. The BPE method is derived in detail in Schienbein et al. (1999), and a method to filter out the effects of waste strength is discussed in Whitney et al. (1996). It is based on the correlation between barometric pressure fluctuations and changes in the waste surface level, which are attributed to expansion and compression of retained gas.

by

The in situ gas volume, $\mathrm{V}_{\mathrm{G}}$, can be estimated from the level-to-pressure correlation, $\mathrm{dL} / \mathrm{dP}$,

$$
V_{G}=-A P_{e f f} d L / d P
$$

where $\mathrm{A}$ is the tank cross-sectional area and $\mathrm{P}_{\text {eff }}$ is the effective pressure at which the gas is stored. The effective pressure can be calculated based on the gas distribution implied by the RGS measurements by assuming each RGS sample represents a point on the gas fraction profile. The gas fraction at the top of the gas-bearing region is assumed to be equal to the gas fraction in the topmost RGS sample, and the gas fraction at the tank bottom is assumed to be equal to that of the lowest RGS sample. Then the average gas fraction in the tank defined for N RGS segments is given by

$$
\bar{\alpha}=\frac{\sum_{\mathrm{i}=1}^{N} \alpha_{\mathrm{i}} \mathrm{H}_{\mathrm{i}}}{\mathrm{L}}
$$

(a) That is, a single measurement represents the mean with an error of 25 to $60 \%$. Though individual measurements have a high uncertainty, the uncertainty of the average is much lower because many data are included. 
where $\alpha_{i}$ is the in situ gas volume fraction in RGS segment $i, L$ is the total height of the gas-bearing region, and the effective heights assigned to each RGS segment, $\mathrm{H}_{\mathrm{i}}$, are defined using Simpson's rule integration as

$$
\begin{aligned}
& \mathrm{H}_{\mathrm{N}}=\mathrm{L}-\frac{1}{2}\left(\mathrm{z}_{\mathrm{N}}+\mathrm{z}_{\mathrm{N}-1}\right), \\
& \mathrm{H}_{\mathrm{i}}=\frac{1}{2}\left(\mathrm{z}_{\mathrm{i}+1}-\mathrm{z}_{\mathrm{i}-1}\right), \quad \text { for } 1<\mathrm{i}<\mathrm{N}, \text { and } \\
& \mathrm{H}_{1}=\frac{1}{2}\left(\mathrm{z}_{2}+\mathrm{z}_{1}\right)
\end{aligned}
$$

The determination of the total height, $L$, depends (somewhat subjectively) on which waste layers were sampled and on the conditions in the tank. For example, in BY-109, $\mathrm{L}$ is taken to be the interstitial liquid level (ILL) because the waste above that level is not saturated with liquid and cannot retain flammable gas, and because the waste above the ILL was not sampled. In many other tanks, cores and temperature measurements indicate a liquid layer that cannot store gas. In these cases, $\mathrm{L}$ is the thickness of the gas-storing layer.

The effective pressure for BPE calculations is determined by (Schienbein et al. 1999)

$$
\mathrm{P}_{\mathrm{eff}}=\frac{\sum_{i=1}^{N} \alpha_{i} \mathrm{H}_{i}}{\sum_{i=1}^{N} \frac{\alpha_{i}}{\mathrm{P}_{i}} \mathrm{H}_{i}}
$$

where $P_{i}$ is the in situ hydrostatic pressure in segment $i$ estimated from the bulk densities of segments above it. The effective $\mathrm{dL} / \mathrm{dP}$ correlation implied by the RGS data can also be calculated by

$$
\frac{\mathrm{dL}}{\mathrm{dP}_{\text {RGS }}}=-\sum_{\mathrm{i}=1}^{N} \frac{\alpha_{i}}{\mathrm{P}_{\mathrm{i}}} \mathrm{H}_{\mathrm{i}}
$$

For the four DSTs in Table 3.4, plus Tank SY-103, the in situ whole-tank gas inventories calculated from VFI and RGS measurements were compared with those calculated with the BPE model. The "VFI\&RGS" gas inventories match the volumes calculated with the BPE model within an error of $\pm 28 \%$ of the "VFI\&RGS" value or smaller (based on Schienbein et al. 1999, Table 2.7.5).

Though the comparison was done only for DSTs, the BPE method can also be applied effectively in other tanks with a sufficiently accurate and frequent surface level measurement (daily readings with an automatic Food Instrument Corporation (FIC) contact probe suffice, hourly readings with an Enraf ${ }^{\circledR}$ buoyancy gauge are best) to provide a clean level-pressure correlation (dL/dP) with reasonably low uncertainty. The level reading must represent the entire waste volume. This requires that the waste be "wet" (i.e., the ILL nearly equal to the surface level) to transmit hydrostatic pressure uniformly and that the level measurement be away from any hard deposits attached to the tank wall. In addition, the waste level should not be affected during the level measurement period of record by operational activities such as mixer pump operation or salt-well pumping. Finally, the BPE inventory is less likely to be correct in tanks that, like A-101 and AX-101, store all their gas in a floating upper layer.

The foregoing discussion indicates that the gas inventory estimated from RGS data in DSTs might be off from $24 \%$ (based on VFI scatter alone) to almost $40 \%$ (Table 3.4). In DSTs, the 
differences between BPE and VFI\&RGS gas inventories are of similar magnitudes, within $28 \%$ of the VFI\&RGS value. Because the waste in the SSTs appears to be much more nonuniform, the uncertainty in the gas inventory is likely higher still. We therefore think that, in an SST whose cores exhibit high lateral variability in the waste and that lacks other information on the total gas volume, the uncertainty in the inventory estimated by RGS data alone should be set at $50 \%$. This pertains to gas in nonconvecting layers; in convective or mechanically mixed layers, an uncertainty of $30 \%$ (the lower end of uncertainties found for DST inventories) is more appropriate (unless measurement uncertainty is higher than $30 \%$ ). (Note that the uncertainty in the BPE volume estimate may also approach $50 \%$.)

The choice between using the RGS and BPE gas inventories for an SST depends on numerous factors. The RGS inventory has the advantage of being based on direct measurements; the BPE inventory has the advantage of being based on global (though indirect), rather than local (although direct). Certain features of the data may, if present, contraindicate use of the RGS inventory:

- RGS data are only from one riser and there are insufficient $x$-ray observations from other risers to allow checking for lateral variability

- the RGS riser is in a part of the tank whose level or surface liquidity are atypical

- the RGS data do not show a consistent pattern of volume fraction variation with depth

- the core extrusion appearance varies greatly from riser to riser

- the RGS data are questionable for some reason.

On the other hand, the BPE gas inventory can be contraindicated if

- the surface level data are not from Enraf measurements

- the Enraf riser is near the tank wall and waste beneath it might be attached to the wall

- there is no surface liquid, or the area under the Enraf riser is dry or otherwise atypical

- the period of record is short.

Only if the RGS gas inventory is more doubtful than the BPE inventory (based on the above criteria) should the BPE method be used to estimate the gas inventory, with the effective gas pressure defined based on RGS data.

\subsection{Review of Assumptions Made in RGS Analysis}

The preceding sections have derived models for interpreting RGS extraction data to calculate the insoluble gas and ammonia content of the original sample and for incorporating those data into estimates of in situ gas volume fractions and inventories. Section 3.8.1 provides a review of the assumptions used in deriving the models. Section 3.8.2 compares the assumptions used in this report with those used in the first RGS report (Shekarriz et al. 1997). In general, the assumptions in the second RGS report, Mahoney et al. (1997), were the same as those in the present report.

Table 3.5 contains a summary of the assumptions listed in Section 3.8.1. The table includes estimates of the effects of the assumptions in terms of bias and scatter that are introduced into the 
Table 3.5. Summary of Major Assumptions in RGS Calculations of Sample Gas Concentrations

\begin{tabular}{|c|c|c|}
\hline Assumption & Bias & Comments \\
\hline $100 \%$ sample recovery & $\begin{array}{l}\text { positive }<10 \% \\
\text { all constituents }\end{array}$ & $\begin{array}{l}\text { A few samples may have higher } \\
\text { overestimation; they are noted in } \\
\text { Section } 4 \text {. }\end{array}$ \\
\hline $\begin{array}{l}\text { Insoluble gas and vapor behaves as } \\
\text { ideal gas }\end{array}$ & & No reason to expect nonideality. \\
\hline $\begin{array}{l}\text { Gas in RGS is well mixed } \\
\text { throughout system }\end{array}$ & $?$ & $\begin{array}{l}\text { Impact unknown but no reason to } \\
\text { expect this assumption to be false. }\end{array}$ \\
\hline $\begin{array}{l}\text { Collector-side condensate model } \\
\text { (Section 3.3) is accurate }\end{array}$ & $\begin{array}{c}\text { direction? } \\
\text { ammonia }<10 \% \\
\text { insol. gas }<5 \%\end{array}$ & \\
\hline $\begin{array}{l}\text { Condensate volume in collector } \\
\text { side is negligible }\end{array}$ & & $\begin{array}{l}\text { Less than } 0.3 \% \text { of collector-side } \\
\text { volume is taken up, trivial effect. }\end{array}$ \\
\hline $\begin{array}{l}\text { Insoluble gas is completely } \\
\text { extracted }\end{array}$ & $\begin{array}{c}\text { negative } \\
\mathrm{N}_{2} \mathrm{O}<20 \% \\
\text { other gas }<10 \%\end{array}$ & \\
\hline $\begin{array}{l}\text { Insignificant amount of ammonia is } \\
\text { lost to sorption }\end{array}$ & $\begin{array}{c}\text { negative } \\
\text { ammonia }<10 \%\end{array}$ & $\begin{array}{l}\text { Decreases extracted ammonia, which } \\
\text { is } 20 \% \text { or less of total. }\end{array}$ \\
\hline $\begin{array}{l}\text { Ammonia vapor reaches } \\
\text { equilibrium shortly after extrusion }\end{array}$ & $\begin{array}{l}\text { direction varies } \\
\text { with tank } \\
<50 \%\end{array}$ & $\begin{array}{l}\text { Size of effect not estimated; } \\
\text { probably less than } 50 \%\end{array}$ \\
\hline $\begin{array}{l}{ }^{15} \mathrm{NH}_{3} / 14 \mathrm{NH}_{3} \text { equilibrium is } \\
\text { reached in the vapor before final } \\
\text { extraction }\end{array}$ & $\begin{array}{l}\text { positive } \\
<100 \% ?\end{array}$ & $\begin{array}{l}\text { Data do not consistently indicate the } \\
\text { large bias value. }\end{array}$ \\
\hline $\begin{array}{l}\text { Sorption does not significantly } \\
\text { affect the }{ }^{15} \mathrm{NH}_{3} / 14 \mathrm{NH}_{3} \text { ratios }\end{array}$ & $\begin{array}{c}\text { usually positive } \\
\text { often } 0 \% \\
<100 \% \\
\text { ammonia }\end{array}$ & $\begin{array}{l}\text { Also increases the scatter in } \\
\text { measurements, because sorption } \\
\text { effect is not consistent. }\end{array}$ \\
\hline
\end{tabular}

RGS results. Because the uncertainty of solubility values and the uncertainties in gas inventories have been discussed in Sections 3.6 and 3.7, they are not included in the table. Positive bias indicates the possibility of overestimation, negative bias underestimation.

\subsubsection{Current Assumptions}

Assumption

The available sampler volume (back to the piston) is filled with tank waste (gas and liquid). That is, 100\% sample recovery is assumed. This assumption (used in Section 3.4) is not necessarily true; it depends on the resistance to movement of the waste during sampling, which may be due either to the cohesive strength of the waste or to drag along the walls of the sampler. This effect might result in the 
waste not filling.the sampler and leaving a void at the top of the sample.(a) However, the assumption of $100 \%$ recovery is unavoidable because, lacking a method for measuring pressure in the sampler and comparing it with in situ pressure, we cannot tell whether the void spaces in X-ray images result from retained gas volume or from incomplete sample recovery. Thus we cannot quantify the impact of this assumption, but we believe it leads to less than $10 \%$ underestimation of gas content in samples with less than complete sample recovery. This statement is based on a semi-quantitative review of $\mathrm{x}$-ray images of samplers and a comparison of their voids with the extracted gas content (see Section 4 for tank-by-tank review).

\title{
Assumption
}

The gas/vapor species behave like ideal gases under the temperatures $\left(25\right.$ to $\left.70^{\circ} \mathrm{C}\right)$ and pressures ( 0.02 to $3 \mathrm{~atm}$ ) applicable to the RGS extraction process and to in situ conditions. This assumption (used in the preceding sections) holds for ammonia vapor as well as for the low-solubility species; ammonia's critical pressure and temperature are $112.5 \mathrm{~atm}$ and $132.5^{\circ} \mathrm{C}$. The reduced pressure $\left(\mathrm{P} / \mathrm{P}_{\mathrm{c}}<0.01\right)$ is so low that the compressibility factor of ammonia is effectively unity.

\begin{abstract}
Assumption
The gas/vapor in the interconnected parts of the RGS system is well mixed such that its composition is the same everywhere. The assumption (used in Sections 3.1 and 3.2) depends on the mixing in both the extractor side and the collector side that comes from sudden volume changes caused by pumping.
\end{abstract}

\section{Assumption}

The water-ammonia equilibrium relations for bulk $\mathrm{NH}_{4} \mathrm{OH}$ solutions correctly predict the behavior of the films of condensate in the collector side. This assumption is used throughout Section 3.3 and has not been tested, so if there is a bias its direction is unknown. The assumption strongly affects the calculation of dissolved $\mathrm{NH}_{3}$ in the collector-side condensate. It also indirectly affects the calculation of insoluble gas and $\mathrm{NH}_{3}$ vapor by affecting the sample water vapor pressure. Condensate ammonia is usually less than $10 \%$ of the total ammonia, because most of the ammonia remains dissolved in the sample. The water vapor pressures calculated using the assumption were generally not far different from those measured for simulants by Norton and Pederson (1994). A comparison of RGS-calculated and simulant-measured water vapor pressures was made by Mahoney et al. (1997) for samples from U-103, S-106, and BY-109, and showed no more than $0.5 \mathrm{kPa}$ error in the calculated pressures. Because collector-side total pressures usually exceeded $20 \mathrm{kPa}$, error in the water vapor pressure probably causes less than $5 \%$ error in the calculated ammonia vapor and insoluble gas.

\section{Assumption \\ The volume of condensate trapped in the collector side is negligible because the density of the liquid is so much greater than that of the vapor. This assumption (used in Section 3.3) is based on calculations that indicate that less than $0.3 \%$ of the collector side volume (or $0.3 \mathrm{~mL}$ ) is taken up by condensate. In addition, experi- mental observations of the transparent walls of the mercury pump showed only a mist of condensate, not droplets or larger amounts.}

(a) Shekarriz A and JD Norton. 1995. Retained Gas Sampler System Analysis. PNLFGP:091595, Pacific Northwest Laboratory, Richland, Washington. 


\title{
Assumption
}

The low-solubility gases are completely extracted from the sample to the collector or the extractor headspace; no significant amount is lost as a result of deposition on equipment surfaces, dissolution in the canister condensate, or dissolution in the waste sample. The mole fraction information for each canister shows the extent to which the assumption of complete gas extraction (e.g., low solubility) used in Sections 3.1-3.4 is consistent with the data. For truly insoluble gases, the relative composition of the insoluble gases (excluding ammonia) will be the same for all canisters of a sample. The results of this comparison are presented in the summary worksheets of the RGS calculation spreadsheets on the CD that accompanies this report. Given the measurement uncertainties, the values for hydrogen, nitrogen, oxygen, and argon are relatively constant as a percentage of the total. However, it does appear that the fractions of nitrous oxide, methane, and the other hydrocarbons increase as the insoluble gases are removed from the sample, indicating the possibility of a solubility effect. Based on a review of the decrease in moles of various gas species with each successive canister of gas extracted, the effect on most insoluble gas species is thought to be underestimated by less than $10 \%$. Nitrous oxide is more affected but expected to be underestimated by less than $20 \%$. A further discussion of extraction completeness can be found in Section 5.2.3 of Mahoney et al. (1997).

\begin{abstract}
Assumption
No significant amount of ammonia is lost from measurement because of deposition on equipment surfaces. This assumption is used in Sections 3.1-3.4. RGS system tests have been made using gas standards with low ammonia concentrations and no water. Under those conditions, approximately $50 \mu \mathrm{mol}$ of ammonia were lost. This finding is order-of-magnitude consistent with the maximum of $20 \mu \mathrm{mol}$ of $\mathrm{NH}_{3}$ that could be held by a monolayer in the apparatus whose surface area is $0.6 \mathrm{~m}^{2}( \pm 50 \%)$. Based on preliminary tests, the ammonia lost to surfaces (in the absence of condensation) would cause no more than a $10 \%$ underestimation for most samples. However, the percentage losses may have been more substantial for canisters with small ammonia contents: unpumped canisters or canisters collected from very low-ammonia samples. In addition, the apparent holdover of $15 \mathrm{NH}_{3}$ in a few samples (Mahoney et al. 1997, Section 5.4.4) is evidence that absorption occurs. The quantity of $15 \mathrm{NH}_{3}$ holdover was $15 \mu \mathrm{mol}$, so the holdover of $15 \mathrm{NH}_{3}$ and ${ }^{14} \mathrm{NH}_{3}$ together must have been higher; this amount is consistent with other findings.
\end{abstract}

\section{Assumption}

The ammonia in the waste sample is assumed to reach equilibrium with the extractor headspace after sample extrusion and before the expansions that result when the extractor is opened to other parts of the system. This assumption, used in Section 3.4, is to some extent inaccurate because pressure-time plots for the extractor side show that after extrusion the pressure seldom reached a steady value before the next expansion. In samples from tanks before Tank SX-106, the extractor-side pressure almost always rises after extrusion. In samples from SX-106 and later, the extractor-side pressure almost always declines after extrusion is complete. The difference may have something to do with the lower extractor temperature maintained for the later tank samples, starting with SX-106. The impact of this error is not yet quantified but probably causes underestimation of ammonia vapor pressures in the early tanks and overestimation in the later tanks. Further study would be needed to quantify the extent of underestimation, but a 50\% bias is thought to be the maximum possible. 


\section{Assumption}

The equilibration of ammonia vapor and solute is substantially complete for both ${ }_{15} \mathrm{NH}_{3}$ and $14 \mathrm{NH}_{3}$ at the time when vapor is extracted. Extractions that were carried out at several different times after adding $15 \mathrm{NH}_{4} \mathrm{OH}$ solution usually showed substantial differences between the $15 \mathrm{~N} / 14 \mathrm{~N}$ ratios measured one or two hours after adding the standard and those measured a day or more after addition. Only small differences were seen between the ratios at one day and those at up to seven days, suggesting that an equilibration time of one day was adequate for the solution standard. However, one sample left for 20 days after the standard was added had a ratio that was twice the value of any other sample from the tank (see Tables 4.10.6, 4.12.7, and 4.13.6). This observation may have been a fluke but raises the possibilities that the required equilibration time is on the order of weeks rather than a day and that failure to equilibrate could cause residual ammonia to be overestimated by a factor of 2 . Further work is needed to document existing data and conduct and analyze ammonia standard test data to quantify the importance of equilibration effects.

\section{Assumption}

Sorption does not affect the $15 \mathrm{~N} / 14 \mathrm{~N}$ ratios measured by mass spectrometry. The ratios, in fact, show a tendency to change (usually to increase) as subsamples are taken from the same canister. In many cases, the increase levels off within three or four subsamples; in some cases, the final subsample gives an isotopic ratio that is a factor of 2 different from the first few subsamples (see Tables 4.12.6 and 4.13.5). This effect could cause a maximum of a factor of 2 error in either direction in ammonia measurements in those samples where only one subsample was taken per extraction step. The variation is attributed to sorption. In addition, the apparent holdover of $15 \mathrm{NH}_{3}$ in some BY-109 samples but not others (Mahoney et al. 1997, Section 5.4.4) is evidence that sorption occurs but is not consistent. The BY-109 holdover produced $15 \mathrm{NH}_{3} / 14 \mathrm{NH}_{3}$ ratios that were between 0.05 and 0.09 when they should have been zero. Holdover affected fewer than half of the BY-109 samples.

\section{Assumption}

Gases are in equilibrium between the gas/vapor and liquid phases under tank conditions, and both phases are at the same temperature. This assumption, which is used in Section 3.6, should hold true for undisturbed waste.

\section{Assumption}

The Schumpe model provides an accurate eslimate of the Henry's Law constants for each gas constituent in salt solution as long as the correct concentrations of ions are used in the waste solution. This assumption is used and discussed in Section 3.6, its uncertainty bracketed by the method detailed there.

\section{Assumption}

The ionic concentration of the waste solution is uniform throughout each tank. This assumption is used in Section 3.6. The concentrations were taken from analyses of drainable liquid from the 1997 core samples and are probably the best concentration data available. The effect on gas volume fraction of nonuniformity in the solution salt concentration is significant only for ammonia and depends on the ammonia concentration in the tank. (Here "significant" means a change that is large compared with the measurement uncertainty.) In a high-ammonia tank like SY-101 (the best example among RGS-sampled tanks), doubling the total salt concentration can increase the gas volume fraction by as much as $50 \%$ because ammonia becomes 
less soluble in the higher-salt solution and thus enters the gas phase, increasing its volume. In a low-ammonia tank, doubling the salt increases the gas volume fraction by no more than $5 \%$ of its value. In both types of tanks, doubling the salt multiplies the ammonia concentration in the vapor phase by a factor of 4 , and halving the concentration has substantially less effect than doubling it. The range of variation of the solubility when the total salt is doubled or halved is a factor of 4 to 16 , depending on the dissolved species.

\section{Assumption}

The ionic concentration of the waste solution in the tank is negligibly different from that measured for tank samples at laboratory temperatures. In fact, temperature has a noticeable effect on the salt solubilities, and samples that are entirely liquid in high-temperature tanks (such as A-101) may contain substantial amounts of solids at laboratory temperature, indicating that cooling causes a decrease in ionic concentrations in solution. The solubilities in water of common waste salts such as sodium nitrate, nitrite, and carbonate increase by $70 \%$ or more, from 25 to $70^{\circ} \mathrm{C}$ (CRC 1975). Because this effect was included in the measurements made for the heterogeneous simulant discussed in Section 3.6.1, the solubility-bracketing assumptions stated there should account for the effect of temperature on ionic concentrations.

\section{Assumption}

The hydrostatic head (in situ pressure) can be calculated with sufficient accuracy by treating each layer as having a uniform density equal to its average density. This assumption is used in Section 3.6. Waste layers are distinguished from one another, for this report, by their thermal behavior (temperature profiles), their physical properties as found in core samples, and their gas retention characteristics. (Tank-specific layering details are presented in Section 4.) A typical error in calculating hydrostatic head might involve having $1 \mathrm{~m}$ less or more of a layer than had been anticipated at a calculated pressure of $1.5 \mathrm{~atm}$. Then the difference in pressure might be $400 \mathrm{~kg} / \mathrm{m}^{3}$ (a typical difference between slurry and liquid densities) times the depth difference times gravity, or about $4 \mathrm{kPa}(0.04 \mathrm{~atm})$. Thus the errors from density and layer depth variation probably cause an error contribution of less than $5 \%$ of the gas volume fraction (which is inversely proportional to pressure).

\section{Assumption}

The pressure experienced by the bubbles is that of atmospheric pressure plus the hydrostatic head of the bulk waste. That is, the bubbles are supporting both the particles and the liquid above them, and the capillary pressure is not significant. (large compared with the uncertainty in the calculated hydrostatic pressure.) This assumption is used in Section 3.6. In the alternative case, the bubbles are confined to the pores of the waste and support only the liquid in the pores; the particles are self-supporting. In this case, bubbles experience hydrostatic pressure from the liquid alone. Because most of the waste in the five tanks is fine-grained and the pores are small, the gas bubbles are expected to be particle-supporting (as assumed in calculations). However, bubbles could be confined to pores in 1) extremely strong waste, 2) near the tank bottom, or 3) more than $50 \mathrm{~cm}$ (20 in.) deep in coarse saltcake (Stewart et al. 1996b, Section 3.1). We do not have enough data on particle size and waste strength to confirm the assumption of particle-supporting bubbles in all cases. But coarse saltcake and very strong wastes are difficult to sample using push mode, and it is unlikely that RGS samples contained these materials. If bubbles in coarse saltcake were not particle-supporting, the in situ gas volume fraction would be underestimated by less than $15 \%$. 


\title{
Assumption
}

All variables are laterally uniform; variation between risers is not accounted for in calculations. This assumption (used in Section 3.7) is necessary because there are too few measurements to allow a sound statistical assessment of the effects of lateral variability. Calculating "alternative" inventories based on each riser alone would have little meaning because for many tanks there would be only one or two samples per layer per riser. Lateral variability estimates are discussed in

Section 3.7.2.

\section{Assumption}

The convective layer is assumed to be well mixed, so temperature and gas concentration (mol/L) are vertically uniform. This assumption (used in Section 3.7) is equivalent to assuming a gas volume fraction that varies with the inverse of depth. In most cases, this assumption is necessary because samples were concentrated in the nonconvecting layer(s) of the tank, where most of the gas is retained, and at most one sample was taken from the convective layer.

\begin{abstract}
Assumption
Concentration within nonconvecting layer varies linearly between the vertical locations at which samples were taken (the elevations of the sampler centers). Again, we have no data to permit any more accurate assumptions than this one (which is used in Section 3.7). VFI results for earlier tanks (Shekarriz et al. 1997) suggest that total gas concentrations (gas volume fractions) may not behave monotonically.
\end{abstract}

In summary, the assumptions under which the low-solubility gas data analysis is conducted are believed to bias the sample gas concentrations on the low side. The total underestimation is probably $10 \%$ or less because of incomplete sample recovery in a few cases, but this cannot be well quantified. Further studies would be needed to put ammonia on the same footing as the lowsolubility gases.

The assumptions in the in situ gas volume fraction method have less than a $\pm 10 \%$ effect on the gas volume fraction in tanks with low ammonia content. However, errors in the ammonia solubility could significantly increase or decrease the gas volume fraction in high-ammonia, high-gas tanks (SY-101 is the most notable example). (Significant here means a change that is large compared with the measurement uncertainty.) The in situ ammonia vapor concentrations are also sensitive to the solubility (proportional to it) in all the tanks measured to date.

The uncertainty in determining the number of moles of each species that were extracted from the sample is a function of the uncertainty in the collector-side pressure measurement, system volume measurement, collector-side temperature measurement, and the mole fraction measurements provided by mass spectrometry. The measurement uncertainties can be found in the sample worksheets in the RGS calculation spreadsheets on the CD that accompanies this report. The cumulative measurement uncertainty is found using the rms approach of Klein and McClintock as described in Holman (1978).

\subsubsection{Assumptions Used in Previous RGS Analyses}

In the first year of the program, certain assumptions that are no longer necessary were made in the RGS analyses that were carried out for Tanks AW-101, AN-105, A-101, AN-104, and AN-103 (Shekarriz et al. 1997): 
- In the current model (Section 3.3), the water vapor pressure in the pumped J canisters is calculated as the water vapor pressure over an ammonia-water solution. In the first-year model, the water was assumed to have the vapor pressure of pure water. The resulting underestimation of gas and ammonia vapor was estimated to be $5 \%$ or less in cases where the in situ gas volume fraction was greater than $2 \%$. Even in samples from convecting layers, which retain much less gas and produce lower total canister pressures, the underestimation of low-solubility gas and ammonia vapor resulting from this assumption was estimated to be $10 \%$ or less.

- In the current model (Sections 3.1 through 3.4), the water vapor pressure for unpumped J canisters is assumed to be the vapor pressure over the waste, which is calculated based on RGS extraction data. In the first-year model, the water vapor pressure over the waste was calculated based on correlations from simulant data (Mahoney and Trent 1995, Equation 6.2 and Table 6.2). This model tended to predict lower vapor pressures than have been calculated from RGS data (Mahoney et al. 1997) and led to an overestimation of the gas and ammonia vapor in the sample. However, these relatively low-pressure unpumped canisters typically do not contribute much of the total extracted gas for a sample, so a typical 10\% error in gas content of an unpumped canister produced an overestimation of only 1 or $2 \%$ in the total sample gas.

- The current model accounts for the gas and ammonia vapor that leave the RGS system in grab samples and partial evacuations. The first-year model did not account for these losses, resulting in an underestimation of less than $2 \%$ of the gas and ammonia vapor in the system.

- In the current model, the part of the extracted ammonia that is dissolved in water condensate on the canister (collector) side of the vacuum pump is accounted for. The first-year model did not include the condensed ammonia, which caused the extracted sample ammonia to be underestimated by a factor between 1.5 and 3 (Mahoney et al. 1997).

- The first-year model assumed, in calculating the residual ammonia, that the ammonia vapor in the extractor was in equilibrium with the dissolved ammonia in the sample at all times. No account was taken of diffusional mass-transfer limitations that make the approach to equilibrium a slow one. The current model either uses ammonia partial pressure to calculate in situ ammonia vapor pressure or measures the residual ammonia by adding an isotopic standard. The first-year model is suspected of having underpredicted the residual ammonia such that the total (residual plus extracted) ammonia could have been considerably underestimated (see Sections 4.1 through 4.5). Further work would be needed to quantify the extent of underestimation.

The experimental procedures used during RGS extractions have also changed since the first year (Shekarriz et al. 1997). Procedures are discussed in more detail on a tank-by-tank basis in Section 4 , but the most noteworthy changes can be summarized here:

- A $15 \mathrm{NH}_{3}$ standard is introduced into the system to allow the residual (postpumping) ammonia in the waste to be determined; the ratio of the isotopic to the normal $14 \mathrm{~N}$ ammonia in the waste is measured by mass spectrometer. A long time is required for equilibration of the isotopic ammonia with the ammonia in the waste, and equilibrium may not have been complete for samples extracted during FY 1998 (Mahoney et al. 1997, Sections 5.4.2 and 5.4.3). The ammonia in those samples 
(from U-103, S-106, BY-109) is suspected to be underestimated by an unknown factor. Holdover of the isotopic ammonia from sample to sample has also appeared (Mahoney et al. 1997, Section 5.4.4).

- The sample no longer undergoes thermal cycling during extraction. Therefore, temperature-related ammonia solubility changes have not been an issue, and the solubility of ammonia can be assumed more confidently not to change during extraction.

- In the third year of the program, FY 1998 (starting with Tank SX-106), a "vapor profile" step was instituted in the extraction. After the initial extraction of most of the gas, a sequence of canisters was collected in which each canister contained only one pump stroke of gas and vapor. The vapor profile results were intended to show how gas and ammonia concentrations changed during a pumping sequence, which could not be seen in the usual extraction where a number of strokes were collected into one canister. Not all pump strokes in the sequences were collected (every third stroke or every tenth, depending on the sample). The contents of the uncollected strokes were evacuated and were therefore estimated by linear interpolation between the collected canisters. Linear interpolation tended to overestimate the gas. The error is estimated to be less than $10 \%$ of the total sample gas, and the additional uncertainty is incorporated in the measurement error band.

- Administrative and scheduling problems made it impossible to carry out PQ canister grab sample analyses after the tanks included in the first report. In that study, these measurements provided direct data for the ammonia partial pressure under laboratory conditions and gave a useful cross-check on ammonia determination.

\subsection{X-Ray Image Analysis}

Analyses of x-ray images are expected to yield several pieces of information that will assist in data interpretation and understanding of the waste characteristics. The most notable parameter that can be extracted from these images is density. In a less quantitative fashion, the phase distribution can be obtained from these images as well. Furthermore, information on where the gas phase is concentrated and how it is distributed, the structure of the solid matrix/particle agglomerates, and the amount of gas can be inferred. Over the course of the RGS program, minor changes were made to the original procedure (that described in Shekarriz et al. 1997) that were documented in the final procedure (Mahoney et al. 1997).

\subsubsection{General Background on X-Ray Image Processing}

The processing approach yields line-of-sight averaged information on the density of the material which fills the sampler. The approach does not offer the ability to obtain local information along the line-of-sight; the system has no "depth perception." As such, the phases might be distributed in many different ways and still produce the same images and results. For instance, we can see that there is a void in the waste and measure its size, but we cannot tell where, front to back, the void is located. Thus the $x$-ray image analysis technique offers a two-dimensional map of phase distributions in the core sampler.

Within half an hour (typically) after sampling, each segment is radiographed using the x-ray imaging system described in Appendix A of Shekarriz et al. (1997), and the radiographs are recorded on video tape. Later, the video for each segment is converted to a grayscale digital image format for analysis. The analysis begins by preparing the air and water standards and extracting the core sample regions of interest from the full-frame video images. Attenuation coefficients for 
the waste and the water standard are then calculated by applying a logarithmic relationship derived from Beer's law (see Shekarriz et al. 1997 for more details). The density (in terms of specific gravity) of the waste is obtained by dividing the attenuation coefficients of the waste by the mean attenuation coefficient for water obtained from the air and water standard sample.

Accurate analysis of x-ray images depends on the invariance of the radiography system between imaging the standards and the waste sample. If either the $\mathrm{x}$-ray source strength or the iris on the $\mathrm{x}$-ray imaging camera are adjusted between the radiography of the standards and completion of the waste radiography, the standards may no longer be valid and analysis may not yield reliable results. The air standard is required to compensate for the attenuation of the steel RGS sampler walls. The water standard is used to derive the waste density from the attenuation coefficients.

The original analysis procedure and its technical basis have been described in detail in Shekarriz et al. (1997), with updates noted in Mahoney et al. (1997). The changes that distinguish the two versions of the procedure are summarized in Table 3.5.

\subsubsection{Guide for Viewing X-Ray Images}

The CD supplied with this report contains a number of $\mathrm{x}$-ray images; this section is provided as a guide to their interpretation. Figure 3.5 shows the image subsegments, which are marked with elevations (in feet) to identify their relative position in the sampler more conveniently . These elevations (which are relative to the sampler bottom) should be referred to when looking at the tank-specific images provided. Note that the lower part of the piston usually appears in the highest subsegment, typically at $1.50 \mathrm{ft}$; and the top of the valve housing usually shows up in the lowest subsegment, at $0.25 \mathrm{ft}$. Also, the cable(s) for the yalve trigger mechanism occasionally show(s) up in the images. Some of the more recent images contain circumferential scribe marks that are located 2 in. apart on the sampler outer wall to aid in locating and identifying waste features. These appear as pale horizontal lines across the whole width of the sampler.

Several additional points have a bearing on the information that can be obtained from the density images. First, only features containing several pixels (a typical pixel is about $0.25 \mathrm{~mm}$ in size) can be interpreted as being real. That is, individual pixels embedded in a matrix of a different color/background do not necessarily imply the presence of very local large density gradients. This is consistent with our earlier estimate that the minimum resolvable feature size was on the order of $0.5 \mathrm{~mm}$. Second, the errors associated with image analysis are greatest near the sampler wall, and in the subsequent reporting of average density, we have therefore elected to discard the first 10 pixel rows on both the right and left sides of the image. This means that effectively only the central $-80 \%$ of the density image is used in the average density measurements, as reported and shown in Section 4 . Therefore, in viewing and interpreting the density images, caution needs to be exercised regarding the density and features present near the walls, even with the images restricted to a central "core," as described above. Third, the accuracy of these reported measurements is estimated to be, on the average, within about $5 \%$ of the true value.

Because this report is intended to both complete and summarize the RGS task, several innovations were introduced to maximize the accessibility of the $x$-ray waste images and to increase their information content. For the former, a CD with all the waste images taken over the four years of tank waste sampling is included with this report. It contains all images that might possess any interesting visual features, as subjectively determined by the analyst. Although a serious effort was made to present all images in a consistent format, this goal was not achieved in practice because of the variability in the stored images and because it was deemed not to be cost-effective to 
Table 3.6. Differences Between Original and Final X-Ray Image Analysis Techniques

\begin{tabular}{|c|c|c|}
\hline Original method/equipment & Final method/equipment & Reason for change \\
\hline $\begin{array}{l}\text { AW-101, AN-105, } \\
\text { A-101, AN-104, } \\
\text { AN-103 }\end{array}$ & $\begin{array}{l}\text { U-103, S-106, } \\
\text { BY-101, BY-109, } \\
\text { SX-106, AX-101, } \\
\text { S-102, S-111, U-109, } \\
\text { SY-101 }\end{array}$ & \\
\hline PC & $\begin{array}{l}\text { Silicon Graphics workstation } \\
\text { with GraphicConverter 2.1.2 } \\
\text { or G3 Macintosh with Micro } \\
\text { Conversions VP-VW4M-PCI } \\
\text { multimedia card }\end{array}$ & Equipment availability \\
\hline Image Pro Plus 2.0 & $\begin{array}{l}\text { NIH Image } 1.62 \mathrm{~b} 7 \mathrm{f} \text { from the } \\
\text { National Institutes of Health(a) }\end{array}$ & $\begin{array}{l}\text { Availability; good image } \\
\text { processing capabilities }\end{array}$ \\
\hline Averaging of eight frames & $\begin{array}{l}\text { Single frame (except for } \\
\text { SX-106, riser } 6 \text {, where } 10- \\
\text { frame averaging was tried) }\end{array}$ & $\begin{array}{l}\text { Image quality not substantially } \\
\text { improved by averaging; } \\
\text { frequently, insufficient jitter- } \\
\text { free frames are available for } \\
\text { any averaging }\end{array}$ \\
\hline $\begin{array}{l}50 \text {-line averaging of air and } \\
\text { water standards }\end{array}$ & $\begin{array}{l}20 \text {-line averaging for air; } 50 \\
\text { for water }\end{array}$ & $\begin{array}{l}\text { Air calibration image size } \\
\text { decreased; it limits averaging } \\
\text { to about } 20 \text { lines }\end{array}$ \\
\hline Creation of composite image & No composite image & $\begin{array}{l}\text { Time-consuming task that } \\
\text { adds no new information }\end{array}$ \\
\hline $\begin{array}{l}\text { Creation and application of } \\
\text { correction matrix }\end{array}$ & Not used & $\begin{array}{l}\text { A separate valid correction } \\
\text { matrix is not available; past } \\
\text { use indicated negligible effect } \\
\text { on results }\end{array}$ \\
\hline $\begin{array}{l}\text { Grayscale images with } \\
\text { inverted density scale }\end{array}$ & $\begin{array}{l}\text { False color and grayscale } \\
\text { images with intensity } \\
\text { proportional to density }\end{array}$ & $\begin{array}{l}\text { Improvement in image } \\
\text { comprehensibility; low-density } \\
\text { bubbles now appear lighter } \\
\text { than the darker, higher-density } \\
\text { waste }\end{array}$ \\
\hline \multicolumn{3}{|c|}{$\begin{array}{l}\text { (a) Analysis performed on a Macintosh computer using the public domain NIH Image 1.62b7f } \\
\text { program (developed at the U.S. National Institutes of Health and available on the Internet at } \\
\text { http://rsb.info.nih.gov/nih-image/). }\end{array}$} \\
\hline
\end{tabular}

recapture the images from the original tapes. The images on the $\mathrm{CD}$ are most often presented in both grayscale and color versions that were pasted into a text document using NIH Image 1.62b7f. They can be individually selected, copied, and pasted into a graphics program such as Adobe Photoshop for additional processing/enhancement by the reader.

Another innovation that was used on Tank SY-101 samples and unfortunately met with only very limited success was the attempt to reconstruct the two-dimensional X-ray images into a single three-dimensional image. Even though two sets of images of each waste sample were taken late in the RGS program, in orthogonal planes, the irregularity of waste features and densities was 


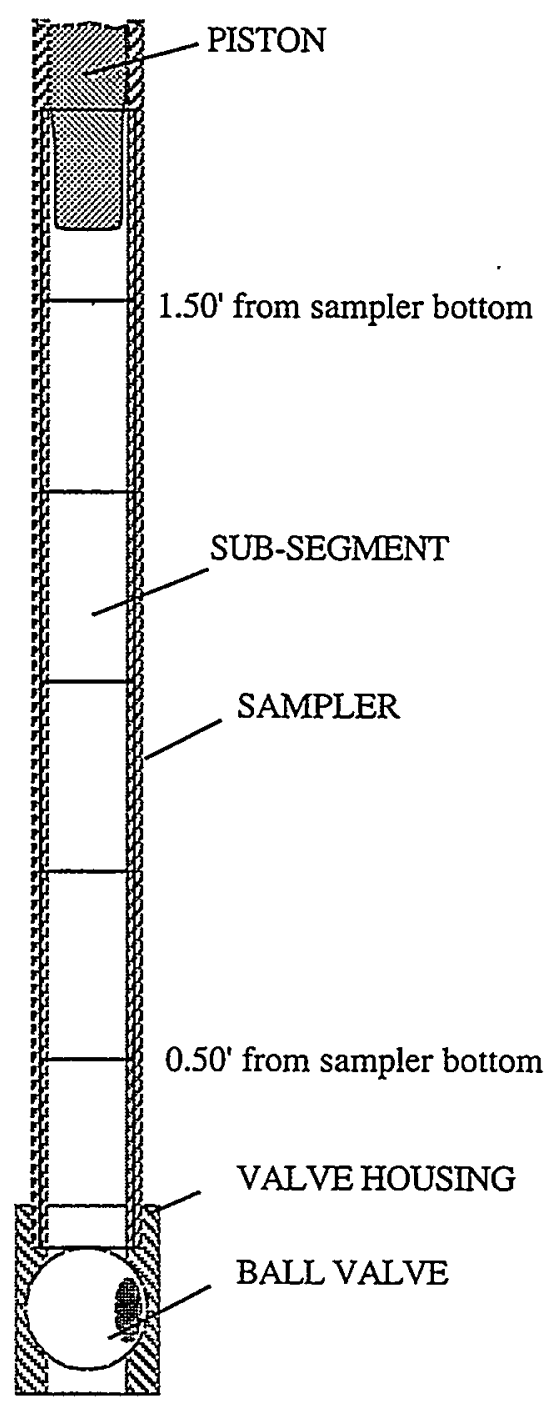

Figure 3.5. Schematic Diagram of X-Ray Subsegments

too great for any mathematical approach to create a meaningful three-dimensional image from only two intersecting planes. However, the additional $\mathrm{x}$-ray images taken in the orthogonal planes were videotaped using a high-resolution charge-coupled device (CCD), and these images frequently show a considerably higher level of detail (e.g., sub-millimeter bubbles) than the standard images.

Yet another enhancement was recalculation of the specific gravities (densities) of many of the waste samples originally documented in the first RGS report (Shekarriz et al. 1997). Some of the densities calculated by the original $\mathrm{x}$-ray analysis procedure, especially for Tanks AN-103 and AN-104, were considerably higher than could reasonably be expected. The recalculations were done with care, using the best available air and water standards that, whenever possible, were matched (air standard) against voids in the waste sampler. The waste images were not recaptured from the original tapes, but stored images were used; these were minimally processed (no image smoothing or contrast enhancements were done) and analyzed. The recalculated specific gravities are generally lower than those originally reported, typically within 10-15\% of the original values, and somewhat more self-consistent. Given the relatively large subjective component in image and calibration selection and their variability, both calculations were believed to be valid within the imposed procedural and equipment constraints. 


\subsection{Tank-by-Tank RGS Results}

This section documents the RGS results from all the tanks that underwent RGS sampling. The RGS sampling program took place during calendar years 1996 (AW-101, AN-105, A-101, AN-104, and AN-103), 1997 (U-103, S-106, BY-101, and BY-109), 1998 (SX-106, AX-101, S-102, S-111, U-109, and SY-101), and 1999 (SY-101). The tank results in this section are presented in the order in which the tanks were sampled.

The gas extraction procedures varied over the years and are described briefly in each tank's subsection. The physical models (algorithms) described in Section 3 were applied to the extraction data to calculate the species concentrations in each sample and then to find the tank inventories. The results of these calculations are found in this section.

There have been two earlier reports covering RGS results. The first, Shekarriz et al. (1997), covered Tanks AW-101, AN-105, A-101, AN-104, and AN-103. The second, Mahoney et al. (1997), covered Tanks U-103, S-106, BY-101, and BY-109. The results given in those reports have been re-examined and revised in several ways for inclusion in this report.

The primary change has been in the interpretation of RGS ammonia data and the comparison of RGS ammonia data with other ammonia measurements. Our later work has led us to believe that the ammonia concentrations reported for Tanks AW-101, AN-105, A-101, AN-104, AN-103, U-103, S-106, and BY-109 in Shekarriz et al. (1997) and Mahoney et al. (1997) were for the most part substantial underestimates. Other revisions include accounting for HHF intrusion, more accurate water vapor estimates, improved data inputs for solubility calculations, and more comparisons between the gas measured by extraction and that visible in the $x$-ray, among many others. The aggregate impact of these changes on composition and gas volume fraction generally ranged between 10 and $30 \%$ of the previously reported values.

The RGS results are presented separately for each tank in Section 4.n, where $n$ is the tank sequence number. For each tank, the sample locations and timing, waste and layer levels and waste densities and types are given. The extraction procedure is briefly described in Section 4.n.1. Then, in Sections 4.n.2 and 4.n.3, the compositions and gas volume fractions are given, along with gas inventory estimates. The features of the waste that were visible in $\mathrm{x}$-ray images of the RGS samplers are described in Sections 4.n.4. Unusual or unprecedented results (when there are any) are highlighted in Sections 4.n.5.

For future reference, note that in some sections of this report the RGS samples are referred to by their riser number and segment (for example, 8-2). In other sections, particularly when comparisons to other tanks' samples are being made, the samples are referred to by tank name and segment (for example, U-109-2). Note also that "old" riser numbers are used in Section 4 for double-shelled tanks (DSTs) to allow easier reference to prior documents. Table 4.1 shows the correspondences between old and new riser numbers for DSTs, and also includes the core numbers for the risers at which RGS samples were taken. Table 4.2 shows the correspondences between riser numbers and core numbers for SSTs, which still employ the old numbering scheme.

The details of the RGS analysis calculations and the inputs to the calculations can be found in the calculations spreadsheets on the CD supplied with this report. The raw data from extraction are also on the CD in text-file and spreadsheet form. These files contain more detail than is appropriate for this report and are provided to support reproducible ancillary calculations. 
Table 4.1. Old and New Riser Numbers and Core Numbers for DSTs

\begin{tabular}{|c|c|c|c|c|c|c|c|}
\hline DST & $\begin{array}{l}\text { Old } \\
\text { Riser }\end{array}$ & $\begin{array}{l}\text { New } \\
\text { Riser }\end{array}$ & $\begin{array}{c}\text { Core } \\
\text { Number }\end{array}$ & DST & $\begin{array}{l}\text { Old } \\
\text { Riser }\end{array}$ & $\begin{array}{l}\text { New } \\
\text { Riser }\end{array}$ & $\begin{array}{c}\text { Core } \\
\text { Number }\end{array}$ \\
\hline \multirow[t]{8}{*}{ AW-101 } & $1 \mathrm{~A}$ & 001 & & \multirow[t]{7}{*}{ AN-103 } & $1 B$ & 003 & \\
\hline & $1 \mathrm{C}$ & 003 & & & $2 \mathrm{~A}$ & 004 & \\
\hline & $2 \mathrm{~A}$ & 004 & & & $4 \mathrm{~A}$ & 006 & \\
\hline & $4 \mathrm{~A}$ & 006 & & & $12 \mathrm{~A}$ & 013 & 166 \\
\hline & $13 \mathrm{~A}$ & 014 & & & $15 \mathrm{~A}$ & 017 & \\
\hline & $15 \mathrm{~A}$ & 017 & & & $16 \mathrm{~B}$ & 018 & \\
\hline & $24 \mathrm{~A}$ & 023 & 132 & & $21 \mathrm{~A}$ & 021 & 167 \\
\hline & $24 \mathrm{~B}$ & 024 & 139 & \multirow[t]{8}{*}{ SY-101 } & $1 \mathrm{C}$ & 001 & \\
\hline \multirow[t]{8}{*}{ AN-105 } & $1 \mathrm{~A}$ & 002 & & & $4 \mathrm{~A}$ & 006 & 257 \\
\hline & $1 B$ & 003 & & & $11 B$ & 011 & \\
\hline & $2 \mathrm{~A}$ & 004 & & & $17 \mathrm{~A}$ & 020 & \\
\hline & $4 \mathrm{~A}$ & 006 & & & 17B & 018 & \\
\hline & 7B & 009 & 153 & & $17 \mathrm{C}$ & 019 & \\
\hline & $12 \mathrm{~A}$ & 013 & 152 & & $22 \mathrm{~A}$ & 021 & 256 \\
\hline & $15 \mathrm{~A}$ & 017 & & & $23 \mathrm{~A}$ & 022 & 255 \\
\hline & $16 \mathrm{~B}$ & 018 & & & & & \\
\hline \multirow[t]{7}{*}{ AN-104 } & $1 \mathrm{~A}$ & 002 & & & & & \\
\hline & 1B & 003 & & & & & \\
\hline & $2 \mathrm{~A}$ & 004 & & & & & \\
\hline & $10 \mathrm{~A}$ & 011 & 163 & & & & \\
\hline & $12 \mathrm{~A}$ & 013 & 164 & & & & \\
\hline & $15 \mathrm{~A}$ & 017 & & & & & \\
\hline & $16 \mathrm{~B}$ & 018 & & & & & \\
\hline
\end{tabular}


Table 4.2. Riser Numbers and Core Numbers for SSTs

\begin{tabular}{|c|c|c|c|c|c|}
\hline SST & Riser & $\begin{array}{c}\text { Core } \\
\text { Number }\end{array}$ & SST & Riser & $\begin{array}{c}\text { Core } \\
\text { Number }\end{array}$ \\
\hline \multirow[t]{2}{*}{ A-101 } & 15 & 154 & \multirow[t]{2}{*}{ SX-106 } & 3 & 20224 \\
\hline & 24 & 156 & & 6 & 223 \\
\hline \multirow[t]{3}{*}{ U-103 } & 2 & 175 & \multirow[t]{2}{*}{$\mathrm{AX}-101$} & 9D & 226 \\
\hline & 7 & 176 & & $9 \mathrm{G}$ & 228 \\
\hline & 13 & 182 & \multirow[t]{3}{*}{ S-102 } & 11 & 125 \\
\hline \multirow[t]{3}{*}{ S-106 } & 7 & 184 & & 14 & 130 \\
\hline & 8 & 183 & & 16 & 232 \\
\hline & 14 & 187 & \multirow[t]{3}{*}{$S-111$} & 6 & 237 \\
\hline \multirow[t]{2}{*}{ BY-101 } & $10 \mathrm{~B}$ & 189 & & 8 & 149 \\
\hline & $10 \mathrm{D}$ & 199 & & 14 & 150 \\
\hline \multirow[t]{2}{*}{ BY-109 } & $10 \mathrm{~B}$ & 203 & \multirow[t]{4}{*}{ U-109 } & 2 & 123 \\
\hline & $12 \mathrm{C}$ & 201 & & 7 & 128 \\
\hline & & & & 8 & 238 \\
\hline & & & & 19 & 124 \\
\hline
\end{tabular}

\subsection{AW-101}

Tank AW-101 was the first tank and the first DST sampled with the RGS. This tank was selected because it exhibited episodic GREs that produced episodic domespace hydrogen concentrations of several thousand ppm. AW-101 was on the FGWL and showed a significant gas volume by the BPE method (discussed in Section 3.7.2). The VFI also measured a significant gas volume fraction the year before RGS sampling (see Section 3.7.2). Most of the tank contents were double-shell slurry feed from the 242-A evaporator (Stewart et al. 1996a).

Push-mode sampling was done in risers 24A and 24B in March and May 1996.(a) Riser $24 \mathrm{~A}$ was sampled because of its proximity to the MIT in riser 15A to correlate the gas composition with local waste temperature. Data from riser $24 \mathrm{~B}$ were expected to be more comparable to the VFI results (Schienbein et al. 1999) taken from neighboring riser 1C in September 1995 (Fig. ure 4.1.1). The approximate locations of various risers are depicted in Figure 4.1.1.

The total depth of waste in Tank AW-101 was approximately $1040 \mathrm{~cm}$ (409 in.); thus, at the time of RGS sampling, AW-101 contained approximately 4,280,000 L (1,130,000 gal) of waste. The RGS risers $24 \mathrm{~A}$ and $24 \mathrm{~B}$ are on the tank periphery. Figure 4.1.2 shows the tank content layering based on Table 2.3.2 of Schienbein et al. (1999). The nonconvective layer was $286 \pm 30 \mathrm{~cm}(112 \pm 12$ in.) in depth, with a convective (supernatant liquid) layer and a $80 \pm 30-\mathrm{cm}$ ( $31 \pm 12$-in.)-thick crust forming the balance of the contents. The elevations of the RGS segments are depicted in Figure 4.1.2.

(a) The sampling scheme may be found in Sampling Plan for Tank 241-AW-101 Retained Gas Sampler Deployment, by JM Bates and R Shekarriz, December 1995. PNLMIT:122195 Rev. 0, Pacific Northwest National Laboratory, Richland, Washington. 


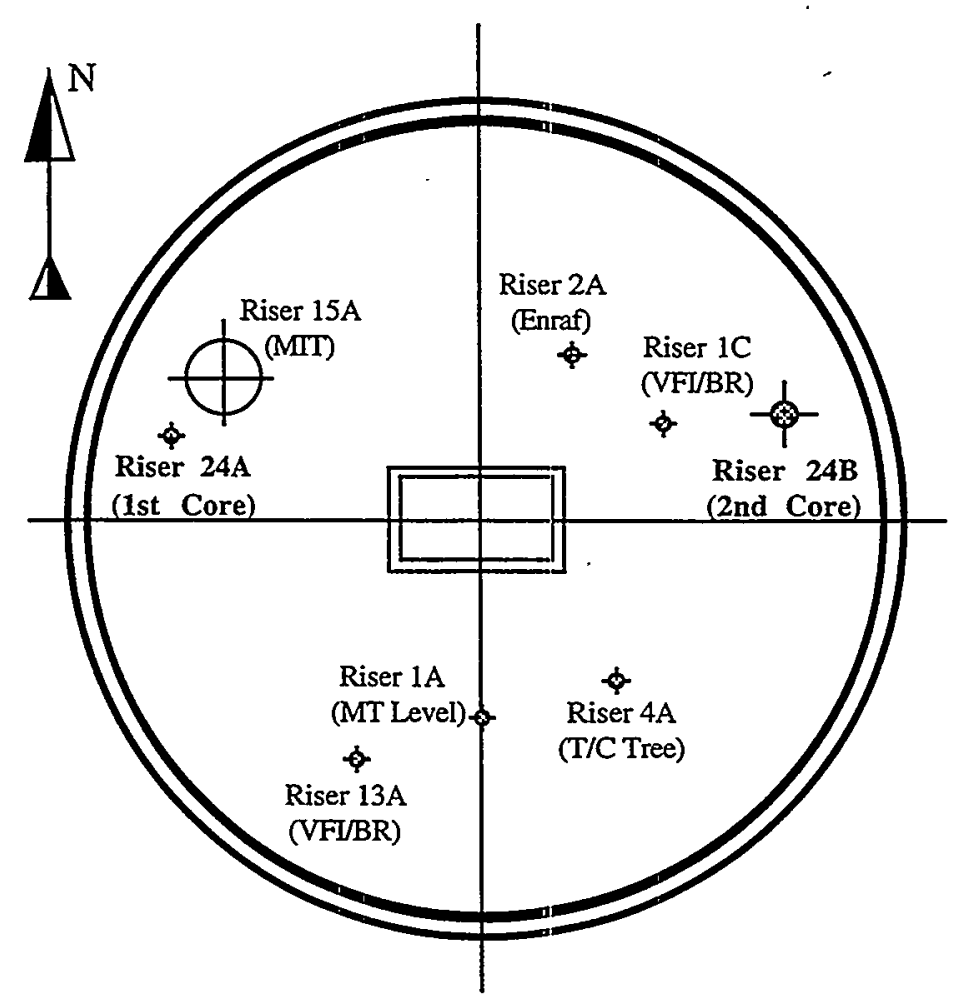

Figure 4.1.1. Schematic Diagram of Riser Locations in Tank AW-101

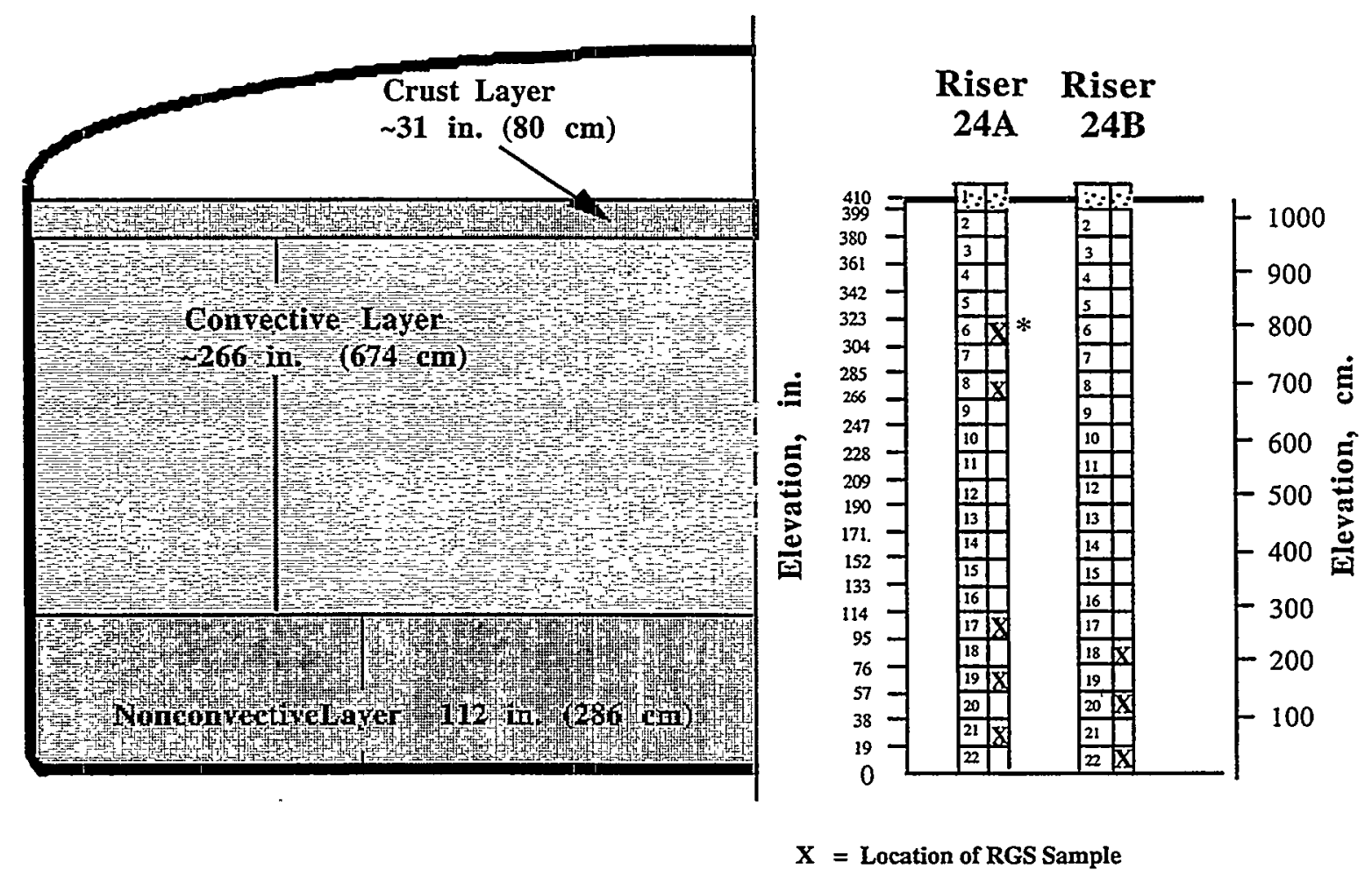

Figure 4.1.2. Diagram of Waste Layering and RGS Sample Elevations for AW-101 
The most recent information on AW-101 layering came from the observations made on the non-RGS samples in the cores from 24A and 24B. The crust was described as dark brown sludge; it occupied as much as 27 inches of core sample. Beneath that were 14 samplers (about 270 in.) of liquid, with 5 to 6 samplers (about 100 in.) of wet salt or sludge at the tank bottom. ("Sludge" is a qualitative term based on core extrusion observations, not chemical analysis. Sludge denotes a core that has a muddy, slimy appearance rather than the gritty, sandy appearance of "salt" materials. "Slurry," another common term, indicates that the core is wet enough to slump under its own weight.)

Two small buoyant-displacement gas-release events (GREs) were near in time to the RGS sampling (Schienbein et al. 1999) and should be considered in interpreting RGS results. The first GRE occurred on February 5, 1996, more than a month before the first RGS samples were taken from riser 24A. The second GRE was on May 14, 1996, after riser 24A sampling but about a week before RGS samples were taken from riser 24B. Hypothetically, the second GRE (although too small to cause a level drop) could have affected the consistency of RGS measurements and resulted in differences between the two sets of RGS measurements. GREs might also have caused differences between RGS values and those measured by VFI in September 1995 (Schienbein et al. 1999), since there were several GREs between the VFI and RGS sampling events.

Densities for the liquid and bulk solids in Tank AW-101 were available from the non-RGS samples taken in 1996 (Baldwin et al. 1995; Benar 1996). Several samples contained drainable liquid. The density of the liquid was $1444 \pm 46 \mathrm{~kg} / \mathrm{m}^{3}$; the crust density (from a single sample) was $1620 \mathrm{~kg} / \mathrm{m}^{3}$; the bulk density of the nonconvective layer samples varied from 1540 to $1600 \mathrm{~kg} / \mathrm{m}^{3}$ with an average and standard deviation of $1569 \pm 26 \mathrm{~kg} / \mathrm{m}^{3}$. Schienbein et al. (1999) stated a density of $1420 \pm 20 \mathrm{~kg} / \mathrm{m}^{3}$ for the convective layer (based on in situ ball rheometry in 1995 ) and $1570 \pm 40 \mathrm{~kg} / \mathrm{m}^{3}$ for the nonconvective layer (based on core samples). All of these measurement methods disturbed the waste and their results should be taken as degassed densities.

For hydrostatic pressure calculations, the liquid density was set at $1420 \mathrm{~kg} / \mathrm{m}^{3}$ and the nonconvective layer was given a degassed bulk density of $1570 \mathrm{~kg} / \mathrm{m}^{3}$. The gassy density of the crust was set equal to that of the liquid below it. Layer depths of $79 \mathrm{~cm}$ ( $31 \mathrm{in}$.), $676 \mathrm{~cm}(266 \mathrm{in}$.), and $284 \mathrm{~cm}$ (112 in.) were used in calculations for the crust, convective, and nonconvective layers.

\subsubsection{Sampling and Extraction Information}

The samples that underwent RGS analysis are listed in Table 4.1.1. (Of the samples shown in Figure 4.1.2, sample 24A-6 was lost in the laboratory due to sampler mishandling, and sample 24B-20 was lost because of sampler valve problems in the hot cell.) Field data, including dose rates and downforce limits, are supplied in Appendix A, which is provided to pull together as many as possible of the data relevant to RGS samples in one document.

Table 4.1.1. Lag Times for Processing RGS Samples from Tank 241-AW-101

\begin{tabular}{||c|c|c|c||}
\hline Sample & Acquisition Date & Processing Date & Lag (days) \\
\hline \hline $24 \mathrm{~A}-8$ & March 15, 1996 & March 26, 1996 & 11 \\
\hline $24 \mathrm{~A}-17$ & March 21, 1996 & March 26, 1996 & 5 \\
\hline $24 \mathrm{~B}-18$ & May 22, 1996 & May 28, 1996 & 6 \\
\hline $24 \mathrm{~A}-19$ & March 22, 1996 & April 1, 1996 & 10 \\
\hline 24A-21 & March 22, 1996 & April 10, 1996 & 19 \\
\hline 24B-22 & May 23, 1996 & June 4, 1996 & 12 \\
\hline
\end{tabular}


Table 4.1.1 also shows the lag times (delay between sample acquisition and processing) for these samples. This information was provided to allow data users to correlate the lag time between sampling and extrusion with the concentrations of the various constituents, to test for decomposition or other chemical reactions. The maximum hold time allowed by the sampling plan is 24 days, based on measured sampler leakage rates during acceptance testing. All the samples were extracted within this time limit.

As discussed in Section 3.5, the HHF used during sampling often enters the sampler in place of some of the waste. HHF is a solution of LiBr; the bromide ion, which is not present in waste, is used as the HHF tracer. Table 4.1.2 shows the volume percentage contamination by HHF of the RGS samples. Most of these samples (excepting 24B-18 and 24B-22) contained bromide below the minimum detection limit (MDL). The measured HHF contamination percentages were used to calculate reduced effective sampler volumes for the RGS samples, with the bromide concentration set equal to the MDL for samples below the limit. (It is conservative to use the MDL for values below it; the conservatism is probably less than $2 \%$.)

Two different extraction procedures were used on the AW-101 samples, but the first several steps were the same in both. (See Figure 3.1 for an RGS system schematic.) After evacuating the whole system and observing the pressure to test for leaks, the operator extruded the RGS sample into the extractor vessel. At this point the vapor space in communication with the sample consisted of the volumes of the extractor vessel and its lines. The sample was briefly stirred. Next, the extractor was opened to the second vessel, to which was connected the first PQ canister. Once a grab sample was collected in the PQ canister, it was closed. The extractor, lines, and second volume were then connected to the pump volume and collector side, which included the collector line volume and one open collection canister. Several strokes of gas and vapor (sometimes called "unbound gas") were pumped from the extractor to the collector, the first collection canister was closed off, and the collector side, second volume, and extractor lines were evacuated.

Next, the extractor and samples were heated to about $50^{\circ} \mathrm{C}$ to drive off "bound gas" and cooled back to hot-cell temperature, and the sample was briefly stirred. A second PQ canister connected to the second volume took a sample and was closed off. Once again, several strokes were pumped from the extractor to the collector, the second collection canister was closed off, and the collector side, second volume, and extractor lines were evacuated. From this point, different procedures were used for the riser 24A and 24B samples.

24A samples: The bulk sample volume determination (BSVD) procedure was performed. Argon was injected into the closed-off second volume, then the second volume opened to the extractor and lines. Pressure measurements and known tare system volumes allowed calculation of

Table 4.1.2. HHF Contamination in AW-101 RGS Samples

\begin{tabular}{||c|c|}
\hline Sample & $\begin{array}{c}\text { HHF Contamination } \\
\text { (vol \%) }\end{array}$ \\
\hline \hline 24A-8 (RGS) & $<2.4$ \\
\hline 24A-17 (RGS) & $<2.4$ \\
\hline 24B-18 (RGS) & 0.8 \\
\hline 24A-19 (RGS) & $<2.4$ \\
\hline 24A-21 (RGS) & $<4.6$ \\
\hline 24B-22 (RGS) & 1.6 \\
\hline
\end{tabular}


the volume of the sample solids and liquid. Next, about $580 \mathrm{cc}$ of water diluent was added to the sample and stirred. A third PQ canister was taken from the collector side, and several strokes of gas and vapor were pumped from the extractor to the collector (to which one open collection canister, the third one, was connected).

24B samples: A third PQ canister was taken from the collector side, and several strokes of gas and vapor were pumped from the extractor to the collector (to which one open collection canister, the third one, was connected). The BSVD procedure was performed last. The 24A procedure was changed because of the large amount of argon and dissolved air gases in the diluent, which contaminated the gas extracted from the sample. Procedural details such as the number of strokes per canister and system volumes can be found in the sample-by-sample worksheets in the AW-101 RGS calculation spreadsheet on the CD supplied with the report.

\subsubsection{Retained Gas Composition}

Table 4.1.3 presents the calculated concentrations of the insoluble gases in each RGS sample taken from AW-101 without corrections for air and argon entrainment, BSVD argon, air leakage, and dissolved air gases added in the diluent. The method used to make the corrections depended on the gas and the step of the extraction procedure in which it appeared. All of the oxygen and argon were subtracted no matter which step had produced them. A number of moles equal to $(3.73) \cdot\left(\mathrm{O}_{2}\right)$ was subtracted from the nitrogen taken in steps before which no diluent was added, consistent with the molar $\mathrm{N}_{2} / \mathrm{O}_{2}$ ratio in atmospheric air, because the oxygen present in these steps was expected to have come from air entrainment during sampling or inleakage during extraction. For riser $24 \mathrm{~A}$ samples, the nitrogen in the third collection canister was estimated to be in the same proportion to hydrogen as in the second canister. This approach was needed because of the large dose of nitrogen in the diluent; the uncertainty in the nitrogen was adjusted to account for this approximation. The corrected concentrations are given in Table 4.1.4.

The large amounts of argon in the samples from riser 24A came from the third canister extracted from each sample, and therefore must have resulted from the BSVD procedure that exposed the sample to argon injection before the third canister was taken. The higher oxygen in the riser 24A samples was also due to the procedure, coming primarily from air gases dissolved in the water diluent added before the third canister. These contamination observations are found in the "Summary" worksheet of the AW-101 RGS calculations spreadsheet on the CD.

Table 4.1.3. Concentrations of Insoluble Constituents ( $\mu \mathrm{mol} / \mathrm{L}$ of waste) in Tank AW-101 Without Correction for Gas Contamination

\begin{tabular}{||c|c|c|c|c|c|c|c|c|c|c|c||}
\hline Sample & $\mathrm{N}_{2}$ & $\mathrm{H}_{2}$ & $\mathrm{~N}_{2} \mathrm{O}$ & $\mathrm{O}_{2}$ & $\mathrm{CH}_{4}$ & $\mathrm{He}$ & $\mathrm{Ar}$ & $\begin{array}{c}\text { Other } \\
\mathrm{NO}_{x}\end{array}$ & $\mathrm{C}_{2} \mathrm{H}_{\mathrm{x}}$ & $\mathrm{C}_{3} \mathrm{H}_{x}$ & $\begin{array}{c}\text { Other } \\
\text { Hyd. }\end{array}$ \\
\hline \hline $24 \mathrm{~A}-8$ & $120 \pm 22$ & $1300 \pm 150$ & $15 \pm 3.0$ & $380 \pm 47$ & $4.7 \pm 1.1$ & (a) & $2500 \pm 360$ & $5.8 \pm 3.8$ & $2.1 \pm 0.8$ & $1.7 \pm 0.5$ & $4.0 \pm 1.5$ \\
\hline $24 \mathrm{~A}-17$ & $620 \pm 51$ & $2600 \pm 230$ & $140 \pm 14$ & $480 \pm 70$ & $38 \pm 3.7$ & (a) & $1300 \pm 240$ & $7.6 \pm 3.3$ & $24 \pm 3.8$ & $7.5 \pm 1.2$ & $32 \pm 6.5$ \\
\hline $24 \mathrm{~B}-18$ & $330 \pm 29$ & $1600 \pm 160$ & $140 \pm 15$ & $130 \pm 20$ & $34 \pm 4.0$ & (a) & $82 \pm 7.4$ & $2.9 \pm 0.4$ & $26 \pm 5.5$ & $6.0 \pm 1.1$ & $37 \pm 8.8$ \\
\hline $24 \mathrm{~A}-19$ & $1900 \pm 100$ & $3500 \pm 250$ & $280 \pm 17$ & $450 \pm 69$ & $61 \pm 3.7$ & (a) & $1100 \pm 200$ & $1.0 \pm 0.5$ & $3.7 \pm 3.9$ & $8.6 \pm 1.0$ & $37 \pm 4.9$ \\
\hline 24A-21 & $1400 \pm 76$ & $4200 \pm 270$ & $410 \pm 26$ & $440 \pm 57$ & $82 \pm 12$ & (a) & $1600 \pm 240$ & $7.2 \pm 4.9$ & $47 \pm 5.3$ & $11 \pm 1.3$ & $51 \pm 5.6$ \\
\hline 24B-22 & $260 \pm 25$ & $1500 \pm 140$ & $280 \pm 37$ & $52 \pm 4.8$ & $42 \pm 7.1$ & (a) & $53 \pm 5.6$ & $0.4 \pm 0.2$ & $38 \pm 12$ & $7.0 \pm 1.7$ & $55 \pm 19$ \\
\hline
\end{tabular}


Table 4.1.4. Concentrations of Insoluble Constituents ( $\mu \mathrm{mol} / \mathrm{L}$ of waste) in Tank AW-101 with Correction for Gas Contamination

\begin{tabular}{||c|c|c|c|c|c|c|c|c|c|c|c||}
\hline Sample & $\mathrm{N}_{2}$ & $\mathrm{H}_{2}$ & $\mathrm{~N}_{2} \mathrm{O}$ & $\mathrm{O}_{2}$ & $\mathrm{CH}_{4}$ & $\mathrm{He}$ & $\mathrm{Ar}$ & $\begin{array}{c}\text { Other } \\
\mathrm{NO}_{x}\end{array}$ & $\mathrm{C}_{2} \mathrm{H}_{\mathrm{x}}$ & $\mathrm{C}_{3} \mathrm{H}_{\mathrm{x}}$ & $\begin{array}{c}\text { Other } \\
\text { Hyd. }\end{array}$ \\
\hline \hline $24 \mathrm{~A}-8$ & $120 \pm 22$ & $310 \pm 110$ & $15 \pm 3.0$ & $0 \pm 47$ & $4.7 \pm 1.1$ & (a) & $0 \pm 360$ & $5.8 \pm 3.8$ & $2.1 \pm 0.8$ & $1.7 \pm 0.5$ & $4.0 \pm 1.5$ \\
\hline $24 \mathrm{~A}-17$ & $620 \pm 51$ & $1200 \pm 130$ & $140 \pm 14$ & $0 \pm 70$ & $38 \pm 3.7$ & (a) & $0 \pm 240$ & $7.6 \pm 3.3$ & $24 \pm 3.8$ & $7.5 \pm 1.2$ & $33 \pm 6.5$ \\
\hline $24 \mathrm{~B}-18$ & $330 \pm 29$ & $1100 \pm 170$ & $140 \pm 15$ & $0 \pm 20$ & $34 \pm 4.0$ & (a) & $0 \pm 7.4$ & $2.9 \pm 0.4$ & $26 \pm 5.5$ & $6.0 \pm 1.1$ & $37 \pm 8.8$ \\
\hline $24 \mathrm{~A}-19$ & $1900 \pm 100$ & $2100 \pm 120$ & $280 \pm 17$ & $0 \pm 69$ & $61 \pm 3.7$ & (a) & $0 \pm 200$ & $1.0 \pm 0.5$ & $3.7 \pm 3.9$ & $8.6 \pm 1.0$ & $37 \pm 4.9$ \\
\hline 24A-21 & $1400 \pm 76$ & $2600 \pm 150$ & $410 \pm 26$ & $0 \pm 57$ & $82 \pm 12$ & (a) & $0 \pm 240$ & $7.2 \pm 4.9$ & $47 \pm 5.3$ & $11 \pm 1.3$ & $51 \pm 5.6$ \\
\hline 24B-22 & $260 \pm 25$ & $1300 \pm 140$ & $280 \pm 37$ & $0 \pm 4.8$ & $42 \pm 7.1$ & (a) & $0 \pm 5.6$ & $0.4 \pm 0.2$ & $38 \pm 12$ & $7.0 \pm 1.7$ & $55 \pm 19$ \\
\hline
\end{tabular}

Table 4.1.5 presents the ammonia measurements. The RGS procedure did not allow determination of the residual or total ammonia, but the post-extrusion and the first PQ canister partial pressures of $\mathrm{NH}_{3}$ over the sample (see Section 3.4.4) were measured at laboratory temperature. The residual ammonia in the samples was also measured by ion-specific electrode (ISE) after the samples were removed from the extractor; as discussed in Section 3.4.2, these ammonia concentrations should be considered lower bounds because of the evaporation of ammonia during sample handling. Ammonia data can be seen in more detail in Appendix C.

Table 4.1.5. Ammonia Data from Tank AW-101 Samples

\begin{tabular}{|c|c|c|c|}
\hline Sample & $\begin{array}{l}\text { Measured } \mathrm{NH}_{3} \\
\text { partial pressure at } \\
\text { about } 24^{\circ} \mathrm{C} \\
\text { (atm) }\end{array}$ & $\begin{array}{c}\text { Total } \mathrm{NH}_{3} \text { from } \\
\text { Post-RGS Residual } \\
\mathrm{NH}_{3}, \text { by ISE (a) } \\
(\mu \mathrm{mol} / \mathrm{L})\end{array}$ & $\begin{array}{c}\text { Best-Estimate } \mathrm{NH}_{3} \\
\text { Concentrations }\end{array}$ \\
\hline $24 \mathrm{~A}-8$ & $0.0038 \pm 0.0019$ & 22000 & \multirow{6}{*}{$\begin{array}{c}\text { The data do not support } \\
\text { conclusive } \mathrm{NH}_{3} \\
\text { concentrations. We } \\
\text { estimate a lower bound of } \\
0.034 \mathrm{M} \text { ( } 580 \mu \mathrm{g} / \mathrm{mL}) \mathrm{NH}_{3} \\
\text { in the liquid of the } \\
\text { nonconvective layer, or } \\
0.031 \text { wt } \% \mathrm{NH}_{3} \text { in the bulk } \\
\text { waste. }\end{array}$} \\
\hline $24 \mathrm{~A}-17$ & $0.0079 \pm 0.0039$ & 23000 & \\
\hline 24B-18 & $0.0041 \pm 0.0020$ & 14000 & \\
\hline $24 \mathrm{~A}-19$ & $0.0108 \pm 0.0054^{(b)}$ & 27000 & \\
\hline $24 \mathrm{~A}-21$ & $0.0103 \pm 0.0051^{(b)}$ & no data & \\
\hline $24 \mathrm{~B}-22$ & $0.0058 \pm 0.0029$ & 13000 & \\
\hline \multicolumn{4}{|c|}{$\begin{array}{l}\text { (a) These total ammonia concentrations are the sum of the post-RGS ISE ammonia residual and } \\
\text { the RGS extracted ammonia. They should be considered lower bounds because of ammonia } \\
\text { evaporation during the sample handling that preceded ISE analysis. } \\
\text { (b) Ammonia partial pressures are based on PQ canister measurements, except for samples 24A- } \\
\text { 19 and 24A-21. No PQ canister data were available, so calculated post-extrusion partial } \\
\text { pressures were used instead. }\end{array}$} \\
\hline
\end{tabular}


The ammonia partial pressure of sample 24A-8 (the only sample in the convective layer) was lower than the pressures of the other samples. No conclusive total ammonia concentration can be calculated, but the lower-bound estimate is roughly $27,000 \mu \mathrm{mol} \mathrm{NH} 3 / \mathrm{L}$ waste. This estimate is based on Sample 24A-19, the maximum total ammonia measurement, which was found by adding the post-RGS ISE measurement of residual ammonia to the RGS-determined extracted ammonia.

Table 4.1.6 contains the composition of the gas/vapor phase in each sample and the integrated average composition in the gas retained in the nonconvective layer. The water vapor is not included in these compositions. The ammonia fractions are derived from the partial pressures shown in Table 4.1.5 extrapolated to vapor pressures at in situ conditions. The sample compositions in the table have been calculated using the in situ solubility method described in Section 3.6.2. As discussed in Section 3.6.1, compositions were calculated for both the lower-bound and upper-bound gas solubilities. Both ends of the composition range are given in Table 4.1.6, together with the measurement uncertainty of each. The average composition of the gas in the nonconvective layer is the result of integrating RGS species concentrations over the waste layer and multiplying those concentrations by the waste volume. The integration method is described in Section 3.7.1.

The samples from riser 24B contained lower mole fractions of $\mathrm{H}_{2}$ and higher mole fractions of $\mathrm{N}_{2}$ than the samples from riser 24A, indicating either lateral spatial variability in the nonconvective layer in AW-101 or temporal variability. This trend was visible even in the compositions of the first canister from each sample, proving that it was not the result of gas contamination during extraction. (These data are in the sample worksheets in the AW-101 calculation spreadsheet on the CD.) There also seemed to be an increase in the $\mathrm{N}_{2} \mathrm{O}$ mole fraction toward the bottom of the tank.

Table 4.1.6. Sample and Overall Average Compositions of Retained Gas in Tank AW-101 with Correction for Gas Contamination(a)

\begin{tabular}{||c|c|c|c|c|c|c||}
\hline \hline Sample & $\mathrm{N}_{2}$ (mol\%) & $\mathrm{H}_{2}$ (mol\%) & $\mathrm{N}_{2} \mathrm{O}(\mathrm{mol} \%)$ & $\mathrm{NH}_{3}$ (mol\%) & $\mathrm{CH}_{4}$ (mol\%) & Other (mol\%) \\
\hline \hline $24 \mathrm{~A}-8$ & $67 \pm 32-69 \pm 33$ & $26 \pm 9.4-25 \pm 9.2$ & $2.3 \pm 0.8-0.8 \pm 0.3$ & $0.6 \pm 0.3-0.2 \pm 0.1$ & $1.0 \pm 0.4$ & $3.0 \pm 1.7-3.3 \pm 1.9$ \\
\hline $24 \mathrm{~A}-17$ & $59 \pm 8.9-61 \pm 9.2$ & $29 \pm 3.9$ & $5.8 \pm 0.9-3.5 \pm 0.5$ & $0.9 \pm 0.4-0.3 \pm 0.2$ & $1.8 \pm 0.3$ & $3.4 \pm 0.8-3.6 \pm 0.8$ \\
\hline $24 \mathrm{~B}-18$ & $67 \pm 14-70 \pm 14$ & $19 \pm 3.1$ & $7.1 \pm 1.2-3.9 \pm 0.7$ & $0.4 \pm 0.2-0.2 \pm 0.1$ & $2.0 \pm 0.4$ & $4.2 \pm 1.1-4.5 \pm 1.2$ \\
\hline $24 \mathrm{~A}-19$ & $47 \pm 4.1-48 \pm 4.1$ & $43 \pm 3.5-44 \pm 3.5$ & $5.8 \pm 0.5-4.3 \pm 0.4$ & $1.1 \pm 0.5-0.4 \pm 0.2$ & $1.4 \pm 0.1$ & $1.9 \pm 0.3-2.0 \pm 0.3$ \\
\hline $24 \mathrm{~A}-21$ & $56 \pm 4.8-58 \pm 4.9$ & $30 \pm 2.5-31 \pm 2.5$ & $8.2 \pm 0.7-6.0 \pm 0.5$ & $0.8 \pm 0.4-0.3 \pm 0.2$ & $1.8 \pm 0.3$ & $2.5 \pm 0.4-2.6 \pm 0.4$ \\
\hline $24 \mathrm{~B}-22$ & $67 \pm 11-72 \pm 12$ & $13 \pm 2.1-14 \pm 2.1$ & $12 \pm 2.2-6.3 \pm 1.1$ & $0.4 \pm 0.2-0.2 \pm 0.1$ & $2.2 \pm 0.4-2.3 \pm 0.5$ & $5.2 \pm 1.8-5.8 \pm 2.0$ \\
\hline $\begin{array}{l}\text { Convective } \\
\text { layer(c) }\end{array}$ & $67 \pm 32-69 \pm 33$ & $26 \pm 9.4-25 \pm 9.2$ & $2.3 \pm 0.8-0.8 \pm 0.3$ & $0.6 \pm 0.3-0.2 \pm 0.1$ & $1.0 \pm 0.4$ & $3.0 \pm 1.7-3.3 \pm 1.9$ \\
\hline $\begin{array}{l}\text { Avg in non- } \\
\text { convective } \\
\text { layer(c) }\end{array}$ & $\begin{array}{l}55 \pm 6.2-57 \pm 6.4 \\
5\end{array}$ & $32 \pm 3.2-33 \pm 3.2$ & $7.5 \pm 0.8-5.1 \pm 0.6$ & $0.9 \pm 0.4-0.3 \pm 0.2$ & $1.7 \pm 0.2$ & $2.8 \pm 0.5-2.9 \pm 0.6$ \\
\hline $\begin{array}{l}\text { (a) Uncertainties on compositions represent only instrument uncertainty. Compositions calculated for both lower- and } \\
\text { upper-bound solubilities. Both ends of composition range are given, lower-bound solubility basis first with instrument } \\
\text { uncertainty on each. Compositions may not sum to } 100 \text { mol\% because of roundoff error. Mole fractions on dry basis and } \\
\text { do not account for water vapor. } \\
\text { (b) Ammonia mole fraction derived from partial pressures in Table 4.1.5 extrapolated to vapor pressure at in situ conditions. } \\
\text { (c) There are too few samples to define the spatial variability of the average gas concentration. }\end{array}$ \\
\hline
\end{tabular}


Nitrogen was high in the convective layer and hydrogen low. This observation is based more on an overview of all convective layer samples from RGS-sampled tanks than on AW-101-24A-8, which has a very broad composition uncertainty because of high air and argon contamination and a small native gas content.

The concentrations and pressures in Tables 4.1.3 and 4.1.4 can also be found in the sample-by-sample worksheets in the AW-101 RGS calculation spreadsheet on the CD. The compositions in Table 4.1.6 are in the "Inventory" worksheet.

\subsubsection{Gas Inventory}

The method by which the in situ gas volume fractions (wet basis) were calculated is given in Section 3.6.2; as for the gas composition, the volume fractions are given as a range from the low gas-solubility value to the high gas-solubility value. The results are presented in Table 4.1.7. Table 4.1.7 also contains the average gas volume fractions in both layers and the average pressure experienced by the gas in the nonconvective layer. The averages are in situ volume averages calculated by Simpson's Rule integration, as described in Section 3.7.1. The corrected gas volume fractions in Table 4.1.7 are consistent with the corrected gas concentrations and compositions in Tables 4.1.4 through 4.1.6. The information in Table 4.1.7 is taken from the "Summary" and "Insitu" worksheets of the AW-101 RGS calculation spreadsheet on the CD.

The "sampler gas volume fraction" is corrected only for inleakage during the extraction process and air gases added in the isotopic solution. It is an attempt to reconstruct the total amount of gas (sample and entrainment) that was present in the sampler during $x$-ray. It is used only for comparison with the X-ray observations of "visible gas fraction" discussed in Section 4.1.4.

Table 4.1.7. In Situ Gas Volume Fractions and Conditions in Tank AW-101

\begin{tabular}{|c|c|c|c|c|c|c|}
\hline Sample & $\begin{array}{l}\text { Sample } \\
\text { central } \\
\text { height } \\
(\mathrm{cm}) \\
\end{array}$ & $\begin{array}{l}\text { Hydro- } \\
\text { static } \\
\text { pressure } \\
\text { (atm) } \\
\end{array}$ & $\begin{array}{l}\text { Calculated } \\
\text { water vapor } \\
\text { pressure } \\
(\mathrm{atm}) \\
\end{array}$ & $\begin{array}{r}\text { Temp } \\
\left({ }^{\circ} \mathrm{C}\right) \\
\end{array}$ & $\begin{array}{c}\text { Corrected gas volume } \\
\text { fraction(a) } \\
\text { (in-tank conditions) }\end{array}$ & $\begin{array}{c}\text { Sampler gas } \\
\text { volume fraction } \\
\text { (in situ conditions, } \\
\text { low solubility) }\end{array}$ \\
\hline $24 \mathrm{~A}-8$ & 700 & 1.46 & 0.020 & 37.5 & $0.008 \pm 0.001-0.007 \pm 0.001$ & 0.015 \\
\hline $24 \mathrm{~A}-17$ & 265 & 2.06 & 0.026 & 40.0 & $0.027 \pm 0.003-0.025 \pm 0.003$ & 0.034 \\
\hline $24 \mathrm{~B}-18$ & 217 & 2.13 & 0.026 & 40.4 & $0.021 \pm 0.004-0.019 \pm 0.004$ & 0.028 \\
\hline $24 \mathrm{~A}-19$ & 169 & 2.20 & 0.025 & 39.7 & $0.052 \pm 0.005-0.050 \pm 0.005$ & 0.057 \\
\hline $24 \mathrm{~A}-21$ & 72.4 & 2.34 & 0.021 & 36.5 & $0.051 \pm 0.005-0.049 \pm 0.005$ & 0.055 \\
\hline $24 \mathrm{~B}-22$ & 24.1 & 2.41 & 0.018 & 33.0 & $0.020 \pm 0.004-0.018 \pm 0.004$ & 0.023 \\
\hline $\begin{array}{c}\text { Convective } \\
\text { layer }\end{array}$ & 673 & & & & $0.008 \pm 0.003-0.007 \pm 0.003$ & \\
\hline $\begin{array}{l}\text { Avg in non- } \\
\text { convective } \\
\text { layer }\end{array}$ & 133 & 2.25 & & & $0.037 \pm 0.018-0.035 \pm 0.018$ & \\
\hline
\end{tabular}


Table 4.1.7 also contains the water vapor pressures that were used for in situ calculations. The water vapor pressures were found by using salt concentrations from Benar (1996) and the temperatures in the table as inputs to Equation 6.2 of Mahoney and Trent (1995), a correlation for water vapor pressure over concentrated homogeneous and non-homogeneous waste simulants. The gas solubilities used the same parameters as inputs to the Schumpe solubility model (see Section 3.6.1). The water vapor pressures and gas solubilities used in calculations can be found in the "In-situ" worksheet of the AW-101 RGS calculation spreadsheet on the CD.

The two samples from the nonconvective layer under riser 24B contained less gas than the three from riser $24 \mathrm{~A}$. As with the difference in gas composition, the difference in gas volume fraction could have indicated lateral spatial variability in the nonconvective layer in AW-101 or temporal variability (perhaps owing to the intervening GRE). No ammonia inventory was calculated for the tank, owing to the absence of total ammonia concentration data for AW-101.

Table 4.1.8 gives various estimates of the STP volume of gas in Tank AW-101, including estimates calculated from RGS data alone. The VFI\&RGS and barometric pressure effect (BPE) inventory values were taken from Schienbein et al. (1999). (The VFI\&RGS inventory is based on both RGS and VFI data.)

The RGS gas inventory in the nonconvective layer was calculated by integrating RGS total gas concentrations over the layer (five data points) and multiplying the average gas concentration by the volume of the waste. The integration method is described in Section 3.7.1. The gas inventory in the convective layer was calculated from a single sample, 24A-8. The RGS volumes in Table 4.1.8 include corrections to remove the contamination gas: entrained air and argon, air leaks during and after extraction, argon from the BSVD procedure, and air gases dissolved in the diluent. The uncertainties on the gas inventories are based on the spatial variability considerations discussed in Section 3.7.2. The information in Table 4.1.8 is taken from the "Inventory" worksheet of the AW-101 RGS calculation spreadsheet on the CD.

Table 4.1.7 also contains the water vapor pressures that were used for in situ calculations. The water vapor pressures were found by using salt concentrations from Benar (1996) and the temperatures in the table as inputs to Equation 6.2 of Mahoney and Trent (1995), a correlation for water vapor pressure over concentrated homogeneous and non-homogeneous waste simulants.

Table 4.1.8. AW-101 Gas Inventory Estimates

\begin{tabular}{|c|c|c|c|c|c|}
\hline \multirow[b]{2}{*}{ Quantity } & \multicolumn{2}{|c|}{ RGS Method } & \multirow{2}{*}{$\begin{array}{c}\text { BPE Method(a) } \\
\text { Tank Total }\end{array}$} & \multicolumn{2}{|c|}{ VFI\&RGS Method(b, c) } \\
\hline & $\begin{array}{c}\text { Nonconvective } \\
\text { Layer }\end{array}$ & $\begin{array}{c}\text { Convective } \\
\text { Layer }\end{array}$ & & $\begin{array}{c}\text { Nonconvective } \\
\text { Layer }\end{array}$ & $\begin{array}{c}\text { Convective } \\
\text { Layer }\end{array}$ \\
\hline $\begin{array}{l}\text { Avg. gas fraction } \\
\text { (low gas solubility) }\end{array}$ & $0.037 \pm 0.018$ & $0.008 \pm 0.003$ & $\begin{array}{c}(\text { tank avg) } \\
0.023 \pm 0.007\end{array}$ & $0.038 \pm 0.004$ & $\begin{array}{c}0.003 \pm \\
0.001\end{array}$ \\
\hline $\begin{array}{l}\text { Gas volume }\left(\mathrm{m}^{3}\right) \\
\text { in situ (wet) }\end{array}$ & $43 \pm 21$ & $21 \pm 7$ & $100 \pm 31$ & $45 \pm 5$ & $10 \pm 2$ \\
\hline STP (wet) & $86 \pm 42$ & $29 \pm 10$ & $162 \pm 51$ & $97 \pm 11$ & $15 \pm 3$ \\
\hline \multicolumn{6}{|c|}{$\begin{array}{l}\text { (a) Barometric pressure method. Includes the crust, for which there were no RGS or VFI data. Table } \\
\text { 2.7.1 of Schienbein et al. (1999) stated the crust gas inventory as } 40 \pm 15 \mathrm{~m}^{3} \text { in situ, or } 41 \pm 15 \mathrm{STP} \mathrm{m}^{3} \text {, } \\
\text { an estimate made by assuming neutral buoyancy. } \\
\text { (b) Based on RGS and VFI data; see Table } 2.7 .3 \text { of Schienbein et al. (1999). } \\
\text { (c) The retained gas inventory calculated from RGS and VFI data is considered the best estimate because } \\
\text { it is based on data from several risers. }\end{array}$} \\
\hline
\end{tabular}


The RGS method gave the highest total gas inventory ( $104 \pm 52 \mathrm{~m}^{3}$ in situ, counting the crust gas) and the VFI\&RGS method the lowest ( $95 \pm 16 \mathrm{~m}^{3}$ in situ, counting the crust gas), with the inventory calculated by the BPE method in between. As Figure 4.1.3 suggests, the RGS nonconvective layer inventory may have been less than that based partly on VFI data because the loss of sample 24B-20 meant that RGS did not capture the highest gas values (at about $100-\mathrm{cm}$ elevation according to VFT data). In addition, the RGS average gas fraction would have been decreased relative to the VFI value by including the small gas volume fraction from $24 \mathrm{~B}-22$, a sample at an elevation too low for VFI to reach. However, the smaller RGS inventory in the nonconvective layer was offset by the larger RGS inventory in the convective layer, so that the RGS total gas inventory was greater than the VFI\&RGS total gas inventory.

The uncertainty in the BPE inventory is large enough that it overlapped both the VFI and RGS estimates. The VFI\&RGS gas inventory is preferable to the BPE inventory because it is based on direct gas measurements from four risers.

Table 4.1 .9 contains the calculated layer inventories of each of the major gases retained in the waste. The gas-phase inventories are based on the layer-average compositions from RGS data (Table 4.1.6) and the best-estimate gas inventories for each layer (VFI\&RGS, Table 4.1.8). The dissolved inventories are based on RGS data alone.

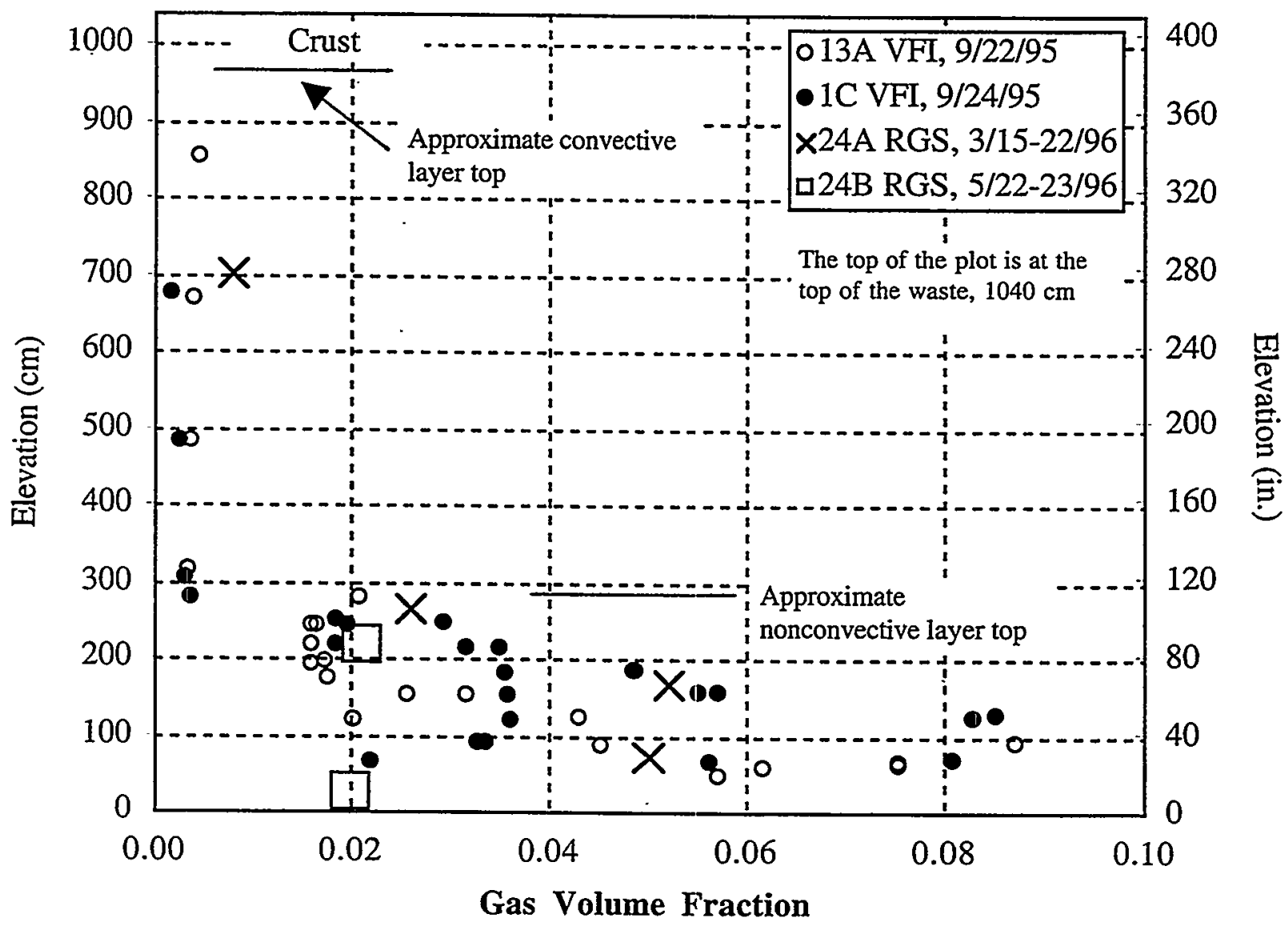

Figure 4.1.3. Gas Volume Fractions Measured by RGS and VFI 
Table 4.1.9. Speciated AW-101 Gas Inventory(a)

\begin{tabular}{||l|c|c||c|c||}
\hline \multirow{3}{*}{ Species } & \multicolumn{2}{|c|}{$\begin{array}{c}\text { Gas-Phase Inventory } \\
\text { (m³ } \text { at STP) }\end{array}$} & \multicolumn{2}{c|}{$\begin{array}{c}\text { Dissolved Inventory } \\
\text { ( } \mathrm{m}^{3} \text { at STP) }\end{array}$} \\
\cline { 2 - 5 } & $\begin{array}{c}\text { Nonconvective } \\
\text { Layer }\end{array}$ & $\begin{array}{c}\text { Convective } \\
\text { Layer }\end{array}$ & $\begin{array}{c}\text { Nonconvective } \\
\text { Layer }\end{array}$ & $\begin{array}{c}\text { Convective } \\
\text { Layer }\end{array}$ \\
\hline $\mathrm{N}_{2}$ & $54-53$ & $10-9.4$ & $0.086-0.91$ & $0.15-0.79$ \\
\hline $\mathrm{H}_{2}$ & $31-30$ & $3.9-3.4$ & $0.14-0.93$ & $0.20-0.67$ \\
\hline $\mathrm{N}_{2} \mathrm{O}$ & $7.2-4.7$ & $0.34-0.12$ & $0.67-3.2$ & $0.28-0.51$ \\
\hline $\mathrm{NH}_{3}$ & $0.83-0.32$ & $0.084-0.033$ & 1000 & 1100 \\
\hline $\mathrm{CH}_{4}$ & 1.6 & $0.15-0.14$ & $0.0048-0.053$ & $0.0041-0.022$ \\
\hline Other & 2.7 & 0.45 & 0 & 0 \\
\hline
\end{tabular}

(a) Gas-phase inventories based on layer-average compositions from RGS data (Table 4.1.6) and best-estimate gas inventories for each layer (VFI\&RGS, Table 4.1.8). Dissolved inventories based on RGS data alone. Uncertainty in each inventory is 50\% based on spatial variability considerations discussed in Section 3.7.2. Inventories given for both lower- and upper-bound solubilities; lower-bound solubility basis first.

Figure 4.1.4 shows sample temperatures, corrected gas volume fractions (Table 4.1.7), and corrected compositions of the low-solubility constituents in the samples from AW-101. The temperatures are from the multifunction instrument tree (MIT) in riser 15A. The compositions represent the mole fraction of the species in the "insoluble" gas; water and ammonia are not included, so the mole fractions are not the same as those in Table 4.1.4. The gas volume fractions and mole fractions in the figure are the values for lower-bound gas solubilities. Figure 4.1.4 also lists the observations from core extrusions of non-RGS samples and from X-rays of RGS samples as a way of tying those observations together with RGS data. The $\mathrm{x}$-ray data are described in more detail in Section 4.1.4.

\subsubsection{X-Ray Results}

Table 4.1.10 summarizes all the available radiography observations from Tank AW-101. The X-ray images themselves can be found on the $\mathrm{CD}$ that accompanies this report. The uncertainties of the visible gas volume fractions that are given in Table 4.1.10 are unknown but (based on the uncertainty in measuring the gas gap from x-ray images) are expected to be larger than the uncertainty in the gas volume fractions calculated from gas extraction. (The "visible" gas volume fraction refers to the gas that has separated from the solids and liquid such that its volume can be calculated based on the image. The uncorrected gas fractions from extraction are used for comparison to reflect the presence of entrained air in the sampler.)

Table 4.1.10 compares $\mathrm{x}$-ray-derived gas volume fractions with extraction-derived gas volume fractions. The comparison shows that sample recovery was close to $100 \%$ for all the samples. This conclusion is based on the fact that the extracted uncorrected gas was a few volume percent more than the visible gas.

Though sample 24B-20 was not successfully extracted, at least as much gas was visible in the $\mathrm{x}$-ray image as in the images of samples that contained 2 to 5 vol\% gas. It is therefore possible that this sample would have shown the same high gas concentrations that were found in VFI measurements at about the same elevation. 
Table 4.1.10. Summary of Observations from X-Ray Images of Tank AW-101

\begin{tabular}{|c|c|}
\hline Segment & Comments/Observations \\
\hline $\begin{array}{l}24 \mathrm{~A}-6 \\
(\mathrm{RGS} \\
2 / 16 / 96)\end{array}$ & $\begin{array}{l}\text { The waste in the image was featureless. There was a gas gap of about } 0.2 \mathrm{~cm}(<0.1 \mathrm{in} \text {.) below } \\
\text { a clean piston, which was fully retracted. The waste surface was flat. The visible gas volume } \\
\text { fraction is } 0.004 \text {. }\end{array}$ \\
\hline $\begin{array}{l}24 \mathrm{~A}-8 \\
(\mathrm{RGS} \\
3 / 15 / 96)\end{array}$ & $\begin{array}{l}\text { The waste in the image was featureless. There was a gas gap of about } 0.2 \mathrm{~cm}(<0.1 \mathrm{in} \text {.) below } \\
\text { a clean piston, which was fully retracted. The waste surface was flat, except for a small bridge } \\
\text { between the surface and the piston. The visible gas volume fraction is } 0.004 \text {. (Compare with } \\
\text { the uncorrected sampler gas volume fraction of } 0.015 \text { in Table } 4.1 .7 \text { obtained by gas extraction.) }\end{array}$ \\
\hline $\begin{array}{l}24 \mathrm{~A}-17 \\
(\mathrm{RGS} \\
3 / 21 / 96)\end{array}$ & $\begin{array}{l}\text { This sample contained a group of } 1 \text { to } 2-\mathrm{mm} \text { diameter bubbles in the bottom inch of the sample } \\
\text { and a few bubbles of similar size near the top. There was a meniscus at the surface and a clean } \\
\text { piston, fully retracted, with a } 0.2-\mathrm{cm}(<0.1 \text {-in.) gas gap below it. The visible gas volume } \\
\text { fraction (not counting bubbles) is } 0.004 \text {. (Compare with the uncorrected sampler gas volume } \\
\text { fraction of } 0.034 \text { in Table } 4.1 .7 \text { obtained by gas extraction.) }\end{array}$ \\
\hline $\begin{array}{l}24 \mathrm{~B}-18 \\
(\mathrm{RGS} \\
5 / 22 / 96)\end{array}$ & $\begin{array}{l}\text { Several 3-mm bubbles in the bottom half of the sampler and a few darker (denser) oblong } \\
\text { features } 1-2 \mathrm{~mm} \text { in size in the top half. No gas gap, piston fully retracted. The visible gas } \\
\text { volume fraction (not counting bubbles) is therefore } 0 \text {. (Compare with the uncorrected sampler } \\
\text { gas volume fraction of } 0.028 \text { in Table } 4.1 .7 \text { obtained by gas extraction.) }\end{array}$ \\
\hline $\begin{array}{l}24 \mathrm{~A}-19 \\
(\mathrm{RGS} \\
3 / 22 / 96)\end{array}$ & $\begin{array}{l}\text { Numerous } 1 \text { to } 2-\mathrm{mm} \text { bubbles and several } 3-\mathrm{mm} \text { bubbles, with one } 6-\mathrm{mm} \text { bubble in the middle } \\
\text { of the sample. No gas gap, piston fully retracted. The visible gas volume fraction (not } \\
\text { counting bubbles) is therefore } 0 \text {. (Compare with the uncorrected sampler gas volume fraction of } \\
0.057 \text { in Table } 4.1 .7 \text { obtained by gas extraction.) }\end{array}$ \\
\hline $\begin{array}{l}24 \mathrm{~B}-20 \\
(\mathrm{RGS} \\
5 / 22 / 96)\end{array}$ & $\begin{array}{l}\text { Many } 1 \text { to } 2-\mathrm{mm} \text { bubbles and several larger oblong bubbles. There were also some slightly } \\
\text { darker (denser) blobs about } 3 \mathrm{~mm} \text { in diameter. Waste surface was flat and piston clean and fully } \\
\text { retracted. } 1.3-\mathrm{cm}(0.5 \text {-in.) gas gap. The visible gas volume fraction (not counting bubbles) is } \\
\text { therefore } 0.011 \text {. }\end{array}$ \\
\hline $\begin{array}{l}24 \mathrm{~A}-21 \\
(\mathrm{RGS} \\
3 / 22 / 96)\end{array}$ & $\begin{array}{l}\text { Numerous } 1 \text { to } 2-\mathrm{mm} \text { bubbles with a few } 3-\mathrm{mm} \text { bubbles. There were more bubbles towards top } \\
\text { of sample. There were also denser features, more of these toward the sample top. No gas gap. } \\
\text { Piston fully retracted. The visible gas volume fraction (not counting bubbles) is therefore } 0 \text {. } \\
\text { (Compare with the uncorrected sampler gas volume fraction of } 0.055 \text { in Table } 4.1 .7 \text { obtained by } \\
\text { gas extraction.) }\end{array}$ \\
\hline $\begin{array}{l}24 \mathrm{~B}-22 \\
(\mathrm{RGS} \\
5 / 23 / 96)\end{array}$ & $\begin{array}{l}\text { There were } 1 \text { to } 2-\mathrm{mm} \text { bubbles throughout, with a few } 2-3 \mathrm{~mm} \text { bubbles in bottom third, one } 3- \\
\text { mm and one } 7 \text {-mm bubble toward top. Some darker features, up to } 5 \mathrm{~mm} \text { across, mostly } \\
\text { oblong. No gas gap. Piston fully retracted. The visible gas volume fraction (not counting } \\
\text { bubbles) is therefore } 0 \text {. (Compare with the uncorrected sampler gas volume fraction of } 0.023 \text { in } \\
\text { Table } 4.1 .7 \text { obtained by gas extraction.) }\end{array}$ \\
\hline
\end{tabular}

Based on our observation of the various segments, bubbles as large as $7 \mathrm{~mm}$ in diameter are clearly present. However, this is not considered to be the upper limit in the size distribution of the bubbles retained within the nonconvective layer in this tank at the time of sampling. Also, close inspection of the images shows that the majority of the visible bubbles were on the order of $1 \mathrm{~mm}$. Whether bubbles smaller than that exist cannot be determined directly from the current density map because of the detection limit dictated by the signal-to-noise ratio, as discussed in . Section 3.9. A preliminary estimate of the volume of visible bubbles in segment 24A-19 shows that more than $90 \%$ of the gas volume was in bubbles below the detectable size. 

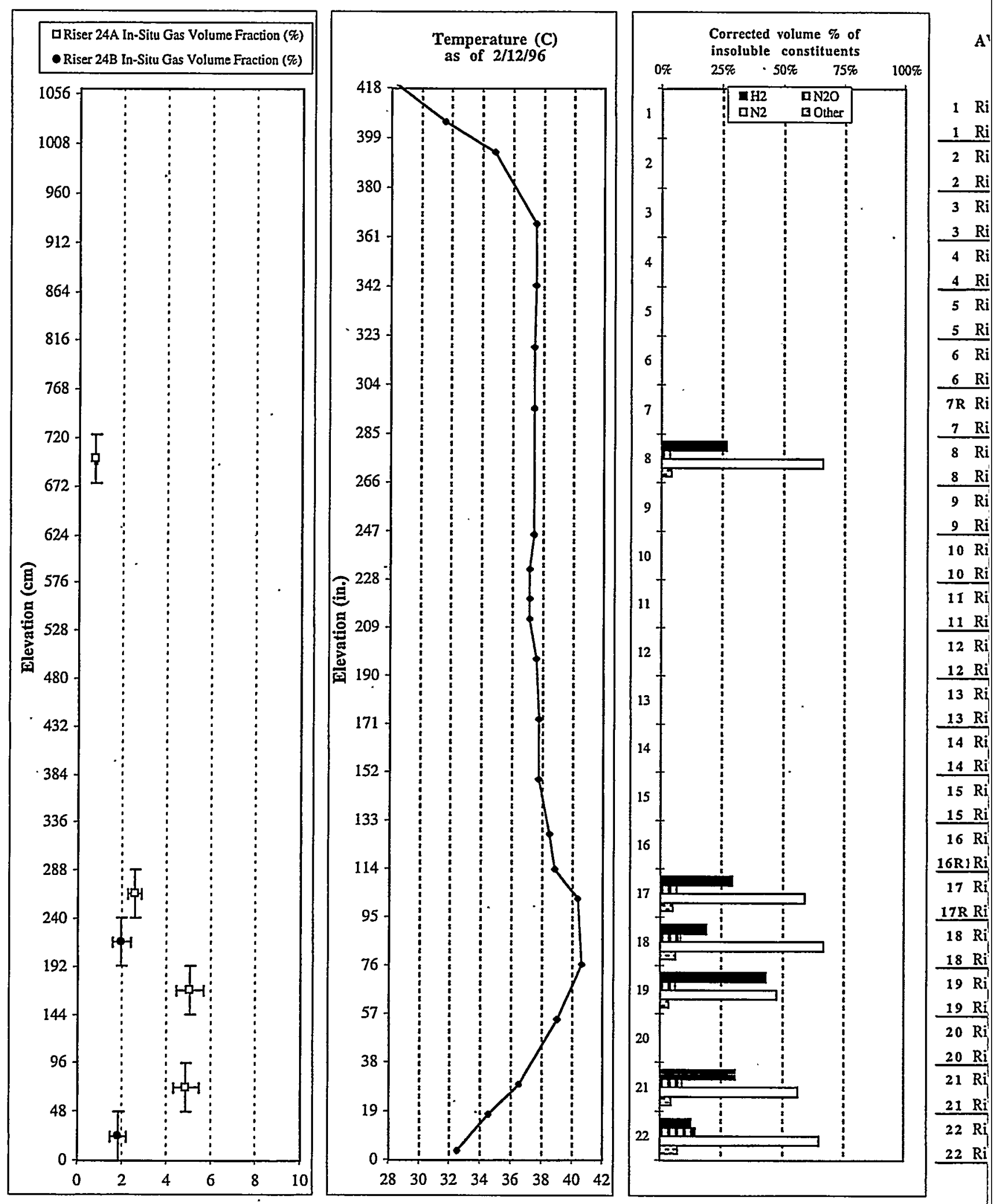

Figure 4.1.4. Gas Volume Fractions, Temperatures, 
01 waste level is $1040 \mathrm{~cm}$ (409 inches).

24A: 13 in. (295 g) dark brown wet sludge; $1.5 \mathrm{R} / \mathrm{hr}$.

24B: 5 in. (107 g) dark gray wet sludge; $1.0 \mathrm{R} / \mathrm{hr}$.

24A: 14 in. (276 g) dark brown wet sludge, $30 \mathrm{ml}(59 \mathrm{~g})$ liquid; $2.4 \mathrm{R} / \mathrm{hr}$.

24B: 8 in. (133 g) brown wet sludge and white salt slurry, $125 \mathrm{~mL}(153 \mathrm{~g}$ ) brown opaque liquid; $2.0 \mathrm{R} / \mathrm{hr}$

24A: 7 in. $(45 \mathrm{~g})$ white salt slurry, $250 \mathrm{~mL}(380 \mathrm{~g})$ liquid; $2.0 \mathrm{R} / \mathrm{hr}$.

24B: 2 in. ( $22 \mathrm{~g}$ ) white salt slurry, $250 \mathrm{~mL}(365 \mathrm{~g})$ yellow opaque liquid; $2.0 \mathrm{R} / \mathrm{hr}$.

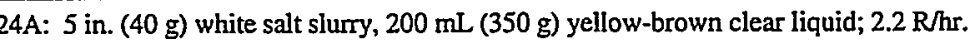

24B: 2 to 3 in. (29 g) white salt siurry, $250 \mathrm{~mL}(378 \mathrm{~g})$ yellow opaque liquid; $2.0 \mathrm{R} / \mathrm{hr}$.

24A: 4 in. $(54 \mathrm{~g}$ ) white salt slurry, $250 \mathrm{~mL}$ ( $365 \mathrm{~g}$ ) yellow-brown opaque liquid; $2.0 \mathrm{R} / \mathrm{hr}$.

24B: 3 in. $(31 \mathrm{~g})$ white salt slumy, $250 \mathrm{~mL}$ ( $384 \mathrm{~g}$ ) yellow opaque liquid; $2.0 \mathrm{R} / \mathrm{hr}$.

24A X-RAY: no features, gas gap $0.2 \mathrm{~cm}$, flat surface.

24B: 2 in. ( $31 \mathrm{~g})$ white/gray salt slurry, $250 \mathrm{~mL}(375 \mathrm{~g})$ yellow clear liquid: $1.5 \mathrm{R} / \mathrm{hr}$.

24A: $2-3$ in. (36 g) white salt slurry, $250 \mathrm{~mL}(365 \mathrm{~g})$ yellow opaque liquid; $2.2 \mathrm{R} / \mathrm{hr}$.

24B: 2 in. ( $38 \mathrm{~g}$ ) white salt slurry, $250 \mathrm{~mL}(356 \mathrm{~g})$ yellow clear liquid: $1.5 \mathrm{R} / \mathrm{hr}$.

24A X-RAY: no features, gas gap $0.2 \mathrm{~cm}$, small bridge between piston and flat surface.

24B: 1 in. (27 g) white sait slurry, $250 \mathrm{~mL}$ ( $367 \mathrm{~g}$ ) yellow clear liquid; $1.9 \mathrm{R} / \mathrm{hr}$.

24A: 6 in. (41 g) white salt slumry, $300 \mathrm{~mL}$ ( $401 \mathrm{~g}$ ) yellow-brown opaque liquid; $2.0 \mathrm{R} / \mathrm{hr}$.

24B: 2 in. (34 g) white salt slurry, $250 \mathrm{~mL}(354 \mathrm{~g})$ yellow clear liquid; $1.9 \mathrm{R} / \mathrm{hr}$.

24A: 4 in. (38 g) white salt slurry, $250 \mathrm{~mL}$ ( $354 \mathrm{~g}$ ) pale yellow opaque liquid; $2.0 \mathrm{R} / \mathrm{hr}$.

24B: 2 in. (28 g) white salt slurry, $250 \mathrm{~mL}$ ( $359 \mathrm{~g}$ ) yellow clear liquid; $1.8 \mathrm{R} / \mathrm{hr}$.

24A: 2 in. (42 g) white salt siurry, $250 \mathrm{~mL}$ ( $354 \mathrm{~g}$ ) yellow opaque liquid; $2.0 \mathrm{R} / \mathrm{hr}$.

24B: 2 in. (24 g) white salt slunry. $250 \mathrm{~mL}(368 \mathrm{~g}$ ) yellow clear liquid; $1.7 \mathrm{R} / \mathrm{hr}$.

24A: 4 in. (31 g) white salt slurry, $250 \mathrm{~mL}$ ( $355 \mathrm{~g}$ ) yellow opaque liquid; $2.0 \mathrm{R} / \mathrm{hr}$.

24B: 1 in. $(20 \mathrm{~g})$ white salt slungy. $250 \mathrm{~mL}(161 \mathrm{~g})$ yellow clear liquid; $1.9 \mathrm{R} / \mathrm{hr}$.

24A: 2.5 in. ( $31 \mathrm{~g}$ ) pale yellow salt slurry, $250 \mathrm{~mL}(377 \mathrm{~g}$ ) yellow clear liquid; $1.8 \mathrm{R} / \mathrm{hr}$.

24B: 2 in. (26 g) white salt slury, $250 \mathrm{~mL}$ ( $369 \mathrm{~g}$ ) yellow clear liquid; $2.1 \mathrm{R} / \mathrm{hr}$.

24A: 8 in. $(54 \mathrm{~g})$ white salt slurry, $250 \mathrm{~mL}(374 \mathrm{~g}$ ) yellow opaque liquid; $1.8 \mathrm{R} / \mathrm{hr}$.

24B: 2 in. (27 g) white salt slumy, $250 \mathrm{~mL}(369 \mathrm{~g})$ yellow clear liquid; $2.0 \mathrm{R} / \mathrm{hr}$.

24A: 2 in. (42 g) white salt slurry, $250 \mathrm{~mL}(368 \mathrm{~g}$ ) yellow opaque liquid; $2.2 \mathrm{R} / \mathrm{hr}$.

24B: 4 in. ( $35 \mathrm{~g}$ ) white salt slurry, $250 \mathrm{~mL}(370 \mathrm{~g})$ yellow clear liquid; $2.1 \mathrm{R} / \mathrm{hr}$.

24A: 7 in. $(52 \mathrm{~g})$ white salt slurry, $250 \mathrm{~mL}(360 \mathrm{~g})$ yellow opaque liquid; $2.5 \mathrm{R} / \mathrm{hr}$.

24B: 2 in. (21 g) white salt slurry, $250 \mathrm{~mL}(374 \mathrm{~g}$ ) yellow opaque liquid; $2.2 \mathrm{R} / \mathrm{hr}$.

24A X-RAY: group of 1 to 2-mm bubbles in bottom inch of sample, surface meniscus, gas gap $0.2 \mathrm{~cm}$.

24B: 15 in. (209 g) dark gray salt slurry, $100 \mathrm{~mL}(157 \mathrm{~g})$ dark gray opaque liquid; $2.0 \mathrm{R} / \mathrm{hr}$.

24A: $19 \mathrm{in}$. (412 g) dark gray wet sludge; $2.0 \mathrm{R} / \mathrm{hr}$.

24B X-RAY: Several 3-mm bubbles in bottom half, a few 1-2 mm oblong dark feanures in top half, no gas gap.

24A X-RAY: numerous 1-2 mm bubbles, several 3-mm, $16-\mathrm{mm}$ in middle, no gas gap.

24B: 19 in. (438 g) gray/black wet sait; $1.7 \mathrm{R} / \mathrm{hr}$.

24A: 19 in. (423 g) dark gray wet sludge; $1.9 \mathrm{R} / \mathrm{hr}$.

24B X-RAY: many 1-2 mm bubbles, several larger oblong bubbles, flat surface, $1.3 \mathrm{~cm}$ gas gap.

24A X-RAY: numerous 1 to $2-\mathrm{mm}$ bubbles, a few 3-mm, more towards top, no gas gap.

24B: 19 in. $(429 \mathrm{~g})$ dark gray wet salt; $1.8 \mathrm{R} / \mathrm{hr}$.

24A: 19 in. (407 g) dark gray wet sludge; $2.2 \mathrm{R} / \mathrm{hr}$.

24B X-RAY: few 2-3 $\mathrm{mm}$ bubbles in bottom third, one 3-mm and one 7-mm bubble toward top, no gas gap.

mpositions, and Observations in Samples from Tank AW-101 
Table 4.1.11 provides a summary of the waste densities that have been calculated from radiographic data for the RGS samples for which air and water standard images were available. The radiographic densities include the gas in the waste and are given for several locations within each sample to show density trends. The overall sample densities were calculated both by the current $\mathrm{x}$-ray analysis method and the original method (described in Section 3.9); the differences give some idea of the sensitivity of results to small variations in method. Table 4.1.11 also includes density measurements made on extruded non-RGS samples (Benar 1996). The radiographic densities exceeded the densities found for the adjacent extruded samples, which is difficult to explain because the radiographic densities included gas and the extruded densities did not.

Table 4.1.11. Densities of AW-101 Samples from Radiography and Core Samples

\begin{tabular}{|c|c|c|c|c|}
\hline $\begin{array}{l}\text { Riser- } \\
\text { Segment } \\
\text { Number }\end{array}$ & $\begin{array}{l}\text { Distance from } \\
\text { Bottom of } \\
\text { Sampler (ft) }\end{array}$ & $\begin{array}{l}\text { Mean Local Density } \\
\text { by Current Method } \\
(\mathrm{g} / \mathrm{cc})\end{array}$ & $\begin{array}{l}\text { Overall Average Sample } \\
\text { Densities } \\
(g / c c)\end{array}$ & $\begin{array}{c}\text { Degassed Density in the non-RGS Cores } \\
\text { above and below the RGS sample } \\
(\mathrm{g} / \mathrm{cc})\end{array}$ \\
\hline \multirow[t]{6}{*}{$24 A-8$} & 1.5 & 1.52 & \multirow{3}{*}{$\begin{array}{l}\text { Current X-ray analysis } \\
\text { method: } \quad 1.59\end{array}$} & density above, $1.39 \mathrm{~g} / \mathrm{cc}$ liquid \\
\hline & 1.25 & 1.60 & & \\
\hline & 1.0 & 1.55 & & \\
\hline & 0.75 & 1.57 & Original x-rav analysis & \\
\hline & 0.5 & 1.60 & method: $\quad 1.42$ (a) & dencity helow 150 alcc linuid \\
\hline & 0.25 & 1.72 & & \\
\hline $24 \mathrm{~A}-17$ & 1.5 & 1.64 & & density above, $1.65 \mathrm{~g} / \mathrm{cc}$ bulk \\
\hline & 1.0 & 1.69 & Current $\mathrm{X}$-ray analysis & \\
\hline & 0.75 & 1.68 & method: 1.68 & \\
\hline & 0.5 & 1.68 & Original $\mathrm{x}$-ray analysis & \\
\hline & 0.25 & 1.7 & method: $\quad 1.58$ (a) & density below, $1.54 \mathrm{~g} / \mathrm{cc}$ bulk \\
\hline $24 \mathrm{~A}-19$ & 1.0 & 1.79 & & density above, $1.54 \mathrm{~g} / \mathrm{cc}$ bulk \\
\hline & 0.75 & 1.79 & Current $\mathrm{X}$-ray analysis & \\
\hline & 0.5 & 1.78 & method: $\quad 1.80$ & \\
\hline & 0.25 & 1.81 & $\begin{array}{l}\text { Unginal } x \text {-ray analysis } \\
\text { method: } \quad 1.60 \text { (a) }\end{array}$ & density below, $1.57 \mathrm{~g} / \mathrm{cc}$ bulk \\
\hline $24 \mathrm{~A}-21$ & 1.5 & 1.83 & & density above, $1.57 \mathrm{~g} / \mathrm{cc}$ bulk \\
\hline & 1.25 & 1.86 & Current $\mathrm{X}$-ray analysis & \\
\hline & 1.0 & 1.88 & method: $\quad 1.90$ & \\
\hline & 0.75 & 1.90 & Original $\mathrm{x}$-ray analysis & \\
\hline & 0.5 & 1.89 & method: $\quad 1.68$ (a) & density below $160 \mathrm{o} / \mathrm{cc}$ bulk \\
\hline & 0.25 & 1.94 & & densily delow, 1.00 g/ce oulk \\
\hline
\end{tabular}

\subsubsection{Other Topics}

\subsubsection{High Local Hydrogen}

In Table 4.1.4, the hydrogen mole fractions for riser 24A (segments 19 and 21) are much higher than 24B (segments 18 and 22). The other insoluble gases, with minor exceptions, do not 
show such a difference. Several explanations are possible; one is the effect of hold times of the samplers from riser $24 \mathrm{~B}$ on leakage of hydrogen through seals and sampler walls.

To determine whether holding time was an issue, the dates the waste samples were taken and the dates the samples were processed in the lab were used to calculate the sample hold times. The results show that the hold times for segments $24 \mathrm{~B}-18$ and $24 \mathrm{~B}-22$ were in fact less than those for segments 24A-19 and 24A-21. In addition, the RGS acceptance test program included leak rate tests during which a gas mixture with $30 \%$ hydrogen was held in the sampler for aging times of 24 to 187 hours without any detectable leakage (Cannon and Knight 1995). Therefore, the leakage of hydrogen (due to extended hold times) does not appear to explain the difference in hydrogen concentration. The most likely explanation is lateral variation in the chemical composition of the waste, which affects the rate of generation of hydrogen.

Hypothetically, a GRE might have caused gas composition variation. But there is no physical reason to predict that GREs affect gas composition because GREs release all the gas constituents together-so there should be no change in the composition of the gas bubbles or pockets. (The void fraction changes, but no gas is preferentially released). Nevertheless, there might have been processes at work that altered the gas composition as a function of residence time. These processes have not been identified, but the change in hydrogen could indicate their existence.

\subsubsection{Drillstring and Domespace Composition Comparison}

The RGS gas-phase composition data (Table 4.1.6) were used to calculate $\mathrm{H}_{2} / \mathrm{N}_{2} \mathrm{O}$ ratios for comparison with ratios from drillstring and domespace grab sample measurements (Table 8.1, Siciliano 1998; Table 4.2, McCain 1999). The results can be seen in Table 4.1.12. While the riser 24A RGS ratios in the nonconvective layer are not far different from the drillstring ratio, the postGRE domespace ratio is higher than any of the other values.

Data from the standard hydrogen monitoring system (SHMS) for the AW-101 dome space show that from July 1, 1998 to July 1, 1999 the hydrogen concentration fluctuated from about 15 to $30 \mathrm{ppm}$, while the nitrous oxide concentration was between 1 and $2 \mathrm{ppm}$ (McCain 1999, Figures D-18 and D-19). These data are consistent with the upper range of ratios in Table 4.1.12, though an average of the point-by-point ratios would be needed to confirm this assessment.

Table 4.1.12. Comparison with Drillstring and Domespace Data(a)

\begin{tabular}{||l|c|}
\hline \multicolumn{1}{|c|}{ Sample } & $\mathrm{H}_{2} / \mathrm{N}_{2} \mathrm{O}$ \\
\hline RGS, 24A-8 & $11-30$ \\
\hline RGS, 24A-17 & $5.0-8.3$ \\
\hline$R G S, 24 \mathrm{~B}-18$ & $2.7-4.8$ \\
\hline$R G S, 24 \mathrm{~A}-19$ & $7.3-9.9$ \\
\hline $\mathrm{RGS}, 24 \mathrm{~A}-21$ & $3.7-5.1$ \\
\hline $\mathrm{RGS}, 24 \mathrm{~B}-22$ & $1.1-2.1$ \\
\hline Drillstring, 2/27/96 & 12 \\
\hline Domespace, after 8/2/95 GRE & 61 \\
\hline $\begin{array}{l}\text { (a) RGS ratios calculated for both lower- and upper-bound solubilities. } \\
\text { Both ends of ratio range are given, lower-bound solubility basis first. }\end{array}$ \\
\hline
\end{tabular}




\subsection{AN-105}

Tank 241-AN-105 (AN-105) was the second tank and the second DST sampled with the RGS. This tank was selected because it was a DST that exhibited episodic GREs. AN-105 was on the FGWL and showed cyclical level drops and episodic domespace hydrogen concentrations of as high as $1.6 \%$ resulting from GREs. Most of the tank contents were double-shell slurry feed (Stewart et al. 1996a).

Push-mode sampling was done in risers 12A and 7B in June 1996.(a) Riser 12A was sampled because of its relative proximity to riser $1 \mathrm{~B}$, where VFI and ball rheometer measurements were made in September 1995 (Figure 4.2.1). Riser 7B was close to the thermocouple tree in riser $4 \mathrm{~A}$, allowing correlation of waste temperatures and gas content. The approximate locations of various risers are depicted in Figure 4.2.1.

The total depth of waste in Tank AN-105 was $1041 \mathrm{~cm}$ (410 in.); at the time of RGS sampling, AN-105 contained approximately 4,280,000 L (1,130,000 gal) of waste. Riser 12A is near the tank center, while riser $7 \mathrm{~B}$ is about half a tank radius from the center. Figure 4.2.2 shows the tank content layering, based on temperature profiles and VFI/ball rheometer data for this tank (Schienbein et al. 1999, Table 2.3.2). The nonconvective layer was $450 \pm 40 \mathrm{~cm}(177 \pm 16 \mathrm{in}$.) in depth, with the balance of the contents being a convective (supernatant liquid) layer of $546 \pm 43 \mathrm{~cm}$ ( $215 \pm 17 \mathrm{in}$.) thickness and a noncontinuous floating crust that (where present) was $45 \pm 10 \mathrm{~cm}$ (18 \pm 4 in.) thick. The elevations of the RGS segments are depicted in Figure 4.2.2.

The most recent information on $\mathrm{AN}-105$ layering came from the observations. made on the non-RGS samples in the cores from 12A and 7B. The topmost sample contained a few inches of salt slurry, possibly the crust. Beneath that were 13 to 14 samplers (250 to 270 in.) of liquid, with 8 to 9 samplers ( 150 to 170 in.) of wet salt or sludge at the tank bottom.

One buoyant displacement GRE occurred on May 30, 1996, about two weeks before RGS sampling began (Schienbein et al. 1999). The GRE caused a waste level drop of $2.4 \mathrm{~cm}$ and might also have caused differences between RGS gas volume fractions and those measured by VFI in December 1995 (Schienbein et al. 1999). Temperature profiles at riser 4A (near RGS riser 7B) showed temperature profile changes down to at least $130 \mathrm{~cm}$ (51 in.) elevation, more than twothirds of the way down into the layer (Schienbein et al. 1999, Figure 2.3.29).

Densities for the liquid and bulk solids in Tank AN-105 were available from the non-RGS samples taken in 1996 (Jo 1997). Several samples contained drainable liquid. The density of the liquid was $1417 \pm 37 \mathrm{~kg} / \mathrm{m}^{3}$; the bulk density of the nonconvective layer samples varied from 1520 to $1660 \mathrm{~kg} / \mathrm{m}^{3}$, with an average and standard deviation of $1578 \pm 42 \mathrm{~kg} / \mathrm{m}^{3}$. Schienbein et al. (1999) state a density of $1400 \pm 60 \mathrm{~kg} / \mathrm{m}^{3}$ for the convective layer (based on in situ ball rheometry in 1995) and $1580 \pm 40 \mathrm{~kg} / \mathrm{m}^{3}$ for the nonconvective layer (based on core samples). All of these measurement methods disturbed the waste, and their results should be taken as degassed densities.

For hydrostatic pressure calculations, the liquid density was set at $1400 \mathrm{~kg} / \mathrm{m}^{3}$, the gassy crust density at the same value as the convective layer supporting it, and the nonconvective layer at a degassed bulk density of $1580 \mathrm{~kg} / \mathrm{m}^{3}$. Depths of $45 \mathrm{~cm}$ (18 in.), $546 \mathrm{~cm}$ (215 in.), and $450 \mathrm{~cm}$ (177 in.) were used in calculations for the crust, convective, and nonconvective layers.

(a) The sampling scheme may be found in Sampling Plan for Tank 241-AN-105 Retained Gas Sampler Deployment, by JR Phillips, R Shekarriz, and JM Bates, March 1996. PNLMIT:030796, Pacific Northwest National Laboratory, Richland, Washington. Also refer to the May 30, 1996 addenda letter to RE Bauer, Westinghouse Hanford Company, from JM Bates, Pacific Northwest National Laboratory, Richland; Washington. 


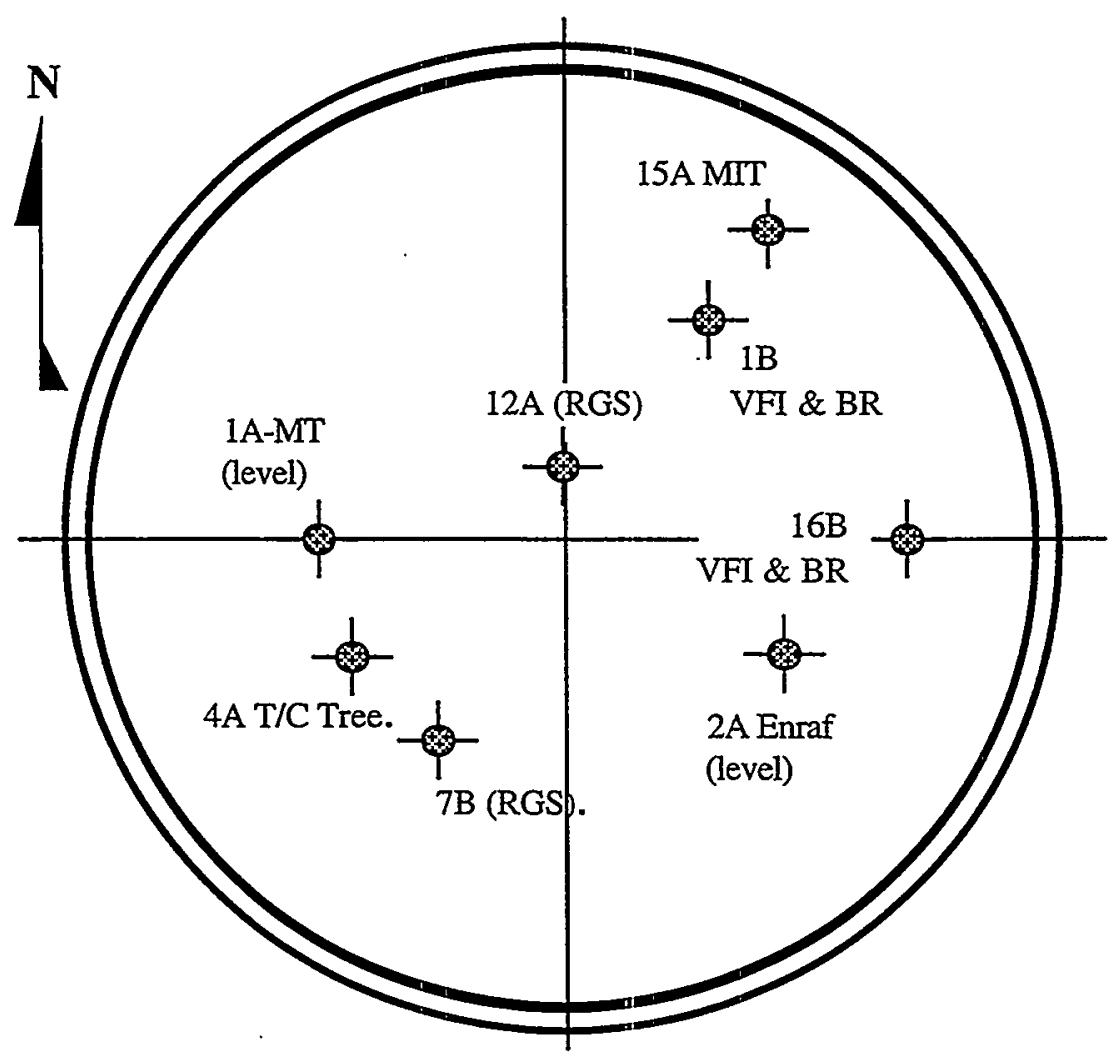

Figure 4.2.1. Schematic Diagram of Riser Locations in Tank AN-105
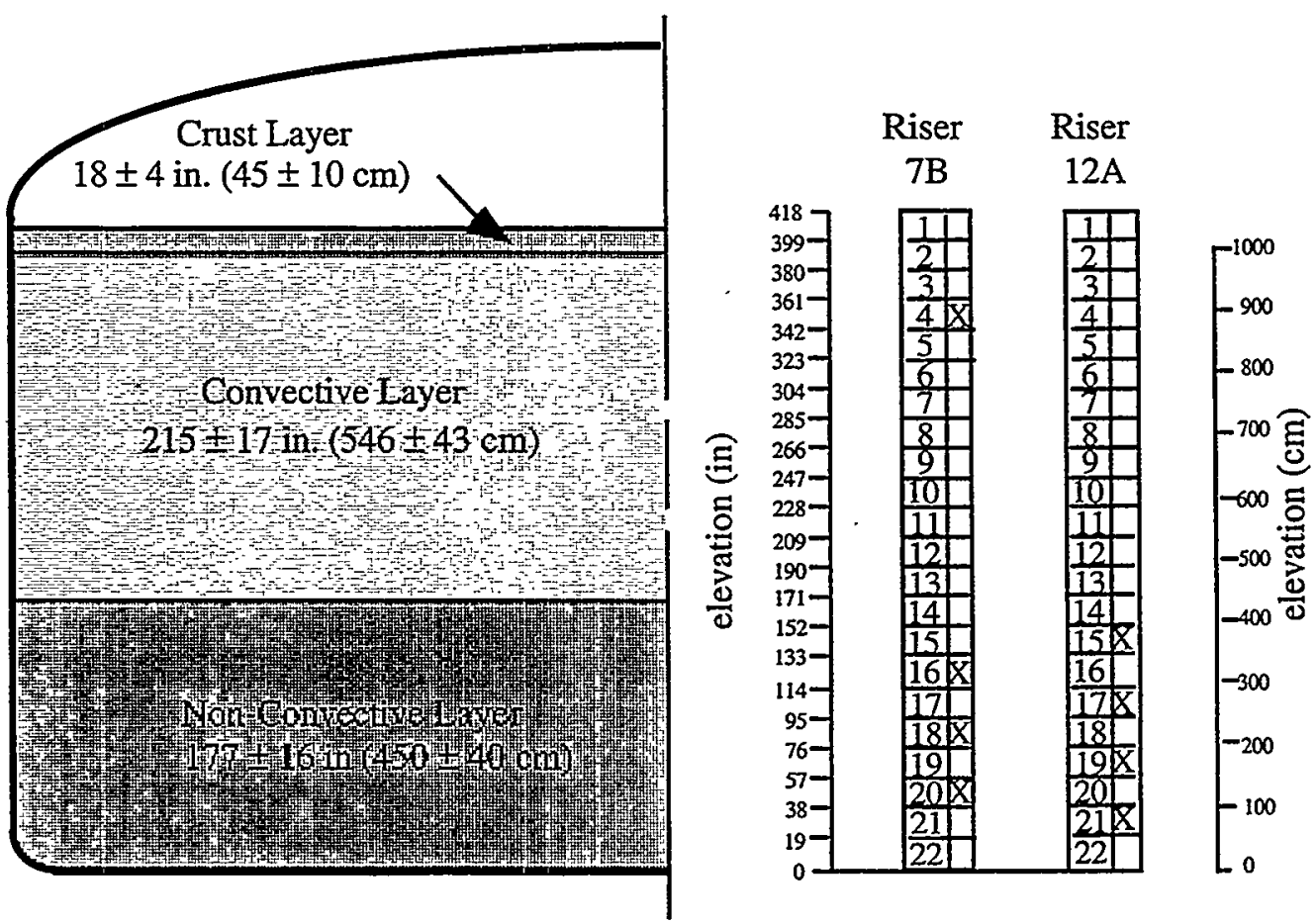

Figure 4.2.2. Diagram of Waste Layering and RGS Sample Elevations for AN-105 


\subsubsection{Sampling and Extraction Information}

The samples that underwent RGS analysis are listed in Table 4.2.1. (Sample 7B-20 was lost because of sampler valve problems.) Field data, including dose rates and downforce limits, are supplied in Appendix A, which is provided to pull together as many as possible of the data relevant to RGS samples into one document.

Table 4.2.1 also shows the lag times (delay between sample acquisition and processing) for these samples. This information was provided to allow data users to correlate the lag time between sampling and extrusion with the concentrations of the various constituents to test for decomposition or other chemical reactions. The maximum hold time allowed by the sampling plan is 24 days, based on measured sampler leakage rates during acceptance testing. The hold time for samples $12 \mathrm{~A}-21$ and $7 \mathrm{~B}-4$ exceeded this limit, but the sample was accepted based on calculations that indicated that the estimated leakage was still negligible.

Table 4.2.2 shows the volume percentage contamination by HHF of the RGS samples. All the measured samples contained bromide below the MDL. The measured HHF contamination percentages were used to calculate reduced effective sampler volumes for the RGS samples for which there were measurements, with the bromide concentration set equal to the MDL for samples below the limit. For the three samples that lacked measurements, the HHF contamination was set to $2.2 \mathrm{vol} \%$, the average of the measured values in AN-105 RGS samples. (It is conservative to use the MDL for values below it; the conservatism is less than $2 \%$.)

Two different extraction procedures were used on the AN-105 samples, but the first several steps were the same in both (see RGS system schematic in Figure 3.1.) After evacuating the whole system and observing the pressure to test for leaks, the operator extruded the RGS sample into the extractor vessel, and the sample was briefly stirred. At this point the vapor space in communication with the sample consisted of the volumes of the extractor vessel and its lines. Next, the extractor was opened to the second vessel, to which was connected a PQ canister. Once a grab sample was collected in the PQ canister, it was closed. The extractor, lines, and second volume were then connected to the pump volume and collector side; the latter included the collector line volume and one open collection canister. Several strokes of gas and vapor (unbound gas) were pumped from the extractor to the collector, after which the first collection canister was closed off. From this point, different procedures were used for the 12A-19 and all other AN-105 samples.

Sample AN-105-12A-19: The collector side, second volume, and extractor lines were not evacuated after the first collection canister was closed off. Several strokes were pumped from the

Table 4.2.1. Lag Times for Processing RGS Samples from Tank 241-AN-105

\begin{tabular}{||c|c|c|c||}
\hline Sample & Acquisition Date & Processing Date & Lag (days) \\
\hline \hline $7 \mathrm{~B}-4$ & June 26,1996 & July 25,1996 & 29 \\
\hline 12A-15 & June 13,1996 & June 18,1996 & 5 \\
\hline $7 \mathrm{~B}-16$ & June 28,1996 & July 10,1996 & 12 \\
\hline 12A-17 & June 14,1996 & June 28,1996 & 14 \\
\hline $7 \mathrm{~B}-18$ & June 28,1996 & July 16,1996 & 18 \\
\hline 12A-19 & June 14,1996 & July 2, 1996 & 18 \\
\hline 12A-21 & June 17,1996 & July 12,1996 & 25 \\
\hline
\end{tabular}


Table 4.2.2. Hydrostatic Head Fluid Contamination in AN-105 RGS Samples

\begin{tabular}{||l|c|}
\hline Sample & $\begin{array}{c}\text { HHF Contamination } \\
\text { (vol \%) }\end{array}$ \\
\hline $7 \mathrm{~B}-4$ (RGS) & $<2.2$ \\
\hline $12 \mathrm{~A}-15$ (RGS) & $<2.7$ \\
\hline $7 \mathrm{~B}-16$ (RGS) & no data \\
\hline $12 \mathrm{~A}-17$ (RGS) & no data \\
\hline 7B-18 (RGS) & $<1.4$ \\
\hline $12 \mathrm{~A}-19$ (RGS) & $<2.7$ \\
\hline $12 \mathrm{~A}-21$ (RGS) & no data \\
\hline
\end{tabular}

extractor to the collector, and the second collection canister was closed off. Then the collector side, second volume, and extractor lines were evacuated. Next, the extractor and samples were heated to $\sim 50^{\circ} \mathrm{C}$ to drive off bound gas, cooled back to hot cell temperature, and briefly stirred. A $\mathrm{PQ}$ canister connected to the extractor vessel took a second sample and was closed off. Several strokes were pumped from the extractor to the collector, and the third collection canister was closed off. Finally, the BSVD procedure was performed: first, argon was injected into the closed-off second volume, which was opened to the extractor and lines. Pressure measurements and known tare system volumes allowed calculation of the volume of sample solids and liquid.

Other AN-105 samples: The collector side, second volume, and extractor lines were evacuated after the first collection canister was closed off. Next, the extractor and samples were heated to about $50^{\circ} \mathrm{C}$ to drive off bound gas, cooled back to hot cell temperature, and briefly stirred. A PQ canister connected to the extractor vessel took a second sample and was closed off. Several strokes were pumped from the extractor to the collector, and the collection canister was closed off. At this point the collector side, second volume, and extractor lines were not evacuated. Several strokes of gas and vapor were pumped from the extractor to the collector (to which one open collection canister, the third one, was connected). The BSVD procedure was performed last.

Procedural details such as the number of strokes per canister and the system volumes are provided in the sample-by-sample worksheets in the AN-105 RGS calculation spreadsheet on the $\mathrm{CD}$ included with this report.

\subsubsection{Retained Gas Composition}

Table 4.2.3 presents the calculated concentrations of the insoluble gases in each RGS sample taken from AN-105 without corrections for air and argon entrainment or air inleakage. The method used to make the corrections depended on the gas. All of the oxygen and argon were subtracted no matter which step had produced them. A number of moles equal to $(3.73) \cdot\left(\mathrm{O}_{2}\right)$ was subtracted from the nitrogen because the oxygen was expected to have come from air entrainment during sampling or inleakage during extraction. Corrected concentrations are given in Table 4.2.4.

The variation in the amount of argon in the AN-105 samples suggests the effect of drillstring purges. These contamination observations are shown in the "Summary" worksheet of the AN-105 RGS calculations spreadsheet on the CD included with the report. 
Table 4.2.3. Concentrations of Insoluble Constituents ( $\mu \mathrm{mol} / \mathrm{L}$ of waste) in Tank AN-105 Without Correction for Gas Contamination

\begin{tabular}{||c|c|c|c|c|c|c|c|c|c|c|c||}
\hline Sample & $\mathrm{N}_{2}$ & $\mathrm{H}_{2}$ & $\mathrm{~N}_{2} \mathrm{O}$ & $\mathrm{O}_{2}$ & $\mathrm{CH}_{4}$ & $\mathrm{He}$ & $\mathrm{Ar}$ & $\begin{array}{c}\text { Other } \\
\mathrm{NO}_{x}\end{array}$ & $\mathrm{C}_{2} \mathrm{H}_{\mathrm{x}}$ & $\mathrm{C}_{3} \mathrm{H}_{\mathrm{x}}$ & Other Hyd. \\
\hline \hline $7 \mathrm{~B}-4$ & $540 \pm 99$ & $91 \pm 18$ & $88 \pm 21$ & $92 \pm 17$ & $5.1 \pm 2.0$ & (a) & $140 \pm 25$ & $3.5 \pm 1.6$ & $2.5 \pm 1.5$ & $1.6 \pm 0.7$ & $3.2 \pm 1.7$ \\
\hline $12 \mathrm{~A}-15$ & $940 \pm 180$ & $79 \pm 13$ & $120 \pm 24$ & $190 \pm 41$ & $5.6 \pm 1.6$ & (a) & $11 \pm 2.1$ & $3.4 \pm 1.5$ & $2.0 \pm 0.8$ & $1.6 \pm 0.5$ & $3.7 \pm 1.2$ \\
\hline $7 \mathrm{~B}-16$ & $660 \pm 100$ & $100 \pm 17$ & $89 \pm 22$ & $77 \pm 12$ & $4.6 \pm 1.8$ & (a) & $110 \pm 17$ & $3.8 \pm 1.7$ & $1.5 \pm 1.1$ & $1.6 \pm 0.8$ & $4.0 \pm 2.1$ \\
\hline $12 \mathrm{~A}-17$ & $1500 \pm 82$ & $3600 \pm 180$ & $640 \pm 45$ & $85 \pm 7.2$ & $34 \pm 4.1$ & (a) & $8.8 \pm 0.9$ & $7.7 \pm 1.0$ & $10 \pm 2.9$ & $2.8 \pm 0.9$ & $16 \pm 4.5$ \\
\hline $7 \mathrm{~B}-18$ & $910 \pm 78$ & $1200 \pm 110$ & $320 \pm 40$ & $62 \pm 6.0$ & $17 \pm 3.3$ & (a) & $54 \pm 5.5$ & $5.9 \pm 1.5$ & $11 \pm 5.1$ & $1.6 \pm 0.5$ & $18 \pm 8.6$ \\
\hline $12 \mathrm{~A}-19$ & $2500 \pm 96$ & $6600 \pm 250$ & $1300 \pm 59$ & $84 \pm 4.0$ & $63 \pm 5.0$ & (a) & $8.9 \pm 0.6$ & $14 \pm 4.1$ & $7.4 \pm 2.2$ & $5.4 \pm 0.9$ & $15 \pm 4.7$ \\
\hline $12 \mathrm{~A}-21$ & $1600 \pm 75$ & $3900 \pm 180$ & $1400 \pm 88$ & $18 \pm 1.4$ & $54 \pm 3.0$ & (a) & $430 \pm 20$ & $15 \pm 1.6$ & $5.1 \pm 1.4$ & $3.4 \pm 0.8$ & $8.1 \pm 2.1$ \\
\hline
\end{tabular}

(a) Below detection limits, which are $0.01 \mathrm{~mol} \%$ or less.

Table 4.2.4. Concentrations of Insoluble Constituents ( $\mu \mathrm{mol} / \mathrm{L}$ of waste) in Tank AN-105 with Correction for Gas Contamination

\begin{tabular}{||c|c|c|c|c|c|c|c|c|c|c|c||}
\hline Sample & $\mathrm{N}_{2}$ & $\mathrm{H}_{2}$ & $\mathrm{~N}_{2} \mathrm{O}$ & $\mathrm{O}_{2}$ & $\mathrm{CH}_{4}$ & $\mathrm{He}$ & $\mathrm{Ar}$ & $\begin{array}{c}\text { Other } \\
\mathrm{NO}_{x}\end{array}$ & $\mathrm{C}_{2} \mathrm{H}_{x}$ & $\mathrm{C}_{3} \mathrm{H}_{x}$ & $\begin{array}{l}\text { Other } \\
\text { Hyd. }\end{array}$ \\
\hline \hline $7 \mathrm{~B}-4$ & $200 \pm 120$ & $91 \pm 18$ & $88 \pm 21$ & $0 \pm 17$ & $5.1 \pm 2.0$ & (a) & $0 \pm 25$ & $3.5 \pm 1.6$ & $2.5 \pm 1.5$ & $1.6 \pm 0.7$ & $3.2 \pm 1.7$ \\
\hline $12 \mathrm{~A}-15$ & $240 \pm 230$ & $79 \pm 13$ & $120 \pm 24$ & $0 \pm 41$ & $5.6 \pm 1.6$ & (a) & $0 \pm 2.1$ & $3.4 \pm 1.5$ & $2.0 \pm 0.8$ & $1.6 \pm 0.5$ & $3.7 \pm 1.2$ \\
\hline $7 \mathrm{~B}-16$ & $380 \pm 110$ & $100 \pm 17$ & $89 \pm 22$ & $0 \pm 12$ & $4.6 \pm 1.8$ & (a) & $0 \pm 17$ & $3.8 \pm 1.7$ & $1.5 \pm 1.1$ & $1.6 \pm 0.8$ & $4.0 \pm 2.1$ \\
\hline $12 \mathrm{~A}-17$ & $1200 \pm 83$ & $3600 \pm 180$ & $640 \pm 45$ & $0 \pm 7.2$ & $34 \pm 4.1$ & (a) & $0 \pm 0.9$ & $7.7 \pm 1.0$ & $10 \pm 2.9$ & $2.8 \pm 0.9$ & $16 \pm 4.5$ \\
\hline $7 \mathrm{~B}-18$ & $680 \pm 79$ & $1200 \pm 110$ & $320 \pm 40$ & $0 \pm 6.0$ & $17 \pm 3.3$ & (a) & $0 \pm 5.5$ & $5.9 \pm 1.5$ & $11 \pm 5.1$ & $1.6 \pm 0.5$ & $18 \pm 8.6$ \\
\hline $12 \mathrm{~A}-19$ & $2200 \pm 320$ & $6600 \pm 250$ & $1300 \pm 59$ & $0 \pm 4.0$ & $63 \pm 5.0$ & (a) & $0 \pm 0.6$ & $14 \pm 4.1$ & $7.4 \pm 2.2$ & $5.4 \pm 0.9$ & $15 \pm 4.7$ \\
\hline $12 \mathrm{~A}-21$ & $1500 \pm 75$ & $3900 \pm 180$ & $1400 \pm 88$ & $0 \pm 1.4$ & $54 \pm 3.0$ & (a) & $0 \pm 20$ & $15 \pm 1.6$ & $5.1 \pm 1.4$ & $3.4 \pm 0.8$ & $8.1 \pm 2.1$ \\
\hline
\end{tabular}

(a) Below detection limits, which are $0.01 \mathrm{~mol} \%$ or less.

Table 4.2.5 presents the ammonia measurements. The RGS procedure did not permit the determination of the residual or total ammonia, but the post-extrusion and first PQ canister partial pressures of $\mathrm{NH}_{3}$ over the sample (see Section 3.4.4) were measured at laboratory temperature. The residual ammonia in a few of the samples was also measured by ISE after the samples were removed from the extractor; as discussed in Section 3.4.2, these ammonia concentrations should be considered lower bounds because of ammonia evaporation during sample handling. Ammonia data can be seen in more detail in Appendix C, Section C.2. No conclusive total ammonia con- centration can be calculated, but the lower-bound estimate was roughly $27,000 \mu \mathrm{mol} \mathrm{NH} / \mathrm{L}$ waste. This estimate is based on a comparison of $\mathrm{AN}-105$ and $\mathrm{AW}-101$ partial pressures; this comparison was useful because AW-101 data included a more complete set of post-RGS ISE ammonia measurements. 
Table 4.2.5. Ammonia Data from Tank AN-105 Samples

\begin{tabular}{|c|c|c|c|}
\hline Sample & $\begin{array}{l}\text { Measured } \mathrm{NH}_{3} \\
\text { partial pressure at } \\
\text { about } 24^{\circ} \mathrm{C} \\
(\mathrm{atm})\end{array}$ & $\begin{array}{c}\text { Total } \mathrm{NH}_{3} \text { from } \\
\text { post-RGS residual } \\
\mathrm{NH}_{3} \text {, by ISE (a) } \\
(\mu \mathrm{mol} / \mathrm{L})\end{array}$ & $\begin{array}{c}\text { Best-Estimate } \mathrm{NH}_{3} \\
\text { Concentrations }\end{array}$ \\
\hline $7 \mathrm{~B}-4$ & $0.0048 \pm 0.0024$ & 2700 & \multirow{7}{*}{$\begin{array}{l}\text { The data do not support } \\
\text { conclusive } \mathrm{NH}_{3} \text { concen- } \\
\text { trations. We estimate a } \\
\text { lower bound of } 0.037 \mathrm{M} \\
(620 \mu \mathrm{mL}) \mathrm{NH}_{3} \text { in the } \\
\text { liquid of the nonconvective } \\
\text { layer, or } 0.032 \text { wt } \% \mathrm{NH}_{3} \text { in } \\
\text { the bulk waste. }\end{array}$} \\
\hline $12 \mathrm{~A}-15$ & $0.0053 \pm 0.0027$ & 12000 & \\
\hline $7 \mathrm{~B}-16$ & $0.0043 \pm 0.0022$ & no data & \\
\hline $12 \mathrm{~A}-17$ & $0.0086 \pm 0.0042^{(b)}$ & no data & \\
\hline $7 \mathrm{~B}-18$ & $0.0057 \pm 0.0029$ & 11000 & \\
\hline $12 \mathrm{~A}-19$ & $0.0070 \pm 0.0034$ & no data & \\
\hline $12 \mathrm{~A}-21$ & $0.0053 \pm 0.0027$ & no data & \\
\hline \multicolumn{4}{|c|}{$\begin{array}{l}\text { (a) These total ammonia concentrations are the sum of the post-RGS ISE ammonia residual and } \\
\text { the RGS extracted ammonia. They should be considered lower bounds because of ammonia } \\
\text { evaporation during the sample handling that preceded ISE analysis. } \\
\text { (b) Ammonia partial pressures are based on PQ canister measurements except for sample } \\
\text { 12A-17. No PQ canister data were available, so the calculated post-extrusion partial pressure was } \\
\text { used instead. }\end{array}$} \\
\hline
\end{tabular}

Table 4.2.6 contains the composition of the gas phase in each sample and the integrated average compositions in the gas retained in the nonconvective layer. The convective layer composition is based on the single sample; the water vapor is not included in these compositions. The ammonia fractions are derived from the partial pressures indicated in Table 4.2.5, extrapolated to vapor pressures at in situ conditions. The sample compositions in the table have been calculated using the in situ solubility method described in Section 3.6.2. As discussed in Section 3.6.1, compositions were calculated for both the lower- and upper-bound gas solubilities. Both ends of the composition range are given in Table 4.2.6, along with the measurement uncertainty on each. The average composition of the gas in the nonconvective layer is the result of integrating RGS species concentrations over the waste layer and multiplying those concentrations by the waste volume. The integration method is described in Section 3.7.1.

Within the uncertainty, there was no definite composition difference between samples from the two risers, though sample 7B-18 appeared lower in hydrogen and higher in nitrogen then vertically adjacent samples $12 \mathrm{~A}-17$ and 12A-19. As in samples from other RGS-sampled tanks, nitrogen was high in the AN-105 convective layer and hydrogen low, though the broad uncertainty makes this interpretation somewhat inconclusive. The top two RGS samples in the nonconvective layer (12A-15 and 7B-16) had the same composition and gas volume fraction as the sample in the convective layer (7B-4), even though core samples showed substantial amounts of solids at and above the level of segment 15 . The absence of retained gas at the top of the nonconvective layer might have resulted from the GRE two weeks before.

The concentrations and pressures in Tables 4.2.3 and 4.2.4 can also be found in the sample-by-sample worksheets in the AN-105 RGS calculation spreadsheet on the CD. The compositions in Table 4.2.6 are in the "Inventory" worksheet on the CD. 


\subsubsection{Gas Inventory}

The method by which the in situ gas volume fractions (wet basis) were calculated is given in Section 3.6.2; as for the gas composition, the volume fractions are given as a range from the low gas-solubility value to the high gas-solubility value. The results are presented in Table 4.2.7. Table 4.2.7 also contains the average gas volume fraction and the average pressure experienced by the gas in the nonconvective layer. The averages are in situ volume averages calculated by Simpson's Rule integration, as described in Section 3.7.1. The corrected gas volume fractions in Table 4.2.7 are consistent with the corrected gas concentrations and compositions in Tables 4.2.4 through 4.2.6. The information in Table 4.2.7 is taken from the "Summary" and "In situ" worksheets of the AN-105 RGS calculation spreadsheet on the enclosed CD.

The "sampler gas volume fraction"-is corrected only for inleakage during the extraction process and air gases added in the isotopic solution. It is an attempt to reconstruct the total amount of gas (sample and entrainment) that was present in the sampler during $\mathrm{X}$-ray and is used only for comparison with $\mathrm{x}$-ray observations of "visible gas fraction" discussed in Section 4.2.4.

Table 4.2.7 also contains the water vapor pressures that were used for in situ calculations. The water vapor pressures were found by using salt concentrations from Jo (1997) and the temperatures in the table as inputs to Equation 6.2 of Mahoney and Trent (1995), a correlation for water vapor pressure over concentrated homogeneous and non-homogeneous waste simulants. The gas solubilities used the same parameters as inputs to the Schumpe solubility model (see Section 3.6.1). The water vapor pressures and gas solubilities used in calculations can be found in the "In situ" worksheet of the AN-105 RGS calculation spreadsheet on the CD.

Table 4.2.6. Sample and Overall Average Compositions of Retained Gas in Tank AN-105 with Correction for Gas Contamination(a)

\begin{tabular}{|c|c|c|c|c|c|c|}
\hline Sample & $\mathrm{N}_{2}(\mathrm{~mol} \%)$ & $\mathrm{H}_{2}^{\prime}(\mathrm{mol} \%)$ & $\mathrm{N}_{2} \mathrm{O}(\mathrm{mol} \%)$ & $\mathrm{NH}_{3}(\mathrm{~mol} \%)$ (b) & $\mathrm{CH}_{4}(\mathrm{~mol} \%)$ & Other (mol\%) \\
\hline $7 \mathrm{~B}-4$ & $57 \pm 41-64 \pm 47$ & $25 \pm 12$ & $13 \pm 6.6-4.6 \pm 2.4$ & $0.8 \pm 0.5-0.4 \pm 0.2$ & $1.4 \pm 0.8$ & $3.0 \pm 2.0-3.9 \pm 2.7$ \\
\hline $12 \mathrm{~A}-15$ & $62 \pm 62-71 \pm 71$ & $19 \pm 13-19 \pm 15$ & $14 \pm 9.7-4.2 \pm 3.2$ & $0.6 \pm 0.5-0.2 \pm 0.2$ & $1.4 \pm 1.0$ & $2.8 \pm 2.2-3.9 \pm 3.3$ \\
\hline 7B-16 & $70 \pm 28-76 \pm 30$ & $18 \pm 5.8$ & $8.5 \pm 3.1-2.9 \pm 1.1$ & $0.4 \pm 0.2-0.2 \pm 0.1$ & $0.8 \pm 0.4$ & $2.0 \pm 1.2-2.5 \pm 1.5$ \\
\hline $12 \mathrm{~A}-17$ & $22 \pm 2.0-23 \pm 2.1$ & $65 \pm 5.2-66 \pm 5.2$ & $11 \pm 1.0-8.8 \pm 0.8$ & $0.9 \pm 0.4-0.4 \pm 0.2$ & $0.6 \pm 0.1$ & $0.7 \pm 0.2$ \\
\hline $7 \mathrm{~B}-18$ & $31 \pm 4.9-33 \pm 5.2$ & $54 \pm 7.7-57 \pm 8.0$ & $12 \pm 2.0-7.6 \pm 1.3$ & $0.7 \pm 0.3-0.3 \pm 0.1$ & $0.8 \pm 0.2$ & $1.6 \pm 0.7-1.8 \pm 0.8$ \\
\hline $12 \mathrm{~A}-19$ & $21 \pm 3.4-22 \pm 3.5$ & $65 \pm 4.9-66 \pm 5.0$ & $12 \pm 1.0-11 \pm 0.9$ & $0.7 \pm 0.3-0.3 \pm 0.1$ & $0.6 \pm 0.06$ & $0.4 \pm 0.1$ \\
\hline $12 \mathrm{~A}-21$ & $22 \pm 1.6-23 \pm 1.7$ & $57 \pm 4.0-59 \pm 4.1$ & $19 \pm 1.6-16 \pm 1.3$ & $0.5 \pm 0.2-0.2 \pm 0.1$ & $0.8 \pm 0.06$ & $0.5 \pm 0.1$ \\
\hline $\begin{array}{c}\text { Convective } \\
\text { layer(c) }\end{array}$ & $57 \pm 41-64 \pm 47$ & $25 \pm 12$ & $13 \pm 6.6-4.6 \pm 2.4$ & $0.8 \pm 0.5-0.4 \pm 0.2$ & $1.4 \pm 0.8$ & $3.0 \pm 2.0-3.9 \pm 2.7$ \\
\hline $\begin{array}{l}\text { Avg in settled } \\
\text { layer(c) }\end{array}$ & $24 \pm 4.0$ & $59 \pm 5.4-62 \pm 5.4$ & $15 \pm 1.5-12 \pm 1.2$ & $0.6 \pm 0.3-0.3 \pm 0.1$ & $0.7 \pm 0.09$ & $0.6 \pm 0.2$ \\
\hline \multicolumn{7}{|c|}{$\begin{array}{l}\text { (a) Uncertainties on compositions represent only instrument error. Compositions were calculated for both lower-and } \\
\text { upper-bound solubilities. Both ends of composition range are given, lower-bound solubility basis first, with instrument } \\
\text { uncertainty on each. Compositions may not sum to } 100 \mathrm{~mol} \% \text { because of roundoff error. Mole fractions are on a dry } \\
\text { basis and do not account for water vapor. } \\
\text { (b) The ammonia mole fraction is derived from the partial pressures indicated in Table } 4.2 .5 \text {, extrapolated to vapor } \\
\text { pressure at in situ conditions. } \\
\text { (c) There are too few samples to define the spatial variability of the average gas concentration. . }\end{array}$} \\
\hline
\end{tabular}


Table 4.2.7. In Situ Gas Volume Fractions and Conditions in Tank AN-105

\begin{tabular}{|c|c|c|c|c|c|c|}
\hline Sample & $\begin{array}{c}\text { Sample } \\
\text { central } \\
\text { height } \\
(\mathrm{cm}) \\
\end{array}$ & $\begin{array}{l}\text { Hydro- } \\
\text { static } \\
\text { pressure } \\
\text { (atm) } \\
\end{array}$ & $\begin{array}{c}\text { Calculated } \\
\text { water vapor } \\
\text { pressure } \\
(\mathrm{atm}) \\
\end{array}$ & \begin{tabular}{|c|} 
Temp. \\
$\left({ }^{\circ} \mathrm{C}\right)$ \\
\end{tabular} & $\begin{array}{c}\text { Corrected gas volume } \\
\text { fraction (a) } \\
\text { (in-tank conditions) }\end{array}$ & $\begin{array}{c}\text { Sampler gas } \\
\text { volume fraction } \\
\text { (in situ conditions, } \\
\text { low solubility) }\end{array}$ \\
\hline $7 \mathrm{~B}-4$ & 893 & 1.20 & 0.034 & 38.1 & $0.008 \pm 0.003-0.006 \pm 0.003$ & 0.018 \\
\hline $12 \mathrm{~A}-15$ & 362 & 1.92 & 0.035 & 38.5 & $0.005 \pm 0.002-0.004 \pm 0.002$ & 0.014 \\
\hline $7 \mathrm{~B}-16$ & 314 & 1.99 & 0.036 & 39.0 & $0.007 \pm 0.002-0.006 \pm 0.002$ & 0.012 \\
\hline $12 \mathrm{~A}-17$ & 265 & 2.06 & 0.024 & 39.5 & $0.069 \pm 0.007-0.066 \pm 0.007$ & 0.074 \\
\hline $7 \mathrm{~B}-18$ & 217 & 2.12 & 0.027 & $\overline{41.1}$ & $0.027 \pm 0.004-0.025 \pm 0.004$ & 0.031 \\
\hline $12 \mathrm{~A}-19$ & 169 & 2.19 & 0.027 & 41.3 & $0.12 \pm 0.008$ & 0.13 \\
\hline $12 \mathrm{~A}-21$ & 72.4 & 2.33 & 0.022 & 38.0 & $0.075 \pm 0.007-0.071 \pm 0.007$ & 0.080 \\
\hline $\begin{array}{c}\text { Convective } \\
\text { layer }\end{array}$ & 608 & 1.45 & & & $0.006 \pm 0.002-0.005 \pm 0.002$ & \\
\hline $\begin{array}{l}\text { Avg in non- } \\
\text { convective } \\
\text { layer }\end{array}$ & 142 & 2.26 & & & $0.052 \pm 0.026-0.049 \pm 0.024$ & \\
\hline $\begin{array}{l}\text { a) Gas volun } \\
\text { Jncertainties } \\
\text { iscussed in } S\end{array}$ & fracti & express & we & $\mathrm{clu}$ & 5 volume contribution of $y$ & lerations \\
\hline
\end{tabular}

No ammonia inventory was calculated for the tank because no total ammonia concentration data exist for AN-105. Table 4.2.8 gives various estimates of the STP volume of gas in AN-105, including estimates calculated from RGS data alone. The VFI\&RGS and BPE inventory values were taken from Schienbein et al. (1999).

The RGS gas inventory in the nonconvective layer was calculated by integrating RGS total gas concentrations over the layer (six data points) and multiplying the average gas concentrations by the volume of the layer. The integration method is described in Section 3.7.1. The convective layer composition is based on the single sample and the convective layer thickness. The RGS volumes in Table 4.2.8 include corrections to remove the contamination gas: entrained air and argon and air leaks during and after extraction. The uncertainties on the gas inventories are based on the spatial variability considerations discussed in Section 3.7.2. The information in Table 4.2.8 is taken from the "Inventory" worksheet of the AN-105 RGS calculation spreadsheet on the CD.

The RGS method gave the highest total gas inventory $\left(134 \pm 48 \mathrm{~m}^{3}\right.$ in situ, counting the crust gas) and the BPE method the lowest, with the inventory calculated by the VFI\&RGS method in between (109 $\pm 19 \mathrm{~m}^{3}$ in situ). As Figure 4.2.3 suggests, the RGS inventory may have been higher than the VFI\&RGS inventory partly because RGS captured relatively high gas contents at an elevation too low for VFI to reach (sample 12A-21). The single high gas sample (12A-19) taken by RGS near the bottom of the VFI elevation range also made the RGS inventory higher. The RGS data suggest but do not confirm that gas volume fractions were lower under riser 7B than under riser $12 \mathrm{~A}$.

The uncertainty in the BPE inventory is large enough that it overlaps both the VFI and RGS estimates. The VFI\&RGS gas inventory is preferable to the BPE inventory because it is based on direct gas measurements from four risers. 
Table 4.2.8. AN-105 Gas Inventory Estimates

\begin{tabular}{||l|c|c|c|c|c||}
\hline \hline \multirow{2}{*}{ Quantity } & \multicolumn{2}{|c|}{ RGS Method } & BPE Method(a) & \multicolumn{2}{c||}{ VFI\&RGS Method(b, c) } \\
\cline { 2 - 6 } & $\begin{array}{c}\text { Nonconvective } \\
\text { Layer }\end{array}$ & $\begin{array}{c}\text { Convective } \\
\text { Layer }\end{array}$ & Tank Total & $\begin{array}{c}\text { Nonconvective } \\
\text { Layer }\end{array}$ & $\begin{array}{c}\text { Convective } \\
\text { Layer }\end{array}$ \\
\hline \hline $\begin{array}{l}\text { Avg gas fraction } \\
\text { (low gas solubility) }\end{array}$ & $0.052 \pm 0.026$ & $0.006 \pm 0.002$ & $0.024 \pm 0.008$ & $0.044 \pm 0.009$ & $0.002 \pm 0.001$ \\
\hline $\begin{array}{l}\text { Gas volume (m3) } \\
\text { in situ (wet) }\end{array}$ & $95 \pm 47$ & $14 \pm 5$ & $105 \pm 36$ & $80 \pm 16$ & $4 \pm 2$ \\
STP (wet) & $186 \pm 93$ & $18 \pm 6$ & $193 \pm 67$ & $140 \pm 40$ & $6 \pm 2$ \\
\hline $\begin{array}{l}\text { (a) The BPE inventory includes the crust, for which there were no RGS or VFI data. Schienbein et al. } \\
\text { (1999) estimated the crust gas inventory as 25 } 10 \mathrm{~m}^{3} \text { in situ, or 25 } \pm 10 \text { STP m3. } \\
\text { (b) Based on VFI and RGS data; see Table 2.7.3 of Schienbein et al. (1999). } \\
\text { (c) The retained gas inventory calculated from RGS and VFI data is considered the best estimate } \\
\text { because it is based on data from several risers. }\end{array}$ \\
\hline
\end{tabular}

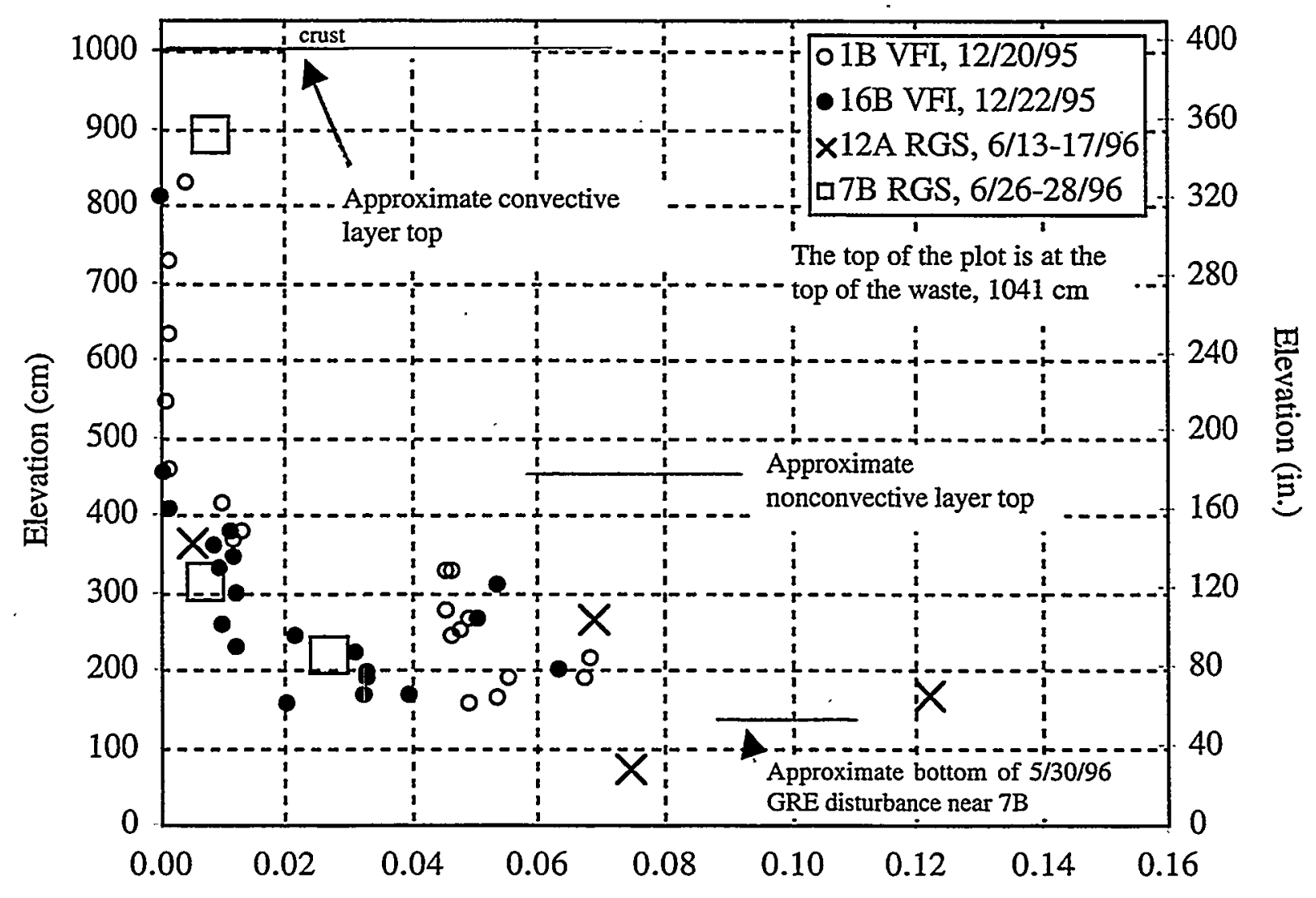

Gas Volume Fraction

Figure 4.2.3. Gas Volume Fractions Measured by RGS and VFI 
Table 4.2.9 contains the calculated layer inventories of each of the major gases retained in the waste. These inventories are based on the layer-average compositions from RGS data (see Table 4.2.6) and the best-estimate gas inventories for each layer (VFI\&RGS, Table 4.2.8).

Figure 4.2.4 shows the sample temperatures, corrected gas volume fractions (Table 4.2.7), and corrected compositions of the low-solubility constituents in the samples from AN-105. The temperatures are from the TC tree in riser 4A. The compositions represent the mole fraction of the species in the "insoluble" gas; water and ammonia are not included, so the mole fractions are not the same as those in Table 4.2.4. The gas volume fractions and mole fractions in the figure are the values for lower-bound gas solubilities. Figure 4.2.4 also lists the observations from core extrusions of non-RGS samples and from x-rays of RGS samples as a way of tying those observations together with RGS data. The $x$-ray data are described in more detail in Section 4.2.4.

\subsubsection{X-Ray Results}

Table 4.2.10 summarizes all the available radiography observations from Tank AN-105. The $\mathrm{x}$-ray images themselves can be found on the $\mathrm{CD}$ that accompanies this report. The uncertainties of the visible gas volume fractions that are given in Table 4.2 .10 are unknown but (based on the uncertainty in measuring the gas gap from $x$-ray images) are expected to be larger than the uncertainties in the gas volume fractions calculated from gas extraction. (The "visible" gas volume fraction refers to the gas that has separated from the solids and liquid such that its volume can be calculated based on the image. The uncorrected gas fractions from extraction are used for comparison to reflect the presence of entrained air in the sampler.)

Table 4.2.9 contains the calculated layer inventories of each of the major gases retained in the waste. The gas-phase inventories are based on the layer-average compositions from RGS data (Table 4.2.6) and the best-estimate gas inventories for each layer (VFI\&RGS, Table 4.2.8). The dissolved inventories are based on RGS data alone.

Table 4.2.9. Speciated AN-105 Gas Inventory(a)

\begin{tabular}{|c|c|c|c|c|}
\hline \multirow[t]{2}{*}{ Species } & \multicolumn{2}{|c|}{$\begin{array}{c}\text { Gas-Phase Inventory } \\
\left(\mathrm{m}^{3} \text { at } \mathrm{STP}\right)\end{array}$} & \multicolumn{2}{|c|}{$\begin{array}{c}\text { Dissolved Inventory } \\
\left(\mathrm{m}^{3} \text { at STP }\right)\end{array}$} \\
\hline & $\begin{array}{c}\text { Nonconvective } \\
\text { Layer }\end{array}$ & $\begin{array}{c}\text { Convective } \\
\text { Layer }\end{array}$ & $\begin{array}{c}\text { Nonconvective } \\
\text { Layer }\end{array}$ & $\begin{array}{c}\text { Convective } \\
\text { Layer }\end{array}$ \\
\hline $\mathrm{N}_{2}$ & $34-33$ & $3.4--3.0$ & $0.14-0.88$ & $0.17--0.54$ \\
\hline $\mathrm{H}_{2}$ & $84-83$ & $1.5-1.2$ & $0.50--2.06$ & $0.23--0.51$ \\
\hline $\mathrm{N}_{2} \mathrm{O}$ & $19-15$ & $0.80-0.22$ & $2.6--6.9$ & $2.1--2.7$ \\
\hline $\mathrm{NH}_{3}$ & $0.95--0.40$ & $0.049--0.017$ & 1500 & 1800 \\
\hline $\mathrm{CH}_{4}$ & $0.97--0.94$ & $0.084-0.068$ & $0.0061--0.034$ & $0.0077-0.024$ \\
\hline Other & 0.92 & 0.18 & 0 & 0 \\
\hline \multicolumn{5}{|c|}{$\begin{array}{l}\text { (a) Gas-phase inventories based on layer-average compositions from RGS data (Table 4.2.6) and } \\
\text { best-estimate gas inventories for each layer (VFI\&RGS, Table } 4.2 .8 \text { ). Dissolved inventories based } \\
\text { on RGS data alone. Uncertainty in each inventory is } 50 \% \text { based on spatial variability consideration } \\
\text { discussed in Section } 3.7 .2 \text {. Inventories given for both lower- and upper-bound solubilities; lower- } \\
\text { bound solubility basis first. }\end{array}$} \\
\hline
\end{tabular}




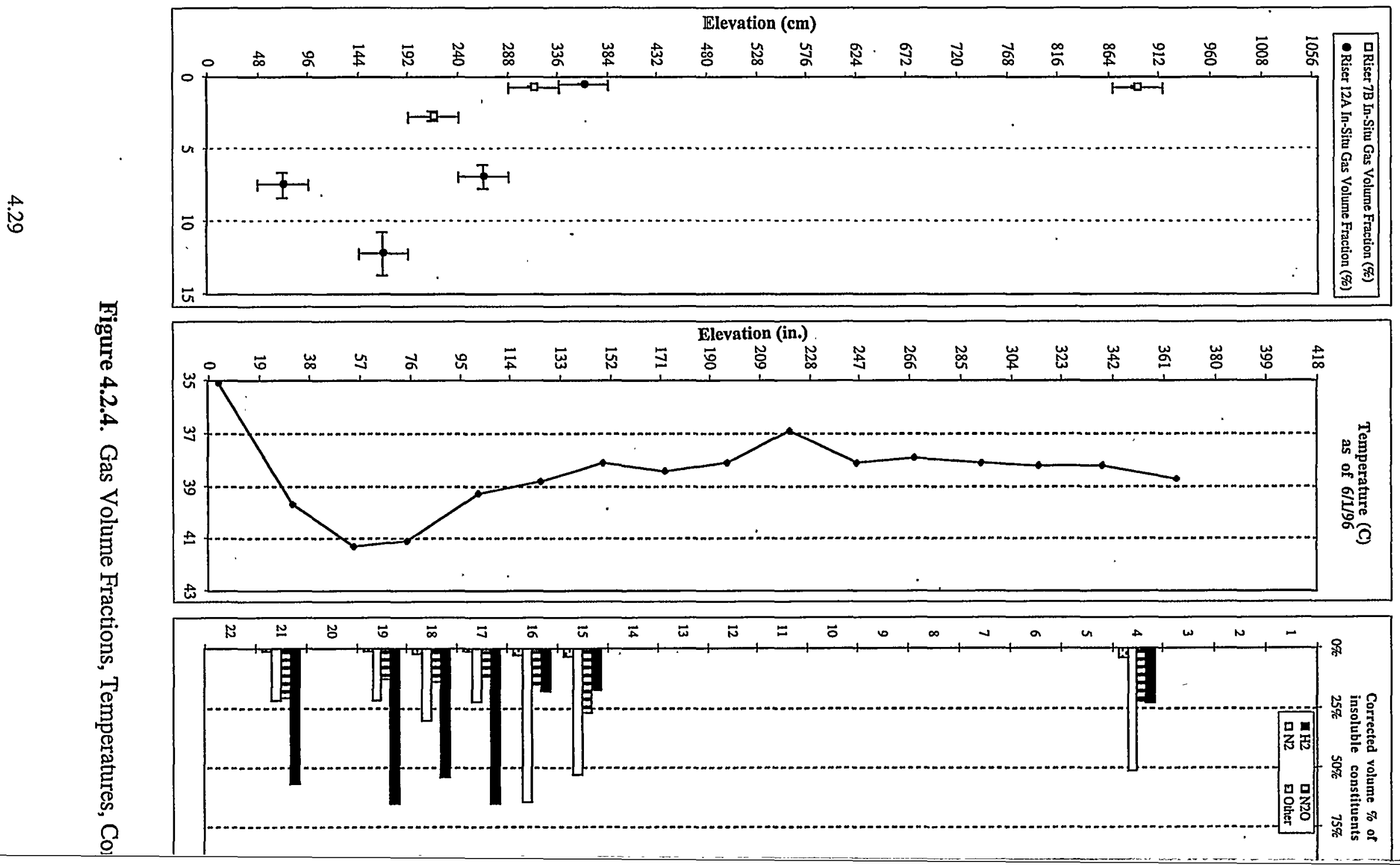


AN-105 waste level is $1041 \mathrm{~cm}$ (410 inches).

1 Riser 12A: 2.5 in. (38 g) light-gray salt slurry, $190 \mathrm{~mL}$ (257 g) green opaque liquid.

1 Riser 7B: a little light-gray salt slurry, $165 \mathrm{~mL}$ (257 g) green opaque liquid.

2 Riser 12A: a liftle light-gray wet salt, $305 \mathrm{~mL}(426 \mathrm{~g})$ green opaque liquid.

2 Riser 7B: a little light-gray salt slurry, $320 \mathrm{~mL}$ ( $423 \mathrm{~g}$ ) green opaque liquid.

3 Riser 12A: a little light-gray wet salt, $280 \mathrm{~mL}(406 \mathrm{~g})$ green opaque liquid.

3 Riser 7B: a little light-gray wet salt, $320 \mathrm{~mL}(367 \mathrm{~g})$ green opaque liquid.

4 Riser 12A: a little light-gray wet salt, $300 \mathrm{~mL}$ ( $429 \mathrm{~g}$ ) green opaque liquid.

4 Riser 7B X-RAY: Featureless, no gas gap under piston.

5 Riser 12A: a little light-gray wet salt, $310 \mathrm{~mL}$ ( $436 \mathrm{~g}$ ) green opaque liquid.

5 Riser 7B: a little light-gray wet salt, $310 \mathrm{~mL}(406 \mathrm{~g})$ green opaque liquid.

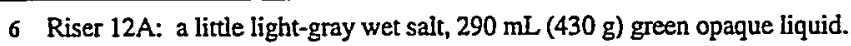

6 Riser 7B: a little light-gray wet salt, $300 \mathrm{~mL}(411 \mathrm{~g})$ green opaque liquid.

7 Riser 12A: a little light-gray wet salt, $290 \mathrm{~mL}(433 \mathrm{~g}$ ) green opaque liquid.

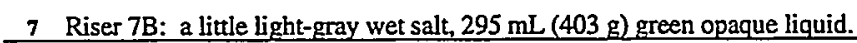

8 Riser 12A: a little light-gray wet salt, $300 \mathrm{~mL}(429 \mathrm{~g}$ ) green opaque liquid.

8 Riser 7B: a litule light-gray wet salt, $290 \mathrm{~mL}$ (427 g) green opaque liquid.

9 Riser 12A: a little light-gray wet salt, $250 \mathrm{~mL}(379 \mathrm{~g})$ green opaque liquid.

9 Riser 7B: a little light-gray wet salt, $300 \mathrm{~mL}(429 \mathrm{~g})$ green opaque liquid.

10 Riser 12A: a little light-gray wet salt, $250 \mathrm{~mL}(392 \mathrm{~g}$ ) green opaque liquid.

10 Riser 7B: a little light-gray wet salt, $290 \mathrm{~mL}(386 \mathrm{~g})$ green opaque liquid.

11 Riser 12A: a little light-gray wet salt, $300 \mathrm{~mL}$ (431 g) green opaque liquid.

11 Riser 7B: a little light-gray wet salt, $290 \mathrm{~mL}(420 \mathrm{~g})$ green opaque liquid.

12 Riser 12A: a little light-gray wet salt, $250 \mathrm{~mL}(380 \mathrm{~g})$ green opaque liquid.

12 Riser 7B: a little light-gray wet salt, $290 \mathrm{~mL}(407 \mathrm{~g})$ green opaque liquid.

13 Riser 12A: a little light-gray wet salt, $250 \mathrm{~mL}(384 \mathrm{~g}$ ) green opaque liquid.

13 Riser 7B: a little light-gray wet salt, $300 \mathrm{~mL}$ (431 g) green opaque liquid.

14 Riser 12A: $235 \mathrm{~g}$ light-gray salt slurry, $90 \mathrm{~mL}(133 \mathrm{~g})$ green opaque liquid, HHF contamination.

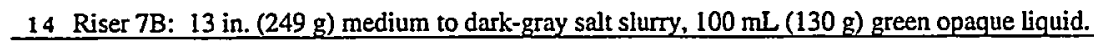

15 Riser 12A X-RAY: A few $1-4 \mathrm{~mm}$ bubbles, more near top, no gas gap, piston fully retracted.

15 Riser 7B: 17 in. ( $379 \mathrm{~g}$ ) gray salt siurry.

16 Riser 12A: 18 in. ( $377 \mathrm{~g}$ ) gray salt slumy.

16 Riser 7B X-RAY: two oblate $5 \mathrm{~mm}$ bubbles, gas gap $0.5 \mathrm{~mm}$, smooth surface, clean fully retracted piston, $2.0 \mathrm{R} / \mathrm{hr}$.

17 Riser 12A X-RAY: Dense 1-5 mm bubbles, 0.75-in. gas gap, rough meniscus surface, small lump on piston.

17 Riser 7B: 19 in. $(421 \mathrm{~g})$ blue-gray wet salt.

18 Riser 12A: 19 in. (384 g) gray wet salt.

18 Riser 7B X-RAY: many 1 to $6 \mathrm{~mm}$ bubbles, vague $0.7 \mathrm{in}$. blob near bottom, no gas gap, $1.8 \mathrm{R} / \mathrm{hr}$.

19 Riser 12A X-RAY: Dense 1 to $3 \mathrm{~mm}$ bubbles, fewer near top, rough surface, 2 in. gas gap, small lump on piston. 19 Riser 7B: 8.5 in. $(244 \mathrm{~g})$ dark-gray wet salt.

20 Riser 12A: 17 in. ( $397 \mathrm{~g}$ ) light to dark gray wet salt and salt slurry.

20 Riser 7B X-RAY: featureless, 1.5 in. gas gap, clean fully retracted piston, slightly rough surface, $1.2 \mathrm{R} / \mathrm{hr}$.

21 Riser 12A X-RAY: Dense 1 to $3 \mathrm{~mm}$ bubbles, two flat voids 0.5 in wide, smaller slits, also partial fracture bubble.

21 Riser 7B: $10 \mathrm{in.}(202 \mathrm{~g})$ gray salt slurry. $120 \mathrm{~mL}(173 \mathrm{~g})$ brown-gray opaque liguid, high HHF contamination.

22 Riser 12A: $13 \mathrm{in}$. (247 g) light to dark gray salt slurry.

22 Riser 7B: 14 in. (257 g) of solids, top 10 in. gray salt-slurry, bottom 4 in. black sludge slurry. 
Table 4.2.10. Summary of Observations from X-Ray Images of Tank AN-105

\begin{tabular}{|c|c|}
\hline Segment & Comments/Observations \\
\hline $\begin{array}{c}\text { 7B-4 } \\
\text { (RGS, } \\
6 / 26 / 96)\end{array}$ & $\begin{array}{l}\text { The waste in the image was featureless. There was no gas gap. The visible gas volume fraction } \\
\text { is therefore } 0 \text {. (Compare with the uncorrected sampler gas volume fraction of } 0.018 \text { in Table } \\
4.2 .7 \text { obtained by gas extraction.) }\end{array}$ \\
\hline $\begin{array}{l}7 \mathrm{~B}-14 \\
\text { (non-RGS, } \\
6 / 27 / 96)\end{array}$ & $\begin{array}{l}\text { The waste in the image was featureless. There was no gas gap. The visible gas volume fraction } \\
\text { is therefore } 0 \text {. }\end{array}$ \\
\hline $\begin{array}{l}12 \mathrm{~A}-15 \\
\text { (RGS, } \\
6 / 13 / 96)\end{array}$ & $\begin{array}{l}\text { A few } 1-4 \mathrm{~mm} \text { bubbles were seen, more of them near the sampler top. The piston was fully } \\
\text { retracted. There was no gas gap. The visible gas volume fraction (not counting bubbles) is } \\
\text { therefore } 0 \text {. (Compare with the uncorrected sampler gas volume fraction of } 0.014 \text { in Table } 4.2 .7 \\
\text { obtained by gas extraction.) }\end{array}$ \\
\hline $\begin{array}{l}\text { 7B-16 } \\
\text { (RGS, } \\
\text { 6/28/96) }\end{array}$ & $\begin{array}{l}\text { One oblate, } 5-\mathrm{mm} \text { bubble was seen near the bottom of the sampler, another near the top. The } \\
\text { gas gap was } 0.5 \mathrm{~mm}(0.02 \mathrm{in} \text {.), the waste surface smooth. The piston was clean and fully } \\
\text { retracted. The visible gas volume fraction (not counting bubbles) is } 0.001 \text {. (Compare with the } \\
\text { uncorrected sampler gas volume fraction of } 0.012 \text { in Table } 4.2 .7 \text { obtained by gas extraction.) }\end{array}$ \\
\hline $\begin{array}{l}12 \mathrm{~A}-17 \\
\text { (RGS, } \\
6 / 14 / 96)\end{array}$ & $\begin{array}{l}\text { Dense } 1-5-\mathrm{mm} \text { bubbles throughout the sample. The gas gap was } 19 \mathrm{~mm}(0.75 \mathrm{in} \text {.). The } \\
\text { surface may have been a meniscus. Small lump of waste on the piston, which was fully } \\
\text { retracted. The visible gas volume fraction (not counting bubbles) is } 0.04 \text {. (Compare with the } \\
\text { uncorrected sampler gas volume fraction of } 0.074 \text { obtained by gas extraction, Table } 4.2 .7 \text {.) }\end{array}$ \\
\hline $\begin{array}{l}\text { 7B-18 (RGS, } \\
6 / 28 / 96)\end{array}$ & $\begin{array}{l}\text { Many } 1 \text { - to } 6-\mathrm{mm} \text { bubbles throughout the sample, and a vague } 13-\mathrm{mm}(0.7-\mathrm{in} \text {.) low-density } \\
\text { blob near the bottom of the sample, possibly a bubble flattened against the side of the sampler. } \\
\text { The piston was fully retracted, and there was no gas gap. The visible gas volume fraction (not } \\
\text { counting bubbles) is therefore } 0 \text {. (Compare with the uncorrected sampler gas volume fraction of } \\
0.031 \text { obtained by gas extraction, Table } 4.2 .7 \text {.) }\end{array}$ \\
\hline $\begin{array}{l}12 \mathrm{~A}-19 \\
\text { (RGS, } \\
6 / 14 / 96)\end{array}$ & $\begin{array}{l}\text { Dense } 1 \text { to } 3 \mathrm{~mm} \text { bubbles, with fewer near the top of the sample. Slightly rough surface, } \\
\text { small lump of waste on piston, which is fully retracted. About a } 5 \text {-cm (2-in.) gas gap, The } \\
\text { visible gas volume fraction (not counting bubbles) is therefore } 0.11 \text {. (Compare with the } \\
\text { uncorrected sampler gas volume fraction of } 0.127 \text { obtained by gas extraction, Table } 4.2 .7 \text {.) }\end{array}$ \\
\hline $\begin{array}{l}\text { 7B-20 } \\
(\mathrm{RGS} \\
6 / 28 / 96)\end{array}$ & $\begin{array}{l}\text { Featureless, with a slightly rough surface. Piston clean and fully retracted. Gas gap about } \\
3.8 \mathrm{~cm} \text { (1.5 in.). The visible gas volume fraction is therefore } 0.08 \text {. }\end{array}$ \\
\hline $\begin{array}{l}12 \mathrm{~A}-21 \\
\text { (RGS, } \\
6 / 17 / 96)\end{array}$ & $\begin{array}{l}\text { Densely packed } 1-\text { to } 3 \text {-mm bubbles throughout the sample. About halfway up were two } \\
\text { flattened bubbles about } 12 \mathrm{~mm}(0.5 \mathrm{in} \text {.) wide by } 5 \mathrm{~mm} \text { ( } 0.2 \mathrm{in} \text {.) high and other smaller slit-like } \\
\text { bubbles. There was also one nearly complete fracture bubble } 0.2 \mathrm{in} \text { high about two-thirds of the } \\
\text { way up. The surface was smooth. There was a little waste on the fully retracted piston. The } \\
\text { gas gap was } 8 \text {-mm ( } 0.3 \text {-in.). The visible gas volume fraction (counting the gas gap, the } \\
\text { fracture, and the flattened bubbles, but not other bubbles) is therefore } 0.03 \text {., Table 4.2.7.) }\end{array}$ \\
\hline
\end{tabular}

Though sample 7B-20 was not successfully extracted, at least 8 vol\% gas was seen in its $\mathrm{x}$-ray. It is therefore likely that this sample, if extracted, would show about the same amount of gas found in samples $12 \mathrm{~A}-17$ and $12 \mathrm{~A}-21$. This observation tends to show that there is little difference between the retained gas in the waste under riser $7 \mathrm{~B}$ and that under riser $12 \mathrm{~A}$. 
Table 4.2.11 summarizes the waste densities calculated from radiographic data for RGS samples for which air and water standard images were available. Radiographic densities include the gas in the waste and are given for several locations within each sample to show trends. The overall sample densities were calculated both by the current $\mathrm{x}$-ray analysis method and the original method (see Section 3.9); the differences give some idea of the sensitivity of results to small variations in method. Table 4.2.11 also includes density measurements made on extruded nonRGS samples (Jo 1997). The radiographic densities equaled or exceeded those found for the adjacent extruded samples, which is difficult to explain because the radiographic densities included gas and the extruded densities did not.

Table 4.2.11. Densities of AN-105 Samples from Radiography and Core Samples

\begin{tabular}{|c|c|c|c|c|}
\hline $\begin{array}{l}\text { Riser- } \\
\text { segment } \\
\text { number }\end{array}$ & $\begin{array}{l}\text { Distance from } \\
\text { bottom of } \\
\text { sampler (ft) }\end{array}$ & $\begin{array}{c}\text { Mean local } \\
\text { density by current } \\
\text { method (g/cc) }\end{array}$ & $\begin{array}{l}\text { Overall average } \\
\text { sample densities } \\
(\mathrm{g} / \mathrm{cc})\end{array}$ & $\begin{array}{l}\text { Degassed density in non-RGS } \\
\text { cores above and below RGS } \\
\text { sample (g/cc) }\end{array}$ \\
\hline $7 B-16$ & & & $\begin{array}{l}\text { Original } x \text {-ray analysis } \\
\text { method: } \quad 1.68 \text { (a) }\end{array}$ & $\begin{array}{l}\text { density above, } 1.54 \mathrm{~g} / \mathrm{cc} \text { bulk } \\
\text { density below, } 1.53 \mathrm{~g} / \mathrm{cc} \text { bulk }\end{array}$ \\
\hline \multirow[t]{5}{*}{$12 \mathrm{~A}-19$} & 1.25 & 1.77 & \multirow{5}{*}{$\begin{array}{l}\text { Current } x \text {-ray analysis } \\
\text { method: } \quad 1.77 \\
\text { Original x-ray analysis } \\
\text { method: } \quad 1.83(\mathrm{a})\end{array}$} & \multirow{5}{*}{ density above, $1.65 \mathrm{~g} / \mathrm{cc}$ bulk } \\
\hline & 1.0 & 1.76 & & \\
\hline & 0.75 & 1.75 & & \\
\hline & 0.5 & 1.81 & & \\
\hline & 0.25 & 1.59 & & \\
\hline \multirow[t]{5}{*}{$7 \mathrm{~B}-20$} & 1.5 & 1.42 & \multirow{5}{*}{$\begin{array}{l}\text { Current } x \text {-ray analysis } \\
\text { method: } \quad 1.56 \\
\text { Original } x-\text { ray analysis } \\
\text { method: } \quad 1.67 \text { (a) }\end{array}$} & \multirow[t]{4}{*}{ density above, $1.56 \mathrm{~g} / \mathrm{cc}$ bulk } \\
\hline & 1.25 & 1.61 & & \\
\hline & 1.0 & 1.60 & & \\
\hline & 0.75 & 1.57 & & \\
\hline & 0.50 & 1.47 & & density below, $1.57 \mathrm{~g} / \mathrm{cc}$ bulk \\
\hline \multirow[t]{6}{*}{$12 \mathrm{~A}-21$} & 1.5 & 1.57 & \multirow{6}{*}{$\begin{array}{l}\text { Current } x \text {-ray analysis } \\
\text { method: } \quad 1.62 \\
\\
\text { Original } x \text {-ray analysis } \\
\text { method: } \quad 1.65 \text { (a) }\end{array}$} & \multirow[t]{6}{*}{ density above, $1.59 \mathrm{~g} / \mathrm{cc}$ bulk } \\
\hline & 1.25 & 1.62 & & \\
\hline & 1.0 & 1.59 & & \\
\hline & 0.75 & 1.60 & & \\
\hline & 0.5 & 1.61 & & \\
\hline & 0.25 & 1.78 & & \\
\hline
\end{tabular}

(a) The densities calculated by the original $x$-ray analysis method were taken from Shekarriz et al. (1997), Table 4.29.

\subsubsection{Domespace Composition Comparison}

The RGS gas-phase composition data (Table 4.2.6) were used to calculate $\mathrm{H}_{2} / \mathrm{N}_{2} \mathrm{O}$ ratios for comparison with ratios from domespace grab sample measurements (McCain 1999, Table 4.5). The results can be seen in Table 4.2.12. The domespace ratios were similar before and after the GRE, and the RGS and domespace ratios were in reasonable agreement. 
Table 4.2.12 Comparison to Domespace Data(a)

\begin{tabular}{|l|c|}
\hline \multicolumn{1}{|c|}{ Sample } & $\mathrm{H}_{2} / \mathrm{N}_{2} \mathrm{O}$ \\
\hline RGS, 7B-4 & $1.8-5.4$ \\
\hline$R G S, 12 \mathrm{~A}-15$ & $1.4-4.6$ \\
\hline RGS, 7B-16 & $2.2-6.3$ \\
\hline$R G S, 12 \mathrm{~A}-17$ & $5.9-7.5$ \\
\hline$R G S, 7 \mathrm{~B}-18$ & $4.5-7.5$ \\
\hline$R G S, 12 \mathrm{~A}-19$ & $5.3-6.1$ \\
\hline RGS, 12A-21 & $3.0-3.8$ \\
\hline Domespace, before 5/30/96 GRE & $4.9,5.0$ \\
\hline $\begin{array}{l}\text { Domespace, after 5/30/96 GRE } \\
\text { (a) RGS ratios were calculated for both lower- and upper- } \\
\text { bound solubilities. Both ends of the ratio range are given in } \\
\text { the table, lower-bound solubility basis first. }\end{array}$ \\
\hline
\end{tabular}

\section{$4.3 \quad$ A-101}

Tank 241-A-101 (A-101) was the third tank and the first SST sampled with the RGS. This tank was selected because it was on the FGWL and showed a significant response to barometric pressure changes (discussed in Section 3.7.2) and high hydrogen and ammonia concentrations in the domespace. Tank A-101 is part of a group of tanks (Cluster 22 in Stewart et al. 1996b) that have fairly high temperatures and high nitrite and TOC concentrations. Historical tank content estimates (HTCE) state that the primary waste stored in A-101 was saltcake resulting from waste from evaporator campaign A1 and that the secondary waste was salt slurry from evaporator campaign A2 (Remund et al. 1995). (This is identical to the HTCE for Tank AX-101, which is also in Cluster 22.)

Push-mode sampling was done in risers 15 and 24 in July 1996.(a) The lateral distance between the two risers was approximately $15 \mathrm{~m}(50 \mathrm{ft})$. The approximate locations of various risers are depicted in Figure 4.3.1.

The total depth of waste in Tank A-101 was approximately $884 \mathrm{~cm}$ (348 in.) at the time of sampling. The 1996 cores showed that the waste was 343 in. deep at riser 24 and 353 in. deep at riser 15, indicating some waste surface irregularity. At the time RGS sampling took place, A-101 contained approximately 3,610,000 L (953,000 gal) of waste (Hodgson et al. 1998). Risers 15 and 24 are more than half a tank radius from the tank center. Figure 4.3.2 shows the tank content layering. The upper nonconvective layer was about $411 \mathrm{~cm}$ (162 in.) in depth, and the more convective lower layer $472 \mathrm{~cm}$ (186 in.) deep, based on core observations, temperature profiles, and a gamma scan (Field 1997). The elevations of the RGS segments are depicted in Figure 4.3.2.

(a) The sampling scheme may be found in Sampling Plan for Tank 241-A-101 Retained Gas Sampler Deployment, by A Shekarriz and JM Bates, June 1996. TWSMIT:061796 Rev. 0, Pacific Northwest National Laboratory, Richland, Washington. 


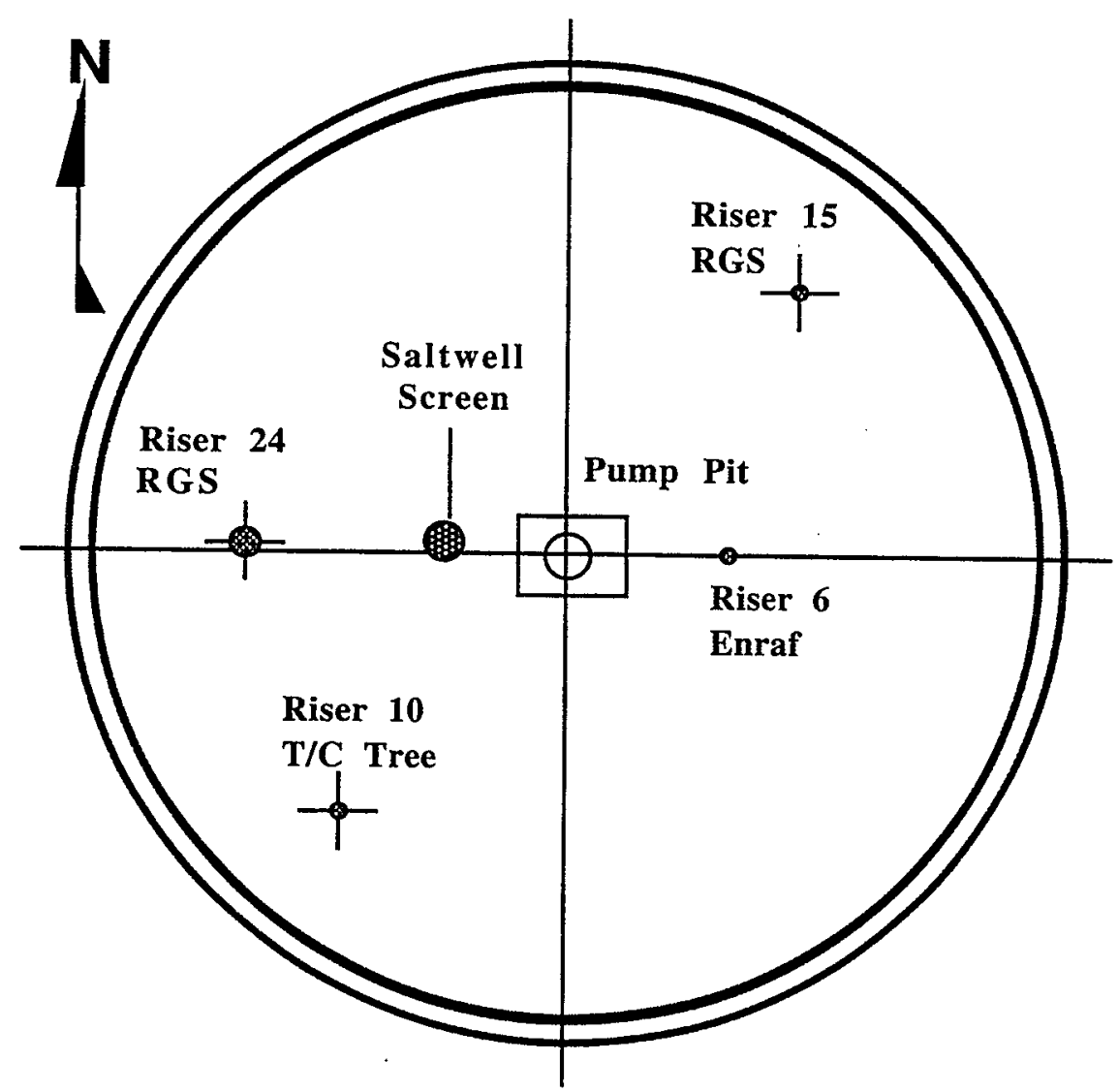

Figure 4.3.1. Schematic Diagram of Riser Locations in Tank A-101
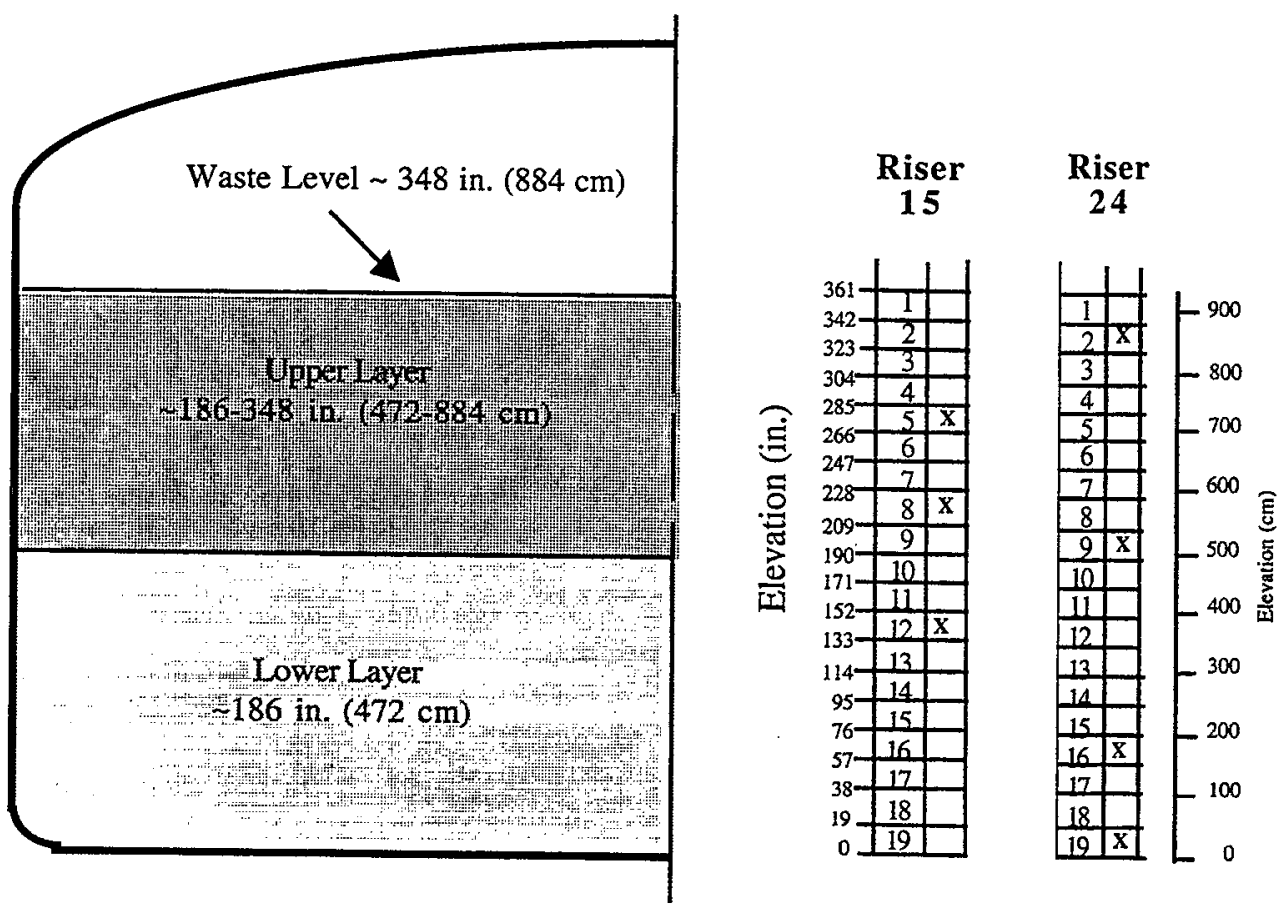

Figure 4.3.2. Diagram of Waste Layering and RGS Sample Elevations for A-101 
Tank A-101 had significantly different thermal characteristics and layering than those reported for DSTs such as AW-101. Based on an extensive analysis, Ogden (1996) surmised the presence of two distinct layers in this tank. The upper layer showed insulating (nonconvective) behavior, while the lower layer had a more uniform temperature associated with its higher conductance (whether in convective or conductive mode). This layering model was consistent with the most recent observations of the sample extrusions, classifying the upper layer material as moist or wet salt and the lower layer as salt slurry or liquid (Field 1997). In this report the lower layer will be referred to as "convective," though convection need not be the only-significant mode of heat transfer.

The 1996 cores (Field 1997) contained dry salt in the top sample, and beneath that eight samplers (about 150 in.) of moist salt. Samples from the bottom 10 samplers contained varying proportions of salt slurry and liquid. ("Salt" is a qualitative term based on core extrusion observations, not chemical analysis; it denotes a core that has a gritty, sandy appearance.) There were no evident differences between samples from the two risers.

Densities for the liquid and bulk solids in Tank A-101 were available from the non-RGS samples taken from the same cores as the RGS samples (Field 1997). Several samples contained drainable liquid with a density of $1444 \pm 23 \mathrm{~kg} / \mathrm{m}^{3}$. The degassed density of the nonconvective layer samples ranged from 1540 to $1750 \mathrm{~kg} / \mathrm{m}^{3}$, with an average and standard deviation of 1678 $\pm 51 \mathrm{~kg} / \mathrm{m}^{3}$; the degassed density of the convective layer samples varied from 1465 to $1518 \mathrm{~kg} / \mathrm{m}^{3}$, with an average and standard deviation of $1498 \pm 17 \mathrm{~kg} / \mathrm{m}^{3}$. These density values were used for in situ inventory and average composition calculations. Layer depths of $424 \mathrm{~cm}$ (167 in.) and $498 \mathrm{~cm}$ (196 in.) were used in calculations for the nonconvective and convective layers.

\subsubsection{Sampling and Extraction Information}

The samples that underwent RGS analysis are listed in Table 4.3.1. Table 4.3.1 also shows the lag times (delay between sample acquisition and processing) for these samples. This information was provided to allow data users to correlate the lag time between sampling and extrusion with the concentrations of the various constituents, to test for decomposition or other chemical reactions. The maximum hold time allowed by the sampling plan is 24 days, based on measured sampler leakage rates during acceptance testing. The hold time for sample 15-5 exceeded this limit, but the sample was accepted based on calculations that indicated the estimated leakage was still negligible compared with the large amount of sample gas. (Field data, including dose rates and downforce limits, are supplied in Appendix A.)

Table 4.3.1. Lag Times for Processing RGS Samples from Tank 241-A-101

\begin{tabular}{||c|c|c|c||}
\hline Sample & Acquisition Date & Processing Date & Lag (days) \\
\hline \hline $24-2$ & July 22, 1996 & August 12, 1996 & 21 \\
\hline $15-5$ & July 12, 1996 & August 7, 1996 & 26 \\
\hline $15-8$ & July 12, 1996 & August 1, 1996 & 20 \\
\hline $24-9$ & July 23, 1996 & August 13, 1996 & 21 \\
\hline $15-12$ & July 17, 1996 & August 9, 1996 & 23 \\
\hline $24-16$ & July 24, 1996 & August 14, 1996 & 21 \\
\hline $24-19$ & July 25, 1996 & August 15, 1996 & 21 \\
\hline
\end{tabular}


As was discussed in Section 4.1 the HHF, a solution of $\mathrm{LiBr}$ used during sampling, often enters the sampler in place of some of the waste. The bromide ion is used as the HHF tracer. Table 4.3.2 shows the volume percentage contamination by HHF of the RGS samples. Most of these samples (except 24-9 and 24-19) contained bromide below the MDL. The measured HHF contamination percentages were used to calculate reduced effective sampler volumes for the RGS samples, with the bromide concentration set equal to the MDL for samples below the limit. (It is conservative to use the MDL for values below it; the conservatism is probably less than $4 \%$.)

Two different extraction procedures were used on the A-101 samples, but the first several steps were the same in both (see the RGS system schematic in Figure 3.1). After evacuating the whole system and observing the pressure to test for leaks, the operator extruded the RGS sample into the extractor vessel and the sample was briefly stirred. At this point, the vapor space in communication with the sample consisted of the volumes of the extractor vessel and its lines. Next, the extractor was opened to the second vessel, to which was connected a "PQ" canister. Once a grab sample was collected in the PQ canister, it was closed. The extractor, lines, and second volume were then connected to the pump volume and collector side; the latter included the collector line volume and one open collection canister. Several strokes of gas and vapor (sometimes called "unbound gas") were pumped from the extractor to the collector, after which the first collection canister was closed off. From this point, different procedures were used for the nonconvective-layer and convective-layer samples, as a result of the high gas content of the nonconvective layer.

Nonconvective-layer samples: The collector side, second volume, and extractor lines were not evacuated after the first collection canister was closed off. Several strokes were pumped from the extractor to the collector, and the second collection canister was closed off. Then the collector side, second volume, and extractor lines were evacuated. Next, the extractor and samples were heated to about $50^{\circ} \mathrm{C}$ to drive off "bound gas," cooled back to hot cell temperature, and briefly stirred. A PQ canister connected to the extractor vessel took a second sample and was closed off. Several strokes were pumped from the extractor to the collector, and the third collection canister was closed off. Finally, the BSVD procedure was performed. Argon was injected into the closedoff second volume, which then was opened to the extractor and lines. Pressure measurements and known tare system volumes allowed calculation of the volume of the sample solids and liquid.

Convective layer samples: The collector side, second volume, and extractor lines were evacuated after the first collection canister was closed off. Next, the extractor and samples were heated to about $50^{\circ} \mathrm{C}$ to drive off "bound gas," cooled back to hot cell temperature, and briefly

Table 4.3.2. Hydrostatic Head Fluid Contamination in A-101 RGS Samples

\begin{tabular}{|l|c|}
\hline Sample & $\begin{array}{c}\text { HHF Contamination } \\
\text { (vol \%) }\end{array}$ \\
\hline \hline $24-2$ (RGS) & $<8.4$ \\
\hline $15-5$ (RGS) & $<3.8$ \\
\hline $15-8$ (RGS) & $<4.1$ \\
\hline $24-9$ (RGS) & 4.5 \\
\hline $15-12$ (RGS) & $<3.6$ \\
\hline $24-16$ (RGS) & $<6.6$ \\
\hline $24-19$ (RGS) & 1.8 \\
\hline
\end{tabular}


stirred. A PQ canister connected to the extractor vessel took a second sample and was closed off. Several strokes were pumped from the extractor to the collector, and the collection canister was closed off. At this point the collector side, second volume, and extractor lines were not evacuated. Several strokes of gas and vapor were pumped from the extractor to the collector (to which one open collection canister, the third one, was connected). The BSVD procedure was performed last. (Procedural details such as the number of strokes per canister and the system volumes are listed on the sample-by-sample worksheets in the A-101 calculation spreadsheet on the enclosed CD.

\subsubsection{Retained Gas Composition}

Table 4.3.3 presents the calculated concentrations of the insoluble gases in each RGS sample taken from A-101 without corrections for air, argon entrainment, or air inleakage. The method used to make the corrections depended on the gas. All of the oxygen and argon were subtracted no matter which step had produced them. A number of moles equal to $(3.73) \cdot\left(\mathrm{O}_{2}\right)$ were subtracted from the nitrogen because the oxygen was expected to have come from air entrainment during sampling or inleakage during extraction. The corrected concentrations are given in Table 4.3.4.

The large amount of oxygen in sample 24-2 probably came from an air leak into the extractor side RGS system in spite of the leak testing that was done before the sampler was attached to the system. Indications of the leak were the increase of $3 \mathrm{kPa}$ in the extractor pressure between the second and third canister, which is more than 10 times the increase for other samples, and the increasing amounts of oxygen in each successive canister. The leak check (performed between 8:57 and 10:35 am on August 12, 1996) had been acceptable, so the leakage (assuming this hypothesis is correct) must have occurred during sample handling (10:45 am to $2: 24 \mathrm{pm}$ the same day). The leak rate must have been

$$
\begin{aligned}
& =\quad\left[\left(3300 \mu \mathrm{mol} \mathrm{O}{ }_{2} / \mathrm{L} \text { waste }\right)(0.281 \mathrm{~L} \text { waste }) /\left(0.21 \mathrm{O}_{2} \text { in air }\right)\right] / 3.7 \mathrm{hr} \\
& =\quad 0.33 \mu \mathrm{mol} \mathrm{air} / \mathrm{s} .
\end{aligned}
$$

Table 4.3.3. Concentrations of Insoluble Constituents ( $\mu \mathrm{mol} / \mathrm{L}$ of waste) in Tank A-101 Without Correction for Gas Contamination

\begin{tabular}{||c|c|c|c|c|c|c|c|c|c|c|c||}
\hline Sample & $\mathrm{N}_{2}$ & $\mathrm{H}_{2}$ & $\mathrm{~N}_{2} \mathrm{O}$ & $\mathrm{O}_{2}$ & $\mathrm{CH}_{4}$ & $\mathrm{He}$ & $\mathrm{Ar}$ & $\begin{array}{c}\text { Other } \\
\mathrm{NO}_{x}\end{array}$ & $\mathrm{C}_{2} \mathrm{H}_{\mathrm{x}}$ & $\mathrm{C}_{3} \mathrm{H}_{\mathrm{x}}$ & $\begin{array}{c}\text { Other } \\
\text { Hyd. }\end{array}$ \\
\hline \hline $24-2$ & $14000 \pm 680$ & $4100 \pm 140$ & $490 \pm 18$ & $3300 \pm 180$ & $27 \pm 5.0$ & (a) & $170 \pm 8.9$ & $6.2 \pm 2.1$ & $10 \pm 1.2$ & $7.5 \pm 1.7$ & $10 \pm 1.7$ \\
\hline $15-5$ & $1400 \pm 65$ & $6000 \pm 280$ & $470 \pm 24$ & $45 \pm 2.7$ & $57 \pm 7.9$ & (a) & $19 \pm 1.6$ & $3.8 \pm 1.2$ & $8.1 \pm 1.5$ & $4.7 \pm 1.2$ & $10 \pm 2.8$ \\
\hline $15-8$ & $1800 \pm 80$ & $7600 \pm 320$ & $550 \pm 25$ & $50 \pm 2.4$ & $68 \pm 5.2$ & (a) & $12 \pm 0.8$ & $8.9 \pm 1.4$ & $9.7 \pm 1.3$ & $4.4 \pm 1.1$ & $7.2 \pm 1.3$ \\
\hline $24-9$ & $2600 \pm 110$ & $7800 \pm 320$ & $550 \pm 26$ & $13 \pm 0.9$ & $94 \pm 7.3$ & (a) & $370 \pm 14$ & $1.2 \pm 0.8$ & $15 \pm 1.2$ & $3.8 \pm 1.2$ & $7.0 \pm 1.6$ \\
\hline $15-12$ & $540 \pm 85$ & $55 \pm 9.4$ & $110 \pm 32$ & $70 \pm 10$ & $14 \pm 6.1$ & (a) & $250 \pm 36$ & $4.5 \pm 2.6$ & $0.4 \pm 0.2$ & $1.6 \pm 1.1$ & $4.6 \pm 2.3$ \\
\hline $24-16$ & $420 \pm 62$ & $73 \pm 11$ & $110 \pm 25$ & $34 \pm 5.4$ & $4.1 \pm 1.0$ & (a) & $400 \pm 57$ & $3.1 \pm 1.7$ & $0.7 \pm 0.3$ & $1.3 \pm 0.9$ & $4.6 \pm 1.8$ \\
\hline $24-19$ & $860 \pm 100$ & $140 \pm 20$ & $130 \pm 25$ & $100 \pm 11$ & $5.0 \pm 0.7$ & (a) & $380 \pm 43$ & $3.0 \pm 1.7$ & $1.1 \pm 0.6$ & $1.4 \pm 1.1$ & $3.8 \pm 1.6$ \\
\hline (a) Below detection limits, which are 0.01 'mol\% or less.
\end{tabular}


Table 4.3.4. Concentrations of Insoluble Constituents ( $\mu \mathrm{mol} / \mathrm{L}$ of waste) in Tank A-101 with Correction for Gas Contamination

\begin{tabular}{||c|c|c|c|c|c|c|c|c|c|c|c||}
\hline Sample & $\mathrm{N}_{2}$ & $\mathrm{H}_{2}$ & $\mathrm{~N}_{2} \mathrm{O}$ & $\mathrm{O}_{2}$ & $\mathrm{CH}_{4}$ & $\mathrm{He}$ & $\mathrm{Ar}$ & $\begin{array}{l}\text { Other } \\
\mathrm{NO}_{x}\end{array}$ & $\mathrm{C}_{2} \mathrm{H}_{x}$ & $\mathrm{C}_{3} \mathrm{H}_{x}$ & $\begin{array}{c}\text { Other } \\
\text { Hyd. }\end{array}$ \\
\hline \hline $24-2$ & $1700 \pm 280$ & $4100 \pm 140$ & $490 \pm 18$ & $0 \pm 180$ & $27 \pm 5.0$ & (a) & $0 \pm 8.9$ & $6.2 \pm 2.1$ & $10 \pm 1.2$ & $7.5 \pm 1.7$ & $10 \pm 1.7$ \\
\hline $15-5$ & $1200 \pm 360$ & $6000 \pm 280$ & $470 \pm 24$ & $0 \pm 2.7$ & $57 \pm 7.9$ & (a) & $0 \pm 1.6$ & $3.8 \pm 1.2$ & $8.1 \pm 1.5$ & $4.7 \pm 1.2$ & $10 \pm 2.8$ \\
\hline $15-8$ & $1600 \pm 530$ & $7600 \pm 320$ & $550 \pm 25$ & $0 \pm 2.4$ & $68 \pm 5.2$ & (a) & $0 \pm 0.8$ & $8.9 \pm 1.4$ & $9.7 \pm 1.3$ & $4.4 \pm 1.1$ & $7.2 \pm 1.3$ \\
\hline $24-9$ & $2500 \pm 470$ & $7800 \pm 320$ & $550 \pm 26$ & $0 \pm 0.9$ & $94 \pm 7.3$ & (a) & $0 \pm 14$ & $1.2 \pm 0.8$ & $15 \pm 1.2$ & $3.8 \pm 1.2$ & $7.0 \pm 1.6$ \\
\hline $15-12$ & $270 \pm 92$ & $55 \pm 9.4$ & $110 \pm 32$ & $0 \pm 10$ & $14 \pm 6.1$ & (a) & $0 \pm 36$ & $4.5 \pm 2.6$ & $0.4 \pm 0.2$ & $1.6 \pm 1.1$ & $4.6 \pm 2.3$ \\
\hline $24-16$ & $290 \pm 65$ & $73 \pm 11$ & $110 \pm 25$ & $0 \pm 5.4$ & $4.1 \pm 1.0$ & (a) & $0 \pm 57$ & $3.1 \pm 1.7$ & $0.7 \pm 0.3$ & $1.3 \pm 0.9$ & $4.6 \pm 1.8$ \\
\hline $24-19$ & $480 \pm 110$ & $140 \pm 20$ & $130 \pm 25$ & $0 \pm 11$ & $5.0 \pm 0.7$ & (a) & $0 \pm 43$ & $3.0 \pm 1.7$ & $1.1 \pm 0.6$ & $1.4 \pm 1.1$ & $3.8 \pm 1.6$ \\
\hline
\end{tabular}

This value is more than 100 times the acceptance limit for the system. Although the inleakage hypothesis matches the data, it is only a hypothesis because it is not clear what could have caused the leak rate to increase so overwhelmingly after the acceptance limit had been met. (The leak rate is tested and confirmed below the limit before each segment is processed.)

Another, less likely explanation is related to the porous structure of the salt material in the 24-2 sampler. The pores might have trapped headspace gas (mostly air) and then become sealed by further salt formation. As the sample was heated the pores reopened, releasing the trapped air.

The samples from riser 24 consistently contained more argon than the riser 15 samples, suggesting that drillstring purges had more effect on the waste in riser 24 . The contamination observations are found in the "Summary" worksheet of the A-101 RGS calculations spreadsheet on the CD.

Table 4.3.5 presents the ammonia measurements. The RGS procedure did not permit the determination of the residual or total ammonia, but the post-extrusion and the first PQ canister partial pressures of $\mathrm{NH}_{3}$ over the sample (see Section 3.4.4) were measured at laboratory temperature. Ammonia was also measured in two salt-well grab samples taken in 1996.

No conclusive total ammonia concentration can be calculated. Based on RGS partial pressure data alone, it is likely that ammonia concentrations in A-101 were two to five times those in AW-101, for which a lower-bound estimate of $27,000 \mu \mathrm{mol} \mathrm{NH}_{3} / \mathrm{L}$ waste was given in Section 4.1.2. However, much lower ammonia concentrations were measured in the salt-well grab samples (see Appendix C, Section C.3 for more details).

Table 4.3.6 contains the composition of the gas/vapor phase in each sample and the integrated average composition in the gas retained in the settled solids. The water vapor is not included in these compositions. The ammonia fractions are derived from the partial pressures indicated in Table 4.3.5 extrapolated to vapor pressures at in situ conditions. The sample compositions in the table have been calculated using the in situ solubility method described in Section 3.5.2. As discussed in Section 3.6.1, compositions were calculated for both the lower-bound and upper-bound gas solubilities. Both ends of the composition range are given in Table 4.3.6, together with the measurement uncertainty on each. The average composition of the gas in each 
Table 4.3.5. Ammonia Data from Tank A-101 Samples

\begin{tabular}{|c|c|c|}
\hline Sample & $\begin{array}{l}\text { Measured } \mathrm{NH}_{3} \text { partial } \\
\text { pressure at about } 24^{\circ} \mathrm{C} \\
\text { (atm) })^{\text {(a) }}\end{array}$ & $\begin{array}{l}\text { RGS Best-Estimate } \mathrm{NH}_{3} \\
\text { Concentrations }\end{array}$ \\
\hline $24-2$ & $0.014 \pm 0.007$ & \multirow{7}{*}{$\begin{array}{l}\text { The data do not support conclusive } \\
\mathrm{NH}_{3} \text { concentrations, since RGS } \\
\text { measurements are much higher } \\
\text { than salt-well grab sample } \\
\text { measurements. We estimate a lower } \\
\text { bound of } 0.08 \mathrm{M}(1400 \mu \mathrm{g} / \mathrm{mL}) \\
\mathrm{NH}_{3} \text { in the liquid, or } 0.06 \mathrm{wt} \% \\
\mathrm{NH}_{3} \text { in the bulk waste, based on } \\
\mathrm{RGS} \text { data alone. }\end{array}$} \\
\hline $15-5$ & $0.018 \pm 0.009$ & \\
\hline $15-8$ & $0.010 \pm 0.005$ & \\
\hline $24-9$ & $0.0089 \pm 0.0044$ & \\
\hline $15-12$ & $0.024 \pm 0.012$ & \\
\hline $24-16$ & $0.027 \pm 0.013$ & \\
\hline $24-19$ & $0.028 \pm 0.014$ & \\
\hline
\end{tabular}

Table 4.3.6. Sample and Overall Average Compositions of Retained Gas in Tank A-101 with Correction for Gas Contamination(a)

\begin{tabular}{|c|c|c|c|c|c|c|}
\hline Sample & $\mathrm{N}_{2}(\mathrm{~mol} \%)$ & $\mathrm{H}_{2}(\mathrm{~mol} \%)$ & $\mathrm{N}_{2} \mathrm{O}(\mathrm{mol} \%)$ & $\mathrm{NH}_{3}(\mathrm{~mol} \%)^{(b)}$ & $\mathrm{CH}_{4}(\mathrm{~mol} \%)$ & Other (mol\%) \\
\hline $24-2$ & $26 \pm 4.8-27 \pm 4.9$ & $63 \pm 5.6-64 \pm 5.4$ & $7.4 \pm 0.7-6.9 \pm 0.6$ & $3.0 \pm 1.3-1.2 \pm 0.5$ & $0.4 \pm 0.1$ & $0.5 \pm 0.1$ \\
\hline $15-5$ & $15 \pm 4.7$ & $72 \pm 8.4-76 \pm 8.0$ & $5.6 \pm 0.7-5.4 \pm 0.6$ & $6.7 \pm 2.7-2.6 \pm 1.0$ & $0.7 \pm 0.1$ & $0.3 \pm 0.1$ \\
\hline $15-8$ & $16 \pm 5.4$ & $74 \pm 8.0-76 \pm 7.8$ & $5.2 \pm 0.6-5.1 \pm 0.5$ & $4.4 \pm 1.6-1.7 \pm 0.6$ & $0.7 \pm 0.08$ & $0.3 \pm 0.06$ \\
\hline $24-9$ & $22 \pm 4.5-23 \pm 4.6$ & $68 \pm 6.2-70 \pm 6.2$ & $4.8 \pm 0.4-4.7 \pm 0.4$ & $3.7 \pm 1.0-1.4 \pm 0.4$ & $0.8 \pm 0.1$ & $0.2 \pm 0.05$ \\
\hline $15-12$ & $58 \pm 27-71 \pm 33$ & $11 \pm 4.2-12 \pm 4.6$ & $17 \pm 7.3-6.7 \pm 2.9$ & $8.1 \pm 4.3-3.1 \pm 1.7$ & $2.9 \pm 1.6-3.3 \pm 1.8$ & $2.4 \pm 1.5-3.3 \pm 2.1$ \\
\hline $24-16$ & $60 \pm 19-72 \pm 23$ & $14 \pm 4.1-15 \pm 4.2$ & $15 \pm 5.0-5.7 \pm 1.8$ & $8.1 \pm 4.0-3.1 \pm 1.6$ & $0.8 \pm 0.3-0.9 \pm 0.3$ & $2.0 \pm 1.1-2.8 \pm 1.5$ \\
\hline 24-19 & $61 \pm 19-71 \pm 22$ & $18 \pm 4.5-19 \pm 4.6$ & $13 \pm 3.7-5.6 \pm 1.6$ & $7.0 \pm 3.5-2.7 \pm 1.3$ & $0.6 \pm 0.2-0.7 \pm 0.2$ & $1.2 \pm 0.7-1.5 \pm 0.9$ \\
\hline $\begin{array}{l}\text { Avg in non- } \\
\text { convective } \\
\text { layer (c) }\end{array}$ & $19 \pm 4.9$ & $70 \pm 7: 3-72 \pm 7.0$ & $5.7 \pm 0.6-5.5 \pm 0.6$ & $4.8 \pm 1.8-1.8 \pm 0.7$ & $0.7 \pm 0.1$ & $0.3 \pm 0.06$ \\
\hline $\begin{array}{l}\text { Avg in } \\
\text { convective } \\
\text { layer (c) }\end{array}$ & $60 \pm 22-71 \pm 27$ & $14 \pm 4.3-15 \pm 4.6$ & $15 \pm 5.4-6.0 \pm 2.2$ & $7.8 \pm 4.0-3.0 \pm 1.5$ & $1.6 \pm 0.7-1.7 \pm 0.8$ & $1.9 \pm 0.9-2.6 \pm 1.2$ \\
\hline \multicolumn{7}{|c|}{$\begin{array}{l}\text { (a) Uncertainties on compositions represent only instrument uncertainty. Compositions calculated for both lower- and upper- } \\
\text { bound solubilities. Both ends of composition range are given, lower-bound solubility basis first with instrument uncertainty } \\
\text { on each. Compositions may not sum to } 100 \mathrm{~mol} \% \text { because of roundoff error. Mole fractions are on a dry basis and do not } \\
\text { account for water vapor. } \\
\text { (b) Ammonia mole fraction derived from partial pressures in Table } 4.3 .5 \text { extrapolated to vapor pressure at in situ conditions. } \\
\text { (c) There are too few samples to define the spatial variability of the average gas concentration. }\end{array}$} \\
\hline
\end{tabular}


layer is the result of integrating RGS species concentrations over the waste layer and multiplying those concentrations by the layer volume. The integration method is described in Section 3.7.1.

The compositions in samples from risers 15 and 24 were the same within uncertainty. Nitrogen was high in the convective layer, as was nitrous oxide, and hydrogen low. The concentrations and pressures in Tables 4.3.3 and 4.3.4 can also be found in the sample-by-sample worksheets in the A-101 RGS calculation spreadsheet on the CD. The compositions in Table 4.3.6 are on the "Inventory" worksheet.

\subsubsection{Gas Inventory}

The method by which the in situ gas volume fractions (wet basis) were calculated is given in Section 3.6.2; as for the gas composition, the volume fractions are given as a range from the low-gas-solubility value to the high-gas-solubility value. The results are presented in Table 4.3.7, which also contains the average gas volume fraction and the average pressure experienced by the gas in each layer. The averages are in situ volume averages calculated by Simpson's Rule integration, as described in Section 3.7.1. The corrected gas volume fractions in Table 4.3.7 are consistent with the corrected gas concentrations and compositions in Tables 4.3.4 through 4.3.6. The information in Table 4.3.7 is taken from the "Summary" and "In situ" worksheets of the A-101 RGS calculation spreadsheet on the CD.

The "sampler gas volume fraction" is corrected only for inleakage during the extraction process and air gases added in the isotopic solution. It is an attempt to reconstruct the total amount of gas (sample and entrainment) that was present in the sampler during $\mathrm{X}$-ray. It is used only for comparison with the $\mathrm{x}$-ray observations of "visible gas fraction" discussed in Section 4.3.4.

Table 4.3.7 also contains the water vapor pressures that were used for in situ calculations. The water vapor pressures were found by using salt concentrations from Field (1997) and the temperatures in the table as inputs to Equation 6.2 of Mahoney and Trent (1995), a correlation for water vapor pressure over concentrated homogeneous and non-homogeneous waste simulants. The gas solubilities used the same parameters as inputs to the Schumpe solubility model (Section 3.5.1). The water vapor pressures and gas solubilities used in calculations can be found in the "In-situ" worksheet of the A-101 RGS calculation spreadsheet on the CD.

The gas content of the nonconvective layer consistently increased as depth increased, with no evident difference between risers. The results showed a high concentration of hydrogen in the nonconvective layer, composing approximately $70 \%$ of the volume of the retained gas. No ammonia inventory was calculated for the tank, owing to the absence of total ammonia concentration data for A-101.

Table 4.3.8 gives various estimates of the STP volume of gas in Tank A-101, including estimates calculated from RGS data alone. The BPE inventory value was found using Eq. 3.6.1, based on the median dL/dP of -0.59 in./in. Hg given by Whitney et al. (1997) and the tank average gas pressure $(136 \mathrm{kPa})$ and elevation $(661 \mathrm{~cm})$ found from RGS results.

The RGS gas inventories in the two layers were calculated by integrating RGS total gas concentrations over the nonconvective layer (four data points) and the convective layer (three data points) and multiplying the average gas concentrations by the volumes of the layers. The integration method is described in Section 3.7.1. The RGS volumes in Table 4.3.8 include corrections to remove the contamination gas: entrained air and argon and air leaks during and after extraction. The uncertainties on the gas inventories are based on the spatial variability considerations discussed in Section 3.7.2. The information in Table 4.3.8 is taken from the "Inventory" worksheet of the A-101 RGS calculation spreadsheet on the CD. 
Table 4.3.7. In Situ Gas Volume Fractions and Conditions in Tank A-101

\begin{tabular}{|c|c|c|c|c|c|c||}
\hline Sample & $\begin{array}{c}\text { Sample } \\
\text { central } \\
\text { height } \\
\text { (cm) }\end{array}$ & $\begin{array}{c}\text { Hydro- } \\
\text { static } \\
\text { pressure } \\
\text { (atm) }\end{array}$ & $\begin{array}{c}\text { Calculated } \\
\text { water vapor } \\
\text { pressure } \\
\text { (atm) }\end{array}$ & $\begin{array}{c}\text { Temp. } \\
\text { ( }{ }^{\circ} \text { C) }\end{array}$ & $\begin{array}{c}\text { Corrected gas volume } \\
\text { fraction(a) } \\
\text { (in-tank conditions) }\end{array}$ & $\begin{array}{c}\text { Sampler gas } \\
\text { volume fraction } \\
\text { (in situ conditions, } \\
\text { low solubility) }\end{array}$ \\
\hline \hline $24-2$ & 845 & 1.05 & 0.024 & 43.0 & $0.16 \pm 0.014$ & 0.29 \\
\hline $15-5$ & 700 & 1.24 & 0.046 & 56.0 & $0.19 \pm 0.021-0.18 \pm 0.021$ & 0.19 \\
\hline $15-8$ & 555 & 1.43 & 0.064 & 62.8 & $0.21 \pm 0.021-0.20 \pm 0.021$ & 0.21 \\
\hline $24-9$ & 507 & 1.49 & 0.066 & 63.5 & $0.22 \pm 0.021-0.21 \pm 0.021$ & 0.23 \\
\hline $15-12$ & 362 & 1.70 & 0.094 & 62.5 & $0.008 \pm 0.003-0.006 \pm 0.003$ & 0.017 \\
\hline $24-16$ & 169 & 1.97 & 0.092 & 62.0 & $0.007 \pm 0.003-0.005 \pm 0.003$ & 0.014 \\
\hline $24-19$ & 24.1 & 2.18 & 0.088 & 61.0 & $0.010 \pm 0.003-0.008 \pm 0.003$ & 0.020 \\
\hline $\begin{array}{c}\text { Avg in non- } \\
\text { convective } \\
\text { layer }\end{array}$ & 652 & 1.30 & & & $0.18 \pm 0.09-0.17 \pm 0.085$ & \\
\hline $\begin{array}{l}\text { Avg in } \\
\text { convective } \\
\text { layer }\end{array}$ & 207 & 1.92 & & & \multicolumn{7}{|l||}{$0.008 \pm 0.003-0.006 \pm 0.003$} & \\
\hline $\begin{array}{l}\text { (a) Gas volume fraction expressed on wet basis, including volume contribution of water vapor. Uncertainties } \\
\text { on layer-average gas volume fractions based on spatial variability considerations (Section 3.7.2). }\end{array}$ \\
\hline
\end{tabular}

The RGS method gave the higher gas inventory ( $334 \pm 159 \mathrm{~m}^{3}$ in situ) and the BPE method the lower. The difference between the two methods for this SST was $27 \%$ of the RGS inventory, which is within the roughly $\pm 30 \%$ range of variability that was shown in Section 3.7.2 to be typical for DST inventories calculated from measurements made by different methods.

Table 4.3.8. A-101 Gas Inventory Estimates

\begin{tabular}{||l|c|c|c||}
\hline \multirow{2}{*}{ Quantity } & \multicolumn{2}{|c|}{ RGS Method(a) } & BPE Method(b) \\
\cline { 2 - 4 } & $\begin{array}{c}\text { Nonconvective } \\
\text { Layer }\end{array}$ & $\begin{array}{c}\text { Convective } \\
\text { Layer }\end{array}$ & Whole Tank \\
\hline \hline $\begin{array}{l}\text { Avg gas fraction } \\
\text { (low gas solubility) }\end{array}$ & $0.18 \pm 0.09$ & $0.008 \pm 0.003$ & $\begin{array}{c}\text { (nonconv. avg) } \\
0.14 \pm 0.035\end{array}$ \\
\hline $\begin{array}{l}\left.\text { Gas volume (m }{ }^{3}\right) \\
\text { in situ (wet) }\end{array}$ & $318 \pm 159$ & $16 \pm 5$ & $245 \pm 61$ \\
STP (wet) & $344 \pm 172$ & $24 \pm 8$ & $270 \pm 67$ \\
\hline $\begin{array}{l}\text { (a) The retained gas inventory calculated from RGS data is considered the best } \\
\text { estimate. } \\
\text { (b) Barometric pressure method. }\end{array}$ \\
\hline
\end{tabular}


We recommend that the RGS gas inventory estimate be used in place of the BPE estimate. No extraction or sample acquisition difficulties cast doubt on the RGS data; there were two RGS risers, spaced well apart, and four good samples within the nonconvective layer; RGS data from the two risers showed a consistent gas volume fraction profile; and core extrusions appeared the same at both risers, leaving little reason to suspect lateral variability of gas. The only sign of lateral variability was the 10 in. waste level difference between risers 15 and 24 . The level data on which the BPE estimate was based are believed to be sound, being derived from Enraf measurements from a riser that is not near the tank wall. With no reason to disqualify either data set and no reason to penalize the RGS data for being local in nature, the direct gas measurements obtained from RGS are preferred to the inferred gas estimate from BPE.

Table 4.3.9 contains the calculated layer inventories of each of the major gases retained in the waste. These inventories are based on the layer-average compositions from RGS data (Table 4.3.6) and the best-estimate gas inventories for each layer (RGS, Table 4.3.8).

Figure 4.3.3 shows the sample temperatures, corrected gas volume fractions (Table 4.3.7), and corrected compositions of the low-solubility constituents in the samples from A-101. The sample temperatures were measured by the TC tree in riser 10 . The compositions represent the mole fraction of the species in the "insoluble" gas; water and ammonia are not included, so the mole fractions are not the same as those in Table 4.3.4. The gas volume fractions and mole fractions in the figure are the values for lower-bound gas solubilities. Figure 4.3.3 also lists the observations from core extrusions of non-RGS samples and from x-rays of RGS samples as a way of tying those observations together with RGS data. The $\mathrm{x}$-ray data are described in more detail in Section 4.3.4.

Table 4.3.9. Speciated A-101 Gas Inventory(a)

\begin{tabular}{|c|c|c|c|c|}
\hline \multirow[t]{2}{*}{ Species } & \multicolumn{2}{|c|}{$\begin{array}{c}\text { Gas-Phase Inventory } \\
\left(\mathrm{m}^{3} \text { at STP) }\right.\end{array}$} & \multicolumn{2}{|c|}{$\begin{array}{l}\text { Dissolved Inventory } \\
\left(\mathrm{m}^{3} \text { at } \mathrm{STP}\right)\end{array}$} \\
\hline & $\begin{array}{c}\text { Nonconvective } \\
\text { Layer }\end{array}$ & $\begin{array}{c}\text { Convective } \\
\text { Layer }\end{array}$ & $\begin{array}{c}\text { Nonconvective } \\
\text { Layer }\end{array}$ & $\begin{array}{c}\text { Convective } \\
\text { Layer }\end{array}$ \\
\hline $\mathrm{N}_{2}$ & $62-61$ & $14-12$ & $0.018-0.16$ & $0.14-1.5$ \\
\hline $\mathrm{H}_{2}$ & 230 & $3.3-2.6$ & $0.23-1.4$ & $0.12-0.77$ \\
\hline $\mathrm{N}_{2} \mathrm{O}$ & $19-18$ & $3.5-1.0$ & $0.24-1.6$ & $1.4-3.9$ \\
\hline $\mathrm{NH}_{3}$ & $16-5.9$ & $1.8-0.52$ & 2000 & 6700 \\
\hline $\mathrm{CH}_{4}$ & 2.1 & $0.36-0.30$ & $0.0010-0.0099$ & $0.0065-0.069$ \\
\hline Other & 1.1 & 0.44 & 0 & 0 \\
\hline \multicolumn{5}{|c|}{$\begin{array}{l}\text { (a) Inventories based on layer-average compositions from RGS data (Table 4.3.6) and } \\
\text { the best-estimate gas inventories for each layer (RGS, Table } 4.3 .8 \text { ). The uncertainty in } \\
\text { each inventory is } 50 \% \text {, based on spatial variability considerations discussed in Section } \\
\text { 3.7.2. Inventories are given for both the lower-bound and upper-bound solubilities; } \\
\text { lower-bound solubility basis is first in the table. }\end{array}$} \\
\hline
\end{tabular}



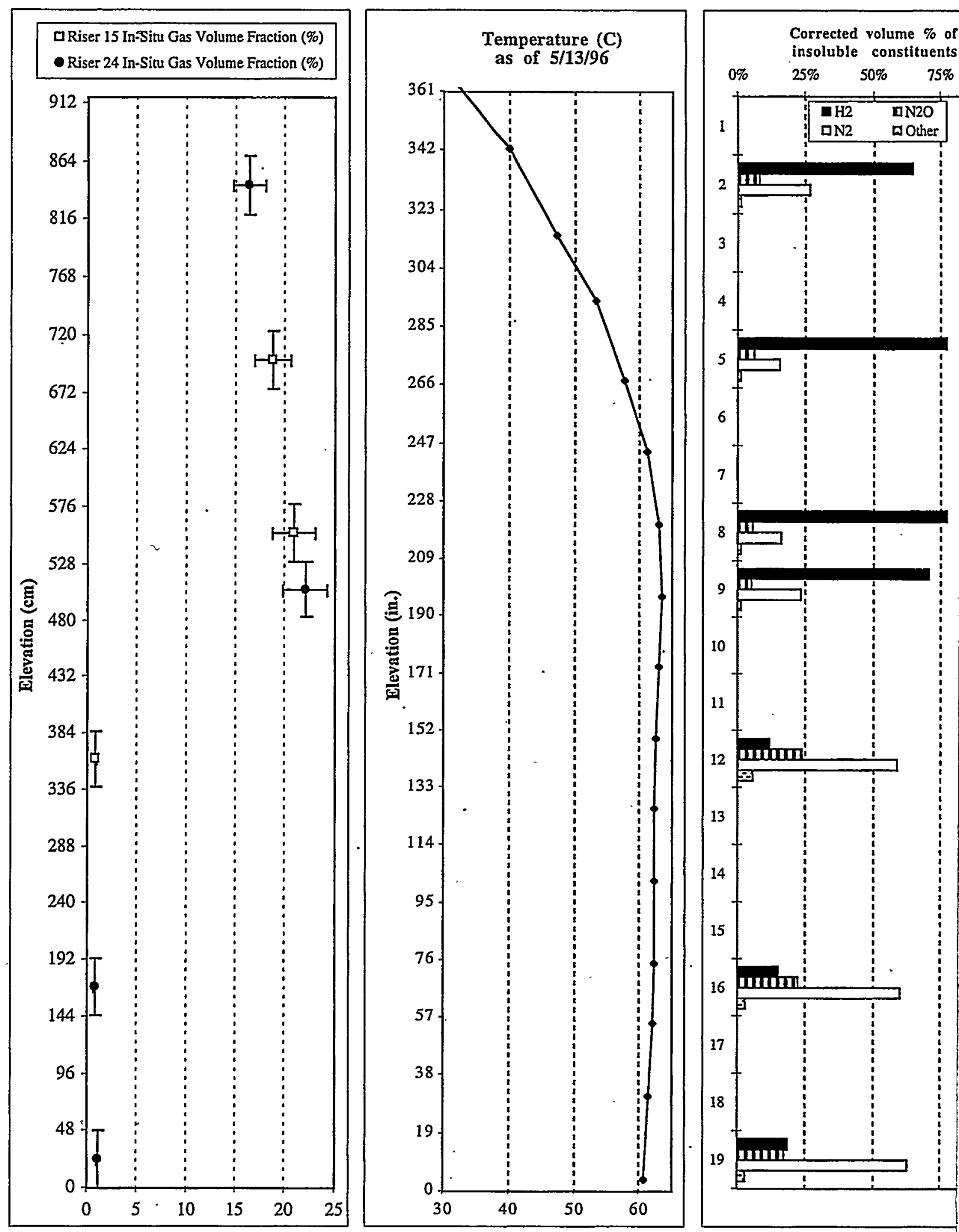

Figure 4.3.3. Gas Volume Fractions, Temperatures, 
A-101 waste level is $884 \mathrm{~cm}$ (348 inches).

1 Riser 15: 11 in. (257 g) yellowish-brown dry salt (top half) and moist salt (bottom half).

1 Riser 24: 1 in. $(33 \mathrm{~g}$ ) green-brown salt slurry, $<5 \mathrm{~mL}$ green-brown opaque liquid.

2 Riser 15: 17 in. (288 g) dark grey moist salt.

2 Riser 24 X-RAY: Rough surface, gas in vague blobs \& twisted cracks, some 1-mm bubbles, mostly in bottom half.

3 Riser 15: 19 in. (427 g) dark grey moist salt.

3 Riser 24: 19 in. ( 426 g) dark gray moist salt.

4 Riser 15: 19 in. (422 g) dark grey moist sait.

4 Riser 24: 18 in. (421 g) dark gray moist salt.

5 Riser 15 X-RAY: Waste across piston, many bubbles $1-4 \mathrm{~mm}$ range, rough flat bubble $0.5 \mathrm{in}$. wide by 0.2 inch tall.

5 Riser 24: 18 in. ( $409 \mathrm{~g})$ dark gray moist salt.

6 Riser 15: 17 in. (406 g) dark grey moist salt.

6 Riser 24: 19 in. $(420 \mathrm{~g})$ dark gray moist salt.

7 Riser 15: 19 in. (411 g) dark grey moist salt.

7 Riser 24: 16 in. (354 g) dark gray moist salt.

8 Riser I5 X-RAY: Many voids, fine bubble structure, large gas gaps (about 4 in. at top, 2 in. at bottom)

8 Riser 24: 19 in (369 g) dark gray moist salt.

9 Riser 15: 19 in. (388 g) dark grey moist salt.

9 Riser 24 X-RAY: Clusters of 1-mm and less bubbles, denser near bottom, rough oval void 0.25 in. tall, 0.7 in. wide.

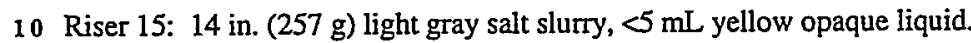

10 Riser 24: 7 in $(152 \mathrm{~g})$ white to gray salt slurry, $110 \mathrm{~mL}(164 \mathrm{~g})$ yellow opaque liquid.

11 Riser 15: Hydrostatic head fluid.

11 Riser 24: $5 \mathrm{in.}(96 \mathrm{~g})$ white salt slurry, $230 \mathrm{~mL}(368 \mathrm{~g})$ green opaque liquid.

12 Riser $15 \mathrm{X}$-RAY: Featureless, 0.5 in. gap under clean piston.

12 Riser 24: 2 in. (101 g) white salt slurry, $230 \mathrm{~mL}$ ( $306 \mathrm{~g}$ ) green opaque liquid.

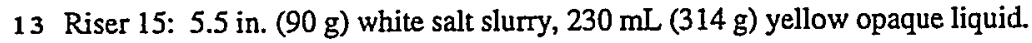

13 Riser 24: $5 \mathrm{in}$. $(70 \mathrm{~g})$ white salt slurry, $250 \mathrm{~mL}(351 \mathrm{~g})$ green opaque liquid.

14 Riser 15: 3 in. ( $52 \mathrm{~g}$ ) white salt slurry, $175 \mathrm{~mL}(248 \mathrm{~g})$ yellow opaque liquid.

14 Riser 24: $3.5 \mathrm{in}$. $(83 \mathrm{~g})$ white salt slurry, $245 \mathrm{~mL}(348 \mathrm{~g})$ yellow opaque liquid.

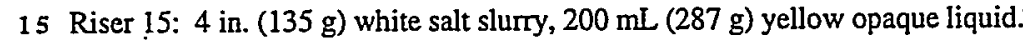

15 Riser 24: 2 in. $(81 \mathrm{~g})$ white salt slurry, $240 \mathrm{~mL}(336 \mathrm{~g})$ green opaque liquid.

16 Riser 15: $6.5 \mathrm{in.} \mathrm{(133} \mathrm{g)} \mathrm{white} \mathrm{salt} \mathrm{slurry,} 205 \mathrm{~mL} \mathrm{(298} \mathrm{g)} \mathrm{yellow} \mathrm{opaque} \mathrm{liquid.}$

16 Riser 24 X-RAY: 0.05 in. gas gap, surface smooth but not well-defined meniscus, featureless. Piston fully retracted.

17 Riser 15: small amount white salt slurry, $300 \mathrm{~mL}$ ( $289 \mathrm{~g})$ white opaque liquid; HHF contamination.

17 Riser 24: 1 in. $(66 \mathrm{~g})$ white salt slurry, $250 \mathrm{~mL}(345 \mathrm{~g})$ green opaque liquid.

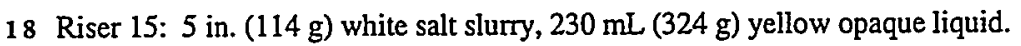

18 Riser 24: 7 in. (118 g) white to light grey salt slurry, $200 \mathrm{~mL}(283 \mathrm{~g})$ green opaque liquid.

19 Riser 15: 3 in. (87 g) white salt slurry, $230 \mathrm{~mL}$ (48 g) green opaque liquid; high HHF contamination.

19 Riser 24 X-RAY: 0.15 in. gas gap, surface smooth but not well-defined meniscus, featureless. Piston fully retracted. 


\subsubsection{X-Ray Results}

Table 4.3.10 summarizes all the available radiography observations from Tank A-101. The $\mathrm{x}$-ray images themselves can be found on the $\mathrm{CD}$ that accompanies this report. The uncertainties of the visible gas volume fractions that are given in Table 4.3.10 are unknown but (based on the uncertainty in measuring the gas gap from $x$-ray images) are expected to be larger than the uncertainties in the gas volume fractions calculated from gas extraction. (The "visible" gas volume fraction refers to the gas that has separated from the solids and liquid such that its volume can be calculated based on the image. The uncorrected gas fractions from extraction are used for comparison to reflect the presence of entrained air in the sampler.)

\section{Table 4.3.10. Summary of Observations from X-Ray Images of Tank A-101}

\begin{tabular}{|c|c|}
\hline Segment & Comments/Observations \\
\hline $\begin{array}{c}24-2 \\
\text { (RGS, } \\
7 / 22 / 96)\end{array}$ & $\begin{array}{l}\text { Gas seldom resolvable into bubbles but observed as vague blobs and twisted cracks. There are } \\
\text { some } 1 \text {-mm bubbles. Most of the gas is in the bottom half. Gas gap } 4 \mathrm{~mm}(0.15 \text { in.), clean, } \\
\text { fully retracted piston. The visible gas volume fraction is } 0.008 \text {. (Compare with the corrected } \\
\text { sampler gas volume fraction of } 0.16 \text { obtained by gas extraction, Table } 4.3 .7 \text {. In this case, } \\
\text { where inleakage during extraction is suspected, the corrected gas fraction is expected to be closer } \\
\text { to the x-rayed gas content than to the uncorrected value.) }\end{array}$ \\
\hline $\begin{array}{c}15-5 \\
(\mathrm{RGS}, \\
7 / 12 / 96)\end{array}$ & $\begin{array}{l}\text { Many bubbles were seen throughout the sample in 1-4-mm-diameter range, often clustered } \\
\text { together. About one-third of the way up from the bottom of the sampler was a rough-surfaced, } \\
\text { gas-filled fracture like a flattened bubble, } 12 \text {-mm ( } 0.5 \text {-in.) wide by } 5 \text {-mm }(0.2 \text {-in.) tall. A } \\
\text { slightly rough surface sloped up toward the sides. Gas gap is } 12-\mathrm{mm}(0.5 \text {-in.), jagged lump of } \\
\text { waste at least } 2 \mathrm{~mm}(0.1 \mathrm{in} \text {.) thick across the piston. The visible gas volume fraction is } 0.03 \text {, } \\
\text { counting the fracture bubble but not other bubbles. (Compare with the uncorrected sampler gas } \\
\text { volume fraction of } 0.19 \text { obtained by gas extraction, Table } 4.3 .7 .)\end{array}$ \\
\hline $\begin{array}{c}15-8 \text {. } \\
(\mathrm{RGS}, \\
7 / 12 / 96)\end{array}$ & $\begin{array}{l}\text { Many bubbles and much fine structure. There is a large gas gap, about } 10 \mathrm{~cm} \text { ( } 4 \text { in.), at the } \\
\text { sampler bottom and another, about } 5 \mathrm{~cm} \text { ( } 2 \text { in., at the top). The visible gas volume fraction } \\
\text { (not counting bubbles) is } 0.3 \text {. Because the } \mathrm{x} \text {-ray tape of this sample was not available for } \\
\text { review in } 1999 \text {, the gas gap measurements were the relatively approximate ones made in } 1996 \text {. } \\
\text { (Compare with the uncorrected sampler gas volume fraction of } 0.21 \text { obtained by gas extraction, } \\
\text { Table 4.3.7.) }\end{array}$ \\
\hline $\begin{array}{c}24-9 \\
(\mathrm{RGS} \\
7 / 23 / 96)\end{array}$ & $\begin{array}{l}\text { Clusters and blobs of } 1-\mathrm{mm} \text { and smaller bubbles throughout, denser toward bottom of sample. } \\
\text { About } 1.5 \text { in. from top of waste, a tilted rough-surfaced oval cavity } 0.25 \text { in. tall by } 0.7 \text { in. wide } \\
\text { with gassy waste around it. A slightly rough surface sloped up toward the sides. Gas gap is } \\
1 \mathrm{~mm}(0.4 \mathrm{in} \text {.); clean, fully retracted piston. The visible gas volume fraction (counting the } \\
\text { cavity but not other bubbles) is } 0.03 \text {. (Compare with the uncorrected sampler gas volume } \\
\text { fraction of } 0.23 \text { obtained by gas extraction, Table } 4.3 .7 .)\end{array}$ \\
\hline $\begin{array}{c}15-12 \\
(\mathrm{RGS} \\
7 / 17 / 96)\end{array}$ & $\begin{array}{l}\text { Featureless, with a } 12-\mathrm{mm}(0.5 \text {-in.) gap under a clean piston. The visible gas volume fraction } \\
\text { is } 0.03 \text {. (Compare with the uncorrected sampler gas volume fraction of } 0.017 \text { obtained by gas } \\
\text { extraction, Table 4.3.7.) }\end{array}$ \\
\hline $\begin{array}{l}24-16 \\
(\mathrm{RGS} \\
7 / 24 / 96)\end{array}$ & $\begin{array}{l}\text { Featureless, with a } 1-\mathrm{mm}(0.05 \text {-in.) gap under a clean, fully retracted piston. Surface smooth } \\
\text { but not a meniscus. The visible gas volume fraction is } 0.003 \text {. (Compare with the uncorrected } \\
\text { sampler gas volume fraction of } 0.014 \text { obtained by gas extraction, Table } 4.3 .7 \text {.) }\end{array}$ \\
\hline $\begin{array}{c}24-19 \\
\text { (RGS, } \\
7 / 25 / 96)\end{array}$ & $\begin{array}{l}\text { Featureless, with a 4-mm }(0.15 \text {-in.) gap under a clean, fully retracted piston. Surface smooth } \\
\text { but not a meniscus. The visible gas volume fraction is } 0.008 \text {. (Compare with the uncorrected } \\
\text { sampler gas volume fraction of } 0.020 \text { obtained by gas extraction, Table 4.3.7.) }\end{array}$ \\
\hline
\end{tabular}


Table 4.3.10 compares x-ray-derived gas volume fractions with extraction-derived gas volume fractions. The comparison shows that sample recovery was close to $100 \%$ for all the samples except possibly sample 15-8. This conclusion is based on the fact that the extracted uncorrected gas for most samples was greater than the visible gas. In the case of sample 15-5, the extracted gas fraction was 0.20 and the visible gas 0.3 according to approximate measurements of the gas gaps. Waste that is visibly bubbly in $\mathrm{x}$-rays typically contains 5 to $10 \%$ gas in addition to what is evident in the gas gap (based on comparing extracted and visible gas fractions). The bubbly A-101 waste contained an unusual 15 to $20 \%$ gas in addition to the gas gap based on samples $24-2,15-5$, and $24-9$. Thus the sample recovery for $15-8$ may have been only 75 to $80 \%$.

The unusual tendency for A-101 waste to retain gas in bubbles in the sampler rather than in a gas gap, and the tendency for those bubbles to appear as slits or irregular structures, may have been signs of unusual waste strength. (However, this observation could be misleading in that a large population of round bubbles could also give the appearance that the gas pockets or bubbles are not round. To resolve this issue, a tomographic $\mathrm{x}$-ray imaging system would be needed to provide projections from several directions and enable the spatial distribution and shape of the gas phase to be determined more accurately.)

Two features that appeared in these images (samples 15-5, 24-9) and that were characteristic of the waste in A-101 were fissures or fractures. However, one may question whether such fractures were present in the tank or were artifacts of the measurement approach produced during sampling. It has been shown that the waste sample is under a compressive stress during sampling.(a) Such a stress field would be acting in the opposite direction from that expected for formation of these fractures. If that stress field was maintained by the waste sample, the fractures would be filled with high-pressure gas that sustained the form of the fractures during sampling.

Such fractures or fissures were not seen when sample A-101-15-5 (not originally a fractured sample) underwent laboratory retained gas expansion tests after mixing (Rassat et al. 1998). However, fractures were seen in some retained gas tests, those using samples of S-102 saltcake and stiff sludge (Gauglitz et al. 1996). The waste adhesion to the sampler piston (sample 15-5) and walls (sample 15-8) was also distinctive and therefore of some interest.

Table 4.3.11 provides a summary of the waste densities that have been calculated from radiographic data for the RGS samples for which air and water standard images were available. The radiographic densities include the gas in the waste. The overall sample densities were calculated by the original method (described in Section 3.9). Table 4.3.11 also includes density measurements made on extruded non-RGS samples (Field 1997). In samples 15-5 and 24-9 the radiographic densities equaled or exceeded the densities found for the adjacent extruded samples, which is difficult to explain because the radiographic densities included substantial gas and the extruded densities did not. The close match between the radiographic and extruded densities of the convective layer low-gas samples $15-12$ and 24-16 is more plausible.

\subsubsection{Domespace Composition Comparison}

The RGS gas-phase composition data (Table 4.3.6) were used to calculate $\mathrm{H}_{2} / \mathrm{N}_{2} \mathrm{O}$ ratios to compare with ratios from domespace grab sample measurements (McCain 1999, Table B-1). The results can be seen in Table 4.3.12. The domespace $\mathrm{H}_{2} / \mathrm{N}_{2} \mathrm{O}$ ratios were not constant over the period from 1995 to 1998 . The more recent lower domespace ratios resembled those found by RGS in the convective layer of A-101.

(a) Shekarriz A and JD Norton. 1995. Retained Gas Sampler System Analysis. PNLFGP:091595, Pacific Northwest Laboratory, Richland, Washington. 
Table 4.3.11. Densities of A-101 Samples from Radiography and Core Samples

\begin{tabular}{||c|c|l||}
\hline $\begin{array}{c}\text { Riser- } \\
\text { Segment } \\
\text { Number }\end{array}$ & $\begin{array}{c}\text { Overall Average } \\
\text { Sample Densities (a) } \\
\text { (g/cc) }\end{array}$ & $\begin{array}{l}\text { Degassed Density in the non-RGS Cores } \\
\text { above and below the RGS Sample } \\
\text { (g/cc) }\end{array}$ \\
\hline $15-5$ & 1.67 & $\begin{array}{l}\text { bulk density above, } 1.69 \mathrm{~g} / \mathrm{cc} \\
\text { bulk density below, } 1.70 \mathrm{~g} / \mathrm{cc}\end{array}$ \\
\hline $15-8$ & 1.46 & $\begin{array}{l}\text { bulk density above, } 1.72 \mathrm{~g} / \mathrm{cc} \\
\text { bulk density below, } 1.70 \mathrm{~g} / \mathrm{cc}\end{array}$ \\
\hline $24-9$ & 1.73 & $\begin{array}{l}\text { bulk density above, } 1.63 \mathrm{~g} / \mathrm{cc} \\
\text { bulk density below, } 1.58 \mathrm{~g} / \mathrm{cc}\end{array}$ \\
\hline $15-12$ & 1.73 & bulk density below, $1.75 \mathrm{~g} / \mathrm{cc}$ \\
\hline $24-16$ & 1.68 & $\begin{array}{l}\text { bulk density above, } 1.74 \mathrm{~g} / \mathrm{cc} \\
\text { bulk density below, } 1.70 \mathrm{~g} / \mathrm{cc}\end{array}$ \\
\hline $\begin{array}{l}\text { (a) The densities were calculated by the original x-ray analysis method and are } \\
\text { taken from Table 4.19 of Shekarriz et al. (1997). }\end{array}$ \\
\hline
\end{tabular}

Table 4.3.12 Comparison with Domespace Data(a)

\begin{tabular}{||l|c||}
\hline \multicolumn{1}{|c|}{ Sample } & $\mathrm{H}_{2} / \mathrm{N}_{2} \mathrm{O}$ \\
\hline$R G S, 24-2$ & $8.4-9.3$ \\
\hline$R G S, 15-5$ & $13-14$ \\
\hline$R G S, 15-8$ & $14-15$ \\
\hline$R G S, 24-9$ & $14-15$ \\
\hline$R G S, 15-12$ & $0.68-1.8$ \\
\hline$R G S, 24-16$ & $0.95-2.7$ \\
\hline$R G S, 24-19$ & $1.4-3.3$ \\
\hline Domespace samples, 8/95 & $6.5,7.4,6.2,6.2$, \\
& $5.9,6.2,5.9$ \\
\hline Domespace sample, 3/19/98 & 2.3 \\
\hline Domespace sample, 5/18/98 & 2.5 \\
\hline Domespace sample, 8/21/98 & 2.2 \\
\hline $\begin{array}{l}\text { (a) RGS ratios were calculated for both lower- and upper-bound } \\
\text { solubilities. Both ends of the ratio range are given, lower-bound } \\
\text { solubility basis first. }\end{array}$ \\
\hline
\end{tabular}




\subsection{AN-104}

Tank 241-AN-104 (AN-104) was the fourth tank and third DST sampled with the RGS. This tank was selected because it was one of the DSTs that exhibited episodic GREs. AN-104 was on the FGWL and showed cyclical level drops and episodic domespace hydrogen concentrations of as high as $0.5 \%$ resulting from GREs. Most of the tank contents were double-shell slurry feed (Stewart et al. 1996a).

Push-mode sampling was done in risers 12A and 10A in August and September 1996.(a) These risers were sampled because they were close to the VFI risers (1B and 16B) and the MIT in riser $15 \mathrm{~A}$, allowing correlation of waste temperatures and gas content. The approximate locations of various risers are depicted in Figure 4.4.1.

The total depth of waste in Tank AN-104 was approximately $979 \mathrm{~cm}$ (385 in.); thus, at the time of RGS sampling, AN-104 contained approximately 4,010,000 L (1,060,000 gal) of waste. Riser 12A is near the tank center, while riser 10A is about three-fourths of a tank radius from the center. Figure 4.4.2 shows the tank content layering at the time, derived from the riser 4A TC tree and riser 15A MIT measured temperature profiles in conjunction with the VFI/ball rheometer data for this tank (Schienbein et al. 1999, Table 2.3.2). The nonconvective layer was $410 \pm 40 \mathrm{~cm}$ (161 \pm 16 in.) in depth, and the balance of the contents were a convective (supernatant liquid) layer of $526 \pm 41 \mathrm{~cm}(207 \pm 16 \mathrm{in}$.) depth and a crust layer of $41 \pm 7 \mathrm{~cm}(16 \pm 3 \mathrm{in}$.) thickness. The elevations of the RGS segments are depicted in Figure 4.4.2.

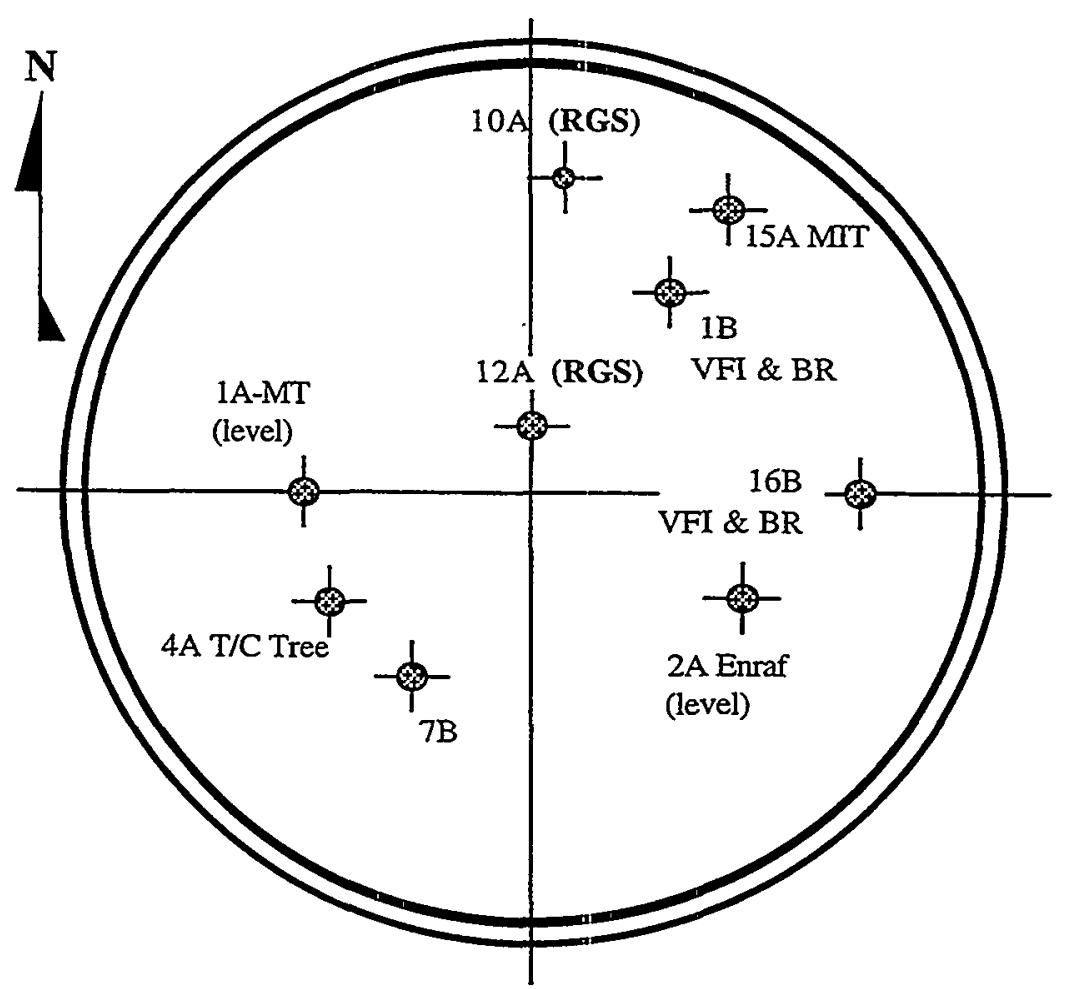

Figure 4.4.1. Schematic Diagram of Riser Locations in Tank AN-104

(a) The sampling scheme may also be found in Sampling Plan for Tank 241-AN-104 Retained Gas Sampler Deployment, by A Shekarriz and JM Bates, April 1996. PNNLMIT:041796, Pacific

Northwest National Laboratory, Richland; Washington. See also the May 30, 1996 addenda letter to RE Bauer, Westinghouse Hanford Company, from JM Bates, Pacific Northwest National Laboratory. 

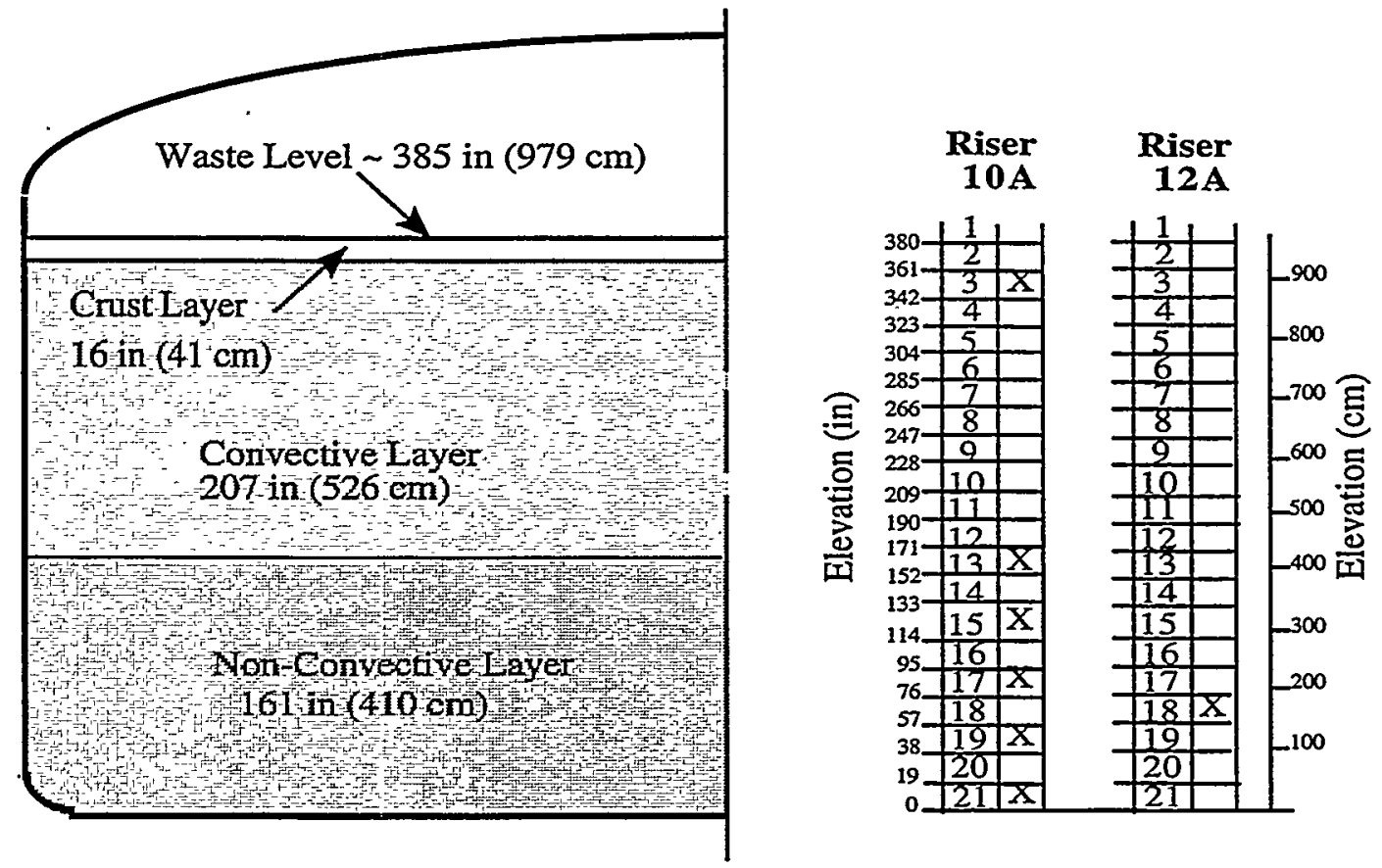

Figure 4.4.2. Diagram of Waste Layering-and RGS Sample Elevations for AN-104

The most recent information on AN-104 layering came from the observations made on the non-RGS samples in the cores from 12A and 10A (Hu et al. 1997). The topmost sample contained 1 to 2 in. of salt slurry. Beneath that was about 11 samplers ( $200 \mathrm{in}$.) of liquid, with eight to nine samplers (160 to 170 in.) of salt slurry, wet salt, or moist salt at the tank bottom.

One small buoyant displacement GRE occurred on May 3, 1996, about a month after the VFI measurements (April 2 and 4,1996) and more than three months before the first RGS samples were taken from riser 12A (Schienbein et al. 1999). This event might have caused a small difference between the VFI and RGS measurements.

Densities for the liquid and bulk solids in AN-104 were available from the non-RGS samples taken in 1996 (Hu et al. 1997). A number of samples contained drainable liquid. The density of the liquid was $1400 \pm 34 \mathrm{~kg} / \mathrm{m}^{3}$; the bulk density of the nonconvective samples varied from 1520 to $1760 \mathrm{~kg} / \mathrm{m}^{3}$, with an average and standard deviation of $1591 \pm 58 \mathrm{~kg} / \mathrm{m}^{3}$. Table 2.7 .1 of Schienbein et al. (1999) gave a density of $1440 \pm 30 \mathrm{~kg} / \mathrm{m}^{3}$ for the convective layer (based on in situ ball rheometry in 1996) and $1580 \pm 50 \mathrm{~kg} / \mathrm{m}^{3}$ for the nonconvective layer (based on core samples). All of these measurement methods disturbed the waste and their results should be taken as degassed densities.

For in situ inventory and average composition calculations, the liquid density was set at $1440 \mathrm{~kg} / \mathrm{m}^{3}$, and the nonconvective layer was given a degassed bulk density of $1580 \mathrm{~kg} / \mathrm{m}^{3}$. The gassy density of the crust was set equal to that of the liquid below it. Layer depths of $41 \mathrm{~cm}$ (16 in.), $526 \mathrm{~cm}$ (207 in.), and $410 \mathrm{~cm}$ (161 in.) were used in hydrostatic pressure calculations for the crust, convective, and nonconvective layers, respectively.

\subsubsection{Sampling and Extraction Information}

The samples that underwent RGS analysis are listed in Table 4.4.1 (sample 10A-19 was lost because of a valving problem during the extraction procedure). Table 4.4.1 also shows the lag 
times (delay between sample acquisition and processing) for these samples. This information was provided to allow data users to correlate the lag time between sampling and extrusion with the concentrations of the various constituents to test for decomposition or other chemical reactions. The maximum hold time allowed by the sampling plan is 24 days, based on measured sampler leakage rates during acceptance testing. The hold time for sample 10A-21 exceeded this limit, but the sample was accepted based on calculations indicating that the estimated leakage was still negligible. Field data, including dose rates and downforce limits, are supplied in Appendix A.

As was discussed in Section 3.5, the HHF used during sampling often enters the sampler in place of some of the waste. The HHF is a solution of $\mathrm{LiBr}$ in which the bromide ion, which is not present in the waste, is used as the HHF tracer. Table 4.4.2 shows the volume percentage contamination of the RGS samples by the HHF. The measured HHF contamination percentages were used to calculate reduced effective sampler volumes for the RGS samples for which there were measurements. Three of the measured samples contained bromide below the MDL. The bromide concentration was set equal to the MDL for sample 10A-3; the other two (10A-17 and $10 \mathrm{~A}-21$ ) had MDLs corresponding to $18 \mathrm{vol} \%$ contamination, too high a value to provide a reasonable estimate of the true HHF contamination. For these samples, the contamination was set at half the MDL; the uncertainty in the contamination fraction was also set to half the MDL so that it would be included in the uncertainty in the RGS results. (It is conservative to use the MDL for values below it; the conservatism is probably less than $6 \%$ even for samples $10 \mathrm{~A}-17$ and $10 \mathrm{~A}-21$.)

Two different extraction procedures were used on the AN-104 samples, but the first several steps were the same in both (see RGS extraction system in Figure 3.1). After evacuating the whole system and observing the pressure to test for leaks, the operator extruded the RGS sample into the extractor vessel, and the sample was briefly stirred. At this point, the vapor space with which the sample was in communication consisted of the volumes of the extractor vessel and its lines. Next, the extractor was opened to the second vessel, to which a PQ canister was connected. Once a grab sample was collected in the PQ canister, it was closed. The extractor, lines, and second volume were then connected to the pump volume and collector side; the latter included the collector line volume and one open collection canister.

From this point, a different procedure were used for sample 10A-21 than for the previous AN-104 samples. This new procedure involved one unpumped canister followed by two pumped canisters. The intention was to use the unpumped canister to capture the initial gas composition, which was expected to be the closest to the in situ composition.

Table 4.4.1. Lag Times for Processing RGS Samples from Tank 241-AN-104

\begin{tabular}{||c|c|c|c||}
\hline Sample & Acquisition Date & Processing Date & Lag (days) \\
\hline \hline 10A-3 & Sept. 9, 1996 & Sept. 18, 1996 & 9 \\
\hline 10A-13 & Sept. 11, 1996 & Sept. 19, 1996 & 8 \\
\hline 10A-15 & Sept. 11, 1996 & Sept. 20, 1996 & 9 \\
\hline 10A-17 & Sept. 11, 1996 & Sept. 23, 1996 & 12 \\
\hline 12A-18 & Aug. 14, 1996 & Aug. 22, 1996 & 8 \\
\hline 10A-21 & Sept. 12, 1996 & Oct. 7, 1996 & 25 \\
\hline
\end{tabular}


Table 4.4.2. Hydrostatic Head Fluid Contamination in AN-104 RGS Samples

\begin{tabular}{|l|c|}
\hline Sample & $\begin{array}{c}\text { HHF Contamination } \\
\text { (vol \%) }\end{array}$ \\
\hline \hline $10 \mathrm{~A}-3$ (RGS) & $<2.4$ \\
\hline $10 \mathrm{~A}-13$ (RGS) & 2.9 \\
\hline $10 \mathrm{~A}-15$ (RGS) & 2.4 \\
\hline $10 \mathrm{~A}-17$ (RGS) & $<8.9$ \\
\hline $12 \mathrm{~A}-18$ (RGS) & 2.4 \\
\hline $10 \mathrm{~A}-21$ (RGS) & $<9.2$ \\
\hline
\end{tabular}

Sample AN-104-10A-21: The first canister, containing a sample of uncompressed (unpumped) gas, was closed off. The second collection canister was opened. Several strokes were pumped from the extractor to the collector, and the second collection canister was closed off. Then the collector side, second volume, and extractor lines were evacuated. Next, the extractor and samples were heated to about $50^{\circ} \mathrm{C}$ to drive off bound gas, cooled back to hot cell temperature, and the sample briefly stirred. A PQ canister connected to the extractor vessel took a second sample and was closed off. Several strokes were pumped from the extractor to the collector, and the third collection canister was closed off. Finally, the BSVD procedure was performed: argon was injected into the closed-off second volume, and then the second volume was opened to the extractor and lines. Pressure measurements and known tare system volumes allowed calculation of the volume of the sample solids and liquid.

Other AN-104 samples: Several strokes of gas and vapor (unbound gas) were pumped from the extractor to the collector, after which the first collection canister was closed off. The collector side, second volume, and extractor lines were evacuated after the first collection canister was closed off. Next, the extractor and samples were heated to about $50^{\circ} \mathrm{C}$ to drive off the bound gas, cooled back to hot cell temperature, and the sample briefly stirred. A PQ canister connected to the extractor vessel took a second sample and was closed off. Several strokes were pumped from the extractor to the collector and the collection canister was closed off. At this point the collector side, second volume, and extractor lines were not evacuated. Several strokes of gas and vapor were pumped from the extractor to the collector (to which one open collection canister, the third one, was connected). The BSVD procedure was performed last.

Procedural details such as the number of strokes per canister and system volumes can be found in the sample-by-sample worksheets in the AN-104 RGS calculation spreadsheet on the CD supplied with this report.

\subsubsection{Retained Gas Composition}

Table 4.4.3 presents the calculated concentrations of the insoluble gases in each RGS sample taken from AN-104 without corrections for air and argon entrainment, or air inleakage. The method used to make the corrections depended on the gas. All of the oxygen and argon were subtracted no matter which step had produced them. A number of moles equal to $(3.73) \cdot\left(\mathrm{O}_{2}\right)$ were subtracted from the nitrogen because the oxygen was expected to have come from air entrainment during sampling or inleakage during extraction. The corrected concentrations are given in Table 4.4.4. 
Table 4.4.3. Concentrations of Insoluble Constituents ( $\mu \mathrm{mol} / \mathrm{L}$ of waste) in Tank AN-104 Without Correction for Gas Contamination

\begin{tabular}{||c|c|c|c|c|c|c|c|c|c|c|c||}
\hline Sample & $\mathrm{N}_{2}$ & $\mathrm{H}_{2}$ & $\mathrm{~N}_{2} \mathrm{O}$ & $\mathrm{O}_{2}$ & $\mathrm{CH}_{4}$ & $\mathrm{He}$ & $\mathrm{Ar}$ & $\begin{array}{c}\text { Other } \\
\mathrm{NO}\end{array}$ & $\mathrm{C}_{2} \mathrm{H}_{\mathrm{x}}$ & $\mathrm{C}_{3} \mathrm{H}_{\mathrm{x}}$ & Other Hyd. \\
\hline \hline $10 \mathrm{~A}-3$ & $690 \pm 130$ & $96 \pm 18$ & $93 \pm 21$ & $130 \pm 23$ & $7.2 \pm 3.4$ & (a) & $8.5 \pm 1.6$ & $2.1 \pm 0.9$ & $1.8 \pm 1.0$ & $1.0 \pm 0.5$ & $4.9 \pm 2.3$ \\
\hline $10 \mathrm{~A}-13$ & $950 \pm 100$ & $630 \pm 71$ & $260 \pm 54$ & $91 \pm 10$ & $19 \pm 6.7$ & (a) & $10 \pm 1.3$ & $3.3 \pm 1.6$ & $8.5 \pm 3.5$ & $1.8 \pm 0.8$ & $15 \pm 6.8$ \\
\hline $10 \mathrm{~A}-15$ & $1400 \pm 140$ & $1700 \pm 170$ & $500 \pm 69$ & $65 \pm 6.7$ & $38 \pm 9.3$ & (a) & $10 \pm 1.4$ & $0.5 \pm 0.3$ & $17 \pm 6.3$ & $2.6 \pm 0.9$ & $26 \pm 12$ \\
\hline $10 \mathrm{~A}-17$ & $3700 \pm 480$ & $1300 \pm 160$ & $690 \pm 95$ & $370 \pm 55$ & $66 \pm 11$ & (a) & $25 \pm 3.9$ & $0.4 \pm 0.3$ & $28 \pm 6.6$ & $4.5 \pm 1.0$ & $35 \pm 12$ \\
\hline $12 \mathrm{~A}-18$ & $2400 \pm 110$ & $2500 \pm 130$ & $900 \pm 69$ & $91 \pm 4.9$ & $62 \pm 9.1$ & (a) & $25 \pm 1.8$ & $0.6 \pm 0.4$ & $29 \pm 9.9$ & $4.7 \pm 1.0$ & $43 \pm 18$ \\
\hline $10 \mathrm{~A}-21$ & $3400 \pm 390$ & $7200 \pm 820$ & $4700 \pm 560$ & $56 \pm 8.8$ & $91 \pm 13$ & (a) & $170 \pm 20$ & $5.2 \pm 2.2$ & $13 \pm 4.0$ & $10 \pm 3.3$ & $23 \pm 7.0$ \\
\hline (a) Below detection limits, which are 0.01 mol\% or less.
\end{tabular}

Table 4.4.4. Concentrations of Insoluble Constituents ( $\mu \mathrm{mol} / \mathrm{L}$ of waste) in Tank AN-104 with Correction for Gas Contamination

\begin{tabular}{||c|c|c|c|c|c|c|c|c|c|c|c||}
\hline Sample & $\mathrm{N}_{2}$ & $\mathrm{H}_{2}$ & $\mathrm{~N}_{2} \mathrm{O}$ & $\mathrm{O}_{2}$ & $\mathrm{CH}_{4}$ & $\mathrm{He}$ & $\mathrm{Ar}$ & $\begin{array}{c}\text { Other } \\
\mathrm{NO}_{x}\end{array}$ & $\mathrm{C}_{2} \mathrm{H}_{x}$ & $\mathrm{C}_{3} \mathrm{H}_{x}$ & Other Hyd. \\
\hline \hline $10 \mathrm{~A}-3$ & $210 \pm 150$ & $96 \pm 18$ & $93 \pm 21$ & $0 \pm 23$ & $7.2 \pm 3.4$ & (a) & $0 \pm 1.6$ & $2.1 \pm 0.9$ & $1.8 \pm 1.0$ & $1.0 \pm 0.5$ & $4.9 \pm 2.3$ \\
\hline $10 \mathrm{~A}-13$ & $610 \pm 100$ & $630 \pm 71$ & $260 \pm 54$ & $0 \pm 10$ & $19 \pm 6.7$ & (a) & $0 \pm 1.3$ & $3.3 \pm 1.6$ & $8.5 \pm 3.5$ & $1.8 \pm 0.8$ & $15 \pm 6.8$ \\
\hline $10 \mathrm{~A}-15$ & $1100 \pm 140$ & $1700 \pm 170$ & $500 \pm 69$ & $0 \pm 6.7$ & $38 \pm 9.3$ & (a) & $0 \pm 1.4$ & $0.5 \pm 0.3$ & $17 \pm 6.3$ & $2.6 \pm 0.9$ & $26 \pm 12$ \\
\hline $10 \mathrm{~A}-17$ & $2300 \pm 420$ & $1300 \pm 160$ & $690 \pm 95$ & $0 \pm 55$ & $66 \pm 11$ & (a) & $0 \pm 3.9$ & $0.4 \pm 0.3$ & $28 \pm 6.6$ & $4.5 \pm 1.0$ & $35 \pm 12$ \\
\hline $12 \mathrm{~A}-18$ & $2000 \pm 110$ & $2500 \pm 130$ & $900 \pm 69$ & $0 \pm 4.9$ & $62 \pm 9.1$ & (a) & $0 \pm 1.8$ & $0.6 \pm 0.4$ & $29 \pm 9.9$ & $4.7 \pm 1.0$ & $43 \pm 18$ \\
\hline $10 \mathrm{~A}-21$ & $3100 \pm 370$ & $7200 \pm 820$ & $4700 \pm 560$ & $0 \pm 8.8$ & $91 \pm 13$ & (a) & $0 \pm .20$ & $5.2 \pm 2.2$ & $13 \pm 4.0$ & $10 \pm 3.3$ & $23 \pm 7.0$ \\
\hline (a) Below detection limits, which are 0.01 mol\% or less.
\end{tabular}

Sample 10A-21 contained more argon than the other samples. The field data (Appendix A) show that a drillstring purge was performed about four hours before sample 10A-19 was acquired, and again about nine hours before sample 10A-21 was acquired. It is interesting that 10A-21 showed the effects of the purge and 10A-19 did not, even though sample 10A-19 was acquired sooner after the purge. Contamination observations are found in the "Summary" worksheet of the AN-104 RGS calculations spreadsheet on the CD.

Table 4.4.5 presents the ammonia measurements. The RGS procedure did not permit the determination of the residual or total ammonia, but the post-extrusion and the first PQ canister partial pressures of $\mathrm{NH}_{3}$ over the sample (see Section 3.4.4) were measured at laboratory temperature. (Ammonia data can be seen in more detail in Section C.4 of Appendix C.)

No conclusive total ammonia concentration can be calculated, but it is likely that the ammonia concentration in Tank AN-104 waste was the same as in Tank AW-101 waste, with a lower bound of $27,000 \mu \mathrm{mol} \mathrm{NH} / \mathrm{L}$ waste. This estimate is based on a comparison of the ammonia partial pressure measurements (see Sections 4.1.2 and C.1 for the AW-101 measurements). 
Table 4.4.5. Ammonia Data from Tank AN-104 Samples ,

\begin{tabular}{|c|c|c|}
\hline Sample & $\begin{array}{l}\text { Measured } \mathrm{NH}_{3} \\
\text { partial pressure at } \\
\text { about } 24^{\circ} \mathrm{C} \\
\text { (atm) }\end{array}$ & $\begin{array}{c}\text { Best-Estimate } \mathrm{NH}_{3} \\
\text { Concentrations }\end{array}$ \\
\hline $10 \mathrm{~A}-3$ & $0.0056 \pm 0.0028$ & \multirow{6}{*}{$\begin{array}{l}\text { The data do not support } \\
\text { conclusive } \mathrm{NH}_{3} \text { concentrations. } \\
\text { We estimate a lower bound of } \\
0.034 \mathrm{M}(580 \mu \mathrm{g} / \mathrm{mL}) \mathrm{NH}_{3} \text { in } \\
\text { the liquid of the nonconvective } \\
\text { layer, or } 0.031 \mathrm{wt} \% \mathrm{NH}_{3} \text { in the } \\
\text { bulk waste. }\end{array}$} \\
\hline $10 \mathrm{~A}-13$ & $0.0043 \pm 0.0022$ & \\
\hline $10 \mathrm{~A}-15$ & $0.021 \pm 0.010$ & \\
\hline $10 \mathrm{~A}-17$ & $0.0073 \pm 0.0036^{(a)}$ & \\
\hline $12 \mathrm{~A}-18$ & $0.0057 \pm 0.0029$ & \\
\hline $10 \mathrm{~A}-21$ & $0.012 \pm 0.006$ (a) & \\
\hline
\end{tabular}

Table 4.4.6 contains the composition of the gas/vapor phase in each sample and the average compositions in the gas retained in the convective and nonconvective layers. The water vapor is not included in these compositions. The ammonia fractions are derived from the partial pressures indicated in Table 4.4.5 extrapolated to vapor pressures at in situ conditions. The sample compositions in the table have been calculated using the in situ solubility method described in Section 3.6.2. As discussed in Section 3.6.1, compositions were calculated for both the lower- and upper-bound gas solubilities. Both ends of the composition range are given in Table 4.4.6 with the measurement uncertainty on each. The average composition of the gas in the nonconvective layer is the result of integrating RGS species concentrations over the layer and multiplying those concentrations by the waste volume. The integration method is described in Section 3.7.1. The single sample in the convective layer (10A-3) was used to calculate its composition.

Within the uncertainty, there was no more composition difference between samples from the two risers than among the samples from riser 10A. Sample 10A-17 was particularly low in hydrogen; sample 10A-21 was high in nitrous oxide, low in nitrogen, and low in methane and other hydrocarbons. As in samples from other RGS-sampled tanks, nitrogen was high in the AN-104 supernatant and low in hydrogen, though the broad uncertainty makes this interpretation somewhat inconclusive.

The concentrations and pressures in Tables 4.4.3 and 4.4.4 can also be found in the sample-by-sample worksheets in the AN-104 RGS calculation spreadsheet on the CD. The compositions in Table 4.4.6 are in the "Inventory" worksheet. 
Table 4.4.6. Sample and Overall Average Compositions of Retained Gas in Tank AN-104 with Correction for Gas Contamination(a)

\begin{tabular}{|c|c|c|c|c|c|c|}
\hline Sample & $\mathrm{N}_{2}(\mathrm{~mol} \%)$ & $\mathrm{H}_{2}(\mathrm{~mol} \%)$ & $\mathrm{N}_{2} \mathrm{O}(\mathrm{mol} \%)$ & $\mathrm{NH}_{3}(\mathrm{~mol} \%)^{(b)}$ & $\mathrm{CH}_{4}(\mathrm{~mol} \%)$ & Other (mol\%) \\
\hline $10 \mathrm{~A}-3$ & $55 \pm 48--63 \pm 57$ & $24 \pm 13--26 \pm 15$ & $15 \pm 8.4--5.2 \pm 3.1$ & $1.0 \pm 0.7--0.4 \pm 0.3$ & $1.8 \pm 1.3--2.0 \pm 1.4$ & $2.6 \pm 1.8--3.3 \pm 2.4$ \\
\hline $10 \mathrm{~A}-13$ & $40 \pm 9.6--44 \pm 10$ & $41 \pm 8.2--44 \pm 8.6$ & $15 \pm 3.8--8.1 \pm 2.1$ & $0.6 \pm 0.2--0.2 \pm 0.1$ & $1.3 \pm 0.5--1.4 \pm 0.5$ & $1.9 \pm 0.9--2.1 \pm 1.0$ \\
\hline $10 \mathrm{~A}-15$ & $32 \pm 5.7--34 \pm 5.9$ & $49 \pm 8.1--52 \pm 8.3$ & $13 \pm 2.5--10 \pm 1.8$ & $3.0 \pm 1.5-1.2 \pm 0.6$ & $1.1 \pm 0.3--1.2 \pm 0.3$ & $1.3 \pm 0.5--1.4 \pm 0.6$ \\
\hline $10 \mathrm{~A}-17$ & $52 \pm 13--54 \pm 13$ & $29 \pm 6.0--30 \pm 6.2$ & $15 \pm 3.1--12 \pm 2.5$ & $1.2 \pm 0.6--0.5 \pm 0.2$ & $1.5 \pm 0.3--1.6 \pm 0.4$ & $1.5 \pm 0.5-1.6 \pm 0.5$ \\
\hline $12 \mathrm{~A}-18$ & $36 \pm 3.1--38 \pm 3.1$ & $45 \pm 3.6--47 \pm 3.7$ & $15 \pm 1.5-12 \pm 1.2$ & $0.8 \pm 0.3--0.3 \pm 0.1$ & $1.1 \pm 0.2$ & $1.4 \pm 0.5$ \\
\hline $10 \mathrm{~A}-21$ & $20 \pm 3.5--21 \pm 3.6$ & $47 \pm 7.8--49 \pm 8.0$ & $30 \pm 5.1--28 \pm 4.7$ & $1.3 \pm 0.7--0.5 \pm 0.3$ & $0.6 \pm 0.1$ & $0.3 \pm 0.1--0.4 \pm 0.3$ \\
\hline $\begin{array}{c}\text { Convective } \\
\text { layer(c) }\end{array}$ & $55 \pm 48--63 \pm 57$ & $24 \pm 13--26 \pm 15$ & $15 \pm 8.4--5.2 \pm 3.1$ & $1.0 \pm 0.7--0.4 \pm 0.3$ & $1.8 \pm 1.3--2.0 \pm 1.4$ & $2.6 \pm 1.8--3.3 \pm 2.4$ \\
\hline $\begin{array}{l}\text { Avg in non- } \\
\text { convective } \\
\text { layer (c) }\end{array}$ & $29 \pm 4.8--31 \pm 5.0$ & $45 \pm 6.9--47 \pm 7.0$ & $23 \pm 3.8--20 \pm 3.3$ & $1.4 \pm 0.7--0.5 \pm 0.3$ & $0.9 \pm 0.2$ & $0.8 \pm 0.3--0.9 \pm 0.3$ \\
\hline \multicolumn{7}{|c|}{$\begin{array}{l}\text { (a) Uncertainties on compositions represent instrument error; compositions calculated for lower- and upper-bound solubilities. } \\
\text { Both ends of composition range given; lower-bound solubility basis first with instrument uncertainty on each. Compositions } \\
\text { may not sum to } 100 \text { mol\% because of roundoff error. Mole fractions are on a dry basis and do not account for water vapor. } \\
\text { (b) Ammonia mole fraction is derived from partial pressures in Table } 4.4 .5 \text { extrapolated to vapor pressure at in situ conditions. } \\
\text { (c) There are too few samples to define the spatial variability of the average gas concentration, }\end{array}$} \\
\hline
\end{tabular}

\subsubsection{Gas Inventory}

The method by which the in situ gas volume fractions (wet basis) were calculated is given in Section 3.6.2; as for the gas composition, the volume fractions are given as a range from the low gas-solubility value to the high gas-solubility value. The results are presented in Table 4.4.7. Table 4.4.7 also contains the average gas volume fraction and the average pressure experienced by the gas in each layer. The averages are in situ volume averages calculated by Simpson's Rule integration, as described in Section 3.7.1. The corrected gas volume fractions in Table 4.4.7 are consistent with the corrected gas concentrations and compositions in Tables 4.4.4 through 4.4.6. The information in Table 4.4.7 is taken from the "Summary" and "In situ" worksheets of the AN-104 RGS calculation spreadsheet on the CD.

The "sampler gas volume fraction" is corrected only for inleakage during the extraction process and air gases added in the isotopic solution. It is an attempt to reconstruct the total amount of gas (sample and entrainment) that was present in the sampler during $x$-ray. It is used only for comparison with the $\mathrm{x}$-ray observations of "visible gas fraction" that are discussed in Section 4.4.4.

Table 4.4.7 also contains the water vapor pressures that were used for in situ calculations. The water vapor pressures were found by using salt concentrations from $\mathrm{Hu}$ et al. (1997) and the temperatures in the table as inputs to Equation 6.2 of Mahoney and Trent (1995), a correlation for water vapor pressure over concentrated homogeneous and non-homogeneous waste simulants. The gas solubilities used the same parameters as inputs to the Schumpe solubility model (see Section 3.6.1). The water vapor pressures and gas solubilities used in calculations can be found in the "In-situ" worksheet of the AN-104 RGS calculation spreadsheet on the CD. No ammonia inventory was calculated for the tank because there were no total ammonia concentration data. 
Table 4.4.7. In Situ Gas Volume Fractions and Conditions in Tank AN-104(a)

\begin{tabular}{|c|c|c|c|c|c|c|}
\hline Sample & $\begin{array}{l}\text { Sample } \\
\text { central } \\
\text { height } \\
(\mathrm{cm}) \\
\end{array}$ & $\begin{array}{l}\text { Hydro- } \\
\text { static } \\
\text { pressure } \\
\text { (atm) } \\
\end{array}$ & $\begin{array}{c}\text { Calculated } \\
\text { water vapor } \\
\text { pressure } \\
(\mathrm{atm}) \\
\end{array}$ & $\begin{array}{l}\text { Temp } \\
\left({ }^{\circ} \mathrm{C}\right)\end{array}$ & $\begin{array}{c}\text { Corrected gas } \\
\text { volume fraction } \\
\text { (in-tank conditions) }\end{array}$ & $\begin{array}{c}\text { Sampler Gas } \\
\text { volume fraction } \\
\text { (in situ conditions, } \\
\text { low solubility) } \\
\end{array}$ \\
\hline $10 \mathrm{~A}-3$ & 893 & 1.12 & 0.036 & 39.0 & $\begin{array}{c}0.009 \pm 0.003 \text { to } \\
0.007 \pm 0.003\end{array}$ & 0.022 \\
\hline $10 \mathrm{~A}-13$ & 410 & 1.78 & 0.029 & 40.5 & $\begin{array}{c}0.022 \pm 0.004 \text { to } \\
0.019 \pm 0.004\end{array}$ & 0.028 \\
\hline $10 \mathrm{~A}-15$ & 314 & 1.92 & 0.037 & 45.6 & $\begin{array}{c}0.048 \pm 0.008 \text { to } \\
0.044 \pm 0.008\end{array}$ & 0.052 \\
\hline $10 \mathrm{~A}-17$ & 217 & 2.06 & 0.043 & 48.4 & $\begin{array}{c}0.058 \pm 0.008 \text { to } \\
0.054 \pm 0.008\end{array}$ & 0.080 \\
\hline $12 \mathrm{~A}-18$ & 169 & 2.12 & 0.042 & 48.0 & $\begin{array}{c}0.071 \pm 0.007 \text { to } \\
0.068 \pm 0.007\end{array}$ & 0.077 \\
\hline $10 \mathrm{~A}-21$ & 24.1 & 2.33 & 0.031 & 42.0 & $\begin{array}{c}0.17 \pm 0.02 \text { to } \\
0.16 \pm 0.02\end{array}$ & 0.18 \\
\hline $\begin{array}{c}\text { Convective } \\
\text { layer }\end{array}$ & 673 & 1.42 & & & $\begin{array}{c}0.005 \pm 0.002 \text { to } \\
0.004 \pm 0.002\end{array}$ & \\
\hline $\begin{array}{l}\text { Avg in non- } \\
\text { convective } \\
\text { layer }\end{array}$ & 119 & 2.20 & & & $\begin{array}{c}0.081 \pm 0.040 \text { to } \\
0.076 \pm 0.038\end{array}$ & \\
\hline
\end{tabular}

Table 4.4.8 gives various estimates of the STP volume of gas in Tank AN-104, including estimates calculated from RGS data alone. The VFI\&RGS and BPE inventory values were taken from Schienbein et al. (1999).

The RGS gas inventory in the nonconvective layer was calculated by integrating RGS total gas concentrations over the layer (five data points) and multiplying the average gas concentration by the layer volume. The integration method is described in Section 3.7.1. The single sample in the convective layer (10A-3) was used to calculate its inventory. The RGS volumes in Table 4.4.8 include corrections to remove the contamination gas: entrained air and argon and air leaks during and after extraction. The uncertainties on the gas inventories are based on the spatial variability considerations discussed in Section 3.7.2. The information in Table 4.4.8 is taken from the "Inventory" worksheet of the AN-104 RGS calculation spreadsheet on the CD.

The RGS method gave the highest total gas inventory $\left(167 \pm 68 \mathrm{~m}^{3}\right.$ in situ, counting the crust gas) and the BPE method the lowest, with the inventory calculated from VFI and RGS data (138 $\pm 14 \mathrm{~m}^{3}$ in situ) in between. As Figure 4.4 .3 suggests, the RGS inventory may have been greater than the VFI\&RGS inventory partly because RGS captured relatively high gas contents at an elevation too low for VFI to reach (sample 10A-21). The match between the RGS and VFI gas fraction measurements suggests that (after three months) there was little remaining effect of the intervening GRE. 
Table 4.4.8. AN-104 Gas Inventory Estimates

\begin{tabular}{|c|c|c|c|c|c|}
\hline \multirow{2}{*}{ Quantity } & \multicolumn{2}{|c|}{ RGS Method } & \multirow{2}{*}{$\begin{array}{c}\text { BPE Method(a) } \\
\text { Tank Total }\end{array}$} & \multicolumn{2}{|c|}{ VFI\&RGS Method $(b, c)$} \\
\hline & $\begin{array}{c}\text { Nonconvective } \\
\text { Layer }\end{array}$ & $\begin{array}{l}\text { Convective } \\
\text { Layer }\end{array}$ & & $\begin{array}{l}\text { Nonconvective } \\
\text { Layer }\end{array}$ & $\begin{array}{c}\text { Convective } \\
\text { Layer }\end{array}$ \\
\hline $\begin{array}{l}\text { Avg gas fraction } \\
\text { (low gas } \\
\text { solubility) }\end{array}$ & $0.081 \pm 0.040$ & $0.005 \pm 0.002$ & $\begin{array}{c}\text { (nonconv. avg) } \\
0.069 \pm 0.023\end{array}$ & $0.066 \pm 0.008$ & $\begin{array}{c}0.004 \pm \\
0.001\end{array}$ \\
\hline $\begin{array}{l}\text { Gas volume }\left(\mathrm{m}^{3}\right) \\
\text { in situ (wet) } \\
\text { STP (wet) }\end{array}$ & $\begin{array}{c}136 \pm 68 \\
257 \pm 128\end{array}$ & $\begin{array}{l}12 \pm 4 \\
15 \pm 5\end{array}$ & $\begin{array}{l}116 \pm 38 \\
218 \pm 72\end{array}$ & $\begin{array}{l}110 \pm 12 \\
186 \pm 13\end{array}$ & $\begin{array}{l}9 \pm 2 \\
12 \pm 2\end{array}$ \\
\hline $\begin{array}{l}\text { (a) Note that the } \mathrm{E} \\
\text { Schienbein et al. } \\
\text { (b) Based on VFI } \\
\text { (c) The retained g }\end{array}$ & $\begin{array}{l}\text { E inventory inc } \\
\text { 99) stated the cr } \\
\text { nd RGS data; se } \\
\text { inventory calcu }\end{array}$ & $\begin{array}{l}\text { udes the crust, } \\
\text { ust gas invent } \\
\text { e Table } 2.7 .3 \\
\text { lated from RG } \\
\text { ers. }\end{array}$ & $\begin{array}{l}\text { which there w } \\
\text { as } 19 \pm 7 \mathrm{~m}^{3} \mathrm{i} \\
\text { schienbein et al } \\
\text { nd VFI data is }\end{array}$ & $\begin{array}{l}\text { no RGS or VF } \\
\text { tu, or } 19 \pm 7 \text { ST } \\
999 \text { ). } \\
\text { isidered the bes }\end{array}$ & $\begin{array}{l}\text { ata. } \\
\mathrm{m}^{3} . \\
\text { timate, it }\end{array}$ \\
\hline
\end{tabular}

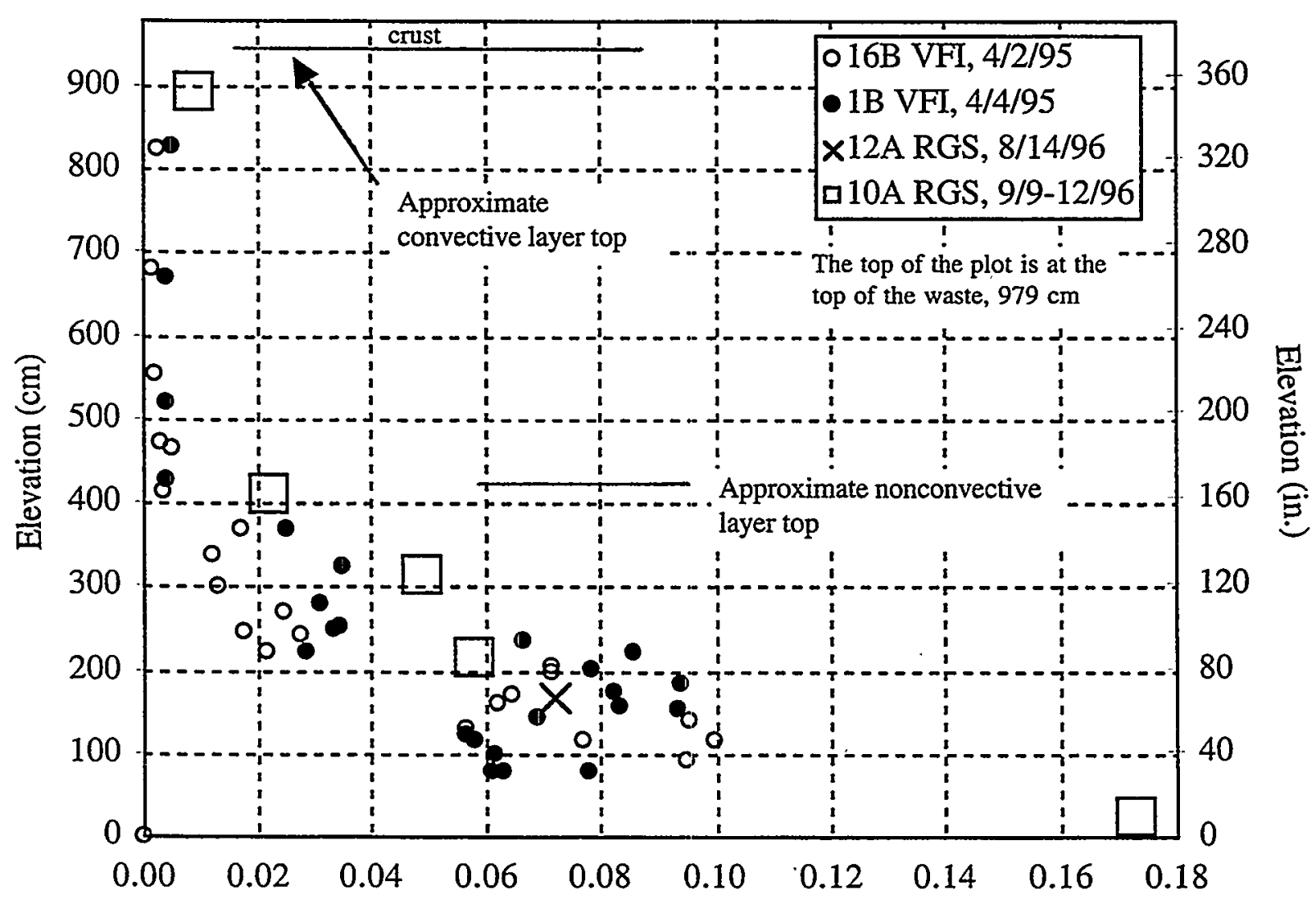

Gas Volume Fraction

Figure 4.4.3. Gas Volume Fractions Measured by RGS and VFI 
The uncertainty in the BPE inventory overlaps the VFI\&RGS but not the RGS estimate. The VFI\&RGS gas inventory is preferable to the other inventories, being based on direct nonconvective layer gas measurements from three risers (plus one convective layer RGS sample from a fourth riser).

Table 4.4 .9 contains the calculated layer inventories of each of the major gases retained in the waste. The gas-phase inventories are based on the layer-average compositions from RGS data (Table 4.4.6) and the best-estimate gas inventories for each layer (VFI\&RGS, Table 4.4.8). The dissolved inventories are based on RGS data alone.

Figure 4.4.4 shows the sample temperatures, corrected gas volume fractions (Table 4.4.7), and corrected compositions of the low-solubility constituents in the samples from Tank AN-104. The temperatures were taken with the MIT in riser 15A. The compositions represent the mole fraction of the species in the insoluble gas; water and ammonia are not included, so the mole fractions are not the same as those in Table 4.4.4. The gas volume fractions and mole fractions in the figure are the values for lower-bound gas solubilities. Figure 4.4 .4 also lists the observations from core extrusions of non-RGS samples and from X-rays of RGS samples as a way of tying those observations together with RGS data. X-ray data are described in detail in Section 4.4.4.

Table 4.4.9. Speciated AN-104 Gas Inventory(a)

\begin{tabular}{||l|c|c||c|c||}
\hline \multirow{2}{*}{ Species } & \multicolumn{2}{|c|}{$\begin{array}{c}\text { Gas-Phase Inventory } \\
\text { (m3 at STP) }\end{array}$} & \multicolumn{2}{c|}{$\begin{array}{c}\text { Dissolved Inventory } \\
\text { (m3 at STP) }\end{array}$} \\
\cline { 2 - 5 } & $\begin{array}{c}\text { Nonconvective } \\
\text { Layer }\end{array}$ & $\begin{array}{c}\text { Convective } \\
\text { Layer }\end{array}$ & $\begin{array}{c}\text { Nonconvective } \\
\text { Layer }\end{array}$ & $\begin{array}{c}\text { Convective } \\
\text { Layer }\end{array}$ \\
\hline $\mathrm{N}_{2}$ & 54 & $6.6-5.9$ & $0.076-0.54$ & $0.11-0.76$ \\
\hline $\mathrm{H}_{2}$ & $83-82$ & $2.9-2.4$ & $0.33-1.5$ & $0.16-0.69$ \\
\hline $\mathrm{N}_{2} \mathrm{O}$ & $43-36$ & $1.8-0.49$ & $2.0-8.6$ & $1.7-3.1$ \\
\hline $\mathrm{NH}_{3}$ & $2.6-0.95$ & $0.13-0.039$ & 2100 & 1800 \\
\hline $\mathrm{CH}_{4}$ & 1.6 & $0.22-0.18$ & $0.0041-0.030$ & $0.0068-0.045$ \\
\hline Other & 1.6 & 0.31 & 0 & 0 \\
\hline $\begin{array}{l}\text { (a) Gas-phase inventories based on layer-average compositions from RGS data (Table 4.4.6) and } \\
\text { best-estimate gas inventories for each layer (VFI\&RGS, Table 4.4.8). Dissolved inventories } \\
\text { based on RGS data alone. The uncertainty in each inventory is 50\%, based on spatial variability } \\
\text { considerations discussed in Section 3.7.2. Inventories are given for both lower-bound and upper- } \\
\text { bound solubilities; lower-bound solubility basis is first in the table. }\end{array}$ \\
\hline
\end{tabular}

\subsubsection{X-Ray Results}

Table 4.4.10 summarizes all the available radiography observations from Tank AN-104. The x-ray images themselves can be found on the CD that accompanies this report. The uncertainties of the visible gas volume fractions that are given in Table 4.4.10 are unknown but (based on the uncertainty in measuring the gas gap from $x$-ray images) are expected to be larger than the uncertainties in the gas volume fractions calculated from gas extraction. (The "visible" gas volume fraction refers to the gas that has separated from the solids and liquid such that its volume can be calculated based on the image. The uncorrected gas fractions from extraction are used for comparison to reflect the presence of entrained air in the sampler.) 
Table 4.4.10. Summary of Observations from X-Ray Images of Tank AN-104

\begin{tabular}{|c|c|}
\hline Segment & Comments/Observations \\
\hline $\begin{array}{l}10 A-2 \\
\text { (non-RGS, } \\
\text { 9/9/96) }\end{array}$ & $\begin{array}{l}\text { The waste in the image was featureless, with a slightly rough surface. Clean, fully retracted } \\
\text { piston. Gas gap } 20 \mathrm{~mm}(0.8 \mathrm{in} \text {.). The visible gas volume fraction is therefore } 0.04 \text {. }\end{array}$ \\
\hline $\begin{array}{l}\text { 10A-3 } \\
\text { (RGS, } \\
\text { 9/9/96) }\end{array}$ & $\begin{array}{l}\text { The waste in the image was featureless, with a smooth meniscus surface. Clean piston, fully } \\
\text { retracted. Gas gap } 3 \mathrm{~mm}(0.13 \mathrm{in} .) \text {. The visible gas volume fraction is therefore } 0.007 \text {. } \\
\text { (Compare with the uncorrected sampler gas volume fraction of } 0.022 \text { obtained by gas extraction, } \\
\text { Table 4.4.7.) }\end{array}$ \\
\hline $\begin{array}{l}10 \mathrm{~A}-13 \\
\text { (RGS, } \\
\text { 9/11/96) }\end{array}$ & $\begin{array}{l}\text { Sparse }<1-\mathrm{mm} \text { bubbles were seen near the bottom of the sampler, with scattered } 1-2 \mathrm{~mm} \\
\text { bubbles in the top half. Slightly rough surface. Clean, fully retracted piston; } 2-\mathrm{mm} \text { ( } 0.1-\mathrm{in} \text {.) } \\
\text { gas gap. The visible gas volume fraction (not counting bubbles) is therefore } 0.005 \text {. (Compare } \\
\text { with the uncorrected sampler gas volume fraction of } 0.028 \text { obtained by gas extraction, Table } \\
\text { 4.4.7.) }\end{array}$ \\
\hline $\begin{array}{l}\text { 10A-14 } \\
\text { (non-RGS, } \\
\text { 9/11/96) }\end{array}$ & $\begin{array}{l}\text { Scattered } 1-2-\mathrm{mm} \text { bubbles, one thin slanted bubble } 0.4 \text { in. long halfway up, and fewer bubbles } \\
\text { toward the top. Slightly rough surface. Clean, fully retracted piston. } 10-\mathrm{mm}(0.4-\mathrm{in} \text {.) gas gap. } \\
\text { The visible gas volume fraction (not counting bubbles) is } 0.02 \text {. }\end{array}$ \\
\hline $\begin{array}{l}\text { 10A-15 } \\
\text { (RGS, } \\
9 / 11 / 96)\end{array}$ & $\begin{array}{l}\text { Moderately dense } 1-2-\mathrm{mm} \text { bubbles, denser in bottom third of the sampler, a few } 3 \text {-mm bubbles. } \\
\text { Near the bottom of the sampler, an irregular void of } 8 \mathrm{~mm}(0.3-\mathrm{in} \text {.). Slightly rough surface, } \\
\text { clean, fully retracted piston. } 2 \text {-mm ( }(0.1-\text {-in.) gas gap. The visible gas volume fraction (not } \\
\text { counting bubbles) is } 0.005 \text {. (Compare with the uncorrected sampler gas volume fraction of } \\
0.052 \text { obtained by gas extraction, Table } 4.4 .7 \text {.) }\end{array}$ \\
\hline $\begin{array}{l}10 A-16(\text { non- } \\
\text { RGS, } 9 / 11 / 96)\end{array}$ & $\begin{array}{l}\text { Dense } 1-3-\mathrm{mm} \text { bubbles and a few } 4-5-\mathrm{mm} \text { bubbles. Smooth, slightly concave surface. A } \\
\text { waste stalactite } 12 \mathrm{~mm}(0.5 \mathrm{in} \text {.) across on a fully retracted piston. } 16-\mathrm{mm}(0.6-\mathrm{in} \text {.) gas gap. } \\
\text { The visible gas volume fraction (not counting bubbles) is therefore } 0.03 \text {. }\end{array}$ \\
\hline $\begin{array}{l}10 \mathrm{~A}-17 \\
\text { (RGS, } \\
9 / 11 / 96)\end{array}$ & $\begin{array}{l}\text { Dense } 1-2-\mathrm{mm} \text { bubbles in bottom half of sampler, one } 4-5-\mathrm{mm} \text { bubble near bottom; dense, } \\
1-3-\mathrm{mm} \text { bubbles in top half. Smooth surface; clean, fully retracted piston; } 4 \text {-mm ( } 0.15 \text {-in.) gas } \\
\text { gap. The visible gas volume fraction (not counting bubbles) is therefore } 0.008 \text {. (Compare with } \\
\text { the corrected sampler gas volume fraction of } 0.058 \text { obtained by gas extraction, Table } 4.4 .7 \text {. In } \\
\text { this case, the corrected gas volume fraction is more appropriate because of air inleakage during } \\
\text { extraction.) }\end{array}$ \\
\hline $\begin{array}{l}10 \mathrm{~A}-18 \\
\text { (non-RGS, } \\
9 / 11 / 96)\end{array}$ & $\begin{array}{l}\text { Dense, 1-3-mm bubbles sometimes grouped into streaks or blobs, fewer near top of sampler. } \\
\text { Smooth, slightly concave surface. An } 8 \text {-mm ( } 0.3 \text {-in.) waste stalactite on a fully retracted piston. } \\
\text { Gas gap about } 2.8 \mathrm{~cm}(1.1 \mathrm{in} \text {.). The visible gas volume fraction (not counting bubbles) is } \\
\text { therefore } 0.06 \text {. }\end{array}$ \\
\hline $\begin{array}{l}10 A-19 \\
\text { (RGS, } \\
9 / 11 / 96)\end{array}$ & $\begin{array}{l}\text { ense, } 1-2-\mathrm{mm} \text { bubbles and a few } 3-\mathrm{mm} \text { bubbles. Smooth surface; clean, fully retracted piston. } \\
\text { 3-mm (0.5-in.) gas gap. The visible gas volume fraction (not counting bubbles) is therefore } \\
03 \text {. }\end{array}$ \\
\hline $\begin{array}{l}10 \mathrm{~A}-20 \\
\text { (non-RGS, } \\
9 / 11 / 96)\end{array}$ & $\begin{array}{l}\text { Dense, } 1-3-\mathrm{mm} \text { bubbles and a few } 4-5-\mathrm{mm} \text { bubbles. Smooth surface; clean, fully retracted } \\
\text { piston. } 3.3-\mathrm{cm} \text { (1.3-in.) gas gap. The visible gas volume fraction (not counting bubbles) is } \\
\text { therefore } 0.07 \text {. }\end{array}$ \\
\hline $\begin{array}{l}10 \mathrm{~A}-21 \\
\text { (RGS, } \\
9 / 12 / 96)\end{array}$ & $\begin{array}{l}\text { Densely packed round or nearly round bubbles of } 2-\mathrm{mm} \text { diameter and less, a few } 3-\mathrm{mm} \text { bubbles. } \\
\text { Fewer bubbles in top half of sampler. Surface is flat, not liquid. Small ( } 0.2 \mathrm{in} \text {.) bit of waste on } \\
\text { fully retracted piston; } 2.4 \text { in. gas gap. The visible gas volume fraction (not counting bubbles) is } \\
0.13 \text {. (Compare with the uncorrected sampler gas volume fraction of } 0.18 \text { obtained by gas } \\
\text { extraction, Table 4.4.7.) }\end{array}$ \\
\hline
\end{tabular}



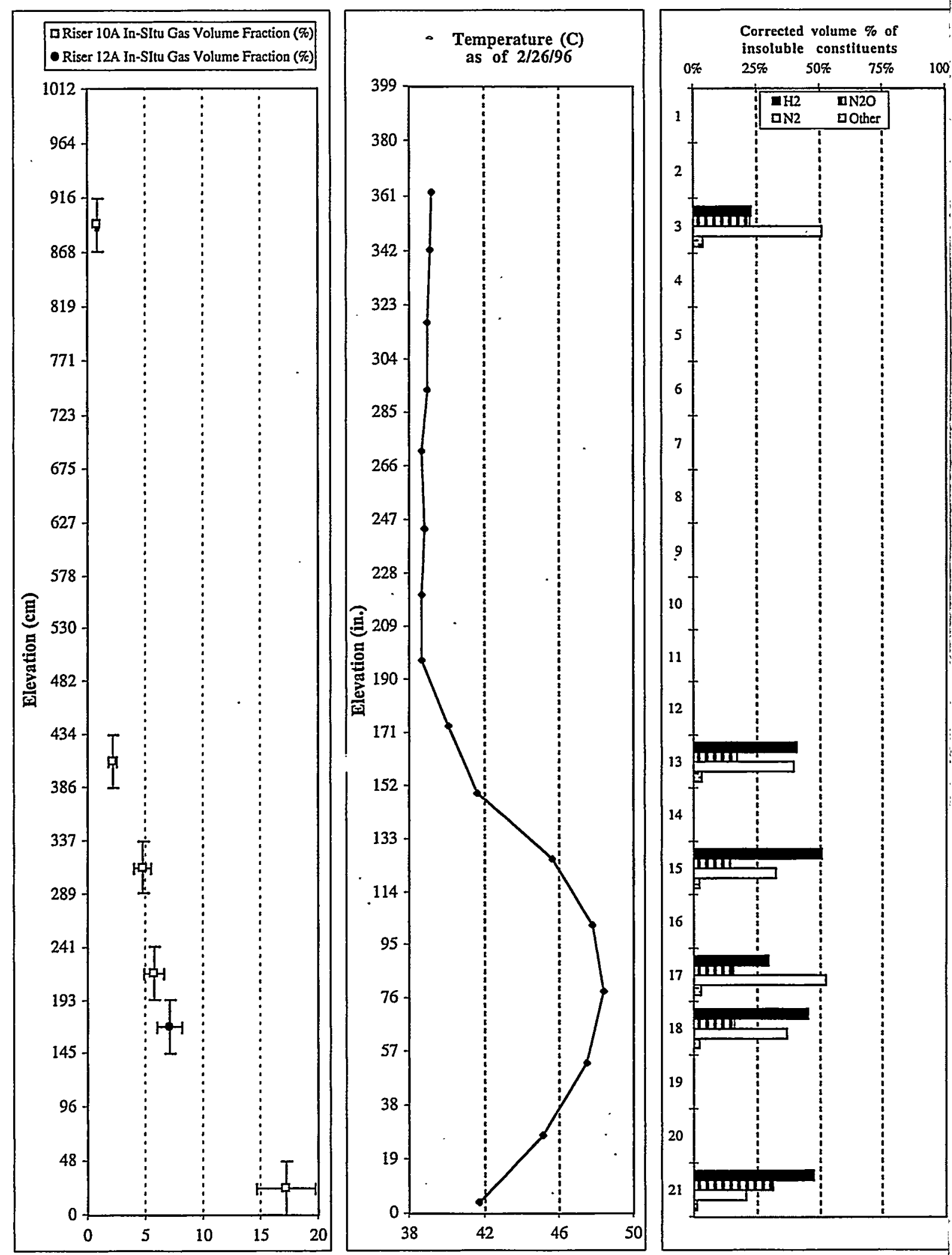

Figure 4.4.4. Gas Volume Fractions, Temperatures, 
AN-104 waste level is $979 \mathrm{~cm}$ (385 inches).

1 Riser 12A: 2 in. (35 g) gray to white salt slunry, $230 \mathrm{~mL}(283 \mathrm{~g}$ ) yellowish-green opaque liquid.

1 Riser 10A: $1 \mathrm{in.}$ (22 g) gray wet salt $<5 \mathrm{~mL}$ green opaque liquid.

2 Riser 12A: $250 \mathrm{~mL}(370 \mathrm{~g}$ ) yellow opaque liquid.

2 Riser 10A: 1 in. $(29 \mathrm{~g})$ gray to white salt slurry, $250 \mathrm{~mL}$ ( $362 \mathrm{~g})$ green opaque liquid. X-ray featureless, $0.8^{\prime \prime}$ gas gap.

3 Riser 12A: $255 \mathrm{~mL}$ (386 g) yellow opaque liquid.

3 Riser 10A X-RAY: Featureless, meniscus surface, gas gap 0.13 in. Clean piston, fully retracted. $2.5 \mathrm{R} / \mathrm{hr}$.

4 Riser 12A: $310 \mathrm{~mL}$ ( $410 \mathrm{~g}$ ) yellow opaque liquid.

4 Riser 10A: $260 \mathrm{~mL}$ (385 g) green opaque liquid.

5 Riser 12A: $315 \mathrm{~mL}$ (436 g) yellow opaque liquid.

5 Riser 10A: $260 \mathrm{~mL}$ ( $399 \mathrm{~g}$ ) yellow opaque liquid.

6 Riser 12A: no sample.

6 Riser 10A: $260 \mathrm{~mL}(374 \mathrm{~g}$ ) green-yellow opaque liquid.

7 Riser 12A: $310 \mathrm{~mL}$ (421 g) yellow opaque liquid.

7 Riser 10A: not available.

8 Riser 12A: $285 \mathrm{~mL}$ (418 g) yellow opaque liquid.

8 Riser 10A: $260 \mathrm{~mL}(391 \mathrm{~g})$ yellow clear liquid.

9 Riser 12A: $300 \mathrm{~mL}$ ( $413 \mathrm{~g}$ ) yellow opaque liquid.

9 Riser 10A: $260 \mathrm{~mL}$ ( $384 \mathrm{~g}$ ) yellow clear liquid.

10 Riser 12A: $245 \mathrm{~mL}$ ( $367 \mathrm{~g}$ ) yellow opaque liquid.

10 Riser 10A: $260 \mathrm{~mL}(390 \mathrm{~g}$ ) yellow opaque liquid.

11 Riser 12A: $315 \mathrm{~mL}$ ( $422 \mathrm{~g}$ ) yellow opaque liquid.

11 Riser 10A: $260 \mathrm{~mL}$ ( $363 \mathrm{~g}$ ) yellow opaque liquid.

12 Riser 12A: $305 \mathrm{~mL}$ (414 g) green opaque liquid.

12 Riser 10A: 5 in. $(78 \mathrm{~g})$ green/white salt slurry, $210 \mathrm{~mL}$ (222 g) green opaque liquid.

13 Riser 12A: 9 in. (92 g) gray to white salt slurry, $230 \mathrm{~mL}(311 \mathrm{~g}$ ) green opaque liquid.

13 Riser 10A X-RAY: Sparse < $1 \mathrm{~mm}$ bubbles bottom, sparse $1-2 \mathrm{~mm}$ bubbles top. $0.1 \mathrm{in}$. gap, clean piston. $1.9 \mathrm{R} / \mathrm{hr}$.

14 Riser 12A: 17 in. (362 g) gray salt slurry.

14 Riser 10A: 17" (326 g) gray salt slurry, $30 \mathrm{~mL}(44 \mathrm{~g})$ yellow-gray opaque liquid. X-ray: $1-2 \mathrm{~mm}$ bubbles, 0.4 " gas gap.

15 Riser 12A: 19 in. ( $370 \mathrm{~g}$ ) gray salt slurry.

15 Riser 10A X-RAY: Dense $1-2 \mathrm{~mm}$ bubbles, one irreg. void $0.3 \mathrm{in}$. size. 0.1 in. gas gap, clean piston. $2.5 \mathrm{R} / \mathrm{hr}$.

16 Riser 12A: 19 in. (392 g) gray wet salt.

16 Riser 10A: 19" (398 g) gray salt slurry. X-ray: dense $1-3 \mathrm{~mm}$ bubbles, few $4-5 \mathrm{~mm}, 0.6^{\prime \prime}$ gap, $0.5^{\prime \prime}$ stalactite. $2.2 \mathrm{R} / \mathrm{hr}$.

17 Riser 12A: 19 in. (392 g) dark gray wet salt.

17 Riser 10A X-RAY: Dense 1-2 mm bubbles bottom, dense 1-3 mm bubbles top. $0.15^{\prime \prime}$ gas gap, clean piston. $2.0 \mathrm{R} / \mathrm{hr}$.

18 Riser 12A X-RAY: not available.

18 Riser 10A: $16.5 \mathrm{in.}(406 \mathrm{~g})$ light gray/green moist salt. X-ray: very dense $1-3 \mathrm{~mm}$ bubbles, $1.1 \mathrm{in} . \mathrm{gap} .1 .9 \mathrm{R} / \mathrm{hr}$.

19 Riser 12A: 19 in. (408 g) gray solids, upper half salt slurry, lower wet salt.

19 Riser 10A X-RAY: Dense 1-2 mm bubbles, a few 3-mm. 0.5 in. gas gap, clean piston. $1.8 \mathrm{R} / \mathrm{hr}$.

20 Riser 12A: 19 in. ( $408 \mathrm{~g}$ ) gray moist salt.

20 Riser 10A: $18 \mathrm{in}$. (388 g) gray salt slurry. X-ray: dense 1-3 mm bubbles, a few $4-5 \mathrm{~mm} .1 .3^{\prime \prime}$ gap. $1.8 \mathrm{R} / \mathrm{hr}$.

21 Riser 12A: no sample.

21 Riser 10A X-RAY: Dense 1-2 mm bubbles, a few 3-mm. Sparser top. $2.4^{\prime \prime}$ gap. $0.2^{\prime \prime}$ bit of piston waste. $2.0 \mathrm{R} / \mathrm{hr}$.

mpositions, and Observations in Samples from AN-104 
Table 4.4.10 compares $\mathrm{x}$-ray-derived gas volume fractions with extraction-derived gas volume fractions. The comparison shows that sample recovery was close to $100 \%$ for all the samples. This conclusion is based on the fact that the extracted uncorrected gas was a few volume percent more than the visible gas.

Though RGS sample 10A-19 was not successfully extracted, at least 3 vol\% gas was seen in its X-ray. It is therefore likely that this sample, if extracted, would have shown about the same amount of gas as was found in samples $10 \mathrm{~A}-15$ or $10 \mathrm{~A}-17$.

Table 4.4.11 provides a summary of the waste densities that have been calculated from radiographic data for the RGS samples for which air and water standard images were available. The radiographic densities include the gas in the waste and are given for several locations within each sample to show density trends. The overall sample densities were calculated by the original method (described in Section 3.9) and also, in two cases, by the current x-ray analysis method. The differences give some idea of the sensitivity of results to small variations in method. Table 4.4.11 also includes density measurements made on extruded non-RGS samples (Hu et al. 1997). The radiographic densities equaled or exceeded the densities found for the adjacent extruded samples, which is difficult to explain because the radiographic densities included gas and the extruded densities did not.

Table 4.4.11. Densities of AN-104 Samples from Radiography and Core Samples

\begin{tabular}{|c|c|c|c|c|}
\hline $\begin{array}{l}\text { Riser } \\
\text { Segment } \\
\text { Number }\end{array}$ & $\begin{array}{l}\text { Distance from } \\
\text { Bottom of } \\
\text { Sampler } \\
\text { (ft) }\end{array}$ & $\begin{array}{l}\text { Mean Local Density } \\
\text { by Current Method } \\
(\mathrm{g} / \mathrm{cc})\end{array}$ & $\begin{array}{l}\text { Overall Average Sample } \\
\text { Densities } \\
(\mathrm{g} / \mathrm{cc})\end{array}$ & $\begin{array}{l}\text { Degassed Density in the non-RGS Cores } \\
\text { Above and Below the RGS Sample } \\
\qquad(\mathrm{g} / \mathrm{cc})\end{array}$ \\
\hline $10 \mathrm{~A}-3$ & & & $\begin{array}{l}\text { Original } \mathrm{x} \text {-ray analysis } \\
\text { method: } \quad 1.41(\mathrm{a})\end{array}$ & $\begin{array}{l}\text { density above, } 1.39 \mathrm{~g} / \mathrm{cc} \text { liquid } \\
\text { density below, } 1.38 \mathrm{~g} / \mathrm{cc} \text { liquid }\end{array}$ \\
\hline $10 \mathrm{~A}-13$ & & & $\begin{array}{l}\text { Original } \mathrm{x} \text {-ray analysis } \\
\text { method: } \quad 1.79(\mathrm{a})\end{array}$ & $\begin{array}{r}\text { density above, } 1.76 \mathrm{~g} / \mathrm{cc} \text { solids } \\
1.46 \mathrm{~g} / \mathrm{cc} \text { liquid } \\
\text { density below, } 1.63 \mathrm{~g} / \mathrm{cc} \text { solids } \\
1.49 \mathrm{~g} / \mathrm{cc} \text { liquid }\end{array}$ \\
\hline \multirow[t]{5}{*}{$10 \mathrm{~A}-15$} & 1.25 & 1.48 & \multirow{5}{*}{$\begin{array}{l}\text { Current } x \text {-ray analysis } \\
\text { method: } \quad 1.57 \\
\text { Original } x \text {-ray analysis } \\
\text { method: } \quad 1.72(\mathrm{a})\end{array}$} & \multirow{5}{*}{$\begin{array}{r}\text { density above, } 1.63 \mathrm{~g} / \mathrm{cc} \text { solids } \\
1.49 \mathrm{~g} / \mathrm{cc} \text { liquid }\end{array}$} \\
\hline & 1.0 & 1.49 & & \\
\hline & 0.75 & 1.58 & & \\
\hline & 0.5 & 1.59 & & \\
\hline & 0.25 & 1.77 & & \\
\hline \multirow[t]{5}{*}{$10 \mathrm{~A}-17$} & 1.25 & 1.84 & \multirow{4}{*}{$\begin{array}{l}\text { Current } x \text {-ray analysis } \\
\text { method: } \quad 1.81\end{array}$} & \multirow[t]{5}{*}{ density above, $1.56 \mathrm{~g} / \mathrm{cc}$ bulk } \\
\hline & 1.0 & 1.79 & & \\
\hline & 0.75 & 1.83 & & \\
\hline & 0.5 & 1.70 & & \\
\hline & 0.25 & 1.98 & $\begin{array}{l}\text { Original } x \text {-ray analysis } \\
\text { method: } \quad 2.09 \text { (a) }\end{array}$ & \\
\hline $10 \mathrm{~A}-19$ & & & $\begin{array}{l}\text { Original } x \text {-ray analysis } \\
\text { method: } \quad 1.74(\mathrm{a})\end{array}$ & $\begin{array}{l}\text { density above, } 1.71 \mathrm{~g} / \mathrm{cc} \text { bulk } \\
\text { density below, } 1.63 \mathrm{~g} / \mathrm{cc} \text { bulk }\end{array}$ \\
\hline $10 \mathrm{~A}-21$ & & & $\begin{array}{l}\text { Original } \mathrm{x} \text {-ray analysis } \\
\text { method: } \quad 1.85(\mathrm{a})\end{array}$ & density above, $1.63 \mathrm{~g} / \mathrm{cc}$ bulk \\
\hline
\end{tabular}




\subsubsection{Drillstring and Domespace Composition Comparison}

The RGS gas-phase composition data (Table 4.4.6) were used to calculate $\mathrm{H}_{2} / \mathrm{N}_{2} \mathrm{O}$ ratios for comparison with ratios from drillstring and domespace grab sample measurements (Siciliano 1998, Table 8.1; McCain 1999, Table 4.4). The results can be seen in Table 4.4.12. While the riser 10A RGS $\mathrm{H}_{2} / \mathrm{N}_{2} \mathrm{O}$ ratios were much the same as the drillstring ratio, the domespace ratios were higher than most of the other values.

Table 4.4.12. Comparison with Drillstring and Domespace Data(a)

\begin{tabular}{|l|c|}
\hline \multicolumn{1}{|c|}{ Sample } & $\mathrm{H}_{2} / \mathrm{N}_{2} \mathrm{O}$ \\
\hline \hline RGS, 10A-3 & $1.6-4.9$ \\
\hline RGS, 10A-13 & $2.8-5.4$ \\
\hline RGS, 10A-15 & $3.7-5.3$ \\
\hline$R G S, 10 \mathrm{~A}-17$ & $2.0-2.6$ \\
\hline RGS, 12A-18 & $3.0-3.7$ \\
\hline RGS, 10A-21 & $1.6-1.7$ \\
\hline Drillstring, riser 10A, 9/12/96 & 3.0 \\
\hline Domespace, 11/9/94 GRE & 8.1 \\
\hline Domespace, 3/27/96 & $6.1,6.4$ \\
\hline Domespace, 8/97 & 5,4 \\
\hline (a) RGS ratios were calculated for both lower- and upper-bound solubilities. Both \\
ends of ratio range are given, lower-bound solubility basis first.
\end{tabular}

\subsection{AN-103}

Tank 241-AN-103 (AN-103) was the fifth tank and the fourth DST sampled with the RGS. This tank was selected because it exhibited episodic GREs. AN-103 was on the FGWL and showed small cyclical level drops resulting from GREs. Most of the tank contents were doubleshell slurry feed (Stewart et al. 1996a).

Push-mode sampling was done in risers 12A and 21A in September 1996.(a) Riser 12A was sampled because it was close to the VFI risers (1B and 16B) and the MIT in riser 15A, allowing correlation of waste temperatures and gas content. The approximate locations of the various risers are depicted in Figure 4.5.1.

The total depth of waste in Tank AN-103 was approximately $884 \mathrm{~cm}$ ( $348 \mathrm{in}$.); thus, at the time of RGS sampling, AN-103 contained approximately 3,590,000 L (958,000 gal) of waste. Riser $12 \mathrm{~A}$ is near the tank center, while riser $21 \mathrm{~A}$ is about three-fourths of a tank radius from the center. Figure 4.5.2 shows the tank content layering derived from the riser 4A TC tree and riser 15A MTT measured temperature profiles in conjunction with the VFI/ball rheometer data for this tank (Schienbein et al. 1999). The nonconvective layer was $378 \pm 7 \mathrm{~cm}$ (149 \pm 3 in.) in depth,

(a) The sampling scheme may be found in Sampling Plan for Tank 241-AN-103 Retained Gas Sampler Deployment, by A Shekarriz and JM Bates. TWSMIT:080196, Pacific Northwest National Laboratory, Richland, Washington, August 1996. 


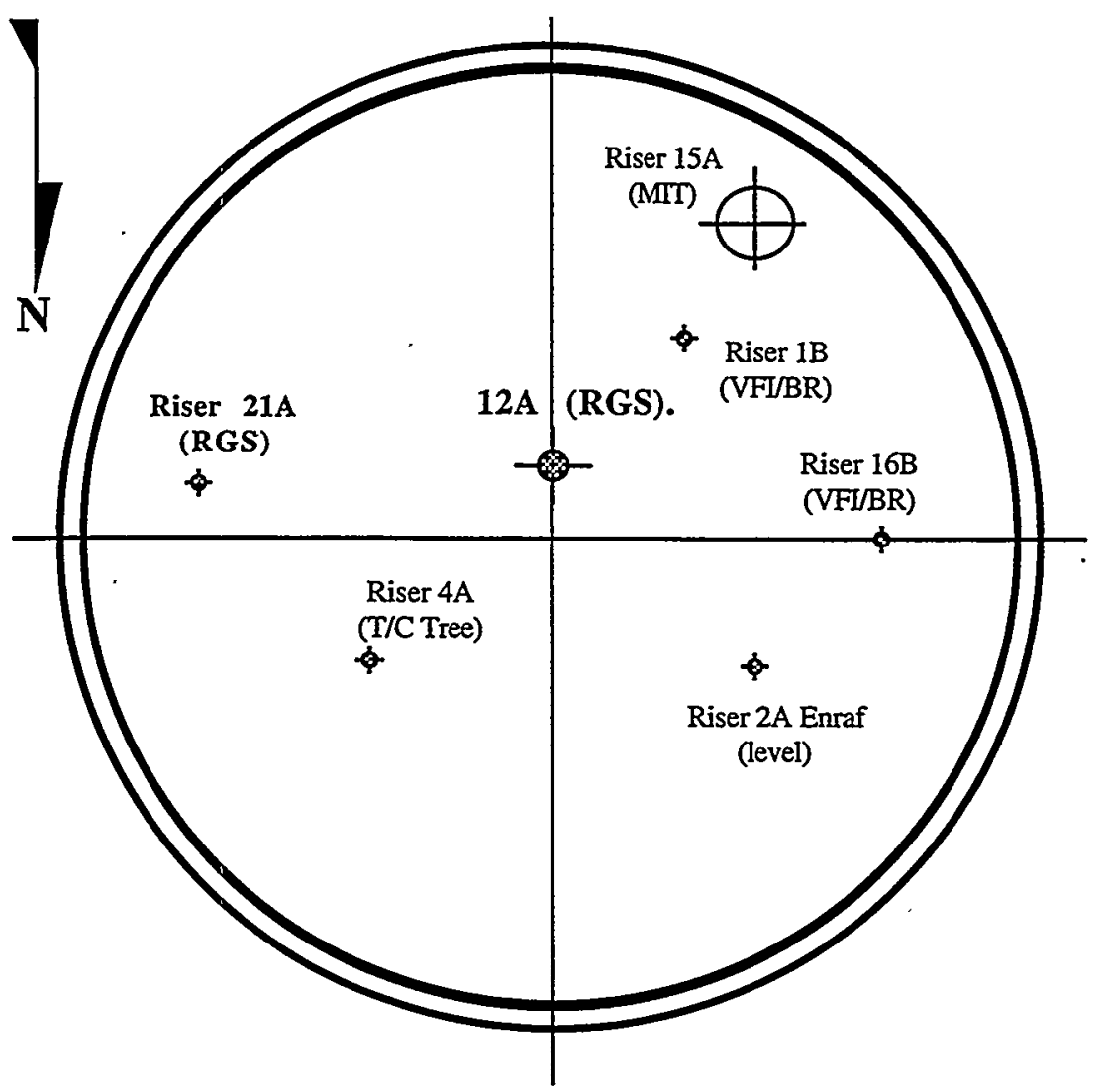

Figure 4.5.1. Schematic Diagram of Riser Locations in Tank AN-103
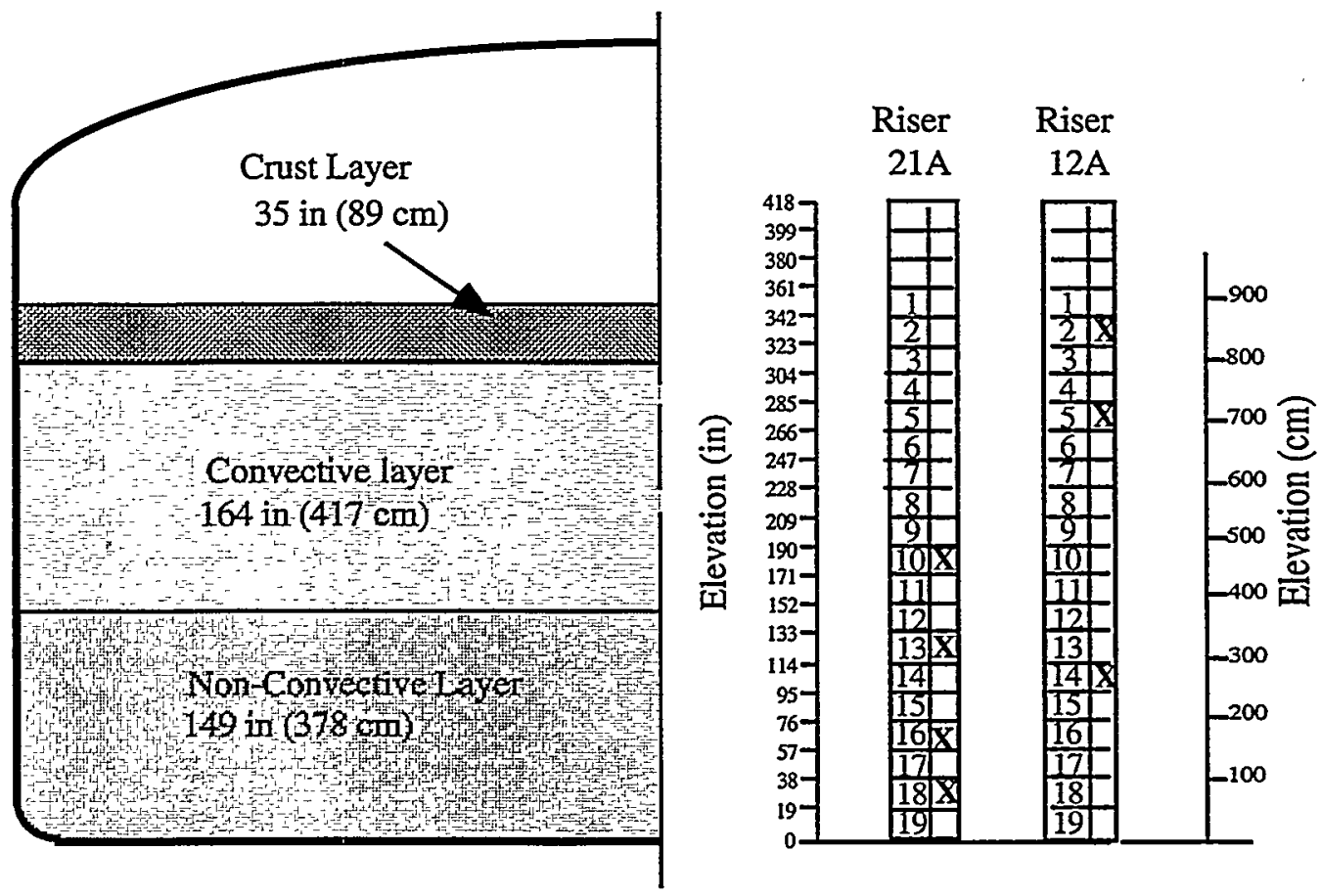

Figure 4.5.2. Diagram of Waste Layering and RGS Sample Elevations for AN-103 
and the balance of the contents were the convective (supernatant liquid) layer of depth $417 \pm 18 \mathrm{~cm}$ (164 \pm 7 in.) and a $89 \pm 16 \mathrm{~cm}$ (35 \pm 6 in.) thick crust. The elevations of the RGS segments are also depicted in Figure 4.5.2.

The most recent information on AN-103 layering came from the observations made on the non-RGS samples in the cores from 12A and 21A. The topmost samples contained 2 to 14 in. of wet or moist salt. Some salt, 6 in. or less, was also evident in the second segment fom each riser. Beneath that was 8 to 10 samplers (150 to 190 in.) of liquid, with 7 to 9 samplers (130 to 160 in.) of salt slurry, wet salt, or moist salt at the tank bottom.

The last buoyant displacement GRE that was relevant to RGS or VFI sampling occurred on August 22, 1995, seven months before the VFI measurements (May 14 and 16, 1996) and more than a year before the first RGS samples were taken from riser 12A (Schienbein et al. 1999). Thus GREs probably did not play a part in the differences between RGS and VFI gas measurements.

Densities for the liquid and bulk solids in Tank AN-103 were available from the non-RGS samples taken in 1996 (Field 1999b). A number of samples contained drainable liquid. The density of the drainable liquid was $1458 \pm 31 \mathrm{~kg} / \mathrm{m}^{3}$. The bulk density of the crust (three samples) varied from 1590 to $1720 \mathrm{~kg} / \mathrm{m}^{3}$, with an average and standard deviation of $1663 \pm 67 \mathrm{~kg} / \mathrm{m}^{3}$. The bulk density of the nonconvective layer samples varied from 1590 to $1930 \mathrm{~kg} / \mathrm{m}^{3}$, with an average and standard deviation of $1736 \pm 108 \mathrm{~kg} / \mathrm{m}^{3}$. Table 2.7 .1 of Schienbein et al. (1999) gave a density of $1530 \pm 50 \mathrm{~kg} / \mathrm{m}^{3}$ for the convective layer (based on in situ ball rheometry in 1996) and $1730 \pm 110 \mathrm{~kg} / \mathrm{m}^{3}$ for the nonconvective layer (based on core samples). All of these measurement methods disturbed the waste, and their results should be taken as degassed densities.

For hydrostatic pressure calculations, the degassed crust density was set at $1663 \mathrm{~kg} / \mathrm{m}^{3}$, the liquid density at $1530 \mathrm{~kg} / \mathrm{m}^{3}$, and the degassed nonconvective layer at $1730 \mathrm{~kg} / \mathrm{m}^{3}$. The gassy crust density (based on the degassed density given above and the RGS crust gas measurement) was calculated to be $1400 \mathrm{~kg} / \mathrm{m}^{3}$, less than required for neutral buoyancy. Layer depths of $89 \mathrm{~cm}$ (35 in.), $417 \mathrm{~cm}$ (164 in.), and $378 \mathrm{~cm}$ (149 in.) were used in calculations for the crust, convective, and nonconvective layers, respectively.

\subsubsection{Sampling and Extraction Information}

The samples that underwent RGS analysis are listed in Table 4.5.1. (Samples 21A-13 and 21A-18 were lost because the sampler valve malfunctioned. The collector pressure data were lost for the final canister of gas extracted from sample 12A-5, but the pressures were approximated and the uncertainty of the results was increased accordingly. The increase in uncertainty was small because the last canister typically contains little gas.) Field data, including dose rates and downforce limits, are supplied in Appendix A.

Table 4.5.1 also shows the lag times (delay between sample acquisition and processing) for these samples. This information was provided to allow data users to correlate the lag time between sampling and extrusion with the concentrations of the various constituents to test for decomposition or other chemical reactions. The maximum hold time allowed by the sampling plan is 24 days based on measured sampler leakage rates during acceptance testing. The hold time for all the samples exceeded this limit, but the samples were accepted based on calculations that indicated the estimated leakage was still negligible.

As was discussed in Section 3.5, the HHF used during sampling often enters the sampler in place of some of the waste. The HHF is a solution of $\mathrm{LiBr}$ in which the bromide ion, which is not present in waste, is used as the HHF tracer. Table 4.5.2 shows the volume percentage contamination by HHF of the RGS samples. The measured HHF contamination percentages were 
Table 4.5.1. Lag Times for Processing RGS Samples from Tank 241-AN-103

\begin{tabular}{||c|c|c|c||}
\hline Sample & Acquisition Date & Processing Date & Lag (days) \\
\hline \hline 12A-2 & Sept. 13, 1996 & Oct. 18, 1996 & 35 \\
\hline 12A-5 & Sept. 13, 1996 & Oct. 22, 1996 & 39 \\
\hline 21A-10 & Sept. 20, 1996 & Oct. 24, 1996 & 34 \\
\hline 12A-14 & Sept. 16,1996 & Oct. 23, 1996 & 37 \\
\hline 21A-16 & Sept. 20, 1996 & Oct. 28, 1996 & 38 \\
\hline
\end{tabular}

used to calculate reduced effective sampler volumes for the RGS samples for which there were measurements. Three of the measured samples contained bromide below the MDL. The bromide concentration was set equal to the MDL for these samples. (It is conservative to use the MDL for values below it; the conservatism is probably less than $2 \%$ for samples $12 \mathrm{~A}-2$ and $21 \mathrm{~A}-10$, and less than $6 \%$ for sample $21 \mathrm{~A}-16$.)

The same procedure was used for all AN-103 samples (see RGS system schematic in Figure 3.1). After evacuating the whole system and observing the pressure for leaks, the operator extruded the RGS sample into the extractor vessel, and the sample was briefly stirred. At this point, the vapor space in communication with the sample consisted of the volumes of the extractor vessel and its lines. Next, the extractor was opened to the second vessel, which was connected to a PQ canister. Once a grab sample was collected in the PQ canister, it was closed. The extractor, lines, and second volume were then connected to the pump volume and collector side; the latter included the collector line volume and one open collection canister. The first canister, containing a sample of uncompressed (unpumped) gas, was closed off. The second collection canister was then opened, several strokes were pumped from the extractor to the collector, and the second collection canister was closed off.

Then the collector side, second volume, and extractor lines were evacuated. Next, the extractor and samples were heated to about $50^{\circ} \mathrm{C}$ to drive off bound gas, cooled back to hot cell temperature, and the sample briefly stirred. A PQ canister connected to the extractor vessel took a second sample and was closed off. Several strokes were pumped from the extractor to the collector, and the third collection canister was closed off. Finally, the BSVD procedure was performed: argon was injected into the closed-off second volume, and the second volume was opened to the extractor and lines. Pressure measurements and known tare system volumes allowed calculation of the volume of the sample solids and liquid.

Table 4.5.2. Hydrostatic Head Fluid Contamination in AN-103 RGS Samples

\begin{tabular}{|c|c|}
\hline Sample & $\begin{array}{c}\text { HHF Contamination } \\
\text { (vol \%) }\end{array}$ \\
\hline \hline $12 \mathrm{~A}-2$ & $<5.0$ \\
\hline $12 \mathrm{~A}-5$ & 4.5 \\
\hline $21 \mathrm{~A}-10$ & $<4.7$ \\
\hline $12 \mathrm{~A}-14$ & 5.4 \\
\hline $21 \mathrm{~A}-16$ & $<10.4$ \\
\hline
\end{tabular}


Procedural details such as the number of strokes per canister and the system volumes can be found in the sample-by-sample worksheets in the AN-103 RGS calculation spreadsheet on the CD provided with this report.

\subsubsection{Retained Gas Composition}

Table 4.5.3 presents the calculated concentrations of the insoluble gases in each RGS sample taken from AN-103 without corrections for air and argon entrainment or air inleakage. The method used to make the corrections depended on the gas. All of the oxygen and argon were subtracted no matter which step had produced them. A number of moles equal to $(3.73) \cdot\left(\mathrm{O}_{2}\right)$ were subtracted from the nitrogen because the oxygen was expected to have come from air entrainment during sampling or inleakage during extraction. Corrected concentrations are given in Table 4.5.4.

Sample 21A-16 contained more argon than the other samples, possibly indicating that an argon drillstring purge was done before the sample was taken. Contamination observations are found in the "Summary" worksheet of the AN-103 RGS calculations spreadsheet on the CD.

Table 4.5.3. Concentrations of Insoluble Constituents ( $\mu \mathrm{mol} / \mathrm{L}$ of waste) in Tank AN-103 Without Correction for Gas Contamination

\begin{tabular}{||c|c|c|c|c|c|c|c|c|c|c|c|}
\hline Sample & $\mathrm{N}_{2}$ & $\mathrm{H}_{2}$ & $\mathrm{~N}_{2} \mathrm{O}$ & $\mathrm{O}_{2}$ & $\mathrm{CH}_{4}$ & $\mathrm{He}$ & $\mathrm{Ar}$ & $\begin{array}{c}\text { Other } \\
\mathrm{NO}_{x}\end{array}$ & $\mathrm{C}_{2} \mathrm{H}_{x}$ & $\mathrm{C}_{3} \mathrm{H}_{x}$ & Other Hyd. \\
\hline \hline $12 \mathrm{~A}-2$ & $2200 \pm 150$ & $4000 \pm 270$ & $450 \pm 30$ & $99 \pm 7.9$ & $41 \pm 3.2$ & (a) & $9.0 \pm 0.7$ & $1.0 \pm 0.6$ & $8.4 \pm 1.4$ & $2.8 \pm 0.8$ & $3.5 \pm 0.8$ \\
\hline $12 \mathrm{~A}-5$ & $750 \pm 140$ & $74 \pm 16$ & $50 \pm 16$ & $130 \pm 23$ & $6.6 \pm 3.6$ & (a) & $8.8 \pm 1.6$ & $2.1 \pm 1.1$ & $0.8 \pm 0.4$ & $1.5 \pm 0.8$ & $0.9 \pm 0.6$ \\
\hline $21 \mathrm{~A}-10$ & $780 \pm 180$ & $77 \pm 17$ & $49 \pm 13$ & $140 \pm 32$ & $4.4 \pm 2.2$ & (a) & $26 \pm 6.0$ & $1.5 \pm 0.8$ & $1.0 \pm 0.4$ & $1.4 \pm 0.7$ & $1.2 \pm 0.7$ \\
\hline $12 \mathrm{~A}-14$ & $2100 \pm 230$ & $2700 \pm 290$ & $250 \pm 30$ & $64 \pm 7.6$ & $34 \pm 6.4$ & (a) & $10 \pm 1.1$ & $1.8 \pm 1.3$ & $9.3 \pm 2.0$ & $3.5 \pm 1.7$ & $4.8 \pm 2.3$ \\
\hline $21 \mathrm{~A}-16$ & $2800 \pm 220$ & $5900 \pm 460$ & $360 \pm 30$ & $12 \pm 2.1$ & $58 \pm 7.6$ & (a) & $76 \pm 6.4$ & $1.5 \pm 0.7$ & $13 \pm 4.0$ & $6.3 \pm 1.3$ & $16 \pm 6.3$ \\
\hline
\end{tabular}

Table 4.5.4. Concentrations of Insoluble Constituents ( $\mu \mathrm{mol} / \mathrm{L}$ of waste) in Tank AN-103 with Correction for Gas Contamination

\begin{tabular}{|c|c|c|c|c|c|c|c|c|c|c|c||}
\hline Sample & $\mathrm{N}_{2}$ & $\mathrm{H}_{2}$ & $\mathrm{~N}_{2} \mathrm{O}$ & $\mathrm{O}_{2}$ & $\mathrm{CH}_{4}$ & $\mathrm{He}$ & $\mathrm{Ar}$ & $\begin{array}{c}\text { Other } \\
\mathrm{NO}_{x}\end{array}$ & $\mathrm{C}_{2} \mathrm{H}_{\mathrm{x}}$ & $\mathrm{C}_{3} \mathrm{H}_{\mathrm{x}}$ & Other Hyd. \\
\hline \hline $12 \mathrm{~A}-2$ & $1800 \pm 150$ & $4000 \pm 270$ & $450 \pm 30$ & $0 \pm 7.9$ & $41 \pm 3.2$ & (a) & $0 \pm 0.7$ & $1.0 \pm 0.6$ & $8.4 \pm 1.4$ & $2.8 \pm 0.8$ & $3.5 \pm 0.8$ \\
\hline $12 \mathrm{~A}-5$ & $260 \pm 160$ & $74 \pm 16$ & $50 \pm 16$ & $0 \pm 23$ & $6.6 \pm 3.6$ & (a) & $0 \pm 1.6$ & $2.1 \pm 1.1$ & $0.8 \pm 0.4$ & $1.5 \pm 0.8$ & $0.9 \pm 0.6$ \\
\hline $21 \mathrm{~A}-10$ & $270 \pm 210$ & $77 \pm 17$ & $49 \pm 13$ & $0 \pm 32$ & $4.4 \pm 2.2$ & (a) & $0 \pm 6.0$ & $1.5 \pm 0.8$ & $1.0 \pm 0.4$ & $1.4 \pm 0.7$ & $1.2 \pm 0.7$ \\
\hline $12 \mathrm{~A}-14$ & $1800 \pm 230$ & $2700 \pm 290$ & $250 \pm 30$ & $0 \pm 7.6$ & $34 \pm 6.4$ & (a) & $0 \pm 1.1$ & $1.8 \pm 1.3$ & $9.3 \pm 2.0$ & $3.5 \pm 1.7$ & $4.8 \pm 2.3$ \\
\hline 21A-16 & $2800 \pm 220$ & $5900 \pm 460$ & $360 \pm 30$ & $0 \pm 2.1$ & $58 \pm 7.6$ & (a) & $0 \pm 6.4$ & $1.5 \pm 0.7$ & $13 \pm 4.0$ & $6.3 \pm 1.3$ & $16 \pm 6.3$ \\
\hline (a) Beiow detection limits, which are 0.01 mol\% or less.
\end{tabular}


Table 4.5.5 presents the ammonia measurements. The RGS procedure did not permit determination of the residual or total ammonia, but the post-extrusion and the first PQ canister partial pressures of $\mathrm{NH}_{3}$ over the sample (see Section 3.4.4) were measured at laboratory temperature. There were no residual ammonia measurements for AN-103 samples. Ammonia data can be seen in more detail in Appendix C (Section C.5 ).

No conclusive total ammonia concentration can be calculated, but it is likely that the ammonia concentration in AN-103 waste was the same as in AW-101 waste, with a lower bound of $27,000 \mu \mathrm{mol} \mathrm{NH} / \mathrm{L}$ waste. This estimate is based on a comparison of the ammonia partial pressure measurements (see Sections 4.1.2 and C.1 for the AW-101 measurements).

Table 4.5.6 contains the composition of the gas phase in each sample and the average compositions in the gas retained in the crust, convective layer, and nonconvective layer. The water vapor is not included in these compositions. The ammonia fractions are derived from the partial pressures indicated in Table 4.5.5 extrapolated to vapor pressures at in situ conditions. The sample compositions in the table have been calculated using the in situ solubility method described in Section 3.6.2. As discussed in Section 3.6.1, compositions were calculated for both the lowerand upper-bound gas solubilities. Both ends of the composition range are given in Table 4.5.6 with the measurement uncertainty on each. The average compositions of the gas in the convective and nonconvective layers is the result of integrating RGS species concentrations over the layer and multiplying those concentrations by the layer volumes. The single sample in the crust was used to obtain the crust gas composition. The integration method is described in Section 3.7.1.

There was little composition difference between samples in the same layer. The composition of the crust gas was the same, within uncertainty, as that of the retained gas in the nonconvective layer. As in samples from other RGS-sampled tanks, nitrogen was high in the AN-103 supernatant and hydrogen low, though the broad uncertainty makes this interpretation somewhat inconclusive.

The concentrations and pressures in Tables 4.5.3 and 4.5.4 can also be found in the sample-by-sample worksheets in the AN-103 RGS calculation spreadsheet on the CD. The compositions in Table 4.5.6 are in the "Inventory" worksheet.

Table 4.5.5. Ammonia Data from Tank AN-103 Samples

\begin{tabular}{|c|c|c|}
\hline Sample & $\begin{array}{l}\text { Measured } \mathrm{NH}_{3} \\
\text { partial pressure at } \\
\text { about } 24^{\circ} \mathrm{C} \\
\text { (atm) }\end{array}$ & $\begin{array}{c}\text { Best-Estimate } \mathrm{NH}_{3} \\
\text { Concentrations }\end{array}$ \\
\hline $12 \mathrm{~A}-2$ & $0.0093 \pm 0.0046$ (a) & \multirow{5}{*}{$\begin{array}{l}\text { The data do not support } \\
\text { conclusive } \mathrm{NH}_{3} \text { concentrations. } \\
\text { We estimate a lower bound of } \\
0.040 \mathrm{M}(680 \mu \mathrm{g} / \mathrm{mL}) \mathrm{NH}_{3} \text { in } \\
\text { the liquid of the nonconvective } \\
\text { layer, or } 0.029 \mathrm{wt} \% \mathrm{NH}_{3} \text { in the } \\
\text { bulk waste. }\end{array}$} \\
\hline $12 A-5$ & $0.0083 \pm 0.0041$ & \\
\hline $21 \mathrm{~A}-10$ & $0.0078 \pm 0.0039$ (a) & \\
\hline $12 \mathrm{~A}-14$ & $0.0076 \pm 0.0038$ & \\
\hline $21 \mathrm{~A}-16$ & $0.0067 \pm 0.0033$ & \\
\hline \multicolumn{3}{|c|}{$\begin{array}{l}\text { (a) Ammonia partial pressures are based on PQ canister measurements for } \\
\text { samples 12A-14 and 21A-16. No PQ canister data were available for } \\
\text { samples } 12 \mathrm{~A}-2,12 \mathrm{~A}-5 \text {, and } 21 \mathrm{~A}-10 \text {, so calculated post-extrusion partial } \\
\text { pressures were used instead. }\end{array}$} \\
\hline
\end{tabular}


Table 4.5.6. Sample and Overall Average Compositions of Retained Gas in Tank AN-103 with Correction for Gas Contamination(a)

\begin{tabular}{|c|c|c|c|c|c|c|}
\hline Sample & $\mathrm{N}_{2}(\mathrm{~mol} \%)$ & $\mathrm{H}_{2}(\mathrm{~mol} \%)$ & $\mathrm{N}_{2} \mathrm{O}(\mathrm{mol} \%)$ & $\mathrm{NH}_{3}(\mathrm{~mol} \%)^{(\mathrm{b})}$ & $\mathrm{CH}_{4}(\mathrm{~mol} \%)$ & Other (mol\%) \\
\hline $12 \mathrm{~A}-2$ & $29 \pm 3.2$ & $62 \pm 6.4-63 \pm 6.4$ & $6.9 \pm 0.7--6.3 \pm 0.6$ & $1.8 \pm 0.8--0.6 \pm 0.3$ & $0.6 \pm 0.07$ & $0.2 \pm 0.05$ \\
\hline $12 \mathrm{~A}-5$ & $68 \pm 54-74 \pm 60$ & $19 \pm 10$ & $8.7 \pm 5.2--2.9 \pm 1.8$ & $1.4 \pm 1.0--0.5 \pm 0.4$ & $1.7 \pm 1.3$ & $1.4 \pm 1.0-1.6 \pm 1.2$ \\
\hline $21 \mathrm{~A}-10$ & $69 \pm 69--75 \pm 75$ & $19 \pm 13$ & $7.9 \pm 5.3--2.3 \pm 1.7$ & $1.1 \pm 0.9--0.4 \pm 0.3$ & $1.1 \pm 0.9$ & $1.4 \pm 1.1--1.6 \pm 1.4$ \\
\hline $12 \mathrm{~A}-14$ & $38 \pm 6.5--39 \pm 6.6$ & $55 \pm 8.8--56 \pm 8.8$ & $4.9 \pm 0.8--4.1 \pm 0.7$ & $1.0 \pm 0.4--0.4 \pm 0.2$ & $0.7 \pm 0.2$ & $0.4 \pm 0.2$ \\
\hline $21 A-16$ & $30 \pm 3.5--31 \pm 3.5$ & $64 \pm 7.2$ & $3.8 \pm 0.4--3.4 \pm 0.4$ & $0.8 \pm 0.4--0.3 \pm 0.1$ & $0.6 \pm 0.1$ & $0.4 \pm 0.2$ \\
\hline Crust layer ${ }^{(c)}$ & $29 \pm 3.2$ & $62 \pm 6.4--63 \pm 6.4$ & $6.9 \pm 0.7--6.3 \pm 0.6$ & $1.8 \pm 0.8--0.6 \pm 0.3$ & $0.6 \pm 0.07$ & $0.2 \pm 0.05$ \\
\hline $\begin{array}{c}\text { Convective } \\
\text { layer(c) }\end{array}$ & $68 \pm 61--75 \pm 68$ & $19 \pm 12$ & $8.3 \pm 5.3--2.6 \pm 1.7$ & $1.2 \pm 0.9--0.5 \pm 0.4$ & $1.4 \pm 1.1$ & $1.4 \pm 0.8--1.6 \pm 0.9$ \\
\hline $\begin{array}{l}\text { Avg in non- } \\
\text { convective } \\
\text { layer(c) }\end{array}$ & $33 \pm 4.3$ & $61 \pm 7.7-62 \pm 7.7$ & $4.2 \pm 0.6--3.6 \pm 0.5$ & $0.9 \pm 0.4--0.3 \pm 0.1$ & $0.6 \pm 0.1$ & $0.4 \pm 0.1$ \\
\hline \multicolumn{7}{|c|}{$\begin{array}{l}\text { (a) Uncertainties on compositions represent only instrument error. Compositions calculated for lower- and upper-bound } \\
\text { solubilities. Both ends of composition range are given, lower-bound solubility basis first with instrument uncertainty on } \\
\text { each. Compositions may not sum to } 100 \mathrm{~mol} \% \text { because of roundoff error. Mole fractions are on a dry basis and do not } \\
\text { account for water vapor. } \\
\text { (b) Ammonia mole fraction derived from partial pressures in Table } 4.5 .5 \text { extrapolated to vapor pressure at in situ conditions. } \\
\text { (c) There are too few samples to define the spatial variability of the average gas concentration. }\end{array}$} \\
\hline
\end{tabular}

\subsubsection{Gas Inventory}

The method by which the in situ gas volume fractions (wet basis) were calculated is given in Section 3.6.2; as for the gas composition, the volume fractions are given as a range from the low gas solubility value to the high gas solubility value. The results are presented in Table 4.5.7, which also contains the average gas volume fraction and the average pressure experienced by the gas in each layer. The averages for the convective and nonconvective layers are in situ volume gas volume fractions in Table 4.5.7 are consistent with the corrected gas concentrations and compositions in Tables 4.5.4 through 4.5.6. The information in Table 4.5.7 is taken from the "Summary" and "In situ" worksheets of the AN-103 RGS calculation spreadsheet on the CD.

The "sampler gas volume fraction" is corrected only for inleakage during the extraction process and air gases added in the isotopic solution. It is an attempt to reconstruct the total amount of gas (sample and entrainment) that was present in the sampler during $\mathrm{X}$-ray. It is used only for comparison with the $\mathrm{X}$-ray observations of "visible gas fraction" that are discussed in Section 4.5.4.

Table 4.5.7 also contains the water vapor pressures that were used for in situ calculations. The water vapor pressures were found by using salt concentrations from Field (1999b) and the temperatures in the table as inputs to Equation 6.2 of Mahoney and Trent (1995), a correlation for averages calculated by Simpson's Rule integration, as described in Section 3.7.1. The corrected water vapor pressure over concentrated homogeneous and non-homogeneous waste simulants. The gas solubilities used the same parameters as inputs to the Schumpe solubility model (see Section 3.6.1). The water vapor pressures and gas solubilities used in calculations can be found in the "In situ" worksheet of the AN-103 RGS calculation spreadsheet on the CD. No ammonia inventory was calculated because there are no total ammonia concentration data for AN-103. 
Table 4.5.7. In Situ Gas Volume Fractions and Conditions in Tank AN-103

\begin{tabular}{|c|c|c|c|c|c|c|}
\hline Sample & $\begin{array}{c}\text { Sample } \\
\text { central } \\
\text { height } \\
(\mathrm{cm})\end{array}$ & $\begin{array}{l}\text { Hydro- } \\
\text { static } \\
\text { Pressure } \\
\text { (atm) }\end{array}$ & $\begin{array}{c}\text { Calculated } \\
\text { water vapor } \\
\text { pressure } \\
\text { (atm) }\end{array}$ & $\begin{array}{l}\text { Temp } \\
\left({ }^{\circ} \mathrm{C}\right)\end{array}$ & $\begin{array}{c}\text { Corrected gas } \\
\text { volume fraction(a) } \\
\text { (in-tank conditions) }\end{array}$ & $\begin{array}{c}\text { Sampler gas } \\
\text { volume fraction } \\
\text { (in situ conditions } \\
\text { low solubility) }\end{array}$ \\
\hline $12 \mathrm{~A}-2$ & 845 & 1.05 & 0.018 & 36.0 & $0.16 \pm 0.014$ & 0.17 \\
\hline $12 \mathrm{~A}-5$ & 700 & 1.26 & 0.034 & 38.7 & $\begin{array}{c}0.008 \pm 0.003 \text { to } \\
0.007 \pm 0.003\end{array}$ & 0.020 \\
\hline $21 \mathrm{~A}-10$ & 458 & 1.61 & 0.036 & 39.6 & $\begin{array}{c}0.006 \pm 0.003 \text { to } \\
0.005 \pm 0.003\end{array}$ & 0.016 \\
\hline $12 \mathrm{~A}-14$ & 265 & 1.90 & 0.021 & 41.9 & $\begin{array}{c}0.067 \pm 0.012 \text { to } \\
0.065 \pm 0.012\end{array}$ & 0.071 \\
\hline $21 \mathrm{~A}-16$ & 169 & 2.05 & 0.020 & 40.6 & $0.12 \pm 0.015$ & 0.12 \\
\hline Crust layer & 839 & 1.06 & & & $\begin{array}{c}0.16 \pm 0.079 \text { to } \\
0.15 \pm 0.076\end{array}$ & \\
\hline $\begin{array}{c}\text { Convective } \\
\text { layer }\end{array}$ & 587 & 1.43 & & & $\begin{array}{c}0.007 \pm 0.003 \text { to } \\
0.006 \pm 0.003\end{array}$ & \\
\hline $\begin{array}{l}\text { Avg in non- } \\
\text { convective } \\
\text { layer }\end{array}$ & 160 & 2.06 & & & $\begin{array}{c}0.092 \pm 0.046 \text { to } \\
0.091 \pm 0.045\end{array}$ & \\
\hline
\end{tabular}

Table 4.5.8 gives various estimates of the STP volume of gas in Tank AN-103, including estimates calculated from RGS data alone. The VFI\&RGS and BPE inventory values were taken from Schienbein et al. (1999).

The RGS gas inventories in the convective and nonconvective layers were calculated by integrating RGS total gas concentrations over each layer (two data points each) and multiplying the average gas concentrations by the volumes of the layers, as described in Section 3.7.1. The single sample in the crust layer (12A-2) was used to calculate its inventory. The RGS volumes in Table 4.5.8 include corrections to remove the contamination gas: entrained air and argon and air leaks during and after extraction. The uncertainties on the gas inventories are based on the spatial variability considerations discussed in Section 3.7.2. The information in Table 4.5.8 is taken from the "Inventory" worksheet of the AN-103 RGS calculation spreadsheet on the CD.

The BPE method gave the highest total gas inventory and the RGS method the lowest (214 $\pm 78 \mathrm{~m}^{3}$ in situ), with the inventory calculated by the VFI\&RGS method ( $230 \pm 24 \mathrm{~m}^{3}$ in situ) in between. As Figure 4.5.3 shows, RGS captured lower gas contents than VFI measurements at the same elevations. The single nonconvective layer sample from riser 12A (sample 12A14) was especially low in gas, perhaps reflecting local conditions. As detailed in Section 4.5.8, gas fractions that were higher than the RGS average value ( 0.12 to 0.19 gas fraction compared with 0.09 ) were visible in the $x$-rays of riser $21 \mathrm{~A}$ samples that were not extracted. These higher 
Table 4.5.8. AN-103 Gas Inventory Estimates

\begin{tabular}{|c|c|c|c|c|c|c|}
\hline \multirow{2}{*}{ Quantity } & \multicolumn{3}{|c|}{ RGS Method } & $\begin{array}{c}\text { BPE } \\
\text { Method(a) }\end{array}$ & \multicolumn{2}{|c|}{ VFI\&RGS Method(b,c) } \\
\hline & $\begin{array}{l}\text { Crust } \\
\text { Layer }\end{array}$ & $\begin{array}{l}\text { Convective } \\
\text { Layer }\end{array}$ & $\begin{array}{c}\text { Nonconvective } \\
\text { Layer }\end{array}$ & Tank Total & $\begin{array}{l}\text { Convective } \\
\text { Layer }\end{array}$ & $\begin{array}{c}\text { Nonconvective } \\
\text { Layer }\end{array}$ \\
\hline $\begin{array}{l}\text { Avg gas fraction } \\
\text { (low gas } \\
\text { solubility) }\end{array}$ & $0.16 \pm 0.079$ & $0.007 \pm 0.003$ & $0.092 \pm 0.046$ & $\begin{array}{c}\text { (tank avg.) } \\
0.075 \pm 0.021\end{array}$ & $0.005 \pm 0.001$ & $0.110 \pm 0.007$ \\
\hline $\begin{array}{l}\text { Gas volume }\left(\mathrm{m}^{3}\right) \\
\text { in situ (wet) }\end{array}$ & $58 \pm 29$ & $12 \pm 4$ & $144 \pm 72$ & $268 \pm 76$ & $8 \pm 2$ & $170 \pm 10$ \\
\hline STP (wet) & $54 \pm 27$ & $15 \pm 5$ & $260 \pm 130$ & $463 \pm 133$ & $11 \pm 3$ & $356 \pm 16$ \\
\hline \multicolumn{7}{|c|}{$\begin{array}{l}\text { (a) Barometric pressure method. Values are taken from Table } 2.7 .5 \text { of Schienbein et al. (1999). } \\
\text { (b) Based on void fraction instrument (VFI) and RGS data; see Table } 2.7 .3 \text { of Schienbein et al. } \\
\text { (1999). There were no VFI data for the crust; however, Schienbein et al. (1999) stated the crust gas } \\
\text { inventory as } 52 \pm 22 \mathrm{~m}^{3} \text { in situ, or } 54 \pm 23 \text { STP } \mathrm{m}^{3} \text {. This inventory is part of the VFI\&RGS total } \\
\text { inventory; the RGS crust inventory is based on RGS crust data alone. } \\
\text { (c) The retained gas inventory calculated by the VFI\&RGS method is considered the best estimate. }\end{array}$} \\
\hline
\end{tabular}

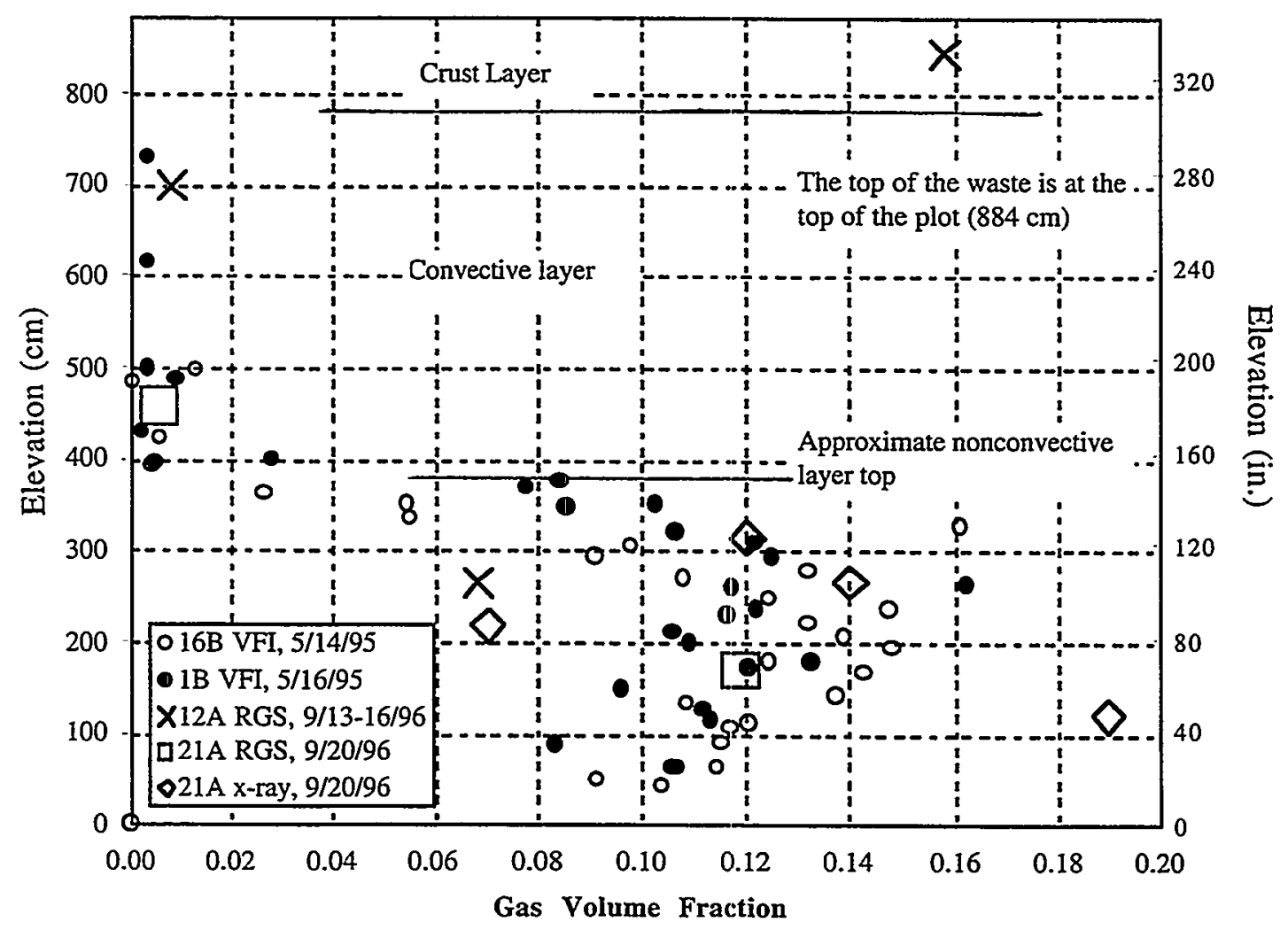

Figure 4.5.3. Gas Volume Fractions Measured by RGS and VFI 
gas samples may explain the difference between RGS and VFI\&RGS inventories. The X-ray visible gas fractions (which typically underestimate the extracted gas fractions) are included in Figure 4.5.3.

The uncertainties of the three inventories (RGS, VFI\&RGS, and BPE) overlap. The VFI\&RGS gas inventory is preferable to the other inventories, being based on direct nonconvective layer gas measurements from four risers.

Table 4.5.9 contains the calculated layer inventories of each of the major gases retained in the waste. The gas-phase inventories are based on the layer-average compositions from RGS data (Table 4.5.6) and the best-estimate gas inventories for each layer (VFI\&RGS, Table 4.5.8). The dissolved inventories are based on RGS data alone.

Figure 4.5.4 shows the sample temperatures, corrected gas volume fractions (Table 4.5.7), and corrected compositions of the low-solubility constituents in the samples from Tank AN-103. The temperatures are based on data from the multi-function instrument tree (MTT) in riser 15A. The compositions represent the mole fraction of the species in the "insoluble" gas; water and ammonia are not included, so the mole fractions are not the same as those in Table 4.5.4. The gas volume fractions and mole fractions in the figure are the values for lower-bound gas solubilities. Figure 4.5.4 also lists the observations from core extrusions of non-RGS samples and from X-rays of RGS samples as a way of tying those observations together with RGS data. The X-ray data are described in more detail in Section 4.5.4.

Table 4.5.9. Speciated AN-103 Gas Inventory(a)

\begin{tabular}{|c|c|c|c|c|c|c|}
\hline \multirow[b]{2}{*}{ Species } & \multicolumn{3}{|c|}{$\begin{array}{c}\text { Gas-Phase Inventory } \\
\left(\mathrm{m}^{3} \text { at STP }\right)\end{array}$} & \multicolumn{3}{|c|}{$\begin{array}{c}\text { Dissolved Inventory } \\
\left(\mathrm{m}^{3} \text { at STP }\right)\end{array}$} \\
\hline & $\begin{array}{l}\text { Crust } \\
\text { Layer }\end{array}$ & $\begin{array}{c}\text { Nonconvective } \\
\text { Layer }\end{array}$ & $\begin{array}{c}\text { Convective } \\
\text { Layer }\end{array}$ & $\begin{array}{l}\text { Crust } \\
\text { Layer }\end{array}$ & $\begin{array}{c}\text { Nonconvective } \\
\text { Layer }\end{array}$ & $\begin{array}{c}\text { Convective } \\
\text { Layer }\end{array}$ \\
\hline $\mathrm{N}_{2}$ & 15 & 120 & $7.5-6.8$ & $0.0048-0.049$ & $0.042-0.56$ & $0.10-0.84$ \\
\hline $\mathrm{H}_{2}$ & 33 & 220 & 2.1-1.7. & $0.036-0.24$ & $0.27-2.3$ & $0.10-0.49$ \\
\hline $\mathrm{N}_{2} \mathrm{O}$ & $3.7-3.3$ & $15-13$ & $0.91-0.24$ & $0.068-0.47$ & $0.29-2.5$ & $0.68-1.4$ \\
\hline $\mathrm{NH}_{3}$ & $0.95-0.34$ & $3.2-1.1$ & 0.14 & 320 & 1000 & 1800 \\
\hline $\mathrm{CH}_{4}$ & 0.34 & 2.3 & $0.16-0.13$ & $0.00019-0.0021$ & $0.0014-0.021$ & $0.0037-0.020$ \\
\hline Other & 0.13 & 1.4 & 0.15 & 0 & 0 & 0 \\
\hline
\end{tabular}

(a) Gas-phase inventories based on layer-average compositions from RGS data (Table 4.5.6) and best-estimate gas inventories for each layer (VFI\&RGS, Table 4.5.8). Dissolved inventories based on RGS data alone. Uncertainty in each inventory is $50 \%$ based on spatial variability considerations discussed in Section 3.7.2. Inventories given for both lower- and upper-bound solubilities; lower-bound solubility basis first.

\subsubsection{X-Ray Results}

Table 4.5.10 compares X-ray- and extraction-derived gas volume fractions. The comparison shows that sample recovery was close to $100 \%$ for all the samples. This conclusion is based on the fact that the extracted uncorrected gas was equal to or a few volume percent more than the visible gas. Though RGS sample 21A-13 was not successfully extracted, a gas fraction of at least 0.12 was seen in its $\mathrm{X}$-ray (however, the leaking sampler valve could have accounted for some part 
Table 4.5.10. Summary of Observations from X-Ray Images of Tank AN-103

\begin{tabular}{|c|c|}
\hline Segment & Comments/Observations \\
\hline $\begin{array}{l}12 \mathrm{~A}-2 \\
\text { (RGS, } \\
9 / 13 / 96)\end{array}$ & $\begin{array}{l}\text { Bottom two-thirds of sampler featureless, then a narrow fracture bubble spanning the sampler, } \\
\text { above that about an inch of waste densely packed with } 2-3-\mathrm{mm} \text { bubbles. Topmost inch slightly } \\
\text { less dense, some bubbles visible. Slightly rough level top surface. Almost clean piston, fully } \\
\text { retracted. Gas gap } 7.6 \mathrm{~cm} \text { ( } 3 \text { in.). The visible gas volume fraction is therefore } 0.16 \text {. (Compare } \\
\text { with uncorrected sampler gas volume fraction of } 0.17 \text { obtained by gas extraction, Table } 4.5 .7 \text {.) }\end{array}$ \\
\hline $\begin{array}{l}12 \mathrm{~A}-3 \\
\text { (non-RGS, } \\
9 / 13 / 96)\end{array}$ & $\begin{array}{l}\text { The waste in the image was featureless. Fully retracted piston. No gas gap. The visible gas } \\
\text { volume fraction is therefore } 0 \text {. }\end{array}$ \\
\hline $\begin{array}{c}12 \mathrm{~A}-4 \\
\text { (non-RGS, } \\
9 / 13 / 96)\end{array}$ & $\begin{array}{l}\text { The waste in the image was featureless. Fully retracted piston. Gas gap was less than } 1 \mathrm{~mm} \text {. The } \\
\text { visible gas volume fraction is therefore } 0.002 \text {. }\end{array}$ \\
\hline $\begin{array}{l}12 \mathrm{~A}-5 \\
(\mathrm{RGS} \\
9 / 13 / 96)\end{array}$ & $\begin{array}{l}\text { The waste in the image was featureless. Fully retracted piston. Gas gap was } 5 \mathrm{~mm}(0.2 \mathrm{in} \text {.). The } \\
\text { visible gas volume fraction is therefore } 0.01 \text {. (Compare with the uncorrected sampler gas volume } \\
\text { fraction of } 0.020 \text { in Table } 4.5 .7 \text { obtained by gas extraction.) }\end{array}$ \\
\hline $\begin{array}{l}21 \mathrm{~A}-13 \text {. } \\
\text { (RGS, } \\
9 / 20 / 96)\end{array}$ & $\begin{array}{l}\text { Thick 1- to 5-mm bubbles; about halfway up was a group of bubbles about } 1 \mathrm{~cm} \text { across. About } \\
\text { two-thirds of the way up was a bullet-shaped, flat-bottomed void about } 15 \mathrm{~mm} \text { ( } 0.6 \mathrm{in} \text {.) across and } \\
2.5 \mathrm{~cm} \text { ( } 1 \text { in.) high. Above that, another arched gap about } 20 \mathrm{~mm}(0.8 \mathrm{in} \text {.) high spanned the } \\
\text { sampler with about an inch of waste between top of void and waste surface. Slightly lumpy } \\
\text { surface; small bit of waste on fully retracted piston. } 3.0-\mathrm{cm} \text { (1.2-in.) gas gap; visible gas volume } \\
\text { fraction (counting large voids but not small bubbles) is } 0.12 \text {. The valve on this sampler leaked. }\end{array}$ \\
\hline $\begin{array}{l}\text { 12A-14 } \\
\text { (RGS, } \\
\text { 9/16/96) }\end{array}$ & $\begin{array}{l}\text { Dense, }<1-\mathrm{mm} \text { bubbles in bottom third of sampler, accompanied by some large bubbles (up to } \\
5 \mathrm{~mm} \text {, possibly flattened against sampler wall) in the center third. In top third, very large bubbles } \\
\text { up to } 15 \mathrm{~mm} \text { across. Slightly rough surface; clean, fully retracted piston. } 1.3 \text {-mm ( } 0.05 \text {-in.) gas } \\
\text { gap. The visible gas volume fraction (not counting bubbles) is therefore } 0.003 \text {. (Compare with } \\
\text { the uncorrected sampler gas volume fraction of } 0.071 \text { obtained by gas extraction, in Table } 4.5 .7 \text {.) }\end{array}$ \\
\hline $\begin{array}{l}21 \mathrm{~A}-14 \\
\text { (non-RGS, } \\
9 / 20 / 96)\end{array}$ & $\begin{array}{l}\text { Dense, } 2-10-\mathrm{mm} \text { bubbles, tending to be larger or in clusters toward the top of the sampler. Flat- } \\
\text { bottomed, ragged fracture bubble across half the sampler width, and at most } 15 \mathrm{~mm}(0.6 \text { in.) tall, } \\
\text { not far below top of waste. Slightly lumpy waste surface, a little waste on piston (which is about } \\
0.3 \text { in short of full retraction). Gas gap about } 6.4 \mathrm{~cm}(2.5 \mathrm{in} .) \text {. The visible gas volume fraction } \\
\text { (not counting bubbles) is therefore } 0.14 \text {. }\end{array}$ \\
\hline $\begin{array}{c}21 \mathrm{~A}-15 \\
\text { (non-RGS, } \\
9 / 20 / 96)\end{array}$ & $\begin{array}{l}\text { Dense, } 1-2-\mathrm{mm} \text { bubbles near bottom, grouped into large bubbles nearer the top of the sampler. } \\
\text { About halfway up, a slanted, thin fracture bubble. Fewer bubbles in the top few inches, slightly } \\
\text { rough waste surface. Small amount waste across most of piston (which is about } 0.3 \text { in short of } \\
\text { full retraction). Gas gap about } 3.0 \mathrm{~cm} \text { ( } 1.2 \text { in.). The visible gas volume fraction (not counting } \\
\text { bubbles) is therefore } 0.07 \text {. }\end{array}$ \\
\hline $\begin{array}{l}21 \mathrm{~A}-16 \\
\text { (RGS, } \\
\text { 9/20/96) }\end{array}$ & $\begin{array}{l}\text { Dense, }<1-\mathrm{mm} \text { bubbles near bottom of sampler. More } 1-3-\mathrm{mm} \text { bubbles, frequently linear, toward } \\
\text { the top; and a number of } 3-\mathrm{mm} \text { bubbles, closely packed, in the top few inches. Fully retracted } \\
\text { piston; no gas gap. The visible gas volume fraction is therefore } 0 \text {. (Compare with the uncorrected } \\
\text { sampler gas volume fraction of } 0.12 \text { obtained by gas extraction, Table } 4.5 .7 . \text { ) }\end{array}$ \\
\hline $\begin{array}{l}21 \mathrm{~A}-17 \\
\text { (non-RGS, } \\
9 / 20 / 96)\end{array}$ & $\begin{array}{l}\text { Thin, linear bubble near bottom of sampler, closely packed } 1-\text { to } 2-\mathrm{mm} \text { bubbles, a group of larger } \\
\text { bubbles (up to } 3 \mathrm{~mm} \text { ) toward top. Slightly rough, level surface, small bit of waste on fully } \\
\text { retracted piston. } 8.9-\mathrm{cm} \text { ( } 3.5 \text {-in.) gas gap; visible gas volume fraction (not counting bubbles) is } \\
\text { therefore } 0.19 \text {. }\end{array}$ \\
\hline
\end{tabular}



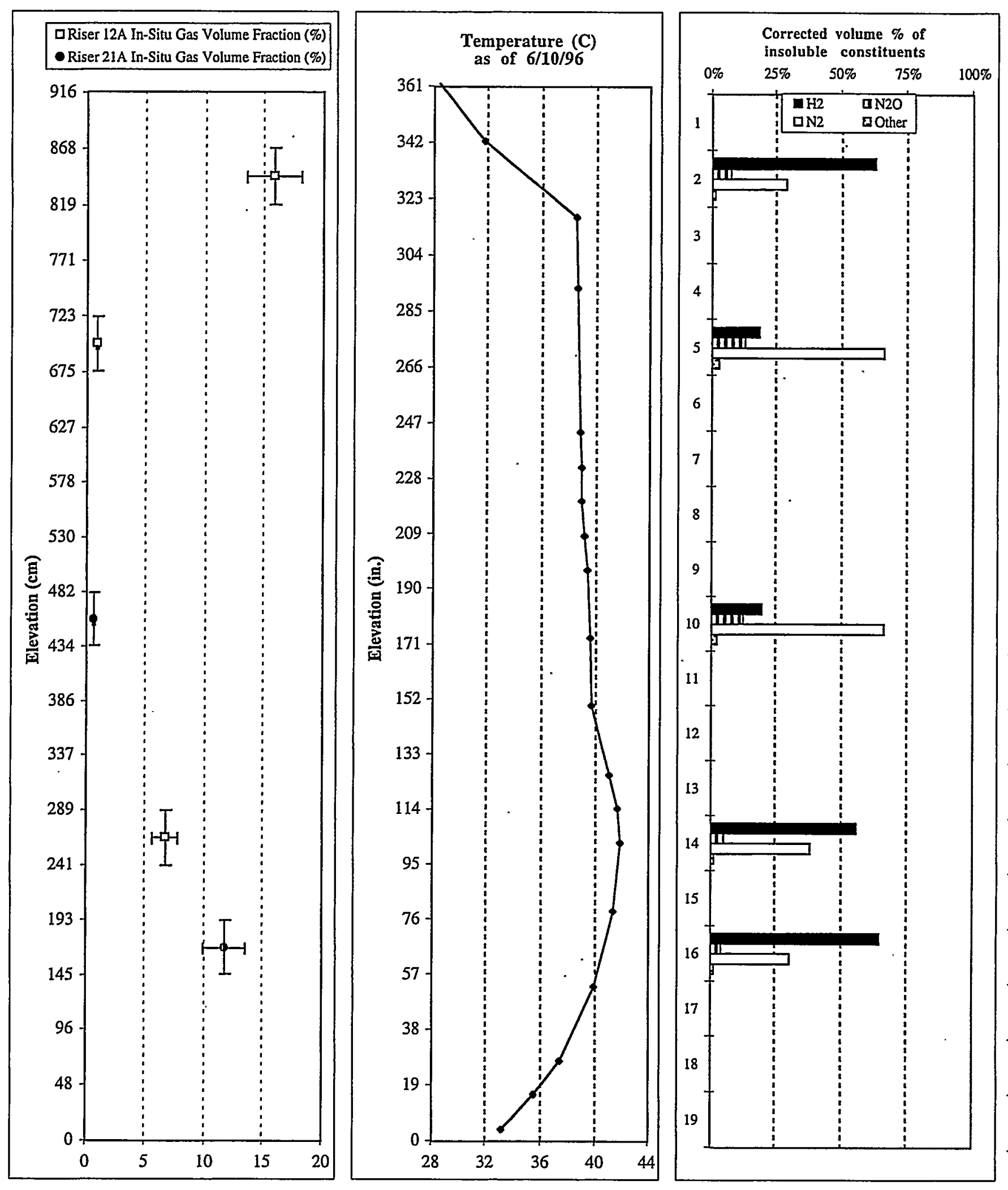

\begin{tabular}{l}
1 \\
\hline 2 \\
2 \\
\hline 3 \\
3 \\
\hline 4 \\
\hline 5 \\
5 \\
\hline 6 \\
6 \\
7 \\
7 \\
\hline 8 \\
\hline 9 \\
9 \\
\hline 1 \\
1 \\
\hline 1 \\
1 \\
\hline 1 \\
1 \\
\hline 1 \\
1 \\
1 \\
\hline 1 \\
1 \\
1 \\
1 \\
1 \\
\hline 1 \\
\hline
\end{tabular}

Figure 4.5.4. Gas Volume fractions, Temperatures, Cor 
$\mathrm{N}-103$ waste level is $884 \mathrm{~cm}$ (348 inches).

ser 12A: 2.5 in. $(58 \mathrm{~g})$ light gray wet salt.

Ser 21A: 14 in. ( $325 \mathrm{~g})$ light gray moist salt.

ser 12A X-RAY: Thin fracture bubble 2 in. down, 2-3 mm dense bubbles above that, featureless below. Gas gap 3 in.

ser 21A: 6 in. (112 g) gray to white salt slurry, $190 \mathrm{~mL}(266 \mathrm{~g}$ ) blue-green opaque liquid.

ser 12A: $260 \mathrm{~mL}(410 \mathrm{~g})$ blue-green opaque liquid. $\mathrm{X}$-ray featureless, no gas gap.

ser 21A: 1 in. ( $31 \mathrm{~g})$ white salt slurry, $250 \mathrm{~mL}(368 \mathrm{~g})$ blue-green opaque liquid.

iser 12A: 2 in. (64 g) white salt slurry, $250 \mathrm{~mL}(369 \mathrm{~g})$ blue-green opaque liquid. X-ray featureless, gas gap $<1 \mathrm{~mm}$.

ser 21 A: 1 in. $(30 \mathrm{~g})$ white salt slurry, $250 \mathrm{~mL}(360 \mathrm{~g})$ blue-green opaque liquid.

iser 12A X-RAY: Featureless, liquid meniscus top, piston nearly clean, gas gap 0.2 in.

iser 21A: 5 in. $(80 \mathrm{~g})$ white salt slurry, $250 \mathrm{~mL}(348 \mathrm{~g}$ ) blue-green opaque liquid.

iser 12A: 2 in. $(63 \mathrm{~g})$ white salt slurry, $250 \mathrm{~mL}(358 \mathrm{~g})$ blue-green opaque liquid.

iser 21A: 3 in. $(75 \mathrm{~g})$ white wet salt, $250 \mathrm{~mL}(349 \mathrm{~g})$ blue-green opaque liquid.

iser 12A: 4 in. $(65 \mathrm{~g})$ white sait slurry, $250 \mathrm{~mL}(369 \mathrm{~g})$ blue-green opaque liquid.

iser 21 A: 2 in. ( $43 \mathrm{~g}$ ) white salt slurry, $250 \mathrm{~mL}(371 \mathrm{~g})$ blue-green opaque liquid.

iser 12A: 4 in. ( $48 \mathrm{~g}$ ) white salt slurry, $250 \mathrm{~mL}(370 \mathrm{~g})$ blue-green opaque liquid.

iser 21A: $1.5 \mathrm{in}$. (45 g) gray salt slurry, $260 \mathrm{~mL}$ ( $378 \mathrm{~g}$ ) blue-green opaque liquid.

iser 12A: 3 in. $(60 \mathrm{~g}$ ) white salt slurry, $260 \mathrm{~mL}(383 \mathrm{~g})$ blue-green opaque liquid.

iser 21A: $5.5 \mathrm{in}$. $(64 \mathrm{~g})$ white salt slurry, $250 \mathrm{~mL}(364 \mathrm{~g}$ ) blue-green opaque liquid.

iser 12A: 2 in. $(58 \mathrm{~g}$ ) white salt slurry, $260 \mathrm{~mL}(388 \mathrm{~g}$ ) blue-green opaque liquid.

iser 21A X-RAY: not available.

iser 12A: 1 in. $(49 \mathrm{~g})$ white salt slurry, $260 \mathrm{~mL}(360 \mathrm{~g})$ blue-green opaque liquid.

iser 21A: 16 in. (375 g) light gray wet salt, $40 \mathrm{~mL}(5 \mathrm{~g})$ gray opaque liquid.

iser 12A: 3 in. $(60 \mathrm{~g})$ white salt slurry, $250 \mathrm{~mL}$ ( $344 \mathrm{~g})$ blue-green opaque liquid.

iser 21A: 18 in. (452 g) light gray salt slurry.

iser 12A: 19 in. $(452 \mathrm{~g})$ light gray wet salt.

iser 21A X-RAY: Two large arched flat-bottomed voids, thick 1 to $5 \mathrm{~mm}$ bubbles. Lumpy surface, 1.2 in. gas gap.

iser 12A X-RAY: Dense < $1 \mathrm{~mm}$ bubbles, some larger (up to $15 \mathrm{~mm}$ ) near top. Slightly rough surface, $1 \mathrm{~mm}$ gas gap.

iser 21A: 15 in. $(406 \mathrm{~g}$ ) light gray wet salt. X-ray dense $2-10 \mathrm{~mm}$ bubbles, flat-bottomed fracture bubble near top.

iser 12A: 19 in. $(457 \mathrm{~g})$ light gray wet salt.

iser 21A: $18 \mathrm{in}$. (445 g) gray moist salt. X-ray dense $1-2 \mathrm{~mm}$ bubbles in clusters, slanted thin fracture bubble.

iser 12A: 19 in. (435 g) light gray moist salt.

iser 21A X-RAY: Dense < $1 \mathrm{~mm}$ bubbles, $1-3 \mathrm{~mm}$ linear voids, dense $3 \mathrm{~mm}$ bubbles near top. No gas gap.

iser 12A: 19 in. (405 g) light gray moist salt.

iser 21A: 14 in. (299 g) light gray moist salt. X-ray 1-2 mm bubbles bottom, 3-mm near top, $3.5 \mathrm{in}$. gas gap.

iser 12A: 19 in. $(416 \mathrm{~g})$ light tan-gray moist salt.

iser 21A X-RAY: not available.

iser 12A: 19 in. (391 g) gray salt slurry.

iser 21A: not available

ositions, and Observations in Samples from Tank AN-103 
of this). High gas was also visible in samples 21A-14 (0.14 gas fraction) and 21A-17 (0.19 gas fraction). These high gas contents, which were not included in the RGS gas inventory (Table 4.5.8), help explain why the RGS gas inventory was lower than the other inventories.

The fracture bubbles and adhering lumps of waste observed in several samples suggest high waste strength. The fact that none of the $12 \mathrm{vol} \%$ of gas that was extracted from sample 21A-16 appeared in the gas gap is also consistent with this interpretation. Gas retention experiments were carried out on an AN-103 composite sample and showed bubbles that were distorted, apparently by waste strength (Rassat et al. 1997).

Table 4.5.11 provides a summary of the waste densities that have been calculated from radiographic data for the RGS samples for which air and water standard images were available.The radiographic densities include the gas in the waste and are given for several locations within

Table 4.5.11. Densities of AN-103 Samples from Radiography and Core Samples

\begin{tabular}{|c|c|c|c|c|}
\hline $\begin{array}{l}\text { Riser- } \\
\text { Segment } \\
\text { Number }\end{array}$ & $\begin{array}{c}\text { Distance } \\
\text { from Bottom } \\
\text { of Sampler } \\
(\mathrm{ft})\end{array}$ & $\begin{array}{c}\text { Mean Local } \\
\text { Density by } \\
\text { Current Method } \\
\text { (g/cc) }\end{array}$ & $\begin{array}{c}\text { Overall Average } \\
\text { Sample Densities } \\
\text { (g/cc) }\end{array}$ & $\begin{array}{c}\text { Degassed Density in non-RGS } \\
\text { Cores above and below RGS } \\
\text { Sample (g/cc) }\end{array}$ \\
\hline \multirow[t]{4}{*}{$12 \mathrm{~A}-2$} & 1.0 & 1.86 & \multirow{4}{*}{$\begin{array}{l}\text { Current } x \text {-ray analysis } \\
\text { method: } \quad 1.88 \\
\text { Original } x \text {-ray analysis } \\
\text { method: } \quad 2.07 \text { (a) }\end{array}$} & \multirow[b]{4}{*}{ density below, $1.47 \mathrm{~g} / \mathrm{cc}$ liquid } \\
\hline & 0.75 & 1.89 & & \\
\hline & 0.5 & 1.94 & & \\
\hline & 0.25 & 1.81 & & \\
\hline \multirow[t]{5}{*}{$12 \mathrm{~A}-5$} & 1.25 & 2.00 & \multirow{5}{*}{$\begin{array}{l}\text { Current } x \text {-ray analysis } \\
\text { method: } \quad 1.99 \\
\\
\text { Original x-ray analysis } \\
\text { method: } \quad 2.12 \text { (a) }\end{array}$} & \multirow[t]{5}{*}{ density above, $1.46 \mathrm{~g} / \mathrm{cc}$ liquid } \\
\hline & 1.0 & 1.91 & & \\
\hline & 0.75 & 1.99 & & \\
\hline & 0.5 & 1.99 & & \\
\hline & 0.25 & 2.15 & & \\
\hline \multirow[t]{5}{*}{$21 \mathrm{~A}-13$} & 1.25 & $0 . \overline{83}$ & \multirow{5}{*}{$\begin{array}{l}\text { Current } x \text {-ray analysis } \\
\text { method: } 1.54 \\
\text { Original } x \text {-ray analysis } \\
\text { method: } \quad 2.01 \text { (a) }\end{array}$} & \multirow[t]{5}{*}{ density above, $1.69 \mathrm{~g} / \mathrm{cc}$ bulk } \\
\hline & 1.0 & 1.45 & & \\
\hline & 0.75 & 1.62 & & \\
\hline & 0.5 & 1.56 & & \\
\hline & 0.25 & 1.46 & & \\
\hline \multirow[t]{6}{*}{$12 \mathrm{~A}-14$} & 1.5 & 1.74 & \multirow{6}{*}{$\begin{array}{l}\text { Current x-ray analysis } \\
\text { method: } 1.53 \\
\text { Original x-ray analysis } \\
\text { method: } \quad 1.85 \text { (a) }\end{array}$} & \multirow[t]{6}{*}{ density above, $1.65 \mathrm{~g} / \mathrm{cc}$ bulk } \\
\hline & 1.25 & 1.45 & & \\
\hline & 1.0 & 1.51 & & \\
\hline & 0.75 & 1.55 & & \\
\hline & 0.5 & 1.60 & & \\
\hline & 0.25 & 1.45 & & \\
\hline \multirow[t]{5}{*}{$21 \mathrm{~A}-16$} & 1.25 & 1.74 & \multirow{5}{*}{$\begin{array}{l}\text { Current } x \text {-ray analysis } \\
\text { method: } \quad 1.71 \\
\\
\text { Original } x \text {-ray analysis } \\
\text { method: } \quad 2.06 \text { (a) }\end{array}$} & \multirow[t]{5}{*}{ density above, $1.88 \mathrm{~g} / \mathrm{cc}$ bulk } \\
\hline & 1.0 & 1.75 & & \\
\hline & 0.75 & 1.70 & & \\
\hline & 0.5 & 1.69 & & \\
\hline & 0.25 & 1.55 & & \\
\hline
\end{tabular}


each sample to show density trends. The overall sample densities were calculated by the original method (described in Section 3.9) and also by the current x-ray analysis method. The differences give some idea of the sensitivity of results to small variations in method. Table 4.5.11 also includes density measurements made on extruded non-RGS samples (Field 1999b). The radiographic densities in two of the samples (12A-2 and 12A-5) greatly exceeded the densities found for the adjacent extruded samples, which is difficult to explain because the radiographic densities included gas and the extruded densities did not. The other three samples showed densities consistent with those measured for extrusions.

\subsubsection{Drillstring and Domespace Composition Comparison}

The RGS gas-phase composition data (Table 4.5.6) were used to calculate $\mathrm{H}_{2} / \mathrm{N}_{2} \mathrm{O}$ ratios for comparison with ratios from drillstring and domespace grab sample measurements (Table 8.1, Siciliano 1998; McCain 1999, Table 4.3). The results can be seen in Table 4.5.12. While the RGS crust $\mathrm{H}_{2} / \mathrm{N}_{2} \mathrm{O}$ ratio was much the same as the drillstring ratio, the GRE domespace ratio was higher than other values and more similar to the ratio for the nonconvective layer RGS samples.

Table 4.5.12 Comparison with Drillstring and Domespace Data(a)

\begin{tabular}{|l|c|}
\hline Sample & $\mathrm{H}_{2} / \mathrm{N}_{2} \mathrm{O}$ \\
\hline \hline RGS, 12A-2 & $9.0-10$ \\
\hline RGS, 12A-5 & $2.2-6.5$ \\
\hline RGS, 21A-10 & $2.5-8.1$ \\
\hline RGS, 12A-14 & $11-14$ \\
\hline RGS, 21A-16 & $17-19$ \\
\hline Drillstring, riser 12A, 9/16/96 & 9.2 \\
\hline Domespace, 8/23/95 GRE & 20 \\
\hline Domespace, 1/29/97 & 10 \\
\hline $\begin{array}{l}\text { (a) RGS ratios calculated for both lower- and upper-bound solubilities. Both } \\
\text { ends of ratio range are given, lower-bound solubility basis first. }\end{array}$ \\
\hline
\end{tabular}




\section{$4.6 \quad \mathrm{U}-103$}

Tank 241-U-103 (U-103) was the sixth tank and the second SST sampled with the RGS. This tank was selected as representing the highest-priority group of SSTs that show evidence of significant gas retention (Stewart et al. 1996b). Tank U-103 is on the FGWL and exhibits high concentrations of $\mathrm{H}_{2}$ and $\mathrm{NH}_{3}$ in the domespace. It is part of a group of tanks (Cluster 13 in Stewart et al. 1996b) that have fairly high radioactivity, fairly low temperatures, high nitrite concentrations, and about $0.1 \%$ TOC. HTCEs.indicate that the primary waste stored in U-103 was saltcake from evaporator campaign S1, and the secondary waste was salt slurry from evaporator campaign S2 (Remund et al. 1995). (This is identical to the HTCE for Tank U-109, which is also in Cluster 13.) Of the RGS-sampled tanks, U-103, SX-106, S-102, and U-109 are all in Cluster 13.

Push-mode sampling was done in risers 7 and 13 in January and April 1997.(a) Sampling was also attempted in riser 2, but the waste (or some object imbedded in it) proved impenetrable. The approximate locations of various risers are depicted in Figure 4.6.1. Risers 2 and 7 are near the tank periphery, while riser 13 is at the center. The elevations of the RGS segments are depicted in Figure 4.6.2.

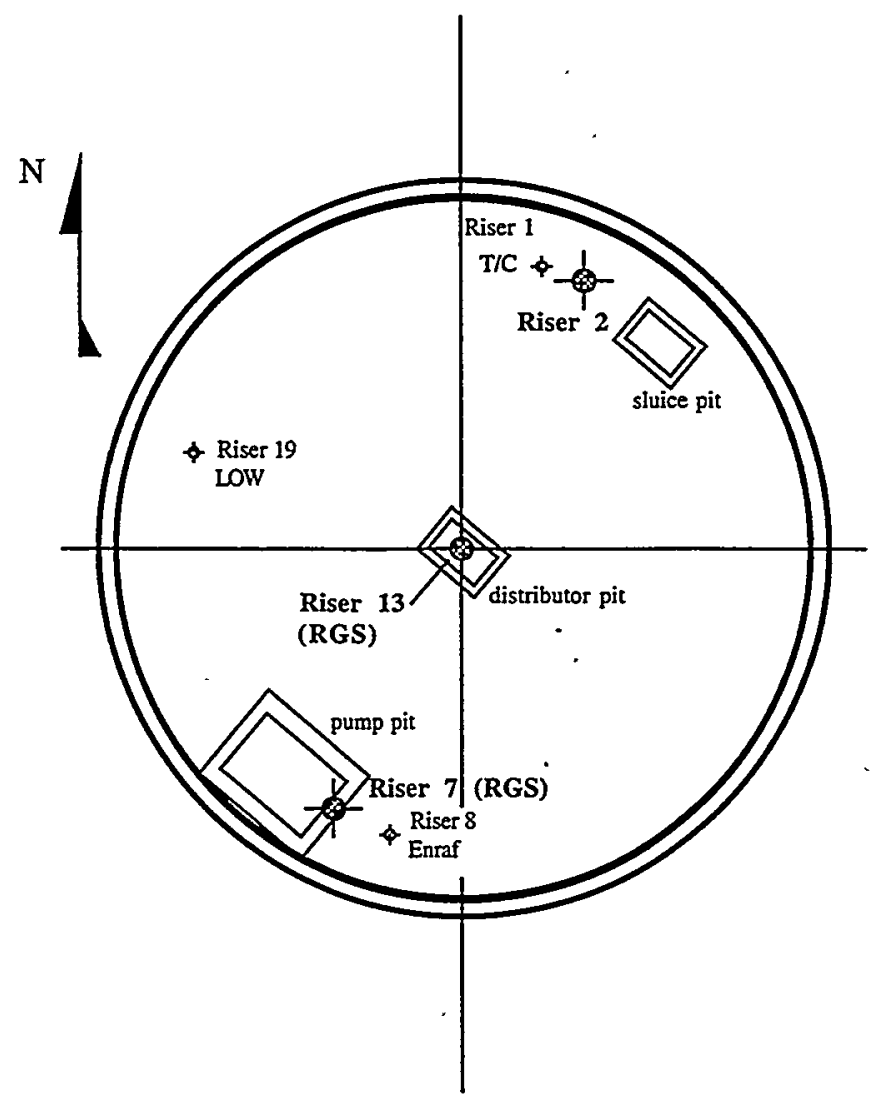

Figure 4.6.1. Schematic Diagram of Riser Locations in Tank U-103

(a) The sampling scheme may be found in Sampling Plan for Tank 241-U-103 Retained Gas Sampler Deployment, by JM Bates and A Shekarriz, October 1996. TWSMIT:091896 Rev. 2, Pacific Northwest National Laboratory, Richland, Washington. 


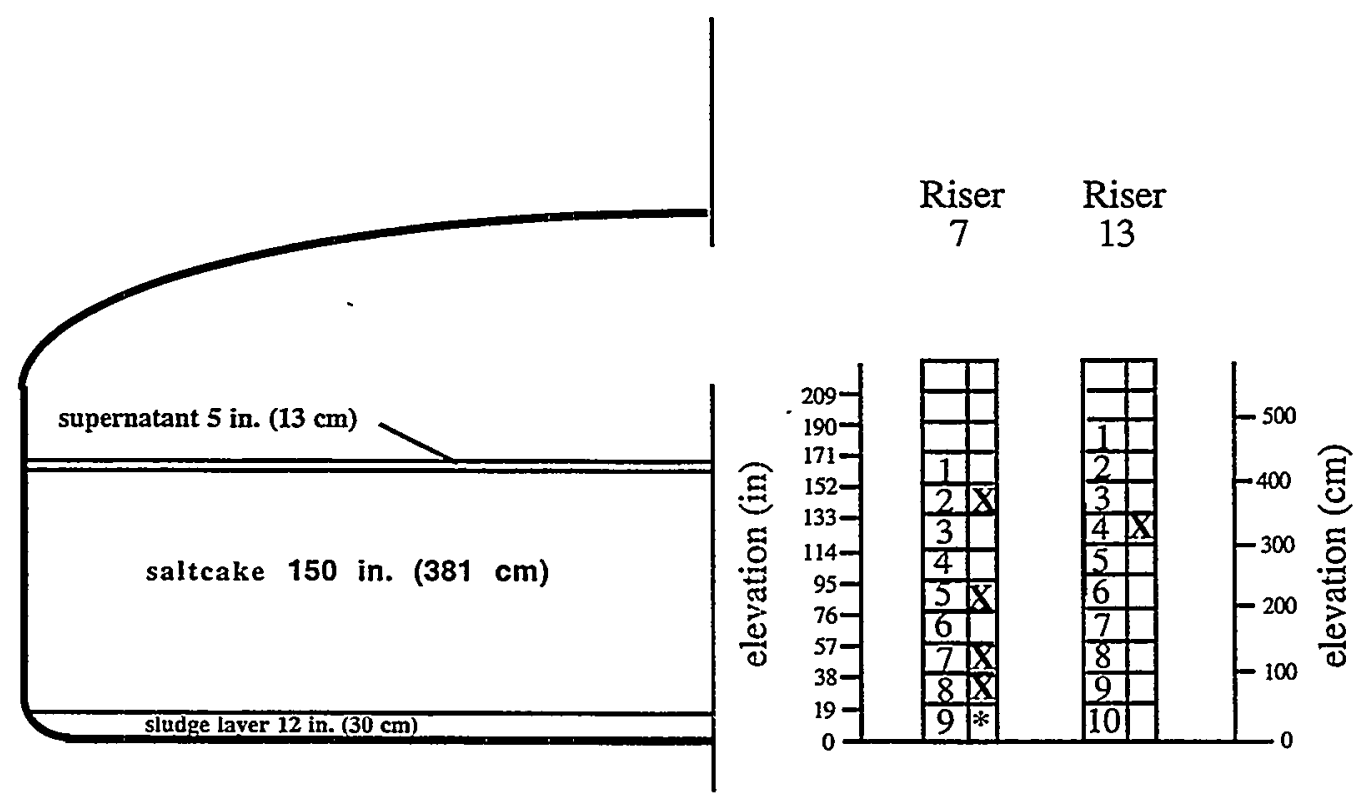

Figure 4.6.2. Diagram of Waste Layering and RGS Sample Elevations for U-103 (only 1.25 in. of riser 7, segment 9 couldbe taken because the waste was too hard or tank bottom reached. Sampling was attempted but abandoned in riser 2 because an obstacle or hard waste blocked the drill string)

The total depth of waste in Tank U-103 in December 1995 was approximately $424 \mathrm{~cm}$ (167 in.), according to manual Enraf readings (Hodgson et al. 1998). At the time of RGS sampling U-103 contained about 1,770,000 L (468,000 gal) of waste. The best-basis inventory (Sasaki 1998) estimated a tank supernatant volume of 54,900 L (14,500 gal), which corresponds to a tank supernatant layer thickness of $13 \mathrm{~cm}$ (5in.). The rest of the tank was filled with saltcake and (at the bottom) sludge, as shown in Figure 4.6.2.

The most recent information on tank content layering comes from core observations made in 1997 (Sasaki 1998). There was no sign of dry crust material in the top segments from risers 2 , 7 , or 13. The thickness of the top layer of liquid or salt slurry varied from zero to three segments over the three risers. (The temperature profile from the TC tree in riser 1 showed no recognizable convective liquid layer, perhaps because the riser is so close to the wall.) Another sign of lateral waste variability was the presence of sludge slurry at the top of riser 13 but not the other risers. Riser 13 also appeared to contain subsurface liquid (segments 8 and 9). Based on the low bromide concentrations in the samples, the liquid in these segments was not an artifact of HHF.

Degassed densities for the liquid and bulk solids in Tank U-103 were available from the non-RGS samples taken in 1997 (Sasaki 1998). Several samples contained drainable liquid with a density of $1422 \pm 78 \mathrm{~kg} / \mathrm{m}^{3}$. The bulk density of the samples from the nonconvective layer (comprising saltcake and sludge) had an average and standard deviation of $1712 \pm 97 \mathrm{~kg} / \mathrm{m}^{3}$. 
These densities were used for hydrostatic pressure calculations. Layer depths of $394 \mathrm{~cm}$ (155 in.) and $30 \mathrm{~cm}$ (12 in.) were used in calculations for the nonconvective and supernatant layers.

\subsubsection{Sampling and Extraction Information}

The samples that underwent RGS analysis are listed in Table 4.6.1. One sample, 13-4, was lost because the sampler valve froze closed after the sample was taken and could only be opened slightly. Some of the gas that leaked out through the valve could be collected, but a complete extraction was impossible. Only $\mathrm{x}$-ray data and insoluble gas composition data could be obtained; these data showed a gas composition and a sampler void space (a rough measure of gas quantity) that were consistent with the findings for segment 7-5. Field data, including dose rates and downforce limits, are supplied in Appendix A.

Table 4.6.1 also shows the lag times (delay between sample acquisition and processing) for these samples. This information was provided to allow data users to correlate the lag time between sampling and extrusion with the concentrations of the various constituents to test for decomposition or other chemical reactions. The maximum hold time allowed by the sampling plan is 24 days based on measured sampler leakage rates during acceptance testing. All the sample hold times were below this limit.

As was discussed in Section 3.5, the HHF tracer often enters the sampler in place of some of the waste. Table 4.6.2 shows the volume percentage contamination by HHF of the RGS samples in riser 7. One of these samples (7-5) contained bromide below the detection limit. Averaging the HHF contamination over all riser 7 RGS samples and treating the below-detection sample as if at the detection limit gave an average contamination of $6.6 \mathrm{vol} \%$. This value was used to calculate a reduced effective sampler volume for RGS sample 7-2, for which there was no bromide data). The actual measured HHF contamination percentages were used for RGS samples 7-7 and 7-8.) (It is conservative to use the MDL for values below it; the conservatism is probably less than 3\%.)

All of these samplers were helium-backfilled and sealed with vacuum grease before they were deployed. This method virtually eliminated air and argon contamination from gases entrained during sampling. The helium backfill results are discussed in detail by Cannon (1997) and summarized in Section 5 of Mahoney et al. (1997).

Three different extraction procedures were used for U-103 samples. (See Figure 3.1 for a schematic of the RGS extraction system.) Isotopic vapor addition was used only on the first U-103 sample that was extracted, sample 7-2. The heating cycle step was not used on samples from U-103 or any subsequently sampled tank; based on experience from the first five tanks, heating was felt to increase the complexity of the procedure without improving results.

Table 4.6.1. Lag Times for Processing RGS Samples from Tank 241-U-103

\begin{tabular}{||c|c|c|c||}
\hline Sample & Acquisition Date & Processing Date & Lag (days) \\
\hline \hline $7-2$ & January 21, 1997 & February 4, 1997 & 14 \\
\hline $7-5$ & January 22, 1997 & February 6, 1997 & 15 \\
\hline $7-7$ & January 22,1997 & February 12, 1997 & 21 \\
\hline $7-8$ & January 22, 1997 & February 13,1997 & 22 \\
\hline $13-4$ & April 2, 1997 & April 23, 1997 & 21 \\
\hline
\end{tabular}


Table 4.6.2. Hydrostatic Head Fluid Contamination in U-103 RGS Samples

\begin{tabular}{|c|c|}
\hline Sample & $\begin{array}{c}\text { HHF Contamination } \\
\text { (vol \%) }\end{array}$ \\
\hline \hline $7-2$ & no data \\
\hline $7-5$ & $<7.3$ \\
\hline $7-7$ & 4.3 \\
\hline $7-8$ & 4.9 \\
\hline
\end{tabular}

Sample 7-2: After evacuating the whole system and observing the pressure to test for leaks, the operator extruded the RGS sample into the extractor vessel and the sample was briefly stirred. At this point the vapor space in communication with the sample consisted of the volumes of the extractor vessel and its lines. Next, the extractor was opened to the second vessel, to which was connected a PQ canister. Once a grab sample was collected in the PQ canister, it was closed. The extractor, lines, and second volume were then connected to the pump volume and collector side; the latter included the collector line volume and one open collection canister. The first canister, containing a sample of uncompressed (unpumped) gas, was closed off. The second collection canister was opened, several strokes were pumped from the extractor to the collector, and the second collection canister was closed off.

Then the collector side was evacuated, but not the second volume and extractor lines. A canister containing $15 \mathrm{NH}_{3}$ vapor was connected to the extractor side and opened to inject isotopic ammonia. Several strokes were pumped from the extractor to the collector and the third collection canister closed off. Finally, the BSVD procedure was performed: argon was injected into the closed-off second volume, and that volume was opened to the extractor and lines. Pressure measurements and known tare system volumes allowed the volume of the sample solids and liquid to be calculated.

Sample 7-5: After evacuating the whole system and observing the pressure to test for leaks, the operator extruded the RGS sample into the extractor vessel, and the sample was briefly stirred. At this point the vapor space in communication with the sample consisted of the volumes of the extractor vessel and its lines. Next, the extractor was opened to the second vessel, to which was connected a PQ canister. Once a grab sample was collected in the PQ canister, it was closed. The extractor, lines and second volume were then connected to the pump volume and collector side; the latter included the collector line volume and two open collection canisters. These first two canisters, containing a sample of uncompressed (unpumped) gas, were closed off. The second collection canister was opened and a second PQ canister taken from the second vessel. Several strokes were pumped from the extractor to the collector, and the second collection canister was closed off. Finally, the BSVD procedure was performed.

Samples 7-7, 7-8: After evacuating the whole system and observing the pressure to test for leaks, the operator extruded the RGS sample into the extractor vessel, and the sample was briefly stirred. At this point the vapor space in communication with the sample consisted of the volumes of the extractor vessel and its lines. Next, the extractor was opened to the second vessel, to which was connected a PQ canister. Once a grab sample was collected in the PQ canister, it was closed. The extractor, lines and second volume were then connected to the pump volume and collector side; the latter included the collector line volume and one open collection canister. This first collection canister was collected unpumped and closed off, and a second one opened. Several strokes were pumped from the extractor to the collector and the second collection canister closed off. The 
third collection canister was opened. Several strokes were pumped from the extractor to the collector and the third collection canister closed off. Finally, the BSVD procedure was performed.

Procedural details such as the number of strokes per canister and the system volumes can be found in the sample-by-sample worksheets in the U-103 RGS calculation spreadsheet on the CD supplied with this report. A very detailed run-through of the procedures can also be found in Appendix A of Mahoney et al. (1997).

\subsubsection{Retained Gas Composition}

Table 4.6.3 presents the calculated concentrations of the insoluble gases in each RGS sample taken from U-103 without corrections for air and argon entrainment, helium backfill, air inleakage, or air added with the isotopic ammonia. The method used to make the corrections depended on the gas. All of the oxygen, argon, and helium were subtracted no matter which step had produced them. A number of moles equal to $(3.73) \cdot\left(\mathrm{O}_{2}\right)$ were subtracted from the nitrogen because the oxygen was expected to have come from air entrainment during sampling or inleakage during extraction. The corrected concentrations are given in Table 4.6.4. Contamination observations can be found in the "Summary" worksheet of the U-103 RGS calculations spreadsheet on the CD.

Table 4.6.5 presents the ammonia measurements. The RGS procedure did not permit determining the residual or total ammonia except for the isotopically measured sample 7-2. The post-extrusion partial pressure of $\mathrm{NH}_{3}$ over the sample (see Section 3.4.4) was measured at laboratory temperature. There were no post-RGS ammonia measurements made by ISE for U-103 samples and, although PQ canisters were taken, their contents were not analyzed.

Table 4.6.3. Concentrations of Insoluble Constituents ( $\mu \mathrm{mol} / \mathrm{L}$ of waste) in Tank U-103 Without Correction for Gas Contamination

\begin{tabular}{||c|c|c|c|c|c|c|c|c|c|c|c||}
\hline \hline Begment & $\mathrm{N}_{2}$ & $\mathrm{H}_{2}$ & $\mathrm{~N}_{2} \mathrm{O}$ & $\mathrm{O}_{2}$ & $\mathrm{CH}_{4}$ & $\mathrm{He}$ & $\mathrm{Ar}$ & $\begin{array}{c}\text { Other } \\
\mathrm{NOx}\end{array}$ & $\mathrm{C}_{2} \mathrm{H}_{x}$ & $\mathrm{C}_{3} \mathrm{H}_{x}$ & $\begin{array}{c}\text { Other } \\
\text { Hyd. }\end{array}$ \\
\hline \hline $7-2$ & $6800 \pm 270$ & $4200 \pm 160$ & $7300 \pm 270$ & $59 \pm 18$ & $73 \pm 4.2$ & $510 \pm 97$ & $12 \pm 1.5$ & $2.2 \pm 1.7$ & $74 \pm 7.0$ & $0.02 \pm 0.02$ & $6.4 \pm 1.7$ \\
\hline $7-5$ & $1600 \pm 64$ & $720 \pm 28$ & $2700 \pm 100$ & $11 \pm 0.4$ & $12 \pm 2.7$ & $400 \pm 16$ & $2.2 \pm 0.2$ & $0 \pm 0$ & $11 \pm 4.4$ & $1.9 \pm 0.9$ & $3.9 \pm 0.9$ \\
\hline $7-7$ & $2600 \pm 110$ & $1500 \pm 64$ & $2100 \pm 87$ & $6.2 \pm 0.3$ & $37 \pm 6.0$ & $510 \pm 21$ & $2.1 \pm 0.1$ & $9.2 \pm 4.6$ & $49 \pm 4.8$ & $0.02 \pm 0.01$ & $5.1 \pm 1.1$ \\
\hline $7-8$ & $1700 \pm 110$ & $1400 \pm 94$ & $1500 \pm 100$ & $10 \pm 0.8$ & $33 \pm 5.5$ & $450 \pm 30$ & $2.7 \pm 0.2$ & $20 \pm 3.9$ & $51 \pm 5.2$ & $0.8 \pm 0.4$ & $5.3 \pm 1.0$ \\
\hline
\end{tabular}

Table 4.6.4. Concentrations of Insoluble Constituents ( $\mu \mathrm{mol} / \mathrm{L}$ of waste) in Tank U-103 with Correction for Gas Contamination

\begin{tabular}{|c|c|c|c|c|c|c|c|c|c|c|c||}
\hline Segment & $\mathrm{N}_{2}$ & $\mathrm{H}_{2}$ & $\mathrm{~N}_{2} \mathrm{O}$ & $\mathrm{O}_{2}$ & $\mathrm{CH}_{4}$ & $\mathrm{He}$ & $\mathrm{Ar}$ & $\begin{array}{c}\text { Other } \\
\mathrm{NOx}\end{array}$ & $\mathrm{C}_{2} \mathrm{H}_{x}$ & $\mathrm{C}_{3} \mathrm{H}_{x}$ & $\begin{array}{c}\text { Other } \\
\text { Hyd. }\end{array}$ \\
\hline \hline $7-2$ & $6600 \pm 270$ & $4200 \pm 160$ & $7300 \pm 270$ & $0 \pm 18$ & $73 \pm 4.2$ & $0 \pm 97$ & $0 \pm 1.5$ & $2.2 \pm 1.7$ & $74 \pm 7.0$ & $0.02 \pm 0.02$ & $6.4 \pm 1.7$ \\
\hline $7-5$ & $1600 \pm 63$ & $720 \pm 28$ & $2700 \pm 100$ & $0 \pm 0.4$ & $12 \pm 2.7$ & $0 \pm 16$ & $0 \pm 0.2$ & $0 \pm 0$ & $11 \pm 4.4$ & $1.9 \pm 0.9$ & $3.9 \pm 0.9$ \\
\hline $7-7$ & $2600 \pm 110$ & $1500 \pm 64$ & $2100 \pm 87$ & $0 \pm 0.3$ & $37 \pm 6.0$ & $0 \pm 21$ & $0 \pm 0.1$ & $9.2 \pm 4.6$ & $49 \pm 4.8$ & $0.02 \pm 0.01$ & $5.1 \pm 1.1$ \\
\hline $7-8$ & $1700 \pm 110$ & $1400 \pm 94$ & $1500 \pm 100$ & $0 \pm 0.8$ & $33 \pm 5.5$ & $0 \pm 30$ & $0 \pm 0.2$ & $20 \pm 3.9$ & $51 \pm 5.2$ & $0.8 \pm 0.4$ & $5.3 \pm 1.0$ \\
\hline
\end{tabular}


Table 4.6.5. Ammonia Data from Tank U-103 Samples

\begin{tabular}{|c|c|c|c|}
\hline Sample & $\begin{array}{c}\text { Isotopically } \\
\text { Measured } \\
\text { Total NH } \\
(\mu \mathrm{mol} / \mathrm{L})\end{array}$ & $\begin{array}{l}\text { Measured } \mathrm{NH}_{3} \\
\text { partial pressure at } \\
\text { about } 24^{\circ} \mathrm{C} \\
(\mathrm{atm})\end{array}$ & Best-Estimate $\mathrm{NH}_{3}$ Concentrations \\
\hline $7-2$ & $2600 \pm 780^{(a)}$ & -0.0063 & \multirow{4}{*}{$\begin{array}{l}\text { The RGS ammonia data are question- } \\
\text { able but suggest a lower bound of } \\
0.084 \mathrm{M}(1400 \mu \mathrm{g} / \mathrm{mL}) \mathrm{NH}_{3} \text { in the } \\
\text { liquid of the nonconvective layer, or } \\
0.056 \text { wt } \% \mathrm{NH}_{3} \text { in the bulk waste. }\end{array}$} \\
\hline $7-5$ & no data & $0.016 \pm 0.008$ (a) & \\
\hline $7-7$ & no data & $0.012 \pm 0.006$ (a) & \\
\hline $7-8$ & no data & $0.0026 \pm 0.0013$ & \\
\hline
\end{tabular}

Sample 7-2 gave a physically impossible negative value for the post-extrusion ammonia partial pressure. In addition, the isotopically determined residual ammonia for sample 7-2 is suspected of being an underestimate because of the very short equilibration time that was allowed (about $0.1 \mathrm{hr}$ ). However, the isotopic value was used to calculate the in situ ammonia for sample 7-2 because no other applicable information was available. Sample 7-8 gave an ammonia partial pressure that was too low to be consistent with the measurements for samples 7-5 and 7-7, particularly considering that all three of these samples produced about the same amount of extracted ammonia. Therefore, for sample 7-8, the average of the ammonia partial pressure from samples 7-5 and 7-8 was used to calculate the in situ ammonia vapor pressure.

No conclusive total ammonia concentration can be calculated, and even estimates are risky because of the difficulties with the data. The ammonia concentration in U-103 waste was about twice that in AW-101 waste, which had a lower bound of $27,000 \mu \mathrm{mol} \mathrm{NH}_{3} / \mathrm{L}$ waste. This estimate is based on a comparison of the ammonia partial pressure measurements (see Sections 4.1.2 and C.1 for the AW-101 measurements). However, the RGS data do not rule out much lower concentrations, and grab sample ammonia measurements also suggest much lower concentrations (see Section C.6).

Table 4.6.6 contains the composition of the gas/vapor phase in each sample and the average composition in the gas retained in the nonconvective layer. The water vapor is not included in these compositions. The ammonia fractions are derived from the data shown in Table 4.6.5, which were used to calculate vapor pressures at in situ conditions. The sample compositions in the table have been calculated using the in situ solubility method described in Section 3.6.2. As discussed in Section 3.6.1, compositions were calculated for both the lower- and upper-bound gas solubilities. Both ends of the composition range are given in Table 4.6.6, along with the measurement uncertainty on each. The average composition of the gas in the nonconvective layer is the result of integrating RGS species concentrations over the layer and multiplying those concentrations by the layer volume. The integration method is described in Section 3.7.1.

Tank U-103 samples contained unusually high $\mathrm{N}_{2} \mathrm{O}$ and low $\mathrm{H}_{2}$, with the highest $\mathrm{H}_{2} / \mathrm{N}_{2} \mathrm{O}$ ratios toward the bottom of the tank. Segment 13-4 is not shown in Table 4.6.6 because a full extraction could not be performed; its $\mathrm{H}_{2} / \mathrm{N}_{2} \mathrm{O}$ ratio (at lower-bound gas solubility) was 1.2, higher 
Table 4.6.6. Sample and Overall Average Compositions of Retained Gas in Tank U-103 with Correction for Gas Contamination(a)

\begin{tabular}{||c|c|c|c|c|c|c||}
\hline Sample & $\mathrm{N}_{2}$ (mol\%) & $\mathrm{H}_{2}$ (mol\%) & $\mathrm{N}_{2} \mathrm{O}$ (mol\%) & $\mathrm{NH}_{3}$ (mol\%) & $\mathrm{CH}_{4}$ (mol\%) & Other (mol\%) \\
\hline \hline $7-2$ & $36 \pm 2.1--37 \pm 2.1$ & $23 \pm 1.3$ & $40 \pm 2.2--39 \pm 2.1$ & $0.1 \pm 0.03--0.05 \pm 0.01$ & $0.4 \pm 0.03$ & $0.5 \pm 0.06$ \\
\hline $7-5$ & $32 \pm 2.0--35 \pm 2.1$ & $14 \pm 0.9--16 \pm 0.9$ & $52 \pm 3.2--48 \pm 2.8$ & $1.8 \pm 0.8--0.9 \pm 0.4$ & $0.2 \pm 0.06--0.3 \pm 0.06$ & $0.3 \pm 0.1-0.4 \pm 0.1$ \\
\hline $7-7$ & $41 \pm 2.6--43 \pm 2.7$ & $24 \pm 1.5--25 \pm 1.5$ & $32 \pm 2.0--29 \pm 1.8$ & $1.2 \pm 0.4--0.6 \pm 0.2$ & $0.6 \pm 0.1$ & $1.0 \pm 0.2$ \\
\hline $7-8$ & $36 \pm 3.6--38 \pm 3.8$ & $30 \pm 3.0--32 \pm 3.2$ & $30 \pm 3.0--26 \pm 2.6$ & $1.3 \pm 0.8--0.6 \pm 0.4$ & $0.6 \pm 0.1--0.8 \pm 0.1$ & $1.6 \pm 0.3--1.8 \pm 0.3$ \\
\hline $\begin{array}{l}\text { Avg in non- } \\
\text { convective } \\
\text { layer(c) }\end{array}$ & $36 \pm 2.3--37 \pm 2.3$ & $23 \pm 1.4$ & $40 \pm 2.4--38 \pm 2.3$ & $0.6 \pm 0.3--0.3 \pm 0.1$ & $0.4 \pm 0.05$ & $0.6 \pm 0.1--0.7 \pm 0.1$ \\
\hline $\begin{array}{l}\text { (a) Uncertainties in composition represent instrument uncertainty; composition calculated for lower- and upper-bound solubilities. } \\
\text { Both ends of the composition range are given, lower-bound solubility basis first with instrument uncertainty on each. Composi- } \\
\text { tions may not sum to 100 mol\% because of roundoff error. Mole fractions are on a dry basis and do not account for water vapor. } \\
\text { (b) Ammonia mole fraction is derived from the data indicated in Table 4.6.5, used to calculate vapor pressure at in situ conditions. } \\
\text { (c) There are too few samples to define the spatial variability of the average gas concentration. }\end{array}$ \\
Tl
\end{tabular}

than the other segments of U-103 but lower than the ratios in samples from many other tanks. The higher ratio in 13-4 was probably the result of incomplete extraction, because the $\mathrm{H}_{2} / \mathrm{N}_{2} \mathrm{O}$ ratio tends to decrease with successive steps in the extraction. The concentrations and pressures in Tables 4.6.3 and 4.6.4 can also be found in the sample-by-sample worksheets in the U-103 RGS calculation spreadsheet on the CD. The compositions in Table 4.6.6 are in the "Inventory" worksheet.

\subsubsection{Gas Inventory}

The method by which the in situ gas volume fractions (wet basis) were calculated is given in Section 3.6.2; as for gas composition, the volume fractions are given as a range from the low gas solubility value to the high gas solubility value. The results are presented in Table 4.6.7, which also contains the average gas volume fraction and average pressure experienced by the gas. The averages are in situ volume averages calculated by Simpson's Rule integration (Section 3.7.1). The corrected gas volume fractions in the table are consistent with the corrected gas concentrations and compositions in Tables 4.6.4 through 4.6.6. The information in Table 4.6.7 is taken from the "Summary" and "In situ" worksheets of the U-103 RGS calculation spreadsheet on the CD.

The "sampler gas volume fraction" is corrected only for inleakage during the extraction process and air gases added in the isotopic solution. It is an attempt to reconstruct the total amount of gas (sample and entrainment) present in the sampler during X-ray and is used only for comparison with the x-ray observations of "visible gas fraction" that are discussed in Section 4.6.4.

Table 4.6.7 also contains the water vapor pressures that were used for in situ calculations. The water vapor pressures were found by using salt concentrations from Sasaki (1998) and the temperatures in the table as inputs to Equation 6.2 of Mahoney and Trent (1995), a correlation for water vapor pressure over concentrated homogeneous and non-homogeneous waste simulants. The gas solubilities used the same parameters as inputs to the Schumpe solubility model (see Section 3.6.1). The water vapor pressures and gas solubilities used in calculations can be found in the "In situ" worksheet of the U-103 RGS calculation spreadsheet on the CD. No ammonia inventory was calculated because there were no total ammonia concentration data for U-103. 
Table 4.6.7. In Situ Gas Volume Fractions and Conditions in Tank U-103(a)

\begin{tabular}{|c|c|c|c|c|c|c||}
\hline Sample & $\begin{array}{c}\text { Sample } \\
\text { central } \\
\text { height } \\
\text { (cm) }\end{array}$ & $\begin{array}{c}\text { Hydro- } \\
\text { static } \\
\text { pressure } \\
\text { (atm) }\end{array}$ & $\begin{array}{c}\text { Calculated } \\
\text { water vapor } \\
\text { pressure } \\
\text { (atm) }\end{array}$ & $\begin{array}{c}\text { Temp } \\
\text { ( }{ }^{\circ} \text { ) }\end{array}$ & $\begin{array}{c}\text { Corrected gas } \\
\text { volume fraction } \\
\text { (in-tank conditions) }\end{array}$ & $\begin{array}{c}\text { Sampler gas volume } \\
\text { fraction } \\
\text { (in situ conditions, } \\
\text { low solubility) }\end{array}$ \\
\hline \hline $7-2$ & 362 & 1.08 & 0.0096 & 27.4 & $\begin{array}{c}0.42 \pm 0.027 \text { to } \\
0.41 \pm 0.026\end{array}$ & 0.43 \\
\hline $7-5$ & 217 & 1.28 & 0.011 & 29.7 & $\begin{array}{c}0.098 \pm 0.008 \text { to } \\
0.088 \pm 0.008\end{array}$ & 0.11 \\
\hline $7-7$ & 121 & 1.41 & 0.011 & 29.8 & $0.11 \pm 0.012$ & 0.12 \\
\hline $7-8$ & 72 & 1.48 & 0.011 & 29.7 & $\begin{array}{c}0.079 \pm 0.010 \text { to } \\
0.073 \pm 0.010\end{array}$ & 0.087 \\
\hline $\begin{array}{l}\text { Noncon- } \\
\text { vective layer }\end{array}$ & 277 & 1.20 & & & $\begin{array}{c}0.19 \pm 0.096 \text { to } \\
0.18 \pm 0.090\end{array}$ & \\
\hline $\begin{array}{l}\text { (a) Gas volume fraction is expressed on a wet basis, including the volume contribution of water vapor. } \\
\text { The uncertainties on the layer-average gas volume fractions are based on spatial variability } \\
\text { considerations discussed in Section 3.7.2. }\end{array}$ \\
\hline
\end{tabular}

Table 4.6.8 gives various estimates of the STP volume of gas in Tank U-103, including estimates calculated from RGS data alone. The RGS gas inventory in the nonconvective layer was calculated by integrating RGS total gas concentrations over the layer (three data points) and multiplying the average gas concentration by the volume of the layer. The integration method is described in Section 3.7.1.

The RGS volumes in Table 4.6.8 include corrections to remove the contamination gas: entrained air and argon, helium backfill, air leaks during and after extraction, and air that came in with the isotopic ammonia vapor. The uncertainty of the gas inventory is based on the spatial variability considerations discussed in Section 3.7.2. The information in Table 4.6.8 is taken from the "Inventory" worksheet of the U-103 RGS calculation spreadsheet on the CD.

Table 4.6.8. U-103 Gas Inventory Estimates

\begin{tabular}{||l|c|c||}
\hline \hline \multirow{2}{*}{ Quantity } & RGS Method & BPE Method(a) \\
\cline { 2 - 3 } & $\begin{array}{c}\text { Nonconvective } \\
\text { Layer }\end{array}$ & Tank Total \\
\hline \hline $\begin{array}{l}\text { Avg. gas fraction (low gas } \\
\text { solubility) }\end{array}$ & $0.19 \pm 0.096$ & $0.11 \pm 0.036$ \\
\hline $\begin{array}{l}\left.\text { Gas volume (m }{ }^{3}\right) \\
\text { in situ (wet) }\end{array}$ & $303 \pm 151$ & $180 \pm 58$ (b) \\
STP (wet) & $330 \pm 165$ & $196 \pm 64$ (b) \\
\hline $\begin{array}{l}\text { (a) Inventory based on average gas pressure calculated from RGS data } \\
\text { and dL/dP of -0.48 } \pm 0.13 \text { in./in.-Hg (Whitney et al. 1997). } \\
\text { (b) Retained gas inventory calculated by BPE method is considered the } \\
\text { better estimate. }\end{array}$ \\
\hline
\end{tabular}


The RGS method gave a much higher gas inventory than the BPE method. The surface level measurement in U-103 is via an Enraf buoyancy gauge in riser 8. This riser is near the tank wall adjacent to riser 7, from which the usable RGS samples were taken. Segments 1 and 3 from riser 7 contained a high fraction of liquid, and no hard saltcake was penetrated at the surface. Riser 13 waste was also moist, although there was no free liquid in the upper segments. We conclude that, though the level gauge is near the tank wall, there was probably no attached "shelf" to prevent it from sensing level fluctuations, and there was free liquid in the vicinity to transmit waste expansion and compression from elsewhere in the tank.

Because of the very large difference between the RGS and BPE predictions, it is worth looking at the long-term surface level rise in U-103 for additional information. The waste surface level rose by 4.4 in. between 1981 and January 1995 (Hodgson et al. 1998). The rise is consistent with a gas volume fraction of 0.03 , matching neither the RGS nor BPE results.

If the high gas fraction measured in sample 7-2 is ignored, the RGS gas inventory is calculated to have been about $150 \mathrm{~m}^{3}$ in situ. While the BPE inventory was higher than that obtained by ignoring the sample 7-2 measurement, it was lower than the RGS inventory in Table 4.6.8, which assumes that the gas profile increases linearly from low gas at the sample 7-5 elevation to high gas at the sample 7-2 elevation. The low inventory indicated by BPE suggests that the high gas fraction measured by RGS in segment 7-2 was to some extent laterally or vertically localized (or both). (See subsection 4.6.5.1 for a detailed discussion of the evidence for high gas in areas other than segment 2 of riser 7. That section also discusses evidence that high-gas waste was not found throughout the layer defined by segments 2 through 4; that is, elevations from $386 \mathrm{~cm}$ [152 in.] down to $241 \mathrm{~cm}$ [95 in.]).

The difference between the RGS inventory (omitting sample 7-2) and the BPE inventory was about $30 \mathrm{~m}^{3}$ of in situ gas. This amount of gas could be accounted for by a continuous layer of waste $20 \mathrm{~cm}$ ( 8 in.) thick containing 0.42 volume fraction of gas (like sample 7-2), with the rest of the waste containing lower gas fractions based on samples 7-5, 7-7, and 7-8. The 8 in. of continuous layer that was calculated to be necessary to explain the BPE inventory was reasonably consistent with the scattered (discontinuous) observations of 5 to 9 in. of gas per sample in the zone between $386 \mathrm{~cm}$ (152 in.) and $241 \mathrm{~cm}$ (95 in.).

We recommend the BPE gas inventory as a better estimate of the U-103 gas inventory than the RGS inventory. Core extrusion observations showed some lateral inhomogeneity in the waste, and a variety of gas observations showed discontinuous high gas in the upper layer. These observations cast doubt on extrapolating the single-riser RGS measurements to the entire tank, whereas there is no reason to regard as non-representative the Enraf measurements on which the BPE estimate is based.

Table 4.6.9 contains the calculated nonconvective layer inventories of each of the major gases retained in the waste. The gas-phase inventory is based on the layer-average composition from RGS data (Table 4.6.6) and the best-estimate gas inventory (BPE, Table 4.6.8). The dissolved inventory is based on RGS data alone.

Figure 4.6.3 shows the sample temperatures, corrected gas volume fractions (Table 4.6.7), and corrected compositions of the low-solubility constituents in the samples from Tank U-103. The temperatures came from the TC tree in riser 1 . The compositions represent the mole fraction of the species in the "insoluble" gas; water and ammonia are not included, so the mole fractions are not the same as those in Table 4.6.4. The gas volume fractions and mole fractions in the figure are the values for lower-bound gas solubilities. Figure 4.6 .3 also lists the observations from core extrusions of non-RGS samples and from x-rays of RGS samples as a way of tying those observations together with RGS data. The x-ray data are described in more detail in Section 4.6.4. 
Table 4.6.9. Speciated U-103 Gas Inventory(a)

\begin{tabular}{|c|c|c|}
\hline \multirow{2}{*}{ Species } & \multicolumn{2}{|c|}{ Nonconvective Layer } \\
\cline { 2 - 3 } & $\begin{array}{c}\text { Gas-Phase Inventory } \\
\left(\mathrm{m}^{3} \text { at STP) }\right.\end{array}$ & $\begin{array}{c}\text { Dissolved Inventory } \\
\left(\mathrm{m}^{3} \text { at STP }\right)\end{array}$ \\
\hline $\mathrm{N}_{2}$ & $71-70$ & $0.051-0.53$ \\
\hline $\mathrm{H}_{2}$ & 44 & $0.099-0.49$ \\
\hline $\mathrm{N}_{2} \mathrm{O}$ & $78-71$ & $3.4-9.9$ \\
\hline $\mathrm{NH}_{3}$ & $1.3-0.54$ & 1800 \\
\hline $\mathrm{CH}_{4}$ & $0.85-0.84$ & $0.0013-0.0092$ \\
\hline Other & 1.3 & 0 \\
\hline $\begin{array}{l}\text { (a) Gas-phase inventories based on layer-average compositions from RGS data (Table 4.6.6) and } \\
\text { best-estimate gas inventory (BPE, Table 4.6.8). Dissolved inventories based on RGS data alone. } \\
\text { Uncertainty in each inventory is 50\% based on spatial variability considerations (Section 3.7.2). } \\
\text { Inventories given for lower- and upper-bound solubilities, lower-bound solubility basis first. }\end{array}$ \\
\hline
\end{tabular}

\subsubsection{X-Ray Results}

Table 4.6.10 summarizes all the available radiography observations from Tank U-103. The $\mathrm{x}$-ray images themselves can be found on the $\mathrm{CD}$ that accompanies this report. The uncertainties of the visible gas volume fractions that are given in Table 4.6.10 are unknown but (based on the uncertainty in measuring the gas gap from $x$-ray images) are expected to be larger than the uncertainties in the gas volume fractions calculated from gas extraction. (The "visible" gas volume fraction refers to the gas that has separated from the solids and liquid such that its volume can be calculated based on the image. The uncorrected gas fractions from extraction are used for comparison to reflect the presence of entrained air in the sampler.)

Table 4.6.10 compares $\mathrm{x}$-ray- and extraction-derived gas volume fractions. The comparison shows that sample recovery was close to $100 \%$ for all the samples except possibly $7-2$. This conclusion is based on the fact that the extracted uncorrected gas was equal to or a few volume percent more than the visible gas, except for sample 7-2. Note that the uncertainty in measuring the large gas gap could explain the difference between extracted and visible gas: when the gas gap is more than 3 or 4 in. it stretches over more than one $x$-ray image and is hard to measure accurately.

The large adhering lumps of waste observed in several samples suggest high waste strength. Gas retention experiments were carried out on an U-103 composite sample, and showed bubbles that were distorted, apparently by "moderate" waste strength (Rassat et al. 1998).

Table 4.6.11 provides a summary of the waste densities that have been calculated from radiographic data for the RGS samples for which air and water standard images were available. Temperatures were taken using the TC tree in riser 1 . The radiographic densities include the gas in the waste, were calculated by the current $\mathrm{x}$-ray analysis method, and are given for several locations within each sample to show density trends. Table 4.5.11 also includes density measurements made on extruded non-RGS samples (Sasaki 1998). (In some cases, densities were also available for RGS segments because the samples underwent further analysis after RGS processing was complete.) The x-ray method gives consistently lower results, even when the extracted gas content of the $\mathrm{x}$-rayed samples is accounted for, and even for the samples that had air/water density profiles. 

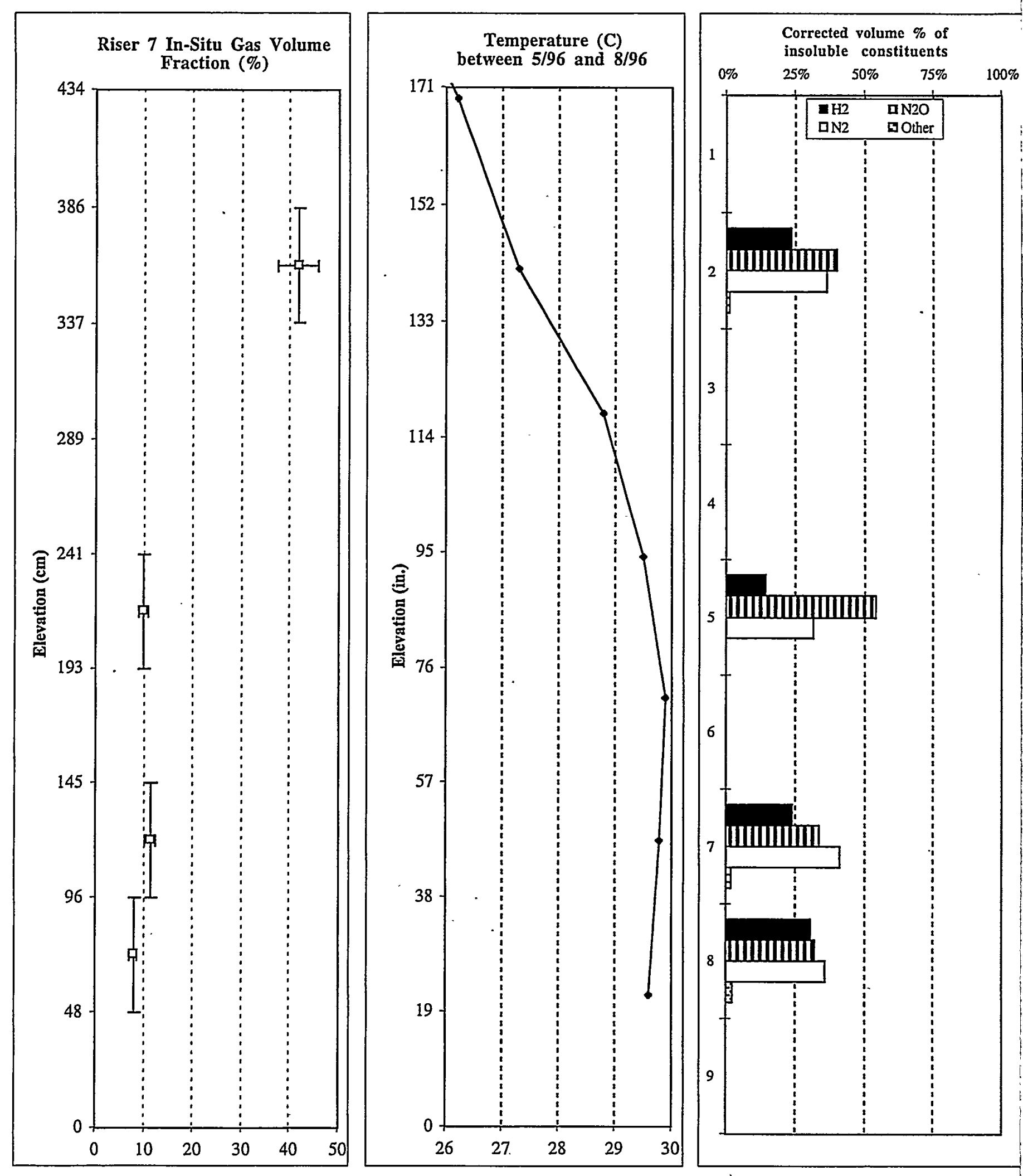

Figure 4.6.3. Gas Volume Fractions, Temperatures, $C Q$ 


\section{U-103 waste level is $424 \mathrm{~cm}$ (167 inches).}

1 Riser 13: (322 g) dark gray-brown sludge slurry, tending to "melt". $1.2 \mathrm{R} / \mathrm{hr}$.

1 Riser 2: 5 in. (97 g) gray salt slurry, $80 \mathrm{~mL}$ (114 g) opaque gray liquid. $0.7 \mathrm{R} / \mathrm{hr}$.

1 Riser 7: 2 in. ( $70 \mathrm{~g})$ dark gray salt slurry, $200 \mathrm{~mL}(259 \mathrm{~g})$ dark gray opaque liquid. $1.5 \mathrm{R} / \mathrm{hr}$.

2 Riser 13: 16 in. ( $314 \mathrm{~g}$ ) dark gray salt slurry. $1 \mathrm{R} / \mathrm{hr}$.

2 Riser 2: 9 in. $(270 \mathrm{~g})$ gray moist salt, $5 \mathrm{~mL}(114 \mathrm{~g})$ opaque brown liquid.

2 Riser 7 X-RAY: Featureless waste, flat top surface. No waste on fully retracted piston, 8.9 in. gas gap. $1.4 \mathrm{R} / \mathrm{hr}$.

3 Riser 13: 17 in. (394 g) dark gray wet salt. $1.2 \mathrm{R} / \mathrm{hr}$.

3

3 Riser 7: 3 in. (53 g) dark gray salt slurry, $250 \mathrm{~mL}(344 \mathrm{~g}$ ) dark gray opaque liquid. HHF contamination. $2.0 \mathrm{R} / \mathrm{hr}$.

4 Riser 13 X-RAY: Many mostly round bubbles up to $10 \mathrm{~mm}$ diam., uneven surface. Gas gap $7 \mathrm{in}$. $0.7 \mathrm{R} / \mathrm{hr}$.

4 Riser 7: 13 in. (336 g) dark gray salt slurry, $20 \mathrm{~mL}$ dark gray opaque liquid. HHF contamination. $1.5 \mathrm{R} / \mathrm{hr}$.

5 Riser 13: 18 in. $(422 \mathrm{~g})$ dark gray moist salt. $1.3 \mathrm{R} / \mathrm{hr}$.

5 Riser $7 \mathrm{X}$-RAY: Many < $2 \mathrm{~mm}$ bubbles, meniscus, 0.6 in. gas gap. Half-width lump waste on piston. $1.5 \mathrm{R} / \mathrm{hr}$.

6 Riser 13: 18 in. ( $457 \mathrm{~g}$ ) dark gray moist salt. $1.3 \mathrm{R} / \mathrm{hr}$.

6 Riser 7: 18 in. $(455 \mathrm{~g})$ gray moist salt. $0.8 \mathrm{R} / \mathrm{hr}$.

7 Riser 13: 18 in. (382 g) dark gray moist salt. $1.6 \mathrm{R} / \mathrm{hr}$.

7 Riser $7 \mathrm{X}$-RAY: Many <2 mm bubbles, lumpy surface, $1.3 \mathrm{in}$. gas gap. Full-width lump waste on piston. $1.3 \mathrm{R} / \mathrm{hr}$.

8 Riser 13: $3 \mathrm{in}$. (84 g) light gray salt slurry, $240 \mathrm{~mL}(332 \mathrm{~g})$ light gray opaque liquid. $2.2 \mathrm{R} / \mathrm{hr}$.

8 Riser 7 X-RAY: Many < $2 \mathrm{~mm}$ bubbles, slanting surface, 1.3 in. gas gap. 1-in. thick lump waste on piston. $1.3 \mathrm{R} / \mathrm{hr}$.

9 Riser 13: 9 in. $(248 \mathrm{~g})$ dark gray moist salt, $120 \mathrm{~mL}(176 \mathrm{~g})$ light gray opaque liquid. HHF contamination. $2.1 \mathrm{R} / \mathrm{hr}$.

10 Riser 13: 11 in. (325 g) dark gray moist salt. $1.2 \mathrm{R} / \mathrm{hr}$. 
Table 4.6.10. Summary of Observations from X-Ray Images of Tank U-103

\begin{tabular}{|c|c|}
\hline Segment & Comments/Observations \\
\hline $\begin{array}{l}\text { 7-2 } \\
\text { (RGS, } \\
1 / 21 / 97)\end{array}$ & $\begin{array}{l}\text { The waste in this sample occupies only the bottom } 24 \mathrm{~cm} \text { ( } 9.3 \mathrm{in} \text {.). The waste is homogeneous in } \\
\text { appearance with a flat surface. No waste was attached to the piston, which was fully retracted. Gas } \\
\text { gap was } 23 \mathrm{~cm}(8.9 \mathrm{in} \text {.). The visible gas fraction is therefore } 0.49 \text {. (Compare with the uncorrected } \\
\text { gas volume fraction of } 0.43 \text { in Table } 4.6 .7 \text {, which was obtained by gas extraction.) }\end{array}$ \\
\hline $\begin{array}{l}13-4 \\
\text { (RGS, } \\
4 / 2 / 97)\end{array}$ & $\begin{array}{l}\text { This sample showed bubble structure, with many mostly round bubbles of diameter up to about } 1 \\
\mathrm{~cm} \text {. It was about } 28 \mathrm{~cm} \text { ( } 11 \text { in) full. The top surface of the waste is uneven. No image of the } \\
\text { piston was available, so it is not known whether waste was attached to it or whether it was fully } \\
\text { retracted. The visible gas volume fraction is } 0.38 \text {, slightly less than that of segment } 7-2 \text {, but there } \\
\text { are no RGS extraction data for comparison. }\end{array}$ \\
\hline $\begin{array}{l}7-5 \\
\text { (RGS, } \\
1 / 22 / 97)\end{array}$ & $\begin{array}{l}\text { This sample contained barely visible gas bubbles, some joined into linear features, and several 3- } \\
\mathrm{mm} \text { bubbles near the sampler top. There is a slightly concave top surface, and a lump of waste } \\
\text { half the width of the piston is attached to the fully retracted piston. The gas gap is } 16 \mathrm{~mm}(0.6 \\
\text { in.). The visible gas volume fraction (not counting bubbles) is therefore } 0.03 \text {. (Compare with } \\
\text { uncorrected gas volume fraction of } 0.11 \text { in Table } 4.6 .7 \text {, which was obtained by gas extraction.) }\end{array}$ \\
\hline $\begin{array}{l}7-7 \\
\text { (RGS, } \\
1 / 22 / 97)\end{array}$ & $\begin{array}{l}\text { This sample contained many }<2 \mathrm{~mm} \text { gas bubbles, some joined into linear features. There is a } \\
\text { lumpy top surface. A lump of waste is attached across the whole width of the piston, which is } 0.4 \\
\text { in. short of full retraction. The gas gap is } 3.3 \mathrm{~cm} \text { ( } 1.3 \text { in.). The visible gas volume fraction (not } \\
\text { counting bubbles) is therefore } 0.07 \text {. (Compare with the uncorrected gas volume fraction of } 0.12 \text { in } \\
\text { Table } 4.6 .7 \text {, which was obtained by gas extraction.) }\end{array}$ \\
\hline $\begin{array}{l}7-8 \\
\text { (RGS, } \\
1 / 22 / 97)\end{array}$ & $\begin{array}{l}\text { This sample contained many }<2 \mathrm{~mm} \text { gas bubbles, some joined into linear features. There is a } \\
\text { slanting top surface. A 1-in thick lump of waste is attached across the whole width of the piston, } \\
\text { which is } 0.7 \text { in. short of full retraction. The gas gap is } 3.3 \mathrm{~cm}(1.3 \text { in.). The visible gas volume } \\
\text { fraction (not counting bubbles) is therefore } 0.07 \text {. (Compare with the uncorrected gas volume } \\
\text { fraction of } 0.087 \text { in Table } 4.6 .7 \text {, which was obtained by gas extraction.) }\end{array}$ \\
\hline
\end{tabular}

\subsubsection{Other Discussions}

\subsubsection{High-Gas Waste}

An unusually large amount of gas was extracted from sample 7-2 of U-103. The in situ gas volume fraction calculated from RGS extraction data was 0.43 for this sample. The high gas content found by extraction was confirmed by the large (approximately 22.9-cm [9-in.]) gap that was seen in the sampler $\mathrm{x}$-rays. The gas in sample 7-2 had much the same composition as that in the other samples. The gas in sample 7-2 contained essentially no oxygen, which was also the case for the other U-103 samples. There is therefore little reason to believe that sample 7-2 was part of a crust that contained large amounts of trapped air or whose pores were open to the air in the domespace. If the top waste at the RGS riser, riser 7, had a closed pore structure that severely restricted gas migration, the high gas content could have accumulated from below. This is not inconsistent with the fact that essentially zero force was required to push the drillstring into the waste at riser 7. A closed pore structure does not necessarily imply great structural strength. (Sampling was discontinued at riser 2, segment 2 (same elevation as sample 7-2) because the waste was impenetrable. In this case, the core is believed to have been blocked not by hard waste but by a submerged object. Therefore, the riser 2 push-force observation neither proves nor disproves the existence of a hard, closed top layer.) However, no hard material was observed in core extrusions from this level.

The high gas content of segment 7-2 was probably not local to riser 7. One piece of evidence for this is the $\mathrm{x}$-ray of the RGS sample from segment 13-4, which had a visible gas 
Table 4.6.11. Densities of U-103 Samples from Radiography and Core Samples

\begin{tabular}{|c|c|c|c|}
\hline $\begin{array}{l}\text { Riser- } \\
\text { Segment } \\
\text { Number }\end{array}$ & $\begin{array}{c}\begin{array}{c}\text { Distance from } \\
\text { Bottom of Sampler } \\
\text { (ft) }\end{array} \\
\end{array}$ & $\begin{array}{c}\text { Calculated Mean Density } \\
\text { by Current Method } \\
(\mathrm{g} / \mathrm{cc})\end{array}$ & $\begin{array}{l}\text { Degassed Density Above and } \\
\text { Below the RGS Sample } \\
\text { (from cores) (g/cc) }\end{array}$ \\
\hline \multirow[t]{3}{*}{$7-2$} & 0.75 & 1.37 & \multirow{3}{*}{$\begin{aligned} \text { density above, } 1.53 \mathrm{~g} / \mathrm{cc} \text { solid } \\
1.42 \mathrm{~g} / \mathrm{cc} \text { liquid } \\
\text { density below, } 1.82 \mathrm{~g} / \mathrm{cc} \text { solid } \\
1.42 \mathrm{~g} / \mathrm{cc} \text { liquid }\end{aligned}$} \\
\hline & 0.50 & 1.44 & \\
\hline & 0.25 & 1.38 & \\
\hline \multirow[t]{3}{*}{$13-4^{(a)}$} & 1.00 & 1.15 & \multirow[t]{3}{*}{ density above, $1.80 \mathrm{~g} / \mathrm{cc}$} \\
\hline & 0.75 & 1.18 & \\
\hline & 0.50 & 1.20 & \\
\hline \multirow[t]{5}{*}{$7-5^{(b)}$} & 1.25 & 1.24 & \multirow[t]{5}{*}{ density above, $1.65 \mathrm{~g} / \mathrm{cc}$} \\
\hline & 1.00 & 1.36 & \\
\hline & 0.75 & 1.55 & \\
\hline & 0.50 & 1.60 & \\
\hline & 0.25 & 1.48 & \\
\hline \multirow[t]{5}{*}{$7-7$} & 1.25 & 1.50 & \multirow[t]{5}{*}{ density above, $1.77 \mathrm{~g} / \mathrm{cc}$} \\
\hline & 1.00 & 1.40 & \\
\hline & 0.75 & 1.30 & \\
\hline & 0.50 & 1.27 & \\
\hline & 0.25 & 1.23 & \\
\hline \multirow[t]{6}{*}{$7-8$} & waste on piston & 1.37 & \multirow[t]{6}{*}{ density above, $1.69 \mathrm{~g} / \mathrm{cc}$} \\
\hline & 1.25 & 1.46 & \\
\hline & 1.00 & 1.34 & \\
\hline & 0.75 & 1.44 & \\
\hline & 0.50 & 1.44 & \\
\hline & 0.25 & 1.40 & \\
\hline
\end{tabular}

volume fraction of 0.38 , not counting numerous bubbles. This sample came from the center of the tank at an elevation about one segment length ( $48 \mathrm{~cm}, 19 \mathrm{in}$.), lower than segment 7-2. (For completeness, it should be noted that segment 13-4 was taken about nine weeks later than the riser 7 samples; the S-106 samples were taken in the interim.)

One of the non-RGS core extrusion observations was also consistent with high gas content in upper-waste locations other than segment 2 of riser 7. (However, non-RGS samplers are not hermetically sealed, and core extrusion length measurements are imprecise, so these observations must be interpreted cautiously.) Sample 7-4 (96 cm or 38 in. higher than sample 7-2) contained only about $14 \mathrm{in}$. of sample, meaning the gas gap could have been 5 in., for a gas fraction of 0.26 . But note that other samples from this level $(13-3,13-5)$ contained 17 or 18 in. of waste out of 
$19 \mathrm{in.} \mathrm{of} \mathrm{sampler} \mathrm{and} \mathrm{so} \mathrm{do} \mathrm{not} \mathrm{suggest} \mathrm{a} \mathrm{complete} \mathrm{layer} \mathrm{of} \mathrm{waste} \mathrm{with} \mathrm{uniformly} \mathrm{high} \mathrm{gas} \mathrm{frac-}$ tions. (The 9 in. of waste in sample 2-2 was the result of difficulties in waste penetration and need not have indicated high gas. The large amount of waste in sample 7-3 is also hard to interpret because of HHF contamination, but it does not seem consistent with high gas.)

Another sign that the high-gas waste near the top was probably present in locations other than the riser 7 area is that when sampling was attempted at riser 2 , segment 2 , unusually high flammable gas concentrations (relative to lower flammability limit [LFL]) were measured in the domespace.(a) Sampling at riser 2, which was the first riser sampled in U-103, was terminated because of obstruction in or by the waste, and there was about a 2-1/2 month delay before riser 7 was sampled. The delay resulted from flammable gas concerns that temporarily halted all the tankintrusive work in the tank farms.

At 1400 hours on October 1, 1996, the two combustible gas meters (CGMs) used by the industrial hygiene organization to sniff the domespace at riser 7 indicated flammable gas concentrations of $10 \%$ (meter \#1) and 13\% (meter \#4) of the LFL. No work was done in the tank on day shift. At 2000 hours, the flammable gas concentration was 12\% of LFL on CGM \#1 and 18\% on CGM \#4. A vapor sample was taken from the domespace at this time (at a riser about $12 \mathrm{~m}$ from the sniff riser); it showed $0.22 \%$ hydrogen, or $5.5 \%$ of LFL. This is consistent with the CGM \#1 reading because the CGMs are calibrated with pentane and are expected to read the hydrogen concentration at least 100\% high (Wilkins and Bauer 1996). At 2140 hours, after core sampling segments 1 and 2 from riser 2, the flammable gas readings were 13\% of LFL on CGM \#1 and $19 \%$ of LFL on CGM \#4. The post-swing-shift calibration showed CGM \#1 was within calibration, but CGM \#4 was about 15\% high. The standard hydrogen monitoring system (SHMS) was out of service and could not provide comparison measurements. Considering that CGM \#4 was out of calibration and CGM \#1 and the domespace sample were roughly consistent, the peak flammable gas concentration in the U-103 dome was about $6 \%$ of LFL in the period of interest.

The flammable gas concentrations seen in U-103 at that time were unusually high (though not hazardous), suggesting that a significant amount of gas was present in a releasable form. The measured high gas concentrations near the top of the waste were a possible source. Note that the peak flammable gas concentrations occurred before as well as during riser 2 core sampling, so it is not clear to what extent the riser 2 operations contributed and to what extent high gas concentrations might have existed in the riser 2 waste.

\subsubsection{Drillstring and Domespace Composition Comparison}

The RGS gas-phase composition data (Table 4.6.6) were used to calculate $\mathrm{H}_{2} / \mathrm{N}_{2} \mathrm{O}$ ratios to compare with those from drillstring and domespace grab sample measurements (Siciliano 1998, Table 8.1; McCain 1999, Table B-26). The results are shown in Table 4.6.12. The RGS $\mathrm{H}_{2} / \mathrm{N}_{2} \mathrm{O}$ ratios agreed closely with one drillstring ratio and the domespace ratios. The drillstring ratios from the January 8, 1997 analysis (which did not agree with RGS data) were calculated from small $\mathrm{H}_{2}$ and $\mathrm{N}_{2} \mathrm{O}$ mole fractions, between $10^{-5}$ and $10^{-4}$, possibly making the ratio less accurate.

(a) Electronic communication from GA Stanton Jr. to LM Sasaki (WHC), October 3, 1996 and from LM Sasaki (LMHC) to LA Mahoney (PNNL), November 7, 1997. Industrial Hygiene Direct Reading Instrument Survey sheets for October 1 1996, sheet ID \#96-1766 and \#96-1985, Westinghouse Hanford Company, Richland, Washington. 
Table 4.6.12. Comparison with Drillstring and Domespace Data(a)

\begin{tabular}{|l|c|}
\hline Sample & $\mathrm{H}_{2} / \mathrm{N}_{2} \mathrm{O}$ \\
\hline \hline$R G S, 7-2$ & $0.58-0.59$ \\
\hline$R G S, 7-5$ & $0.28-0.32$ \\
\hline$R G S, 7-7$ & $0.75-0.85$ \\
\hline$R G S, 7-8$ & $0.75-0.86$ \\
\hline Drillstring, riser 2, 10/1/96 & 0.60 \\
\hline Drillstring, riser 2, 1/8/97 & $2.1,1.8$ \\
\hline Domespace, 7/95 & $0.95,0.67,0.75,0.72,0.73$, \\
& $0.70,0.71$ \\
\hline Domespace, 10/22/97 & 0.70 \\
\hline $\begin{array}{l}\text { (a) RGS ratios calculated for both lower- and upper-bound solubilities. Both } \\
\text { ends of ratio range given, lower-bound solubility basis first. }\end{array}$ \\
\hline
\end{tabular}

\subsection{S-106}

Tank 241-S-106 (S-106) was the seventh tank and the third SST sampled with the RGS. This tank was selected for RGS sampling for its uniquely high barometric pressure response and continuing rapid surface level rise, both of which indicate a large and increasing volume of retained gas. It is part of a group of tanks (Cluster 20 in Stewart et al. 1996b) that have high radioactivity, moderate nitrite concentrations $(0.1-0.16 \mathrm{M})$, and low TOC $(0.03-0.05 \%)$. HTCEs indicate that the primary waste stored in S-106 was saltcake from evaporator campaign S1, and the secondary waste was aluminum cladding Redox wastes from 1961 to 1967 (Remund et al. 1995). Of the RGS-sampled tanks, S-111 is also in Cluster 20.

Push-mode sampling was carried out in risers 8 and 7 in February 1997.(a) Sampling was also attempted in riser 14, but the waste (or some object imbedded in it) proved impenetrable. The approximate locations of various risers are depicted in Figure 4.7.1. Risers 7 and 8 are both near the center of the tank, while riser 14 is at the periphery. The elevations of the RGS segments are depicted in Figure 4.7.2, as is the tank content layering.

The Tank Characterization Plan (Homi 1995) describes S-106 as a passively ventilated, sound SST awaiting interim stabilization. Tank S-106 has a capacity of about 750,000 gal and contains about $1,810,000 \mathrm{~L}$ (479,000 gal) of waste, of which $201,000 \mathrm{~L}(53,000 \mathrm{gal})$ are supernatant liquid (Field 1999a).

Automatic FIC surface level measurements through riser 3 showed an increasing trend in waste level from about 1989 to 1993. Riser 4 liquid observation well (LOW) measurements showed a relatively stable liquid level from March 1989 through 1993. The best available data showed that the total depth of waste at the locations of risers 7 and 8 was about $460 \mathrm{~cm}(181 \mathrm{in}$.) at the time of sampling (see Brevick et al. 1994). Waste depth at the location of riser 14 was estimated as $\sim 235$ in., indicating a nonuniform waste surface level consistent with a high crust around

(a) The sampling scheme may be found in Sampling Plan for Tank 241-S-106 Retained Gas Sampler Deployment, by JM Bates and A Shekarriz, January 1997. TWSFG:97.20, Pacific Northwest National Laboratory, Richland, Washington. 


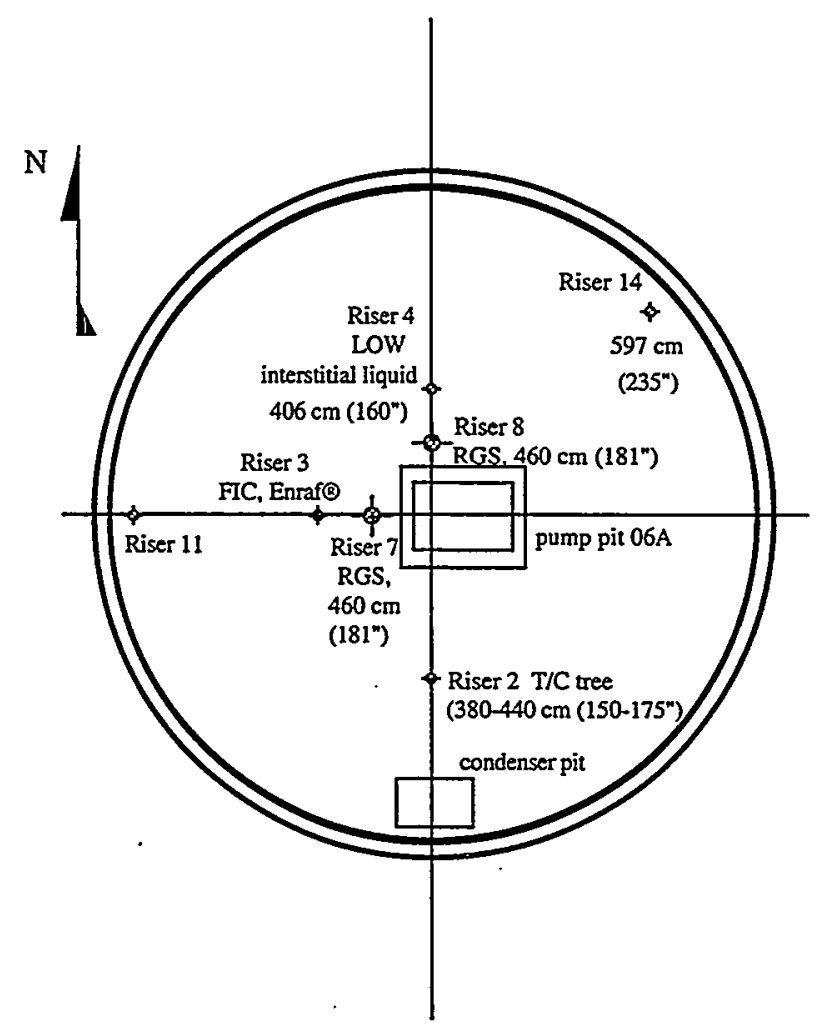

Figure 4.7.1. Schematic Diagram of Riser Locations in Tank S-106

the periphery of the tank, as shown in Figure 4.7.2. A further indication of waste surface level variations was that surface level measurements by the FIC in riser 3 indicated levels of $~ 181$ in. The best-basis inventory of 479,000 gal of waste was based on a uniform waste level of 181 in. (Field 1999a).

Densities for the liquid and bulk solids in Tank S-106 were available from the non-RGS samples taken in 1997 (Field 1999a). Several samples contained drainable liquid with a density of $1422 \pm 55 \mathrm{~kg} / \mathrm{m}^{3}$. The degassed bulk density of the nonconvective layer (saltcake) had an average and standard deviation of $1775 \pm 119 \mathrm{~kg} / \mathrm{m}^{3}$ (four data points). The peripheral crust above $181 \mathrm{in}$. elevation was not considered in calculating the hydrostatic pressures.

These densities were used for pressure calculations. Layer thickness of $49 \mathrm{~cm}$ (19.3 in.) and $411 \mathrm{~cm}$ (161.7 in.) were used in calculations for the supernatant and nonconvective layers, treating the two as being of uniform thickness throughout the tank. The supernatant layer thickness is based on the best-basis supernatant inventory of $201,000 \mathrm{~L} \mathrm{(53,000} \mathrm{gal)} \mathrm{and} \mathrm{the} 22.9-\mathrm{m}$ (75-ft) tank diameter.

The most recent information on tank content layering, derived from the core observations made in 1997 (Field 1999a), is presented in Figure 4.7.3. Note the strongly varying extents and levels of the layers at risers 7 and 8 . This is especially remarkable because risers 7 and 8 are near each other in the center portion of the tank. According to these observations, a substantial solids fraction was probably present in all four of the RGS samples that were taken successfully (samples $7-3,7-5,8-6$, and 8-10). The upper layer, which was predominantly liquid, was not RGSsampled because of sampler failures. 


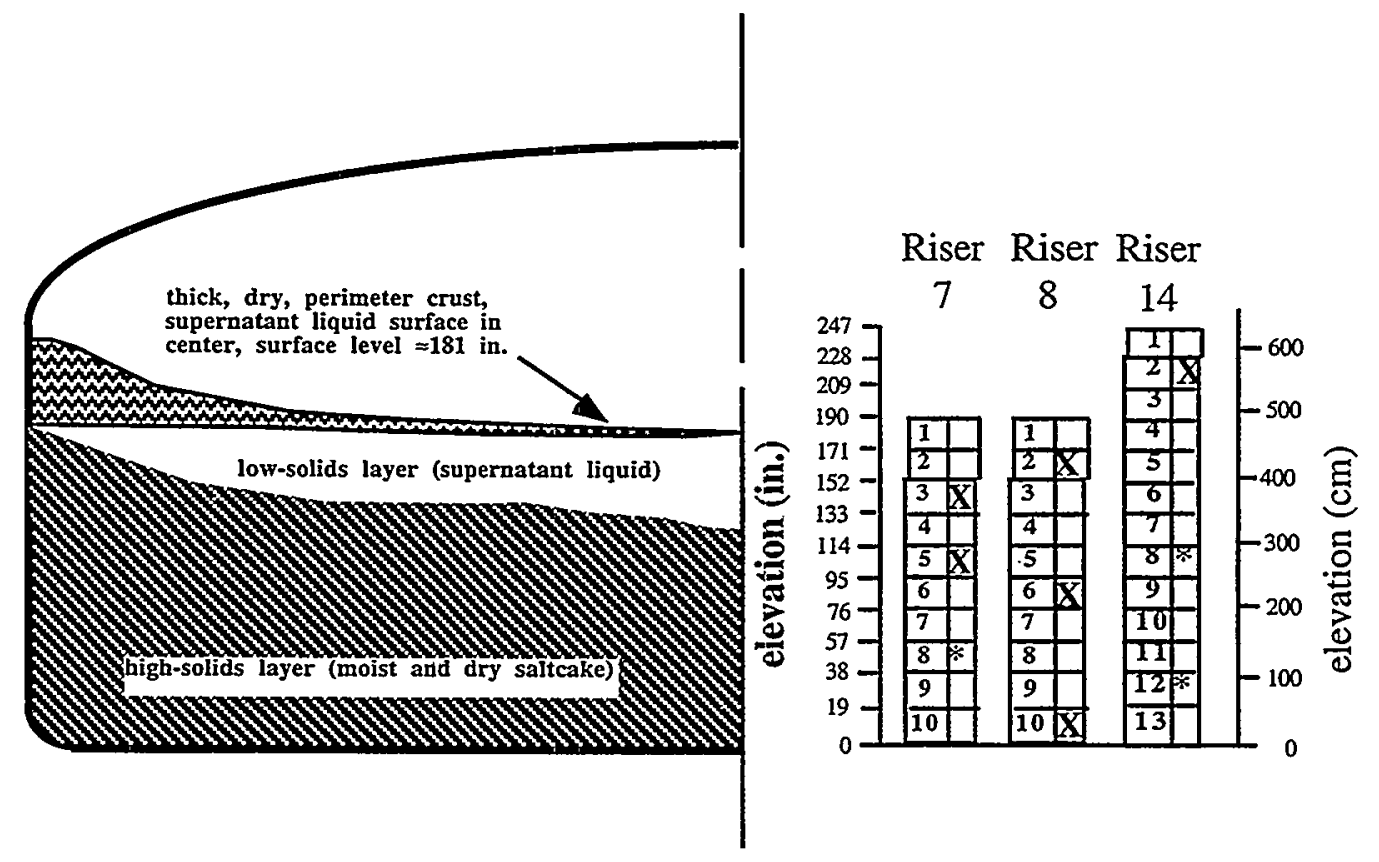

Figure 4.7.2. Diagram of Waste Layering and RGS Sample Elevations for Tank S-106 (segments marked with * could not be taken because the waste was too hard or tank bottom reached. Sampling was attempted but abandoned in riser 14 because an obstacle or hard waste blocked the drillstring.)

\subsubsection{Sampling and Extraction Information}

The samples that underwent RGS analysis are listed in Table 4.7.1. Two samples, 8-2 and 14-2, were discarded because the sampler valves had not closed completely. As a result of these difficulties, no samples were taken successfully in the peripheral crust at riser 14 or in the supernatant layer that apparently occupied the topmost two to five segments of risers 7 and 8 . The valve springs were subsequently changed to exert stronger valve closure force in future sampling. Field data, including dose rates and downforce limits, are supplied in Appendix A.

Table 4.7.1 also shows the lag times (delay between sample acquisition and processing) for these samples. This information was provided to allow data users to correlate the lag time between sampling and extrusion with the concentrations of the various constituents to test for decomposition or other chemical reactions. The maximum hold time allowed by the sampling plan is 24 days, based on measured sampler leakage rates during acceptance testing. All the sample hold times were less than or equal to this limit.

As was discussed in Section 3.5, the HHF used during sampling often enters the sampler in place of some of the waste. Table 4.7.2 shows the volume percentage contamination by HHF of samples for which bromide measurements were made (these did not include any RGS samples). Some of these non-RGS samples (7-1, 7-2, 8-1, 8-5, 8-8, and 8-9) contained bromide below the 

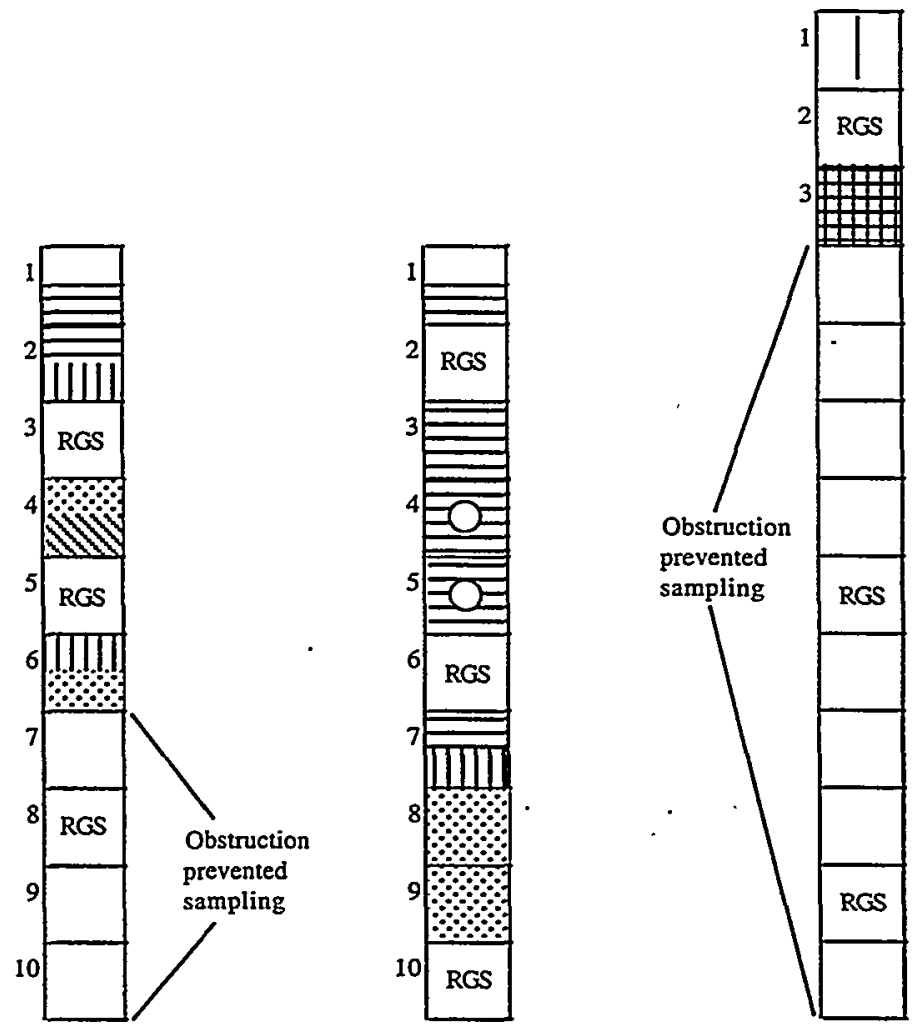

Physical Character (based on qualitative observationś)

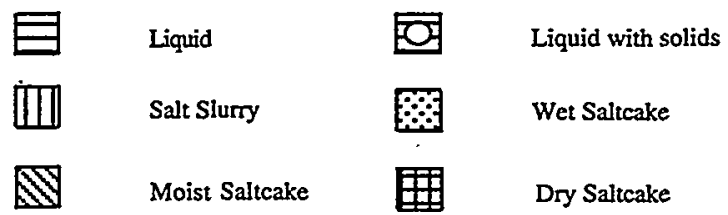

Figure 4.7.3. Recent Core Profile for Selected Push-Mode Sampling Risers in Tank S-106(a)

Table 4.7.1. Lag Times for Processing RGS Samples from Tank 241-S-106

\begin{tabular}{||c|c|c|c|}
\hline Sample & Acquisition Date & Processing Date & Lag (days) \\
\hline \hline $7-3$ & February 24, 1997 & March 20,1997 & 24 \\
\hline $7-5$ & February 25, 1997 & March 18, 1997 & 21 \\
\hline $8-6$ & February 18, 1997 & March 11, 1997 & 21 \\
\hline $8-10$ & February 21, 1997 & March 13,1997 & 20 \\
\hline
\end{tabular}

(a) Personal communication, AP Mousel (LATA) to LA Mahoney (PNNL). May 23, 1997. "S-106 PMCS Core Profile." File S106.CRD, revised April 4, 1997. Los Alamos Technical Associates, Richland, Washington. 
Table 4.7.2. Hydrostatic Head Fluid Contamination in S-106 Non-RGS Samples

\begin{tabular}{|c|c|}
\hline Sample & $\begin{array}{c}\text { HHF Contamination } \\
\text { (vol\%) }\end{array}$ \\
\hline $7-1$ & $<2.6$ \\
\hline $7-2$ & $<3.6$ \\
\hline $7-4$ & 5.4 \\
\hline $8-1$ & $<3.2$ \\
\hline $8-3$ & 4.6 \\
\hline $8-4$ & 11.5 \\
\hline $8-5$ & $<10.1$ \\
\hline $8-7$ & 17.4 \\
\hline $8-8$ & $<2.8$ \\
\hline $8-9$ & $<5.2$ \\
\hline
\end{tabular}

detection limit. Averaging the HHF contamination over the samples and treating the belowdetection samples as if at the detection limit gave an average contamination of $1139 \mu \mathrm{g} / \mathrm{g}$, or $9.1 \mathrm{vol} \%$. This value was used to calculate a reduced effective sampler volume for the RGS samples. (It is conservative to use the MDL for values below it; the conservatism is probably less than 7\%, based on HHF measurements in non-RGS samples.)

All of these samplers were helium-backfilled and sealed with vacuum grease before they were deployed. This method virtually eliminated air and argon contamination from gases entrained during sampling. The helium backfill results are discussed in detail by Cannon (1997) and summarized in Section 5 of Mahoney et al. (1997).

The same extraction procedure was used on all of the S-106 samples. No PQ canisters were taken for S-106 samples or those from any subsequent tanks. (See Figure 3.1 for a schematic of the RGS extraction system.)

After evacuating the whole system and observing the pressure to test for leaks, the operator extruded the RGS sample into the extractor vessel, and the sample was briefly stirred. At this point the vapor space in communication with the sample consisted of the volumes of the extractor vessel, its lines, the second volume, the pump volume, and the collector side; the latter included the collector line volume and two open collection canisters. These first canisters, containing a sample of uncompressed (unpumped) gas, were closed off. The second collection canister was opened. Several strokes were pumped from the extractor to the collector, and the second collection canister was closed off.

Then the collector side and the second volume and extractor lines were evacuated. A canister containing ${ }^{15 \mathrm{NH}_{3}}$ vapor was connected to the extractor side and opened to inject isotopic ammonia. Several strokes were pumped from the extractor to the collector, and the third collection canister was closed off. Finally, the BSVD procedure was performed: argon was injected into the closed-off second volume, and the second volume was opened to the extractor and lines. Pressure measurements and known tare system volumes allowed calculation of the volume of the sample solids and liquid. 
Procedural details such as the number of strokes per canister and the system volumes can be found in the sample-by-sample worksheets in the S-106 RGS calculation spreadsheet on the CD supplied with this report. A very detailed run-through of the procedures can also be found in Appendix A of Mahoney et al. (1997).

\subsubsection{Retained Gas Composition}

Table 4.7.3 presents the calculated concentrations of the insoluble gases in each RGS sample taken from S-106 without corrections for air and argon entrainment, helium backfill, air inleakage, or air added with the isotopic ammonia. The method used to make the corrections depended on the gas. All of the oxygen, argon, and helium were subtracted no matter which step had produced them. A number of moles equal to (3.73) $\left(\mathrm{O}_{2}\right)$ was subtracted from the nitrogen because the oxygen was expected to have come from air entrainment during sampling or inleakage during extraction. The corrected concentrations are given in Table 4.7.4. Contamination observations are in the "Summary" worksheet of the S-106 RGS calculations spreadsheet on the CD.

Table 4.7.5 presents the ammonia measurements. The residual ammonia was determined isotopically, and the total ammonia (calculated from the isotopic residual) is included in the table. The post-extrusion partial pressure of $\mathrm{NH}_{3}$ over the sample (see Section 3.4.4) was measured at laboratory temperature and is included in Table 4.7.5. No other ammonia measurements were made on RGS samples.

Table 4.7.3. Concentrations of Insoluble Constituents ( $\mu \mathrm{mol} / \mathrm{L}$ of waste) in Tank S-106 Without Correction for Gas Contamination

\begin{tabular}{|c|c|c|c|c|c|c|c|c|c|c|c||}
\hline Segment & $\mathrm{N}_{2}$ & $\mathrm{H}_{2}$ & $\mathrm{~N}_{2} \mathrm{O}$ & $\mathrm{O}_{2}$ & $\mathrm{CH}_{4}$ & $\mathrm{He}$ & $\mathrm{Ar}$ & $\begin{array}{c}\text { Other } \\
\mathrm{NOx}\end{array}$ & $\mathrm{C}_{2} \mathrm{H}_{x}$ & $\mathrm{C}_{3} \mathrm{H}_{x}$ & $\begin{array}{c}\text { Other } \\
\text { Hyd. }\end{array}$ \\
\hline \hline $7-3$ & $1500 \pm 98$ & $2600 \pm 150$ & $380 \pm 23$ & $18 \pm 10$ & $18 \pm 6.9$ & $520 \pm 37$ & $12 \pm 1.2$ & $0.8 \pm 0.6$ & $5.3 \pm 4.4$ & $2.2 \pm 0.6$ & $2.4 \pm 1.0$ \\
\hline $7-5$ & $1400 \pm 140$ & $3400 \pm 160$ & $820 \pm 41$ & $35 \pm 29$ & $0.5 \pm 0.4$ & $510 \pm 28$ & $4.7 \pm 1.8$ & $3.7 \pm 3.1$ & $11 \pm 6.6$ & $6.6 \pm 3.3$ & $7.5 \pm 3.6$ \\
\hline $8-6$ & $1100 \pm 110$ & $2700 \pm 240$ & $470 \pm 54$ & $24 \pm 1.3$ & $22 \pm 9.0$ & $420 \pm 37$ & $5.4 \pm 1.2$ & $5.2 \pm 4.5$ & $20 \pm 16$ & $9.8 \pm 9.0$ & $3.1 \pm 1.6$ \\
\hline $8-10$ & $2500 \pm 250$ & $6300 \pm 200$ & $1100 \pm 39$ & $80 \pm 76$ & $16 \pm 1.0$ & $540 \pm 20$ & $6.2 \pm 3.6$ & $3.1 \pm 1.3$ & $18 \pm 15$ & $10 \pm 7.5$ & $2.0 \pm 1.4$ \\
\hline
\end{tabular}

Table 4.7.4. Concentrations of Insoluble Constituents ( $\mu \mathrm{mol} / \mathrm{L}$ of waste) in Tank S-106 with Correction for Gas Contamination

\begin{tabular}{||c|c|c|c|c|c|c|c|c|c|c|c||}
\hline Segment & $\mathrm{N}_{2}$ & $\mathrm{H}_{2}$ & $\mathrm{~N}_{2} \mathrm{O}$ & $\mathrm{O}_{2}$ & $\mathrm{CH}_{4}$ & $\mathrm{He}$ & $\mathrm{Ar}$ & $\begin{array}{c}\text { Other } \\
\mathrm{NOx}\end{array}$ & $\mathrm{C}_{2} \mathrm{H}_{\mathrm{x}}$ & $\mathrm{C}_{3} \mathrm{H}_{\mathrm{x}} \cdot$ & $\begin{array}{c}\text { Other } \\
\text { Hyd. }\end{array}$ \\
\hline \hline $7-3$ & $1400 \pm 100$ & $2600 \pm 150$ & $380 \pm 23$ & $0 \pm 10$ & $18 \pm 6.9$ & $0 \pm 37$ & $0 \pm 1.2$ & $0.8 \pm 0.6$ & $5.3 \pm 4.4$ & $2.2 \pm 0.6$ & $2.4 \pm 1.0$ \\
\hline $7-5$ & $1200 \pm 170$ & $3400 \pm 160$ & $820 \pm 41$ & $0 \pm 29$ & $0.5 \pm 0.4$ & $0 \pm 28$ & $0 \pm 1.8$ & $3.7 \pm 3.1$ & $11 \pm 6.6$ & $6.6 \pm 3.3$ & $7.5 \pm 3.6$ \\
\hline $8-6$ & $1000 \pm 110$ & $2700 \pm 240$ & $470 \pm 54$ & $0 \pm 1.3$ & $22 \pm 9.0$ & $0 \pm 37$ & $0 \pm 1.2$ & $5.2 \pm 4.5$ & $20 \pm 16$ & $9.8 \pm 9.0$ & $3.1 \pm 1.6$ \\
\hline $8-10$ & $2200 \pm 380$ & $6300 \pm 200$ & $1100 \pm 39$ & $0 \pm 76$ & $16 \pm 1.0$ & $0 \pm 20$ & $0 \pm 3.6$ & $3.1 \pm 1.3$ & $18 \pm 15$ & $10 \pm 7.5$ & $2.0 \pm 1.4$ \\
\hline
\end{tabular}


Table 4.7.5. Ammonia Data from Tank S-106 Samples

\begin{tabular}{|c|c|c|c|}
\hline Sample & $\begin{array}{l}\text { Isotopically } \\
\text { Measured } \\
\text { Total } \mathrm{NH}_{3} \\
(\mu \mathrm{mol} / \mathrm{L})\end{array}$ & $\begin{array}{l}\text { Measured } \mathrm{NH}_{3} \\
\text { partial pressure at } \\
\text { about } 24^{\circ} \mathrm{C} \\
\text { (atm) }\end{array}$ & $\begin{array}{c}\text { Best-Estimate } \mathrm{NH}_{3} \\
\text { Concentrations }\end{array}$ \\
\hline $7-3$ & $980 \pm 380$ & $0.0043 \pm 0.0022$ (a) & \multirow{4}{*}{$\begin{array}{l}\text { The data do not support } \\
\text { conclusive } \mathrm{NH}_{3} \text { concentrations. } \\
\text { We estimate a lower bound of } \\
0.025 \mathrm{M}(430 \mu \mathrm{g} / \mathrm{L}) \mathrm{NH}_{3} \text { in the } \\
\text { liquid of the nonconvective layer, } \\
\text { or } 0.015 \mathrm{wt} \% \mathrm{NH}_{3} \text { in the bulk } \\
\text { waste. }\end{array}$} \\
\hline $7-5$ & $6200 \pm 1800$ & $0.0045 \pm 0.0023^{(a)}$ & \\
\hline 8-6 & $1800 \pm 650$ & $0.0073 \pm 0.0036$ (a) & \\
\hline $8-10$ & $1700 \pm 560$ & $0.0041 \pm 0.0021$ & \\
\hline
\end{tabular}

The ammonia measurement made by isotopic ammonia vapor injection is believed to underestimate the ammonia in S-106 samples because of the very short equilibration times (ranging from 0.1 to 2 hours). Accordingly, the post-extrusion $\mathrm{NH}_{3}$ partial pressures were used to calculate the in situ ammonia vapor pressures.

No conclusive total ammonia concentration can be calculated, but it is likely that the ammonia concentration in S-106 waste was about half that in AW-101 waste, which had a lower bound of $27,000 \mu \mathrm{mol} \mathrm{NH}_{3} / \mathrm{L}$ waste. This estimate is based on a comparison of the ammonia partial pressure measurements (see Sections 4.1.2 and C.1 for the AW-101 measurements). The estimate is consistent with but greater than the lower-bound ammonia concentrations found in grab samples and non-RGS core extrusions. Concentrations in the pool of supernatant could have been much lower. (See Appendix C, Section C.7 for more detail on the ammonia data.)

Table 4.7.6 contains the composition of the gas/vapor phase in each sample and the average composition in the gas retained in the nonconvective layer. The water vapor is not included in these compositions. The ammonia fractions are derived from the data in Table 4.7.5, which were used to calculate vapor pressures at in situ conditions. The sample compositions in the table have been calculated using the in situ solubility method described in Section 3.6.2. As discussed in Section 3.6.1, compositions were calculated for both the lower- and upper-bound gas solubilities. Both ends of the composition range are given in Table 4.7.6 along with the measurement uncertainty on each. The average compositions of the gas in the nonconvective layer is the result of integrating RGS species concentrations over the layer and multiplying those concentrations by the layer volume. No RGS samples were successfully taken from the supernatant or peripheral crust layers. The integration method is described in Section 3.7.1. The retained gas in the S-106 samples was of nearly the same composition in all the samples, despite the variation between risers that was seen in core extrusions (Figure 4.7.3).

The concentrations and pressures in Tables 4.7.3 and 4.7.4 can also be found in the sample-by-sample worksheets in the S-106 RGS calculation spreadsheet on the CD. The compositions in Table 4.7.6 are in the "Inventory" worksheet. 
Table 4.7.6. Sample and Overall Average Compositions of Retained Gas in Tank S-106 with Correction for Gas Contamination(a)

\begin{tabular}{|c|c|c|c|c|c|c|}
\hline Sample & $\mathrm{N}_{2}(\mathrm{~mol} \%)$ & $\mathrm{H}_{2}(\mathrm{~mol} \%)$ & $\mathrm{N}_{2} \mathrm{O}(\mathrm{mol} \%)$ & $\mathrm{NH}_{3}(\mathrm{~mol} \%)^{(b)}$ & $\mathrm{CH}_{4}(\mathrm{~mol} \%)$ & Other (mol\%) \\
\hline $7-3$ & $32 \pm 3.1--33 \pm 3.2$ & $59 \pm 5.0-60 \pm 5.1$ & $7.9 \pm 0.7--6.4 \pm 0.6$ & $0.3 \pm 0.2--0.2 \pm 0.1$ & $0.4 \pm 0.2$ & $0.2 \pm 0.2$ \\
\hline $7-5$ & $23 \pm 3.6$ & $62 \pm 5.4--64 \pm 5.6$ & $14 \pm 1.2--12 \pm 1.0$ & $0.3 \pm 0.2--0.2 \pm 0.1$ & $0.01 \pm 0.01$ & $0.5 \pm 0.3$ \\
\hline $8-6$ & $25 \pm 3.6--26 \pm 3.7$ & $63 \pm 8.6--65 \pm 8.8$ & $10 \pm 1.6--7.8 \pm 1.2$ & $0.6 \pm 0.3--0.3 \pm 0.1$ & $0.5 \pm 0.2$ & $0.9 \pm 0.7$ \\
\hline $8-10$ & $23 \pm 4.2--24 \pm 4.3$ & $65 \pm 4.9--66 \pm 5.0$ & $11 \pm 0.8--9.5 \pm 0.7$ & $0.3 \pm 0.1-0.1 \pm 0.06$ & $0.2 \pm 0.02$ & $0.4 \pm 0.3$ \\
\hline $\begin{array}{l}\text { Avg in non- } \\
\text { convective } \\
\text { layer(c) }\end{array}$ & $25 \pm 3.7--26 \pm 3.8$ & $63 \pm 5.7--64 \pm 5.8$ & $11 \pm 1.0--9.0 \pm 0.9$ & $0.4 \pm 0.2--0.2 \pm 0.1$ & $0.3 \pm 0.08$ & $0.5 \pm 0.3$ \\
\hline \multicolumn{7}{|c|}{$\begin{array}{l}\text { (a) Uncertainties on compositions represent instrument uncertainty; compositions calculated for lower- and upper-bound } \\
\text { solubilities. Both ends of composition range given, lower-bound solubility basis first with instrument uncertainty on } \\
\text { each; compositions may not sum to } 100 \text { mol\% because of roundoff error; mole fractions on dry basis, do not account } \\
\text { for water vapor. } \\
\text { (b) Ammonia mole fraction derived from data in Table } 4.7 .5 \text { used to calculate vapor pressure at in situ conditions. } \\
\text { (c) There are too few samples to define the spatial variability of the average gas concentration. }\end{array}$} \\
\hline
\end{tabular}

\subsubsection{Gas Inventory}

The method by which the in situ gas volume fractions (wet basis) were calculated is given in Section 3.6.2; as for the gas composition, the volume fractions are given as a range from the low gas solubility value to the high gas solubility value. The results are presented in Table 4.7.7, which also contains the average gas volume fraction and the average pressure experienced by the gas in the nonconvective layer. The averages for the nonconvective layer are in situ volume averages calculated by Simpson's Rule integration, as described in Section 3.7.1. The corrected gas volume fractions in Table 4.7.7 are consistent with the corrected gas concentrations and compositions in Tables 4.7.4 through 4.7.6. The information in Table 4.7.7 is taken from the "Summary" and "In situ" worksheets of the S-106 RGS calculation spreadsheet on the CD.

The "sampler gas volume fraction" is corrected only for inleakage during the extraction process and air gases added in the isotopic solution in an attempt to reconstruct the total amount of gas (sample and entrainment) that was present in the sampler during X-ray. It is used only for comparison with the $\mathrm{x}$-ray observations of "visible gas fraction" that are discussed in Section 4.7.4.

Table 4.7.7 also contains the water vapor pressures that were used for in situ calculations. The water vapor pressures were found by using salt concentrations from Field (1999a) and the temperatures in the table as inputs to Equation 6.2 of Mahoney and Trent (1995), a correlation for water vapor pressure over concentrated homogeneous and non-homogeneous waste simulants. The gas solubilities used the same parameters as inputs to the Schumpe solubility model (Section 3.6.1). The water vapor pressures and gas solubilities used in calculations are found in the "In situ" worksheet of the S-106 RGS calculation spreadsheet on the CD. No ammonia inventory was calculated because there were no total ammonia concentration data for S-106. 
Table 4.7.7. In Situ Gas Volume Fractions and Conditions in Tank S-106(a)

\begin{tabular}{||c|c|c|c|c|c|c||}
\hline Sample & $\begin{array}{c}\text { Sample } \\
\text { central } \\
\text { height } \\
(\mathrm{cm})\end{array}$ & $\begin{array}{c}\text { Hydro- } \\
\text { static } \\
\text { pressure } \\
(\mathrm{atm})\end{array}$ & $\begin{array}{c}\text { Calculated } \\
\text { water vapor } \\
\text { pressure } \\
(\mathrm{atm})\end{array}$ & $\begin{array}{c}\text { Temp } \\
\left({ }^{\circ} \mathrm{C}\right)\end{array}$ & $\begin{array}{c}\text { Corrected gas } \\
\text { volume fraction } \\
\text { (in-tank conditions) }\end{array}$ & $\begin{array}{c}\text { Sampler gas } \\
\text { volume fraction } \\
\text { (in situ conditions, } \\
\text { low solubility) }\end{array}$ \\
\hline \hline $7-3$ & 362 & 1.14 & 0.0078 & 24.9 & $\begin{array}{c}0.097 \pm 0.009 \text { to } \\
0.094 \pm 0.009\end{array}$ & 0.11 \\
\hline $7-5$ & 265 & 1.29 & 0.0081 & 25.4 & $0.10 \pm 0.010$ & 0.12 \\
\hline $8-6$ & 217 & 1.36 & 0.0084 & 26.1 & $\begin{array}{c}0.077 \pm 0.008 \text { to } \\
0.073 \pm 0.008\end{array}$ & 0.086 \\
\hline $8-10$ & 24 & 1.66 & 0.0083 & 25.8 & $0.14 \pm 0.012$ & 0.16 \\
\hline $\begin{array}{c}\text { Avg in non- } \\
\text { convective } \\
\text { layer }\end{array}$ & 164 & 1.45 & & & $0.10 \pm 0.052$ & \\
\hline
\end{tabular}

(a) The gas volume fraction is expressed on a wet basis, including the volume contribution of water vapor. The uncertainties on the layer-average gas volume fractions are based on spatial variability considerations discussed in Section 3.7.2.

Table 4.7.8 gives various estimates of the STP volume of gas in Tank S-106, including estimates calculated from RGS data alone. The RGS gas inventory in the nonconvective layer was calculated by integrating RGS total gas concentrations over the layer (four data points) and multiplying the average concentration by the volume of the layer. The integration method is described in Section 3.7.1. The RGS volumes in Table 4.7.8 include corrections to remove the contamination gas: entrained air and argon, helium backfill, air leaks during and after extraction, and air that came in with the isotopic ammonia vapor. The uncertainties in the gas inventories are based on the spatial variability considerations discussed in Section 3.7.2. The information in Table 4.7.8 is taken from the "Inventory" worksheet of the S-106 RGS calculation spreadsheet on the CD.

Table 4.7.8. S-106 Gas Inventory Estimates

\begin{tabular}{||l|c|c||}
\hline \multirow{2}{*}{ Quantity } & RGS Method & BPE Method(a) \\
\cline { 2 - 3 } & $\begin{array}{c}\text { Nonconvective Layer } \\
\text { Without Crust }\end{array}$ & Tank Total \\
\hline \hline $\begin{array}{l}\text { Avg gas fraction (low } \\
\text { gas solubility) }\end{array}$ & $0.10 \pm 0.052$ & $0.23 \pm 0.09$ \\
\hline $\begin{array}{l}\text { Gas volume (m3) } \\
\text { in situ (wet) }\end{array}$ & $169 \pm 85$ & $411 \pm 166$ \\
STP (wet) & $224 \pm 112$ & $543 \pm 219$ \\
\hline $\begin{array}{l}\text { (a) Barometric pressure method. Inventory based on average gas pressure } \\
\text { calculated from RGS data and dL/dP of -0.91 } \pm 0.36 \text { in./in.-Hg (Whitney et al. } \\
\text { 1997). Neither inventory can be selected as a best-estimate because of the } \\
\text { unusual waste configuration. }\end{array}$ \\
\hline
\end{tabular}


The RGS method ( $169 \pm 85 \mathrm{~m}^{3}$ in situ) gave less than half the gas inventory that was calculated by the BPE method. The RGS measurements did not include the gas in the peripheral crust or the supernatant. The gas inventory in the supernatant would have been negligible based on experience with other tanks. The gas inventory in the peripheral crust is unknown but could have comprised domespace air to a large extent. Segment 1 (the top segment) from peripheral riser 14 appeared frothy, possibly fissured in its x-ray image, while segment 2 from riser 14 contained very dry crumbly salt. Such a material would not be expected to retain waste gas. However, the (probably wetter) waste farther down would have retained gas.

The surface level measurement in S-106 is made via an Enraf buoyancy gauge in riser 3. This riser is in the central part of the tank near risers 7 and 8, from which the usable RGS samples were taken. This central area is a supernatant pool of about $4.6 \mathrm{~m}(15 \mathrm{ft})$ radius, as described by Field (1999a, Appendix D). Segments 1 and 2 from riser 7 and segments 1, 3, 4, and 5 from riser 8 contained a high fraction of liquid, and the sampler did not penetrate hard material at the surface. Level data for this tank were recorded hourly, which enables the most precise determination of the $\mathrm{dL} / \mathrm{dP}$ correlation. In a typical tank waste configuration, because the level gauge is in the central portion of the tank and there is evidence of a liquid layer to transmit waste expansion/compression, the BPE method could be confidently applied. However, this unusual tank waste configuration posed a unique problem of interpretation.

The difference between the inventories calculated by the RGS and BPE methods can be explained by either (or both) of two possible situations. First, the liquid whose surface level was measured by Enraf was in contact with interstitial gas fractions that were much higher than those measured in the central portion of the tank to which RGS sampling was limited. (These high gas fractions might have been related to the higher waste level at the tank periphery.) Or, second, the liquid in the "bowl" in the center of the tank was displaced by gas under and all around the sides of the bowl so the liquid surface response to gas volume changes caused by barometric pressure changes was amplified. This requires liquid to be driven out of the bottom of the bowl by gas expansion, and its rising surface to not soak back rapidly into the upper "walls" of the bowl.

Because of the very large difference between the RGS and BPE predictions, it is worth looking at the long-term surface level rise in S-106 for additional information. The waste surface level (measured at riser 3) rose steadily by more than 17 in. between 1981 and September 1995. If an additional $9 \mathrm{in}$. rise is allowed for the gas inventory as of 1981, and $15 \mathrm{in}$. for estimated evaporation since 1981 (Hodgson et al. 1998), the overall rise of 41 in. is consistent with a gas volume fraction of 0.26 . The surface level rise estimate could be also affected by amplification (though it is a long-term measurement, and the amplification would be reduced by liquid soaking back into the waste).

Because the two inventories are very different and both open to question-the RGS inventory because both risers are centrally located and the waste shows strong signs of lateral variability, and the BPE inventory because of the unusual waste configuration-neither can be recommended as a "best estimate." Further information would be required to develop a best estimate.

Table 4.7.9 contains the calculated nonconvective layer inventories of each of the major gases retained in the waste. These inventories are based on the layer-average composition from RGS data (Table 4.7.6) and the RGS gas inventory (Table 4.7.8). The inventories should not be considered a best estimate; they are more likely to be a lower bound. 
Table 4.7.9. Speciated S-106 Gas Inventory(a)

\begin{tabular}{||c|c|c||}
\hline \multirow{2}{*}{ Species } & \multicolumn{2}{|c|}{ Nonconvective Layer (without crust) } \\
\cline { 2 - 3 } & $\begin{array}{c}\text { Gas-Phase Inventory } \\
\left(\mathrm{m}^{3} \text { at STP) }\right.\end{array}$ & $\begin{array}{c}\text { Dissolved Inventory } \\
\text { (m³ at STP) }\end{array}$ \\
\hline $\mathrm{N}_{2}$ & $55-54$ & $0.062-0.37$ \\
\hline $\mathrm{H}_{2}$ & 140 & $0.41-1.7$ \\
\hline $\mathrm{N}_{2} \mathrm{O}$ & $24-19$ & $1.6-6.1$ \\
\hline $\mathrm{NH}_{3}$ & $0.78-0.36$ & 850 \\
\hline $\mathrm{CH}_{4}$ & $0.56-0.55$ & $0.0014-0.0087$ \\
\hline Other & 1.0 & 0 \\
\hline $\begin{array}{l}\text { (a) Inventories based on layer-average compositions from RGS data (Table 4.7.6) } \\
\text { and gas inventory (RGS, Table 4.7.8). Uncertainty in each inventory is 50\% based } \\
\text { on spatial variability considerations (Section 3.7.2). Inventories given for lower- } \\
\text { and upper-bound solubilities, lower-bound solubility basis first. }\end{array}$ \\
\hline
\end{tabular}

Figure 4.7.4 shows the sample temperatures, corrected gas volume fractions (Table 4.7.7), and corrected compositions of the low-solubility constituents in the samples from Tank S-106. The temperatures came from the TC tree in riser 2. The compositions represent the mole fraction of the species in the "insoluble" gas; water and ammonia are not included, so the mole fractions are not the same as those in Table 4.7.4. The gas volume fractions and mole fractions in the figure are the values for lower-bound gas solubilities. Figure 4.7.4 also lists the observations from core extrusions of non-RGS samples and from $x$-rays of RGS samples as a way of tying those observations together with RGS data. The x-ray data are described in more detail in Section 4.7.4.

\subsubsection{X-Ray Results}

Table 4.7.10 summarizes all the available radiography observations from Tank S-106. The $\mathrm{x}$-ray images themselves can be found on the $\mathrm{CD}$ that accompanies this report. The uncertainties of the visible gas volume fractions that are given in Table 4.7.10 are unknown but (based on the uncertainty in measuring the gas gap from $x$-ray images) are expected to be larger than the uncertainties in the gas volume fractions calculated from gas extraction. (The "visible" gas volume fraction refers to the gas that has separated from the solids and liquid such that its volume can be calculated based on the image. The uncorrected gas fractions from extraction are used for comparison to reflect the presence of entrained air in the sampler.)

Table 4.7.10 compares $\mathrm{x}$-ray-derived gas volume fractions with extraction-derived gas volume fractions. The comparison shows that sample recovery was close to $100 \%$ for all the samples. This conclusion is based on the fact that the extracted uncorrected gas was equal to or a few volume percent more than the visible gas.

Though RGS samples 8-2 and 14-2 were not successfully extracted, gas was seen in their $\mathrm{x}$-rays. Only a small amount of gas was seen in sample 8-2. Sample 14-2 appears to have had high gas content, but it is also possible that the sample recovery was not complete. Unfortunately, the sample was compromised, and it was therefore impossible to determine how much of the apparent gas volume in the sampler was waste gas. Sample 14-1 contained no liquid or solids, so it is possible that sample 14-2 was the top waste and partly filled with air. However, there is 

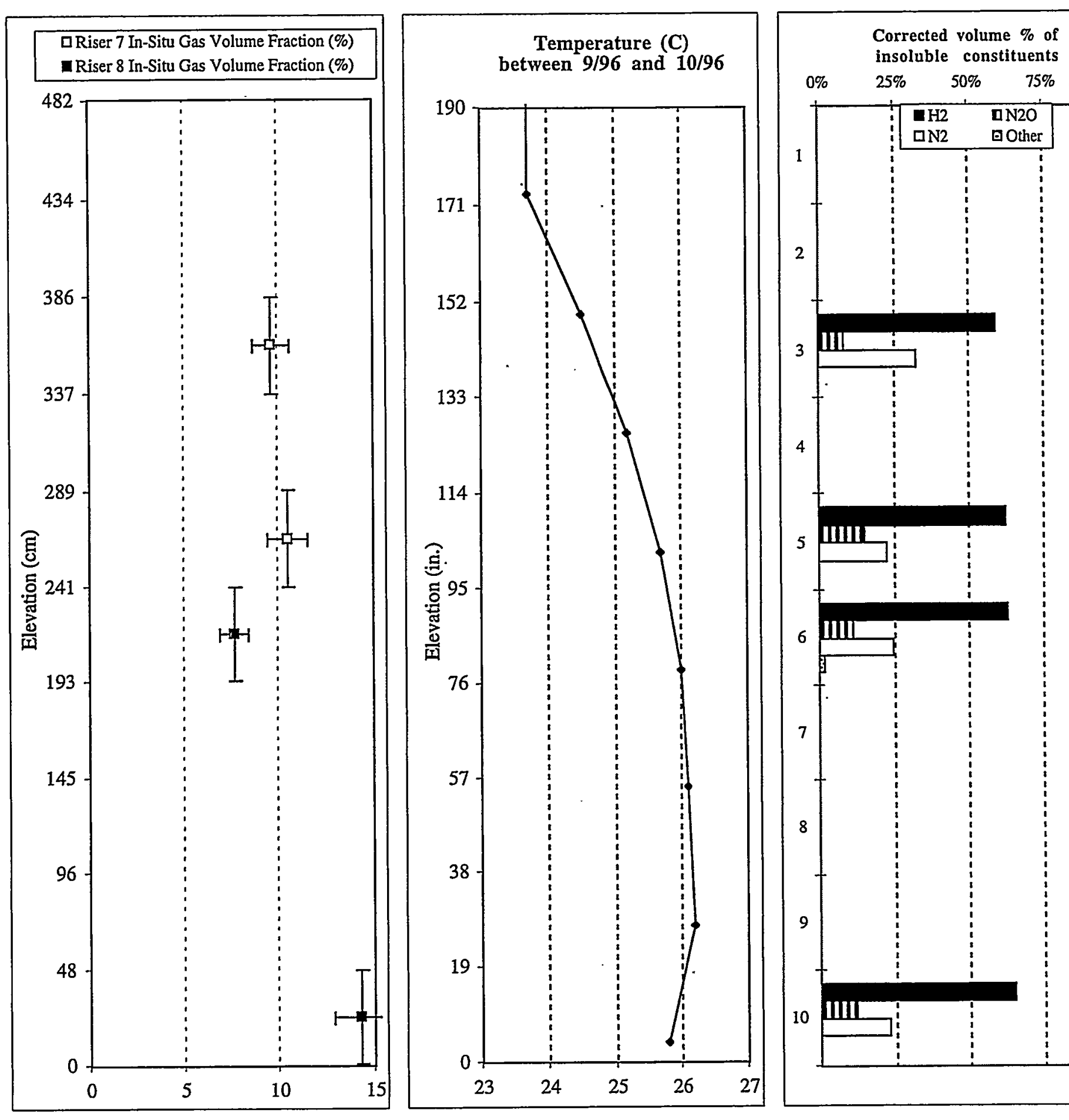

Figure 4.7.4. Gas Volume Fractions, Temperatures, $C$ 


\section{S-106 waste level is $460 \mathrm{~cm}$ (181 inches).}

2 Riser 14 X-RAY: much void, lumpy or frothy surface. Clean piston, 10 in. gas gap. $15 \mathrm{mR} / \mathrm{hr}$.

3 Riser 14: 2 in. (15 g) gray and white very dry crumbly salt.

1 Riser 8: Less than measurable white crystals, $310 \mathrm{~mL}$ ( $413 \mathrm{~g}$ ) clear yellow liquid.

1 Riser 7: Less than measurable white crystals, $250 \mathrm{~mL}$ ( $344 \mathrm{~g}$ ) clear yellow liquid.

2 Riser 8 X-RAY: Featureless, flat surface, no waste on fully-retracted piston. Gas gap 0.7 in. $0.8 \mathrm{R} / \mathrm{hr}$.

2 Riser 7: 3 in. (79 g) black, green and white salt slurry, $230 \mathrm{~mL}(303 \mathrm{~g}$ ) green opaque liquid.

3 - Riser 8: Less than measurable white crystals, $310 \mathrm{~mL}$ ( $427 \mathrm{~g}$ ) opaque yellow liquid.

3 Riser 7 X-RAY: 1- to 2-mm bubbles bottom 6 in., no features above. Flat surface, clean piston, 1.7 in. gas gap.

4 Riser 8: 1 in. (22 g) dark green wet salt, $250 \mathrm{~mL}$ (341 g) opaque dark green liquid. HHF contamination.

4 Riser 7: 19 in. (427 g) solids, top half dark gray wet salt with some large crystals, bottom half green-gray moist salt.

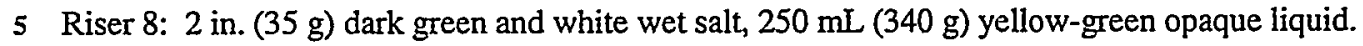

5 Riser 7 X-RAY: one worm-like void ( $2 \mathrm{~mm}$ wide, $7 \mathrm{~mm}$ long), scattered $<1 \mathrm{~mm}$ bubbles mostly near top. No gas gap.

6 Riser 8 X-RAY: Up to 3-mm voids in bottom 6 in., no features above. Flat surface, bare piston, gas gap 1.1 in.

6 Riser 7: 3 in. (102 g) dark- or green-gray salt slurry, $140 \mathrm{~mL}$ (202 g) dark-green opaque liquid. HHF contamination.

7 Riser 8: 3 in. (67 g) dark green wet salt, $190 \mathrm{~mL}$ (286 g) dark-green opaque liquid. HHF contamination.

8 Riser 8: 19 in. (428 g) green-gray wet salt.

9 Riser 8: 19 in. (438 g) green-gray wet salt.

10 Riser 8 X-RAY: Several bullet-shaped voids, some 1-cm, one sampler-spanning. Separated liquid. Gas gap 1.5 in. 
Table 4.7.10. Summary of Observations from X-Ray Images of Tank S-106

\begin{tabular}{|c|c|}
\hline Segment & Comments/Observations \\
\hline $\begin{array}{l}14-2 \\
\text { (RGS, } \\
3 / 20 / 97)\end{array}$ & $\begin{array}{l}\text { Only } 21 \mathrm{~cm} \text { ( } 8 \text { in.) of waste, showing much bubble structure (shaped rather like gas around } \\
\text { lumps of sand) and also some higher-density blobs. The top waste surface is lumpy or frothy, } \\
\text { definitely not a liquid meniscus. There is no waste attached to the piston, which is fully } \\
\text { retracted. The gas gap is } 25 \mathrm{~cm} \text { ( } 10 \text { in.), for a visible gas (or void) volume fraction of } 0.55 \text {. } \\
\text { (Because the sampler valve had not fully closed and the sample was compromised, there is no } \\
\text { extraction-based gas volume fraction for comparison.) }\end{array}$ \\
\hline $\begin{array}{c}8-2 \\
\text { (RGS, } \\
2 / 12 / 97) \\
\end{array}$ & $\begin{array}{l}\text { Completely featureless, with a flat meniscus and no waste hanging down from the piston. } \\
\text { Piston is fully retracted. The gas gap is } 1.7 \mathrm{~cm}(0.7 \mathrm{in} \text {.), for a visible volume fraction of } 0.04 \text {. }\end{array}$ \\
\hline $\begin{array}{l}7-3 \\
(\mathrm{RGS} \\
2 / 24 / 97)\end{array}$ & $\begin{array}{l}\text { Mostly featureless, with a flat meniscus and no waste hanging down from the piston. In the } \\
\text { lower third of the sampler, a few barely perceptible lower-density features, probably gas bubbles; } \\
\text { round, about } 4 \mathrm{~mm} \text { in diameter. The piston was fully retracted. The gas gap under the piston is } \\
4.3 \mathrm{~cm}(1.7 \text { in.), for a visible volume fraction of } 0.09 \text {. (Compare with the uncorrected gas } \\
\text { volume fraction of } 0.11 \text { obtained by gas extraction, Table } 4.7 .7 \text {.) }\end{array}$ \\
\hline $\begin{array}{l}7-5 \\
\text { (RGS, } \\
2 / 25 / 97)\end{array}$ & $\begin{array}{l}\text { Mostly featureless, with the sampler completely filled (no gas gap). One irregular (worm-like) } \\
\text { lower-density feature, probably a bubble, about } 2 \mathrm{~mm} \text { wide by } 7 \mathrm{~mm} \text { long. Piston fully } \\
\text { retracted. The visible volume fraction is } 0 \text {. (Compare with the uncorrected gas volume fraction } \\
\text { of } 0.12 \text { obtained by gas extraction Table } 4.7 .7 \text {.) }\end{array}$ \\
\hline $\begin{array}{l}8-6 \\
\text { (RGS, } \\
2 / 18 / 97)\end{array}$ & $\begin{array}{l}\text { Several definite lower- and higher-density features (oval, about } 4 \mathrm{~mm} \text { diameter) in one image in } \\
\text { the lower third of the sampler; otherwise featureless. The lower-density features are probably } \\
\text { bubbles; the nature of the higher-density features is unknown. The meniscus is flat and there is } \\
\text { no waste attached to the piston. The gas gap is } 2.8 \mathrm{~cm}(1.1 \mathrm{in} \text {.), for a visible volume fraction } \\
\text { of } 0.06 \text {. (Compare with the uncorrected gas volume fraction of } 0.086 \text { obtained by gas extraction, } \\
\text { Table 4.7.7.) }\end{array}$ \\
\hline $\begin{array}{l}8-10 \\
\text { (RGS, } \\
2 / 21 / 97)\end{array}$ & $\begin{array}{l}\text { Several large ( } 1 \mathrm{~cm} \text { and larger), flat-bottomed, bullet-shaped gas features. Also, in the lower } \\
\text { third of the sampler, a gas gap all the way across the width (though probably not across its } \\
\text { whole depth). This large gas space is also bullet-shaped and flat-bottomed. Its base appears to } \\
\text { be a layer (about } 3 \mathrm{~cm} \text { deep) of waste with a slightly lower density than most of the sample. } \\
\text { The bottom of this possibly liquid layer is also flat, and beneath it is the denser (and more } \\
\text { typical) waste. There is also a thin layer (about } 5 \mathrm{~mm} \text { deep) of "liquid" at the top of the waste. } \\
\text { Both its upper interface (with gas) and its lower one (with denser material) are flat. } \\
\text { The gas gap at the top of the waste is about } 3.8 \mathrm{~cm} \text { ( } 1.5 \mathrm{in} \text { ), giving a gap volume fraction of } \\
0.08 \text {. The embedded bullet-shaped bubbles add roughly } 0.02 \text { volume fraction of gas, bringing the } \\
\text { visible gas volume fraction to about } 0.10 \text {. (Compare with the uncorrected gas volume fraction } \\
\text { of } 0.16 \text { in Table } 4.7 .7 \text {, obtained by gas extraction Table } 4.7 .7 \text {.) }\end{array}$ \\
\hline
\end{tabular}

another possibility. Because the sample was taken near the top of the tank in a riser near the tank wall, like sample 7-2 of U-103, we can speculate that sample 14-2 of S-106 contained a high gas fraction, like sample 7-2 of U-103. If there were higher gas fractions in the waste in the tank periphery than in the center, it would explain why RGS (whose successful samples were taken only in the center) estimated a lower gas inventory for S-106 than did the BPE method (a less localized approach).

Sample 7-5, uniquely, contains no gas gap and only one bubble in the waste. Thus its visible gas volume fraction is zero. However, its uncorrected gas volume fraction was 0.117 (based on gas extraction). This suggests that the gas was predominantly present either in solution 
or in bubbles too small to be recognized in the $\mathrm{x}$-ray ( $0.5 \mathrm{~mm}$ or less). Recall that sample 7-5 was also unique in having an ammonia concentration that was higher than that in the other samples. However, the relationship between these observations is unclear.

Sample 8-10 also shows unique behavior. None of the other samples showed any sign of separation of the solids and liquids, but in sample 8-10 liquid layers appear to have formed under the gas gaps. It is possible that the liquid layers came into existence because the solids settled out of them (forming, for example, the flat bottom of the liquid layer that underlies the largest visible bubble). Some of the liquid could also have come from drainage from the solids above the larger bubbles. However, such drainage can be questioned because core extrusion observations showed no drainable liquid in the two samples above this one.

Table 4.7.11 provides a summary of the waste densities that have been calculated from radiographic data for the RGS samples for which air and water standard images were available. The radiographic densities include the gas in the waste, were calculated by the current $x$-ray analysis method, and are given for several locations within each sample to show density trends. Table 4.7.11 also includes density measurements made on extruded non-RGS samples (Field 1998). The two methods give reasonably consistent results for four of the five samples considering the differences expected because of the effect of retained gas on the radiographic densities of the RGS samples, the fact that RGS are adjacent to standard core samples rather than collocated, and the partial unavailability of $\mathrm{x}$-ray calibration records. Sample 8-10 appears to be underexposed and therefore to give an inaccurately high range of densities.

\subsubsection{Drillstring Composition Comparison}

The RGS gas-phase composition data (Table 4.7.6) were used to calculate $\mathrm{H}_{2} / \mathrm{N}_{2} \mathrm{O}$ ratios for comparison with ratios from drillstring measurements (Siciliano 1998, Table 8.1). Domespace grab samples (McCain 1999, Table B-12) all contained $\mathrm{N}_{2} \mathrm{O}$ below the detection limit, so dome space ratios were not calculated. The ratios can be seen in Table 4.7.12. The $\mathrm{RGS} \mathrm{H}_{2} / \mathrm{N}_{2} \mathrm{O}$ ratios agreed closely with two of the three drillstring ratios. The riser 8 drillstring ratio (which did not agree with RGS data) was calculated from an $\mathrm{N}_{2} \mathrm{O}$ mole fraction that was quite small, $0.003 \mathrm{vol} \%$, possibly making the ratio less accurate.

Table 4.7.11. Densities of S-106 Samples from Radiography and Core Samples

\begin{tabular}{|c|c|c|c|}
\hline $\begin{array}{l}\text { Riser- } \\
\text { Segment } \\
\text { Number }\end{array}$ & $\begin{array}{l}\text { Distance From } \\
\text { bottom of sampler } \\
\text { (ft) }\end{array}$ & $\begin{array}{c}\text { Calculated Mean Density } \\
\text { by Current Method } \\
(\mathrm{g} / \mathrm{cc})\end{array}$ & $\begin{array}{l}\text { Degassed Density Above and } \\
\text { Below the RGS Sample } \\
\text { (from cores) (g/cc) }\end{array}$ \\
\hline \multirow[t]{2}{*}{ 14-2(a) } & 0.50 & 0.89 & \multirow{2}{*}{$\begin{array}{l}\text { no liquids or solids collected } \\
\text { above } \\
\text { dry crumbly salt below }\end{array}$} \\
\hline & 0.25 & 0.94 & \\
\hline \multirow[t]{5}{*}{$8-2$} & 1.25 & 1.29 & \multirow[t]{5}{*}{ density above, $1.41 \mathrm{~g} / \mathrm{cc}$ (liquid) } \\
\hline & 1.00 & 1.38 & \\
\hline & 0.75 & 1.46 & \\
\hline & 0.50 & 1.44 & \\
\hline & 0.25 & 1.35 & \\
\hline
\end{tabular}


Table 4.7.11 (contd)

\begin{tabular}{|c|c|c|c|}
\hline $\begin{array}{l}\text { Riser- } \\
\text { Segment } \\
\text { Number }\end{array}$ & $\begin{array}{l}\text { Distance From } \\
\text { bottom of sampler } \\
\text { (ft) }\end{array}$ & $\begin{array}{c}\text { Calculated Mean Density } \\
\text { by Current Method } \\
(\mathrm{g} / \mathrm{cc})\end{array}$ & $\begin{array}{l}\text { Degassed Density Above and } \\
\text { Below the RGS Sample } \\
\text { (from cores) }(\mathrm{g} / \mathrm{cc})\end{array}$ \\
\hline \multirow[t]{5}{*}{$7-3$} & 1.25 & 1.46 & \multirow[t]{5}{*}{ density above, $1.39 \mathrm{~g} / \mathrm{cc}$ (liquid) } \\
\hline & 1.00 & 1.47 & \\
\hline & 0.75 & 1.49 & \\
\hline & 0.50 & 1.56 & \\
\hline & 0.25 & 1.56 & \\
\hline \multirow[t]{5}{*}{$7-5$} & 1.25 & 1.60 & \multirow[t]{5}{*}{ density above, $1.81 \mathrm{~g} / \mathrm{cc}$ (solids) } \\
\hline & 1.00 & 1.51 & \\
\hline & 0.75 & 1.37 & \\
\hline & 0.50 & 1.39 & \\
\hline & 0.25 & 1.33 & \\
\hline \multirow[t]{5}{*}{$8-6(b)$} & 1.25 & 1.48 & \multirow[t]{5}{*}{ density above, $1.47 \mathrm{~g} / \mathrm{cc}$ (liquid) } \\
\hline & 1.00 & 1.48 & \\
\hline & 0.75 & 1.50 & \\
\hline & 0.50 & 1.69 & \\
\hline & 0.25 & 1.36 & \\
\hline \multirow[t]{5}{*}{$8-10$ (b) } & 1.25 & 2.30 & \multirow{5}{*}{ density above, $1.64 \mathrm{~g} / \mathrm{cc}$} \\
\hline & 1.00 & 2.27 & \\
\hline & 0.75 & 2.23 & \\
\hline & 0.50 & 1.89 & \\
\hline & 0.25 & 2.27 & \\
\hline \multicolumn{4}{|c|}{$\begin{array}{l}\text { (a) This image was unusually blurry. } \\
\text { (b) These segments lacked standard air/water density profiles; used data from segment } 8-2 \text {, taken at almost the } \\
\text { same time. Segment 8-10 is probably underexposed. }\end{array}$} \\
\hline
\end{tabular}

Table 4.7.12. Comparison with Drillstring Data(a)

\begin{tabular}{||l|c||}
\hline \multicolumn{1}{|c|}{ Sample } & $\mathrm{H}_{2} / \mathrm{N}_{2} \mathrm{O}$ \\
\hline \hline RGS, 7-3 & $7.4-9.4$ \\
\hline RGS, 7-5 & $4.4-5.5$ \\
\hline RGS, 8-6 & $6.2-8.3$ \\
\hline RGS, 8-10 & $6.0-7.0$ \\
\hline Drillstring, riser 7, 2/25/97 & 4.0 \\
\hline Drillstring, riser 7, 2/25/97 & 6.3 \\
\hline Drillstring, riser 8, 2/18/97 & 22 \\
\hline $\begin{array}{l}\text { (a) RGS ratios calculated for lower- and upper-bound solubilities. Both ends } \\
\text { of ratio range given in table, lower-bound solubility basis first. }\end{array}$ \\
\hline
\end{tabular}




\subsection{BY-101}

Tank BY-101 was the eighth tank and the fourth SST sampled with the RGS. The methods of RGS sampler preparation, sample acquisition, and sample gas extraction were the same as those used for U-103, S-106, and BY-109, the other tanks that were sampled in FY 1997.

Two samples were taken from BY-101: riser 10B, segment 3, and riser 10D, segment 2 . Further sampling was impossible because of obstructions at both risers. The samples contained no retained gas and provided no useful information on the composition or quantity of retained gas. Following are the supporting evidence and additional details:

- Mass spectrometry analyses showed that only air and helium were present in the two BY-101 samples. The typical retained waste gases hydrogen, nitrous oxide, methane, and ammonia were found in quantities of less than $0.01 \mathrm{~mol} \%$ each.

- $\quad \mathrm{X}$-rays showed no visible waste in the samplers, (a) although the samples were taken well below the waste surface level of $345 \mathrm{~cm}$ (136 in.). The observations made during RGS system cleanup, which was carried out after the attempted gas extraction, also confirmed that the samplers contained no solid or liquid waste.

- Helium was present at about 1 mol\% in the samples, representing roughly $3 \mathrm{cc}$ (at STP) of gas trapped in the sampler's leading edge. This quantity of helium is consistent with the findings for other samples taken with samplers backfilled with helium.

When the samples were extruded into the evacuated RGS system (whose total volume was $1575 \mathrm{cc}$ ), the resulting pressures were $20.04 \mathrm{kPa}$ for segment $10 \mathrm{D}-2$ and $20.42 \mathrm{kPa}$ for segment 10B-3. Applying the ideal gas law shows that the sample pressure (in the 308-cc sampler volume) must have been $103 \mathrm{kPa}$, or roughly $1 \mathrm{~atm}$. This atmospheric pressure, in conjunction with the composition of the sample gas, suggests that the samples contained domespace gas.

\subsection{BY-109}

Tank 241-BY-109 (BY-109) was the ninth tank and fifth SST sampled with the RGS. An important factor in choosing this tank for RGS sampling was to assess the effect of salt-well pumping on gas retention. BY-109 had its supernatant liquid removed in 1985 and was partially saltwell pumped in 1996, not long before sampling, leaving the interstitial liquid level (ILL) about 20 in. below the waste surface. BY-109 is part of a group of tanks (Cluster 15 in Stewart et al. $1996 \mathrm{~b}$ ) that have fairly high radioactivity, nitrite concentrations around $0.25 \mathrm{M}$, and low TOC (about $0.05 \%$ ). HTCEs indicate that the primary waste stored in BY-109 was saltcake from evaporator campaign BY, and the secondary waste was metals from the bismuth phosphate process (1944 to 1951) (Remund et al. 1995).

Push-mode sampling was carried out in risers 12C and 10B in June 1997.(b) The approximate locations of various risers are depicted in Figure 4.9.1. Risers $10 \mathrm{~B}$ and $12 \mathrm{C}$ are both

(a) Mousel A (LATA). 1997. Sampling field log spreadsheet "tk1data.xls," received by LA Mahoney (PNNL) on June 26, 1997. Los Alamos Technical Associates, Richland, Washington.

(b) The sampling scheme may be found in Sampling Plan for Tank 241-BY-109 Retained Gas Sampler Deployment, by HC Reid, JM Bates, and A Shekarriz, May 1997. TWSFG:97.42, Pacific Northwest National Laboratory, Richland, Washington. 


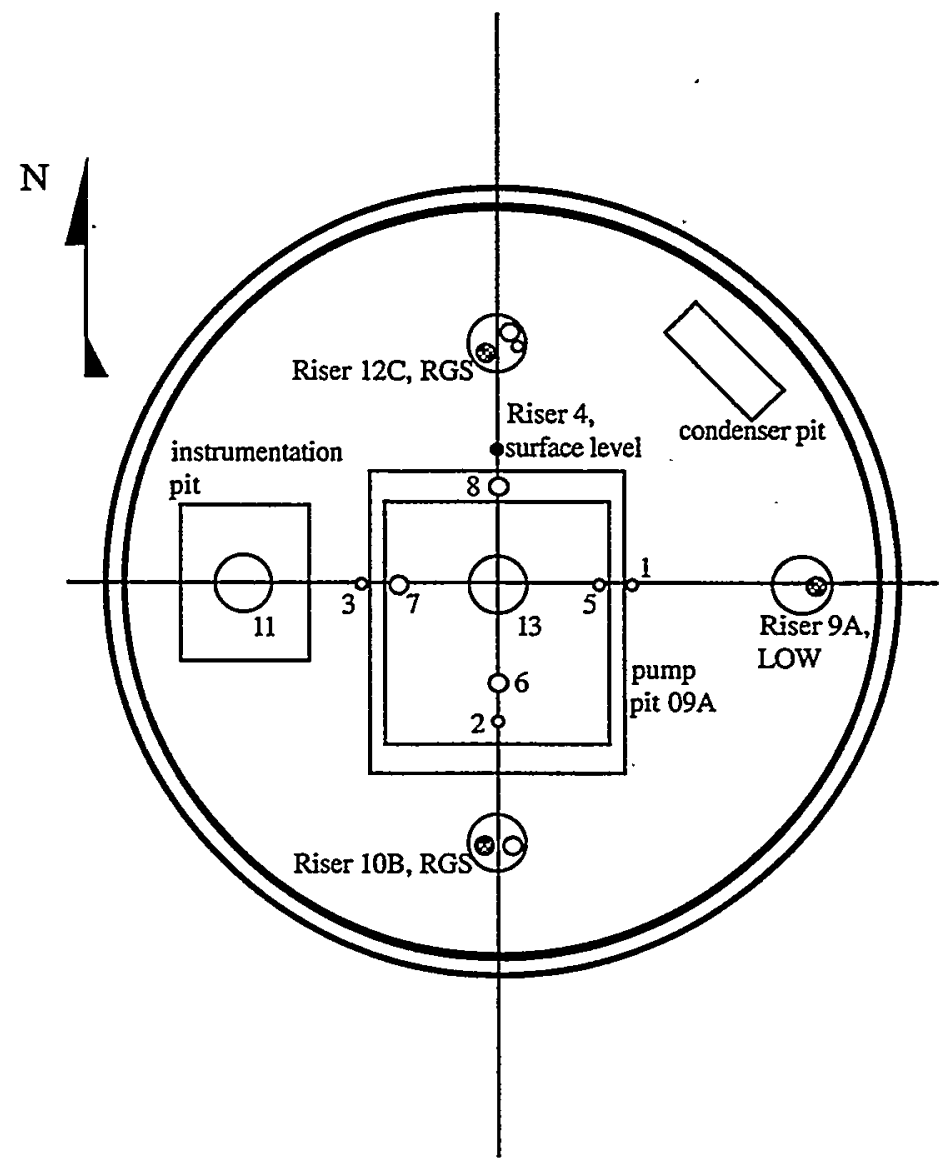

Figure 4.9.1. Schematic Diagram of Riser Locations in Tank BY-109

roughly two-thirds of a tank radius from the tank center in opposite directions. The elevations of the RGS segments are depicted in Figure 4.9.2, as is the tank content layering.

Consort et al. (1996) describe Tank BY-109 as a sound SST. Interim stabilization was completed in June 1985. BY-109 has a capacity of about 758,000 gal and, at the time of sampling, contained about 1,600,000 L (423,000 gal) of waste, of which 1,290,000 L (340,000 gal) was saltcake and 314,000 L (83,000 gal) was sludge-type waste. Salt-well pumping carried out in 1996 removed the supernatant liquid, but about 121,000 L (32,000 gal) of interstitial liquid remained in the saltcake and sludge at the time of sampling. Of the waste, about 1,070,000 L $(282,000 \mathrm{gal})$ lay below the ILL.

Surface level measurements through riser 4, as catalogued on the Tank Characterization Database, showed that the level had remained steady, with readings fluctuating between 357.6 and $348.2 \mathrm{~cm}$ (140.8 and 137.1 in.). Riser 9A LOW measurements showed an IIL of $279.1 \mathrm{~cm}$ (109.9 in.) as of January 21, 1997. Zipcord measurements taken March 6, 1997 indicated waste depth under riser 10B as $342.6 \mathrm{~cm}$ (134.9 in.).(a) For riser $12 \mathrm{C}$, the second riser selected for core sampling, zipcord measurements indicated a waste depth of $311.6 \mathrm{~cm}$ (122.7 in.). (Both zipcord measurements are referenced to tank bottom at tank center.) RGS sampling locations for each riser are shown in relation to the tank contents profile in Figure 4.9.2.

(a) cc:Mail communication, BC Simpson (LMHC) to JM Bates (PNNL), April 16, 1997. 


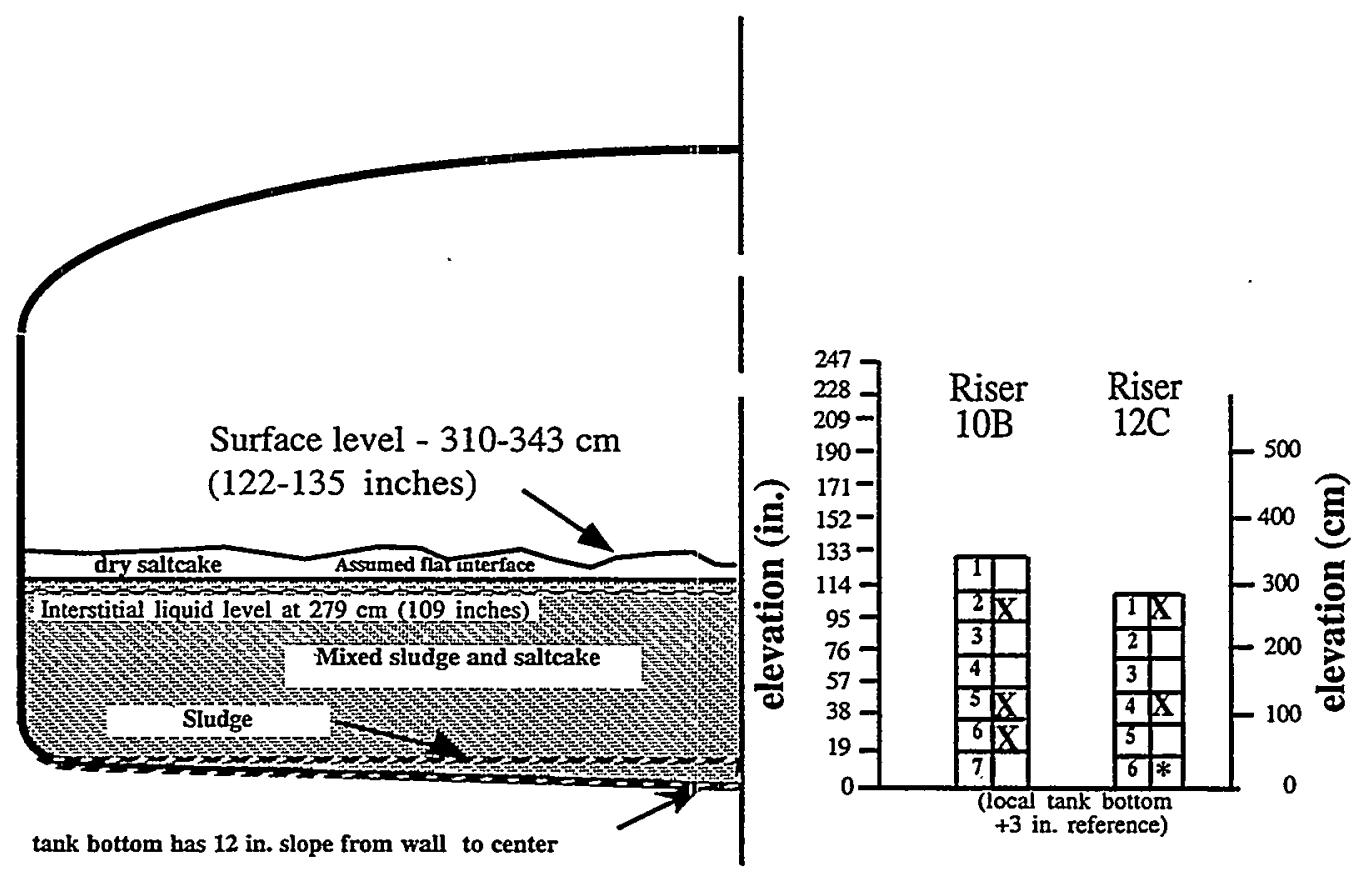

Figure 4.9.2. Diagram of Waste Layering and RGS Sample Elevations for BY-109 (segments marked with a * could not be taken because the waste was too hard or the tank bottom was reached)

The most recent information on tank content layering (derived from the core observations made in 1997) is presented in Figure 4.9.3 (Jo 1998). Note the variation in the nature of the layers at risers $12 \mathrm{C}$ and $10 \mathrm{~B}$. According to these observations, sludge slurry probably made up much or all of the three RGS samples that were taken successfully (riser $12 \mathrm{C}$, segment 4, and riser $10 \mathrm{~B}$, segments 5 and 6). It should be noted that the RGS samples were observed after RGS gas extraction, which could have homogenized or dried them or otherwise modified their appearance.

Temperature data were not available for Tank BY-109. The TC tree in the tank became unusable sometime during the late 1970s and was not replaced after it malfunctioned. Tran (1993), the standard source for TC elevation data, does not give locations for this TC tree. The most recent historical temperature datum (from TC\#1 in 1991, before salt-well pumping) gave a local temperature of $87.2^{\circ} \mathrm{F}$. This temperature is used for all the in situ calculations throughout the tank because no recent temperature profile data are available.

Densities for the liquid and bulk solids in Tank BY-109 were available from the non-RGS samples taken in 1997 (Jo 1998). Only one sample contained drainable liquid; its density was $1500 \mathrm{~kg} / \mathrm{m}^{3}$. The degassed bulk density of the zone above the ILL was based on a single data point of $1820 \mathrm{~kg} / \mathrm{m}^{3}$, and the zone below the $\Pi \mathrm{LL}$ had a degassed density with an average and standard deviation of $1696 \pm 65 \mathrm{~kg} / \mathrm{m}^{3}$ (three data points). These densities were used for hydrostatic pressure calculations. Layer depths of $64 \mathrm{~cm}$ (25 in.) and $279 \mathrm{~cm}$ (110 in.) were used in calculations for the zones above and below the ILL. 

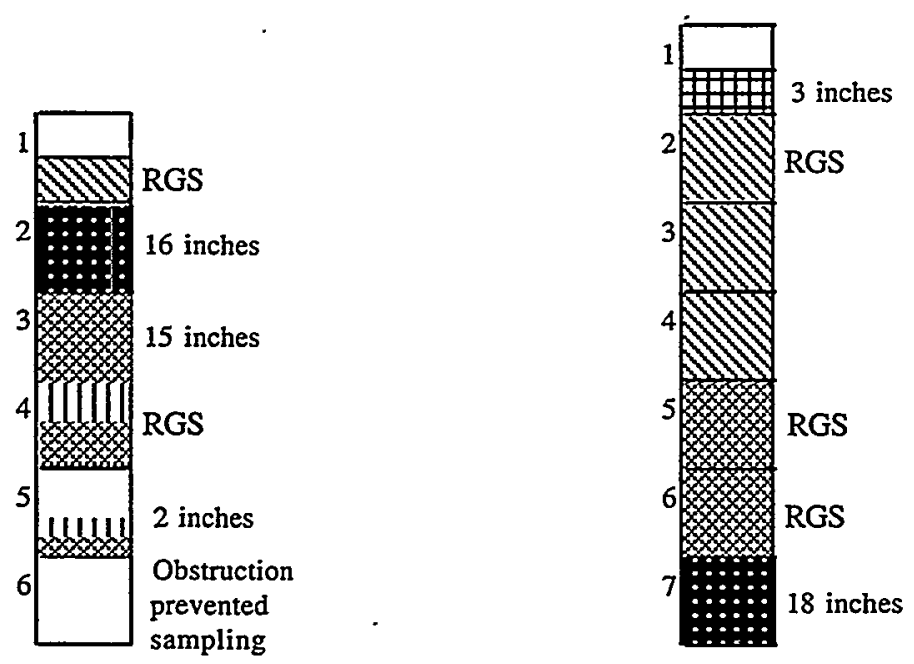

\section{Physical Character}

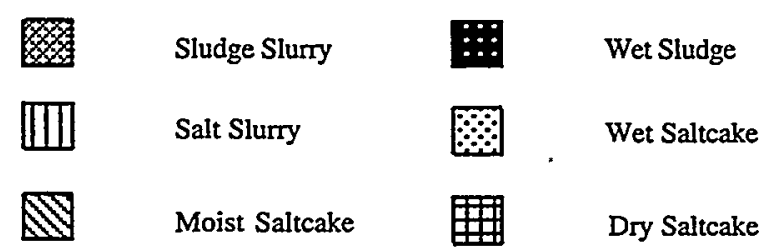

Figure 4.9.3. Recent Core Profiles for the Push-Mode Sampling Risers in Tank BY-109

\subsubsection{Sampling and Extraction Information}

The samples that underwent RGS analysis are listed in Table 4.9.1. Field data, including dose rates and downforce limits, are supplied in Appendix A.

For the first seven tanks, up through S-106, the predominant cause of sampler loss had been the failure of the sampler ball valve to close (Mahoney et al. 1997). The ball valve spring was therefore changed to exert more closure force. After that change, a previously unseen type of sampler failure occurred in BY-109 samples: the sampler piston did not fully retract, or so much . force was needed to retract the piston that the piston seals were damaged and the sample compromised. Further sampler changes were made to avoid this difficulty.

Two BY-109 samples, 12C-1 and 10B-2, were discarded because the piston seals were damaged, allowing post-sampling leakage to compromise the samples. As a result of these difficulties, no samples were taken successfully in the zone of waste above the ILL. The successful samples were of reduced volume because the sampler piston was not fully retracted. The reduced effective sampler volumes and increased uncertainty in the sampler volume were taken into account in calculating gas concentrations in the waste. Piston positions were found by examining $\mathrm{x}$-ray images (Section 4.9.4). 
Table 4.9.1. Lag Times for Processing RGS Samples from Tank BY-109

\begin{tabular}{|c|c|c|c||}
\hline Sample & Acquisition Date & Processing Date & Lag (days) \\
\hline \hline 12C-4 & June 12,1997 & July 1, 1997 & 19 \\
\hline 10B-5 & June 16,1997 & July 11, 1997 & 25 \\
\hline 10B-6 & June 17, 1997 & July 16,1997 & 29 \\
\hline
\end{tabular}

Table 4.9.1 also shows the lag times for these samples. This information was provided to allow data users to correlate the lag time between sampling and extrusion with the concentrations of time allowed by the sampling plan is 24 days based on measured sampler leakage rates during acceptance testing. The hold times for samples 10B-5 and 10B-6 exceeded this limit, but the samples were accepted based on calculations that indicated the estimated leakage was still negligible.

As was discussed in Section 3.5, the HHF used during sampling often enters the sampler in place of some of the waste. Table 4.9.2 shows the volume percentage contamination by HHF of RGS and non-RGS samples from BY-109. Some of these samples (12C-4, 12C-5, 10B-3, 10B-4, 10B-5, 10B-6, and 10B-7) contained bromide below the detection limit. The actual measured HHF contamination percentages (or, rather, the upper bounds set by the detection limit) were used for RGS samples 12C-4, 10B-5, and 10B-6. (It is conservative to use the MDL for values below it; the conservatism is probably less than $5 \%$.)

Table 4.9.2. Hydrostatic Head Fluid Contamination in BY-109 Samples

\begin{tabular}{|c|c||}
\hline Sample & $\begin{array}{c}\text { HHF Contamination } \\
\text { (vol\%) }\end{array}$ \\
\hline 12C-1 (RGS) & 15.7 \\
\hline $12 \mathrm{C}-2$ & 15.7 \\
\hline $12 \mathrm{C}-3$ & no data \\
\hline 12C-4 (RGS) & $<8.0$ \\
\hline $12 \mathrm{C}-5$ & $<6.2$ \\
\hline $10 \mathrm{~B}-1$ & 10.7 \\
\hline 10B-2 (RGS) & 16.7 \\
\hline $10 \mathrm{~B}-3$ & $<5.7$ \\
\hline $10 \mathrm{~B}-4$ & $<9.2$ \\
\hline 10B-5 (RGS) & $<6.4$ \\
\hline 10B-6 (RGS) & $<6.4$ \\
\hline 10B-7 & $<11.3$ \\
\hline \hline
\end{tabular}


All of these samplers.were helium-backfilled and sealed with vacuum grease before they were deployed. This method virtually eliminated air and argon contamination from gases entrained during sampling. The helium backfill results are discussed in detail by Cannon (1997) and summarized in Section 5 of Mahoney et al. (1997). A different extraction procedure was used for each of the three BY-109 samples. (See Figure 3.1 for a schematic of the RGS extraction system.)

Sample 12C-4: After evacuating the whole system and observing the pressure to test for leaks, the operator extruded the RGS sample into the extractor vessel, and the sample was briefly stirred. At this point the vapor space in communication with the sample consisted of the extractor vessel, its lines, the second volume, the pump volume, and the collector side; the latter included the collector line volume and one open collection canister. The first canister, containing a sample of uncompressed (unpumped) gas, was closed off. The second collection canister was opened. Several strokes were pumped from the extractor to the collector, and the second collection canister was closed off. Then the collector side was evacuated. The BSVD procedure was performed: helium was injected into the closed-off second volume, and then the second volume was opened to the extractor and lines. Pressure measurements and known tare system volumes allowed calculation of the volume of the sample solids and liquid.

A canister containing $15 \mathrm{NH}_{3}$ vapor was connected to the extractor side and opened to inject isotopic ammonia. An equilibration period of 139 hours was allowed, during which the extractor vessel mixer was running. The mixer was turned off. The canister used for adding the standard was left open to take an extractor-side grab sample. Several strokes were pumped from the extractor to the collector. The third set of two collection canisters was closed off, as was the standard canister.

The collector pressure data (except for the beginning and end pressures) were lost for the post-isotopic canisters of gas extracted from sample $12 \mathrm{C}-4$, but the pressures were approximated and the uncertainty of the results was increased accordingly. The increase in uncertainty was small because the last canister typically contains little gas.

Sample 10B-5: After evacuating the whole system and observing the pressure to test for leaks, the operator extruded the RGS sample into the extractor vessel and the sample was briefly stirred. At this point the vapor space in communication with the sample consisted of the extractor vessel, its lines, the second volume, the pump volume, and the collector side; the latter included the collector line volume and one open collection canister. Several strokes were pumped from the extractor to the collector, and the first collection canister was closed off. The second set of two collection canisters was opened.

Then the collector side and the second volume and extractor lines were evacuated. A canister containing $15 \mathrm{NH}_{3}$ vapor was connected to the extractor side and opened to inject isotopic ammonia. An equilibration period of 91 hours was allowed, during which the extractor vessel mixer was running slowly. The mixer was turned off. The canister used for adding the standard was left open to take an extractor-side grab sample. Several strokes were pumped from the extractor to the collector. The second set of two collection canisters was closed off, as was the standard canister. Finally, the BSVD procedure was performed.

Sample 10B-6: After evacuating the whole system and observing the pressure to test for leaks, the operator extruded the RGS sample into the extractor vessel and the sample was briefly stirred. At this point the vapor space in communication with the sample consisted of the extractor vessel, its lines, the second volume, the pump volume, and the collector side; the latter included the collector line volume and one open collection canister. The first canister, containing a sample of uncompressed (unpumped) gas, was closed off. The second collection canister was opened. Several strokes were pumped from the extractor to the collector, and the second collection canister was closed off. The second set of three collection canisters was opened. 
Then the collector side and the second volume and extractor lines were evacuated. A canister containing $15 \mathrm{NH}_{3}$ vapor was connected to the extractor side and opened to inject isotopic ammonia. An equilibration period of 21.5 hours was allowed, during which the extractor vessel mixer was running slowly. The mixer was turned off. The canister used for adding the standard was left open to take an extractor-side grab sample. Several strokes were pumped from the extractor to the collector. The second set of three collection canisters was closed off, as was the standard canister. Finally, the BSVD procedure was performed.

Procedural details such as the number of strokes per canister and the system volumes can be found in the sample-by-sample worksheets in the BY-109 RGS calculation spreadsheet on the $\mathrm{CD}$ included with this report. A very detailed run-through of the procedures can also be found in Appendix A of Mahoney et al. (1997).

\subsubsection{Retained Gas Composition}

Table 4.9.3 presents the calculated concentrations of the insoluble gases in each RGS sample taken from BY-109 without corrections for air and argon entrainment, helium backfill, air inleakage, air added with the isotopic ammonia, or BSVD helium. The method used to make the corrections depended on the gas. All of the oxygen, argon, and helium were subtracted no matter which step had produced them. A number of moles equal to $(3.73) \cdot\left(\mathrm{O}_{2}\right)$ were subtracted from the nitrogen because the oxygen was expected to have come from air entrainment during sampling or inleakage during extraction. The corrected concentrations are given in Table 4.9.4.

Sample 12C-4 contained unusual amounts of helium and oxygen contamination. The excess helium appeared in the last set of canisters and came from the BSVD procedure that preceded the final post-isotopic extraction. The air came from air inleakage into the collector through an open line during the isotopic equilibration period; this diagnosis was based on the gradual increase of the collector pressure from zero to $3.38 \mathrm{kPa}$. Contamination observations can be found in the "Summary" worksheet of the BY-109 RGS calculations spreadsheet on the CD.

Table 4.9.3. Concentrations of Insoluble Constituents ( $\mu \mathrm{mol} / \mathrm{L}$ of waste) in Tank BY-109 Without Correction for Gas Contamination

\begin{tabular}{|c|c|c|c|c|c|c|c|c|c|c|c||}
\hline Segment & $\mathrm{N}_{2}$ & $\mathrm{H}_{2}$ & $\mathrm{~N}_{2} \mathrm{O}$ & $\mathrm{O}_{2}$ & $\mathrm{CH}_{4}$ & $\mathrm{He}$ & $\mathrm{Ar}$ & $\begin{array}{c}\text { Other } \\
\mathrm{NOx}\end{array}$ & $\mathrm{C}_{2} \mathrm{H}_{x}$ & $\mathrm{C}_{3} \mathrm{H}_{x}$ & $\begin{array}{c}\text { Other } \\
\text { Hyd. }\end{array}$ \\
\hline \hline $12 \mathrm{C}-4$ & $2300 \pm 140$ & $1200 \pm 41$ & $750 \pm 27$ & $250 \pm 53$ & $34 \pm 4.7$ & $1600 \pm 250$ & $31 \pm 5.0$ & $0.7 \pm 0.5$ & $30 \pm 5.1$ & $6.3 \pm 0.8$ & $38 \pm 6.0$ \\
\hline $10 \mathrm{~B}-5$ & $1600 \pm 140$ & $2400 \pm 140$ & $800 \pm 44$ & $66 \pm 38$ & $32 \pm 3.5$ & $460 \pm 27$ & $12 \pm 2.3$ & $0.8 \pm 0.6$ & $33 \pm 5.3$ & $5.8 \pm 1.2$ & $43 \pm 12$ \\
\hline $10 \mathrm{~B}-6$ & $1800 \pm 140$ & $3900 \pm 230$ & $1200 \pm 210$ & $59 \pm 41$ & $61 \pm 3.6$ & $380 \pm 23$ & $17 \pm 2.7$ & $1.0 \pm 0.7$ & $92 \pm 9.6$ & $10 \pm 2.6$ & $81 \pm 21$ \\
\hline
\end{tabular}

Table 4.9.4. Concentrations of Insoluble Constituents ( $\mu \mathrm{mol} / \mathrm{L}$ of waste) in Tank BY-109 with Correction for Gas Contamination

\begin{tabular}{||c|c|c|c|c|c|c|c|c|c|c|c||}
\hline Segment & $\mathrm{N}_{2}$ & $\mathrm{H}_{2}$ & $\mathrm{~N}_{2} \mathrm{O}$ & $\mathrm{O}_{2}$ & $\mathrm{CH}_{4}$ & $\mathrm{He}$ & $\mathrm{Ar}$ & $\begin{array}{l}\text { Other } \\
\mathrm{NOx}\end{array}$ & $\mathrm{C}_{2} \mathrm{H}_{x}$ & $\mathrm{C}_{3} \mathrm{H}_{x}$ & $\begin{array}{c}\text { Other } \\
\text { Hyd. }\end{array}$ \\
\hline \hline $12 \mathrm{C}-4$ & $1400 \pm 230$ & $1200 \pm 41$ & $750 \pm 27$ & $0 \pm 53$ & $34 \pm 4.7$ & $0 \pm 250$ & $0 \pm 5.0$ & $0.7 \pm 0.5$ & $30 \pm 5.1$ & $6.3 \pm 0.8$ & $38 \pm 6.0$ \\
\hline $10 \mathrm{~B}-5$ & $1300 \pm 200$ & $2400 \pm 140$ & $800 \pm 44$ & $0 \pm 38$ & $32 \pm 3.5$ & $0 \pm 27$ & $0 \pm 2.3$ & $0.8 \pm 0.6$ & $33 \pm 5.3$ & $5.8 \pm 1.2$ & $43 \pm 12$ \\
\hline $10 \mathrm{~B}-6$ & $1600 \pm 210$ & $3900 \pm 230$ & $1200 \pm 210$ & $0 \pm 41$ & $61 \pm 3.6$ & $0 \pm 23$ & $0 \pm 2.7$ & $1.0 \pm 0.7$ & $92 \pm 9.6$ & $10 \pm 2.6$ & $81 \pm 21$ \\
\hline
\end{tabular}


Table 4.9.5 presents the ammonia measurements. The residual ammonia was determined isotopically, and the total ammonia (calculated from the isotopic residual) is included in the table. The post-extrusion partial pressure of $\mathrm{NH}_{3}$ over the sample (see Section 3.4.4) was measured at laboratory temperature and is included in Table 4.9.5. No other ammonia measurements were made on RGS samples. The ammonia measurement made by isotopic ammonia vapor injection may have underestimated the ammonia in BY-109 samples in spite of longer equilibration times than had been used for samples from U-103 or S-106. Accordingly, the post-extrusion $\mathrm{NH}_{3}$ partial pressures were used to calculate the in situ ammonia vapor pressures.

No conclusive total ammonia concentration can be calculated, but it is likely that the ammonia concentration in Tank BY-109 waste was one-third to one-half that in Tank AW-101 waste, which had a lower bound of $27,000 \mu \mathrm{mol} \mathrm{NH} / \mathrm{L}$ waste. This estimate is based on a comparison of the ammonia partial pressure measurements (see Sections 4.1.2 and C.1 for the AW-101 measurements). The estimate based on partial pressures is closely consistent with the total ammonia concentrations that were found isotopically (see Appendix C, Section C.9 for more details on ammonia data.)

Table 4.9.6 contains the composition of the gas/vapor phase in each sample and the average composition in the gas retained in the zone below the $\mathrm{LL}$. The water vapor is not included in these compositions. The ammonia fractions are derived from the data indicated in Table 4.9.5, which were used to calculate vapor pressures at in situ conditions. The sample compositions in the table have been calculated using the in situ solubility method described in Section 3.6.2. As discussed in Section 3.6.1, compositions were calculated for both the lower-bound and upper-bound gas solubilities. Both ends of the composition range are given in Table 4.9.6, along with the measurement uncertainty on each. The average composition of the gas below the IIL is the result of integrating RGS species concentrations over the waste zone and multiplying those concentrations by the zone volume. No RGS samples were successfully taken from zone above the ILL. The integration method is described in Section 3.7.1.

The retained gas in the BY-109 samples was of different composition in sample $12 \mathrm{C}-4$ than in either of the $10 \mathrm{~B}$ riser samples. This suggests spatial variability but is not conclusive because there were so few samples. The concentrations and pressures in Tables 4.9 .3 and 4.9 .4 can also be found in the sample-by-sample worksheets in the BY-109 RGS calculation spreadsheet on the CD. The compositions in Table 4.9.6 are in the "Inventory" worksheet.

Table 4.9.5. Ammonia Data from Tank BY-109 Samples

\begin{tabular}{|c|c|c|c|}
\hline Sample & $\begin{array}{l}\text { Isotopically } \\
\text { Measured } \\
\text { Total NH } \\
(\mu \mathrm{mol} / \mathrm{L})\end{array}$ & $\begin{array}{l}\text { Measured } \mathrm{NH}_{3} \\
\text { partial pressure at } \\
\text { about } 24^{\circ} \mathrm{C} \\
(\mathrm{atm})\end{array}$ & $\begin{array}{l}\text { Best-Estimate } \mathrm{NH}_{3} \\
\text { Concentrations }\end{array}$ \\
\hline $12 C-4$ & $9300 \pm 1400$ & $0.0038 \pm 0.0019$ (a) & \multirow{3}{*}{$\begin{array}{l}\text { The data do not support conclusive } \\
\mathrm{NH}_{3} \text { concentrations. We estimate a } \\
\text { lower bound of } 0.014 \mathrm{M}(250 \mu \mathrm{g} / \mathrm{L} \\
\mathrm{NH}_{3} \text { in the liquid of the zone below } \\
\text { the ILL or. } 0.011 \mathrm{wt} \% \mathrm{NH}_{3} \text { in the } \\
\text { bulk waste. }\end{array}$} \\
\hline $10 \mathrm{~B}-5$ & $14000 \pm 1300$ & $0.0030 \pm 0.0015$ (a) & \\
\hline $10 \mathrm{~B}-6$ & $6300 \pm 520$ & $0.0035 \pm 0.0018$ (a) & \\
\hline
\end{tabular}


Table 4.9.6. Sample and Overall Average Compositions of Retained Gas in Tank BY-109 with Correction for Gas Contamination(a)

\begin{tabular}{||c|c|c|c|c|c|c||}
\hline Sample & $\mathrm{N}_{2}$ (mol\%) & $\mathrm{H}_{2}(\mathrm{~mol} \%)$ & $\mathrm{N}_{2} \mathrm{O}(\mathrm{mol} \%)$ & $\mathrm{NH}_{3}(\mathrm{~mol} \%)$ & $\mathrm{CH}_{4}(\mathrm{~mol} \%)$ & Other (mol\%) \\
\hline \hline $12 \mathrm{C}-4$ & $40 \pm 7.9--41 \pm 8.2$ & $35 \pm 3.6--36 \pm 3.7$ & $21 \pm 2.2--19 \pm 2.0$ & $0.4 \pm 0.2--0.2 \pm 0.08$ & $1.0 \pm 0.2$ & $2.2 \pm 0.4--2.3 \pm 0.4$ \\
\hline $10 \mathrm{~B}-5$ & $29 \pm 5.0$ & $52 \pm 5.5$ & $17 \pm 1.7--16 \pm 1.6$ & $0.3 \pm 0.1--0.1 \pm 0.05$ & $0.7 \pm 0.1$ & $1.8 \pm 0.4$ \\
\hline $10 \mathrm{~B}-6$ & $23 \pm 3.8$ & $56 \pm 6.4-57 \pm 6.4$ & $17 \pm 3.4--16 \pm 3.2$ & $0.3 \pm 0.1--0.1 \pm 0.06$ & $0.9 \pm 0.1$ & $2.6 \pm 0.6--2.7 \pm 0.0$ \\
\hline $\begin{array}{l}\text { Avg in zone } \\
\text { below } \\
\mathrm{ML}(\mathrm{c})\end{array}$ & $28 \pm 5.0--29 \pm 5.1$ & $50 \pm 5.5--51 \pm 5.6$ & $18 \pm 2.5--17 \pm 2.3$ & $0.3 \pm 0.1--0.1 \pm 0.06$ & $0.8 \pm 0.1--0.9 \pm 0.1$ & $2.3 \pm 0.4$ \\
\hline
\end{tabular}

(a) Uncertainties on compositions represent only instrument uncertainty. Compositions calculated for lower- and upperbound solubilities. Both ends of composition range given, lower-bound solubility basis first, with instrument uncertainty on each. Compositions may not sum to $100 \mathrm{~mol} \%$ because of roundoff error. Mole fractions on a dry basis and do not account for water vapor.

(b) Ammonia mole fraction derived from data in Table 4.9.5, used to calculate vapor pressure at in situ conditions.

(c) There are too few samples to define the spatial variability of the average gas concentration.

\subsubsection{Gas Inventory}

The method by which the in situ gas volume fractions (wet basis) were calculated is given in Section 3.6.2; as for the gas composition, the volume fractions are given as a range from the low gas solubility value to the high gas solubility value. The results are presented in Table 4.9.7, which also contains the average gas volume fraction and the average pressure experienced by the gas in the zone below the ILL. The averages for the zone below the ILL are in situ volume averages calculated by Simpson's Rule integration, as described in Section 3.7.1. The corrected gas volume fractions in the table are consistent with the corrected gas concentrations and compositions in Tables 4.9.4 through 4.9.6. The information in Table 4.9.7 is taken from the "Summary" and "In situ" worksheets of the BY-109 RGS calculation spreadsheet on the CD.

The "sampler gas volume fraction" is corrected only for inleakage during the extraction process and air gases added in the isotopic solution. It is an attempt to reconstruct the total amount of gas (sample and entrainment) that was present in the sampler during $\mathrm{x}$-ray. It is used only for comparison with $\mathrm{x}$-ray observations of "visible gas fraction" that are discussed in Section 4.9.4.

Table 4.9.7 also contains the water vapor pressures that were used for in situ calculations. The water vapor pressures were found by using salt concentrations from Jo (1998) and the temperatures in the table as inputs to Equation 6.2 of Mahoney and Trent (1995), a correlation for water vapor pressure over concentrated homogeneous and non-homogeneous waste simulants. The gas solubilities used the same parameters as inputs to the Schumpe solubility model (Section 3.6.1). The water vapor pressures and gas solubilities used in calculations can be found in the "In situ" worksheet of the BY-109 RGS calculation spreadsheet on the CD. No ammonia inventory was calculated because there were no total ammonia concentration data for BY-109.

Table 4.9.8 estimates the STP volume of gas below the ILL in BY-109 calculated from RGS data alone. The RGS gas inventory in the zone below the ILL was calculated by integrating RGS total gas concentrations over the zone (three data points) and multiplying the average gas concentration by the volume of the zone. Because samples 12C-4 and 10B-6 were at the same elevation, their gas fractions were averaged to represent a single datum. The integration method is described in Section 3.7.1. The RGS volumes in Table 4.9.8 include corrections to remove the contamination gas: entrained air and argon, helium backfill, air leaks during and after extraction, air that came in with the isotopic ammonia vapor, and helium from the BSVD procedure. The 
Table 4.9.7. In Situ Gas Volume Fractions and Conditions in Tank BY-109

\begin{tabular}{||c|c|c|c|c|c|c||}
\hline Sample & $\begin{array}{c}\text { Sample } \\
\text { central } \\
\text { height } \\
(\mathrm{cm})\end{array}$ & $\begin{array}{c}\text { Hydrostatic } \\
\text { pressure } \\
\text { (atm) }\end{array}$ & $\begin{array}{c}\text { Calculated } \\
\text { water vapor } \\
\text { pressure } \\
(\mathrm{atm})\end{array}$ & $\begin{array}{c}\text { Temp } \\
\left({ }^{\circ} \mathrm{C}\right)\end{array}$ & $\begin{array}{c}\text { Corrected gas } \\
\text { volume fraction(a) } \\
\text { (in-tank conditions) }\end{array}$ & $\begin{array}{c}\text { Sampler gas } \\
\text { volume fraction } \\
\text { (in situ conditions, } \\
\text { low solubility) }\end{array}$ \\
\hline \hline $12 \mathrm{C}-4$ & 121 & 1.34 & 0.014 & 30.7 & $\begin{array}{c}0.064 \pm 0.004 \text { to } \\
0.062 \pm 0.004\end{array}$ & 0.11 \\
\hline $10 \mathrm{~B}-5$ & 121 & 1.34 & 0.014 & 30.7 & $\begin{array}{c}0.087 \pm 0.008 \text { to } \\
0.085 \pm 0.008\end{array}$ & 0.10 \\
\hline 10B-6 & 72.4 & 1.41 & 0.014 & 30.7 & $0.12 \pm 0.010$ & 0.14 \\
\hline $\begin{array}{l}\text { Avg in zone } \\
\text { below ILL }\end{array}$ & 120 & 1.34 & & & $\begin{array}{c}0.095 \pm 0.047 \text { to } \\
0.093 \pm 0.046\end{array}$ & \\
\hline $\begin{array}{l}\text { (a) Gas volume fraction is expressed on a wet basis, including the volume contribution of water vapor. } \\
\text { The uncertainties on the layer-average gas volume fractions are based on spatial variability } \\
\text { considerations discussed in Section 3.7.2. }\end{array}$ \\
\hline
\end{tabular}

uncertainties on the gas inventories are based on the spatial variability considerations discussed in Section 3.7.2. The information in Table 4.9.8 is taken from the "Inventory" worksheet of the BY-109 RGS calculation spreadsheet on the CD.

The gas inventory calculation for BY-109 (below the ILL) is based solely on RGS data; the BPE method is not applicable in this tank, and surface level rise yields no information because of salt-well pumping. The $\mathrm{x}$-ray of non-RGS sample 12C-3R1 showed a 9-in. gas gap, for a visible gas fraction of nearly 0.50 ; however, this sample was not well sealed, and leakage could have caused the large gap. Core extrusions of non-RGS samples 12C-2, 10B-3, and 10B-4 showed sample lengths of 16, 19, and $19 \mathrm{in}$., respectively. The latter two samples were consistent with RGS measurements, the former (implying a 3-in. gas gap and a visible gas fraction of 0.16 ) suggested higher gas in one sample. However, non-RGS samplers are not hermetically sealed and extrusion length measurements are not precise, so these non-RGS observations must be interpreted with caution. Because so few measurements were available in this tank and they cover only the lower half of the waste, the assigned 50\% uncertainty may be too low; however, there is enough consistency among RGS measurements and non-RGS indications to provide some support for the inventory.

Table 4.9.9 contains the calculated inventories of each of the major gases retained in the portion of the waste that was below the ILL. These inventories are based on the layer-average composition from RGS data (Table 4.9.6) and the RGS gas inventory (Table 4.9.8).

Figure 4.9.4 shows the sample corrected gas volume fractions (Table 4.9.7), and corrected compositions of the low-solubility constituents in the samples from Tank BY-109. A sample temperature profile was not available. The compositions represent the mole fraction of the species in the "insoluble" gas; water and ammonia are not included, so the mole fractions are not the same as those in Table 4.9.4. The gas volume fractions and mole fractions in the figure are the values for lower-bound gas solubilities. Figure 4.9.4 also lists the observations from core extrusions of non-RGS samples and from X-rays of RGS samples as a way of tying those observations together with RGS data. The x-ray data are described in more detail in Section 4.9.4. 
Table 4.9.8. BY-109 Gas Inventory Estimates(a)

\begin{tabular}{|l|c|}
\hline \multicolumn{1}{|c|}{ Quantity } & RGS Method \\
\cline { 2 - 3 } & Zone below ILL \\
\hline \hline Avg gas fraction (low gas solubility) & $0.095 \pm 0.047$ \\
\hline Gas volume (m ${ }^{3}$ ) in situ (wet) & $101 \pm 50$ \\
STP (wet) & $122 \pm 61$ \\
\hline $\begin{array}{l}\text { (a) The 50\% uncertainty may be too low considering the } \\
\text { small number of measurements and the lateral variability } \\
\text { observed in core extrusions. }\end{array}$ \\
\hline
\end{tabular}

Table 4.9.9. Speciated BY-109 Gas Inventory(a)

\begin{tabular}{|c|c|c|}
\hline \multirow[b]{2}{*}{ Species } & \multicolumn{2}{|c|}{ Zone Below ILL } \\
\hline & $\begin{array}{c}\text { Gas-Phase Inventory } \\
\left(\mathrm{m}^{3} \text { at STP }\right)\end{array}$ & $\begin{array}{c}\text { Dissolved Inventory } \\
\left(\mathrm{m}^{3} \text { at } \mathrm{STP}\right)\end{array}$ \\
\hline $\mathrm{N}_{2}$ & 34 & $0.012-0.084$ \\
\hline $\mathrm{H}_{2}$ & 60 & $0.074-0.34$ \\
\hline $\mathrm{N}_{2} \mathrm{O}$ & $22-20$ & $0.52-2.5$ \\
\hline $\mathrm{NH}_{3}$ & $0.38-0.17$ & 240 \\
\hline $\mathrm{CH}_{4}$ & 1.0 & $0.00063-0.0045$ \\
\hline Other & 2.8 & 0 \\
\hline \multicolumn{3}{|c|}{$\begin{array}{l}\text { (a) Inventory based on layer-average compositions from RGS data (Table 4.9.6) and } \\
\text { best-estimate gas inventory (RGS, Table 4.9.8). Uncertainty in each inventory is } \\
50 \% \text { based on spatial variability considerations (Section 3.7.2). Inventories given } \\
\text { for lower- and upper-bound solubilities, lower-bound solubility basis first. }\end{array}$} \\
\hline
\end{tabular}

\subsubsection{X-Ray Results}

Table 4.9.10 summarizes all the available radiography observations from Tank BY-109. The $\mathrm{x}$-ray images themselves can be found on the $\mathrm{CD}$ that accompanies this report. The uncertainties of the visible gas volume fractions that are given in Table 4.9.10 are unknown but (based on the uncertainty in measuring the gas gap from x-ray images) are expected to be larger than the uncertainties in the gas volume fractions calculated from gas extraction. (The "visible" gas volume fraction refers to the gas that has separated from the solids and liquid such that its volume can be calculated based on the image. The uncorrected gas fractions from extraction are used for comparison to reflect the presence of entrained air in the sampler.) 

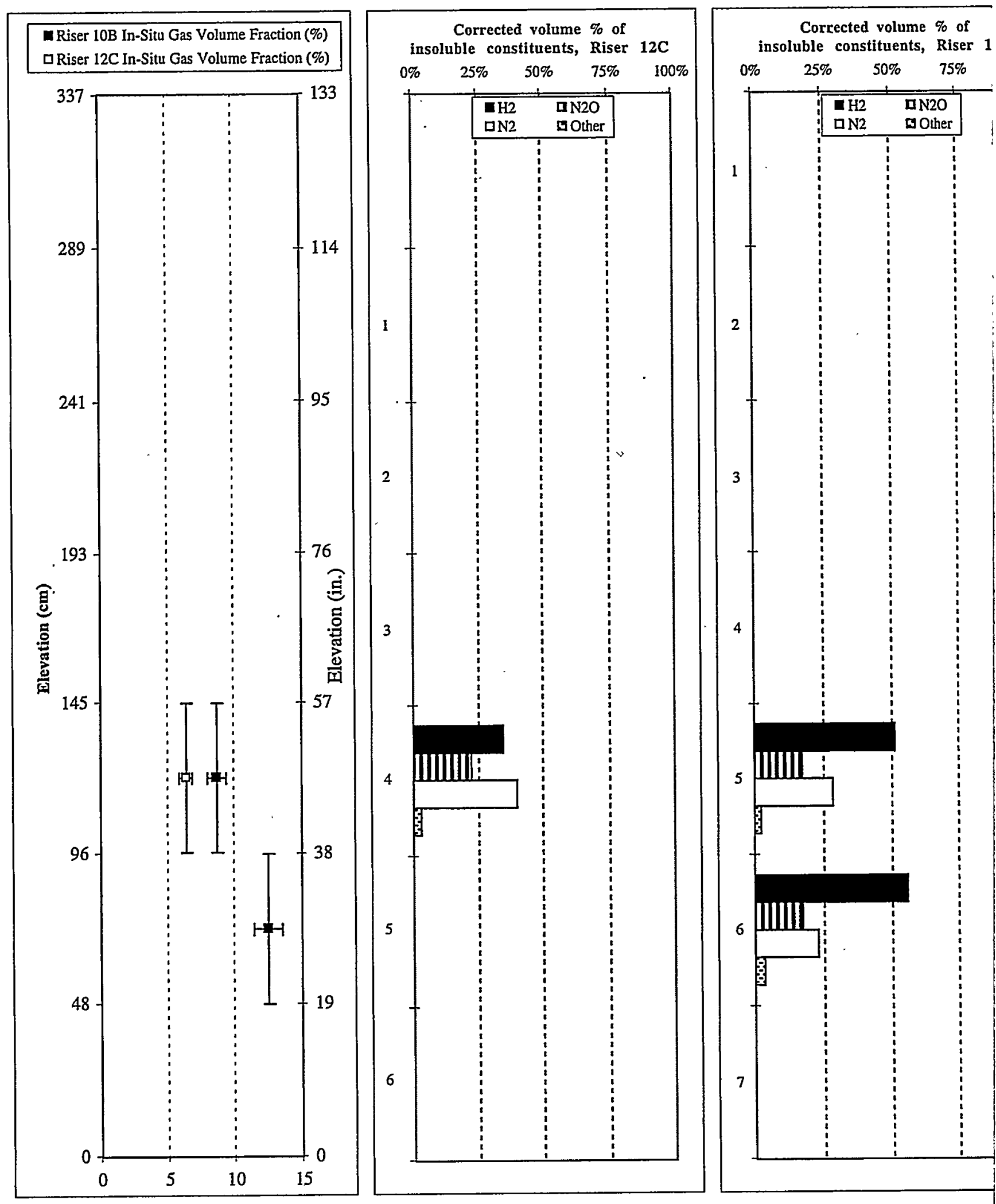

Figure 4.9.4. Gas Volume Fractions, Compositi 
BY-109 waste level is $310-343 \mathrm{~cm}$ (122-135 inches).

1 Riser 10B: 3 in. (89 g) gray and white dry salt crystals.

1 Riser 12C X-RAY: About 3 in. waste, fissured gas structure. Piston fully retracted, small waste on it. $35 \mathrm{mR} / \mathrm{hr}$.

2 Riser 10B X-RAY: Number of bubbles $2 \mathrm{~mm}$ and less near bottom, flat surface, 9 in. gas gap. $70 \mathrm{mR} / \mathrm{hr}$.

2 Riser 12C: 16 in. (351 g) brown wet sludge, some granular material in lower part. $0.44 \mathrm{R} / \mathrm{hr}$.

3 Riser 10B: $19 \mathrm{in.}(466 \mathrm{~g})$ gray moist salt, more granular in lower part. $0.41 \mathrm{R} / \mathrm{hr}$.

3R1 Riser 12C: $15 \mathrm{in.}(340 \mathrm{~g})$ gray moist salt, $30 \mathrm{~mL}$ (39 g) opaque yellow-green liquid. X-RAY: string of 3-mm bubbles.

4 Riser 10B: 19 in. (453 g) gray moist salt. $0.47 \mathrm{R} / \mathrm{hr}$.

4 Riser 12C X-RAY: 1-mm or less bubbles, denser striations. No gas gap, piston 2.3 in. from the stops. $0.35 \mathrm{R} / \mathrm{hr}$.

5 Riser 10B X-RAY: Fracture bubble $6 \mathrm{~mm}$ thick, above it swirls of denser waste. Piston 0.25 in. from stops. $0.31 \mathrm{R} / \mathrm{hr}$.

5 Riser 12C: 2 in. (117 g) brown sludge slurry mixed with gray salt slurry, $100 \mathrm{~mL}$ (146 g) opaque yellow-brown liquid.

6 Riser 10B X-RAY: Fracture bubble $6 \mathrm{~mm}$ thick, above it swirls of denser waste. Piston 0.75 in. from stops. $0.28 \mathrm{R} / \mathrm{hr}$.

6 Riser 12C: N/A.

7 Riser 10B: $18 \mathrm{in}$. (502 g) solids, upper half creamy tan wet sludge, lower half creamy white wet sludge. $0.65 \mathrm{R} / \mathrm{hr}$. 
Table 4.9.10. Summary of Observations from X-Ray Images of Tank BY-109

\begin{tabular}{|c|c|}
\hline Segment & Comments/Observations \\
\hline $\begin{array}{l}12 \mathrm{C}-1 \text { (RGS, } \\
6 / 6 / 97)\end{array}$ & $\begin{array}{l}\text { Only about } 3 \text { in. of waste was recovered, showing a fissured gas structure; the sampler was } \\
\text { virtually empty. No waste was attached to the piston, which was fully retracted. }\end{array}$ \\
\hline $\begin{array}{l}\text { 10B-2 } \\
\text { (RGS, } \\
6 / 16 / 97)\end{array}$ & $\begin{array}{l}\text { The recovered waste fills only about half the sampler volume. Dense } 2-\mathrm{mm} \text { and smaller } \\
\text { bubbles appear in the lowest third of the sampler. The waste becomes less dense toward the top } \\
\text { and has a flat top surface. There is a small lump of waste on the piston, which is fully retracted } \\
\text { The gas gap is } 23 \mathrm{~cm}(9 \mathrm{in} \text {.). It is possible that this gas volume region represents gas trapped } \\
\text { in the waste, but the more likely explanation for its existence, given the known piston seal } \\
\text { problem, is incomplete sample recovery and/or gas leakage. }\end{array}$ \\
\hline $\begin{array}{l}12 \mathrm{C}-3 \mathrm{R} 1 \\
\text { (non-RGS, } \\
6 / 10 / 97 \text { ) }\end{array}$ & $\begin{array}{l}\text { This sample contained many gas bubbles and a "string" of bubbles of about } 3 \mathrm{~mm}(0.13 \mathrm{in} \text {.) } \\
\text { diameter evident in the lower portion. Density gradients are also present. There is a flat top } \\
\text { surface. The piston is } 1.7 \text { in. short of full retraction. The gas gap is } 2.8 \mathrm{~cm} \text { (1.1 in.). The } \\
\text { visible gas volume fraction (not counting bubbles) is therefore } 0.07 \text {. }\end{array}$ \\
\hline $\begin{array}{l}12 \mathrm{C}-4 \\
\text { (RGS, } \\
6 / 12 / 97)\end{array}$ & $\begin{array}{l}\text { The lower waste is fairly homogeneous but becomes increasingly less so toward the top. } \\
\text { Some lighter areas/bubbles are visible in the middle third of the sample, with many dark/dense } \\
\text { striations and swirls evident in the upper third. The light/bubble features are typically about } \\
1 \mathrm{~mm} \text { in dimension, or mottled with gas too small for resolution. The dark regions typically } \\
\text { extend across the whole sampler. The piston is about } 1 \mathrm{~cm} \text { ( } 2.3 \text { in.) from full retraction. The } \\
\text { waste extends all the way to the piston, giving a visible gas volume fraction of } 0 \text {. (Compare to } \\
\text { the corrected gas volume fraction of } 0.064 \text { in Table } 4.9 .7 \text { obtained by gas extraction. The } \\
\text { corrected gas fraction was used because gas contamination added during extraction made up most } \\
\text { of the correction.) }\end{array}$ \\
\hline $\begin{array}{l}\text { 10B-5 } \\
\text { (RGS, } \\
6 / 16 / 97)\end{array}$ & $\begin{array}{l}\text { The lower half of the waste shows low-density material, probably gas, including a single } \\
\text { large gas "bubble" that appears to span the entire sampler diameter but is relatively flat, being } \\
\text { only about } 0.6 \mathrm{~cm}(0.25 \mathrm{in} \text {.) or so high. The waste higher up contains decreasing amounts of } \\
\text { low-density material (with gas too small for resolution), containing instead numerous, large } \\
\text { "swirls" of darker/denser material. The waste extends all the way to the piston. The piston is } \\
0.25 \text { in. from full retraction. The visible gas volume fraction (based on the large bubble) is } \\
0.01 \text {. (Compare with the uncorrected gas volume fraction of } 0.10 \text { in Table } 4.9 .7 \text { obtained by } \\
\text { gas extraction.) }\end{array}$ \\
\hline $\begin{array}{l}\text { 10B-6 } \\
\text { (RGS, } \\
6 / 17 / 97)\end{array}$ & $\begin{array}{l}\text { The waste was very similar to sample 10B-5. It also contains lower-density material and has } \\
\text { a large single "bubble" of } 0.6 \mathrm{~cm} \text { thickness about mid-way up the sample, but it contains fewer } \\
\text { "swirls" than 10B- } 5 \text { and has some low-density material in the upper sampler region. A flat } \\
\text { meniscus is visible at the waste/gas interface. There is no waste attached to the piston, which is } \\
\text { fully retracted. The gas gap was } 4.6 \mathrm{~cm} \text { (1.8 in.). The visible gas volume fraction (based on the } \\
\text { large bubble and the gas gap) is } 0.12 \text {. (Compare with the uncorrected gas volume fraction of } \\
0.14 \text { in Table } 4.9 .7 \text { obtained by gas extraction.) }\end{array}$ \\
\hline
\end{tabular}

Table 4.9.10 compares $\mathrm{x}$-ray-derived gas volume fractions with extraction-derived gas volume fractions. The comparison shows that sample recovery was close to $100 \%$ for all the samples once incomplete piston retraction was accounted for. This conclusion is based on the fact that the extracted uncorrected gas was equal to or a few volume percent more than the visible gas. 
Table 4.9.11 provides a summary of the waste densities that have been calculated from radiographic data for the RGS samples for which air and water standard images were available. The radiographic densities include the gas in the waste, were calculated by the current $\mathrm{x}$-ray analysis method, and are given for several locations within each sample to show density trends.

Table 4.9.11. Densities of BY-109 Samples, from Radiography and Core Samples

\begin{tabular}{|c|c|c|c|}
\hline $\begin{array}{l}\text { Riser- Segment } \\
\text { Number }\end{array}$ & $\begin{array}{c}\text { Distance } \\
\text { from bottom } \\
\text { of sampler, } \\
(\mathrm{ft})\end{array}$ & $\begin{array}{l}\text { Calculated } \\
\text { Mean } \\
\text { Density, } \\
\text { (g/cc) } \\
\end{array}$ & $\begin{array}{l}\text { Degassed Density Above and Below } \\
\text { the RGS Sample (from cores) } \\
\text { (g/cc) }\end{array}$ \\
\hline $12 \mathrm{C}-1$ (a) & 0.25 & $\mathrm{n} / \mathrm{a}^{(\mathrm{b})}$ & density below, 1.85 \\
\hline \multirow[t]{3}{*}{$10 \mathrm{~B}-2$} & 0.75 & 1.46 & \multirow{3}{*}{$\begin{array}{l}\text { density above, } \mathrm{n} / \mathrm{a} \\
\text { density, } 1.66 \\
\text { density below, } 1.72\end{array}$} \\
\hline & 0.50 & 1.55 & \\
\hline & 0.25 & 1.47 & \\
\hline \multirow[t]{5}{*}{$12 \mathrm{C}-3 \mathrm{R} 1^{(\mathrm{b})}$} & 1.25 & 1.41 & \multirow[t]{5}{*}{ density above, 1.85} \\
\hline & 1.00 & 1.47 & \\
\hline & 0.75 & 1.63 & \\
\hline & 0.50 & 1.57 & \\
\hline & 0.25 & 1.55 & \\
\hline \multirow[t]{5}{*}{$12 \mathrm{C}-4(\mathrm{c})$} & 1.25 & 2.21 & \multirow[t]{5}{*}{ density above, 1.69} \\
\hline & 1.00 & 1.85 & \\
\hline & 0.75 & 1.60 & \\
\hline & 0.50 & 1.65 & \\
\hline & 0.25 & 1.44 & \\
\hline \multirow[t]{6}{*}{$10 \mathrm{~B}-5$ (d) } & 1.50 & 1.64 & \multirow[t]{6}{*}{ density above, 1.68} \\
\hline & 1.25 & 1.56 & \\
\hline & 1.00 & 1.03 & \\
\hline & 0.75 & 1.46 & \\
\hline & 0.50 & 1.43 & \\
\hline & 0.25 & 1.37 & \\
\hline \multirow[t]{5}{*}{ 10B-6(a) } & 1.25 & 1.57 & \multirow[t]{5}{*}{ density above, 1.61} \\
\hline & 1.00 & 1.67 & \\
\hline & 0.75 & 1.64 & \\
\hline & 0.50 & 1.59 & \\
\hline & 0.25 & 1.47 & \\
\hline \multicolumn{4}{|c|}{$\begin{array}{l}\text { (a) Not enough waste was in the sampler for a meaningful density estimate. } \\
\text { (b) Flattening visible at top of the standard air density profile. } \\
\text { (c) Segment lacked standard air/water density profiles, so profiles from segment 12C-3R1 were used } \\
\text { (d) The quality of the } x \text {-ray images is uneven, with varying exposure and several images containing } \\
\text { large bright areas outside the sampler. These areas affect the overall brightness level and make } \\
\text { densities less accurate, as can be seen at the 1-ft level (which is overexposed). }\end{array}$} \\
\hline
\end{tabular}


Table 4.9.11 also includes density measurements made on extruded non-RGS samples (Jo 1998). The two methods give reasonably consistent results, considering the differences that can be expected because of the effect of retained gas on the radiographic densities of the RGS samples, the fact that RGS are adjacent to standard core samples rather than collocated, and the partial unavailability of $\mathrm{x}$-ray calibration records.

\subsubsection{Drillstring and Domespace Composition Comparison}

The RGS gas-phase composition data (Table 4.9.6) were used to calculate $\mathrm{H}_{2} / \mathrm{N}_{2} \mathrm{O}$ ratios to compare with ratios from drillstring and domespace grab sample measurements (Siciliano 1998, Table 8.1; McCain 1999, Table B-8). The results can be seen in Table 4.9.12. While the RGS crust $\mathrm{H}_{2} / \mathrm{N}_{2} \mathrm{O}$ ratios in riser $10 \mathrm{~B}$ were much the same as the drillstring ratio and the September 1995 domespace ratio, the October 1995 domespace ratios were much higher. A BY-109 salt-well pumping campaign was carried on intermittently between May 31, 1994 and October 16, 1995 (Caley et al. 1996), so the different domespace ratios could have been related to changes in the pumping status.

Table 4.9.12. Comparison with Drillstring and Domespace Data(a)

\begin{tabular}{|l|c|}
\hline Sample & $\mathrm{H}_{2} / \mathrm{N}_{2} \mathrm{O}$ \\
\hline \hline RGS, 12C-4 & $1.6-1.8$ \\
\hline RGS, 10B-5 & $3.1-3.4$ \\
\hline RGS, 10B-6 & $3.2-3.4$ \\
\hline Drillstring, 6/17/97 & 3.4 \\
\hline Domespace, 9/22/95 & 3.8 \\
\hline Domespace, 10/2 - 10/13/95 & $10,14,13$ \\
\hline $\begin{array}{l}\text { (a) RGS ratios were calculated for both lower- and upper- } \\
\text { bound solubilities. } \text { Both ends of the ratio range are given in } \\
\text { the table, lower-bound solubility basis first. }\end{array}$ \\
\hline
\end{tabular}




\section{$4.10 \quad S X-106$}

Tank 241-SX-106 (SX-106) was the tenth tank and sixth SST sampled with the RGS. This tank was selected as representing the highest-priority group of SSTs that show evidence of significant gas retention (Stewart et al. 1996b). Tank SX-106 is on the FGWL and exhibits high $\mathrm{dL} / \mathrm{dP}$ and level rise and a moderate concentration of $\mathrm{NH}_{3}$ in the domespace. Tank SX-106 is part of a group of tanks (Cluster 13 in Stewart et al. 1996b) that have fairly high radioactivity, fairly low temperatures, high nitrite concentrations, and about $0.1 \%$ TOC. HTCEs indicate that the primary waste stored in SX-106 was salt slurry from evaporator campaign S2 and the secondary waste was saltcake from evaporator campaign S1 (Remund et al. 1995). '(This is identical to the HTCE for Tank S-102, which is also in Cluster 13.) Of the RGS-sampled tanks, U-103, SX-106, S-102, and U-109 are all in Cluster 13.

Push-mode sampling was done in riser 6 in October to November 1997 and in riser 3 in December 1997.(a) The approximate locations of various risers are depicted in Figure 4.10.1. Risers 6 and 3 are both about one-third of the tank radius from the center of the tank. The elevations of the RGS segments are depicted in Figure 4.10.2. The figure also shows the tank content layering at the time of sampling, with a thin crust overlying supernatant of $188 \mathrm{~cm}(74 \mathrm{in}$.) depth. Beneath that was $316 \mathrm{~cm}$ (126 in.) of salt slurry and sludge (toward the bottom of the tank).

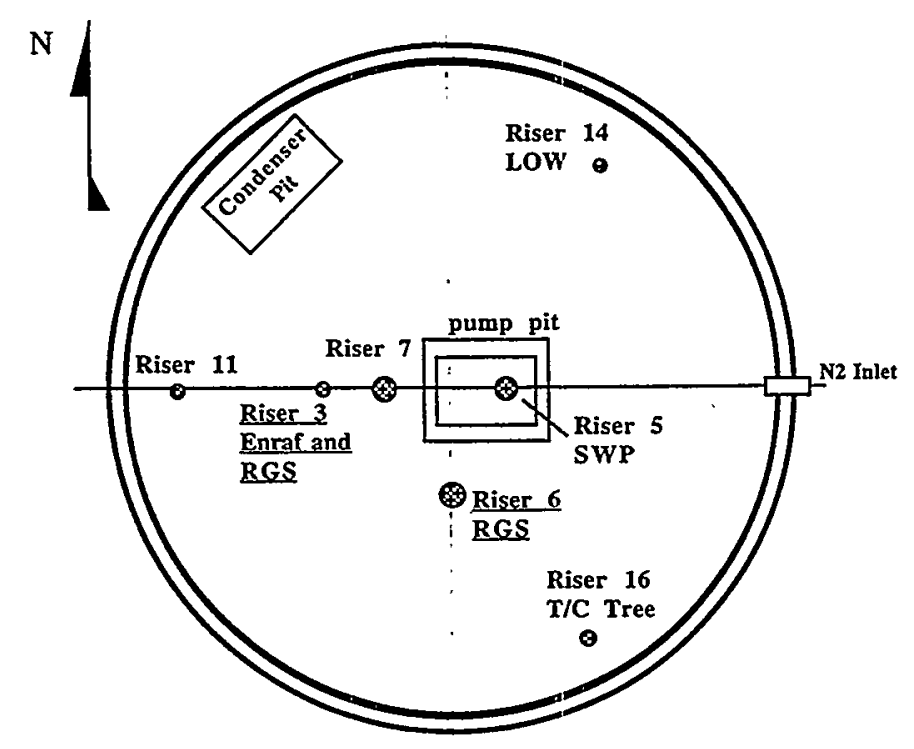

Figure 4.10.1. Schematic Diagram of Riser Locations in Tank SX-106

(a) The sampling scheme may be found in Sampling Plan for Tank 241-SX-106 Retained Gas Sampler Deployment, by JM Bates, October 1997. TWSFG97.72 Rev. 1, Pacific Northwest National Laboratory, Richland, Washington. 


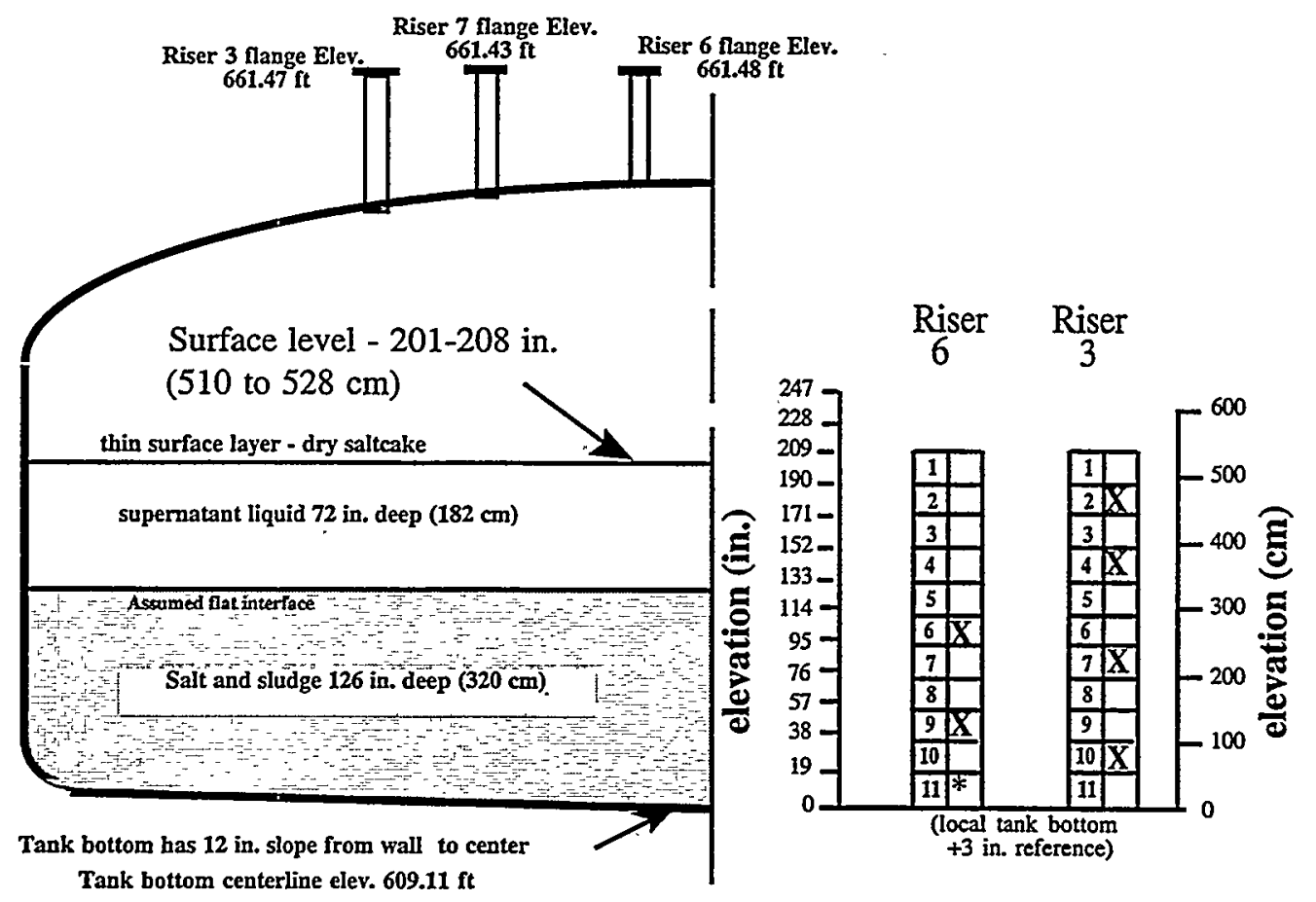

Figure 4.10.2. Diagram of Waste Layering and RGS Sample Elevations for SX-106 (segments with * could not be taken because the waste was too hard)

Field (1998) describes Tank SX-106 as a partially isolated, sound SST containing about $2,040,000 \mathrm{~L}$ (538,000 gal) of waste, including $813,000 \mathrm{~L}(215,000 \mathrm{gal})$ of liquid. At the time of sampling, the total depth of waste in Tank SX-106 was 510 to $528 \mathrm{~cm}$ (201 to 208 in.) at risers 6 and 3. Neutron scans in the riser $14 \mathrm{LOW}$ indicated liquid at the surface.

The most recent information on SX-106 layering comes from the core observations made in 1997 (Field 1998). There was no sign of crust in segment 1 at either riser 3 or riser 6 . The thickness of the top layer of supernatant liquid was between four and five segments at risers 6 and 3 . Most of the nonconvective bottom layer was salt or salt slurry, though sludge or sludge/salt slurry was seen in the range of segments 6 through 8 of both risers.

Densities for the liquid and bulk solids in SX-106 were available from the non-RGS samples taken in 1997 (Field 1998). Several samples contained drainable liquid. The density of drainable liquid varied from 1400 to $1450 \mathrm{~kg} / \mathrm{m}^{3}$, with an average and standard deviation of 1415 $\pm 19 \mathrm{~kg} / \mathrm{m}^{3}$. The bulk density of the samples with high solids concentrations varied from 1590 to $1790 \mathrm{~kg} / \mathrm{m}^{3}$, with an average and standard deviation of $1686 \pm 57 \mathrm{~kg} / \mathrm{m}^{3}$. The gassy density of the crust was set equal to that of the supernatant supporting it for hydrostatic pressure calculations.

For in situ inventory and average composition calculations, the liquid density was set at $1415 \mathrm{~kg} / \mathrm{m}^{3}$, and the lower nonconvective layer was given a degassed bulk density of $1686 \mathrm{~kg} / \mathrm{m}^{3}$. The crust was given a thickness of $16 \mathrm{~cm}(6.5 \mathrm{in}$.) and the supernatant a thickness of $182 \mathrm{~cm}$ (72 in.). The supernatant depth is based on the estimate of $813,000 \mathrm{~L}(215,000 \mathrm{gal})$ of liquid in SX-106 in 1997, and matches with the liquid thickness observed in the core extrusions. The nonconvective layer depth was set at $320 \mathrm{~cm}$ (126 in.). 


\subsubsection{Sampling and Extraction Information}

The samples that underwent RGS analysis are listed in Table 4.10.1. Because of mechanical problems, sample 6-6 was taken by "slurping" the waste into the sampler (sucking the waste in by pressure differential without moving the sampler down through the waste). Subsequently, segment 6-6A was taken in the same location by the standard method. Another mechanical problem occurred while sample 3-10 was being taken. The piston was not pulled back as the sampler was pushed in, so the drill string was retracted and the procedure repeated correctly. All three of these samples should be considered as having been taken from disturbed waste; they may not be comparable with other samples. The retry, sample 6-6A, is probably the least representative of the three disturbed-waste samples. Field data, including dose rates and downforce limits, are supplied in Appendix A.

Table 4.10.1 also shows the lag times (delay between sample acquisition and processing) for these samples. This information was provided to allow data users to correlate the lag time between sampling and extrusion with the concentrations of the various constituents to test for decomposition or other chemical reactions. The maximum hold time allowed by the sampling plan is 24 days based on measured sampler leakage rates during acceptance testing. The hold time for four of the samples (6-6, 6-9, 3-7, and 3-10) exceeded this limit, but the samples were accepted based on calculations that indicated the estimated leakage was still negligible. For some of these samples $(6-9,3-7$, and 3-10), long lag times resulted from delays to devise new procedures or from facility shutdowns and repairs.

As was discussed in Section 3.5, the HHF used during sampling often enters the sampler in place of some of the waste. The volume percentage contamination by the HHF of the RGS samples is shown in Table 4.10.2. All but one of these RGS samples (6-6A) contained bromide below the detection limit. The measured value or (where necessary) the MDLs were used to calculate reduced effective sampler volumes for the RGS samples. (It is conservative to use the MDL for values below it; the conservatism is probably less than $3 \%$.)

All of these samplers were helium-backfilled and sealed with vacuum grease before they were deployed. This method virtually eliminated air and argon contamination from gases entrained during sampling. The helium backfill results are discussed in detail by Cannon (1997) and summarized in Section 5 of Mahoney et al. (1997). The same extraction procedure was used on all of the SX-106 samples except 3-10. However, the procedure before the post-isotopic extractions was the same for all samples. (Figure 3.1 is a schematic of the RGS extraction system.)

Table 4.10.1. Lag Times for Processing RGS Samples from Tank 241-SX-106

\begin{tabular}{|c|c|c|c||}
\hline Sample & Acquisition Date & Processing Date & Lag (days) \\
\hline \hline $3-2$ & December 3,1997 & December 15, 1997 & 12 \\
\hline $3-4$ & December 9,1997 & December 18, 1997 & 9 \\
\hline $6-6$ & October 30, 1997 & November 24, 1997 & 25 \\
\hline $6-6 \mathrm{~A}$ & October 30, 1997 & November 20,1997 & 21 \\
\hline $3-7$ & December 12,1997 & January 16, 1998 & 35 \\
\hline $6-9$ & November 7, 1997 & December 10,1997 & 33 \\
\hline $3-10$ & December 12,1997 & January 23,1998 & 42 \\
\hline
\end{tabular}


Table 4.10.2. Hydrostatic Head Fluid Contamination in SX-106 RGS Samples

\begin{tabular}{|c|c|}
\hline Sample & $\begin{array}{c}\text { HHF Contamination } \\
\text { (vol\%) }\end{array}$ \\
\hline $3-2$ & $<2.2$ \\
\hline $3-4$ & $<2.2$ \\
\hline $6-6$ & $<4.5$ \\
\hline $6-6 \mathrm{~A}$ & 6.5 \\
\hline $3-7$ & $<2.9$ \\
\hline $6-9$ & $<6.7$ \\
\hline $3-10$ & $<2.7$ \\
\hline
\end{tabular}

After evacuating the whole system and observing the pressure to test for leaks, the operator extruded the RGS sample into the extractor vessel, and the sample was briefly stirred. At this point the vapor space in communication with the sample consisted of the volumes of the extractor vessel, its lines, the second volume, the pump volume, and the collector side; the latter included the collector line volume and an open collection canister. This first canister, containing a sample of uncompressed (unpumped) gas, was closed off. The second set of two collection canisters was opened. Several strokes were pumped from the extractor to the collector, and the second collection canisters were closed off. Then the collector side and the extractor lines were evacuated. (The second volume was never used during the extraction process for SX-106 samples.)

The vapor profile procedure was then performed. The first pump stroke and every third stroke thereafter were collected in single canisters, one stroke per canister. On the intermediate strokes the gas was pumped to the collector lines with no canister attached. The collector lines were evacuated after every pump stroke, whether or not the pumped gas was collected in a canister. The total gas and vapor pumped from the extractor to the collector during this procedure was calculated by interpolating linearly between collected canisters to find the contents of the pump strokes that were not collected. At the end of the procedure, the first post-isotopic set of canisters was attached to the collector lines and opened, and both the extractor lines and the collector side were evacuated. Next, $300 \mathrm{~mL}$ of $0.02 \mathrm{M} 15 \mathrm{NH}_{4} \mathrm{OH}$ solution was added to the sample in the extractor and stirred. From this point, the two procedures diverged.

Sample 3-10: After a relatively short time the extractor was opened to the collector, and a single unpumped canister took a sample and was closed off. Then several strokes were pumped from the extractor to the collector, where three parallel open canisters were connected. This first post-isotopic set of canisters was closed off, the second set was attached and opened, and the collector side was evacuated. More time was allowed for equilibration, and then the same steps were repeated, collecting one unpumped canister followed by three pumped canisters. Finally, the BSVD procedure was performed: argon was injected into the closed-off second volume and the second volume then opened to the extractor and lines. Pressure measurements and known tare system volumes allowed calculation of the volume of the sample solids and liquid.

All other samples: After a relatively short time, several strokes were pumped from the extractor to the collector. The first post-isotopic set of three canisters was closed off, the second set of three was attached and opened, and the collector side was evacuated. More time was allowed for equilibration, then several strokes were pumped from the extractor to the collector. The second set of three post-isotopic canisters was closed off. Finally, the BSVD procedure was performed. 
In processing sample 3-2, vacuum was accidentally applied just after the first canister of the vapor profile procedure. The error caused some loss of gas; an attempt was made to compensate, and the uncertainty of the gas measurement was increased. Based on experience with other samples, we expect any gas underestimation resulting from the loss to be less than $10 \%$.

Procedural details such as the number of strokes per canister and the system volumes can be found in the sample-by-sample worksheets in the SX-106 RGS calculation spreadsheet on the $\mathrm{CD}$ included with this report.

\subsubsection{Retained Gas Composition}

Table 4.10.3 lists the calculated concentrations of the insoluble gases in the SX-106 RGS samples without corrections for air and argon entrainment, helium backfill, air inleakage, or air dissolved in the isotopic standard solution. The method used to make the corrections depended on the gas. All of the oxygen, argon, and helium were subtracted no matter which step had produced them. A number of moles equal to $(3.73) \cdot\left(\mathrm{O}_{2}\right)$ were subtracted from the nitrogen because the oxygen was expected to have come from air entrainment during sampling or inleakage during extraction. Because a substantial amount of dissolved air gases entered the extractor with the $15 \mathrm{NH}_{4} \mathrm{OH}$ solution, all the nitrogen present in the gas collected after the solution addition was subtracted. Thus it was assumed that more than $95 \%$ of the original retained nitrogen had already been collected, as was the case for hydrogen. The corrected concentrations are given in Table 4.10.4.

Sample 3-7 shows the effects of a substantial air leak into the system. The $\mathrm{O}_{2}$ measurements established that the leak lasted from the beginning of the extraction process to the end. The leak must have been into the extractor, based on the extractor pressure increase of $5 \mathrm{kPa}$ that occurred during the 24 hours between the first and second sets of post-isotopic canisters. In addition, higher argon levels in some samples, particularly 6-9, suggest that argon drillstring purges were performed. These contamination observations can be found in the "Summary" worksheet of the SX-106 RGS calculations spreadsheet on the CD.

Table 4.10.3. Concentrations of Insoluble Constituents ( $\mu \mathrm{mol} / \mathrm{L}$ of waste) in Tank SX-106 Without Correction for Gas Contamination

\begin{tabular}{|c|c|c|c|c|c|c|c|c|c|c|c|}
\hline $\begin{array}{l}\text { Seg- } \\
\text { ment }\end{array}$ & $\mathrm{N}_{2}$ & $\mathrm{H}_{2}$ & $\mathrm{~N}_{2} \mathrm{O}$ & $\mathrm{O}_{2}$ & $\mathrm{CH}_{4}$ & $\mathrm{He}$ & $\mathrm{Ar}$ & $\begin{array}{l}\text { Other } \\
\text { NOx }\end{array}$ & $\mathrm{C}_{2} \mathrm{H}_{\mathrm{x}}$ & $\mathrm{C}_{3} \mathrm{H}_{\mathrm{x}}$ & $\begin{array}{l}\text { Other } \\
\text { Hyd. }\end{array}$ \\
\hline $3-2$ & $960 \pm 46$ & $13 \pm 0.8$ & $27 \pm 2.1$ & $470 \pm 21$ & $0.7 \pm 0.3$ & $270 \pm 15$ & $99 \pm 4.6$ & (a) & $0.4 \pm 0.2$ & (a) & $0.5 \pm 0.2$ \\
\hline $3-4$ & $350 \pm 40$ & $18 \pm 2.5$ & $40 \pm 10$ & $140 \pm 15$ & $0.7 \pm 0.3$ & $340 \pm 25$ & $72 \pm 12$ & (a) & $0.3 \pm 0.1$ & (a) & $0.5 \pm 0.2$ \\
\hline $6-6(b)$ & $2700 \pm 120$ & $2400 \pm 140$ & $870 \pm 61$ & $290 \pm 15$ & $89 \pm 13$ & $12 \pm 1.1$ & $65 \pm 3.6$ & (a) & $27 \pm 11$ & $14 \pm 7.5$ & $6.4 \pm 2.8$ \\
\hline $6-6 A^{(b)}$ & $2900 \pm 370$ & $1100 \pm 61$ & $500 \pm 44$ & $460 \pm 28$ & $57 \pm 19$ & $430 \pm 24$ & $43 \pm 2.7$ & $1.0 \pm 1.0$ & $10 \pm 3.7$ & $9.8 \pm 4.8$ & $8.8 \pm 4.5$ \\
\hline $3-7$ & $\begin{array}{c}17000 \pm \\
6300 \\
\end{array}$ & $7700 \pm 270$ & $4400 \pm 860$ & $\begin{array}{c}7100 \pm \\
4600\end{array}$ & $82 \pm 4.7$ & $380 \pm 15$ & $210 \pm 56$ & $5.7 \pm 5.7$ & $24 \pm 10$ & $0.6 \pm 0.6$ & $10 \pm 5.9$ \\
\hline $6-9$ & $4600 \pm 170$ & $12000 \pm 370$ & $3600 \pm 130$ & $690 \pm 58$ & $74 \pm 29$ & $520 \pm 18$ & $240 \pm 42$ & (a) & $8.7 \pm 3.1$ & $36 \pm 15$ & $12 \pm 8.0$ \\
\hline $3-10(b)$ & $5700 \pm 190$ & $8500 \pm 260$ & $5400 \pm 190$ & $570 \pm 27$ & $110 \pm 6.2$ & $540 \pm 19$ & $22 \pm 1.4$ & (a) & $51 \pm 13$ & (a) & $9.9 \pm 5.5$ \\
\hline
\end{tabular}


Table 4.10.4. Concentrations of Insoluble Constituents ( $\mu \mathrm{mol} / \mathrm{L}$ of waste) in Tank SX-106 with Correction for Gas Contamination

\begin{tabular}{|c|c|c|c|c|c|c|c|c|c|c|c||}
\hline Segment & $\mathrm{N}_{2}$ & $\mathrm{H}_{2}$ & $\mathrm{~N}_{2} \mathrm{O}$ & $\mathrm{O}_{2}$ & $\mathrm{CH}_{4}$ & $\mathrm{He}$ & $\mathrm{Ar}$ & $\begin{array}{c}\text { Other } \\
\mathrm{NOx}\end{array}$ & $\mathrm{C}_{2} \mathrm{H}_{\mathrm{x}}$ & $\mathrm{C}_{3} \mathrm{H}_{\mathrm{x}}$ & $\begin{array}{c}\text { Other } \\
\text { Hyd. }\end{array}$ \\
\hline \hline $3-2$ & $32 \pm 3.9$ & $13 \pm 0.8$ & $27 \pm 2.1$ & $0 \pm 21$ & $0.7 \pm 0.3$ & $0 \pm 15$ & $0 \pm 4.6$ & (a) & $0.4 \pm 0.2$ & (a) & $0.5 \pm 0.2$ \\
\hline $3-4$ & $52 \pm 17$ & $18 \pm 2.5$ & $40 \pm 10$ & $0 \pm 15$ & $0.7 \pm 0.3$ & $0 \pm 25$ & $0 \pm 12$ & (a) & $0.3 \pm 0.1$ & (a) & $0.5 \pm 0.2$ \\
\hline $6-6^{(\mathrm{b})}$ & $1100 \pm 130$ & $2400 \pm 140$ & $870 \pm 61$ & $0 \pm 15$ & $89 \pm 13$ & $0 \pm 1.1$ & $0 \pm 3.6$ & (a) & $27 \pm 11$ & $14 \pm 7.5$ & $6.4 \pm 2.8$ \\
\hline $6-6 \mathrm{~A}^{\text {(b) }}$ & $400 \pm 58$ & $1100 \pm 61$ & $500 \pm 44$ & $0 \pm 28$ & $57 \pm 19$ & $0 \pm 24$ & $0 \pm 2.7$ & $1.0 \pm 1.0$ & $10 \pm 3.7$ & $9.8 \pm 4.8$ & $8.8 \pm 4.5$ \\
\hline $3-7$ & $3000 \pm 1300$ & $7700 \pm 270$ & $4400 \pm 860$ & $0 \pm 4600$ & $82 \pm 4.7$ & $0 \pm 15$ & $0 \pm 56$ & $5.7 \pm 5.7$ & $24 \pm 10$ & $0.6 \pm 0.6$ & $10 \pm 5.9$ \\
\hline $6-9$ & $3600 \pm 370$ & $12000 \pm 370$ & $3600 \pm 130$ & $0 \pm 58$ & $74 \pm 29$ & $0 \pm 18$ & $0 \pm 42$ & (a) & $8.7 \pm 3.1$ & $36 \pm 15$ & $12 \pm 8.0$ \\
\hline $3-10^{\text {(b) }}$ & $4100 \pm 400$ & $8500 \pm 260$ & $5400 \pm 190$ & $0 \pm 27$ & $110 \pm 6.2$ & $0 \pm 19$ & $0 \pm 1.4$ & (a) & $51 \pm 13$ & (a) & $9.9 \pm 5.5$ \\
\hline
\end{tabular}

(a) Below detection limits, which are $0.01 \mathrm{~mol} \%$ or less.

(b) For samples 6-6 and 3-10, waste was disturbed because of sampling difficulties. Sample 6-6A was the second attempt at sampling the segment 6 elevation.

Table 4.10.5 presents the ammonia measurements. The residual ammonia was determined isotopically, and the total ammonia (calculated from the isotopic residual) is included in the table. The post-extrusion partial pressure of $\mathrm{NH}_{3}$ over the sample (see Section 3.4.4) was measured at chilled extractor temperature and is included in Table 4.10.5. The residual ammonia in the samples was also measured by ISE after the samples were removed from the extractor. The post-RGS ISE measurements can be found in Appendix C, Table C.10.1.

The four different types of measurements of ammonia in the supernatant layer did not give consistent results. We have used the isotopic RGS ammonia concentrations to calculate the ammonia in the in situ vapor, though we think it is possible that they were overestimated by about a factor of 2 (see Appendix C, Section C.10 for more detail). Despite the uncertainties, the RGS measurements show that the ammonia concentrations in SX-106 supernatant were at least a factor of 4 lower than the ammonia concentrations in the interstitial liquid of the nonconvective layer.

There were three types of ammonia measurements in the nonconvective layer, isotopic, partial-pressure, and post-RGS ISE, all consistent. Most of the isotopic RGS measurements gave concentrations of 60,000 to $75,000 \mu \mathrm{mol} \mathrm{NH} 3 / \mathrm{L}$ waste, with one higher value from sample 6-6. The average and standard deviation of the isotopically measured ammonia concentrations over samples $6-6,6-6 \mathrm{~A}, 3-7,6-9$, and 3-10 were 78,000 $\pm 19,000 \mu \mathrm{mol} / \mathrm{L}$ of waste. This corresponds to an average of $0.18 \mathrm{wt} \% \mathrm{NH}_{3}$ in the bulk waste, or $2500 \mu \mathrm{g} \mathrm{NH} / \mathrm{mL}$ of liquid $\left(0.15 \mathrm{M} \mathrm{NH}_{3}\right)$, at the time of sampling. See Appendix C, Section C.10 for more detail.

Table 4.10.6 contains the composition of the gas/vapor phase in each sample and the average compositions in the gases retained in the supernatant and nonconvective layers. The water vapor is not included in these compositions; the ammonia fractions are derived from the data in Table 4.10.5, which were used to calculate vapor pressures at in situ conditions. The sample compositions in the table have been calculated using the in situ solubility method described in Section 3.6.2. As discussed in Section 3.6.1, compositions were calculated for both the lower- and upper-bound gas solubilities. Both ends of the range are given in Table 4.10 .6 with the 
Table 4.10.5. Ammonia Data from Tank SX-106 Samples

\begin{tabular}{||c|c|c|c||}
\hline Sample & $\begin{array}{c}\text { Isotopically Measured } \\
\text { Total } \mathrm{NH}_{3} \text { (a) } \\
(\mu \mathrm{mol} / \mathrm{L})\end{array}$ & $\begin{array}{c}\text { Measured } \mathrm{NH}_{3} \text { Partial } \\
\text { Pressure at about } 12{ }^{\circ} \mathrm{C} \\
(\mathrm{atm})\end{array}$ & $\begin{array}{c}\text { RGS } \mathrm{NH}_{3} \text { Concentrations } \\
\text { in the Liquid } \\
(\mathrm{M})\end{array}$ \\
\hline \hline $3-2$ & $25000 \pm 3200$ & $0.0024 \pm 0.0012$ & $420 \mu \mathrm{g} / \mathrm{mL}(0.025 \mathrm{M})$ \\
\hline $3-4$ & $23000 \pm 2000$ & $0.0021 \pm 0.0010$ & $400 \mu \mathrm{g} / \mathrm{mL}(0.023 \mathrm{M})$ \\
\hline $6-6$ & $110000 \pm 10000$ & $0.0067 \pm 0.0033$ & $3000 \mu \mathrm{g} / \mathrm{mL}(0.18 \mathrm{M})$ \\
\hline $6-6 \mathrm{~A}$ & $70000 \pm 6100$ & $0.0039 \pm 0.0020$ & $1800 \mu \mathrm{g} / \mathrm{mL}(0.11 \mathrm{M})$ \\
\hline $3-7$ & $72000 \pm 32000$ & $0.013 \pm 0.065$ & $2500 \mu \mathrm{g} / \mathrm{mL}(0.15 \mathrm{M})$ \\
\hline $6-9$ & $61000 \pm 8800$ & $0.016 \pm 0.008$ & $2300 \mu \mathrm{g} / \mathrm{mL}(0.14 \mathrm{M})$ \\
\hline $3-10$ & $75000 \pm 10000$ & $0.012 \pm 0.006$ & $2700 \mu \mathrm{g} / \mathrm{mL}(0.16 \mathrm{M})$ \\
\hline (a) These data were used to calculate in situ ammonia vapor pressures. \\
\hline
\end{tabular}

measurement uncertainty on each. The average composition of the gas in the nonconvective layer is the result of integrating RGS species concentrations over the layer and multiplying those concentrations by the layer volume. The integration method is described in Section 3.7.1. The average composition of gas in the supernatant layer is an arithmetic average of the two samples.

Table 4.10.6. Sample and Overall Average Compositions of Retained Gas in SX-106 with Correction for Gas Contamination(a)

\begin{tabular}{|c|c|c|c|c|c|c||}
\hline \hline Sample & $\mathrm{N}_{2}$ (mol\%) & $\mathrm{H}_{2}$ (mol\%) & $\mathrm{N}_{2} \mathrm{O}$ (mol\%) & $\mathrm{NH}_{3}$ (mol\%) & $\mathrm{CH}_{4}$ (mol\%) & Other (mol\%) \\
\hline \hline $3-2$ & $60 \pm 10--74 \pm 13$ & $22 \pm 2.9--18 \pm 2.6$ & $14 \pm 1.9--2.4 \pm 0.4$ & $0.7 \pm 0.1--0.2 \pm 0.05$ & $1.3 \pm 0.5--1.1 \pm 0.4$ & $1.8 \pm 0.9--4.4 \pm 2.3$ \\
\hline $3-4$ & $62 \pm 26--77 \pm 33$ & $19 \pm 6.0--17 \pm 5.6$ & $16 \pm 6.0--3.0 \pm 1.2$ & $0.6 \pm 0.2--0.2 \pm 0.06$ & $0.9 \pm 0.4--0.8 \pm 0.4$ & $1.0 \pm 0.5--1.8 \pm 0.9$ \\
\hline $6-6$ & $23 \pm 3.3--25 \pm 3.5$ & $51 \pm 5.1--53 \pm 5.4$ & $18 \pm 2.0-17 \pm 1.8$ & $5.1 \pm 0.6--1.9 \pm 0.2$ & $1.9 \pm 0.3-2.0 \pm 0.3$ & $1.0 \pm 0.5--1.1 \pm 0.5$ \\
\hline $6-6 \mathrm{~A}$ & $19 \pm 3.3--21 \pm 3.6$ & $51 \pm 5.6--55 \pm 6.1$ & $23 \pm 3.0--19 \pm 2.4$ & $3.0 \pm 0.4-1.1 \pm 0.2$ & $2.7 \pm 0.9--3.0 \pm 1.0$ & $1.4 \pm 0.6--1.5 \pm 0.8$ \\
\hline $3-7$ & $19 \pm 8.7--20 \pm 9.0$ & $48 \pm 8.5--50 \pm 8.4$ & $27 \pm 7.2--28 \pm 7.1$ & $4.5 \pm 2.2--1.7 \pm 0.8$ & $0.5 \pm 0.1$ & $0.2 \pm 0.1--0.3 \pm 0.1$ \\
\hline $6-9$ & $18 \pm 2.0$ & $60 \pm 3.6--62 \pm 3.5$ & $17 \pm 1.1$ & $4.0 \pm 0.6--1.5 \pm 0.2$ & $0.4 \pm 0.1$ & $0.3 \pm 0.1$ \\
\hline $3-10$ & $21 \pm 2.4--22 \pm 2.5$ & $45 \pm 2.7--46 \pm 2.7$ & $28 \pm 1.8--29 \pm 1.7$ & $4.6 \pm 0.7--1.7 \pm 0.2$ & $0.6 \pm 0.05$ & $0.3 \pm 0.1$ \\
\hline $\begin{array}{l}\text { Avg in } \\
\text { supernatant(c) }\end{array}$ & $61 \pm 20--76 \pm 25$ & $21 \pm 4.8--18 \pm 4.4$ & $15 \pm 4.2--2.8 \pm 0.8$ & $0.6 \pm 0.1--0.2 \pm 0.05$ & $0.9 \pm 0.4$ & $1.3 \pm 0.6--2.7 \pm 1.4$ \\
\hline $\begin{array}{l}\text { Avg in non- } \\
\text { convective } \\
\text { layer(c) }\end{array}$ & $20 \pm 4.0--21 \pm 4.0$ & $51 \pm 4.7--53 \pm 4.5$ & $24 \pm 2.7$ & $4.4 \pm 0.9--1.6 \pm 0.4$ & $0.6 \pm 0.1$ & $0.3 \pm 0.1$ \\
\hline $\begin{array}{l}\text { (a) Uncertainties on compositions represent instrument uncertainty. Compositions calculated for lower- and upper-bound } \\
\text { solubilities. Both ends of composition range are given, Iower-bound solubility basis first with instrument uncertainty on each. } \\
\text { Compositions may not sum to 100 mol\% because of roundoff error; mole fractions on dry basis, do not account for water } \\
\text { vapor. } \\
\text { (b) Ammonia mole fraction derived from data in Table 4.10.5 used to calculate vapor pressure at in situ conditions. } \\
\text { (c) There are too few samples to define the spatial variability of the average gas concentration. }\end{array}$ \\
\hline
\end{tabular}


The retained gas in the SX-106 samples was of nearly the same composition in all the samples within a layer. The samples from the nonconvective layer under riser 6 appears to have contained less $\mathrm{N}_{2} \mathrm{O}$ than those from the nonconvective layer under riser 3, but the difference is nearly within the uncertainty.

The concentrations and pressures in Tables 4.10.3 and 4.10.4 can also be found in the sample-by-sample worksheets in the SX-106 RGS calculation spreadsheet on the CD. The compositions in Table 4.10.6 are in the "Inventory" worksheet.

\subsubsection{Gas Inventory}

The method by which the in situ gas volume fractions (wet basis) were calculated is given in Section 3.6.2; as for the gas composition, the volume fractions are given as a range from the low-gas-solubility value to the high-gas-solubility value. The results are shown in Table 4.10.7, which also contains the average gas volume fraction and average pressure experienced by the gas in the two layers. The average for the nonconvective layer is an in situ volume average calculated by Simpson's Rule integration as described in Section 3.7.1. The average for the supernatant layer is an arithmetic average of the two samples. The corrected gas volume fractions in Table 4.10.7 are consistent with the corrected gas concentrations and compositions in Tables 4.10.4, 4.10.5, and 4.10.6. The information in Table 4.10.7 is taken from the "Summary" and "In situ" worksheets of the SX-106 RGS calculation spreadsheet on the CD.

The "sampler gas volume fraction" is corrected only for inleakage during the extraction process and air gases added in the isotopic solution. It is an attempt to reconstruct the total amount of gas (sample and entrainment) that was present in the sampler during x-ray. It is used only for comparison with the $\mathrm{x}$-ray observations of "visible gas fraction" discussed in Section 4.10.4.

Table 4.10.7 also contains the water vapor pressures that were used for in situ calculations. The water vapor pressures were found by using salt concentrations from Field (1998) and the temperatures in the table as inputs to Equation 6.2 of Mahoney and Trent (1995), a correlation for water vapor pressure over concentrated homogeneous and nonhomogeneous waste simulants. The gas solubilities used the same parameters as inputs to the Schumpe solubility model (see Section 3.6.1). The water vapor pressures and gas solubilities used in calculations can be found in the "In situ" worksheet of the SX-106 RGS calculation spreadsheet on the CD.

The six ammonia concentration measurements in SX-106 (leaving out sample 6-6A, which was taken from disturbed waste) integrate to a total (vapor and dissolved) ammonia inventory that would have had an STP volume of $2700 \mathrm{~m}^{3}\left(94,000 \mathrm{ft}^{3}\right)$, of which $84 \%$ was in the interstitial liquid in the nonconvective layer. The uncertainty on the ammonia inventory was at least $\pm 70 \%$.

Table 4.10.8 gives estimates of the STP volume of gas in Tank SX-106 including some calculated from RGS data alone. The RGS gas inventory in the nonconvective layer was calculated by integrating RGS total gas concentrations over the layer (four data points, omitting sample 6-6A) and multiplying the average gas concentration by the volume of the layer. The integration method is described in Section 3.7.1. The inventory in the supernatant layer was based on the arithmetic average gas concentration. The RGS volumes in Table 4.10.8 include corrections to remove the contamination gas: entrained air and argon, helium backfill, air leaks during and after extraction, and air dissolved in the isotopic standard.

The RGS method gave 70\% more gas inventory than was calculated by the BPE method, although the RGS inventory did not include the gas in the thin crust. The uncertainties on the gas inventories are based on the spatial variability considerations discussed in Section 3.7.2. The information in Table 4.10.8 is taken from the "Inventory" worksheet of the SX-106 RGS calculation spreadsheet on the $C D$. 
Table 4.10.7. In Situ Gas Volume Fractions and Conditions in Tank SX-106

\begin{tabular}{|c|c|c|c|c|c|c|}
\hline Sample & $\begin{array}{l}\text { Sample } \\
\text { Central } \\
\text { Height } \\
\text { (cm) }\end{array}$ & \begin{tabular}{|c|} 
Hydro- \\
static \\
Pressure \\
(atm)
\end{tabular} & $\begin{array}{l}\text { Calculated } \\
\text { Water Vapor } \\
\text { Pressure } \\
\text { (atm) }\end{array}$ & $\begin{array}{c}\text { Temp } \\
\left({ }^{\circ} \mathrm{C}\right)\end{array}$ & $\begin{array}{c}\text { Corrected Gas Volume } \\
\text { Fraction(a) } \\
\text { (in-tank conditions) }\end{array}$ & $\begin{array}{l}\text { Sampler Gas } \\
\text { Volume Fraction } \\
\text { (in situ conditions, } \\
\text { low solubility) }\end{array}$ \\
\hline $3-2$ & 458 & 1.08 & 0.022 & 30.6 & $\begin{array}{c}0.001 \pm 0.0004 \text { to } \\
0.0005 \pm 0.0004\end{array}$ & 0.007 \\
\hline $3-4$ & 362 & 1.22 & 0.022 & 30.6 & $\begin{array}{c}0.002 \pm 0.0007 \text { to } \\
0.0009 \pm 0.0006\end{array}$ & 0.008 \\
\hline $6-6$ & 265 & 1.34 & 0.017 & 36.9 & $0.089 \pm 0.010$ to $0.084 \pm 0.010$ & 0.091 \\
\hline $6-6 A$ & 265 & 1.34 & 0.017 & 36.9 & $0.040 \pm 0.006$ to $0.037 \pm 0.006$ & 0.049 \\
\hline $3-7$ & 217 & 1.40 & 0.019 & 39.4 & $0.30 \pm 0.11$ to $0.28 \pm 0.11$ & 0.40 \\
\hline $6-9$ & 121 & 1.51 & 0.020 & 40.3 & $0.35 \pm 0.022$ to $0.34 \pm 0.022$ & 0.37 \\
\hline $3-10$ & 72 & 1.57 & 0.020 & 40.8 & $0.32 \pm 0.020$ to $0.30 \pm 0.019$ & 0.33 \\
\hline $\begin{array}{c}\text { Avg in } \\
\text { supernatant(b) }\end{array}$ & 406 & 1.15 & & . & $\begin{array}{l}0.002 \pm 0.001 \text { to } \\
0.0007 \pm 0.0005\end{array}$ & \\
\hline $\begin{array}{l}\text { Avg in non- } \\
\text { convective } \\
\text { layer(b) }\end{array}$ & 133 & 1.50 & & & $0.26 \pm 0.13$ to $0.25 \pm 0.125$ & \\
\hline
\end{tabular}

The long-term surface level rise can also be considered as a sign of substantial retained gas volume: the waste surface rose a net $52 \mathrm{~cm}$ (20.5 in.) between 1981 and January 1996 (Hodgson et al. 1998). This rise implies an in situ gas accumulation equivalent to 0.19 gas in situ volume fraction in the nonconvective layer, but may underestimate the gas by not accounting for evaporation losses and gas present in the tank before 1981.

One explanation for the mismatch between the BPE gas inventory estimates and the RGS estimate might be that the high-gas region sampled by RGS did not extend over the entire tank; however, this explanation is not consistent with the presence of high gas volume fractions at both of the sampled risers. Another hypothesis is that the SX-106 waste strength (Section 4.10.5.1) was great enough to restrict the short-term dynamic response of the waste level to the gas volume changes caused by barometric pressure variation. If this were the case, BPE would have tended to underestimate the gas inventory.

Some points argue against the RGS inventory and in favor of the BPE inventory. The RGS data were taken only in risers near the tank center and might have missed a (hypothetical) low-gas zone further out toward the tank periphery. Several RGS samples in the nonconvective layer (6-6, 3-7, and 3-10) suffered from increased uncertainty because of sample acquisition problems, air inleakage during extraction, and lag times slightly longer than prescribed. In addition, the presence of a supernatant layer, covered by only a thin crust, should have made the waste level (and so the BPE method) a good indicator of the total gas inventory of the tank. However, the consistency of the high-gas RGS results (both in terms of gas volume fraction and composition) and the backup provided by non-RGS core extrusion observations (see the further discussion in Section 4.10.5.1), leads us to recommend the RGS inventory as the better estimate of gas in SX-106 at the time of sampling. 
Table 4.10.8. SX-106 Gas Inventory Estimates

\begin{tabular}{||l|c|c|c|}
\hline \multirow{2}{*}{ Quantity } & \multicolumn{2}{|c|}{ RGS Method } & BPE Method(a) \\
\cline { 2 - 4 } & $\begin{array}{c}\text { Supernatant } \\
\text { Layer }\end{array}$ & $\begin{array}{c}\text { Nonconvective } \\
\text { Layer }\end{array}$ & Tank Total \\
\hline $\begin{array}{l}\text { Avg. gas fraction } \\
\text { (low gas solubility) }\end{array}$ & $0.002 \pm 0.001$ & $0.26 \pm 0.13$ & $\begin{array}{c}0.16 \pm 0.04 \\
\text { (average over } \\
\text { nonconvective layer) }\end{array}$ \\
\hline $\begin{array}{l}\text { Gas volume (m3) } \\
\text { in situ (wet) }\end{array}$ & $1.2 \pm 0.6(\mathrm{~b})$ & $326 \pm 163(\mathrm{~b})$ & $192 \pm 52$ \\
STP (wet) & $1.2 \pm 0.6(\mathrm{~b})$ & $426 \pm 213(\mathrm{~b})$ & $252 \pm 68$ \\
\hline
\end{tabular}

(a) Barometric pressure method. Inventory based on the average gas pressure calculated from RGS data, and on dL/dP of $-0.41 \pm 0.10$ in./in.-Hg (Whitney et al. 1997).

(b) Retained gas inventory calculated from RGS data considered the better estimate.

Table 4.10.9 contains the calculated layer inventories of each of the major gases retained in the waste. These inventories are based on the layer-average compositions from RGS data (Table 4.10.6) and the best-estimate gas inventories for each layer (RGS, Table 4.10.8).

Figure 4.10.3 shows sample temperatures, corrected gas volume fractions (Table 4.10.7), and corrected compositions of the low-solubility constituents in the samples from Tank SX-106. The compositions represent the mole fraction of the species in the "insoluble" gas; water and ammonia are not included, so the mole.fractions are not the same as those in Table 4.10.6. The gas volume fractions and mole fractions in the figure are the values for lower-bound gas solubilities. Figure 4.10.3 also lists the observations from core extrusions of non-RGS samples and from X-rays of RGS samples as a way of tying those observations together with RGS data. The $\mathrm{x}$-ray data are described in more detail in Section 4.10.4.

Table 4.10.9. Speciated SX-106 Gas Inventory(a)

\begin{tabular}{|c|c|c|c|c|}
\hline \multirow[b]{2}{*}{ Species } & \multicolumn{2}{|c|}{$\begin{array}{c}\text { Gas-Phase Inventory } \\
\left(\mathrm{m}^{3} \text { at STP) }\right.\end{array}$} & \multicolumn{2}{|c|}{$\begin{array}{c}\text { Dissolved Inventory } \\
\left(\mathrm{m}^{3} \text { at } \mathrm{STP}\right)\end{array}$} \\
\hline & $\begin{array}{c}\text { Nonconvective } \\
\text { Layer }\end{array}$ & $\begin{array}{c}\text { Supernatant } \\
\text { Layer }\end{array}$ & $\begin{array}{c}\text { Nonconvective } \\
\text { Layer }\end{array}$ & $\begin{array}{c}\text { Supernatant } \\
\text { Layer }\end{array}$ \\
\hline $\mathrm{N}_{2}$ & 84 & $0.74-0.44$ & $0.0076-0.084$ & $0.025-0.33$ \\
\hline $\mathrm{H}_{2}$ & 210 & $0.25-0.10$ & $0.073-0.50$ & $0.032-0.18$ \\
\hline $\mathrm{N}_{2} \mathrm{O}$ & $101-97$ & $0.18-0.016$ & $0.50-3.9$ & $0.43-0.60$ \\
\hline $\mathrm{NH}_{3}$ & $19-6.6$ & $0.0072-0.0012$ & 2200 & 440 \\
\hline $\mathrm{CH}_{4}$ & 2.5 & $0.012-0.0052$ & $0.00059-0.0070$ & $0.00081-0.0081$ \\
\hline Other & 1.4 & 0.015 & 0 & 0 \\
\hline
\end{tabular}




\subsubsection{X-Ray Results}

Table 4.10.10 summarizes all the available radiography observations from Tank SX-106. The $\mathrm{X}$-ray images themselves can be found on the CD that accompanies this report. The uncertainties of the visible gas volume fractions that are given in Table 4.10.10 are unknown but (based on the uncertainty in measuring the gas gap from x-ray images) are expected to be larger than the uncertainties in the gas volume fractions calculated from gas extraction. (The "visible" gas volume fraction refers to the gas that has separated from the solids and liquid such that its volume can be calculated based on the image. The uncorrected gas fractions from extraction are used for comparison to reflect the presence of entrained air in the sampler.)

Table 4.10.10 compares $x$-ray-derived and extraction-derived gas volume fractions. The comparison shows that sample recovery was close to $100 \%$ for all the samples. This conclusion is based on the fact that the extracted uncorrected gas was equal to or a few volume percent more than the visible gas. Samples 3-7 and 6-9 were exceptions, showing slightly more visible than extracted gas, but the difference is judged to be well within the uncertainty of the visible gas estimation method.

Table 4.10 .11 provides a summary of the waste densities that have been calculated from radiographic data for the RGS samples for which air and water standard images were available. The radiographic densities include the gas in the waste, were calculated by the current X-ray analysis method, and are given for several locations within each sample to show density trends. Table 4.10.11 also includes density measurements made on extruded non-RGS samples (Field 1998). The x-ray method gives results that are often consistent with those found by laboratory analysis of adjacent samples.

\subsubsection{Other Discussions}

\section{High Gas Fractions}

Unusually large amounts of gas were extracted from samples 3-7, 6-9, and 3-10 of Tank SX-106. The in situ gas volume fractions calculated from RGS extraction data were greater than or equal to 0.3 for these samples. The high gas content found by extraction was confirmed by the large gaps that were seen in the sampler x-rays for the same RGS samples.

The gas in samples 6-9, 3-7, and 3-10 was calculated to be enough to reduce the gassy bulk density of the gas-containing waste below the density of the overlying supernatant liquid. In addition, non-RGS samples 6-7, 6-8, 6-10, 3-8, and 3-9 each contained 14.5 in. or less of solids, as shown in Figure 4.10.3. These low solids contents may indicate there were 4.5 or more inches of gas in these samplers (evidence of gas fractions exceeding 0.25 like those of the RGS samples). However, the non-RGS samplers are not hermetically sealed and the core extrusion length is not a precise measurement, so non-RGS "gas gaps" must be interpreted with caution.

This situation poses the question of why a rollover had not occurred. One possibility is that the waste above the high-gas region-for example, the relatively low-gas waste in sample 6-6was dense enough to hold down the layer as a whole. Another hypothesis is that SX-106 waste had unusually high strength and adhesion to the tank walls (as suggested by the presence of waste stalactites, seen in SX-106 sample X-rays) and therefore remained in place after the buoyant density was reached and until gas percolation began. A high amount of percolation would be expected at such high gas volume fractions. Once the channels formed, the subsequently generated gas would be released as it was generated, as has happened in laboratory-scale experiments with tank wastes containing gas volume fractions from 0.25 to 0.5 (Gauglitz et al. 1996; Rassat et al. 1997). If this somewhat speculative hypothesis is correct, rollover might not have been possible in SX-106 in spite of the low gassy density in the nonconvective layer. 

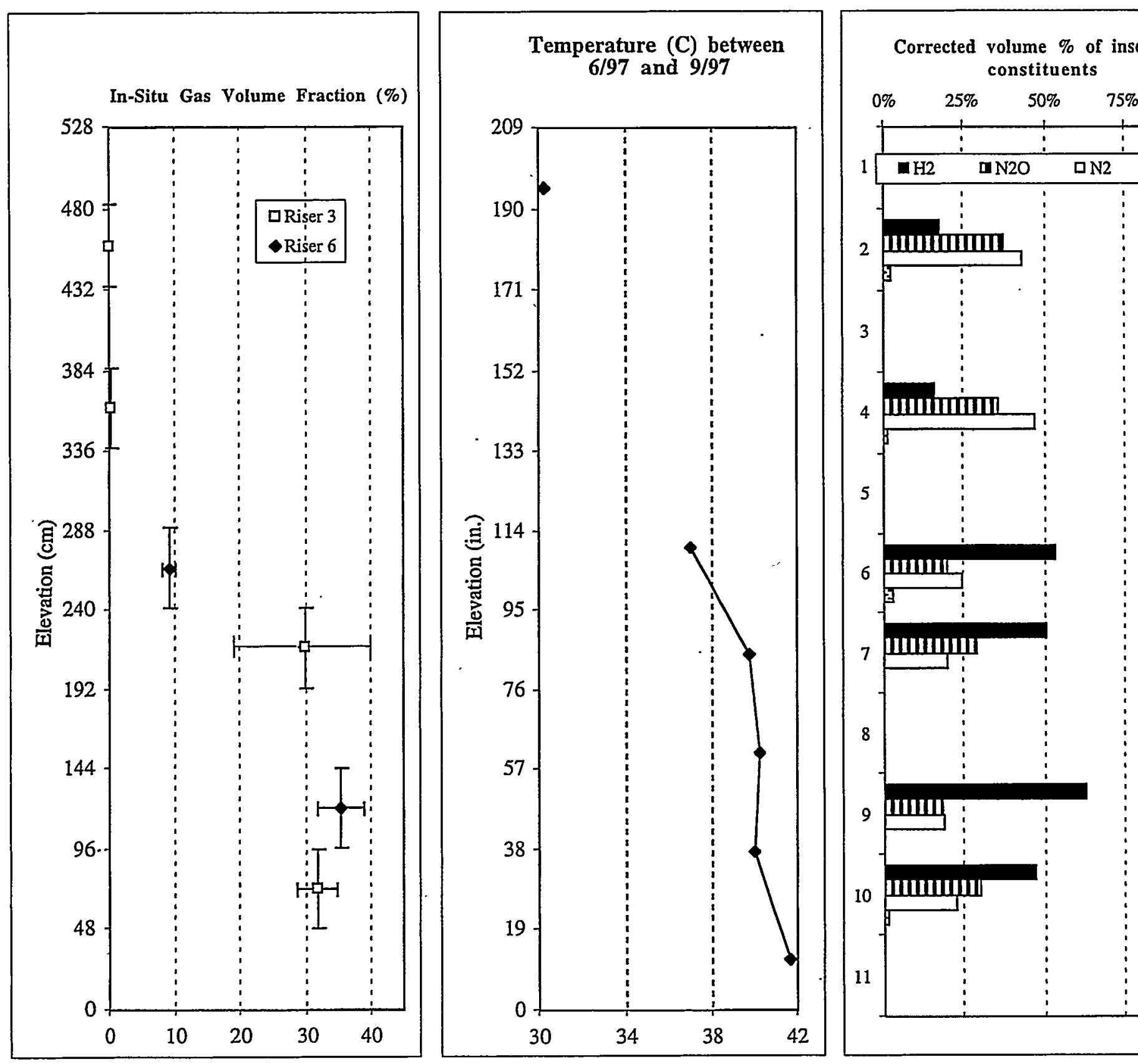

Figure 4.10.3. Volume Fractions, Temperatures, Con 


\section{Waste level in SX-106 is 503 to $526 \mathrm{~cm}$ (198 to 207 inches).}

1 Riser 6: $250 \mathrm{~mL}$ (329 g) pale yellow liquid, 3 in. (39 g) pale yellow wet salt.

1 Riser 3: $250 \mathrm{~mL}$ ( $356 \mathrm{~g}$ ) pale yellow liquid, 3 in. $(45 \mathrm{~g})$ white salt slurry.

2 Riser 6: $250 \mathrm{~mL}$ (325 g) pale yellow liquid, 3 in. (57 g) pale yellow wet sait.

2 Riser 3 X-RAY: No gap below piston, a few bubbles at piston, meniscus ripples. $1.8 \mathrm{R} / \mathrm{hr}$.

3 Riser 6: $250 \mathrm{~mL}$ ( $354 \mathrm{~g}$ ) pale yellow liquid, 4 in. $(72 \mathrm{~g})$ pale yellow wet salt.

3 Riser 3: $250 \mathrm{~mL}$ ( $366 \mathrm{~g}$ ) pale yellow liquid, 2 in. ( $35 \mathrm{~g}$ ) white wet salt.

4 Riser 6: $270 \mathrm{~mL}$ ( $380 \mathrm{~g}$ ) grey-green liquid, $1.5 \mathrm{in}$. (32 g) gray salt slurry.

4 Riser 3 X-RAY: 0.1 in. gas gap below piston, no bubbles, waste stalactite, meniscus ripples.

5 Riser 6: $250 \mathrm{~mL}$ (363 g) green-brown liquid, 1 in. (45 g) gray salt slumy.

5 Riser 3: $110 \mathrm{~mL}(136 \mathrm{~g})$ dark gray liquid, 2 in. $(12 \mathrm{~g})$ white salt siurry, 12 in. (263 g) brown salt/sludge slurry.

6 Riser $6 \mathrm{X}$-RAY: 0.4 in. gap below piston, no adhering waste, many bubbles bottom part. $1.6 \mathrm{R} / \mathrm{hr}$.

6 Riser 3: $55 \mathrm{~mL}(79 \mathrm{~g})$ dark gray liquid, $16 \mathrm{in}$. (326 g) brown salt/sludge slurry.

7 Riser 6: 14 in. (394 g) wet black sludge.

7 Riser 3 X-RAY: 5.8 in. gas gap below piston, many bubbles lower part.

8 Riser 6: 2 in. (97 g) wet black sludge, 8 in. (245 g) gray salt slurry.

8 Riser 3: 13 in. $(316 \mathrm{~g})$ gray wet salt.

9 Riser 6X-RAY: 6.8 in. gap below piston, waste stalactite, many bubbles throughout. $0.8 \mathrm{R} / \mathrm{hr}$.

9 Riser 3: 6 in. $(130 \mathrm{~g})$ gray wet salt, 8.5 in. $(156 \mathrm{~g})$ gray dry salt.

10 Riser 6: 9 in. (235 g) moist dark brown salt, 2.5 in. ( $57 \mathrm{~g}$ ) dark brown salt slurry.

10 Riser 3 X-RAY: 5.6 in. gas gap below piston, many bubbles throughout, waste stalactite.

11 Riser 6: No sample.

11 Riser 3: 16 in. (427 g) gray wet salt. 
Table 4.10.10. Summary of Observations from X-Ray Images of Tank SX-106

\begin{tabular}{|c|c|}
\hline Segment & Comments/Observations \\
\hline $\begin{array}{l}3-2 \\
\text { (RGS, } \\
12 / 3 / 97)\end{array}$ & $\begin{array}{l}\text { No visible features in this sample. The waste top surface is liquid (rippled when sampler was } \\
\text { moved) with a few bubbles resting against the piston. Other than that, there is no gap between } \\
\text { waste and piston, which was fully retracted. The visible gas fraction is therefore } 0 \text {. (Compare } \\
\text { with the uncorrected gas volume fraction of } 0.007 \text { obtained by gas extraction, Table } 4.10 .7 \text {.) }\end{array}$ \\
\hline $\begin{array}{l}3-4 \\
\text { (RGS, } \\
12 / 9 / 97)\end{array}$ & $\begin{array}{l}\text { No visible features in this sample. The waste top surface is liquid (rippled when sampler was } \\
\text { moved) with a } 0.2-\mathrm{cm}(0.1-\text { in.) gas gap between waste and piston. A small waste bridge crosses } \\
\text { the gap at one point. Piston is fully retracted; visible gas volume fraction is } 0.005 \text {. (Compare } \\
\text { with the uncorrected gas volume fraction of } 0.008 \text { obtained by gas extraction, Table } 4.10 .7 \text {.) }\end{array}$ \\
\hline $\begin{array}{c}\text { 6-6 } \\
\text { (RGS, } \\
\text { 10/30/97) }\end{array}$ & $\begin{array}{l}\text { This sample contained many gas bubbles in the 2--to 4-mm range throughout the sample. } \\
\text { There is a flat top surface and a gas gap below the fully retracted piston, from which a large } \\
\text { lump of waste has become detached and is hanging above the waste top surface, apparently } \\
\text { supported by adhesion to the sampler walls. Together, the gas gaps above and below the } \\
\text { detached stalactite add to about } 2.1 \mathrm{~cm} \text { ( } 0.8 \text { in.) The visible gas volume fraction (not counting } \\
\text { bubbles and excluding the volume of the "floating" stalactite) is therefore } 0.04 \text {. (Compare with } \\
\text { uncorrected gas volume fraction of } 0.091 \text { obtained by gas extraction, Table 4.10.7.) }\end{array}$ \\
\hline $\begin{array}{l}\text { 6-6A } \\
\text { (RGS, } \\
\text { 10/30/97) }\end{array}$ & $\begin{array}{l}\text { This sample contained gas bubbles in the } 2 \text {-mm size range in the bottom third of the sample, } \\
\text { nothing visible above. There is a flat top surface and no waste attached to the fully retracted } \\
\text { piston, with a } 1.1-\mathrm{cm}(0.45 \text {-in.) gas gap below the piston. The visible gas volume fraction (not } \\
\text { counting bubbles) is therefore } 0.02 \text {. (Compare with the uncorrected gas volume fraction of } \\
0.049 \text { obtained by gas extraction, Table } 4.10 .7 \text {.) }\end{array}$ \\
\hline $\begin{array}{c}3-7 \\
\text { (RGS, } \\
12 / 12 / 97)\end{array}$ & $\begin{array}{l}\text { Numerous gas bubbles in the 2- to 3-mm range near the bottom of the sample, fewer or none } \\
\text { visible toward the top of the waste. The bottom of the piston was not visible in the image. } \\
\text { The waste top surface is flat, with a 15-cm (5.8-in.) gas gap between it and the piston. The } \\
\text { visible gas volume fraction (not counting bubbles and assuming no waste on the piston) is } \\
\text { therefore } 0.32 \text {. (Compare with the corrected gas volume fraction of } 0.30 \text { obtained by gas } \\
\text { extraction, Table } 4.10 .7 \text {. In this case the corrected gas fraction is more appropriate for } \\
\text { comparison because of air inleakage during extraction.) }\end{array}$ \\
\hline $\begin{array}{c}6-9 \\
\text { (RGS, } \\
11 / 7 / 97)\end{array}$ & $\begin{array}{l}\text { This sample contained a great many gas bubbles throughout in the } 2-\text { to } 3 \text {-mm range. There is } \\
\text { a flat top surface and a } 17-\mathrm{cm} \text { ( } 6.8 \text {-in.) gas gap below the piston to which a lump of waste is } \\
\text { attached. The visible gas volume fraction (not counting bubbles, and excluding the volume of } \\
\text { the waste on the piston) is therefore } 0.37 \text {. (Compare with the uncorrected gas volume fraction } \\
\text { of } 0.37 \text { obtained by gas extraction, Table } 4.10 .7 \text {.) }\end{array}$ \\
\hline $\begin{array}{c}\text { (RGS, } \\
12 / 12 / 97)\end{array}$ & $\begin{array}{l}\text { This sample contained a great many gas bubbles throughout in the } 2-\text { to } 3 \text {-mm range. There is } \\
\text { a flat top surface and a } 15-\mathrm{cm} \text { ( } 5.8 \text {-in.) gas gap below the piston to which a lump of waste is } \\
\text { attached. The visible gas volume fraction (not counting bubbles and excluding the volume of } \\
\text { the waste on the piston) is therefore } 0.32 \text {. (Compare with the uncorrected gas volume fraction } \\
\text { of } 0.33 \text { obtained by gas extraction, Table 4.10.7.) }\end{array}$ \\
\hline
\end{tabular}


Table 4.10.11. Densities of SX-106 Samples from Radiography and Core Samples

\begin{tabular}{|c|c|c|c|}
\hline $\begin{array}{l}\text { Riser- } \\
\text { Segment } \\
\text { Number }\end{array}$ & $\begin{array}{l}\text { Distance from } \\
\text { bottom of sampler } \\
\text { (ft) }\end{array}$ & $\begin{array}{c}\text { Calculated Mean Density } \\
\text { by Current Method } \\
(\mathrm{g} / \mathrm{cc})\end{array}$ & $\begin{array}{l}\text { Degassed Density above and } \\
\text { below the RGS Sample } \\
\text { (from cores) (g/cc) }\end{array}$ \\
\hline \multirow[t]{5}{*}{$3-2$} & 1.25 & $1.47(\mathrm{a})$ & \multirow{5}{*}{$\begin{array}{r}\text { density above, } 1.56 \mathrm{~g} / \mathrm{cc} \text { bulk } \\
1.45 \mathrm{~g} / \mathrm{cc} \text { liquid }\end{array}$} \\
\hline & 1.00 & 1.43 & \\
\hline & 0.75 & 1.49 & \\
\hline & 0.50 & 1.42 & \\
\hline & 0.25 & 1.36 & \\
\hline \multirow[t]{5}{*}{$3-4$} & 1.25 & $0.68(a)$ & \multirow{5}{*}{$\begin{array}{r}\text { density above, } 1.61 \mathrm{~g} / \mathrm{cc} \text { bulk } \\
1.41 \mathrm{~g} / \mathrm{cc} \text { liquid }\end{array}$} \\
\hline & 1.00 & 1.34 & \\
\hline & 0.75 & 1.34 & \\
\hline & 0.50 & 1.40 & \\
\hline & 0.25 & 1.20 & \\
\hline \multirow[t]{5}{*}{$6-6$} & 1.25 & $1.33(\mathrm{a})$ & \multirow{5}{*}{$\begin{array}{r}\text { density above, } 1.44 \mathrm{~g} / \mathrm{cc} \text { bulk } \\
1.43 \mathrm{~g} / \mathrm{cc} \text { liquid }\end{array}$} \\
\hline & 1.00 & 1.50 & \\
\hline & 0.75 & 1.50 & \\
\hline & 0.50 & 1.51 & \\
\hline & 0.25 & 1.37 & \\
\hline \multirow[t]{5}{*}{$6-6 A^{(b)}$} & 1.25 & $1.72(a)$ & \multirow{5}{*}{$\begin{array}{r}\text { density above, } 1.44 \mathrm{~g} / \mathrm{cc} \text { bulk } \\
1.43 \mathrm{~g} / \mathrm{cc} \text { liquid }\end{array}$} \\
\hline & 1.00 & 1.81 & \\
\hline & 0.75 & 1.85 & \\
\hline & 0.50 & 1.85 & \\
\hline & 0.25 & 1.76 & \\
\hline \multirow[t]{4}{*}{$3-7$} & 1.00 & $1.13(a)$ & \multirow{4}{*}{$\begin{array}{r}\text { density above, } 1.68 \mathrm{~g} / \mathrm{cc} \text { bulk } \\
1.43 \mathrm{~g} / \mathrm{cc} \text { liquid }\end{array}$} \\
\hline & 0.75 & 1.72 & \\
\hline & 0.50 & 1.75 & \\
\hline & 0.25 & 1.68 & \\
\hline \multirow[t]{4}{*}{$6-9$} & 1.02 & $1.65(\mathrm{a})$ & \multirow[t]{4}{*}{ density above, $1.69 \mathrm{~g} / \mathrm{cc}$ bulk } \\
\hline & 0.75 & 1.68 & \\
\hline & 0.50 & 1.72 & \\
\hline & 0.25 & 1.57 & \\
\hline \multirow[t]{4}{*}{$3-10$} & 1.00 & $0.64(a)$ & \multirow[t]{4}{*}{ density above, $1.72 \mathrm{~g} / \mathrm{cc}$ bulk } \\
\hline & 0.75 & 1.35 & \\
\hline & 0.50 & 1.21 & \\
\hline & 0.25 & 1.21 & \\
\hline
\end{tabular}




\subsubsection{Drillstring and Domespace Composition Comparison}

The RGS gas-phase composition data (Table 4.10.6) were used to calculate $\mathrm{H}_{2} / \mathrm{N}_{2} \mathrm{O}$ ratios for comparison with ratios from drillstring and domespace grab sample measurements (Siciliano 1998, Table 8.1; McCain 1999, Table B-22). The results can be seen in Table 4.10.12. The RGS, drillstring, and domespace $\mathrm{H}_{2} / \mathrm{N}_{2} \mathrm{O}$ ratios were all in good agreement. Higher domespace ratios were measured in September and October 1995-all but one were in the range of 4 to 5 (the exception was a single ratio of 13 ).

Table 4.10.12. Comparison with Drillstring and Domespace Data(a)

\begin{tabular}{|l|c|}
\hline \multicolumn{1}{|c|}{ Sample } & $\mathrm{H}_{2} / \mathrm{N}_{2} \mathrm{O}$ \\
\hline \hline $\mathrm{RGS}, 3-2$ & $1.6-7.5$ \\
\hline $\mathrm{RGS}, 3-4$ & $1.2-5.7$ \\
\hline $\mathrm{RGS}, 6-6$ & $2.8-3.1$ \\
\hline $\mathrm{RGS}, 6-6 \mathrm{~A}$ & $2.2-2.9$ \\
\hline $\mathrm{RGS}, 3-7$ & 1.8 \\
\hline $\mathrm{RGS}, 6-9$ & 3.5 \\
\hline $\mathrm{RGS}, 3-10$ & 1.6 \\
\hline Drillstring, riser 6, 10/29/97 & 2.5 \\
\hline Domespace, 8/25/97 & 3.3 \\
\hline Domespace, 11/13/97 & 1.5 \\
\hline Domespace, 2/19/98 & 0.45 \\
\hline $\begin{array}{l}\text { (a) RGS ratios were calculated for both lower- and upper- } \\
\text { bound solubilities. Both ends of the ratio range are given in } \\
\text { the table, lower-bound solubility basis first. }\end{array}$ \\
\hline
\end{tabular}




\subsection{AX-101}

Tank 241-AX-101 (AX-101) was the eleventh tank and the seventh SST sampled with the RGS. Tank AX-101 is on the FGWL and is part of a group of tanks (Cluster 22 in Stewart et al. 1996b) that have fairly high temperatures and high nitrite and TOC concentrations. HTCEs state that the primary waste stored in AX-101 was saltcake resulting from waste from evaporator campaign $A 1$, and that the secondary waste was salt slurry from evaporator campaign A2 (Remund et al. 1995). (This is identical to the HTCE for Tank A-101, which is also in Cluster 22 and is compared with AX-101 later in this report.)

Push-mode sampling was performed in riser 9D of Tank AX-101 in January 1998 and in riser $9 \mathrm{G}$ in February 1998. (a) The approximate locations of various risers are depicted in Figure 4.11.1. These risers are near the wall of the tank and about 90 degrees apart. The elevation of the RGS segment is depicted in Figure 4.11.2, as is the tank content layering.

AX-101 was described by Brevick et al. (1997) as a sound, partially isolated SST, identified on the FGWL. The tank is actively ventilated and has a capacity of about $1,000,000 \mathrm{gal}$. At the time of sampling, AX-101 contained about $2,870,000 \mathrm{~L}$ (757,000 gal) of waste. No supernatant liquid was present, but there was a lower liquid layer containing $1,460,000 \mathrm{~L}$ ( $387,000 \mathrm{gal})$ of liquid (Hodgson et al. 1998).

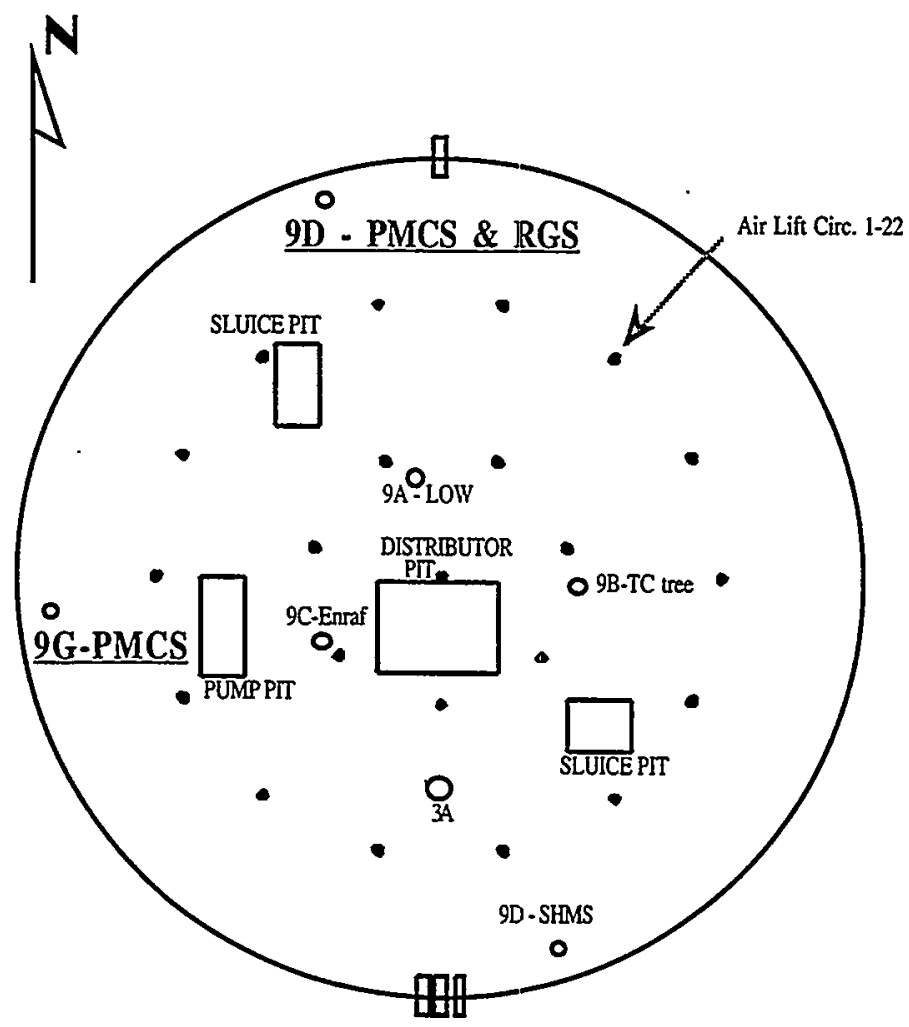

Figure 4.11.1. Schematic Diagram of Riser Locations in Tank AX-101

(a) The sampling scheme may be found in Sampling Plan for Tank 241-AX-101 Retained Gas Sampler Deployment, by JM Bates, November 1997. TWSFG98.05 Rev. 0, Pacific Northwest National Laboratory, Richland, Washington. 


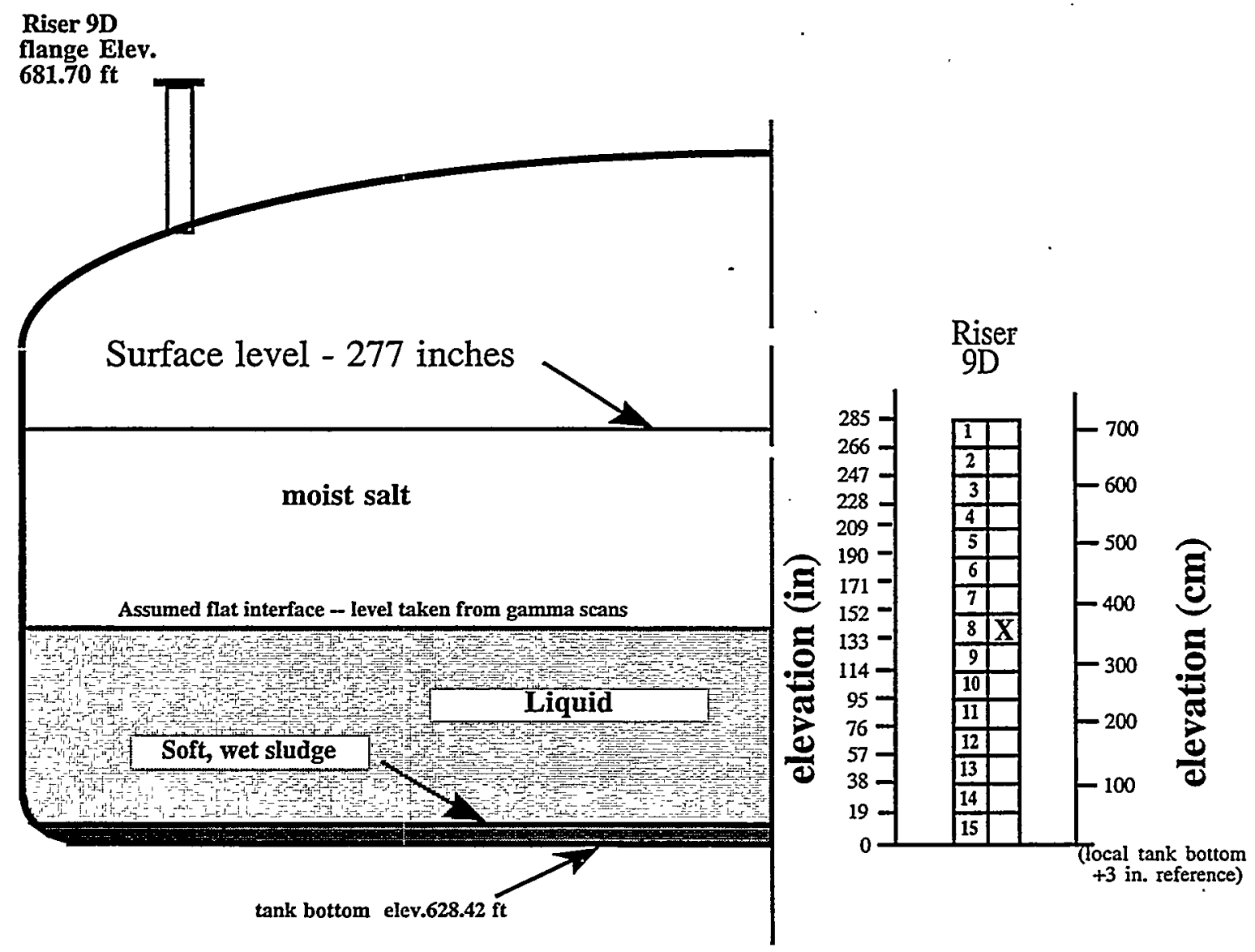

Figure 4.11.2. Diagram of Waste Layering and RGS Sample Elevations for AX-101

The waste levels at risers 9D and 9G were $704 \mathrm{~cm}$ (277 in.) and $708 \mathrm{~cm}$ (278.5 in.), respectively, based on zipcord measurements. Enraf measurements in riser 9C, near the tank center, gave a waste level of $704 \mathrm{~cm}$. Neutron scans in the riser 9A LOW indicated liquid at the surface, but these readings were considered questionable. The location of the interface between the convective and nonconvective layers was $250 \mathrm{~cm}$ (98 in) at riser $9 \mathrm{G}$ (based on core samples), $230 \mathrm{~cm}$ (91 in.) at riser 9D (based on core samples), and $360 \mathrm{~cm}$ (142 in.) at riser 9A (based on the gamma scan) (Hodgson et al. 1998).

The most recent information on AX-101 layering came from the core observations made in 1998 (Esch 1998). All of the material in segments 2 through 10 of riser 9D was gray moist salt, often described as "pitted" in texture (probably indicating numerous bubbles). Segments 11R, 12, 13,14 , and 15 of riser 9D contained primarily yellow liquid, typically combined with 2 to 7 in. of white to dark grey solids with a salt-slurry texture. There appeared to be a small amount of settled solids at the tank bottom. The cores from riser $9 \mathrm{G}$ were nearly identical to the $9 \mathrm{D}$ cores. No sludge was seen in any of the samples, though historical information predicted its presence. The cores and temperature profiles were indicative of an upper, nonconvective layer and a lower, convective layer of uniform temperature.

Although gamma-scan data showed the bottom of the nonconvective layer at $360 \mathrm{~cm}$ (142 in.) elevation, the $\mathrm{X}$-rays of the cores from risers $9 \mathrm{D}$ and $9 \mathrm{G}$ (Section 4.11.4) clearly showed retained gas bubbles as far down as the bottom of segment 10 of riser $9 \mathrm{D}$ at $241 \mathrm{~cm}$ (95 in.) elevation. This observation suggests that the bottom of the nonconvective layer was irregular. 
Densities for the liquid and bulk solids in Tank AX-101 were available from the non-RGS samples taken in 1998 (Esch 1998). Three samples contained drainable liquid. The density of the liquid was $1413 \pm 137 \mathrm{~kg} / \mathrm{m}^{3}$. The degassed bulk density of the nonconvective layer had an average and standard deviation of $1683 \pm 50 \mathrm{~kg} / \mathrm{m}^{3}$ and the lower convective layer had an average and standard deviation of $1653 \pm 100 \mathrm{~kg} / \mathrm{m}^{3}$.

These densities were used for hydrostatic pressure calculations. Layer thicknesses of $343 \mathrm{~cm}$ (135 in.) and $360 \mathrm{~cm}(142 \mathrm{in}$.) were used in calculations for the nonconvective and convective layers. There were no gas measurements in the convective or settled solids layers, so pressure calculations in the settled solids and the thickness of the settled layer were not necessary.

\subsubsection{Sampling and Extraction Information}

The sample that underwent RGS analysis is described in Table 4.11.1. Only one sample was taken; there were no samples from the convective layer or from the settled solids at the bottom of the tank. Field data, including dose rates and downforce limits, are supplied in Appendix A.

Table 4.11.1 also shows the lag time (delay between sample acquisition and processing) for this sample. This information was provided to allow data users to correlate the lag time between sampling and extrusion with the concentrations of the various constituents to test for decomposition or other chemical reactions. The maximum hold time allowed by the sampling plan is 24 days, based on measured sampler leakage rates during acceptance testing. The hold time for sample 9D-8 exceeded this limit, but the sample was accepted based on calculations that indicated the estimated leakage was still negligible compared with the large amount of sample gas.

Table 4.11.1. Lag Times for Processing RGS Sample from Tank AX-101

\begin{tabular}{|c|c|c|c|}
\hline Sample & Acquisition Date & Processing Date & Lag (days) \\
\hline \hline 9D-8 & January 9, 1998 & February 9, 1998 & 31 \\
\hline
\end{tabular}

As was discussed in Section 3.5, the HHF often enters the sampler in place of some of the waste. Table 4.11.2 shows the volume percentage contamination by HHF of RGS sample 9D-8. The MDL was used to calculate the reduced effective sampler volume for the RGS sample. (It is conservative to use the MDL for values below it; the conservatism is probably less than $2 \%$.)

This sampler was helium-backfilled and sealed with vacuum grease before it was deployed. This method virtually eliminated air and argon contamination from gases entrained during sampling. The helium backfill results are discussed in detail by Cannon (1997) and summarized in Section 5 of Mahoney et al. (1997).

After evacuating the whole system and observing the pressure to test for leaks, the operator extruded sample 9D-8 into the extractor vessel, and the sample was briefly stirred (see the RGS system schematic in Figure 3.1). At this point the vapor space in communication with the sample consisted of the volumes of the extractor vessel, its lines,the pump volume, and the collector side; including the collector line volume and an open collection canister. This first canister, containing a sample of uncompressed (unpumped) gas, was closed off. The second set of two collection canisters was opened; several strokes were pumped from the extractor to the collector, and that set of collection canisters was closed off. The collector side and the extractor lines were evacuated. (The second volume was never used during the extraction process for this sample.) 
The vapor profile procedure was then performed. The first pump stroke and every third stroke thereafter were collected in single canisters, with only one stroke per canister. On the intermediate strokes, the gas was pumped to the collector lines with no canister attached. The collector lines were evacuated after every pump stroke, whether or not the pumped gas was collected in a canister. The total gas and vapor pumped from the extractor to the collector during this procedure was calculated by interpolating linearly between collected canisters to find the contents of the pump strokes that were not collected. At the end of the procedure, the first post-isotopic set of three canisters was attached to the collector lines and opened, and both the extractor lines and the collector side were evacuated.

Next, $300 \mathrm{~mL}$ of $0.02 \mathrm{M} 15 \mathrm{NH}_{4} \mathrm{OH}$ solution was added to the sample in the extractor and stirred. After 1.7 hours, several strokes were pumped from the extractor to the collector. The first post-isotopic set of three canisters was closed off, the second set of three was attached and opened, and the collector side was evacuated. More time was allowed for equilibration until 24 hours had passed since the standard was added. Then several strokes were pumped from the extractor to the collector, and the second set of post-isotopic canisters was closed off. Finally, the BSVD procedure was performed: argon was injected into the closed-off second volume and the second volume opened to the extractor and lines. Pressure measurements and known tare system volumes allowed calculation of the volume of the sample solids and liquid.

The collector pressure data (except for the beginning and end pressures) were lost for the post-isotopic canisters of gas extracted from sample 9D-8 at the later equilibration time, but the pressures were approximated and the uncertainty of the results was increased accordingly. The increase in uncertainty was small because the last canister set typically contains little gas.

Procedural details such as the number of strokes per canister and the system volumes are found in the sample-by-sample worksheets in the AX-101 RGS calculation spreadsheet on the CD supplied with this report.

Table 4.11.2. Hydrostatic Head Fluid Contamination in AX-101 RGS Sample

\begin{tabular}{|c|c|}
\hline Sample & $\begin{array}{c}\text { HHF Contamination } \\
\text { (vol\%) }\end{array}$ \\
\hline 9D-8 & $<3.2$ \\
\hline
\end{tabular}

\subsubsection{Retained Gas Composition}

Table 4.11.3 presents the calculated concentrations of the insoluble gases in the AX-101 RGS sample without corrections for air and argon entrainment, helium backfill, air inleakage, or air dissolved in the isotopic standard solution. The method used to make the corrections depended on the gas. All of the oxygen, argon, and helium were subtracted no matter which step had produced them. A number of moles equal to $(3.73) \cdot\left(\mathrm{O}_{2}\right)$ was subtracted from the nitrogen because the oxygen was expected to have come from air entrainment during sampling or inleakage during extraction. Because a substantial amount of dissolved air gases entered the extractor with the $15 \mathrm{NH}_{4} \mathrm{OH}$ solution, all the nitrogen present in the gas collected after the solution addition was subtracted. Thus, it was assumed that more than $95 \%$ of the original retained nitrogen had already been collected, as was the case for hydrogen. The corrected concentrations are in Table 4.11.4. Contamination observations can be found in the "Summary" worksheet of the AX-101 RGS calculations spreadsheet on the $\mathrm{CD}$. 
Table 4.11.3. Concentrations of Insoluble Constituents ( $\mu \mathrm{mol} / \mathrm{L}$ of waste) in Tank AX-101 Without Correction for Gas Contamination

\begin{tabular}{|c|c|c|c|c|c|c|c|c|c|c|c|}
\hline $\begin{array}{c}\text { Seg- } \\
\text { ment }\end{array}$ & $\mathrm{N}_{2}$ & $\mathrm{H}_{2}$ & $\mathrm{~N}_{2} \mathrm{O}$ & $\mathrm{O}_{2}$ & $\mathrm{CH}_{4}$ & $\mathrm{He}$ & $\mathrm{Ar}$ & $\begin{array}{c}\text { Other } \\
\text { NOx }\end{array}$ & $\mathrm{C}_{2} \mathrm{H}_{x}$ & $\mathrm{C}_{3} \mathrm{H}_{x}$ & $\begin{array}{c}\text { Other } \\
\text { Hyd. }\end{array}$ \\
\hline \hline 9D-8 & $5100 \pm 240$ & $5500 \pm 250$ & $1100 \pm 51$ & $330 \pm 85$ & $220 \pm 13$ & $420 \pm 19$ & $26 \pm 2.1$ & (a) & $41 \pm 17$ & (a) & $21 \pm 11$ \\
\hline
\end{tabular}

(a) Below detection limits, which are $0.01 \mathrm{~mol} \%$ or less.

Table 4.11.4. Concentrations of Insoluble Constituents ( $\mu \mathrm{mol} / \mathrm{L}$ of waste) in Tank AX-101 with Correction for Gas Contamination

\begin{tabular}{|c|c|c|c|c|c|c|c|c|c|c|c|}
\hline $\begin{array}{c}\text { Seg- } \\
\text { ment }\end{array}$ & $\mathrm{N}_{2}$ & $\mathrm{H}_{2}$ & $\mathrm{~N}_{2} \mathrm{O}$ & $\mathrm{O}_{2}$ & $\mathrm{CH}_{4}$ & $\mathrm{He}$ & $\mathrm{Ar}$ & $\begin{array}{l}\text { Other } \\
\text { NOx }\end{array}$ & $\mathrm{C}_{2} \mathrm{H}_{\mathrm{x}}$ & $\mathrm{C}_{3} \mathrm{H}_{\mathrm{x}}$ & $\begin{array}{l}\text { Other } \\
\text { Hyd. }\end{array}$ \\
\hline \hline 9D-8 & $1500 \pm 200$ & $5500 \pm 250$ & $1100 \pm 51$ & $0 \pm 85$ & $220 \pm 13$ & $0 \pm 19$ & $0 \pm 2.1$ & (a) & $41 \pm 17$ & (a) & $21 \pm 11$ \\
\hline
\end{tabular}

(a) Below detection limits, which are $0.01 \mathrm{~mol} \%$ or less.

Table 4.11.5 presents ammonia measurements. The residual ammonia was determined isotopically, and the total ammonia (calculated from the isotopic residual) is included in the table. The post-extrusion partial pressure of $\mathrm{NH}_{3}$ over the sample (see Section 3.4.4) was measured at chilled extractor temperature and is included in the table. No other ammonia measurements were made on RGS samples.

Table 4.11.5. Ammonia Data from Tank AX-101 Sample

\begin{tabular}{|c|c|c|c||}
\hline \hline Sample & $\begin{array}{c}\text { Isotopically } \\
\text { Measured } \\
\text { Total } \mathrm{NH}_{3} \\
(\mu \mathrm{mol} / \mathrm{L})\end{array}$ & $\begin{array}{c}\text { Measured } \mathrm{NH}_{3} \\
\text { partial pressure at } \\
\text { about } 12^{\circ} \mathrm{C} \\
\text { (atm) }\end{array}$ & $\begin{array}{c}\text { RGS } \mathrm{NH}_{3} \text { Concentration } \\
\text { in the Liquid }\end{array}$ \\
\hline \hline $9 \mathrm{D}-8$ & $139000 \pm 30000^{(2)}$ & $0.014 \pm 0.007$ & $4100 \mu \mathrm{g} / \mathrm{mL}(0.24 \mathrm{M})$ \\
\hline (a) This datum was used to calculate in situ ammonia vapor pressure. \\
\hline
\end{tabular}

Ammonia was estimated from the RGS measurement of partial pressure and calculated from isotopic results. A comparison with grab sample data taken in 1997 was also made. (For details, see Section C.11 of Appendix C.) The ammonia estimate based on partial pressure comparison (about $80,000 \mu \mathrm{mol} \mathrm{NH} \mathrm{N}_{3} / \mathrm{L}$ waste) is consistent with the ammonia concentrations found in the saltwell grab samples $(40,000$ to $65,000 \mu \mathrm{mol} \mathrm{NH} / \mathrm{L}$ waste) if the latter are considered lower bounds. The estimate is low compared with the ammonia found by isotopic measurement of the RGS sample, but the uncertainties of the estimates and the measurement overlap. It is possible that the RGS-measured ammonia is an upper bound, but the comparison data show that it cannot have overestimated the ammonia concentration by more than a factor of 2 . 
Table 4.11.6 contains the composition of the gas/vapor phase in the sample. The water vapor is not included in this composition. The ammonia fraction is derived from the datum indicated in Table 4.11.5, which was used to calculate vapor pressures at in situ conditions. The sample compositions in the table have been calculated using the in situ solubility method described in Section 3.6.2. As discussed in Section 3.6.1, compositions were calculated for both the lowerbound and upper-bound gas solubilities. Both ends of the composition range are given in the table with the measurement uncertainty on each. No RGS samples were taken from the convective layer or settled solids. The integration method is described in Section 3.7.1.

Table 4.11.6. Sample Composition of Retained Gas in Tank AX-101 with Correction for Gas Contamination(a)

\begin{tabular}{|c|c|c|c|c|c|c|}
\hline Sample & $\mathrm{N}_{2}(\mathrm{~mol} \%)$ & $\mathrm{H}_{2}(\mathrm{~mol} \%)$ & $\mathrm{N}_{2} \mathrm{O}(\mathrm{mol} \%)$ & $\mathrm{NH}_{3}(\mathrm{~mol} \%)(\mathrm{b})$ & $\mathrm{CH}_{4}(\mathrm{~mol} \%)$ & $\begin{array}{l}\text { Other } \\
\text { (mol\%) }\end{array}$ \\
\hline $9 D-8$ & $\begin{array}{c}16 \pm 2.6 \\
\text { to } 18 \pm 2.7\end{array}$ & $\begin{array}{l}60 \pm 5.5 \\
\text { to } 64 \pm 5.4\end{array}$ & $11 \pm 1.0$ & $\begin{array}{c}9.1 \pm 2.1 \\
\text { to } 3.5 \pm 0.8\end{array}$ & $\begin{array}{c}2.4 \pm 0.2 \\
\text { to } 2.5 \pm 0.2\end{array}$ & $0.7 \pm 0.3$ \\
\hline \multicolumn{7}{|c|}{$\begin{array}{l}\text { (a) Uncertainties on composition represent instrument uncertainty. Composition calculated for lower- and upper- } \\
\text { bound solubilities. Both ends of range given, lower-bound solubility basis first with instrument uncertainty on } \\
\text { each. Composition may not sum to } 100 \mathrm{~mol} \% \text { because of roundoff error. Mole fractions on a dry basis, do not } \\
\text { account for water vapor. } \\
\text { (b) Ammonia mole fraction derived from data in Table } 4.11 .5 \text { used to calculate vapor pressure at in situ conditions. }\end{array}$} \\
\hline
\end{tabular}

The concentrations and pressures in Tables 4.11.3 and 4.11.4 can also be found in the sample-by-sample worksheets in the AX-101 RGS calculation spreadsheet on the CD. The compositions in Table 4.11.6 are in the "Inventory" worksheet.

\subsubsection{Gas Inventory}

The method by which the in situ gas volume fraction (wet basis) was calculated is given in Section 3.6.2; as for the gas composition, the volume fraction is given as a range from the lowgas-solubility value to the high-gas-solubility value. The results are presented in Table 4.11.7. The corrected gas volume fractions in Table 4.11.7 are consistent with the corrected gas concentrations and compositions in Tables 4.11.4 through 4.11.6. The information in Table 4.11.7 is from the "Summary" and "In situ" worksheets of the AX-101 RGS calculation spreadsheet on the CD.

The "sampler gas volume fraction" is corrected only for inleakage during the extraction process and air gases added in the isotopic solution. It is an attempt to reconstruct the total amount of gas (sample and entrainment) present in the sampler during $\mathrm{x}$-ray. It is used only for comparison with the x-ray observations of "visible gas fraction" that are discussed in Section 4.11.4.

Table 4.11.7 also contains the water vapor pressure that was used for in situ calculations. The water vapor pressure was found by using the salt concentration from Esch (1998) and the temperature in the table as inputs to Equation 6.2 of Mahoney and Trent (1995), a correlation for water vapor pressure over concentrated homogeneous and non-homogeneous waste simulants. The gas solubilities used the same parameters as inputs to the Schumpe solubility model (Section 3.6.1). The water vapor pressure and gas solubilities used in calculations can be found in the "In situ" worksheet of the AX-101 RGS calculation spreadsheet on the CD.

The BPE method could not be used for Tank AX-101. According to the Enraf instrument in riser $9 \mathrm{C}$, there was no correlation between level fluctuations and barometric pressure (Hodgson 
et al. 1998). It has been suggested that the Enraf data do not represent the tank level behavior well because riser $9 \mathrm{C}$ is only one or two feet from the dry well and the air lift circulator so that the waste under 9C is effectively pinned in place. However, the long-term surface level rise (January 1981 to January 1995) was also nearly zero, though the FIC instrument that measured the level was probably not in the same riser as the Enraf (Hodgson et al. 1998). Whatever the reason, the single RGS measurement and the x-ray information (discussed in Section 4.11.4) are the only available data that demonstrate high gas content in AX-101.

An examination of the X-ray and extraction data for all the RGS-sampled tanks shows that the gas gap visible in $\mathrm{X}$-rays almost always underestimates the amount of gas found by RGS extraction. Based on all the samples in which the waste is visibly bubbly, the extracted gas volume fraction is $0.07 \pm 0.07$ volume fraction greater than the gas visible on the $x$-ray. Judging by the 0.17 volume fraction difference between the x-ray and extracted gas in sample 9D-8 (Table 4.11.8), the all-tanks average difference of 0.07 underestimated the difference for $\mathrm{AX}-101$. (Much the same could be said for tank A-101, as shown in Table 4.3.10; three of the four samples from its nonconvective layer showed extracted gas volume fractions that were $0.15,0.16$, and 0.2 volume fraction greater than the $\mathrm{X}$-ray estimates. However, in the fourth case, the extracted fraction was 0.09 volume fraction less. Tank A-101 is relevant here because it is in the same HTCE cluster as AX-101 and has the same unusual upper nonconvective layer.)

A gas inventory in the nonconvective layer was calculated by integrating the $\mathrm{x}$-ray gas volume fractions (and the single contamination-corrected RGS datum) over the layer (eight data points) and multiplying the average by the volume of the layer. The integration method is described in Section 3.7.1. This inventory, which must be regarded as a minimum inventory, as presented in Table 4.11.8. The information in Table 4.11.8 is taken from the "Inventory" worksheet of the A-101 RGS calculation spreadsheet on the CD.

For comparison, the gas inventory in the nonconvective layer of Tank A-101 was (per Table 4.3.8) $318 \mathrm{~m}^{3}$ in situ ( $344 \mathrm{~m}^{3}$ at STP). The maximum measured gas volume fraction in A-101 was 0.22 , in the bottommost sample of the nonconvective layer, and the nonconvective layer thickness in A-101 was $412 \mathrm{~cm}$ (162 in.). Scale the A-101 nonconvective layer inventory by the ratio of the $A X-101$ and $A-101$ gas volume fractions in the bottom segment $(0.18 / 0.22)$ and by the ratio of the layer thickness $(343 \mathrm{~cm} / 412 \mathrm{~cm})$; the result is $217 \mathrm{~m}^{3}$ in situ $\left(234 \mathrm{~m}^{3}\right.$ at STP). The comparability of the scaled A-101 inventory and the AX-101 minimum inventory does not provide a quantitative idea of the uncertainty or the true inventory but gives some reassurance that the minimum inventory is not wildly out of line. Note that the scaled AX-101 inventory is to some extent minimized because it uses the lower value of layer thickness based on gamma scans, which is only two-thirds of the higher value consistent with core extrusion and $\mathrm{x}$-ray observations.

Table 4.11.9 contains the minimum gas-phase inventory of each major gas retained in the nonconvective layer of AX-101. This inventory is based on the single measured composition from

Table 4.11.7. In Situ Gas Volume Fraction and Conditions in Tank AX-101

\begin{tabular}{||c|c|c|c|c|c|c||}
\hline \hline Sample & $\begin{array}{c}\text { Sample } \\
\text { Central } \\
\text { Height }\end{array}$ & $\begin{array}{c}\text { Hydro- } \\
\text { static } \\
\text { Pressure } \\
\text { (atm) }\end{array}$ & $\begin{array}{c}\text { Calculated } \\
\text { Water Vapor } \\
\text { Pressure } \\
(\mathrm{atm})\end{array}$ & $\begin{array}{c}\text { Temp } \\
\left({ }^{\circ} \mathrm{C}\right)\end{array}$ & $\begin{array}{c}\text { Corrected Gas Volume } \\
\text { Fraction(a) } \\
\text { (in-tank conditions) }\end{array}$ & $\begin{array}{c}\text { Sampler Gas } \\
\text { Volume Fraction } \\
\text { (in situ conditions, } \\
\text { low solubility) }\end{array}$ \\
\hline \hline $9 \mathrm{D}-8$ & 362 & 1.46 & 0.043 & 53.1 & $\begin{array}{c}0.17 \pm 0.013 \text { to } \\
0.16 \pm 0.013\end{array}$ & 0.18 \\
\hline
\end{tabular}


Table 4.11.8. AX-101 Minimum Gas Inventory Estimates(a)

\begin{tabular}{||l|c||}
\hline \multicolumn{1}{|c|}{ Quantity } & RGS Method \\
\cline { 2 - 2 } & Nonconvective Layer \\
\hline \hline MINIMUM \\
Avg gas fraction (low gas solubility) & 0.10 \\
\hline $\begin{array}{l}\text { MINIMUM } \\
\text { Gas volume (m3) in situ (wet) }\end{array}$ & 186 \\
STP (wet) & 195 \\
\hline $\begin{array}{l}\text { (a) Uncertainty in the inventory is unknown but (following the same } \\
\text { logic as for other SSTs, given in Section 3.7.2) is at least 50\%. }\end{array}$ \\
\hline
\end{tabular}

RGS data (Table 4.11.6) and the minimum gas inventory (Table 4.11.8). There were not enough data to justify calculating the dissolved gas inventories and the variation of inventories because of the uncertainty in the gas solubilities.

Figure 4.11.3 shows the temperature profile, corrected gas volume fraction (Table 4.11.7), and corrected composition of the low-solubility constituents in the sample from Tank AX-101. The temperatures were measured by the TC tree in riser 9B. The composition represents the mole fraction of the species in the insoluble gas; water and ammonia are not included, so the mole fractions are not the same as those in Table 4.11.6. The gas volume fraction and mole fractions in the figure are the values for lower-bound gas solubilities. Figure 4.11.3 also lists the observations from core extrusions of non-RGS samples and $x$-rays of RGS samples as a way of tying those observations together with RGS data. X-ray data are described in more detail in Section 4.11.4.

Table 4.11.9. Speciated Minimum AX-101 Gas Inventory(a)

\begin{tabular}{||l|c|}
\hline \multirow{2}{*}{ Species } & \begin{tabular}{c} 
Nonconvective Layer \\
\cline { 2 - 3 }
\end{tabular} \\
\hline $\mathrm{N}_{2}$ & $\begin{array}{c}\text { Minimum Gas-Phase Inventory } \\
\text { (m³ at STP) }\end{array}$ \\
\hline $\mathrm{H}_{2}$ & 31 \\
\hline $\mathrm{N}_{2} \mathrm{O}$ & 110 \\
\hline $\mathrm{NH}_{3}$ & 21 \\
\hline $\mathrm{CH}_{4}$ & 17 \\
\hline Other & 4.4 \\
\hline $\begin{array}{l}\text { (a) Inventories based on single measured composition from RGS data } \\
\text { (Table 4.11.6) and minimum free gas inventory (Table 4.11.8). } \\
\text { Uncertainty in each inventory is unknown but (following the same } \\
\text { logic as for other SSTs (Section 3.7.2) is at least 50\%. The } \\
\text { inventories are given only for the lower-bound gas solubilities. }\end{array}$ \\
\hline
\end{tabular}




\subsubsection{X-Ray Results}

Table 4.11.10 summarizes all the available radiography observations from Tank AX-101. The $\mathrm{x}$-ray images themselves can be found on the $\mathrm{CD}$ that accompanies this report. The uncertainties of the visible gas volume fractions given in Table 4.11.10 are unknown but (based on the uncertainty in measuring the gas gap from $\mathrm{x}$-ray images) are expected to be larger than the uncertainties in the gas volume fractions calculated from gas extraction. (The "visible" gas volume fraction refers to the gas that has separated from the solids and liquid such that its volume can be calculated based on the image. The uncorrected gas fractions from extraction are used for comparison to reflect the presence of entrained air in the sampler.)

Table 4.11.10 compares the $\mathrm{x}$-ray-derived and extraction-derived gas volume fractions. The comparison shows that sample recovery was close to $100 \%$. This conclusion is based on the fact that the uncorrected extracted gas was greater than the visible gas.

$\mathrm{X}$-rays of the riser 9D samples show that the top three-fourths of the tank, segments 1 through 10, held enough solids to contain a substantial amount of bubble structure. Segments 11 through 14 of riser 9D were primarily liquid, although some bubble structure appeared in the bottom $8 \mathrm{in}$. of segment 14 (whose bottom was $22 \mathrm{in.} \mathrm{above} \mathrm{the} \mathrm{bottom} \mathrm{of} \mathrm{the} \mathrm{tank).} \mathrm{A} \mathrm{similar}$ trend was seen in riser 9G, with bubble structure in segments 2 and 9 ( 3 through 8 were not $\mathrm{x}$-rayed) and no visible features in segments 10 through 15 . It therefore appears that an upper gasretaining layer extended from about 269 to $318 \mathrm{~cm}$ (106 to 125 in.) elevation to the top of the waste. Beneath this was a layer that retained little gas, whose top was between 269 and $318 \mathrm{~cm}$ (106 and 125 in.) and whose bottom was about $76 \mathrm{~cm}$ (30 in.) above the center of the tank bottom. Beneath that layer, there may have been another gas-retaining layer of irregular thickness, as suggested by bubble structure seen at the bottom of segment 14 of riser 9D but not in samples $9 \mathrm{G}-14$ and $9 \mathrm{G}-15$.

The gas in the AX-101 RGS sample was found (by extraction) to occupy $17 \pm 1.3$ vol\% of the waste under in situ conditions ( $1.46 \mathrm{~atm}$ pressure and $53^{\circ} \mathrm{C}$ ). X-rays of this sample showed bubble structure throughout the depth of the waste, with bubbles of $2 \mathrm{~mm}$ and less in size, some joined or distorted bubble shapes, and a lumpy waste surface. The gas gap at the top of the waste was about $0.2 \mathrm{in}$. and was partly bridged by waste; the gas gap volume fraction was about $1 \mathrm{vol} \%$. This gas fraction was much less than the gas volume found by extraction, indicating that nearly all of the gas (about $16 \mathrm{vol} \%$ ) was in the bubble structure. It is unusual for so much gas to be in the bubble structure rather than the gas gap.

The amount of gas in the RGS sample appeared typical of waste in the nonconvective layer of Tank AX-101, as can be seen from the sample $\mathrm{X}$-ray descriptions in Table 4.11.10. Consider segments 2 and 10 of riser 9D as two non-RGS examples of the waste in the nonconvective layer and sample 9D-14 (one segment up from the bottom) as representative of the lower convective layer. Both samples 9D-2 and 9D-10 contained more bubble structure than was in the RGS sample, including bubbles of about 8-mm diameter (9D-10) and a sampler-spanning bubble (9D-2). By contrast, sample 9D-14 contained some bubble structure (2-mm diameter or less) in the bottom third of the sampler but nothing discernible above that point. 

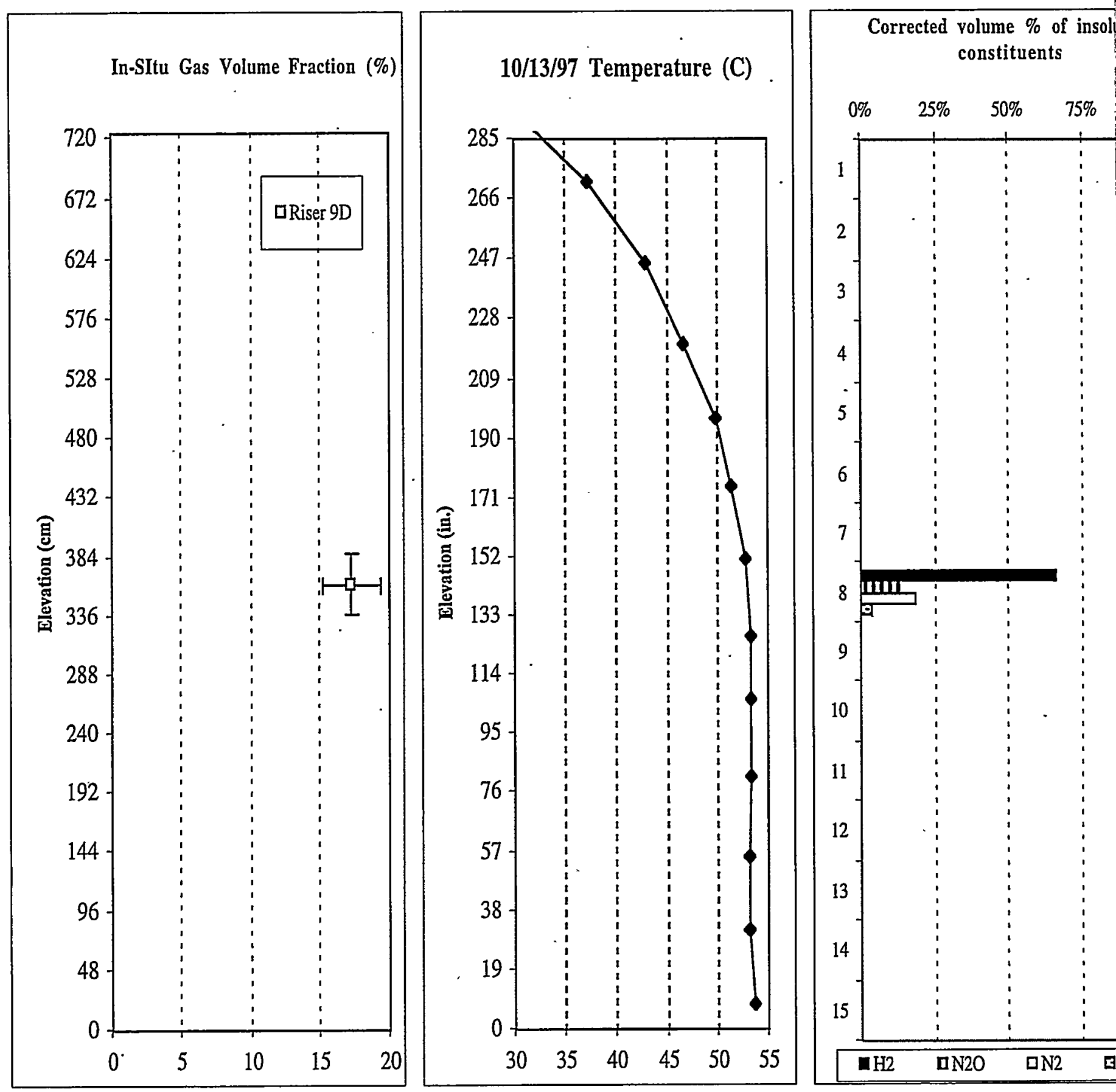

Figure 4.11.3. Sample Temperatures, Corrected Gas Volumes, and 
Waste level is $704 \mathrm{~cm}$ (277 inches).

1 Riser 9D: 3 in. (65 g) white/gray dry salt, $25 \mathrm{~g}$ yellow-brown liquid. X-RAY: 3 in. waste, many dense features.

1

2 Riser 9D: 18 in. (392 g) gray moist salt. X-RAY: large voids, one fracture, 1.3 in gas gap.

2 Riser 9G: 16 in. $(360 \mathrm{~g}$ ) pitted gray moist salt. X-RAY: $3-6 \mathrm{~mm}$ voids, 3 in. gas gap.

3 Riser 9D: 18 in. (419 g) pitted gray moist salt. X-RAY: Many large bubbles. 2 in. gas gap.

3 Riser 9G: 19 in. (417 g) pitted gray moist salt.

4 Riser 9D: 18 in. (426 g) pitted gray moist salt. X-RAY: Many small/medium bubbles.

4 Riser 9G: 19 in. (437 g) pitted gray moist salt.

5 Riser 9D: 18 in. ( $430 \mathrm{~g}$ ) pitted gray moist salt. X-RAY: Some small/medium bubbles, fewer near top.

5 Riser 9G: 19 in. (414 g) pitted gray moist salt.

6 Riser 9D: 19 in. (454 g) pitted gray moist salt. X-RAY: Large to medium bubbles, 1 in. gas gap.

6 Riser 9G: 18 in. (423 g) pitted gray moist salt, few white flakes.

7 Riser 9D: 19 in. (427 g) pitted gray moist salt, crumbly. X-RAY: Medium bubbles, one fracture, 1 in. gas gap.

7 Riser 9G: 5 in. $(260 \mathrm{~g})$ pitted white/gray wet salt, $123 \mathrm{~g}$ gray opaque liquid.

8 Riser 9D X-RAY: Many bubbles, bridged 0.2-in. gas gap.

8 Riser 9G: 19 in. (438 g) pitted gray moist salt, white crystals intermixed.

9 Riser 9D: 18 in. $(445 \mathrm{~g})$ pitted gray moist salt.

9 Riser 9G: 19 in. (449 g) pitted gray moist salt. X-RAY: Up to 1-cm voids, one fracture, bridged 0.3-in gas gap.

10 Riser 9D: 18 in. (438 g) pitted gray moist salt, moister than above. X-RAY: Many large bubbles, 1 in. gas gap.

10 Riser 9G: 4 in. (144 g) bluegray salt slurry, $252 \mathrm{~g}$ bluegray liquid. X-RAY: Featureless, bridged 0.9-in gas gap.

11R Riser 9D: 6 in. (146 g) white/gray salt slurry, $191 \mathrm{~g}$ light-yellow liquid. X-RAY: Featureless, 1 in. gas gap.

11 Riser 9G: 4 in. (144 g) white salt slurry, $237 \mathrm{~g}$ clear yellow liquid. X-RAY: Featureless, 0.1-in. gas gap.

12 Riser 9D: 7 in. (167 g) white salt slurry, $233 \mathrm{~g}$ opaque light-yellow liquid. X-RAY: Featureless, 0.4-in. gas gap.

12 Riser 9G: 5 in. (155 g) white salt slurry, $272 \mathrm{~g}$ clear yellow liquid. X-RAY: Featureless, 0.2-in. gas gap.

13 Riser 9D: 5 in. (118 g) white salt slurry, $295 \mathrm{~g}$ light-yellow liquid. X-RAY: Featureless, bridged 0.2-in. gas gap.

13 Riser 9G: 5 in. (159 g) white salt slurry, $266 \mathrm{~g}$ clear yellow liquid. X-RAY: Featureless, 0.2-in. gas gap.

14 Riser 9D: 7 in. (154 g) white/gray salt slurry, $174 \mathrm{~g}$ opaque light-yellow liquid. X-RAY: Featureless, 0.4-in. gap.

14 Riser 9G: 3 in. (113 g) white/gray salt slurry, $230 \mathrm{~g}$ clear yellow liquid. X-RAY: Featureless, 0.9-in. gas gap.

15 Riser 9D: 2 in. (29 g) white/black salt slurry, $38 \mathrm{~g}$ opaque light-yellow liquid.

15 Riser 9G: 5 in. (138 g) pale yellow salt slurry, $234 \mathrm{~g}$ clear yellow liquid. X-RAY: Featureless, 0.25-in. gas gap. 
Table 4.11.10. Summary of Observations from X-Ray Images of Tank AX-101

\begin{tabular}{|c|c|c|c|}
\hline \begin{tabular}{|c|} 
Riser- \\
Segment \\
\end{tabular} & Observations & \begin{tabular}{|c|} 
Riser- \\
Segment \\
\end{tabular} & Observations \\
\hline 9D-1 & $\begin{array}{l}\text { Only } 3 \text { in. of sample recovered. Many darker (denser) } \\
\text { features visible, flat top surface. }\end{array}$ & & \\
\hline $9 \mathrm{D}-2$ & $\begin{array}{l}\text { Many large voids, including one that spans the } \\
\text { sampler diameter. The gas gap at top is about } 1.3 \\
\text { in., with some lumpiness of the top waste surface. } \\
\text { No waste on piston. }\end{array}$ & $9 \mathrm{G}-2$ & $\begin{array}{l}\text { Several large voids ( }>6 \mathrm{~mm} \text { dia) halfway up sample; } \\
\text { many small features }(3 \mathrm{~mm} \text { and less) above and below. } \\
\text { Jagged surface with low-density feature that resembles } \\
\text { flat crystal. Gas gap }-3 \text { in. below clean piston. }\end{array}$ \\
\hline $9 D-3$ & $\begin{array}{l}\text { Many large bubbles evenly distributed in sample; } 2 \\
\text { in. gas gap below a clean piston. }\end{array}$ & & (x-ray not available) \\
\hline $9 \mathrm{D}-4$ & $\begin{array}{l}\text { Many small/medium sized bubbles; otherwise like } \\
\text { segment } 3 .\end{array}$ & & (x-ray not available) \\
\hline $9 D-5$ & $\begin{array}{l}\text { Like segment } 4 \text {, but fewer bubbles; they are } \\
\text { especially few at the top of the sample. }\end{array}$ & & (x-ray not available) \\
\hline 9D-6 & $\begin{array}{l}\text { Well-distributed bubbles, some large. A solids } \\
\text { "molehill" at the top waste surface, with a 1-in. gas } \\
\text { gap below the piston. }\end{array}$ & & (x-ray not available) \\
\hline $9 D-7$ & $\begin{array}{l}\text { Mostly slightly smaller bubbles than in segment } 6 \\
\text { but contains a sampler-spanning bubble. Solids } \\
\text { "piling" (lumpiness) evident at top and a 1-in. gas } \\
\text { gap below the piston. }\end{array}$ & & ( $x$-ray not available) \\
\hline \multirow[t]{2}{*}{$\begin{array}{l}\text { 9D-8 } \\
\text { (RGS, } \\
1 / 9 / 98)\end{array}$} & $\begin{array}{l}\text { Many well-distributed bubbles. Small waste "bridge" } \\
\text { crosses } 0.2 \text {-in. gas gap above lumpy top surface of } \\
\text { waste. The piston was fully retracted. Visible gas } \\
\text { volume fraction was } 0.01 \text {; compare to the uncorrected } \\
\text { gas volume fraction of } 0.18 \text { found by extraction. }\end{array}$ & & (x-ray not available) \\
\hline & (x-ray not available) & $9 \mathrm{G}-9$ & $\begin{array}{l}\text { Many large voids (up to } 6 \mathrm{~mm} \text { ), with a few larger ones } \\
\text { (up to } 1 \mathrm{~cm} \text { ), one of angular shape crossing most of } \\
\text { sampler. 'Gas gap about } 0.3 \text { in. high and } 0.5 \text { in. wide; } \\
\text { rest is waste bridge between piston and waste surface. }\end{array}$ \\
\hline $9 \mathrm{D}-10$ & $\begin{array}{l}\text { Numerous large bubbles near sampler bottom, } \\
\text { becoming fewer in the middle, then increasing toward } \\
\text { the top. Gas gap is about } 1 \text { in., the top waste surface } \\
\text { flat but uneven. }\end{array}$ & $9 \mathrm{G}-10$ & \begin{tabular}{|l|} 
No structure or bubbles discernible. Gas gap about \\
$0.9 \mathrm{in}$. high and $0.56 \mathrm{in}$. wide; rest is waste bridge \\
between piston and waste surface. Top 0.4 in. of \\
waste either lumpy, lower density, or coating on wall.
\end{tabular} \\
\hline $9 \mathrm{D}-11$ & $\begin{array}{l}\begin{array}{l}\text { No features discernible. It appears to be all liquid, } \\
\text { with a gas gap of about } 1 \text { in. }\end{array} \\
\end{array}$ & $9 \mathrm{G}-11$ & \begin{tabular}{|l|} 
No features discernible. Top surface is flat with liquid \\
meniscus. Gas gap 0.12 in., under clean piston. \\
\end{tabular} \\
\hline $9 \mathrm{D}-12$ & $\begin{array}{l}\text { Like segment } 11 \text {. Ripples were observed at the waste } \\
\text { top. The gas gap was about } 0.4 \text { in. }\end{array}$ & $9 \mathrm{G}-12$ & $\begin{array}{l}\text { No structure or bubbles discemible; top surface flat } \\
\text { with liquid meniscus; gas gap } \sim 0.2 \text { in.,under clean } \\
\text { piston. }\end{array}$ \\
\hline $9 \mathrm{D}-13$ & $\begin{array}{l}\text { Like segment } 11 \text {, but smaller gas gap and some waste } \\
\text { bridging between the piston and the waste surface. }\end{array}$ & $9 \mathrm{G}-13$ & $\begin{array}{l}\text { No structure or bubbles discernible. Top surface is flat } \\
\text { with liquid meniscus. Gas gap is about } 0.2 \text { in., under } \\
\text { clean piston. }\end{array}$ \\
\hline \multirow[t]{2}{*}{$9 D-14$} & $\begin{array}{l}\text { Bubble structure visible in the bottom } 8 \text { in. of this } \\
\text { sample, with homogeneous (possibly liquid) waste } \\
\text { above. Gas gap is about } 0.5 \text { in. }\end{array}$ & $9 \mathrm{G}-14$ & $\begin{array}{l}\text { No structure or bubbles discemible. Top surface is flat } \\
\text { with liquid meniscus. Gas gap is about } 0.88 \text { in. under } \\
\text { piston with a } 0.12 \text {-in. wide piece of waste on it. }\end{array}$ \\
\hline & & 9G-15 & $\begin{array}{l}\text { No structure or bubbles discernible. Top surface is flat } \\
\text { with liquid meniscus. Gas gap is about } 0.25 \text { in., under } \\
\text { piston with a } 0.25 \text { in. wide piece of waste on it. }\end{array}$ \\
\hline
\end{tabular}


The gas gap volume fractions from these $x$-rayed non-RGS samples were 0.07 (9D-2), 0.05 (9D-10), and 0.02 (9D-14). The gap in sample 9D-14 amounted to about $5 \mathrm{cc}$ of gas, an amount that could have come from air/argon entrainment during sampling but (considering the structure in the bottom of the sample) could also have come from the waste itself. There was more gas present in the gas gaps of these three $\mathrm{x}$-rayed samples than was consistent with entrainment. But some of the liquid samples ( $9 \mathrm{G}-11,-12$ and -13 ) may have contained only entrained gas.

There is no reason to believe that any of the x-rayed samples from the nonconvective layer contained much less gas than the RGS sample, 9D-8. Table 4.11.10 gives descriptions of all of the X-rayed samples from both risers of Tank AX-101. Based on these X-rays-which included at least as much bubble structure as the RGS sample and often larger gas gaps-and the large amount of gas in the bubble structure of the RGS sample, we think it is possible that much of the nonconvective layer in Tank AX-101 contained gas fractions of 10 to $20 \mathrm{vol} \%$ (at in situ conditions).

Table 4.11.11 provides a summary of the waste densities that have been calculated from radiographic data for RGS sample 9D-8. The radiographic densities 1) include the gas in the waste, 2) were calculated by the current $x$-ray method, and 3) are given for several locations within each sample to show density trends. Table 4.11.11 also includes density measurements made on extruded non-RGS samples (Esch 1998). The radiographic densities in the sample equaled or exceeded the densities found for the adjacent extruded samples, which is difficult to explain because the radiographic densities included gas and the extruded densities did not. Note that the $\mathrm{X}$-ray analysis depended on standard air/water density profiles that were taken four days after sample 9D-8 was x-rayed; some instrument drift could have occurred in the interim.

\subsubsection{Domespace Composition Comparison}

The RGS gas-phase composition data (Table 4.11.6) were used to calculate an $\mathrm{H}_{2} / \mathrm{N}_{2} \mathrm{O}$ ratio for comparison with ratios from domespace grab sample measurements (McCain 1999, Table B-3). The results can be seen in Table 4.11.12. With one exception, the domespace $\mathrm{H}_{2} / \mathrm{N}_{2} \mathrm{O}$ ratios were all higher than the RGS ratio. The opposite was true for Tank A-101, despite the other similarities of A-101 and AX-101.

Table 4.11.11. Density of AX-101 Sample from Radiography and Core Samples

\begin{tabular}{|c|c|c|c|}
\hline $\begin{array}{l}\text { Riser- } \\
\text { Segment } \\
\text { Number }\end{array}$ & $\begin{array}{c}\begin{array}{c}\text { Distance From } \\
\text { bottom of sampler } \\
\text { (ft) }\end{array} \\
\end{array}$ & $\begin{array}{c}\text { Calculated Mean Density } \\
\text { by Current Method } \\
(\mathrm{g} / \mathrm{cc})\end{array}$ & $\begin{array}{c}\text { Degassed Density above and below } \\
\text { RGS Sample (from cores) } \\
\text { (g/cc) }\end{array}$ \\
\hline \multirow[t]{6}{*}{ 9D-8(a) } & 1.50 & 1.83 & \multirow[t]{6}{*}{ density above, $1.62 \mathrm{~g} / \mathrm{cc}$ (bulk) } \\
\hline & 1.25 & 1.85 & \\
\hline & 1.00 & 1.80 & \\
\hline & 0.75 & 1.81 & \\
\hline & 0.50 & 1.77 & \\
\hline & 0.25 & 1.66 & \\
\hline
\end{tabular}


Table 4.11.12 Comparison with Domespace Data(a)

\begin{tabular}{|c|c|}
\hline Sample & $\mathrm{H}_{2} / \mathrm{N}_{2} \mathrm{O}$ \\
\hline RGS, 9D-8 & $5.3--5.7$ \\
\hline Domespace, $8 / 3-8 / 15 / 95$ & $\begin{array}{c}9.5,1.3,11,8.8, \\
11,7.8,11\end{array}$ \\
\hline \multicolumn{2}{|c|}{$\begin{array}{l}\text { (a) RGS ratio was calculated for both lower- and upper-bound } \\
\text { solubilities. Both ends of the ratio range are given in the } \\
\text { table, lower-bound solubility basis first. }\end{array}$} \\
\hline
\end{tabular}

\subsection{S-102}

Tank 241-S-102 (S-102) was the twelfth tank and the eighth SST sampled with the RGS. This tank was selected as representing the highest-priority group of SSTs that show evidence of significant gas retention (Stewart et al. 1996b). Tank S-102 is on the FGWL, exhibiting high level rise and response to barometric pressure changes (see Section 4.12.3) and high concentrations of $\mathrm{H}_{2}$ and $\mathrm{NH}_{3}$ in the headspace. Tank S-102 is part of a group of tanks (Cluster 13 in Stewart et al. 1996b) that have fairly high radioactivity, fairly low temperatures, high nitrite concentrations, and about $0.1 \%$ TOC. Historical tank content estimates indicate that the primary waste stored in S-102 was salt slurry from evaporator campaign S2 and that the secondary waste was saltcake from evaporator campaign S1 (Remund et al. 1995). (This is identical to the HTCE estimate for Tank SX-106, which is also in Cluster 13.) Of the RGS-sampled tanks, U-103, SX-106, S-102, and U-109 are all in Cluster 13.

Push-mode sampling was done in riser 16 in March and April 1998.(a) The approximate locations of various risers are depicted in Figure 4.12.1. Riser 16 is about three-fourths of the tank radius from the center of the tank. The elevations of the RGS segments are depicted in Figure 4.12.2. The figure also shows the tank content layering.

Brevick et al. (1994) describe Tank S-102 as a partially isolated, sound SST. At the time of sampling S-102 contained about 2,080,000 L (549,000 gal) of waste, including 742,000 L $(196,000$ gal) of pumpable interstitial liquid and no supernatant (Hodgson et al. 1998). The total depth of waste in Tank S-102 was 510 to $528 \mathrm{~cm}$ (201 to $208 \mathrm{in}$.) at risers 11 and 14, where core samples were taken between January and March 1996 (Fritts 1998). Neutron scans in the riser 14 LOW indicated liquid near the waste surface. Risers 11 and 14 are near the tank periphery, as is riser 16.

The most recent past information on S-102 layering comes from the core observations made in 1996 (Fritts 1998). Despite the documented absence of supernatant, liquid was found in segment 1 at riser 16; its bromide concentration was low, indicating that the liquid was not the product of contamination by HHF. The top half or more of the waste depth was primarily saltcake, but sludge or sludge/salt slurry was present in the bottom three segments of both risers.

Densities for the liquid and bulk solids in S-102 were available from cores taken in 1996 (Fritts 1998). Three samples containing drainable liquid were taken. The density of drainable

(a) The sampling scheme may be found in Sampling Plan for Tank 241-S-102 Retained Gas Sampler Deployment, by JM Bates, December 1997. TWSFG98.20 Rev. 0, Pacific Northwest National Laboratory, Richland, Washington. 


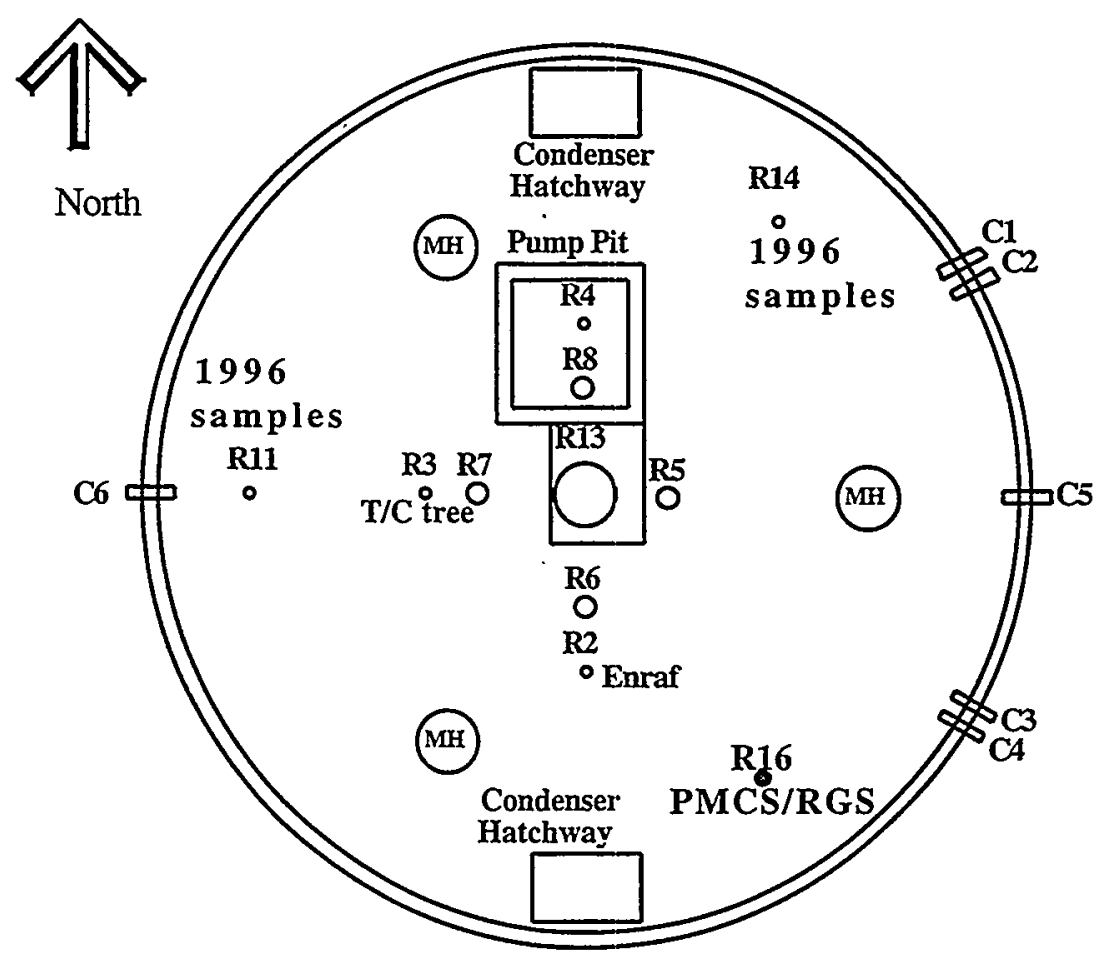

Figure 4.12.1. Schematic Diagram of Riser Locations in Tank S-102

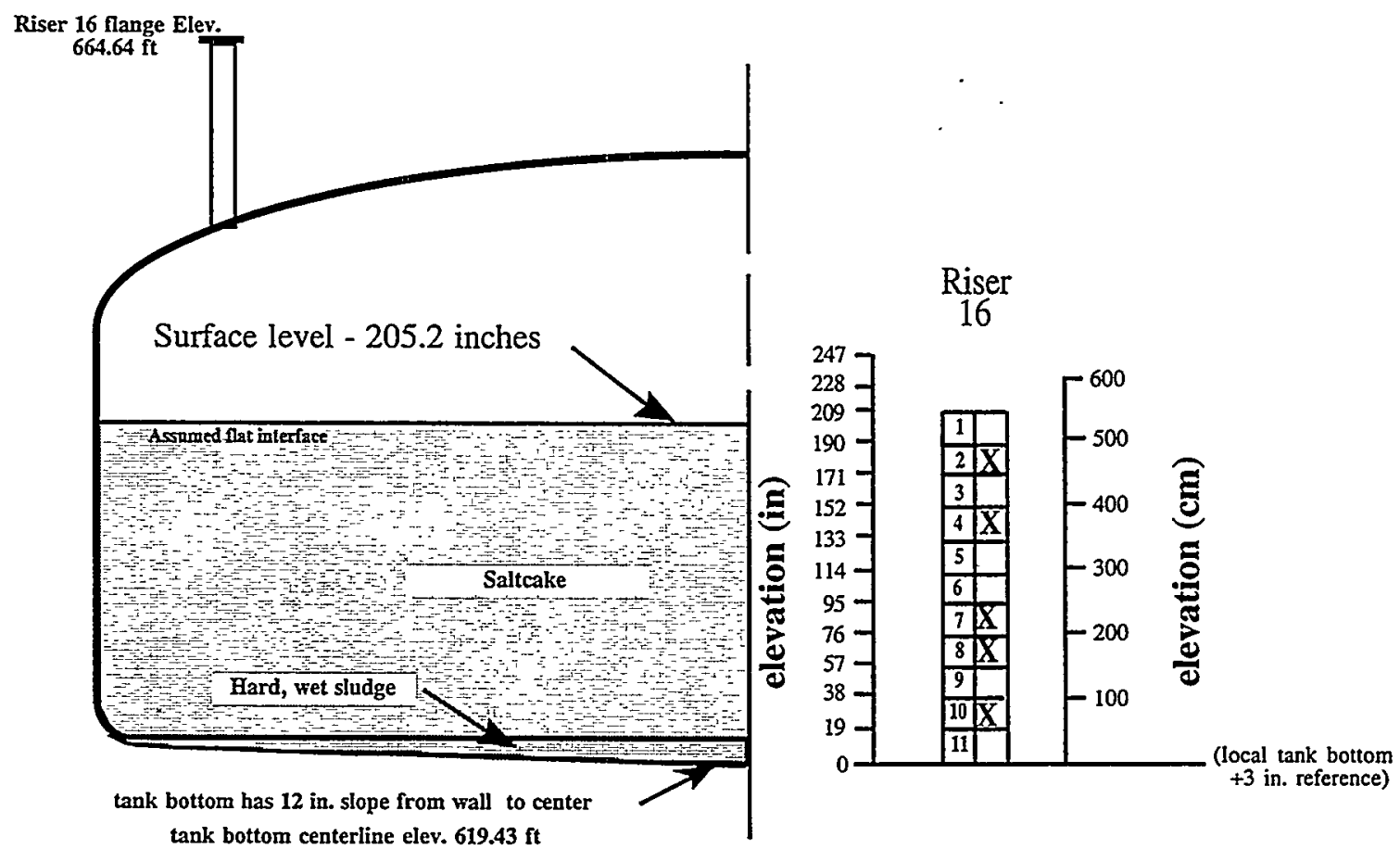

Figure 4.12.2. Diagram of Waste Layering and RGS Sample Elevations for S-102 
liquid varied from 1415 to $1470 \mathrm{~kg} / \mathrm{m}^{3}$ with average and standard deviation of $1434 \pm 31 \mathrm{~kg} / \mathrm{m}^{3}$. The bulk density of the samples with high solids concentrations varied from 1440 to $1920 \mathrm{~kg} / \mathrm{m}^{3}$ with an average and standard deviation of $1684 \pm 136 \mathrm{~kg} / \mathrm{m}^{3}$.

For hydrostatic pressure calculations, the liquid density was set at $1434 \mathrm{~kg} / \mathrm{m}^{3}$ and the bulk tank waste was given a degassed bulk density of $1684 \mathrm{~kg} / \mathrm{m}^{3}$. A waste depth of $521 \mathrm{~cm}(205 \mathrm{in}$.) was used in calculations.

\subsubsection{Sampling and Extraction Information}

The samples that underwent RGS analysis are listed in Table 4.12.1. Sample 16-8, shown in Figure 4.12.2, was taken successfully, but the sampler valve jammed closed and could not be opened for analysis. Because of sampling problems with segment 16-4, segment 16-4R was taken in the same location and should be considered as having been taken from disturbed waste; it may not be comparable with other samples. Field data, including dose rates and downforce limits, are supplied in Appendix A.

Table 4.12.1 also shows the lag times (delay between sample acquisition and processing) for these samples. This information was provided to allow data users to correlate the lag time between sampling and extrusion with the concentrations of the various constituents, to test for decomposition or other chemical reactions. The maximum hold time allowed by the sampling plan is 24 days, based on measured sampler leakage rates during acceptance testing. The hold time for one sample (16-10) exceeded this limit, but the sample was accepted based on calculations that indicated the estimated leakage was still negligible.

As discussed in Section 3.5, the HHF used during sampling often enters the sampler in place of some of the waste. Table 4.12.2 shows the volume percentage contamination by HHF of the RGS samples. These values were used to calculate reduced effective sampler volumes for the RGS samples. The bromide concentration was set equal to the MDL for sample 16-10 and equal to the measured value for sample 16-2. (It is conservative to use the MDL for values below it; the conservatism is probably less than $2 \%$.) A third sample (16-7) was below the detection limit, which corresponded to $12 \mathrm{vol} \%$ contamination, too high to provide a reasonable estimate of the true HHF contamination. For this sample, the contamination was set at half the MDL; the uncertainty in the contamination fraction was also set to half the MDL so it would be included in the uncertainty in the RGS results. There was no bromide measurement for the fourth sample (16-4R); its contamination fraction was set equal to $4.6 \mathrm{vol} \%$, the average of the other RGS samples.

Table 4.12.1. Lag Times for Processing RGS Samples from Tank 241-S-102

\begin{tabular}{|c|c|c|c||}
\hline Sample & Acquisition Date & Processing Date & Lag (days) \\
\hline \hline $16-2$ & March 5, 1998 & March 12, 1998 & 7 \\
\hline $16-4 \mathrm{R}^{\text {(a) }}$ & March 6, 1998 & March 19, 1998 & 13 \\
\hline $16-7$ & March 18, 1998 & April 6, 1998 & 19 \\
\hline $16-10$ & April 2, 1998 & April 27, 1998 & 25 \\
\hline (a) Sample 16-4R was the second attempt at sampling the segment 4 elevation. \\
\hline
\end{tabular}


All of these samplers were helium-backfilled and sealed with vacuum grease before they were deployed. This method virtually eliminated air and argon contamination from gases entrained during sampling. The helium backfill results are discussed in detail by Cannon (1997) and summarized in Section 5 of Mahoney et al. (1997).

Two different extraction procedures were used on the S-102 samples. The first, with two post-isotopic extractions, was used on 16-2 and 16-4R. The second, with four post-isotopic extractions, was used on 16-7 and 16-10. The procedure used before the post-isotopic extractions was the same for all samples. (See Figure 3.1 for a schematic of the RGS extraction system.)

After evacuating the whole system and observing the pressure to test for leaks, the operator extruded the RGS sample into the extractor vessel, and the sample was stirred briefly. At this point the vapor space in communication with the sample consisted of the volumes of the extractor vessel, its lines, the second volume, the pump volume, and the collector side; the latter included the collector line volume and an open collection canister. This first canister, containing a sample of uncompressed (unpumped) gas, was closed off. The second set of two (or, for 16-10, three) collection canisters was opened. Several strokes were pumped from the extractor to the collector, and the second collection canisters were closed off. Then the collector side was evacuated. (The second volume was never used during the extraction process for S-102 samples.)

The vapor profile procedure was then performed. The first pump stroke and every third stroke thereafter were collected in single canisters with only one stroke per canister. On the intermediate strokes, the gas was pumped to the collector lines with no canister attached. The collector lines were evacuated after every pump stroke whether or not the pumped gas was collected in a canister. The total gas and vapor pumped from the extractor to the collector during this procedure was calculated by interpolating linearly between collected canisters to find the contents of the pump strokes that were not collected. At the end of the procedure, the first post-isotopic set of canisters was attached to the collector lines and opened and both the extractor lines and the collector side were evacuated. From this point, the two procedures diverged.

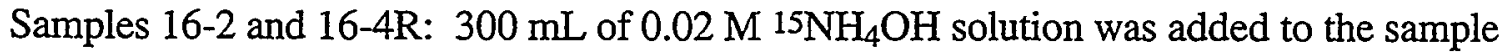
in the extractor and stirred. After a relatively short time, several strokes were pumped from the extractor to the collector. The first post-isotopic set of three canisters was closed off, the second set of three was attached and opened, and the collector side and extractor lines were evacuated. Further time was allowed for equilibration, and then several strokes were pumped from the extractor to the collector. The second post-isotopic set of three canisters was closed off. Finally, the BSVD procedure was performed: argon was injected into the closed-off second volume, and the second volume was opened to the extractor and lines. Pressure measurements and known tare system volumes allowed calculation of the volume of the sample solids and liquid.

Table 4.12.2. Hydrostatic Head Fluid Contamination in S-102 RGS Samples

\begin{tabular}{||c|c|}
\hline Sample & $\begin{array}{c}\text { HHF Contamination } \\
\text { (vol\%) }\end{array}$ \\
\hline $16-2$ & 5.0 \\
\hline $16-4 \mathrm{R}$ & no data \\
\hline $16-7$ & $<6.1$ \\
\hline $16-10$ & $<2.8$ \\
\hline
\end{tabular}


Samples 16-7 and 16-10: $300 \mathrm{~mL}$ of $0.04 \mathrm{M} 15 \mathrm{NH}_{4} \mathrm{OH}$ solution were added to the sample in the extractor and stirred. After a relatively short time, several strokes were pumped from the extractor to the collector. The first post-isotopic set of three canisters was closed off, the second set of three was attached and opened, and the collector side and extractor lines were evacuated. More time was allowed for equilibration, then several strokes were pumped from the extractor to the collector. The second post-isotopic set of three canisters was closed off, the third set of three was attached and opened, and the collector side and extractor lines were evacuated. More time was allowed for equilibration, then several strokes were pumped from the extractor to the collector. The third post-isotopic set of three canisters was closed off, the fourth set of three was attached and opened, and the collector side and extractor lines were evacuated. More time was allowed for equilibration, then several strokes were pumped from the extractor to the collector. The fourth post-isotopic set of three canisters was closed off. Finally, the BSVD procedure was performed.

For sample 16-10, more than one aliquot (subsample) was drawn from each post-isotopic canister to provide more opportunity for the isotopic ratios to converge. In all previous samples (including those from SX-106, AX-101, BY-109, S-106, and U-103) the isotopic ratio was based on only one aliquot per canister. Procedural details such as the number of strokes per canister and the system volumes are in the sample-by-sample worksheets in the S-102 RGS calculation spreadsheet on the CD supplied with this report.

\subsubsection{Retained Gas Composition}

Table 4.12.3 presents the calculated concentrations of the insoluble gases in the S-102 RGS samples without corrections for air and argon entrainment, helium backfill, air inleakage, or air dissolved in the isotopic standard solution. The method used to make the corrections depended on the gas. All of the oxygen, argon, and helium were subtracted no matter which step had produced them. A number of moles equal to $(3.73) \cdot\left(\mathrm{O}_{2}\right)$ were subtracted from the nitrogen because the oxygen was expected to have come from air entrainment during sampling or inleakage during extraction. Because a substantial amount of dissolved air gases entered the extractor with the $15 \mathrm{NH}_{4} \mathrm{OH}$ solution, all the nitrogen present in the gas collected after the solution addition was subtracted. Thus it was assumed that more than $95 \%$ of the original retained nitrogen had already been collected, as was the case for hydrogen. The corrected concentrations are in Table 4.12.4.

Sample 16-2 showed the effects of a substantial air leak into the system. The $\mathrm{O}_{2}$ measurements established that the leak lasted from the beginning of the extraction process to the end. The leak must have been into the extractor, based on the extractor pressure increase of $3 \mathrm{kPa}$ that

Table 4.12.3. Concentrations of Insoluble Constituents ( $\mu \mathrm{mol} / \mathrm{L}$ of waste) in Tank S-102 Without Correction for Gas Contamination

\begin{tabular}{|c|c|c|c|c|c|c|c|c|c|c|c||}
\hline Segment & $\mathrm{N}_{2}$ & $\mathrm{H}_{2}$ & $\mathrm{~N}_{2} \mathrm{O}$ & $\mathrm{O}_{2}$ & $\mathrm{CH}_{4}$ & $\mathrm{He}$ & $\mathrm{Ar}$ & $\begin{array}{c}\text { Other } \\
\mathrm{NOx}\end{array}$ & $\mathrm{C}_{2} \mathrm{H}_{x}$ & $\mathrm{C}_{3} \mathrm{H}_{x}$ & $\begin{array}{c}\text { Other } \\
\text { Hyd. }\end{array}$ \\
\hline \hline $16-2$ & $17000 \pm 2000$ & $5100 \pm 150$ & $3700 \pm 120$ & $2800 \pm 530$ & $59 \pm 6.3$ & $560 \pm 21$ & $170 \pm 25$ & (a) & $7.2 \pm 3.0$ & (a) & $6.1 \pm 1.4$ \\
\hline $16-4 \mathrm{R}(\mathrm{b})$ & $1800 \pm 73$ & $1100 \pm 46$ & $1300 \pm 80$ & $310 \pm 19$ & $8.1 \pm 2.5$ & $450 \pm 19$ & $17 \pm 0.7$ & (a) & $6.1 \pm 2.6$ & (a) & $2.0 \pm 1.5$ \\
\hline $16-7$ & $9200 \pm 640$ & $4200 \pm 300$ & $6800 \pm 490$ & $1500 \pm 110$ & $58 \pm 8.7$ & $520 \pm 38$ & $71 \pm 5.1$ & (a) & $5.4 \pm 2.1$ & (a) & $6.5 \pm 3.7$ \\
\hline $16-10$ & $4000 \pm 190$ & $3200 \pm 150$ & $1900 \pm 91$ & $340 \pm 25$ & $53 \pm 3.9$ & $1.6 \pm 0.3$ & $27 \pm 1.8$ & (a) & $28 \pm 6.0$ & (a) & $12 \pm 5.0$ \\
\hline
\end{tabular}


Table 4.12.4. Concentrations of Insoluble Constituents ( $\mu \mathrm{mol} / \mathrm{L}$ of waste) in Tank S-102 with Correction for Gas Contamination

\begin{tabular}{||c|c|c|c|c|c|c|c|c|c|c|c||}
\hline \hline Segment & $\mathrm{N}_{2}$ & $\mathrm{H}_{2}$ & $\mathrm{~N}_{2} \mathrm{O}$ & $\mathrm{O}_{2}$ & $\mathrm{CH}_{4}$ & $\mathrm{He}$ & $\mathrm{Ar}$ & $\begin{array}{c}\text { Other } \\
\text { NOx }\end{array}$ & $\mathrm{C}_{2} \mathrm{H}_{x}$ & $\mathrm{C}_{3} \mathrm{H}_{x}$ & $\begin{array}{c}\text { Other } \\
\text { Hyd. }\end{array}$ \\
\hline \hline $16-2$ & $5200 \pm 520$ & $5100 \pm 150$ & $3700 \pm 120$ & $0 \pm 530$ & $59 \pm 6.3$ & $0 \pm 21$ & $0 \pm 25$ & (a) & $7.2 \pm 3.0$ & (a) & $6.1 \pm 1.4$ \\
\hline $16-4 \mathrm{R}(\mathrm{b})$ & $1100 \pm 110$ & $1100 \pm 46$ & $1300 \pm 80$ & $0 \pm 19$ & $8.1 \pm 2.5$ & $0 \pm 19$ & $0 \pm 0.7$ & (a) & $6.1 \pm 2.6$ & (a) & $2.0 \pm 1.5$ \\
\hline $16-7$ & $4500 \pm 540$ & $4200 \pm 300$ & $6800 \pm 490$ & $0 \pm 110$ & $58 \pm 8.7$ & $0 \pm 38$ & $0 \pm 5.1$ & (a) & $5.4 \pm 2.1$ & (a) & $6.5 \pm 3.7$ \\
\hline $16-10$ & $2100 \pm 270$ & $3200 \pm 150$ & $1900 \pm 91$ & $0 \pm 25$ & $53 \pm 3.9$ & $0 \pm 0.3$ & $0 \pm 1.8$ & (a) & $28 \pm 6.0$ & (a) & $12 \pm 5.0$ \\
\hline
\end{tabular}

(a) Below detection limits, which are $0.01 \mathrm{~mol} \%$ or less.

(b) For sample 16-4R waste was disturbed because the sample was the second attempt at sampling the segment 4 elevation.

occurred during the 70 hours between the first and second sets of post-isotopic canisters. Contamination observations can be found in the "Summary" worksheet of the S-102 RGS calculations spreadsheet on the $\mathrm{CD}$.

Table 4.12.5 presents the ammonia measurements. The residual ammonia was determined isotopically, and the total ammonia (calculated from the isotopic residual) is included in the table. The post-extrusion partial pressure of $\mathrm{NH}_{3}$ over the sample (see Section 3.4.4) was measured at the chilled extractor temperature and is also included. The residual ammonia in the samples was also measured by ISE after the samples were removed from the extractor. Some ammonia measurements were also made on grab samples. The post-RGS ISE measurements can be found in Appen$\operatorname{dix} C$ (Table C.12.1). The grab sample results are also discussed there.

The isotopic measurements of residual ammonia did not produce consistent results for the four S-102 samples. The two samples taken at the highest elevation gave high values of residual ammonia because the measured isotopic ratios did not converge on a value within each set of three post-isotopic canisters; therefore the isotopic results are considered suspect and were not used to calculate in situ ammonia (see Section C.12 for details). The post-RGS ISE residual ammonia measurements were used instead, with a high uncertainty attached to them to reflect the possibility of ammonia losses during handling. Normally, the post-extrusion ammonia partial pressures would be preferred, but in this case, one pressure (for 16-2) was considered suspect because of air inleakage and the other pressure (for 16-4) was a physically impossible negative value (apparently because of a high calculated water vapor pressure over the sample).

Table 4.12.5. Ammonia Data from Tank S-102 Samples

\begin{tabular}{|c|c|c|c|}
\hline Sample & $\begin{array}{c}\text { Isotopically } \\
\begin{array}{c}\text { Measured Total } \mathrm{NH}_{3} \\
(\mu \mathrm{mol} / \mathrm{L})\end{array}\end{array}$ & $\begin{array}{l}\text { Measured } \mathrm{NH}_{3} \text { partial } \\
\text { pressure at about } 12^{\circ} \mathrm{C} \\
\text { (atm) }\end{array}$ & $\begin{array}{c}\text { RGS NH} H_{3} \text { Concentrations in the } \\
\text { Liquid }\end{array}$ \\
\hline $16-2$ & $298000 \pm 97000$ & $0.0037 \pm 0.0019$ & \multirow{4}{*}{$\begin{array}{l}\text { The RGS ammonia data are question } \\
\text { able but suggest a concentration of } \\
0.10 \mathrm{M}(1700 \mu \mathrm{g} / \mathrm{mL}) \mathrm{NH}_{3} \text { in the } \\
\text { interstitial liquid, or } 0.072 \mathrm{wt} \% \mathrm{NH}_{3} \\
\text { in the bulk waste. }\end{array}$} \\
\hline $16-4 \mathrm{R}$ & $67000 \pm 45000$ & -0.0019 & \\
\hline $16-7$ & $34000 \pm 9100^{(a)}$ & $0.011 \pm 0.0053$ & \\
\hline $16-10$ & $38000 \pm 9100^{(a)}$ & $0.0063 \pm 0.0032$ & \\
\hline
\end{tabular}


These and other inconsistencies in the RGS ammonia data are such that it could be misleading to calculate an average ammonia concentration for Tank S-102. In addition, the grab sample ammonia measurements are in most cases substantially lower than the RGS values. However, most of the data suggest a concentration of no less than $\sim 15,000 \mu \mathrm{mol} \mathrm{NH} / 2$ waste and no more than $\sim 70,000 \mu \mathrm{mol} \mathrm{NH}_{3} / \mathrm{L}$ waste at the time of sampling.

Table 4.12.6 contains the composition of the gas/vapor phase in each sample and the average compositions in the gases retained in the waste. The water vapor is not included in these compositions. The ammonia fractions are derived from the data in Table 4.12.5, which were used to calculate vapor pressures at in situ conditions. The sample compositions in the table were calculated using the in situ solubility method described in Section 3.6.2. As discussed in Section 3.6.1, compositions were calculated for both lower- and upper-bound gas solubilities. Both ends of the composition range are given in Table 4.12 .6 with the measurement uncertainty on each. The average composition of the gas in the waste is the result of integrating RGS species concentrations over the waste and multiplying those concentrations by the waste volume. The integration method is described in Section 3.7.1. The concentrations and pressures in Tables 4.12.3 and 4.12.4 can also be found in the sample-by-sample worksheets in the S-102 RGS calculation spreadsheet on the CD. The compositions in Table 4.12.6 are shown in the "Inventory" worksheet.

Table 4.12.6. Sample and Overall Average Compositions of Retained Gas in Tank S-102 with Correction for Gas Contamination(a)

\begin{tabular}{|c|c|c|c|c|c|c|}
\hline Sample & $\mathrm{N}_{2}(\mathrm{~mol} \%)$ & $\mathrm{H}_{2}(\mathrm{~mol} \%)$ & $\mathrm{N}_{2} \mathrm{O}(\mathrm{mol} \%)$ & $\mathrm{NH}_{3}(\mathrm{~mol} \%)^{(b)}$ & $\mathrm{CH}_{4}(\mathrm{~mol} \%)$ & Other (mol\%) \\
\hline $16-2$ & $37 \pm 4.4$ & $36 \pm 2.4--37 \pm 2.4$ & $26 \pm 1.8--25 \pm 1.7$ & $0.5 \pm 0.4-0.2 \pm 0.2$ & $0.4 \pm 0.05$ & $0.09 \pm 0.03$ \\
\hline $16-4 R$ & $31 \pm 4.1--35 \pm 4.5$ & $32 \pm 2.8--36 \pm 3.0$ & $35 \pm 3.4--28 \pm 2.7$ & $1.1 \pm 0.8--0.4 \pm 0.3$ & $0.2 \pm 0.07--0.3 \pm 0.08$ & $0.2 \pm 0.1--0.3 \pm 0.1$ \\
\hline $16-7$ & $29 \pm 4.2-30 \pm 4.4$ & $27 \pm 3.1--28 \pm 3.1$ & $42 \pm 4.8--41 \pm 4.6$ & $1.3 \pm 0.4--0.6 \pm 0.2$ & $0.4 \pm 0.06$ & $\begin{array}{c}0: 07 \pm 0.03 \text { to } \\
0.08 \pm 0.03\end{array}$ \\
\hline $16-10$ & $29 \pm 4.3--31 \pm 4.6$ & $43 \pm 3.8--45 \pm 4.0$ & $26 \pm 2.2--22 \pm 2.0$ & $1.0 \pm 0.3-0.4 \pm 0.1$ & $0.7 \pm 0.08--0.8 \pm 0.08$ & $0.6 \pm 0.2$ \\
\hline $\begin{array}{l}\text { Avg in } \\
\operatorname{tank}(\mathrm{c})\end{array}$ & $32 \pm 4.3--33 \pm 4.4$ & $33 \pm 3.0--34 \pm 3.0$ & $33 \pm 3.3--32 \pm 3.0$ & $1.0 \pm 0.3--0.4 \pm 0.1$ & $0.4 \pm 0.06$ & $0.2 \pm 0.06$ \\
\hline \multicolumn{7}{|c|}{$\begin{array}{l}\text { (a) Uncertainties represent instrument uncertainty; compositions calculated for lower-and upper-bound solubilities; both } \\
\text { ends of composition range given; lower-bound solubility basis first with the instrument uncertainty on each. Compositions } \\
\text { may not sum to } 100 \text { mol\% because of roundoff error; mole fractions are on dry basis and do not account for water vapor. } \\
\text { (b) Ammonia mole fraction is derived from the data in Table } 4.12 .5 \text { used to calculate vapor pressure at in situ conditions. } \\
\text { (c) There are too few samples to define spatial variability of average gas concentration. }\end{array}$} \\
\hline
\end{tabular}

\subsubsection{Gas Inventory}

The method by which the in situ gas volume fractions (wet basis) were calculated is given in Section 3.6.2; as for the gas composition, the volume fractions are given as a range from the low gas-solubility value to the high gas-solubility value. Table 4.12 .7 presents the results. This table also contains the average gas volume fraction and the average pressure experienced by the gas in the waste. The average is an in situ volume average calculated by Simpson's Rule integration, as described in Section 3.7.1. The corrected gas volume fractions in Table 4.12.7 are consistent with the corrected gas concentrations and compositions in Tables 4.12.4, 4.12.5, and 4.12.6. The information in Table 4.12.7 is taken from the "Summary" and "In situ" worksheets of the S-102 RGS calculation spreadsheet on the CD. 
Table 4.12.7. In Situ Gas Volume Fractions and Conditions in Tank S-102

\begin{tabular}{|c|c|c|c|c|c|c||}
\hline Sample & $\begin{array}{c}\text { Sample } \\
\text { Height } \\
(\mathrm{cm})\end{array}$ & $\begin{array}{c}\text { Hydrostatic } \\
\text { Pressure } \\
(\mathrm{atm})\end{array}$ & $\begin{array}{c}\text { Calculated } \\
\text { Water Vapor } \\
\text { Pressure } \\
(\mathrm{atm})\end{array}$ & $\begin{array}{c}\text { Temp } \\
\left({ }^{\circ} \mathrm{C}\right)\end{array}$ & $\begin{array}{c}\text { Sampler Gas } \\
\text { Corrected Gas } \\
\text { Volume Fraction } \\
\text { (in-tank conditions) }\end{array}$ & $\begin{array}{c}\text { Volume Fraction } \\
\text { (in situ conditions, } \\
\text { low solubility) }\end{array}$ \\
\hline \hline $16-2$ & 458 & 1.08 & 0.017 & 35.0 & $0.33 \pm 0.043$ & 0.36 \\
\hline $16-4 \mathrm{R}$ & 362 & 1.19 & 0.019 & 36.9 & $\begin{array}{c}0.075 \pm 0.007 \text { to } \\
0.066 \pm 0.007\end{array}$ & 0.087 \\
\hline $16-7$ & 217 & 1.37 & 0.022 & 39.7 & $\begin{array}{c}0.30 \pm 0.019 \text { to } \\
0.29 \pm 0.019\end{array}$ & 0.31 \\
\hline $16-10$ & 72.4 & 1.55 & 0.022 & 40.0 & $0.12 \pm 0.011$ & 0.12 \\
\hline $\begin{array}{l}\text { Avg in } \\
\text { tank(b) }\end{array}$ & 310 & 1.26 & & & $0.26 \pm 0.13$ & \\
\hline $\begin{array}{l}\text { (a) gas volume fraction expressed on wet basis, including volume contribution of water vapor. } \\
\text { Uncertainties on average gas volume fractions based on spatial variability considerations (Section 3.7.2.) }\end{array}$ \\
\hline
\end{tabular}

The "sampler gas volume fraction" is corrected only for inleakage during the extraction process and air gases added in the isotopic solution. It is an attempt to reconstruct the total amount of gas (sample and entrainment) present in the sampler during $\mathrm{X}$-ray. It is used only for comparison with the $\mathrm{x}$-ray observations of "visible gas fraction" that are discussed in Section 4.12.4.

Table 4.12.7 also contains the water vapor pressures that were used for in situ calculations. The water vapor pressures were found by using salt concentrations from Fritts (1998) and the temperatures in the table as inputs to Equation 6.2 of Mahoney and Trent (1995), a correlation for water vapor pressure over concentrated homogeneous and non-homogeneous waste simulants. The gas solubilities used the same parameters as inputs to the Schumpe solubility model (Section 3.6.1). The water vapor pressures and gas solubilities used in calculations can be found in the "In situ" worksheet of the S-102 RGS calculation spreadsheet on the CD. Because of the inconsistencies in the ammonia data, no ammonia inventory was calculated for Tank S-102.

Table 4.12.8 gives various estimates of the STP volume of gas in Tank S-102, including estimates calculated from RGS data alone. The RGS gas inventory in the waste was calculated by integrating RGS total gas concentrations over the layer (three data points, omitting 16-4R because it was the second sample attempt in the same location) and multiplying the average gas concentration by the volume of the waste. The integration method is described in Section 3.7.1. The RGS volumes in Table 4.12.8 include corrections to remove the contamination gas: entrained air and argon, helium backfill, air leaks during and after extraction, and air dissolved in the isotopic standard. The uncertainties on the gas inventories are based on the spatial variability considerations discussed in Section 3.7.2. The information in Table 4.12.8 is taken from the "Inventory" worksheet of the S-102 RGS calculation spreadsheet on the CD.

The RGS method gave twice as much gas inventory as was calculated by the BPE method. The long-term surface level rise can be considered an indication of retained gas volume: the waste surface rose a net $20 \mathrm{~cm}$ ( 8 in.) between 1981 and September 1995 (Hodgson et al. 1998), with a pre-1981 level rise of $65 \mathrm{~cm}$ (25 in.). The total waste rise is consistent with an in situ gas volume fraction of 0.19 , midway between the BPE estimate and the RGS estimate.

Both the BPE and RGS methods use data that are not beyond question. The absence of supernatant casts some doubt on the waste level response (dL/dP) as an indicator of the total gas inventory of the tank by the BPE method. In addition, the $\mathrm{dL} / \mathrm{dP}$ value cited by Hodgson et al. 
Table 4.12.8. S-102 Gas Inventory Estimates

\begin{tabular}{|c|c|c|}
\hline \multirow[b]{2}{*}{ Quantity } & RGS Method & BPE Method(a) \\
\hline & Tank Total & Tank Total \\
\hline $\begin{array}{l}\text { Avg. gas fraction } \\
\text { (low gas solubility) }\end{array}$ & $0.26 \pm 0.13$ & $0.13 \pm 0.04$ \\
\hline $\begin{array}{l}\text { Gas volume (m3) } \\
\text { in situ (wet) }\end{array}$ & $544 \pm 272$ (b) & $267 \pm 75$ \\
\hline STP (wet) & $601 \pm 300$ (b) & $294 \pm 83$ \\
\hline \multicolumn{3}{|c|}{$\begin{array}{l}\text { (a) Barometric pressure method. The inventory is based on the average } \\
\text { gas pressure calculated from RGS data, and on the Q-fit value } \mathrm{dL} / \mathrm{dP} \text { of } \\
0.68 \pm 0.18 \text { in./in.-Hg (Hodgson et al. } 1998 \text { )). } \\
\text { (b) The retained gas inventory calculated by the RGS method is } \\
\text { considered the better estimate. }\end{array}$} \\
\hline
\end{tabular}

(1998) was based on the FIC level gauge rather than the preferred Enraf instrument. The RGS data, on the other hand, were subject to increased uncertainty because of air inleakage (sample 16-2) and incomplete piston retraction (samples 16-7 and 16-10). It is also not clear whether sample 16-4R should have been included in the inventory as a true lower-gas point, or omitted (as it was) for coming from disturbed waste.

One explanation for the mismatch between the BPE gas inventory estimates and the RGS estimate might be that the high-gas region did not extend over the entire tank but was local to the area sampled by RGS, in the periphery of the tank. Risers 11 and 14, from which apparent highgas samples were taken in 1996, were also near the periphery and so did not rule out different conditions toward the tank center. Another possibility is that the gas in the tank waste was "syringed" into some of the RGS samples in disproportionately high amounts. However, the mechanism by which such "gas syringing" could occur is speculative at best.

Although the RGS data were taken from only one riser, the high-gas results were consistently confirmed by a number of non-RGS samples from two other risers (see discussion in Section 4.12.5.1 for more details). These consistent observations, and the fact that the BPE inventory was based on FIC measurements of a waste level that might not have been free liquid, cause us to recommend that the RGS inventory be considered the better estimate of the S-102 gas inventory.

Table 4.12.9 contains the calculated waste inventories of each of the major gases retained in the waste. These inventories are based on the average composition from RGS data (Table 4.12.6) and the best-estimate gas inventory (RGS, Table 4.12.8).

Figure 4.12.3 shows the sample temperatures, the corrected gas volume fractions (Table 4.12.7), and the corrected compositions of the low-solubility constituents in the samples from Tank S-102. The temperatures were measured by the TC tree installed in riser 3; no TCs were available in the waste above the 100 -in. level. The compositions represent the mole fraction of the species in the "insoluble" gas; water and ammonia are not included, so the mole fractions are not the same as those in Table 4.12.6. The gas volume fractions and mole fractions in the figure are the values for lower-bound gas solubilities. Figure 4.12.3 also lists the observations from core extrusions of non-RGS samples and from $x$-rays of RGS samples as a way of tying those observations together with RGS data. The $\mathrm{x}$-ray data are described in more detail in Section 4.12.4. 
Table 4.12.9. Speciated S-102 Gas Inventory(a)

\begin{tabular}{|c|c|c|}
\hline \multirow[b]{2}{*}{ Species } & \multicolumn{2}{|c|}{ Whole Tank } \\
\hline & $\begin{array}{l}\text { Gas-Phase Inventory } \\
\left(\mathrm{m}^{3} \text { at } \mathrm{STP}\right)\end{array}$ & $\begin{array}{l}\text { Dissolved Inventory } \\
\quad\left(\mathrm{m}^{3} \text { at STP }\right)\end{array}$ \\
\hline $\mathrm{N}_{2}$ & 190 & $0.069-0.51$ \\
\hline $\mathrm{H}_{2}$ & 190 & $0.23-1.2$ \\
\hline $\mathrm{N}_{2} \mathrm{O}$ & $200-180$ & $3.6-19$ \\
\hline $\mathrm{NH}_{3}$ & $5.7-2.3$ & 1300 \\
\hline $\mathrm{CH}_{4}$ & 2.6 & $0.0021-0.016$ \\
\hline Other & 0.93 & 0 \\
\hline \multicolumn{3}{|c|}{$\begin{array}{l}\text { (a) Inventories based on layer-average compositions from RGS data } \\
\text { (Table 4.12.6) and best-estimate gas inventories for each layer (RGS, } \\
\text { Table 4.12.8). Uncertainty in each inventory is } 50 \% \text { based on spatial } \\
\text { variability considerations (Section 3.7.2). Inventories given for lower- } \\
\text { and upper-bound solubilities; lower-bound solubility basis first. }\end{array}$} \\
\hline
\end{tabular}

\subsubsection{X-Ray Results}

Table 4.12.10 summarizes all the available radiography observations from Tank S-102. The $\mathrm{x}$-ray images themselves can be found on the $\mathrm{CD}$ that accompanies this report. The uncertainties of the visible gas volume fractions that are given in Table 4.12.10 are unknown but (based on the uncertainty in measuring the gas gap from x-ray images) are expected to be larger than the uncertainties in the gas volume fractions calculated from gas extraction.

Table 4.12.10 compares $\mathrm{x}$-ray-derived gas volume fractions with extraction-derived gas volume fractions. The comparison shows that sample recovery was close to $100 \%$ for all the samples. This conclusion is based on the fact that the extracted uncorrected gas was equal to or a few volume percent more than the visible gas. Sample 16-2 was nominally an exception, showing slightly more visible than extracted gas, but the difference is judged to be well within the uncertainty of the visible gas estimation method.

The major peculiarities of S-102 waste, as observed from $\mathrm{x}$-ray images, were waste stalactites and fracture bubbles (usually about one-third of the way up the sampler). Sample 16-11, a non-RGS sample, appeared to contain an annulus of gas around a solid core for about 2 in. of its length. Since the core was $\mathrm{x}$-rayed from only one direction, the appearance of an annulus may be misleading; the narrow strip of solids could have been stuck to one wall of the sampler. These observations indicate waste with substantial cohesive strength and adhesion to the sampler. Note also that sample 16-7 was measured by extraction to contain much more gas (volume fraction of 0.31 ) than was visible on the $\mathrm{x}$-ray (volume fraction of 0.09 ).

Table 4.12.11 provides a summary of the waste densities that have been calculated from radiographic data for the RGS samples for which air and water standard images were available. The radiographic densities 1) include the gas in the waste, 2) were calculated by the current $\mathrm{x}$-ray analysis method, and 3) are given for several locations within each sample to show density trends. No density data were available from non-RGS core samples in riser 16 for comparison. For many samples the $\mathrm{x}$-ray images were underexposed, making the radiographic densities overestimates. 

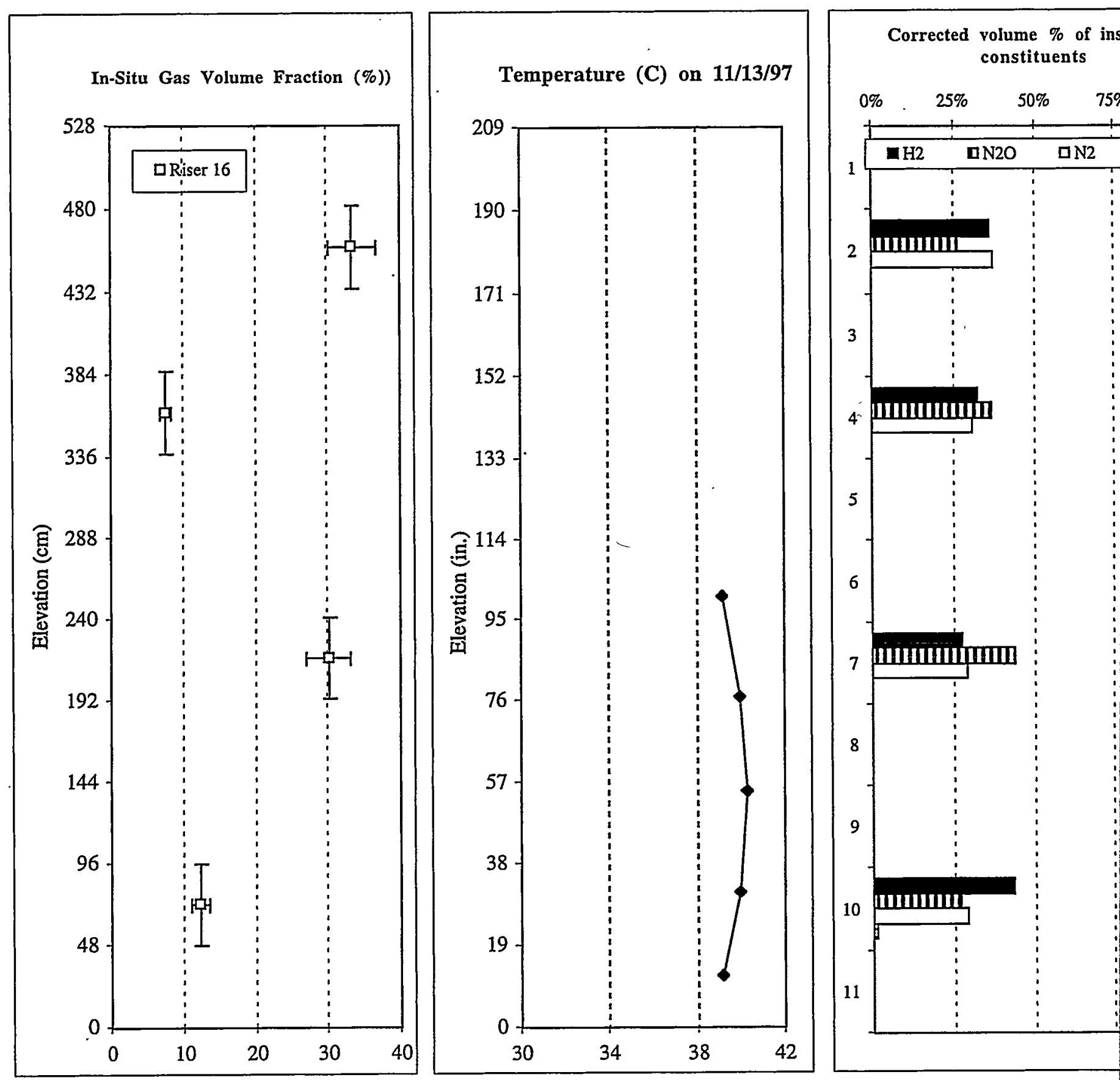

Figure 4.12.3. Gas Volume Fractions, Temperatures, $\mathrm{C}$ 
Waste level is 510 to $528 \mathrm{~cm}$ (201 to 208 inches).

1 Riser 16: $250 \mathrm{~mL}$ (351 g) yellow liquid, small amount of solids. X-RAY: Featureless, 0.1 " gap at piston.

1 Riser 11: no sample.

Riser 14: void.

2 Riser 16 X-RAY: 7.2" gap below piston, many 3-mm bubbles in bottom third of sampler.

2 Riser 11: 6 in. (142 g) brown and white saltcake, $250 \mathrm{mR} / \mathrm{hr}$.

2 Riser 14: 8 in. $(140 \mathrm{~g})$ gray saltcake, $260 \mathrm{mR} / \mathrm{hr}$.

3 Riser 16: $<5 \mathrm{~mL}$ gray liquid, 12 in. ( $405 \mathrm{~g}$ ) gray \& white wet salt. X-RAY: many <2-mm bubbles, 2" gap at piston.

3 Riser 11: $12 \mathrm{in}$. (224 g) gray saltcake, $400 \mathrm{mR} / \mathrm{hr}$, difficulty extruding.

3 Riser 14: $10 \mathrm{in}$. $(245 \mathrm{~g})$ gray saltcake, $700 \mathrm{mR} / \mathrm{hr}$.

4R Riser 16 X-RAY: 0.7" gas gap at clean piston, 20-mm bubble and smaller ones, liquid layer.

4 Riser 11: $15 \mathrm{in}$. (316 g) gray saltcake, $500 \mathrm{mR} / \mathrm{hr}$, difficulty extruding.

4 Riser 14: $10 \mathrm{in}$. (185 g) gray and white crumbly saltcake, $700 \mathrm{mR} / \mathrm{hr}$.

5 Riser 16: void.

5 Riser 11: 14 in. (317 g) gray saltcake, $700 \mathrm{mR} / \mathrm{hr}$.

5 Riser 14: $30 \mathrm{~mL}(44 \mathrm{~g})$ yellow-gray liquid, $12 \mathrm{in.}(349 \mathrm{~g})$ gray sludge/saltcake, $1.6 \mathrm{R} / \mathrm{hr}$.

6 Riser 16: No sample.

6 Riser 11: 13 in. (285 g) gray saltcake, $1.1 \mathrm{R} / \mathrm{hr}$.

6 Riser 14: 6 in. (134 g) gray sludge/saltcake, $1.3 \mathrm{R} / \mathrm{hr}$.

7 Riser 16X-RAY: 1.5" gap below piston, many $<5$-mm bubbles in bottom third of sampler.

7 Riser 11: $70 \mathrm{~mL}(88 \mathrm{~g})$ gray liquid, $14 \mathrm{in}$. $(321 \mathrm{~g})$ gray damp saltcake, $1.3 \mathrm{R} / \mathrm{hr}$.

7 Riser 14: 4 in. (85 g) blue-gray damp saltcake, $600 \mathrm{mR} / \mathrm{hr}$.

8 Riser 16X-RAY: 4.5" gap under 0.8"-thick piston-stalactite; near bottom, a bubble spans sampler.

8 Riser 11: 6 in. (193 g) gray solids, 5 in. $(78 \mathrm{~g}$ ) brown and white damp saltcake, 1.6 R/hr.

8 Riser 14: 6 in. (124 g) dark gray damp saltcake, 11 in. $(286 \mathrm{~g})$ gray \& black saltcake \& sludge. $800 \mathrm{mR} / \mathrm{hr}$.

9 Riser 16: 17 in. (480 g) gray wet salt. X-RAY: several <2-mm bubbles, one 10-mm wide $\times 2 \mathrm{~mm}$, liquid layer.

9 Riser 11: 19 in. (462 g) black soft sludge, $1.1 \mathrm{R} / \mathrm{hr}$.

9 Riser 14: 21 inches $(479 \mathrm{~g})$ wet black sludge, $1.0 \mathrm{R} / \mathrm{hr}$.

10 Riser 16 X-RAY: large fracture bubbles near bottom, some <2-mm bubbles, no gap at piston.

10 Riser 11: 17 in. (409 g) black soft sludge, $900 \mathrm{mR} / \mathrm{hr}$.

10 Riser 14: $43 \mathrm{~mL}(43 \mathrm{~g})$ black opaque liquid, $16 \mathrm{in}$. $(398 \mathrm{~g})$ black sludge, $1.4 \mathrm{R} / \mathrm{hr}$.

11 Riser 16: 13 in. (335 g) black moist salt. X-RAY: 2.4" gap at piston, annular gas region near top, <2-mm bubbles.

11 Riser 11: $<5 \mathrm{~mL}(13 \mathrm{~g})$ clear light yellow liquid, $10 \mathrm{in}$. $(300 \mathrm{~g})$ gray saltcake, $1.6 \mathrm{R} / \mathrm{hr}$.

11 Riser 14: 9 in. $(179 \mathrm{~g})$ black sludge/saltcake, $1.3 \mathrm{R} / \mathrm{hr}$. 
Table 4.12.10. Summary of Observations from X-Ray Images of Tank S-102

\begin{tabular}{|c|c|}
\hline Segment & Comments/Observations \\
\hline $\begin{array}{l}16-1 \\
\text { (non-RGS, } \\
3 / 5 / 98)\end{array}$ & $\begin{array}{l}\text { The image was featureless. There was a gas gap of about } 0.25 \mathrm{~cm}(0.1 \mathrm{in} \text {.) below a clean } \\
\text { piston. The visible gas volume fraction is } 0.005 \text {. }\end{array}$ \\
\hline $\begin{array}{l}16-2 \\
\text { (RGS, } \\
3 / 5 / 98)\end{array}$ & $\begin{array}{l}\text { This sample contained many gas bubbles of about } 3-\mathrm{mm} \text { diameter in the bottom third of the } \\
\text { sample but no features visible above, suggesting liquid in the upper portion. The piston is not } \\
\text { visible. There is a flat top surface and a gas gap of } 18 \mathrm{~cm}(7.2 \mathrm{in} \text {.). The visible gas volume } \\
\text { fraction (not counting bubbles) is therefore } 0.40 \text {. (Compare with the corrected sampler gas } \\
\text { volume fraction of } 0.33 \text { in Table } 4.12 .7 \text { obtained by gas extraction. Because of air inleakage } \\
\text { during extraction, the corrected gas fraction is more appropriate for comparison.) }\end{array}$ \\
\hline $\begin{array}{l}16-3 \\
\text { (non-RGS, } \\
\text { 3/5/98) }\end{array}$ & $\begin{array}{l}\text { Contained numerous gas bubbles of } 1 \text { - to } 2 \text {-mm size in the bottom } 3 / 4 \text { of the sample. There } \\
\text { appears to be liquid (featureless material of slightly lower density) above } 1.2 \mathrm{ft} \text { from the sampler } \\
\text { bottom. There is a flat top surface, a small amount of waste attached to the piston, and a } 5.1- \\
\mathrm{cm} \text { (2-in.) gas gap below it. Visible gas volume fraction (not counting bubbles) is thus } 0.11 \text {. }\end{array}$ \\
\hline $\begin{array}{l}16-4 \mathrm{R}(\mathrm{a}) \\
\text { (RGS, } \\
3 / 5 / 98)\end{array}$ & $\begin{array}{l}\text { Near the bottom were several large voids, including one about } 20 \mathrm{~mm} \text { across, spanning more } \\
\text { than half the sampler. Above that are several } 1 \text { - to } 5 \text {-mm voids. There appears to be liquid } \\
\text { (featureless material of slightly lower density) above } 1.25 \mathrm{ft} \text { above the sampler bottom. There } \\
\text { is a flat top surface and a } 1.8-\mathrm{cm} \text { ( } 0.7 \text {-in.) gas gap below the piston, which is clean. The visible } \\
\text { gas volume fraction (not counting bubbles) is therefore } 0.04 \text {. (Compare with the sampler gas } \\
\text { volume fraction of } 0.087 \text { in Table } 4.12 .7 \text { obtained by gas extraction.) }\end{array}$ \\
\hline $\begin{array}{l}16-7 \\
(\mathrm{RGS} \\
3 / 27 / 98)\end{array}$ & $\begin{array}{l}\text { Contained several gas bubbles } 1-\text { to } 5 \text {-mm in size in the bottom } 2 / 3 \text { of the sample. A flat top } \\
\text { surface and a } 3.8-\mathrm{cm} \text { (1.5-in.) gas gap exist below the piston, on which there is a small amount } \\
\text { of waste. The sampler piston was } 1.5 \text { to } 2 \text { in. short of being fully retracted, giving an effective } \\
\text { sample length of } 16.4 \text { in. The visible gas volume fraction (not counting bubbles) is thus } 0.09 \text {. } \\
\text { A sampler gas volume fraction of } 0.31 \text { (Table } 4.12 .7 \text { ) was obtained by gas extraction. }\end{array}$ \\
\hline $\begin{array}{c}16-8 \\
(\mathrm{RGS} \\
3 / 27 / 98)\end{array}$ & $\begin{array}{l}\text { Contained several gas bubbles in the } 1-\text { to } 10 \text {-mm size range in the bottom } 2 / 3 \text { of the sample } \\
\text { and a sampler-spanning void (about } 1.5 \mathrm{~cm} \text { thick) about one-third of the way up with lumpy top } \\
\text { and bottom. There is a lumpy top surface and a 11.4-cm (4.5-in.) gas gap below the large waste } \\
\text { stalactite ( } 2.0 \mathrm{~cm} \text { thick) attached to the piston. The sampler piston was } 0.25 \text { in. short of being } \\
\text { fully retracted, giving an effective sample length of } 18 \text { in. The visible gas volume fraction } \\
\text { (including the sampler-spanning bubble but not the smaller ones) is thus } 0.28 \text {. }\end{array}$ \\
\hline $\begin{array}{l}16-9 \\
\text { (non-RGS, } \\
4 / 3 / 98)\end{array}$ & $\begin{array}{l}\text { Several } 1-\text { to } 2-\mathrm{mm} \text { voids about halfway up and, near the top, a } 2-\mathrm{mm} \text { thick by } 10-\mathrm{mm} \text { long } \\
\text { slit-like horizontal void. The top surface is liquid. The gas gap is } 0.5 \mathrm{~cm}(0.2 \mathrm{in} .) \text {, and there is } \\
\text { a barely visible amount of waste on the piston. The visible gas volume fraction is } 0.01 \text {. }\end{array}$ \\
\hline $\begin{array}{l}16-10 \\
\text { (RGS, } \\
4 / 3 / 98)\end{array}$ & $\begin{array}{l}\text { Contained many gas bubbles in the } 1-\text { to } 5 \text {-mm size range as well as two sampler-spanning } \\
\text { voids (the larger one about } 1.5 \mathrm{~cm} \text { thick) about } 1 / 3 \text { of the way up with lumpy tops and } \\
\text { bottoms. There is no gas gap below the piston. The sampler piston was } 1.0 \text { in. short of being } \\
\text { fully retracted, giving an effective sample length of } 17.2 \text { in. The visible gas volume fraction } \\
\text { (including the sampler-spanning bubbles but not the smaller ones) is about } 0.05 \text {. A sampler } \\
\text { gas volume fraction of } 0.12 \text { (in Table } 4.12 .7 \text { ) was obtained by gas extraction. }\end{array}$ \\
\hline $\begin{array}{l}16-11 \\
\text { (non-RGS, } \\
4 / 3 / 98)\end{array}$ & $\begin{array}{l}\text { Several 1- to } 2 \text {-mm voids near the bottom. About halfway up, there appears to be an irregular } \\
\text { annular gas gap surrounding a solid core; the region is about } 4.8 \mathrm{~cm}(1.9 \mathrm{in} \text {.) high, and the gas } \\
\text { annulus an average of } 0.3 \mathrm{~cm}(0.12 \text { in) thick. The top surface is lumpy. The gas gap is } 6.1 \mathrm{~cm} \\
\text { ( } 2.4 \text { in.), and there is a small amount of waste on the piston. The visible gas volume fraction } \\
\text { is about } 0.15 \text {, counting the annulus and the top gas gap but not the bubbles. }\end{array}$ \\
\hline
\end{tabular}


Table 4.12.11. Densities of S-102 Samples from Radiography

\begin{tabular}{|c|c|c|}
\hline $\begin{array}{l}\text { Riser Segment } \\
\text { Number }\end{array}$ & $\begin{array}{l}\text { Distance from Bottom } \\
\text { of Sampler } \\
(\mathrm{ft})\end{array}$ & $\begin{array}{c}\text { Calculated Mean Density } \\
\text { by Current Method } \\
\text { (g/cc) }\end{array}$ \\
\hline \multirow[t]{6}{*}{$16-1$} & 1.50 & $1.65(\mathrm{a})$ \\
\hline & 1.25 & 1.54 \\
\hline & 1.00 & 1.52 \\
\hline & 0.75 & 1.52 \\
\hline & 0.50 & 1.51 \\
\hline & 0.25 & 1.58 \\
\hline \multirow[t]{3}{*}{$16-2$} & 0.75 & 1.72 (a) \\
\hline & 0.50 & 1.80 \\
\hline & 0.25 & 1.91 \\
\hline \multirow[t]{5}{*}{$16-3$} & 1.25 & $1.35(\mathrm{a})$ \\
\hline & 1.00 & 1.75 \\
\hline & 0.75 & 1.69 \\
\hline & 0.50 & 1.70 \\
\hline & 0.25 & 1.65 \\
\hline \multirow[t]{5}{*}{$16-4 \mathrm{R}^{(\mathrm{b})}$} & 1.50 & $1.71(\mathrm{a})$ \\
\hline & 1.25 & 1.93 \\
\hline & 1.00 & 2.18 \\
\hline & 0.75 & 2.10 \\
\hline & 0.50 & 1.96 \\
\hline \multirow[t]{4}{*}{$16-7$} & 1.00 & 1.79(a) \\
\hline & 0.75 & 1.95 \\
\hline & 0.50 & 1.90 \\
\hline & 0.25 & 1.88 \\
\hline \multirow[t]{5}{*}{$16-8$ (b) } & 1.25 & $0.68(\mathrm{a})$ \\
\hline & 1.00 & 1.94 \\
\hline & 0.75 & 2.13 \\
\hline & 0.50 & 1.84 \\
\hline & 0.25 & 1.89 \\
\hline \multirow[t]{6}{*}{$16-9(b, c)$} & 1.50 & 2.09 (a) \\
\hline & 1.25 & 2.22 \\
\hline & 1.00 & 2.25 \\
\hline & 0.75 & 2.20 \\
\hline & 0.50 & 3.05 \\
\hline & 0.25 & 2.45 \\
\hline
\end{tabular}


Table 4.12.11 (contd)

\begin{tabular}{||c|c|c||}
\hline $\begin{array}{c}\text { Riser Segment } \\
\text { Number }\end{array}$ & $\begin{array}{c}\text { Distance from Bottom } \\
\text { of Sampler } \\
\text { (ft) }\end{array}$ & $\begin{array}{c}\text { Calculated Mean Density } \\
\text { by Current Method } \\
\text { (g/cc) }\end{array}$ \\
\hline \multirow{3}{*}{$16-10^{(\mathrm{c})}$} & 1.25 & $1.75(\mathrm{a})$ \\
\cline { 2 - 3 } & 1.00 & 1.71 \\
\cline { 2 - 3 } & 0.75 & 1.71 \\
\cline { 2 - 3 } & 0.50 & 1.31 \\
\hline $16-11^{(\mathrm{c})}$ & 0.25 & 1.11 \\
\cline { 2 - 3 } & 1.00 & $1.45(\mathrm{a})$ \\
\cline { 2 - 3 } & 0.75 & 1.72 \\
\cline { 2 - 3 } & 0.50 & 1.76 \\
\hline & 0.25 & 1.91 \\
\hline \multirow{2}{*}{$\begin{array}{l}\text { (a) The topmost averages may be decreased by including the gas gap } \\
\text { beneath the piston in the averaging process. } \\
\text { (b) This segment was somewhat underexposed (dark relative to the standard } \\
\text { profiles). } \\
\text { (c) This segment lacked standard air/water density profiles; used data from } \\
\text { segment 16-8. }\end{array}$} \\
\hline
\end{tabular}

\subsubsection{Other Discussions}

\subsubsection{High Gas Fractions}

Unusually large amounts of gas were extracted from samples 16-2 and 16-7 of S-102. The in situ gas volume fractions calculated from RGS extraction data were about 0.3 for these samples. The high gas content found by extraction was consistent with the large gap that was seen in the sampler $\mathrm{x}$-ray for sample 16-2, though a large gap was not seen in sample 16-7. In addition, RGS sample 16-8, which did not undergo RGS extraction, contained a large amount of visible gas (a gas volume fraction of 0.28). The non-RGS samples 16-3 and 16-11 showed visible gas of, respectively, 0.11 and 0.15 volume fraction. (However, non-RGS samplers are not hermetically sealed, so leakage could have affected the gas volume fraction.)

Large gas gaps (short extrusion lengths) were also seen in many of the 1996 non-RGS core samples taken from risers 11 and 14 of Tank S-102. In the region sampled by segments 2 through 8 , extrusion lengths of 6 to 15 inches were seen, possibly indicating gas fractions of 0.20 or higher. Sample 11-2 had a 6-in. sample length, while sample 14-2 had an 8-in. sample length; these short samples suggest gas fractions at least as high as the 0.3 measured by extraction in RGS sample 16-2. The evidence suggests that a high gas fraction could have been present through the segment-2 level of S-102.

It is unclear whether the retaken RGS sample 16-4R represented a true lower-gas region or whether it contained less gas because the waste had been locally disturbed. When Tank SX-106 was sampled, sample SX-106-6-6 contained about 0.09 gas and the retaken sample SX-106-6-6A contained 0.04 gas (Table 4.10 .7 ), strongly suggesting the possibility of gas loss by disturbance. However, areas of lower gas fraction were observed in undisturbed samples from S-102: for example, the non-RGS sample 16-9 contained a visible gas volume fraction of only 0.01 . 
Waste "stalactites" hung from the sampler piston in one sample (16-10), and in a few cases fracture bubbles that spanned the sampler appeared in the middle of samples 16-8 and 16-10. These observations suggest high waste strength and cohesiveness. Gauglitz and Aikin (1997) estimated that S-102 samples 14-9 and 14-10, which were extruded as long unbroken pieces, had high shear strengths of 1000 to $2000 \mathrm{kPa}$.

\subsubsection{Drillstring and Domespace Composition Comparison}

The RGS gas-phase composition data (Table 4.12.6) were used to calculate $\mathrm{H}_{2} / \mathrm{N}_{2} \mathrm{O}$ ratios for comparison with ratios from drillstring and domespace grab sample measurements (Siciliano 1998, Table 8.1; McCain 1999, Table B-11). The results can be seen in Table 4.12.12. There was little agreement between the $\mathrm{RGS} \mathrm{H}_{2} / \mathrm{N}_{2} \mathrm{O}$ ratios and the drillstring or domespace ratios. The ratios from RGS samples 16-2 and 16-7 (both of which had high gas volume fractions) were not far different from the riser 14 drillstring ratio. However, of the RGS samples, only 16-4R (the sample from disturbed waste) resembled the low domespace ratios or the drillstring ratio from January 12, 1996 (almost certainly associated with riser 11 sampling, based on the timing).

Table 4.12.12 Comparison with Drillstring and Domespace Data(a)

\begin{tabular}{|l|c||}
\hline \multicolumn{1}{|c|}{ Sample } & $\mathrm{H}_{2} / \mathrm{N}_{2} \mathrm{O}$ \\
\hline \hline RGS, 16-2 & 9.6 \\
\hline RGS, 16-4R & $0.94-1.3$ \\
\hline RGS, 16-7 & 8.9 \\
\hline RGS, 16-10 & 4.3 \\
\hline Drillstring, 1/12/96 & 1.7 \\
\hline Drillstring, riser 14, 2/9/96 & 12 \\
\hline Domespace, 8/4 - 8/11/95 & $1.5,1.3,1.5$, \\
& $1.3,1.4,1.4$, \\
& $0.9,1.4,1.4$ \\
\hline Domespace, 1/21/99 & 0.91 \\
\hline $\begin{array}{l}\text { (a) RGS ratios were calculated for both lower- and upper- } \\
\text { bound solubilities. Both ends of the ratio range are given in } \\
\text { he table, lower-bound solubility basis first. }\end{array}$ \\
\hline
\end{tabular}




\subsection{S-111}

Tank 241-S-111 (S-111) was the thirteenth tank and the ninth SST sampled with the RGS. This tank was selected to represent a group of SSTs that show evidence of significant gas retention (Stewart et al. 1996b). S-111 is on the FGWL for exhibiting high response to barometric pressure changes (discussed in Section 3) and having high concentrations of $\mathrm{H}_{2}$ in the headspace. Tank S-111 (like S-106) is part of a group of tanks (Cluster 20 in Stewart et al. 1996) that have high radioactivity, moderate nitrite concentrations, and less than $0.05 \%$ TOC. HTCEs indicate that the primary waste stored in S-111 was saltcake from evaporator campaign S1 and that the secondary waste was redox waste generated between 1952 and 1958 (Remund et al. 1995). (This is similar to the HTCE estimate for Tank S-106, whose primary waste was saltcake from evaporator campaign $S 1$ and whose secondary waste was aluminum cladding redox waste generated between 1961 and 1967.)

Push-mode sampling was done in riser 6 in April 1998.(a) Riser 8 had been successfully sampled in May 1996, and a partial core was taken at riser 14 at the same time (Steen 1996b). The approximate locations of various risers are depicted in Figure 4.13.1. Riser 6 is roughly a third of the tank radius from the center. Figure 4.13.2 depicts the elevations of the RGS segments. This figure also shows the tank content layering, with supernatant liquid of varying depth overlying saltcake and sludge (at the bottom of the tank).

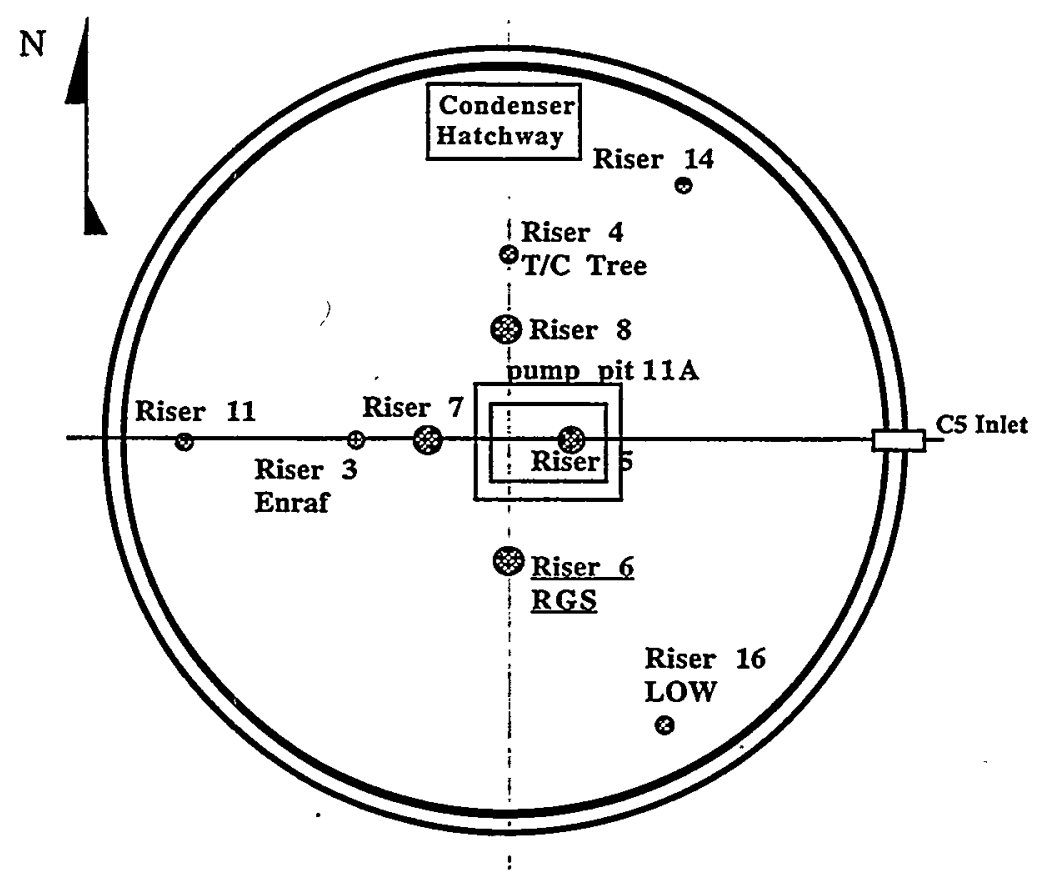

Figure 4.13.1. Schematic Diagram of Riser Locations in Tank S-111

(a) The sampling scheme may be found in Sampling Plan for Tank 241-S-111 Retained Gas Sampler Deployment, by JM Bates, March 1998. TWSFG98.34 Rev. 0, Pacific Northwest National Laboratory, Richland, Washington. 


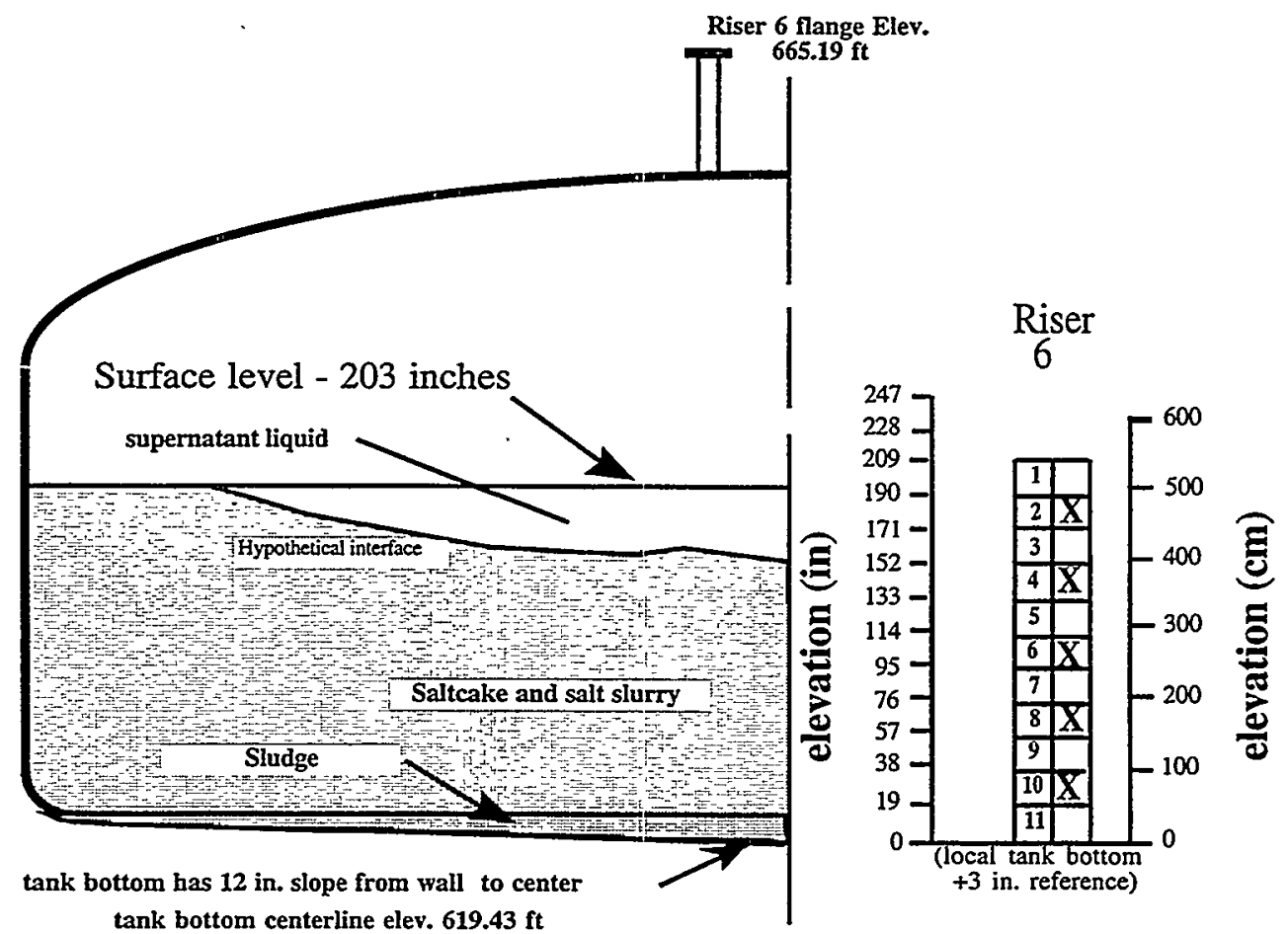

Figure 4.13.2. Diagram of Waste Layering and RGS Sample Elevations for S-111

Brevick et al. (1994) describe S-111 as a partially isolated, sound SST. At the time of sampling Tank S-111 contained about 2,040,000 L (540,000 gal) of waste, including 37,800 L $(10,000 \mathrm{gal})$ of supernatant (Hodgson et al. 1998). The total depth of waste in Tank S-111 was 516 to $518 \mathrm{~cm}$ (203 to $204 \mathrm{in}$.) at riser 3 . Neutron scans in the riser $16 \mathrm{LOW}$ indicated liquid at the waste surface. Riser 3 is about one-third of a tank radius from the center of the tank, like riser 6 , while riser 16 is near the tank periphery.

The most recent prior information on S-111 layering comes from the core observations made in 1996 (Steen 1996). The thickness of the top layer of supernatant liquid was about three segments at riser 8, about a third of a radius out from the tank center. (The 1998 core samples at riser 6 also showed at least three segments of liquid at the top of the waste.) At riser 14, near the tank periphery, no supernatant was evident. The nonconvective layer in the tank was described as moist salt, wet salt, or salt slurry; only one sample (8-4) was described as sludge.

The reported $37,800 \mathrm{~L}$ (10,000 gal) of supernatant would form a layer $10 \mathrm{~cm}$ (4 in.) deep if spread uniformly over the entire tank. However, liquid was demonstrably deeper than 4 in. at risers near the center: 109 to $122 \mathrm{~cm}$ (43 to $48 \mathrm{in}$.), based on core extrusions and accounting for the partial segment at the top of the waste. No liquid was seen at one riser near the periphery (riser 14), but at another peripheral riser (riser 16) liquid was reported to be at the surface. On this evidence, it is likely that the supernatant liquid was of irregular depth, possibly deeper at the center, and was not present over the entire waste surface.

Densities for the liquid and bulk solids in Tank S-111 were available from cores taken in 1996 (Steen 1996) and from non-RGS samples taken in 1998.(a) The density of drainable liquid

(a) Transmittal from FH Steen (RFSHI) to LA Mahoney (PNNL) of preliminary data for Core 237 from Tank S-111, July 8, 1998. Rust Federal Services of Hanford, Inc., Richland, Washington. 
varied from 1348 to $1367 \mathrm{~kg} / \mathrm{m}^{3}$ (three samples), with an average and standard deviation of 1359 $\pm 10 \mathrm{~kg} / \mathrm{m}^{3}$. The bulk density of the samples with high solids concentrations varied from 1470 to $1870 \mathrm{~kg} / \mathrm{m}^{3}$, with an average and standard deviation of $1648 \pm 112 \mathrm{~kg} / \mathrm{m}^{3}$.

For hydrostatic pressure calculations, the liquid density was set at $1359 \mathrm{~kg} / \mathrm{m}^{3}$, and the remainder of the tank waste was given a degassed bulk density of $1648 \mathrm{~kg} / \mathrm{m}^{3}$. A supernatant depth of $10 \mathrm{~cm}(4 \mathrm{in}$.) and a nonconvective layer depth of $505 \mathrm{~cm}$ (199 in.) were used in calculations.

\subsubsection{Sampling and Extraction Information}

The samples that underwent RGS analysis are listed in Table 4.13.1. Field data, including dose rates and downforce limits, are supplied in Appendix A. Table 4.13.1 also shows the lag times (delay between sample acquisition and processing) for these samples. This information was provided to allow data users to correlate the lag time between sampling and extrusion with the concentrations of the various constituents to test for decomposition or other chemical reactions. The long lag times for several of the samples were the result of laboratory procedural reviews and a building-wide stand-down at the 222-S Laboratory, where the RGS analysis was carried out. The effect of the long lag times on the samples is discussed in detail in Section 4.13.5.2. The conclusion is that the lag time changed the gas composition by less than the measurement uncertainty and that there is less than $10 \%$ potential underestimation of gas volume fraction resulting from the long lag times.

As was discussed in Section 3.5, the HHF used during sampling often enters the sampler in place of some of the waste. There were no bromide data for the RGS samples, and the nonRGS samples from the S-111 core taken in 1998 were all below the MDL, which in many cases was $1020 \mu \mathrm{g} / \mathrm{g}$.(a) For lack of other information, the RGS samples were assumed to have a bromide concentration of $1020 \mu \mathrm{g} / \mathrm{g}$; this was about $5.7 \mathrm{vol} \%$ HHF. This assumption is probably conservative, and (based on HHF data for other tanks) the conservatism is expected to be less than $3 \%$.

Table 4.13.1. Lag Times for Processing RGS Samples from Tank S-111

\begin{tabular}{||c|c|c|c||}
\hline \hline Sample & Acquisition Date & Processing Date & Lag (days) \\
\hline \hline $6-2$ & April 8, 1998 & May 11, 1998 & 33 \\
\hline $6-4$ & April 9, 1998 & July 21, 1998 & 103 \\
\hline $6-6$ & April 9, 1998 & July 25, 1998 & 107 \\
\hline $6-8$ & April 9, 1998 & July 29, 1998 & 111 \\
\hline $6-10$ & April 10, 1998 & September 20, 1998 & 132 \\
\hline
\end{tabular}

(a) Transmittal from FH Steen (RFSHI) to LA Mahoney (PNNL) of preliminary data for Core 237 from Tank S-111, July 8, 1998. Rust Federal Services of Hanford, Inc., Richland, Washington. 
All of these samplers were helium-backfilled and sealed with vacuum grease before they were deployed. This method virtually eliminated air and argon contamination from gases entrained during sampling. The helium backfill results are discussed in detail by Cannon (1997) and summarized in Section 5 of Mahoney et al. (1997). Sample 6-2 showed signs of an air leak in the RGS system (in spite of the pre-extrusion system leak check). Subsequent testing confirmed the leak, which was corrected by replacing some seals before the next sample was processed. In addition, there was an unexplained large argon contamination in sample 6-6. This latter topic is discussed more fully in Section 4.13.5.1.

Two different extraction procedures were used on the S-111 samples. (See Figure 3.1 for a schematic of the RGS extraction system.) The first procedure, with four post-isotopic extractions, was used on 6-2. The second, with one post-isotopic extractions, was used on the other four samples; schedule and lag-time constraints precluded using an equilibration time greater than one or two days. The procedure before the post-isotopic extractions was substantially the same for all samples.

After evacuating the whole system and observing the pressure to test for leaks, the operator extruded the RGS sample into the extractor vessel, and the sample was briefly stirred. At this point the vapor space in communication with the sample consisted of the volumes of the extractor vessel, its lines, the second volume, the pump volume, and the collector side; the latter included the collector line volume and an open collection canister. This first canister, containing a sample of uncompressed (unpumped) gas, was closed off. The second set of two collection canisters was opened. Several strokes were pumped from the extractor to the collector, and the second collection canisters were closed off. Then the collector side was evacuated. (The second volume was never used during the extraction process for S-111 samples.)

The vapor profile procedure was then performed. The first pump stroke and every third stroke thereafter (for 6-2) or every ninth stroke thereafter (for the other samples) were collected in single canisters, with only one stroke per canister. On the intermediate strokes the gas was pumped to the collector lines, with no canister attached. The collector lines were evacuated after every pump stroke whether or not the pumped gas was collected in a canister. The total gas and vapor pumped from the extractor to the collector during this procedure was calculated by interpolating linearly between collected canisters to find the contents of the pump strokes that were not collected. At the end of the procedure, the first post-isotopic set of canisters was attached to the collector lines and opened and both the extractor lines and the collector side were evacuated. From this point, the two procedures diverged.

Sample 6-2: $300 \mathrm{~mL}$ of $0.04 \mathrm{M} 15 \mathrm{NH}_{4} \mathrm{OH}$ solution was added to the sample in the extractor and stirred. After a relatively short time, several strokes were pumped from the extractor to the collector. The first post-isotopic set of three canisters was closed off, the second set of three was attached and opened, and the collector side and extractor lines were evacuated. Further time was allowed for equilibration, and then several strokes were pumped from the extractor to the collector. The second post-isotopic set of three canisters was closed off, the third set of three was attached and opened, and the collector side and extractor lines were evacuated. Further time was allowed for equilibration, and then several strokes were pumped from the extractor to the collector. The third post-isotopic set of three canisters was closed off, the fourth set of three was attached and opened, and the collector side and extractor lines were evacuated. More time was allowed for equilibration, and then several strokes were pumped from the extractor to the collector. The fourth post-isotopic set of three canisters was closed off. Finally, the BSVD procedure was performed: argon was injected into the closed-off second volume, and then the second volume was opened to the extractor and lines. Pressure measurements and known tare system volumes allowed the volume of the sample solids and liquid to be calculated. 


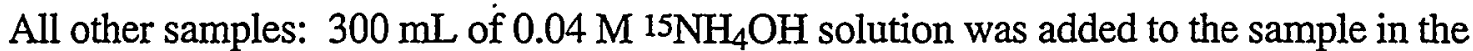
extractor and stirred. After about 24 hours, several strokes were pumped from the extractor to the collector. The first (and only) post-isotopic set of three canisters was closed off. The BSVD procedure was performed. For all the S-111 samples, three to five aliquots (subsamples) were drawn from each post-isotopic canister to provide more opportunity for the isotopic ratios to converge. Procedural details such as number of strokes per canister and system volumes are in the sample-by-sample worksheets in the S-111 RGS calculation spreadsheet on the CD.

\subsubsection{Retained Gas Composition}

Table 4.13.2 presents the calculated concentrations of the insoluble gases in the S-111 RGS samples without corrections for air and argon entrainment, helium backfill, air inleakage, or air dissolved in the isotopic standard solution. The method used to make the corrections depended on the gas. All of the oxygen, argon, and helium were subtracted no matter which step had produced them. A number of moles equal to $(3.73) \cdot\left(\mathrm{O}_{2}\right)$ were subtracted from the nitrogen because the oxygen was expected to have come from air entrainment during sampling or inleakage during extraction. Because a substantial amount of dissolved air gases entered the extractor with the $15 \mathrm{NH}_{4} \mathrm{OH}$ solution, all the nitrogen present in the gas collected after the solution addition was subtracted. Thus it was assumed that more than $95 \%$ of the original retained nitrogen had already been collected, as it was for hydrogen. Corrected concentrations are given in Table 4.13.3.

Sample 6-6 contained more than 70\% argon, the source of which is unknown (the possible sources, all of which are contradicted by evidence, are discussed in Section 4.13.5.1). The argon could, in theory, have been introduced from drillstring purge gas and could have taken the place of waste in a significant volume of the sampler. Therefore, the effective (argon-free) volume of the sampler might have been much less than the nominal sampler volume, which was used to calculate the gas concentrations for sample 6-6 in Tables 4.13.2 and 4.13.3. If the volume occupied by the argon was excluded from the effective sampler volume, the corrected gas concentrations for sample 6-6 would roughly double. As a result, the uncertainty in the upward direction is much greater than that in the downward direction, as shown in Table 4.13.3. Contamination observations can be found in the "Summary" worksheet of the S-111 RGS calculations spreadsheet on the CD.

In four of the five samples from S-111, the amount of helium present was found to be equivalent to the air/argon entrainment that had been contained in samples taken from tanks sampled previously (Shekarriz et al. 1997; Mahoney et al. 1997). In sample 6-6, however, only about $1 \mathrm{cc}$ of helium was present, half or less of the usual values. The reason for this is unknown but may be related to the large amount of argon found in the sample.

Table 4.13.2. Concentrations of Insoluble Constituents ( $\mu \mathrm{mol} / \mathrm{L}$ of waste) in Tank S-111 Without Correction for Gas Contamination

\begin{tabular}{||c|c|c|c|c|c|c|c|c|c|c|c||}
\hline \hline $\begin{array}{c}\text { Seg- } \\
\text { ment }\end{array}$ & $\mathrm{N}_{2}$ & $\mathrm{H}_{2}$ & $\mathrm{~N}_{2} \mathrm{O}$ & $\mathrm{O}_{2}$ & $\mathrm{CH}_{4}$ & $\mathrm{He}$ & $\mathrm{Ar}$ & $\begin{array}{l}\text { Other } \\
\mathrm{NO}_{x}\end{array}$ & $\mathrm{C}_{2} \mathrm{H}_{x}$ & $\mathrm{C}_{3} \mathrm{H}_{x}$ & $\begin{array}{c}\text { Other } \\
\text { Hyd. }\end{array}$ \\
\hline \hline $6-2$ & $770 \pm 130$ & $24 \pm 2.9$ & $24 \pm 6.4$ & $120 \pm 32$ & $1.2 \pm 0.5$ & $340 \pm 24$ & $10 \pm 1.8$ & (a) & $0.7 \pm 0.5$ & (a) & $2.9 \pm 2.0$ \\
\hline $6-4$ & $2100 \pm 470$ & $1600 \pm 550$ & $580 \pm 93$ & $320 \pm 96$ & $20 \pm 2.8$ & $580 \pm 190$ & $37 \pm 5.5$ & (a) & $5.7 \pm 0.7$ & (a) & $3.7 \pm 0.8$ \\
\hline $6-6$ & $2400 \pm 100$ & $3300 \pm 160$ & $860 \pm 55$ & $260 \pm 15$ & $43 \pm 5.2$ & $160 \pm 8.2$ & $19000 \pm 930$ & (a) & $14 \pm 5.9$ & (a) & $14 \pm 7.1$ \\
\hline $6-8$ & $4100 \pm 210$ & $7900 \pm 540$ & $1500 \pm 120$ & $500 \pm 30$ & $73 \pm 6.9$ & $610 \pm 41$ & $21 \pm 1.3$ & (a) & $11 \pm 4.8$ & (a) & $9.6 \pm 5.8$ \\
\hline $6-10$ & $2700 \pm 130$ & $11000 \pm 500$ & $1400 \pm 78$ & $190 \pm 12$ & $39 \pm 5.0$ & $500 \pm 30$ & $14 \pm 1.1$ & (a) & $6.1 \pm 3.5$ & (a) & $6.3 \pm 4.1$ \\
\hline
\end{tabular}


Table 4.13 .4 presents the ammonia measurements. The residual ammonia was determined isotopically, and the total ammonia (calculated from the isotopic residual) is included in the table. The post-extrusion partial pressure of $\mathrm{NH}_{3}$ over the sample (see Section 3.4.4) was measured at the chilled extractor temperature and is also included. The residual ammonia in S-111 samples was not measured by post-RGS ion-specific electrode methods. Other ammonia measurements were made on non-RGS core samples. The details of these data can be found in Section C.13 of Appendix C.

The various ammonia measurements were not consistent. For lack of more definitive information, the RGS isotopic measurements were used to calculate the ammonia mole fractions in the in situ vapor. Considering the discrepancies, it would be difficult to say more than that the ammonia concentration of the supernatant was probably between 4,000 and $13,000 \mu \mathrm{mol} \mathrm{NH}_{3} / \mathrm{L}$ liquid. There was also no agreement between the non-RGS and RGS measurements for the samples in the nonconvective layer (samples 6-4 through 6-10).

Table 4.13.3. Concentrations of Insoluble Constituents ( $\mu \mathrm{mol} / \mathrm{L}$ of waste) in Tank S-111 with Correction for Gas Contamination

\begin{tabular}{|c|c|c|c|c|c|c|c|c|c|c|c||}
\hline $\begin{array}{c}\text { Seg- } \\
\text { ment }\end{array}$ & $\mathrm{N}_{2}$ & $\mathrm{H}_{2}$ & $\mathrm{~N}_{2} \mathrm{O}$ & $\mathrm{O}_{2}$ & $\mathrm{CH}_{4}$ & $\mathrm{He}$ & $\mathrm{Ar}$ & $\begin{array}{c}\text { Other } \\
\mathrm{NO}_{x}\end{array}$ & $\mathrm{C}_{2} \mathrm{H}_{x}$ & $\mathrm{C}_{3} \mathrm{H}_{x}$ & $\begin{array}{c}\text { Other } \\
\text { Hyd. }\end{array}$ \\
\hline \hline $6-2$ & $330 \pm 180$ & $24 \pm 2.9$ & $24 \pm 6.4$ & $0 \pm 32$ & $1.2 \pm 0.5$ & $0 \pm 24$ & $0 \pm 1.8$ & (a) & $0.7 \pm 0.5$ & (a) & $2.9 \pm 2.0$ \\
\hline $6-4$ & $1200 \pm 590$ & $1600 \pm 550$ & $580 \pm 93$ & $0 \pm 96$ & $20 \pm 2.8$ & $0 \pm 190$ & $0 \pm 5.5$ & (a) & $5.7 \pm 0.7$ & (a) & $3.7 \pm 0.8$ \\
\hline $6-6$ & $\begin{array}{r}1500+1500 \\
-160\end{array}$ & $\begin{array}{r}3300+3300 \\
-160\end{array}$ & $\begin{array}{c}860+860 \\
-55\end{array}$ & $0 \pm 15$ & $\begin{array}{r}43+43 \\
-5.2\end{array}$ & $0 \pm 8.2$ & $0 \pm 930$ & (a) & $\begin{array}{r}14+14 \\
-5.9\end{array}$ & (a) & $\begin{array}{r}14+14 \\
-7.1\end{array}$ \\
\hline $6-8$ & $2300 \pm 270$ & $7900 \pm 540$ & $1500 \pm 120$ & $0 \pm 30$ & $73 \pm 6.9$ & $0 \pm 41$ & $0 \pm 1.3$ & (a) & $11 \pm 4.8$ & (a) & $9.6 \pm 5.8$ \\
\hline $6-10$ & $2300 \pm 250$ & $11000 \pm 500$ & $1400 \pm 78$ & $0 \pm 12$ & $39 \pm 5.0$ & $0 \pm 30$ & $0 \pm 1.1$ & (a) & $6.1 \pm 3.5$ & (a) & $6.3 \pm 4.1$ \\
\hline
\end{tabular}

Table 4.13.4. Ammonia Data from Tank S-111 Samples

\begin{tabular}{|c|c|c|c|}
\hline Sample & $\begin{array}{c}\text { Isotopically Measured } \\
\text { Total } \mathrm{NH}_{3} \\
(\mu \mathrm{mol} / \mathrm{L})\end{array}$ & $\left|\begin{array}{c}\text { Measured } \mathrm{NH}_{3} \text { partial } \\
\text { pressure at about } 12^{\circ} \mathrm{C} \\
(\mathrm{atm})\end{array}\right|$ & $\begin{array}{c}\text { RGS } \mathrm{NH}_{3} \text { Concentrations in the } \\
\text { Liquid }\end{array}$ \\
\hline $6-2$ & $13000 \pm 8600(a)$ & $0.0020 \pm 0.0010$ & $\begin{array}{l}0.013 \mathrm{M}(220 \mu \mathrm{g} / \mathrm{mL}) \mathrm{NH}_{3} \text { in } \\
\text { supernatant; non-RGS } \\
\text { measurements are much lower }\end{array}$ \\
\hline $6-4$ & $55000 \pm 18000^{(a)}$ & -0.0058 & \multirow{4}{*}{$\begin{array}{l}\text { The RGS ammonia data in the } \\
\text { nonconvective layer are question } \\
\text { able but suggest a concentration } \\
\text { of } 0.09 \mathrm{M} \text { (1500 } \mu \mathrm{g} / \mathrm{mL}) \mathrm{NH}_{3} \text { in } \\
\text { the interstitial liquid, or } 0.065 \\
\text { wt\% } \mathrm{NH}_{3} \text { in the bulk waste. }\end{array}$} \\
\hline $6-6$ & $\begin{aligned} 58000 & +58000(a) \\
& -17000\end{aligned}$ & $0.0079 \pm 0.0039$ & \\
\hline $6-8$ & $35000 \pm 10000^{(a)}$ & $0.0066 \pm 0.0033$ & \\
\hline $6-10$ & $82000 \pm 26000^{(a)}$ & $0.010 \pm 0.005$ & \\
\hline
\end{tabular}

(a) These data were used to calculate in situ ammonia vapor pressures (Section C.13 has details). 
Non-RGS ammonia measurements in the nonconvective layer were below the detection limit of about $18,000 \mu \mathrm{mol} \mathrm{NH}_{3} / \mathrm{L}$ of waste. Leaving out the physically impossible negative ammonia partial pressure measured for sample 6-4, the partial pressures suggested concentrations somewhat less than twice those in Tank AW-101 waste, which had a lower bound of $27,000 \mu \mathrm{mol}$ $\mathrm{NH}_{3} / \mathrm{L}$ waste. This approximate estimate of 45,000 to $60,000 \mu \mathrm{mol} \mathrm{NH}_{3} / \mathrm{L}$ waste is very roughly consistent with the isotopic measurements but is not consistent with the non-RGS measurements.

The inconsistencies in the ammonia data are such that it could be misleading to calculate an average ammonia concentration for Tank S-111. The data ranged widely from a lower bound of $4,000 \mu \mathrm{mol} \mathrm{NH} / \mathrm{L}$ liquid (supernatant) to an upper bound of $80,000 \mu \mathrm{mol} \mathrm{NH}_{3} / \mathrm{L}$ waste (nonconvective layer).

Table 4.13.5 contains the composition of the gas/vapor phase in each sample and the integrated average composition for S-111. The water vapor is not included in these compositions. The ammonia fractions are derived from the data in Table 4.13.4, which were used to calculate vapor pressures at in situ conditions. The sample compositions in the table have been calculated using the in situ solubility method described in Section 3.6.2. As discussed in Section 3.6.1, compositions were calculated for both the lower-bound and upper-bound gas solubilities. Both ends of the composition range are given in Table 4.13.5 with the measurement uncertainties on each. The average composition of the gas in the nonconvective layer is the result of integrating RGS species concentrations over the waste layer and multiplying them by the layer volume. The integration method is described in Section 3.7.1. The composition of the gas in the supernatant layer was taken to be equal to that in sample 6-2, the only sample located entirely within the layer.

Because the corrected composition of sample 6-6 is the ratio of non-argon gases, it is reliable even though the concentration of ammonia and the gas volume fraction are highly uncertain. Because the absolute concentrations of gases and ammonia are needed to calculate the tank average

Table 4.13.5. Sample and Overall Average Compositions of Retained Gas in Tank S-111 with Correction for Gas Contamination(a)

\begin{tabular}{||c|c|c|c|c|c|c||}
\hline Sample & $\mathrm{N}_{2}$ (mol\%) & $\mathrm{H}_{2}$ (mol\%) & $\mathrm{N}_{2} \mathrm{O}$ (mol\%) & $\mathrm{NH}_{3}$ (mol\%) $^{(\mathrm{b})}$ & $\mathrm{CH}_{4}$ (mol\%) & Other (mol\%) \\
\hline \hline $6-2$ & $90 \pm 68--91 \pm 69$ & $6.4 \pm 3.4--6.1 \pm 3.3$ & $2.2 \pm 1.3--0.9 \pm 0.6$ & $0.1 \pm 0.1$ & $0.3 \pm 0.2$ & $\begin{array}{c}1.0 \pm 0.9 \\
1.2 \pm 1.0\end{array}$ \\
\hline $6-4$ & $36 \pm 22--38 \pm 23$ & $48 \pm 24--50 \pm 25$ & $14 \pm 5.7-11 \pm 4.5$ & $0.7 \pm 0.4--0.4 \pm 0.2$ & $0.6 \pm 0.2$ & $0.3 \pm 0.1$ \\
\hline $6-6$ & $26 \pm 3.4--27 \pm 3.5$ & $58 \pm 5.0--59 \pm 5.1$ & $14 \pm 1.3--12 \pm 1.2$ & $1.0 \pm 0.3--0.6 \pm 0.2$ & $0.8 \pm 0.1$ & $0.5 \pm 0.2$ \\
\hline $6-8$ & $20 \pm 2.8$ & $67 \pm 7.1--68 \pm 7.1$ & $12 \pm 1.4-11 \pm 1.3$ & $0.6 \pm 0.2--0.4 \pm 0.1$ & $0.6 \pm 0.08$ & $0.2 \pm 0.1$ \\
\hline $6-10$ & $16 \pm 2.0$ & $73 \pm 5.7--74 \pm 5.6$ & $9.3 \pm 0.8--8.8 \pm 0.7$ & $1.4 \pm 0.4-0.8 \pm 0.2$ & $0.3 \pm 0.04$ & $0.2 \pm 0.1$ \\
\hline $\begin{array}{l}\text { Avg in } \\
\text { liquid(c) }\end{array}$ & $90 \pm 68--91 \pm 69$ & $6.4 \pm 3.4--6.1 \pm 3.3$ & $2.2 \pm 1.3--0.9 \pm 0.6$ & $0.1 \pm 0.1$ & $0.3 \pm 0.2$ & $1.0 \pm 0.9$ to \\
\hline $\begin{array}{l}\text { Avg in non- } \\
\text { convective } \\
\text { layer(c) }\end{array}$ & $21 \pm 5.6--22 \pm 5.7$ & $66 \pm 10-67 \pm 11$ & $11 \pm 1.8--10 \pm 1.6$ & $1.0 \pm 0.3-0.5 \pm 0.2$ & $0.5 \pm 0.08$ & $0.2 \pm 0.07$ \\
\hline
\end{tabular}


composition, sample 6-6 was not included in the average. Its composition was about halfway between the compositions of the adjacent samples, so its omission had little effect on the average.

The concentrations and pressures in Tables 4.13.2 and 4.13.3 can also be found in the sample-by-sample worksheets in the S-111 RGS calculation spreadsheet on the CD. The compositions in Table 4.13 .5 are in the "Inventory" worksheet.

\subsubsection{Gas Inventory}

The method by which the in situ gas volume fractions (wet basis) were calculated is given in Section 3.6.2; for gas composition, the volume fractions are given as a range from the low gassolubility value to the high gas-solubility value. The results are presented in Table 4.13.6, which also contains the average gas volume fraction and the average pressure experienced by the gas in the nonconvective layer. The average is an in situ volume average calculated by Simpson's Rule integration, as described in Section 3.7.1. The corrected gas volume fractions in Table 4.13.6 are consistent with the corrected gas concentrations and compositions in Tables 4.13.2, 4.13.3, and 4.13.5. The information in Table 4.13.6 is taken from the "Summary" and "In situ" worksheets of the S-111 RGS calculation spreadsheet on the CD.

The "sampler gas volume fraction" is corrected only for inleakage during extraction and when air gases added in the isotopic solution. It is an attempt to reconstruct the total amount of gas (sample and entrainment) that was present in the sampler during X-ray and is used only for comparison with the $\mathrm{x}$-ray observations of "visible gas fraction" discussed in Section 4.13.4.

Table 4.13.6 also contains the water vapor pressures that were used for in situ calculations. The water vapor pressures were found by using salt concentrations from Steen (1996) and the temperatures in the table as inputs to Equation 6.2 of Mahoney and Trent (1995), a correlation for

Table 4.13.6. In Situ Gas Volume Fractions and Conditions in Tank S-111

\begin{tabular}{||c|c|c|c|c|c|c||}
\hline Sample & $\begin{array}{c}\text { Sample } \\
\text { Hentral } \\
\text { (cm) }\end{array}$ & $\begin{array}{c}\text { Hydro- } \\
\text { static } \\
\text { Pressure } \\
\text { (atm) }\end{array}$ & $\begin{array}{c}\text { Calculated } \\
\text { Water Vapor } \\
\text { Pressure } \\
\text { (atm) }\end{array}$ & $\begin{array}{c}\text { Temp } \\
\text { ( } \mathrm{C} \text { ) }\end{array}$ & $\begin{array}{c}\text { Corrected Gas Volume } \\
\text { Fraction(a) } \\
\text { (in-tank conditions) }\end{array}$ & $\begin{array}{c}\text { Sampler Gas } \\
\text { Volume Fraction } \\
\text { (in situ conditions, } \\
\text { low solubility) }\end{array}$ \\
\hline \hline $6-2$ & 458 & 1.08 & 0.019 & 26.4 & $0.008 \pm 0.002$ to $0.007 \pm 0.002$ & 0.024 \\
\hline $6-4$ & 362 & 1.21 & 0.015 & 28.5 & $0.070 \pm 0.021$ to $0.066 \pm 0.021$ & 0.082 \\
\hline $6-6$ & 265 & 1.34 & 0.013 & 30.7 & $0.15 \pm 0.05$ (b) & 0.44 \\
\hline $6-8$ & 169 & 1.47 & 0.013 & 31.6 & $0.20 \pm 0.029$ & 0.21 \\
\hline $6-10$ & 72 & 1.60 & 0.013 & 31.7 & $0.23 \pm 0.032$ to $0.22 \pm 0.032$ & 0.24 \\
\hline $\begin{array}{l}\text { Avg in } \\
\text { supernatant(c) }\end{array}$ & 510 & 1.01 & & & $0.008 \pm 0.003$ to $0.007 \pm 0.002$ & \\
\hline $\begin{array}{l}\text { Avg in non- } \\
\text { convective } \\
\text { layer(c) }\end{array}$ & 184 & 1.45 & & & $0.15 \pm 0.075$ to $0.14 \pm 0.070$ & \\
\hline $\begin{array}{l}\text { (a) The gas volume fraction is expressed on a wet basis, including the volume contribution of water vapor. } \\
\text { (b) The corrected value given is halfway between the void fractions that are calculated with and without excluding the } \\
\text { argon volume from the effective sampler volume. } \\
\text { (c) The uncertainties on the layer-average gas volume fractions are based on spatial variability considerations discussed } \\
\text { in Section 3.7.2. }\end{array}$ \\
\hline
\end{tabular}


water vapor pressure over concentrated homogeneous and nonhomogeneous waste simulants. The gas solubilities used the same parameters as inputs to the Schumpe solubility model (Section 3.6.1). The water vapor pressures and gas solubilities used in calculations can be found in the "In situ" worksheet of the S-111 RGS calculation spreadsheet on the CD. Because of the inconsistencies in the ammonia data, no ammonia inventory was calculated for Tank S-111.

Table 4.13.7 gives various estimates of the STP volume of gas in Tank S-111, including estimates calculated from RGS data alone. The RGS gas inventory in the nonconvective layer was calculated by integrating RGS total gas concentrations over the layer (three data points, omitting sample 6-6 because of the uncertainty caused by the argon intrusion) and multiplying the average gas concentration by the volume of the layer. The integration method is described in Section 3.7.1. The gas inventory in the supernatant layer was calculated from the single sample in that layer. The RGS volumes in Table 4.13.7 include corrections to remove the contamination gas: entrained air and argon, helium backfill, air leaks during and after extraction, and air dissolved in the isotopic standard. The uncertainties on the gas inventories are based on the spatial variability considerations discussed in Section 3.7.2. The information in Table 4.13.7 is taken from the "Inventory" worksheet of the S-111 RGS calculation spreadsheet on the CD.

The gas inventories calculated by the RGS and BPE methods are within $4 \%$ of each other. It is interesting that the two inventory estimates should be so close, considering that there were only three data points in the RGS integration and that the measured waste level fluctuations could have been biased high (as for S-106) by a supernatant layer that did not cover the entire waste surface. Most of the 1996 non-RGS samples from riser 8 contained 17 to 19 inches of sample, suggesting gas fractions no greater than 0.15 (and often less). However, the core extrusion observations are imprecise measures of gas fraction. We recommend that the RGS gas inventory estimate be used as the better estimate of the retained gas in Tank S-111 at the time of sampling.

It is possible that the gas volume fraction was roughly 0.2 all the way through the nonconvective layer (at least at riser 6). As stated in the footnote to Table 4.13.6, if the argon volume is excluded from the effective sampler volume in sample 6-6, the gas volume fraction of that sample is about 0.2 . In addition, the 0.066 gas volume fraction in sample 6-4 appeared (based on the X-ray) to be in the bottom third of the sampler. It is likely that only the bottom third of the sampler contained solids and the rest was supernatant liquid (as in sample 6-2). If so, then the

Table 4.13.7. S-111 Gas Inventory Estimates

\begin{tabular}{|c|c|c|c|}
\hline \multirow{2}{*}{ Quantity } & \multicolumn{2}{|c|}{ RGS Method } & BPE Method(a) \\
\hline & $\begin{array}{l}\text { Supernatant } \\
\text { Layer }\end{array}$ & $\begin{array}{c}\text { Nonconvective } \\
\text { Layer }\end{array}$ & Tank Total \\
\hline $\begin{array}{l}\text { Avg. gas fraction } \\
\text { (low gas solubility) }\end{array}$ & $0.008 \pm 0.003$ & $0.15 \pm 0.075$ & $0.15 \pm 0.034$ \\
\hline $\begin{array}{l}\text { Gas volume }\left(\mathrm{m}^{3}\right) \\
\text { in situ (wet) }\end{array}$ & $0.3 \pm 0.1$ (b) & $293 \pm 146(b)$ & $303 \pm 67$ \\
\hline STP (wet) & $0.3 \pm 0.1(b)$ & $382 \pm 196(b)$ & $393 \pm 88$ \\
\hline $\begin{array}{l}\text { (a) Barometric press } \\
\text { pressure calculated fr } \\
\text { in./in.-Hg (Whitney e } \\
\text { (b) The retained gas } \\
\text { better estimate. }\end{array}$ & $\begin{array}{l}\text { ethod. The in } \\
\text { GS data, and } \\
\text { (997). } \\
\text { tory calculate }\end{array}$ & $\begin{array}{l}\text { ry is based on } \\
\text { the RGS metho }\end{array}$ & $\begin{array}{l}\text { average gas } \\
-0.67 \pm 0.13 \\
\text { considered the }\end{array}$ \\
\hline
\end{tabular}


volume fraction in the high-solids third of sampler 6-4 was about 0.2 . An in situ gas volume fraction of 0.2 throughout the nonconvective layer, which is an average of $505 \mathrm{~cm}$ (199 in.) thick, corresponds to an in situ gas volume of $410 \mathrm{~m}^{3}$.

Table 4.13.8 contains the calculated layer inventories of each of the major gases retained in the waste. These inventories are based on the layer-average compositions from RGS data (Table 4.13.5) and the best-estimate gas inventories (RGS, Table 4.13.7).

Figure 4.13.3 shows sample temperatures, corrected gas volume fractions (Table 4.13.6), and corrected compositions of the low-solubility constituents in the samples from Tank S-111. The temperatures were measured by the thermocouple (TC) tree in riser 4 . The compositions represent the mole fraction of the species in the "insoluble" gas; water and ammonia are not included, so the mole fractions are not the same as those in Table 4.13.5. The gas volume fractions and mole fractions in the figure are the values for lower-bound gas solubilities. Figure 4.13 .3 also lists the observations from core extrusions of non-RGS samples and from X-rays of RGS samples as a way of tying those observations together with RGS data. The x-ray data are described in more detail in Section 4.13.4.

Table 4.13.8. Speciated S-111 Gas Inventory(a)

\begin{tabular}{|c|c|c|c|c|}
\hline \multirow[b]{2}{*}{ Species } & \multicolumn{2}{|c|}{$\begin{array}{c}\text { Gas-Phase Inventory } \\
\left(\mathrm{m}^{3} \text { at STP }\right)\end{array}$} & \multicolumn{2}{|c|}{$\begin{array}{c}\text { Dissolved Inventory } \\
\left(\mathrm{m}^{3} \text { at STP }\right)\end{array}$} \\
\hline & $\begin{array}{c}\text { Nonconvective } \\
\text { Layer }\end{array}$ & $\underset{\text { Layer }}{\text { Supernatant }}$ & $\begin{array}{c}\text { Nonconvective } \\
\text { Layer }\end{array}$ & $\begin{array}{c}\text { Supernatant } \\
\text { Layer }\end{array}$ \\
\hline $\mathrm{N}_{2}$ & $81-80$ & $0.27-0.24$ & $0.12-0.49$ & $0.0097-0.037$ \\
\hline $\mathrm{H}_{2}$ & 250 & $0.019-0.016$ & $0.70-2.0$ & $0.0018-0.0048$ \\
\hline $\mathrm{N}_{2} \mathrm{O}$ & $43-37$ & $0.0064-0.0023$ & $3.2-8.5$ & $0.014-0.018$ \\
\hline $\mathrm{NH}_{3}$ & $3.6-2.0$ & $0.00041-0.00020$ & 2500 & 11 \\
\hline $\mathrm{CH}_{4}$ & 1.8 & $0.00091-0.00074$ & $0.0046-0.018$ & $0.000065-0.00023$ \\
\hline Other & 0.60 & 0.0030 & 0 & 0 \\
\hline \multicolumn{5}{|c|}{$\begin{array}{l}\text { (a) Inventories based on layer-average compositions from RGS data (Table 4.13.5) and best- } \\
\text { estimate gas inventories for each layer (RGS, Table 4.13.7). Uncertainty in each inventory is } \\
50 \% \text { based on spatial variability considerations (Section 3.7.2). Inventories given for lower- and } \\
\text { upper-bound solubilities; lower-bound solubility basis first. }\end{array}$} \\
\hline
\end{tabular}

\subsubsection{X-Ray Results}

Table 4.13.9 summarizes all the available radiography observations from Tank S-111. The $\mathrm{x}$-ray images themselves can be found on the CD that accompanies this report. The uncertainties of the visible gas volume fractions that are given in Table 4.13.9 are unknown but (based on the uncertainty in measuring the gas gap from x-ray images) are expected to be larger than the uncertainties in the gas volume fractions calculated from gas extraction. (The "visible" gas volume fraction refers to the gas that has separated from the solids and liquid such that its volume can be calculated based on the image. The uncorrected gas fractions from extraction are used for comparison to reflect the presence of entrained air in the sampler.) 


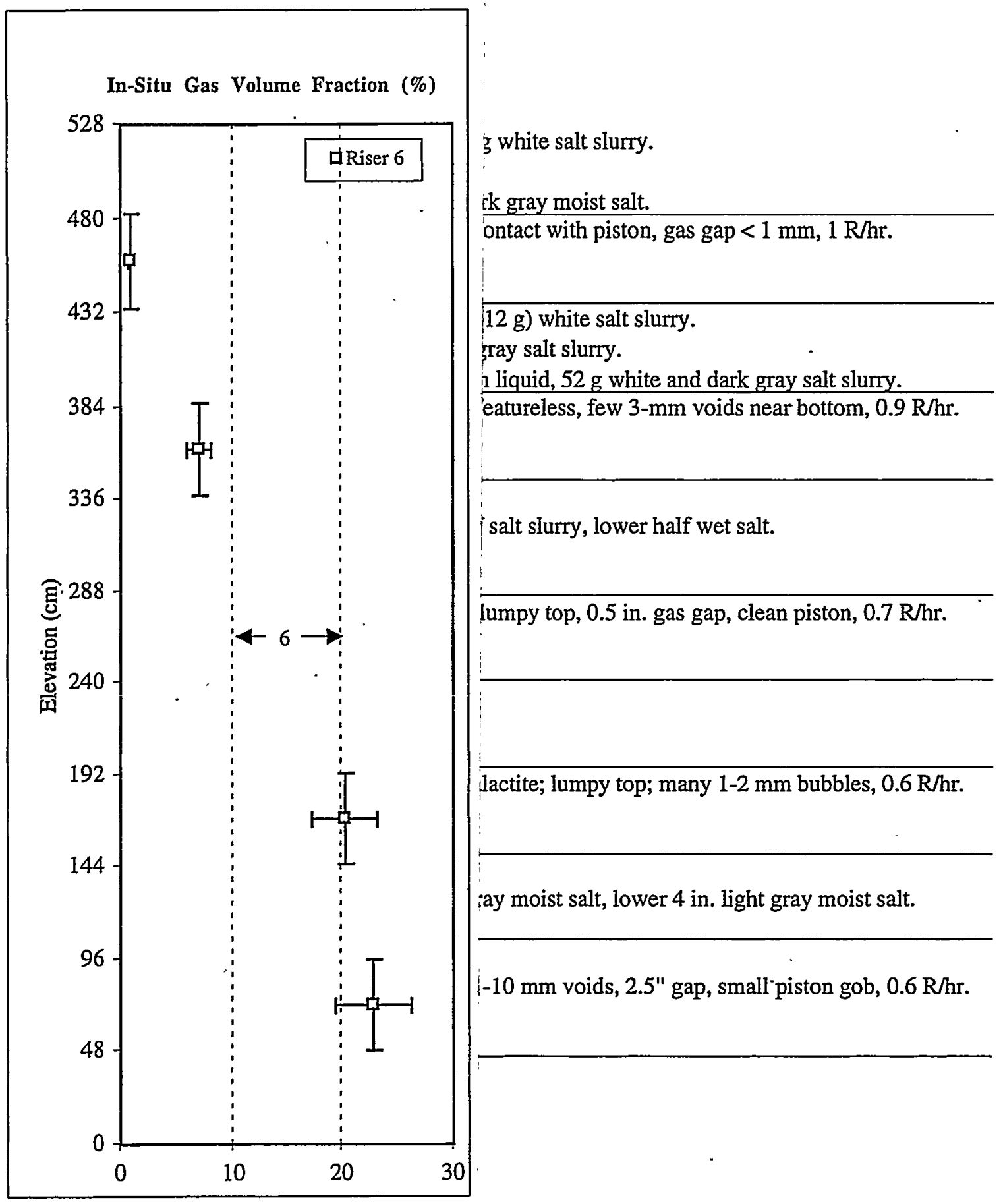



Table 4.13.9. Summary of Observations from X-Ray Images of Tank S-111

\begin{tabular}{|c|c|}
\hline Segment & Comments/Observations \\
\hline $\begin{array}{l}6-2 \\
\text { (RGS, } \\
4 / 8 / 98)\end{array}$ & $\begin{array}{l}\text { The image was featureless. There was a gas gap of about } 0.1 \mathrm{~cm}(0.04 \mathrm{in} \text {.) below a clean } \\
\text { piston. The visible gas volume fraction is } 0.002 \text {. (Compare with the sampler gas volume } \\
\text { fraction of } 0.024 \text { in Table } 4.13 .6 \text { obtained by gas extraction.) }\end{array}$ \\
\hline $\begin{array}{l}6-4 \\
\text { (RGS, } \\
4 / 9 / 98)\end{array}$ & $\begin{array}{l}\text { This sample contained several } 1-3 \mathrm{~mm} \text { darker (denser) features and several gas bubbles of } \\
1 \text { to } 3 \mathrm{~mm} \text { diameter in the bottom } 2 \text { inches of the sample but no features visible above, } \\
\text { suggesting liquid in most of the upper portion. There is a flat top surface and a gas gap of } 2.0 \\
\mathrm{~cm}(0.8 \text { in.). The piston is clean. The visible gas volume fraction (not counting bubbles) is } \\
\text { therefore } 0.04 \text {. (Compare with the sampler gas yolume fraction of } 0.082 \text { in Table } 4.13 .6 \\
\text { obtained by gas extraction.) }\end{array}$ \\
\hline $\begin{array}{c}6-6 \\
\text { (RGS, } \\
4 / 9 / 98)\end{array}$ & $\begin{array}{l}\text { This sample contained numerous curved-slit voids of } 2 \text { to } 5 \text {-mm size throughout the sample, and } \\
\text { one } 5-\mathrm{mm} \text { round bubble near the top. There is a lumpy top surface and a clean piston, with a } \\
1.3-\mathrm{cm}(0.5 \text {-in.) gas gap below it. The visible gas volume fraction (not counting bubbles) is } \\
\text { therefore } 0.03 \text {. (Compare with the values in Table } 4.13 .6 \text { obtained by gas extraction: } \\
\text { uncorrected sampler gas volume fraction of } 0.44 \text {, or the corrected value of } 0.15 \pm 0.05 \text {.) }\end{array}$ \\
\hline $\begin{array}{c}6-8 \\
\text { (RGS, } \\
4 / 9 / 98)\end{array}$ & $\begin{array}{l}\text { This sample contained numerous curved-slit voids of } 2 \text { to } 5 \text {-mm size throughout the sample, } \\
\text { with one long void ( } 1 \text { to } 2 \mathrm{~mm} \text { by } 10 \mathrm{~mm} \text { ) near the bottom. Near the top of the sample, all the } \\
\text { bubbles are in the } 1 \text { to } 2-\mathrm{mm} \text { range. There is a lumpy top surface, a } 6-\mathrm{mm}(0.25 \mathrm{in} \text {.) thick } \\
\text { stalactite across about half of the piston, with a } 2.4-\mathrm{cm} \text { (1-in.) gas gap below it. The visible } \\
\text { gas volume fraction (not counting bubbles) is therefore } 0.05 \text {. (Compare with the sampler gas } \\
\text { volume fraction of } 0.21 \text { in Table } 4.13 .6 \text { obtained by gas extraction.) }\end{array}$ \\
\hline $\begin{array}{l}6-10 \\
\text { (RGS, } \\
4 / 10 / 98)\end{array}$ & $\begin{array}{l}\text { There are two full-diameter voids (or fracture bubbles), one near the bottom, the other about } \\
\text { halfway up. Both voids have rough steeply sloped tops, and the upper one has separated liquid } \\
\text { (a slightly less dense flat-surfaced layer) at the bottom There are many } 1 \text { to } 3 \text {-mm voids toward } \\
\text { the middle of the sample, with } 3 \text { to } 10-\mathrm{mm} \text { voids near the bottom. A layer of separated liquid is } \\
\text { at the top of the waste and there is a } 6.4-\mathrm{cm} \text { ( } 2.5 \text {-in.) gas gap below the piston, which has a } \\
\text { small gob of waste attached to it and is } 0.3 \text { in short of full retraction. The visible gas volume } \\
\text { fraction (including the full-diameter bubbles but not the smaller ones) is } 0.20 \text {. (Compare with } \\
\text { the sampler gas volume fraction of } 0.24 \text { in Table } 4.13 .6 \text { obtained by gas extraction.) }\end{array}$ \\
\hline
\end{tabular}

Table 4.13.9 compares $\mathrm{x}$-ray-derived gas volume fractions with extraction-derived gas volume fractions. The comparison shows that sample recovery was close to $100 \%$ for all the samples. This conclusion is based on the fact that the extracted uncorrected gas was equal to or a few volume percent more than the visible gas.

The major peculiarities of S-111 waste, as observed from X-ray images, were slit-shaped bubbles and, in segment 6-10, two fracture bubbles spanning the sampler diameter. Both of the fracture bubbles had sloped tops. The bottom of the lower fracture could not be observed, but the bottom of the upper one was a flat layer of separated liquid. There was also a layer of separated liquid (about $6 \mathrm{~mm}$ deep) at the top of the waste sample. In addition, two of the S-111 samples had small amounts of waste (less than $6 \mathrm{~mm}$, or $0.25 \mathrm{in}$.) stuck to the piston. These observations indicate waste that was cohesive and, to a lesser extent, adhesive. The separated liquid layer on the waste surface above fracture bubbles suggests that, for some reason, liquid was not free to drain from the top into the bubbles. 
Sample 6-6 was measured by extraction to contain much more gas (volume fraction of 0.44 ) than is visible on the $\mathrm{x}$-ray (volume fraction of 0.03 ). Most of this gas was argon. Leaving out the argon, the gas volume fraction present (based on the entire sampler volume) was about 0.1 . This value is not inconsistent with the $\mathrm{x}$-ray observation of 0.03 , considering that sample 6-8 (with only trace argon) had a visible gas volume fraction of 0.05 and an extracted gas volume fraction of 0.20 . Aside from having unusually large amounts of gas concealed in the matrix, samples 6-6 and 6-8 were also similar in the form the gas took: a small gas gap and voids in the shape of curved slits.

Sample 6-10 contained flat-surfaced less-dense features that suggested the presence of separated liquid. This sample apparently contained considerably more liquid than the 1996 nonRGS samples 8-9, 8-10, and 8-11, which were described as moist or dry salt. This higher moisture level was consistent with the higher ammonia concentration measured in 6-10, since ammonia is believed to be present primarily in sample liquid.

Table 4.13.10 is a summary of the waste densities that have been calculated from radiographic data for the RGS samples for which air and water standard images were available. The radiographic densities include the gas in the waste, were calculated by the current $\mathrm{x}$-ray analysis method, and are given for several locations within each sample to show density trends. The table also includes density measurements made on extruded non-RGS samples (Steen 1996). In sample 6-2, the radiographic densities were much higher than would be expected, considering the evidence that the segment contained liquid. Improbably high radiographic densities-were also found in part of sample 6-10.

To get a rough estimate of the gas in the matrix in sample 6-8, the densities of the darkest (densest) parts in the $\mathrm{x}$-ray images were calculated. These maximum densities were between 1.8 and $1.9 \mathrm{~g} / \mathrm{cc}$ and probably represent the densities of low-gas portions of the waste. By comparison, the mean densities. in the image, which include the average gas content of sample 6-8, were 1.67 to $1.76 \mathrm{~g} / \mathrm{cc}$ (Table 4.13.10). The gas volume fraction in the matrix that was estimated by comparing the mean and maximum densities was 7 to $12 \mathrm{vol} \%$ in sample 6-8. This estimate is consistent with the 0.15 volume fraction difference between the extracted-basis gas volume fraction of 0.20 and the visible-gap gas volume fraction of 0.05 .

\subsubsection{Other Discussions}

\subsubsection{High Argon Content}

Mass spectrometric analysis of the gas in sample 6-6 of S-111 found that the gas contained more than $70 \mathrm{~mol} \%$ argon (about $96 \mathrm{cc}$ of argon at in situ conditions). Because there are strong reasons to believe that argon is not generated in or native to tank waste, we looked for an external source for the argon. Every source considered was contradicted by one or more observations, and as yet no plausible explanation has been found.

The argon-to-oxygen ratio was greater than 5000, much too high to agree with atmospheric air as an argon source. One possible source was drill string purge gas, which is argon. The Industrial Hygiene records do not contain enough information to either confirm or deny that the drill string was purged before acquisition of sample 6-6. (This level of detail is not a formal requirement.) Furthermore, the $x$-ray images of sample 6-6 showed only 0.03 volume fraction of gas. It is nearly inconceivable that the true gas volume fraction could have been as high as 0.42 (the value including argon, Table 4.13 .6 ) with only 0.03 volume fraction being visible. Therefore it is 
Table 4.13.10. Densities of S-111 Samples from Radiography

\begin{tabular}{|c|c|c|c|}
\hline \begin{tabular}{|l|} 
Riser- \\
Segment \\
Number \\
\end{tabular} & $\begin{array}{c}\text { Distance from } \\
\text { Bottom of Sampler } \\
\text { (ft) }\end{array}$ & $\begin{array}{c}\begin{array}{c}\text { Calculated Mean Density } \\
\text { by Current Method } \\
(\mathrm{g} / \mathrm{cc})\end{array} \\
\end{array}$ & $\begin{array}{l}\text { Degassed Density Above and } \\
\text { Below the RGS Sample } \\
\text { (from cores) (g/cc) }\end{array}$ \\
\hline \multirow[t]{6}{*}{$6-2(a)$} & 1.50 & $2.26(\mathrm{~b})$ & \multirow{6}{*}{$\begin{aligned} & \text { density above, } 1.60 \mathrm{~g} / \mathrm{cc} \text { bulk } \\
& 1.43 \mathrm{~g} / \mathrm{cc} \text { liquid }\end{aligned}$} \\
\hline & 1.25 & 2.17 & \\
\hline & 1.00 & 2.15 & \\
\hline & 0.75 & 2.18 & \\
\hline & 0.50 & 2.02 & \\
\hline & 0.25 & 2.13 & \\
\hline \multirow[t]{6}{*}{$6-4$} & 1.42 & $1.44(\mathrm{~b})$ & \multirow{6}{*}{$\begin{aligned} & \text { density above, } 1.47 \mathrm{~g} / \mathrm{cc} \text { bulk } \\
& 1.44 \mathrm{~g} / \mathrm{cc} \text { liquid }\end{aligned}$} \\
\hline & 1.26 & 1.56 & \\
\hline & 1.00 & 1.60 & \\
\hline & 0.75 & 1.51 & \\
\hline & 0.50 & 1.62 & \\
\hline & 0.25 & --- & \\
\hline \multirow[t]{5}{*}{$6-6$} & 1.25 & 1.56 (b) & \multirow[t]{5}{*}{ density above, $1.52 \mathrm{~g} / \mathrm{cc}$ bulk } \\
\hline & 1.00 & 1.50 & \\
\hline & 0.75 & 1.51 & \\
\hline & 0.50 & 1.52 & \\
\hline & 0.25 & 1.56 & \\
\hline \multirow[t]{5}{*}{$6-8$} & 1.25 & 1.76 & \multirow[t]{5}{*}{ density above, $1.64 \mathrm{~g} / \mathrm{cc}$ bulk } \\
\hline & 1.00 & 1.70 & \\
\hline & 0.75 & 1.70 & \\
\hline & 0.50 & 1.66 & \\
\hline & 0.25 & 1.67 & \\
\hline \multirow[t]{5}{*}{$6-10$} & 1.25 & 1.68 (c) & \multirow[t]{5}{*}{ density above, $1.61 \mathrm{~g} / \mathrm{cc}$ bulk } \\
\hline & 1.00 & $1.86(\mathrm{c})$ & \\
\hline & 0.75 & 2.21 & \\
\hline & 0.50 & 2.07 & \\
\hline & 0.25 & 1.32 & \\
\hline
\end{tabular}

hard to credit that the argon could have been introduced before the x-ray was taken. (We have also confirmed that the images we were using for sample 6-6 really were images of sample 6-6, and that the casks used to ship the RGS samplers to the 222-S facility are not pressurized and use an air, not an argon, atmosphere.) 
Another possible source of argon was the RGS argon used for BSVD. This standard part of the RGS procedure introduces argon to pressurize the system; the change in pressure and the known system vessel volumes are used to find the volume of solids and liquids in the sample. However, this procedure (typically carried out only after extraction is complete) was not performed at all for sample 6-6 because the pressure transducer needed for it was not operating. An even stronger argument is the fact that the pressure measurements before and during extraction (measurements taken every 10 seconds) did not show any anomalies. The collector canister pressures were zero before extraction began, indicating that no argon or other gas was present. The extractor (sample vessel) pressures declined steadily during extraction, with no pressure increase to indicate gas injection. Neither collector nor extractor pressures showed any unexplained increases.

\subsubsection{Long Lag Times}

Procedural difficulties at the 222-S Laboratory caused delays in processing the RGS samples from Tank S-111. The lag times (delays between sample acquisition and sample processing) are shown in Table 4.13.1.

The RGS Acceptance Test Report (Cannon 1996) described three possible forms of sample deterioration that could occur over long lag times. The first, hydrogen seal permeation, would cause a change in the composition of the sample as hydrogen was lost. The second, air inleakage, would change both the composition and the amount of gas. The third, sample gas outleakage, would change the amount of gas in the sample but not the composition (no preferential leakage of any species was found during acceptance testing). In-sampler reaction is a fourth possible form of sample deterioration.

The experiments carried out during RGS acceptance testing (Cannon 1996) showed no measurable change in the hydrogen mole fraction for up to 76 days of sample aging. The apparent scatter in measurement was about $\pm 1 \mathrm{~mol} \% \mathrm{H}_{2}$. Because the change due to aging was less than $1 \mathrm{~mol} \% \mathrm{H}_{2}$ over 76 days, it is likely that the $\mathrm{S}-111$ lag times (as much as 132 days) would change the hydrogen mole fraction by less than $2 \mathrm{~mol}_{2} \mathrm{H}_{2}$, the acceptance criterion. For comparison, a theoretical conservative estimate of hydrogen loss rates was calculated to be $0.044 \%$ of the hydrogen inventory per day (at $100^{\circ} \mathrm{F}$ ). This leads to an estimate of $5.8 \%$ loss of hydrogen inventory over 132 days. Assuming the gas in an S-111 sampler is $66 \mathrm{~mol}^{\circ} \mathrm{H}_{2}$ (the average composition) before hydrogen loss, the gas after hydrogen loss would have a hydrogen fraction of $(0.66)(1-0.058) /(1-[0.66][0.058])$ or $0.646 \mathrm{~mol}_{0} \mathrm{H}_{2}$. The change in composition is less than the acceptance criterion by the conservative theoretical prediction as well as by extrapolation of measured data.

Air inleakage during an extended lag time is not a concern because air is corrected for in the data analysis. In any case, there was no visible correlation between the oxygen concentration and the lag time; only trace amounts of oxygen were present in the S-111 samples with lag times longer than 100 days.

The third lag-time concern is loss of sample gases. All samplers are leak-tested with helium before use. According to Cannon (1996), the average leak rate has been about $5 \times 10^{-7} \mathrm{~atm} / \mathrm{cc} / \mathrm{sec}$ at a pressure difference of $2 \mathrm{~atm}$. This corresponds to an out-leakage of about $0.043 \mathrm{STP} \mathrm{mL} /$ day (again, at a pressure difference of $2 \mathrm{~atm}$ ). The maximum pressure difference for $\mathrm{S}-111$ samplers, based on a maximum hydrostatic head of $1.6 \mathrm{~atm}$, is about $0.6 \mathrm{~atm}$. Assuming that the leak rate is proportional to the square root of the pressure difference, as for orifice flow, the estimated leak rate for the S-111 samplers is about (0.043 STP mL/day)(0.6/2) $)^{1 / 2}$, or $0.024 \mathrm{STP} \mathrm{mL} /$ day. At this rate, the amount of gas lost in 33 days (the lag time for sample 6-2) is $0.8 \mathrm{STP} \mathrm{mL}$; at 103 days (the lag time for sample 6-4), it is $2.5 \mathrm{STP} \mathrm{mL}$; and at 132 days (the longest lag time), it is 3.2 STP mL. These losses can be compared with the measured gas content of the samples: $7 \mathrm{cc}$ in 
$6-2,26 \mathrm{cc}$ in $6-4$, and 39 to $95 \mathrm{cc}$ in the other S-111 samples. The possible loss of gas is $10 \%$ or less of the original gas in all these cases. This loss is within the uncertainties of the gas volume fractions (Table 4.13.6). The maximum loss constitutes about 1 vol\% of the sampler volume.

Finally, there is a possibility that reaction might occur in the waste and change the gas composition, especially if inleaked oxygen is present as a reactant. Experiments with waste from Tank AN-105 found that the half-life for $\mathrm{O}_{2}$ removal at $25^{\circ} \mathrm{C}$ was 210 days, that 0.16 mole of $\mathrm{H}_{2}$ was formed per mole of $\mathrm{O}_{2}$ reacted, and that the production of $\mathrm{N}_{2}$ and $\mathrm{N}_{2} \mathrm{O}$ was $5 \%$ or less of the $\mathrm{H}_{2}$ production (Person 1998). The 76-day sampler test carried out during acceptance testing (Cannon 1996) found an inleaked oxygen concentration of only $0.047 \mathrm{~mol} \%$, showing that 0.23 $\mathrm{mL}$ of air (at STP) had inleaked. Assuming that the inleakage is proportional to the lag time and that AN-105 waste reaction rates hold for S-111 waste, the amount of hydrogen produced over a 132-day period would be $0.012 \mathrm{~mL}$ at STP. This amount of $\mathrm{H}_{2}$ generation would cause a composition error of less than $2 \%$ of the hydrogen mole fraction in $1 \mathrm{cc}$ of retained gas sample. An error of this magnitude is less than the acceptance criterion.

\subsubsection{Domespace Composition Comparison}

The RGS gas-phase composition data (Table 4.13.5) were used to calculate $\mathrm{H}_{2} / \mathrm{N}_{2} \mathrm{O}$ ratios to compare with ratios from domespace grab sample measurements (McCain 1999, Table B-15). The results can be seen in Table 4.13.11. The domespace $\mathrm{H}_{2} / \mathrm{N}_{2} \mathrm{O}$ ratio from October 22, 1997 was higher than the RGS ratios and than most other domespace ratios (though another equally high domespace ratio was measured on August 29,1997). The later domespace ratios were in good agreement with the RGS ratios in the upper part of the waste.

Table 4.13.11 Comparison with Domespace Data(a)

\begin{tabular}{|l|c|}
\hline Sample & $\mathrm{H}_{2} / \mathrm{N}_{2} \mathrm{O}$ \\
\hline RGS, 6-2 & $2.9-6.8$ \\
\hline RGS, 6-4 & $3.3-4.5$ \\
\hline RGS, 6-6 & $4.1-4.9$ \\
\hline RGS, 6-8 & $5.6-6.1$ \\
\hline RGS, 6-10 & $7.8-8.4$ \\
\hline Domespace, 10/22/97 & 13 \\
\hline Domespace, 1/23/98 & 4.8 \\
\hline Domespace, 4/17/98 & 4.4 \\
\hline Domespace, 8/19/98 & 3 \\
\hline $\begin{array}{l}\text { (a) RGS ratios were calculated for both lower- and upper- } \\
\text { bound solubilities. Both ends of the ratio range are given in } \\
\text { the table, lower-bound solubility basis first. }\end{array}$ \\
\hline
\end{tabular}




\section{$4.14 \quad U-109$}

Tank 241-U-109 (U-109) was the fourteenth tank and the tenth SST sampled with the RGS. This tank was selected to represent a group of SSTs that show evidence of significant gas retention (Stewart et al. 1996b). U-109 is on the FGWL and exhibits high response to barometric pressure changes, high concentrations of $\mathrm{H}_{2}$ in the headspace, and some level growth. U-109 is part of a group of tanks (Cluster 13 in Stewart et al. 1996) that have fairly high radioactivity, fairly low temperatures, high nitrite concentrations, and about $0.1 \%$ TOC. HTCEs indicate that the primary waste stored in U-109 was saltcake from evaporator campaign S1 and that the secondary waste was salt slurry from evaporator campaign S2 (Remund et al. 1995). (This is nearly identical to the HTCEs for Tanks U-103, SX-106, and S-102, which are also in Cluster 13.)

Push-mode sampling was done in riser 8 in April 1998.(a) Risers 2, 7, and 19 had been successfully sampled in December 1995 through January 1996 (Baldwin 1996). The approximate locations of various risers are depicted in Figure 4.14.1; risers 2, 7, 8, and 19 are near the tank periphery. The elevations of the RGS segments are depicted in Figure 4.14.2. The figure also shows the tank content layering, with supernatant liquid of varying depth overlying saltcake.

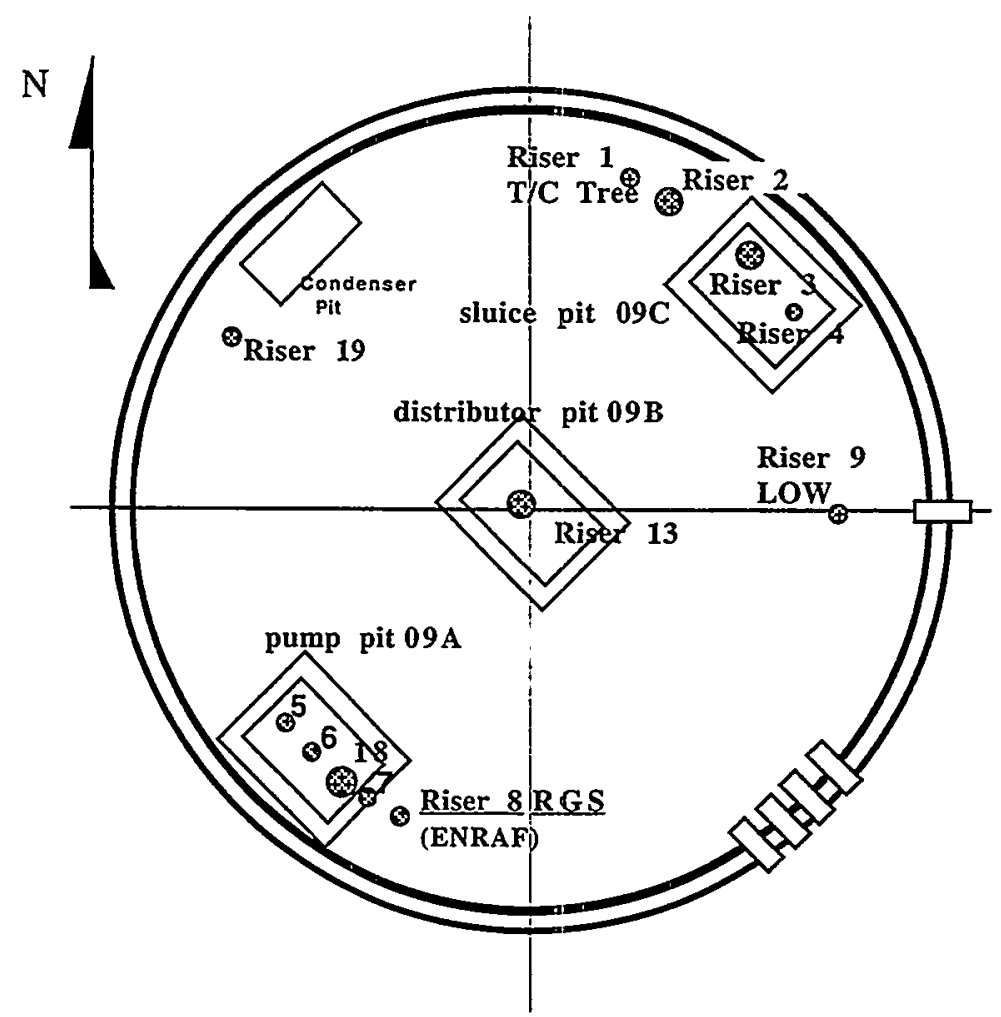

Figure 4.14.1. Schematic Diagram of Riser Locations in Tank U-109

(a) The sampling scheme may be found in Sampling Plan for Tank 241-U-109 Retained Gas Sampler Deployment, by JM Bates, April 1998. TWSFG98.42 Rev. 0, Pacific Northwest National Laboratory, Richland, Washington. 


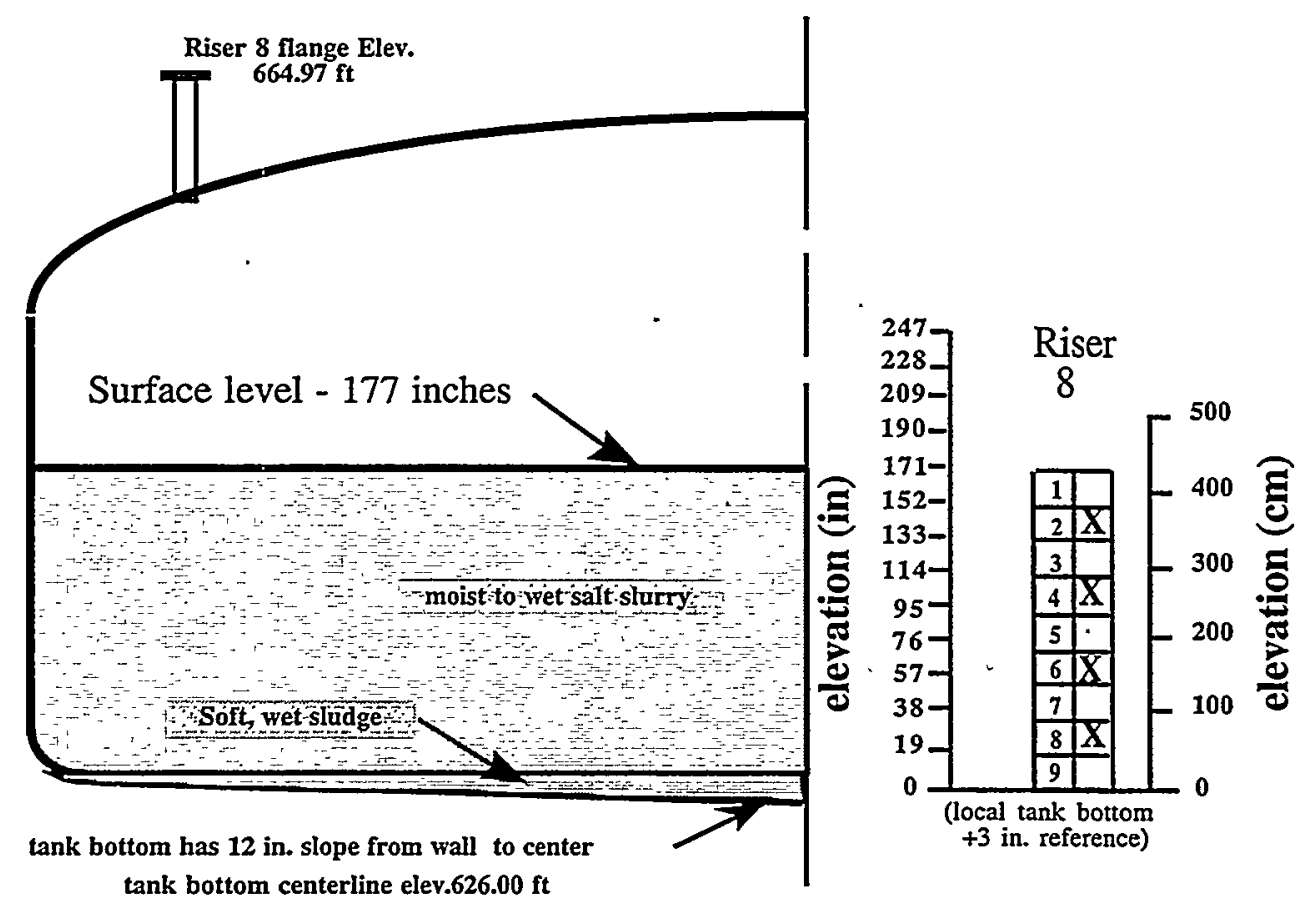

Figure 4.14.2. Diagram of Waste Layering and RGS Sample Elevations for U-109

Brevick et al. (1994) describe U-109 as a partially isolated, sound SST. Tank U-109 contained about 1,750,000 L (463,000 gal) of waste, including 71,900 L (19,000 gal) of supernatant (Hodgson et al. 1998). At the time of sampling, the total depth of waste in Tank U-109 was 434 to $450 \mathrm{~cm}$ (170 to $177 \mathrm{in}$.); it was $450 \mathrm{~cm}$ deep at riser 8 . Neutron scans in the riser $9 \mathrm{LOW}$ indicated liquid at the waste surface. Riser 9 is about three-fourths of a tank radius from the center of the tank, not far from the tank periphery.

The most recent information on U-109 layering came from the core observations made in 1996 (Baldwin 1996) in risers 2, 7, and 19 (all near the tank periphery). No supernatant layer was evident in any of the samples from those risers, or in the 1998 samples from riser 8. (Drainable liquid was found in 199.8 sample 8-1, but this was the result of contamination with hydrostatic head fluid.) The solid layer in the tank was described as moist salt, wet salt, or salt slurry, in some cases with a putty-like consistency. No sludge was observed.

The reported 71,900 L (19,000 gal) of supernatant would form a layer $17 \mathrm{~cm}$ ( 7 in.) deep if spread uniformly over the entire tank. However, no liquid was seen at any of the sampled risers, all of which were near the periphery. At another peripheral riser (riser 9) liquid was reported to be at the surface. On this evidence, it is likely that the supernatant liquid was of irregular depth, possibly deeper at the center, and was not present over the entire waste surface.

Densities for the liquid and bulk solids in Tank U-109 were available from cores taken in 1996 (Baldwin 1996) and from non-RGS samples taken in 1998.(a) Only one sample contained drainable liquid. Although this liquid came in part from hydrostatic head fluid, it was the only liquid sample that was at all germane to Tank U-109, and its ion concentrations and density were typical of interstitial liquid from other tanks. It was therefore used to represent the interstitial liquid

(a) Transmittal from FH Steen (RFSHI) to LA Mahoney (PNNL) of preliminary data for Core 238 from Tank U-109, July 8, 1998. Rust Federal Services of Hanford, Inc., Richland, Washington. 
for gas solubility and hydrostatic pressure calculations. The density of the liquid was $1474 \mathrm{~kg} / \mathrm{m}^{3}$; the bulk density of the samples with high solids concentrations varied from 1270 to $1835 \mathrm{~kg} / \mathrm{m}^{3}$, with an average and standard deviation of $1646 \pm 144 \mathrm{~kg} / \mathrm{m}^{3}$.

For hydrostatic pressure calculations, the liquid density was set at $1474 \mathrm{~kg} / \mathrm{m}^{3}$, and the remainder of the tank waste was given a degassed bulk density of $1646 \mathrm{~kg} / \mathrm{m}^{3}$. A supernatant layer depth of $17 \mathrm{~cm}$ (7 in.) and a nonconvective layer depth of $432 \mathrm{~cm}$ (170 in.) were used in calculations.

\subsubsection{Sampling and Extraction Information}

The samples that underwent RGS analysis are listed in Table 4.14.1. Field data, including dose rates and downforce limits, are supplied in Appendix A. Table 4.14.1 also shows the lag times (delay between sample acquisition and processing) for these samples. This information was provided to allow data users to correlate the lag time between sampling and extrusion with the concentrations of the various constituents to test for decomposition or other chemical reactions. The long lag times experienced by several of the samples were the result of laboratory procedural reviews and a building-wide stand-down at the 222-S Laboratory, where the RGS analysis was carried out. The effect of the long lag times on the samples was discussed in detail for S-111 samples in Section 4.13.5.2. The conclusions were that 1) the lag time caused a change in the gas composition that was less than the measurement uncertainty and 2) there is less than $10 \%$ potential underestimation of gas volume fraction resulting from the long lag times.

As was discussed in Section 3.5, the HHF used during sampling often enters the sampler in place of some of the waste. There were no bromide data for the RGS samples, and the nonRGS samples from the U-109 core taken in 1998 were about half above and half below the MDL, which in many cases was $1250 \mu \mathrm{g} / \mathrm{g}$.(a) The RGS samples were assumed to have a bromide concentration of $1250 \mu \mathrm{g} / \mathrm{g}$ for lack of other information; this was about $6.1 \mathrm{vol} \% \mathrm{HHF}$. This assumption is probably conservative, and (based on HHF data for other tanks) the conservatism is expected to be less than $3 \%$.

All of these samplers were helium-backfilled and sealed with vacuum grease before they were deployed. This method virtually eliminated air and argon contamination from gases entrained during sampling. The helium backfill results are discussed in detail by Cannon (1997) and summarized in Section 5 of Mahoney et al. (1997). Samples 8-4 and 8-8 showed signs of an air leak into the RGS system (in spite of the pre-extrusion system leak check). We corrected for the inleaked air in the usual way.

Table 4.14.1. Lag Times for Processing RGS Samples from Tank 241-U-109

\begin{tabular}{||c|c|c|c||}
\hline Sample & Acquisition Date & Processing Date & Lag (days) \\
\hline \hline $8-2$ & April 22, 1998 & August 28, 1998 & 128 \\
\hline $8-4$ & April 23, 1998 & September 3, 1998 & 133 \\
\hline $8-6$ & April 27, 1998 & September 10, 1998 & 136 \\
\hline $8-8$ & April 28, 1998 & September 21, 1998 & 146 \\
\hline
\end{tabular}

(a) Transmittal from FH Steen (RFSHI) to LA Mahoney (PNNL) of preliminary data for Core 237 from Tank U-109, July 8, 1998. Rust Federal Services of Hanford, Inc., Richland, Washington. 
The same extraction procedure was used on all of the U-109 samples. The procedure included only one post-isotopic extraction; schedule and lag-time constraints precluded using an equilibration time greater than one day (see Figure 3.1 for a schematic of the RGS extraction system).

After evacuating the whole system and observing the pressure to test for leaks, the operator extruded the RGS sample into the extractor vessel, and the sample was briefly stirred. At this point, the vapor space in communication with the sample consisted of the volumes of the extractor vessel, its lines, the second volume, the pump volume, and the collector side; the latter included the collector line volume and an open collection canister. This first canister, containing a sample of uncompressed (unpumped) gas, was closed off. The second set of two collection canisters was opened. Several strokes were pumped from the extractor to the collector, and the second collection canisters were closed off. Then the collector side was evacuated. (The second volume was never used during the extraction process for U-109 samples.)

The vapor profile procedure was then performed. The first pump stroke and every third stroke thereafter (for 8-2) or every ninth stroke thereafter (for the other samples) were collected in single canisters, with only one stroke per canister. On the intermediate strokes, the gas was pumped to the collector lines with no canister attached. The collector lines were evacuated after every pump stroke, whether or not the pumped gas was collected in a canister. The total gas and vapor pumped from the extractor to the collector during this procedure was calculated by interpolating linearly between collected canisters to find the contents of the pump strokes that were not collected. At the end of the procedure, the first post-isotopic set of canisters was attached to the collector lines and opened, and both the extractor lines and the collector side were evacuated.

Three-hundred milliliters of $0.04 \mathrm{M} 15 \mathrm{NH}_{4} \mathrm{OH}$ solution was added to the sample in the extractor and stirred. After 16 to 22 hours, several strokes were pumped from the extractor to the collector. The first (and only) post-isotopic set of three canisters was closed off. Finally, the BSVD procedure was performed: argon was injected into the closed-off second volume, and then the second volume was opened to the extractor and lines. Pressure measurements and known tare system volumes allowed calculation of the volume of the sample solids and liquid.

In processing sample 8-6, vacuum was applied to the extractor (causing a loss of gas), and the data file from the vapor profile step was lost. An attempt was made to compensate for the gas that went unmeasured, and the uncertainty of the gas measurement was increased. Based on experience with other samples from U-109, we expect any gas underestimation resulting from the loss to be less than $15 \%$ of the total.

For all the U-109 samples, three to seven aliquots (subsamples) were drawn from each post-isotopic canister to provide more opportunity for the isotopic ratios to converge. Procedural details such as the number of strokes per canister and the system volumes can be found in the sample-by-sample worksheets in the U-109 RGS calculation spreadsheet on the CD included with this report.

\subsubsection{Retained Gas Composition}

Table 4.14.2 presents the calculated concentrations of the insoluble gases in the U-109 RGS samples without corrections for air and argon entrainment, helium backfill, air inleakage, or air dissolved in the isotopic standard solution. The method used to make the corrections depended on the gas. All of the oxygen, argon, and helium were subtracted no matter which step had produced them. A number of moles equal to $(3.73) \cdot\left(\mathrm{O}_{2}\right)$ were subtracted from the nitrogen because the oxygen was expected to have come from air entrainment during sampling or inleakage during 
Table 4.14.2. Concentrations of Insoluble Constituents ( $\mu \mathrm{mol} / \mathrm{L}$ of waste) in Tank U-109 Without Correction for Gas Contamination

\begin{tabular}{||c|c|c|c|c|c|c|c|c|c|c|c||}
\hline Segment & $\mathrm{N}_{2}$ & $\mathrm{H}_{2}$ & $\mathrm{~N}_{2} \mathrm{O}$ & $\mathrm{O}_{2}$ & $\mathrm{CH}_{4}$ & $\mathrm{He}$ & $\mathrm{Ar}$ & $\begin{array}{c}\text { Other } \\
\mathrm{NO}_{x}\end{array}$ & $\mathrm{C}_{2} \mathrm{H}_{\mathrm{x}}$ & $\mathrm{C}_{3} \mathrm{H}_{\mathrm{x}}$ & $\begin{array}{c}\text { Other } \\
\text { Hyd. }\end{array}$ \\
\hline \hline $8-2$ & $4600 \pm 380$ & $1800 \pm 140$ & $3400 \pm 430$ & $260 \pm 16$ & $48 \pm 5.2$ & $560 \pm 44$ & $20 \pm 1.9$ & (a) & $14 \pm 4.7$ & (a) & $19 \pm 13$ \\
\hline $8-4$ & $32000 \pm 1400$ & $2700 \pm 240$ & $4100 \pm 230$ & $7000 \pm 340$ & $69 \pm 6.2$ & $450 \pm 39$ & $340 \pm 17$ & (a) & $23 \pm 5.4$ & (a) & $14 \pm 5.4$ \\
\hline $8-6$ & $6900 \pm 590$ & $2300 \pm 200$ & $2200 \pm 270$ & $380 \pm 80$ & $89 \pm 9.6$ & $450 \pm 43$ & $22 \pm 4.0$ & (a) & $38 \pm 14$ & (a) & $16 \pm 11$ \\
\hline $8-8$ & $13000 \pm 440$ & $4600 \pm 160$ & $3200 \pm 120$ & $910 \pm 180$ & $130 \pm 10$ & $590 \pm 24$ & $49 \pm 5.2$ & (a) & $55 \pm 10$ & (a) & $9.4 \pm 2.3$ \\
\hline
\end{tabular}

extraction. Because a substantial amount of dissolved air gases entered the extractor with the $15 \mathrm{NH}_{4} \mathrm{OH}$ solution, all the nitrogen in the gas collected after the solution addition was subtracted. Thus it was assumed that more than $95 \%$ of the original retained nitrogen had already been collected, as was the case for hydrogen. The corrected concentrations are given in Table 4.14.3.

Samples 8-4 and 8-8 were affected by air leaks. Judging by the canisters in which high air first appeared, the leak began after the vapor profile step in the sample extraction and continued during the vapor profile step for sample 8-8. Contamination observations can be found in the "Summary" worksheet of the U-109 RGS calculations spreadsheet on the CD.

Table 4.14.4 presents the ammonia measurements. The residual ammonia was determined isotopically, and the total ammonia (calculated from the isotopic residual) is included in the table. The post-extrusion partial pressure of $\mathrm{NH}_{3}$ over the sample (see Section 3.4.4) was measured at chilled extractor temperature and is included in Table 4.14.4. The residual ammonia in U-109 samples was not measured by post-RGS ISE methods. Other ammonia measurements were taken by ISE on non-RGS samples from riser 8. See Section C.14 of Appendix C for more details on the ammonia data.

The various ammonia measurements were somewhat difficult to reconcile, but most of the data were approximately consistent. For lack of more definitive information, the RGS isotopic measurements were used to calculate the ammonia mole fractions in the in situ vapor.

Table 4.14.3. Concentrations of Insoluble Constituents ( $\mu \mathrm{mol} / \mathrm{L}$ of waste) in Tank U-109 with Correction for Gas Contamination

\begin{tabular}{|c|c|c|c|c|c|c|c|c|c|c|c||}
\hline Segment & $\mathrm{N}_{2}$ & $\mathrm{H}_{2}$ & $\mathrm{~N}_{2} \mathrm{O}$ & $\mathrm{O}_{2}$ & $\mathrm{CH}_{4}$ & $\mathrm{He}$ & $\mathrm{Ar}$ & $\begin{array}{c}\text { Other } \\
\mathrm{NO}_{x}\end{array}$ & $\mathrm{C}_{2} \mathrm{H}_{\mathrm{x}}$ & $\mathrm{C}_{3} \mathrm{H}_{x}$ & $\begin{array}{c}\text { Other } \\
\text { Hyd. }\end{array}$ \\
\hline \hline $8-2$ & $3700 \pm 490$ & $1800 \pm 140$ & $3400 \pm 430$ & $0 \pm 16$ & $48 \pm 5.2$ & $0 \pm 44$ & $0 \pm 1.9$ & (a) & $14 \pm 4.7$ & (a) & $19 \pm 13$ \\
\hline $8-4$ & $4300 \pm 560$ & $2700 \pm 240$ & $4100 \pm 230$ & $0 \pm 340$ & $69 \pm 6.2$ & $0 \pm 39$ & $0 \pm 17$ & (a) & $23 \pm 5.4$ & (a) & $14 \pm 5.4$ \\
\hline $8-6$ & $3400 \pm 670$ & $2300 \pm 200$ & $2200 \pm 270$ & $0 \pm 80$ & $89 \pm 9.6$ & $0 \pm 43$ & $0 \pm 4.0$ & (a) & $38 \pm 14$ & (a) & $16 \pm 11$ \\
\hline $8-8$ & $8900 \pm 1100$ & $4600 \pm 160$ & $3200 \pm 120$ & $0 \pm 180$ & $130 \pm 10$ & $0 \pm 24$ & $0 \pm 5.2$ & (a) & $55 \pm 10$ & (a) & $9.4 \pm 2.3$ \\
\hline a) Below detection limits, which are 0.01 mol\% or less.
\end{tabular}


Table 4.14.4. Ammonia Data from Tank U-109 Samples

\begin{tabular}{|c|c|c|c|}
\hline Sample & $\begin{array}{c}\text { Isotopically Measured } \\
\text { Total } \mathrm{NH}_{3} \\
(\mu \mathrm{mol} / \mathrm{L})\end{array}$ & $\begin{array}{c}\text { Post-extrusion } \mathrm{NH}_{3} \\
\text { partial pressure } \\
\text { (atm) }\end{array}$ & $\begin{array}{l}\mathrm{RGS} \mathrm{NH}_{3} \text { Concentrations in the } \\
\text { Liquid }\end{array}$ \\
\hline $8-2$ & $35000 \pm 11000(\mathrm{a})$ & -0.00053 & $930 \mu \mathrm{g} / \mathrm{mL}(0.054 \mathrm{M})$ \\
\hline $8-4$ & $88000 \pm 36000^{(a)}$ & $0.0017 \pm 0.0008$ & $2400 \mu \mathrm{g} / \mathrm{mL}(0.14 \mathrm{M})$ \\
\hline $8-6$ & $44000 \pm 13000^{(a)}$ & $0.0056 \pm 0.0028$ & $1100 \mu \mathrm{g} / \mathrm{mL}(0.067 \mathrm{M})$ \\
\hline $8-8$ & $44000 \pm 15000^{(a)}$ & $0.0058 \pm 0.0028$ & $1300 \mu \mathrm{g} / \mathrm{mL}(0.079 \mathrm{M})$ \\
\hline
\end{tabular}

(a) These data were used to calculate in situ ammonia vapor pressures.

It is likely that the ammonia concentration of the U-109 nonconvective layer was between 25,000 and $45,000 \mu \mathrm{mol} \mathrm{NH} / / \mathrm{L}$ waste. The average and standard deviation of the ammonia concentrations over samples $8-2,8-6$, and $8-8$ were $41,000 \pm 5,600 \mu \mathrm{mol} / \mathrm{L}$ waste. This average ammonia concentration in the bulk waste corresponded to $0.077 \mathrm{wt} \% \mathrm{NH}_{3}$ in the bulk waste, or $0.065 \mathrm{M} \mathrm{NH}_{3}$ in the liquid ( $1100 \mu \mathrm{g} \mathrm{NH}_{3} / \mathrm{mL}$ liquid).

Table 4.14.5 contains the composition of the gas/vapor phase in each sample and the integrated average composition for Tank U-109. The water vapor is not included in these compositions. The ammonia fractions are derived from the data in Table 4.14.4 that were used to calculate vapor pressures at in situ conditions. The sample compositions in the table have been calculated using the in situ solubility method (Section 3.6.2). As discussed in Section 3.6.1, compositions were calculated for both lower- and upper-bound gas solubilities. Both ends of the range are given in Table 4.14.5 with the measurement uncertainty on each. The average composition of the gas in the nonconvective layer is the result of integrating RGS species concentrations over the layer and multiplying by the layer volume. The integration method is described in Section 3.7.1.

Table 4.14.5. Sample and Overall Average Compositions of Retained Gas in Tank U-109 with Correction for Gas Contamination(a)

\begin{tabular}{|c|c|c|c|c|c|c||}
\hline Sample & $\mathrm{N}_{2}$ (mol\%) & $\mathrm{H}_{2}$ (mol\%) & $\mathrm{N}_{2} \mathrm{O}$ (mol\%) & $\mathrm{NH}_{3}$ (mol\%) & $\mathrm{CH}_{4}$ (mol\%) & Other (mol\%) \\
\hline \hline $8-2$ & $42 \pm 7.6$ to $44 \pm 7.9$ & $20 \pm 2.9$ to $21 \pm 3.0$ & $36 \pm 6.4$ to $34 \pm 5.9$ & $0.6 \pm 0.2$ to $0.3 \pm 0.1$ & $0.5 \pm 0.1$ to $0.6 \pm 0.1$ & $0.4 \pm 0.2$ \\
\hline $8-4$ & $38 \pm 6.3$ to $40 \pm 6.5$ & $24 \pm 3.1$ to $25 \pm 3.2$ & $35 \pm 4.0$ to $33 \pm 3.8$ & $1.5 \pm 0.6$ to $0.8 \pm 0.3$ & $0.6 \pm 0.08$ & $0.3 \pm 0.1$ \\
\hline $8-6$ & $43 \pm 11$ to $44 \pm 11$ & $28 \pm 4.9$ to $30 \pm 5.2$ & $26 \pm 5.1$ to $24 \pm 4.6$ & $0.7 \pm 0.2$ to $0.4 \pm 0.1$ & $1.1 \pm 0.2$ to $1.2 \pm 0.2$ & $0.7 \pm 0.3$ \\
\hline $8-8$ & $52 \pm 7.5$ to $53 \pm 7.6$ & $27 \pm 2.4$ to $28 \pm 2.4$ & $19 \pm 1.6$ to $18 \pm 1.6$ & $0.7 \pm 0.2$ to $0.4 \pm 0.1$ & $0.8 \pm 0.09$ & $0.4 \pm 0.08$ \\
\hline $\begin{array}{l}\text { Avg in non- } \\
\text { convective } \\
\text { layer(c) }\end{array}$ & $46 \pm 7.7$ to $47 \pm 8.0$ & $25 \pm 3.0$ to $26 \pm 3.1$ & $27 \pm 3.6$ to $25 \pm 3.4$ & $0.9 \pm 0.3$ to $0.4 \pm 0.2$ & $0.7 \pm 0.1$ & $0.4 \pm 0.2$ \\
\hline $\begin{array}{l}\text { (a) Uncertainties on compositions represent instrument uncertainty. Compositions calculated for lower- and upper-bound } \\
\text { solubilities. Both ends of composition range are given, lower-bound solubility basis first with instrument uncertainty on each: }\end{array}$ \\
$\begin{array}{l}\text { Compositions may not sum to } 100 \text { mol\% because of roundoff error; mole fractions on dry basis, do not account for water } \\
\text { vapor. } \\
\text { (b) Ammonia mole fraction derived from data in Table 4.14.4 used to calculate vapor pressure at in situ conditions. } \\
\text { (c) There are too few samples to define spatial variability of average gas concentration. }\end{array}$ \\
\hline
\end{tabular}


The concentrations and pressures in Tables 4.14.2 and 4.14.3 can also be found in the sample-by-sample worksheets in the U-109 RGS calculation spreadsheet on the CD. The compositions in Table 4.14.5 are in the "Inventory" worksheet.

\subsubsection{Gas Inventory}

The method by which the in situ gas volume fractions (wet basis) were calculated is given in Section 3.6.2; for the gas composition, the volume fractions are given as a range from the low gas-solubility value to the high gas-solubility value. The results are presented in Table 4.14.6. Table 4.14.6 also contains the average gas volume fraction and the average pressure experienced by the gas in the nonconvective layer. The average is an in situ volume average calculated by Simpson's Rule integration, as described in Section 3.7.1. The corrected gas volume fractions in Table 4.14.6 are consistent with the corrected gas concentrations and compositions in Tables 4.14.2, 4.14.3, and 4.14.5. The information in Table 4.14.6 is taken from the "Summary" and "In situ" worksheets of the U-109 RGS calculation spreadsheet on the CD.

The "sampler gas volume fraction" is corrected only for inleakage during the extraction process and air gases added in the isotopic solution. It is an attempt to reconstruct the total amount of gas (sample and entrainment) that was present in the sampler during $\mathrm{X}$-ray. It is used only for comparison with the $\mathrm{x}$-ray observations of "visible gas fraction" discussed in Section 4.14.4.

Table 4.14.6 also contains the water vapor pressures that were used for in situ calculations. The water vapor pressures were found by using salt concentrations from Baldwin (1996) and the temperatures in the table as inputs to Equation 6.2 of Mahoney and Trent (1995), a correlation for water vapor pressure over concentrated homogeneous and nonhomogeneous waste simulants. The gas solubilities used the same parameters as inputs to the Schumpe solubility model (Section 3.6.1). The water vapor pressures and gas solubilities used in calculations can be found in the "In situ" worksheet of the U-109 RGS calculation spreadsheet on the CD.

Table 4.14.6. In Situ Gas Volume Fractions and Conditions in Tank U-109

\begin{tabular}{|c|c|c|c|c|c|c||}
\hline Sample & $\begin{array}{c}\text { Sample } \\
\text { Central } \\
\text { Height }\end{array}$ & $\begin{array}{c}\text { Hydro- } \\
\text { static } \\
\text { Pressure- } \\
(\mathrm{atm})\end{array}$ & $\begin{array}{c}\text { Calculated } \\
\text { Water Vapor } \\
\text { Pressure } \\
(\mathrm{atm})\end{array}$ & $\begin{array}{c}\text { Temperature } \\
\left({ }^{\circ} \mathrm{C}\right)\end{array}$ & $\begin{array}{c}\text { Corrected Gas } \\
\text { Volume Fraction (a) } \\
\text { (in-tank conditions) }\end{array}$ & $\begin{array}{c}\text { Sampler Gas } \\
\text { Volume Fraction } \\
\text { (in situ conditions, } \\
\text { low solubility) }\end{array}$ \\
\hline \hline $8-2$ & 362 & 1.11 & 0.010 & 24.2 & $\begin{array}{c}0.20 \pm 0.025 \text { to } \\
0.19 \pm 0.025\end{array}$ & 0.21 \\
\hline $8-4$ & 265 & 1.21 & 0.012 & 26.9 & $\begin{array}{c}0.23 \pm 0.022 \text { to } \\
0.22 \pm 0.022\end{array}$ & 0.25 \\
\hline $8-6$ & 169 & 1.32 & 0.013 & 28.3 & $\begin{array}{c}0.15 \pm 0.010 \text { to } \\
0.14 \pm 0.010\end{array}$ & 0.16 \\
\hline $8-8$ & 72 & 1.42 & 0.013 & 27.5 & $\begin{array}{c}0.30 \pm 0.018 \text { to } \\
0.29 \pm 0.018\end{array}$ & 0.31 \\
\hline $\begin{array}{l}\text { Avg in non- } \\
\text { convective } \\
\text { layer(b) }\end{array}$ & 202 \\
\hline $\begin{array}{l}\text { (a) Gas volume fraction is expressed on a wet basis, including the volume contribution of water vapor. } \\
\text { (b) Uncertainties on layer-average gas volume fractions are based on spatial variability considerations } \\
\text { discussed in Section 3.7.2. }\end{array}$ & 1.28 & & $\begin{array}{l}0.22 \pm 0.11 \text { to } \\
0.21 \pm 0.10\end{array}$ \\
\hline
\end{tabular}


The four ammonia concentration measurements in U-109 integrated to a total (vapor and dissolved) ammonia inventory that would have had an STP volume of $1900 \mathrm{~m}^{3}\left(69,000 \mathrm{ft}^{3}\right)$. The uncertainty on the ammonia inventory was at least $70 \%$.

Table 4.14.7 gives various estimates of the STP volume of gas in Tank U-109, including estimates calculated from RGS data alone. The RGS gas inventory in the nonconvective layer was calculated by integrating RGS total gas concentrations over the nonconvective layer (four data points) and multiplying the average gas concentration by the volume of the waste. The integration method is described in Section 3.7.1. No samples were taken in the supernatant liquid, so its inventory (almost certainly negligible) is not calculated. The RGS volumes in Table 4.14.7 include corrections to remove the contamination gas: entrained air and argon, helium backfill, air leaks during and after extraction, and air dissolved in the isotopic standard. The uncertainties in the gas inventories are based on the spatial variability considerations discussed in Section 3.7.2. The information in Table 4.14.7 is taken from the "Inventory" worksheet of the U-109 RGS calculation spreadsheet on the CD.

The gas inventory calculated by the BPE method was less than half of the RGS estimate. The surface level rise (SLR) can also be considered as an indication of retained gas volume; the waste surface rose a net $4.4 \mathrm{~cm}$ (1.7 in.) between 1981 and December 1995 (Hodgson et al. 1998), which implied an in situ gas accumulation smaller than that predicted by BPE.

Both the BPE and RGS methods employ data that are not beyond question. The lack of complete supernatant coverage casts some doubt on the waste level response (dL/dP) as an indicator of the total gas inventory of the tank by the BPE method. Note that the Enraf instrument was located in the RGS riser, where no supernatant was found. The RGS data, on the other hand, were subject to increased uncertainty because of long lag times (though this effect should be negligible), data loss (sample 8-6), and incomplete piston retraction (sample 8-10).

One explanation for the mismatch between the BPE gas inventory estimates and the RGS estimate might be that the high-gas region did not extend over the entire tank but was local to the area sampled by RGS, in the periphery of the tank. Risers 2,7 , and 19, from which apparent high-gas samples were taken in 1996, were also near the periphery and so did not rule out different conditions toward the tank center.

Table 4.14.7. U-109 Gas Inventory Estimates

\begin{tabular}{|l|c|c||}
\hline \multirow{2}{*}{ Quantity } & RGS Method & BPE Method(a) \\
\cline { 2 - 3 } & $\begin{array}{c}\text { Nonconvective } \\
\text { Layer }\end{array}$ & Tank Total \\
\hline \hline $\begin{array}{l}\text { Avg. gas fraction (low } \\
\text { gas solubility) }\end{array}$ & $0.22 \pm 0.11$ & $0.090 \pm 0.039$ \\
\hline $\begin{array}{l}\left.\text { Gas volume (m }{ }^{3}\right) \\
\text { in situ (wet) }\end{array}$ & $377 \pm 189(\mathrm{~b})$ & $152 \pm 67$ \\
$\begin{array}{l}\text { STP (wet) } \\
\text { (a) Barometric pressure method; inventory based on average gas } \\
\text { pressure calculated from RGS data and on median dL/dP of }-0.38 \\
\pm 0.16 \text { in./in.-Hg (Whitney et al. 1997). } \\
\text { (b) Retained gas inventory calculated from RGS data is } \\
\text { considered the better estimate. }\end{array}$ \\
\hline
\end{tabular}


Although the RGS data were taken from only one riser, the high-gas results were partially confirmed by a number of non-RGS samples from three other risers (risers 2, 7, and 19). Sample lengths between 6 and 19 inches were seen during core extrusion. Over all, in the samples from these three risers that were between segments 2 and 9 inclusive and contained 10 inches or more of sample, the average apparent gas volume fraction was 0.17 , between the RGS and BPE values. However, this value is somewhat qualitative because of possible leakage from the non-RGS samplers and imprecision of sample length measurement. Considering all the evidence, we consider the RGS gas inventory to be the better estimate of the gas in Tank U-109 at the time of sampling. The reason for the much lower level-based inventory measures is unknown.

Table 4.14.8 contains the nonconvective layer inventories of each of the major gases retained in the waste. This inventory is based on the layer-average composition from RGS data (Table 4.14.5) and the best-estimate gas inventory (RGS, Table 4.14.7). No samples were taken in the supernatant liquid, so its inventory (almost certainly negligible) was not calculated.

Figure 4.14.4 shows sample temperatures, corrected gas volume fractions (Table 4.14.6), and corrected compositions of the low-solubility constituents in the samples from Tank U-109. The temperatures were measured by the TC tree in riser 1 . The compositions represent the mole fraction of the species in the insoluble gas; water and ammonia are not included so are not the same as those in Table 4.14.5. The gas volume fractions and mole fractions in the figure are the values for lower-bound gas solubilities. Figure 4.14.4 also lists the observations from core extrusions of non-RGS samples and from x-rays of RGS samples as a way of tying those observations together with RGS data. The x-ray data are described in more detail in Section 4.14.4.

Table 4.14.8. Speciated U-109 Gas Inventory

\begin{tabular}{|c|c|c||}
\hline \multirow{2}{*}{ Species } & \multicolumn{2}{|c|}{ Nonconvective Layer } \\
\cline { 2 - 3 } & $\begin{array}{c}\text { Gas-Phase Inventory } \\
\left(\mathrm{m}^{3} \text { at STP) }\right.\end{array}$ & $\begin{array}{c}\text { Dissolved Inventory } \\
\left(\mathrm{m}^{3} \text { at STP) }\right.\end{array}$ \\
\hline $\mathrm{N}_{2}$ & 200 & $0.11-0.57$ \\
\hline $\mathrm{H}_{2}$ & 110 & $0.18-0.64$ \\
\hline $\mathrm{N}_{2} \mathrm{O}$ & $120-110$ & $4.4-16$ \\
\hline $\mathrm{NH}_{3}$ & $3.8-1.9$ & 1900 \\
\hline $\mathrm{CH}_{4}$ & 3.2 & $0.0036-0.019$ \\
\hline Other & 1.8 & 0 \\
\hline $\begin{array}{l}\text { (a) Inventories based on layer-average compositions from RGS data (Table 4.14.5) and best- } \\
\text { estimate gas inventories for each layer (RGS, Table 4.14.7). Uncertainty in each inventory } \\
\text { is 50\% based on spatial variability considerations (Section 3.7.2). Inventories given for } \\
\text { lower- and upper-bound solubilities; lower-bound solubility basis first. }\end{array}$ \\
\hline
\end{tabular}

\subsubsection{X-Ray Results}

Table 4.14.9 summarizes all the available radiography observations from Tank U-109. The $\mathrm{x}$-ray images themselves can be found on the CD that accompanies this report. The uncertainties of the visible gas volume fractions that are given in Table 4.14 .9 are unknown but are expected to be larger than those in the gas volume fractions calculated from gas extraction. 

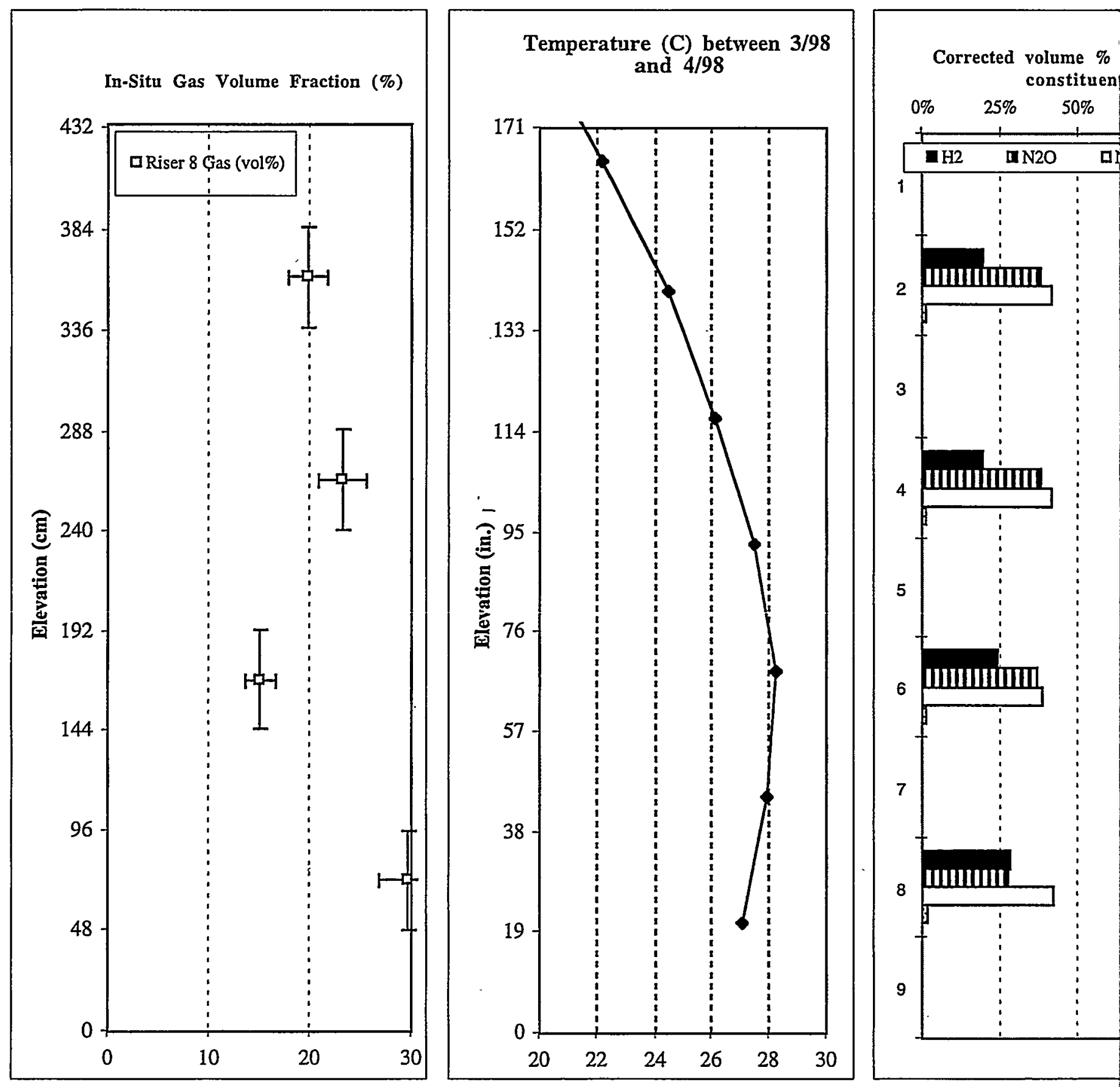

Figure 4.14.3. Gas Volume Fractions, Temperatures, C 
insoluble

Waste level is 434 to $450 \mathrm{~cm}$ (170 to 177 inches).

1 Riser 2: 2 to 3 in. (79 g) gray wet and moist salt; larger crystals near top.

1 Riser 8: $80 \mathrm{~mL}$ (118 g) opaque gray liquid, 3 in. (89 g) dark-gray salt slurry.

1 Riser 7: 3 in. $(70 \mathrm{~g})$ crumbly gray wet salt.

2 Riser 2: 10 in. (293 g) gray moist salt, fine crystals

2 Riser $8 \mathrm{X}$-RAY: Some 1-4 mm bubbles, top $0.5 \mathrm{in}$. separated liquid, clean piston, 2.2 in. gap, $1.5 \mathrm{R} / \mathrm{hr}$.

2 Riser 19: 2 in (26 g) black moist to dry salt.

2 Riser 7: 6 in. (144 g) bluish-gray moist salt.

3 Riser 2: 18 in. (340 g) gray to bluish-gray moist salt.

3 Riser 8: 15 in. (373 g) dark gray wet salt.

3 Riser 19: black liquid, gray solids, heavily contaminated by hydrostatic head fluid.

3 Riser 7: $10 \mathrm{in.}(216 \mathrm{~g})$ bluish-gray moist salt.

4 Riser 2: 16 in. (409 g) bluish-gray moist salt.

4 Riser 8 X-RAY: Many vague 1-2 mm bubbles, $0.2 \mathrm{in}$. sep. liquid, clean piston, $2.7 \mathrm{in}$. gap, $1.0 \mathrm{R} / \mathrm{hr}$.

4 Riser 19: 9 in. (246 g) bluish-gray dry granular salt.

4 Riser 7: 14 in. (314 g) dark-gray wet salt.

5 Riser 8: 19 in. (463 g) gray moist sait.

5 Riser 19: 18 in. (474 g) whitish-gray smooth moist salt.

5 Riser 7: 7 in. $(175 \mathrm{~g})$ dark-gray salt.

6 Riser 2: 19 in. (506 g) gray putty-like moist salt.

6 Riser 8 X-RAY: Elongated voids $2-10 \mathrm{~mm}$ long, lumpy top, small gob on piston, $1.4 \mathrm{in}$. gap, $1.0 \mathrm{R} / \mathrm{hr}$.

6 Riser 19: 19 in. (477 g) gray putty-like moist salt.

6 Riser 7: 16 in. (381 g) dark-gray wet salt.

7 Riser 2: 14 in. (394 g) bluish-gray moist salt.

7 Riser 8: 18 in. ( $440 \mathrm{~g}$ ) white-flecked gray moist salt.

7 Riser 19: 17.5 in. (448 g) gray putty-like moist salt.

7 Riser 7: 18 in. (403 g) dark-gray moist salt.

8 Riser 2: 16 in. (454 g) bluish-gray moist salt.

8 Riser 8 X-RAY: Few vague bubbles, flat top surface, nearly clean piston, 5.5 in gap, $0.8 \mathrm{R} / \mathrm{hr}$.

8 Riser 19: 16 in. (445 g) gray putty-like moist salt.

8 Riser 7: 17 in. $(440 \mathrm{~g})$ dark-gray salt.

9 Riser 2: 16 in. (420 g) moist sait, upper part gray to dark gray, lower part yellow.

9 Riser 8: 16 in. (412 g) total moist salt, varying shades of gray, some white-flecked.

9 Riser 19: 12 in. $(250 \mathrm{~g})$ solids; upper black smooth salt, lower gray pitted salt.

9 Riser 7: 9 in. (229 g) brown to dark-gray salt with white flecks.

positions, and Observations in Samples from Tank U-109 
Table 4.14.9. Summary of Observations from X-Ray Images of Tank U-109

\begin{tabular}{|c|c|}
\hline Segment & Comments/Observations \\
\hline $\begin{array}{c}8-2 \\
(\mathrm{RGS} \\
4 / 22 / 98)\end{array}$ & $\begin{array}{l}\text { The image contained some very vague light features and a few recognizable bubbles in the } 1 \text { to } \\
4-\mathrm{mm} \text {-diameter range. There was a gas gap of about } 5.6 \mathrm{~cm}(2.2 \mathrm{in} \text {.) below a clean piston and } \\
\text { a separated liquid layer about } 1.2 \mathrm{~cm} \text { thick. The visible gas volume fraction is } 0.12 \text {. (Compare } \\
\text { with the sampler gas volume fraction of } 0.21 \text { in Table } 4.14 .6 \text { obtained by gas extraction.) }\end{array}$ \\
\hline $\begin{array}{l}8-4 \\
\text { (RGS, } \\
4 / 23 / 98)\end{array}$ & $\begin{array}{l}\text { Numerous voids in the } 1 \text { to } 2 \mathrm{~mm} \text { diameter range. There is a separated liquid layer about } 0.5 \mathrm{~cm} \\
\text { thick at the top and a gas gap of about } 6.8 \mathrm{~cm} \text { ( } 2.7 \text { in.). The piston is clean. The effective } \\
\text { sampler length is only } 18.1 \text { in. because the piston is } 0.1 \text { in. from full retraction. The visible } \\
\text { gas volume fraction (not counting bubbles) is therefore } 0.15 \text {. (Compare with the sampler gas } \\
\text { volume fraction of } 0.25 \text { in Table } 4.14 .6 \text { obtained by gas extraction.) }\end{array}$ \\
\hline $\begin{array}{l}8-6 \\
\text { (RGS, } \\
4 / 27 / 98)\end{array}$ & $\begin{array}{l}\text { This sample contained numerous vaguely visible elongated voids of } 2 \text { to } 10 \text {-mm size throughout } \\
\text { the sample. There is a lumpy top surface and a piston with a small gob of adhering waste, with } \\
\text { a } 3.6-\mathrm{cm} \text { ( } 1.4 \text {-in.) gas gap below it. The effective sampler length is only } 18.1 \text { in. because the } \\
\text { piston is } 0.1 \text { in. from full retraction. The visible gas volume fraction (not counting bubbles) is } \\
\text { therefore } 0.08 \text {. (Compare with the sampler gas volume fraction of } 0.16 \text { in Table } 4.14 .6 \\
\text { obtained by gas extraction.) }\end{array}$ \\
\hline $\begin{array}{c}8-8 \\
(\mathrm{RGS} \\
4 / 28 / 98)\end{array}$ & $\begin{array}{l}\text { This sample contained only a few vague bubbles; much of the waste was featureless. There is a } \\
\text { flat top surface and a nearly clean piston with a } 14-\mathrm{cm} \text { ( } 5.5 \text {-in.) gas gap below it. The effective } \\
\text { sampler length is only } 14.7 \text { in. because the piston is } 3.5 \text { in. from full retraction. The visible } \\
\text { gas volume fraction (not counting bubbles) is therefore } 0.37 \text {. (Compare with the sampler gas } \\
\text { volume fraction of } 0.31 \text { in Table } 4.14 .6 \text { obtained by gas extraction.) }\end{array}$ \\
\hline
\end{tabular}

Table 4.14.9 compares $\mathrm{x}$-ray-derived and extraction-derived gas volume fractions. The comparison shows that sample recovery was close to $100 \%$ for samples 8-2, 8-4, and 8-6; sample 8-8 may have been only 85 to $90 \%$ recovered. This rough estimate is based on the fact that a larger gap was observed in the $\mathrm{x}$-ray than would have been present at the in situ pressure, based on the extracted gas.

The major peculiarity of U-109 waste, as observed from X-ray images, was that relatively large amounts of gas could be present in an "invisible" form. Sample 8-6 contained roughly 15 vol\% gas in waste that showed only vaguely visible bubbles (when they were visible at all). A more typical gas volume fraction for gas in the waste is 5 to $10 \mathrm{vol} \%$, and in such cases the waste is usually visibly bubbly. It is possible that 5 to $10 \mathrm{vol} \%$ of gas was generated during the long lag time and was not visible on the x-ray because it didn't exist yet. However, the other samples from the tank did not contain unusual amounts of invisible gas (or generated gas, depending on the hypothesis).

In addition, such a high rate of gas generation is not consistent with that observed in similar tank wastes under similar laboratory conditions. The "invisible" gas amounts to about 7 vol\%, or $700 \mu \mathrm{mol}$ hypothetically generated in 136 days at about $28^{\circ} \mathrm{C}$, with no radiation other than that generated by the sample itself (and possibly by samples stored nearby). Waste samples from Tank AN-105 generated $1.66 \mu \mathrm{mol} / \mathrm{L}$ waste/day at $35^{\circ} \mathrm{C}$ under self-irradiation conditions similar to those experienced by the RGS samples (Person 1998). The AN-105 samples would have produced only $70 \mu \mathrm{mol}$ of gas from $0.3 \mathrm{~L}$ of waste (the sample volume) over 136 days. 
Table 4.14.10 provides a summary of the waste densities that have been calculated from radiographic data for the RGS samples for which air and water standard images were available. The radiographic densities include the gas in the waste, were calculated by the current $\mathrm{x}$-ray analysis method, and are given for several locations within each sample to show density trends. Table 4.14.10 also includes density measurements made on extruded non-RGS samples.

Table 4.14.10. Densities of U-109 Samples from Radiography

\begin{tabular}{|c|c|c|c|}
\hline $\begin{array}{l}\text { Riser- } \\
\text { Segment } \\
\text { Number }\end{array}$ & $\begin{array}{l}\text { Distance from } \\
\text { Bottom of Sampler } \\
\text { (ft) }\end{array}$ & $\begin{array}{c}\text { Calculated Mean Density } \\
\text { by Current Method } \\
(\mathrm{g} / \mathrm{cc})\end{array}$ & $\begin{array}{c}\text { Degassed Density above and } \\
\text { below the RGS Sample } \\
\text { (from cores) } \\
\text { (g/cc) }\end{array}$ \\
\hline \multirow[t]{5}{*}{$8-2$ (a) } & $\begin{array}{l}1.25 \\
\end{array}$ & 1.40 & \multirow{5}{*}{$\begin{array}{r}\text { density above, } 1.78 \mathrm{~g} / \mathrm{cc} \text { bulk } \\
1.47 \mathrm{~g} / \mathrm{cc} \mathrm{liquid}\end{array}$} \\
\hline & 1.00 & 1.39 & \\
\hline & 0.75 & 1.38 & \\
\hline & 0.50 & 1.40 & \\
\hline & 0.25 & 1.28 & \\
\hline \multirow[t]{5}{*}{$8-4$} & 1.25 & 1.60 & \multirow[t]{5}{*}{ density above, $1.72 \mathrm{~g} / \mathrm{cc}$ bulk } \\
\hline & 1.00 & 1.59 & \\
\hline & 0.75 & 1.62 & \\
\hline & 0.50 & 1.63 & \\
\hline & 0.25 & 1.43 & \\
\hline \multirow[t]{5}{*}{$8-6$} & 1.25 & 1.43 & \multirow[t]{5}{*}{ density above, $1.59 \mathrm{~g} / \mathrm{cc}$ bulk } \\
\hline & 1.00 & 1.43 & \\
\hline & 0.75 & 1.41 & \\
\hline & 0.50 & 1.48 & \\
\hline & 0.25 & 1.36 & \\
\hline \multirow[t]{3}{*}{$8-8$} & 0.76 & 1.27 & \multirow[t]{3}{*}{ density above, $1.43 \mathrm{~g} / \mathrm{cc}$ bulk } \\
\hline & 0.50 & 1.12 (a) & \\
\hline & 0.25 & $0.81(a)$ & \\
\hline
\end{tabular}

\subsubsection{Drillstring and Domespace Composition Comparison}

The RGS gas-phase composition data (Table 4.14.5) were used to calculate $\mathrm{H}_{2} / \mathrm{N}_{2} \mathrm{O}$ ratios for comparison with ratios from drillstring and domespace grab sample measurements (Siciliano 1998, Table 8.1; McCain 1999, Table B-30). The results can be seen in Table 4.14.11. The RGS $\mathrm{H}_{2} / \mathrm{N}_{2} \mathrm{O}$ ratios agreed with many of the drillstring or domespace ratios. However, there also were some much higher ratios measured in both the drillstring (December 28, 1995 during the 1996 sampling campaign) and the domespace (May 9, 1996). 
Table 4.14.11. Comparison with Drillstring and Domespace Data(a)

\begin{tabular}{|l|c|}
\hline \hline Sample & $\mathrm{H}_{2} / \mathrm{N}_{2} \mathrm{O}$ \\
\hline \hline RGS, 8-2 & $0.55-0.62$ \\
\hline $\mathrm{RGS}, 8-4$ & $0.67-0.74$ \\
\hline $\mathrm{RGS}, 8-6$ & $1.1-1.2$ \\
\hline $\mathrm{RGS}, 8-8$ & $1.5-1.6$ \\
\hline Drillstring, 12/28/95 & 5.1 \\
\hline Drillstring, riser 2, 1/4/96 & 1.2 \\
\hline Domespace, 2/7/96 & $0.77,0.79$ \\
\hline Domespace, 5/9/96 & $8.3,8.0$ \\
\hline Domespace, 3/18/98 & 0.57 \\
\hline Domespace, 5/20/98 & 0.65 \\
\hline $\begin{array}{l}\text { (a) RGS ratios were calculated for both lower- and upper- } \\
\text { bound solubilities. Both ends of the ratio range are given in } \\
\text { the table, lower-bound solubility basis first. }\end{array}$ \\
\hline
\end{tabular}

\subsection{SY-101}

Tank 241-SY-101 (SY-101) was the fifteenth tank and the fifth DST sampled with the RGS. SY-101 was sampled as part of a program to determine the causes and significance of recent unexplained level rises that have resulted in declaration of a new USQ (Waste Surface Level Growth in 241-SY-101, USQ No. TF-97-0975). SY-101 has undergone intense study since 1990, when periodic large level drops indicated that episodic gas releases were occurring. Domespace gas monitoring and computational simulations indicated that some of these releases contained enough flammable gas to cause tank damage if the gas were ignited. The releases proved to be the result of gases generated in the nonconvective layer at the tank bottom; when enough buoyant gas had accumulated, portions of the waste broke free and rose, releasing the gas they contained and disrupting the crust at the waste surface.

In July 1993, a mixer pump was installed to mitigate the buoyancy-induced releases by dispersing the nonconvective layer. While mixer pump operations prevented buoyant displacement GREs, the absence of the periodic crust breakup provided by GREs allowed the crust to grow. This growth was first detected in 1996 and was recognized as a potential safety issue in late 1997.

RGS was part of the logic path prescribed by the Level Growth Issue Task Team(a) to investigate an observed level growth phenomenon. This logic called for deployment of the VFI prior to deployment of the RGS core sampling. Should the VFI not completely describe retained gas behavior consistent with the observed level growth, the RGS was to be deployed with an emphasis on gathering information on possible gas retention in the crust layers of SY-101.

(a) Bates JM, RE Bauer, CE Hanson, MG Hunn, GD Johnson, NW Kirch, DC Larsen, and CM Welch. February 1998. Internal Hanford report, Task Team Report on Level Growth Issue in 241-SY-101. 
Push-mode RGS sampling was performed in riser 23A in November and December 1998 and in riser 22A in January 1999.(a) A subsequent core was taken from riser 4A in February 1999. While the waste under riser 23A had never been disturbed by sampling, riser $4 \mathrm{~A}$ had been used for VFI measurements and in situ rheometry in early 1995 (Schienbein et al. 1999), and riser 22A was the location of the Window $C$ core samples in May 1991. The approximate locations of various risers are shown in Figure 4.15.1.

Tank SY-101 is a sound DST with active ventilation and a capacity of $1,160,000$ gallons. A summary of Tank SY-101 conditions and parameters is extracted from Field (1998):

\section{Waste Parameters}

- Volume: 4,263,000 L (1,126,000 gal) concentrated complexant waste; tank had three layers in 1991; convective layer of $2,370,000 \mathrm{~L}(626,000 \mathrm{gal})$, nonconvective layer of $1,670,000 \mathrm{~L}(442,000 \mathrm{gal})$ and crust of $220,000 \mathrm{~L}$ (58,000 gal); layers no longer exist due to mixer pump installation (and operation)

- Waste Types: 564,000 gal 242-S Evaporator saltcake, 544,000 gal B Plant cesium and strontium recovery process waste, plus lance water and miscellaneous

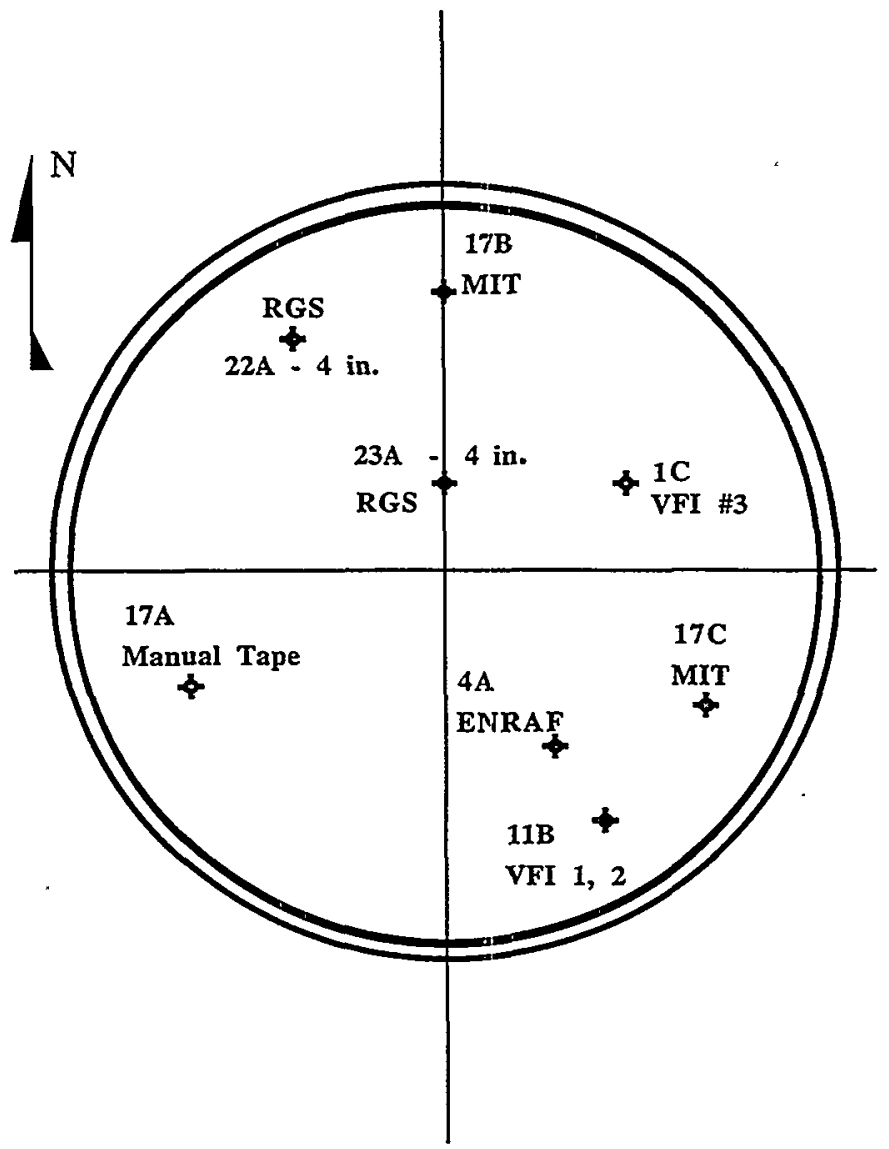

Figure 4.15.1. Schematic Diagram of Riser Locations in Tank SY-101

(a) The sampling scheme can be found in Sampling Plan for Tank 241-SY-101 Retained Gas Sampler Deployment, by JM Bates, February 1999. Letter report TWSFG98.75 Rev. 2, Pacific Northwest National Laboratory, Richland, Washington. 
- $\quad$ Maximum temperature on March 1, 1998: $48^{\circ} \mathrm{C}\left(118^{\circ} \mathrm{F}\right)$; on August $1,1999: 51^{\circ} \mathrm{C}\left(123^{\circ} \mathrm{F}\right)$

- Heat Load: $40,400 \mathrm{Btu} / \mathrm{hr}$ based on radionuclides that generate heat

- $\quad$ Sample Events: grab samples, 1986; crust samples, November 1990; crust samples and push core samples, May 1991; push mode core samples, December 1991; VFI operation, December 1994 and January 1995; ball rheometer, March and April 1995; VFI operation, June, July, and September 1998; push mode core samples, November to December 1998, January 1999, March 1999.

- $\quad$ Significant Results: Primary analytes, sodium, nitrate, nitrite, hydroxide, carbonate

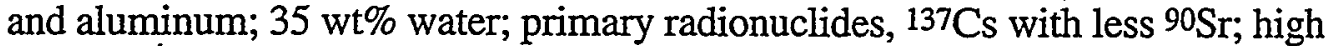
exotherms $(>480 \mathrm{~J} / \mathrm{g})$.

Surface level measurements taken through risers $1 \mathrm{~A}$ and $1 \mathrm{C}$, as catalogued.in the Tank Characterization Database, showed the level increasing at an accelerating rate in 1998 with readings approaching $417 \mathrm{in}$ : (the level readings at riser $1 \mathrm{~A}$ were the reportable waste surface levels) as of September 1, 1998 (referenced to tank bottom-elevation 617.34 ft.) (Figure 4.15.2 ).(a) The waste level continued to rise during the period over which RGS samples were taken.

Figure 4.15.3 shows the approximate tank content layering, with a thick crust and a mixed slurry layer above a settled solids layer at the bottom of the tank. The crust layer was taken to include the high-gas region that at that time was found at the bottom of the crust, postulated to be a

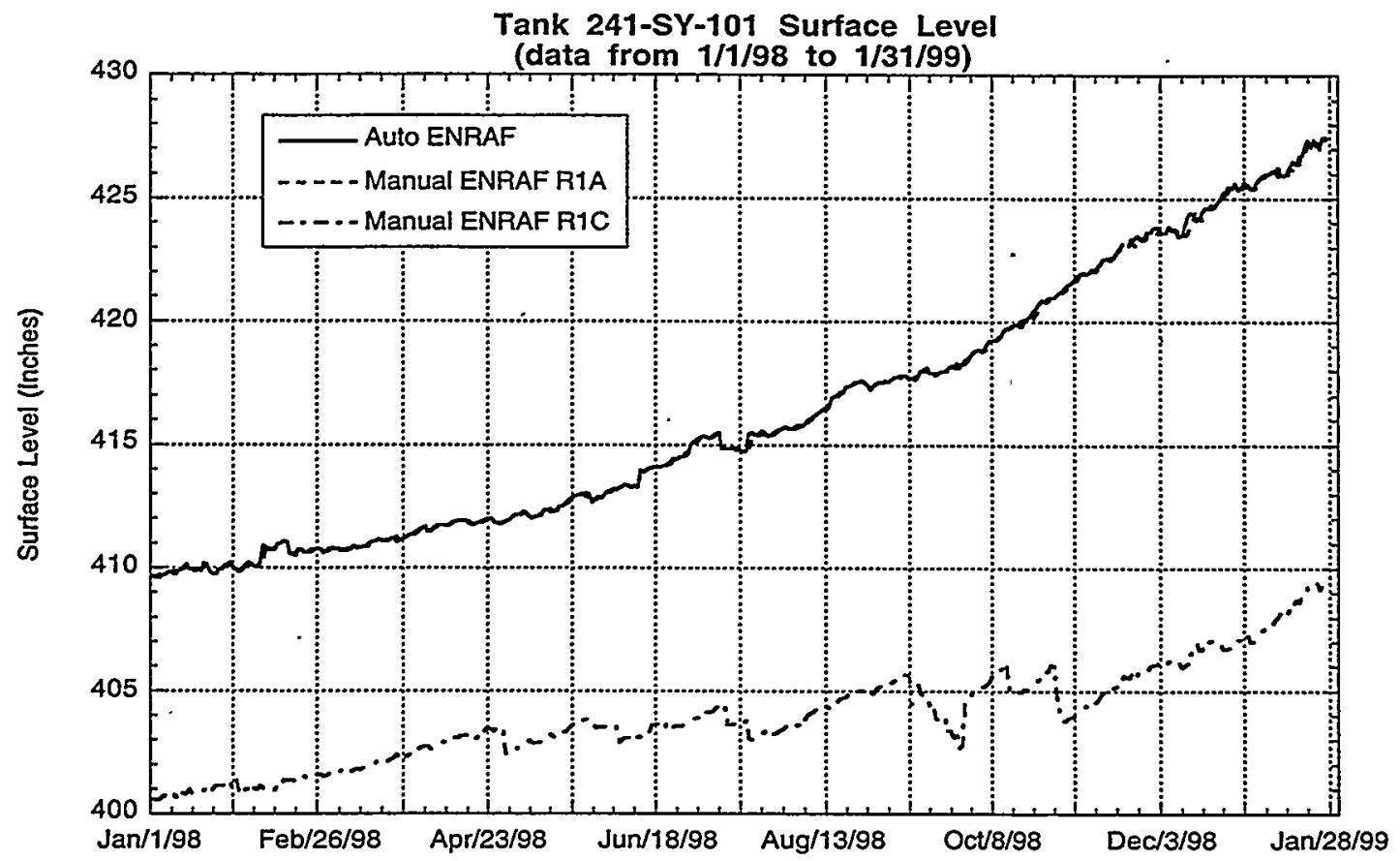

Figure 4.15.2. Waste Level Data from Tank SY-101

(a) Fluor Daniel Northwest Inc. 1997. HTCE Supporting Documents (Appendix C and F). HNF-SD-WM-ER-314-319 Rev. 1, Fluor Daniel Northwest Inc., Richland, Washington. 


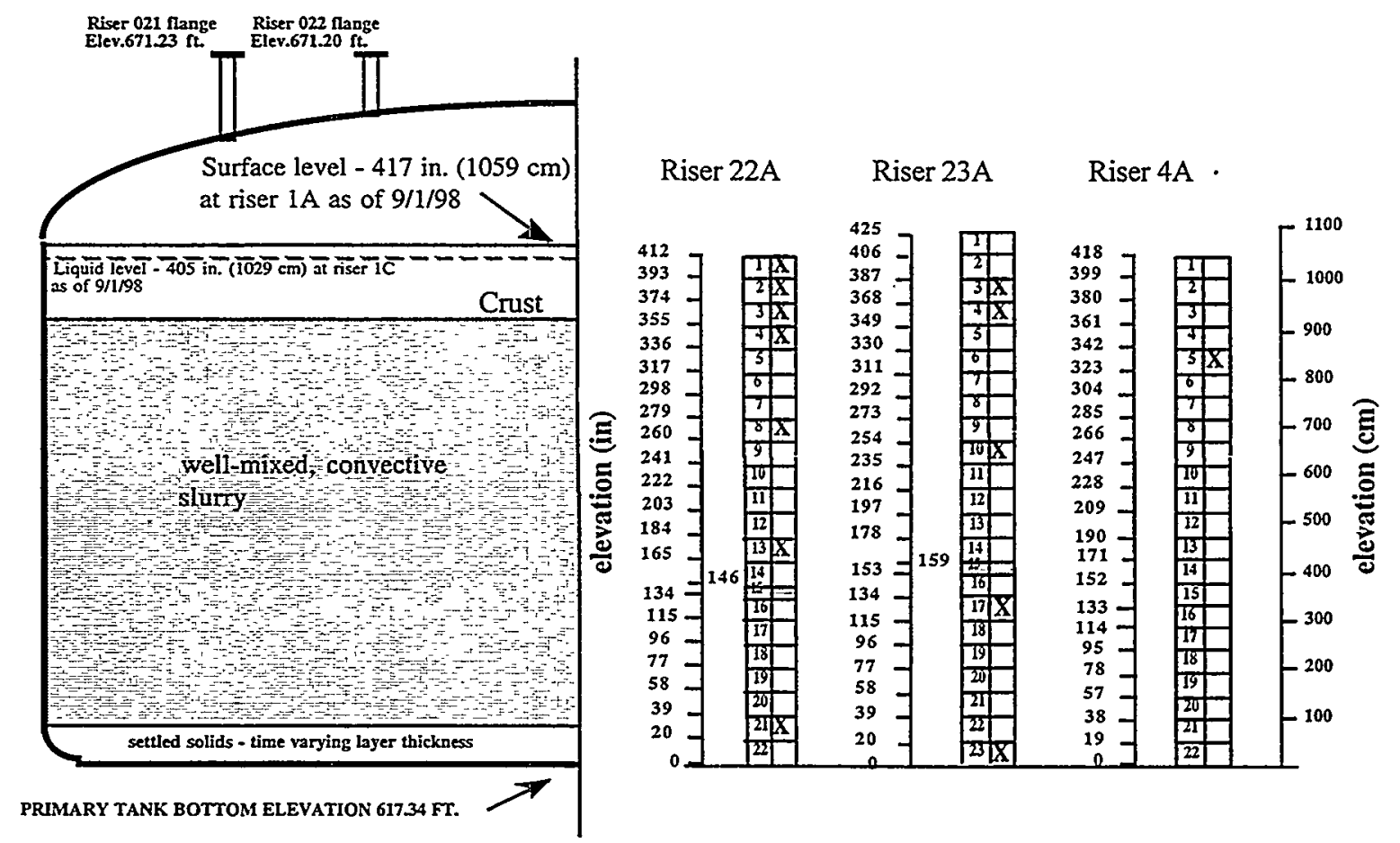

Figure 4.15.3. Tank 241-SY-101 Estimated Waste Layer Structure and Chart of RGS Sample Elevations for Risers 22A, 23A, and 4A. The short gray segments are those of partial length that were used to adjust the core length to match surface level. Segments are referenced to local tank bottom.

"bubble slurry" layer lying beneath the crust proper. (However, the actual gas morphology was unknown.) Because of mixing pump operations, the thickness of the settled layer varied with time and location. The elevations of the RGS segments are depicted in Figure 4.15.3.

For in situ inventory and average composition calculations, the degassed, solids-free density of the liquid was set at $1500 \mathrm{~kg} / \mathrm{m}^{3}$, consistent with Meyer et al. (1997), and the crust layer was given a degassed bulk density of $1750 \pm 44 \mathrm{~kg} / \mathrm{m}^{3}$ based on preliminary data from 1998 and 1999 cores.(a) Because no distinct settled solids layer was found in the cores, the settled layer was given the same degassed density as the mixed-layer liquid, $1596 \pm 14 \mathrm{~kg} / \mathrm{m}^{3}$. The liquid density is consistent with the value of $1600 \pm 30 \mathrm{~kg} / \mathrm{m}^{3}$ found by ball rheometry in 1995 (Schienbein et al. 1999).

Cores 255 and 256 (risers 23A and 22A) showed different crust thicknesses, with the crust proper and "bubble-slurry" jointly occupying three 19-in. segments of Core 255 and four segments of Core 256. The waste heights (measured by zipcord)(b) were also different at the dates and locations of the two cores. Therefore, for Core 255 (at riser 23A) the crust layer thickness was set at $145 \mathrm{~cm}$ (57 in.) for hydrostatic pressure and gas inventory calculations, and the mixed slurry and "settled" layer thickness at $902 \mathrm{~cm}$ (355 in.). For Core 256 (at riser 22A), the crust layer was assumed $193 \mathrm{~cm}$ (76 in.) thick, and the mixed slurry and "settled" layer $881 \mathrm{~cm}(347 \mathrm{in}$.) thick.

(a) Transmittal from FH Steen to LA Mahoney (PNNL) of data to be included in an SY-101 Tank Characterization Report (in preparation), April 27, 1999. Lockheed Martin Hanford Corporation, Richland, Washington.

(b) Transmittal from JG Douglas (Cogema) to LA Mahoney (PNNL) of a spreadsheet containing sample elevations and recoveries, April 1999. COGEMA Engineering Corp., Richland, Washington. 
The different crust base elevations at risers $23 \mathrm{~A}$ and $22 \mathrm{~A}$ were based on the RGS X-ray observations. These observations were consistent with other, more generally applicable crust thickness assessments that have been made on the basis of validation temperature profiles and neutron scans.(a)

\subsubsection{Sampling and Extraction Information}

The samples that underwent RGS analysis are listed in Table 4.15.1. Field data, including dose rates and downforce limits, are supplied in Appendix A. Table 4.15.1 includes the elapsed time between mixer pump runs and sample acquisition. Two times are given for each sample: the top time is the hours since the last pump run that was aimed within five degrees of the riser angle; the bottom time is the hours since the most recent pump run (wherever aimed).

Table 4.15.1. Acquisition and Processing Schedule for RGS Samples from Tank SY-101

\begin{tabular}{|c|c|c|c|c|}
\hline Sample & Acquisition Date & Processing Start Date & Lag (days) & Time Since Last Pumping \\
\hline $23 \mathrm{~A}-1$ & November 9, 1998 & December 8, 1998 & 29 & $\begin{array}{l}183 \mathrm{hr}\left(\text { since pump at } 95^{\circ}\right) \\
\left.42 \mathrm{hr} \text { (since last pump, at } 185^{\circ}\right)\end{array}$ \\
\hline $23 \mathrm{~A}-2$ & November 9, 1998 & November 18, 1998 & 9 & $\begin{array}{l}\left.184 \mathrm{hr} \text { (since pump at } 95^{\circ}\right) \\
\left.43 \mathrm{hr} \text { (since last pump, at } 185^{\circ}\right)\end{array}$ \\
\hline $22 A-3$ & January 6, 1999 & February 3, 1999 & 28 & $\begin{array}{l}\left.356 \mathrm{hr} \text { (since pump at } 50^{\circ}\right) \\
42 \mathrm{hr}\left(\text { since lașt pump, at } 65^{\circ}\right)\end{array}$ \\
\hline $23 \mathrm{~A}-3$ & November 10,1998 & November 23, 1998 & 13 & $\begin{array}{l}185 \mathrm{hr}\left(\text { since pump at } 95^{\circ}\right) \\
\left.45 \mathrm{hr} \text { (since last pump, at } 185^{\circ}\right)\end{array}$ \\
\hline $22 \mathrm{~A}-4$ & January 7,1999 & March 2, 1999 & 54 & $\begin{array}{l}\left.371 \mathrm{hr} \text { (since pump at } 50^{\circ}\right) \\
\left.57 \mathrm{hr} \text { (since last pump, at } 155^{\circ}\right)\end{array}$ \\
\hline $23 \mathrm{~A}-4$ & November 10,1998 & December 2, 1998 & 22 & $\begin{array}{l}191 \mathrm{hr}\left(\text { since pump at } 95^{\circ}\right) \\
50 \mathrm{hr} \text { (since last pump, at } 185^{\circ} \text { ) }\end{array}$ \\
\hline $4 A-5$ & March 8, 1999 & March 29, 1999 & 21 & $\begin{array}{l}\left.129 \mathrm{hr} \text { (since pump at } 50^{\circ}\right) \\
\left.27 \mathrm{hr} \text { (since last pump, at } 110^{\circ}\right)\end{array}$ \\
\hline $23 \mathrm{~A}-8$ & November 18,1998 & December 15,1998 & 27 & $\begin{array}{l}390 \mathrm{hr} \text { (since pump at } 95^{\circ} \text { ) } \\
9 \mathrm{hr} \text { (since last pump, at } 50^{\circ} \text { ) }\end{array}$ \\
\hline $22 \mathrm{~A}-10$ & January 12, 1999 & March 15, 1999 & 62 & $\begin{array}{l}501 \mathrm{hr}\left(\text { since pump at } 50^{\circ}\right) \\
\left.49 \mathrm{hr} \text { (since last pump, at } 125^{\circ}\right)\end{array}$ \\
\hline $23 A-13$ & November 19, 1998 & December 30, 1998 & 41 & $\begin{array}{l}406 \mathrm{hr}\left(\text { since pump at } 95^{\circ}\right) \\
\left.24 \mathrm{hr} \text { (since last pump, at } 50^{\circ}\right)\end{array}$ \\
\hline $22 \mathrm{~A}-17$ & January 13, 1999 & April 26, 1999 & 103 & $\begin{array}{l}524 \mathrm{hr}\left(\text { since pump at } 50^{\circ}\right) \\
71 \mathrm{hr}\left(\text { since last pump, at } 125^{\circ}\right)\end{array}$ \\
\hline $23 \mathrm{~A}-21$ & December 17, 1998 & January 15, 1999 & 29 & $\begin{array}{l}\left.177 \mathrm{hr} \text { (since pump at } 95^{\circ}\right) \\
105 \mathrm{hr} \text { (since last pump, at } 155^{\circ} \text { ) }\end{array}$ \\
\hline $22 \mathrm{~A}-23$ & January 18, 1999 & May 4, 1999 & 106 & $\begin{array}{l}\left.573 \mathrm{hr} \text { (since pump at } 50^{\circ}\right) \\
121 \mathrm{hr} \text { (since last pump, at } 125^{\circ} \text { ) }\end{array}$ \\
\hline
\end{tabular}

(a) Stewart CW, PA Meyer, and BL Deatherage. July 1999. Assessment of the Recent Crust Base Evolution in SY-101. TWS99.46 Rev. 1, Pacific Northwest National Laboratory, Richland, Washington. 
Table 4.15.1 also shows the lag times (delay between sample acquisition and processing) for these samples. This information was provided to allow data users to correlate the lag time between sampling and extrusion with the concentrations of the various constituents, to test for decomposition or other chemical reactions. The long lag times experienced by several of the samples were the result, in part, of the long ammonia equilibration times between measurements. The effect of the long lag times on the samples was discussed in detail for S-111 samples in Section 4.13.5.2. The conclusion was that lag times longer than those incurred for SY-101 samples changed the gas composition by less than the measurement uncertainty and that there is substantially less than $10 \%$ potential underestimation of gas volume fraction resulting from the long lag times.

Of the samples shown in Table 4.15.1, sample 23A-4 gases were lost during processing in the laboratory when vacuum was inadvertently applied during the initial extrusion of the sample. Samples 23A-2 and 23A-3 both suffered some loss of pressure data as a result of overwritten files or a too-slow data acquisition rate. The logbook supplied the most essential information, and the uncertainty was increased to account for the remaining data loss.

As was discussed in Section 3.5, the HHF used during sampling often enters the sampler in place of some of the waste. There were no bromide data for the RGS samples. The bromide concentrations in the non-RGS samples averaged out to about 7 vol\% contamination, whether the samples that were below the MDL were included or not.(a) Thus the RGS samples were assumed to have been contaminated with $7.0 \mathrm{vol} \% \mathrm{HHF}$. Based on HHF measurements in samples from other tanks, this estimate should not produce more than $5 \%$ conservatism.

All of these samplers were helium-backfilled and sealed with vacuum grease before they were deployed. This method virtually eliminated air and argon contamination from gases entrained during sampling. The helium backfill results are discussed in detail by Cannon (1997) and summarized in Section 5 of Mahoney et al. (1997). Sample 22A-4 showed signs of air leaks into the RGS processing system (in spite of the pre-extrusion system leak check). The inleaked air was corrected for in the usual way.

Two different extraction procedures were used on the SY-101 samples. The first included only one post-isotopic extraction; the second included two. All the samples from riser 22A used the second, more complete procedure, as did samples 4A-5, 23A-1, and 23A-21. Most of the samples from riser $23 \mathrm{~A}$ used the first procedure. The two procedures were identical before the isotopic solution was added. (See Figure 3.1 for a schematic of the RGS extraction system.)

After evacuating the whole system and observing the pressure to test for leaks, the operator extruded the RGS sample into the extractor vessel, and the sample was briefly stirred. At this point, the sample was in communication with a vapor space consisting of the volumes of the extractor vessel, its lines, the second volume, the pump volume, and the collector side; the latter included the collector line volume and an open collection canister. This first canister, containing a sample of uncompressed (unpumped) gas, was closed off and the second set of three collection canisters opened. Several strokes were pumped from the extractor to the collector, and the second set of collection canisters was closed off. (In sample 22A-4 there was so much gas that it was necessary to collect an extra set of three canisters after the usual second set was full.) Then the collector side was evacuated. (The second volume was never used during the extraction process for SY-101 samples.)

The vapor profile procedure was then performed. The first pump stroke and every ninth stroke thereafter were collected in single canisters, with only one stroke per canister. On the inter-

(a) Transmittal from FH Steen (RFSHI) to LA Mahoney (PNNL) of preliminary data for Core 237 from Tank SY-101, July 1998. Rust Federal Services of Hanford, Inc., Richland; Washington. 
mediate strokes the gas was pumped to the collector lines, with no canister attached. The collector lines were evacuated after every pump stroke, whether or not the pumped gas was collected in a canister. The total gas and vapor pumped from the extractor to the collector during this procedure was calculated by interpolating linearly between collected canisters to find the contents of the pump strokes that were not collected. At the end of the procedure, the first post-isotopic set of canisters was attached to the collector lines and opened, and both the extractor lines and the collector side were evacuated.

For most riser $23 \mathrm{~A}$ samples: $300 \mathrm{~mL}$ of $0.04 \mathrm{M} 15 \mathrm{NH}_{4} \mathrm{OH}$ solution was added to the sample in the extractor and stirred. After 20 to 115 hours, several strokes were pumped from the extractor to the collector. The first (and only) post-isotopic set of three canisters was closed off. Finally, the BSVD procedure was performed: argon was injected into the closed-off second volume and the second volume opened to the extractor and lines. Pressure measurements and known tare system volumes allowed calculation of the volume of the sample solids and liquid.

For riser 22A samples 4A-5, 23A-1, and 23A-21: $300 \mathrm{~mL}$ of $0.04 \mathrm{M} 15 \mathrm{NH}_{4} \mathrm{OH}$ solution were added to the sample in the extractor and stirred. After 22 to 26 hours, several strokes were pumped from the extractor to the collector. The first post-isotopic set of three canisters was closed off. The second set of post-isotopic set of canisters was attached to the collector lines and opened, and both the extractor lines and the collector side were evacuated. After a few more days, several strokes were pumped from the extractor to the collector. The second post-isotopic set of three canisters was closed off, and the BSVD procedure was performed.

For all the SY-101 samples, four to seven aliquots (subsamples) were drawn from each post-isotopic canister to provide more opportunity for the isotopic ratios to converge. Sample 22A-4 underwent further processing(a) to ascertain the organic constituents making up the nonmethane hydrocarbons that the usual RGS method lumps together as "Other Hydrocarbons." This processing and the results are discussed in Appendix B.

Procedural details like the number of strokes per canister and the system volumes can be found in the sample-by-sample worksheets in the SY-101 RGS calculation spreadsheet on the CD supplied with this report.

\subsubsection{Retained Gas Composition}

Table 4.15.2 presents the calculated concentrations of the insoluble gases in the SY-101 RGS samples without corrections for air and argon entrainment, helium backfill, air inleakage, or air dissolved in the isotopic standard solution. The method used to make the corrections depended on the gas. All of the oxygen, argon, and helium were subtracted no matter which step had produced them. A number of moles equal to $(3.73) \cdot\left(\mathrm{O}_{2}\right)$ was subtracted from the nitrogen because the oxygen was expected to have come from air entrainment during sampling or inleakage during extraction. Because a substantial amount of dissolved air gases entered the extractor with the $15 \mathrm{NH}_{4} \mathrm{OH}$ solution, all the nitrogen in the gas collected after the solution addition was subtracted. Thus it was assumed that more than $95 \%$ of the original retained nitrogen had already been collected, as was the case for hydrogen. The corrected concentrations are given in Table 4.15.3.

Samples 23A-1 and 22A-4 both contained unusual amounts of air. The high oxygen in sample $23 \mathrm{~A}-1$ was part of the sample and was probably air contained in open pores of the crust (see Section 4.15.5.2). Judging by pressure data and contamination measurements, the oxygen in sample $22 \mathrm{~A}-4$ originated in accidental air inleakage into the collector side of the RGS system

(a) Evans JC, AV Mitroshkov, LA Mahoney. 1999. SY-101 Retained Gas Vapor GC/MS Analysis. Letter report TWS99.31, Pacific Northwest National Laboratory, Richland, Washington. 
Table 4.15.2. Concentrations of Insoluble Constituents ( $\mu \mathrm{mol} / \mathrm{L}$ of waste) in Tank SY-101 Without Correction for Gas Contamination

\begin{tabular}{||c|c|c|c|c|c|c|c|c|c|c|c|}
\hline Sample & $\mathrm{N}_{2}$ & $\mathrm{H}_{2}$ & $\mathrm{~N}_{2} \mathrm{O}$ & $\mathrm{O}_{2}$ & $\mathrm{CH}_{4}$ & $\mathrm{He}$ & $\mathrm{Ar}$ & $\begin{array}{l}\text { Other } \\
\mathrm{NO}_{x}\end{array}$ & $\mathrm{C}_{2} \mathrm{H}_{x}$ & $\mathrm{C}_{3} \mathrm{H}_{x}$ & Other Hyd \\
\hline \hline $23 \mathrm{~A}-1$ & $10000 \pm 700$ & $1700 \pm 130$ & $1800 \pm 140$ & $1400 \pm 96$ & $62 \pm 6.1$ & $270 \pm 22$ & $88 \pm 6.3$ & (a) & $48 \pm 5.7$ & (a) & $7.1 \pm 2.9$ \\
\hline $23 \mathrm{~A}-2$ & $6600 \pm 490$ & $5600 \pm 450$ & $3500 \pm 280$ & $230 \pm 22$ & $92 \pm 9.5$ & $620 \pm 53$ & $17 \pm 2.4$ & (a) & $130 \pm 45$ & (a) & $12 \pm 6.4$ \\
\hline $22 \mathrm{~A}-3$ & $7100 \pm 480$ & $4700 \pm 360$ & $3500 \pm 270$ & $280 \pm 21$ & $89 \pm 21$ & $570 \pm 51$ & $20 \pm 2.0$ & (a) & $49 \pm 22$ & (a) & $11 \pm 5.4$ \\
\hline $23 \mathrm{~A}-3$ & $7300 \pm 310$ & $10000 \pm 550$ & $4600 \pm 210$ & $280 \pm 19$ & $140 \pm 13$ & $610 \pm 37$ & $18 \pm 2.0$ & (a) & $120 \pm 20$ & (a) & $12 \pm 6.0$ \\
\hline $22 \mathrm{~A}-4$ & $14000 \pm 460$ & $11000 \pm 420$ & $5300 \pm 190$ & $1900 \pm 75$ & $180 \pm 16$ & $460 \pm 26$ & $93 \pm 5.3$ & (a) & $120 \pm 11$ & (a) & $8.5 \pm 2.9$ \\
\hline 4A-5 & $2400 \pm 490$ & $1100 \pm 150$ & $720 \pm 130$ & $200 \pm 64$ & $30 \pm 6.8$ & $540 \pm 80$ & $23 \pm 6.4$ & (a) & $17 \pm 5.5$ & (a) & $13 \pm 7.9$ \\
\hline $23 \mathrm{~A}-8$ & $1600 \pm 210$ & $460 \pm 79$ & $460 \pm 92$ & $72 \pm 13$ & $26 \pm 8.8$ & $560 \pm 94$ & $16 \pm 3.2$ & (a) & $13 \pm 5.8$ & (a) & $13 \pm 4.1$ \\
\hline $22 \mathrm{~A}-10$ & $2200 \pm 210$ & $560 \pm 130$ & $540 \pm 120$ & $280 \pm 19$ & $27 \pm 5.9$ & $140 \pm 34$ & $22 \pm 2.2$ & (a) & $13 \pm 4.8$ & (a) & $13 \pm 2.9$ \\
\hline 23A-13 & $2000 \pm 210$ & $550 \pm 130$ & $540 \pm 130$ & $240 \pm 18$ & $22 \pm 6.0$ & $620 \pm 150$ & $19 \pm 2.7$ & (a) & $14 \pm 5.7$ & (a) & $14 \pm 7.7$ \\
\hline $22 \mathrm{~A}-17$ & $2400 \pm 200$ & $590 \pm 93$ & $620 \pm 100$ & $300 \pm 20$ & $29 \pm 5.2$ & $430 \pm 69$ & $21 \pm 2.1$ & (a) & $14 \pm 4.4$ & (a) & $11 \pm 6.0$ \\
\hline 23A-21 & $2400 \pm 250$ & $600 \pm 120$ & $560 \pm 100$ & $240 \pm 17$ & $32 \pm 7.2$ & $380 \pm 80$ & $23 \pm 2.9$ & (a) & $15 \pm 4.9$ & (a) & $13 \pm 6.7$ \\
\hline 22A-23 & $2600 \pm 280$ & $770 \pm 130$ & $730 \pm 150$ & $330 \pm 28$ & $32 \pm 6.8$ & $430 \pm 78$ & $22 \pm 2.4$ & (a) & $17 \pm 6.6$ & (a) & $13 \pm 6.5$ \\
\hline (a) Below detection limits, which are 0.01 mol\% or less. & & & & & & \\
\hline
\end{tabular}

Table 4.15.3. Concentrations of Insoluble Constituents ( $\mu \mathrm{mol} / \mathrm{L}$ of waste) in Tank SY-101 with Correction for Gas Contamination

\begin{tabular}{||c|c|c|c|c|c|c|c|c|c|c|c||}
\hline $\begin{array}{l}\text { Sam- } \\
\text { ple }\end{array}$ & $\mathrm{N}_{2}$ & $\mathrm{H}_{2}$ & $\mathrm{~N}_{2} \mathrm{O}$ & $\mathrm{O}_{2}$ & $\mathrm{CH}_{4}$ & $\mathrm{He}$ & $\mathrm{Ar}$ & $\begin{array}{l}\text { Other } \\
\mathrm{NO}_{x}\end{array}$ & $\mathrm{C}_{2} \mathrm{H}_{x}$ & $\mathrm{C}_{3} \mathrm{H}_{x}$ & $\begin{array}{l}\text { Other } \\
\text { Hyd. }\end{array}$ \\
\hline \hline $23 \mathrm{~A}-1$ & $3700 \pm 570$ & $1700 \pm 130$ & $1800 \pm 140$ & $0 \pm 96$ & $62 \pm 6.1$ & $0 \pm 22$ & $0 \pm 6.3$ & (a) & $48 \pm 5.7$ & (a) & $7.1 \pm 2.9$ \\
\hline $23 \mathrm{~A}-2$ & $4400 \pm 530$ & $5600 \pm 450$ & $3500 \pm 280$ & $0 \pm 22$ & $92 \pm 9.5$ & $0 \pm 53$ & $0 \pm 2.4$ & (a) & $130 \pm 45$ & (a) & $12 \pm 6.4$ \\
\hline $22 \mathrm{~A}-3$ & $4200 \pm 520$ & $4700 \pm 360$ & $3500 \pm 270$ & $0 \pm 21$ & $89 \pm 21$ & $0 \pm 51$ & $0 \pm 2.0$ & (a) & $49 \pm 22$ & (a) & $11 \pm 5.4$ \\
\hline $23 \mathrm{~A}-3$ & $5900 \pm 600$ & $10000 \pm 550$ & $4600 \pm 210$ & $0 \pm 19$ & $140 \pm 13$ & $0 \pm 37$ & $0 \pm 2.0$ & (a) & $120 \pm 20$ & (a) & $12 \pm 6.0$ \\
\hline $22 \mathrm{~A}-4$ & $6800 \pm 740$ & $11000 \pm 420$ & $5300 \pm 190$ & $0 \pm 75$ & $180 \pm 16$ & $0 \pm 26$ & $0 \pm 5.3$ & (a) & $120 \pm 11$ & (a) & $8.5 \pm 2.9$ \\
\hline $4 \mathrm{~A}-5$ & $780 \pm 170$ & $1100 \pm 150$ & $720 \pm 130$ & $0 \pm 64$ & $30 \pm 6.8$ & $0 \pm 80$ & $0 \pm 6.4$ & (a) & $17 \pm 5.5$ & (a) & $13 \pm 7.9$ \\
\hline $23 \mathrm{~A}-8$ & $880 \pm 200$ & $460 \pm 79$ & $460 \pm 92$ & $0 \pm 13$ & $26 \pm 8.8$ & $0 \pm 94$ & $0 \pm 3.2$ & (a) & $13 \pm 5.8$ & (a) & $13 \pm 4.1$ \\
\hline 22A-10 & $670 \pm 200$ & $560 \pm 130$ & $540 \pm 120$ & $0 \pm 19$ & $27 \pm 5.9$ & $0 \pm 34$ & $0 \pm 2.2$ & (a) & $13 \pm 4.8$ & (a) & $13 \pm 2.9$ \\
\hline 23A-13 & $700 \pm 190$ & $550 \pm 130$ & $540 \pm 130$ & $0 \pm 18$ & $22 \pm 6.0$ & $0 \pm 150$ & $0 \pm 2.7$ & (a) & $14 \pm 5.7$ & (a) & $14 \pm 7.7$ \\
\hline 22A-17 & $680 \pm 160$ & $590 \pm 93$ & $620 \pm 100$ & $0 \pm 20$ & $29 \pm 5.2$ & $0 \pm 69$ & $0 \pm 2.1$ & (a) & $14 \pm 4.4$ & (a) & $11 \pm 6.0$ \\
\hline 23A-21 & $800 \pm 210$ & $600 \pm 120$ & $560 \pm 100$ & $0 \pm 17$ & $32 \pm 7.2$ & $0 \pm 80$ & $0 \pm 2.9$ & (a) & $15 \pm 4.9$ & (a) & $13 \pm 6.7$ \\
\hline 22A-23 & $850 \pm 210$ & $770 \pm 130$ & $730 \pm 150$ & $0 \pm 28$ & $32 \pm 6.8$ & $0 \pm 78$ & $0 \pm 2.4$ & (a) & $17 \pm 6.6$ & (a) & $13 \pm 6.5$ \\
\hline (a) Below detection imits, which are 0.01 mol\% or less. \\
\hline
\end{tabular}


during the initial pumped gas extraction. Contamination observations can be found in the "Summary" worksheet of the SY-101 RGS calculations spreadsheet on the CD.

Table 4.15.4 presents the ammonia measurements. The residual ammonia was determined isotopically, and the total ammonia (calculated from the isotopic residual) is included in the table. The post-extrusion partial pressure of $\mathrm{NH}_{3}$ over the sample (see Section 3.4.4) was measured at chilled extractor temperature and is included in Table 4.15.4. The residual ammonia in SY-101 samples was not measured by post-RGS ISE methods.

A number of other ammonia measurements have also been made on SY-101 samples. These include composites of core samples taken in May and December 1991 (Windows C and E) and measurements made on the non-RGS core extrusion samples taken during 1998 and 1999.

Most of the various ammonia measurements could be reconciled easily. In general, the isotopic ammonia measurements for SY-101 appeared to be of higher quality and more consistent than in earlier tanks. The RGS isotopic measurements were used to calculate the ammonia mole fractions in the in situ vapor. The ammonia concentration of the SY-101 waste (excluding the topmost segment of the crust) was between 140,000 and $200,000 \mu \mathrm{mol} \mathrm{NH}_{3} / \mathrm{L}$ of waste. The average and standard deviation of the RGS ammonia concentrations over all the samples except $23 \mathrm{~A}-1$ were $166,000 \pm 24,000 \mu \mathrm{mol} / \mathrm{L}$ waste. This ammonia concentration in the bulk waste corresponds to $0.24 \mathrm{wt} \% \mathrm{NH}_{3}$ in the bulk waste, or $4600 \mu \mathrm{g} \mathrm{NH} / \mathrm{mL}$ liquid $\left(0.27 \mathrm{M} \mathrm{NH}_{3}\right)$.

Table 4.15.4. Ammonia Data from Tank SY-101 Samples

\begin{tabular}{||c|c|c|c||}
\hline Sample & $\begin{array}{c}\text { Isotopically } \\
\text { Measured } \\
\text { Total } \mathrm{NH}_{3}(\mathrm{a})\end{array}$ & $\begin{array}{c}\text { Measured } \mathrm{NH}_{3} \\
\text { Partial Pressure } \\
\text { at about } 12^{\circ} \mathrm{C} \\
(\text { atm })\end{array}$ & $\begin{array}{c}\text { RGS } \mathrm{NH}_{3} \text { Concentrations } \\
\text { in the Liquid }\end{array}$ \\
\hline \hline $23 \mathrm{~A}-1$ & $81000 \pm 19000$ & $0.023 \pm 0.011$ & $2500 \mu \mathrm{g} / \mathrm{mL}(0.14 \mathrm{M})$ \\
\hline $23 \mathrm{~A}-2$ & $160000 \pm 32000$ & $0.032 \pm 0.016$ & $6300 \mu \mathrm{g} / \mathrm{mL}(0.37 \mathrm{M})$ \\
\hline $22 \mathrm{~A}-3$ & $93000 \pm 26000$ & $0.034 \pm 0.017$ & $4600 \mu \mathrm{g} / \mathrm{mL}(0.27 \mathrm{M})$ \\
\hline $23 \mathrm{~A}-3$ & $150000 \pm 35000$ & $0.032 \pm 0.016$ & $7800 \mu \mathrm{g} / \mathrm{mL}(0.46 \mathrm{M})$ \\
\hline $22 \mathrm{~A}-4$ & $130000 \pm 38000$ & $0.030 \pm 0.015(\mathrm{~b})$ & $10000 \mu \mathrm{g} / \mathrm{mL}(0.61 \mathrm{M})$ \\
\hline $4 \mathrm{~A}-5$ & $120000 \pm 34000$ & $0.035 \pm 0.017$ & $3500 \mu \mathrm{g} / \mathrm{mL}(0.21 \mathrm{M})$ \\
\hline $23 \mathrm{~A}-8$ & $140000 \pm 27000$ & $0.036 \pm 0.018$ & $2800 \mu \mathrm{g} / \mathrm{mL}(0.16 \mathrm{M})$ \\
\hline $22 \mathrm{~A}-10$ & $160000 \pm 45000$ & $0.039 \pm 0.019$ & $4100 \mu \mathrm{g} / \mathrm{mL}(0.24 \mathrm{M})$ \\
\hline $23 \mathrm{~A}-13$ & $200000 \pm 49000$ & $0.033 \pm 0.016$ & $4000 \mu \mathrm{g} / \mathrm{mL}(0.23 \mathrm{M})$ \\
\hline $22 \mathrm{~A}-17$ & $120000 \pm 34000$ & $0.034 \pm 0.017$ & $3200 \mu \mathrm{g} / \mathrm{mL}(0.19 \mathrm{M})$ \\
\hline $23 \mathrm{~A}-21$ & $180000 \pm 39000$ & $0.035 \pm 0.017$ & $3600 \mu \mathrm{g} / \mathrm{mL}(0.21 \mathrm{M})$ \\
\hline 22A-23 & $130000 \pm 36000$ & $0.033 \pm 0.016$ & $3400 \mu \mathrm{g} / \mathrm{mL}(0.20 \mathrm{M})$ \\
\hline (a) These data were used to calculate in situ ammonia vapor pressures. \\
\hline
\end{tabular}


The ammonia concentrations in the liquid were very high in samples $23 \mathrm{~A}-3$ and $22 \mathrm{~A}-4$, which were in the high-gas region referred to as the "bubble slurry." We suspect these high concentrations of being exaggerated by the low calculated liquid volume fraction in this part of the waste and by underestimated gas solubilities. Note that Table 4.15.4 shows the dissolved ammonia concentrations calculated for the lower-bound gas solubilities. At the upper-bound gas solubilities, the dissolved ammonia concentrations for $23 \mathrm{~A}-3$ and $22 \mathrm{~A}-4$ were calculated to be, respectively, $0.34 \mathrm{M}$ (rather than the low-solubility value of $0.46 \mathrm{M}$ ) and $0.42 \mathrm{M}$ (rather than the low-solubility value of $0.61 \mathrm{M}$ ). Using upper-bound solubilities had little effect on the calculated concentrations of dissolved ammonia in any of the other samples because the fraction of the gas volume that depended on ammonia vapor was so much smaller for those samples than for the bubble-slurry samples.

Table 4.15.5 contains the composition of the gas/vapor phase in each sample and the integrated average composition for the crust and mixed slurry layers in Tank SY-101. The water vapor is not included in these compositions. The ammonia fractions are derived from the data indicated in Table 4.15.4, which were used to calculate vapor pressures at in situ conditions. The sample compositions in the table have been calculated using the in situ solubility method described

\section{Table 4.15.5. Sample and Overall Average Compositions of Retained Gas in} Tank SY-101 with Correction for Gas Contamination(a)

\begin{tabular}{|c|c|c|c|c|c|c|}
\hline Sample & $\mathrm{N}_{2}(\mathrm{~mol} \%)$ & $\mathrm{H}_{2}(\mathrm{~mol} \%)$ & $\mathrm{N}_{2} \mathrm{O}(\mathrm{mol} \%)$ & $\mathrm{NH}_{3}(\mathrm{~mol} \%)^{(b)}$ & $\mathrm{CH}_{4}(\mathrm{~mol} \%)$ & Other (mol\%) \\
\hline $23 \mathrm{~A}-1$ & $47 \pm 9.4-51 \pm 9.9$ & $22 \pm 3.2-23 \pm 3.3$ & $23 \pm 3.4$ & $6.1 \pm 1.6-2.0 \pm 0.5$ & $0.8 \pm 0.1$ & $0.7 \pm 0.2-0.8 \pm 0.2$ \\
\hline $22 \mathrm{~A}-3$ & $30 \pm 4.9-33 \pm 5.2$ & $33 \pm 4.4-37 \pm 4.6$ & $25 \pm 3.3-26 \pm 3.3$ & $11 \pm 2.7-3.5 \pm 0.8$ & $0.6 \pm 0.2-0.7 \pm 0.2$ & $0.4 \pm 0.2-0.5 \pm 0.2$ \\
\hline $23 \mathrm{~A}-3$ & $21 \pm 3.2-27 \pm 3.4$ & $37 \pm 4.4-46 \pm 4.3$ & $17 \pm 2.0-20 \pm 1.8$ & $24 \pm 6.1-5.8 \pm 1.4$ & $0.5 \pm 0.07-0.6 \pm 0.07$ & $0.5 \pm 0.1-0.6 \pm 0.1$ \\
\hline $23 \mathrm{~A}-8$ & $45 \pm 14-52 \pm 16$ & $23 \pm 6.4-27 \pm 7.4$ & $22 \pm 6.4-15 \pm 4.5$ & $7.3 \pm 2.1-2.3 \pm 0.7$ & $1.3 \pm 0.5-1.5 \pm 0.6$ & $1.3 \pm 0.8-1.6 \pm 0.9$ \\
\hline $22 \mathrm{~A}-10$ & $34 \pm 13-42 \pm 16$ & $28 \pm 9.7-34 \pm 12$ & $26 \pm 8.5-18 \pm 6.1$ & $9.9 \pm 3.3-3.2 \pm 1.0$ & $1.4 \pm 0.4-1.6 \pm 0.6$ & $1.3 \pm 0.6-1.7 \pm 0.8$ \\
\hline $23 A-13$ & $36 \pm 13-44 \pm 17$ & $28 \pm 9.6-33 \pm 12$ & $25 \pm 8.8-17 \pm 5.8$ & $8.3 \pm 2.9-2.7 \pm 0.9$ & $1.1 \pm 0.4-1.4 \pm 0.5$ & $1.4 \pm 0.8-1.8 \pm 1.0$ \\
\hline $22 \mathrm{~A}-17$ & $34 \pm 10-42 \pm 13$ & $29 \pm 7.3-35 \pm 8.9$ & $28 \pm 7.2-18 \pm 4.6$ & $6.0 \pm 1.7-1.9 \pm 0.6$ & $1.4 \pm 0.4-1.8 \pm 0.5$ & $1.2 \pm 0.6-1.6 \pm 0.7$ \\
\hline $\begin{array}{l}23 \mathrm{~A} \text { avg } \\
\text { in mixed } \\
\text { slurry(c) }\end{array}$ & $40 \pm 14-48 \pm 16$ & $26 \pm 8.1-31 \pm 9.5$ & $24 \pm 7.4-16 \pm 4.9$ & $7.1 \pm 2.2-2.3 \pm 0.7$ & $1.3 \pm 0.5-1.6 \pm 0.6$ & $1.4 \pm 0.7-1.7 \pm 0.9$ \\
\hline $\begin{array}{l}22 \mathrm{~A} \text { avg } \\
\text { in crust(b) }\end{array}$ & $27 \pm 4.5-33 \pm 4.9$ & $32 \pm 3.9-39 \pm 3.9$ & $19 \pm 2.4-22 \pm 2.4$ & $21 \pm 5.1-4.8 \pm 1.3$ & $0.6 \pm 0.1-0.7 \pm 0.1$ & $0.4 \pm 0.1-0.5 \pm 0.1$ \\
\hline $\begin{array}{l}22 \mathrm{~A} \text { avg } \\
\text { in mixed } \\
\text { slurry(c) }\end{array}$ & $32 \pm 11-39 \pm 13$ & $31 \pm 8.5-37 \pm 10$ & $26 \pm 7.5-18 \pm 5.2$ & $7.8 \pm 2.3-2.5 \pm 0.8$ & $1.3 \pm 0.4-1.6 \pm 0.5$ & $1.2 \pm 0.6-1.5 \pm 0.7$ \\
\hline \multicolumn{7}{|c|}{$\begin{array}{l}\text { (a) Uncertainties on compositions represent instrument uncertainty; compositions calculated for lower- and upper-bound solubilities. } \\
\text { Both ends of composition range given, lower-bound solubility basis first with instrument uncertainty on each. Compositions may } \\
\text { not sum to } 100 \mathrm{~mol} \% \text { because of roundoff error. Mole fractions on dry basis and do not account for water vapor. } \\
\text { (b) Ammonia mole fraction derived from data in Table } 4.15 .4 \text { used to calculate vapor pressure at in situ conditions. } \\
\text { (c) There are too few samples to define the spatial variability of the average gas concentration. }\end{array}$} \\
\hline
\end{tabular}


in Section 3.6.2. As discussed in Section 3.6.1, compositions were calculated for both the lowerand upper-bound gas solubilities. Both ends of the composition range are given in Table 4.15.5, together with the measurement uncertainty on each. The average composition of the gas in each layer is the result of integrating RGS species concentrations over the layer and multiplying those concentrations by the layer volume. The integration method is described in Section 3.7.1.

Sample 4A-5 was integrated with the samples from riser $22 \mathrm{~A}$ to obtain the average mixed slurry composition because it was closer to them in time. In addition, because riser $22 \mathrm{~A}$ samples only included the lower part of the crust, sample $23 \mathrm{~A}-1$ was included in the riser-22A crust integration as an assumed value at the top surface of the crust.

At the low-solubility end of the range, the solubility model calculated unusually high mole fractions of $\mathrm{NH}_{3}$ in the in situ vapor for samples $23 \mathrm{~A}-3$ and $22 \mathrm{~A}-4,24$ and $28 \mathrm{~mol} \%$. These high values resulted from two features of the samples: the high ammonia Henry's Law constant calculated for the liquid and the low volume fraction of liquid in the sample (low because the gas-phase volume fraction is so high). The low-solubility estimate of the ammonia vapor pressure was probably 50 to $60 \%$ higher than the true value, based on the data at $50^{\circ} \mathrm{C}$ shown in Figure 3.3. If we increase the Henry's Law constant for ammonia by $50 \%$ to give a better estimate (more in accord with Figure 3.3), the ammonia mole fraction in sample 23A-3 drops from 24 to 13 mol\%. The change in mole fraction is more than proportional to the change in vapor pressure because of the effect that ammonia has on the gas volume fraction, which in tum drives the liquid volume fraction.

The liquid volume fraction was calculated from the degassed bulk waste density, the solid density, the liquid density, and the gas volume fraction. The lower the liquid fraction for a given measured amount of ammonia, the higher the concentration of ammonia in the liquid and the higher the vapor pressure. The higher the vapor pressure, the more ammonia must be distributed into the vapor phase, which drives the gas volume fraction higher and the liquid fraction lower. Therefore, under the proper conditions (unusually high ammonia together with unusually high "insoluble" gas), the calculation of the ammonia vapor pressure can be sensitive to the liquid fraction. A higher value for liquid fraction in the region at the bottom of the crust represented by samples 23A-3 and 22A-4 would produce a lower ammonia vapor pressure, other things being held constant. For example, if there were no solids in this region, the liquid volume fraction in the degassed waste would be 100 rather than $88 \mathrm{vol \%}$, and the ammonia mole fraction (at the lowsolubility end of the range) would be reduced from 24 to $20 \mathrm{~mol} \%$ in sample 23A-3. Again, the relative change in mole fraction is greater than the relative change in liquid fraction.

Based on these considerations, we think that the ammonia concentration in the gas retained at the bottom of the crust was probably closer to $12-17 \mathrm{~mol} \%$ ammonia than to either end of the range in the table. The average of low- and high-solubility estimates is the most plausible value. The concentrations and pressures in Tables 4.15.2 and 4.15.3 can also be found in the sample-bysample worksheets in the SY-101 RGS calculation spreadsheet on the CD. The compositions in Table 4.15.5 are in the "Inventory" worksheet.

\subsubsection{Gas Inventory}

The method by which the in situ gas volume fractions (wet basis) were calculated is given in Section 3.6.2; for gas composition, the volume fractions are given as a range from the low-gassolubility value to the high-gas-solubility value. The results are presented in Table 4.15.6, which also contains the average gas volume fraction and the average pressure experienced by the gas in each layer. The average is an in situ volume average calculated by Simpson's Rule integration, as described in Section 3.7.1. The corrected gas volume fractions in Table 4.15.6 are consistent with 
Table 4.15.6. In Situ Gas Volume Fractions and Conditions in Tank SY-101

\begin{tabular}{||c|c|c|c|c|c|c||}
\hline \hline Sample & $\begin{array}{c}\text { Sample } \\
\text { Central } \\
\text { (cm) }\end{array}$ & $\begin{array}{c}\text { Hydro- } \\
\text { static } \\
\text { Pressure } \\
\text { (atm) }\end{array}$ & $\begin{array}{c}\text { Calculated } \\
\text { Water Vapor } \\
\text { Pressure } \\
\text { (atm) }\end{array}$ & $\begin{array}{c}\text { Temp } \\
\text { ( }{ }^{\circ} \text { ) }\end{array}$ & $\begin{array}{c}\text { Corrected Gas Volume } \\
\text { Fraction(a) } \\
\text { (in-tank conditions) }\end{array}$ & $\begin{array}{c}\text { Sampler Gas } \\
\text { Volume Fraction } \\
\text { (in situ conditions, } \\
\text { low solubility) }\end{array}$ \\
\hline \hline $23 \mathrm{~A}-1$ & 1022 & 1.02 & 0.015 & 38.3 & $0.20 \pm 0.015-0.18 \pm 0.015$ & 0.33 \\
\hline $23 \mathrm{~A}-2$ & 974 & 1.08 & 0.018 & 41.1 & $0.40 \pm 0.030-0.34 \pm 0.029$ & 0.42 \\
\hline $22 \mathrm{~A}-3$ & 959 & 1.13 & 0.017 & 40.6 & $0.33 \pm 0.027-0.30 \pm 0.027$ & 0.34 \\
\hline $23 \mathrm{~A}-3$ & 925 & 1.13 & 0.030 & 45.0 & $0.65 \pm 0.039-0.52 \pm 0.039$ & 0.66 \\
\hline $22 \mathrm{~A}-4$ & 911 & 1.18 & 0.029 & 44.4 & $0.72 \pm 0.07-0.567 \pm 0.037$ & 0.73 \\
\hline $4 \mathrm{~A}-5$ & 845 & 1.27 & 0.035 & 48.3 & $0.062 \pm 0.018-0.051 \pm 0.017$ & 0.074 \\
\hline $23 \mathrm{~A}-8$ & 684 & 1.48 & 0.035 & 48.2 & $0.036 \pm 0.012-0.030 \pm 0.012$ & 0.047 \\
\hline $22 \mathrm{~A}-10$ & 621 & 1.61 & 0.035 & 48.2 & $0.033 \pm 0.011-0.026 \pm 0.010$ & 0.036 \\
\hline $23 \mathrm{~A}-13$ & 443 & 1.84 & 0.034 & 48.1 & $0.029 \pm 0.009-0.022 \pm 0.009$ & 0.038 \\
\hline $22 \mathrm{~A}-17$ & 316 & 2.07 & 0.034 & 48.1 & $0.026 \pm 0.007-0.020 \pm 0.007$ & 0.032 \\
\hline $23 \mathrm{~A}-21$ & 75 & 2.39 & 0.033 & 47.2 & $0.023 \pm 0.007-0.018 \pm 0.007$ & 0.031 \\
\hline 22A-23 & 28 & 2.50 & 0.029 & 44.4 & $0.026 \pm 0.008-0.020 \pm 0.008$ & 0.032 \\
\hline $\begin{array}{c}\text { Avg in } \\
\text { 23A crust(b) }\end{array}$ & 953 & 1.10 & & & $0.40 \pm 0.20-0.34 \pm 0.17$ & \\
\hline $\begin{array}{c}\text { Avg in } \\
\text { 23A mixed } \\
\text { slurry(b) }\end{array}$ & 445 & 1.84 & & & $0.029 \pm 0.014-0.023 \pm 0.014$ & \\
\hline $\begin{array}{c}\text { Avg in } \\
\text { 22A crust(b) }\end{array}$ & 942 & 1.15 & & & $0.52 \pm 0.26-0.44 \pm 0.22$ & \\
\hline $\begin{array}{c}\text { Avg in } \\
\text { 22A mixed } \\
\text { slurry(b) }\end{array}$ & 455 & 1.88 & & & $0.031 \pm 0.015-0.025 \pm 0.015$ & \\
\hline $\begin{array}{l}\text { (a) Gas volume fraction expressed on a wet basis, including the volume contribution of water vapor. } \\
\text { (b) Uncertainties on layer-average gas volume fractions based on spatial variability considerations } \\
\text { discussed in Section 3.7.2. }\end{array}$ & & & & & & \\
\hline
\end{tabular}

the corrected gas concentrations and compositions in Tables 4.15.2, 4.15.3, and 4.15.5. The information in Table 4.15.6 is taken from the "Summary" and "In situ" worksheets of the SY-101 RGS calculation spreadsheet on the $C D$.

The "sampler gas volume fraction" is corrected only for inleakage during the extraction process and air gases added in the isotopic solution. It is an attempt to reconstruct the total amount of gas (sample and entrainment) that was present in the sampler during $\mathrm{X}$-ray. It is used only for comparison with the $\mathrm{x}$-ray observations of "visible gas fraction" discussed in Section 4.15.4.

Table 4.15.6 also contains the water vapor pressures that were used for in situ calculations. The water vapor pressures were found by using salt concentrations from Reynolds (1993) and the temperatures in the table as inputs to Equation 6.2 of Mahoney and Trent (1995), a correlation for water vapor pressure over concentrated homogeneous and non-homogeneous waste simulants. The gas solubilities used the same parameters as inputs to the Schumpe solubility model (Section 3.6.1). The water vapor pressures and gas solubilities used in calculations can be found in the "In situ" worksheet of the SY-101 RGS calculation spreadsheet on the CD. 
The ammonia concentration measurements in SY-101 integrate to a total (vapor and dissolved) ammonia inventory that would have had an STP volume of $16000 \mathrm{~m}^{3}\left(560,000 \mathrm{ft}^{3}\right)$. Of this, 10 to $15 \%$ was in the crust (including the "bubble slurry" at the bottom). The uncertainty on the ammonia inventory was at least $70 \%$.

Table 4.15.7 gives estimates of the STP volumes of gas in Tank SY-101 calculated from RGS data and from VFI data (Stewart et al. 1998). The RGS gas inventory in each layer was calculated by integrating RGS total gas concentrations over the layer and multiplying the average gas concentration by the volume of the layer. (The integration method is described in Section 3.7.1.) The RGS volumes in Table 4.15.7 include corrections to remove the contamination gas: entrained air and argon, helium backfill, air leaks during and after extraction, and air dissolved in the isotopic standard. The uncertainties on the gas inventories are based on the spatial variability considerations discussed in Section 3.7.2. The information in Table 4.15.7 is taken from the "Inventory" worksheet of the SY-101 RGS calculation spreadsheet on the CD.

The RGS-derived crust gas inventories for the two risers differed from each other for two major reasons. First, the crust was observed to be 30\% thicker at riser 22A in January 1999 than at riser 23A in November 1998. Second, a higher gas fraction was measured in the crust bottom at $22 \mathrm{~A}$ than at $23 \mathrm{~A}$. Also, note that no crust-top RGS samples were taken in riser $22 \mathrm{~A}$; therefore, sample $23 \mathrm{~A}-1$ was included in the riser-22A crust integration as an assumed value at the top surface of the crust.

The RGS gas inventories are larger than those measured by the VFI. This is true for both the crust and mixed slurry layers. The differences between the RGS and VFI crust gas inventories were within the uncertainties and were exaggerated by using the low-solubility RGS estimates. However, it is likely that the higher RGS inventory in the crust under riser 23A did in fact represent an increase in the gas inventory after VFI measurements, consistent with other observations. The higher RGS gas inventory in the mixed slurry layer almost certainly indicated an increase in the gas, as corroborated by measured changes in the mixer pump power consumption and discharge pressure over the period in question.

Table 4.15.7. SY-101 Gas Inventory Estimates

\begin{tabular}{|c|c|c|c|c|c|c|}
\hline \multirow{3}{*}{ Quantity } & \multicolumn{4}{|c|}{ RGS Method } & \multicolumn{2}{|c|}{ VFI Method } \\
\hline & \multicolumn{2}{|c|}{ Crust } & \multicolumn{2}{|c|}{ Mixed Slurry } & \multirow[b]{2}{*}{$\begin{array}{c}\text { Crust } \\
6 / 98 \& \text { \& 9/98 }\end{array}$} & \multirow[b]{2}{*}{$\begin{array}{c}\text { Mixed Slurry } \\
7 / 98 \& \text { \& } 9 / 98\end{array}$} \\
\hline & $\begin{array}{c}\text { Riser 23A } \\
11 / 98 \\
\end{array}$ & $\begin{array}{c}\text { Riser } 22 \mathrm{~A} \\
1 / 99 \\
\end{array}$ & $\begin{array}{c}\text { Riser } 23 \mathrm{~A} \\
11 / 98-12 / 98 \\
\end{array}$ & $\begin{array}{c}\text { Risers 22A, 4A } \\
1 / 99 \& 3 / 99 \\
\end{array}$ & & \\
\hline $\begin{array}{l}\text { Avg gas fraction } \\
\text { (low gas } \\
\text { solubility) }\end{array}$ & $0.40 \pm 0.20$ & $0.52 \pm 0.26$ & $0.029 \pm 0.014$ & $0.031 \pm 0.015$ & $0.30 \pm 0.04$ & $0.013 \pm 0.001$ \\
\hline $\begin{array}{l}\text { Gas volume } \\
\left(\mathrm{m}^{3}\right) \text { in situ } \\
\text { (wet) }\end{array}$ & & $424 \pm 212$ & $108 \pm 54$ & $116 \pm 58$ & $145 \pm 24$ & $48 \pm 5$ \\
\hline STP (wet) & $233 \pm 116$ & $420 \pm 210$ & $169 \pm 84$ & $185 \pm 92$ & $149 \pm 24$ & $84 \pm 9$ \\
\hline
\end{tabular}


Values of dL/dP were calculated for Tank SY-101 during those few several-day intervals in November and December 1998 when there was no pumping to disturb the waste level.(a) The $\mathrm{dL} / \mathrm{dP}$ ranged widely, from -0.21 to -1.0 in./in. $\mathrm{Hg}$; this variability indicates that the $\mathrm{dL} / \mathrm{dP}$ could have been unreliable, perhaps because of vertical motion of bubbles suspended by the mixer pump. The median of the $\mathrm{dL} / \mathrm{dP}$ values was $-0.67 \pm 0.26 \mathrm{in}$./in. Hg. Assuming an average gas pressure of $1.33 \mathrm{~atm}$ based on RGS data from riser 23A, the total in situ gas volume in Tank SY-101 is calculated (from the $\mathrm{dL} / \mathrm{dP}$ ) to have been $277 \pm 111 \mathrm{~m}^{3}$ as of November-December 1998. Thus it was in reasonable agreement with the total gas inventory of $353 \pm 176 \mathrm{~m}^{3}$ calculated from riser 23A RGS data, which were taken in the same period.

The total gas inventories calculated for SY-101 were $193 \pm 24 \mathrm{~m}^{3}$ in situ from the summer 1998 VFI data, $353 \pm 133 \mathrm{~m}^{3}$ in situ from riser 23A RGS data (November and December 1998), and $540 \pm 220 \mathrm{~m}^{3}$ in situ from riser 22A/4A RGS data (January 1999). Of the two sets of RGS inventories, we recommend the ones calculated from riser $23 \mathrm{~A}$ data as the better estimate of the tank gas inventory between November 1998 and January 1999. Both RGS inventories contain enough uncertainty to be reconcilable with the measured level, but the $23 \mathrm{~A}$ inventory provides a better match to our current understanding of SY-101 behavior. The average gas volume fractions for crust and mixed slurry were used as inputs to the buoyancy model described by Stewart et al. (1999, Section 2). The 23A data resulted in a predicted waste level of 434 in. on February 8, 1999 while the 22A/4A data gave a predicted level of $441 \mathrm{in}$; the measured value was 428 in.(b) The above-mentioned RGS inventories were all based on the lower-bound gas solubilities; both the sets of inventories are lower at the upper-bound solubilities, and give a better match to the waste level.

Table 4.15.8 contains the calculated layer inventories of each of the major gases retained in the waste. These inventories are based on the layer-average compositions from RGS data (Table 4.15.5) and the best-estimate gas inventories for November 1998 through January 1999 (RGS riser 23A, Table 4.15.7).

Table 4.15.8. Speciated SY-101 Gas Inventories(a)

\begin{tabular}{|c|c|c|c|c|}
\hline \multirow[b]{2}{*}{ Species } & \multicolumn{2}{|c|}{$\begin{array}{c}\text { Gas-Phase Inventory } \\
\left(\mathrm{m}^{3} \text { at STP) }\right.\end{array}$} & \multicolumn{2}{|c|}{$\begin{array}{c}\text { Dissolved Inventory } \\
\left(\mathrm{m}^{3} \text { at STP }\right)\end{array}$} \\
\hline & $\begin{array}{l}\text { Crust } \\
\text { Layer }\end{array}$ & $\begin{array}{c}\text { Mixed Slurry } \\
\text { Layer }\end{array}$ & $\begin{array}{l}\text { Crust } \\
\text { Layer } \\
\end{array}$ & $\begin{array}{c}\text { Mixed Slurry } \\
\text { Layer }\end{array}$ \\
\hline $\mathrm{N}_{2}$ & 62 & $66-64$ & $0.0045-0.078$ & $0.11-1.8$ \\
\hline $\mathrm{H}_{2}$ & 77 & $44-41$ & $0.014-0.16$ & $0.28-2.7$ \\
\hline $\mathrm{N}_{2} \mathrm{O}$ & $44-42$ & $39-21$ & $0.15-1.8$ & $3.3-22$ \\
\hline $\mathrm{NH}_{3}$ & $43-9.4$ & $12-3.1$ & 1700 & 14000 \\
\hline $\mathrm{CH}_{4}$ & 1.3 & $2.2-2.1$ & $0.00015-0.0029$ & $0.0064-0.12$ \\
\hline Other & 1.4 & 2.3 & 0 & 0 \\
\hline \multicolumn{5}{|c|}{$\begin{array}{l}\text { (a) Inventories based on layer-average compositions from RGS data (Table } 4.15 .5 \text { ) and best-estimate gas inventories } \\
\text { for each layer (RGS, Table 4.15.7). Uncertainty in each inventory is } 50 \% \text { based on spatial variability consideration } \\
\text { (Section 3.7.2). Inventories given for lower- and upper-bound solubilities; lower-bound solubility basis first. }\end{array}$} \\
\hline
\end{tabular}

(a) Chen G. 1999. "Re-Evaluation of SY-101 Gas Volume Using the Barometric Pressure Effect Method." Notes supplied on March 2, 1999 to LA Mahoney, Pacific Northwest National Laboratory, Richland, Washington.

(b) CW Stewart (PNNL), personal communication to LA Mahoney (PNNL), September 28, 1999, buoyancy model spreadsheet. 
Figure 4.15.4 compares RGS and VFI data. The increase in the measured gas fractions in both layers can be seen in the figure. Both the VFI and RGS data indicated that the gas volume fraction tended to be higher toward the bottom of the crust layer. Ammonia had a strong effect on the gas volume fractions just under the crust, as seen in the effect that the gas solubility assumptions have on the gas volume fraction. This effect may be exaggerated by the tendency of the gas solubility model to overstate the vapor pressure of ammonia and by a possible underestimate of the liquid volume fraction in this region. Nonetheless, sample $22 \mathrm{~A}-4$ contained 0.53 volume fraction of insoluble gas alone, and sample 23A-3 contained 0.49 volume fraction insoluble gas.

RGS data gave no way to tell whether the high gas fractions measured in samples $22 \mathrm{~A}-4$ and $23 \mathrm{~A}-3$ represented a gas volume fraction of about 0.5 spread evenly over an 18.2 -in. span (the RGS sample length) or, conceivably, a higher gas volume fraction in part of the sample averaged with lower fractions in the rest. The x-ray images provide information only on the bubble structure after the sample has been disturbed; a high-gas-fraction structure would be expected to collapse during sample acquisition before the $x$-ray.

Figure 4.15.5 shows sample temperatures, corrected gas volume fractions (Table 4.15.6), and corrected compositions of the low-solubility constituents in the samples from riser $23 \mathrm{~A}$ of Tank SY-101. The temperatures were measured at the MIT at riser 17B. The compositions represent the mole fraction of the species in the "insoluble" gas; water and ammonia are not included, so the mole fractions are not the same as those in the table. The gas volume fractions and mole fractions in the figure are the values for lower-bound gas solubilities. Figure 4.15.5 also lists the observations from core extrusions of non-RGS samples and from X-rays of RGS samples as a way of tying those observations together with RGS data. Figure 4.15.6 provides the same types of information for the RGS samples from risers 22A and 4A of SY-101. The X-ray data are described in more detail in Section 4.15.4.

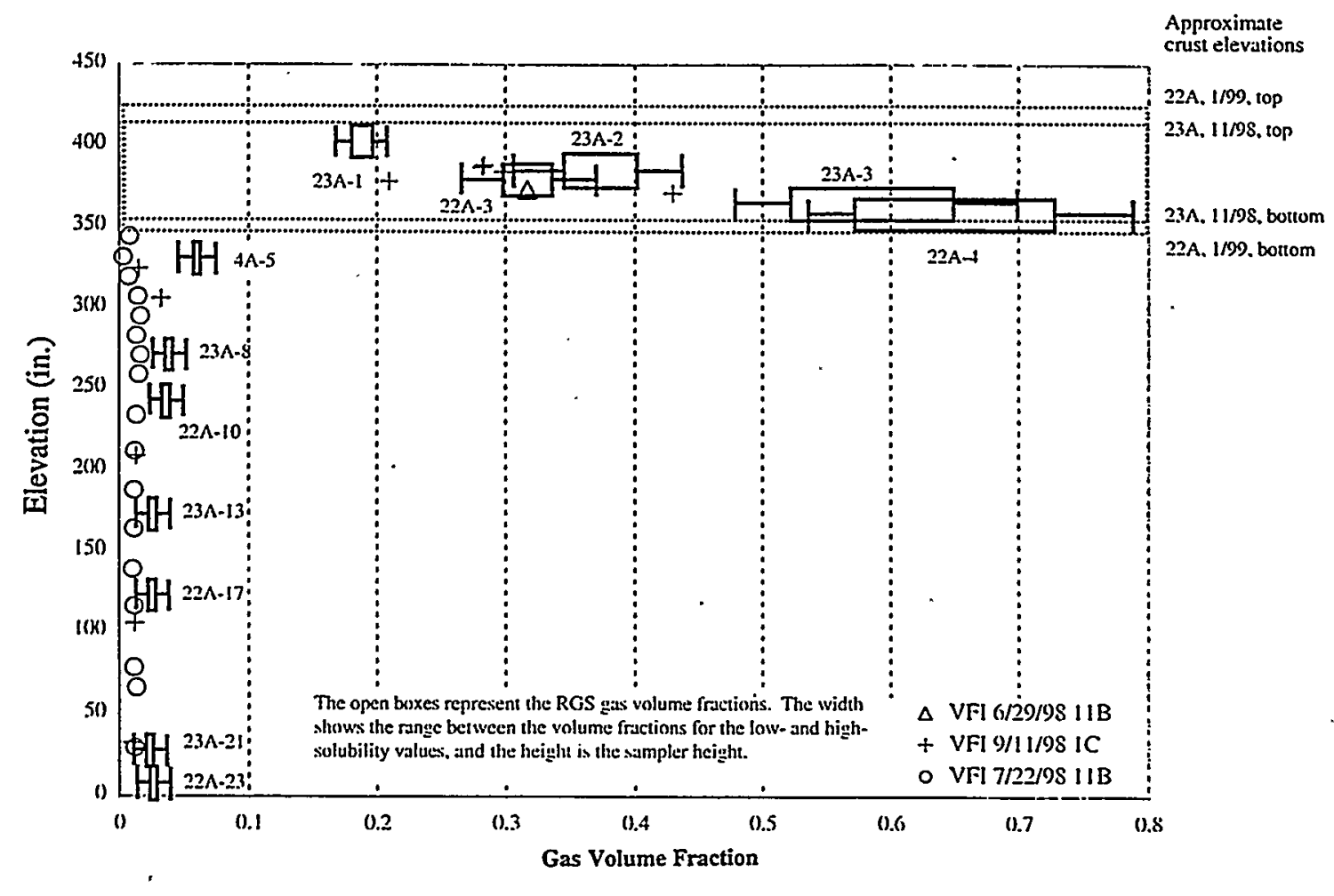

Figure 4.15.4. 1998 and 1999 RGS and VFI Data for SY-101 


\subsubsection{X-Ray Results}

Table 4.15.9 summarizes all the available radiography observations from Tank SY-101. The $\mathrm{x}$-ray images themselves can be found on the $\mathrm{CD}$ that accompanies this report. The uncertainties of the visible gas volume fractions that are given in Table 4.15.9 are unknown but (based on the uncertainty of gas gap measurement) are expected to be larger than those in the gas volume fractions calculated from gas extraction.

Table 4.15.9 compares $\mathrm{x}$-ray-derived gas volume fractions with extraction-derived gas volume fractions. The comparison shows that sample recovery was close to $100 \%$ for all the RGS samples. This conclusion is based on the observation that more gas was extracted than was visible in the $\mathrm{x}$-rays.

Table 4.15.9 compares $\mathrm{x}$-ray-derived and extraction-derived gas volume fractions. In the cases where the piston was more than 0.5 in. from full retraction (samples 23A-1, 23A-2, 23A-3, and 22A-3), there is some doubt about whether the piston could have been at a different location when the sampler ball valve was closed than at the time the $\mathrm{x}$-ray was taken. If the piston moved between those times, the gas gap in the $\mathrm{x}$-ray would not be equal to the gas gap when the sample was taken. In these cases, it is, difficult to meaningfully compare the visible gas volume fraction with the extracted gas volume fraction. The failure to fully retract the piston was not seen in any significant degree in samples from any previous tank except for BY-109. The complications introduced by incomplete piston retraction are discussed further in Section 4.15.5.

Table 4.15.10 summarizes the waste densities that have been calculated from radiographic data and densities in neighboring extruded samples. Radiographic densities include the gas in the waste and are given for several locations within each sample to show trends. The densities of many samples were calculated to be $1.9 \mathrm{~g} / \mathrm{cc}$ or more, which seems unreasonably high because none of the extruded samples show such high values. The reason for the high densities calculated from x-rays is unknown. Table 4.15.10 also includes density measurements made on extruded non-RGS samples.

\subsubsection{Other Discussions}

\subsubsection{Incomplete Piston Retraction}

X-rays showed that several SY-101 RGS samples had incompletely retracted pistons. The incomplete retraction occurred in association with the piston cycling that was needed because of sampler retrieval problems. Sampler modifications were made after the first core (that taken in riser 23A), and no further sampler retrieval problems arose. An incompletely retracted piston suggests one of two possibilities. One, an incomplete sample was taken-i.e., the sample at in situ pressure occupied less than a full sample length $(46 \mathrm{~cm}$ or $18.2 \mathrm{in}$.). Two, a full-length sample was taken, but piston cycling compressed the sample gas to more than in situ pressure.

RGS processing provided additional information that was used to determine the piston displacement (nominal minus true sample length) that corresponded to in situ pressure. The last RGS process step is the BSVD, in which argon is injected into the second volume vessel, its pressure in this known volume measured, and the second volume vessel opened to the extractor volume. The pressure after opening the valve is measured. The ideal gas law then permits the volume of the extractor minus that of the diluted sample to be calculated and the diluted sample volume found. The volume of the original sample is assumed to be the volume of the diluted sample minus the volume of the diluent (the isotopic standard solution). The uncertainty introduced by assuming linear addition of volumes is unknown. 

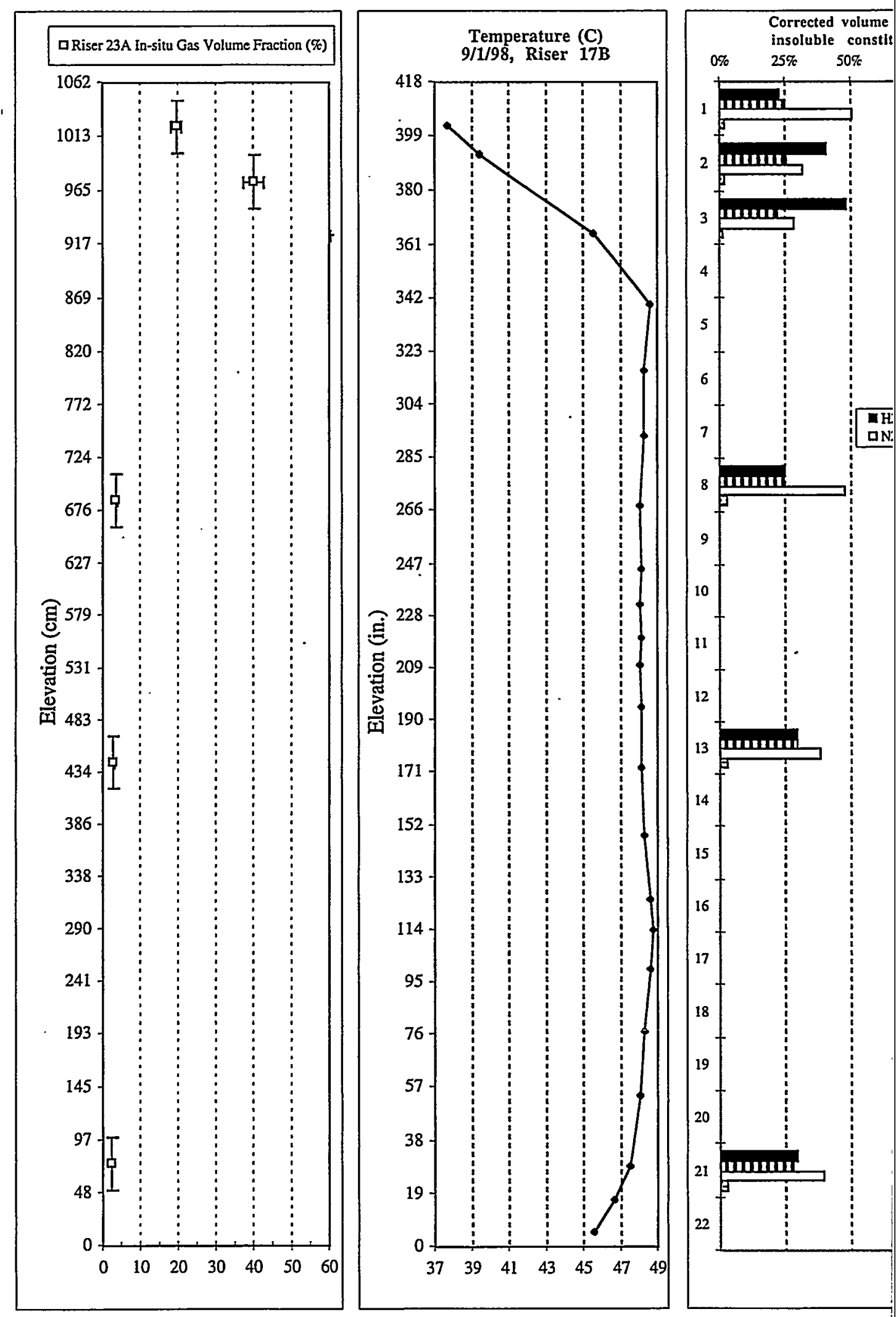

Figure 4.15.5. Sample Temperatures, Corrected Gas Volumes, and C 
Waste level at riser $23 \mathrm{~A}$ was $1046 \mathrm{~cm}$ (412 inches) by zipcord as of November 1998:

Riser 23A samples were 13" lower than Riser 22A samples of same segment ID\#.

1

Riser 23A X-RAY: Narrow fracture bubble, many 2-5 mm bubbles, possibly flattened surface, waste on piston.

2 Riser 23A X-RAY: Large fracture bubble, few vague 2-3 mm bubbles, steep rough surface, little waste on piston.

2

3 Riser 23A X-RAY: Many 3-5 mm bubbles, steep rough surface, $1 \times 2 \mathrm{~cm}$ oval void by wall, little waste on piston.

3

4 Riser 23A X-RAY: Little structure, single bubble visible 4 in. down, slightly lumpy surface, almost no gas gap.

4

5 Riser 23A: $<5 \mathrm{~mL}$ gray opaque liquid, $18 \mathrm{in}$. (472 g) gray unpitted easily-sheared moist salt.

$\mathbf{5}$

6 Riser 23A X-RAY: Thin raft of gassy solids at top.

6 Riser 23A: $<5 \mathrm{~mL}$ gray opaque liquid, $18 \mathrm{in}$. (441 g) gray unpitted easily-sheared moist salt.

Riser 23A X-RAY: Rising gas bubble observed, about I inch of gassy solids at top.

Riser 23A: <10 mL gray-brown opaque liquid, 18 in. (470 g) gray-brown unpitted easily-sheared wet salt.

9

10 Riser 23A: <10 mL gray-brown opaque liquid, 18 in. (464 g) gray-brown unpitted easily-sheared wet salt. 10

11 Riser 23A: <10 mL gray-brown opaque liquid, 18 in. (453 g) gray-brown unpitted easily-sheared wet salt.

11

12 Riser 23A: <10 mL gray-brown opaque liquid, $17 \mathrm{in.} \mathrm{(458} \mathrm{g)} \mathrm{gray-brown} \mathrm{unpitted} \mathrm{easily-sheared} \mathrm{wet} \mathrm{salt.}$

12

13

13

14 Riser 23A: <10 mL gray-brown opaque liquid, $17 \mathrm{in}$. (463 g) gray-brown unpitted easily-sheared wet salt. 14

15 Riser 23A: <10 mL gray opaque liquid, $18 \mathrm{in}$. (462 g) gray unpitted easily-sheared moist salt.

15

16 Riser 23A X-RAY: $1-\mathrm{mm}$ bubble about 2 in. down, otherwise featureless, gas gap 1-2 mm.

16 Riser 23A: <10 mL gray opaque liquid, $18.5 \mathrm{in}$. (459 g) gray unpitted easily-sheared wet sait.

17 Riser 23A: $15 \mathrm{~mL}$ gray opaque liquid, 18 in. (453 g) gray unpitted easily-sheared wet salt.

17

18 Riser 23A X-RAY: Featureless, slightly rough surface, gas gap 1-2 mm.

18 Riser 23A: $20 \mathrm{~mL}$ gray opaque liquid, $18.5 \mathrm{in}$. (449 g) gray unpitted easily-sheared wet salt.

19 Riser 23A X-RAY: Few isolated 1-2 mm bubbles in bottom half, slightly rough surface, gas gap 1-2 mm.

19 Riser 23A: $10 \mathrm{~mL}$ gray opaque liquid, $18.5 \mathrm{in}$. (455 g) gray unpitted easily-sheared wet salt.

20 Riser 23A: $10 \mathrm{~mL}$ gray opaque liquid, 19 in. (471 g) gray unpitted easily-sheared wet salt.

20

21 Riser 23A X-RAY: Some isolated 1-2 mm bubbles, one perhaps oblong, no gas gap.

21

22 Riser 23A: 10-mL gray opaque liquid, 17 in. (449 g) gray unpitted easily-sheared wet salt.

22 


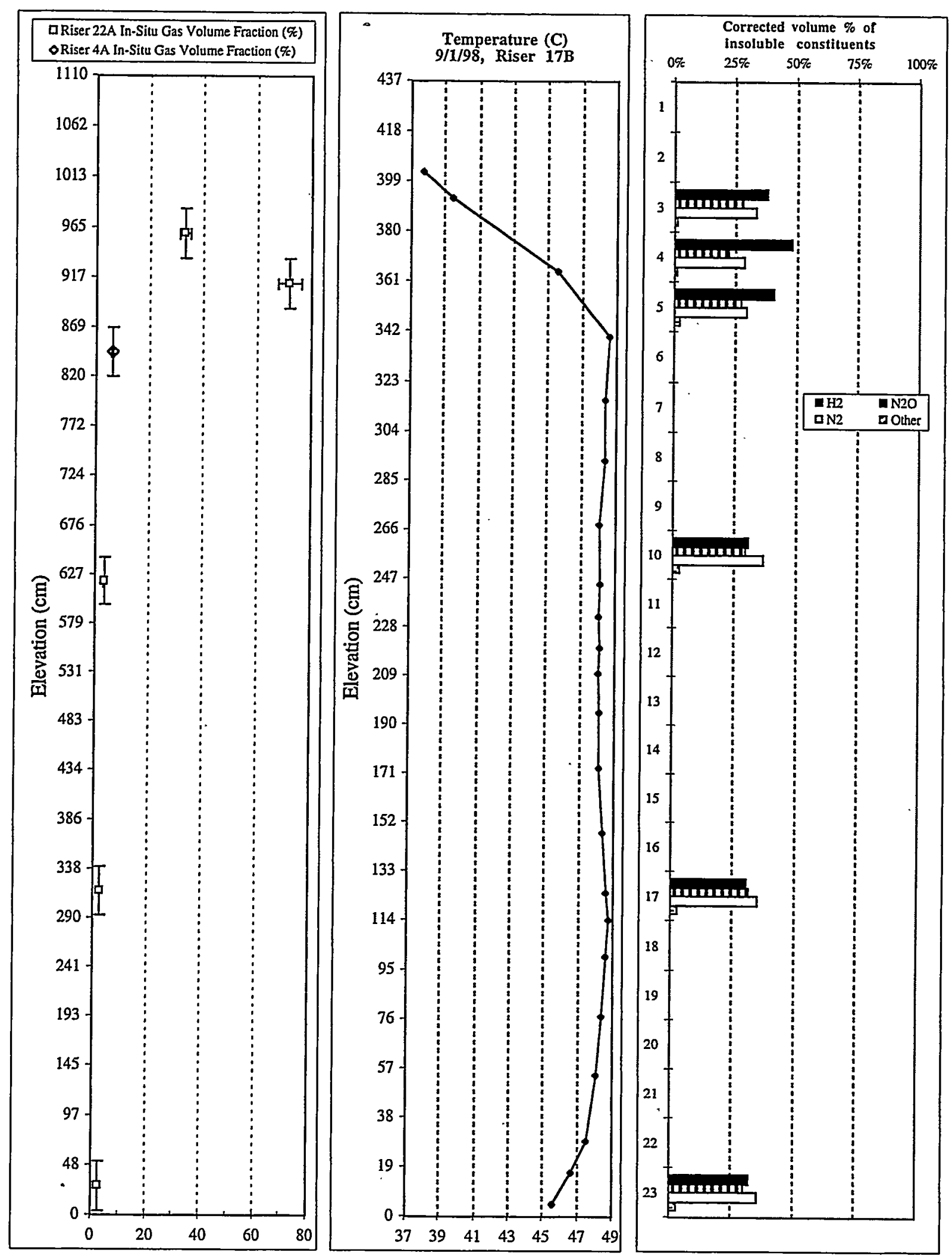

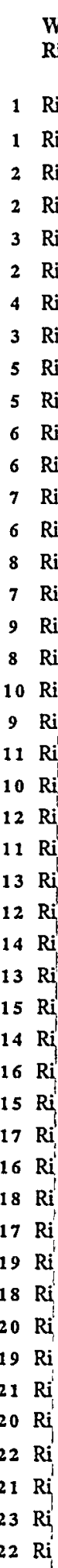

Figure 4.15.6. Gas Volume Fractions, Temperatures, Compos 
level at riser $22 \mathrm{~A}$ was $1080 \mathrm{~cm}$ (425 inches) as of January 1999.

4A samples were $7 "$, and Riser 23A 13", lower than Riser 22A samples of same segment ID\#.

2A X-RAY: Low-density plumes and swirls, dense 1-3 mm bubbles at bottom, gas gap $3.5 \mathrm{in}$; lumpy surface.

2A: 15 in. ( $326 \mathrm{~g}$ ) pale yellow moist salt with a gray outer surface; large gap after first 5 inches.

2A X-RAY: Many $1-3 \mathrm{~mm}$ bubbles; gas blobs up to $6 \mathrm{~mm}$ against wall; 0.5 in. waste on piston; gas gap 4.8 in. :2A: 15 in. (342 g) gray moist salt. Riser 4A Seg. 1: 9 in. (194 g) very pitted black/gray/white dry salt.

I2A X-RAY: Clouds of 1-mm bubbles; few 5-mm gas blobs on wall; lumpy surface; waste on piston; gap 4.3 in. AA: 15 in. (333 g) gray moist salt.

:2A X-RAY: Clouds of 1-mm bubbles; 3-5 mm gas blobs; lumpy surface, little waste on piston; gas gap $6.7 \mathrm{in}$. A: 14 in. (271 g) gray moist salt.

12A X-RAY: 1-2 mm bubbles, denser near top. Slightly rough surface; gas gap $3.5 \mathrm{in}$.; small waste on piston

22A: 14.5 in. (342 g) gray wet salt. Riser 4A Seg. 4: 15.5 in. $(267 \mathrm{~g}$ ) gray moist salt, X-RAY: $1-6 \mathrm{~mm}$ bubbles.

22A X-RAY: Few vague bubbles, $1 \mathrm{~mm}$ or less. Slightly rough surface; gas gap $0.2 \mathrm{in}$; clean piston.

32A: 19 in. (471 g) gray wet salt. Riser 4A Seg. 5 X-RAY: gas-mottled top, few $<2 \mathrm{~mm}$ bubbles, 0.2 in. gap.

22A: $<10 \mathrm{~mL}$ liquid, 17 in. ( $433 \mathrm{~g})$ gray wet sait.

IA: 16 in. (453 g) gray wet salt.

22A: $<10 \mathrm{~mL}$ liquid, 17 in. ( $443 \mathrm{~g}$ ) gray wet salt.

4A: 18 in. (435 g) gray wet salt.

22A: $<10 \mathrm{~mL}$ liquid, 18 in. (439 g) gray wet salt.

1A: 19 in. (454 g) gray wet salt.

22A X-RAY: Featureless; clean piston; no gas gap.

4A: $15 \mathrm{~mL}$ opaque gray liquid, 17 in. $(441 \mathrm{~g})$ gray wet salt.

22A: $<10 \mathrm{~mL}$ liquid, 19 in. $(460 \mathrm{~g})$ gray wet salt.

4A: $15 \mathrm{~mL}$ opaque gray liquid, 16 in. (449 g) gray wet salt.

22A: $<\mathrm{mL}$ liquid, 18 in. $(460 \mathrm{~g})$ gray wet salt.

4A: $10 \mathrm{~mL}$ opaque gray liquid, $17.5 \mathrm{in}$. (442 g) gray wet salt.

22A: $<5 \mathrm{~mL}$ liquid, $19 \mathrm{in}$. ( $465 \mathrm{~g}$ ) gray wet salt.

$4 A: 18$ in. (428 g) gray wet salt.

22A: $<10 \mathrm{~mL}$ opaque gray liquid, 19 in. (466 g) gray wet salt. 4A: $20 \mathrm{~mL}$ opaque gray liquid, 18 in. (434 g) gray wet salt.

22A: $20 \mathrm{~mL}$ opaque gray liquid, $17.5 \mathrm{in}$. (454 g) gray wet sait.

4A: $10 \mathrm{~mL}$ opaque gray liquid, 17 in. $(456 \mathrm{~g})$ gray wet salt.

22A: $25 \mathrm{~mL}$ opaque gray liquid, $18.5 \mathrm{in}$. (448 g) gray wet salt.

4A: $<5 \mathrm{~mL}$ opaque gray liquid, $19 \mathrm{in}$. ( $455 \mathrm{~g}$ ) gray wet salt.

22A X-RAY: Featureless except for two rising bubbles, 1-mm and 3-mm diameters; clean piston; no gas gap.

4A: $<5 \mathrm{~mL}$ opaque gray liquid, 19 in. ( $451 \mathrm{~g}$ ) gray wet salt.

22A: $20 \mathrm{~mL}$ opaque gray liquid, $18 \mathrm{in}$. (457 g) gray wet salt.

4A: $<5 \mathrm{~mL}$ opaque gray liquid, 19 in. ( $456 \mathrm{~g}$ ) gray wet salt.

22A: $<5 \mathrm{~mL}$ opaque gray liquid, $18.5 \mathrm{in}$. (477 g) gray wet salt.

4A: $10 \mathrm{~mL}$ opaque gray liquid, 19 in. ( $456 \mathrm{~g}$ ) gray wet salt.

22A: 18.5 in. $(481 \mathrm{~g})$ gray wet salt.

4A: $10 \mathrm{~mL}$ opaque gray liquid, 18 in. (447 g) gray wet salt.

22A: $<5 \mathrm{~mL}$ opaque gray liquid, $18.5 \mathrm{in}$. (467 g) gray wet salt.

4A: $<10 \mathrm{~mL}$ opaque gray liquid, $19 \mathrm{in}$. ( $452 \mathrm{~g}$ ) gray wet salt.

22A: $15 \mathrm{~mL}$ opaque gray liquid, 18 in. (463 g) gray wet salt.

4A: $10 \mathrm{~mL}$ opaque gray liquid, 19 in. (465 g) gray wet salt.

22A X-RAY: Featureless; clean piston; no gas gap.

4A: $20 \mathrm{~mL}$ opaque gray liquid, $18 \mathrm{in}$. (454 g) gray wet salt.

ons, and Observations in Samples from Tank SY-101, Risers 22A and 4A 
Table 4.15.9. Summary of Observations from X-Ray Images of Tank SY-101

\begin{tabular}{|c|c|}
\hline Segment & Comments/Observations \\
\hline $\begin{array}{c}22 \mathrm{~A}-1 \\
\text { (non-RGS, } \\
1 / 5 / 99 \text { ) }\end{array}$ & $\begin{array}{l}\text { Less-dense areas (not resolvable into individual bubbles) in plumes reach about } 5 \mathrm{~cm}(2 \mathrm{in} \text {.) } \\
\text { down from the top and } 5 \mathrm{~cm} \text { ( } 2 \text { in.) up from bottom of the sample. Adjacent to the plumes are } \\
\text { zones with sparse scattering of bubbles less than } 2 \mathrm{~mm} \text { in size, and at the center of the sample } \\
\text { (between the low-gas zones) were blurs and swirls of gassy material with no resolvable bubbles. } \\
\text { Densely packed } 1-\text { to } 3 \text {-mm bubbles at bottom. Top surface lumpy, a little waste on the piston. } \\
\text { Piston fully retracted with a gas gap of } 8.9 \mathrm{~cm}(3.5 \mathrm{in} \text {.). Visible gas volume fraction is } 0.19 \text {. }\end{array}$ \\
\hline $\begin{array}{c}23 \mathrm{~A}-1 \\
\text { (RGS, } \\
11 / 9 / 98)\end{array}$ & $\begin{array}{l}\text { Substantial bubble structure, mostly in } 2 \text { - to } 5 \text {-mm-diameter range. Bubbles largest in middle } \\
\text { of sample, some clustered into partial fracture bubble. Top of waste slightly sloped, smeared. } \\
\text { No visible free liquid. Gas gap of } \sim 8.4 \mathrm{~cm} \text { ( } 3.3 \mathrm{in} \text {.) below piston with } \sim 0.6 \mathrm{~cm} \text { sampler gas } \\
\text { volume fraction of } 0.33 \text {, including air, in Table } 4.15 .6 \text { obtained by gas extraction. Extracted } \\
\text { insoluble gases alone would have been volume fraction of } 0.31 \text {; the rest was ammonia.) }\end{array}$ \\
\hline $\begin{array}{c}22 \mathrm{~A}-2 \\
\text { (non-RGS, } \\
1 / 6 / 99 \text { ) }\end{array}$ & $\begin{array}{l}\text { A mottling of } 1 \text { - to } 3-\mathrm{mm} \text { bubbles in the top } 7.6 \mathrm{~cm} \text { ( } 3 \text { in.) of waste; below that, about } 3.8 \mathrm{~cm} \\
\text { (1.5 in.) of waste with large blobs of gas, up to } 6 \mathrm{~mm} \text { in size, apparently not spherical but } \\
\text { spread across the sampler wall. Below that, more of the same structure as at the top of the } \\
\text { waste. There was a rough top surface and } 1 \text { to } 1.2 \mathrm{~cm}(0.4 \text { to } 0.5 \text { in.) of slightly bubbly waste } \\
\text { on the piston, which was about } 0.5 \mathrm{~cm}(0.2 \text { in.) from being fully retracted. The gas gap was } \\
12 \mathrm{~cm} \text { ( } 4.8 \text { in.). The visible gas volume fraction is } 0.27 \text {. }\end{array}$ \\
\hline $\begin{array}{c}23 \mathrm{~A}-2 \\
\text { (RGS, } \\
11 / 9 / 98)\end{array}$ & $\begin{array}{l}\text { Little bubble structure except a fracture bubble about } 1.2 \mathrm{~cm}(0.5 \mathrm{in}) \text { near bottom. A few } 2 \text { - to } \\
\text { 3-mm bubbles vaguely visible. Both fracture bubble surfaces and top surface of waste rough and } \\
\text { steeply sloped. Piston has small gob of adhering waste with } 4.8-\mathrm{cm} \text { (1.9-in.) gas gap below it. } \\
\text { Piston not fully retracted; was } 6.6 \mathrm{~cm} \text { ( } 2.6 \text { in.) from stops. Visible gas volume fraction } 0.12 \text {. } \\
\text { (Compare with sampler gas volume fraction of } 0.42 \text { in Table } 4.15 .6 \text { obtained by gas extraction. } \\
\text { Extracted insoluble gases alone would be volume fraction of } 0.35 \text {; the rest was ammonia.) }\end{array}$ \\
\hline $\begin{array}{c}22 \mathrm{~A}-3 \\
\text { (RGS, } 1 / 6 / 99)\end{array}$ & $\begin{array}{l}\text { Densely scattered } 1 \text {-mm bubbles, seldom resolvable into individual bubbles. A few blobs of gas } \\
\text { of } 5 \text {-mm ( } 0.2-\mathrm{in} \text {.) size, probably on the sampler wall, near the top. There was a very lumpy top } \\
\text { surface with more lumps smeared on the sampler wall and a waste stalactite covering about half } \\
\text { the piston, which was about } 1.8 \mathrm{~cm}(0.7 \text { in.) from being fully retracted. The gas gap was } \\
11 \mathrm{~cm}(4.3 \text { in.). The visible gas volume fraction is } 0.24 \text {. (Compare with the sampler gas } \\
\text { volume fraction of } 0.33 \text { total in Table } 4.15 .6 \text { obtained by gas extraction. Extracted insoluble } \\
\text { gases alone would have been a volume fraction of } 0.31 \text {; the rest was ammonia.) }\end{array}$ \\
\hline $\begin{array}{c}\text { (RGS, } \\
11 / 10 / 98)\end{array}$ & $\begin{array}{l}\text { Substantial bubble structure, mostly in } 3-\text { to } 5 \text {-mm-diameter range. A thin fracture bubble } \\
\text { separated the top inch from the waste below it. A large, roughly oval void about } 2 \text {-cm long by } \\
1 \text {-cm wide stretched vertically about halfway up the sampler. Both fracture bubble surfaces and } \\
\text { top surface of waste were rough and steeply sloped. No visible free liquid. Gas gap of } \sim 16.8 \\
\mathrm{~cm}(6.6 \text { in.) below a piston with a small gob of waste on it. Piston not fully retracted; it was } \\
3.6 \mathrm{~cm} \text { ( } 1.4 \text { in.) from the stops. The visible gas volume fraction is } 0.39 \text {. (Compare with } \\
\text { sampler gas volume fraction of } 0.65 \text { total in Table } 4.15 .6 \text { obtained by gas extraction. Extracted } \\
\text { insoluble gases alone would have been a volume fraction of } 0.49 \text {; the rest was ammonia.) }\end{array}$ \\
\hline $\begin{array}{l}\text { (RGS, } \\
\text { 1/7/99) }\end{array}$ & $\begin{array}{l}\text { Densely scattered } 1-\mathrm{mm} \text { bubbles, seldom resolvable into individual bubbles. A few blobs of gas } \\
\text { of } 3 \text { to } 5-\mathrm{mm}(0.1 \text { to } 0.2-\mathrm{in} \text {.) size near top. Lumpy top surface, small gob of waste on piston, } \\
\text { which was } \sim 0.5 \mathrm{~cm}(0.2 \mathrm{in} \text {.) from being fully retracted. Gas gap was } 17 \mathrm{~cm}(6.7 \mathrm{in} \text {.). Visible } \\
\text { gas volume fraction is } 0.37 \text {. (Compare with sampler gas volume fraction of } 0.72 \text { total in } \\
\text { Table } 4.15 .6 \text { obtained by gas extraction. Extracted insoluble gases alone would be volume } \\
\text { fraction of } 0.53 \text {, the rest was ammonia.) }\end{array}$ \\
\hline
\end{tabular}


Table 4.15.9 (contd)

\begin{tabular}{|c|c|}
\hline Segment & Comments/Observations \\
\hline $\begin{array}{l}23 \mathrm{~A}-4 \\
(\text { lost RGS, } \\
11 / 10 / 98)\end{array}$ & $\begin{array}{l}\text { No features visible except a vague round void about } 10 \mathrm{~cm} \text { ( } 4 \text { in.) from top of sample. Slightly } \\
\text { sloping lumpy top surface and nearly clean piston with } 0.5-\mathrm{cm}(0.2 \text {-in.) gas gap below it. } \\
\text { Piston fully retracted; visible gas volume fraction (not counting.bubbles) is therefore } 0.01 \text {. }\end{array}$ \\
\hline $\begin{array}{l}\text { 4A-4 } \\
\text { (non-RGS, } \\
\text { 3/8/99) }\end{array}$ & $\begin{array}{l}\text { Mottled with } 1-\text { to } 3-\mathrm{mm} \text { bubbles that are most dense near the bottom; in bottom and middle, a } \\
\text { few large blobs of gas up to } 6 \mathrm{~mm} \text { in size, apparently not spherical but spread across the } \\
\text { sampler wall. There was a sloped, lumpy top surface. Piston almost clean and fully retracted. } \\
\text { The gas gap was } 12 \mathrm{~cm} \text { ( } 4.6 \mathrm{in} \text {.); the visible gas volume fraction is } 0.25 \text {. }\end{array}$ \\
\hline $\begin{array}{l}22 \mathrm{~A}-5 \\
\text { (non-RGS, } \\
1 / 8 / 99)\end{array}$ & $\begin{array}{l}1-2-\mathrm{mm} \text { bubbles near bottom, much more densely packed toward the top. A slightly rough top } \\
\text { surface and a gas gap of } 8.9 \mathrm{~cm}(3.5 \mathrm{in} \text {.). A small amount of waste on the piston, which was } \\
\text { fully retracted. The visible gas volume fraction is } 0.19 \text {. }\end{array}$ \\
\hline $\begin{array}{l}4 \mathrm{~A}-5 \\
\text { (RGS, } \\
3 / 8 / 99)\end{array}$ & $\begin{array}{l}\text { Mottled, foamy; } 4 \text {-mm bubble in top centimeter or so; remainder featureless but for } \sim 12 \text { bubbles } \\
\text { less than } 2 \mathrm{~mm} \text { diameter, mostly (apparently) on sampler centerline. Slightly rough flat } \\
\text { surface; clean, fully retracted piston. Gas gap } 0.5 \mathrm{~cm}(0.2 \text { in.); visible gas volume fraction } 0.01 \\
\text { (compare with sampler gas volume fraction of } 0.07 \text { in Table } 4.15 .6 \text { obtained by gas extraction). }\end{array}$ \\
\hline $\begin{array}{l}22 \mathrm{~A}-6 \\
\text { (non-RGS, } \\
1 / 8 / 99)\end{array}$ & $\begin{array}{l}\text { A few vaguely visible bubbles of } 1 \mathrm{~mm} \text { or less diameter. Slightly rough top surface and gas } \\
\text { gap of } 0.5 \mathrm{~cm} \text { ( } 0.2 \text { in.). Piston clean and fully retracted; visible gas volume fraction is } 0.01 \text {. }\end{array}$ \\
\hline $\begin{array}{l}23 \mathrm{~A}-8 \\
(\mathrm{RGS} \\
11 / 18 / 98)\end{array}$ & $\begin{array}{l}\text { No features except two rising bubbles (3-mm diameter) and slightly rough top surface with } \sim 0.6 \\
\mathrm{~cm}(0.25 \mathrm{in} \text {.) of less dense waste below it, possibly solids floated by bubbles too fine to see. } \\
\text { Gas gap } 1 \mathrm{~mm} \text { or less; piston fully retracted. Visible gas volume fraction less than } 0.005 \text {. } \\
\text { (Compare with sampler gas volume fraction of } 0.05 \text { in Table } 4.15 .6 \text { obtained by gas extraction.) }\end{array}$ \\
\hline $\begin{array}{l}22 \mathrm{~A}-10 \\
\text { (RGS, } \\
1 / 12 / 99)\end{array}$ & $\begin{array}{l}\text { Featureless in standard-resolution images; no gas gap. Many bubbles less than } 1 \text {-mm diameter } \\
\text { visible in high-resolution images. Piston fully retracted. Visible gas volume fraction } 0 \\
\text { (compare with sampler gas volume fraction of } 0.04 \text { in Table } 4.15 .6 \text { obtained by gas extraction.) }\end{array}$ \\
\hline $\begin{array}{l}23 A-16 \\
\text { (non-RGS, } \\
12 / 16 / 98)\end{array}$ & $\begin{array}{l}\text { No features visible except } 1-\mathrm{mm} \text { bubble about } 5 \mathrm{~cm} \text { ( } 2 \mathrm{in} \text {.) from the sample top. Gas gap was } \\
1 \text { to } 2 \mathrm{~mm} \text {. The piston was fully retracted. Visible gas volume fraction less than } 0.005 \text {. }\end{array}$ \\
\hline $\begin{array}{l}22 \mathrm{~A}-17 \\
(\mathrm{RGS} \\
1 / 13 / 99)\end{array}$ & $\begin{array}{l}\text { Featureless except for two rising bubbles, } 1-\mathrm{mm} \text { and } 3-\mathrm{mm} \text { diameters, with no gas gap. Piston } \\
\text { was fully retracted. The visible gas volume fraction is } 0 \text {. (Compare with the sampler gas } \\
\text { volume fraction of } 0.03 \text { in Table } 4.15 .6 \text { obtained by gas extraction.) }\end{array}$ \\
\hline $\begin{array}{l}\text { 23A-18 } \\
\text { (non-RGS, } \\
12 / 16 / 98)\end{array}$ & $\begin{array}{l}\text { No features visible. The top surface is slightly rough, possibly solids. The gas gap was } 1 \text { to } \\
2 \mathrm{~mm} \text {. The piston was fully retracted. The visible gas volume fraction is less than } 0.005 \text {. }\end{array}$ \\
\hline $\begin{array}{l}23 A-19 \\
\text { (non-RGS, } \\
12 / 17 / 98)\end{array}$ & $\begin{array}{l}\text { Several bubbles of } 1-\text { to } 2-\mathrm{mm} \text { diameter vaguely visible. The top surface is slightly rough, } \\
\text { possibly solids. The gas gap was } 1 \text { to } 2 \mathrm{~mm} \text {. The piston was fully retracted. The visible gas } \\
\text { volume fraction is less than } 0.005 \text {. }\end{array}$ \\
\hline $\begin{array}{l}23 \mathrm{~A}-21 \\
(\mathrm{RGS} \\
12 / 17 / 98)\end{array}$ & $\begin{array}{l}\text { Two bubbles, } 1 \text { to } 2 \mathrm{~mm} \text { diameter vaguely visible. Top surface slightly rough, possibly solids. } \\
\text { No gas gap. Piston fully retracted. Visible gas volume fraction is } 0 \text {. (Compare with sampler } \\
\text { gas volume fraction of } 0.03 \text { in Table } 4.15 .6 \text { obtained by gas extraction.) }\end{array}$ \\
\hline $\begin{array}{l}22 \mathrm{~A}-23 \\
\text { (RGS, } \\
1 / 18 / 99)\end{array}$ & $\begin{array}{l}\text { Featureless in standard-resolution images, no gas gap. Many bubbles less than } 1 \text {-mm diameter } \\
\text { visible in high-resolution images. Piston fully retracted. Visible gas volume fraction is } 0 \text {. } \\
\text { (Compare with sampler gas volume fraction of } 0.03 \text { in Table } 4.15 .6 \text { obtained by gas extraction.) }\end{array}$ \\
\hline
\end{tabular}


Table 4.15.10. Densities of SY-101 Samples from Radiography

\begin{tabular}{|c|c|c|c|}
\hline $\begin{array}{l}\text { Riser and } \\
\text { Segment Number }\end{array}$ & $\begin{array}{l}\text { Distance from } \\
\text { Bottom of Sample } \\
\text { (ft) }\end{array}$ & $\begin{array}{c}\text { Calculated } \\
\text { Mean Density } \\
(\mathrm{g} / \mathrm{cc}) \\
\end{array}$ & $\begin{array}{l}\text { Degassed. Density in Extruded Samples } \\
\text { above and below RGS Sample (g/cc) }\end{array}$ \\
\hline \multirow[t]{4}{*}{$22 \mathrm{~A}-1$} & 1.25 & 1.45 & \multirow{4}{*}{ density of this sample: $1.73 \mathrm{~g} / \mathrm{cc}$, bulk } \\
\hline & 1.00 & 1.88 & \\
\hline & 0.75 & 1.63 & \\
\hline & 0.50 & 1.93 & \\
\hline \multirow[t]{3}{*}{$23 \mathrm{~A}-1$} & 0.75 & 2.51 & \\
\hline & 0.50 & 3.04 & \\
\hline & 0.25 & 2.89 & \\
\hline \multirow[t]{4}{*}{$22 \mathrm{~A}-2$} & 1.50 & 1.60 (b) & \multirow{4}{*}{ density of this sample: $1.72 \mathrm{~g} / \mathrm{cc}$, bulk } \\
\hline & 1.00 & 1.54 (b) & \\
\hline & 0.75 & $1.61(\mathrm{~b})$ & \\
\hline & 0.50 & 1.68 (b) & \\
\hline \multirow[t]{4}{*}{$23 \mathrm{~A}-2$ (a) } & 1.00 & 1.74 & \\
\hline & 0.75 & 1.82 & \\
\hline & 0.50 & 1.35 & \\
\hline & 0.25 & 1.48 & \\
\hline \multirow[t]{4}{*}{$22 \mathrm{~A}-3$} & 1.00 & 1.03 & \multirow{4}{*}{ density above, $1.72 \mathrm{~g} / \mathrm{cc}$ bulk } \\
\hline & 0.75 & 1.27 & \\
\hline & 0.50 & 1.36 & \\
\hline & 0.25 & 1.39 & \\
\hline \multirow[t]{2}{*}{$23 \mathrm{~A}-3$} & 0.75 & 1.64 & \\
\hline & 0.50 & 1.67 & \\
\hline \multirow[t]{3}{*}{$22 \mathrm{~A}-4$} & 0.90 & 1.28 & \multirow{3}{*}{ density below, $1.59 \mathrm{~g} / \mathrm{cc}$ bulk } \\
\hline & 0.75 & 1.57 & \\
\hline & 0.50 & 1.62 & \\
\hline \multirow[t]{5}{*}{$23 \mathrm{~A}-4$} & 1.50 & 1.77 (b) & \multirow{5}{*}{ density below, $1.56 \mathrm{~g} / \mathrm{cc}$ bulk } \\
\hline & 1.25 & 1.86 & \\
\hline & 1.00 & 1.85 & \\
\hline & 0.75 & 1.79 & \\
\hline & 0.50 & 1.79 & \\
\hline \multirow[t]{4}{*}{$4 \mathrm{~A}-4$} & 1.25 & 0.98 & \\
\hline & 1.00 & 1.72 & \\
\hline & 0.75 & 1.73 & \\
\hline & 0.50 & 1.71 & \\
\hline \multirow{5}{*}{$4 \mathrm{~A}-5$} & 1.50 & 1.99 (b) & \\
\hline & 1.25 & 2.00 & \\
\hline & 1.00 & 2.02 (b) & \\
\hline & 0.75 & 2.00 (b) & \\
\hline & 0.50 & 1.98 (b) & \\
\hline
\end{tabular}


Table 4.15.10 (contd)

\begin{tabular}{|c|c|c|c|}
\hline $\begin{array}{c}\text { Riser and } \\
\text { Segment Number }\end{array}$ & $\begin{array}{c}\text { Distance from } \\
\text { Bottom of Sample } \\
\text { (ft) }\end{array}$ & $\begin{array}{c}\text { Calculated } \\
\text { Mean Density } \\
(\mathrm{g} / \mathrm{cc}) \\
\end{array}$ & $\begin{array}{c}\text { Degassed Density in Extruded Samples } \\
\text { above and below RGS Sample (g/cc) }\end{array}$ \\
\hline \multirow[t]{5}{*}{$23 \mathrm{~A}-8$} & 1.50 & 2.14 & \multirow[b]{5}{*}{ density below, $1.54 \mathrm{~g} / \mathrm{cc}$ bulk } \\
\hline & 1.25 & 2.05 & \\
\hline & 1.00 & 2.15 & \\
\hline & 0.75 & 2.08 & \\
\hline & 0.50 & 1.80 & \\
\hline \multirow[t]{5}{*}{$22 \mathrm{~A}-10$} & 1.50 & $2.04(\mathrm{~b})$ & \multirow[t]{5}{*}{ density above, $1.59 \mathrm{~g} / \mathrm{cc}$ bulk } \\
\hline & 1.25 & 1.93 & \\
\hline & 1.00 & 1.93 & \\
\hline & 0.75 & 1.91 & \\
\hline & 0.50 & 1.91 & \\
\hline \multirow[t]{5}{*}{$22 \mathrm{~A}-17$} & 1.50 & 1.92 (b) & \multirow[t]{5}{*}{ density above, $1.59 \mathrm{~g} / \mathrm{cc}$ bulk } \\
\hline & 1.25 & 1.86 & \\
\hline & 1.00 & 1.85 & \\
\hline & 0.75 & 1.92 & \\
\hline & 0.50 & 1.91 & \\
\hline \multirow[t]{5}{*}{$23 \mathrm{~A}-21$} & 1.50 & 1.33 & \multirow[t]{5}{*}{ density above, $1.62 \mathrm{~g} / \mathrm{cc}$ bulk } \\
\hline & 1.25 & 1.21 & \\
\hline & 1.00 & 1.18 & \\
\hline & 0.75 & 1.22 & \\
\hline & 0.50 & 1.83 & \\
\hline \multirow[t]{5}{*}{$22 \mathrm{~A}-23$} & 1.50 & 2.10 (b) & \multirow[t]{5}{*}{ density above, $1.62 \mathrm{~g} / \mathrm{cc}$ bulk } \\
\hline & 1.25 & 1.95 & \\
\hline & 1.00 & 1.97 & \\
\hline & 0.75 & 1.93 & \\
\hline & 0.50 & 1.94 & \\
\hline
\end{tabular}

Given the bulk (no-gas) sample volume from BSVD and the volume at in situ pressure and temperature of the gas extracted by the RGS process the original sample volume and effective sampler length (sampler length minus in situ piston displacement) can be calculated. Table 4.15.11 gives the BSVD volume, the RGS-based in situ piston displacement, and the piston displacement observed in the $\mathrm{x}$-ray for all the samples in which incomplete piston retraction was seen. The table uses the convention that a piston displacement of zero is fully retracted (to the piston stops) and a positive piston displacement is the distance by which the piston fell short of full retraction.

In Table 4.15.11, the in situ displacements are where the piston stopped while the sample was being taken at in situ pressure. The more positive the displacement value, the smaller the effective total sample volume. The post-cycling displacements show where the piston stuck after being acted on by external atmospheric pressure, friction, and internal pressure. 
Table 4.15.11. Calculated and Observed Piston Retraction(a)

\begin{tabular}{|c|c|c|c|c|}
\hline \multirow[t]{2}{*}{ Sample } & \multirow{2}{*}{$\begin{array}{l}\text { Degassed } \\
\text { sample volume } \\
\text { (cc), by BSVD }\end{array}$} & \multicolumn{2}{|c|}{$\begin{array}{c}\text { In situ piston displacement } \\
\text { calculated from BSVD and RGS } \\
\text { (in.) }\end{array}$} & \multirow{2}{*}{$\begin{array}{l}\text { Post-cycling piston } \\
\text { displacement } \\
\text { observed in x-ray } \\
\text { (in.) }\end{array}$} \\
\hline & & Low solubility & High solubility & \\
\hline $23 \mathrm{~A}-1$ & 208 & 0.3 & 0.4 & 3.0 \\
\hline $23 \mathrm{~A}-2$ & 183 & 1.1 & 1.5 & 2.6 \\
\hline $22 \mathrm{~A}-3$ & 208 & 0.3 & 0.6 & 0.7 \\
\hline $23 \mathrm{~A}-3$ & 156 & -1.0 & -0.2 & 1.4 \\
\hline $22 \mathrm{~A}-4$ & 145 & -1.1 & -0.2 & 0.2 \\
\hline $4 \mathrm{~A}-5$ & 304 & -1.0 & -1.0 & 0 \\
\hline $23 \mathrm{~A}-8$ & 302 & -0.5 & -0.5 & 0 \\
\hline $22 \mathrm{~A}-10$ & 305 & -0.5 & -0.5 & 0 \\
\hline $23 \mathrm{~A}-13$ & 303 & -0.4 & -0.4 & 0 \\
\hline $22 \mathrm{~A}-17$ & 314 & -1.0 & -1.0 & 0 \\
\hline $23 \mathrm{~A}-21$ & 312 & -0.8 & -0.8 & 0 \\
\hline $22 \mathrm{~A}-23$ & 294 & 0.3 & 0.3 & 0 \\
\hline
\end{tabular}

As a result of unusual difficulties in pulling the pintle rod, the piston was cycled during removal of the first several RGS samplers from SY-101. The cycling could have led to driving the piston down into the sampler with as much as $50 \mathrm{lb}$ force after the ball valve was closed. Had this happened, the compression of the gas would have pushed the piston up, while atmospheric pressure and piston friction would have resisted upward motion, leaving the piston incompletely retracted. As an example, suppose the in situ sample pressure was $1.03 \mathrm{~atm}$ and there was 6 in. of gas volume before piston cycling. (These conditions represent sample 23A-1.) After cycling, the force resisting upward motion of the piston (retraction) would be 1 atm acting on a 1.125 in.diameter area, plus friction. The piston friction has been estimated at 3 to $4 \mathrm{lb}$.(a) These forces amount to a total resisting pressure of roughly $1.2 \mathrm{~atm}$, greater than in situ pressure, so some compression of the sample is possible. The slight compression would reduce the gas gap by $(1-1.03 / 1.2)$ ( 6 in.), or 0.85 in., so the post-cycling piston displacement would be 0.85 in. greater than the pre-cycling (in situ) displacement.

The more gas in the sampler, the lower the in situ pressure; the higher the piston friction, the larger the difference can be between the in situ and post-cycling displacements. When samples contain very little gas, as in the mixed slurry layer, no perceptible piston displacement difference should be seen. When the in situ pressure is greater than the pressure of the resisting forces, the piston should be fully retracted (pushed up against its stops).

Consider the positive pre-cycling displacements in Table 4.15.11. All are less than the post-cycling displacements, as is expected, but the difference between in situ and post-cycling displacements is larger than piston friction can account for. In sample 23A-1, piston friction could cause a 0.85 -in. difference, but $2.7 \mathrm{in}$. is found. It is possible that the in situ friction was greater than the estimate of 3 to $4 \mathrm{lb}$ or that the estimate of the degassed sample volume was inaccurate.

(a) Graves DB. 1999. Personal communication with LA Mahoney (PNNL) concerning approximate piston friction force, June 14 1999. COGEMA Engineering Corporation, Richland, Washington. 
Note that in two cases, samples 22A-17 and 23A-21, the BSVD method measured degassed sample volumes that were greater than the sampler volume at full piston retraction. This suggests that, possibly because of dilution density-change effects, the BSVD method can overestimate the degassed sample volume by as much as 1 in. of sampler length. This effect could account for most of the negative pre-cycling piston displacements in the table. (Negative piston displacements are not physically possible because the piston cannot go back farther than the stops.)

The negative piston displacements calculated for samples $23 \mathrm{~A}-2$ and $23 \mathrm{~A}-3$ may also result from another effect. We suspect that the in situ sample gas volumes (and so the sampler volumes required to contain them) were overestimated by RGS because the Schumpe model underestimates ammonia solubility and thus overestimates the moles of ammonia in the in situ retained gas. This solubility effect is strong in samples $23 \mathrm{~A}-2$ and $22 \mathrm{~A}-3$ because the low liquid fraction (high insoluble gas fraction) increased the ammonia concentration in the liquid. That increased the vapor pressure, accentuating the volume contributed by ammonia vapor. The solubility effect is weaker in the other samples in Table 4.15.11 (as can be seen by comparing the low-and high-solubility estimates in the table) and is negligible in the mixed slurry samples with their small retained gas volumes.

\subsubsection{Air in the Crust}

The oxygen in sample 23A-1 (the top of the crust) was consistent with the sample containing $33 \mathrm{cc}$ of air at STP, equivalent to 1.9 in. of sampler length occupied by air alone. There is no way to determine whether this amount of air came directly from the dome space or whether it was present in the pores of the crust.

It is possible to estimate the depth to which the crust pores were open to air on the assumption that the crust pores were the only source of air. Air plus waste gas made up $88 \mathrm{cc}$ of the $307-\mathrm{cc}$ volume. If this $29 \%$ gas volume fraction was evenly distributed throughout the sampler, the $33 \mathrm{cc}$ of air would contribute the gas in 33/88 of the sampler length, or about 7 in. This value (estimated from a sample taken in November 1998) is in reasonable agreement with the estimate of about 12 in. of "freeboard" that was made from July 1998 Enraf data (Stewart et al. 1998).

As some reviewers have suggested, argon might also be used as a tracer gas to determine the amount of air in the waste. This method has both advantages and disadvantages compared with using oxygen as a tracer. Argon's advantage is that it does not react with the waste. Oxygen does react, but at sample storage temperatures and dose rates, the reaction consumes only negligible amounts of oxygen (Person 1998). However, the oxygen reaction rate could be faster in the in situ pores of the top of the crust.

Argon's disadvantage is that it is used for a drillstring purge when high-drillstring $\mathrm{H}_{2}$ is measured, and it is also used in the RGS system to measure the bulk sample volume. There is no evidence (in the form of purge log entries) that the drillstring was purged before taking sample 23A-1 or any other SY-101 RGS sampler. This is not sufficient evidence, though, because not all purges are logged.(a) Argon is introduced to the RGS system only after the gas extraction is complete. Evacuating the system before the next sample is handled should protect against argon contamination from this source, but some type of holdover on the system surfaces may occur.

To check argon versus oxygen, the $\mathrm{Ar} / \mathrm{O}_{2}$ ratios were calculated for each sample at each step of processing. For most samples, the ratios were between 0.15 and 1 before the isotopic solution was added and between 0.05 and 0.15 after. The $\mathrm{Ar} / \mathrm{O}_{2}$ value in the atmosphere is 0.044 . There was one sample, 22A-4, in which the collected gas was inadvertently contaminated with air during RGS processing; for this step and this sample, the ratio was 0.046 , as would be expected.

(a) Thielges JR. May 25, 1999. "RGS Field Data Summary for FY99." Letter NHC-9953549, Numatec Hanford Corporation, Richland, Washington. 
If the high $\mathrm{Ar} / \mathrm{O}_{2}$ ratios during the pre-isotopic steps were the result of oxygen reaction, then two-thirds or more of the oxygen would need to have reacted, which is unlikely. If the argon were an accurate tracer for air in the samples, there would have been 3 to $5 \mathrm{cc}$ of air in some samples in which $\mathrm{x}$-rays showed no visible gas. The latter is not a very strong counter-argument, though, because gas is frequently present even though invisible on the $\mathrm{x}$-ray. Any substantial reaction of oxygen is considered implausible based on laboratory measurements of oxygen kinetics, but it cannot be excluded given the high ratios in most of the SY-101 RGS samples.

In sample 23A-1, though, the pre-isotopic $\mathrm{Ar} / \mathrm{O}_{2}$ ratios were between 0.06 and 0.09 , in the same range as most post-isotopic measurements. This does not suggest that any substantial oxygen reaction has occurred either in the in situ crust pores or during the 29-day lag time before the sample was analyzed. (The other samples whose lag times were 29 days or less all showed considerably higher pre-isotopic $\mathrm{Ar} / \mathrm{O}_{2}$ ratios than did $23 \mathrm{~A}-1$-another reason to suspect that oxygen reaction is not the major cause of the high ratios.)

\subsubsection{Domespace Composition Comparison}

The RGS gas-phase composition data (Table 4.15.5) were used to calculate $\mathrm{H}_{2} / \mathrm{N}_{2} \mathrm{O}$ and $\mathrm{H}_{2} / \mathrm{NH}_{3}$ ratios for comparison with selected ratios from domespace gas monitoring measurements (McCain 1999, Table 4-17). The results are shown in Table 4.15.12. The $\mathrm{RGS} \mathrm{H}_{2} / \mathrm{N}_{2} \mathrm{O}$ ratios from the mixed slurry layer and the top of the waste tended to match the domespace ratios. The gas retained in the crust proper and the "bubble slurry" layer presented a different ratio, higher in hydrogen.

Table 4.15.12 Comparison with Domespace Data(a)

\begin{tabular}{||l|c|c||}
\hline \multicolumn{1}{|c|}{ Sample } & $\mathrm{H}_{2} / \mathrm{N}_{2} \mathrm{O}$ & $\mathrm{H}_{2} / \mathrm{NH}_{3}$ \\
\hline \hline $\mathrm{RGS}, 23 \mathrm{~A}-1$ & $0.92-1.0$ & $3.5-12$ \\
\hline $\mathrm{RGS}, 23 \mathrm{~A}-2$ & 1.6 & $2.0-7.8$ \\
\hline $\mathrm{RGS}, 22 \mathrm{~A}-3$ & 1.4 & $2.9-10$ \\
\hline $\mathrm{RGS}, 23 \mathrm{~A}-3$ & 2.2 & $1.6-7.9$ \\
\hline $\mathrm{RGS}, 22 \mathrm{~A}-4$ & 2.2 & $1.2-7.0$ \\
\hline $\mathrm{RGS}, 4 \mathrm{~A}-5$ & $1.5-2.1$ & $3.3-12$ \\
\hline $\mathrm{RGS}, 23 \mathrm{~A}-8$ & $1.1-1.8$ & $3.2-11$ \\
\hline $\mathrm{RGS}, 22 \mathrm{~A}-10$ & $1.1-1.9$ & $2.8-11$ \\
\hline $\mathrm{RGS}, 23 \mathrm{~A}-13$ & $1.1-2.0$ & $3.3-12$ \\
\hline $\mathrm{RGS}, 22 \mathrm{~A}-17$ & $1.0-2.0$ & $4.9-18$ \\
\hline $\mathrm{RGS}, 23 \mathrm{~A}-21$ & $1.2-2.3$ & $5.1-19$ \\
\hline $\mathrm{RGS}, 22 \mathrm{~A}-23$ & $1.2-2.2$ & $6.9-25$ \\
\hline Domespace after 6/26/93 GRE & 0.96 & 2.4 \\
\hline Domespace, 11/10/97. & 0.60 & 0.68 \\
\hline Domespace, 3/29/98 & 0.92 & 0.38 \\
\hline Domespace, 12/9/98 & 1.2 & 0.45 \\
\hline Domespace, 6/22/99 & 1.2 & 0.27 \\
\hline $\begin{array}{l}\text { (a) RGS ratios were calculated for both lower- and upper-bound solubilities. Both } \\
\text { ends of the ratio range are given in the table, lower-bound solubility basis first. }\end{array}$ \\
\hline
\end{tabular}


The $\mathrm{H}_{2} / \mathrm{NH}_{3}$ ratios in the domespace have generally been substantially lower than those in the RGS measurements. One explanation would be that ammonia is released by evaporation from liquid surfaces as well as by gas releases. However, the last GRE had a higher $\mathrm{H}_{2} / \mathrm{NH}_{3}$ ratio than the steady-state domespace measurements in and after November 1997. The GRE created such a large amount of wetted area that evaporative ammonia should have given lower ratios for the GRE.

\subsubsection{Miscellaneous}

Judging by the higher gas volume fraction, higher hydrogen, and lower nitrogen in sample $4 \mathrm{~A}-5$, it was probably a mixture of a little bubble slurry (typified by samples $22 \mathrm{~A}-4$ and $23 \mathrm{~A}-3$ ) with the true mixed slurry. It is also possible that the amount and composition of the mixed slurry gas changed between January and March 1999; this cannot be ruled out with only one RGS sample from riser 4A. In this context, it is worth noting that the high gas gaps typical of the region at the crust bottom (bubble slurry) in risers 23A and 22A did not appear in the X-rays of riser 4A samples, suggesting a difference in gas behavior at the time and/or location of sampling riser $4 \mathrm{~A}$.

Sample 23A-8 was centered on an elevation of $684 \mathrm{~cm}$ (269 in.), close to the pump intake level, and was taken only nine hours after a pump run. All other samples were taken 24 hours or more after the last pump run, and the time since the last pump run had no apparent effect on the sample contents. Sample 23A-8 was lower in ammonia and had a different insoluble gas composition than the other samples from the mixed slurry layer. Although this single data point is not statistically significant, it poses the possibility that there could be some connection between the composition of 23A-8 and its proximity in time and elevation to the pump inflow. 


\subsection{Conclusions}

The quantitative results obtained from the RGS are summarized in this section. With additional evidence from various sources discussed throughout the report, the results support the conclusions that are explained in the following sections.

\subsection{RGS Gas Inventories -- Conclusions}

- The best estimate for the retained gas inventory in the nonconvective layers. in DSTs is obtained from the combination of RGS and VFI data. The RGS nonconvective layer gas inventories computed from RGS data alone for AW-101 and AN-103 were within $30 \%$ of the VFI\&RGS inventory. The RGS nonconvective layer gas inventory for AN-104 was $38 \%$ higher than the VFI\&RGS value, largely because of a single high-gas sample in a region near the tank bottom that could not be sampled by the VFI. The same was true for AN-105, whose RGS nonconvective layer gas inventory was 33\% higher than the VFI\&RGS value. On the other hand, the RGS missed the higher-gas regions in AW-101 and AN-103, which makes the RGS-only nonconvective layer inventories, respectively, $11 \%$ and $27 \%$ low compared to the VFI\&RGS values.

- Auxiliary information was often needed to estimate SST inventories because there were too few RGS samples to represent the tank. This information included x-ray observations on non-RGS samples, core extrusion lengths measured for non-RGS samples, and inventories given by the barometric BPE method. In several SSTs, $x$-rays and extrusions of non-RGS samples provided information that supported the RGS inventory, rather than the BPE inventory, as the better estimate of the tank retained gas.

- The gas inventories derived from RGS data were within $40 \%$ of the BPE inventories for six of the eleven RGS-sampled tanks for which the BPE method was appropriate. Those six tanks were AW-101, AN-105, A-101, AN-104, AN-103, and S-111. The RGS (or VFI\&RGS) and BPE inventories are compared in Table 5.1. The largest differences between the two types of inventories were seen for the SSTs.

Table 5.1. Comparison with BPE Retained Gas Inventories

\begin{tabular}{||c|c||c|c||}
\hline \multicolumn{2}{|c|}{ DSTs } & \multicolumn{2}{c|}{ SSTs } \\
\hline Tank & (BPE-VFI\&RGS)/BPE & Tank & (BPE-RGS)/BPE \\
\hline \hline AW-101 & $6 \%$ & A-101 & $-36 \%$ \\
\hline AN-105 & $11 \%$ & U-103 & $-68 \%$ \\
\hline AN-104 & $0.5 \%$ & S-106 & $59 \%$ \\
\hline AN-103 & $9 \%$ & SX-106 & $-69 \%$ \\
\hline \hline & & S-102 & $-104 \%$ \\
\hline & & S-111 & $3 \%$ \\
\hline & & U-109 & $-148 \%$ \\
\hline
\end{tabular}




\subsection{RGS Gas Volume Fractions-Conclusions}

- The RGS method gave retained gas volume fraction measurements with low uncertainty. The uncertainty caused by gas solubility and extraction measurement was usually less than $\pm 15 \%$ for samples from nonconvective waste. RGS and VFI data typically agreed well (AW-101, AN-105, AN-104, and AN-103), validating the low calculated uncertainty.

- Convective waste consistently had less than 0.01 volume fraction of free (undissolved) gas, while almost all wet nonconvective waste contained gas volume fractions of 0.02 to 0.3 or more. No high-gas samples were taken from liquid waste, though SY-101 mechanically-mixed slurry samples contained gas volume fractions of 0.02 to 0.03 .

\subsection{RGS Gas Volume Fractions-Key Observations}

- The calculated gas volume fractions were usually not sensitive to the ammonia solubility or the ammonia concentration, even though the in situ gas volume fraction was calculated using a gas solubility model with a substantial uncertainty. The crust region of SY-101 waste was an exception because of the combination of unusually high ammonia and gas concentrations. For a few of these samples, the gas volume fraction uncertainty due to solubility uncertainty was greater than or equal to that from measurement uncertainty.

- The measurement uncertainty of RGS determinations of in situ retained gas volume fraction was generally less than $\pm 15 \%$. The accuracy of the gas volume fraction calculation depended in part on extracting all gas from the sample, which required extraction into a series of canisters. Comparisons of the amount of gas extracted in the last canister with that extracted in the first canister typically showed no sign that substantial gas remained unextracted. However, the possible underestimation of the measured low-solubility gas concentration is considered to be less than 10\%, as discussed by Mahoney et al. (1997).

- Unusually high gas volume fractions ( 0.3 or more) were found in samples from the nonconvective layers of several tanks. In SY-101 and U-103, gas fractions greater than 0.4 were found near the waste surface. In SY-101, the gas had accumulated in the bottom third of the floating crust layer; the retention mechanism in the U-103 sample is not known. In SX-106 and U-109, gas fractions from 0.3 to 0.4 were found near the bottom of the tank. In S-102, high-gas samples were found both near tank bottom and near the waste surface.

- Gas volume fractions were consistently less than 0.01 in convective waste layers characterized by high liquid content (under in situ conditions) and nearly constant temperature from top to bottom. However, the mixed slurry layer in SY-101, which is mixed by periodic pumping rather than by convection, was found to contain 0.02 to 0.03 gas volume fraction.

- Substantial gas fractions can be retained in wastes after salt-well pumping. Samples taken below the interstitial liquid level (LL) from BY-109, a tank that had been pumped not long before RGS sampling, had in situ gas fractions of roughly 0.10 .

\subsection{RGS Gas Compositions-Conclusions}

- The RGS method gave retained gas compositions with low uncertainty for samples containing more than 0.02 gas volume fraction. In those samples, mole fraction uncertainties were typically within $\pm 20 \%$ of that measured. Drillstring sample and domespace $\mathrm{H}_{2} / \mathrm{N}_{2} \mathrm{O}$ ratios frequently matched the ratios from RGS data, providing some corroboration. 
- Gas composition in convective and nonconvective wastes was consistently distinctly different, with the nonconvective waste gas containing more $\mathrm{H}_{2}$ and less $\mathrm{N}_{2}$. There was substantial tank-to-tank variation in the composition of gas in the nonconvective wastes, but the composition was typically relatively uniform within a tank.

- The free (undissolved) gases retained in tank wastes can have substantial nonflammable components. Nitrogen, an inert gas, was found to be a major component of the retained gas (between 15 and $67 \mathrm{vol} \%$ ) in the gas retained in nonconvective wastes. Only Tanks A-101, SX-106, and S-111 contained nonconvecting wastes in which mole fractions of $\mathrm{N}_{2}$ of less than $20 \mathrm{~mol} \%$ were found in the retained gas.

\subsection{RGS Gas Compositions-Key Observations}

- The compositions of gases in the convective layers of the sampled tanks almost always showed $\mathrm{N}_{2}$ in the range of 55 to $70 \mathrm{~mol} \%$. The single exception was a sample from the top of Tank S-111, in which the gas was $90 \mathrm{~mol} \% \mathrm{~N}_{2}$. The hydrogen in convective-layer gas was always less than or equal to $25 \mathrm{~mol} \%$. The mechanically mixed slurry layer in SY-101, however, contained gas that was 30 to $40 \mathrm{~mol} \% \mathrm{~N}_{2}$ and 25 to $30 \mathrm{~mol} \% \mathrm{H}_{2}$. Note that these results are consistent across all the tanks in spite of the large measurement uncertainty bands that were caused by the small amount of gas present in convective wastes.

- The composition of retained gases in the gas-retaining layers of RGS-sampled tanks showed considerable diversity. Some nonconvective wastes retained gas that was more than 60 mol\% hydrogen (A-101, AN-105, AN-103, S-106, AX-101, and S-111). Only A-101 and S-111 gave samples with hydrogen greater than $70 \mathrm{~mol} \% \mathrm{H}_{2}$ in the retained gas. Other wastes retained $20 \mathrm{~mol} \%$ or more nitrous oxide, including AN-104, U-103, SX-106, S-102, U-109, and SY-101. U-103 waste held substantially more nitrous oxide than any other, about $40 \mathrm{~mol} \%$ in the retained gas. AW-101 was the only tank in which high $\mathrm{N}_{2}$ (50 mol\% or more) was measured in the nonconvective waste; it also contained more methane and other hydrocarbons than the gas in any other measured tank waste.

- For wastes with gas volume fractions of 0.02 or more, such as nonconvective wastes, more than $45 \%$ of the $\mathrm{N}_{2} \mathrm{O}$ (often much more) and more than $90 \%$ of the $\mathrm{H}_{2}, \mathrm{~N}_{2}$, and $\mathrm{CH}_{4}$ were calculated to be present in the free (undissolved) gas phase. In convective wastes (with less than 0.01 gas volume fraction), as much as $90 \%$ of the $\mathrm{N}_{2} \mathrm{O}$ and $25 \%$ of the $\mathrm{H}_{2}$, $\mathrm{N}_{2}$, and $\mathrm{CH}_{4}$ were calculated to be dissolved in the liquid.

- Solubility uncertainty had a large effect only on the composition of gas from convective layer wastes; when the volume fraction was more than 0.02 , solubility effects were usually within the bounds of measurement uncertainty. The solubility uncertainty affected the composition of gas from convective layer waste because there was so little gas that a large fraction of it went in or out of solution according to solubility. Ammonia and nitrous oxide evidenced the greatest relative changes in mole fraction as a result of solubility variation.

\subsection{RGS Ammonia Measurements-Observations and Conclusions}

Throughout the RGS program, ammonia has been the gas constituent most difficult to measure. In the last two years, an isotopic ammonia solution standard was used for (SX-106, AX-101, S-102, S-111, U-109, and SY-101). Before that, an isotopic vapor standard was tried for S-106 and BY-109. In the earliest RGS efforts, mass-balance methods were used to calculate 
ammonia in AW-101, A-101, AN-105, AN-104, AN-103, and U-103. All of these methods were hampered by the slowness with which the ammonia in solution in the samples equilibrated with the ammonia vapor in the RGS extraction system headspace. The measurement challenges made early RGS ammonia measurements insufficient to determine ammonia concentrations.

The earlier RGS reports (Shekarriz et al. 1997; Mahoney et al. 1997) gave ammonia concentrations for AW-101, AN-105, A-101, AN-104, AN-103, U-103, S-106, and BY-109. Those ammonia concentrations were for the most part underestimates (as a result of slow equilibration) and should not be used. The ammonia concentrations found for later tanks (SX-106, AX-101, S-102, S-111, U-109, and SY-101) by an isotopic standard solution addition are believed to be more accurately measured. Nevertheless, some doubt remains about the results obtained by isotopic solution addition. The ammonia concentrations measured by the isotopic solution method frequently disagree with other measurements (which themselves can be subject to question). Ongoing standards tests should resolve many of these questions. But because of these doubts, this report reviewed and compared four types of ammonia data, not all of which were available for every tank:

- Ammonia measurements made on RGS samples by isotopic solution addition as part of the RGS extraction

- Ammonia partial pressures over RGS samples just after extrusion into the extractor; in some cases, there were direct partial pressure measurements, and in almost all cases ammonia partial pressures could be calculated by subtracting water and gas pressures from the total extractor pressure

- ISE measurements of the ammonia remaining in the RGS samples after extraction (these are termed "post-RGS" measurements)

- ISE measurements of the ammonia in non-RGS samples, which could include core extrusions, salt-well grab samples, or supernatant grab samples.

The data sets from AW-101, AN-105, A-101, AN-104, AN-103, and U-103 included only ammonia partial pressures and, in some cases, post-RGS and grab-sample ammonia measurements. These data did not allow any precise determination of the ammonia concentrations, but indications were that $\mathrm{AW}-101, \mathrm{AN}-105, \mathrm{AN}-104$, and AN-103 all had similar ammonia concentrations with a lower bound of 20,000 to $30,000 \mu \mathrm{mol} \mathrm{NH} 3 / \mathrm{L}$ waste. The upper bounds on these concentrations probably could not have been greater than double the lower bounds and were probably less. This conclusion is based on the upper and lower bounds found for samples that had isotopic measurements of ammonia as well as ammonia partial pressure measurements. Tank A-101 waste probably had two to five times the ammonia concentration of AW-101 based on a comparison of ammonia partial pressures. On the same approximate basis, Tank U-103 waste probably contained twice the ammonia concentration of AW-101.

The ammonia concentrations found using isotopic vapor standards for Tank S-106 waste are believed to have been considerably underestimated by Mahoney et al. (1997). Ammonia partial pressures and a salt-well grab sample both supported the estimate of an ammonia concentration for S-106 that was about half that for AW-101. The BY-109 ammonia concentration appeared to be lower than that in S-106. In BY-109, the isotopic measurements agreed with the estimates based on ammonia partial pressure, perhaps because a longer equilibration time made the isotopic measurements more accurate.

The isotopic solution method was used to measure ammonia in the samples from SX-106, AX-101, S-102, S-111, U-109, and SY-101. The SY-101 measurements appear to be the best determined, based on comparison with partial pressures and measurements in non-ISE core extru- 
sions. This improved reliability was obtained because SY-101 waste contained more ammonia than any other measured tank waste, which reduced inaccuracies related to sorption losses, and because essentially the same standard extraction procedure was used on all the samples. Most of the SY-101 ammonia concentrations were between 140,000 and $200,000 \mu \mathrm{mol} \mathrm{NH}_{3} / \mathrm{L}$ waste.

The ammonia concentrations in the SX-106 nonconvective layer, the single AX-101 sample, and the U-109 samples were considered reliably characterized by the isotopic solution method. The quality of characterization was assessed based on a reasonably close match between the isotopic results and other types of ammonia measurements. The ammonia concentration in the SX-106 nonconvective layer was between 60,000 and $100,000 \mu \mathrm{mol} \mathrm{NH}_{3} / \mathrm{L}$ waste; that in the AX-101 sample was between 80,000 and $140,000 \mu \mathrm{mol} \mathrm{NH}_{3} / \mathrm{L}$ waste; and that in $\mathrm{U}-109$-was between 25,000 and $45,000 \mu \mathrm{mol} \mathrm{NH}_{3} / \mathrm{L}$ waste.

The ammonia concentrations in the SX-106 convective layer, S-102, and S-111 were not reliably characterized in that various types of measurements did not agree. The SX-106 supernatant could have contained between 1,000 and $25,000 \mu \mathrm{mol} \mathrm{NH}_{3} / \mathrm{L}$ waste; measurements were scattered over that range. Both the isotopic and partial pressure measurements from Tank S-102 included unusual amounts of scatter and non-physical values. The S-111 data had similar though less pronounced problems. However, there were no plausible data that suggested ammonia concentrations outside the range of 15,000 to $70,000 \mu \mathrm{mol} \mathrm{NH} / \mathrm{L}$ waste for $\mathrm{S}-102$, or outside the range of 4,000 to $80,000 \mu \mathrm{mol} \mathrm{NH}_{3} / \mathrm{L}$ waste for $\mathrm{S}-111$.

\subsection{RGS X-Ray Analyses-Conclusions}

The gas volume fraction estimates made from RGS sample $\mathrm{x}$-rays almost always underestimated the amount of gas found by extraction. There were only three exceptions to this rule among the 53 extracted samples in whose $x$-rays more than one or two bubbles were visible. The average and standard deviation for the amount by which extracted gas exceeded visible gas was $0.07 \pm 0.08$ (gas volume fraction). The maximum such difference was 0.36 gas volume fraction.

The x-rays were of limited usefulness for sample density calculation because of variations in exposure and the fact that the necessary air/water standard density profiles were lacking for a number of samples. There was no consistent bias in the sample densities estimated from the $x$-rays compared with the densities obtained for adjacent samples by laboratory analysis.

\subsection{RGS Performance Summary}

The overall success rate for RGS sampling was $85 \%$. Of 88 samples that were taken, six were lost because of sampler valve leaks, two because the sampler valve froze closed, two because of piston seal leaks, and three because of extraction procedure errors. The overall sample loss rate was the same for DSTs as for SSTs.

\subsection{Summary of RGS Results}

Table 5.2 contains the compositions and gas volume fractions for all RGS samples.Each mole fraction and gas volume fraction is expressed as a range between two values, each of which has its own \pm uncertainty. The first number in the range is the value for the lower-bound estimate of gas solubility, and the second number is the value for the higher-bound solubility estimate. The \pm uncertainty on each of those numbers represents the measurement uncertainty. 
Table 5.2. In Situ Compositions and Volume Fractions of Retained Gas from RGS Data ${ }^{(\mathrm{a})}$

\begin{tabular}{|c|c|c|c|c|c|c|c|c|}
\hline \multirow{2}{*}{$\begin{array}{l}\text { Tank and Samplc } \\
\text { (or layer) }\end{array}$} & \multicolumn{6}{|c|}{ Mole Percent of Constituent in Bubbles } & \multirow{2}{*}{$\begin{array}{l}\text { In Situ Gas Volume } \\
\text { (percent) }\end{array}$} & \multirow{2}{*}{$\begin{array}{l}\text { Elevation } \\
(\mathrm{cm}, \text { in.) }\end{array}$} \\
\hline & $\mathrm{H}_{2}$ & $\mathrm{~N}_{2}$ & $\mathrm{~N}_{2} \mathrm{O}$ & $\mathrm{CH}_{4}$ & $\mathrm{NH}_{3}$ & Other & & \\
\hline & $26 \pm 9.4$ to $25 \pm 9.2$ & $67 \pm 32$ to $69 \pm 33$ & $2.3 \pm 0.8$ to $0.8 \pm 0.3$ & $1.0 \pm 0.4$ & $0.6 \pm 0.3$ to $0.2 \pm 0.1$ & $3.0 \pm 1.7$ to $3.3 \pm 1.9$ & $0.8 \pm 0.1$ to $0.7 \pm 0.1$ & $700,276.5$ \\
\hline AW-101-24A-17 & $29 \pm 3.9$ & $59 \pm 8.9$ to $61 \pm 9.2$ & $5.8 \pm 0.9$ to $3.5 \pm 0.5$ & $1.8 \pm 0.3$ & $0.9 \pm 0.4$ to $0.3 \pm 0.2$ & $3.4 \pm 0.8$ to $3.6 \pm 0.8$ & $2.7 \pm 0.3$ to $2.5 \pm 0.3$ & $265,104.5$ \\
\hline$A W-101-24 \mathrm{~B}-18$ & $19 \pm 3.1$ & $67 \pm 14$ to $70 \pm 14$ & $7.1 \pm 1.2$ to $3.9 \pm 0.7$ & $2.0 \pm 0.4$ & $0.4 \pm 0.2$ to $0.2 \pm 0.1$ & $4.2 \pm 1.1$ to $4.5 \pm 1.2$ & $2.1 \pm 0.4$ to $1.9 \pm 0.4$ & $217,86.5$ \\
\hline AW-101-24A-19 & $43 \pm 3.5$ to $44 \pm 3.5$ & $47 \pm 4.1$ to $48 \pm 4.1$ & $5.8 \pm 0.5$ to $4.3 \pm 0.4$ & $1.4 \pm 0.1$ & $1.1 \pm 0.5$ to $0.4 \pm 0.2$ & $1.9 \pm 0.3$ to $2.0 \pm 0.3$ & $5.2 \pm 0.5$ to $5.0 \pm 0.5$ & $169,66.5$ \\
\hline$A W-101-24 A-21$ & $30 \pm 2.5$ to $31 \pm 2.5$ & $56 \pm 4.8$ to $58 \pm 4.9$ & $8.2 \pm 0.7$ to $6.0 \pm 0.5$ & $1.8 \pm 0.3$ & $0.8 \pm 0.4$ to $0.3 \pm 0.2$ & $2.5 \pm 0.4$ to $2.6 \pm 0.4$ & $5.1 \pm 0.5$ to $4.8 \pm 0.5$ & $72.3,36.5$ \\
\hline $\mathrm{AW}-101-24 \mathrm{~B}-22$ & $13 \pm 2.1$ to $14 \pm 2.1$ & $67 \pm 11$ to $72 \pm 12$ & $12 \pm 2.2$ to $6.3 \pm 1.1$ & $2.2 \pm 0.4$ to $2.3 \pm 0.5$ & $0.4 \pm 0.2$ to $0.2 \pm 0.1$ & $5.2 \pm 1.8$ to $5.8 \pm 2.0$ & $2.0 \pm 0.4$ to $1.8 \pm 0.4$ & $24.1,9.5$ \\
\hline$A W-101-C^{(b)}$ & $26 \pm 9.4$ to $25 \pm 9.2$ & $67 \pm 32$ to $69 \pm 33$ & $2.3 \pm 0.8$ to $0.8 \pm 0.3$ & $1.0 \pm 0.4$ & $0.6 \pm 0.3$ to $0.2 \pm 0.1$ & $3.0 \pm 1.7$ to $3.3 \pm 1.9$ & $0.8 \pm 0.3$ to $0.7 \pm 0.3$ & 673,265 \\
\hline AW-101-NC & $32 \pm 3.2$ to $33 \pm 3.2$ & $55 \pm 6.2$ to $57 \pm 6.3$ & $7.5 \pm 0.8$ to $5.1 \pm 0.6$ & $1.7 \pm 0.2$ & $0.9 \pm 0.4$ to $0.3 \pm 0.2$ & $2.8 \pm 0.5$ to $2.9 \pm 0.6$ & $3.7 \pm 1.8$ to $3.5 \pm 1.8$ & 133,52 \\
\hline $\mathrm{AN}-105-7 \mathrm{~B}-4$ & $25 \pm 12$ & $57 \pm 41$ to $64 \pm 47$ & $13 \pm 6.6-4.6 \pm 2.4$ & $1.4 \pm 0.8$ & $0.8 \pm 0.5$ to $0.4 \pm 0.2$ & $3.0 \pm 2.0$ to $3.9 \pm 2.7$ & $0.8 \pm 0.3$ to $0.6 \pm 0.3$ & $893,351.5$ \\
\hline AN-105-12A-15 & $19 \pm 13$ to $19 \pm 15$ & $62 \pm 62$ to $71 \pm 71$ & $14 \pm 9.7-4.2 \pm 3.2$ & $1.4 \pm 1.0$ & $0.6 \pm 0.5$ to $0.2 \pm 0.2$ & $2.8 \pm 2.2$ to $3.9 \pm 3.3$ & $0.5 \pm 0.2$ to $0.4 \pm 0.2$ & $362,142.5$ \\
\hline \begin{tabular}{|l|}
$\mathrm{AN}-105-7 \mathrm{~B}-16$ \\
\end{tabular} & $18 \pm 5.8$ & $70 \pm 28$ to $76 \pm 30$ & $8.5 \pm 3.1$ to $2.9 \pm 1.1$ & $0.8 \pm 0.4$ & $0.4 \pm 0.2$ to $0.2 \pm 0.1$ & $2.0 \pm 1.2$ to $2.5 \pm 1.5$ & $0.7 \pm 0.2$ to $0.6 \pm 0.2$ & $314,123.5$ \\
\hline AN-105-12A-17 & $65 \pm 5.2$ to $66 \pm 5.2$ & $22 \pm 2.0$ to $23 \pm 2.1$ & $11 \pm 1.0$ to $8.8 \pm 0.8$ & $0.6 \pm 0.1$ & $0.9 \pm 0.4$ to $0.4 \pm 0.2$ & $0.7 \pm 0.2$ & $6.9 \pm 0.7$ to $6.6 \pm 0.7$ & $265,104.5$ \\
\hline AN-105-7B-18 & $54 \pm 7.7$ to $57 \pm 8.0$ & $31 \pm 4.9$ to $33 \pm 5.2$ & $12 \pm 2.0$ to $7.6 \pm 1.3$ & $0.8 \pm 0.2$ & $0.7 \pm 0.3$ to $0.3 \pm 0.1$ & $1.6 \pm 0.7$ to $1.8 \pm 0.8$ & $2.7 \pm 0.4$ to $2.5 \pm 0.4$ & $217,85.5$ \\
\hline AN-105-12A-19 & $65 \pm 4.9$ to $66 \pm 5.0$ & $21 \pm 3.4$ to $22 \pm 3.5$ & $12 \pm 1.0$ to $11 \pm 0.9$ & $0.6 \pm 0.06$ & $0.7 \pm 0.3$ to $0.3 \pm 0.1$ & $0.4 \pm 0.1$ & $12 \pm 0.8$ & $169,66.5$ \\
\hline AN-105-12A-21 & $57 \pm 4.0$ to $59 \pm 4.1$ & $22 \pm 1.6$ to $23 \pm 1.7$ & $19 \pm 1.6$ to $16 \pm 1.3$ & $0.8 \pm 0.06$ & $0.5 \pm 0.2$ to $0.2 \pm 0.1$ & $0.5 \pm 0.1$ & $7.5 \pm 0.7$ to $7.1 \pm 0.7$ & $72.4,28.5$ \\
\hline $\mathrm{AN}-105-\mathrm{C}$ & $25 \pm 12$ & $57 \pm 41$ to $64 \pm 47$ & $13 \pm 6.6$ to $4.6 \pm 2.4$ & $1.4 \pm 0.8$ & $0.8 \pm 0.5$ to $0.4 \pm 0.2$ & $3.0 \pm 2.0$ to $3.9 \pm 2.7$ & $0.6 \pm 0.2$ to $0.5 \pm 0.2$ & 608,239 \\
\hline$A N-105-N C$ & $59 \pm 5.4$ to $62 \pm 5.4$ & $24 \pm 4.0$ & $15 \pm 1.5$ to $12 \pm 1.2$ & $0.7 \pm 0.09$ & $0.6 \pm 0.3$ to $0.3 \pm 0.1$ & $0.6 \pm 0.2$ & $5.2 \pm 2.6$ to $4.9 \pm 2.4$ & 142,56 \\
\hline A-101-24-2 & $63 \pm 5.6$ to $64 \pm 5.4$ & $26 \pm 4.8$ to $27 \pm 4.9$ & $7.4 \pm 0.7$ to $6.9 \pm 0.6$ & $0.4 \pm 0.1$ & $3.0 \pm 1.3$ to $1.2 \pm 0.5$ & $0.5 \pm 0.1$ & $16 \pm 1.4$ & $845,332.5$ \\
\hline A-101-15-5 & $72 \pm 8.4$ to $76 \pm 8.0$ & $15 \pm 4.7$ & $5.6 \pm 0.7$ to $5.4 \pm 0.6$ & $0.7 \pm 0.1$ & $6.7 \pm 2.7$ to $2.6 \pm 1.0$ & $0.3 \pm 0.1$ & $19 \pm 2.1$ to $18 \pm 2.1$ & $700,275.5$ \\
\hline A-101-15-8. & $74 \pm 8.0$ to $76 \pm 7.8$ & $16 \pm 5.4$ & $5.2 \pm 0.6$ to $5.1 \pm 0.5$ & $0.7 \pm 0.08$ & $4.4 \pm 1.6$ to $1.7 \pm 0.6$ & $0.3 \pm 0.06$ & $21 \pm 2.1$ to $20 \pm 2.1$ & $555,218.5$ \\
\hline $\mathrm{A}-101-24-9$ & $68 \pm 6.2$ to $70 \pm 6.2$ & $22 \pm 4.5$ to $23 \pm 4.6$ & $4.8 \pm 0.4$ to $4.7 \pm 0.4$ & $0.8 \pm 0.1$ & $3.7 \pm 1.0$ to $1.4 \pm 0.4$ & $0.2 \pm 0.05$ & $22 \pm 2.1$ to $21 \pm 2.1$ & $507,199.5$ \\
\hline$A-101-15-12$ & $11 \pm 4.2$ to $12 \pm 4.6$ & $58 \pm 27$ to $71 \pm 33$ & $17 \pm 7.3$ to $6.7 \pm 2.9$ & $2.9 \pm 1.6$ to $3.3 \pm 1.8$ & $8.1 \pm 4.3$ to $3.1 \pm 1.7$ & $2.4 \pm 1.5$ to $3.3 \pm 2.1$ & $0.8 \pm 0.3$ to $0.6 \pm 0.3$ & $362,142.5$ \\
\hline A-101-24-16 & $14 \pm 4.1$ to $15 \pm 4.2$ & $60 \pm 19$ to $72 \pm 23$ & $15 \pm 5.0$ to $5.7 \pm 1.8$ & $0.8 \pm 0.3$ to $0.9 \pm 0.3$ & $8.1 \pm 4.0$ to $3.1 \pm 1.6$ & $2.0 \pm 1.1$ to $2.8 \pm 1.5$ & $0.7 \pm 0.3$ to $0.5 \pm 0.3$ & $169,66.5$ \\
\hline$A-101-24-19$ & $18 \pm 4.5$ to $19 \pm 4.6$ & $61 \pm 19$ to $71 \pm 22$ & $13 \pm 3.7$ to $5.6 \pm 1.6$ & $0.6 \pm 0.2$ to $0.7 \pm 0.2$ & $7.0 \pm 3.5$ to $2.7 \pm 1.3$ & $1.2 \pm 0.7$ to $1.5 \pm 0.9$ & $1.0 \pm 0.3$ to $0.8 \pm 0.3$ & $24.1,9.5$ \\
\hline $\mathrm{A}-101-\mathrm{NC}$ & $70 \pm 7.3$ to $72 \pm 7.0$ & $19 \pm 4.9$ & $5.7 \pm 0.6$ to $5.5 \pm 0.5$ & $0.7 \pm 0.1$ & $4.8 \pm 1.8$ to $1.8 \pm 0.57$ & $0.3 \pm 0.06$ & $18 \pm 9.0$ to $17 \pm 8.5$ & 652,257 \\
\hline$A-101-C$ & $14 \pm 4.3$ to $15 \pm 4.6$ & $60 \pm 22$ to $71 \pm 27$ & $15 \pm 5.4$ to $6.0 \pm 2.2$ & $1.6 \pm 0.7$ to $1.7 \pm 0.8$ & $7.8 \pm 4.0$ to $3.0 \pm 1.5$ & $1.9 \pm 0.9$ to $2.6 \pm 1.2$ & $0.8 \pm 0.3$ to $0.6 \pm 0.3$ & 207,81 \\
\hline & & & & & & & & \\
\hline
\end{tabular}


Table 5.2. (contd)

\begin{tabular}{|c|c|c|c|c|c|c|c|c|}
\hline \multirow{2}{*}{$\begin{array}{l}\text { Tank and Sample } \\
\text { (or layer) }\end{array}$} & \multicolumn{6}{|c|}{ Mole Percent of Constituent in Bubbles } & \multirow{2}{*}{$\begin{array}{c}\text { In Situ Gas Volume } \\
\text { (percent) }\end{array}$} & \multirow{2}{*}{$\begin{array}{c}\text { Elevation } \\
\text { (cm, in.) }\end{array}$} \\
\hline & $\mathrm{H}_{2}$ & $\mathrm{~N}_{2}$ & $\mathrm{~N}_{2} \mathrm{O}$ & $\mathrm{CH}_{4}$ & $\mathrm{NH}_{3}$ & Other & & \\
\hline AN-104-10A-3 & $24 \pm 13$ to $26 \pm 15$ & $55 \pm 48$ to $63 \pm 57$ & $15 \pm 8.4$ to $5.2 \pm 3.1$ & $1.8 \pm 1.3$ to $2.0 \pm 1.4$ & $1.0 \pm 0.7$ to $0.4 \pm 0.3$ & $2.6 \pm 1.8$ to $3.3 \pm 2.4$ & $0.9 \pm 0.3$ to $0.7 \pm 0.3$ & $893,351.5$ \\
\hline $\mathrm{AN}-104-10 \mathrm{~A}-13$ & $41 \pm 8.2$ to $44 \pm 8.6$ & $40 \pm 9.6$ to $44 \pm 10$ & $15 \pm 3.8$ to $8.1 \pm 2.1$ & $1.3 \pm 0.5$ to $1.4 \pm 0.5$ & $0.6 \pm 0.2$ to $0.2 \pm 0.1$ & $1.9 \pm 0.9$ to $2.1 \pm 1.0$ & $2.2 \pm 0.4$ to $1.9 \pm 0.4$ & $410,161.5$ \\
\hline AN-104-10A-15 & $49 \pm 8.1$ to $52 \pm 8.3$ & $32 \pm 5.7$ to $34 \pm 5.9$ & $13 \pm 2.5$ to $10 \pm 1.8$ & $1.1 \pm 0.3$ to $1.2 \pm 0.3$ & $3.0 \pm 1.5$ to $1.2 \pm 0.6$ & $1.3 \pm 0.5$ to $1.4 \pm 0.6$ & $4.8 \pm 0.8$ to $4.4 \pm 0.8$ & $314,123.5$ \\
\hline $\mathrm{AN}-104-10 \mathrm{~A}-17$ & $29 \pm 6.0$ to $30 \pm 6.2$ & $52 \pm 13$ to $54 \pm 13$ & $15 \pm 3.1$ to $12 \pm 2.5$ & $1.5 \pm 0.3$ to $1.6 \pm 0.3$ & $1.2 \pm 0.6$ to $0.5 \pm 0.2$ & $1.5 \pm 0.5$ to $1.6 \pm 0.5$ & $5.8 \pm 0.8$ to $5.4 \pm 0.8$ & $217,85.5$ \\
\hline $\mathrm{AN}-104-12 \mathrm{~A}-18$ & $45 \pm 3.6$ to $47 \pm 3.7$ & $36 \pm 3.1$ to $38 \pm 3.1$ & $15 \pm 1.5$ to $12 \pm 1.2$ & $1.1 \pm 0.2$ & $0.8 \pm 0.3$ to $0.3 \pm 0.1$ & $1.4 \pm 0.5$ & $7.1 \pm 0.7$ to $6.8 \pm 0.7$ & $169,66.5$ \\
\hline $\mathrm{AN}-104-10 \mathrm{~A}-21$ & $47 \pm 7.8$ to $49 \pm 8.0$ & $20 \pm 3.5$ to $21 \pm 3.6$ & $30 \pm 5.1$ to $28 \pm 4.7$ & $0.6 \pm 0.1$ & $1.3 \pm 0.7$ to $0.5 \pm 0.3$ & $0.3 \pm 0.1$ to $0.4 \pm 0.1$ & $17 \pm 1.9$ to $16 \pm 1.9$ & $24.1,9.5$ \\
\hline$A N-104-C$ & $24 \pm 13$ to $26 \pm 15$ & $55 \pm 48$ to $63 \pm 57$ & $15 \pm 8.4$ to $5.2 \pm 3.1$ & $1.8 \pm 1.3$ to $2.0 \pm 1.4$ & $1.0 \pm 0.7$ to $0.4 \pm 0.3$ & $2.6 \pm 1.8$ to $3.3 \pm 2.4$ & $0.5 \pm 0.2$ to $0.4 \pm 0.2$ & 673,265 \\
\hline$A N-104-N C$ & $45 \pm 6.9$ to $47 \pm 7.0$ & $29 \pm 4.8$ to $31 \pm 5.0$ & $23 \pm 3.8$ to $20 \pm 3.3$ & $0.9 \pm 0.2$ & $1.4 \pm 0.7$ to $0.5 \pm 0.3$ & $0.8 \pm 0.3$ to $0.9 \pm 0.3$ & $8.1 \pm 4.0$ to $7.6 \pm 3.8$ & 119,47 \\
\hline AN-103-12A-2 & $62 \pm 6.4$ to $63 \pm 6.4$ & $29 \pm 3.2$ & $6.9 \pm 0.7$ to $6.3 \pm 0.6$ & $0.6 \pm 0.07$ & $1.8 \pm 0.8$ to $0.6 \pm 0.3$ & $0.2 \pm 0.05$ & $16 \pm 1.4$ & $845,332.5$ \\
\hline AN-103-12A-5 & $19 \pm 10$ & $68 \pm 54$ to $74 \pm 60$ & $8.7 \pm 5.2$ to $2.9 \pm 1.8$ & $1.7 \pm 1.3$ & $1.4 \pm 1.0$ to $0.5 \pm 0.4$ & $1.4 \pm 1.0$ to $1.6 \pm 1.2$ & $0.8 \pm 0.3$ to $0.7 \pm 0.3$ & $700,275.5$ \\
\hline AN-103-21A-10 & $19 \pm 13$ & $69 \pm 69$ to $75 \pm 75$ & $7.9 \pm 5.3$ to $2.3 \pm 1.7$ & $1.1 \pm 0.9$ & $1.1 \pm 0.9$ to $0.4 \pm 0.3$ & $1.4 \pm 1.1$ to $1.6 \pm 1.4$ & $0.6 \pm 0.3$ to $0.5 \pm 0.3$ & $458,180.5$ \\
\hline AN-103-12A-14 & $55 \pm 8.8$ to $56 \pm 8.8$ & $38 \pm 6.5$ to $39 \pm 6.6$ & $4.9 \pm 0.8$ to $4.1 \pm 0.7$ & $0.7 \pm 0.2$ & $1.0 \pm 0.4$ to $0.4 \pm 0.2$ & $0.4 \pm 0.2$ & $6.7 \pm 1.2$ to $6.5 \pm 1.2$ & $265,104.5$ \\
\hline $\mathrm{AN}-103-21 \mathrm{~A}-16$ & $64 \pm 7.2$ & $30 \pm 3.5$ to $31 \pm 3.5$ & $3.8 \pm 0.4$ to $3.4 \pm 0.4$ & $0.6 \pm 0.1$ & $0.8 \pm 0.4$ to $0.3 \pm 0.1$ & $0.4 \pm 0.2$ & $12 \pm 1.5$ & $169,66.5$ \\
\hline AN-103 crust & $62 \pm 6.4$ to $63 \pm 6.4$ & $29 \pm 3.2$ & $6.9 \pm 0.7$ to $6.3 \pm 0.6$ & $0.6 \pm 0.07$ & $1.8 \pm 0.8$ to $0.6 \pm 0.3$ & $0.2 \pm 0.05$ & $16 \pm 7.9$ to $15 \pm 7.6$ & 839,330 \\
\hline$A N-103-C$ & $19 \pm 12$ & $68 \pm 61$ to $75 \pm 68$ & $8.3 \pm 5.3$ to $2.6 \pm 1.7$ & $1.4 \pm 1.1$ & $1.2 \pm 0.9$ to $0.5 \pm 0.4$ & $1.4 \pm 0.8$ to $1.6 \pm 0.9$ & $0.7 \pm 0.3$ to $0.6 \pm 0.3$ & 587,231 \\
\hline AN-103-NC & $61 \pm 7.7$ to $62 \pm 7.7$ & $33 \pm 4.3$ & $4.2 \pm 0.6$ to $3.6 \pm 0.5$ & $0.6 \pm 0.1$ & $0.9 \pm 0.4$ to $0.3 \pm 0.1$ & $0.4 \pm 0.1$ & $9.2 \pm 4.6$ to $9.1 \pm 4.5$ & 160,63 \\
\hline U-103-7-2 & $23 \pm 1.3$ & $36 \pm 2.1$ to $37 \pm 2.1$ & $40 \pm 2.2$ to $39 \pm 2.1$ & $0.4 \pm 0.03$ & $\begin{array}{c}0.1 \pm 0.03 \text { to } \\
0.05 \pm 0.01\end{array}$ & $0.5 \pm 0 . \dot{0} 6$ & $42 \pm 2.7$ to $41 \pm 2.6$ & $362,142.5$ \\
\hline U-103-7-5 & $14 \pm 0.9$ to $16 \pm 0.9$ & $32 \pm 2.0$ to $35 \pm 2.1$ & $52 \pm 3.2$ to $48 \pm 2.8$ & $0.2 \pm 0.06$ to $0.3 \pm 0.06$ & $1.8 \pm 0.8$ to $0.9 \pm 0.4$ & $0.3 \pm 0.1$ to $0.4 \pm 0.1$ & $9.8 \pm 0.8$ to $8.8 \pm 0.8$ & $217,85.5$ \\
\hline U-103-7-7 & $24 \pm 1.5$ to $25 \pm 1: 5$ & $41 \pm 2.6$ to $43 \pm 2.7$ & $32 \pm 2.0$ to $29 \pm 1.8$ & $0.6 \pm 0.1$ & $1.2 \pm 0.4$ to $0.6 \pm 0.2$ & $1.0 \pm 0.2$ & $11 \pm 1.2$ & $121,47.5$ \\
\hline U-103-7-8 & $30 \pm 3.0$ to $32 \pm 3.2$ & $36 \pm 3.6$ to $38 \pm 3.8$ & $30 \pm 3.0$ to $26 \pm 2.6$ & $0.6 \pm 0.1$ to $0.8 \pm 0.1$ & $1.3 \pm 0.8$ to $0.6 \pm 0.4$ & $1.6 \pm 0.3$ to $1.8 \pm 0.3$ & $7.9 \pm 1.0$ to $7.3 \pm 1.0$ & $72,28.5$ \\
\hline $\mathrm{U}-103-\mathrm{NC}$ & $23 \pm 1.4$ & $36 \pm 2.3$ to $37 \pm 2.3$ & $40 \pm 2.4$ to $38 \pm 2.3$ & $0.4 \pm 0.05$ & $0.6 \pm 0.3$ to $0.3 \pm 0.1$ & $0.6 \pm 0.1$ to $0.7 \pm 0.1$ & $19 \pm 9.6$ to $18 \pm 9.0$ & 277,109 \\
\hline$S-106-7-3$ & $59 \pm 5.0$ to $60 \pm 5.1$ & $32 \pm 3.1$ to $33 \pm 3.2$ & $7.9 \pm 0.7$ to $6.4 \pm 0.6$ & $0.4 \pm 0.2$ & $0.3 \pm 0.2$ to $0.2 \pm 0.1$ & $0.2 \pm 0.2$ & $9.7 \pm 0.9$ to $9.4 \pm 0.9$ & $362,142.5$ \\
\hline$S-106-7-5$ & $62 \pm 5.4$ to $64 \pm 5.6$ & $23 \pm 3.6$ & $14 \pm 1.2$ to $12 \pm 1.0$ & $0.01 \pm 0.01$ & $0.3 \pm 0.2$ to $0.2 \pm 0.1$ & $0.5 \pm 0.3$ & $10 \pm 1.0$ & $265,104.5$ \\
\hline$S-106-8-6$ & $63 \pm 8.6$ to $65 \pm 8.8$ & $25 \pm 3.6$ to $26 \pm 3.7$ & $10 \pm 1.6$ to $7.8 \pm 1.2$ & $0.5 \pm 0.2$ & $0.6 \pm 0.3$ to $0.3 \pm 0.1$ & $0.9 \pm 0.7$ & $7.7 \pm 0.8$ to $7.3 \pm 0.8$ & $217,85.5$ \\
\hline S-106-8-10 & $65 \pm 4.9$ to $66 \pm 5.0$ & $23 \pm 4.2$ to $24 \pm 4.3$ & $11 \pm 0.8$ to $9.5 \pm 0.7$ & $0.2 \pm 0.02$ & $0.3 \pm 0.1$ to $0.1 \pm 0.06$ & $0.4 \pm 0.3$ & $14 \pm 1.2$ & $24,9.5$ \\
\hline S-106-NC & $63 \pm 5.7$ to $64 \pm 5.9$ & $25 \pm 3.7$ to $26 \pm 3.8$ & $11 \pm 1.0$ to $9.0 \pm 0.9$ & $0.3 \pm 0.08$ & $0.4 \pm 0.2$ to $0.2 \pm 0.1$ & $0.5 \pm 0.3$ & $10 \pm 5.2$ & 164,64 \\
\hline
\end{tabular}


Table 5.2. (contd)

\begin{tabular}{|c|c|c|c|c|c|c|c|c|}
\hline \multirow{2}{*}{$\begin{array}{l}\text { Tank and Sample } \\
\text { (or layer) }\end{array}$} & \multicolumn{6}{|c|}{ Mole Percent of Constituent in Bubbles (water vapor excluded) } & \multirow{2}{*}{$\begin{array}{c}\text { In Situ Gas Volume } \\
\text { (percent) }\end{array}$} & \multirow{2}{*}{$\begin{array}{c}\text { Elcvation } \\
(\mathrm{cm}, \text { in.) }\end{array}$} \\
\hline & $\mathrm{H}_{2}$ & $\mathrm{~N}_{2}$ & $\mathrm{~N}_{2} \mathrm{O}$ & $\mathrm{CH}_{4}$ & $\mathrm{NH}_{3}$ & Other & & \\
\hline BY $-109-12 C-4$ & $35 \pm 3.6$ to $36 \pm 3.7$ & $40 \pm 7.9$ to $41 \pm 8.2$ & $21 \pm 2.2$ to $19 \pm 2.0$ & $1.0 \pm 0.2$ & $0.4 \pm 0.2$ to $0.2 \pm 0.1$ & $2.2 \pm 0.4$ to $2.3 \pm 0.4$ & $6.4 \pm 0.4$ to $6.2 \pm 0.4$ & $121,47.5$ \\
\hline BY-109-10B-5 & $52 \pm 5.5$ & $29 \pm 5.0$ & $17 \pm 1.7$ to $16 \pm 1.6$ & $0.7 \pm 0.1$ & $0.3 \pm 0.1$ to $0.1 \pm 0.05$ & $1.8 \pm 0.4$ & $8.7 \pm 0.8$ to $8.5 \pm 0.8$ & $121,47.5$ \\
\hline BY-109-10B-6 & $56 \pm 6.4$ to $57 \pm 6.4$ & $23 \pm 3.8$ & $17 \pm 3.4$ to $16 \pm 3.2$ & $0.9 \pm 0.1$ & $0.3 \pm 0.1$ to $0.1 \pm 0.06$ & $2.6 \pm 0.6$ to $2.7 \pm 0.6$ & $12 . \pm 1.0$ & $72,28.5$ \\
\hline BY-109 below ILL & $50 \pm 5.5$ to $51 \pm 5.6$ & $28 \pm 5.0$ to $29 \pm 5.1$ & $18 \pm 2.5$ to $17 \pm 2.3$ & $0.8 \pm 0.1$ to $0.9 \pm 0.1$ & $0.3 \pm 0.1$ to $0.1 \pm 0.06$ & $2.3 \pm 0.4$ & $9.5 \pm 4.7$ to $9.3 \pm 4.6$ & 120,47 \\
\hline$S X-106-3-2$ & $22 \pm 2.9$ to $18 \pm 2.6$ & $60 \pm 10$ to $74 \pm 13$ & $14 \pm 1.9$ to $2.4 \pm 0.4$ & $1.3 \pm 0.5$ to $1.1 \pm 0.4$ & $0.7 \pm 0.1$ to $0.2 \pm 0.05$ & $1.8 \pm 0.9$ to $4.4 \pm 2.3$ & $0.1 \pm 0.04$ to $0.05 \pm 0.04$ & $458,180.5$ \\
\hline SX-106-3-4 & $19 \pm 6.0$ to $17 \pm 5.6$ & $62 \pm 26$ to $77 \pm 33$ & $16 \pm 6.0$ to $3.0 \pm 1.2$ & $0.9 \pm 0.4$ to $0.8 \pm 0.4$ & $0.6 \pm 0.2$ to $0.2 \pm 0.06$ & $1.0 \pm 0.5$ to $1.8 \pm 0.9$ & $0.2 \pm 0.07$ to $0.09 \pm 0.06$ & $362,142.5$ \\
\hline$S X-106-6-6$ & $51 \pm 5.1$ to $53 \pm 5.4$ & $23 \pm 3.3$ to $25 \pm 3.5$ & $18 \pm 2.0$ to $17 \pm 1.8$ & $1.9 \pm 0.3$ to $2.0 \pm 0.3$ & $5.1 \pm 0.6$ to $1.9 \pm 0.2$ & $1.0 \pm 0.5$ to $1.1 \pm 0.5$ & $8.9 \pm 1.0$ to $8.4 \pm 1.0$ & $265,104.5$ \\
\hline$S X-106-6-6 A$ & $51 \pm 5.6$ to $55 \pm 6.1$ & $19 \pm 3.3$ to $21 \pm 3.6$ & $23 \pm 3.0$ to $19 \pm 2.4$ & $2.7 \pm 0.9$ to $3.0 \pm 1.0$ & $3.0 \pm 0.4$ to $1.1 \pm 0.2$ & $1.4 \pm 0.6$ to $1.5 \pm 0.8$ & $4.0 \pm 0.6$ to $3.7 \pm 0.6$ & $265,104.5$ \\
\hline SX-106-3-7 & $48 \pm 8.5$ to $50 \pm 8.4$ & $19 \pm 8.7$ to $20 \pm 9.0$ & $27 \pm 7.2$ to $28 \pm 7.1$ & $0.5 \pm 0.1$ & $4.5 \pm 2.2$ to $1.7 \pm 0.8$ & $0.2 \pm 0.1$ to $0.3 \pm 0.1$ & $30 \pm 11$ to $28 \pm 11$ & $217,85.5$ \\
\hline SX-106-6-9 & $60 \pm 3.6$ to $62 \pm 3.5$ & $18 \pm 2.0$ & $17 \pm 1.1$ & $0.4 \pm 0.1$ & $4.0 \pm 0.6$ to $1.5 \pm 0.2$ & $0.3 \pm 0.1$ & $35 \pm 2.2$ to $34 \pm 2.2$ & $121,47.5$ \\
\hline SX-106-3-10 & $45 \pm 2.7$ to $46 \pm 2.7$ & $21 \pm 2.4$ to $22 \pm 2.5$ & $28 \pm 1.8$ to $29 \pm 1.7$ & $0.6 \pm 0.05$ & $4.6 \pm 0.7$ to $1.7 \pm 0.2$ & $0.3 \pm 0.1$ & $32 \pm 2.0$ to $30 \pm 1.9$ & $72,28.5$ \\
\hline SX-106-C & $21 \pm 4.8$ to $18 \pm 4.4$ & $61 \pm 20$ to $76 \pm 25$ & $15 \pm 4.2$ to $2.8 \pm 0.8$ & $0.9 \pm 0.4$ & $0.6 \pm 0.1$ to $0.2 \pm 0.05$ & $1.3 \pm 0.6$ to $2.7 \pm 1.4$ & $0.2 \pm 0.1$ to $0.07 \pm 0.05$ & 406,160 \\
\hline SX-106-NC & $51 \pm 4.7$ to $53 \pm 4.5$ & $20 \pm 4.0$ to $21 \pm 4.0$ & $24 \pm 2.7$ & $0.6 \pm 0.1$ & $4.4 \pm 0.9$ to $1.6 \pm 0.4$ & $0.3 \pm 0.1$ & $26 \pm 13$ to $25 \pm 12.5$ & 133,52 \\
\hline AX-101-9D-8 & $60 \pm 5.5$ to $64 \pm 5.4$ & $16 \pm 2.6$ to $18 \pm 2.7$ & $11 \pm 1.1$ & $2.4 \pm 0.2$ to $2.5 \pm 0.2$ & $9.2 \pm 2.1$ to $3.5 \pm 0.8$ & $0.7 \pm 0.3$ & $17 \pm 1.3$ to $16 \pm 1.3$ & $362,142.5$ \\
\hline & $\cdot$ & & & & & & & \\
\hline S-102-16-2 & $36 \pm 2.4$ to $37 \pm 2.4$ & $37 \pm 4.4$ & $26 \pm 1.8$ to $25 \pm 1.7$ & $0.4 \pm 0.05$ & $0.5 \pm 0.4$ to $0.2 \pm 0.2$ & $0.09 \pm 0.03$ & $33 \pm 4.3$ & $458,180.5$ \\
\hline$S-102-16-4 R$ & $32 \pm 2.8$ to $36 \pm 3.0$ & $31 \pm 4.1$ to $35 \pm 4.5$ & $35 \pm 3.4$ to $28 \pm 2.7$ & $0.2 \pm 0.07$ to $0.3 \pm 0.08$ & $1.1 \pm 0.8$ to $0.4 \pm 0.3$ & $0.2 \pm 0.1$ to $0.3 \pm 0.1$ & $7.5 \pm 0.7$ to $6.6 \pm 0.7$ & $362,142.5$ \\
\hline S-102-16-7 & $27 \pm 3.1$ to $28 \pm 3.1$ & $29 \pm 4.2$ to $30 \pm 4.4$ & $42 \pm 4.8$ to $41 \pm 4.6$ & $0.4 \pm 0.06$ & $1.3 \pm 0.4$ to $0.6 \pm 0.2$ & $\begin{array}{c}0.07 \pm 0.03 \text { to } \\
0.08 \pm 0.03\end{array}$ & $30 \pm 1.9$ to $29 \pm 1.9$ & $217,85.5$ \\
\hline$S-102-16-10$ & $43 \pm 3.8$ to $45 \pm 4.0$ & $29 \pm 4.3$ to $31 \pm 4.6$ & $26 \pm 2.2$ to $22 \pm 2.0$ & $0.7 \pm 0.08$ to $0.8 \pm 0.08$ & $1.0 \pm 0.3$ to $0.4 \pm 0.1$ & $0.6 \pm 0.2$ & $12 \pm 1.1$ & $72,28.5$ \\
\hline S-102 tank avg. & $33 \pm 3.0$ to $34 \pm 3.0$ & $32 \pm 4.3$ to $33 \pm 4.4$ & $33 \pm 3.3$ to $32 \pm 3.0$ & $0.4 \pm 0.06$ & $1.0 \pm 0.3$ to $0.4 \pm 0.1$ & $0.2 \pm 0.06$ & $26 \pm 13$ & 310,122 \\
\hline$S-111-6-2$ & $6.4 \pm 3.4$ to $6.1 \pm 3.3$ & $90 \pm 68$ to $91 \pm 69$ & $2.2 \pm 1.3$ to $0.9 \pm 0.6$ & $0.3 \pm 0.2$ & $0.1 \pm 0.1$ & $1.0 \pm 0.9$ to $1.2 \pm 1.0$ & $0.8 \pm 0.2$ to $0.7 \pm 0.2$ & $458,180.5$ \\
\hline$S-111-6-4$ & $48 \pm 24$ to $50 \pm 25$ & $36 \pm 22$ to $38 \pm 23$ & $14 \pm 5.7$ to $11 \pm 4.5$ & $0.6 \pm 0.2$ & $0.7 \pm 0.4$ to $0.4 \pm 0.2$ & $0.3 \pm 0.1$ & $7.0 \pm 2.1$ to $6.6 \pm 2.1$ & $362,142.5$ \\
\hline$S-111-6-6$ & $58 \pm 5.0$ to $59 \pm 5.1$ & $26 \pm 3.4$ to $27 \pm 3.5$ & $14 \pm 1.3$ to $12 \pm 1.2$ & $0.8 \pm 0.1$ & $1.0 \pm 0.3$ to $0.6 \pm 0.2$ & $0.5 \pm 0.2$ & $15 \pm 5$ & $265,104.5$ \\
\hline$S-111-6-8$ & $67 \pm 7.1$ to $68 \pm 7.1$ & $20 \pm 2.8$ & $12 \pm 1.4$ to $11 \pm 1.3$ & $0.6 \pm 0.08$ & $0.6 \pm 0.2$ to $0.4 \pm 0.1$ & $0.2 \pm 0.1$ & $20 \pm 2.9$ & $169,66.5$ \\
\hline$S-111-6-10$ & $73 \pm 5.7$ to $74 \pm 5.6$ & $16 \pm 2.0$ & $9.3 \pm 0.8$ to $8.8 \pm 0.7$ & $0.3 \pm 0.04$ & $1.4 \pm 0.4$ to $0.8 \pm 0.2$ & $0.2 \pm 0.1$ & $23 \pm 3.2$ to $22 \pm 3.2$ & $72,28.5$ \\
\hline $\mathrm{S}-111-\mathrm{C}$ & $6.4 \pm 3.4$ to $6.1 \pm 3.3$ & $90 \pm 68$ to $91 \pm 69$ & $2.2 \pm 1.3$ to $0.9 \pm 0.6$ & $0.3 \pm 0.2$ & $0.1 \pm 0.1$ & $1.0 \pm 0.9$ to $1.2 \pm 1.0$ & $0.8 \pm 0.3$ to $0.7 \pm 0.2$ & 510,201 \\
\hline S-111-NC & $66 \pm 10$ to $67 \pm 11$ & $21 \pm 5.6$ to $22 \pm 5.7$ & $11 \pm 1.8$ to $10 \pm 1.6$ & $0.5 \pm 0.08$ & $1.0 \pm 0.3$ to $0.5 \pm 0.2$ & $0.2 \pm 0.07$ & $15 \pm 7.5$ to $14 \pm 7.0$ & 184,72 \\
\hline
\end{tabular}


Table 5.2. (contd)

\begin{tabular}{|c|c|c|c|c|c|c|c|c|}
\hline \multirow{2}{*}{$\begin{array}{l}\text { Tank and Sample } \\
\text { (or layer) }\end{array}$} & \multicolumn{6}{|c|}{ Mole Percent of Constituent in Bubbles (water vapor excluded) } & \multirow{2}{*}{$\begin{array}{c}\text { In Situ Gas Volume } \\
\text { (percent) }\end{array}$} & \multirow{2}{*}{$\begin{array}{c}\text { Elevation } \\
\text { (cm, in.) }\end{array}$} \\
\hline & $\mathrm{H}_{2}$ & $\mathrm{~N}_{2}$ & $\mathrm{~N}_{2} \mathrm{O}$ & $\mathrm{CH}_{4}$ & $\mathrm{NH}_{3}$ & Other & & \\
\hline U-109-8-2 & $20 \pm 2.9$ to $21 \pm 3.0$ & $42 \pm 7.6$ to $44 \pm 7.9$ & $36 \pm 6.4$ to $34 \pm 5.9$ & $0.5 \pm 0.1$ to $0.6 \pm 0.1$ & $0.6 \pm 0.2$ to $0.3 \pm 0.1$ & $0.4 \pm 0.2$ & $20 \pm 2.5$ to $19 \pm 2.5$ & $362,142.5$ \\
\hline U-109-8-4 & $24 \pm 3.1$ to $25 \pm 3.2$ & $38 \pm 6.3$ to $40 \pm 6.5$ & $35 \pm 4.0$ to $33 \pm 3.8$ & $0.6 \pm 0.08$ & $1.5 \pm 0.6$ to $0.8 \pm 0.3$ & $0.3 \pm 0.1$ & $23 \pm 2.2$ to $22 \pm 2.2$ & $265,104.5$ \\
\hline U-109-8-6 & $28 \pm 4.9$ to $30 \pm 5.2$ & $43 \pm 11$ to $44 \pm 11$ & $26 \pm 5.1$ to $24 \pm 4.6$ & $1.1 \pm 0.2$ to $1.2 \pm 0.2$ & $0.7 \pm 0.2$ to $0.4 \pm 0.1$ & $0.7 \pm 0.3$ & $15 \pm 1.0$ to $14 \pm 1.0$ & $169,66.5$ \\
\hline U-109-8-8 & $27 \pm 2.4$ to $28 \pm 2.4$ & $52 \pm 7.5$ to $53 \pm 7.6$ & $19 \pm 1.6$ to $18 \pm 1.6$ & $0.8 \pm 0.09$ & $0.7 \pm 0.2$ to $0.4 \pm 0.1$ & $0.4 \pm 0.08$ & $30 \pm 1.8$ to $29 \pm 1.8$ & $72,28.5$ \\
\hline $\mathrm{U}-109-\mathrm{NC}$ & $25 \pm 3.0$ to $26 \pm 3.1$ & $46 \pm 7.7$ to $47 \pm 8.0$ & $27 \pm 3.6$ to $25 \pm 3.4$ & $0.7 \pm 0.1$ & $0.9 \pm 0.3$ to $0.4 \pm 0.2$ & $0.4 \pm 0.2$ & $22 \pm 11$ to $21 \pm 10$ & 202,80 \\
\hline$S Y-101-23 A-1$ & $22 \pm 3.2$ to $23 \pm 3.3$ & $47 \pm 9.4$ to $51 \pm 9.9$ & $23 \pm 3.4$ & $0.8 \pm 0.1$ & $6.1 \pm 1.6$ to $2.0 \pm 0.5$ & $0.7 \pm 0.2$ to $0.8 \pm 0.2$ & $19.5 \pm 1.5$ to $18.2 \pm 1.5$ & 1022,402 \\
\hline SY-101-23A-2 & $34 \pm 4.8$ to $39 \pm 5.1$ & $27 \pm 4.4$ to $31 \pm 4.8$ & $21 \pm 3.0$ to $24 \pm 3.0$ & $0.6 \pm 0.1$ & $17 \pm 3.9$ to $5.0 \pm 1.2$ & $0.8 \pm 0.3$ to $1.0 \pm 0.4$ & $39.9 \pm 3.0$ to $34.5 \pm 2.9$ & $974,383.5$ \\
\hline$S Y-101-22 A-3$ & $33 \pm 4.4$ to $37 \pm 4.6$ & $30 \pm 4.9$ to $33 \pm 5.2$ & $25 \pm 3.3$ to $26 \pm 3.3$ & $0.6 \pm 0.2$ to $0.7 \pm 0.2$ & $11 \pm 2.7$ to $3.5 \pm 0.8$ & $0.4 \pm 0.2$ to $0.5 \pm 0.2$ & $32.8 \pm 2.7$ to $29.5 \pm 2.7$ & $959,377.5$ \\
\hline SY-101-23A-3 & $37 \pm 4.4$ to $46 \pm 4.3$ & $21 \pm 3.2$ to $27 \pm 3.4$ & $17 \pm 2.0$ to $20 \pm 1.8$ & $0.5 \pm 0.07$ to $0.6 \pm 0.07$ & $24 \pm 6.1$ to $5.8 \pm 1.4$ & $0.5 \pm 0.1$ to $0.6 \pm 0.1$ & $64.8 \pm 3.9$ to $52.0 \pm 3.9$ & $926,364.5$ \\
\hline$S Y-101-22 A-4$ & $34 \pm 3.9$ to $45 \pm 3.5$ & $20 \pm 3.1$ to $27 \pm 3.5$ & $16 \pm 1.8$ to $20 \pm 1.6$ & $0.5 \pm 0.07$ to $0.7 \pm 0.08$ & $28 \pm 7.3$ to $6.4 \pm 1.6$ & $\begin{array}{c}0.4 \pm 0.06 \text { to } \\
0.5 \pm 0.06\end{array}$ & $72 \pm 7$ to $56.7 \pm 3.7$ & $911,358.5$ \\
\hline$S Y-101-4 A-5$ & $36 \pm 8.5$ to $42 \pm 9.8$ & $27 \pm 7.7$ to $32 \pm 9.0$ & $24 \pm 6.1$ to $20 \pm 5.2$ & $1.0 \pm 0.3$ to $1.2 \pm 0.4$ & $11 \pm 3.0$ to $3.5 \pm 0.9$ & $1.0 \pm 0.5$ to $1.2 \pm 0.6$ & $6.2 \pm 1.8$ to $5.1 \pm 1.7$ & $845,332.5$ \\
\hline SY-101-23A-8 & $23 \pm 6.4$ to $27 \pm 7.4$ & $45 \pm 14$ to $52 \pm 16$ & $22 \pm 6.4$ to $15 \pm 4.5$ & $1.3 \pm 0.5$ to $1.5 \pm 0.6$ & $7.3 \pm 2.1$ to $2.3 \pm 0.7$ & $1.3 \pm 0.8$ to $1.6 \pm 0.9$ & $3.6 \pm 1.2$ to $3.0 \pm 1.2$ & $685,269.5$ \\
\hline$S Y-101-22 A-10$ & $28 \pm 9.7$ to $34 \pm 12$ & $34 \pm 13$ to $42 \pm 16$ & $26 \pm 8.5$ to $18 \pm 6.1$ & $1.4 \pm 0.4$ to $1.6 \pm 0.6$ & $9.9 \pm 3.3$ to $3.2 \pm 1.0$ & $1.3 \pm 0.6$ to $1.7 \pm 0.8$ & $3.3 \pm 1.1$ to $2.6 \pm 1.0$ & $621,244.5$ \\
\hline$S Y-101-23 A-13$ & $28 \pm 9.6$ to $33 \pm 12$ & $36 \pm 13$ to $44 \pm 17$ & $25 \pm 8.8$ to $17 \pm 5.8$ & $1.1 \pm 0.4$ to $1.4 \pm 0.5$ & $8.3 \pm 2.9$ to $2.7 \pm 0.9$ & $1.4 \pm 0.8$ to $1.8 \pm 1.0$ & $2.9 \pm 0.9$ to $2.2 \pm 0.9$ & $443,174.5$ \\
\hline$S Y-101-22 A-17$ & $29 \pm 7.3$ to $35 \pm 8.9$ & $34 \pm 10$ to $42 \pm 13$ & $28 \pm 7.2$ to $18 \pm 4.6$ & $1.4 \pm 0.4$ to $1.8 \pm 0.5$ & $6.0 \pm 1.7$ to $1.9 \pm 0.6$ & $1.2 \pm 0.6$ to $1.6 \pm 0.7$ & $2.6 \pm 0.7$ to $2.0 \pm 0.7$ & $316,124.5$ \\
\hline SY-101-23A-21 & $29 \pm 8.6$ to $33 \pm 10$ & $38 \pm 13$ to $47 \pm 16$ & $24 \pm 7.1$ to $14 \pm 4.3$ & $1.5 \pm 0.5$ to $1.8 \pm 0.6$ & $5.6 \pm 1.7$ to $1.8 \pm 0.6$ & $1.4 \pm 0.6$ to $1.7 \pm 0.8$ & $2.3 \pm 0.7$ to $1.8 \pm 0.7$ & $75,29.5$ \\
\hline SY-101-22A-23 & $31 \pm 8.6$ to $37 \pm 10$ & $35 \pm 11$ to $42 \pm 14$ & $27 \pm 7.9$ to $17 \pm 4.8$ & $1.3 \pm 0.4$ to $1.5 \pm 0.5$ & $4.5 \pm 1.4$ to $1.4 \pm 0.4$ & $1.2 \pm 0.6$ to $1.5 \pm 0.8$ & $2.6 \pm 0.8$ to $2.0 \pm 0.8$ & 28,11 \\
\hline $\begin{array}{l}\text { SY-101-23A } \\
\text { crust }\end{array}$ & $34 \pm 4.4$ to $40 \pm 4.5$ & $27 \pm 4.5$ to $32 \pm 4.9$ & $19 \pm 2.5$ to $22 \pm 2.4$ & $0.6 \pm 0.08$ to $0.7 \pm 0.09$ & $19 \pm 4.7$ to $4.9 \pm 1.2$ & $0.6 \pm 0.2$ to $0.7 \pm 0.2$ & $\begin{array}{r}40 \pm 20 \text { to } 34 \pm 17 \\
-\end{array}$ & 953,375 \\
\hline SY-101-23A liq. & $26 \pm 8.1$ to $31 \pm 9.5$ & $40 \pm 14$ to $48 \pm 16$ & $24 \pm 7.4$ to $16 \pm 4.9$ & $1.3 \pm 0.5$ to $1.6 \pm 0.6$ & $7.1 \pm 2.2$ to $2.3 \pm 0.7$ & $1.4 \pm 0.7$ to $1.7 \pm 0.9$ & $2.9 \pm 1.4$ to $2.3 \pm 1.4$ & 445,175 \\
\hline $\begin{array}{l}S Y-101-22 A \\
\text { crust }\end{array}$ & $32 \pm 3.9$ to $39 \pm 3.9$ & $27 \pm 4.5$ to $33 \pm 4.9$ & $19 \pm 2.4$ to $22 \pm 2.4$ & $0.6 \pm 0.1$ to $0.7 \pm 0.1$ & $21 \pm 5.1$ to $4.8 \pm 1.3$ & $0.4 \pm 0.1$ to $0.5 \pm 0.1$ & $52 \pm 26$ to $44 \pm 22$ & 942,371 \\
\hline SY-101-22A liq. & $31 \pm 8.5$ to $37 \pm 10$ & $32 \pm 11$ to $39 \pm 13$ & $26 \pm 7.5$ to $18 \pm 5.2$ & $1.3 \pm 0.4$ to $1.6 \pm 0.5$ & $7.8 \pm 2.3$ to $2.5 \pm 0.8$ & $1.2 \pm 0.6$ to $1.5 \pm 0.7$ & $3.1 \pm 1.5$ to $2.5 \pm 1.5$ & 451,177 \\
\hline
\end{tabular}




\subsection{References}

\subsection{Cited References}

AIChE. 1984. Perry's Chemical Engineers' Handbook, 6th Ed. Edited by RH Perry, DW Green, and JO Maloney, American Institute of Chemical Engineers. McGraw-Hill Book Company, New York.

Baldwin JH. 1996. Final Report for Tank 241-U-109, Rotary and Push Mode Cores 123, 124, and 128. WHC-SD-WM-DP-181 Rev. 1, Westinghouse Hanford Company, Richland, Washington.

Baldwin JH, LC Amato, and TT Tran. 1995. Tank Characterization Report for Double-Shell Tank 241-AW-101. WHC-SD-WM-ER-470 Rev. 0, Westinghouse Hanford Company, Richland, Washington.

Benar, CJ. 1996b. Tank 241-AW-101, Cores 132 and 139 Analytical Results for the Final Report. WHC-SD-WM-DP-192, Rev 0, Rust Federal Services of Hanford Inc., Richland, Washington.

Brevick CH, LA Gaddis, and WW Pickett. 1994. Historical Tank Content Estimate for the Southwest Quadrant of the Hanford 200 West Area. WHC-SD-WM-ER-352 Rev. 0, Westinghouse Hanford Company, Richland, Washington.

Brevick CH, LA Gaddis, and WW Pickett. 1997. Historical Tank Content Estimate for the Northeast Quadrant of the Hanford 200E Area. WHC-SD-WM-ER-349, Rev. 1b, Westinghouse Hanford Company, Richland, Washington.

Caley SM, LA Mahoney, and PA Gauglitz. 1996. Summary of Tank Information Relating Salt Well Pumping to Flammable Gas Safety Issues. PNNL-11335, Pacific Northwest National Laboratory, Richland, Washington.

Cannon NS and RC Knight. November 1995. Retained Gas Sampler System Acceptance Test Report. WHC-SD-WM-ATR-137 Rev 0, Westinghouse Hanford Company, Richland, Washington.

Cannon NS. 1996. Retained Gas Sampler System Acceptance Test Report. WHC-SD-WMATR-137 Rev. 1, Westinghouse Hanford Company, Richland, Washington.

Cannon NS. March 1997. Retained Gas Sampler Interface Volume. HNF-SD-WM-CN-092, SGN Eurisys Services Corporation, Richland, Washington.

Consort SD, KL Ewer, JW Funk, RG Hale, GA Lisle, and CV Salois. 1996. Historical Tank Content Estimate for the Northeast Quadrant of the Hanford 200 East Area. WHC-SD-WM-ER349 Rev. 1, Westinghouse Hanford Company, Richland, Washington.

CRC. 1975. Handbook of Chemistry and Physics, 56th Edition. CRC Press, Cleveland, Ohio.

Esch R. 1998. Tank 241-AX-101, Cores 226 and 228, Analytical Results for the Final Report. HNF-SD-WM-DP-300, Rev. 0, Waste Management of Hanford, Inc., Richland, Washington.

Field JG. 1997. Tank Characterization Report for Single-Shell Tank 241-A-101. HNF-SD-WMER-673 Rev. 0, Lockheed Martin Hanford Corporation, Richland, Washington. 
Field JG. 1998. Tank Characterization Report for Single-Shell Tank 241-SX-106. HNF-SDWM-ER-645 Rev. 0, Lockheed Martin Hanford Corporation, Richland, Washington.

Field JG. 1999a. Tank Characterization Report for Single-Shell Tank 241-S-106. HNF-SDWM-ER-714 Rev. 1, Lockheed Martin Hanford Corporation, Richland, Washington.

Field JG. 1999b. Tank Characterization Report for Double-Shell Tank 241-AN-103. HNF-SDWM-ER-702 Rev. 0D, Lockheed Martin Hanford Corporation, Richland, Washington.

Fritts LL. March 1998. Final Report for Tank 241-S-102, Push Mode Cores 125 and 130. WHC-SD-WM-DP-179 Rev. 1B, Waste Management of Hanford, Inc., Richland, Washington.

Gauglitz PA, SD Rassat, PR Bredt, JH Konynenbelt, SM Tingey, and DP Mendoza. 1996. Mechanisms of Gas Bubble Retention and Release: Results for Hanford Waste Tanks 241-S-102, 241-SY-103, and Single-Shell Tank Simulants. PNNL-11298, Pacific Northwest National Laboratory, Richland, Washington.

Gauglitz PA and JT Aikin. 1997. Waste Behavior Düring Horizontal Extrusions: Effect of Waste Strength for Bentonite and Kaolin/Ludox Simulants and Strength Estimates for Wastes from Hanford Waste Tanks 241-SY-103, AW-101, AN-103, and S-102. PNNL-11706, Pacific Northwest National Laboratory, Richland, Washington.

Hermann C, I Dewes, and A Schumpe. 1995. "The estimation of gas solubilities in salt solutions." Chem. Eng. Sci., 50:1673-4.

Hodgson KM, RP Anantatmula, SA Barker, KD Fowler, JD Hopkins, JA Lechelt, DA Reynolds, DC Hedengren, RE Stout, and RT Winward. 1998. Evaluation of Hanford Tanks for Trapped Gas. WHC-SD-WM-ER-526 Rev. 1C, Westinghouse Hanford Company, Richland, Washington.

Holman JP. 1978. Experimental Methods for Engineers. McGraw-Hill Book Company, New York.

Homi CS. August 1995. Tank 241-S-106 Tank Characterization Plan. WHC-SD-WM-TP-389 Rev. 0, Westinghouse Hanford Company, Richland, Washington.

Hu TA, LW Shelton Jr, and TL Welsh. 1997. Tank Characterization Report for Double-Shell Tank 24I-AN-104. HNF-SD-WM-ER-690 Rev. 0, Lockheed Martin Hanford Corporation, Richland, Washington.

Jo J. 1997. Tank Characterization Report for Double-Shell Tank 241-AN-105. HNF-SD-WMER-678 Rev. 0, Lockheed Martin Hanford Corporation, Richland, Washington.

Jo J. 1998. Tank Characterization Report for Single-Shell Tank 241-BY-109. HNF-SD-WMER-648 Rev. 1, Lockheed Martin Hanford Corporation, Richland, Washington.

Mahoney LA, ZI Antoniak, and JM Bates. 1997. Composition and Quantities of Retained Gas Measured in Hanford Waste Tanks 241-U-103, S-106, BY-101, and BY-109. PNNL-11777, Pacific Northwest National Laboratory, Richland, WA 99352.

Mahoney LA and DS Trent. 1995. Correlation Models for Waste Tank Sludges and Slurries. PNL-10695, Pacific Northwest Laboratory, Richland, Washington. 
McCain DJ. 1999. Results of Vapor Space Monitoring of Flammable Gas Watch List Tanks. HNF-SD-WM-TI-797 Rev. 4, Lockheed Martin Hanford Corp., Richland, Washington.

Meyer PA, ME Brewster, SA Bryan, G Chen, LR Pederson, CW Stewart, and G Terrones. 1997. Gas Retention and Release Behavior in Hanford Double Shell Waste Tanks. PNNL-11536 Rev. 1, Pacific Northwest National Laboratory, Richland, Washington.

Norton JD and LR Pederson. 1994. Ammonia in Simulated Hanford Double-Shell Tank Wastes: Solubility and Effects on Surface Tension. PNL-10173, Pacific Northwest Laboratory, Richland, Washington.

Norton JD and LR Pederson. 1995. Solubilities of Gases in Simulated Tank 241-SY-10I Wastes. PNL-10785, Pacific Northwest Laboratory, Richland, Washington.

Ogden DM. March 1996. Thermal Hydraulic Behavior of Tank A-101. WHC-SD-WM-ER-555, Rev. 0, Westinghouse Hanford Company, Richland, Washington.

Person JC. 1998. Gas Generation in Tank 241-AN-105 Waste With and Without Oxygen Reactions. HNF-2038 Rev. 0, Numatec Hanford Corporation, Richland, WA.

Prausnitz JM, RN Lichtenthaler, and EG de Azevedo. 1986. Molecular Thermodynamics of Fluid-Phase Equilibria, 2nd Ed. Prentice-Hall, Inc., Englewood Cliffs, New Jersey.

Rassat SD, PA Gauglitz, PR Bredt, LA Mahoney, SV Forbes, and SM Tingey. 1997. Mechanisms of Gas Bubble Retention and Release: Experimental Results for Hanford Waste Tanks 241-AW-101 and 241-AN-103. PNNL-11642, Pacific Northwest National Laboratory, Richland, Washington.

Rassat SD, SM Caley, PR Bredt, PA Gauglitz, DE Rinehart, and SV Forbes. 1998. Mechanisms of Gas Retention and Release: Experimental Results for Hanford Single-Shell Waste Tanks 241$A-101,241-S-106$, and 241-U-103. PNNL-11981, Pacific Northwest National Laboratory, Richland, Washington.

Reid RC, JM Prausnitz, and BE Poling. 1987. The Properties of Gases and Liquids, 4th Ed. McGraw-Hill, New York.

Remund KM, CM Anderson, and BC Simpson. 1995. Hanford Single-Shell Tank Grouping Study. PNL-10749, Pacific Northwest National Laboratory, Richland, Washington.

Reynolds DA. 1993. Tank 101-SY Window E Core Sample: Interpretation of Results. WHC-EP-0628, Westinghouse Hanford Company, Richland, Washington.

Sasaki LM. 1998. Tank Characterization Report for Single-Shell Tank 241-U-103. HNF-SDWM-ER-712 Rev. 0, Lockheed Martin Hanford Corporation, Richland, Washington.

Schienbein LA, SA Bryan, DL Lessor, PA Meyer and CW Stewart. 1999. Gas Retention and Release Behavior in Hanford Double-Shell Tanks. PNNL-11536 Rev. 2. Pacific Northwest National Laboratory, Richland, Washington.

Shekarriz A, DR Rector, LA Mahoney, MA Chieda, JM Bates, RE Bauer, NS Cannon, BE Hey, CG Linschooten, FJ Reitz, and ER Siciliano. 1997. Composition and Quantities of Retained Gas Measured in Hanford Waste Tanks 241-AW-101, A-101, AN-105, AN-104, and AN-103. PNNL-11450 Rev. 1, Pacific Northwest National Laboratory, Richland, Washington. 
Siciliano ER. 1998. Drill String Gas Data. HNF-1754, Fluor Daniel Northwest, Inc., Richland, Washington.

Steen FH. 1996. Tank 241-S-111, Cores 149 and 150 Analytical Results for the Final Report. WHC-SD-WM-DP-195 Rev. 1, Rust Federal Services of Hanford, Inc., Richland, Washington.

Stewart CW, JM Alzheimer, ME Brewster, G Chen, RE Mendoza, HC Reid, CL Shepard, and G Terrones. 1996a. In Situ Rheology and Gas Volume in Hanford Double-Shell Waste Tanks. PNL-11296, Pacific Northwest Laboratory, Richland, Washington.

Stewart CW, ME Brewster, PA Gauglitz, LA Mahoney, PA Meyer, KP Recknagle, and HC Reid. 1996b. Gas Retention and Release Behavior in Hanford Single-Shell Waste Tanks.

PNNL-11391, Pacific Northwest National Laboratory, Richland, Washington.

Stewart CW, JM Alzheimer, G Chen, and PA Meyer. 1998. In Situ Void Fraction and Gas Volume in Hanford Tank 241-SY-101 as Measured with the Void Fraction Instrument. PNNL12033, Pacific Northwest National Laboratory, Richland, Washington.

Stewart CW, SD Rassat, and JH Sukamto. 1999. Effects of Back-Dilution on Bouyancy of the Tank 241-SY-101 Crust Layer. PNNL-13040, Pacific Northwest National Laboratory, Richland, Washington.

Tran TT. 1993. Thermocouple Status: Single Shell and Double Shell Waste Tanks. WHC-SDWM-TI-553 Rev. 0, Westinghouse Hanford Company, Richland, Washington.

Webb BJ. 1994. Summary Report on the Design of the Retained Gas Sampler System. WHC-SO-WM-ER-387, Westinghouse Hanford Company, Richland, Washington. Temperatures from $273 \mathrm{~K}$ to $363 \mathrm{~K}$. AIChE J., Vol. 42, No. 1, pp. 298-300.

Whitney PD, PA Meyer, NE Wilkins, F Gao, and AG Wood. 1996. Flammable Gas Data Evaluation Progress Report. PNNL-11373, Pacific Northwest National Laboratory, Richland, Washington.

Whitney PD, G Chen, PA Gauglitz, PA Meyer, and NE Miller. 1997. Estimating Retained Gas Volumes in the Hanford Waste Tanks Using Waste Level Measurements. PNNL-11693, Pacific Northwest National Laboratory, Richland, Washington.

Wilkins NE and RE Bauer. 1996. Test Evaluation of Industrial Hygiene Hand Held Combustible Gas Monitor. WHC-SD-WM-TRP-256 Rev. 0, Westinghouse Hanford Company, Richland, Washington.

\subsection{Bibliography}

Bryan SA and LR Pederson. 1996. Thermal and Combined Thermal and Radiolytic Reactions Involving Nitrous Oxide, Hydrogen, Nitrogen, and Ammonia in Contact with Tank 241-SY-101 Simulated Waste. PNNL-10748, Pacific Northwest National Laboratory, Richland, Washington.

Gauglitz, PA, LA Mahoney, DP Mendoza, and MC Miller. 1994. Mechanisms of Gas Bubble Retention. PNL-10120, Pacific Northwest National Laboratory, Richland, Washington. 
Hardy DB. 1998. Final Report for Tank 241-S-102, Push Mode Core 125 and 131. WHC-SDWM-DP-179 Rev. 1B, Waste Management of Hanford Inc., Richland, Washington.

Hey BE. January 1996. Test Plan for the Authentic Application of the Retained Gas Sampler System. WHC-SD-WM-TP-423 Rev. 0, Westinghouse Hanford Company, Richland, Washington.

Hey BE. January 1996. Retained Gas Sampler System Operation. LT-160-101 BU, Westinghouse Hanford Company, Richland, Washington.

Peurrung LM, LA Mahoney, CW Stewart, PA Gauglitz, LR Pederson, SA Bryan, and CL Shepard. 1998. Flammable Gas Issues in Double-Contained Receiver Tanks. PNNL-11836 Rev. 2, Pacific Northwest National Laboratory, Richland, Washington.

Schumpe A. 1993. The Estimation of Gas Solubilities in Salt Solutions. Chem. Eng. Sci., Vol. 48, p. 153.

Shekarriz A. 1994. Retained Gas Sampler Flow Visualization Guide. PNL-10138, Pacific Northwest Laboratory, Richland, Washington.

Shekarriz A and JD Norton. 1995. Retained Gas Sampler System Analysis. PNLFGP:091595, Pacific Northwest National Laboratory, Richland, Washington.

Weisenberger S and A Schumpe. 1996. Estimation of Gas Solubilities in Salt Solutions at Temperatures from $273 \mathrm{~K}$ to $363 \mathrm{~K}$. AIChE J., 42(1):298-300. 


\section{Appendix A \\ Field Data for RGS Tanks}




\section{Appendix A \\ Field Data for RGS Tanks}

The data in this appendix were derived from field records. Summaries of the information obtained from these field records (and believed relevant to RGS) are given for individual tanks in the following tables. The information is organized by year and grouped by tank in the order in which they were sampled.

In the tables, the column heading, "Time and Date of Sampling," indicates when the sampler ball valve was closed (by pulling the pintle rod), thus acquiring the sample. Usually, the sampler would be removed from the tank within 15 to 30 minutes after the ball valve was closed, although in some cases, the sampler was left overnight (or longer) in the tank and retrieved later. The column entitled, "Max Force to Unseat Sampler," indicates the time when sampler removal from the tank began.

Purge gas date and times were usually provided on the Industrial Hygiene Direct Reading Instrument Survey; but these data were not the responsibility of Industrial Hygiene, and there are probably times when drill string purges were performed that were not recorded on the DRIs. 
- 


\section{FY 1996 Field Data for RGS Tanks AW-101, AN-105, A-101, AN-104, and $\mathrm{AN}-103$}

(in chronological sampling order) 
-

• 
Table A.1. FY 1996 RGS Summary Data Sheet

Tank AW-101 Riser 24A

\begin{tabular}{|c|c|c|c|c|c|c|c|c|}
\hline $\begin{array}{c}\text { Sampler Serial } \\
\text { Number } \\
* * * * * * * * * * * * * * \\
\text { Cask Serial } \\
\text { Number } \\
* * * * * * * * * * * * * * \\
\text { Sample Number }\end{array}$ & $\begin{array}{l}\text { Time and Date } \\
\text { of Sampling } \\
\text { (just after pintle } \\
\text { rod has been } \\
\text { pulled) }\end{array}$ & $\begin{array}{l}\text { Max Force to } \\
\text { Unseat Sampler } \\
\text { (initiation of } \\
\text { sampler removal } \\
\text { from tank) } \\
\text { Time } \\
\text { Date }\end{array}$ & $\begin{array}{c}\text { Purge Gas } \\
\text { (a) } \\
\text { DSP = Drill } \\
\text { String Purge } \\
* * * * * * * * * * * \\
\text { Time } \\
\text { Date }\end{array}$ & $\begin{array}{c}\text { Amount of } \\
\text { Hydrostatic } \\
\text { Head Fluid } \\
\text { Added }\end{array}$ & $\begin{array}{l}\text { Radiation } \\
\text { Dose Rate } \\
\text { Reading } \\
\text { Through Drill } \\
\text { String }\end{array}$ & $\begin{array}{l}\text { Strip Chart } \\
\text { Downforce } \\
\text { Max Load } \\
\text { Avg Load } \\
\text { Duration }\end{array}$ & $\begin{array}{l}\text { Procedure } \\
\text { Version and } \\
\text { Procedural } \\
\text { Exceptions }\end{array}$ & $\begin{array}{c}\text { X-Ray } \\
\text { Observation } \\
\text { Notes }\end{array}$ \\
\hline $\begin{array}{l}\text { RGS-009 seg } 6 \\
\text { SN 19-G } \\
96-067\end{array}$ & $\begin{array}{l}2-16-96 \\
12: 45 \mathrm{hr}\end{array}$ & $\begin{array}{c}65 \mathrm{lb} \\
1300 \mathrm{hr}\end{array}$ & & $1300 \mathrm{~mL}$ & $1.8 \mathrm{R}$ & . & $\begin{array}{c}\text { TO-80-503 } \\
\text { Rev. C-2 }\end{array}$ & Valve stuck \\
\hline $\begin{array}{l}\text { RGS-014 seg } 8 \\
\text { SN } 73 \\
96-069\end{array}$ & $\begin{array}{l}3-15-96 \\
14: 00 \mathrm{hr} \\
\end{array}$ & $\begin{array}{c}65 \mathrm{lb} \\
14: 22 \mathrm{hr}\end{array}$ & & $1300 \mathrm{~mL}$ & $2.0 \mathrm{R}$ & & $\begin{array}{l}\text { TO-80-503. } \\
\text { Rev. C-3 }\end{array}$ & 19 in. of sample \\
\hline $\begin{array}{l}\text { RGS-003 seg } 17 \\
\text { SN-58 } \\
96-078\end{array}$ & $\begin{array}{l}3-21-96 \\
04: 30 \mathrm{hr}\end{array}$ & $\begin{array}{c}71 \mathrm{lb} \\
04: 57 \mathrm{hr} \\
.\end{array}$ & & $1300 \mathrm{~mL}$ & $2 R$ & & $\begin{array}{l}\text { T0-80-503 } \\
\text { Rev. C-3 }\end{array}$ & 19 in. of sample \\
\hline $\begin{array}{l}\text { RGS-012 seg } 19 \\
1004 \mathrm{C} \\
96-080\end{array}$ & $\begin{array}{l}3-22-96 \\
09: 52 \mathrm{hr}\end{array}$ & $\begin{array}{l}68.8 \mathrm{lb} \\
10: 14 \mathrm{hr}\end{array}$ & & $1300 \mathrm{~mL}$ & $2 \mathrm{R}$ & . & $\begin{array}{l}\text { T0-80-503 } \\
\text { Rev. C-3 }\end{array}$ & 19 in. of sample \\
\hline $\begin{array}{l}\text { RGS-011 seg } 21 \\
76 \\
96-082\end{array}$ & $\begin{array}{l}3-22-96 \\
12: 45 \mathrm{hr}\end{array}$ & $\begin{array}{l}65.0 \mathrm{lb} \\
12: 59 \mathrm{hr}\end{array}$ & & $1300 \mathrm{~mL}$ & $1.9 \mathrm{R}$ & & $\begin{array}{l}\text { T0-80-503 } \\
\text { Rev. C-3 }\end{array}$ & 19 in. of sample \\
\hline
\end{tabular}


Table A.2. FY 1996 RGS Summary Data Sheet

Tank AW-101 Riser 24-B

\begin{tabular}{|c|c|c|c|c|c|c|c|c|}
\hline $\begin{array}{c}\text { Sampler Serial } \\
\text { Number } \\
* * * * * * * * * * * * * * \\
\text { Cask Serial } \\
\text { Number } \\
* * * * * * * * * * * * * * \\
\text { Sample Number }\end{array}$ & $\begin{array}{c}\text { Time and Date } \\
\text { of Sampling } \\
\text { (just after } \\
\text { pintle rod } \\
\text { has been } \\
\text { pulled) }\end{array}$ & $\begin{array}{l}\text { Max Force to } \\
\text { Unseat Sampler } \\
\text { (initiation of } \\
\text { sampler removal } \\
\text { from tank) } \\
\text { Time } \\
\text { Date }\end{array}$ & $\begin{array}{c}\text { Purge Gas }{ }^{j(a)} \\
* * * * * * * * * * * \\
\text { DSP = Drill } \\
\text { String Purge } \\
* * * * * * * * * * * \\
\text { Time } \\
\text { Date }\end{array}$ & $\begin{array}{c}\text { Amount of } \\
\text { Hydrostatic } \\
\text { Head Fluid } \\
\text { Added }\end{array}$ & $\begin{array}{l} \\
\text { Radiation Dose } \\
\text { Rate Reading } \\
\text { Through Drill } \\
\text { String }\end{array}$ & $\begin{array}{l}\text { Strip Chart } \\
\text { Downforce } \\
\text { Max Load } \\
\text { Avg Load } \\
\text { Duration }\end{array}$ & $\begin{array}{l}\text { Procedure } \\
\text { Version and } \\
\text { Procedural } \\
\text { Exceptions }\end{array}$ & $\begin{array}{c}\text { X-Ray } \\
\text { Observation } \\
\text { Notes }\end{array}$ \\
\hline $\begin{array}{l}\text { RGS-013 seg } 18 \\
1014 C \\
96-152\end{array}$ & $\begin{array}{l}5-22-96 \\
10: 12 \cdot \mathrm{hr}\end{array}$ & $\begin{array}{c}95 \mathrm{lb} \\
5-22-96 \\
10: 29 \mathrm{br}\end{array}$ & & $1500 \mathrm{~mL}$ & $1.8 \mathrm{R}$ & & $\begin{array}{l}\text { T0-80-503 } \\
\text { Rev. C-4 }\end{array}$ & $\begin{array}{l}\text { Drill string } \\
\text { pulled- } \\
\text { Newly installed } \\
19 \text { in. of sample }\end{array}$ \\
\hline $\begin{array}{l}\text { RGS-004 seg } 20 \\
\text { SN-76 } \\
96-154\end{array}$ & $\begin{array}{l}5-22-96 \\
13: 02 \mathrm{hr}\end{array}$ & $\begin{array}{c}114 \mathrm{lb} \\
5-23-96 \\
10: 11 \mathrm{hr}\end{array}$ & & $1500 \mathrm{~mL}$ & $1.8 \mathrm{R}$ & & $\begin{array}{l}\text { T0-80-503 } \\
\text { Rev. C-4 }\end{array}$ & $\begin{array}{l}19 \text { in. of sample } \\
\text { During } \\
\text { processing, valve } \\
\text { found to not be } \\
\text { completely } \\
\text { closed. } \\
\text { Discarded. }\end{array}$ \\
\hline $\begin{array}{l}\text { RGS-002 seg } 22 \\
\text { C2008 } \\
96-156\end{array}$ & $\begin{array}{l}5-23-96 \\
12: 49 \text { hr }\end{array}$ & $\begin{array}{c}101 \mathrm{lb} \\
10: 11 \mathrm{hr} \\
5-24-96 \\
\end{array}$ & & $1500 \mathrm{~mL}$ & $1.7 \mathrm{R}$ & & $\begin{array}{l}\text { T0-80-503 } \\
\text { Rev. C-4 }\end{array}$ & 19 in. of sample \\
\hline
\end{tabular}


Table A.3. FY 1996 RGS Summary Data Sheet

Tank AN-105 Riser 12A

\begin{tabular}{|c|c|c|c|c|c|c|c|c|}
\hline $\begin{array}{c}\begin{array}{c}\text { Sampler Serial } \\
\text { Number } \\
* * * * * * * * * * * * * *\end{array} \\
\text { Cask Serial } \\
\text { Number } \\
\text { **************} \\
\text { Sample Number } \\
\end{array}$ & $\begin{array}{c}\text { Time and Date } \\
\text { of Sampling } \\
\text { (just after } \\
\text { pintle rod } \\
\text { has been } \\
\text { pulled) } \\
\end{array}$ & $\begin{array}{l}\text { Max Force to } \\
\text { Unseat Sampler } \\
\text { (initiation of } \\
\text { sampler removal } \\
\text { from tank) } \\
\text { Time } \\
\text { Date }\end{array}$ & $\begin{array}{c}\text { Purge Gas } \\
* * * * * * * * * * * \\
\text { DSP }=\text { Drill } \\
\text { String Purge } \\
* * * * * * * * * * * \\
\text { Time } \\
\text { Date }\end{array}$ & $\begin{array}{c}\text { Amount of } \\
\text { Hydrostatic } \\
\text { Head Fluid } \\
\text { Added }\end{array}$ & $\begin{array}{l}\text { Radiation } \\
\text { Dose Rate } \\
\text { Reading } \\
\text { Through Drill } \\
\text { String }\end{array}$ & $\begin{array}{l}\text { Strip Chart } \\
\text { Downforce } \\
\text { Max Load } \\
\text { Avg Load } \\
\text { Duration }\end{array}$ & $\begin{array}{l}\text { Procedure } \\
\text { Version- } \\
\text { and } \\
\text { Procedural } \\
\text { Exceptions }\end{array}$ & $\begin{array}{c}\text { X-Ray } \\
\text { Observation } \\
\text { Notes }\end{array}$ \\
\hline $\begin{array}{l}\text { RGS-008 seg } 15 \\
\text { C-1041 } \\
96-267\end{array}$ & $\begin{array}{l}\text { 6-13-96 } \\
02: 50 \mathrm{hr}\end{array}$ & $\begin{array}{c}112 \mathrm{lb} \\
6-13-96 \\
10: 52-96\end{array}$ & & $1500 \mathrm{~mL}$ & $1.5 \mathrm{R}$ & & $\begin{array}{l}\text { T0-80-503 } \\
\text { Rev. C-5 }\end{array}$ & 19 in. of sample \\
\hline $\begin{array}{l}\text { RGS-006 seg } 17 \\
\text { SN-67 } \\
96-269\end{array}$ & $\begin{array}{l}6-13-96 \\
22: 17 \mathrm{hr}\end{array}$ & $\begin{array}{l}.118 \mathrm{lb} \\
6-14-96 \\
01: 07 \mathrm{hr}\end{array}$ & & $1500 \mathrm{~mL}$ & $1.8 \mathrm{R}$ & & $\begin{array}{l}\text { T0-80-503 } \\
\text { Rev. C-5 }\end{array}$ & \\
\hline $\begin{array}{l}\text { RGS-023 seg } 19 \\
\text { SN-64 } \\
96-271\end{array}$ & $\begin{array}{l}6-14-96 \\
04: 01 \mathrm{hr}\end{array}$ & $\begin{array}{c}112 \mathrm{lb} \\
6-14-96 \\
04: 22 \\
\end{array}$ & & $1500 \mathrm{~mL}$ & $1.7 \mathrm{R}$ & $\cdot$ & $\begin{array}{l}\text { T0-80-503 } \\
\text { Rev. C-5 }\end{array}$ & 19 in. of sample \\
\hline $\begin{array}{l}\text { RGS-018 seg } 21 \\
\text { C-2014 } \\
96-273\end{array}$ & $\begin{array}{l}6-17-96 \\
03: 21 \mathrm{hr}\end{array}$ & $\begin{array}{c}112 \mathrm{lb} \\
6-17-96 \\
18: 30 \mathrm{hr}\end{array}$ & & $1500 \mathrm{~mL}$ & $1.6 \mathrm{R}$ & & $\begin{array}{l}\text { T0-80-503 } \\
\text { Rev. C-5 }\end{array}$ & 19 in. sample \\
\hline
\end{tabular}


Table A.4. FY 1996 RGS Summary Data Sheet

Tank AN-105 Riser 7B

\begin{tabular}{|c|c|c|c|c|c|c|c|c|}
\hline 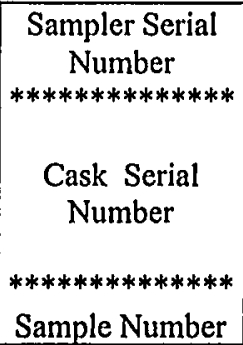 & $\begin{array}{l}\text { Time and Date } \\
\text { of Sampling } \\
\text { (just after } \\
\text { pintle rod } \\
\text { has been } \\
\text { pulled) }\end{array}$ & $\begin{array}{l}\text { Max Force to } \\
\text { Unseat Sampler } \\
\text { (initiation of } \\
\text { sampler removal } \\
\text { from tank) } \\
\text { Time } \\
\text { Date }\end{array}$ & $\begin{array}{c}\text { Purge Gas }{ }^{(\mathrm{a})} \\
* * * * * * * * * * \\
\text { DSP = Drill } \\
\text { String Purge } \\
* * * * * * * * * * \\
\text { Time } \\
\text { Date }\end{array}$ & $\begin{array}{c}\text { Amount of } \\
\text { Hydrostatic } \\
\text { Head Fluid } \\
\text { Added }\end{array}$ & $\begin{array}{l}\text { Radiation } \\
\text { Dose Rate } \\
\text { Reading } \\
\text { Through Drill } \\
\text { String }\end{array}$ & $\begin{array}{l}\text { Strip Chart } \\
\text { Downforce } \\
\text { Max Load } \\
\text { Avg Load } \\
\text { Duration }\end{array}$ & $\begin{array}{l}\text { Procedure } \\
\text { Version and } \\
\text { Procedural } \\
\text { Exceptions }\end{array}$ & $\begin{array}{c}\text { X-Ray } \\
\text { Observation } \\
\text { Notes }\end{array}$ \\
\hline $\begin{array}{l}\text { RGS-016 seg } 4 \\
\text { C-1025 } \\
96-278 \\
\end{array}$ & $\begin{array}{l}6-26-96 \\
02: 51 \mathrm{hr}\end{array}$ & $\begin{array}{c}116 \mathrm{lb} \\
6-26-96 \\
03: 00 \mathrm{hr}\end{array}$ & & $1500 \mathrm{~mL}$ & $2.0 \mathrm{R}$ & & $\begin{array}{l}\text { T0-80-503 } \\
\text { Rev. C-5 }\end{array}$ & 19 in. sample \\
\hline $\begin{array}{l}\text { RGS-015 seg } 16 \\
\text { C-2008 } \\
96-290\end{array}$ & $\begin{array}{l}6-26-96 \\
19: 52 \mathrm{hr}\end{array}$ & $\begin{array}{c}117 \mathrm{lb} \\
6-27-96 \\
01: 00 \mathrm{hr}\end{array}$ & & $1500 \mathrm{~mL}$ & $2.0 \mathrm{R}$ & & $\begin{array}{l}\text { T0-80-503 } \\
\text { Rev. C-5 }\end{array}$ & 19 in. sample \\
\hline $\begin{array}{l}\text { RGS-019 seg } 18 \\
\text { SN-77 } \\
96-292 \\
\end{array}$ & $\begin{array}{l}6-28-96 \\
03: 11 \mathrm{hr}\end{array}$ & $\begin{array}{c}123 \mathrm{lb} \\
04: 49 \mathrm{hr} \\
6-28-96 \\
\end{array}$ & & $1500 \mathrm{~mL}$ & $1.8 \mathrm{R}$ & & $\begin{array}{l}\text { T0-80-503 } \\
\text { Rev. C-5 }\end{array}$ & 19 in. sample \\
\hline $\begin{array}{l}\text { RGS-024 seg } 20 \\
\text { SN-76 } \\
96-294 \\
\end{array}$ & $\begin{array}{c}6-28-96 \\
06: 30\end{array}$ & $\begin{array}{c}134 \mathrm{lb} \\
6-28-96 \\
09: 38 \mathrm{hr} \\
\end{array}$ & & $1500 \mathrm{~mL}$ & $1.2 \mathrm{R}$ & & $\begin{array}{l}\text { T0-80-503 } \\
\text { Rev. C-5 }\end{array}$ & Valve open \\
\hline
\end{tabular}


Table A.5. FY 1996 RGS Summary Data Sheet

Tank A-101 Riser 15

\begin{tabular}{|c|c|c|c|c|c|c|c|c|}
\hline $\begin{array}{c}\text { Sampler Serial } \\
\text { Number } \\
* * * * * * * * * * * * * * \\
\\
\text { Cask Serial } \\
\text { Number } \\
* * * * * * * * * * * * * * \\
\text { Sample Number }\end{array}$ & $\begin{array}{l}\text { Time and Date } \\
\text { of Sampling } \\
\text { (just after } \\
\text { pintle rod } \\
\text { has been } \\
\text { pulled) }\end{array}$ & $\begin{array}{l}\text { Max Force to } \\
\text { Unseat Sampler } \\
\text { (initiation of } \\
\text { sampler removal } \\
\text { from tank) } \\
\text { Time } \\
\text { Date }\end{array}$ & $\begin{array}{c}\text { Purge Gas } \\
* * * * * * * * * * * \\
\text { DSP = Drill } \\
\text { String Purge } \\
* * * * * * * * * * * \\
\text { Time } \\
\text { Date }\end{array}$ & $\begin{array}{c}\text { Amount of } \\
\text { Hydrostatic } \\
\text { Head Fluid } \\
\text { Added }\end{array}$ & $\begin{array}{l}\text { Radiation } \\
\text { Dose Rate } \\
\text { Reading } \\
\text { Through Drill } \\
\text { String }\end{array}$ & $\begin{array}{l}\text { Strip Chart } \\
\text { Downforce } \\
\text { Max Load } \\
\text { Avg Load } \\
\text { Duration }\end{array}$ & $\begin{array}{l}\text { Procedure } \\
\text { Version } \\
\quad \text { and } \\
\text { Procedural } \\
\text { Exceptions }\end{array}$ & $\begin{array}{c}\text { X-Ray } \\
\text { Observation } \\
\text { Notes }\end{array}$ \\
\hline $\begin{array}{l}\text { RGS-021 seg } 5 \\
\text { SN-64 } \\
96-301\end{array}$ & $\begin{array}{l}7-11-96 \\
22: 43 \mathrm{hr}\end{array}$ & $\begin{array}{l}120 \mathrm{lb} \\
7-12-96 \\
03: 17 \mathrm{hr}\end{array}$ & . & $1500 \mathrm{~mL}$ & $1.3 \mathrm{R}$ & & $\begin{array}{l}\text { T0-80-503 } \\
\text { Rev. C-5 }\end{array}$ & 19 in. sample \\
\hline $\begin{array}{l}\text { RGS- } 025 \operatorname{seg} 8 \\
\text { C-2009 } \\
96-304\end{array}$ & $\begin{array}{l}7-12-96 \\
18: 02 \mathrm{hr}\end{array}$ & $\begin{array}{c}111 \mathrm{lb} \\
7-12-96 \\
18: 55 \mathrm{hr} \\
\end{array}$ & & $\begin{array}{l}\text { No volume } \\
\text { recorded }\end{array}$ & $900 \mathrm{mR}$ & & $\begin{array}{l}\text { T0-80-503 } \\
\text { Rev. C-5 }\end{array}$ & 12 in. of recovery \\
\hline $\begin{array}{l}\text { RGS-026 seg } 12 \\
31-G \\
96-308\end{array}$ & $\begin{array}{l}7-17-96 \\
05: 39 \mathrm{hr}\end{array}$ & $\begin{array}{c}124 \mathrm{lb} \\
7-17-96 \\
05: 55 \mathrm{hr}\end{array}$ & $\cdot$ & $\begin{array}{c}1500 \mathrm{~mL} \\
19 \mathrm{in.} \mathrm{sample}\end{array}$ & $2.0 \mathrm{R}$ & & $\begin{array}{l}\text { T0-80-503 } \\
\text { Rev. C-5 }\end{array}$ & $\cdots$ \\
\hline
\end{tabular}


Table A.6. FY 1996 RGS Summary Data Sheet

\section{Tank A-101 Riser 24}

\begin{tabular}{|c|c|c|c|c|c|c|c|c|}
\hline 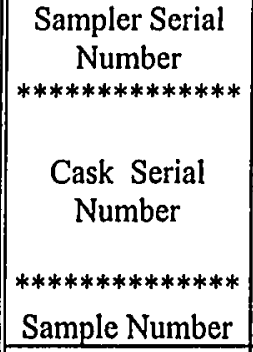 & $\begin{array}{l}\text { Time and Date } \\
\text { of Sampling } \\
\text { (just after } \\
\text { pintle rod } \\
\text { has been } \\
\text { pulled) }\end{array}$ & $\begin{array}{l}\text { Max Force to } \\
\text { Unseat Sampler } \\
\text { (initiation of } \\
\text { sampler removal } \\
\text { from tank) } \\
\text { Time } \\
\text { Date }\end{array}$ & $\begin{array}{c}\text { Purge Gas } \\
* * * * * * * * * * * \\
\text { DSP = Drill } \\
\text { String Purge } \\
* * * * * * * * * * \\
\text { Time } \\
\text { Date } \\
\end{array}$ & $\begin{array}{c}\text { Amount of } \\
\text { Hydrostatic } \\
\text { Head Fluid } \\
\text { Added }\end{array}$ & $\begin{array}{l}\text { Radiation } \\
\text { Dose Rate } \\
\text { Reading } \\
\text { Through Drill } \\
\text { String }\end{array}$ & $\begin{array}{l}\text { Strip Chart } \\
\text { Downforce } \\
\text { Max Load } \\
\text { Avg Load } \\
\text { Duration }\end{array}$ & $\begin{array}{l}\text { Procedure } \\
\text { Version and } \\
\text { Procedural } \\
\text { Exceptions }\end{array}$ & $\begin{array}{c}\text { X-Ray } \\
\text { Observation } \\
\text { Notes }\end{array}$ \\
\hline $\begin{array}{l}\text { RGS-022 seg } 2 \\
10-G \\
96-337 \\
\end{array}$ & $\begin{array}{l}7-22-96 \\
03: 05 \mathrm{hr}\end{array}$ & $\begin{array}{c}195 \mathrm{lb} \\
7-22-96 \\
03: 30 \mathrm{hr} \\
\end{array}$ & & $1500 \mathrm{~mL}$ & $1.3 \mathrm{R}$ & & $\begin{array}{l}\text { T0-80-503 } \\
\text { Rev. C-6 }\end{array}$ & Full sampler \\
\hline $\begin{array}{l}\text { RGS-031 seg } 9 \\
\text { SN-19G } \\
96-344 \\
\end{array}$ & $\begin{array}{l}7-23-96 \\
03: 26 \mathrm{hr}\end{array}$ & $\begin{array}{c}117 \mathrm{lb} \\
7-23-96 \\
03: 40 \mathrm{hr} \\
\end{array}$ & & $1500 \mathrm{~mL}$ & $1.3 \mathrm{R}$ & & $\begin{array}{l}\text { T0-80-503 } \\
\text { Rev. C-6 }\end{array}$ & 19 in. sample \\
\hline $\begin{array}{l}\text { RGS-032 seg } 16 \\
\text { SN-61 } \\
96-351\end{array}$ & $\begin{array}{l}7-24-96 \\
19: 44 \mathrm{hr}\end{array}$ & $\begin{array}{c}95 \mathrm{lb} \\
7-24-96 \\
20: 32 \mathrm{hr}\end{array}$ & & $1500 \mathrm{~mL}$ & $1.8 \mathrm{R}$ & & $\begin{array}{l}\text { T0-80-503 } \\
\text { Rev. C-6 }\end{array}$ & $\begin{array}{l}19 \text { in., mostly } \\
\text { liquid }\end{array}$ \\
\hline $\begin{array}{l}\text { RGS-030 seg } 19 \\
\text { C-1019 } \\
96-335 \\
\end{array}$ & $\begin{array}{l}7-25-96 \\
03: 10 \mathrm{hr}\end{array}$ & $\begin{array}{c}137 \mathrm{lb} \\
7-25-96 \\
03: 30 \mathrm{hr} \\
\end{array}$ & & $1500 \mathrm{~mL}$ & $1.7 \mathrm{R}$ & $\cdot$ & $\begin{array}{l}\text { T0-80-503 } \\
\text { Rev. C-6 }\end{array}$ & $\begin{array}{l}\text { X-ray shows } \\
\text { liquid. HBD went } \\
\text { off } 8 \text { in. into } \\
\text { stroke }\end{array}$ \\
\hline
\end{tabular}


Table A.7. FY 1996 RGS Summary Data Sheet

Tank AN-104 Riser 12A

\begin{tabular}{|c|c|c|c|c|c|c|c|c|}
\hline $\begin{array}{c}\text { Sampler Serial } \\
\text { Number } \\
* * * * * * * * * * * * * * \\
\text { Cask Serial } \\
\text { Number } \\
* * * * * * * * * * * * * * \\
\text { Sample Number }\end{array}$ & $\begin{array}{c}\text { Time and Date } \\
\text { of Sampling } \\
\text { (just after } \\
\text { pintle rod } \\
\text { has been } \\
\text { pulled) }\end{array}$ & $\begin{array}{l}\text { Max Force to } \\
\text { Unseat Sampler } \\
\text { (initiation of } \\
\text { sampler removal } \\
\text { from tank) } \\
\text { Time } \\
\text { Date }\end{array}$ & 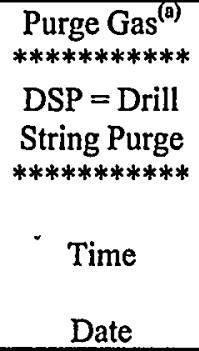 & $\begin{array}{c}\text { Amount of } \\
\text { Hydrostatic } \\
\text { Head Fluid } \\
\text { Added }\end{array}$ & $\begin{array}{l}\text { Radiation } \\
\text { Dose Rate } \\
\text { Reading } \\
\text { Through Drill } \\
\text { String }\end{array}$ & $\begin{array}{l}\text { Strip Chart } \\
\text { Downforce } \\
\text { Max Load } \\
\text { Avg Load } \\
\text { Duration }\end{array}$ & $\begin{array}{c}\text { Procedure } \\
\text { Version and } \\
\text { Procedural } \\
\text { Exceptions }\end{array}$ & $\begin{array}{c}\text { X-Ray } \\
\text { - Observation } \\
\text { Notes }\end{array}$ \\
\hline $\begin{array}{l}\text { RGS-029 seg } 18 \\
\text { C-1049 } \\
96-435\end{array}$ & $\begin{array}{l}8-14-96 \\
02: 06 \mathrm{hr}\end{array}$ & $\begin{array}{c}110 \mathrm{lb} \\
8-14-96 \\
02: 45 \mathrm{hr}\end{array}$ & & $1500 \mathrm{~mL}$ & $2 \mathrm{R}$ & & $\begin{array}{l}\text { T0-80-503 } \\
\text { Rev. C-7 }\end{array}$ & $\begin{array}{l}1 / 2 \text { in. to } 5 / 8 \text { in } \\
\text { air pocket } \\
\text { between piston } \\
\text { and sample. } \\
\text { Many gas } \\
\text { bubbles noted. }\end{array}$ \\
\hline & & & & & & & & \\
\hline & & & & & & & & \\
\hline & & & & & & & & \\
\hline
\end{tabular}


Table A.8. FY 1996 RGS Summary Data Sheet

\section{Tank AN-104 Riser 10A}

\begin{tabular}{|c|c|c|c|c|c|c|c|c|}
\hline $\begin{array}{c}\text { Sampler Serial } \\
\text { Number } \\
* * * * * * * * * * * * * * \\
\\
\text { Cask Serial } \\
\text { Number } \\
* * * * * * * * * * * * * \\
\text { Sample Number }\end{array}$ & $\begin{array}{l}\text { Time and Date } \\
\text { of Sampling } \\
\text { (just after } \\
\text { pintle rod } \\
\text { has been } \\
\text { pulled) }\end{array}$ & $\begin{array}{c}\text { Max Force to } \\
\text { Unseat Sampler } \\
\text { (initiation of } \\
\text { sampler removal } \\
\text { from tank) } \\
\text { Time } \\
\text { Date }\end{array}$ & $\begin{array}{c}\text { Purge Gas }{ }^{(\mathrm{a})} \\
* * * * * * * * * * * \\
\text { DSP = Drill } \\
\text { String Purge } \\
* * * * * * * * * * * \\
\text { Time } \\
\text { Date }\end{array}$ & $\begin{array}{c}\text { Amount of } \\
\text { Hydrostatic } \\
\text { Head Fluid } \\
\text { Added }\end{array}$ & $\begin{array}{l}\text { Radiation } \\
\text { Dose Rate } \\
\text { Reading } \\
\text { Through Drill } \\
\text { String }\end{array}$ & $\begin{array}{l}\text { Strip Chart } \\
\text { Downforce } \\
\text { Max Load } \\
\text { Avg Load } \\
\text { Duration }\end{array}$ & $\begin{array}{l}\text { Procedure } \\
\text { Version and } \\
\text { Procedural } \\
\text { Exceptions }\end{array}$ & $\begin{array}{c}\text { X-Ray } \\
\text { Observation } \\
\text { Notes }\end{array}$ \\
\hline $\begin{array}{l}\text { RGS-034 seg } 3 \\
23-G \\
96-399\end{array}$ & $\begin{array}{l}9-9-96 \\
11: 30 \mathrm{hr}\end{array}$ & $\begin{array}{c}138 \mathrm{lb} \\
9.9-96 \\
11: 50 \mathrm{hr} \\
\end{array}$ & & $1500 \mathrm{~mL}$ & $2.5 \mathrm{R}$ & & $\begin{array}{l}\text { T0-80-503 } \\
\text { Rev. C-8 }\end{array}$ & Full sampler \\
\hline $\begin{array}{l}\text { RGS-13 seg } 13 \\
\text { SN-73 } \\
96-409 \\
\end{array}$ & $\begin{array}{l}9-10-96 \\
21: 47 \mathrm{hr}\end{array}$ & $\begin{array}{c}80 \mathrm{lb} \\
9-11-96 \\
00: 58 \mathrm{hr} \\
\end{array}$ & & $1500 \mathrm{~mL}$ & $1.9 \mathrm{R}$ & & $\begin{array}{l}\text { T0-80-503 } \\
\text { Rev. C-8 }\end{array}$ & 19 in. sample \\
\hline $\begin{array}{l}\text { RGS-035 seg } 15 \\
\text { SN-14-G } \\
96-411\end{array}$ & $\begin{array}{l}9-11-96 \\
09: 50 \mathrm{hr}\end{array}$ & $\begin{array}{c}81 \mathrm{lb} \\
9-11-96 \\
10: 05 \mathrm{hr}\end{array}$ & & $1500 \mathrm{~mL}$ & $2.5 \mathrm{R}$ & & $\begin{array}{l}\text { T0-80-503 } \\
\text { Rev. C-8 }\end{array}$ & Full sampler \\
\hline $\begin{array}{l}\text { RGS-036 seg } 17 \\
\text { C-2011 } \\
96-413 \\
\end{array}$ & $\begin{array}{l}9-11-96 \\
12: 20 \mathrm{hr}\end{array}$ & $\begin{array}{c}79 \mathrm{lb} \\
9-11-96 \\
16: 49 \mathrm{hr} \\
\end{array}$ & & $1500 \mathrm{~mL}$ & $2 R$ & & $\begin{array}{l}\text { T0-80-503 } \\
\text { Rev. C-8 }\end{array}$ & Full sampler \\
\hline $\begin{array}{l}\text { C-2015 } \\
96-415\end{array}$ & $\begin{array}{l}9-11-96 \\
21: 29 \mathrm{hr}\end{array}$ & $\begin{array}{c}78 \mathrm{lb} \\
9-11-96 \\
21: 44 \mathrm{hr}\end{array}$ & $\begin{array}{l}\text { Purge } \\
\text { 17:55 hr }\end{array}$ & $1500 \mathrm{~mL}$ & $1.8 \mathrm{R}$ & & $\begin{array}{l}\text { T0-80-503 } \\
\text { Rev. C-8 }\end{array}$ & $\begin{array}{l}\text { X-ray showed } \\
\text { full sampler }\end{array}$ \\
\hline
\end{tabular}


Table A.9. FY 1996 RGS Summary Data Sheet

Tank AN-104 Riser 10A

\begin{tabular}{|c|c|c|c|c|c|c|c|c|}
\hline $\begin{array}{c}\text { Sampler Serial } \\
\text { Number } \\
* * * * * * * * * * * * * * \\
\text { Cask Serial } \\
\text { Number } \\
* * * * * * * * * * * * * * \\
\text { Sample Number }\end{array}$ & $\begin{array}{l}\text { Time and Date } \\
\text { of Sampling } \\
\text { (just after } \\
\text { pintle rod } \\
\text { has been } \\
\text { pulled) }\end{array}$ & $\begin{array}{l}\text { Max Force to } \\
\text { Unseat Sampler } \\
\text { (initiation of } \\
\text { sampler removal } \\
\text { from tank) } \\
\text { Time } \\
\text { Date }\end{array}$ & $\begin{array}{c}\text { Purge Gas } \\
* * * * * * * * * * \\
\text { DSP = Drill } \\
\text { String Purge } \\
* * * * * * * * * * * \\
\text { Time } \\
\text { Date }\end{array}$ & $\begin{array}{c}\text { Amount of } \\
\text { Hydrostatic } \\
\text { Head Fluid } \\
\text { Added }\end{array}$ & $\begin{array}{l}\text { Radiation } \\
\text { Dose Rate } \\
\text { Reading } \\
\text { Through Drill } \\
\text { String }\end{array}$ & $\begin{array}{l}\text { Strip Chart } \\
\text { Downforce } \\
\text { Max Load } \\
\text { Avg Load } \\
\text { Duration }\end{array}$ & $\begin{array}{l}\text { Procedure } \\
\text { Version } \\
\text { and } \\
\text { Procedural } \\
\text { Exceptions }\end{array}$ & $\begin{array}{c}\text { X-Ray } \\
\text { Observation } \\
\text { Notes }\end{array}$ \\
\hline $\begin{array}{l}\text { RGS-037 seg } 21 \\
1004-C \\
96-417\end{array}$ & $\begin{array}{l}9-12-96 \\
14: 32 \mathrm{hr}\end{array}$ & $\begin{array}{c}107 \mathrm{lb} \\
9-12-96 \\
17: 00 \mathrm{hr}\end{array}$ & $\begin{array}{c}\text { Purge } \\
05: 20 \mathrm{hr}\end{array}$ & Not recorded & $2.0 \mathrm{R}$ & • & $\begin{array}{l}\text { T0-80-503 } \\
\text { Rev. C-8 }\end{array}$ & $\begin{array}{l}\text { Not complete } \\
\text { (19 in.) stroke. } \\
\text { HBD tripped at } \\
18 \text { in. } \\
\text { High LFL } \\
\text { recorded. } \\
16 \text { in. of sample }\end{array}$ \\
\hline & & & & & & & & \\
\hline & & & & & & & & \\
\hline
\end{tabular}


Table A.10. FY 1996 RGS Summary Data Sheet

\section{Tank AN-103 Riser 12A}

\begin{tabular}{|c|c|c|c|c|c|c|c|c|}
\hline $\begin{array}{c}\text { Sampler Serial } \\
\text { Number } \\
* * * * * * * * * * * * * * \\
\\
\text { Cask Serial } \\
\text { Number } \\
* * * * * * * * * * * * * * \\
\text { Sample Number }\end{array}$ & $\begin{array}{l}\text { Time and Date } \\
\text { of Sampling } \\
\text { (just after } \\
\text { pintle rod } \\
\text { has been } \\
\text { pulled) }\end{array}$ & $\begin{array}{l}\text { Max Force to } \\
\text { Unseat Sampler } \\
\text { (initiation of } \\
\text { sampler removal } \\
\text { from tank) } \\
\text { Time } \\
\text { Date }\end{array}$ & $\begin{array}{c}\text { Purge Gas } \\
* * * * * * * * * * * \\
\text { DSP = Drill } \\
\text { String Purge } \\
* * * * * * * * * * * \\
\text { Time } \\
\text { Date } \\
\end{array}$ & $\begin{array}{c}\text { Amount of } \\
\text { Hydrostatic } \\
\text { Head Fluid } \\
\text { Added }\end{array}$ & $\begin{array}{c}\text { Radiation } \\
\text { Dose Rate } \\
\text { Reading } \\
\text { Through Drill } \\
\text { String }\end{array}$ & $\begin{array}{l}\text { Strip Chart } \\
\text { Downforce } \\
\text { Max Load } \\
\text { Avg Load } \\
\text { Duration }\end{array}$ & $\begin{array}{l}\text { Procedure } \\
\text { Version and } \\
\text { Procedural } \\
\text { Exceptions }\end{array}$ & $\begin{array}{c}\text { X-Ray } \\
\text { Observation } \\
\text { Notes }\end{array}$ \\
\hline $\begin{array}{l}\text { RGS-052 seg } 2 \\
\text {. } \\
96-446 \\
\end{array}$ & $\begin{array}{l}9-13-96 \\
18: 54 \mathrm{hr}\end{array}$ & $\begin{array}{c}9-13-96 \\
18: 58\end{array}$ & & No data & & & $\begin{array}{l}\text { T0-80-503 } \\
\text { Rev. C-8 }\end{array}$ & \\
\hline $\begin{array}{l}\text { RGS-050 seg } 5 \\
96-449\end{array}$ & $\begin{array}{l}9-13-96 \\
21: 12 \mathrm{hr}\end{array}$ & $\begin{array}{l}9-13-96 \\
21: 17 \mathrm{hr}\end{array}$ & & No data & & & $\begin{array}{l}\text { T0-80-503 } \\
\text { Rev. C-8 }\end{array}$ & \\
\hline $\begin{array}{l}\text { RGS-042 seg } 14 \\
\text { C- } 1016 \\
96-458\end{array}$ & $\begin{array}{l}9-14-96 \\
16: 28 \mathrm{hr}\end{array}$ & $\begin{array}{l}9-16-96 \\
01: 22 \mathrm{hr}\end{array}$ & & No data & $2.2 \mathrm{R}$ & & $\begin{array}{l}\text { T0-80-503 } \\
\text { Rev. C-8 }\end{array}$ & $\begin{array}{l}\text { Lots of gas } \\
\text { bubbles at the } \\
\text { top of the } \\
\text { sampler, none at } \\
\text { the bottom. }\end{array}$ \\
\hline
\end{tabular}


Table A.11. FY 1996 RGS Summary Data Sheet

\section{Tank AN-103 Riser 21A}

\begin{tabular}{|c|c|c|c|c|c|c|c|c|}
\hline $\begin{array}{c}\text { Sampler Serial } \\
\text { Number } \\
* * * * * * * * * * * * * \\
\\
\text { Cask Serial } \\
\text { Number } \\
* * * * * * * * * * * * * \\
\\
\text { Sample Number }\end{array}$ & $\begin{array}{l}\text { Time and Date } \\
\text { of Sampling } \\
\text { (just after } \\
\text { pintle rod } \\
\text { has been } \\
\text { pulled) } \\
\end{array}$ & $\begin{array}{l}\text { Max Force to } \\
\text { Unseat Sampler } \\
\text { (initiation of } \\
\text { sampler removal } \\
\text { from tank) } \\
\text { Time } \\
\text { Date } \\
\end{array}$ & $\begin{array}{c}\text { Purge Gas } \\
* * * * * * * * * \\
\text { DSP }=\text { Drill } \\
\text { String Purge } \\
* * * * * * * * * * * \\
\text { Time } \\
\text { Date } \\
\end{array}$ & $\begin{array}{c}\text { Amount of } \\
\text { Hydrostatic } \\
\text { Head Fluid } \\
\text { Added }\end{array}$ & $\begin{array}{l}\text { Radiation } \\
\text { Dose Rate } \\
\text { Reading } \\
\text { Through Drill } \\
\text { String } \\
\end{array}$ & $\begin{array}{l}\text { Strip Chart } \\
\text { Downforce } \\
\text { Max Load } \\
\text { Avg Load } \\
\text { Duration }\end{array}$ & $\begin{array}{l}\text { Procedure } \\
\text { Version } \\
\text { and } \\
\text { Procedural } \\
\text { Exceptions }\end{array}$ & $\begin{array}{c}\text { X-Ray } \\
\text { Observation } \\
\text { Notes }\end{array}$ \\
\hline $\begin{array}{l}\text { RGS-048 seg } 10 \\
6-G \\
96-473 \\
\end{array}$ & $\begin{array}{l}9-20-96 \\
02: 13 \mathrm{hr}\end{array}$ & $\begin{array}{l}9-20-96 \\
02: 30 \mathrm{hr}\end{array}$ & & No volume data & $2.9 \mathrm{R}$ & & $\begin{array}{l}\text { T0-80-503 } \\
\text { Rev. C-8 }\end{array}$ & $\begin{array}{l}\text { Full sampler } \\
\text { - }\end{array}$ \\
\hline $\begin{array}{l}\text { RGS-040 seg } 13 \\
\text { C-2007 } \\
96-476\end{array}$ & $\begin{array}{l}9-20-96 \\
06: 53 \mathrm{hr}\end{array}$ & $\begin{array}{l}9-20-96 \\
09: 43 \mathrm{hr}\end{array}$ & & No volume data & $1.8 \mathrm{R}$ & & $\begin{array}{l}\text { T0-80-503 } \\
\text { Rev. C-8 }\end{array}$ & $\begin{array}{l}17 \text { in. of sample } \\
\text { Found to be } \\
\text { leaking during } \\
\text { processing; } \\
\text { discarded. }\end{array}$ \\
\hline $\begin{array}{l}\text { RGS-045 seg } 16 \\
\text { SN-74 } \\
96-479 \\
\end{array}$ & $\begin{array}{l}9-20-96 \\
18: 50 \mathrm{hr}\end{array}$ & $\begin{array}{l}9-20-96 \\
18: 57 \mathrm{hr}\end{array}$ & & No volume data & $1.8 \mathrm{R}$ & & $\begin{array}{l}\text { T0-80-503 } \\
\text { Rev. C-8 }\end{array}$ & 19 in. of sample \\
\hline $\begin{array}{l}\text { RGS-033 seg } 18 \\
1037 \\
96-481 \\
\end{array}$ & $9-23-96$ & $9-23-96$ & & No volume data & $80 \mathrm{mR}$ & & $\begin{array}{l}\text { T0-80-503 } \\
\text { Rev. C-8 }\end{array}$ & $\begin{array}{l}\text { Valve open, } \\
\text { pintle rod } \\
\text { removal } \\
\text { problem. } \\
\text { Sample empty. }\end{array}$ \\
\hline
\end{tabular}





\section{FY 1997 Field Data for RGS Tanks U-103, S-106, BY-101, and BY-109 \\ (in chronological sampling order)}


Table A.12. FY 1997 RGS Field Data Summary Sheet

Tank U-103 Riser 7

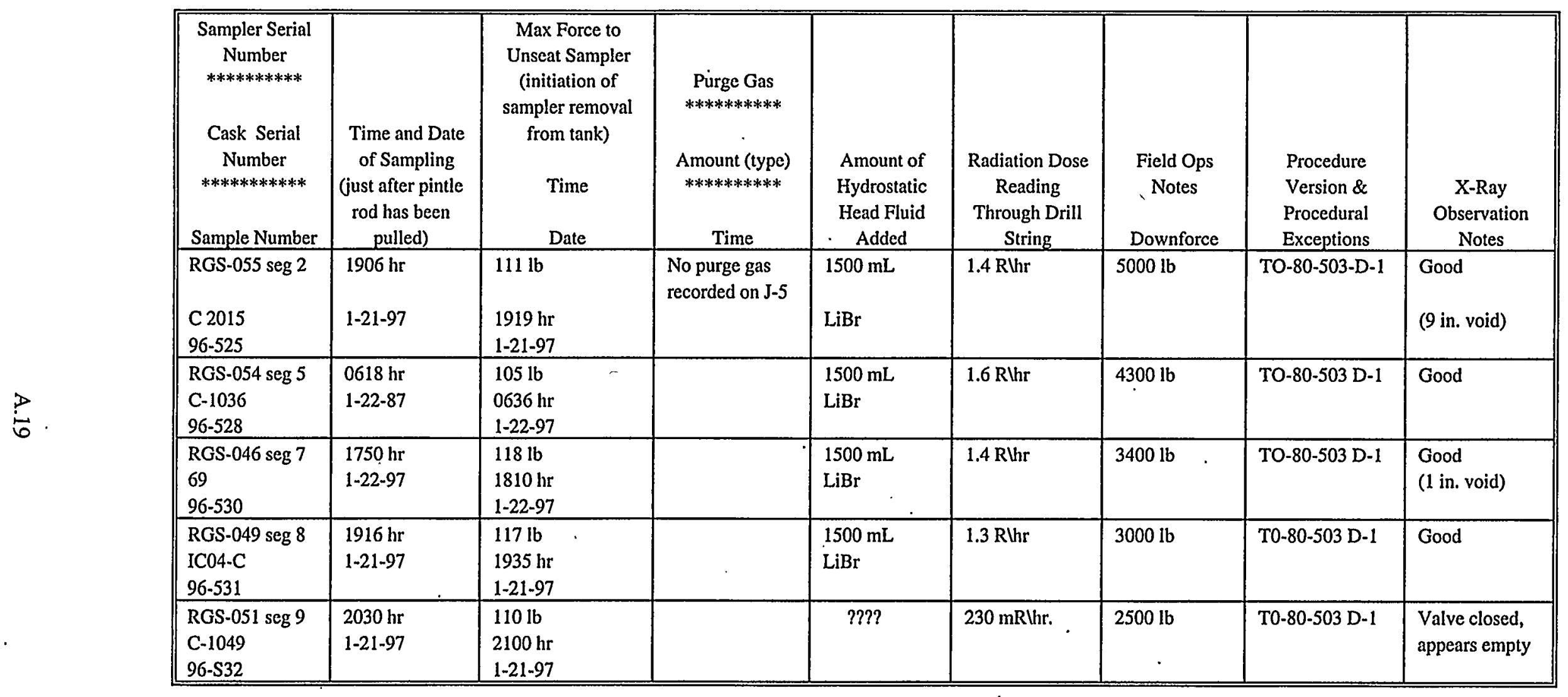


Table A.13. FY 1997 RGS Field Data Summary Sheet

Tank U-103 Riser 13

\begin{tabular}{|c|c|c|c|c|c|c|c|c|}
\hline $\begin{array}{c}\text { Sampler Serial } \\
\text { Number } \\
* * * * * * * * * * * * \\
\text { Cask Serial } \\
\text { Number } \\
* * * * * * * * * * * \\
\text { Sample Number }\end{array}$ & $\begin{array}{l}\text { Time and Date } \\
\text { of Sampling } \\
\text { (just after } \\
\text { pintle rod has } \\
\text { been pulled) }\end{array}$ & $\begin{array}{l}\text { Max Force to } \\
\text { Unseat Sampler } \\
\text { (initiation of } \\
\text { sampler removal } \\
\text { from tank) } \\
\text { Time } \\
\text { Date }\end{array}$ & 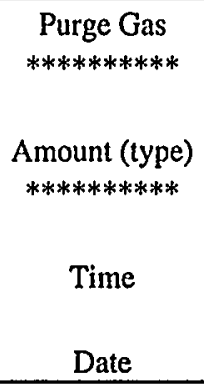 & $\begin{array}{l}\text { Amount of } \\
\text { Hyddrostatic } \\
\text { Head Fluid } \\
\text { Added }\end{array}$ & $\begin{array}{l}\text { Radiation Dose } \\
\text { Reading } \\
\text { Through Drill } \\
\text { String } \\
\end{array}$ & $\begin{array}{l}\text { Field Ops } \\
\text { Notes } \\
\text { Downforce }\end{array}$ & $\begin{array}{l}\text { Procedure } \\
\text { Version and } \\
\text { Procedural } \\
\text { Exceptions } \\
\end{array}$ & $\begin{array}{c}\text { X-Ray } \\
\text { Observation } \\
\text { Notes } \\
\end{array}$ \\
\hline $\begin{array}{l}\text { RGS-057 seg } 4 \\
23 \\
97-020\end{array}$ & $\begin{array}{l}1319 \mathrm{hr} \\
4-2-97\end{array}$ & $\begin{array}{c}182 \mathrm{lb} \\
\\
1323 \mathrm{hr} \\
4-2-97\end{array}$ & $\begin{array}{l}\text { No purge gas } \\
\text { recorded on J- } \\
5\end{array}$ & $\begin{array}{c}1500 \mathrm{~mL} \\
\mathrm{LiBr}\end{array}$ & 700 mRlhr. & $4000 \mathrm{lb}$ & T0-80-503 E-0 & $\begin{array}{l}10 \mathrm{in.} \mathrm{of} \\
\text { sample }\end{array}$ \\
\hline
\end{tabular}


Table A.14. FY 1997 RGS Field Data Summary Sheet

Tank S-106 Riser 7

\begin{tabular}{|c|c|c|c|c|c|c|c|c|}
\hline $\begin{array}{c}\begin{array}{c}\text { Sampler Serial } \\
\text { Number } \\
(\mathrm{a})\end{array} \\
* * * * * * * * * * * * \\
\text { Cask Serial } \\
\text { Number } \\
* * * * * * * * * * * \\
\text { Sample Number }\end{array}$ & $\begin{array}{l}\text { Time and Date } \\
\text { of Sampling } \\
\text { (just after } \\
\text { pintle rod has } \\
\text { been pulled) }\end{array}$ & $\begin{array}{l}\text { Max Force to } \\
\text { Unseat Sampler } \\
\text { (initiation of } \\
\text { sampler removal } \\
\text { from tank) } \\
\text { Time } \\
\text { Date }\end{array}$ & $\begin{array}{c}\text { Purge Gas } \\
* * * * * * * * * * * \\
\text { Amount (type) } \\
* * * * * * * * * * * \\
\text { Time } \\
\text { Date } \\
\end{array}$ & $\begin{array}{c}\text { Amount of } \\
\text { Hydrostatic } \\
\text { Head Fluid } \\
\text { Added }\end{array}$ & $\begin{array}{l}\text { Radiation Dose } \\
\text { Reading } \\
\text { Through Drill } \\
\text { String }\end{array}$ & $\begin{array}{l}\text { Field Ops } \\
\text { Notes } \\
\text { Downforce }\end{array}$ & $\begin{array}{l}\text { Procedure } \\
\text { Version and } \\
\text { Procedural } \\
\text { Exceptions }\end{array}$ & $\begin{array}{c}\text { X-Ray } \\
\text { Observation } \\
\text { Notes }\end{array}$ \\
\hline $\begin{array}{l}\text { RGS-063 seg } 3 \\
10-6 \\
97-40\end{array}$ & $\begin{array}{l}2000 \mathrm{hr} \\
2-24-97\end{array}$ & $\begin{array}{c}170 \mathrm{lb} \\
2020 \mathrm{hr} \\
2-24-97\end{array}$ & $\begin{array}{c}\text { No J-5 data } \\
\text { logged on } \\
2-24-97\end{array}$ & $\begin{array}{c}1500 \mathrm{~mL} \\
\mathrm{LiBr}\end{array}$ & $1.2 \mathrm{R} \mathrm{khr}$ & $3500 \mathrm{lb}$ & $\mathrm{T} 0-80-503 \mathrm{E}-0$ & Good \\
\hline $\begin{array}{l}\text { RGS-062 seg } 5 \\
69 \\
97-38\end{array}$ & $\begin{array}{l}0325 \mathrm{hr} \\
2-25-97\end{array}$ & $\begin{array}{c}171 \mathrm{lb} \\
0355 \mathrm{hr} \\
2-25-97 \\
\end{array}$ & $\begin{array}{l}\text { No J-5 data } \\
\text { logged on } \\
2-25-97\end{array}$ & $\begin{array}{c}1500 \mathrm{~mL} \\
\mathrm{LiBr}\end{array}$ & $1.2 \mathrm{R} h \mathrm{hr}$ & $2800 \mathrm{lb}$ & T0-80-503 E-0 & Good \\
\hline
\end{tabular}


Table A.15. RGS Field Data Summary Sheet

Tank S-106 Riser 8

\begin{tabular}{|c|c|c|c|c|c|c|c|c|}
\hline $\begin{array}{c}\text { Sampler Serial } \\
\text { Number } \\
* * * * * * * * * * \\
\text { Cask Serial } \\
\text { Number } \\
* * * * * * * * * * * \\
\text { Sample Number }\end{array}$ & $\begin{array}{l}\text { Time and Date } \\
\text { of Sampling } \\
\text { (just after } \\
\text { pintle rod has } \\
\text { been pulled) }\end{array}$ & $\begin{array}{c}\text { Max Force to } \\
\text { Unseat Sampler } \\
\text { (initiation of } \\
\text { sampler removal } \\
\text { from tank) } \\
\text { Time } \\
\text { Date }\end{array}$ & $\begin{array}{c}\text { Purge Gas } \\
* * * * * * * * * * * \\
\text { Amount (type) } \\
* * * * * * * * * * * \\
\text { Time } \\
\text { Date } \\
\end{array}$ & $\begin{array}{c}\text { Amount of } \\
\text { Hydrostatic } \\
\text { Head Fluid } \\
\text { Added } \\
\end{array}$ & $\begin{array}{c}\text { Radiation Dose } \\
\text { Reading } \\
\text { Through Drill } \\
\text { String } \\
\end{array}$ & $\begin{array}{l}\text { Field Ops } \\
\text { Notes } \\
\text { Downforce }\end{array}$ & $\begin{array}{c}\text { Procedure } \\
\text { Version and } \\
\text { Procedural } \\
\text { Exceptions } \\
\end{array}$ & $\begin{array}{c}\text { X-Ray } \\
\text { Observation } \\
\text { Notes } \\
\end{array}$ \\
\hline $\begin{array}{l}\text { RGS-044 seg } 2 \\
\text { C } 2018 \\
97-31\end{array}$ & $\begin{array}{l}2010 \mathrm{hr} \\
2-12-97\end{array}$ & $\begin{array}{c}180 \mathrm{lb} \\
2020 \mathrm{hr} \\
2-12-97 \\
\end{array}$ & $\begin{array}{c}\text { No purge gas } \\
\text { datả }\end{array}$ & $\begin{array}{c}1500 \mathrm{~mL} \\
\mathrm{LiBr}\end{array}$ & $800 \mathrm{mRhhr}$ & $3800 \mathrm{lb}$ & T0-80-503 E-0 & $\begin{array}{l}\text { Valve open? } \\
\text { full }\end{array}$ \\
\hline $\begin{array}{l}\text { RGS-061 seg } 6 \\
68 \\
97-31 \\
\end{array}$ & $\begin{array}{l}0410 \mathrm{hr} \\
\cdot \\
2-18-97\end{array}$ & $\begin{array}{c}180 \mathrm{lb} \\
\\
0420 \mathrm{hr} \\
2-18-97 \\
\end{array}$ & $\begin{array}{c}\text { No purge gas } \\
\text { data }\end{array}$ & $\begin{array}{c}1500 \mathrm{~mL} \\
\mathrm{LiBr}\end{array}$ & $900 \mathrm{mR} \backslash \mathrm{hr}$ & $2500 \mathrm{lb}$ & T0-80-503 E-0 & Good \\
\hline $\begin{array}{l}\text { RGS-056 seg } 10 \\
60 \\
97-35\end{array}$ & $\begin{array}{l}0313 \mathrm{hr} \\
2-21-97\end{array}$ & \begin{tabular}{|l}
$233 \mathrm{lb}$ \\
$2045 \mathrm{hr}$ \\
$2-21-97$
\end{tabular} & $\begin{array}{c}\text { Argon purge } \\
\text { done on swing } \\
2-21-97\end{array}$ & None & I Rlhr & $1700 \mathrm{lb}$ & T0-80-503 E-0 & Good \\
\hline
\end{tabular}


Table A.16. FY 1997 RGS Field Data Summary Sheet

\section{Tank S-106 Riser 14}

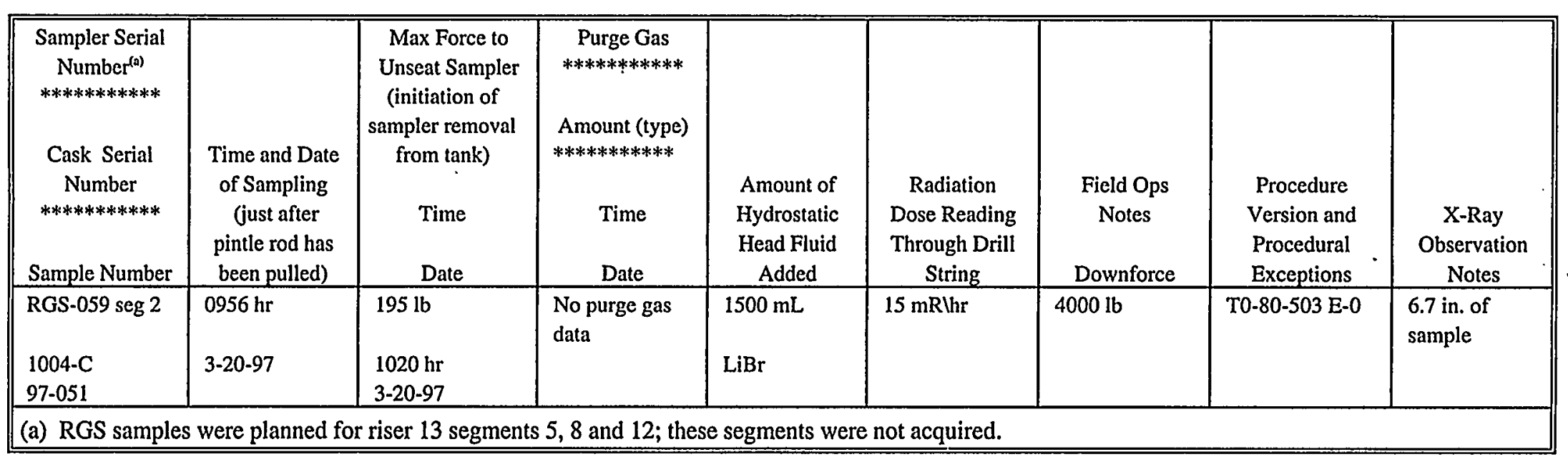


Table A.17. FY 1997 RGS Field Data Summary Sheet

Tank BY-101 Riser 10B

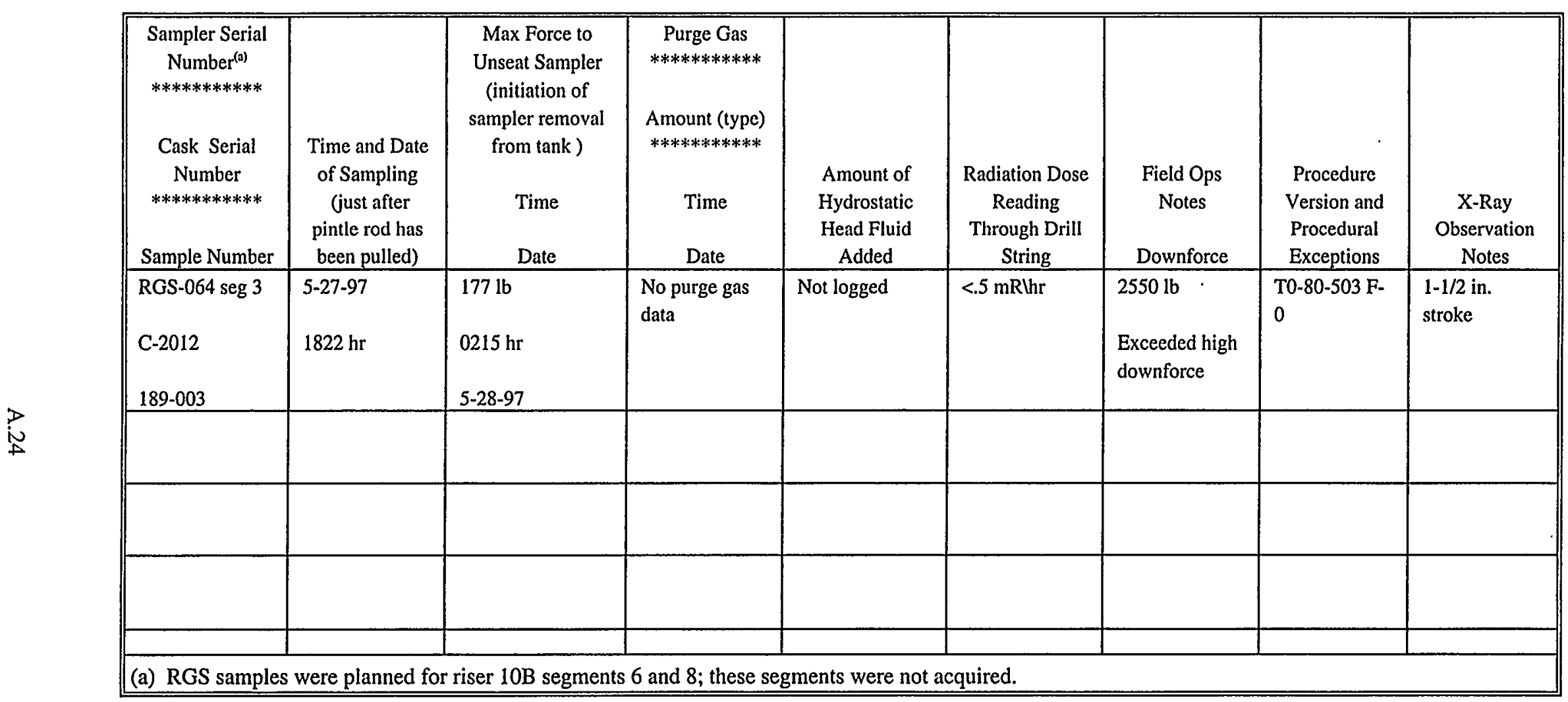


Table A.18. FY 1997 RGS Field Data Summary Sheet

Tank BY-101 Riser 10D

\begin{tabular}{|c|c|c|c|c|c|c|c|c|}
\hline $\begin{array}{c}\begin{array}{c}\text { Sampler Serial } \\
\text { Number } \\
r^{(a)} \\
* * * * * * * * * * *\end{array} \\
\text { Cask Serial } \\
\text { Number } \\
* * * * * * * * * * * \\
\text { Sample Number }\end{array}$ & $\begin{array}{c}\text { Time and Date } \\
\text { of Sampling } \\
\text { (just after } \\
\text { pintle rod has } \\
\text { been pulled) }\end{array}$ & $\begin{array}{c}\text { Max Force to } \\
\text { Unseat Sampler } \\
\text { (initiation of } \\
\text { sampler removal } \\
\text { from tank) } \\
\text { Time } \\
\text { Date }\end{array}$ & 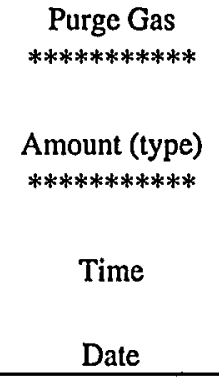 & $\begin{array}{c}\text { Amount of } \\
\text { Hydrostatic } \\
\text { Head Fluid } \\
\text { Added }\end{array}$ & $\begin{array}{l}\text { Radiation Dose } \\
\text { Reading } \\
\text { Through Drill . } \\
\text { String } \\
\end{array}$ & $\begin{array}{l}\text { Field Ops } \\
\text { Notes } \\
\text { Downforce }\end{array}$ & $\begin{array}{c}\text { Procedure } \\
\text { Version and } \\
\text { Procedural } \\
\text { Exceptions } \\
\end{array}$ & $\begin{array}{c}\text { X-Ray } \\
\text { Observation } \\
\quad \text { Notes } \\
\end{array}$ \\
\hline $\begin{array}{l}\text { RGS-067 seg } 2 \\
\text { C-1028 } \\
199-02 \\
\end{array}$ & $\begin{array}{l}1858 \mathrm{hr} \\
5-28-97\end{array}$ & $\begin{array}{l}234 \mathrm{lb} \\
1907 \mathrm{hr} \\
5-28-97 \\
\end{array}$ & $\begin{array}{l}\text { No purge gas } \\
\text { data }\end{array}$ & $\begin{array}{l}1500 \mathrm{~mL} \\
\mathrm{LiBr}\end{array}$ & $4 \mathrm{mRlhr}$ & $2900 \mathrm{lb}$ & T0-80-503 F-0 & Empty \\
\hline & & & & & & & & \\
\hline & & & & & & & & \\
\hline (a) RGS sampl & re planne & 10D segm & and $7 ;$ these & ents were $n$ & uuired. & & & \\
\hline
\end{tabular}




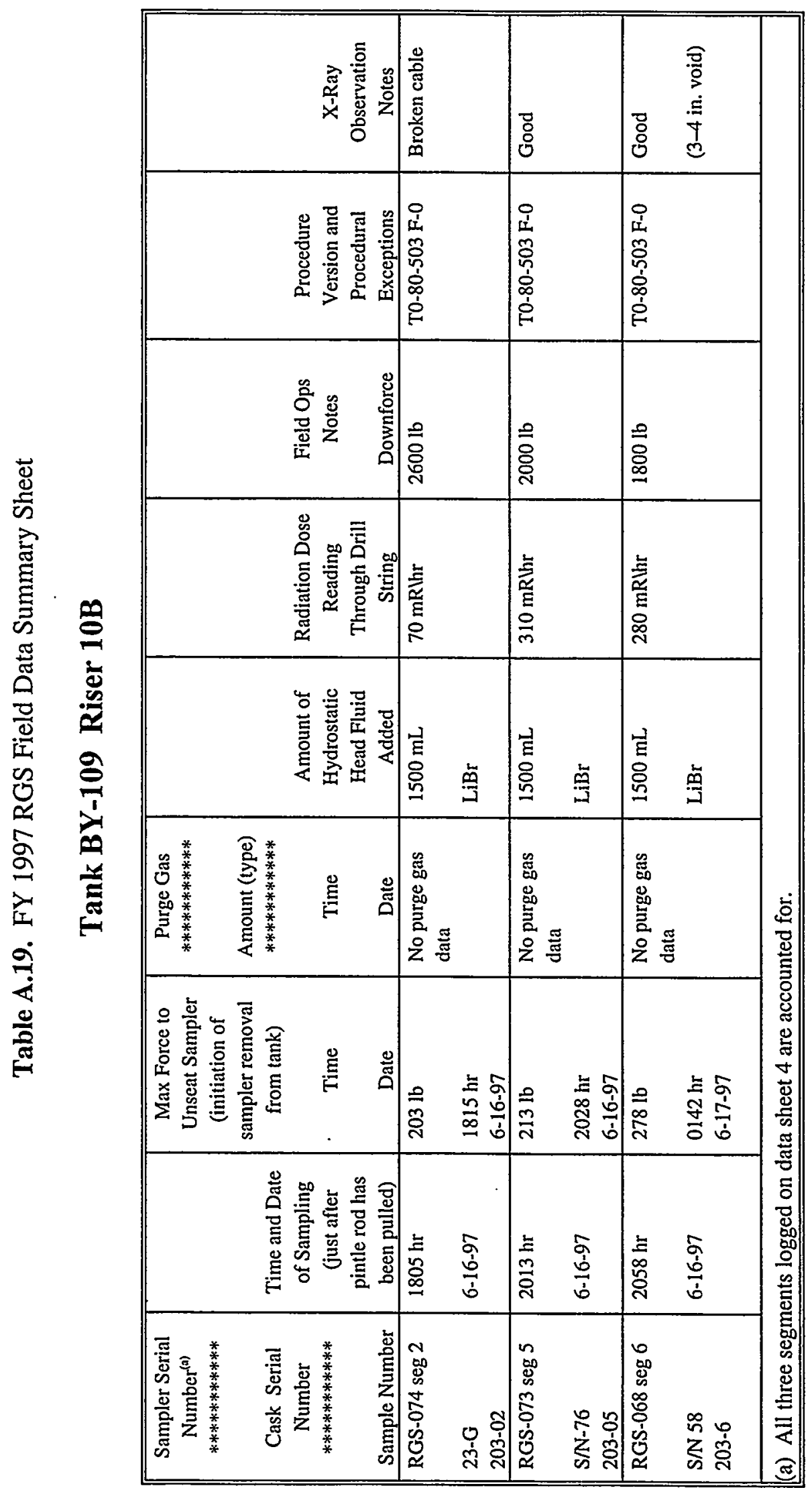


Table A.20. FY 1997 RGS Field Data Summary Sheet

Tank BY-109 Riser 12C

\begin{tabular}{|c|c|c|c|c|c|c|c|c|}
\hline 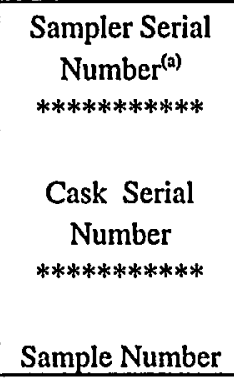 & $\begin{array}{c}\text { Time and Date } \\
\text { of Sampling } \\
\text { (just after } \\
\text { pintle rod has } \\
\text { been pulled) }\end{array}$ & $\begin{array}{l}\text { Max Force to } \\
\text { Unseat Sampler } \\
\text { (initiation of } \\
\text { sampler removal } \\
\text { from tank) } \\
\text { Time } \\
\text { Date }\end{array}$ & $\begin{array}{c}\text { Purge Gas } \\
* * * * * * * * * * * \\
\text { Amount (type) } \\
* * * * * * * * * * * \\
\text { Time } \\
\text { Date } \\
\end{array}$ & $\begin{array}{c}\text { Amount of } \\
\text { Hydrostatic } \\
\text { Head Fluid } \\
\text { Added } \\
\end{array}$ & $\begin{array}{c}\text { Radiation Dose } \\
\text { Reading } \\
\text { Through Drill } \\
\text { String } \\
\end{array}$ & $\begin{array}{c}\text { Field Ops } \\
\text { Notes } \\
\text { Downforce }\end{array}$ & $\begin{array}{l}\text { Procedure } \\
\text { Version and } \\
\text { Procedural } \\
\text { Exceptions } \\
\end{array}$ & $\begin{array}{c}\text { X-Ray } \\
\text { Observation } \\
\text { Notes } \\
\end{array}$ \\
\hline $\begin{array}{l}\text { RGS-066 seg } 1 \\
18 G \\
2101-4 \\
\end{array}$ & $\begin{array}{l}1039 \mathrm{hr} \\
6-6-97\end{array}$ & $\begin{array}{c}190 \mathrm{lb} \\
\\
\\
1309 \mathrm{hr} \\
6-6-97 \\
\end{array}$ & $\begin{array}{c}\text { No purge gas } \\
\text { data }\end{array}$ & $\begin{array}{c}1488 \mathrm{~mL} \\
\mathrm{LiBr}\end{array}$ & $42 \mathrm{mR}$ hhr & $2700 \mathrm{lb}$ & T0-80-503 F-0 & $\begin{array}{l}\text { Only } \\
2-3 \text { in. of } \\
\text { sample }\end{array}$ \\
\hline $\begin{array}{l}\text { RGS-070 seg } 4 \\
77 \\
201-4\end{array}$ & $\begin{array}{l}1504 \mathrm{hr} \\
6-12-97\end{array}$ & $\begin{array}{l}172 \mathrm{lb} \\
\\
1803 \mathrm{hr} \\
6-12-97\end{array}$ & $\begin{array}{l}\text { No purge gas } \\
\text { data }\end{array}$ & $\begin{array}{c}1500 \mathrm{~mL} \\
\mathrm{LiBr}\end{array}$ & $350 \mathrm{mRVhr}$ & $2000 \mathrm{lb}$ & T0-80-503 F-0 & $\begin{array}{l}\text { Good (less } \\
\text { than 3-4 in.) }\end{array}$ \\
\hline
\end{tabular}




-


FY 1998 Field Data for RGS Tanks

\section{SX-106, AX-101, S-102, S-111 and U- 109}

(in chronological sampling order) 
$+$

. 
Table A.21. FY 1998 RGS Summary Data Sheet

Tank SX-106 Riser 6

\begin{tabular}{|c|c|c|c|c|c|c|c|c|}
\hline $\begin{array}{c}\text { Sampler Serial } \\
\text { Number } \\
* * * * * * * * * * * \\
\\
\text { Cask Serial } \\
\text { Number } \\
* * * * * * * * * * \\
\text { Sample Number }\end{array}$ & $\begin{array}{l}\text { Time and } \\
\text { Date } \\
\text { of Sampling } \\
\text { (just after } \\
\text { pintle rod has } \\
\text { been pulled) }\end{array}$ & $\begin{array}{l}\text { Max Force to } \\
\text { Unseat Sampler } \\
\text { (initiation of } \\
\text { sampler removal } \\
\text { from tank) } \\
\text { Time } \\
\text { Date }\end{array}$ & $\begin{array}{c}\text { Purge Gas } \\
* * * * * * * * * \\
\text { DSP = Drill } \\
\text { String Purge } \\
* * * * * * * * * * \\
\text { Time } \\
\text { Date }\end{array}$ & $\begin{array}{c}\text { Amount of } \\
\text { Hydrostatic } \\
\text { Head Fluid } \\
\text { Added }\end{array}$ & $\begin{array}{c}\text { Radiation } \\
\text { Dose Rate } \\
\text { Reading } \\
\text { Through Drill } \\
\text { String }\end{array}$ & $\begin{array}{l}\text { Strip Chart } \\
\text { Downforce } \\
\text { Max Load } \\
\text { Avg Load } \\
\text { Duration }\end{array}$ & $\begin{array}{c}\text { Procedure } \\
\text { Version and } \\
\text { Procedural } \\
\text { Exceptions }\end{array}$ & $\begin{array}{c}\text { X-Ray } \\
\text { Observation } \\
\text { Notes }\end{array}$ \\
\hline $\begin{array}{l}\text { RGS-079 seg } 6 \\
22 \mathrm{G} \\
223-06\end{array}$ & $\mathrm{NA}$ & $\begin{array}{l}197 \mathrm{lb} \\
1734 \\
10-28-97 \\
\end{array}$ & $\begin{array}{l}\text { No DSP } \\
\text { recorded }\end{array}$ & $\begin{array}{l}1500 \mathrm{~mL} \\
0.3 \mathrm{M} \mathrm{LiBr}\end{array}$ & $1.6 \mathrm{R} / \mathrm{hr}$ & NA & $\begin{array}{l}\text { TO-80-503 } \\
\text { F4 }\end{array}$ & $\begin{array}{l}\text { Approximately } \\
17.7 \text { in. of } \\
\text { sample }\end{array}$ \\
\hline $\begin{array}{l}\text { RGS-058 seg 6A } \\
59 \\
223-06 \mathrm{~A}\end{array}$ & $\begin{array}{l}1816 \\
10-28-97\end{array}$ & $\begin{array}{l}205 \mathrm{lb} \\
1845 \\
10-28-97\end{array}$ & $\begin{array}{l}\text { No DSP } \\
\text { recorded }\end{array}$ & $\begin{array}{l}1500 \mathrm{~mL} \\
0.3 \mathrm{M} \mathrm{LiBr}\end{array}$ & $1.7 \mathrm{R} / \mathrm{hr}$ & $\begin{array}{l}404 \mathrm{lb} \\
289 \mathrm{lb} \\
183 \mathrm{sec}\end{array}$ & $\begin{array}{l}\text { TO-80-503 } \\
\text { F4 }\end{array}$ & $\begin{array}{l}\text { Full sampler } \\
\text { (18.2 in. of } \\
\text { sample) }\end{array}$ \\
\hline $\begin{array}{l}\text { RGS-080 seg } 9 \\
70 \\
223-09\end{array}$ & $\begin{array}{l}2110 \\
10-28-97\end{array}$ & $\begin{array}{l}200 \mathrm{lb} \\
1810 \\
10-29-97\end{array}$ & $\begin{array}{l}\text { No DSP } \\
\text { recorded }\end{array}$ & $\begin{array}{l}1500 \mathrm{~mL} \\
0.3 \mathrm{M} \mathrm{LiBr}\end{array}$ & $800 \mathrm{mR} / \mathrm{hr}$ & $\begin{array}{l}519 \mathrm{lb} \\
289 \mathrm{lb} \\
195 \mathrm{sec}\end{array}$ & $\begin{array}{l}\text { TO-80-503 } \\
\text { F4 }\end{array}$ & $\begin{array}{l}\text { Approximately } \\
12 \text { in. of sample }\end{array}$ \\
\hline $\begin{array}{l}\text { RGS-075 seg } 11 \\
4 G \\
223-011\end{array}$ & $\begin{array}{l}1755 \\
10-31-97\end{array}$ & $\begin{array}{l}205 \mathrm{lb} \\
1241 \\
11-21-97\end{array}$ & $\begin{array}{l}\text { DSP @ 1925 } \\
10-30-97 \\
\\
\text { DSP @ 1705 } \\
10-31-97 \\
\end{array}$ & NA & $150 \mathrm{mR} / \mathrm{hr}$ & $\begin{array}{l}577 \mathrm{lb} \\
346 \mathrm{lb}\end{array}$ & $\begin{array}{l}\text { TO-80-503 } \\
\text { F4 }\end{array}$ & $\begin{array}{l}\text { This segment } \\
\text { was lost }\end{array}$ \\
\hline
\end{tabular}


Table A.21 (contd)

Tank SX-106 Riser 3

\begin{tabular}{|c|c|c|c|c|c|c|c|c|}
\hline $\begin{array}{c}\text { Sampler Serial } \\
\text { Number } \\
* * * * * * * * * * * * \\
\text { Cask Serial } \\
\text { Number } \\
* * * * * * * * * * * \\
\text { Sample Number }\end{array}$ & $\begin{array}{l}\text { Time and } \\
\text { Date } \\
\text { of Sampling } \\
\text { (just after } \\
\text { pintle rod } \\
\text { has been } \\
\text { pulled) }\end{array}$ & $\begin{array}{l}\text { Max Force to } \\
\text { Unseat Sampler } \\
\text { (initiation of } \\
\text { sampler removal } \\
\text { from tank) } \\
\text { Time } \\
\text { Date }\end{array}$ & $\begin{array}{c}\text { Purge Gas }{ }^{(\mathrm{a})} \\
* * * * * * * * * * \\
\text { DSP = Drill } \\
\text { String Purge } \\
* * * * * * * * * \\
\text { Time } \\
\text { Date } \\
\end{array}$ & $\begin{array}{c}\text { Amount of } \\
\text { Hydrostatic } \\
\text { Head Fluid } \\
\text { Added } \\
\end{array}$ & $\begin{array}{c}\text { Radiation Dose } \\
\text { Rate Reading } \\
\text { Through Drill } \\
\text { String } \\
\end{array}$ & $\begin{array}{l}\text { Strip Chart } \\
\text { Downforce } \\
\text { Max Load } \\
\text { Avg Load } \\
\text { Duration }\end{array}$ & $\begin{array}{l}\text { Procedure } \\
\text { Version and } \\
\text { Procedural } \\
\text { Exceptions } \\
\end{array}$ & $\begin{array}{c}\text { X-Ray } \\
\text { Observation } \\
\text { Notes }\end{array}$ \\
\hline $\begin{array}{l}\text { RGS-072 seg } 2 \\
57 \\
224-02\end{array}$ & $\begin{array}{l}2002 \\
12-02-97\end{array}$ & $\begin{array}{l}238 \mathrm{lb} \\
2025 \\
12-02-97\end{array}$ & $\begin{array}{l}\text { No DSP } \\
\text { recorded }\end{array}$ & $\begin{array}{l}1500 \mathrm{~mL} \\
0.3 \mathrm{M} \mathrm{LiBr}\end{array}$ & $<0.5 \mathrm{mR} / \mathrm{hr}$ & $\mathrm{NA}$ & $\begin{array}{l}\text { TO-80-503 } \\
\text { F4 }\end{array}$ & $\begin{array}{l}\text { Full sample } \\
\text { (18.2 in. } \\
\text { of sample) }\end{array}$ \\
\hline $\begin{array}{l}\text { RGS-060 seg. } 4 \\
22 \mathrm{G} \\
224-04\end{array}$ & $\begin{array}{l}2109 \\
12-05-97\end{array}$ & $\begin{array}{l}258 \mathrm{lb} \\
1017 \\
12-08-97\end{array}$ & $\begin{array}{l}\text { No DSP } \\
\text { recorded }\end{array}$ & $\begin{array}{l}1500 \mathrm{~mL} \\
0.3 \mathrm{M} \mathrm{LiBr}\end{array}$ & $\begin{array}{l}<0.5 \mathrm{mR} / \mathrm{hr} \\
\text { at Grapple }\end{array}$ & NA & $\begin{array}{l}\text { TO-80-503 } \\
\text { F4 }\end{array}$ & $\begin{array}{l}\text { Approximately } \\
17.9 \text { in. } \\
\text { of sample }\end{array}$ \\
\hline $\begin{array}{l}\text { RGS-071 seg } 7 \\
4-G \\
224-10\end{array}$ & $\begin{array}{l}1850 \\
12-08-97\end{array}$ & $\begin{array}{l}200 \mathrm{lb} \\
1858\end{array}$ & $\begin{array}{l}\text { No DSP } \\
\text { recorded }\end{array}$ & $\begin{array}{l}1500 \mathrm{~mL} \\
0.3 \mathrm{M} \mathrm{LiBr}\end{array}$ & $20 \mathrm{mR} / \mathrm{hr}$ & $\mathrm{NA}$ & $\begin{array}{l}\text { TO-80-503 } \\
\text { F4 }\end{array}$ & $\begin{array}{l}\text { Approximately } \\
13 \text { in. of } \\
\text { sample }\end{array}$ \\
\hline $\begin{array}{l}\text { RGS-077 seg } 10 \\
1030 \\
224-10\end{array}$ & $\begin{array}{l}1055 \\
12-09-97\end{array}$ & $\begin{array}{l}\text { Lost pintle rod } \\
\text { grip } \\
\text { Repeated effort } \\
\text { as below }\end{array}$ & $\begin{array}{l}\text { No DSP } \\
\text { recorded }\end{array}$ & NA & NA & $\mathrm{NA}$ & $\begin{array}{l}\text { TO-80-503 } \\
\text { F4 }\end{array}$ & $\begin{array}{l}\text { Lost pintle rod } \\
\text { grip - repeated } \\
\text { test }\end{array}$ \\
\hline $\begin{array}{l}\text { RGS-077 seg } 10 \\
1030 \\
224-10\end{array}$ & $\begin{array}{l}1043 \\
12-10-97\end{array}$ & $\begin{array}{l}219 \mathrm{lb} \\
1106 \\
12-10-97\end{array}$ & $\begin{array}{l}\text { No DSP } \\
\text { recorded }\end{array}$ & $\begin{array}{l}1500 \mathrm{~mL} \\
0.3 \mathrm{M} \mathrm{LiBr}\end{array}$ & $2-50 \mathrm{mR} / \mathrm{hr}$ & $\overline{N A}$ & $\begin{array}{l}\text { TO-80-503 } \\
\text { F4 }\end{array}$ & $\begin{array}{l}\text { Approximately } \\
13.5 \mathrm{in.} \\
\text { of sample }\end{array}$ \\
\hline
\end{tabular}


Table A.22. FY 1998 RGS Summary Data Sheet

\section{Tank AX-101 Riser 9d}

\begin{tabular}{|c|c|c|c|c|c|c|c|c|}
\hline $\begin{array}{c}\text { Sampler Serial } \\
\text { Number } \\
* * * * * * * * * * * * * \\
\\
\text { Cask Serial } \\
\text { Number } \\
* * * * * * * * * * * * * \\
\text { Sample Number }\end{array}$ & $\begin{array}{l}\text { Time and Date } \\
\text { of Sampling } \\
\text { (just after pintle } \\
\text { rod has been } \\
\text { pulled) }\end{array}$ & $\begin{array}{l}\text { Max Force to } \\
\text { Unseat Sampler } \\
\text { (initiation of } \\
\text { sampler removal } \\
\text { from tank) } \\
\text { Time } \\
\text { Date }\end{array}$ & $\begin{array}{c}\text { Purge Gas }{ }^{(\mathrm{a})} \\
* * * * * * * * * * \\
\text { DSP }=\text { Drill } \\
\text { String Purge } \\
* * * * * * * * * \\
\text { Time } \\
\text { Date }\end{array}$ & $\begin{array}{c}\text { Amount of } \\
\text { Hydrostatic } \\
\text { Fluid } \\
\text { Added }\end{array}$ & $\begin{array}{c}\text { Radiation Dose } \\
\text { Rate Reading } \\
\text { Through Drill } \\
\text { String }\end{array}$ & $\begin{array}{l}\text { Strip Chart } \\
\text { Downforce } \\
\text { Max Load } \\
\text { Avg Load } \\
\text { Duration }\end{array}$ & $\begin{array}{l}\text { Procedure } \\
\text { Version and } \\
\text { Procedural } \\
\text { Exceptions }\end{array}$ & $\begin{array}{c}\text { X-Ray } \\
\text { Observation } \\
\text { Notes }\end{array}$ \\
\hline $\begin{array}{l}\text { RGS-078 seg } 8 \\
1013-C \\
226-08\end{array}$ & $\begin{array}{l}1935 \\
01-09-98 \\
\end{array}$ & $\begin{array}{l}1950 \\
01-09-98\end{array}$ & $\begin{array}{l}\text { No DSP } \\
\text { recorded }\end{array}$ & $\begin{array}{l}1500 \mathrm{~mL} \\
0.3 \mathrm{M} \mathrm{LiBr}\end{array}$ & $1.5 \mathrm{R} / \mathrm{hr}$ & $390 \mathrm{lb}$ & TO-80-503-F4 & $\begin{array}{l}\text { Full sampler } \\
\text { (18.2 in. of } \\
\text { sample) }\end{array}$ \\
\hline & & & & & & & & \\
\hline & & & & & & & & \\
\hline & & & & & & & & \\
\hline
\end{tabular}


Table A.23. FY 1998 RGS Summary Data Sheet

Tank S-102 Riser 16

\begin{tabular}{|c|c|c|c|c|c|c|c|c|}
\hline $\begin{array}{c}\text { Sampler Serial } \\
\text { Number } \\
* * * * * * * * * * * * * \\
\text { Cask Scrial } \\
\text { Number } \\
\text { *************** } \\
\text { Sample Number }\end{array}$ & $\begin{array}{l}\text { Time and Date } \\
\text { of Sampling } \\
\text { (just after } \\
\text { pintle rod has } \\
\text { been pulled) } \\
\end{array}$ & $\begin{array}{c}\text { Max Force to } \\
\text { Unseat Sampler } \\
\text { (initiation of } \\
\text { sampler removal } \\
\text { from tank) } \\
\text { Time } \\
\text { Date } \\
\end{array}$ & $\begin{array}{c}\text { Purge Gas(a) } \\
* * * * * * * * * * * \\
\text { DSP }=\text { Drill } \\
\text { String Purge } \\
* * * * * * * * * * * \\
\text { Time } \\
\text { Date } \\
\end{array}$ & $\begin{array}{c}\text { Amount of } \\
\text { Hydrostatic } \\
\text { Head Fluid } \\
\text { Added } \\
\end{array}$ & $\begin{array}{c}\text { Radiation } \\
\text { Dose Rate } \\
\text { Reading } \\
\text { Through Drill } \\
\text { String } \\
\end{array}$ & $\begin{array}{l}\text { Strip Chart } \\
\text { Downforce } \\
\text { Max Load } \\
\text { Avg Load } \\
\text { Duration } \\
\end{array}$ & $\begin{array}{c}\text { Procedure } \\
\text { Version and } \\
\text { Procedural } \\
\text { Exceptions } \\
\end{array}$ & $\begin{array}{c}\text { X-Ray } \\
\text { Observation } \\
\text { Notes } \\
\end{array}$ \\
\hline $\begin{array}{l}\text { RGS-083 seg } 2 \\
31 G \\
232-2 \\
\end{array}$ & $\begin{array}{l}1722 \\
3-5-98\end{array}$ & $\begin{array}{l}219 \mathrm{lb} \\
1755 \mathrm{hr} \\
3-5-98 \\
\end{array}$ & $\begin{array}{l}\text { No DSP } \\
\text { recorded }\end{array}$ & $\begin{array}{l}1500 \mathrm{~mL} \\
\mathrm{LiBr}\end{array}$ & $800 \mathrm{mR} / \mathrm{hr}$ & $\mathrm{NA}$ & $\begin{array}{l}\text { TO-080-503 } \\
\text { G-0 }\end{array}$ & II in. of sample \\
\hline $\begin{array}{l}\text { RGS-085 seg } 4 \\
\text { C-2013 } \\
232-4\end{array}$ & $\begin{array}{l}2000 \mathrm{hr} \\
3-5-98\end{array}$ & $\begin{array}{l}2010 \mathrm{hr} \\
3-5-98\end{array}$ & $\begin{array}{l}\text { No DSP } \\
\text { recorded }\end{array}$ & $\begin{array}{l}1500 \mathrm{~mL} \\
\mathrm{LiBr}\end{array}$ & $27 \mathrm{mR} / \mathrm{hr}$ & $\mathrm{NA}$ & $\begin{array}{l}\text { TO-080-503 } \\
\text { G0 }\end{array}$ & $\begin{array}{l}\text { ball valve } \\
\text { problem, } \\
\text { sampler empty }\end{array}$ \\
\hline $\begin{array}{l}\text { RGS-076 seg } 4 R^{(b)} \\
\text { C2000 } \\
232-4 R\end{array}$ & $\begin{array}{l}0952 \mathrm{hr} \\
3-6-98\end{array}$ & $\begin{array}{l}1208 \mathrm{hr} \\
3-6-98\end{array}$ & $\begin{array}{l}\text { No DSP } \\
\text { recorded }\end{array}$ & $\begin{array}{l}1500 \mathrm{~mL} \\
\mathrm{LiBr}\end{array}$ & $600 \mathrm{mR} / \mathrm{hr}$ & $\mathrm{NA}$ & $\begin{array}{l}\text { TO-080-503 } \\
\text { GO }\end{array}$ & 18.in. of sample \\
\hline $\begin{array}{l}\text { RGS-086 seg } 6 \\
67 \\
232-6 \\
\end{array}$ & $\begin{array}{l}1915 \\
3-6-98\end{array}$ & N/A & $\begin{array}{l}\text { No DSP } \\
\text { recorded }\end{array}$ & N/A & N/A & $\begin{array}{l}1674 \mathrm{lb} \\
866 \mathrm{lb} \\
544 \mathrm{sec} \\
\end{array}$ & $\begin{array}{l}\text { TO-080-503 } \\
\text { GO }\end{array}$ & No sample \\
\hline $\begin{array}{l}\text { RGS-084 seg } 7^{(0)} \\
\text { C-1051 } \\
232-7\end{array}$ & $\begin{array}{l}1817 \\
3-18-98\end{array}$ & $\begin{array}{l}1830 \\
3-18-98\end{array}$ & $\begin{array}{l}\text { No DSP } \\
\text { recorded }\end{array}$ & $\begin{array}{l}1500 \mathrm{~mL} \\
\mathrm{LiBr}\end{array}$ & $1 \mathrm{R} / \mathrm{hr}$ & $\begin{array}{l}1385 \mathrm{lb} \\
\\
693 \mathrm{lb} \\
664 \mathrm{sec} \text { in } \\
3 \text { runs } \\
\end{array}$ & $\begin{array}{l}\text { TO-080-503 } \\
\text { GO }\end{array}$ & $15 \mathrm{in.}$ of sample \\
\hline $\begin{array}{l}\text { (a) Drill string pu } \\
\text { (b) Segment 4R r } \\
\text { (c) Segment } 7 \text { rep }\end{array}$ & segment 4 ( & problem) at & ent 4 loca & & & & & \\
\hline
\end{tabular}


Table A.23 (contd)

\section{Tank S-102 Riser 16}

\begin{tabular}{|c|c|c|c|c|c|c|c|c|}
\hline $\begin{array}{c}\text { Sampler Serial } \\
\text { Number } \\
* * * * * * * * * * * * \\
\\
\text { Cask Serial } \\
\text { Number } \\
* * * * * * * * * * * * \\
\text { Sample Number }\end{array}$ & $\begin{array}{c}\text { Time and } \\
\text { Date of } \\
\text { Sampling } \\
\text { (just after } \\
\text { pintle rod has } \\
\text { been pulled) }\end{array}$ & $\begin{array}{l}\text { Max Force to } \\
\text { Unseat } \\
\text { Sampler } \\
\text { (initiation of } \\
\text { sampler } \\
\text { removal from } \\
\text { tank) }\end{array}$ & $\begin{array}{c}\text { Purge Gas }{ }^{(\mathrm{a})} \\
* * * * * * * * * * * * \\
\text { DSP = Drill } \\
\text { String Purge } \\
* * * * * * * * * * * * \\
\text { Time } \\
\text { Date }\end{array}$ & $\begin{array}{l}\text { Amount of } \\
\text { Hydrostatic } \\
\text { Head Fluid } \\
\text { Added }\end{array}$ & $\begin{array}{l}\text { Radiation } \\
\text { Dose Rate } \\
\text { Reading } \\
\text { Through Drill } \\
\text { String }\end{array}$ & $\begin{array}{l}\text { Strip Chart } \\
\text { Downforce } \\
\text { Max Load } \\
\text { Avg Load } \\
\text { Duration }\end{array}$ & $\begin{array}{l}\text { Procedure } \\
\text { Version and } \\
\text { Procedural } \\
\text { Exceptions }\end{array}$ & $\begin{array}{c}\text { X-Ray } \\
\text { Observation } \\
\text { Notes }\end{array}$ \\
\hline $\begin{array}{l}\text { RGS-087 seg } 8 \\
1006-C \\
232-8\end{array}$ & $\begin{array}{l}2100 \\
03-18-98\end{array}$ & $\begin{array}{l}297 \mathrm{lb} \\
2115 \\
03-18-98\end{array}$ & $\begin{array}{l}\text { No DSP } \\
\text { recorded }\end{array}$ & $\begin{array}{l}1500 \mathrm{~mL} \\
\mathrm{LiBr}\end{array}$ & $1 \mathrm{R} / \mathrm{hr}$ & $\begin{array}{l}981 \mathrm{lb} \\
693 \mathrm{lb} \\
250 \mathrm{sec}\end{array}$ & $\begin{array}{l}\text { TO-080-503 } \\
\text { G-0 }\end{array}$ & $\begin{array}{l}12 \text { in. of } \\
\text { sample }\end{array}$ \\
\hline $\begin{array}{l}\text { RGS-081 seg } 10 \\
\text { C-2002 } \\
232-10\end{array}$ & $\begin{array}{l}1850 \\
04-02-98 \\
\end{array}$ & $\begin{array}{l}220 \mathrm{lb} \\
1857 \\
04-02-98\end{array}$ & $\begin{array}{l}\text { DSP @ } 1740 \\
03-27-98 \\
\text { DSP @ } 1230 \\
03-31-98\end{array}$ & $\begin{array}{l}1500 \mathrm{~mL} \\
\mathrm{LiBr}\end{array}$ & $1 \mathrm{R} / \mathrm{hr}$ & $\begin{array}{l}1212 \mathrm{lb} \\
866 \mathrm{lb} \\
410 \mathrm{sec}\end{array}$ & $\begin{array}{l}\text { TO-080-503 } \\
\text { G-1 }\end{array}$ & $\begin{array}{l}19 \text { in. of } \\
\text { sample }\end{array}$ \\
\hline
\end{tabular}


Table A.24. FY 1998 RGS Summary Data Sheet

Tank S-111 Riser 6

\begin{tabular}{|c|c|c|c|c|c|c|c|c|}
\hline $\begin{array}{c}\text { Sampler Serial } \\
\text { Number } \\
* * * * * * * * * * * * \\
\text { Cask Serial } \\
\text { Number } \\
* * * * * * * * * * * * * \\
\text { Sample Number }\end{array}$ & $\begin{array}{l}\text { Time and Date } \\
\text { of Sampling } \\
\text { (just after } \\
\text { pintle rod has } \\
\text { been pulled) }\end{array}$ & $\begin{array}{l}\text { Max Force to } \\
\text { Unseat Sampler } \\
\text { (initiation of } \\
\text { sampler removal } \\
\text { from tank) } \\
\text { Time } \\
\text { Date }\end{array}$ & $\begin{array}{c}\text { Purge Gas }{ }^{(\mathfrak{a})} \\
* * * * * * * * * * * \\
\text { DSP = Drill } \\
\text { String Purge } \\
* * * * * * * * * * * \\
\text { Time } \\
\text { Date } \\
\end{array}$ & $\begin{array}{c}\text { Amount of } \\
\text { Hydrostatic } \\
\text { Head fluid } \\
\text { Added } \\
\end{array}$ & $\begin{array}{c}\text { Radiation } \\
\text { Dose Rate } \\
\text { Reading } \\
\text { Through Drill } \\
\text { String } \\
\end{array}$ & $\begin{array}{l}\text { Strip Chart } \\
\text { Downforce } \\
\text { Max Load } \\
\text { Avg Load } \\
\text { Duration }\end{array}$ & $\begin{array}{l}\text { Procedure } \\
\text { Version and } \\
\text { Procedural } \\
\text { Exceptions } \\
\end{array}$ & $\begin{array}{c}\text { X-Ray } \\
\text { Observation } \\
\text { Notes }\end{array}$ \\
\hline $\begin{array}{l}\text { RGS-088 seg } 2 \\
\text { C-1040 } \\
237-02\end{array}$ & $\begin{array}{l}1030 \\
04-08-98 \\
\end{array}$ & $\begin{array}{l}212 \mathrm{lb} \\
1035 \\
04-08-98 \\
\end{array}$ & $\begin{array}{l}\text { No DSP } \\
\text { recorded }\end{array}$ & $\begin{array}{l}1700 \mathrm{~mL} \\
0.3 \mathrm{M} \mathrm{LiBr}\end{array}$ & I R/hr & $\begin{array}{l}1270 \mathrm{lb} \\
693 \mathrm{lb} \\
306 \mathrm{sec} \\
\end{array}$ & TO-80-503G-1 & $\begin{array}{l}\text { Sampler } \\
\text { appears full } \\
\text { (approximately } \\
18.2 \text { in. of } \\
\text { sample) }\end{array}$ \\
\hline $\begin{array}{l}\text { RGS-082 seg } 4 \\
\text { C-1027 } \\
237-04\end{array}$ & $\begin{array}{l}2210 \\
04-08-98\end{array}$ & $\begin{array}{l}0923 \\
04-09-98 \\
\end{array}$ & $\begin{array}{l}\text { No DSP } \\
\text { recorded }\end{array}$ & $\begin{array}{l}1700 \mathrm{~mL} \\
0.3 \mathrm{M} \mathrm{LiBr}\end{array}$ & $900 \mathrm{mR} / \mathrm{hr}$ & $\begin{array}{l}695 \mathrm{lb} \\
462 \mathrm{lb} \\
275 \mathrm{sec}\end{array}$ & TO-80-503G-1 & $\begin{array}{l}\text { About } 17.4 \mathrm{in} . \\
\text { of sample }\end{array}$ \\
\hline $\begin{array}{l}\text { RGS-089 seg } 6 \\
24 G \\
237-06\end{array}$ & $\begin{array}{l}1715 \\
04-09-98\end{array}$ & $\begin{array}{l}190 \mathrm{lb} \\
1726 \\
04-09-98\end{array}$ & $\begin{array}{l}\text { No DSP } \\
\text { recorded }\end{array}$ & $\begin{array}{l}1700 \mathrm{~mL} \\
0.3 \mathrm{M} \mathrm{LiBr}\end{array}$ & $700 \mathrm{mR} / \mathrm{hr}$ & $420 \mathrm{lb}$ & TO-80-503G-1 & $\begin{array}{l}\text { About } 17.7 \text { in. } \\
\text { of sample }\end{array}$ \\
\hline $\begin{array}{l}\text { RGS-091 seg } 8 \\
\begin{array}{l}\text { C-2013 } \\
237-08\end{array}\end{array}$ & $\begin{array}{l}2146 \\
04-09-98\end{array}$ & $\begin{array}{l}207 \mathrm{lb} . \\
2203 \\
04-09-98\end{array}$ & $\begin{array}{l}\text { No DSP } \\
\text { recorded }\end{array}$ & $\begin{array}{l}2000 \mathrm{~mL} \\
0.3 \mathrm{M} \mathrm{LiBr}\end{array}$ & $600 \mathrm{mR} / \mathrm{hr}$ & $540 \mathrm{lb}$ & TO-80-503G-1 & $\begin{array}{l}\text { About } 17.4 \mathrm{in} . \\
\text { of sample }\end{array}$ \\
\hline $\begin{array}{l}\text { RGS-094 seg } 10 \\
\text { C-2007 } \\
237-10\end{array}$ & $\begin{array}{l}1025 \\
04-10-98\end{array}$ & $\begin{array}{l}373 \mathrm{lb} \\
1030 \\
04-10-98 \\
\end{array}$ & $\begin{array}{l}\text { DSP @ } 0838 \\
04-10-98\end{array}$ & $\begin{array}{l}2 \mathrm{gal} \\
0.3 \mathrm{M} \mathrm{LiBr}\end{array}$ & $600 \mathrm{mR} / \mathrm{hr}$ & $960 \mathrm{lb}$ & TO-80-503G-1 & $\begin{array}{l}\text { About } 15 \text { in. of } \\
\text { sample }\end{array}$ \\
\hline
\end{tabular}


Table A.25. FY 1998 RGS Summary Data Sheet

Tank U-109 Riser 8

\begin{tabular}{|c|c|c|c|c|c|c|c|c|}
\hline $\begin{array}{c}\text { Sampler Serial } \\
\text { Number } \\
* * * * * * * * * * * * * \\
\\
\text { Cask Serial } \\
\text { Number } \\
* * * * * * * * * * * * * \\
\text { Sample Number }\end{array}$ & $\begin{array}{l}\text { Time and } \\
\text { Date } \\
\text { of Sampling } \\
\text { (just after } \\
\text { pintle rod has } \\
\text { been pulled) }\end{array}$ & $\begin{array}{l}\text { Max Force to } \\
\text { Unseat Sampler } \\
\text { (initiation of } \\
\text { sampler removal } \\
\text { from tank) } \\
\text { Time } \\
\text { Date }\end{array}$ & $\begin{array}{c}\text { Purge Gas } \\
* * * * * * * *) \\
\text { DSP = Drill } \\
\text { String Purge } \\
* * * * * * * * * * * \\
\text { Time } \\
\text { Date }\end{array}$ & $\begin{array}{c}\text { Amount of } \\
\text { Hydrostatic } \\
\text { Head Fluid } \\
\text { Added }\end{array}$ & $\begin{array}{c}\text { Radiation } \\
\text { Dose Rate } \\
\text { Reading } \\
\text { Through } \\
\text { Drill } \\
\text { String }\end{array}$ & $\begin{array}{l}\text { Strip Chart } \\
\text { Downforce } \\
\text { Max Load } \\
\text { Avg Load } \\
\text { Duration }\end{array}$ & $\begin{array}{c}\text { Procedure } \\
\text { Version and } \\
\text { Procedural } \\
\text { Exceptions }\end{array}$ & $\begin{array}{c}\text { X-Ray } \\
\text { Observation } \\
\text { Notes }\end{array}$ \\
\hline $\begin{array}{l}\text { RGS-092 seg } 2 \\
\text { C-1028 } \\
238-02 \\
\end{array}$ & $\begin{array}{l}2205 \\
04-22-98 \\
\end{array}$ & $\begin{array}{l}213 \mathrm{lb} \\
2223 \\
04-22-98\end{array}$ & $\begin{array}{l}\text { No DSP } \\
\text { recorded }\end{array}$ & $\begin{array}{l}1500 \mathrm{~mL} \\
0.3 \mathrm{M} \mathrm{LiBr}\end{array}$ & $1.5 \mathrm{R} / \mathrm{hr}$ & $\mathrm{NA}$ & $\begin{array}{l}\text { TO-80-503G- } \\
l\end{array}$ & $\begin{array}{l}\text { About } 16 \text { in. } \\
\text { of sample }\end{array}$ \\
\hline $\begin{array}{l}\text { RGS-093 seg } 4 \\
27 \mathrm{G} \\
238-04\end{array}$ & $\begin{array}{l}1034 \\
04-23-98\end{array}$ & $\begin{array}{l}188 \mathrm{lb} \\
1046 \\
04-23-98\end{array}$ & $\begin{array}{l}\text { No DSP } \\
\text { recorded }\end{array}$ & $\begin{array}{l}1500 \mathrm{~mL} \\
0.3 \mathrm{M} \mathrm{LiBr}\end{array}$ & $1 \mathrm{R} / \mathrm{hr}$ & $930 \mathrm{lb}$ & $\begin{array}{l}\text { TO-80-503G- } \\
1\end{array}$ & $\begin{array}{l}\text { About } 15.5 \\
\text { in. of sample }\end{array}$ \\
\hline $\begin{array}{l}\text { RGS-096 seg } 6 \\
7 G \\
238-06\end{array}$ & 1027 & $\begin{array}{l}230 \mathrm{lb} \\
1039 \\
04-27-98\end{array}$ & $\begin{array}{l}\text { No DSP } \\
\text { recorded }\end{array}$ & $\begin{array}{l}1500 \mathrm{~mL} \\
0.3 \mathrm{M} \mathrm{LiBr}\end{array}$ & $1 \mathrm{R} / \mathrm{hr}$ & $1080 \mathrm{lb}$ & $\begin{array}{l}\text { TO-80-503G- } \\
1\end{array}$ & $\begin{array}{l}\text { About } 16.5 \\
\text { in. of sample }\end{array}$ \\
\hline $\begin{array}{l}\text { RGS-095 seg } 8 \\
\text { C-1047 } \\
238-08\end{array}$ & $\begin{array}{l}0939 \\
04-28-98\end{array}$ & $\begin{array}{l}0951 \\
04-28-98\end{array}$ & $\begin{array}{l}\text { No DSP } \\
\text { recorded }\end{array}$ & $\begin{array}{c}1500 \mathrm{~mL} \\
0.3 \mathrm{M} \mathrm{LiBr}\end{array}$ & $800 \mathrm{mR} / \mathrm{hr}$ & $630 \mathrm{lb}$ & $\begin{array}{l}\text { TO-80-503G- } \\
1\end{array}$ & $\begin{array}{l}\text { About } 9 \mathrm{in} . \\
\text { of } \\
\text { sample }\end{array}$ \\
\hline
\end{tabular}



RGS Field Data for Tank 241-SY-101 

Table A.26. SY-101 Summary Data Sheet

Riser 022 (23A)

\begin{tabular}{|c|c|c|c|c|c|c|c|c|}
\hline 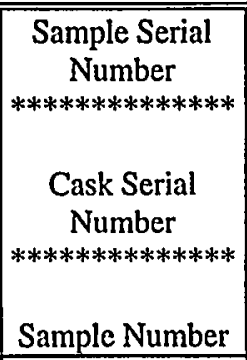 & $\begin{array}{c}\text { Time and Date } \\
\text { of Sampling } \\
\text { (just after } \\
\text { pintle rod } \\
\text { has been } \\
\text { pulled) }\end{array}$ & \begin{tabular}{|c|} 
Max Force to \\
Unseat Sampler \\
(initiation of \\
sampler removal \\
from tank) \\
Time \\
Date \\
\end{tabular} & $\begin{array}{c}\text { Purge Gas }{ }^{(a)} \\
* * * * * * * * * * * \\
\text { DSP }=\text { Drill } \\
\text { String Purge } \\
* * * * * * * * * * \\
\text { Time } \\
\text { Date } \\
\end{array}$ & $\begin{array}{c}\text { Amount of } \\
\text { Hydrostatic } \\
\text { Head Fluid } \\
\text { Added } \\
\end{array}$ & $\begin{array}{c}\text { Radiation } \\
\text { Dose Rate } \\
\text { Reading } \\
\text { Through Drill } \\
\text { String } \\
\text { (R/hr) } \\
\end{array}$ & $\begin{array}{l}\text { Strip Chart } \\
\text { Downforce } \\
\text { Max Load } \\
\text { Avg Load } \\
\text { Duration } \\
\end{array}$ & $\begin{array}{c}\text { Procedure } \\
\text { Version and } \\
\text { Procedural } \\
\text { Exceptions } \\
\end{array}$ & $\begin{array}{c}\text { X-Ray } \\
\text { Observation } \\
\text { Notes } \\
\end{array}$ \\
\hline $\begin{array}{l}\text { RGS 103 seg } 1 \\
\text { C-1042 } \\
255-01\end{array}$ & $\begin{array}{l}19: 30 \mathrm{hr} \\
11-09-98\end{array}$ & $\begin{array}{c}83 \mathrm{lb} \\
19: 45 \mathrm{hr} \\
11-09-98\end{array}$ & & $1750 \mathrm{~mL} \mathrm{LiBr}$ & 1.8 & & T0-080-504 A2 & 9 in. of sample \\
\hline $\begin{array}{l}\text { RGS-099 seg } 2 \\
2 G \\
255-02\end{array}$ & $\begin{array}{l}20: 40 \mathrm{hr} \\
11-09-98\end{array}$ & $\begin{array}{c}79 \mathrm{lb} \\
20: 55 \mathrm{hr} \\
11-09-98\end{array}$ & & $1750 \mathrm{~mL} \mathrm{LiBr}$ & 1.5 & & T0-080-504 A2 & $\sim 12$ in. of sample \\
\hline $\begin{array}{l}\text { RGS-101 seg } 3 \\
16 G \\
255-03\end{array}$ & $\begin{array}{l}21: 50 \mathrm{hr} \\
11-09-98\end{array}$ & $\begin{array}{c}77 \mathrm{lb} \\
01: 49 \mathrm{hr} \\
11-10-98\end{array}$ & & $1750 \mathrm{~mL} \mathrm{LiBr}$ & 1.9 & ' & T0-080-504 A2 & $\begin{array}{l}\sim 11 \text { in. of } \\
\text { sample. many } \\
\text { bubbles, some } \\
\text { large }\end{array}$ \\
\hline $\begin{array}{l}\text { RGS-105 seg } 4 \\
\text { C } 2014 \\
255-04\end{array}$ & $\begin{array}{c}03: 39 \mathrm{hr} \\
11-10-98\end{array}$ & $\begin{array}{c}80 \mathrm{lb} \\
04: 06 \mathrm{hr} \\
11-10-98\end{array}$ & & $1750 \mathrm{~mL} \mathrm{LiBr}$ & 3 & & T0-080-504 A2 & $\begin{array}{l}\text { Full sampler, } \\
\text { little structure, } \\
\text { few bubbles } \\
\text { visible }\end{array}$ \\
\hline $\begin{array}{l}97-570 \text { seg } 5 \\
74 \\
255-05\end{array}$ & $\begin{array}{c}18: 00 \mathrm{hr} \\
11-10-98\end{array}$ & $\begin{array}{c}78 \mathrm{lb} \\
13: 43 \mathrm{hr} \\
11-11-98\end{array}$ & & $1750 \mathrm{~mL} \mathrm{LiBr}$ & 2.5 & & T0-080-504 A2 & $\begin{array}{l}\text { No x-ray } \\
\text { taken }\end{array}$ \\
\hline
\end{tabular}


Table A.26 (contd)

\begin{tabular}{|c|c|c|c|c|c|c|c|c|}
\hline $\begin{array}{c}\text { Sampler Serial } \\
\text { Number } \\
* * * * * * * * * * * * * * * * \\
\text { Cask Serial } \\
\text { Number } \\
* * * * * * * * * * * * * * * \\
\text { Sample Number }\end{array}$ & $\begin{array}{l}\text { Time and Date } \\
\text { of Sampling } \\
\text { (just after pintle } \\
\text { rod has been } \\
\text { pulled) }\end{array}$ & $\begin{array}{c}\text { Max Force to } \\
\text { Unseat Sampler } \\
\text { (initiation of } \\
\text { sampler removal } \\
\text { from tank) } \\
\text { Time } \\
\text { Date }\end{array}$ & $\begin{array}{c}\text { Purge Gas } \\
* * * * * * * * * * * * \\
\text { DSP }=\text { Drill } \\
\text { String Purge } \\
* * * * * * * * * * * \\
\text { Time } \\
\text { Date }\end{array}$ & $\begin{array}{c}\text { Amount of } \\
\text { Hydrostatic } \\
\text { Head Fluid } \\
\text { Added }\end{array}$ & $\begin{array}{c}\text { Radiation } \\
\text { Dose Rate } \\
\text { Reading } \\
\text { Through Drill } \\
\text { String } \\
\text { (R/hr) }\end{array}$ & $\begin{array}{l}\text { Strip Chart } \\
\text { Downforce } \\
\text { Max Load } \\
\text { Avg Load } \\
\text { Duration }\end{array}$ & $\begin{array}{l}\text { Procedure } \\
\text { Version and } \\
\text { Procedural } \\
\text { Exceptions }\end{array}$ & $\begin{array}{l}\text { X-Ray } \\
\text { Observation } \\
\text { Notes }\end{array}$ \\
\hline $\begin{array}{l}97-346103 \text { seg } 6 \\
1024 \\
255-06\end{array}$ & $\begin{array}{c}18: 00 \mathrm{hr} \\
11-11-98\end{array}$ & $\begin{array}{c}72 \mathrm{lb} \\
18: 07 \mathrm{hr} \\
11-11-98\end{array}$ & & $1750 \mathrm{~mL} \mathrm{LiBr}$ & 2.4 & & T0-080-504 A2 & Full sampler \\
\hline $\begin{array}{l}\text { RGS-098 seg } 7 \\
1027 \\
255-07\end{array}$ & \multicolumn{8}{|c|}{$\begin{array}{l}\text { SAMPLER } \\
\text { DESTROYED }\end{array}$} \\
\hline $\begin{array}{l}\text { RGS-104 seg } 8 \\
1027 \\
255-08 \\
\end{array}$ & $\begin{array}{c}10: 45 \mathrm{hr} \\
11-18-98\end{array}$ & $\begin{array}{c}86 \mathrm{lb} \\
11: 00 \mathrm{hr} \\
11-18-98\end{array}$ & & $1750 \mathrm{~mL} \mathrm{LiBr}$ & 1.8 & & T0-080-504 A2 & $\begin{array}{l}\text { Sampler full, top } \\
\text { third had moving } \\
\text { bubbles }\end{array}$ \\
\hline $\begin{array}{l}97-571 \text { seg } 9 \\
\text { SN-73 } \\
255-09\end{array}$ & $\begin{array}{l}18: 37 \mathrm{hr} \\
11-18-98\end{array}$ & $\begin{array}{c}82 \mathrm{lb} \\
18: 59 \mathrm{hr} \\
11-18-98\end{array}$ & & $1750 \mathrm{~mL} \mathrm{LiBr}$ & 2.8 & & T0-080-504 A2 & $\begin{array}{l}19 \text { in. of sample } \\
\text { gas bubbles } \\
\text { observed }\end{array}$ \\
\hline $\begin{array}{l}97-573 \text { seg } 10 \\
\text { SN } 71 \\
255-10\end{array}$ & $\begin{array}{l}20: 10 \mathrm{hr} \\
11-18-98\end{array}$ & $\begin{array}{c}81 \mathrm{lb} \\
20: 29 \mathrm{hr} \\
11-18-98\end{array}$ & & $1750 \mathrm{~mL} \mathrm{LiBr}$ & 2.8 & & T0-080-504 A2 & 19 in. of sample \\
\hline
\end{tabular}


Table A.26 (contd)

\begin{tabular}{|c|c|c|c|c|c|c|c|c|}
\hline \begin{tabular}{|c|} 
Sampler Serial \\
Number \\
$* * * * * * * * * * * * * *$ \\
\\
Cask Serial \\
Number \\
$* * * * * * * * * * * * *$ \\
\\
Sample Number \\
\end{tabular} & $\begin{array}{l}\text { Time and Date } \\
\text { of Sampling } \\
\text { (just after } \\
\text { pintle rod } \\
\text { has been } \\
\text { pulled) } \\
\end{array}$ & $\begin{array}{c}\text { Max Force to } \\
\text { Unseat Sampler } \\
\text { (initiation of } \\
\text { sampler removal } \\
\text { from tank) } \\
\text { Time } \\
\text { Date } \\
\end{array}$ & $\begin{array}{c}\text { Purge Gas } \\
* * * * * * * * * * * * \\
\text { DSP }=\text { Drill } \\
\text { String Purge } \\
* * * * * * * * * * * * \\
\text { Time } \\
\text { Date } \\
\end{array}$ & $\begin{array}{c}\text { Amount of } \\
\text { Hydrostatic } \\
\text { Head Fluid } \\
\text { Added } \\
\end{array}$ & $\begin{array}{l}\text { Radiation } \\
\text { Dose Rate } \\
\text { Reading } \\
\text { Through Drill } \\
\text { String } \\
\\
\text { (R/hr) } \\
\end{array}$ & $\begin{array}{l}\text { Strip Chart } \\
\text { Downforce } \\
\text { Max Load } \\
\text { Avg Load } \\
\text { Duration } \\
\end{array}$ & $\begin{array}{l}\text { Procedure } \\
\text { Version and } \\
\text { Procedural } \\
\text { Exceptions }\end{array}$ & $\begin{array}{c}\text { X-Ray } \\
\text { Observation } \\
\text { Notes } \\
\end{array}$ \\
\hline $\begin{array}{l}97-324 \text { seg } 11 \\
\text { C } 1040 \\
255-11\end{array}$ & $\begin{array}{l}21: 30 \mathrm{hr} \\
11-18-98\end{array}$ & $\begin{array}{c}72 \mathrm{lb} \\
01: 10 \mathrm{hr} \\
11-19-98\end{array}$ & & $1750 \mathrm{~L} \mathrm{LiBr}$ & 2 & & T0-080-504 A2 & $18 \mathrm{in}$. of sample \\
\hline $\begin{array}{l}97-597 \text { seg } 12 \\
22-G \\
255-12\end{array}$ & $\begin{array}{l}02: 00 \mathrm{hr} \\
11-19-98\end{array}$ & $\begin{array}{c}70 \mathrm{lb} \\
02: 10 \mathrm{hr} \\
11-19-98\end{array}$ & & $1750 \mathrm{~mL} \mathrm{LiBr}$ & 2.1 & & T0-080-504 A2 & Full sampler \\
\hline \begin{tabular}{|l} 
RGS-100 seg 13 \\
$5 G$ \\
$255-13$ \\
\end{tabular} & $\begin{array}{l}02: 50 \mathrm{hr} \\
11-19-98\end{array}$ & $\begin{array}{c}71 \mathrm{lb} \\
03: 00 \mathrm{hr}\end{array}$ & & $1750 \mathrm{~mL} \mathrm{LiBr}$ & 2 & & T0-080-504 A2 & $\begin{array}{l}\mathrm{X} \text {-ray and } \\
\text { radiation dose } \\
\text { rate conflict }\end{array}$ \\
\hline \begin{tabular}{|l}
$97-323$ seg 14 \\
SN-64 \\
$255-14$ \\
\end{tabular} & $\begin{array}{l}03: 50 \mathrm{hr} \\
11-19-98\end{array}$ & $\begin{array}{c}70 \mathrm{lb} \\
04: 05 \mathrm{hr} \\
11-19-98\end{array}$ & & $1750 \mathrm{~mL} \mathrm{LiBr}$ & 2 & & T0-080-504 A2 & Full sampler \\
\hline $\begin{array}{l}97-576 \operatorname{seg} 15 \\
\text { SN 30G } \\
255-15\end{array}$ & $\begin{array}{l}04: 50 \mathrm{hr} \\
11-19-98\end{array}$ & $\begin{array}{c}82 \mathrm{lb} \\
21: 47 \mathrm{hr} \\
11-19-98\end{array}$ & & $1750 \mathrm{~mL} \mathrm{LiBr}$ & 2.9 & . & T0-080-504 A2 & $\begin{array}{l}\text { Full sampler } \\
\text { bubbles observed }\end{array}$ \\
\hline Orill string $\mathrm{p}$ & - are not alu & recorded on the & strial Hua & PI a curyay ra & & & & \\
\hline
\end{tabular}


Table A.26 (contd)

\begin{tabular}{|c|c|c|c|c|c|c|c|c|}
\hline 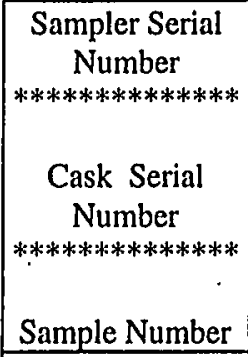 & $\begin{array}{c}\text { Time and Date } \\
\text { of Sampling } \\
\text { (just after pintle } \\
\text { rod has been } \\
\text { pulled) }\end{array}$ & $\begin{array}{c}\text { Max Force to } \\
\text { Unseat Sampler } \\
\text { (initiation of sampler } \\
\text { removal from tank) } \\
\text { Time } \\
\text { Date }\end{array}$ & 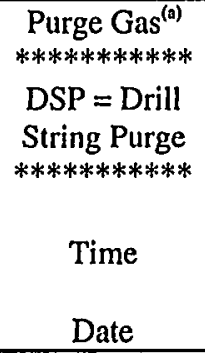 & $\begin{array}{c}\text { Amount of } \\
\text { Hydrostatic } \\
\text { Head Fluid } \\
\text { Added }\end{array}$ & $\begin{array}{c}\text { Radiation Dose } \\
\text { Rate Reading } \\
\text { Through Drill } \\
\text { String } \\
\text { (R/hr) }\end{array}$ & $\begin{array}{l}\text { Strip Chart } \\
\text { Downforce } \\
\text { Max Load } \\
\text { Avg Load } \\
\text { Duration } \\
\end{array}$ & $\begin{array}{l}\text { Procedure } \\
\text { Version and } \\
\text { Procedural } \\
\text { Exceptions }\end{array}$ & $\begin{array}{c}\text { X-Ray } \\
\text { Observation } \\
\text { Notes }\end{array}$ \\
\hline $\begin{array}{l}97-584 \text { seg } 15 A \\
\text { C } 1017 \\
255-15 A\end{array}$ & $\begin{array}{c}01: 15 \mathrm{hr} \\
11-20-98\end{array}$ & $\begin{array}{c}75 \mathrm{lb} \\
13: 58 \mathrm{hr} \\
11-23-98\end{array}$ & & $1750 \mathrm{~mL} \mathrm{LiBr}$ & 2.7 & & T0-080-504 A2 & No notes \\
\hline $\begin{array}{l}97-561 \text { seg } 16 \\
24-G \\
255-16 \\
\end{array}$ & $\begin{array}{c}10: 30 \mathrm{hr} \\
12-16-98\end{array}$ & $\begin{array}{c}86 \mathrm{lb} \\
10: 37 \mathrm{hr} \\
12-16-98\end{array}$ & & $1750 \mathrm{~mL} \mathrm{LiBr}$ & 3 & & T0-080-504 A2 & $19 \mathrm{in.}$ \\
\hline $\begin{array}{l}97-569 \text { seg } 17 \\
14-G \\
255-17 \\
\end{array}$ & $\begin{array}{c}11: 00 \mathrm{hr} \\
12-16-98\end{array}$ & $\begin{array}{c}78 \mathrm{lb} \\
13: 20 \mathrm{hr} \\
12-16-98\end{array}$ & & $1750 \mathrm{~mL} \mathrm{LiBr}$ & 2.8 & & T0-080-504 A2 & Full sampler \\
\hline $\begin{array}{l}97-582 \operatorname{seg} 18 \\
\text { C-1032 } \\
255-18\end{array}$ & $\begin{array}{c}13: 52 \mathrm{hr} \\
12-16-98\end{array}$ & $\begin{array}{c}73 \mathrm{lb} \\
14: 00 \mathrm{hr} \\
12-16-98\end{array}$ & & $1750 \mathrm{~mL} \mathrm{LiBr}$ & 2.9 & & T0-080-504 A2 & $19 \mathrm{in.}$ \\
\hline $\begin{array}{l}97-325 \text { seg } 19 \\
\text { C } 1018 \\
255-19\end{array}$ & $\begin{array}{c}14: 05 \mathrm{hr} \\
12-17-98\end{array}$ & $\begin{array}{c}72 \mathrm{lb} \\
21: 05 \mathrm{hr} \\
12-17-98\end{array}$ & & $1750 \mathrm{~mL} \mathrm{LiBr}$ & 2.9 & & T0-080-504 A2 & $19 \mathrm{in}$. of sample \\
\hline
\end{tabular}


Table A.26 (contd)

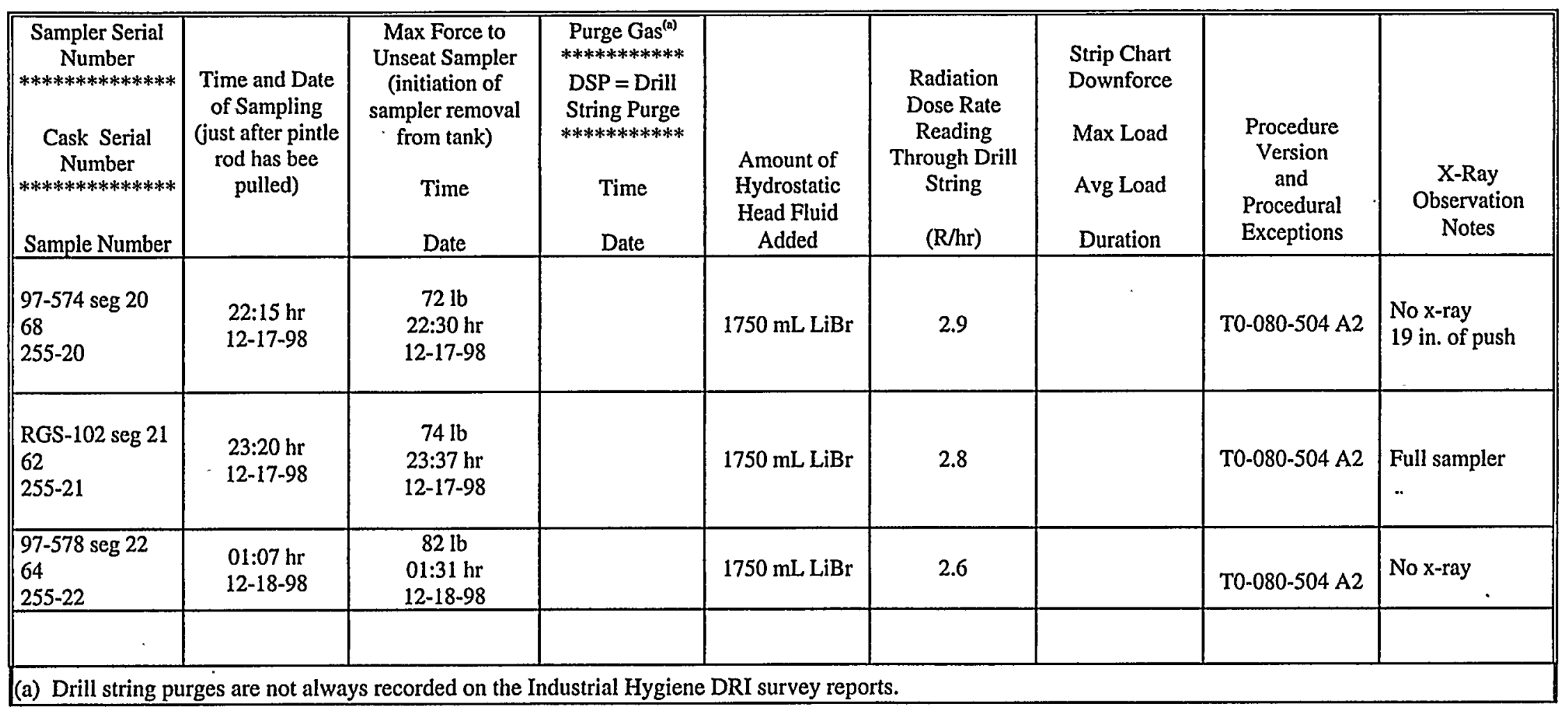


Table A.27. SY-101 RGS Summary Data Sheet

\section{Riser 021 (22A)}

\begin{tabular}{|c|c|c|c|c|c|c|c|c|}
\hline $\begin{array}{c}\text { Sampler Serial } \\
\text { Number } \\
* * * * * * * * * * * * * * \\
\text { Cask Serial } \\
\text { Number } \\
\text { *************** } \\
\text { Sample Number }\end{array}$ & $\begin{array}{l}\text { Time and Date } \\
\text { of Sampling } \\
\text { (just after pintle } \\
\text { rod has been } \\
\text { pulled) }\end{array}$ & $\begin{array}{l}\text { Max Force to } \\
\text { Unseat Sampler } \\
\text { (initiation of } \\
\text { sampler removal } \\
\text { from tank) } \\
\text { Time } \\
\text { Date }\end{array}$ & $\begin{array}{c}\text { Purge Gas }{ }^{(a)} \\
* * * * * * * * * * * \\
\text { DSP = Drill } \\
\text { String Purge } \\
* * * * * * * * * * * \\
\text { Time } \\
\text { Date }\end{array}$ & $\begin{array}{c}\text { Amount of } \\
\text { Hydrostatic } \\
\text { Head Fluid } \\
\text { Added }\end{array}$ & $\begin{array}{l}\text { Radiation Dose } \\
\text { Rate Reading } \\
\text { Through Drill } \\
\text { String }\end{array}$ & $\begin{array}{l}\text { Strip Chart } \\
\text { Downforce } \\
\text { Max Load } \\
\text { Avg Load } \\
\text { Duration }\end{array}$ & $\begin{array}{l}\text { Procedure } \\
\text { Version and } \\
\text { Procedural } \\
\text { Exceptions }\end{array}$ & $\begin{array}{c}\text { X-Ray } \\
\text { Observation } \\
\text { Notes }\end{array}$ \\
\hline $\begin{array}{l}\text { RGS-106, seg } 1 \\
\text { C-1040 } \\
256-01\end{array}$ & $\begin{array}{l}20: 37 \mathrm{hr} \\
01-05-99\end{array}$ & $\begin{array}{c}76 \mathrm{lb} \\
21: 00 \mathrm{hr} \\
01 / 05 / 99 \\
\end{array}$ & - & $1750 \mathrm{~mL} \mathrm{LiBr}$ & $1.6 \mathrm{R}$ & & $\begin{array}{c}\text { T0-080-504 } \\
\text { A-4 }\end{array}$ & $\begin{array}{l}\text { No gas bubbles } \\
14 \mathrm{in} \text {. of sample }\end{array}$ \\
\hline $\begin{array}{l}\text { RGS-107 seg } 2 \\
\text { C-1242 } \\
256-02 \\
\end{array}$ & $\begin{array}{l}14: 15 \mathrm{hr} \\
01 / 06 / 99\end{array}$ & $\begin{array}{c}76 \mathrm{lb} \\
14: 37 \mathrm{hr} \\
01 / 06 / 99 \\
\end{array}$ & & $1750 \mathrm{~mL} \mathrm{LiBr}$ & $1.9 \mathrm{R}$ & & $\begin{array}{c}\mathrm{T} 0-080-504 \\
\mathrm{~A}-4\end{array}$ & $14 \mathrm{in}$. of sample \\
\hline $\begin{array}{l}\text { RGS-110 seg } 3 \\
\text { C-2014 } \\
256-03\end{array}$ & $\begin{array}{l}19: 45 \mathrm{hr} \\
01 / 06 / 99\end{array}$ & $\begin{array}{c}76 \mathrm{lb} \\
19: 58 \mathrm{hr} \\
01 / 06 / 99\end{array}$ & & $1750 \mathrm{~mL} \mathrm{LiBr}$ & $1.7 \mathrm{R}$ & & $\begin{array}{c}\text { T0-080-504 } \\
\text { A-4 }\end{array}$ & $\begin{array}{l}\text { Some gas } \\
\text { pockets } \\
14 \text { in. of } \\
\text { sample }\end{array}$ \\
\hline $\begin{array}{l}\text { RGS-109 seg } 4 \\
6-G \\
256-04\end{array}$ & $\begin{array}{l}10: 22 \mathrm{hr} \\
01-07-99\end{array}$ & $\begin{array}{c}80 \mathrm{lb} \\
10: 37 \mathrm{hr} \\
01-07-99\end{array}$ & & $1750 \mathrm{~mL} \mathrm{LiBr}$ & $1.8 \mathrm{R}$ & & $\begin{array}{c}\text { T0-080-504 } \\
\text { A-4 }\end{array}$ & $\begin{array}{l}19 \mathrm{in} \text {. of push } \\
\text { waste on outside } \\
\text { of sampler }\end{array}$ \\
\hline $\begin{array}{l}97-266 \text { seg } 5 \\
22-G \\
256-05\end{array}$ & $\begin{array}{l}10: 27 \mathrm{hr} \\
01-08-99\end{array}$ & $\begin{array}{c}78 \mathrm{lb} \\
13: 30 \mathrm{hr} \\
01-08-99\end{array}$ & $\begin{array}{l}1-7-99 \\
1920 \mathrm{hr}\end{array}$ & $1750 \mathrm{~mL} \mathrm{LiBr}$ & $2.7 \mathrm{R}$ & & $\begin{array}{c}\text { T0-080-504 } \\
\text { A-4 }\end{array}$ & $\begin{array}{l}\text { Waste on bottom } \\
\text { of sampler }\end{array}$ \\
\hline
\end{tabular}


Table A.27 (contd)

\begin{tabular}{|c|c|c|c|c|c|c|c|c|}
\hline $\begin{array}{c}\text { Sampler Serial } \\
\text { Number } \\
* * * * * * * * * * * * * * \\
\\
\text { Cask Serial } \\
\text { Number } \\
* * * * * * * * * * * * * \\
\text { Sample Number }\end{array}$ & $\begin{array}{l}\text { Time and Date } \\
\text { of Sampling } \\
\text { (just after } \\
\text { pintle rod } \\
\text { has been } \\
\text { pulled) }\end{array}$ & $\begin{array}{l}\text { Max Force to } \\
\text { Unseat Sampler } \\
\text { (initiation of } \\
\text { sampler removal } \\
\text { from tank) } \\
\text { Time } \\
\text { Date } \\
\end{array}$ & $\begin{array}{c}\text { Purge Gas }{ }^{()^{2}} \\
* * * * * * * * * * * \\
\text { DSP = Drill } \\
\text { String Purge } \\
* * * * * * * * * * * * \\
\text { Time } \\
\text { Date } \\
\end{array}$ & $\begin{array}{c}\text { Amount of } \\
\text { Hydrostatic } \\
\text { Head Fluid } \\
\text { Added }\end{array}$ & $\begin{array}{l}\text { Radiation } \\
\text { Dose Rate } \\
\text { Reading } \\
\text { Through Drill } \\
\text { String }\end{array}$ & $\begin{array}{l}\text { Strip Chart } \\
\text { Downforce } \\
\text { Max Load } \\
\text { Avg Load } \\
\text { Duration } \\
\end{array}$ & $\begin{array}{l}\text { Procedure } \\
\text { Version and } \\
\text { Procedural } \\
\text { Exceptions }\end{array}$ & $\begin{array}{c}\text { X-Ray } \\
\text { Observation } \\
\text { Notes }\end{array}$ \\
\hline $\begin{array}{l}97-522 \text { seg } 6 \\
\text { C-1017 } \\
256-06\end{array}$ & $\begin{array}{c}18: 05 \mathrm{hr} \\
01-08-99\end{array}$ & $\begin{array}{c}75 \mathrm{lb} \\
18: 10 \mathrm{hr} \\
01-08-99\end{array}$ & $\begin{array}{c}1-8-99 \\
13: 05 \mathrm{hr} \\
13: 45 \mathrm{hr} \\
14: 13 \mathrm{hr} \\
14: 35 \mathrm{hr} \\
\end{array}$ & $1750 \mathrm{~mL} \mathrm{LiBr}$ & $2.9 \mathrm{R}$ & & $\begin{array}{c}\text { T0-080-504 } \\
\text { A-4 }\end{array}$ & Full sampler \\
\hline $\begin{array}{l}97-254 \operatorname{seg} 7 \\
30-G \\
256-07\end{array}$ & $\begin{array}{c}11: 05 \mathrm{hr} \\
01-12-99\end{array}$ & $\begin{array}{c}95 \mathrm{lb} \\
11: 17 \mathrm{hr} \\
01-12-99\end{array}$ & $\begin{array}{l}1-8-99 \\
17: 40 \mathrm{hr} \\
18: 20 \mathrm{hr}\end{array}$ & $1750 \mathrm{~mL} \mathrm{LiBr}$ & $2.4 \mathrm{R}$ & & $\begin{array}{c}\text { T0-080-504 } \\
\mathrm{A}-4\end{array}$ & No notes \\
\hline $\begin{array}{l}97-051 \text { seg } 8 \\
\text { SN-1037 } \\
256-08\end{array}$ & $\begin{array}{c}12: 00 \mathrm{hr} \\
01-12-99\end{array}$ & $\begin{array}{c}74 \mathrm{lb} \\
12: 25 \mathrm{hr} \\
01-12-99\end{array}$ & & $1750 \mathrm{~mL} \mathrm{LiBr}$ & $2.5 \mathrm{R}$ & $\cdot$ & $\underset{\mathrm{A}-4}{\mathrm{~T} 0-080-504}$ & No notes \\
\hline $\begin{array}{l}97-062 \text { seg } 9 \\
\text { SN-73 } \\
256-09 \\
\end{array}$ & $\begin{array}{c}13: 10 \mathrm{hr} \\
01-12-99\end{array}$ & $\begin{array}{c}94 \mathrm{lb} \\
20: 37 \mathrm{hr} \\
01-12-99\end{array}$ & & $1750 \mathrm{~mL} \mathrm{LiBr}$ & $2.8 \mathrm{R}$ & & $\begin{array}{c}\text { T0-080-504 } \\
\text { A-4 }\end{array}$ & $\begin{array}{l}\text { No } x \text {-ray } \\
19 \text { in. push }\end{array}$ \\
\hline $\begin{array}{l}\text { RGS-090 seg } 10 \\
\text { SN-74 } \\
256-10\end{array}$ & $\begin{array}{l}21: 30 \mathrm{hr} \\
01-12-99\end{array}$ & $\begin{array}{c}75 \mathrm{lb} \\
21: 42 \mathrm{hr} \\
01-12-99\end{array}$ & & $1750 \mathrm{~mL} \mathrm{LiBr}$ & $3.0 \mathrm{R}$ & & $\begin{array}{c}\mathrm{T} 0-080-504 \\
\mathrm{~A}-4\end{array}$ & $19 \mathrm{in.} \mathrm{of} \mathrm{sample}$ \\
\hline
\end{tabular}


Table A.27 (contd)

\begin{tabular}{|c|c|c|c|c|c|c|c|c|}
\hline $\begin{array}{c}\text { Sampler Serial } \\
\text { Number } \\
* * * * * * * * * * * * * \\
\text { Cask Serial } \\
\text { Number } \\
* * * * * * * * * * * * * \\
\text { Sample Number }\end{array}$ & $\begin{array}{l}\text { Time and Date } \\
\text { of Sampling } \\
\text { (just after } \\
\text { pintle rod has } \\
\text { been pulled) }\end{array}$ & $\begin{array}{l}\text { Max Force to } \\
\text { Unscat Sampler } \\
\text { (initiation of } \\
\text { sampler removal } \\
\text { from tank) } \\
\text { Time } \\
\text { Date }\end{array}$ & $\begin{array}{c}\text { Purge Gas } \\
* * * * * * * * * * * \\
\text { DSP = Drill } \\
\text { String Purge } \\
* * * * * * * * * * * \\
\text { Time } \\
\text { Date } \\
\end{array}$ & $\begin{array}{c}\text { Amount of } \\
\text { Hydrostatic } \\
\text { Head Fluid } \\
\text { Added }\end{array}$ & $\begin{array}{c}\text { Radiation } \\
\text { Dose Rate } \\
\text { Reading } \\
\text { Through Drill } \\
\text { String } \\
\end{array}$ & $\begin{array}{l}\text { Strip Chart } \\
\text { Downforce } \\
\text { Max Load } \\
\text { Avg Load } \\
\text { Duration }\end{array}$ & $\begin{array}{l}\text { Procedure } \\
\text { Version } \\
\text { and } \\
\text { Procedural } \\
\text { Exceptions } \\
\end{array}$ & $\begin{array}{c}\text { X-Ray } \\
\text { Observation } \\
\text { Notes }\end{array}$ \\
\hline $\begin{array}{l}97-544 \operatorname{seg} 11 \\
\text { C-2015 } \\
256-11\end{array}$ & $\begin{array}{l}23: 00 \mathrm{hr} \\
01-12-99\end{array}$ & $\begin{array}{c}78 \mathrm{lb} \\
23: 34 \mathrm{hr} \\
01-12-99\end{array}$ & & $1750 \mathrm{~mL} \mathrm{LiBr}$ & $2.7 \mathrm{R}$ & & $\begin{array}{c}\text { T0-080-504 } \\
\text { A-4 }\end{array}$ & $\begin{array}{l}19 \text { in. of push } \\
\text { no x-ray }\end{array}$ \\
\hline $\begin{array}{l}97-254 \operatorname{seg} 12 \\
\text { SN-71 } \\
256-12\end{array}$ & $\begin{array}{c}00: 25 \mathrm{hr} \\
01-13-99\end{array}$ & $\begin{array}{c}72 \mathrm{lb} \\
00: 40 \mathrm{hr} \\
01-13-99\end{array}$ & & $1750 \mathrm{~mL} \mathrm{LiBr}$ & $2.8 \mathrm{R}$ & & $\begin{array}{c}\text { T0-080-504 } \\
\text { A-4 }\end{array}$ & $\begin{array}{l}19 \text { in. of push } \\
\text { no } x \text {-ray }\end{array}$ \\
\hline $\begin{array}{l}97-543 \operatorname{seg} 13 \\
3-G \\
256-13\end{array}$ & $\begin{array}{c}01: 25 \mathrm{hr} \\
01-13-99\end{array}$ & $\begin{array}{c}78 \mathrm{lb} \\
01: 38 \mathrm{hr} \\
01-13-99\end{array}$ & & $1750 \mathrm{~mL} \mathrm{LiBr}$ & $2.8 \mathrm{R}$ & & $\begin{array}{c}\text { T0-080-504 } \\
\text { A-4 }\end{array}$ & $\begin{array}{l}19 \mathrm{in.} \text { of push } \\
\text { no } \mathrm{x} \text {-ray }\end{array}$ \\
\hline $\begin{array}{l}97-503 \operatorname{seg} 14 \\
5-\mathrm{G} \\
256-14\end{array}$ & $\begin{array}{c}02: 20 \mathrm{hr} \\
01-13-99\end{array}$ & $\begin{array}{c}79 \mathrm{lb} \\
09: 20 \mathrm{hr} \\
01-13-99\end{array}$ & & $1750 \mathrm{~mL} \mathrm{LiBr}$ & $2.5 \mathrm{R}$ & & $\begin{array}{c}\text { T0-080-504 } \\
\text { A-4 }\end{array}$ & No notes \\
\hline $\begin{array}{l}97-211 \text { seg } 15 \\
\text { C-1024 } \\
256-15\end{array}$ & $\begin{array}{c}09: 40 \mathrm{hr} \\
01-13-99\end{array}$ & $\begin{array}{c}78 \mathrm{lb} \\
10: 05 \mathrm{hr} \\
01-13-99\end{array}$ & & $1750 \mathrm{~mL} \mathrm{LiBr}$ & $2.5 \mathrm{R}$ & & $\begin{array}{c}\text { T0-080-504 } \\
\mathrm{A}-4\end{array}$ & No notes \\
\hline
\end{tabular}


Table A.27 (contd)

\begin{tabular}{|c|c|c|c|c|c|c|c|c|}
\hline \begin{tabular}{|c|} 
Sampler Serial \\
Number \\
$* * * * * * * * * * * * * * *$ \\
\\
Cask Serial \\
Number \\
$* * * * * * * * * * * * * *$ \\
Sample Number \\
\end{tabular} & $\begin{array}{c}\text { Time and Date } \\
\text { of Sampling } \\
\text { (just after pintle } \\
\text { rod has been } \\
\text { pulled) }\end{array}$ & $\begin{array}{c}\text { Max Force to } \\
\text { Unseat Sampler } \\
\text { (initiation of } \\
\text { sampler removal } \\
\text { from tank) } \\
\text { Time } \\
\text { Date } \\
\end{array}$ & $\begin{array}{c}\text { Purge Gas }{ }^{(2)} \\
* * * * * * * * * * * \\
\text { DSP }=\text { Drill } \\
\text { String Purge } \\
* * * * * * * * * * * \\
\text { Time } \\
\text { Date } \\
\end{array}$ & $\begin{array}{c}\text { Amount of } \\
\text { Hydrostatic } \\
\text { Head Fluid } \\
\text { Added }\end{array}$ & $\begin{array}{l}\text { Radiation } \\
\text { Dose Rate } \\
\text { Reading } \\
\text { Through Drill } \\
\text { String }\end{array}$ & $\begin{array}{l}\text { Strip Chart } \\
\text { Downforce } \\
\text { Max Load } \\
\text { Avg Load } \\
\text { Duration }\end{array}$ & $\begin{array}{c}\text { Procedure } \\
\text { Version and } \\
\text { Procedural } \\
\text { Exceptions }\end{array}$ & $\begin{array}{c}\text { X-Ray } \\
\text { Observation } \\
\text { Notes }\end{array}$ \\
\hline $\begin{array}{l}97-358 \text { seg } 16 \\
68 \\
256-16 \\
\end{array}$ & $\begin{array}{c}10: 20 \mathrm{hr} \\
01-13-99\end{array}$ & $\begin{array}{c}76 \mathrm{lb} \\
19: 03 \mathrm{hr} \\
01-13-99\end{array}$ & & $1750 \mathrm{~mL} \mathrm{LiBr}$ & $2.7 \mathrm{R}$ & & $\begin{array}{c}\text { T0-080-504 } \\
\text { A-4 }\end{array}$ & $\begin{array}{l}\text { No } x \text {-ray } \\
19 \text { in. push }\end{array}$ \\
\hline $\begin{array}{l}\text { RGS-108 seg } 17 \\
2 \mathrm{G} \\
256-17 \\
\end{array}$ & $\begin{array}{c}19: 44 \mathrm{hr} \\
01-13-99\end{array}$ & $\begin{array}{c}78 \mathrm{lb} \\
20: 02 \mathrm{hr} \\
01-13-99\end{array}$ & & $1750 \mathrm{~mL} \mathrm{LiBr}$ & $2.8 \mathrm{R}$ & & $\begin{array}{c}\text { T0-080-504 } \\
\mathrm{A}-4\end{array}$ & $\begin{array}{l}19 \text { in. of sample } \\
\text { some bubbles }\end{array}$ \\
\hline $\begin{array}{l}97-543 \text { seg } 18 \\
\text { C-1027 } \\
256-18 \\
\end{array}$ & $\begin{array}{c}21: 34 \mathrm{hr} \\
01-13-99\end{array}$ & $\begin{array}{c}78 \mathrm{lb} \\
21: 51 \mathrm{hr} \\
01-013-99\end{array}$ & & $1750 \mathrm{~mL} \mathrm{LiBr}$ & $2.8 \mathrm{R}$ & $\begin{array}{c}\text { Hit high } \\
\text { downforce at } \\
\text { 18-in. stroke }\end{array}$ & $\begin{array}{c}\text { T0-080-504 } \\
\text { A-4 }\end{array}$ & $\begin{array}{l}18 \mathrm{in} \text {. of stroke } \\
\text { waste on sampler } \\
\text { bottom }\end{array}$ \\
\hline $\begin{array}{l}97-536 \text { seg } 19 \\
64 \\
256-19 \\
\end{array}$ & $\begin{array}{c}09: 35 \mathrm{hr} \\
01-15-99\end{array}$ & $\begin{array}{c}73 \mathrm{lb} \\
09: 50 \mathrm{hr} \\
01-15-99\end{array}$ & & $1750 \mathrm{~mL} \mathrm{LiBr}$ & $2 . .3 \mathrm{R}$ & & $\begin{array}{c}\mathrm{T} 0-080-504 \\
\mathrm{~A}-4\end{array}$ & Full sampler \\
\hline $\begin{array}{l}97-545 \operatorname{seg} 20 \\
C-1018 \\
256-20\end{array}$ & $\begin{array}{c}10: 35 \mathrm{hr} \\
01-15-99\end{array}$ & $\begin{array}{c}73 \mathrm{lb} \\
10: 50 \mathrm{hr} \\
01-15-99\end{array}$ & & $1750 \mathrm{~mL} \mathrm{LiBr}$ & $2.2 \mathrm{R}$ & & $\underset{\mathrm{A}-4}{\mathrm{~T} 0-080-504}$ & Full sampler \\
\hline
\end{tabular}


Table A.27 (contd)

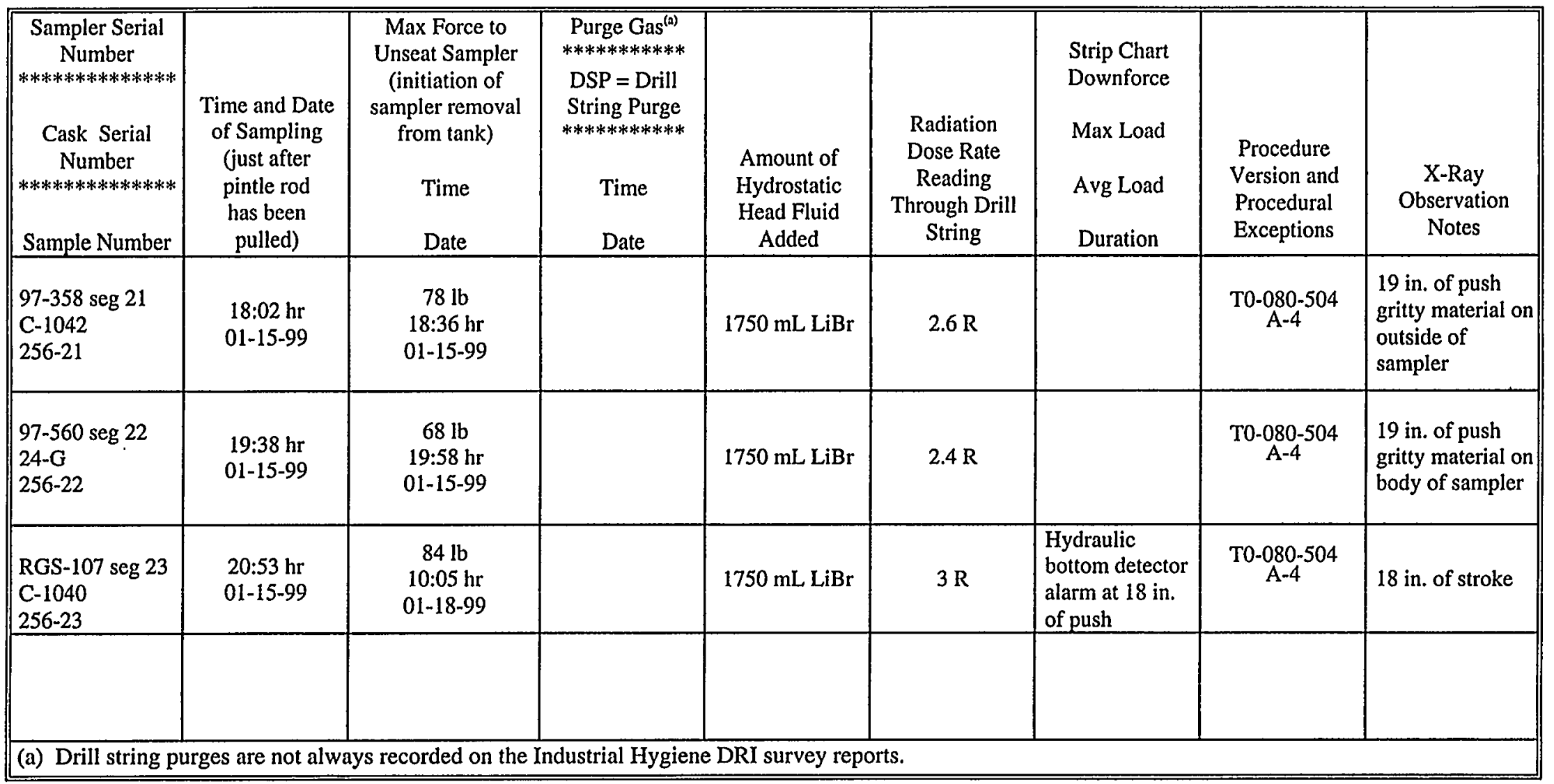


Table A.28. SY-101 RGS Summary Data Sheet

Riser 006 (4A or 4)

\begin{tabular}{|c|c|c|c|c|c|c|c|c|}
\hline $\begin{array}{c}\text { Sampler Serial } \\
\text { Number } \\
* * * * * * * * * * \\
\\
\text { Cask Serial } \\
\text { Number } \\
* * * * * * * * * * \\
\text { Sample Number }\end{array}$ & $\begin{array}{l}\text { Time and Date } \\
\text { of Sampling } \\
\text { (just after } \\
\text { pintle rod } \\
\text { has been } \\
\text { pulled) }\end{array}$ & $\begin{array}{c}\text { Max Force to } \\
\text { Unseat Sampler } \\
\text { (initiation of } \\
\text { sampler removal } \\
\text { from tank) } \\
\text { Time } \\
\text { Date }\end{array}$ & $\begin{array}{c}\text { Purge Gas } \\
* * * * * * * * * * * * * \\
\text { DSP = Drill } \\
\text { String Purge } \\
* * * * * * * * * * * \\
\text { Time } \\
\text { Date }\end{array}$ & $\begin{array}{c}\text { Amount of } \\
\text { Hydrostatic } \\
\text { Head Fluid } \\
\text { Added }\end{array}$ & $\begin{array}{c}\text { Radiation } \\
\text { Dose Rate } \\
\text { Reading } \\
\text { Through Drill } \\
\text { String }\end{array}$ & $\begin{array}{l}\text { Strip Chart } \\
\text { Downforce } \\
\text { Max Load } \\
\text { Avg Load } \\
\text { Duration }\end{array}$ & $\begin{array}{l}\text { Procedure } \\
\text { Version } \\
\text { and } \\
\text { Procedural } \\
\text { Exceptions }\end{array}$ & $\begin{array}{c}\text { X-Ray } \\
\text { Observation } \\
\text { Notes }\end{array}$ \\
\hline $\begin{array}{l}97-417 \text { seg } 1 \\
\text { SN-14G } \\
257-01 \\
\end{array}$ & $\begin{array}{l}3-8-99 \\
13: 00 \mathrm{hr}\end{array}$ & $\begin{array}{c}86 \mathrm{lb} \\
3-8-99 \\
13: 14 \mathrm{hr}\end{array}$ & & $1750 \mathrm{~mL} \mathrm{LiBr}$ & $500 \mathrm{mR}$ & & $\begin{array}{c}\text { TO-80-504 } \\
\text { Rev. A-6 }\end{array}$ & - \\
\hline $\begin{array}{l}97-295 \text { seg } 2 \\
66 \\
257-02 \\
\end{array}$ & $\begin{array}{l}3-8-99 \\
13: 59 \mathrm{hr}\end{array}$ & $\begin{array}{c}86 \mathrm{lb} \\
3-8-99 \\
14: 16 \mathrm{hr} \\
\end{array}$ & & $1750 \mathrm{~mL} \mathrm{LiBr}$ & $1.6 \mathrm{R}$ & & $\begin{array}{c}\text { TO-80-504 } \\
\text { Rev. A-6 }\end{array}$ & \\
\hline $\begin{array}{l}97-268 \operatorname{seg} 3 \\
\text { SN } 20-G \\
257-03\end{array}$ & $\begin{array}{c}3-8-99 \\
14: 54 \mathrm{hr}\end{array}$ & $\begin{array}{c}83 \mathrm{lb} \\
3-8.99 \\
20: 40 \mathrm{hr}\end{array}$ & & $1750 \mathrm{~mL} \mathrm{LiBr}$ & $1.6 \mathrm{R}$ & & $\begin{array}{c}\text { TO-80-504 } \\
\text { Rev. A-6 }\end{array}$ & \\
\hline $\begin{array}{l}\text { RGS-111 seg } 4 \\
\text { C-2011 } \\
257-04 \\
\end{array}$ & $\begin{array}{l}3-8-99 \\
21: 40 \mathrm{hr}\end{array}$ & $\begin{array}{c}80 \mathrm{lb} \\
3-8-99 \\
21: 54 \mathrm{hr} \\
\end{array}$ & & $1750 \mathrm{~mL} \mathrm{LiBr}$ & $1.8 \mathrm{R}$ & & $\begin{array}{c}\text { T0-80-504 } \\
\text { Rev. A-6 }\end{array}$ & \\
\hline $\begin{array}{l}\text { RGS-112 seg } 5 \\
\text { C-1028 } \\
275-5\end{array}$ & $\begin{array}{l}3-8-99 \\
23: 15 \mathrm{hr}\end{array}$ & $\begin{array}{c}74 \mathrm{lb} \\
3-8-99 \\
23: 26 \mathrm{hr}\end{array}$ & & $1750 \mathrm{~mL} \mathrm{LiBr}$ & $2.3 \mathrm{R}$ & & $\begin{array}{c}\text { T0-80-504 } \\
\text { Rev. A-6 }\end{array}$ & $\begin{array}{l}19 \text { in. of sample } \\
\text { and gas bubbles }\end{array}$ \\
\hline
\end{tabular}


Table A. 28 (contd)

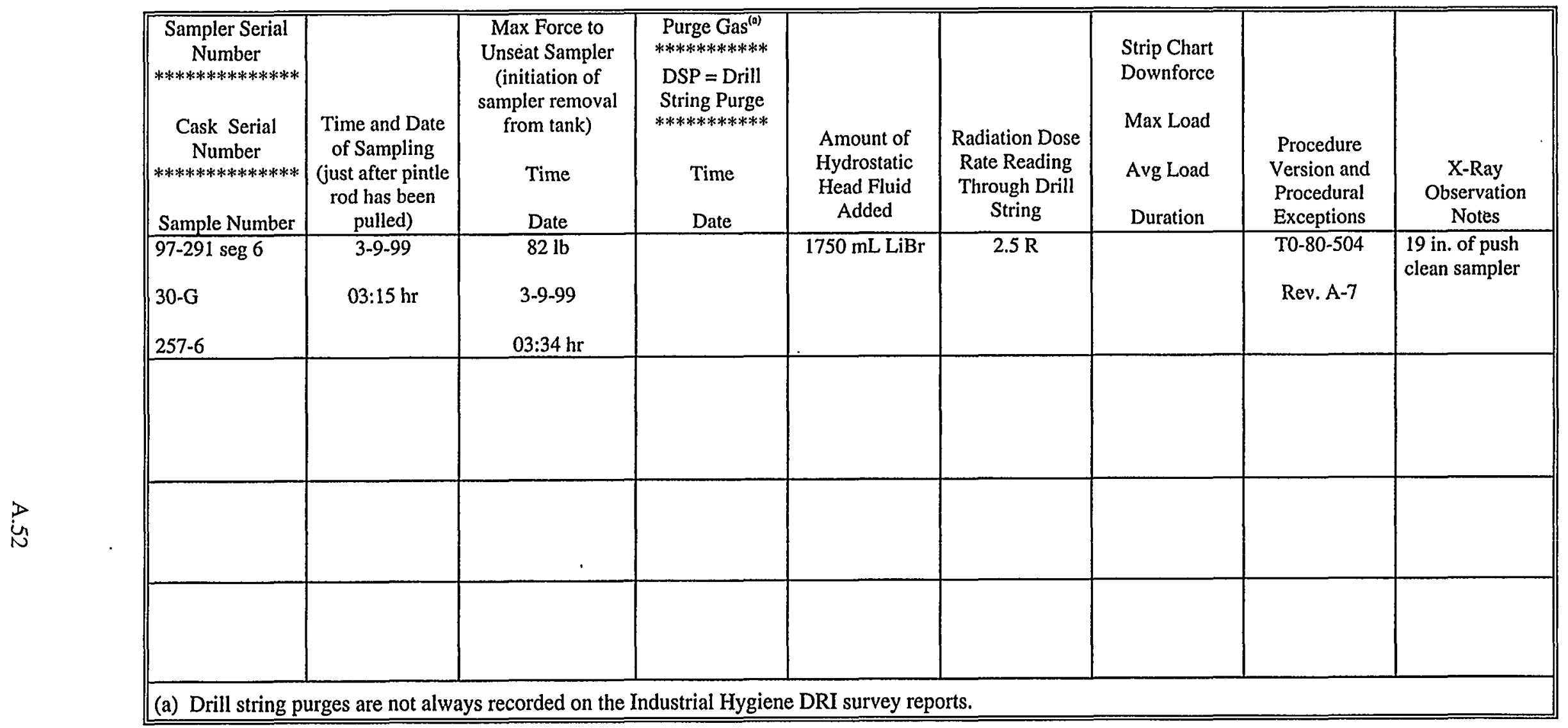


Appendix B

\section{Organic Speciation}




\section{Appendix B}

\section{Organic Speciation}

\section{Conclusions}

The individual organic compounds that were most concentrated in the retained gas were calculated to be at concentrations of $300 \mathrm{ppmv}$ or less in the SY-101 crust and bubbly slurry or $2000 \mathrm{ppmv}$ or less in the SY-101 mixed slurry. (There were no detailed speciation data for the gas in the mixed slurry, so the mixed slurry organic concentrations were extrapolated from bubbly slurry values and should be recognized as extrapolations.) The organic compounds included butane, 1-butanol, 2-butene, and 1,3-butadiene (all firmly identified), and the tentatively identified compounds 2-butene and propane. 1,3-butadiene is of particular interest because it is prominent in the State of Washington Toxic Air Pollutant requirements and has not previously been reported as a major constituent in Tank 241-SY-101 (SY-101) or any other Hanford tank. The sample also contained many more organic compounds, which are listed in Table B.1.

\section{Experimental Procedure}

The RGS core sample 021-4 was acquired through riser 021 of SY-101 (which was riser $22 \mathrm{~A}$ in the previous nomenclature). The sample was taken from the high-gas bubbly slurry under the crust on January 7, 1999; RGS processing began on March 1, 1999. After the sample was extruded into the pre-evacuated RGS system, the Hoke cylinder C-0 (volume $90.5 \mathrm{cc}$ ) was filled with sample gas and vapor at a system pressure of $10.75 \mathrm{kPa}$. Subsequently, five strokes of a mercury displacement pump filled cylinders $\mathrm{C}-\mathrm{A}, \mathrm{C}-\mathrm{B}$, and $\mathrm{C}-\mathrm{C}$ (volumes $75.8,39.2$, and $27 \mathrm{cc}$, respectively) with sample gas and vapor pressurized by pumping to $96.03 \mathrm{kPa}$. These three canisters were filled in parallel and are known to contain liquid water and dissolved ammonia as well as ammonia and water vapor. Aliquots were taken from each of the four cylinders (C-0 through C-C) for mass-spectrometric analysis of the overall gas composition: permanent gases, $\mathrm{C}_{2} \mathrm{H}_{x}$, other hydrocarbons, ammonia, and water vapor. More detail on the part of the analysis summarized above, which is standard RGS procedure, can be found in Section 4.15 of the main report.

During the week of April 7, 1999, the remaining gas in cylinders C-0 through C-C was quantitatively transferred to 150 -cc canisters and diluted with nitrogen to avoid above-range organic concentrations. The diluted gas was then transferred to 2.7-L canisters to obtain the lower pressure required for measurement and analyzed for organic content by GC/MS analysis using cryogenic preconcentration. ${ }^{(a)}$

(a) Evans JC, AV Mitroshkov, and LA Mahoney. 1999. SY-101 Retained Gas Vapor GC/MS Analysis. TWS99.31, Pacific Northwest National Laboratory, Richland, Washington. 


\section{Results}

The organic compounds that were measured in the bubbly-slurry gases from the four cylinders can be seen in Table B.1. The table separates the target compounds, those that were compared against a 67-compound vapor standard from the tentatively identified compounds (TICs). Of the latter, 1,3-butadiene was reviewed against a subsequently run vapor standard and should be regarded as positively—not tentatively-identified.

\section{Interpretation Issues}

The object of the organic compound measurements was to estimate the organic contents of the retained gas in Tank SY-101 and to determine to what extent the measured speciation can be considered representative of the retained gas in the tank.

First, there is the question of sampling location. Sample 021-4 was drawn from the highgas region of the tank. The hydrogen and ammonia concentrations in this zone are unusually high compared with samples from elsewhere in the tank, and the "other hydrocarbons" (as measured by standard RGS procedure) are low. These observations suggest that the high-gas region may not represent other parts of the tank very closely.

Second, the RGS extraction process and system was not designed to measure trace quantities of sorbable materials and therefore did not minimize the sorption surface area. It is expected that sorption would preferentially remove the more polar and high molecular weight species from the sample stream during RGS extraction.

One clue was seen to possible sorption effects. Although the gases from cylinders C-A, $\mathrm{C}-\mathrm{B}$, and $\mathrm{C}-\mathrm{C}$ ought to have shown identical composition, having been drawn off from the core sample in parallel, they in fact showed a consistent trend to substantially lower concentrations. $\mathrm{C}-\mathrm{C}$ was lower than $\mathrm{C}-\mathrm{B}$ and $\mathrm{C}-\mathrm{B}$ lower than $\mathrm{C}-\mathrm{A}$. The cylinders were handled in the sequence $\mathrm{C}-0, \mathrm{C}-\mathrm{A}, \mathrm{C}-\mathrm{B}, \mathrm{C}-\mathrm{C}$, so it is possible that some effect of handling caused the concentration changes. However, no mechanism based on handling sequence has been identified. It seems likelier that the difference in cylinder volumes (C-A, $75.8 \mathrm{cc}$; C-B, $39.2 \mathrm{cc} ; \mathrm{C}-\mathrm{C}, 27 \mathrm{cc}$ ) is responsible because it would lead to different ratios of cylinder surface-area-to-gas volume.

Third, note that some organic compounds may have remained substantially dissolved in the samples, either in the brine (ethanol, methanol) or in small amounts of oil, possibly micelles. In these cases, we would expect the gas in the collection cylinders to be enriched in water and in all the soluble compounds that would have kept coming out of solution during pumping. This would be similar to the behavior that is displayed by ammonia, a very soluble species; it would lead to relative understatement of any insoluble compounds or permanent gases. The analysts stated that "All four samples showed vapor compositions heavily weighted toward low molecular weight, relatively volatile compounds. This observation contrasts with extensive past sampling in the tank headspace, which typically shows a much more abundant mixture of heavier compounds. The difference may be associated with the collection procedure itself, which is quite likely to have caused significant fractionation in a manner consistent with the observed results." 


\section{Table B.1. Calculated Organic Speciation of Retained Gas in Crust and Bubbly Slurry}

\begin{tabular}{|c|c|}
\hline Target Compounds & ppmv in retained gas \\
\hline Ethanol & 265 \\
\hline Methanol & 259 \\
\hline Butane & 103 \\
\hline 1-Butanol & 73 \\
\hline Pyridine & 44 \\
\hline Propanol & 44 \\
\hline Tetrahydrofuran & 7.3 \\
\hline Tetradecane & 7.2 \\
\hline Pentane & 6.9 \\
\hline Acetone & 6.1 \\
\hline 2-Butanone & 2.9 \\
\hline Tridecane & 2.8 \\
\hline Benzene & 1.9 \\
\hline Heptane & 1.6 \\
\hline Undecane & 1.4 \\
\hline Hexane & 1.3 \\
\hline Dodecane & 1.3 \\
\hline Toluene & 1.1 \\
\hline Acetonitrile & 0.98 \\
\hline Decane & 0.75 \\
\hline Methylene Chloride & 0.70 \\
\hline Cyclohexane & 0.69 \\
\hline 4-Methyl-2-Pentanone & 0.50 \\
\hline 1,2,4-Trichlorobenzene & 0.48 \\
\hline Cyclohexanone & 0.29 \\
\hline Chloroethane & 0.26 \\
\hline p/m-Xylene & 0.24 \\
\hline Chloromethane & 0.23 \\
\hline Vinyl Chloride & 0.21 \\
\hline Octane & 0.16 \\
\hline 1,2-dichloro-1,1,2,2-tetrafluoroethane & 0.15 \\
\hline Propanenitrile & 0.14 \\
\hline Bromoform & 0.13 \\
\hline Bromomethane & 0.11 \\
\hline Hexanenitrile & 0.11 \\
\hline Pentanenitile & 0.10 \\
\hline Butanenitrile & 0.10 \\
\hline Nonane & 0.10 \\
\hline 1,1-Dichloroethene & 0.08 \\
\hline cis-1,2-Dichloroethene & 0.07 \\
\hline Trichlorofluoromethane & 0.07 \\
\hline Dichlorodifluoromethane & 0.06 \\
\hline Ethylbenzene & 0.06 \\
\hline 1,2-Dibromoethane & 0.06 \\
\hline 1,2-Dichloropropane & 0.05 \\
\hline o-Xylene & 0.05 \\
\hline Tetrachloroethylene & 0.05 \\
\hline 1,1,2,2-Tetrachloroethane & 0.05 \\
\hline 1,1,1-Trichloroethane & 0.05 \\
\hline Chlorobenzene & 0.05 \\
\hline 1,2-Dichloroethane & 0.04 \\
\hline Carbon Tetrachloride & 0.04 \\
\hline Chloroform & 0.04 \\
\hline 1,2,4-Trimethylbenzene & 0.04 \\
\hline cis-1,3-Dichloropropene & 0.04 \\
\hline 1,1,2-trichloro-1,2,2-trifluoroethane & 0.04 \\
\hline Styrene & 0.04 \\
\hline 1,1,2-Trichloroethane & 0.04 \\
\hline 1,1-Dichloroethane & 0.04 \\
\hline Trichloroethene & 0.04 \\
\hline trans-1,3-Dichloropropene & 0.03 \\
\hline 1-Ethyl-2-Methyl-Benzene & 0.03 \\
\hline 1,3,5-Trimethylbenzene & 0.03 \\
\hline 1,3-Dichlorobenzene & 0.02 \\
\hline 1,4-Dichlorobenzene & 0.02 \\
\hline 1,2-Dichlorobenzene & 0.02 \\
\hline
\end{tabular}

Tentatively Identified Compounds ppmv in retained gas Propene 249

Methylamine 206

Propane 152

2-Butene, (E)- 151

1,3-Butadiene 109

Cyclopropane 49

1-Butene 48

1-Propene, 2-methyl- 36

Ethylamine 31

Dimethyl ether 21

Butane, 2-methyl- 13

Propane, 2,2-dimethyl-

Isopropyl Alcohol $\quad 10$

Aziridine, l-methyl-

1-Propyne 7.8

2-Propanol, 2-methyl-

I-Butene, 2-methyl- $\quad 4.5$

3.5

2-Butene, 2-methyl- 3.4

Ethanol, 2-butoxy- 3.4

3.2

Methanamine, N-methyl- $\quad 2.8$

Silane, methoxytrimethyl-

l-Pentene 2.2

Pentane, 2-methyl- $\quad 2.0$

Butane, 2,2-dimethyl- $\quad 2.0$

N-Nitrosodimethylamine 1.9

Methane, nitro- 1.9

Pentane, 3-methyl- $\quad 1.7$

1-Propanol, 2,2-dimethyl- $\quad 1.5$

Pyrazine 1.4

3-Heptanol 11.2

1-Hexene 1.02

2-Butanol, (. +/-.) 1.01

Cyclopropane, 1,2-dimethyl-, cis- $\quad 1.00$

Cyclotetrasiloxane, octamethyl- $\quad 0.99$

Propane, 2-methyl-2-nitro- $\quad 0.97$

Cyclohexane, methyl- $\quad 0.84$

2-Propenenitrile $\quad 0.76$

Carbon disulfide $\quad 0.75$

Disiloxare, hexamethyl- $\quad 0.68$

2-Butanol 0.66

Furan, tetrahydro-2,2,5,5-tetramethyl- $\quad 0.59$

Silanol, trimethyl- $\quad 0.53$

2-Butenal 0.51

Cyclopentane, methyl- $\quad 0.47$

2-Butanamine, (.t/-.)- $\quad 0.42$

Heptane, 3-methylene- $\quad-0.39$

2-Heptene

Heptane, 3-methyl- . $\quad 0.38$

1-Propanamine $\quad 0.37$

Propanamide 0.36

2-Octene, (Z)- $\quad 0.23$

Hexane, 3-methyl- $\quad 0.19$

Amylene Hydrate $\quad 0.19$

0.16

3-Heptanol, 3-methyl- $\quad 0.15$

Silane, butoxytrimethyl- $\quad 0.15$

2-Hexanone

Hexane, 2,3,4-trimethyl- $\quad 0.12$

Octanenitrile 0.10

7-Azabicyclo[4.1.0]heptane, 2-methyl- $\quad 0.09$

(Z)-3-Heptene $\quad 0.07$

1-Hexanol, 2-ethyl- $\quad 0.07$

2-Heptanone $\quad 0.03$

* 1,3-butadiene received detailed review and should be considered conclusively identified. 
Finally, there is a significant difference between the fraction of the retained gas that was found to be "Other Hydrocarbons" by the standard RGS procedure and the fraction of nonmethane, non- $\mathrm{C}_{2} \mathrm{H}_{\mathrm{x}}$ hydrocarbons found by organic speciation analysis. Segment $021-4$ was found to contain $0.03 \mathrm{~mol} \%$ "other hydrocarbons" by the mass-spectrometry methods typically used for RGS but $0.05,0.09,0.10$; or $0.13 \mathrm{~mol} \%$ non-methane, non- $\mathrm{C}_{2} \mathrm{H}_{\mathrm{x}}$ hydrocarbons by speciation analysis. One possible explanation is that subsampling during the RGS procedure drew off primarily the organic compounds in the vapor phase in the cylinder. There may also have been organics dissolved in the condensed water on the cylinder walls; certainly there is substantial ammonia in the condensate. Both condensate and gas would have been transferred to the canisters used for speciation analysis, based on the transfer procedures used. Under these conditions, more moles of hydrocarbon would be measured by the speciation analysis than by the RGS analysis.

\section{Retained Gas Speciation}

While Table B.1 gives the composition calculated for the retained gas in the crust and bubbly slurry, Table B.2 gives the same information for the retained gas in the mixed slurry. Here the speciation for the bubbly slurry was used to speciate the "other hydrocarbons" found in the mixed slurry gases. The compounds with the highest concentrations are at less than $300 \mathrm{ppm}$ in the crust and bubbly slurry gas and less than $2000 \mathrm{ppm}$ in the mixed slurry retained gas. The concentrations in the tables came from retained gas speciation calculations that were based on the following assumptions:

- The speciation profile is complete. This is known not to be the case; many compounds cannot be measured reliably by a GC/MS, including formaldehyde, acetaldehyde, ethane, ethene, and acetylene. Little or nothing is known about the presence of these compounds in tank gases, and they are omitted from this analysis.

- The speciation profile represents all the retained gas in the tank. The accuracy of this assumption is unknown. It seems likely that some trace organic compounds are underestimated because they were lost to wall sorption in the RGS system, while others (particularly the water-soluble and low molecular-weight ones) are overrepresented.

The following bases were used in the calculations:

- We used the maximum concentration of each species that was measured in any of the cylinders to obtain the speciation profile. The total concentration of speciated hydrocarbons came to $0.16 \mathrm{~mol} \%$.

- We used a total concentration of $0.20 \mathrm{~mol} \%$ non-methane non- $\mathrm{C}_{2} \mathrm{H}_{\mathrm{x}}$ hydrocarbons to represent the composition of the gas in the crust and bubble slurry layers and a total concentration of $1.5 \mathrm{~mol} \%$ for the gas retained in the mixed slurry layer. These values are double the maximum measurement of "Other Hydrocarbons" from standard RGS in each layer. The factor of 2 is used as a safety factor. 


\section{Table B.2. Extrapolated Organic Speciation of Retained Gas in Mixed Slurry}

\begin{tabular}{|c|c|}
\hline Target Compounds & ppmv in retained gas \\
\hline Ethanol & 1990 \\
\hline Methanol & 1943 \\
\hline Butane & 772 \\
\hline I-Butanol & 547 \\
\hline Pyridine & 328 \\
\hline Propanol & 327 \\
\hline Tetrahydrofuran & 55 \\
\hline Tetradecane & 54 \\
\hline Pentane & 51 \\
\hline Acetone & 46 \\
\hline 2-Butanone & 22 \\
\hline Tridecane & 21 \\
\hline Benzene & 14 \\
\hline Heptane & 12 \\
\hline Undecane & 11 \\
\hline Hexane & 9.9 \\
\hline Dodecane & 9.4 \\
\hline Toluene & 7.9 \\
\hline Acetonitrile & 7.4 \\
\hline Decane & 5.6 \\
\hline Methylene Chloride & 5.2 \\
\hline Cyclohexane & 5.2 \\
\hline 4-Methyl-2-Pentanone & 3.8 \\
\hline 1,2,4-Trichlorobenzene & 3.6 \\
\hline Cyclohexanone & 2.2 \\
\hline Chloroethane & 1.9 \\
\hline $\mathrm{p} / \mathrm{m}$-Xylene & 1.8 \\
\hline Chloromethane & 1.7 \\
\hline Vinyl Chloride & 1.6 \\
\hline Octane & 1.2 \\
\hline 1,2-dichloro-1,1,2,2-tetrafluoroethane & 1.1 \\
\hline Propanenitrile & 1.0 \\
\hline Bromoform & 1.0 \\
\hline Bromomethane & 0.82 \\
\hline Hexanenitrile & 0.80 \\
\hline Pentanenitrile & 0.76 \\
\hline Butanenitrile & 0.74 \\
\hline Nonane & 0.72 \\
\hline 1,1-Dichloroethene & 0.59 \\
\hline cis-1,2-Dichloroethene & 0.53 \\
\hline Trichlorofluoromethane & 0.52 \\
\hline Dichlorodifluoromethane & 0.46 \\
\hline Ethylbenzene & 0.43 \\
\hline 1,2-Dibromoethane & 0.42 \\
\hline 1,2-Dichloropropane & 0.40 \\
\hline o-Xylene & 0.40 \\
\hline Tetrachloroethylene & 0.38 \\
\hline 1,1,2,2-Tetrachloroethane & 0.38 \\
\hline 1,1,1-Trichloroethane & 0.36 \\
\hline Chlorobenzene & 0.35 \\
\hline 1,2-Dichloroethane & 0.33 \\
\hline Carbon Tetrachloride & 0.33 \\
\hline Chloroform & 0.33 \\
\hline 1,2,4-Trimethylbenzene & 0.32 \\
\hline cis-1,3-Dichloropropene & 0.32 \\
\hline 1,1,2-trichloro-1,2,2-trifluoroethane & 0.32 \\
\hline Styrene & 0.32 \\
\hline 1,1,2-Trichloroethane & 0.31 \\
\hline 1,1-Dichloroethane & 0.30 \\
\hline Trichloroethene & 0.27 \\
\hline trans-1,3-Dichloropropene & 0.24 \\
\hline 1-Ethyl-2-Methyl-Benzene & 0.22 \\
\hline 1,3,5-Trimethylbenzene & 0.22 \\
\hline 1,3-Dichlorobenzene & 0.18 \\
\hline 1,4-Dichlorobenzene & 0.18 \\
\hline 1,2-Dichlorobenzene & 0.18 \\
\hline
\end{tabular}

\begin{tabular}{|c|c|}
\hline Tentatively Identified Compounds & ppmv in retained gas \\
\hline Propene & 1869 \\
\hline Methylamine & 1547 \\
\hline Propane & 1138 \\
\hline 2-Butene, (E)- & 1136 \\
\hline 1,3-Butadiene & $816^{*}$ \\
\hline Cyclopropane & 371 \\
\hline l-Butene & 358 \\
\hline 1-Propene, 2-methyl- & 268 \\
\hline Ethylamine & 229 \\
\hline Dimethyl ether & 158 \\
\hline Butane, 2-methyl- & 96 \\
\hline Propane, 2,2-dimethyl- & 94 \\
\hline Isopropyl Alcohol & 77 \\
\hline Aziridine, 1-methyl- & 60 \\
\hline 1-Propyne & 58 \\
\hline 2-Propanol, 2-methyl- & 55 \\
\hline 1-Butene, 2-methyl- & 34 \\
\hline Cyclobutane & 26 \\
\hline 2-Butene, 2-methyl- & 25 \\
\hline Ethanol, 2-butoxy- & 25 \\
\hline 1,4-Dioxane & 24 \\
\hline Methanamine, N-methyl- & 21 \\
\hline Silane, methoxytrimethyl- & 17 \\
\hline 1-Pentene & 17 \\
\hline Pentane, 2-methyl- & 15 \\
\hline Butane, 2,2-dimethyl- & 15 \\
\hline N-Nitrosodimethylamine & 14 \\
\hline Methane, nitro- & 14 \\
\hline Pentane, 3-methyl- & 12 \\
\hline 1-Propanol, 2,2-dimethyl- & 11 \\
\hline Pyrazine & 10 \\
\hline 3-Heptanol & 9.2 \\
\hline 1-Hexene & 7.6 \\
\hline 2-Butanol, (.+/-.)- & 7.6 \\
\hline Cyclopropane, 1,2-dimethyl-, cis- & 7.5 \\
\hline Cyclotetrasiloxane, octamethyl- & 7.4 \\
\hline Propane, 2-methyl-2-nitro- & 7.3 \\
\hline Cyclohexane, methyl- & 6.3 \\
\hline 2-Propenenitrile & 5.7 \\
\hline Carbon disulfide & 5.7 \\
\hline Disiloxane, hexamethyl- & 5.1 \\
\hline 2-Butanol & 5.0 \\
\hline Furan, tetrahydro-2,2,5,5-tetramethyl- & 4.4 \\
\hline Silanol, trimethyl- & 4.0 \\
\hline 2-Butenal & 3.8 \\
\hline Cyclopentane, methyl- & 3.5 \\
\hline 2-Butanamine, $(++/-)$. & 3.1 \\
\hline Heptane, 3-methylene- & 3.0 \\
\hline 2-Heptene & 2.9 \\
\hline Heptane, 3-methyl- & 2.8 \\
\hline 1-Propanamine & 2.8 \\
\hline Propanamide . & 2.7 \\
\hline 2-Octene, $(Z)$. & 1.7 \\
\hline Hexane, 3-methyl- & 1.4 \\
\hline Amylene Hydrate & 1.4 \\
\hline 3-Heptene & 1.2 \\
\hline 3-Heptanol, 3-methyl- & 1.1 \\
\hline Silane, butoxytrimethyl- & 1.1 \\
\hline 2-Hexanone & 0.92 \\
\hline Hexane, 2,3,4-trimethyl- & 0.88 \\
\hline Octanenitrile & 0.76 \\
\hline 7-Azabicyclo[4.1.0]heptane, 2-methyl- & 0.70 \\
\hline (Z)-3-Heptene & 0.53 \\
\hline 1-Hexanol, 2-ethyl- & 0.49 \\
\hline 2-Heptanone & 0.22 \\
\hline
\end{tabular}

* 1,3-butadiene received detailed review and should be considered conclusively identified. 


\section{Gas Inventories and Releases}

The SY-101 gas inventories calculated from the most recent RGS data were $420 \mathrm{STP} \mathrm{m}^{3}$ in the crust and bubble slurry and $185 \mathrm{STP} \mathrm{m}^{3}$ in the mixed slurry (see Table 4.15.8). It follows that each of the most concentrated organic compounds in the retained gas is present at a volume of less than $0.13 \mathrm{~m}^{3}$ in the crust and bubbly slurry and less than $0.4 \mathrm{~m}^{3}$ in the mixed slurry. Of course, all but 10 of the compounds are present in concentrations that are $20 \%$ or less of the calculated maximum values.

The concentrations of organic compounds in the dome space after a gas release depend on the release fraction and rate and on the available dome space. The maximum possible dome space concentration of any single compound, for a total instantaneous release of $0.4 \mathrm{~m}^{3}$ into a dome space of $760 \mathrm{~m}^{3}$ (waste surface at $439 \mathrm{in}$.), would be $530 \mathrm{ppmv}$. Most release scenarios would produce much lower dome space concentrations than this. 


\section{Appendix C}

Detailed Retained Gas Sampler $\mathrm{NH}_{3}$ Results 


\section{Appendix C}

\section{Detailed RGS $\mathrm{NH}_{3}$ Results}

This document gives a detailed description of the ammonia measurements that were considered in making the ammonia estimates summarized in Section 4 of the main report. Like Section 4, this appendix is arranged with one subsection for each tank sampled by the retained gas sampler (RGS), with the tanks presented in the same sequence as they were sampled.

\section{C.1 AW-101}

Table C.1.1 presents the ammonia measurements. The "Extracted $\mathrm{NH}_{3}$ " column gives the total moles of ammonia that were pumped as vapor from the samples into the collection canisters, expressed as the moles of $\mathrm{NH}_{3}$ per liter of original waste (including gas, solids, and liquid volume). The procedure did not permit the determination of the residual or total ammonia, but the post-extrusion and the first $\mathrm{PQ}$ canister partial pressures of $\mathrm{NH}_{3}$ over the sample (Section 3.4.4) were measured at laboratory temperature and are also in Table C.1.1, together with the measurement temperatures. The residual ammonia in the samples was also measured by ion-specific electrode (ISE) after the samples were removed from the extractor; as discussed in Section 3.4.2, these ammonia concentrations should be considered lower bounds because of ammonia evaporation during sample handling.

The ammonia partial pressure of sample 24A-8 (the only sample in the convective layer) appears to be lower than the pressures of the other samples, based on the PQ canister partial pressures. No conclusive total ammonia concentration can be calculated, but it seems likely that the lower-bound estimate is roughly $27,000 \mu \mathrm{mol} \mathrm{NH} 3 / \mathrm{L}$ waste. This estimate is based on Sample $24 \mathrm{~A}-19$, the maximum total ammonia measurement, which was found by adding the post-RGS ISE measurement of residual ammonia to the RGS-determined extracted ammonia.

Table C.1.1. Ammonia Data from Tank AW-101 Samples

\begin{tabular}{|c|c|c|c|c|c|}
\hline Sample & $\begin{array}{c}\text { Extracted } \\
\mathrm{NH}_{3} \\
(\mu \mathrm{mol} / \mathrm{L})\end{array}$ & $\begin{array}{l}\text { Post-extrusion } \\
\mathrm{NH}_{3} \text { partial } \\
\text { pressure } \\
\text { (atm) }\end{array}$ & $\begin{array}{c}\text { 1st } \mathrm{PQ} \text { canister } \\
\mathrm{NH}_{3} \text { partial } \\
\text { pressure } \\
\text { (atm) }\end{array}$ & $\begin{array}{c}\text { Measure- } \\
\text { ment } \\
\text { temperature } \\
\left({ }^{\circ} \mathrm{C}\right)\end{array}$ & $\begin{array}{c}\text { Total } \mathrm{NH}_{3} \text { from } \\
\text { post-RGS residual } \\
\mathrm{NH}_{3}, \text { by ISE (a) } \\
(\mu \mathrm{mol} / \mathrm{L})\end{array}$ \\
\hline $24 \mathrm{~A}-8$ & $2100 \pm 360$ & $0.0063 \pm 0.0031$ & $0.0038 \pm 0.0019^{(b)}$ & 22.6 & 22000 \\
\hline $24 \mathrm{~A}-17$ & $4700 \pm 670$ & $0.0084 \pm 0.0042$ & $0.0079 \pm 0.0039$ (b) & 23.0 & 23000 \\
\hline $24 B-18$ & $6400 \pm 800$ & $0.0080 \pm 0.0040$ & $0.0041 \pm 0.0020^{(b)}$ & 24.6 & 14000 \\
\hline $24 A-19$ & $4600 \pm 610$ & $0.0108 \pm 0.0054^{(b)}$ & no data & 23.7 & 27000 \\
\hline $24 \mathrm{~A}-21$ & $3900 \pm 550$ & $0.0103 \pm 0.0051^{(b)}$ & no data & 24.1 & no data \\
\hline $24 B-22$ & $7200 \pm 920$ & $0.0078 \pm 0.0039$ & $0.0058 \pm 0.0029(b)$ & 23.9 & 13000 \\
\hline
\end{tabular}


A lower-bound concentration of $27,000 \mu \mathrm{mol} \mathrm{NH}_{3} / \mathrm{L}$ waste in the nonconvective layer corresponds to $0.034 \mathrm{M}(580 \mu \mathrm{g} / \mathrm{mL})$ in the liquid, or $0.031 \mathrm{wt} \%$ of the bulk waste. (The conversion of $\mathrm{mol} / \mathrm{L}$ waste to liquid concentrations assumed a gas volume fraction of 0.05 in the waste and a solids volume fraction of 0.17 in the degassed waste, as shown in the AW-101 calculations spreadsheet on the CD supplied with this report.) The concentration of ammonia in the convective layer liquid could be lower, but there is no reason to expect it to be less than half of the concentration in the nonconvective layer liquid.

\section{C.2 AN-105}

Table C.2.1 presents the ammonia measurements. The "Extracted $\mathrm{NH}_{3}$ " column gives the total moles of ammonia that were pumped as vapor from the samples into the collection canisters expressed as the moles of $\mathrm{NH}_{3}$ per liter of original waste (including gas, solids, and liquid volume). The procedure did not permit determination of the residual or total ammonia, but the postextrusion and first PQ canister partial pressures of $\mathrm{NH}_{3}$ over the sample (see Section 3.4.4) were measured at laboratory temperature and are in the table along with the measurement temperatures. The residual ammonia in a few of the samples was also measured by ISE after the samples were removed from the extractor; as discussed in Section 3.4.2, these ammonia concentrations should be considered lower bounds because of ammonia evaporation during sample handling.

No conclusive total ammonia concentration can be calculated, but it seems likely that the lower-bound estimate is roughly $27,000 \mu \mathrm{mol} \mathrm{NH}_{3} / \mathrm{L}$ waste. This estimate is based on a comparison with AW-101 data, which included a more complete set of post-RGS ISE ammonia measurements. The partial pressures over the AN-105 samples are slightly less than or equal to those over the AW-101 waste (Table C.1.1). Some of the post-RGS ISE ammonia values for AW-101 were as low as 13,000 or $14,000 \mu \mathrm{mol} \mathrm{NH} / \mathrm{L}$ waste (similar to $\mathrm{AN}-105$ samples $12 \mathrm{~A}-15$ and $7 \mathrm{~B}-18$ ), while the maximum post-RGS ISE value was $27,000 \mu \mathrm{mol} \mathrm{NH} \mathrm{N}_{3} / \mathrm{L}$ waste.

Table C.2.1. Ammonia Data from Tank AN-105 Samples

\begin{tabular}{|c|c|c|c|c|c|}
\hline Sample & $\begin{array}{c}\text { Extracted } \\
\mathrm{NH}_{3} \\
(\mu \mathrm{mol} / \mathrm{L})\end{array}$ & $\begin{array}{l}\text { Post-extrusion } \\
\mathrm{NH}_{3} \text { partial } \\
\text { pressure } \\
\quad(\mathrm{atm})\end{array}$ & $\begin{array}{l}\text { 1st PQ canister } \\
\mathrm{NH}_{3} \text { partial } \\
\text { pressure } \\
\text { (atm) }\end{array}$ & $\begin{array}{l}\text { Measure- } \\
\text { ment } \\
\text { temperature } \\
\left({ }^{\circ} \mathrm{C}\right)\end{array}$ & $\begin{array}{l}\text { Total } \mathrm{NH}_{3} \text { from } \\
\text { post-RGS residual } \\
\mathrm{NH}_{3}, \text { by ISE (a) } \\
(\mu \mathrm{mol} / \mathrm{L})\end{array}$ \\
\hline $7 B-4$ & $2300 \pm 510$ & $0.0059 \pm 0.0030$ & $0.0048 \pm 0.0024(\mathrm{~b})$ & 24.0 & 2700 \\
\hline $12 \mathrm{~A}-15$ & $2700 \pm 520$ & $0.0055 \pm 0.0028$ & $0.0053 \pm 0.0027^{(b)}$ & 24.3 & 12000 \\
\hline $7 \mathrm{~B}-16$ & $2100 \pm 480$ & $0.0052 \pm 0.0026^{(c)}$ & $0.0043 \pm 0.0022^{(b)}$ & 24.3 & no data \\
\hline $12 \mathrm{~A}-17$ & $4000 \pm 660$ & $0.0086 \pm 0.0042^{(b)}$ & no data & 25.0 & no data \\
\hline $7 \mathrm{~B}-18$ & $3800 \pm 650$ & $0.0065 \pm 0.0032$ & $0.0057 \pm 0.0029$ (b) & 24.1 & 11000 \\
\hline $12 \mathrm{~A}-19$ & $6500 \pm 1000$ & $0.0089 \pm 0.0044$ & $0.0070 \pm 0.0034^{(b)}$ & 24.3 & no data \\
\hline $12 \mathrm{~A}-21$ & $2900 \pm 570$ & $0.0060 \pm 0.0038$ & $0.0053 \pm 0.0027^{(b)}$ & 24.2 & no data \\
\hline \multicolumn{6}{|c|}{$\begin{array}{l}\text { (a) Total ammonia concentrations are the sum of post-RGS ISE ammonia residual and RGS extracted ammonia. They } \\
\text { should be considered lower bounds because of ammonia evaporation during sample handling preceding ISE analysis. } \\
\text { (b) These ammonia partial pressures were used to calculate in situ ammonia vapor pressures. } \\
\text { (c) This value is questionable because the extractor pressure showed an unusual profile. }\end{array}$} \\
\hline
\end{tabular}


A lower-bound concentration of $27,000 \mu \mathrm{mol} \mathrm{NH} / \mathrm{L}$ waste in the nonconvective layer corresponds to $0.037 \mathrm{M}(620 \mu \mathrm{g} / \mathrm{mL})$ in the liquid, or $0.032 \mathrm{wt} \%$ of the bulk waste. (The conversion of $\mathrm{mol} / \mathrm{L}$ waste to liquid concentrations assumed a gas volume fraction of 0.08 in the waste and a solids volume fraction of 0.20 in the degassed waste, as shown in the AN-105 calculations spreadsheet on the CD supplied with this report.) The concentration of ammonia in the convective layer liquid could be lower, but there is no reason to expect it to be less than two-thirds of the concentration in the nonconvective layer liquid.

\section{C.3 A-101}

Table C.3.1 presents the ammonia measurements. The "Extracted $\mathrm{NH}_{3}$ " column gives the total moles of ammonia that were pumped as vapor from the samples into the collection canisters, expressed as the moles of $\mathrm{NH}_{3}$ per liter of original waste (including gas, solids, and liquid volume). The procedure did not permit the determination of the residual or total ammonia, but the post-extrusion and the first PQ canister partial pressures of $\mathrm{NH}_{3}$ over the sample (see Section 3.4.4) were measured at laboratory temperature and are also in Table C.3.1 with the measurement temperatures.

For comparison, Table C.3.1 also includes ammonia measurements from salt-well grab samples taken from the top and the middle of the waste in 1996 (Steen 1996), in which ammonia was measured by ion-specific electrode (ISE). The ammonia measurements were made on subsamples of liquid pipetted from the grab samples at laboratory temperature and gave concentrations of 129 and $508 \mu \mathrm{g} \mathrm{NH} / 3 \mathrm{~mL}$ of sample liquid for the top and middle samples. Both samples had been liquid when taken, but under lab conditions there was less than 5 vol\% solids in the top sample and about $30 \mathrm{vol} \%$ centrifuged solids in the mid-waste sample. Assuming the centrifuged solids were one-third interstitial liquid by volume, then the ammonia concentrations (corrected back to original liquid volume) were about 125 and $406 \mu \mathrm{g} \mathrm{NH} / \mathrm{mL}$ of original liquid.

Table C.3.1. Ammonia Data from Tank A-101 Samples

\begin{tabular}{|c|c|c|c|c|c|}
\hline Sample & $\begin{array}{l}\text { Extracted } \\
\mathrm{NH}_{3} \\
(\mu \mathrm{mol} / \mathrm{L} \\
\text { waste) }\end{array}$ & $\begin{array}{l}\text { Post-extrusion } \\
\mathrm{NH}_{3} \text { partial } \\
\text { pressure } \\
\text { (atm) }\end{array}$ & $\begin{array}{c}\text { First PQ canister } \\
\mathrm{NH}_{3} \text { partial pressure } \\
\text { (atm) }\end{array}$ & $\begin{array}{c}\text { Measurement } \\
\text { temperature } \\
\left({ }^{\circ} \mathrm{C}\right)\end{array}$ & $\begin{array}{l}\text { Salt-well grab } \\
\text { sample } \mathrm{NH}_{3} \text {, by } \\
\text { ISE (a) } \\
(\mu \mathrm{mol} / \mathrm{L} \text { liq })\end{array}$ \\
\hline $24-2$ & $6300 \pm 610$ & $0.036 \pm 0.018^{(b)}$ & $0.014 \pm 0.007$ (c) & 24.8 & \multirow{2}{*}{7400} \\
\hline $15-5$ & $15000 \pm 1100$ & $0.021 \pm 0.010$ & $0.018 \pm 0.009$ (c) & 23.8 & \\
\hline $15-8$ & $13000 \pm 1100$ & $0.016 \pm 0.008$ (d) & $0.010 \pm 0.005$ (c) & 24.1 & \\
\hline $24-9$ & $17000 \pm 1100$ & $0.027 \pm 0.014$ & $0.0089 \pm 0.0044(c)$ & 24.1 & \multirow{2}{*}{24000} \\
\hline $15-12$ & $22000 \pm 1400$ & $0.019 \pm 0.0096$ & $0.024 \pm 0.012$ (c) & 24.2 & \\
\hline $24-16$ & $20000 \pm 1400$ & $0.019 \pm 0.0097$ & $0.027 \pm 0.013(c)$ & 23.4 & \\
\hline $24-19$ & $20000 \pm 1300$ & $0.022 \pm 0.011$ & $0.028 \pm 0.014(c)$ & 23.5 & \\
\hline \multicolumn{6}{|c|}{$\begin{array}{l}\text { (a) Ammonia concentrations measured in grab samples may be low because of ammonia evaporation from liquid in } \\
\text { the salt well or evaporation during sample handling. } \\
\text { (b) This value is questionable because of the effects of air inleakage. } \\
\text { (c) These ammonia partial pressures were used to calculate in situ ammonia vapor pressures. } \\
\text { (d) This value is questionable because the extractor pressure showed an unusual profile. }\end{array}$} \\
\hline
\end{tabular}


Note that the ammonia concentrations measured in these grab samples might not be strictly comparable to those in RGS samples. The ammonia in the grab sample could be lowered relative to that in RGS core samples by one or both of two mechanisms. There could have been ammonia evaporation from the sample during sample handling, as for core extrusions. There could also have been in situ ammonia evaporation from the salt-well liquid that decreased its ammonia concentration relative to that in the less-exposed interstitial liquid sampled by RGS.

No conclusive total ammonia concentration can be calculated. However, based on the partial pressures of ammonia measured in A-101 and AW-101, it seems likely that ammonia concentrations in A-101 are two to five times those in AW-101, for which a lower-bound estimate of $27,000 \mu \mathrm{mol} \mathrm{NH} / \mathrm{L}$ waste was given in Section C.1. The partial pressures found by the two methods agree that the ammonia partial pressures in the upper nonconvective layer are higher than those in the lower convective layer, though the difference falls within the $50 \%$ uncertainty. Thus the ammonia concentration in the nonconvective layer may have a lower bound of $50,000 \mu \mathrm{mol}$ $\mathrm{NH}_{3} / \mathrm{L}$ waste, while the lower bound in the convective layer may be closer to $100,000 \mu \mathrm{mol}$ $\mathrm{NH}_{3} / \mathrm{L}$ waste.

A lower-bound concentration of $50,000 \mu \mathrm{mol} \mathrm{NH} / \mathrm{L}$ waste in the nonconvective layer corresponds to $0.084 \mathrm{M}(1400 \mu \mathrm{g} / \mathrm{mL})$ in the liquid, or $0.062 \mathrm{wt} \%$ of the bulk waste. (The conversion of $\mathrm{mol} / \mathrm{L}$ waste to liquid concentrations assumed a gas volume fraction of 0.18 in the waste and a solids volume fraction of 0.27 in the degassed waste, as shown in the A-101 calculations spreadsheet on the CD supplied with this report.) This lower bound is a factor of 3 to 10 times the concentrations found in salt-well grab samples.

A lower-bound concentration of $100,000 \mu \mathrm{mol} \mathrm{NH} 3 / \mathrm{L}$ waste in the convective layer corresponds to $0.11 \mathrm{M}(1800 \mu \mathrm{g} / \mathrm{mL})$ in the liquid; or $0.11 \mathrm{wt} \%$ of the bulk waste. (The conversion of $\mathrm{mol} / \mathrm{L}$ waste to liquid concentrations assumed a gas volume fraction of 0.008 in the waste and a solids volume fraction of 0.06 in the degassed waste, as shown in the spreadsheet.)

The available data are not easily reconcilable, but there is no reason to expect ammonia concentrations of less than $0.024 \mathrm{M}$ in the liquid (based on the higher salt-well grab sample). The lower-bound RGS values suggest concentrations of $0.08 \mathrm{M}$ or more may be present, particularly in the tank's lower layer.

\section{C.4 AN-104}

Table C.4.1 presents the ammonia measurements. The "Extracted $\mathrm{NH}_{3}$ " column gives the total moles of ammonia that were pumped as vapor from the samples into the collection canisters, expressed as the moles of $\mathrm{NH}_{3}$ per liter of original waste (including gas, solids, and liquid volume). The procedure did not permit the determination of the residual or total ammonia, but the post-extrusion and the first PQ canister partial pressures of $\mathrm{NH}_{3}$ over the sample (Section 3.4.4) were measured at laboratory temperature and are also included along with the measurement temperatures. There were no residual ammonia measurements for AN-104 samples except for two (samples 10A-17 and 10A-21) whose residual ammonia concentrations were found to be below the MDL of 80,000 to $90,000 \mu \mathrm{mol} \mathrm{NH}_{3} / \mathrm{L}$.

No conclusive total ammonia concentration can be calculated, but it seems likely that the ammonia concentration in AN-104 waste is about the same as in AW-101 waste, with a lower bound of $27,000 \mu \mathrm{mol} \mathrm{NH} / \mathrm{L}$ waste. This estimate is based on a comparison of the ammonia partial pressure measurements (see Sections 4.1.2 and C.1 for the AW-101 measurements). 
Table C.4.1. Ammonia Data from Tank AN-104 Samples

\begin{tabular}{||c|c|c|c|c||}
\hline Sample & $\begin{array}{c}\text { Extracted } \\
\mathrm{NH}_{3} \\
(\mu \mathrm{mol} / \mathrm{L})\end{array}$ & $\begin{array}{c}\text { Post-extrusion } \mathrm{NH}_{3} \\
\text { partial pressure } \\
(\mathrm{atm})\end{array}$ & $\begin{array}{c}1 \text { st PQ canister } \\
\mathrm{NH}_{3} \text { partial pressure } \\
(\mathrm{atm})\end{array}$ & $\begin{array}{c}\text { Measurement } \\
\text { temperature } \\
\left({ }^{\circ} \mathrm{C}\right)\end{array}$ \\
\hline \hline $10 \mathrm{~A}-3$ & $2800 \pm 580$ & $0.0062 \pm 0.0031$ & $0.0056 \pm 0.0028$ (a) & 24.2 \\
\hline $10 \mathrm{~A}-13$ & $5200 \pm 780$ & $0.0067 \pm 0.0034$ & $0.0043 \pm 0.0022$ (a) & 24.1 \\
\hline $10 \mathrm{~A}-15$ & $4500 \pm 780$ & $0.0083 \pm 0.0042$ & $0.021 \pm 0.010$ (a) & 24.1 \\
\hline $10 \mathrm{~A}-17$ & $3600 \pm 810$ & $0.0073 \pm 0.0036$ (a, b) & no data & 24.1 \\
\hline $12 \mathrm{~A}-18$ & $7600 \pm 950$ & $0.01 \pm 0.005$ & $0.0057 \pm 0.0029$ (a) & 24.1 \\
\hline $10 \mathrm{~A}-21$ & $2500 \pm 730$ & $0.012 \pm 0.006$ (a) & no data & 24.1 \\
\hline $\begin{array}{l}\text { (a) These ammonia partial pressures were used to calculate in situ ammonia vapor pressures. } \\
\text { (b) This value is questionable because of the effects of air inleakage. }\end{array}$ \\
\hline
\end{tabular}

\section{C.5 AN-103}

Table C.5.1 presents the ammonia measurements. The "Extracted $\mathrm{NH}_{3}$ " column gives the total moles of ammonia that were pumped as vapor from the samples into the collection canisters, expressed as the moles of $\mathrm{NH}_{3}$ per liter of original waste (including gas, solids, and liquid volume). The procedure did not permit determination of the residual or total ammonia, but the postextrusion and the first $\mathrm{PQ}$ canister partial pressures of $\mathrm{NH}_{3}$ over the sample (see Section 3.4.4) were measured at laboratory temperature and are also in Table C.5.1, together with the measurement temperatures. There were no residual ammonia measurements for $\mathrm{AN}-103$ samples.

Table C.5.1. Ammonia Data from Tank AN-103 Samples

\begin{tabular}{||l|c|c|c|c||}
\hline Sample & $\begin{array}{c}\text { Extracted } \\
\mathrm{NH}_{3} \\
(\mu \mathrm{mol} / \mathrm{L})\end{array}$ & $\begin{array}{c}\text { Post-extrusion } \mathrm{NH}_{3} \\
\text { partial pressure (atm) }\end{array}$ & $\begin{array}{c}1^{\text {st }} \mathrm{PQ} \text { canister } \\
\mathrm{NH}_{3} \text { partial pressure } \\
\text { (atm) }\end{array}$ & $\begin{array}{c}\text { Measurement } \\
\text { temperature } \\
\left({ }^{\circ} \mathrm{C}\right)\end{array}$ \\
\hline \hline $12 \mathrm{~A}-2$ & $3700 \pm 710$ & $0.0093 \pm 0.0046(\mathrm{a})$ & no data & 24.1 \\
\hline $12 \mathrm{~A}-5$ & $3200 \pm 470$ & $0.0083 \pm 0.0041$ (a, b) & no data & 23.8 \\
\hline $21 \mathrm{~A}-10$ & $3100 \pm 690$ & $0.0078 \pm 0.0039$ (a) & no data & 23.8 \\
\hline $12 \mathrm{~A}-14$ & $6100 \pm 980$ & $0.010 \pm 0.005$ & $0.0076 \pm 0.0038$ (a) & 23.9 \\
\hline $21 \mathrm{~A}-16$ & $4500 \pm 940$ & $0.0083 \pm 0.0041$ & $0.0067 \pm 0.0033$ (a) & 23.9 \\
\hline $\begin{array}{l}\text { (a) These ammonia partial pressures were used to calculate in situ ammonia vapor pressures. } \\
\text { (b) This value is suspect because pressure data were lost for the 3rd canister. }\end{array}$ \\
\hline
\end{tabular}


No conclusive total ammonia concentration can be calculated, but it seems likely that the ammonia concentration in AN-103 waste is about the same as in AW-101 waste, with a lower bound of $27,000 \mu \mathrm{mol} \mathrm{NH}_{3} / \mathrm{L}$ waste. This estimate is based on a comparison of the ammonia partial pressure measurements (see Sections 4.1.2 and C.1 for the AW-101 measurements).

A lower-bound concentration of $27,000 \mu \mathrm{mol} \mathrm{NH} / \mathrm{L}$ waste in the nonconvective layer corresponds to $0.040 \mathrm{M}(680 \mu \mathrm{g} / \mathrm{mL})$ in the liquid, or $0.029 \mathrm{wt} \%$ of the bulk waste. (The conversion of $\mathrm{mol} / \mathrm{L}$ waste to liquid concentrations assumed a gas volume fraction of 0.09 in the waste and a solids volume fraction of 0.26 in the degassed waste, as shown in the AN-103 calculations spreadsheet on the CD supplied with this report.) There is no evidence that the ammonia concentration in the convective layer is lower than that in the non-convective layer.

\section{C.6 U-103}

Table C.6.1 presents the ammonia measurements. The "Extracted $\mathrm{NH}_{3}$ " column gives the total moles of ammonia that were pumped as vapor from the samples into the collection canisters, expressed as the moles of $\mathrm{NH}_{3}$ per liter of original waste (including gas, solids, and liquid volume). The procedure did not permit the determination of the residual or total ammonia except for the isotopically measured sample 7-2. The post-extrusion partial pressure of $\mathrm{NH}_{3}$ over the sample (see Section 3.4.4) was measured at laboratory temperature and is also in Table C.6.1 with the measurement temperatures. There were no post-RGS ammonia measurements made by ISE for U-103 samples, and although PQ canisters were taken, their contents were not analyzed.

Sample 7-2 gave a physically impossible negative value for the post-extrusion ammonia partial pressure. The gas content of the sample was high (over 0.40 volume fraction), and the ammonia content may have been low (judging by the small amount of extracted ammonia). The ammonia partial pressure is calculated by subtracting the water vapor pressure and the gas pressure (based on the extracted gas) from the total pressure. Thus, when large amounts of gas and small

Table C.6.1. Ammonia Data from Tank U-103 Samples

\begin{tabular}{||c|c|c|c|c||}
\hline Sample & $\begin{array}{c}\text { Extracted } \mathrm{NH}_{3} \\
(\mu \mathrm{mol} / \mathrm{L})\end{array}$ & $\begin{array}{c}\text { Isotopically measured } \\
\text { residual } \mathrm{NH}_{3} \\
(\mu \mathrm{mol} / \mathrm{L})\end{array}$ & $\begin{array}{c}\text { Post-extrusion } \mathrm{NH}_{3} \\
\text { partial pressure } \\
(\mathrm{atm})\end{array}$ & $\begin{array}{c}\text { Measurement } \\
\text { temperature } \\
\left({ }^{\circ} \mathrm{C}\right)\end{array}$ \\
\hline \hline $7-2$ & $580 \pm 310$ & $2000 \pm 720$ (a) & -0.0063 (b) & 24.2 \\
\hline $7-5$ & $12000 \pm 1800$ & no data & $0.016 \pm 0.008$ (a) & 24.1 \\
\hline $7-7$ & $22000 \pm 2300$ & no data & $0.012 \pm 0.006$ (a) & 24.2 \\
\hline $7-8$ & $15000 \pm 1500$ & no data & $0.0026 \pm 0.0013$ (c) & 24.2 \\
\hline $\begin{array}{l}\text { (a) These data were used to calculate in situ ammonia vapor pressures. } \\
\text { (b) This physically impossible negative pressure is believed to be the result of a low ammonia vapor } \\
\text { pressure. } \\
\text { (c) This inconsistently low partial pressure was not used to calculate the in situ ammonia vapor pressure } \\
\text { for sample 7-8. Instead, the average of the partial pressures for samples 7-5 and 7-7 was used. }\end{array}$ \\
\hline
\end{tabular}


amounts of ammonia are present, error in the gas pressure (an overestimate) could mean that the small ammonia vapor pressure would be overwhelmed and a negative ammonia vapor pressure would be calculated. A small decrease in the gas content, less than $10 \%$ of the gas, would have been enough to give a small positive ammonia partial pressure for sample 7-2.

The isotopically determined residual ammonia for sample 7-2 is suspected of being an underestimate because of the very short equilibration time that was allowed (about $0.1 \mathrm{hr}$ ). A tooshort equilibration time would have meant that too much $15 \mathrm{NH}_{3}$ was left in the vapor phase, giving too high a ratio of $15 \mathrm{NH}_{3} / 14 \mathrm{NH}_{3}$ and consequently underestimating the residual $14 \mathrm{NH}_{3}$. However, the isotopic value was used to calculate the in situ ammonia for sample 7-2 because no other applicable information was available.

Sample 7-8 gave an ammonia partial pressure that was too low to be consistent with the measurements for samples 7-5 and 7-7, particularly considering that all three of these samples produced about the same amount of extracted ammonia. The low value may be explained by the fact that the extractor pressure was increasing four or more times as rapidly at the time of measurement for sample 7-8 as for samples 7-5 and 7-7, so that the ammonia partial pressure was probably farther from equilibration. Therefore, for sample 7.-8, the average of the ammonia partial pressure from samples 7-5 and 7-8 was used to calculate the in situ ammonia partial pressure.

March 1999 non-RGS ammonia measurements were also available for Tank U-103.(a) The measurements were made on liquid decanted from grab samples that contained some solids. One of the three samples gave both primary and duplicate measurements of less than $50 \mu \mathrm{g} \mathrm{NH} / \mathrm{mL}$ (the minimum detection limit); one gave a primary measurement of $68.6 \mu \mathrm{g} \mathrm{NH} / \mathrm{mL}$ and a duplicate of less than $50 \mu \mathrm{g} \mathrm{NH} / \mathrm{mL}$; and the third gave a primary measurement of less than $50 \mu \mathrm{g}$ $\mathrm{NH}_{3} / \mathrm{mL}$ and a duplicate of $73.6 \mu \mathrm{g} \mathrm{NH} / \mathrm{mL}$. These concentrations are all much lower than the RGS estimate for interstitial liquid deep in the waste but are higher than the RGS isotopic measurement of ammonia in sample 7-2.

No conclusive total ammonia concentration can be calculated, and even estimates are risky because about half the data indicate very low ammonia partial pressures or concentrations and the other half indicate moderately high partial pressures. It seems possible that the ammonia concentration in U-103 waste was about twice that in Tank AW-101 waste, which had a lower bound of $27,000 \mu \mathrm{mol} \mathrm{NH} 3 / \mathrm{L}$ waste. This estimate is based on a comparison of the ammonia partial pressure measurements (see Sections 4.1.2 and C.1 for the AW-101 measurements).

A lower-bound concentration of $50,000 \mu \mathrm{mol} \mathrm{NH} 3 / \mathrm{L}$ waste in the nonconvective layer corresponds to $0.084 \mathrm{M}(1400 \mu \mathrm{g} / \mathrm{mL})$ in the liquid, or $0.056 \mathrm{wt} \%$ of the bulk waste. (The conversion of $\mathrm{mol} / \mathrm{L}$ waste to liquid concentrations assumed a gas volume fraction of 0.11 in the waste and a solids volume fraction of 0.33 in the degassed waste, as shown in the U-103 calculations spreadsheet on the CD supplied with this report.) There are no RGS data for ammonia concentration in the thin supernatant layer.

\section{C.7 S-106}

Table C.7.1 presents the RGS ammonia measurements. The "Extracted $\mathrm{NH}_{3}$ " column gives the total moles of ammonia that were pumped as vapor from the samples into the collection canisters, expressed as the moles of $\mathrm{NH}_{3}$ per liter of original waste (including gas, solids, and liquid volume). The residual ammonia was determined isotopically and is included in the table.

(a) TWINS database, samples 3U-99-1, 3U-99-2, and 3U-99-3; samples' ID numbers were S99T000537, S99T000546, and S99T000548. 
The post-extrusion partial pressure of $\mathrm{NH}_{3}$ over the sample (see Section 3.4.4) was measured at laboratory temperature and is also included in the table along with the measurement temperatures. No other ammonia measurements were made on RGS samples.

The table also includes ammonia measured by ISE in drainable liquid from non-RGS core samples from risers 7 and 8.(a) The concentrations were originally reported per volume of liquid, but for Table C.7.1 have been converted to a volume-waste basis. The core extrusions showed that non-RGS samples 7-1, 7-2, 8-1, and 8-4 were largely composed of liquid (though in the case of 8-4 some of the liquid was hydrostatic head fluid). For these samples, $1 \mathrm{~L}$ of liquid was taken to equal $1 \mathrm{~L}$ of waste. The other, lower-elevation samples were taken to contain only 0.50 volume fraction liquid, so that $0.5 \mathrm{~L}$ liquid equaled $1 \mathrm{~L}$ waste. (Liquid volume fractions used in RGS calculations in the S-106 spreadsheet supplied with this report are about 0.50 .)

Ammonia was also measured by (ISE) in a 1992 grab-sample taken from the liquid at the bottom of the salt-well screen.(b) This was a completely liquid sample, containing less than 10 vol\% bulk solids even under laboratory conditions. The concentration of $14,000 \mu \mathrm{mol} \mathrm{NH} / \mathrm{L}$ liquid would correspond to a concentration of roughly $7,000 \mu \mathrm{mol} \mathrm{NH}_{3} / \mathrm{L}$ waste in the nonconvective layer, assuming the volume fraction of liquid in the layer is about 0.5 .

Table C.7.1. Ammonia Data from Tank S-106 Samples

\begin{tabular}{|c|c|c|c|c|c|}
\hline Sample & $\begin{array}{c}\text { Extracted } \\
\mathrm{NH}_{3} \\
(\mu \mathrm{mol} / \mathrm{L})\end{array}$ & $\begin{array}{l}\text { Isotopically } \\
\text { measured } \\
\text { residual } \mathrm{NH}_{3} \\
(\mu \mathrm{mol} / \mathrm{L})\end{array}$ & $\begin{array}{c}\text { Non-RGS } \\
\text { core sample } \\
\mathrm{NH}_{3} \\
(\mu \mathrm{mol} / \mathrm{L})\end{array}$ & $\begin{array}{c}\text { Post-extrusion } \mathrm{NH}_{3} \\
\text { partial pressure } \\
\text { (atm) }\end{array}$ & $\begin{array}{l}\text { Measurement } \\
\text { temperature } \\
\left({ }^{\circ} \mathrm{C}\right)\end{array}$ \\
\hline $\begin{array}{l}8-1 \\
7-1 \\
\end{array}$ & & & $\begin{array}{l}1500 \\
<590 \\
\end{array}$ & & \\
\hline $7-2$ & & & $<290$ & & \\
\hline $7-3$ & $120 \pm 120$ & $860 \pm 260$ & $\begin{array}{c}1700 \\
\text { (Seg. 8-3) }\end{array}$ & $0.0043 \pm 0.0022^{(a)}$ & 25.0 \\
\hline $8-4$ & & & 9400 & & \\
\hline $7-5$ & $1800 \pm 480$ & $4500 \pm 1300$ & $\begin{array}{c}11000 \\
\text { (Seg. 8-5) }\end{array}$ & $0.0045 \pm 0.0023(a)$ & 25.0 \\
\hline $8-6$ & $390 \pm 230$ & $1400 \pm 420$ & $\begin{array}{c}6100 \\
(\text { Seg. } 7-6)\end{array}$ & $\begin{array}{c}0.0073 \pm \\
0.0036(\mathrm{a}, \mathrm{b})\end{array}$ & 24.2 \\
\hline $8-7$ & & & 5500 & & \\
\hline $8-10$ & $600 \pm 230$ & $1100 \pm 330$ & & $0.0041 \pm 0.0021^{(a)}$ & 22.1 \\
\hline
\end{tabular}

(a) TWINS database, samples with ID numbers S98T002575, S99T002576, S99T002577, S99T002578, S99T002579, S99T002580, S99T002581, and S99T002582.

(b) Sutey MJ. December 4, 1995. "Waste Compatibility Assessment of Tanks 241-S-101, 241-S103, 241-S-106, 241-S-107, 241-S-108, 241-S-109, and 241-S-110 Waste with Tank 241-SY-102 Waste via DCRT 244-S." Memo 7724-95-030 to SJ Rifaey, Westinghouse Hanford Company, Richland, Washington. 
November 1998 non-RGS ammonia measurements were also available for tank S-106.(a) The ISE measurements were made on liquid grab samples. Concentrations were consistently above the minimum detection limits of $55 \mu \mathrm{g} \mathrm{NH} / \mathrm{mL}$ and ranged from $131 \mu \mathrm{g} \mathrm{NH} / \mathrm{mL}$ to $240 \mu \mathrm{g}$ $\mathrm{NH}_{3} / \mathrm{mL}$. These concentrations are equivalent to a range of 7,700 to $14,000 \mu \mathrm{mol} \mathrm{NH} / \mathrm{L}$ liquid. Taking the liquid fraction of 0.5 into account, the range would correspond to about 3,800 to 7,000 $\mu \mathrm{mol} \mathrm{NH}_{3} / \mathrm{L}$ waste in the nonconvective layer.

Note that the ammonia concentration measured in the grab samples might not be strictly comparable to the concentrations in RGS samples. The ammonia in the grab sample liquid could be lowered relative to that in RGS core sample liquid by one or both of two mechanisms. There could have been ammonia evaporation from the sample during sample handling, as for core extrusions, or there could have been in situ ammonia evaporation from the salt-well or supernatant liquid that decreased its ammonia concentration relative to that in the less-exposed interstitial liquid sampled by RGS. The core extrusions would also be subject to ammonia loss from handling. In general, measurements on extrusions and grab samples are likely to be lower bounds, as is also discussed in Section 3.4.2.

The ammonia measurement made by isotopic ammonia vapor injection is believed to underestimate the ammonia in S-106 samples because of the very short equilibration times (ranging from 0.1 to 2 hours). A too-short equilibration time would mean that too much $15 \mathrm{NH}_{3}$ was left in the vapor phase, giving too high a ratio of $15 \mathrm{NH}_{3} / 14 \mathrm{NH}_{3}$ and consequently underestimating the residual $14 \mathrm{NH}_{3}$. Accordingly, the post-extrusion $\mathrm{NH}_{3}$ partial pressures were used to calculate the in situ ammonia vapor pressures.

A comparison of the ammonia partial pressure measurements indicates that the ammonia concentration in S-106 waste was about half that in AW-101 waste, which had a lower bound of $27,000 \mu \mathrm{mol} \mathrm{NH} / \mathrm{L}$ waste (see Sections 4.1.2 and C.1 for the AW-101 measurements). The grab samples, on the other hand, indicate a lower bound of 7,000 to $11,000 \mu \mathrm{mol} \mathrm{NH}_{3} / \mathrm{L}$ waste (where some of the scatter undoubtedly comes from uncertainty in the liquid volume fraction). While these two sets of lower-bound estimates differ by $30 \%$ or more, they do both suggest that the ammonia concentrations in S-106 were relatively low -- but not as low as those found by the isotopic method (which were probably underestimates).

Based on RGS data (partially confirmed by other sources), a lower-bound concentration of $13,000 \mu \mathrm{mol} \mathrm{NH} / 2$ waste is taken to have existed in the nonconvective layer. This concentration corresponds to $0.025 \mathrm{M}(430 \mu \mathrm{g} / \mathrm{mL})$ in the interstitial liquid, or $0.015 \mathrm{wt} \%$ of the bulk waste. (The conversion of mol/L waste to liquid concentrations assumed a gas volume fraction of 0.14 in the waste and a solids volume fraction of 0.40 in the degassed waste, as shown in the S-106 calculations spreadsheet on the CD supplied with this report.)

\section{C.8 BY-101}

No RGS samples from BY-101 were successfully extracted, so there are no RGS ammonia estimates for this tank.

(a) TWINS database, samples with ID numbers S98T003258, S99T003259, and S99T003260. 


\section{C.9 BY-109}

Table C.9.1 presents the ammonia measurements. The "Extracted $\mathrm{NH}_{3}$ " column gives the total moles of ammonia that were pumped as vapor from the samples into the collection canisters expressed as the moles of $\mathrm{NH}_{3}$ per liter of original waste (including gas, solids, and liquid volume). The residual ammonia was determined isotopically and is included in the table. The post-extrusion partial pressure of $\mathrm{NH}_{3}$ over the sample (see Section 3.4.4) was measured at laboratory temperature and is included in Table C.9.1 together with the measurement temperatures. No other ammonia measurements were made on RGS samples.

The ammonia measurement made by isotopic ammonia vapor injection may have underestimated the ammonia in BY-109 samples in spite of the longer equilibration times (ranging from 21.5 to 139 hours). A too-short equilibration time would have meant that too much $15 \mathrm{NH}_{3}$ was left in the vapor phase, giving too high a ratio of $15 \mathrm{NH}_{3} / 14 \mathrm{NH}_{3}$ and consequently underestimating the residual ${ }^{14} \mathrm{NH}_{3}$. Accordingly, the post-extrusion $\mathrm{NH}_{3}$ partial pressures were used to calculate the in situ ammonia vapor pressures.

No conclusive total ammonia concentration can be calculated, but it seems likely that the ammonia concentration in Tank BY-109 waste is one-third to one-half that in Tank AW-101 waste, which had a lower bound of $27,000 \mu \mathrm{mol} \mathrm{NH}_{3} / \mathrm{L}$ waste. This estimate is based on a comparison of the ammonia partial pressure measurements (see Sections 4.1.2 and C.1 for the AW-101 measurements). The estimate is closely consistent with the total ammonia concentrations of 9000 to $14000 \mu \mathrm{mol} \mathrm{NH} 3 / \mathrm{L}$ waste that were found isotopically for samples $12 \mathrm{C}-4$ and $10 \mathrm{~B}-5$, which were allowed 139 and 91 hours equilibration respectively, with mixing. The total ammonia concentration of $6300 \mu \mathrm{mol} \mathrm{NH} / \mathrm{L}$ waste that was found isotopically for sample $10 \mathrm{~B}-6$ is the lowest of the three, perhaps because of the shorter equilibration time of 21.5 hours.

Based on RGS data, a lower-bound concentration of $10,000 \mu \mathrm{mol} \mathrm{NH}_{3} / \mathrm{L}$ waste is taken to have existed in the waste below the interstitial liquid level (ILL). This concentration corresponds to $0.014 \underline{\mathrm{M}}(250 \mu \mathrm{g} / \mathrm{mL})$ in the interstitial liquid, or $0.011 \mathrm{wt} \%$ of the bulk waste. (The conversion of $\mathrm{mol} / \mathrm{L}$ waste to liquid concentrations assumed a gas volume fraction of 0.09 in the waste and a solids volume fraction of 0.24 in the degassed waste, as shown in the BY-109 calculations spreadsheet on the CD supplied with this report.)

Table C.9.1. Ammonia Data from Tank BY-109 Samples

\begin{tabular}{||c|c|c|c|c||}
\hline Sample & $\begin{array}{c}\text { Extracted } \\
\mathrm{NH}_{3} \\
(\mu \mathrm{mol} / \mathrm{L})\end{array}$ & $\begin{array}{c}\text { Isotopically measured } \\
\text { residual } \mathrm{NH}_{3} \\
(\mu \mathrm{mol} / \mathrm{L})\end{array}$ & $\begin{array}{c}\text { Post-extrusion } \mathrm{NH}_{3} \\
\text { partial pressure } \\
(\mathrm{atm})\end{array}$ & $\begin{array}{c}\text { Measurement } \\
\text { temperature } \\
\left({ }^{\circ} \mathrm{C}\right)\end{array}$ \\
\hline \hline 12C-4 & $1000 \pm 480$ & $8300 \pm 1300$ & $0.0038 \pm 0.0019(\mathrm{a})$ & 24.7 \\
\hline 10B-5 & $2000 \pm 560$ & $12000 \pm 1200$ & $0.0030 \pm 0.0015(\mathrm{a})$ & 23.9 \\
\hline 10B-6 & $1400 \pm 490$ & $4900 \pm 190$ & $0.0035 \pm 0.0018(\mathrm{a})$ & 24.1 \\
\hline (a) These data were used to calculate in situ ammonia vapor pressures. \\
\hline
\end{tabular}




\section{C.10 SX-106}

Table C.10.1 presents the ammonia measurements. The "Extracted $\mathrm{NH}_{3}$ " column gives the total moles of ammonia that were pumped as vapor from the samples into the collection canisters, expressed as the moles of $\mathrm{NH}_{3}$ per liter of original waste (including gas, solids, and liquid volume). The residual ammonia was determined isotopically and is included in the table. The postextrusion partial pressure of $\mathrm{NH}_{3}$ over the sample (see Section 3.4.4) was measured at laboratory temperature and is included in Table C.10.1 with the measurement temperatures. The residual ammonia in the samples was also measured by ISE after the samples were removed from the extractor; as discussed in Section 3.4.2, these ammonia concentrations should be considered lower bounds on the concentrations in the samples because of ammonia evaporation during sample handling.

For comparison, the table also presents the ammonia measured by ISE in a $1998 \mathrm{grab}$ sample taken from the supernatant liquid.(a) The concentration of $1100 \mu \mathrm{mol} \mathrm{NH} / \mathrm{L}$ liquid is on the same basis as the RGS ammonia measurements in the supernatant layer, where "per L waste" and "per L liquid" are the same. Note that the ammonia concentration measured in this grab sample may be an underestimate of the concentration in the supernatant. There could have been ammonia evaporation from the sample during sample handling, as for core extrusions.

Table C.10.1. Ammonia Data from Tank SX-106 Samples

\begin{tabular}{|c|c|c|c|c|c|c|}
\hline Sample & $\begin{array}{c}\text { Extracted } \mathrm{NH}_{3} \\
(\mu \mathrm{mol} / \mathrm{L})\end{array}$ & $\begin{array}{c}\text { Isotopically } \\
\text { measured } \\
\text { residual } \mathrm{NH}_{3} \\
(\mu \mathrm{mol} / \mathrm{L})\end{array}$ & $\begin{array}{l}\text { Total } \mathrm{NH}_{3} \text { from } \\
\text { post-RGS } \\
\text { residual by } \\
\text { ISE(a) } \\
(\mu \mathrm{mol} / \mathrm{L})\end{array}$ & $\begin{array}{l}\text { Supernatant } \\
\text { grab sample } \\
(\mu \mathrm{mol} / \mathrm{L} \text { liq })\end{array}$ & $\begin{array}{c}\text { Post-extrusion } \\
\mathrm{NH}_{3} \text { partial } \\
\text { pressure } \\
\text { (atm) }\end{array}$ & $\begin{array}{c}\text { Measurement } \\
\text { temperature } \\
\left({ }^{\circ} \mathrm{C}\right)\end{array}$ \\
\hline $3-2$ & $25 \pm 13(b)$ & $25000 \pm 3200(b)$ & 10000 & \multirow{2}{*}{1100} & $\begin{array}{c}0.0024 \pm \\
0.0012\end{array}$ & 12.3 \\
\hline $3-4$ & $12 \pm 8.7^{(b)}$ & $23000 \pm 2000^{(b)}$ & 2500 & & $\begin{array}{c}0.0021 \pm \\
0.0010\end{array}$ & 12.3 \\
\hline $6-6$ & $8100 \pm 600^{(b)}$ & $\begin{array}{l}100000 \pm \\
10000(b)\end{array}$ & 58000 & & $\begin{array}{c}0.0067 \pm \\
0.0033\end{array}$ & 12.0 \\
\hline $6-6 A$ & $4500 \pm 350^{(b)}$ & $66000 \pm 6100^{(b)}$ & 46000 & & $\begin{array}{c}0.0039 \pm \\
0.0020\end{array}$ & 12.4 \\
\hline $3-7$ & $9000 \pm 770^{(b)}$ & $63000 \pm 32000^{(b)}$ & 38000 & & $\begin{array}{l}0.013 \pm \\
0.065(c)\end{array}$ & 11.9 \\
\hline $6-9$ & $15000 \pm 1100^{(b)}$ & $46000 \pm 8700^{(b)}$ & 42000 & & $0.016 \pm 0.008$ & 12.2 \\
\hline $3-10$ & $16000 \pm 850^{(b)}$ & $59000 \pm 10000^{(b)}$ & 52000 & & $0.012 \pm 0.006$ & 11.9 \\
\hline \multicolumn{7}{|c|}{$\begin{array}{l}\text { (a) These total ammonia concentrations are the sum of the post-RGS ISE ammonia residual and the RGS extracted ammonia. } \\
\text { They should be considered lower bounds because of ammonia evaporation during the sample handling that preceded ISE } \\
\text { analysis. } \\
\text { (b) These data were used to calculate in situ ammonia vapor pressures. } \\
\text { (c) This value is suspect because of air inleakage. }\end{array}$} \\
\hline
\end{tabular}

(a) TWINS Database, sample S98T002675, 6SX-98-1. 
The four different types of measurements of ammonia in the supernatant layer did not give consistent results. We have used the isotopic RGS ammonia concentrations to calculate the ammonia in the in situ vapor, though for the following reasons we think it is possible that they were overestimated by about a factor of 2:

- The isotopic RGS measurements (samples 3-2 and 3-4) gave the highest concentrations, about $25,000 \mu \mathrm{mol} \mathrm{NH} / \mathrm{L}$ liquid. There is a possibility that equilibration was not complete, although sample 3-4 (with a 3-day equilibration time) gave about the same result as sample 3-2 (with a 1-day equilibration time) (see Table C.10.2.) A too-short equilibration time would have meant that too little $15 \mathrm{NH}_{3}$ had reached the vapor phase, giving too low a ratio of $15 \mathrm{NH}_{3} / 14 \mathrm{NH}_{3}$ and consequently overestimating the residual $14 \mathrm{NH}_{3}$.

- The supernatant ammonia concentrations based on post-RGS ISE were the next highest values at 2,500 and $10,000 \mu \mathrm{mol} \mathrm{NH} / \mathrm{L}$ liquid. These are likely to be lower bounds because of ammonia evaporation during post-RGS handling. Substantial evaporation is believed to have occurred from these samples; the post-RGS ISE measurements gave ammonia contents that were less (10\% and $70 \%$ less) than the amount of ammonia added in the standard, which is not physically possible without losses.

- The supernatant ammonia concentration was also estimated approximately at $10,000 \mu \mathrm{mol}$ $\mathrm{NH}_{3} / \mathrm{L}$ waste from the post-extrusion ammonia partial pressures (which were consistent with one another). These partial pressures were upper bounds based on the unusually rapid rate of decrease of the extractor pressure at the time of measurement. The pressures were about one-half to one-third of those found for AW-101 waste, which had a lower bound of $27,000 \mu \mathrm{mol} \mathrm{NH} / \mathrm{L}$ liquid. To make the comparison, the SX-106 partial pressure was adjusted upward by $80 \%$, to $0.4 \mathrm{kPa}$, to account for the temperature difference between SX-106 and AW-101 measurements (12 and $24^{\circ} \mathrm{C}$ respectively). The temperature adjustment was based on the Schumpe model of ammonia solubility, which is accurate within $20 \%$ at temperatures below $30^{\circ} \mathrm{C}$ (see Figure 3.3 and the accompanying discussion in Section 3.6.1).

- The lowest measured ammonia concentration in the supernatant was that in the grab sample (only a single sample). Here again, sample handling losses could make this measurement a lower bound, though losses would not normally be expected to cause a factor of 5 or more difference from other higher measurements. On the other hand, there was a factor of 4 difference between the two post-RGS ISE measurements in the supernatant liquid, and that difference probably came from handling losses.

- The ammonia extracted from the supernatant samples (3-2 and 3-4) was much less than that extracted from the others. The very low extraction could indicate much lower ammonia concentrations in these samples. This explanation is contradicted by the observation that, after the isotopic solution is added, every sample shows very little extracted ammonia no matter how high the extracted ammonia was before adding the solution. Thus the amount of ammonia extracted does not always correlate with the total ammonia. We do not have an explanation for this, but we note that low extraction often coincides with high liquid content and was seen only for samples from SX-106 and later tanks, where the extraction process chilled the samples to $10-15^{\circ} \mathrm{C}$.

It is useful in assessing the quality of isotopic $\mathrm{NH}_{3}$ measurements to review the isotopic ratios measured for each canister of gas. For SX-106, three canisters of gas were collected in parallel at each equilibration time; the contents of all three should have had the same isotopic ratio. Table C.10.3 shows that the three canisters rarely gave the same ratio and that there was often a 
Table C.10.2. Residual Ammonia Measurements versus Equilibration Time

\begin{tabular}{|c|c|c|c|c|c||}
\hline \multirow{3}{*}{ Sample } & \multicolumn{5}{|c|}{$\begin{array}{c}\text { Estimated residual }{ }^{14} \mathrm{NH}_{3} \text { ( } \mu \text { mol) in sample } \\
\text { at approximate times after isotopic addition }\end{array}$} \\
\cline { 2 - 6 } & $1-2 \mathrm{hr}$ & 1 day & 2 days & 3 days & 6 days \\
\hline $3-2$ & 8700 & 7500 & & & \\
\hline $3-4$ & 9800 & & & 7000 & \\
\hline $6-6$ & 31000 & & & & 30000 \\
\hline $6-6 \mathrm{~A}$ & 27000 & & & 19000 & \\
\hline $3-7$ & 38000 & 19000 & & & \\
\hline $6-9$ & 29000 & 13000 & & & \\
\hline $3-10$ & 43000 & & & 18000 & \\
\hline
\end{tabular}

consistent trend as canisters from the same sample were successively measured. The trend was usually upward (toward lower estimates of residual ammonia). It was suspected that the changing ratio measurements were caused by ammonia sorption, but the exact mechanism was not determined.

The samples from the supernatant layer showed ratios that appeared to still be rising at the second equilibration time (the one used for residual ammonia calculations). That is, if more subsamples had been taken from the canisters, the ratios might have leveled out (as was seen for some later tanks' samples) higher ratios might have been measured and lower residual ammonia calculated. However, multiple subsampling was not being done at the time SX-106 samples were processed. In addition, SX-106 sample measurements allowed only a short pump-down to clear sorbed ammonia between canister sets from different samples or equilibration times, so different canister sets run in sequence might have influenced each others' ratios.

There were three types of ammonia measurements in the nonconvective layer, all consistent. Most of the isotopic RGS measurements gave concentrations of 60,000 to $75,000 \mu \mathrm{mol}$ $\mathrm{NH}_{3} / \mathrm{L}$ waste, with one higher value from sample 6-6.

The post-RGS ISE results from nonconvective layer samples ranged from 40,000 to $60,000 \mu \mathrm{mol}$ of $\mathrm{NH}_{3} / \mathrm{L}$ waste, a difference that could be accounted for by handling losses. The post-extrusion partial pressures (when raised by $80 \%$ to account for measurement temperature differences) suggest concentrations somewhat less than twice those in Tank AW-101 waste, which had a lower bound of $27,000 \mu \mathrm{mol} \mathrm{NH} / \mathrm{L}$ waste. This approximate estimate of 45,000 to $60,000 \mu \mathrm{mol} \mathrm{NH}_{3} / \mathrm{L}$ of waste is consistent with the other measurements as a lower bound.

Referring to Table C.10.3, samples 6-6, 6-6A, and 6-9 gave (at the second equilibration time) isotopic ratios that seemed to have leveled out to relatively constant values. Sample 3-10 ratios were probably still rising, and sample 3-7 ratios were scattered and difficult to measure because of the large air inleakage. The several samples from the nonconvective layer gave similar values (except for 6-6), whether or not the ratios were still trending upward. It seems likely that, because of the ratio trending, the isotopic method overestimated the residual ammonia in SX-106. However, the consistency of isotopic results with post-RGS ISE and partial-pressure results suggests that the overestimation of nonconvective layer ammonia was not substantial. 
Table C.10.3. Isotopic Ratios and Measurement Sequences

\begin{tabular}{|c|c|c|c|c|c|}
\hline \multirow[b]{2}{*}{ Sample } & \multirow{2}{*}{$\begin{array}{l}\text { Isotopic } \\
\text { equilibra- } \\
\text { tion time } \\
\end{array}$} & \multirow{2}{*}{$\begin{array}{l}\text { Length of preceding } \\
\text { mass-spec } \\
\text { pumpdown }\end{array}$} & \multicolumn{3}{|c|}{${ }^{15} \mathrm{NH}_{3} /{ }^{14} \mathrm{NH}_{3}$ ratio measured by mass spec } \\
\hline & & & $1^{\text {st }}$ canister & $2^{\text {nd }}$ canister & $3^{\text {rd }}$ canister \\
\hline $3-2$ & $1 \mathrm{hr}$ & $5 \mathrm{~min}$. & 0.48 & 0.66 & 0.64 \\
\hline $3-2$ & 1 day & $5 \mathrm{~min}$. & 0.60 & 0.71 & 0.75 \\
\hline $3-4$ & $2 \mathrm{hr}$ & $5 \mathrm{~min}$. & $0.72^{(a)}$ & 0.65 (a) & 0.36 (a) \\
\hline $3-4$ & 3 days & $5 \mathrm{~min}$. & 0.76 (a) & $0.76(a)$ & $0.81(\mathrm{a})$ \\
\hline $6-6$ & $2 \mathrm{hr}$ & $5 \mathrm{~min}$. & 0.137 & 0.184 & 0.181 \\
\hline $6-6$ & 6 days & $5 \mathrm{~min}$. & 0.194 & 0.173 & 0.194 \\
\hline $6-6 \mathrm{~A}$ & $1 \mathrm{hr}$ & $5 \mathrm{~min}$. & 0.160 & 0.190 & 0.212 \\
\hline $6-6 \mathrm{~A}$ & 3 days & 5 min. & 0.271 & 0.296 & 0.303 \\
\hline $3-7$ & $2 \mathrm{hr}$ & $5 \mathrm{~min}$. & $-\ldots$ (a) & $0.074(\mathrm{a})$ & 0.15 (a) \\
\hline $3-7$ & 1 day & $5 \mathrm{~min}$. & $\ldots$-.a) & $\ldots$ (a) & 0.30 (a) \\
\hline $6-9$ & $1 \mathrm{hr}$ & $5 \mathrm{~min}$. & 0.179 & 0.191 & 0.195 \\
\hline $6-9$ & 1 day & $5 \mathrm{~min}$. & 0.289 & 0.407 & 0.432 \\
\hline $3-10$ & $2 \mathrm{hr}$ & $5 \mathrm{~min}$. & 0.13 & 0.13 & 0.13 \\
\hline $3-10$ & 3 days & $5 \mathrm{~min}$. & 0.22 & 0.28 & 0.32 \\
\hline
\end{tabular}

The average and standard deviation of the isotopically determined ammonia concentrations over samples 6-6, 6-6A, 3-7, 6-9, and 3-10 were 78,000 $\pm 19,000 \mu \mathrm{mol} / \mathrm{L}$ of waste. This corresponds to an average of $0.18 \mathrm{wt} \% \mathrm{NH}_{3}$ in the bulk waste, or $0.15 \mathrm{M}(2500 \mu \mathrm{g} \mathrm{NH} / \mathrm{mL})$ in the liquid, at the time of sampling. The gas and solid volume fractions of each sample were used to calculate the ammonia concentration in the liquid for the sample; those volume fractions can be seen in the "In Situ" worksheet of the SX-106 spreadsheet on the CD supplied with this report.

Table C.10.2 shows the way in which the residual ammonia measurements from SX-106 samples varied with equilibration time. The data make it clear that further equilibration occurred after the first hour or two, but it is not clear whether more than 24 hours was required. The higher ammonia concentration found for sample 6-6 seems unlikely to have resulted from incomplete equilibration, as its equilibration time was longer than that of any other sample (Table C.10.2).

\section{C.11 AX-101}

Table C.11.1 presents ammonia measurements. The "Extracted $\mathrm{NH}_{3}$ " column gives the total moles of ammonia that were pumped as vapor from the sample into the collection canisters expressed as the moles of $\mathrm{NH}_{3}$ per liter of original waste (including gas, solids, and liquid volumes). The residual ammonia was determined isotopically, and is also included in the table. The post-extrusion partial pressure of $\mathrm{NH}_{3}$ over the sample (see Section 3.4.4) was measured at laboratory temperature and is included in the table as are the measurement temperatures. No other ammonia measurements were made on RGS samples. 
Table C.11.1. Ammonia Data from Tank AX-101 Sample

\begin{tabular}{|c|c|c|c|c|c||}
\hline Sample & $\begin{array}{c}\text { Extracted } \mathrm{NH}_{3} \\
(\mu \mathrm{mol} / \mathrm{L})\end{array}$ & $\begin{array}{c}\text { Isotopically measured } \\
\text { residual } \mathrm{NH}_{3} \\
(\mu \mathrm{mol} / \mathrm{L})\end{array}$ & $\begin{array}{c}\text { Salt-well grab } \\
\text { sample } \\
(\mu \mathrm{mol} / \mathrm{L} \text { liq })\end{array}$ & $\begin{array}{c}\text { Post-extrusion } \\
\mathrm{NH}_{3} \text { partial } \\
\text { pressure (atm) })\end{array}$ & $\begin{array}{c}\text { Measurement } \\
\text { temperature } \\
\left({ }^{\circ} \mathrm{C}\right)\end{array}$ \\
\hline \hline $\begin{array}{c}\text { level of } \\
\text { seg 2 }\end{array}$ & & 108000 & & \\
\hline $\begin{array}{c}\text { level. of } \\
\text { seg 4 }\end{array}$ & & & 66000 & & \\
\hline 9D-8 & $19000 \pm 1000^{(a)}$ & $120000 \pm 30000^{(a)}$ & 84000 & $0.014 \pm 0.007$ & 11.9 \\
\hline (a) These data were used to calculate in situ ammonia vapor pressures. \\
\hline
\end{tabular}

For comparison, Table C.11.1 also includes ammonia measurements from the liquid drained from salt-well grab samples taken in 1997 (Esch 1997), in which ammonia was measured by ISE. These samples contained 9 to 18 vol\% bulk solids under laboratory conditions. A concentration of 66,000 to $108,000 \mu \mathrm{mol} \mathrm{NH} 3 / \mathrm{L}$ liquid, for example, would correspond to a concentration of roughly 40,000 to $65,000 \mu \mathrm{miol} \mathrm{NH} / \mathrm{L}$ waste in the nonconvective layer, assuming the volume fraction of liquid in the layer is about $60 \mathrm{vol} \%$. (The liquid volume fraction used in RGS calculations for sample 9D-8 is 0.57, as can be seen in the "In Situ" worksheet in the AX-101 calculations spreadsheet on the CD supplied with this report.)

Note that the ammonia concentrations measured in these grab samples might not be strictly comparable to those in RGS samples. The ammonia in the grab sample could have been lowered relative to that in RGS core samples by one or both of two mechanisms. There could have been ammonia evaporation from the sample during sample handling, as for core extrusions, or in situ ammonia evaporation from the salt-well liquid that decreased its ammonia concentration relative to that in the less-exposed interstitial liquid sampled by RGS.

Partial pressures indicate that the ammonia concentration in the AX-101 waste sample was roughly three times that in Tank AW-101 waste, which had a lower bound of $27,000 \mu \mathrm{mol} \mathrm{NH} / \mathrm{L}$ waste. This estimate is based on a comparison of the ammonia partial pressure measurements (see Sections 4.1.2 and C.1 for the AW-101 measurements). To make the comparison, the AX-101 partial pressure was adjusted upward by $80 \%$, to $2.6 \mathrm{kPa}$, to account for the temperature difference between AX-101 and AW-101 measurements. The temperature adjustment was based on the Schumpe model of ammonia solubility, which is accurate within $20 \%$ at temperatures below $30^{\circ} \mathrm{C}$ (see Figure 3.3 and the accompanying discussion in Section 3.6.1).

The ammonia estimate based on partial pressure comparison is consistent with the ammonia concentrations found in the salt-well grab samples, if the latter are considered lower bounds. The estimate seems somewhat low compared with the ammonia found by extraction and isotopic measurement of the RGS sample, but the uncertainties of the estimate and the isotopic measurement overlap.

The possibility that the mixture of isotopic standard and sample had not reached vaporliquid equilibrium cannot be ruled out, even though the residual ammonia measured isotopically after 1.7 hours of equilibration was within $3 \%$ of that measured after 24 hours (Table C.11.2). If equilibration was incomplete, the residual ammonia would have been overestimated because a less than equilibrium amount of the $15 \mathrm{NH}_{3}$ would have been in the sampled vapor. The isotopic ratio 
trend appeared to have leveled off for the canisters taken at the second equilibration time, so sorption effects were probably small. Despite these favorable indications, it remains possible that the RGS-measured ammonia was an upper bound.

The isotopically determined ammonia concentration of sample 9D-8 was $139,000 \pm$ $30,000 \mathrm{mmol} / \mathrm{L}$ of waste. This corresponds to $0.17 \mathrm{wt} \% \mathrm{NH}_{3}$ in the bulk waste, or $0.24 \mathrm{M}$ ( $4100 \mathrm{mg} \mathrm{NH} / \mathrm{mL}$ ) in the liquid, at the time of sampling (see Table C.11.3). The gas and solid volume fraction of the sample was used to calculate the ammonia concentration in the liquid; those volume fractions can be seen in the "In Situ" worksheet of the AX-101 calculation spreadsheet on the CD supplied with this report.

Table C.11.2. Residual Ammonia Measurements versus Equilibration Time

\begin{tabular}{||c|c|c|c|c|c|}
\hline \multirow{2}{*}{ Sample } & \multicolumn{5}{|c|}{$\begin{array}{c}\text { Estimated residual } \\
\text { at approximate times after isotopic addition }\end{array}$} \\
\cline { 2 - 6 } & $1-2 \mathrm{hr}$ & 1 day & 2 days & 3 days & 6 days \\
\hline 9D-8 & 37000 & 36000 & & & \\
\hline
\end{tabular}

Table C.11.3. Isotopic Ratios and Measurement Sequences

\begin{tabular}{|c|c|c|c|c|c|}
\hline \multirow[b]{2}{*}{ Sample } & \multirow{2}{*}{$\begin{array}{c}\text { Isotopic } \\
\text { equilibration } \\
\text { time } \\
\end{array}$} & \multirow{2}{*}{$\begin{array}{c}\text { Length of preceding } \\
\text { mass-spec } \\
\text { pumpdown }\end{array}$} & \multicolumn{3}{|c|}{${ }^{15} \mathrm{NH}_{3} / 14 \mathrm{NH}_{3}$ ratio measured by mass spec } \\
\hline & & & $1^{\text {st }}$ canister & $2^{\text {nd }}$ canister & $3^{\text {rd }}$ canister \\
\hline $9 \mathrm{D}-8$ & $2 \mathrm{hr}$ & $5 \mathrm{~min}$. & 0.089 & 0.11 & 0.153 \\
\hline 9D-8 & 1 day & $5 \mathrm{~min}$. & 0.142 & 0.157 & 0.158 \\
\hline
\end{tabular}

\section{C.12 S-102}

Table C.12.1 presents the RGS ammonia measurements. The "Extracted $\mathrm{NH}_{3}$ " column gives the total moles of ammonia that were pumped as vapor from the samples into the collection canisters expressed as the moles of $\mathrm{NH}_{3}$ per liter of original waste (including gas, solids, and liquid volume). The residual ammonia was determined isotopically and is included in the table. The post-extrusion partial pressure of $\mathrm{NH}_{3}$ over the sample (see Section 3.4.4) was measured at laboratory temperature and is included with the measurement temperatures. The residual ammonia in the samples was also measured by ISE after the samples were removed from the extractor; as discussed in Section 3.4.3, these ammonia concentrations should be considered lower bounds on the concentrations in the samples because of ammonia evaporation during sample handling. 
Table C.12.1. Ammonia Data from Tank S-102 Samples

\begin{tabular}{||c|c|c|c|c|c||}
\hline \hline Sample & $\begin{array}{c}\text { Extracted NH } \\
(\mu \mathrm{mol} / \mathrm{L})\end{array}$ & $\begin{array}{c}\text { Isotopically } \\
\text { measured } \\
\text { residual NH } \\
(\mu \mathrm{mol} / \mathrm{L})\end{array}$ & $\begin{array}{c}\text { Total } \mathrm{NH}_{3} \text { from } \\
\text { post-RGSS residual } \\
\text { by ISE(a) } \\
(\mu \mathrm{mol} / \mathrm{L})\end{array}$ & $\begin{array}{c}\text { Post-extrusion } \\
\mathrm{NH}_{3} \text { partial } \\
\text { pressure } \\
\text { (atm) }\end{array}$ & $\begin{array}{c}\text { Measurement } \\
\text { temperature } \\
\left({ }^{\circ} \mathrm{C}\right)\end{array}$ \\
\hline \hline $16-2$ & $340 \pm 170$ & $298000 \pm 97000$ & $13000^{(\mathrm{b})}$ & $0.0037 \pm 0.0019(\mathrm{c})$ & 11.8 \\
\hline $16-4 \mathrm{R}$ & $160 \pm 120$ & $67000 \pm 45000$ & $36000^{(\mathrm{b})}$ & -0.0019 & 12.1 \\
\hline $16-7$ & $3400 \pm 480^{(\mathrm{b})}$ & $31000 \pm 9000^{(\mathrm{b})}$ & 43000 & $0.011 \pm 0.0053$ & 12.1 \\
\hline $16-10$ & $7000 \pm 1000^{(\mathrm{b})}$ & $31000 \pm 9000^{(\mathrm{b})}$ & 48000 & $0.0063 \pm 0.0032$ & 12.0 \\
\hline $\begin{array}{l}\text { (a) The total ammonia concentrations are the sum of post-RGS ISE ammonia residual and RGS-extracted ammonia and } \\
\text { should be considered lower bounds because ammonia evaporated during sample handling preceding ISE analysis. } \\
\text { (b) These data were used to calculate in situ ammonia vapor pressures. } \\
\text { (c) This value is suspect because of air inleakage. }\end{array}$ \\
\hline
\end{tabular}

The non-RGS measurements of ammonia in S-102 include ISE measurements made on the liquid in non-RGS core sample 16-1(a) and on grab samples taken in 1998.(b) Core sample 16-1 contained $1700 \mu \mathrm{mol} / \mathrm{L}$ liquid, considerably lower than any RGS-measured value. However, liquid at the waste top, exposed to the domespace, would be expected to be lower in ammonia than interstitial liquid.

The liquid drained from the S-102 grab samples consistently gave low ammonia, less than the MDL of $1300 \mu \mathrm{mol} / \mathrm{L}$ liquid. However, the water-digested solids from the grab samples contained concentrations ranging from 122 to $475 \mu \mathrm{g} / \mathrm{g}$ solids, or 12,000 to $42,000 \mu \mathrm{mol} / \mathrm{L}$ solids. (Note that the "solids" were not dry but included interstitial liquid.) One possible explanation for the difference between the ammonia concentration in the drainable liquid from the grab samples and that in the bulk solids is that more ammonia was lost from the liquid during handling because the liquid had an exposed surface and was not "protected" in the pores of a matrix.

In general, the ammonia concentration measured in the grab samples might not be strictly comparable to the concentrations in RGS samples. The ammonia in the grab sample liquid could be lowered relative to that in RGS core sample liquid by either or both of two mechanisms. There could have been ammonia evaporation from the sample during sample handling, as for core extrusions. There could also have been in situ ammonia evaporation from the salt-well or supernatant liquid that decreased its ammonia concentration relative to that in the less-exposed interstitial liquid sampled by RGS. The core extrusions would also be subject to ammonia loss from handling. In general, measurements on extrusions and grab samples are likely to be lower bounds, as is also discussed in Section 3.4.2.

The RGS isotopic measurements of residual ammonia did not produce internally consistent results for the four S-102 samples. The ammonia concentrations determined by the isotopic method for samples 16-7 and 16-10 were lower than those determined by the post-RGS ISE method, even though the latter is suspected of underestimating residual ammonia because of handling losses and the former is suspected of overestimating ammonia because of insufficient

(a) TWINS database, sample with ID number S98T002516.

(b) TWINS database, samples with ID numbers S98T003209W, S98T003210W, S98T003211W, S98T003202, S98T003206, and S98T003198. 
isotopic equilibration and sorption trending. (Table C.12.2 suggests that for these two samples equilibration was not complete within two days and does not make it clear whether complete equilibration had been reached at seven days. The one-day value is overestimated by about $50 \%$ compared with the seven- or eight-day value. In addition, Table C.12.3 shows that at the final equilibration times the isotopic ratios may still have been rising for samples 16-7 and 16-10.)

The two samples taken at the highest elevation both gave high values of residual ammonia. On these samples, the measured isotopic ratios did not converge on a value within each set of three post-isotopic canisters (Table C.12.3). Therefore the isotopic results are considered suspect and were not used to calculate in situ ammonia. The post-RGS ISE residual ammonia measurements were used instead, with a high uncertainty attached to them to reflect the possibility of ammonia losses during handling. Normally the post-extrusion ammonia partial pressures would have been preferred, but in the case of these two samples, one pressure (for 16-2) was considered suspect because of air inleakage and the other pressure (for 16-4) was a physically impossible negative value (apparently because of a high calculated water vapor pressure over the sample).

The differences between the isotopic and post-RGS ISE methods are barely within the measurement uncertainty of the isotopic method. The post-extrusion partial pressures of these two samples (when raised by $80 \%$ to account for measurement temperature differences) suggest concentrations somewhat less than twice those in Tank AW-101 waste, which had a lower bound of $27,000 \mu \mathrm{mol} \mathrm{NH} / \mathrm{L}$ waste. This approximate estimate of 45,000 to $60,000 \mu \mathrm{mol} \mathrm{NH} / \mathrm{L}$ waste is high compared with the isotopic and post-RGS measurements, though the latter data lie within the $50 \%$ uncertainty of the partial pressure measurements. One of the grab-sample solids ammonia measurements fell near the lower end of the 45,000 to $60,000 \mu \mathrm{mol} \mathrm{NH}_{3} / \mathrm{L}$ waste range, though the other two were substantially lower.

The inconsistencies in the ammonia data are such that it could be misleading to calculate an average ammonia concentration for Tank S-102. However, most of the data suggest a concentration of no less than $\sim 15,000 \mu \mathrm{mol} \mathrm{NH}_{3} / \mathrm{L}$ waste and no more than $\sim 70,000 \mu \mathrm{mol} \mathrm{NH} / \mathrm{L}$ waste. A very approximate concentration of $50,000 \mu \mathrm{mol} \mathrm{NH} / / \mathrm{L}$ waste is taken to have existed in the $S-102$ waste. This concentration corresponds to $0.10 \mathrm{M}(1700 \mu \mathrm{g} / \mathrm{mL})$ in the interstitial liquid, or $0.072 \mathrm{wt} \%$ of the bulk waste. (The conversion of $\mathrm{mol} / \mathrm{L}$ waste to liquid concentrations assumed a gas volume fraction of 0.30 in the waste and a solids volume fraction of 0.29 in the degassed waste, as shown in the S-102 calculations spreadsheet on the CD supplied with this report.)

Table C.12.2. Residual Ammonia Measurements versus Equilibration Time

\begin{tabular}{|c|c|c|c|c|c|c||}
\hline \multirow{3}{*}{ Sample } & \multicolumn{6}{|c||}{$\begin{array}{c}\text { Estimated residual }{ }^{14} \mathrm{NH}_{3} \text { ( } \mu \text { mol) in sample } \\
\text { at approximate times after isotopic addition }\end{array}$} \\
\cline { 2 - 7 } & $2.5 \mathrm{hr}$ & 1 day & 2 days & 4 days & 7 days & 8 days \\
\hline $16-2$ & & 48000 & & 87000 & & \\
\hline $16-4 \mathrm{R}$ & & 2300000 & & 20000 & & \\
\hline $16-7$ & 29000 & 13000 & 12000 & & 8100 & \\
\hline $16-10$ & 11000 & 10000 & 9600 & & & 8700 \\
\hline
\end{tabular}


Table C.12.3. Isotopic Ratios and Measurement Sequences

\begin{tabular}{|c|c|c|c|c|c|}
\hline \multirow[b]{2}{*}{ Sample } & \multirow{2}{*}{$\begin{array}{c}\text { Isotopic } \\
\text { equilibration } \\
\text { time }\end{array}$} & \multirow{2}{*}{$\begin{array}{l}\text { Length of preceding } \\
\text { mass-spec } \\
\text { pumpdown }\end{array}$} & \multicolumn{3}{|c|}{${ }^{15} \mathrm{NH}_{3} /{ }^{14} \mathrm{NH}_{3}$ ratio measured by mass spec } \\
\hline & & & $1^{\text {st }}$ canister & $2^{\text {nd }}$ canister & $3^{\text {rd }}$ canister \\
\hline$S-102-2$ & 1 day & $5 \mathrm{~min}$. & 0.31 (a) & $0.10(a)$ & 0.12 (a) \\
\hline$S-102-2$ & 4 days & $5 \mathrm{~min}$. & 0.05 (a) & $0.066^{(a)}$ & $\ldots-$ (a) \\
\hline S-102-4R & 1 day & $5 \mathrm{~min}$. & $\ldots$-.(a) & $0.0027(\mathrm{a})$ & $0.0048(a)$ \\
\hline S-102-4R & 4 days & $5 \mathrm{~min}$. & $0.0052^{(a)}$ & 0.33 (a) & 0.29 (a) \\
\hline S-102-7 & $2.5 \mathrm{hr}$ & $5 \min$. & --- (a) & $0.44(a)$ & 0.40 (a) \\
\hline$S-102-7$ & 1 day & $5 \mathrm{~min}$. & 0.65 & 0.78 & 0.89 \\
\hline S-102-7 & 2 days & $5 \mathrm{~min}$. & 0.87 (a) & $0.91(\mathrm{a})$ & 1.00 \\
\hline$S-102-7$ & 7 days & $5 \mathrm{~min}$. & 1.28 & 1.37 & 1.48 \\
\hline \multirow[t]{3}{*}{ S-102-10 } & \multirow[t]{3}{*}{$2.5 \mathrm{hr}$} & overnight & 0.847 & 0.864 & 0.855 \\
\hline & & $1 \mathrm{hr}$ & 1.018 & 1.034 & 1.035 \\
\hline & & overnight & 1.105 & 1.126 & 1.074 \\
\hline \multirow[t]{2}{*}{ S-102-10 } & \multirow[t]{2}{*}{1 day } & $5 \mathrm{~min}$. & 0.931 & 1.011 & 1.084 \\
\hline & & overnight & 1.149 & 1.196 & 1.177 \\
\hline \multirow[t]{2}{*}{ S-102-10 } & \multirow[t]{2}{*}{2 days } & overnight & 0.673 & 0.773 & 0.870 \\
\hline & & overnight & 1.131 & 1.165 & 1.253 \\
\hline \multirow[t]{2}{*}{ S-102-10 } & \multirow[t]{2}{*}{8 days } & weekend & 1.070 & 1.240 & 1.300 \\
\hline & & $2 \mathrm{hr}$ & 1.308 & 1.388 & 1.426 \\
\hline
\end{tabular}

(a) There was less than $1 \mathrm{~mol} \%$ total ammonia in the composition measured by mass spectrometry; these ratios may be less accurate because of the low signal-to-noise ratio.

\section{C.13 S-111}

Table C.13.1 presents the ammonia measurements. The "Extracted $\mathrm{NH}_{3}$ " column gives the total moles of ammonia that were pumped as vapor from the samples into the collection canisters expressed as the moles of $\mathrm{NH}_{3}$ per liter of original waste (including gas, solids, and liquid volume). The residual ammonia, determined isotopically, is also included in the table. The postextrusion partial pressure of $\mathrm{NH}_{3}$ over the sample (see Section 3.4.4) was measured at laboratory temperature and is included in Table C.13.1 as are the measurement temperatures. The residual ammonia in S-111 samples was not measured by post-RGS ISE methods. Other measurements of ammonia in S-111 were also available from the 1998 non-RGS samples in riser 6 and from some of the riser 8 samples taken in 1996.(a) These measurements were made from extruded cores, some from drainable liquid and some from water digests of the solids portion of samples.

The various ammonia measurements were not consistent. For lack of more definitive information, the RGS isotopic measurements were used to calculate the ammonia mole fractions in the in situ vapor.

(a) TWINS database, samples S98T001359W, S98T001360W, S98T001361W, S98T001376W, S98T001377W, S98T001382, S98T001383, S96T003695, S96T003697, and S96T005969. 
Table C.13.1. Ammonia Data from Tank S-111 Samples

\begin{tabular}{|c|c|c|c|c|c|}
\hline Sample & $\begin{array}{c}\text { Extracted } \\
\mathrm{NH}_{3} \\
(\mu \mathrm{mol} / \mathrm{L})\end{array}$ & $\begin{array}{l}\text { Isotopically } \\
\text { Measured } \\
\text { Residual NH} \\
(\mu \mathrm{mol} / \mathrm{L})\end{array}$ & $\begin{array}{l}\text { Total } \mathrm{NH}_{3} \text { from } \\
\text { core sample, by ISE(a) } \\
(\mu \mathrm{mol} / \mathrm{L})\end{array}$ & $\begin{array}{c}\text { Post-extrusion } \\
\mathrm{NH}_{3} \text { partial } \\
\text { pressure } \\
\text { (atm) }\end{array}$ & $\begin{array}{c}\text { Measure- } \\
\text { ment } \\
\text { temperature } \\
\left({ }^{\circ} \mathrm{C}\right)\end{array}$ \\
\hline & & & $\begin{array}{c}<94000 \mu \mathrm{mol} / \mathrm{L} \text { waste } \\
\text { (sample } 6-1, \text { lower half solids) } \\
330 \mu \mathrm{mol} / \mathrm{L} \text { liquid } \\
\text { (sample } 6-1, \text { drainable liquid) } \\
2800 \mu \mathrm{mol} / \mathrm{L} \text { liquid } \\
\text { (sample } 8-1, \text { drainable liquid, 1996) }\end{array}$ & & \\
\hline \multirow[t]{2}{*}{$6-2$} & $74 \pm 59(b)$ & $13000 \pm 8600(b)$ & $\begin{array}{l}4000 \mu \mathrm{mol} / \mathrm{L} \text { liquid } \\
\text { (sample } 8-2, \text { drainable liquid, 1996) }\end{array}$ & $0.0020 \pm 0.0010$ (c) & 12.3 \\
\hline & & & $\begin{array}{c}<86000 \mu \mathrm{mol} / \mathrm{L} \text { waste } \\
\text { (sample 6-3, lower half solids) } \\
1300 \mu \mathrm{mol} / \mathrm{L} \text { liquid } \\
\text { (sample 6-3, drainable liquid) } \\
2200 \mu \mathrm{mol} / \mathrm{L} \text { liquid } \\
\text { (sample } 8-3 \text {, drainable liquid, } 1996 \text { ) }\end{array}$ & & \\
\hline \multirow[t]{2}{*}{$6-4$} & $121 \pm 93(b)$ & $55000 \pm 18000(b)$ & & -0.0058 & 11.7 \\
\hline & & & $\begin{array}{c}<18000 \mu \mathrm{mol} / \mathrm{L} \text { waste } \\
\text { (sample } 6-5, \text { lower half solids) }\end{array}$ & & \\
\hline \multirow[t]{2}{*}{$6-6$} & $\begin{array}{c}5100+5100(b) \\
-480\end{array}$ & $\begin{array}{c}53000+53000(b) \\
-17000\end{array}$ & & $\begin{array}{l}0.0079 \pm \\
0.0039(\mathrm{~d})\end{array}$ & 11.5 \\
\hline & & & $\begin{array}{c}<19000 \mu \mathrm{mol} / \mathrm{L} \text { waste } \\
\text { (sample 6-7, lower half solids) }\end{array}$ & & \\
\hline \multirow[t]{2}{*}{$6-8$} & $3100 \pm 360(b)$ & $32000 \pm 10000$ (b) & & $0.0066 \pm 0.0033$ & 12.0 \\
\hline & & & $\begin{array}{c}<19000 \mu \mathrm{mol} / \mathrm{L} \text { waste } \\
\text { (sample } 6-9, \text { lower half solids) }\end{array}$ & & \\
\hline $6-10$ & $2800 \pm 680(b)$ & $79000 \pm 26000(b)$ & & $0.010 \pm 0.005$ & 11.8 \\
\hline \multicolumn{6}{|c|}{$\begin{array}{l}\text { (a) These total ammonia concentrations should be considered lower bounds because of ammonia evaporation during } \\
\text { the sample handling that preceded ISE analysis. } \\
\text { (b) These data were used to calculate in situ ammonia vapor pressures. } \\
\text { (c) This value is suspect because of air inleakage. } \\
\text { (d) This value is suspect because of a large intrusion of argon. }\end{array}$} \\
\hline
\end{tabular}

The RGS sample taken at the highest elevation, sample 6-2, which was supernatant liquid, gave a lower value of residual ammonia by the isotopic method than other RGS samples. The isotopic ratios (measured after seven days of equilibration, as shown in Table C.13.2) converged acceptably on a value between 3.1 and 3.3, as can be seen in Table C.13.3. The 13,000 $\mu \mathrm{mol}$ $\mathrm{NH}_{3} / \mathrm{L}$ waste (or per liter of liquid) is considerably higher than any of the ammonia concentrations measured in the drainable liquids from non-RGS samples, which contained 330 to $4,000 \mu \mathrm{mol}$ $\mathrm{NH}_{3} / \mathrm{L}$ liquid. The post-extrusion partial pressure of this sample (when raised by $80 \%$ to account for measurement temperature differences) suggests a concentration one-half to one-third of that in 
Table C.13.2. Residual Ammonia Measurements versus Equilibration Time

\begin{tabular}{||c|c|c|c|c|c||}
\hline \hline \multirow{2}{*}{ Sample } & \multicolumn{5}{|c||}{ Estimated residual ${ }^{14} \mathrm{NH}_{3}(\mu \mathrm{mol})$ in sample } \\
& at approximate times after isotopic addition \\
\hline & $2 \mathrm{hr}$ & 1 day & 2 days & 7 days & 20 days \\
\hline $6-2$ & 8400 & 3900 & 3000 & 3800 & \\
\hline $6-4$ & & 16000 & & & \\
\hline $6-6$ & & & 15000 & & \\
\hline $6-8$ & & & & & 9300 \\
\hline $6-10$ & & 23000 & & & \\
\hline
\end{tabular}

AW-101 waste, which had a lower bound of $27,000 \mu \mathrm{mol} \mathrm{NH} 3 / \mathrm{L}$ waste. This estimate is more consistent with the isotopic ammonia measurement than with non-RGS measurements, unless the latter lost half or more of their ammonia by evaporation. Considering the discrepancies, it would be difficult to say more than that the ammonia concentration of the supernatant was probably between 4,000 and $13,000 \mu \mathrm{mol} \mathrm{NH}_{3} / \mathrm{L}$ liquid.

There is no agreement between the non-RGS and RGS measurements for the samples in the nonconvective layer (samples 6-4 through 6-10), though in all cases the isotopic ratios had converged. Three of the RGS samples from this layer had a one- or two-day equilibration time; the

Table C.13.3. Isotopic Ratios and Measurement Sequences

\begin{tabular}{|c|c|c|c|c|c|}
\hline \multirow[b]{2}{*}{ Sample } & \multirow{2}{*}{$\begin{array}{l}\text { Isotopic } \\
\text { equilibra- } \\
\text { tion time }\end{array}$} & \multirow{2}{*}{$\begin{array}{l}\text { Length of } \\
\text { preceding } \\
\text { mass-spec } \\
\text { pumpdown }\end{array}$} & \multicolumn{3}{|c|}{${ }^{15} \mathrm{NH}_{3} /{ }^{14} \mathrm{NH}_{3}$ ratio measured by mass spectrometry } \\
\hline & & & $1^{\text {st }}$ canister & $2^{\text {nd }}$ canister & $3^{\text {rd }}$ canister \\
\hline $6-2$ & $2 \mathrm{hr}$ & $6 \mathrm{hr}$ or more & $\begin{array}{l}3.2^{(\mathrm{a}, \mathrm{b})}, 1.5^{(\mathrm{a})}, 1.3^{(\mathrm{a})} \\
(6 \mathrm{hr} \text { pumpdown })\end{array}$ & $\begin{array}{l}1.9^{(b)}, 1.9,1.9,1.7,1.5 \\
\text { (overnight pumpdown) }\end{array}$ & $\begin{array}{l}1.7^{(\mathrm{b})}, 1.7,1.6,1.5 \\
(6 \mathrm{hr} \text { pumpdown })\end{array}$ \\
\hline $6-2$ & 1 day & $6 \mathrm{hr}$ or more & $\begin{array}{c}2.8^{(a, b)}, 2.5(a), 2.7^{(a)} \\
\text { (overnight pumpdown) }\end{array}$ & $\begin{array}{c}3.0(\mathrm{~b}), 2.4,3.0 \\
(6 \mathrm{hr} \text { pumpdown })\end{array}$ & $\begin{array}{c}3.7(\mathrm{~b}), 2.9,3.1 \\
\text { (overnight pumpdown) }\end{array}$ \\
\hline $6-2$ & 2 days & $1 \mathrm{hr}$ or more & $\begin{array}{c}2.93,3.65,3.94 \\
\text { (overnight pumpdown) }\end{array}$ & $\begin{array}{c}2.65,3.31,3.88 \\
\text { (2 hr pumpdown) }\end{array}$ & $\begin{array}{c}3.07,3.76,4.17 \\
(1 \mathrm{hr} \text { pumpdown })\end{array}$ \\
\hline $6-2$ & 7 days & $5 \mathrm{~min}$. & $2.2,2.6,3.1$ & $2.5,2.9,3.1$ & $3.0,3.1,3.3$ \\
\hline $6-4(a)$ & 1 day & $5 \mathrm{~min}$. & $\begin{array}{c}0.41,0.54,0.46,0.36, \\
0.49,0.37\end{array}$ & $\begin{array}{cc}0.52, & 0.52,0.66,0.69 \\
& 0.65,0.70\end{array}$ & $\begin{array}{c}0.66,0.55,0.67,0.70 \\
0.68,0.77\end{array}$ \\
\hline $6-6(a)$ & 2 days & $5 \mathrm{~min}$. & $\begin{array}{c}0.35,0.47,0.55,0.61 \\
0.65,0.73\end{array}$ & $0.50,0.64,0.66,0.67$ & $\begin{array}{c}0.53,0.70,0.71,0.75 \\
0.73,0.78\end{array}$ \\
\hline $6-8$ & 20 days & $5 \mathrm{~min}$. & $\begin{array}{c}0.59,0.78,0.94,1.07 \\
1.16,1.22,1.27\end{array}$ & $\begin{array}{l}1.12,1.16,1.25,1.16 \\
1.21,1.21,1.22,1.29 \\
\end{array}$ & $\begin{array}{c}1.29,1.33,1.24,1.32 \\
1.28,1.29,1.31 \\
\end{array}$ \\
\hline $6-10(a)$ & 1 day & $5 \mathrm{~min}$. & $\begin{array}{c}0.17,0.33,0.38,0.46 \\
0.47,0.52\end{array}$ & $\begin{array}{c}0.44,0.49,0.50,0.52 \\
0.52,0.54 ; 0.54\end{array}$ & $\begin{array}{c}0.42,0.45,0.48,0.53 \\
0.53,0.53\end{array}$ \\
\hline \multicolumn{6}{|c|}{$\begin{array}{l}\text { (a) There was less than } 1 \text { mol\% total ammonia in the composition measured by mass spectrometry for these } \\
\text { samples; these ratios may be less accurate because of the low signal-to-noise ratio. } \\
\text { (b) The ammonia mass peaks were just above the detection limit for the first gas aliquots taken from each } \\
\text { canister, but increased with further gas removal. }\end{array}$} \\
\hline
\end{tabular}


fourth equilibrated for about 20 days because of a facility stand-down. There is a factor of 2 difference between the residual ammonia found at the shorter and longer equilibration times, as shown in Table C.13.2. There is no way to know whether the difference resulted from more complete equilibration or some artifact of the long delay.

Even the lower concentration measured at the 20-day equilibration time, 35,000 $\mu \mathrm{mol}$ $\mathrm{NH}_{3} / \mathrm{L}$ waste in sample 6-8, is not as low as the concentrations measured in the non-RGS samples-all of which were below the detection limit of about $18,000 \mu \mathrm{mol} \mathrm{NH} / 2 / \mathrm{L}$ of waste. Leaving out the physically impossible negative ammonia partial pressure measured for sample 6-4, the partial pressures (accounting for measurement temperature differences) suggest concentrations somewhat less than twice those in Tank AW-101 waste, which had a lower bound of 27,000 $\mu \mathrm{mol}$ $\mathrm{NH}_{3} / \mathrm{L}$ waste. This partial-pressure-based approximate estimate of 45,000 to $60,000 \mu \mathrm{mol} \mathrm{NH}_{3} / \mathrm{L}$ waste is consistent with the isotopic measurements (see Table C.13.3), if those made at one- or two-day times are considered to be upper limits. Taken together, the various RGS measurements indicate ammonia concentrations between 35,000 and $85,000 \mu \mathrm{mol} \mathrm{NH} / \mathrm{L}$ waste, but are not consistent with the much-lower non-RGS measurements.

The inconsistencies in the ammonia data are such that it could be misleading to calculate an average ammonia concentration for Tank S-111. However, most of the data suggest a concentration of no less than $\sim 4,000 \mu \mathrm{mol} \mathrm{NH} / \mathrm{L}$ waste and no more than $\sim 13,000 \mu \mathrm{mol} \mathrm{NH}_{3} / \mathrm{L}$ waste in the supernatant liquid, and no less than $\sim 35,000 \mu \mathrm{mol} \mathrm{NH_{3 }} / \mathrm{L}$ waste and no more than $\sim 80,000$ $\mu \mathrm{mol} \mathrm{NH}_{3} / \mathrm{L}$ waste in the nonconvective layer.

A very approximate concentration of $50,000 \mu \mathrm{mol} \mathrm{NH} / \mathrm{L}$ waste is taken to have existed in the S-11 nonconvective layer. This concentration corresponds to $0.090 \mathrm{M}(1500 \mu \mathrm{g} / \mathrm{mL})$ in the interstitial liquid, or $0.065 \mathrm{wt} \%$ of the bulk waste. (The conversion of $\mathrm{mol} / \mathrm{L}$ waste to liquid concentrations assumed a gas volume fraction of 0.20 in the waste and a solids volume fraction of 0.31 in the degassed waste (shown in the S-111 spreadsheet on the CD supplied with this report).

\section{C.14 U-109}

Table C.14.1 presents the ammonia measurements. The "Extracted $\mathrm{NH}_{3}$ " column gives the total moles of ammonia that were pumped as vapor from the samples into the collection canisters, expressed as the moles of $\mathrm{NH}_{3}$ per liter of original waste (including gas, solids, and liquid volume). The residual ammonia was determined isotopically and is included in the table. The postextrusion partial pressure of $\mathrm{NH}_{3}$ over the sample (see Section 3.4.4) was measured at laboratory temperature and is included in Table C.14.1 with the measurement temperatures. The residual ammonia in U-109 samples was not measured by post-RGS ion-specific electrode methods.

Other measurements of ammonia in U-109 were also available from the 1998 non-RGS samples in riser 8.(a) These measurements were made from extruded cores, drainable liquid, and water digests of the solids portion of samples. Almost all of these measurements gave ammonia concentrations below the MDL. Two non-RGS samples, 8-3 and 8-7, each gave one value above the MDL and one (the duplicate measurement) below it.

The various ammonia measurements were somewhat difficult to reconcile. For lack of more definitive information, the RGS isotopic measurements were used to calculate the ammonia mole fractions in the in situ vapor.

(a) TWINS database, samples S98T001428W, S98T001429W, S98T001430W, S98T001431W, S98T001432W, S98T001440, and S98T001452W. 
Table C.14.1. Ammonia Data from Tank U-109 Samples

\begin{tabular}{|c|c|c|c|c|c|}
\hline Sample & $\begin{array}{c}\text { Extracted } \\
\mathrm{NH}_{3} \\
(\mu \mathrm{mol} / \mathrm{L}) \\
\end{array}$ & $\begin{array}{l}\text { Isotopically } \\
\text { measured } \\
\text { residual } \mathrm{NH}_{3} \\
(\mu \mathrm{mol} / \mathrm{L}) \\
\end{array}$ & $\begin{array}{c}\text { Total } \mathrm{NH}_{3} \text { from } \\
\text { core sample, by ISE (a) } \\
(\mu \mathrm{mol} / \mathrm{L})\end{array}$ & $\begin{array}{c}\text { Post-extrusion } \\
\mathrm{NH}_{3} \text { partial } \\
\text { pressure } \\
\text { (atm) }\end{array}$ & $\begin{array}{l}\text { Measurement } \\
\text { temp } \\
\left({ }^{\circ} \mathrm{C}\right) \\
\end{array}$ \\
\hline & & & $\begin{array}{l}<21000 \mu \mathrm{mol} / \mathrm{L} \text { waste } \\
\text { (sample } 8-1, \text { lower half, } \\
\text { both primary and duplicate) } \\
<290 \mu \mathrm{mol} / \mathrm{L} \text { waste } \\
\text { (sample } 8-1, \text { drainable liquid) }\end{array}$ & & \\
\hline \multirow[t]{2}{*}{$8-2$} & $2900 \pm 300(b)$ & $32000 \pm 11000$ (b) & & -0.00053 & 11.9 \\
\hline & & & $\begin{array}{l}<19000 \mu \mathrm{mol} / \mathrm{L} \text { waste } \\
\text { (sample } 8-3 \text {, } \\
\text { lower half, primary) } \\
37000 \mu \mathrm{mol} / \mathrm{L} \text { waste } \\
\text { (sample } 8-3 \text {, } \\
\text { lower half, duplicate) } \\
\end{array}$ & & \\
\hline \multirow[t]{2}{*}{$8-4$} & $\begin{array}{l}4400 \pm \\
1400(b)\end{array}$ & $83000 \pm 36000(b)$ & & $\begin{array}{l}0.0017 \pm \\
0.0008(\mathrm{c})\end{array}$ & 12.0 \\
\hline & & & $\begin{array}{l}<19000 \mu \mathrm{mol} / \mathrm{L} \text { waste } \\
\text { (sample } 8-5, \text { lower half, } \\
\text { both primary and duplicate) }\end{array}$ & & \\
\hline \multirow[t]{2}{*}{$8-6$} & $\begin{array}{l}5800 \pm \\
4600(\text { b) } \\
\end{array}$ & $39000 \pm 12000(b)$ & & $\begin{array}{c}0.0056 \pm \\
0.0028\end{array}$ & 11.8 \\
\hline & - & . & $\begin{array}{l}25000 \mu \mathrm{mol} / \mathrm{L} \text { waste } \\
\text { (sample } 8-7 \text {, } \\
\text { lower half, primary) } \\
<19000 \mu \mathrm{mol} / \mathrm{L} \text { waste } \\
\text { (sample } 8-7 \text {, } \\
\text { lower half, duplicate) } \\
\end{array}$ & & \\
\hline \multirow[t]{2}{*}{$8-8$} & $560 \pm 110^{(b)}$ & $43000 \pm 15000(b)$ & & $\begin{array}{c}0.0058 \pm \\
0.0028\end{array}$ & 11.5 \\
\hline & & & $\begin{array}{l}<95000 \mu \mathrm{mol} / \mathrm{L} \text { waste } \\
\text { (sample } 8-9, \\
\text { 2nd and 3rd quarters, } \\
\text { both primary and duplicate) }\end{array}$ & . & \\
\hline \multicolumn{6}{|c|}{$\begin{array}{l}\text { (a) These total ammonia concentrations should be considered lower bounds because of ammonia evaporation during the } \\
\text { sample handling that preceded ISE analysis. } \\
\text { (b) These data were used to calculate in situ ammonia vapor pressures. } \\
\text { (c) This value is suspect because of air inleakage. }\end{array}$} \\
\hline
\end{tabular}

- Of the four RGS samples, three gave concentrations of 35,000 to $45,000 \mu \mathrm{mol} \mathrm{NH}_{3} / \mathrm{L}$ waste and the fourth (sample 8-4) gave double that value, according to the isotopic method. It is probably not a coincidence that sample 8-4 was the only one with ammonia peaks near the detection limit and the only one whose isotopic ratios did not converge (Table C.14.2). Thus the ammonia measurement for sample 8-4 may not be reliable. Note that all the RGS sample equilibration times were similar, between 16 and $22 \mathrm{hr}$, so the data do not allow any examination of the effect of equilibration time. 
Table C.14.2. Isotopic Ratios and Measurement Sequences

\begin{tabular}{|c|c|c|c|c|c|}
\hline \multirow[b]{2}{*}{ Sample } & \multirow{2}{*}{$\begin{array}{c}\text { Isotopic } \\
\text { equilibration } \\
\text { time }\end{array}$} & \multirow{2}{*}{$\begin{array}{l}\text { Length of } \\
\text { preceding } \\
\text { mass-spec } \\
\text { pumpdown }\end{array}$} & \multicolumn{3}{|c|}{${ }^{15} \mathrm{NH}_{3} /{ }^{14} \mathrm{NH}_{3}$ ratio measured by mass spectrometry } \\
\hline & & & 1st canister & $2^{\text {nd }}$ canister & 3rd canister \\
\hline $8-2(a)$ & 1 day & $5 \mathrm{~min}$. & $\begin{array}{c}0.14,0.43,0.82,0.97 \\
0.98,1.02,1.09\end{array}$ & $\mid \begin{array}{c}0.98,1.12,1.17,1.15 \\
1.28,1.18,1.30\end{array}$ & $\begin{array}{c}1.09,1.30,1.35,1.28 \\
1.32,1.29,1.31\end{array}$ \\
\hline $8-4(a, b)$ & 1 day & $5 \mathrm{~min}$. & $0.98,0.70,0.51,0.54$ & $\begin{array}{c}0.64,0.75,0.34 \\
0.48,0.48\end{array}$ & $\begin{array}{c}0.50, \\
0.29,0.50,0.60 \\
0.47,0.45\end{array}$ \\
\hline $8-6(a)$ & 1 day & $5 \mathrm{~min}$. & $\begin{array}{c}0.57,0.76,0.98,0.93, \\
1.02,0.95\end{array}$ & $\begin{array}{c}0.98,1.03,0.98 \\
1.09,0.93\end{array}$ & $1.09,1.06,1.09$ \\
\hline $8-8(a)$ & 1 day & $5 \mathrm{~min}$. & $\begin{array}{c}0.45,0.59,0.76,0.90 \\
0.95,1.03\end{array}$ & $\begin{array}{c}1.03,1.23,1.10,1.13, \\
1.18,1.23\end{array}$ & $1.19,1.18,1.16,1.16$ \\
\hline
\end{tabular}

- Because the non-RGS ammonia measurements were not closely replicated, it is hard to know what reliance to place on them. The two measurements that were above the MDL (which in both cases was roughly 20,000 ) were 25,000 and $37,000 \mu \mathrm{mol} \mathrm{NH} 3 / \mathrm{L}$ waste (samples $8-3$ and $8-7$ ). These concentrations are reasonably consistent with the isotopic measurements if taken as lower bounds.

- Only two samples (8-6 and 8-8) had post-extrusion ammonia partial pressures that were not suspect. These partial pressures (when raised by $80 \%$ to account for measurement temperature differences) suggest a concentration about the same as that in AW-101 waste, which had a lower bound of $27,000 \mu \mathrm{mol} \mathrm{NH} / \mathrm{L}$ waste. This estimate is consistent with the isotopic ammonia measurements and the two above-the-limit non-RGS ammonia measurements, though a little low. It is not consistent with most of the non-RGS measurements, unless the latter subsamples lost enough ammonia by evaporation to fall below the MDL.

Considering the above information, it seems likely that the ammonia concentration of the U-109 nonconvective layer was between 25,000 and $45,000 \mu \mathrm{mol} \mathrm{NH} 3 / \mathrm{L}$ waste. The only evidence against that conclusion is that of the core samples that were below the MDL of roughly $20,000 \mu \mathrm{mol} \mathrm{NH} / 3 / \mathrm{L}$ waste. The average and standard deviation of the isotopically measured ammonia concentrations over samples $8-2,8-6$, and $8-8$ were $41,000 \pm 5,600 \mu \mathrm{mol} / \mathrm{L}$ waste. This ammonia concentration in the bulk waste corresponds to $0.077 \mathrm{wt} \% \mathrm{NH}_{3}$ in the bulk waste, or $1100 \mu \mathrm{g} \mathrm{NH}_{3} / \mathrm{mL}$ liquid. (The gas volume fraction of each sample and a solid volume fraction of 0.21 in the degassed waste were used to calculate the ammonia concentration in the liquid.)

\section{C.15 SY-101}

Table C.15.1 presents the ammonia measurements. The "Extracted $\mathrm{NH}_{3}$ " column gives the total moles of ammonia that were pumped as vapor from the samples into the collection canisters expressed as the moles of $\mathrm{NH}_{3}$ per liter of original waste (including gas, solids, and liquid volume). The residual ammonia was determined isotopically, and is also included in the table. The post-extrusion partial pressure of $\mathrm{NH}_{3}$ over the sample (see Section 3.4.4) was measured at 
Table C.15.1. Ammonia Data from Tank SY-101 Samples

\begin{tabular}{||c|c|c|c|c||}
\hline Sample & $\begin{array}{c}\text { Extracted } \mathrm{NH}_{3}(\mathrm{a}) \\
(\mu \mathrm{mol} / \mathrm{L})\end{array}$ & $\begin{array}{c}\text { Isotopically } \\
\text { measured } \\
\text { residual } \mathrm{NH}_{3}(\mathrm{a}) \\
(\mu \mathrm{mol} / \mathrm{L})\end{array}$ & $\begin{array}{c}\text { Post-Extrusion } \\
\mathrm{NH}_{3} \text { partial } \\
\text { pressure } \\
(\mathrm{atm})\end{array}$ & $\begin{array}{c}\text { Measurement } \\
\text { temperature } \\
\left({ }^{\circ} \mathrm{C}\right)\end{array}$ \\
\hline \hline $23 \mathrm{~A}-1$ & $17000 \pm 1700$ & $64000 \pm 18000$ & $0.023 \pm 0.011$ & 11.4 \\
\hline $23 \mathrm{~A}-2$ & $42000 \pm 2900$ & $112000 \pm 32000$ & $0.032 \pm 0.016$ & 11.4 \\
\hline $22 \mathrm{~A}-3$ & $33000 \pm 2200$ & $93000 \pm 26000$ & $0.034 \pm 0.017$ & 12.0 \\
\hline $23 \mathrm{~A}-3$ & $42000 \pm 1800$ & $106000 \pm 34000$ & $0.032 \pm 0.016$ & 11.2 \\
\hline $22 \mathrm{~A}-4$ & $27000 \pm 1300$ & $130000 \pm 38000$ & $0.030 \pm 0.015(\mathrm{~b})$ & 12.0 \\
\hline $4 \mathrm{~A}-5$ & $48000 \pm 1500$ & $120000 \pm 34000$ & $0.035 \pm 0.017$ & 11.9 \\
\hline $23 \mathrm{~A}-8$ & $48000 \pm 1500$ & $91000 \pm 27000$ & $0.036 \pm 0.018$ & 11.9 \\
\hline $22 \mathrm{~A}-10$ & $44000 \pm 1400$ & $160000 \pm 45000$ & $0.039 \pm 0.019$ & 12.4 \\
\hline $23 \mathrm{~A}-13$ & $45000 \pm 1300$ & $160000 \pm 48000$ & $0.033 \pm 0.016$ & 12.3 \\
\hline 22A-17 & $39000 \pm 1300$ & $120000 \pm 34000$ & $0.034 \pm 0.017$ & 12.0 \\
\hline 23A-21 & $41000 \pm 1300$ & $140000 \pm 39000$ & $0.035 \pm 0.017$ & 12.4 \\
\hline 22A-23 & $46000 \pm 2900$ & $130000 \pm 36000$ & $0.033 \pm 0.016$ & 11.8 \\
\hline $\begin{array}{l}\text { (a) These data were used to calculate in situ ammonia vapor pressures. } \\
\text { (b) This value is suspect because of air inleakage. }\end{array}$ \\
\hline
\end{tabular}

laboratory temperature and is included in Table C.15.1, together with the measurement temperatures. The residual ammonia in SY-101 samples was not measured by post-RGS ISE methods.

Composites of core samples taken in May and December 1991 (Windows C and E) were found to contain, respectively, $0.26 \mathrm{wt} \%$ and $0.19 \mathrm{wt} \%$ of ammonia (Kubic 1994). These values were considered lower bounds on the ammonia concentration because as much as $50 \%$ of the ammonia might have evaporated from the extruded samples before analysis.

Other measurements of ammonia in SY-101 were also available from the 1999 non-RGS samples in riser 4A.(a) These measurements were made from extruded cores, typically from water digests of the solids portion of samples. Almost all of these measurements gave ammonia concentrations below the MDL, which in most cases was about $0.49 \% \mathrm{NH}_{3}$ (expressed as weight percent of the bulk sample). Some of the non-RGS samples (4A-7, 4A-8, 4A-13, and 4A-20) each gave one value above the MDL and one (the duplicate measurement) below it; the values above the MDL were, respectively, $0.59 \%, 0.57 \%, 0.11 \%$, and $0.78 \% \mathrm{NH}_{3}$. Only sample $4 \mathrm{~A}-19$ and the composite of segments $1-4$ from riser $4 \mathrm{~A}$ gave both primary and duplicate measurements above the MDL. These concentrations were, respectively, $1.6 \mathrm{wt} \% \mathrm{NH}_{3}$ and $4.1 \mathrm{wt} \% \mathrm{NH}_{3} .(\mathrm{b})$ These measurements are considered suspect, in part because the individual samples used in making the crust composite all gave ammonia measurements below the minimum detection limit.

(a) TWINS database, samples S99T000525-8, S99T000677-88, and S99T000734-8.

(b) Personal e-mail communication from JM Conner (LMHC) to LA Mahoney (PNNL), September 13, 1999, regarding ammonia measurements in SY-101 samples. 
After more than two months of storage, sample 4A-16 contained $67 \mathrm{~g}$ of decantable liquid that had separated from the sample over time. This liquid was analyzed and found to contain $407.5 \mu \mathrm{g}$ $\mathrm{NH}_{3} / \mathrm{mL}$ liquid. This concentration was less than $10 \%$ of the value estimated by RGS and much less than the ammonia found in 1991, suggesting that losses might have occurred during storage. Most of the various ammonia measurements could be reconciled easily. For lack of more definitive information, the RGS isotopic measurements were used to calculate the ammonia mole fractions in the in situ vapor.

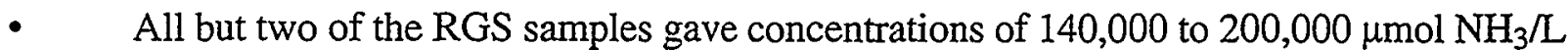
of waste, according to the isotopic method. The sample from the top of the crust (sample $23 \mathrm{~A}-1$ ) gave a lower value of $81,000 \mu \mathrm{mol} \mathrm{NH}_{3} / \mathrm{L}$ of waste, perhaps because it was drier (less saturated with ammonia-containing liquid) than the other samples. Ammonia evaporation could also have played a part. Sample 22A-3 also had lower than average ammonia, $130,000 \mu \mathrm{mol} \mathrm{NH} / \mathrm{L}$ of waste.

- There was little evidence that equilibration was incomplete; rather, most of the samples showed increases in the measured residual ammonia as equilibration time increased (Table C.15.2). Those increases were within the uncertainty of the residual ammonia measurement. The isotopic ratios showed excellent convergence (Table C.15.3). In general, the isotopic ammonia measurements for SY-101 appear to be of higher quality and more consistent than in earlier tanks.

- $\quad$ Because the 1999 non-RGS ammonia measurements were so scattered and the MDL so high, it is hard to know what reliance to place on them. The 1999 non-RGS data included values that were lower and higher than the RGS values. (Most of the latter were in a range from $0.15 \mathrm{wt} \% \mathrm{NH}_{3}$ to $0.25 \mathrm{wt} \% \mathrm{NH}_{3}$.)

Table C.15.2. Residual Ammonia Measurements versus Equilibration Time

\begin{tabular}{|c|c|c|c|c|c|c|}
\hline Sample & \multicolumn{6}{|c|}{$\begin{array}{l}\text { Estimated residual }{ }^{14} \mathrm{NH}_{3} \text { ( } \mu \mathrm{mol} \text { ) in sample } \\
\text { at approximate times after isotopic addition }\end{array}$} \\
\hline & 1 day & 2 days & 4 days & 5 days & 6 days & 7 days \\
\hline $23 \mathrm{~A}-1$ & 33000 & & & 18000 & & \\
\hline $23 \mathrm{~A}-2$ & 30000 & & & & & \\
\hline $22 \mathrm{~A}-3$ & 31000 & & 26000 & & & \\
\hline $23 \mathrm{~A}-3$ & 30000 & & & & & \\
\hline $22 \mathrm{~A}-4$ & 38000 & & & & 38000 & \\
\hline $4 A-5$ & 34000 & & & & 36000 & \\
\hline $23 \mathrm{~A}-8$ & & & & 26000 & & \\
\hline $22 \mathrm{~A}-10$ & 34000 & & & & 46000 & \\
\hline $23 \mathrm{~A}-13$ & & & 44000 & & & \\
\hline $22 \mathrm{~A}-17$ & 29000 & & & & & 35000 \\
\hline $23 \mathrm{~A}-21$ & 37000 & & & & & 40000 \\
\hline $22 \mathrm{~A}-23$ & 30000 & & & 34000 & & \\
\hline
\end{tabular}


Table C.15.3. Isotopic Ratios and Measurement Sequences

\begin{tabular}{|c|c|c|c|c|c|}
\hline \multirow[b]{2}{*}{ Sample } & \multirow{2}{*}{$\begin{array}{c}\text { Isotopic } \\
\text { equilibration } \\
\text { time } \\
\end{array}$} & \multirow{2}{*}{$\begin{array}{c}\text { Length of } \\
\text { preceding mass- } \\
\text { spec pumpdown }\end{array}$} & \multicolumn{3}{|c|}{${ }^{15} \mathrm{NH}_{3} / 14 \mathrm{NH}_{3}$ ratio measured by mass spectrometry } \\
\hline & & & 1st canister & $2^{\text {nd }}$ canister & $3^{\text {rd }}$ canister \\
\hline \multirow[t]{2}{*}{$23 \mathrm{~A}-1$} & 1 day & $5 \mathrm{~min}$. & $\begin{array}{c}0.23(\mathrm{a}), 0.27,0.28 \\
0.31,0.32,0.33,0.32\end{array}$ & $\begin{array}{c}0.34,0.35,0.38,0.36 \\
0.37,0.38 \\
\end{array}$ & $\begin{array}{c}0.36(\mathrm{a}), 0.34,0.34 \\
0.35,0.36,0.36\end{array}$ \\
\hline & 5 days & $5 \mathrm{~min}$. & $\begin{array}{c}0.62,0.64,0.66,0.65 \\
0.69 \\
\end{array}$ & $\begin{array}{c}0.59,0.58,0.56,0.57 \\
0.56,0.57 \\
\end{array}$ & $\begin{array}{cc}0.65,0.67,0.67, & 0.68 \\
0.68 & \\
\end{array}$ \\
\hline $23 \mathrm{~A}-2$ & 1 day & $5 \mathrm{~min}$. & $\begin{array}{c}0.39,0.39,0.39,0.39 \\
0.39 \\
\end{array}$ & $0.4,0.4,0.4,0.4,0.39$ & $\begin{array}{c}0.39,0.4,0.41,0.39 \\
0.4\end{array}$ \\
\hline \multirow[t]{2}{*}{$22 \mathrm{~A}-3$} & 1 day & $5 \mathrm{~min}$. & $\begin{array}{c}0.3,0.3,0.36,0.37 \\
0.38,0.38\end{array}$ & $0.4,0.4,0.4,0.39,0.39$ & $\begin{array}{c}0.38,0.37,0.38,0.39 \\
0.39\end{array}$ \\
\hline & 4 days & $5 \mathrm{~min}$. & $\begin{array}{c}0.44,0.45,0.46,0.46 \\
0.46\end{array}$ & $\begin{array}{c}0.46,0.46,0.47,0.46 \\
0.46 \\
\end{array}$ & $\begin{array}{c}0.46,0.45,0.46,0.46, \\
0.47 . \\
\end{array}$ \\
\hline $23 \mathrm{~A}-3$ & 1 day & $5 \mathrm{~min}$. & $\begin{array}{c}0.23(\mathrm{a}), 0.25,0.26 \\
0.27,0.26,0.26\end{array}$ & $\begin{array}{c}0.37,0.38,0.39,0.39 \\
0.37 \\
\end{array}$ & $0.4,0.39,0.39,0.4,0.4$ \\
\hline \multirow[t]{2}{*}{$22 \mathrm{~A}-4$} & 1 day & $5 \mathrm{~min}$. & $\begin{array}{c}0.23(\mathrm{a}), 0.26,0.27 \\
0.31,0.32,0.32 \\
\end{array}$ & $\begin{array}{c}0.34(\mathrm{a}), 0.32,0.33 \\
0.32,0.3,0.33 \\
\end{array}$ & $\begin{array}{c}0.27(\mathrm{a}), 0.33,0.38 \\
0.33,0.37,0.31 \\
\end{array}$ \\
\hline & 6 days & $5 \mathrm{~min}$. & $\begin{array}{c}0.31,0.31,0.31,0.32 \\
0.31 \\
\end{array}$ & $|0.31,0.3,0.3,0.31,0.3|$ & $0.31,0.33,0.35,0.35$ \\
\hline \multirow[t]{2}{*}{$4 \mathrm{~A}-5$} & I day & $5 \mathrm{~min}$ & $\begin{array}{c}0.29(\mathrm{a}), 0.32,0.33 \\
0.33,0.33\end{array}$ & $\begin{array}{c}0.33,0.34,0.34,0.33 \\
0.34\end{array}$ & $\begin{array}{c}0.33,0.35,0.36,0.35 \\
0.36 \\
\end{array}$ \\
\hline & 6 days & $5 \mathrm{~min}$. & $\begin{array}{c}0.33,0.32,0.33,0.33 \\
0.33\end{array}$ & $\begin{array}{c}0.33,0.33,0.33,0.34 \\
0.33\end{array}$ & $\begin{array}{c}0.34,0.33,0.34,0.34, \\
0.34 \\
\end{array}$ \\
\hline $23 \mathrm{~A}-8$ & 5 days & $5 \mathrm{~min}$. & $\begin{array}{c}0.29(\mathrm{a}), 0.34,0.39 \\
0.41,0.41,0.42 \\
\end{array}$ & $\begin{array}{c}0.4,0.41,0.44,0.45 \\
0.45,0.45 \\
\end{array}$ & \begin{tabular}{cc|}
0.46, & $0.45,0.45,0.46$ \\
$0.46,0.46$ \\
\end{tabular} \\
\hline \multirow[t]{2}{*}{$22 \mathrm{~A}-10$} & 1 day & 5 min. & $\begin{array}{c}0.26,0.31,0.31,0.33 \\
0.33,0.33 \\
\end{array}$ & $\begin{array}{c}0.34(\mathrm{a}), 0.35,0.35 \\
0.35,0.35 \\
\end{array}$ & $\begin{array}{c}0.34(\mathrm{a}), 0.35,0.35 \\
0.34,0.36 \\
\end{array}$ \\
\hline & 6 days & $5 \mathrm{~min}$. & $\begin{array}{c}0.26,0.25,0.25,0.24 \\
0.24\end{array}$ & $\begin{array}{c}0.26,0.26,0.26,0.26 \\
0.26\end{array}$ & $\begin{array}{c}0.26,0.26,0.26,0.26, \\
0.26\end{array}$ \\
\hline $23 \mathrm{~A}-13$ & 4 days & $5 \mathrm{~min}$. & $\begin{array}{c}0.17,0.18,0.19,0.19 \\
0.19,0.19\end{array}$ & $\begin{array}{c}0.23,0.24,0.25,0.26 \\
0.26,0.26 \\
\end{array}$ & $\begin{array}{c}0.27,0.27,0.27,0.27, \\
0.27 \\
\end{array}$ \\
\hline \multirow[t]{2}{*}{$22 \mathrm{~A}-17$} & 1 day & $5 \mathrm{~min}$. & $\begin{array}{c}0.22,0.28,0.31,0.34, \\
0.33,0.33 \\
\end{array}$ & $\begin{array}{c}0.35,0.35,0.37,0.39 \\
0.41,0.4\end{array}$ & $\begin{array}{c}0.42,0.4,0.4,0.41 \\
0.41\end{array}$ \\
\hline & 7 days & $5 \mathrm{~min}$. & $\begin{array}{c}0.32,0.33,0.34,0.34 \\
0.35\end{array}$ & $\begin{array}{c}0.34,0.35,0.33,0.33 \\
0.35\end{array}$ & $\begin{array}{c}0.34,0.34,0.34,0.34 \\
0.35 \\
\end{array}$ \\
\hline \multirow[t]{2}{*}{$23 A-21$} & 1 day & $5 \mathrm{~min}$. & $\begin{array}{c}0.29(\mathrm{a}), 0.3,0.3,0.31 \\
0.31\end{array}$ & $\begin{array}{c}0.3,0.27,0.3,0.29 \\
0.29,0.28 \\
\end{array}$ & $\begin{array}{ll}0.32, & 0.31,0.31,0.34 \\
. \quad 0.33,0.35 \\
\end{array}$ \\
\hline & 7 days & $5 \mathrm{~min}$. & $0.29,0.29,0.29,0.29$ & $0.29,0.29,0.3,0.3,0.3$ & $0.3,0.3,0.3,0.3$ \\
\hline \multirow[t]{2}{*}{$22 \mathrm{~A}-23$} & 1 day & $5 \mathrm{~min}$. & $\begin{array}{c}0.3,0.32,0.36,0.37 \\
0.38,0.38,0.39 \\
\end{array}$ & $0.4,0.4,0.4,0.4,0.4$ & $0.4,0.4,0.4,0.4,0.4$ \\
\hline & 5 days & $5 \mathrm{~min}$. & $\begin{array}{c}0.36,0.35,0.35,0.35 \\
0.35\end{array}$ & $\begin{array}{c}0.36,0.35,0.35,0.35 \\
0.35\end{array}$ & $\begin{array}{c}0.35,0.35,0.35,0.34 \\
0.35\end{array}$ \\
\hline
\end{tabular}


- The composites from Windows $\mathrm{C}$ and $\mathrm{E}$ had ammonia concentrations that were closely consistent with the 1999 RGS measurements.

- $\quad$ All of the RGS samples except 23A-1 had post-extrusion ammonia partial pressures between 3.2 and $4.0 \mathrm{kPa}$, much higher than in any other tank sampled with RGS. These partial pressures (when raised by $80 \%$ to account for measurement temperature differences) suggest a concentration six to eight times that in Tank AW-101 waste, which had a lower bound of $27,000 \mu \mathrm{mol} \mathrm{NH} / \mathrm{L}$ waste. This estimate is consistent with the isotopic ammonia measurements and the non-RGS ammonia measurements from Windows $\mathrm{C}$ and $\mathrm{E}$ but not with the non-RGS measurements from 1999.

Considering the above information, it seems likely that the ammonia concentration of the SY-101 waste (excluding the top of the crust) was between 140,000 and $200,000 \mu \mathrm{mol} \mathrm{NH} / \mathrm{L}$ of waste. The average and standard deviation of the RGS ammonia concentrations over all the samples except $23 \mathrm{~A}-1$ were $166,000 \pm 24,000 \mu \mathrm{mol} / \mathrm{L}$ waste. This ammonia concentration in the bulk waste corresponds to $0.24 \mathrm{wt} \% \mathrm{NH}_{3}$ in the bulk waste, or $4600 \mu \mathrm{g} \mathrm{NH} / \mathrm{mL}$ liquid. (The gas volume fraction of each sample and solid volume fractions based on the degassed waste densities were used to calculate the dissolved ammonia concentration in the liquid. The solid volume fraction was calculated from the bulk and liquid densities given in Section 4.15.1 and an assumed solid density of $2300 \mathrm{~kg} / \mathrm{m}^{3}$.)

\section{References}

Esch R. 1997. Tank 241-AX-101, Grab Samples, IAX-97-1 Through 1AX-97-3 Analytical Results for the Final Report. HNF-SD-WM-DP-268, Rev. 0, Waste Management of Hanford, Inc., Richland, Washington.

Kubic WL Jr. 1994. Dissolved Ammonia in Hanford Site Waste Tank 241-SY-101. LA-UR-94-4235 (revised), Los Alamos National Laboratory, Los Alamos, New Mexico.

Steen FH. 1996. Waste Compatibility and Final Report for Tank 241-A-101, Grab Samples IA96-1, IA-96-2, and IA-96-3. WHC-SD-WM-DP-186 Rev. 0, Westinghouse Hanford Company, Richland, Washington. 


\section{Distribution}

No. of

Copies

Offsite

2 DOE Office of Scientific and Technical Information

H. Babad

2540 Cordoba Ct.

Richland, WA 99352

M. J. Barnes

Savannah River Site

Aiken, SC 29802

D. O. Campbell

102 Windham Road

Oak Ridge, TN 37830

C. W. Forsberg

Oak Ridge National Laboratory

P.O. Box 2008, MS-6495

Oak Ridge, TN 37831-6495

B. C. Hudson

P.O. Box 271

Lindsborg, KS 67456

M. S. Kazimi

Massachusetts Institute

of Technology

Department of Nuclear Engineering

77 Massachusetts Avenue

Cambridge, MA 02139
No. of

Copies

J. L. Kovach

P.O. Box 29151

70000 Huntley Road

Columbus, OH 43229

T. S. Kress

102-B Newridge Road

Oak Ridge, TN 37830

W. L. Kubic

Los Alamos National Laboratory

P.O. Box 1663

Los Alamos, NM 87545

T. E. Larson

2711 Walnut St.

Los Alamos, NM 87545

D. A. Powers

Sandia National Laboratory

Nuclear Facilities Safety Department

MS-0744

Albuquerque, NM 87185-0744

W. R. Rossen

University of Texas at Austin

Department of Petroleum/Geosystems

Engineering

Austin, TX 78712

S. E. Slezak

806 Hermosa NE

Albuquerque, NM 87110 
No. of

Copies

Onsite

3 DOE Office of River Protection

C. A. Groendyke

G. W. Rosenwald

J.-S. Shuen

S7 -54

S7-54

S7-54

37 PHMC Team

R.P. Anantatmula

S. A. Barker

W. B. Barton

R. E. Bauer (3)

R. J. Cash

N.S. Cannon

A.F. Choho

J.M. Conner

M. E. Dahl

J.G. Field

L.A. Fort

J. M. Grigsby

K. M. Hall

B. E. Hey

D.C. Hedengren

K. M. Hodgson

T. A. Hu

J. W. Hunt

G. D. Johnson (3)

N. W. Kirch

C. E. Leach
R1-30

R2-11

R2-11

G1-54

S7-73

L6-38

R3-73

R2-11

L6-38

R2-11

R2-11

R1-49

R2-12

T6-07

R2-11

R2-11

R2-11

R2-12

S7-73

R2-11

R1-49
No. of

Copies

C.G. Linschooten

S7-12

L. L. Lockrem

T6-07

D.J. McCain

R2-11

S.G. McKinney

R2-12

J. C. Person

T6-07

J.H. Rasmussen

R2-12

R. E. Raymond

G1-54

D. A. Reynolds

R2-11

L.M. Sasaki

R2-12

B.C. Simpson

R2-11

L. M. Stock

S7-73

J. E. Van Beek

S2-48

41 Pacific Northwest National Laboratory

Z. I. Antoniak

K7-15

J. M. Bates

K7-15

B. O. Barnes

P8-50

S. Q. Bennett

K7-90

J. W. Brothers (10)

K9-20

S. A. Bryan

P7-25

J. A. Fort

K7-15

P. A. Gauglitz

K6-28

J. L. Huckaby

K6-80

L. A. Mahoney (10)

K7-15

P. A. Meyer

$\mathrm{K} 7-15$

L. R. Pederson

$\mathrm{K} 2-44$

L.M. Peurrung

S. D. Rassat

K. P. Recknagle

K6-24

K6-28

K7-15

D. R. Rector

K7-15

C. W. Stewart

K7-15

G. Terrones

K7-15

Information Release (5)

K1-06 